\title{
Charge-Transfer Transitions in Protein Circular Dichroism Calculations
}

\section{Supporting Information}

\section{The Matrix Method}

The Hamiltonian, $H$, in the matrix method is constructed as follows:

Equation 1

$$
H=\left(\begin{array}{cccc}
E_{n \pi^{*}}^{1} & V_{n \pi^{*} \pi \pi^{*}}^{11} & V_{n \pi^{*} n \pi^{*}}^{12} & V_{n \pi^{*} \pi \pi^{*}}^{12} \\
V_{n \pi^{*} \pi \pi^{*}}^{11} & E_{\pi \pi^{*}}^{1} & V_{n \pi^{*} \pi \pi^{*}}^{12} & V_{\pi \pi^{*} \pi \pi^{*}}^{12} \\
V_{n \pi^{*} n \pi^{*}}^{21} & V_{n \pi^{*} \pi \pi^{*}}^{21} & E_{n \pi^{*}}^{2} & V_{n \pi^{*} \pi \pi^{*}}^{22} \\
V_{n \pi^{*} \pi \pi^{*}}^{21} & V_{\pi \pi^{*} \pi \pi^{*}}^{21} & V_{n \pi^{*} \pi \pi^{*}}^{22} & E_{\pi \pi^{*}}^{2}
\end{array}\right)
$$

The diagonal elements, $E$, are the excitation energies of the transitions. The off-diagonal elements, $V$, are the couplings between transitions, which depend on the transition densities, $\rho$, and the distances, $r$, between them:

Equation 2

$$
V_{i 0 a ; j 0 b}=\iint \frac{\rho_{i 0 a}\left(r_{i}\right) \rho_{j 0 b}\left(r_{j}\right)}{r_{12}} \approx \sum_{s} \sum_{t} \frac{q_{s} q_{t}}{r_{s t}}
$$

The calculations can be simplified by using the monopole approximation, which represents each transition density as a set of point charges, $q$. Diagonalization of $H$ gives a set of eigenvalues and eigenvectors that produce the transition properties of the protein.

In the original formulation of the matrix method, the excited states of proteins are described as linear combinations of electronic configurations involving single excitations. Gilbert and Hirst ${ }^{10}$ have shown that charge-transfer transitions can be cast as a specific type of double excitation. In our calculations, we only consider this subset of double excitations and not all possible double excitations. There is some precedent for the extension of configuration lists with subsets of a type of excitation, for example, the use of perturbative triples in $\operatorname{CCSD}(\mathrm{T})$.

The matrix method considers only electrostatic interactions between chromophores. Introducing charge-transfer between chromophores augments this, by introducing a non-electrostatic interchromophore interaction. To accommodate such an interaction within the framework of the matrix method, we use charge-transfer chromophores that span two peptide groups and only have transition properties, making no contribution to the ground-state electronic structure of the protein.

Elements of the Hamiltonian matrix involving charge-transfer transitions are calculated using equations similar to Equation 2. For example, the coupling between a charge-transfer transition and the ground state is given by:

Equation 3

$$
V_{m a ;(k l) \hat{c}}=\sum_{i \neq(k l)}^{M} V_{i 00 ;(k l) a \hat{c}}
$$

where $k$ and $l$ are the residues involved in the charge transfer. In this work $l=k+1$, because we only consider charge transfer between adjacent peptide groups. Care must be taken in modeling the interactions between charge-transfer transition and a local transition on the same peptide group:

Equation 4

$$
V_{0 ;(k l) \hat{c}}=\sum_{i \neq(k l)}^{M} V_{i 00 ;(k l) 0 \hat{c}}
$$


Successive charge-transfer chromophores also overlap, but the coupling between them cannot be calculated using Equation 2, because the point charges in the two chromophores overlap. Interactions of this type could only be modeled by ab initio calculations on tripeptides, which are impractical, due to the large conformational space and the computational cost of the ab initio calculation at each geometry. Therefore, these interactions are neglected.

Proteins used in this study and their pdb codes.

\begin{tabular}{|c|c|c|c|c|c|}
\hline Insulin & 4ins & Xylanase & $1 \mathrm{en} x$ & Lactate dehydrogenase & 9ldt \\
\hline Lipase & 3lip & Transferrin & $11 f g$ & Human serum albumin & 1 ao6 \\
\hline$\alpha$-amylase & lbag & $\alpha$-lactalbumin & $1 \mathrm{f} 6 \mathrm{~s}$ & $\beta$-Lactoglobulin & $1 \mathrm{~b} 8 \mathrm{e}$ \\
\hline Myoglobin & 1wla & Ovalbumin & lova & Staphylococcal nuclease & 1ey0 \\
\hline Papain & 9pap & Hemoglobin & $1 \mathrm{~g} 08$ & Glucose isomerase & load \\
\hline Avidin & lave & Subtilisin A & $1 \mathrm{sbc}$ & Ribonuclease $\mathrm{A}$ & $1 \mathrm{fs} 3$ \\
\hline Catalase & 7 cat & Cytochrome c & $1 \mathrm{hrc}$ & Carbonic anhydrase & $1 \mathrm{~g} 6 \mathrm{v}$ \\
\hline Pepsin & 4pep & Conalbumin & lovt & $\alpha$-chymotrypsinogen & $2 \mathrm{cga}$ \\
\hline Lysozyme & 1hel & Thioredoxin & $2 \operatorname{trx}$ & Concanavalin A & $2 \mathrm{ctv}$ \\
\hline Peroxidase & 1atj & Trypsinogen & $1 \operatorname{tgn}$ & Soybean trypsin inhibitor & 1avu \\
\hline Elastase & 3est & & & & \\
\hline
\end{tabular}

\section{Complete references 18 and 19.}

(18) Andersson, K.; Barysz, M.; Bernhardsson, A.; Blomberg, M. R. A.; Carissan, Y.; Cooper, D. L.; Cossi, M.; Fleig, T.; Fülscher, M. P.; Gagliardi, L.; de Graaf, C.; Hess, B. A.; Karlström, G.; Lindh, R.; Malmqvist, P.-Å; Neogrády, P.; Olsen, J.; Roos, B. O.; Schimmelpfennig, B.; Schütz, M.; Seijo, L.; Serrano-Andrés, L.; Siegbahn, P. E. M.; Stålring, J.; Thorsteinsson, T.; Veryazov, V.; Wierzbowska, M.; Widmark, P.-O.; MOLCAS, version 5.2; Lund University, 2001.

(19) Amos, R. D.; Bernhardsson, A.; Berning, A.; Celani, P.; Cooper, D. L.; Deegan, M. J. O.; Dobbyn, A. J.; Eckert, F.; Hampel, C.; Hetzer, G.; Knowles, P. J.; Korona, T.; Lindh, R.; Lloyd, A. W.; McNicholas, S. J.; Manby, F. R.; Meyer, W.; Mura, M. E.; Nicklass, A.; Palmieri, P.; Pitzer, R.; Rauhut, G.; Schütz, M.; Schumann, U.; Stoll, H.; Stone, A. J.; Tarroni, R.; Thorsteinsson, T.; Werner., H.-J.; Molpro, a package of ab initio programs, version 2002.1; University of Birmingham: Birmingham, UK, 2002. 
Properties of the charge-tranfer chromophores.

Permanent and transition dipole moments in Debye, energies in $\mathrm{cm}^{-1}$ and positions of atom centres and point charges in $\AA$.

- CT01009A-

\begin{tabular}{|c|c|c|c|c|}
\hline 2.3281 & 0.1647 & -0.0078 & 1.0000 & $\# \mathrm{Cl}$ \\
\hline 2.5980 & 1.3728 & -0.0828 & 1.0000 & $\begin{array}{lll}\# & 01\end{array}$ \\
\hline 1.0437 & -0.2763 & 0.0210 & 1.0000 & $\# \mathrm{~N} 1$ \\
\hline-1.3568 & -0.1312 & 0.0102 & 1.0000 & $\# \mathrm{C} 2$ \\
\hline-1.3786 & -1.3677 & 0.0932 & 1.0000 & $\# \mathrm{~N} 2$ \\
\hline-2.4858 & 0.6143 & -0.0404 & 1.0000 & $\# \mathrm{O}_{2}$ \\
\hline
\end{tabular}

\&TRANSITION $1->\ldots$ 64

\begin{tabular}{|c|c|}
\hline \multirow{2}{*}{\multicolumn{2}{|c|}{ 45087. \# Energy 1->2 }} \\
\hline & $-0.0183 \quad-0.1611$ \\
\hline-0.2191 & -0.7849 \\
\hline 2.27520919 & 0.11177704 \\
\hline 2.27520919 & 0.11177704 \\
\hline 2.27520919 & 0.21761249 \\
\hline 2.27520919 & 0.21761249 \\
\hline 2.38104463 & 0.11177704 \\
\hline 2.38104463 & 0.11177704 \\
\hline 2.38104463 & 0.21761249 \\
\hline 2.38104463 & 0.21761249 \\
\hline-1.40967047 & -0.18411981 \\
\hline-1.40967047 & -0.18411981 \\
\hline-1.40967047 & -0.07828436 \\
\hline-1.40967047 & -0.07828436 \\
\hline-1.30383503 & -0.18411981 \\
\hline-1.30383503 & -0.18411981 \\
\hline-1.30383503 & -0.07828436 \\
\hline-1.30383503 & -0.07828436 \\
\hline 2.54510117 & 1.31986856 \\
\hline 2.54510117 & 1.31986856 \\
\hline 2.54510117 & 1.42570400 \\
\hline 2.54510117 & 1.42570400 \\
\hline 2.65093660 & 1.31986856 \\
\hline 2.65093660 & 1.31986856 \\
\hline 2.65093660 & 1.42570400 \\
\hline 2.65093660 & 1.42570400 \\
\hline-1.43156683 & -1.42060065 \\
\hline-1.43156683 & -1.42060065 \\
\hline-1.43156683 & -1.31476510 \\
\hline-1.43156683 & -1.31476510 \\
\hline-1.32573140 & -1.42060065 \\
\hline-1.32573140 & -1.42060065 \\
\hline-1.32573140 & -1.31476510 \\
\hline-1.32573140 & -1.31476510 \\
\hline 0.99076527 & -0.32923394 \\
\hline 0.99076527 & -0.32923394 \\
\hline 0.99076527 & -0.22339852 \\
\hline 0.99076527 & -0.22339852 \\
\hline 1.09660077 & -0.32923394 \\
\hline 1.09660077 & -0.32923394 \\
\hline 1.09660077 & -0.22339852 \\
\hline 1.09660077 & -0.22339852 \\
\hline-2.53873253 & 0.56140047 \\
\hline-2.53873253 & 0.56140047 \\
\hline-2.53873253 & 0.66723585 \\
\hline-2.53873253 & 0.66723585 \\
\hline-2.43289709 & 0.56140047 \\
\hline-2.43289709 & 0.56140047 \\
\hline-2.43289709 & 0.66723585 \\
\hline-2.43289709 & 0.66723585 \\
\hline 0.74471217 & -1.31334066 \\
\hline 0.74471217 & -1.31334066 \\
\hline
\end{tabular}

1.000 \# Electr. mom., scale-fac
\# Magnetic mom.

$-0.06075061$

$-41.07429886$

$0.04508484 \quad 36.09272003$

$-0.06075061 \quad 40.82384872$

$0.04508484-35.92380524$

$\begin{array}{rr}-0.06075061 & 43.28390884 \\ 0.04508484 & -38.62309647\end{array}$

$0.04508484 \quad-38.62309647$

$-0.06075061 \quad-43.10685349$

$0.04508484 \quad 38.49382019$

$-0.04267391 \quad-4.54160309$

$0.06316154 \quad 4.60974264$

$\begin{array}{rr}-0.04267391 & 4.64513254\end{array}$

$0.06316154 \quad-4.82243299$

$-0.04267391$

0.06316154

$-0.04267391$

0.06316154

$-0.13571967$

$-0.02988423$

$-0.13571967$

$-0.02988423$

$-0.13571967$

$-0.02988423$

$-0.13571967$

$-0.02988423$

0.04030320

0.14613864

0.04030320

0.14613864

0.04030320

0.14613864

0.04030320

0.14613864

$-0.03195595$

0.07387950

$-0.03195595$

0.07387950

$-0.03195595$

0.07387950

$-0.03195595$

0.07387950

$-0.09333258$

0.01250287

$-0.09333258$

0.01250287

$-0.09333258$

0.01250287

$-0.09333258$

0.01250287

0.02794744

0.13378289
4.76534128

$-4.82977009$

$-4.84077644$

5.01270914

5.61699057

$-6.74295759$

$-12.21906853$

13.09038734

$-2.74566317$

3.84523773

9.54930973

$-10.40014458$

0.09744275

$-0.11600212$

0.50347388

$-0.47518063$

$-0.02506382$

0.04585206

$-0.59067440$

0.55936462

$-0.77296895$

0.19240575

$-0.74139625$

0.98523372

3.20605564

$-2.11771464$

$-1.26700330$

0.59326309

0.99599177

$-1.20718300$

$-0.85971290$

1.05377734

$-1.08406806$

1.35098290

0.90329260

$-1.14823258$

$-0.75649840$

0.55145943 


0.74471217
0.74471217
0.85054761
0.85054761
0.85054761
0.85054761
-2.46623826
-2.46623826
-2.46623826
-2.46623826
-2.36040306
-2.36040306
-2.36040306
-2.36040306

64 46170 .
$-1.20750511$

$-1.20750511$

$-1.31334066$

$-1.31334066$

$-1.20750511$

$-1.20750511$

1.56936514

1.56936514

1.67520046

1.67520046

1.56936514

1.56936514

1.67520046

1.67520046

$-0.0068$

$-0.0538$

2. 27520919

2. 27520919

2. 27520919

2. 27520919

2.38104463

2. 38104463

2. 38104463

2.38104463

$-1.40967047$

$-1.40967047$

$-1.40967047$

$-1.40967047$

$-1.30383503$

$-1.30383503$

$-1.30383503$

$-1.30383503$

2. 54510117

2. 54510117

2. 54510117

2. 54510117

2.65093660

2.65093660

2.65093660

2.65093660

$-1.43156683$

$-1.43156683$

$-1.43156683$

$-1.43156683$

$-1.32573140$

$-1.32573140$

$-1.32573140$

$-1.32573140$

0.99076527

0.99076527

0.99076527

0.99076527

1. 09660077

1.09660077

1.09660077

1.09660077

$-2.53873253$

$-2.53873253$

$-2.53873253$

$-2.53873253$

$-2.43289709$

$-2.43289709$

$-2.43289709$

$-2.43289709$

0.74471217

0.74471217

\# Energy 1->3

$\begin{array}{ll}0.0143 & 0.1692\end{array}$

$0.9301 \quad-0.0617$

0.11177704

0.11177704

0.21761249

0.21761249

0.11177704

0.11177704

0.21761249

0.21761249

$-0.18411981$

$-0.18411981$

$-0.07828436$

$-0.07828436$

$-0.18411981$

$-0.18411981$

$-0.07828436$

$-0.07828436$

1. 31986856

1. 31986856

1. 42570400

1. 42570400

1. 31986856

1. 31986856

1. 42570400

1. 42570400

$-1.42060065$

$-1.42060065$

$-1.31476510$

$-1.31476510$

$-1.42060065$

$-1.42060065$

$-1.31476510$

$-1.31476510$

$-0.32923394$

$-0.32923394$

$-0.22339852$

$-0.22339852$

$-0.32923394$

$-0.32923394$

$-0.22339852$

$-0.22339852$

0.56140047

0.56140047

0.66723585

0.66723585

0.56140047

0.56140047

0.66723585

0.66723585

$-1.31334066$

$-1.31334066$

$\begin{array}{rr}0.02794744 & 1.06996894 \\ 0.13378289 & -0.73756510 \\ 0.02794744 & 1.41013539 \\ 0.13378289 & -1.09383011 \\ 0.02794744 & -2.00513506 \\ 0.13378289 & 1.51830792 \\ -0.15553895 & 0.15858892 \\ -0.04970350 & -0.19270399 \\ -0.15553895 & -0.05264948 \\ -0.04970350 & 0.07657776 \\ -0.15553895 & -0.12588938 \\ -0.04970350 & 0.16830038 \\ -0.15553895 & 0.02356306 \\ -0.04970350 & -0.05324495 \\ & \end{array}$

1.000 \# Electr. mom., scale-fac

$-0.06075061$

0.04508484

$-0.06075061$

0.04508484

$-0.06075061$

0.04508484

$-0.06075061$

0.04508484

$-0.04267391$

0.06316154

$-0.04267391$

0.06316154

$-0.04267391$

0.06316154

$-0.04267391$

0.06316154

$-0.13571967$

$-0.02988423$

$-0.13571967$

$-0.02988423$

$-0.13571967$

$-0.02988423$

$-0.13571967$

$-0.02988423$

0.04030320

0.14613864

0.04030320

0.14613864

0.04030320

0.14613864

0.04030320

0.14613864

$-0.03195595$

0.07387950

$-0.03195595$

0.07387950

$-0.03195595$

0.07387950

$-0.03195595$

0.07387950

$-0.09333258$

0.01250287

$-0.09333258$

0.01250287

$-0.09333258$

0.01250287

$-0.09333258$

0.01250287

0.02794744

0.13378289
7.80312395

$-7.44534159$

$-7.65318823$

7.30483341

$-7.52743673$

7.19455433

7. 42637682

$-7.10051441$

$-32.01524734$

32.31226730

33.04950333

$-33.10401154$

32.62303925

$-32.96739578$

$-33.59871674$

33.71694565

1.06162286

$-0.95962262$

$-0.59695995$

0.52508408

$-1.21962547$

1. 13039720

0.71917522

$-0.65786344$

8.23012543

$-7.83764553$

$-1.62411273$

1. 15400302

$-8.20188999$

7.81907749

1. 65001738

$-1.19227064$

7.64998865

$-8.30631256$

$-7.09606600$

7.79338455

$-7.00623846$

7.60046911

6.39896917

$-7.04267931$

8.29012680

$-8.88395405$

$-6.61282110$

7.32017994

$-8.82738018$

9.28167915

6.74016809

$-7.31887150$

0.34502918

$-0.43408900$ 


\begin{tabular}{|c|c|c|c|}
\hline 0.74471217 & -1.20750511 & 0.02794744 & -0.56363666 \\
\hline 0.74471217 & -1.20750511 & 0.13378289 & 0.69883996 \\
\hline 0.85054761 & -1.31334066 & 0.02794744 & -0.28095195 \\
\hline 0.85054761 & -1.31334066 & 0.13378289 & 0.35002807 \\
\hline 0.85054761 & -1.20750511 & 0.02794744 & 0.45503640 \\
\hline 0.85054761 & -1.20750511 & 0.13378289 & -0.56105858 \\
\hline-2.46623826 & 1.56936514 & -0.15553895 & 1.28167629 \\
\hline-2.46623826 & 1.56936514 & -0.04970350 & -1.61860895 \\
\hline-2.46623826 & 1.67520046 & -0.15553895 & -0.68214250 \\
\hline-2.46623826 & 1.67520046 & -0.04970350 & 0.92587054 \\
\hline-2.36040306 & 1.56936514 & -0.15553895 & -0.83066535 \\
\hline-2.36040306 & 1.56936514 & -0.04970350 & 1.13909483 \\
\hline-2.36040306 & 1.67520046 & -0.15553895 & 0.37651587 \\
\hline-2.36040306 & 1.67520046 & -0.04970350 & -0.59988606 \\
\hline 5403 & \# Energy 1->4 & & \\
\hline 3.6232 & $1.4716 \quad-0.0909$ & $1.000 \quad \#$ & Electr. mom., scale-fac \\
\hline 0.0238 & -0.1147 & \# & Magnetic mom. \\
\hline 2.27520919 & 0.11177704 & -0.06075061 & -6.90116978 \\
\hline 2.27520919 & 0.11177704 & 0.04508484 & 1.75767112 \\
\hline 2.27520919 & 0.21761249 & -0.06075061 & 7.11415815 \\
\hline 2.27520919 & 0.21761249 & 0.04508484 & -1.38973320 \\
\hline 2.38104463 & 0.11177704 & -0.06075061 & 5.43748426 \\
\hline 2.38104463 & 0.11177704 & 0.04508484 & -1.46922302 \\
\hline 2.38104463 & 0.21761249 & -0.06075061 & -5.47101402 \\
\hline 2.38104463 & 0.21761249 & 0.04508484 & 1.12455988 \\
\hline-1.40967047 & -0.18411981 & -0.04267391 & -11.23878384 \\
\hline-1.40967047 & -0.18411981 & 0.06316154 & 1.25693965 \\
\hline-1.40967047 & -0.07828436 & -0.04267391 & 14.13043499 \\
\hline-1.40967047 & -0.07828436 & 0.06316154 & -1.34472382 \\
\hline-1.30383503 & -0.18411981 & -0.04267391 & 8.80578804 \\
\hline-1.30383503 & -0.18411981 & 0.06316154 & -4.12962627 \\
\hline-1.30383503 & -0.07828436 & -0.04267391 & -11.35169315 \\
\hline-1.30383503 & -0.07828436 & 0.06316154 & 3.73296404 \\
\hline 2.54510117 & 1.31986856 & -0.13571967 & -0.61119068 \\
\hline 2.54510117 & 1.31986856 & -0.02988423 & -2.40248513 \\
\hline 2.54510117 & 1.42570400 & -0.13571967 & 0.45585302 \\
\hline 2.54510117 & 1.42570400 & -0.02988423 & 1.84340227 \\
\hline 2.65093660 & 1.31986856 & -0.13571967 & 0.07988201 \\
\hline 2.65093660 & 1.31986856 & -0.02988423 & 2.15432930 \\
\hline 2.65093660 & 1.42570400 & -0.13571967 & -0.01755885 \\
\hline 2.65093660 & 1.42570400 & -0.02988423 & -1.74962461 \\
\hline-1.43156683 & -1.42060065 & 0.04030320 & -4.10874033 \\
\hline-1.43156683 & -1.42060065 & 0.14613864 & 4.04919767 \\
\hline-1.43156683 & -1.31476510 & 0.04030320 & 4.85444355 \\
\hline-1.43156683 & -1.31476510 & 0.14613864 & -4.67887211 \\
\hline-1.32573140 & -1.42060065 & 0.04030320 & 3.30771661 \\
\hline-1.32573140 & -1.42060065 & 0.14613864 & -4.73805475 \\
\hline-1.32573140 & -1.31476510 & 0.04030320 & -3.44786000 \\
\hline-1.32573140 & -1.31476510 & 0.14613864 & 5.46799660 \\
\hline 0.99076527 & -0.32923394 & -0.03195595 & -8.05048084 \\
\hline 0.99076527 & -0.32923394 & 0.07387950 & 5.09236050 \\
\hline 0.99076527 & -0.22339852 & -0.03195595 & 8.78905296 \\
\hline 0.99076527 & -0.22339852 & 0.07387950 & -4.35518885 \\
\hline 1.09660077 & -0.32923394 & -0.03195595 & 9.29727173 \\
\hline 1.09660077 & -0.32923394 & 0.07387950 & -5.62672663 \\
\hline 1.09660077 & -0.22339852 & -0.03195595 & -9.85534954 \\
\hline 1.09660077 & -0.22339852 & 0.07387950 & 4.83365107 \\
\hline-2.53873253 & 0.56140047 & -0.09333258 & -3.11366200 \\
\hline-2.53873253 & 0.56140047 & 0.01250287 & -4.93999195 \\
\hline-2.53873253 & 0.66723585 & -0.09333258 & 4.96372414 \\
\hline-2.53873253 & 0.66723585 & 0.01250287 & 4.37577915 \\
\hline-2.43289709 & 0.56140047 & -0.09333258 & 1.95241714 \\
\hline-2.43289709 & 0.56140047 & 0.01250287 & 7.21290398 \\
\hline-2.43289709 & 0.66723585 & -0.09333258 & -4.23598337 \\
\hline-2.43289709 & 0.66723585 & 0.01250287 & -7.02376223 \\
\hline 0.74471217 & -1.31334066 & 0.02794744 & -1.85990810 \\
\hline 0.74471217 & -1.31334066 & 0.13378289 & 2.11217332 \\
\hline
\end{tabular}




\begin{tabular}{|c|c|c|c|}
\hline 0.74471217 & -1.20750511 & 0.02794744 & 2.37862277 \\
\hline 0.74471217 & -1.20750511 & 0.13378289 & -2.78968549 \\
\hline 0.85054761 & -1.31334066 & 0.02794744 & 2.05110765 \\
\hline 0.85054761 & -1.31334066 & 0.13378289 & -2.29275537 \\
\hline 0.85054761 & -1.20750511 & 0.02794744 & -2.66050768 \\
\hline 0.85054761 & -1.20750511 & 0.13378289 & 3.03242540 \\
\hline-2.46623826 & 1.56936514 & -0.15553895 & -3.19949675 \\
\hline-2.46623826 & 1.56936514 & -0.04970350 & 1.11690962 \\
\hline-2.46623826 & 1.67520046 & -0.15553895 & 2.62947202 \\
\hline-2.46623826 & 1.67520046 & -0.04970350 & -1.10291636 \\
\hline-2.36040306 & 1.56936514 & -0.15553895 & 2.83879399 \\
\hline-2.36040306 & 1.56936514 & -0.04970350 & -0.20452853 \\
\hline-2.36040306 & 1.67520046 & -0.15553895 & -2.35662723 \\
\hline-2.36040306 & 1.67520046 & -0.04970350 & 0.46843940 \\
\hline 5271 & \# Energy 1->5 & & \\
\hline 1.1733 & $\begin{array}{ll}-3.5820 & 0.2392\end{array}$ & 1.000 & Electr. mom., scale-fac \\
\hline-0.0112 & -0.2403 & \# & Magnetic mom. \\
\hline 2.27520919 & 0.11177704 & -0.06075061 & 17.95178223 \\
\hline 2.27520919 & 0.11177704 & 0.04508484 & 0.47478238 \\
\hline 2.27520919 & 0.21761249 & -0.06075061 & -17.49017334 \\
\hline 2.27520919 & 0.21761249 & 0.04508484 & -2.07485700 \\
\hline 2.38104463 & 0.11177704 & -0.06075061 & -14.45499706 \\
\hline 2.38104463 & 0.11177704 & 0.04508484 & 0.62514490 \\
\hline 2.38104463 & 0.21761249 & -0.06075061 & 13.71105099 \\
\hline 2.38104463 & 0.21761249 & 0.04508484 & 0.71858525 \\
\hline-1.40967047 & -0.18411981 & -0.04267391 & -2.67748189 \\
\hline-1.40967047 & -0.18411981 & 0.06316154 & -2.78744030 \\
\hline-1.40967047 & -0.07828436 & -0.04267391 & 3.19260931 \\
\hline-1.40967047 & -0.07828436 & 0.06316154 & 2.33364749 \\
\hline-1.30383503 & -0.18411981 & -0.04267391 & 1.56158161 \\
\hline-1.30383503 & -0.18411981 & 0.06316154 & 2.19488502 \\
\hline-1.30383503 & -0.07828436 & -0.04267391 & -2.01993394 \\
\hline-1.30383503 & -0.07828436 & 0.06316154 & -1.79329693 \\
\hline 2.54510117 & 1.31986856 & -0.13571967 & -1.46731043 \\
\hline 2.54510117 & 1.31986856 & -0.02988423 & 10.42683315 \\
\hline 2.54510117 & 1.42570400 & -0.13571967 & 1.28198063 \\
\hline 2.54510117 & 1.42570400 & -0.02988423 & -7.89608145 \\
\hline 2.65093660 & 1.31986856 & -0.13571967 & 2.30990505 \\
\hline 2.65093660 & 1.31986856 & -0.02988423 & -8.69299126 \\
\hline 2.65093660 & 1.42570400 & -0.13571967 & -1.99874747 \\
\hline 2.65093660 & 1.42570400 & -0.02988423 & 6.83653259 \\
\hline-1.43156683 & -1.42060065 & 0.04030320 & -0.89498526 \\
\hline-1.43156683 & -1.42060065 & 0.14613864 & -0.10458699 \\
\hline-1.43156683 & -1.31476510 & 0.04030320 & 1.09921348 \\
\hline-1.43156683 & -1.31476510 & 0.14613864 & 0.19712284 \\
\hline-1.32573140 & -1.42060065 & 0.04030320 & 0.47672051 \\
\hline-1.32573140 & -1.42060065 & 0.14613864 & 0.09682512 \\
\hline-1.32573140 & -1.31476510 & 0.04030320 & -0.44562736 \\
\hline-1.32573140 & -1.31476510 & 0.14613864 & -0.20732982 \\
\hline 0.99076527 & -0.32923394 & -0.03195595 & 12.63207626 \\
\hline 0.99076527 & -0.32923394 & 0.07387950 & -1.13696599 \\
\hline 0.99076527 & -0.22339852 & -0.03195595 & -12.27402687 \\
\hline 0.99076527 & -0.22339852 & 0.07387950 & -0.93728089 \\
\hline 1.09660077 & -0.32923394 & -0.03195595 & -16.56740952 \\
\hline 1.09660077 & -0.32923394 & 0.07387950 & 2.01886153 \\
\hline 1.09660077 & -0.22339852 & -0.03195595 & 15.65645313 \\
\hline 1.09660077 & -0.22339852 & 0.07387950 & 0.19125348 \\
\hline-2.53873253 & 0.56140047 & -0.09333258 & -4.13560438 \\
\hline-2.53873253 & 0.56140047 & 0.01250287 & 1.03671896 \\
\hline-2.53873253 & 0.66723585 & -0.09333258 & 4.72899342 \\
\hline-2.53873253 & 0.66723585 & 0.01250287 & -1.36469543 \\
\hline-2.43289709 & 0.56140047 & -0.09333258 & 4.27946091 \\
\hline-2.43289709 & 0.56140047 & 0.01250287 & -0.44937965 \\
\hline-2.43289709 & 0.66723585 & -0.09333258 & -5.02578354 \\
\hline-2.43289709 & 0.66723585 & 0.01250287 & 0.65387166 \\
\hline 0.74471217 & -1.31334066 & 0.02794744 & 2.67983484 \\
\hline 0.74471217 & -1.31334066 & 0.13378289 & -2.79105043 \\
\hline
\end{tabular}




$\begin{array}{rr}0.74471217 & -1.20750511 \\ 0.74471217 & -1.20750511 \\ 0.85054761 & -1.31334066 \\ 0.85054761 & -1.31334066 \\ 0.85054761 & -1.20750511 \\ 0.85054761 & -1.20750511 \\ -2.46623826 & 1.56936514 \\ -2.46623826 & 1.56936514 \\ -2.46623826 & 1.67520046 \\ -2.46623826 & 1.67520046 \\ -2.36040306 & 1.56936514 \\ -2.36040306 & 1.56936514 \\ -2.36040306 & 1.67520046 \\ -2.36040306 & 1.67520046 \\ 58475 . & \# \text { Energy 1->6 }\end{array}$

64

$\begin{array}{lll}-0.2257 & -1.1836 & 0.0819\end{array}$

$\begin{array}{lll}-0.0058 & -0.0105 & -0.1905\end{array}$

2.27520919

0.11177704

2. 27520919

0.11177704

2. 27520919

0.21761249

2.27520919

0.21761249

2.38104463

0.11177704

2. 38104463

0.11177704

2. 38104463

0.21761249

2.38104463

$-1.40967047$

$-1.40967047$

$-1.40967047$

$-1.40967047$

$-1.30383503$

$-1.30383503$

$-1.30383503$

$-1.30383503$

2. 54510117

2. 54510117

2. 54510117

2. 54510117

2.65093660

2.65093660

2.65093660

2.65093660

$-1.43156683$

$-1.43156683$

$-1.43156683$

$-1.43156683$

$-1.32573140$

$-1.32573140$

0.21761249

$-0.18411981$

$-0.18411981$

$-0.07828436$

$-0.07828436$

$-0.18411981$

$-0.18411981$

$-0.07828436$

$-0.07828436$

1. 31986856

1. 31986856

1. 42570400

1. 42570400

1. 31986856

1. 31986856

1. 42570400

1. 42570400

$-1.42060065$

$-1.42060065$

$-1.31476510$

$-1.31476510$

$-1.42060065$

$-1.42060065$

$-1.32573140$

$-1.32573140$

0.99076527

0.99076527

0.99076527

0.99076527

1. 09660077

1.09660077

1.09660077

1.09660077

$-2.53873253$

$-2.53873253$

$-2.53873253$

$-2.53873253$

$-2.43289709$

$-2.43289709$

$-2.43289709$

$-2.43289709$

0.74471217

$-1.31476510$

$-1.31476510$

$-0.32923394$

$-0.32923394$

$-0.22339852$

$-0.22339852$

$-0.32923394$

$-0.32923394$

$-0.22339852$

$-0.22339852$

0.56140047

0.56140047

0.66723585

0.66723585

0.56140047

0.56140047

0.66723585

0.66723585

0.74471217

$-1.31334066$

$-1.31334066$

$\begin{array}{rr}0.02794744 & -4.04377556 \\ 0.13378289 & 3.88907623 \\ 0.02794744 & -3.52594948 \\ 0.13378289 & 3.46829534 \\ 0.02794744 & 5.23627043 \\ 0.13378289 & -4.77917814 \\ -0.15553895 & -1.91827321 \\ -0.04970350 & 1.52045405 \\ -0.15553895 & 1.50405693 \\ -0.04970350 & -1.20039821 \\ -0.15553895 & 1.91426086 \\ -0.04970350 & -1.29415870 \\ -0.15553895 & -1.49787128 \\ -0.04970350 & 1.04850054 \\ & \end{array}$

1.000 \# Electr. mom., scale-fac

$-0.06075061$

0.04508484

$-0.06075061$

0.04508484

$-0.06075061$

0.04508484

$-0.06075061$

0.04508484

$-0.04267391$

0.06316154

$-0.04267391$

0.06316154

$-0.04267391$

0.06316154

$-0.04267391$

0.06316154

$-0.13571967$

$-0.02988423$

$-0.13571967$

$-0.02988423$

$-0.13571967$

$-0.02988423$

$-0.13571967$

$-0.02988423$

0.04030320

0.14613864

0.04030320

0.14613864

0.04030320

0.14613864

0.04030320

0.14613864

$-0.03195595$

0.07387950

$-0.03195595$

0.07387950

$-0.03195595$

0.07387950

$-0.03195595$

0.07387950

$-0.09333258$

0.01250287

$-0.09333258$

0.01250287

$-0.09333258$

0.01250287

$-0.09333258$

0.01250287

0.02794744

0.13378289
4.67680502

$-4.28662443$

$-5.15555239$

4. 72392702

$-3.65085173$

3.22308040

4. 04695845

$-3.56304145$

$-15.95936012$

6.56223249

17.41057777

$-7.18016911$

14.66236687

$-6.66330433$

$-15.99604130$

7.12614870

2.09671330

$-2.27355862$

$-1.62530494$

1. 65332377

$-1.77901459$

1.83237076

$-1.32741284$

$-2.80498147$

1. 46042478

3.62726474

$-1.88875306$

2.44107819

$-1.49218321$

$-3.05168176$

1.90498745

8.73708153

$-3.59457278$

$-8.50956917$

3. 43514752

$-9.65071201$

4.63885689

9.42798519

$-4.43323755$

$-1.22049034$

3.17963243

1. 77741623

$-3.41759586$

1.33381331

$-3.47862244$

$-2.08837771$

3. 76228762

1. 93232739

$-1.25091255$
1. 37972784 


\begin{tabular}{|c|c|c|c|}
\hline 0.74471217 & -1.20750511 & 0.02794744 & -2.78861070 \\
\hline 0.74471217 & -1.20750511 & 0.13378289 & 1.78639483 \\
\hline 0.85054761 & -1.31334066 & 0.02794744 & -2.05149841 \\
\hline 0.85054761 & -1.31334066 & 0.13378289 & 1.47310781 \\
\hline 0.85054761 & -1.20750511 & 0.02794744 & 2.93960643 \\
\hline 0.85054761 & -1.20750511 & 0.13378289 & -2.07416010 \\
\hline-2.46623826 & 1.56936514 & -0.15553895 & -0.50030649 \\
\hline-2.46623826 & 1.56936514 & -0.04970350 & 1.02381611 \\
\hline-2.46623826 & 1.67520046 & -0.15553895 & 0.28263801 \\
\hline-2.46623826 & 1.67520046 & -0.04970350 & -0.62790149 \\
\hline-2.36040306 & 1.56936514 & -0.15553895 & 0.66174495 \\
\hline-2.36040306 & 1.56936514 & -0.04970350 & -1.15323699 \\
\hline-2.36040306 & 1.67520046 & -0.15553895 & -0.39485028 \\
\hline-2.36040306 & 1.67520046 & -0.04970350 & 0.71264577 \\
\hline 64501 & \# Energy 1->7 & & \\
\hline 0.0018 & -0.0148 & 1.000 & Electr. mom., scale-fac \\
\hline 0.0601 & -0.1013 & \# & Magnetic mom. \\
\hline 2.27520919 & 0.11177704 & -0.06075061 & -5.48151064 \\
\hline 2.27520919 & 0.11177704 & 0.04508484 & 5.35152960 \\
\hline 2.27520919 & 0.21761249 & -0.06075061 & 5.60183191 \\
\hline 2.27520919 & 0.21761249 & 0.04508484 & -5.41537142 \\
\hline 2.38104463 & 0.11177704 & -0.06075061 & 4.83924770 \\
\hline 2.38104463 & 0.11177704 & 0.04508484 & -4.76872683 \\
\hline 2.38104463 & 0.21761249 & -0.06075061 & -5.00477839 \\
\hline 2.38104463 & 0.21761249 & 0.04508484 & 4.89101887 \\
\hline-1.40967047 & -0.18411981 & -0.04267391 & 3.84103775 \\
\hline-1.40967047 & -0.18411981 & 0.06316154 & -4.23288822 \\
\hline-1.40967047 & -0.07828436 & -0.04267391 & -3.45395970 \\
\hline-1.40967047 & -0.07828436 & 0.06316154 & 3.86121416 \\
\hline-1.30383503 & -0.18411981 & -0.04267391 & -4.17828989 \\
\hline-1.30383503 & -0.18411981 & 0.06316154 & 4.56844664 \\
\hline-1.30383503 & -0.07828436 & -0.04267391 & 3.72992754 \\
\hline-1.30383503 & -0.07828436 & 0.06316154 & -4.13229752 \\
\hline 2.54510117 & 1.31986856 & -0.13571967 & -3.01965261 \\
\hline 2.54510117 & 1.31986856 & -0.02988423 & 2.91053295 \\
\hline 2.54510117 & 1.42570400 & -0.13571967 & 3.23308897 \\
\hline 2.54510117 & 1.42570400 & -0.02988423 & -3.16355658 \\
\hline 2.65093660 & 1.31986856 & -0.13571967 & 2.88409376 \\
\hline 2.65093660 & 1.31986856 & -0.02988423 & -2.81220651 \\
\hline 2.65093660 & 1.42570400 & -0.13571967 & -3.03847671 \\
\hline 2.65093660 & 1.42570400 & -0.02988423 & 2.99771357 \\
\hline-1.43156683 & -1.42060065 & 0.04030320 & 0.03059113 \\
\hline-1.43156683 & -1.42060065 & 0.14613864 & -0.11005454 \\
\hline-1.43156683 & -1.31476510 & 0.04030320 & -0.48328251 \\
\hline-1.43156683 & -1.31476510 & 0.14613864 & 0.58644944 \\
\hline-1.32573140 & -1.42060065 & 0.04030320 & -0.05214289 \\
\hline-1.32573140 & -1.42060065 & 0.14613864 & 0.13381433 \\
\hline-1.32573140 & -1.31476510 & 0.04030320 & 0.51758736 \\
\hline-1.32573140 & -1.31476510 & 0.14613864 & -0.62275022 \\
\hline 0.99076527 & -0.32923394 & -0.03195595 & -5.55259132 \\
\hline 0.99076527 & -0.32923394 & 0.07387950 & 5.55094767 \\
\hline 0.99076527 & -0.22339852 & -0.03195595 & 5.03296947 \\
\hline 0.99076527 & -0.22339852 & 0.07387950 & -4.99476099 \\
\hline 1.09660077 & -0.32923394 & -0.03195595 & 5.19011736 \\
\hline 1.09660077 & -0.32923394 & 0.07387950 & -5.12432003 \\
\hline 1.09660077 & -0.22339852 & -0.03195595 & -4.67527723 \\
\hline 1.09660077 & -0.22339852 & 0.07387950 & 4.57401180 \\
\hline-2.53873253 & 0.56140047 & -0.09333258 & -0.28324065 \\
\hline-2.53873253 & 0.56140047 & 0.01250287 & 0.42627603 \\
\hline-2.53873253 & 0.66723585 & -0.09333258 & 0.14813197 \\
\hline-2.53873253 & 0.66723585 & 0.01250287 & -0.28927860 \\
\hline-2.43289709 & 0.56140047 & -0.09333258 & 0.15198369 \\
\hline-2.43289709 & 0.56140047 & 0.01250287 & -0.31132099 \\
\hline-2.43289709 & 0.66723585 & -0.09333258 & 0.01497444 \\
\hline-2.43289709 & 0.66723585 & 0.01250287 & 0.13958666 \\
\hline 0.74471217 & -1.31334066 & 0.02794744 & -0.38749328 \\
\hline 0.74471217 & -1.31334066 & 0.13378289 & 0.38390788 \\
\hline
\end{tabular}




\begin{tabular}{|c|c|c|c|}
\hline 0.74471217 & -1.20750511 & 0.02794744 & 0.63160646 \\
\hline 0.74471217 & -1.20750511 & 0.13378289 & -0.62883264 \\
\hline 0.85054761 & -1.31334066 & 0.02794744 & 0.25437725 \\
\hline 0.85054761 & -1.31334066 & 0.13378289 & -0.23659185 \\
\hline 0.85054761 & -1.20750511 & 0.02794744 & -0.44799146 \\
\hline 0.85054761 & -1.20750511 & 0.13378289 & 0.42544699 \\
\hline-2.46623826 & 1.56936514 & -0.15553895 & -0.00603617 \\
\hline-2.46623826 & 1.56936514 & -0.04970350 & 0.04873122 \\
\hline-2.46623826 & 1.67520046 & -0.15553895 & 0.00801974 \\
\hline-2.46623826 & 1.67520046 & -0.04970350 & -0.03775623 \\
\hline-2.36040306 & 1.56936514 & -0.15553895 & -0.07445864 \\
\hline-2.36040306 & 1.56936514 & -0.04970350 & 0.02937622 \\
\hline-2.36040306 & 1.67520046 & -0.15553895 & 0.04791313 \\
\hline-2.36040306 & 1.67520046 & -0.04970350 & -0.01660842 \\
\hline 7539 & \# Energy 1->8 & & \\
\hline 0.8524 & -0.6656 & 1.000 & Electr. mom., scale-fac \\
\hline 0.0171 & -0.0405 & -0.0 & Magnetic mom. \\
\hline 2.27520919 & 0.11177704 & -0.06075061 & $1 \quad-11.71134663$ \\
\hline 2.27520919 & 0.11177704 & 0.04508484 & 6.71749306 \\
\hline 2.27520919 & 0.21761249 & -0.06075061 & 11.57118416 \\
\hline 2.27520919 & 0.21761249 & 0.04508484 & -5.14685917 \\
\hline 2.38104463 & 0.11177704 & -0.06075061 & 10.74480820 \\
\hline 2.38104463 & 0.11177704 & 0.04508484 & -6.89142752 \\
\hline 2.38104463 & 0.21761249 & -0.06075061 & -10.41268063 \\
\hline 2.38104463 & 0.21761249 & 0.04508484 & 5.70588827 \\
\hline-1.40967047 & -0.18411981 & -0.04267391 & -1.35506260 \\
\hline-1.40967047 & -0.18411981 & 0.06316154 & -1.90478539 \\
\hline-1.40967047 & -0.07828436 & -0.04267391 & 1.91900098 \\
\hline-1.40967047 & -0.07828436 & 0.06316154 & 1.60732114 \\
\hline-1.30383503 & -0.18411981 & -0.04267391 & 1.26176560 \\
\hline-1.30383503 & -0.18411981 & 0.06316154 & 2.16059494 \\
\hline-1.30383503 & -0.07828436 & -0.04267391 & -1.74974096 \\
\hline-1.30383503 & -0.07828436 & 0.06316154 & -1.79759324 \\
\hline 2.54510117 & 1.31986856 & -0.13571967 & 0.38088119 \\
\hline 2.54510117 & 1.31986856 & -0.02988423 & -4.04164839 \\
\hline 2.54510117 & 1.42570400 & -0.13571967 & -0.67689461 \\
\hline 2.54510117 & 1.42570400 & -0.02988423 & 3.33630061 \\
\hline 2.65093660 & 1.31986856 & -0.13571967 & -0.50602698 \\
\hline 2.65093660 & 1.31986856 & -0.02988423 & 2.90866017 \\
\hline 2.65093660 & 1.42570400 & -0.13571967 & 0.76923507 \\
\hline 2.65093660 & 1.42570400 & -0.02988423 & -2.52609205 \\
\hline-1.43156683 & -1.42060065 & 0.04030320 & 0.84969413 \\
\hline-1.43156683 & -1.42060065 & 0.14613864 & -1.27191889 \\
\hline-1.43156683 & -1.31476510 & 0.04030320 & -0.52893662 \\
\hline-1.43156683 & -1.31476510 & 0.14613864 & 1.10639703 \\
\hline-1.32573140 & -1.42060065 & 0.04030320 & -0.76839119 \\
\hline-1.32573140 & -1.42060065 & 0.14613864 & 1.36852944 \\
\hline-1.32573140 & -1.31476510 & 0.04030320 & 0.32814345 \\
\hline-1.32573140 & -1.31476510 & 0.14613864 & -1.15777552 \\
\hline 0.99076527 & -0.32923394 & -0.03195595 & -5.41478205 \\
\hline 0.99076527 & -0.32923394 & 0.07387950 & 1.38147163 \\
\hline 0.99076527 & -0.22339852 & -0.03195595 & 5.02816248 \\
\hline 0.99076527 & -0.22339852 & 0.07387950 & -0.86538762 \\
\hline 1.09660077 & -0.32923394 & -0.03195595 & 6.73903561 \\
\hline 1.09660077 & -0.32923394 & 0.07387950 & -1.90425968 \\
\hline 1.09660077 & -0.22339852 & -0.03195595 & -6.21504450 \\
\hline 1.09660077 & -0.22339852 & 0.07387950 & 1.07812941 \\
\hline-2.53873253 & 0.56140047 & -0.09333258 & -0.76397151 \\
\hline-2.53873253 & 0.56140047 & 0.01250287 & 0.59354544 \\
\hline-2.53873253 & 0.66723585 & -0.09333258 & 0.98377675 \\
\hline-2.53873253 & 0.66723585 & 0.01250287 & -0.65348399 \\
\hline-2.43289709 & 0.56140047 & -0.09333258 & 0.58897227 \\
\hline-2.43289709 & 0.56140047 & 0.01250287 & -0.55022097 \\
\hline-2.43289709 & 0.66723585 & -0.09333258 & -0.85271269 \\
\hline 289709 & 0.66723585 & 0.01250287 & 0.57746053 \\
\hline 0.74471217 & -1.31334066 & 0.02794744 & -1.12129009 \\
\hline 0.74471 & -1.31334066 & 0.13378289 & 0.7046608 \\
\hline
\end{tabular}




$\begin{array}{ll}0.74471217 & -1.20750511 \\ 0.74471217 & -1.20750511 \\ 0.85054761 & -1.31334066 \\ 0.85054761 & -1.31334066 \\ 0.85054761 & -1.20750511 \\ 0.85054761 & -1.20750511 \\ -2.46623826 & 1.56936514 \\ -2.46623826 & 1.56936514 \\ -2.46623826 & 1.67520046 \\ -2.46623826 & 1.67520046 \\ -2.36040306 & 1.56936514 \\ -2.36040306 & 1.56936514 \\ -2.36040306 & 1.67520046 \\ -2.36040306 & 1.67520046 \\ & \text { \#5849. Energy 1->9 }\end{array}$

64

$\begin{array}{rr}0.02794744 & 1.33445203 \\ 0.13378289 & -0.59278834 \\ 0.02794744 & 1.42081332 \\ 0.13378289 & -0.87807035 \\ 0.02794744 & -1.82233918 \\ 0.13378289 & 0.91323030 \\ -0.15553895 & -0.19280320 \\ -0.04970350 & 0.10810955 \\ -0.15553895 & 0.12102319 \\ -0.04970350 & -0.05456750 \\ -0.15553895 & 0.15351707 \\ -0.04970350 & -0.06421594 \\ -0.15553895 & -0.08676271 \\ -0.04970350 & 0.01762242\end{array}$
$\begin{array}{lrr}0.5177 & 0.9538 & -0.0674 \\ 0.0020 & -0.0155 & -0.1045\end{array}$

2. 27520919

2. 27520919

2. 27520919

2. 27520919

2.38104463

2.38104463

2.38104463

2. 38104463

$-1.40967047$

$-1.40967047$

$-1.40967047$

$-1.40967047$

$-1.30383503$

$-1.30383503$

$-1.30383503$

$-1.30383503$

2. 54510117

2. 54510117

2. 54510117

2. 54510117

2.65093660

2.65093660

2.65093660

2.65093660

$-1.43156683$

$-1.43156683$

$-1.43156683$

$-1.43156683$

$-1.32573140$

$-1.32573140$

$-1.32573140$

$-1.32573140$

0.99076527

0.99076527

0.99076527

0.99076527

1. 09660077

1.09660077

1.09660077

1.09660077

$-2.53873253$

$-2.53873253$

$-2.53873253$

$-2.53873253$

$-2.43289709$

$-2.43289709$

$-2.43289709$

$-2.43289709$

0.74471217

0.74471217
0.11177704

0.11177704

0.21761249

0.21761249

0.11177704

0.11177704

0.21761249

0.21761249

$-0.18411981$

$-0.18411981$

$-0.07828436$

$-0.07828436$

$-0.18411981$

$-0.18411981$

$-0.07828436$

$-0.07828436$

1. 31986856

1. 31986856

1. 42570400

1. 42570400

1. 31986856

1. 31986856

1. 42570400

1. 42570400

$-1.42060065$

$-1.42060065$

$-1.31476510$

$-1.31476510$

$-1.42060065$

$-1.42060065$

$-1.31476510$

$-1.31476510$

$-0.32923394$

$-0.32923394$

$-0.22339852$

$-0.22339852$

$-0.32923394$

$-0.32923394$

$-0.22339852$

$-0.22339852$

0.56140047

0.56140047

0.66723585

0.66723585

0.56140047

0.56140047

0.66723585

0.66723585

$-1.31334066$

$-1.31334066$
0.06316154

0.14613864

\begin{abstract}
1.000 \# Electr. mom., scale-fac \# Magnetic mom.
\end{abstract}

$-0.06075061$

0.04508484

$-0.06075061$

0.04508484

$-0.06075061$

0.04508484

$-0.06075061$

0.04508484

$-0.04267391$

0.06316154

$-0.04267391$

0.06316154

$-0.04267391$

$-0.04267391$

0.06316154

$-0.13571967$

$-0.02988423$

$-0.13571967$

$-0.02988423$

$-0.13571967$

$-0.02988423$

$-0.13571967$

$-0.02988423$

0.04030320

0.14613864

0.04030320

0.14613864

0.04030320

0.04030320

0.14613864

$-0.03195595$

0.07387950

$-0.03195595$

0.07387950

$-0.03195595$

0.07387950

$-0.03195595$

0.07387950

$-0.09333258$

0.01250287

$-0.09333258$

0.01250287

$-0.09333258$

0.01250287

$-0.09333258$

0.01250287

0.02794744

0.13378289

4.65949821

$-2.21367240$

$-4.73359823$

1. 28587806

$-4.31808043$

2.40655732

4. 29540062

$-1.75207829$

$-4.95175457$

0.74117941

5. 23603249

$-0.22713147$

4.43446684

$-0.24003322$

$-4.53519297$

0.01811815

0.11850081

2.09164762

$-0.12059030$

$-1.50530982$

0.02618812

$-1.50195098$

$-0.02007390$

1. 11862540

$-0.18616094$

0.15949123

0.31019932

$-0.31306675$

0.27587321

0.21206468

$-0.56376165$

$-0.12523875$

2. 02151561

$-0.15465701$

$-1.82875431$

$-0.12110656$

$-2.19594765$

$-0.13394164$

2. 06159234

0.48770759

$-2.12168241$

$-0.51774895$

2. 40602469

0.76161224

2. 48719382

0.01740502

$-2.88307405$

$-0.43041709$

0.60902870

$-0.15366936$ 


\begin{abstract}
0.74471217
0.74471217

0.85054761

0.85054761

0.85054761

0.85054761

$-2.46623826$

$-2.46623826$

$-2.46623826$

$-2.46623826$

$-2.36040306$

$-2.36040306$

$-2.36040306$

$-2.36040306$
\end{abstract}

\&TRANSITION $2->$. .
$-1.20750511$
$-1.20750511$
$-1.31334066$
$-1.31334066$
$-1.20750511$
$-1.20750511$
1.56936514
1.56936514
1.67520046
1.67520046
1.56936514
1. 56936514
1.67520046
1.67520046

$$
\begin{array}{cc}
0 . \quad \# \text { Energy } & 2->3 \\
-0.3840 & 0.0244 \\
-0.0006 & -0.0063
\end{array}
$$

-0.0311
-0.0002

2. 27520919

2. 27520919

2. 27520919

2. 27520919

2.38104463

2. 38104463

2. 38104463

2.38104463

$-1.40967047$

$-1.40967047$

$-1.40967047$

$-1.40967047$

$-1.30383503$

$-1.30383503$

$-1.30383503$

$-1.30383503$

2. 54510117

2. 54510117

2. 54510117

2.54510117

2.65093660

2.65093660

2.65093660

2.65093660

$-1.43156683$

$-1.43156683$

$-1.43156683$

$-1.43156683$

$-1.32573140$

$-1.32573140$

$-1.32573140$

$-1.32573140$

0.99076527

0.99076527

0.99076527

0.99076527

1. 09660077

1.09660077

1.09660077

1. 09660077

$-2.53873253$

$-2.53873253$

$-2.53873253$

$-2.53873253$

$-2.43289709$

$-2.43289709$

$-2.43289709$

$-2.43289709$

0.74471217
0.11177704

0.11177704

0.21761249

0.21761249

0.11177704

0.11177704

0.21761249

0.21761249

$-0.18411981$

$-0.18411981$

$-0.07828436$

$-0.07828436$

$-0.18411981$

$-0.18411981$

$-0.07828436$

$-0.07828436$

1. 31986856

1. 31986856

1. 42570400

1. 42570400

1. 31986856

1.31986856

1. 42570400

1. 42570400

$-1.42060065$

$-1.42060065$

$-1.31476510$

$-1.31476510$

$-1.42060065$

$-1.42060065$

$-1.31476510$

$-1.31476510$

$-0.32923394$

$-0.32923394$

$-0.22339852$

$-0.22339852$

$-0.32923394$

$-0.32923394$

$-0.22339852$

$-0.22339852$

0.56140047

0.56140047

0.66723585

0.66723585

0.56140047

0.56140047

0.66723585

0.66723585

$-1.31334066$

$$
\begin{array}{r}
0.02794744 \\
0.13378289 \\
0.02794744 \\
0.13378289 \\
0.02794744 \\
0.13378289 \\
-0.15553895 \\
-0.04970350 \\
-0.15553895 \\
-0.04970350 \\
-0.15553895 \\
-0.04970350 \\
-0.15553895 \\
-0.04970350
\end{array}
$$

$$
\begin{array}{r}
-0.82784843 \\
0.16688229 \\
-0.62684822 \\
0.05490256 \\
0.85628486 \\
-0.04655139 \\
-0.32961124 \\
-0.29244989 \\
0.30467394 \\
0.12404846 \\
0.41617346 \\
0.27573267 \\
-0.36584508 \\
-0.10265119
\end{array}
$$

\begin{abstract}
1.000 \# Electr. mom., scale-fac \# Magnetic mom.
\end{abstract}

$\begin{array}{rr}-0.06075061 & 4.23570871 \\ 0.04508484 & 1.29491365 \\ -0.06075061 & -4.57676315 \\ 0.04508484 & -1.06756592 \\ -0.06075061 & -3.44174790 \\ 0.04508484 & -1.46770191 \\ -0.06075061 & 3.71360636 \\ 0.04508484 & 1.35438275 \\ -0.04267391 & -2.15621829 \\ 0.06316154 & 1.50014055 \\ -0.04267391 & 1.95778620 \\ 0.06316154 & -1.55932367 \\ -0.04267391 & 1.35289681\end{array}$

0.06316154

$-0.04267391$

0.06316154

$-0.13571967$

$-0.02988423$

$-0.13571967$

$-0.02988423$

$-0.13571967$

$-0.02988423$

$-0.13571967$

$-0.02988423$

0.04030320

0.14613864

0.04030320

0.14613864

0.04030320

0.14613864

0.04030320

0.14613864

$-0.03195595$

0.07387950

$-0.03195595$

0.07387950

$-0.03195595$

0.07387950

$-0.03195595$

0.07387950

$-0.09333258$

0.01250287

$-0.09333258$

0.01250287

$-0.09333258$

0.01250287

$-0.09333258$

0.01250287

0.02794744

$-0.88728231$

$-1.22382200$

0.95124733

1. 27751279

0.82302511

$-1.09007156$

$-0.61696118$

$-1.02733707$

$-1.05202413$

0.89082551

0.78362310

$-1.02446961$

0.42692509

1.32181287

$-0.51018709$

0.69185340

$-0.16572125$

$-0.88678467$

0.18211994

3. 84087133

$-1.00822222$

$-3.68970728$

1. 18869662

$-4.31635952$

1. 22415161

4.12700605

$-1.40441370$

$-1.33686507$

1. 38307798

1.48392820

$-1.61578321$

1.59349656

$-1.27863824$

$-1.78756285$

1.54543579

0.56797665 


\subsection{7 \\ 0.74471217 \\ 0.74471217 \\ 0.85054761 \\ 0.85054761 \\ 0.85054761 \\ 0.85054761 \\ $-2.46623826$ \\ $-2.46623826$ \\ $-2.46623826$ \\ $-2.46623826$ \\ $-2.36040306$ \\ $-2.36040306$ \\ $-2.36040306$ \\ $-2.36040306$}

64

0.0268

0.1170

2. 27520919

2. 27520919

2. 27520919

2. 27520919

2.38104463

2.38104463

2. 38104463

2. 38104463

$-1.40967047$

$-1.40967047$

$-1.40967047$

$-1.40967047$

$-1.30383503$

$-1.30383503$

$-1.30383503$

$-1.30383503$

2. 54510117

2. 54510117

2. 54510117

2. 54510117

2.65093660

2.65093660

2.65093660

2.65093660

$-1.43156683$

$-1.43156683$

$-1.43156683$

$-1.43156683$

$-1.32573140$

$-1.32573140$

$-1.32573140$

$-1.32573140$

0.99076527

0.99076527

0.99076527

0.99076527

1. 09660077

1.09660077

1.09660077

1. 09660077

$-2.53873253$

$-2.53873253$

$-2.53873253$

$-2.53873253$

$-2.43289709$

$-2.43289709$

$-2.43289709$

$-2.43289709$

0.74471217
$-1.31334066$

$-1.20750511$

$-1.20750511$

$-1.31334066$

$-1.31334066$

$-1.20750511$

$-1.20750511$

1.56936514

1.56936514

1.67520046

1.67520046

1.56936514

1.56936514

1.67520046

1.67520046

0. \# Energy 2->4

$\begin{array}{lr}0.0260 & 0.0805 \\ 0.4194 & -0.0305\end{array}$

0.11177704

0.11177704

0.21761249

0.21761249

0.11177704

0.11177704

0.21761249

0.21761249

$-0.18411981$

$-0.18411981$

$-0.07828436$

$-0.07828436$

$-0.18411981$

$-0.18411981$

$-0.07828436$

$-0.07828436$

1. 31986856

1.31986856

1. 42570400

1. 42570400

1.31986856

1.31986856

1. 42570400

1. 42570400

$-1.42060065$

$-1.42060065$

$-1.31476510$

$-1.31476510$

$-1.42060065$

$-1.42060065$

$-1.31476510$

$-1.31476510$

$-0.32923394$

$-0.32923394$

$-0.22339852$

$-0.22339852$

$-0.32923394$

$-0.32923394$

$-0.22339852$

$-0.22339852$

0.56140047

0.56140047

0.66723585

0.66723585

0.56140047

0.56140047

0.66723585

0.66723585

$-1.31334066$
0.13378289

0.02794744

0.13378289

0.02794744

0.13378289

0.02794744

0.13378289

$-0.15553895$

$-0.04970350$

$-0.15553895$

$-0.04970350$

$-0.15553895$

$-0.04970350$

$-0.15553895$

$-0.04970350$

1.000

$-0.06075061$

Electr. mom.,

0.04508484

$-0.06075061$

0.04508484

$-0.06075061$

0.04508484

$-0.06075061$

0.04508484

$-0.04267391$

0.06316154

$-0.04267391$

0.06316154

$-0.04267391$

0.06316154

$-0.04267391$

0.06316154

$-0.13571967$

$-0.02988423$

$-0.13571967$

$-0.02988423$

$-0.13571967$

$-0.02988423$

$-0.13571967$

$-0.02988423$

0.04030320

0.14613864

0.04030320

0.14613864

0.04030320

0.14613864

0.04030320

0.14613864

$-0.03195595$

0.07387950

$-0.03195595$

0.07387950

$-0.03195595$

0.07387950

$-0.03195595$

0.07387950

$-0.09333258$

0.01250287

$-0.09333258$

0.01250287

$-0.09333258$

0.01250287

$-0.09333258$

0.01250287

0.02794744
$-0.26237112$

$-0.78291488$

0.43504754

$-0.65503067$

0.34065419

0.90576571

$-0.53483748$

$-0.30794352$

0.45758978

0.21033946

$-0.31677344$

0.35017550

$-0.38049430$

$-0.24061520$

0.25992107 etic mom.

-3.21707559
3.66525412
3.63733697
-4.12250900
3.56822348
-3.93273568
-3.98671746
4.38347101
0.58045435

$-0.51139724$

$-0.39433908$

0.33207035

$-0.49776185$

0.42371523

0.30063134

$-0.23374061$

1. 38535047

$-1.11757588$

0.15769538

$-0.38809711$

$-1.61886072$

1.37791026

0.10274916

0.10548931

0.15280408

$-0.14283799$

0.12680548

$-0.13724715$

$-0.12506513$

0.11433760

$-0.16349088$

0.17514053

$-0.86595321$

1.23223495

0.77340388

$-1.10263658$

1. 13939500

$-1.53446412$

$-0.95315707$

1.31289279

$-0.05217111$

0.06759544

$-0.08397532$

0.06804368

$-0.00856185$

$-0.01314502$

0.17455219

$-0.15217444$

0.02584879 


\begin{tabular}{|c|c|c|c|}
\hline 0.74471217 & -1.31334066 & 0.13378289 & 0.02955652 \\
\hline 0.74471217 & -1.20750511 & 0.02794744 & -0.03997838 \\
\hline 0.74471217 & -1.20750511 & 0.13378289 & -0.03879608 \\
\hline 0.85054761 & -1.31334066 & 0.02794744 & 0.08476138 \\
\hline 0.85054761 & -1.31334066 & 0.13378289 & -0.13391390 \\
\hline 0.85054761 & -1.20750511 & 0.02794744 & -0.12895404 \\
\hline 0.85054761 & -1.20750511 & 0.13378289 & 0.20016204 \\
\hline-2.46623826 & 1.56936514 & -0.15553895 & 0.04205130 \\
\hline-2.46623826 & 1.56936514 & -0.04970350 & -0.03986583 \\
\hline-2.46623826 & 1.67520046 & -0.15553895 & -0.02756901 \\
\hline-2.46623826 & 1.67520046 & -0.04970350 & 0.02610737 \\
\hline-2.36040306 & 1.56936514 & -0.15553895 & -0.09952853 \\
\hline-2.36040306 & 1.56936514 & -0.04970350 & 0.09533390 \\
\hline-2.36040306 & 1.67520046 & -0.15553895 & 0.06493360 \\
\hline-2.36040306 & 1.67520046 & -0.04970350 & -0.06201565 \\
\hline & \# Energy 2->5 & & \\
\hline-0.0064 & -0.0337 & 1.000 & lectr. mom., \\
\hline-0.0402 & -0.1677 & $\#$ & Magnetic mom. \\
\hline 2.27520919 & 0.11177704 & -0.06075061 & 7.41419220 \\
\hline 2.27520919 & 0.11177704 & 0.04508484 & -8.86566544 \\
\hline 2.27520919 & 0.21761249 & -0.06075061 & -8.61340809 \\
\hline 2.27520919 & 0.21761249 & 0.04508484 & 10.17537403 \\
\hline 2.38104463 & 0.11177704 & -0.06075061 & -8.45775223 \\
\hline 2.38104463 & 0.11177704 & 0.04508484 & 9.65756512 \\
\hline 2.38104463 & 0.21761249 & -0.06075061 & 9.65448475 \\
\hline 2.38104463 & 0.21761249 & 0.04508484 & -10.95124245 \\
\hline-1.40967047 & -0.18411981 & -0.04267391 & 1.90684259 \\
\hline-1.40967047 & -0.18411981 & 0.06316154 & -2.14948487 \\
\hline-1.40967047 & -0.07828436 & -0.04267391 & -2.44032669 \\
\hline-1.40967047 & -0.07828436 & 0.06316154 & 2.65449429 \\
\hline-1.30383503 & -0.18411981 & -0.04267391 & -2.11376715 \\
\hline-1.30383503 & -0.18411981 & 0.06316154 & 2.38839388 \\
\hline-1.30383503 & -0.07828436 & -0.04267391 & 2.62989640 \\
\hline-1.30383503 & -0.07828436 & 0.06316154 & -2.87235022 \\
\hline 2.54510117 & 1.31986856 & -0.13571967 & -3.93468904 \\
\hline 2.54510117 & 1.31986856 & -0.02988423 & 3.12269950 \\
\hline 2.54510117 & 1.42570400 & -0.13571967 & -0.69470447 \\
\hline 2.54510117 & 1.42570400 & -0.02988423 & 1.38878429 \\
\hline 2.65093660 & 1.31986856 & -0.13571967 & 4.67723036 \\
\hline 2.65093660 & 1.31986856 & -0.02988423 & -3.94341612 \\
\hline 2.65093660 & 1.42570400 & -0.13571967 & -0.12224441 \\
\hline 2.65093660 & 1.42570400 & -0.02988423 & -0.50713032 \\
\hline-1.43156683 & -1.42060065 & 0.04030320 & 0.02504485 \\
\hline-1.43156683 & -1.42060065 & 0.14613864 & -0.09976745 \\
\hline-1.43156683 & -1.31476510 & 0.04030320 & 0.04542044 \\
\hline-1.43156683 & -1.31476510 & 0.14613864 & 0.0567104 \\
\hline-1.32573140 & -1.42060065 & 0.04030320 & -0.06585922 \\
\hline-1.32573140 & -1.42060065 & 0.14613864 & 0.15101281 \\
\hline-1.32573140 & -1.31476510 & 0.04030320 & 0.007995 \\
\hline-1.32573140 & -1.31476510 & 0.14613864 & -0.12374059 \\
\hline 0.99076527 & -0.32923394 & -0.03195595 & 1.63186443 \\
\hline 0.99076527 & -0.32923394 & 0.07387950 & -2.72056913 \\
\hline 0.99076527 & -0.22339852 & -0.03195595 & -1.49354863 \\
\hline 0.99076527 & -0.22339852 & 0.07387950 & 2.46389508 \\
\hline 1.09660077 & -0.32923394 & -0.03195595 & -2.37855053 \\
\hline 1.09660077 & -0.32923394 & 0.07387950 & 3.57304049 \\
\hline 1.09660077 & -0.22339852 & -0.03195595 & 1.994 \\
\hline 1.09660077 & -0.22339852 & 0.07387950 & -3.07338119 \\
\hline-2.53873253 & 0.56140047 & -0.09333258 & -1.2671715 \\
\hline-2.53873253 & 0.56140047 & 0.01250287 & 1.248 \\
\hline-2.53873253 & 0.66723585 & -0.09333258 & 1.1979155 \\
\hline-2.53873253 & 0.66723585 & 0.01250287 & -1.17163420 \\
\hline-2.43289709 & 0.56140047 & -0.09333258 & 1.5585974 \\
\hline-2.43289709 & 0.56140047 & 0.01250287 & -1.53 \\
\hline-2.43289709 & 0.66723585 & -0.09333258 & -1.4619193 \\
\hline-2.43289709 & 0.66723585 & 0.01250287 & 1.4298828 \\
\hline 0.74471217 & -1.31334066 & 0.02794744 & -0.1209147 \\
\hline
\end{tabular}




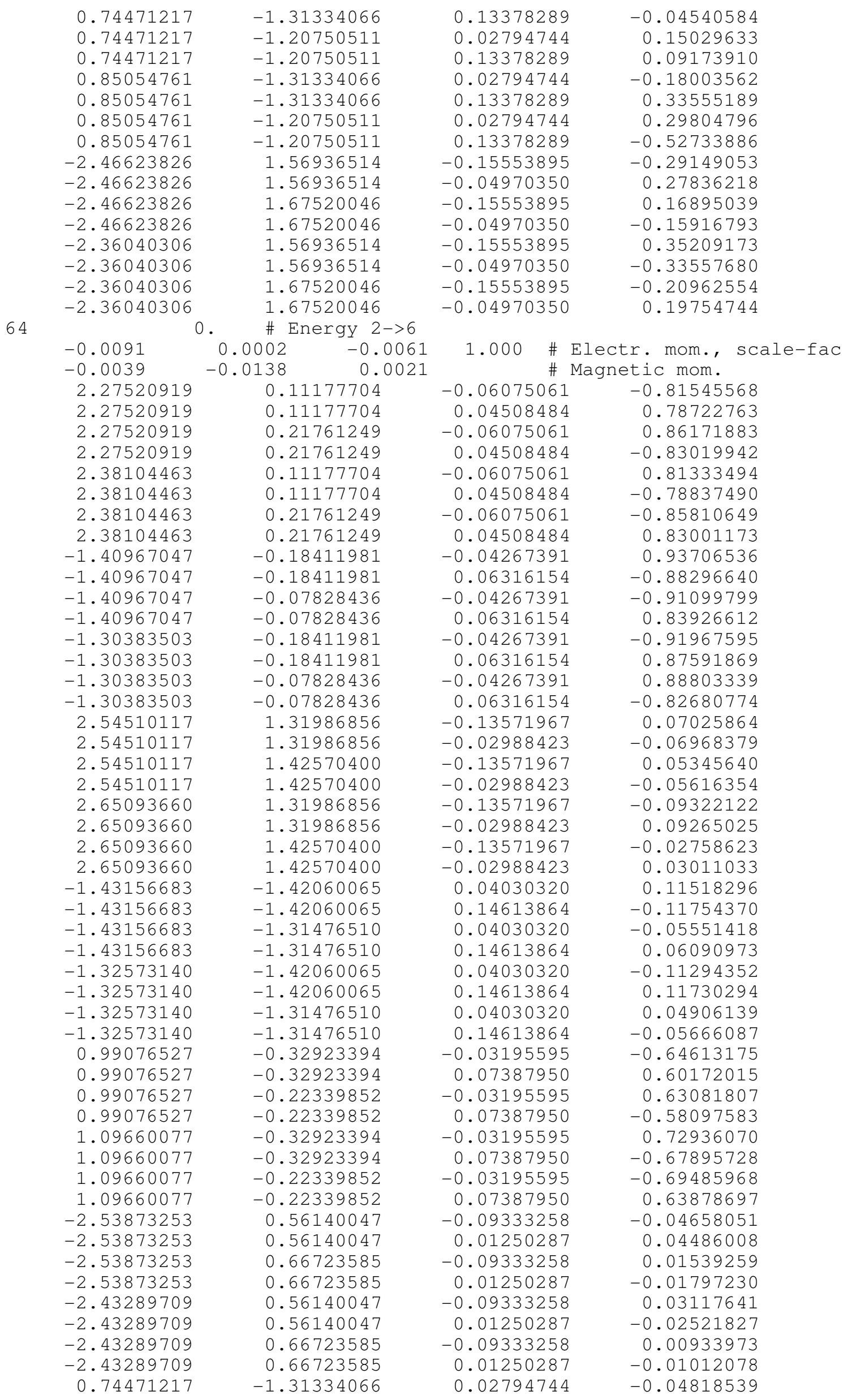




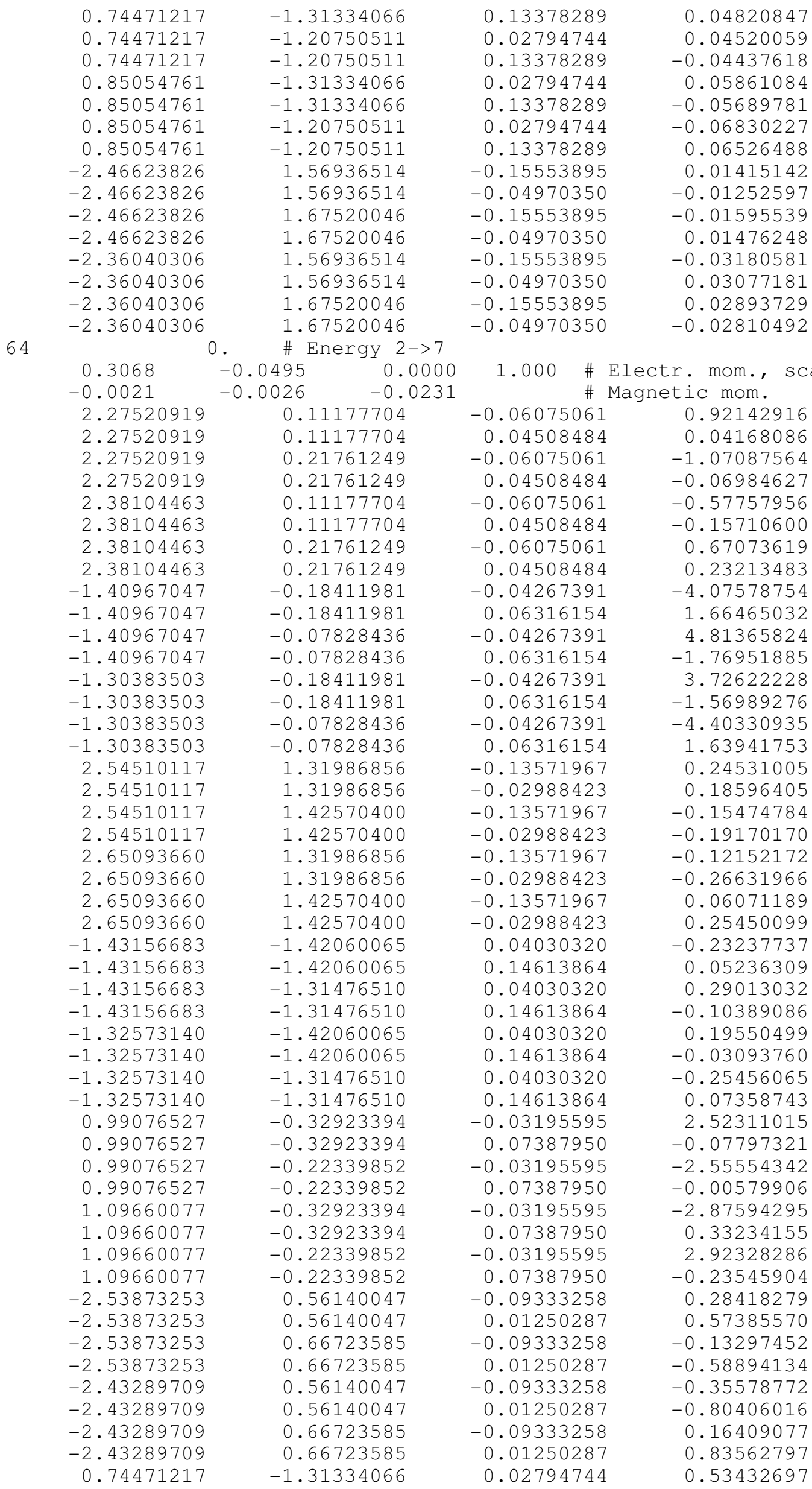




\subsection{7 \\ 0.74471217 \\ 0.74471217 \\ 0.85054761 \\ 0.85054761 \\ 0.85054761 \\ 0.85054761 \\ $-2.46623826$ \\ $-2.46623826$ \\ $-2.46623826$ \\ $-2.46623826$ \\ $-2.36040306$ \\ $-2.36040306$ \\ $-2.36040306$ \\ $-2.36040306$}

64
0

$\begin{array}{rr}\text { \# Energy } 2->8 \\ 0.1704 & -0.0456 \\ -0.1792 & -0.0164\end{array}$

$\begin{array}{rrr}0.7290 & 0.1704 & -0.0456 \\ -0.0265 & -0.1792 & -0.0164\end{array}$

2.27520919

2. 27520919

2. 27520919

2.27520919

2.38104463

2.38104463

2. 38104463

2.38104463

$-1.40967047$

$-1.40967047$

$-1.40967047$

$-1.40967047$

$-1.30383503$

$-1.30383503$

$-1.30383503$

$-1.30383503$

2.54510117

2. 54510117

2.54510117

2.54510117

2.65093660

2.65093660

2.65093660

2.65093660

$-1.43156683$

$-1.43156683$

$-1.43156683$

$-1.43156683$

$-1.32573140$

$-1.32573140$

$-1.32573140$

$-1.32573140$

0.99076527

0.99076527

0.99076527

0.99076527

1.09660077

1.09660077

1.09660077

1.09660077

$-2.53873253$

$-2.53873253$

$-2.53873253$

$-2.53873253$

$-2.43289709$

$-2.43289709$

$-2.43289709$

$-2.43289709$

0.74471217
$-1.31334066$

$-1.20750511$

$-1.20750511$

$-1.31334066$

$-1.31334066$

$-1.20750511$

$-1.20750511$

1.56936514

1.56936514

1.67520046

1.67520046

1.56936514

1.56936514

1.67520046

1.67520046

0.11177704

0.11177704

0.21761249

0.21761249

0.11177704

0.11177704

0.21761249

0.21761249

$-0.18411981$

$-0.18411981$

$-0.07828436$

$-0.07828436$

$-0.18411981$

$-0.18411981$

$-0.07828436$

$-0.07828436$

1.31986856

1.31986856

1. 42570400

1. 42570400

1.31986856

1.31986856

1. 42570400

1.42570400

$-1.42060065$

$-1.42060065$

$-1.31476510$

$-1.31476510$

$-1.42060065$

$-1.42060065$

$-1.31476510$

$-1.31476510$

$-0.32923394$

$-0.32923394$

$-0.22339852$

$-0.22339852$

$-0.32923394$

$-0.32923394$

$-0.22339852$

$-0.22339852$

0.56140047

0.56140047

0.66723585

0.66723585

0.56140047

0.56140047

0.66723585

0.66723585

$-1.31334066$
0.13378289

0.02794744

0.13378289

0.02794744

0.13378289

0.02794744

0.13378289

$-0.15553895$

$-0.04970350$

$-0.15553895$

$-0.04970350$

$-0.15553895$

$-0.04970350$

$-0.15553895$

$-0.04970350$

1.000 \# Electr. mom., scale-fac \# Magnetic mom.

$-0.06075061$

0.04508484

$-0.06075061$

0.04508484

$-0.06075061$

0.04508484

$-0.06075061$

0.04508484

$-0.04267391$

0.06316154

$-0.04267391$

0.06316154

$-0.04267391$

0.06316154

$-0.04267391$

0.06316154

$-0.13571967$

$-0.02988423$

$-0.13571967$

$-0.02988423$

$-0.13571967$

$-0.02988423$

$-0.13571967$

$-0.02988423$

0.04030320

0.14613864

0.04030320

0.14613864

0.04030320

0.14613864

0.04030320

0.14613864

$-0.03195595$

0.07387950

$-0.03195595$

0.07387950

$-0.03195595$

0.07387950

$-0.03195595$

0.07387950

$-0.09333258$

0.01250287

$-0.09333258$

0.01250287

$-0.09333258$

0.01250287

$-0.09333258$

0.01250287

0.02794744
$-0.38425547$

$-0.77856624$

0.54199845

$-0.60014993$

0.45665243

0.86453342

$-0.63725692$

0.13601115

$-0.03284562$

$-0.15060681$

0.08835680

$-0.12060663$

$-0.03469012$

0.14566757

$-0.05060776$
$-15.06507587$

12.60274315

16.37056732

$-13.92827702$

14.74876881

$-12.35821438$

$-15.93778896$

13.54257584

2.93089628

$-2.02449179$

$-2.63907576$

1.96154904

$-2.70267129$

1.62865818

2.43229890

$-1.60986507$

0.06871671

$-0.74346030$

1.20383620

$-0.59005737$

$-0.37510031$

1.13982260

$-0.88148069$

0.21366553

0.78529763

$-0.39077172$

$-1.05447328$

0.48862925

$-0.69647413$

0.22246134

0.94475311

$-0.28555295$

$-6.70350504$

5.53747416

6.93908739

$-5.81595039$

7.81428289

$-6.63456345$

$-8.05547142$

6.94058228

0.28088897

$-0.91230291$

$-0.20552631$

0.90395558

$-0.60081303$

1.17574561

0.53838247

$-1.20813382$

$-0.98186624$ 


\subsection{7 \\ 0.74471217 \\ 0.74471217 \\ 0.85054761 \\ 0.85054761 \\ 0.85054761 \\ 0.85054761 \\ $-2.46623826$ \\ $-2.46623826$ \\ $-2.46623826$ \\ $-2.46623826$ \\ $-2.36040306$ \\ $-2.36040306$ \\ $-2.36040306$ \\ $-2.36040306$}

64
$-1.31334066$

$-1.20750511$

$-1.20750511$

$-1.31334066$

$-1.31334066$

$-1.20750511$

$-1.20750511$

1.56936514

1.56936514

1.67520046

1.67520046

1.56936514

1.56936514

1.67520046

1.67520046

0 . \# Energy 2->9

$0.0026-0.0042 \quad 0.0365$

0.0078

2.27520919

2.27520919

2.27520919

2.27520919

2.38104463

2. 38104463

2. 38104463

2.38104463

$-1.40967047$

$-1.40967047$

$-1.40967047$

$-1.40967047$

$-1.30383503$

$-1.30383503$

$-1.30383503$

$-1.30383503$

2.54510117

2.54510117

2.54510117

2.54510117

2.65093660

2.65093660

2.65093660

2.65093660

$-1.43156683$

$-1.43156683$

$-1.43156683$

$-1.43156683$

$-1.32573140$

$-1.32573140$

$-1.32573140$

$-1.32573140$

0.99076527

0.99076527

0.99076527

0.99076527

1.09660077

1.09660077

1.09660077

1.09660077

$-2.53873253$

$-2.53873253$

$-2.53873253$

$-2.53873253$

$-2.43289709$

$-2.43289709$

$-2.43289709$

$-2.43289709$

0.74471217
$0.1655 \quad-0.0112$

0.11177704

0.11177704

0.21761249

0.21761249

0.11177704

0.11177704

0.21761249

0.21761249

$-0.18411981$

$-0.18411981$

$-0.07828436$

$-0.07828436$

$-0.18411981$

$-0.18411981$

$-0.07828436$

$-0.07828436$

1.31986856

1.31986856

1. 42570400

1. 42570400

1.31986856

1.31986856

1. 42570400

1.42570400

$-1.42060065$

$-1.42060065$

$-1.31476510$

$-1.31476510$

$-1.42060065$

$-1.42060065$

$-1.31476510$

$-1.31476510$

$-0.32923394$

$-0.32923394$

$-0.22339852$

$-0.22339852$

$-0.32923394$

$-0.32923394$

$-0.22339852$

$-0.22339852$

0.56140047

0.56140047

0.66723585

0.66723585

0.56140047

0.56140047

0.66723585

0.66723585

$-1.31334066$
0.13378289

0.02794744

0.13378289

0.02794744

0.13378289

0.02794744

0.13378289

$-0.15553895$

$-0.04970350$

$-0.15553895$

$-0.04970350$

$-0.15553895$

$-0.04970350$

$-0.15553895$

$-0.04970350$
0.83273822

1.39122069

$-1.19424832$

1.11489689

$-0.98207444$

$-1.57362783$

1.39795041

$-0.26178461$

$-0.02661303$

0.22442918

$-0.01152052$

0.16339055

0.11160943

$-0.14999519$

$-0.05104633$
1.000 \# Electr. mom., scale-fac
\# Magnetic mom.

$-0.06075061$

0.04508484

$-0.06075061$

0.04508484

$-0.06075061$

0.04508484

$-0.06075061$

0.04508484

$-0.04267391$

0.06316154

$-0.04267391$

0.06316154

$-0.04267391$

0.06316154

$-0.04267391$

0.06316154

$-0.13571967$

$-0.02988423$

$-0.13571967$

$-0.02988423$

$-0.13571967$

$-0.02988423$

$-0.13571967$

$-0.02988423$

0.04030320

0.14613864

0.04030320

0.14613864

0.04030320

0.14613864

0.04030320

0.14613864

$-0.03195595$

0.07387950

$-0.03195595$

0.07387950

$-0.03195595$

0.07387950

$-0.03195595$

0.07387950

$-0.09333258$

0.01250287

$-0.09333258$

0.01250287

$-0.09333258$

0.01250287

$-0.09333258$

0.01250287

0.02794744
6.07198763

$-6.07979345$

$-6.68310404$

6.72644949

$-6.32503128$

6.30728292

6.89069462

$-6.90383482$

$-2.01122022$

2. 02328253

2.18294859

$-2.19064999$

2.17556977

$-2.19610643$

$-2.35145974$

2.36574674

$-1.16718209$

1.07280540

0.23841754

$-0.15008686$

1.18788469

$-1.10661066$

$-0.29483178$

0.21674591

$-0.14651601$

0.16394320

$-0.00988609$

$-0.01739869$

0.15858676

$-0.18033281$

$-0.00956108$

0.04254156

1.46150100

$-1.59047282$

$-1.70266044$

1.83846653

$-1.79890740$

1.94337070

2. 09279227

$-2.24493885$

0.00136857

$-0.04457918$

0.02538197

0.01689273

$-0.10952236$

0.16056451

0.07557425

$-0.12634557$

0.20941047 


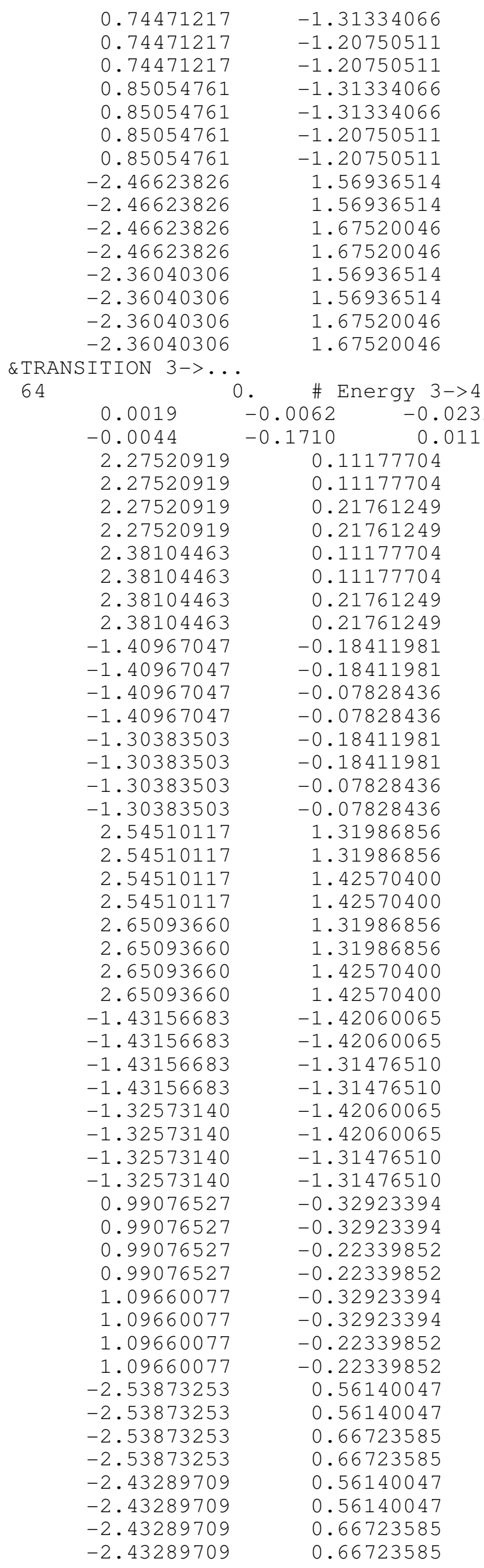

0.13378289

0.02794744

0.13378289

0.02794744

0.13378289

0.02794744

0.13378289

$-0.15553895$

$-0.04970350$

$-0.15553895$

$-0.04970350$

$-0.15553895$

$-0.04970350$

$-0.15553895$

$-0.04970350$
-0.21723370
-0.33422360
0.34341338
-0.22874571
0.23598424
0.35129753
-0.36027643
0.02861025
-0.04406414
0.00978706
0.00136141
-0.05409913
0.07185626
0.00614197
-0.01898793
1.000 \# Electr. mom., scale-fac \# Magnetic mom.

$-0.06075061$

0.04508484

$-0.06075061$

0.04508484

$-0.06075061$

0.04508484

$-0.06075061$

0.04508484

$-0.04267391$

0.06316154

$-0.04267391$

0.06316154

$-0.04267391$

0.06316154

$-0.04267391$

0.06316154

$-0.13571967$

$-0.02988423$

$-0.13571967$

$-0.02988423$

$-0.13571967$

$-0.02988423$

$-0.13571967$

$-0.02988423$

0.04030320

0.14613864

0.04030320

0.14613864

0.04030320

0.14613864

0.04030320

0.14613864

$-0.03195595$

0.07387950

$-0.03195595$

0.07387950

$-0.03195595$

0.07387950

$-0.03195595$

0.07387950

$-0.09333258$

0.01250287

$-0.09333258$

0.01250287

$-0.09333258$

0.01250287

$-0.09333258$

0.01250287
$-1.17802131$

1. 12599862

1.08321929

$-1.01020551$

0.95853633

$-0.91943753$

$-0.87753135$

0.82112026

15.59917355

$-15.55491543$

$-16.01064110$

15.93017578

$-15.29283810$

15.33284187

15.48385239

$-15.47936344$

$-0.48481345$

0.44865611

$-0.39687291$

0.46587729

$-0.43637237$

$-0.40430072$

0.38338909

1.42772913

$-1.59663403$

2. 38811851

$-2.11566734$

$-1.23830867$

1. 44011819

$-2.61540055$

2. 30209923

$-1.91500866$

1.81149566

1.68694079

$-1.57748687$

1. 58026958

$-1.44636655$

$-1.36409843$

1.22604966

$-6.74751949$

6.92459869

4.60485744

$-4.75546217$

7.49557781

$-7.74345255$

$-4.82974434$

5.05127430
0.42326561 


\begin{tabular}{|c|c|c|c|}
\hline 0.74471217 & -1.31334066 & 0.02794744 & 0.01724154 \\
\hline 0.74471217 & -1.31334066 & 0.13378289 & -0.03924852 \\
\hline 0.74471217 & -1.20750511 & 0.02794744 & -0.00124442 \\
\hline 0.74471217 & -1.20750511 & 0.13378289 & 0.04838345 \\
\hline 0.85054761 & -1.31334066 & 0.02794744 & -0.04002938 \\
\hline 0.85054761 & -1.31334066 & 0.13378289 & 0.07110581 \\
\hline 0.85054761 & -1.20750511 & 0.02794744 & 0.03477518 \\
\hline 0.85054761 & -1.20750511 & 0.13378289 & -0.09218175 \\
\hline-2.46623826 & 1.56936514 & -0.15553895 & -0.79844350 \\
\hline-2.46623826 & 1.56936514 & -0.04970350 & 0.77586776 \\
\hline-2.46623826 & 1.67520046 & -0.15553895 & 0.43358994 \\
\hline-2.46623826 & 1.67520046 & -0.04970350 & -0.41523474 \\
\hline-2.36040306 & 1.56936514 & -0.15553895 & 0.38263497 \\
\hline-2.36040306 & 1.56936514 & -0.04970350 & -0.37548026 \\
\hline-2.36040306 & 1.67520046 & -0.15553895 & -0.17321660 \\
\hline-2.36040306 & 1.67520046 & -0.04970350 & 0.16670801 \\
\hline & \# Energy 3->5 & & \\
\hline 0.0099 & -0.0105 & 1.000 & Electr. mom., scale-fac \\
\hline 0.0008 & 0.0242 & \# & Magnetic mom. \\
\hline 2.27520919 & 0.11177704 & -0.06075061 & -0.96121365 \\
\hline 2.27520919 & 0.11177704 & 0.04508484 & 1.05075884 \\
\hline 2.27520919 & 0.21761249 & -0.06075061 & 1.01324260 \\
\hline 2.27520919 & 0.21761249 & 0.04508484 & -1.10403371 \\
\hline 2.38104463 & 0.11177704 & -0.06075061 & 0.96584749 \\
\hline 2.38104463 & 0.11177704 & 0.04508484 & -1.04008102 \\
\hline 2.38104463 & 0.21761249 & -0.06075061 & -1.02362204 \\
\hline 2.38104463 & 0.21761249 & 0.04508484 & 1.09931362 \\
\hline-1.40967047 & -0.18411981 & -0.04267391 & 4.82708120 \\
\hline-1.40967047 & -0.18411981 & 0.06316154 & -4.78056192 \\
\hline-1.40967047 & -0.07828436 & -0.04267391 & -4.95909691 \\
\hline-1.40967047 & -0.07828436 & 0.06316154 & 4.89685297 \\
\hline-1.30383503 & -0.18411981 & -0.04267391 & -4.66123343 \\
\hline-1.30383503 & -0.18411981 & 0.06316154 & 4.64286041 \\
\hline-1.30383503 & -0.07828436 & -0.04267391 & 4.70841169 \\
\hline-1.30383503 & -0.07828436 & 0.06316154 & -4.67206812 \\
\hline 2.54510117 & 1.31986856 & -0.13571967 & 0.17313842 \\
\hline 2.54510117 & 1.31986856 & -0.02988423 & -0.12140605 \\
\hline 2.54510117 & 1.42570400 & -0.13571967 & 0.16541176 \\
\hline 2.54510117 & 1.42570400 & -0.02988423 & -0.21078309 \\
\hline 2.65093660 & 1.31986856 & -0.13571967 & -0.24070315 \\
\hline 2.65093660 & 1.31986856 & -0.02988423 & 0.19341409 \\
\hline 2.65093660 & 1.42570400 & -0.13571967 & -0.09308757 \\
\hline 2.65093660 & 1.42570400 & -0.02988423 & 0.13482133 \\
\hline-1.43156683 & -1.42060065 & 0.04030320 & 0.72285920 \\
\hline-1.43156683 & -1.42060065 & 0.14613864 & -0.77196544 \\
\hline-1.43156683 & -1.31476510 & 0.04030320 & 0.62955707 \\
\hline-1.43156683 & -1.31476510 & 0.14613864 & -0.54773313 \\
\hline-1.32573140 & -1.42060065 & 0.04030320 & -0.65718460 \\
\hline-1.32573140 & -1.42060065 & 0.14613864 & 0.71717173 \\
\hline-1.32573140 & -1.31476510 & 0.04030320 & -0.71159750 \\
\hline-1.32573140 & -1.31476510 & 0.14613864 & 0.61638457 \\
\hline 0.99076527 & -0.32923394 & -0.03195595 & -0.85332942 \\
\hline 0.99076527 & -0.32923394 & 0.07387950 & 0.88522333 \\
\hline 0.99076527 & -0.22339852 & -0.03195595 & 0.77853185 \\
\hline 0.99076527 & -0.22339852 & 0.07387950 & -0.80181718 \\
\hline 1.09660077 & -0.32923394 & -0.03195595 & 0.87895685 \\
\hline 1.09660077 & -0.32923394 & 0.07387950 & -0.90970272 \\
\hline 1.09660077 & -0.22339852 & -0.03195595 & -0.77776784 \\
\hline 1.09660077 & -0.22339852 & 0.07387950 & 0.80012274 \\
\hline-2.53873253 & 0.56140047 & -0.09333258 & -2.45133638 \\
\hline-2.53873253 & 0.56140047 & 0.01250287 & 2.50447297 \\
\hline-2.53873253 & 0.66723585 & -0.09333258 & 1.70222843 \\
\hline-2.53873253 & 0.66723585 & 0.01250287 & -1.74763966 \\
\hline-2.43289709 & 0.56140047 & -0.09333258 & 2.69749379 \\
\hline-2.43289709 & 0.56140047 & 0.01250287 & -2.77248621 \\
\hline-2.43289709 & 0.66723585 & -0.09333258 & -1.76396871 \\
\hline-2.43289709 & 0.66723585 & 0.01250287 & 1.83166516 \\
\hline
\end{tabular}




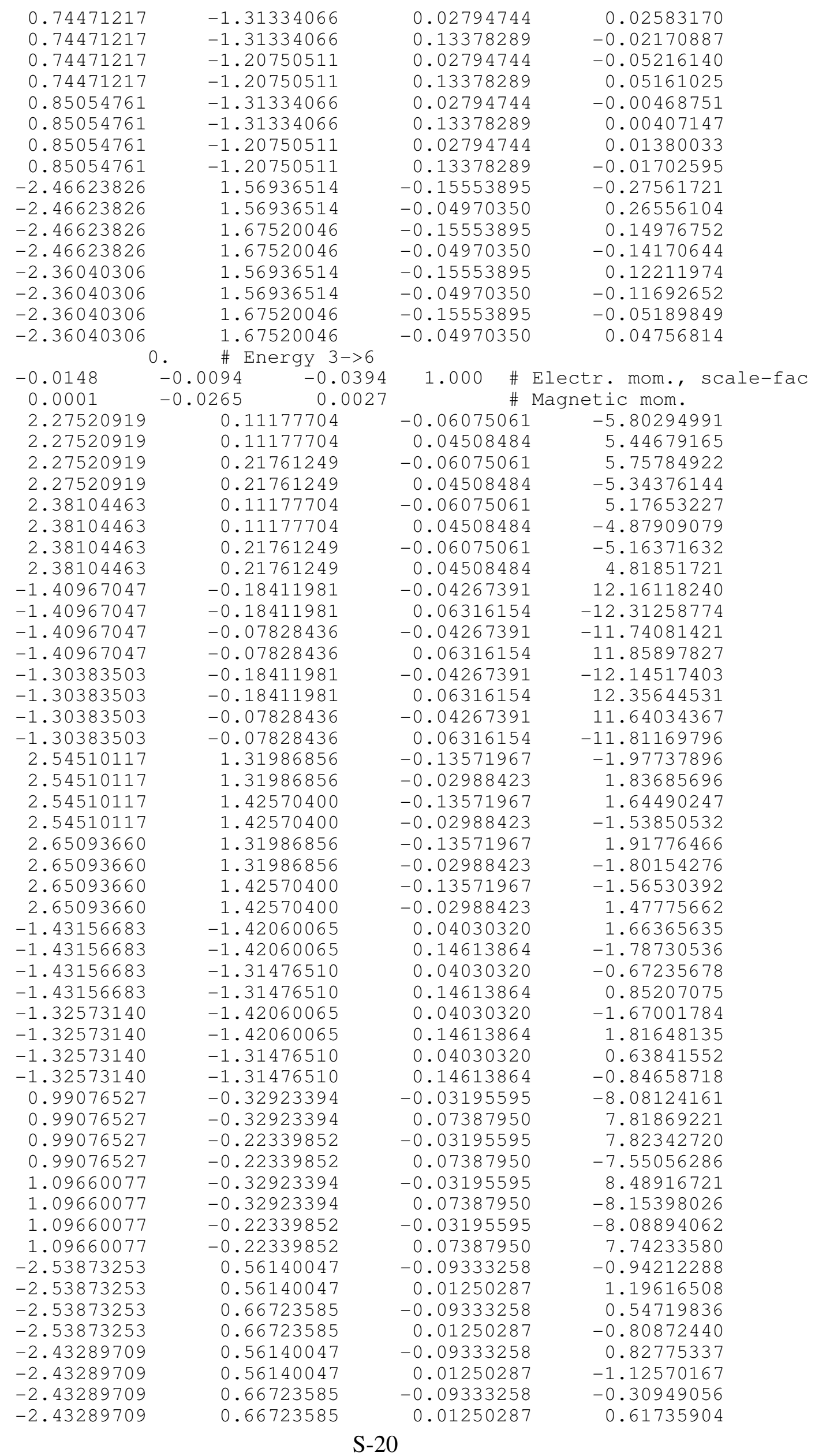




\subsection{7 \\ 0.74471217 \\ 0.74471217 \\ 0.74471217 \\ 0.85054761 \\ 0.85054761 \\ 0.85054761 \\ 0.85054761 \\ $-2.46623826$ \\ $-2.46623826$ \\ $-2.46623826$ \\ $-2.46623826$ \\ $-2.36040306$ \\ $-2.36040306$ \\ $-2.36040306$ \\ $-2.36040306$}

64

$-0.0010$

2. 27520919

2.27520919

2.27520919

2. 27520919

2.38104463

2. 38104463

2.38104463

2.38104463

$-1.40967047$

$-1.40967047$

$-1.40967047$

$-1.40967047$

$-1.30383503$

$-1.30383503$

$-1.30383503$

$-1.30383503$

2. 54510117

2.54510117

2. 54510117

2.54510117

2.65093660

2.65093660

2.65093660

2.65093660

$-1.43156683$

$-1.43156683$

$-1.43156683$

$-1.43156683$

$-1.32573140$

$-1.32573140$

$-1.32573140$

$-1.32573140$

0.99076527

0.99076527

0.99076527

0.99076527

1.09660077

1.09660077

1.09660077

1.09660077

$-2.53873253$

$-2.53873253$

$-2.53873253$

$-2.53873253$

$-2.43289709$

$-2.43289709$

$-2.43289709$

$-2.43289709$
$-1.31334066$

$-1.31334066$

$-1.20750511$

$-1.20750511$

$-1.31334066$

$-1.31334066$

$-1.20750511$

$-1.20750511$

1.56936514

1.56936514

1.67520046

1.67520046

1.56936514

1.56936514

1.67520046

1.67520046

\# Energy 3-> 7

$\begin{array}{lr}0.2982 & -0.0191 \\ 0.0041 & 0.0362\end{array}$

0.11177704

0.11177704

0.21761249

0.21761249

0.11177704

0.11177704

0.21761249

0.21761249

$-0.18411981$

$-0.18411981$

$-0.07828436$

$-0.07828436$

$-0.18411981$

$-0.18411981$

$-0.07828436$

$-0.07828436$

1.31986856

1.31986856

1. 42570400

1.42570400

1.31986856

1. 31986856

1. 42570400

1. 42570400

$-1.42060065$

$-1.42060065$

$-1.31476510$

$-1.31476510$

$-1.42060065$

$-1.42060065$

$-1.31476510$

$-1.31476510$

$-0.32923394$

$-0.32923394$

$-0.22339852$

$-0.22339852$

$-0.32923394$

$-0.32923394$

$-0.22339852$

$-0.22339852$

0.56140047

0.56140047

0.66723585

0.66723585

0.56140047

0.56140047

0.66723585

0.66723585
0.02794744

0.13378289

0.02794744

0.13378289

0.02794744

0.13378289

0.02794744

0.13378289

$-0.15553895$

$-0.04970350$

$-0.15553895$

$-0.04970350$

$-0.15553895$

$-0.04970350$

$-0.15553895$

$-0.04970350$
$-0.58052284$

0.55082828

0.53653252

$-0.48979729$

0.49033687

$-0.44596645$

$-0.50585645$

0.44025257

0.15151566

$-0.10468544$

$-0.21282728$

0.18314537

$-0.35749885$

0.30234301

0.36874709

$-0.33363408$
\# Magnetic mom.

0.06075061
0.04508484

$-0.06075061$

0.04508484

$-0.06075061$

0.04508484

$-0.06075061$

0.04508484

$-0.04267391$

0.06316154

$-0.04267391$

0.06316154

$-0.04267391$

0.06316154

$-0.04267391$

0.06316154

$-0.13571967$

$-0.02988423$

$-0.13571967$

$-0.02988423$

$-0.13571967$

$-0.02988423$

$-0.13571967$

$-0.02988423$

0.04030320

0.14613864

0.04030320

0.14613864

0.04030320

0.14613864

0.04030320

0.14613864

$-0.03195595$

0.07387950

$-0.03195595$

0.07387950

$-0.03195595$

0.07387950

$-0.03195595$

0.07387950

$-0.09333258$

0.01250287

$-0.09333258$

0.01250287

$-0.09333258$

0.01250287

$-0.09333258$

0.01250287
$-1.79869723$

$-1.29215860$

1.90942752

1.36895740

1.66589153

1.00594473

$-1.76277256$

$-1.05982840$

2.77822661

$-1.62940276$

$-2.69403625$

1. 78911519

$-2.32780385$

1.23556471

2. 27491379

$-1.41604209$

$-0.12361011$

$-1.31405032$

0.13049881

0.93904632

0.15451501

1.15745735

$-0.15323117$

$-0.80604625$

0.94618124

$-0.38486665$

$-1.21134412$

0.47505921

$-0.74246353$

0.21842861

0.93527532

$-0.26729059$

1.19707549

$-0.81637293$

$-1.11810696$

1.00738430

$-0.77652568$

0.86420459

0.74873972

$-1.04162300$

0.65198541

$-1.05145812$

$-0.72589654$

1.19020939

$-0.82008296$

1.02796984

0.92791599

$-1.20515633$ 


\subsection{7 \\ 0.74471217 \\ 0.74471217 \\ 0.74471217 \\ 0.85054761 \\ 0.85054761 \\ 0.85054761 \\ 0.85054761 \\ $-2.46623826$ \\ $-2.46623826$ \\ $-2.46623826$ \\ $-2.46623826$ \\ $-2.36040306$ \\ $-2.36040306$ \\ $-2.36040306$ \\ $-2.36040306$}

64

$0.2016 \quad 0$

0.0052

2.27520919

2.27520919

2. 27520919

2. 27520919

2.38104463

2.38104463

2.38104463

2.38104463

$-1.40967047$

$-1.40967047$

$-1.40967047$

$-1.40967047$

$-1.30383503$

$-1.30383503$

$-1.30383503$

$-1.30383503$

2. 54510117

2.54510117

2. 54510117

2.54510117

2.65093660

2.65093660

2.65093660

2.65093660

$-1.43156683$

$-1.43156683$

$-1.43156683$

$-1.43156683$

$-1.32573140$

$-1.32573140$

$-1.32573140$

$-1.32573140$

0.99076527

0.99076527

0.99076527

0.99076527

1.09660077

1.09660077

1.09660077

1.09660077

$-2.53873253$

$-2.53873253$

$-2.53873253$

$-2.53873253$

$-2.43289709$

$-2.43289709$

$-2.43289709$

$-2.43289709$
$-1.31334066$

$-1.31334066$

$-1.20750511$

$-1.20750511$

$-1.31334066$

$-1.31334066$

$-1.20750511$

$-1.20750511$

1.56936514

1.56936514

1.67520046

1.67520046

1.56936514

1.56936514

1.67520046

1.67520046

\# Energy 3->8

$\begin{array}{lr}0.1150 & 0.0032 \\ 0.0385 & -0.0746\end{array}$

0.11177704

0.11177704

0.21761249

0.21761249

0.11177704

0.11177704

0.21761249

0.21761249

$-0.18411981$

$-0.18411981$

$-0.07828436$

$-0.07828436$

$-0.18411981$

$-0.18411981$

$-0.07828436$

$-0.07828436$

1.31986856

1.31986856

1. 42570400

1.42570400

1.31986856

1. 31986856

1. 42570400

1. 42570400

$-1.42060065$

$-1.42060065$

$-1.31476510$

$-1.31476510$

$-1.42060065$

$-1.42060065$

$-1.31476510$

$-1.31476510$

$-0.32923394$

$-0.32923394$

$-0.22339852$

$-0.22339852$

$-0.32923394$

$-0.32923394$

$-0.22339852$

$-0.22339852$

0.56140047

0.56140047

0.66723585

0.66723585

0.56140047

0.56140047

0.66723585

0.66723585
0.02794744

0.13378289

0.02794744

0.13378289

0.02794744

0.13378289

0.02794744

0.13378289

$-0.15553895$

$-0.04970350$

$-0.15553895$

$-0.04970350$

$-0.15553895$

$-0.04970350$

$-0.15553895$

$-0.04970350$

1

-0.06075061
0.04508484

\# Electr. mom., scale-fac \# Magnetic mom.

$-0.06075061$

0.04508484

$-0.06075061$

0.04508484

$-0.06075061$

0.04508484

$-0.04267391$

0.06316154

$-0.04267391$

0.06316154

$-0.04267391$

0.06316154

$-0.04267391$

0.06316154

$-0.13571967$

$-0.02988423$

$-0.13571967$

$-0.02988423$

$-0.13571967$

$-0.02988423$

$-0.13571967$

$-0.02988423$

0.04030320

0.14613864

0.04030320

0.14613864

0.04030320

0.14613864

0.04030320

0.14613864

$-0.03195595$

0.07387950

$-0.03195595$

0.07387950

$-0.03195595$

0.07387950

$-0.03195595$

0.07387950

$-0.09333258$

0.01250287

$-0.09333258$

0.01250287

$-0.09333258$

0.01250287

$-0.09333258$

0.01250287
0.20484149

$-0.00306443$

$-0.37606114$

$-0.01017189$

$-0.08430153$

$-0.04922506$

0.19298305

0.07833640

0.09481240

$-0.33850724$

$-0.04935159$

0.22618178

$-0.14609873$

0.33661541

0.08783086

$-0.22594078$
1.07815659

$-0.58051336$

$-1.10912919$

0.64261800

$-1.02037275$

0.59643584

1.05201852

$-0.66658455$

0.61989754

1.31711912

$-1.41991711$

$-1.05190313$

$-0.39235348$

$-1.32308888$

1.11336255

1.10197914

0.02756500

0.14052376

$-0.20197345$

0.07138744

0.01507476

$-0.13968185$

0.15456609

$-0.05264066$

0.14702640

$-0.02825443$

$-0.50847346$

0.37018728

$-0.15836310$

0.05873717

0.51584381

1.22349048

$-0.87816930$

$-1.12179267$

1.06625760

$-0.94859713$

0.67460489

0.85801226

$-0.86722392$

$-1.30777133$

0.69418931

1.18271399

$-0.69407642$

1.48589611

$-0.60195023$

$-1.32679737$

0.58483350
$-0.40553719$ 


\begin{tabular}{|c|c|c|c|}
\hline 0.74471217 & -1.31334066 & 0.02794744 & 0.13140175 \\
\hline 0.74471217 & -1.31334066 & 0.13378289 & 0.07595045 \\
\hline 0.74471217 & -1.20750511 & 0.02794744 & -0.18501087 \\
\hline 0.74471217 & -1.20750511 & 0.13378289 & -0.09514760 \\
\hline 0.85054761 & -1.31334066 & 0.02794744 & -0.06109976 \\
\hline 0.85054761 & -1.31334066 & 0.13378289 & -0.13762292 \\
\hline 0.85054761 & -1.20750511 & 0.02794744 & 0.08621697 \\
\hline 0.85054761 & -1.20750511 & 0.13378289 & 0.17896217 \\
\hline-2.46623826 & 1.56936514 & -0.15553895 & -0.35266846 \\
\hline-2.46623826 & 1.56936514 & -0.04970350 & 0.26754010 \\
\hline-2.46623826 & 1.67520046 & -0.15553895 & 0.30036676 \\
\hline-2.46623826 & 1.67520046 & -0.04970350 & -0.24548683 \\
\hline-2.36040306 & 1.56936514 & -0.15553895 & 0.35535172 \\
\hline-2.36040306 & 1.56936514 & -0.04970350 & -0.21058416 \\
\hline-2.36040306 & 1.67520046 & -0.15553895 & -0.30611226 \\
\hline-2.36040306 & 1.67520046 & -0.04970350 & 0.21061058 \\
\hline & \# Energy 3->9 & & \\
\hline-0.0011 & -0.0006 & 1.000 & Electr. mom., scale-fac \\
\hline 0.0249 & -0.0112 & $\#$ & Magnetic mom. \\
\hline 2.27520919 & 0.11177704 & -0.06075061 & -0.05473729 \\
\hline 2.27520919 & 0.11177704 & 0.04508484 & -0.01522021 \\
\hline 2.27520919 & 0.21761249 & -0.06075061 & 0.07084170 \\
\hline 2.27520919 & 0.21761249 & 0.04508484 & -0.02273195 \\
\hline 2.38104463 & 0.11177704 & -0.06075061 & 0.21447825 \\
\hline 2.38104463 & 0.11177704 & 0.04508484 & -0.14350691 \\
\hline 2.38104463 & 0.21761249 & -0.06075061 & -0.22566478 \\
\hline 2.38104463 & 0.21761249 & 0.04508484 & 0.17153993 \\
\hline-1.40967047 & -0.18411981 & -0.04267391 & -9.61777020 \\
\hline-1.40967047 & -0.18411981 & 0.06316154 & 9.84235573 \\
\hline-1.40967047 & -0.07828436 & -0.04267391 & 8.59456444 \\
\hline-1.40967047 & -0.07828436 & 0.06316154 & -8.89664078 \\
\hline-1.30383503 & -0.18411981 & -0.04267391 & 9.91127014 \\
\hline-1.30383503 & -0.18411981 & 0.06316154 & -10.14476395 \\
\hline-1.30383503 & -0.07828436 & -0.04267391 & -8.96642971 \\
\hline-1.30383503 & -0.07828436 & 0.06316154 & 9.26658058 \\
\hline 2.54510117 & 1.31986856 & -0.13571967 & 0.57738262 \\
\hline 2.54510117 & 1.31986856 & -0.02988423 & -0.57051188 \\
\hline 2.54510117 & 1.42570400 & -0.13571967 & -0.67381889 \\
\hline 2.54510117 & 1.42570400 & -0.02988423 & 0.67092723 \\
\hline 2.65093660 & 1.31986856 & -0.13571967 & -0.50960982 \\
\hline 2.65093660 & 1.31986856 & -0.02988423 & 0.50946343 \\
\hline 2.65093660 & 1.42570400 & -0.13571967 & 0.60756928 \\
\hline 2.65093660 & 1.42570400 & -0.02988423 & -0.60995024 \\
\hline-1.43156683 & -1.42060065 & 0.04030320 & 0.15887538 \\
\hline-1.43156683 & -1.42060065 & 0.14613864 & -0.07227819 \\
\hline-1.43156683 & -1.31476510 & 0.04030320 & -1.63572407 \\
\hline-1.43156683 & -1.31476510 & 0.14613864 & 1.50223243 \\
\hline-1.32573140 & -1.42060065 & 0.04030320 & -0.19782259 \\
\hline-1.32573140 & -1.42060065 & 0.14613864 & 0.09791911 \\
\hline-1.32573140 & -1.31476510 & 0.04030320 & 1.67940807 \\
\hline-1.32573140 & -1.31476510 & 0.14613864 & -1.52899277 \\
\hline 0.99076527 & -0.32923394 & -0.03195595 & 2.59064531 \\
\hline 0.99076527 & -0.32923394 & 0.07387950 & -2.56708407 \\
\hline 0.99076527 & -0.22339852 & -0.03195595 & -2.54496717 \\
\hline 0.99076527 & -0.22339852 & 0.07387950 & 2.47884440 \\
\hline 1.09660077 & -0.32923394 & -0.03195595 & -2.23204446 \\
\hline 1.09660077 & -0.32923394 & 0.07387950 & 2.19120002 \\
\hline 1.09660077 & -0.22339852 & -0.03195595 & 2.19950938 \\
\hline 1.09660077 & -0.22339852 & 0.07387950 & -2.11678123 \\
\hline-2.53873253 & 0.56140047 & -0.09333258 & -3.20266175 \\
\hline-2.53873253 & 0.56140047 & 0.01250287 & 2.99493051 \\
\hline-2.53873253 & 0.66723585 & -0.09333258 & 3.04036856 \\
\hline-2.53873253 & 0.66723585 & 0.01250287 & -2.85463834 \\
\hline-2.43289709 & 0.56140047 & -0.09333258 & 3.53485107 \\
\hline-2.43289709 & 0.56140047 & 0.01250287 & -3.24108529 \\
\hline-2.43289709 & 0.667235 & 9333258 & -3.33068538 \\
\hline-2.43289709 & 0.66723585 & 0.01250287 & 3.06342912 \\
\hline
\end{tabular}




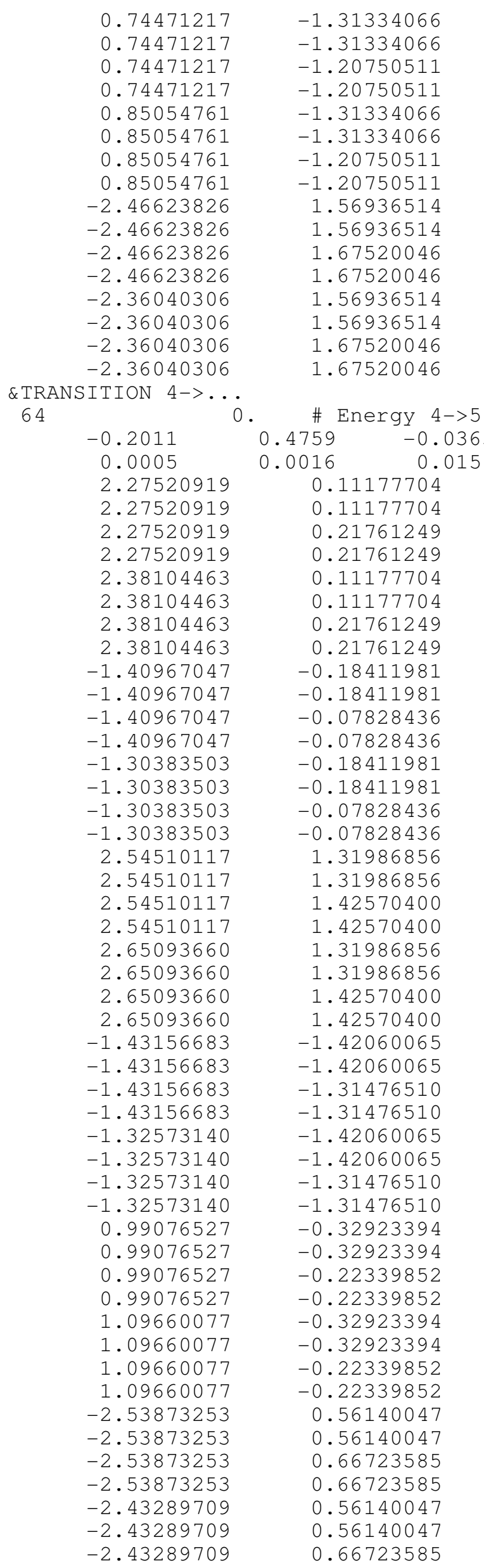

0.74471217

7447121

0.7447121

0.85054761

0.85054761

$-2.46623826$

$-2.36040306$

$-2.36040306$

$-2.36040306$

2.27520919

2.27520919

2.38104463

-1.409670

$-1.40967047$

$-1.30383503$

2. 54510117

2. 54510117

2.65093660

$-1.43156683$

$-1.43156683$

$-1.32573140$

$-1.32573140$

0.99076527

0.99076527

.99076527

$-2.53873253$

$-2.43289709$
$-0.2011$

1.09660077

$$
\begin{array}{r}
0.02794744 \\
0.13378289 \\
0.02794744 \\
0.13378289 \\
0.02794744 \\
0.13378289 \\
0.02794744 \\
0.13378289 \\
-0.15553895 \\
-0.04970350 \\
-0.15553895 \\
-0.04970350 \\
-0.15553895 \\
-0.04970350 \\
-0.15553895 \\
-0.04970350
\end{array}
$$

0.31790376

$-0.44213566$

0.43199682

$-0.24422131$

0.23210108

0.33902109

$-0.32141471$

$-0.61255056$

0.59217948

0.47397581

$-0.46167755$

0.65128559

$-0.61360389$

$-0.49945650$

0.47501773
$-0.31148607$
\# Energy 4->5

$$
0.4759 \quad-0.0363
$$

0.11177704

0.11177704

0.11177704

0.11177704

0.21761249

0.21761249

$-0.07828436$

$-0.07828436$

$-0.18411981$

$-0.07828436$

1.31986856

1.31986856

1. 42570400

1.42570400

1.42570400

1. 42570400

$-1.42060065$

$-1.31476510$

$-1.31476510$

$-1.42060065$

$-1.31476510$

$-0.32923394$

$-0.32923394$

$-0.22339852$

0.56140047

0.56140047

0.66723585

0.56140047

0.66723585
0.21761249
1.000 \# Electr. mom., scale-fac \# Magnetic mom.

$\begin{array}{ll}-0.06075061 & -1.93619084\end{array}$

$0.04508484 \quad 3.83992076$

$-0.06075061 \quad 2.11936069$

$0.04508484 \quad-3.75807095$

$\begin{array}{rr}-0.06075061 & 2.33527684 \\ 0.04508484 & -3.03430510\end{array}$

$0.04508484 \quad-3.03430510$

$-0.06075061 \quad-2.58865333$

$0.04508484 \quad 3.16884708$

$\begin{array}{rr}-0.04267391 & 4.19604588\end{array}$

$0.06316154-4.70579386$

$\begin{array}{ll}-0.04267391 & -3.86488318\end{array}$

$0.06316154 \quad 4.81063032$

$-0.04267391$

0.06316154

$-0.04267391$

0.06316154

$-0.13571967$

$-0.02988423$

$-0.13571967$

$-0.02988423$

$-0.13571967$

$-0.02988423$

$-0.13571967$

$-0.02988423$

0.04030320

0.14613864

0.04030320

0.14613864

0.04030320

0.14613864

0.04030320

0.14613864

$-0.03195595$

0.07387950

$-0.03195595$

0.07387950

$-0.03195595$

0.07387950

$-0.03195595$

0.07387950

$-0.09333258$

0.01250287

$-0.09333258$

0.01250287

$-0.09333258$

0.01250287

$-0.09333258$

$-3.76836610$

4.63371515

3.37429357

$-4.80538654$

$-0.85761356$

1.24154568

0.75089073

$-1.03681791$

0.92459309

$-1.37391317$

$-0.77477860$

1.15519309

1.41174173

$-0.96044362$

$-1.82078695$

1.20145202

$-1.37966812$

0.80056745

1. 75645864

$-1.02838886$

2.62085629

1.50382710

$-2.29967308$

$-0.81506759$

$-2.82464504$

$-1.82369041$

2. 42486072

1.01096094

0.49790591

3.81555295

$-0.86908764$

$-3.80533814$

$-0.64218372$

$-3.89744139$

1.11903477 


-2.43289709
0.74471217
0.74471217
0.74471217
0.74471217
0.85054761
0.85054761
0.85054761
0.85054761
-2.46623826
-2.46623826
-2.46623826
-2.46623826
-2.36040306
-2.36040306
-2.36040306
-2.36040306

64
0.66723585

$-1.31334066$

$-1.31334066$

$-1.20750511$

$-1.20750511$

$-1.31334066$

$-1.31334066$

$-1.20750511$

$-1.20750511$

1.56936514

1.56936514

1.67520046

1.67520046

1.56936514

1.56936514

1.67520046

1.67520046

0. \# Energy 4->6

$\begin{array}{lll}0.0755 & -0.1316 & 0.0044\end{array}$

$-0.0010$

2.27520919

2. 27520919

2. 27520919

2. 27520919

2.38104463

2.38104463

2.38104463

2.38104463

$-1.40967047$

$-1.40967047$

$-1.40967047$

$-1.40967047$

$-1.30383503$

$-1.30383503$

$-1.30383503$

$-1.30383503$

2. 54510117

2. 54510117

2.54510117

2.54510117

2.65093660

2.65093660

2.65093660

2.65093660

$-1.43156683$

$-1.43156683$

$-1.43156683$

$-1.43156683$

$-1.32573140$

$-1.32573140$

$-1.32573140$

$-1.32573140$

0.99076527

0.99076527

0.99076527

0.99076527

1.09660077

1.09660077

1.09660077

1.09660077

$-2.53873253$

$-2.53873253$

$-2.53873253$

$-2.53873253$

$-2.43289709$

$-2.43289709$

$-2.43289709$
0.01250287

0.02794744

0.13378289

0.02794744

0.13378289

0.02794744

0.13378289

0.02794744

0.13378289

$-0.15553895$

$-0.04970350$

$-0.15553895$

$-0.04970350$

$-0.15553895$

$-0.04970350$

$-0.15553895$

$-0.04970350$

1.000 \# Electr. mom., scale-fac

$-0.06075061$

0.04508484

$-0.06075061$

0.04508484

$-0.06075061$

0.04508484

$-0.06075061$

0.04508484

$-0.04267391$

0.06316154

$-0.04267391$

0.06316154

$-0.04267391$

0.06316154

$-0.04267391$

0.06316154

$-0.13571967$

$-0.02988423$

$-0.13571967$

$-0.02988423$

$-0.13571967$

$-0.02988423$

$-0.13571967$

$-0.02988423$

0.04030320

0.14613864

0.04030320

0.14613864

0.04030320

0.14613864

0.04030320

0.14613864

$-0.03195595$

0.07387950

$-0.03195595$

0.07387950

$-0.03195595$

0.07387950

$-0.03195595$

0.07387950

$-0.09333258$

0.01250287

$-0.09333258$

0.01250287

$-0.09333258$

0.01250287

$-0.09333258$

3.97065783

0.47020450

0.17371175

$-0.77806270$

$-0.19603021$

$-0.55299419$

$-0.16928840$

0.89141440

0.19542752

0.00273054

0.76107168

$-0.04541884$

$-0.48131517$

$-0.08878330$

$-0.80572557$

0.11249029

0.49756658 Magnetic mom.

$-2.17569709$

$-1.12624085$

2. 10203171

1. 56951714

1.66915321

1.10550523

$-1.55248463$

$-1.51439285$

$-2.16524839$

1. 20096684

1.77976131

$-0.92533320$

2. 61501336

$-1.05586135$

$-2.21957755$

0.86723185

0.27307329

$-1.85561717$

$-0.24821389$

1.44618630

$-0.44263399$

1. 69966841

0.39030236

$-1.36118150$

$-0.81088138$

0.97377616

1. 01463592

$-1.19385529$

0.80836886

$-1.02855432$

$-0.99618137$

1. 25068831

$-2.59579015$

$-0.40988815$

2. 42095470

0.70273203

2. 60352111

0.41295218

$-2.48042178$

$-0.80080324$

0.62896788

0.73847055

$-0.88300705$

$-0.51941705$

$-0.54198575$

$-1.14235187$

0.83988136 


-2.43289709
0.74471217
0.74471217
0.74471217
0.74471217
0.85054761
0.85054761
0.85054761
0.85054761
-2.46623826
-2.46623826
-2.46623826
-2.46623826
-2.36040306
-2.36040306
-2.36040306
-2.36040306

64
0.66723585

$-1.31334066$

$-1.31334066$

$-1.20750511$

$-1.20750511$

$-1.31334066$

$-1.31334066$

$-1.20750511$

$-1.20750511$

1.56936514

1.56936514

1.67520046

1.67520046

1.56936514

1. 56936514

1.67520046

1.67520046

0 . \# Energy 4->7
0.01250287

0.02794744

0.13378289

0.02794744

0.13378289

0.02794744

0.13378289

0.02794744

0.13378289

$-0.15553895$

$-0.04970350$

$-0.15553895$

$-0.04970350$

$-0.15553895$

$-0.04970350$

$-0.15553895$

$-0.04970350$

$$
\begin{array}{r}
0.92754358 \\
-0.41234705 \\
0.36615837 \\
0.56511575 \\
-0.50451005 \\
0.38113230 \\
-0.40154356 \\
-0.51478976 \\
0.54870546 \\
0.46464276 \\
-0.21500786 \\
-0.36340213 \\
0.17884609 \\
-0.42162490 \\
0.09986459 \\
0.32972002 \\
-0.09624370
\end{array}
$$
$-0.0028$
0.0046
$-0.0200$
2. 27520919
0.0185
$-0.0039$
2.27520919
2. 27520919
2. 27520919
2. 38104463
2. 38104463
2.38104463
2. 38104463
$-1.40967047$
$-1.40967047$
$-1.40967047$
$-1.40967047$
$-1.30383503$
$-1.30383503$
$-1.30383503$
$-1.30383503$
2. 54510117
2. 54510117
2. 54510117
2. 54510117
2.65093660
2.65093660
2.65093660
2. 65093660
$-1.43156683$
$-1.43156683$
$-1.43156683$
$-1.43156683$
$-1.32573140$
$-1.32573140$
$-1.32573140$
$-1.32573140$
0.99076527
0.99076527
0.99076527
0.99076527
1. 09660077
1. 09660077
1.09660077
1.09660077
$-2.53873253$
$-2.53873253$
$-2.53873253$
$-2.53873253$
$-2.43289709$
$-2.43289709$
$-2.43289709$
0.11177704
1.000
$-0.06075061$
0.04508484
$-0.06075061$
0.04508484
$-0.06075061$
0.04508484
$-0.06075061$
0.04508484
$-0.04267391$
0.06316154
$-0.04267391$
0.06316154
$-0.04267391$
0.06316154
$-0.04267391$
0.06316154
$-0.13571967$
$-0.02988423$
$-0.13571967$
$-0.02988423$
$-0.13571967$
$-0.02988423$
$-0.13571967$
$-0.02988423$
0.04030320
0.14613864
0.04030320
0.14613864
0.04030320
0.14613864
0.04030320
0.14613864
$-0.03195595$
0.07387950
$-0.03195595$
0.07387950
$-0.03195595$
0.07387950
$-0.03195595$
0.07387950
$-0.09333258$
0.01250287
$-0.09333258$
0.01250287
$-0.09333258$
0.01250287
$-0.09333258$ Magnetic mom.
1.83372474
$-1.56081581$
$-1.73415470$
1. 48114407
$-1.97443140$
1. 71465099
1.87290514
$-1.62762320$
$-1.04038978$
0.96419048
1.16753531
$-1.06611216$
0.91649425
$-0.84454173$
$-1.04216290$
0.94686973
$-0.54830348$
0.60158122
1. 49142003
$-1.53931737$
0.23268931
$-0.29090619$
$-1.17703509$
1. 22849703
0.10344915
$-0.09017809$
$-0.22139853$
0.20306458
$-0.11425810$
0.09880529
0.24291094
$-0.22177435$
$-1.07723570$
1. 03202260
1. 04178286
$-0.95681751$
0.90439135
$-0.88642853$
$-0.86641294$
0.80131537
0.91089714
$-0.87056518$
$-0.76715630$
0.73176736
$-0.97655499$
0.92485702
0.79457653

Electr. mom., scale-fac 


-2.43289709
0.74471217
0.74471217
0.74471217
0.74471217
0.85054761
0.85054761
0.85054761
0.85054761
-2.46623826
-2.46623826
-2.46623826
-2.46623826
-2.36040306
-2.36040306
-2.36040306
-2.36040306

64
0.66723585

$-1.31334066$

$-1.31334066$

$-1.20750511$

$-1.20750511$

$-1.31334066$

$-1.31334066$

$-1.20750511$

$-1.20750511$

1.56936514

1.56936514

1.67520046

1.67520046

1.56936514

1. 56936514

1.67520046

1.67520046

0 . \# Energy 4->8

$\begin{array}{lll}-0.8354 & -0.2215 & 0.0260\end{array}$

$-0.0111$

2. 27520919

2.27520919

2. 27520919

2. 27520919

2. 38104463

2. 38104463

2.38104463

2. 38104463

$-1.40967047$

$-1.40967047$

$-1.40967047$

$-1.40967047$

$-1.30383503$

$-1.30383503$

$-1.30383503$

$-1.30383503$

2. 54510117

2.54510117

2. 54510117

2. 54510117

2.65093660

2.65093660

2.65093660

2.65093660

$-1.43156683$

$-1.43156683$

$-1.43156683$

$-1.43156683$

$-1.32573140$

$-1.32573140$

$-1.32573140$

$-1.32573140$

0.99076527

0.99076527

0.99076527

0.99076527

1. 09660077

1.09660077

1.09660077

1.09660077

$-2.53873253$

$-2.53873253$

$-2.53873253$

$-2.53873253$

$-2.43289709$

$-2.43289709$

$-2.43289709$

$\begin{array}{rr}0.01250287 & -0.74933684 \\ 0.02794744 & -0.09354643 \\ 0.13378289 & 0.09707699 \\ 0.02794744 & 0.18590051 \\ 0.13378289 & -0.19609746 \\ 0.02794744 & 0.04518769 \\ 0.13378289 & -0.05634285 \\ 0.02794744 & -0.11427640 \\ 0.13378289 & 0.13490260 \\ -0.15553895 & 0.10903569 \\ -0.04970350 & -0.09959896 \\ -0.15553895 & -0.07606132 \\ -0.04970350 & 0.06910491 \\ -0.15553895 & -0.07379234 \\ -0.04970350 & 0.06307655 \\ -0.15553895 & 0.05287193 \\ -0.04970350 & -0.04507277\end{array}$

1.000 \# Electr. mom., scale-fac \# Magnetic mom.

$-0.06075061-3.13981009$

$0.04508484 \quad 2.46696043$

$\begin{array}{ll}-0.06075061 & 3.35747027\end{array}$

$0.04508484 \quad-2.51261759$

$\begin{array}{ll}-0.06075061 & 3.40190506\end{array}$

$0.04508484 \quad-3.01212311$

$-0.06075061 \quad-3.61209416$

$0.04508484 \quad 3.13768578$

$-0.04267391-1.75087643$

$0.06316154 \quad-0.33504725$

$\begin{array}{ll}-0.04267391 & 1.90505934\end{array}$

$0.06316154 \quad 0.43182334$

$\begin{array}{ll}-0.04267391 & 1.60005248\end{array}$

$0.06316154 \quad 0.20764005$

$-0.04267391-1.76076066$

$0.06316154 \quad-0.30885136$

$-0.13571967 \quad-0.19477791$

$-0.02988423 \quad 0.24945556$

$-0.13571967 \quad-0.07595847$

$\begin{array}{ll}-0.02988423 & 0.00543160\end{array}$

$\begin{array}{ll}-0.13571967 & 0.37483925\end{array}$

$-0.02988423 \quad-0.42594877$

$-0.13571967$

$-0.02988423$

0.04030320

0.14613864

0.04030320

0.14613864

0.04030320

0.14613864

0.04030320

0.14613864

$-0.03195595$

0.07387950

$-0.03195595$

0.07387950

$-0.03195595$

0.07387950

$-0.03195595$

0.07387950

$-0.09333258$

0.01250287

$-0.09333258$

0.01250287

$-0.09333258$

0.01250287

$-0.09333258$

$-0.06517382$

0.12028315

$-0.46539220$

0.23850530

0.51212198

$-0.20629822$

0.44910249

$-0.21805827$

$-0.46256813$

0.16179578

$-0.68502498$

$-0.49454355$

1. 09178722

0.60986644

0.84958303

0.55631912

$-1.30876637$

$-0.67882019$

$-0.32225457$

1. 45131767

0.28340366

$-1.39892697$

0.51661295

$-1.64315403$

$-0.49455172$ 


-2.43289709
0.74471217
0.74471217
0.74471217
0.74471217
0.85054761
0.85054761
0.85054761
0.85054761
-2.46623826
-2.46623826
-2.46623826
-2.46623826
-2.36040306
-2.36040306
-2.36040306
-2.36040306

64
0.66723585

$-1.31334066$

$-1.31334066$

$-1.20750511$

$-1.20750511$

$-1.31334066$

$-1.31334066$

$-1.20750511$

$-1.20750511$

1.56936514

1.56936514

1.67520046

1.67520046

1.56936514

1. 56936514

1.67520046

1.67520046

0 . \# Energy 4->9

$\begin{array}{lll}-0.4574 & 0.2547 & -0.0155\end{array}$

$-0.0005$

2. 27520919

2. 27520919

2. 27520919

2.27520919

2. 38104463

2.38104463

2.38104463

2. 38104463

$-1.40967047$

$-1.40967047$

$-1.40967047$

$-1.40967047$

$-1.30383503$

$-1.30383503$

$-1.30383503$

$-1.30383503$

2.54510117

2. 54510117

2.54510117

2. 54510117

2.65093660

2.65093660

2.65093660

2.65093660

$-1.43156683$

$-1.43156683$

$-1.43156683$

$-1.43156683$

$-1.32573140$

$-1.32573140$

$-1.32573140$

$-1.32573140$

0.99076527

0.99076527

0.99076527

0.99076527

1. 09660077

1. 09660077

1.09660077

1. 09660077

$-2.53873253$

$-2.53873253$

$-2.53873253$

$-2.53873253$

$-2.43289709$

$-2.43289709$

$-2.43289709$
0.01250287

0.02794744

0.13378289

0.02794744

0.13378289

0.02794744

0.13378289

0.02794744

0.13378289

$-0.15553895$

$-0.04970350$

$-0.15553895$

$-0.04970350$

$-0.15553895$

$-0.04970350$

$-0.15553895$

$-0.04970350$

1.000 \# Electr. mom., scale-fac
\# Magnetic mom.

$-0.06075061$

0.04508484

$-0.06075061$

0.04508484

$-0.06075061$

0.04508484

$-0.06075061$

0.04508484

$-0.04267391$

0.06316154

$-0.04267391$

0.06316154

$-0.04267391$

0.06316154

$-0.04267391$

0.06316154

$-0.13571967$

$-0.02988423$

$-0.13571967$

$-0.02988423$

$-0.13571967$

$-0.02988423$

$-0.13571967$

$-0.02988423$

0.04030320

0.14613864

0.04030320

0.14613864

0.04030320

0.14613864

0.04030320

0.14613864

$-0.03195595$

0.07387950

$-0.03195595$

0.07387950

$-0.03195595$

0.07387950

$-0.03195595$

0.07387950

$-0.09333258$

0.01250287

$-0.09333258$

0.01250287

$-0.09333258$

0.01250287

$-0.09333258$
1. 59451342

0.04977009

$-0.08040554$

0.06830334

$-0.03988791$

$-0.04524969$

0.08032504

0.00661022

$-0.05196261$

0.36385420

0.02165079

$-0.24384181$

0.12107686

$-0.43127635$

$-0.07180314$

0.29264581
$-0.04094598$

$$
\begin{array}{r}
-0.19085769 \\
-0.69254488 \\
0.21714170 \\
0.75825894 \\
-0.01656942 \\
0.83965921 \\
0.00564497 \\
-0.94438380 \\
4.18099165 \\
-6.15194702 \\
-3.84068298 \\
6.01459885 \\
-4.70082378 \\
5.51227379 \\
4.32653189 \\
-5.46234369 \\
0.00336288 \\
-0.56973064 \\
-0.01630481 \\
0.46480587 \\
-0.09444942 \\
0.57967299 \\
0.09304771 \\
-0.47175664 \\
1.14899552 \\
-1.34890640 \\
-1.37742782 \\
1.68787265 \\
-1.10343540 \\
1.31373966 \\
1.35625637 \\
-1.63021481 \\
-1.96936703 \\
-0.00998406 \\
2.03601146 \\
0.01860347 \\
2.27058077 \\
-0.11966943 \\
-2.29471016 \\
0.14216074 \\
-1.195437135 \\
4.85858822 \\
0.91450232 \\
-4.82528830 \\
-09253228
\end{array}
$$

\section{S-28}




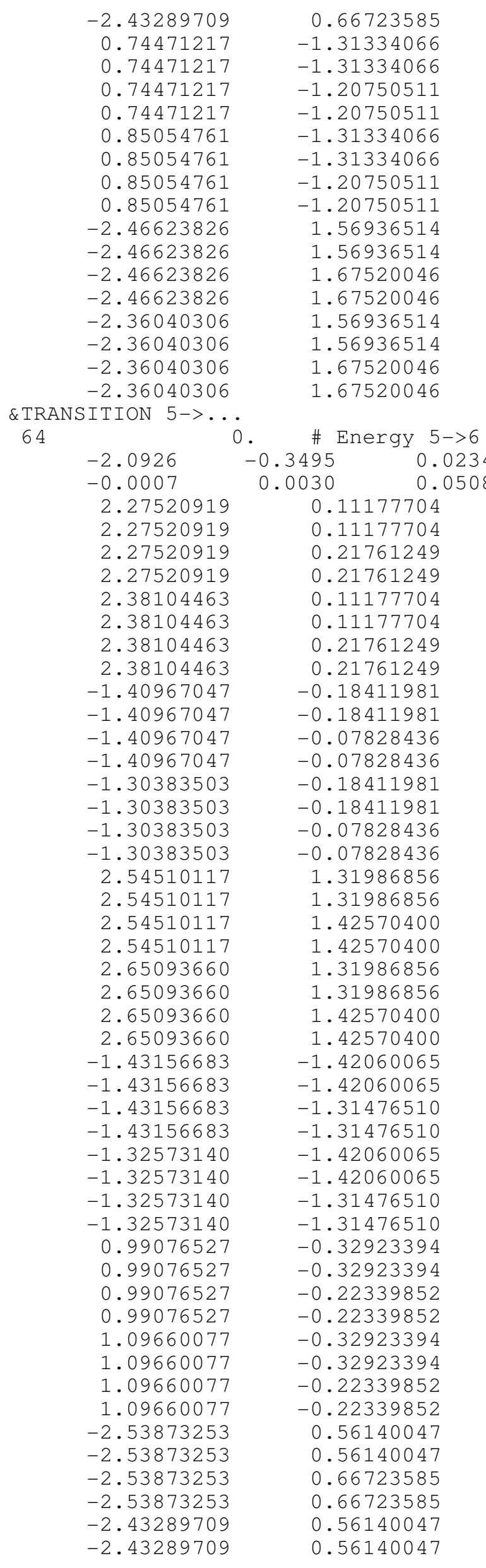

TRANSITION 5->...

0.01250287
0.02794744
0.13378289
0.02794744
0.13378289
0.02794744
0.13378289
0.02794744
0.13378289
-0.15553895
-0.04970350
-0.15553895
-0.04970350
-0.15553895
-0.04970350
-0.15553895
-0.04970350

4.63816357

$-0.77290893$

0.26102272

1.09603715

$-0.36280265$

0.81279492

$-0.28647244$

$-1.15535700$

0.39693877

$-0.38903168$

1.62577796

0.27298516

$-1.15951097$

0.43587360

$-1.65718997$

$-0.31487983$

1.19403386 


-2.43289709
-2.43289709
0.74471217
0.74471217
0.74471217
0.74471217
0.85054761
0.85054761
0.85054761
0.85054761
-2.46623826
-2.46623826
-2.46623826
-2.46623826
-2.36040306
-2.36040306
-2.36040306
-2.36040306

64
0.66723585
0.66723585
-1.31334066
-1.31334066
-1.20750511
-1.20750511
-1.31334066
-1.31334066
-1.20750511
-1.20750511
1.56936514
1.56936514
1.67520046
1.67520046
1.56936514
1.56936514
1.67520046
1.67520046
$\#$ Energy $5->7$
$020 \quad-0.0035$
071

$\begin{array}{lll}-0.0211 & -0.0020 & -0.0035\end{array}$

0.0324

2.27520919

2.27520919

2.27520919

2.27520919

2.38104463

2.38104463

2.38104463

2.38104463

$-1.40967047$

$-1.40967047$

$-1.40967047$

$-1.40967047$

$-1.30383503$

$-1.30383503$

$-1.30383503$

$-1.30383503$

2.54510117

2.54510117

2.54510117

2. 54510117

2.65093660

2.65093660

2.65093660

2.65093660

$-1.43156683$

$-1.43156683$

$-1.43156683$

$-1.43156683$

$-1.32573140$

$-1.32573140$

$-1.32573140$

$-1.32573140$

0.99076527

0.99076527

0.99076527

0.99076527

1.09660077

1.09660077

1.09660077

1.09660077

$-2.53873253$

$-2.53873253$

$-2.53873253$

$-2.53873253$

$-2.43289709$

$-2.43289709$

0.11177704

0.11177704

0.21761249

0.21761249

0.11177704

0.11177704

0.21761249

0.21761249

$-0.18411981$

$-0.18411981$

$-0.07828436$

$-0.07828436$

$-0.18411981$

$-0.18411981$

$-0.07828436$

$-0.07828436$

1.31986856

1.31986856

1. 42570400

1. 42570400

1.31986856

1.31986856

1. 42570400

1. 42570400

$-1.42060065$

$-1.42060065$

$-1.31476510$

$-1.31476510$

$-1.42060065$

$-1.42060065$

$-1.31476510$

$-1.31476510$

$-0.32923394$

$-0.32923394$

$-0.22339852$

$-0.22339852$

$-0.32923394$

$-0.32923394$

$-0.22339852$

$-0.22339852$

0.56140047

0.56140047

0.66723585

0.66723585

0.56140047

0.56140047

-0.09333258
0.01250287
0.02794744
0.13378289
0.02794744
0.13378289
0.02794744
0.13378289
0.02794744
0.13378289
-0.15553895
-0.04970350
-0.15553895
-0.04970350
-0.15553895
-0.04970350
-0.15553895
-0.04970350

$-0.05005179$

1.29310417

0.48278907

$-0.24879147$

$-0.73287463$

0.37208655

$-0.58844894$

0.33184001

0.87604809

$-0.48228541$

0.01457863

0.11296988

$-0.05456574$

$-0.02182908$

$-0.00695383$

$-0.17691521$

0.05585313

0.05597752

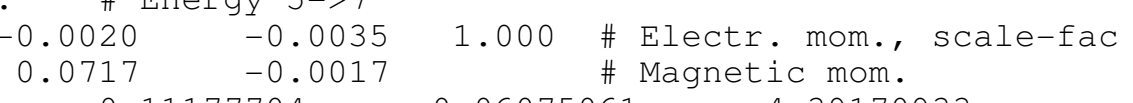
1.000 \# Electr. mom., scale-fac \# Magnetic mom.

$-0.06075061$

0.04508484

$-0.06075061$

0.04508484

$-0.06075061$

0.04508484

$-0.06075061$

0.04508484

$-0.04267391$

0.06316154

$-0.04267391$

0.06316154

$-0.04267391$

0.06316154

$-0.04267391$

0.06316154

$-0.13571967$

$-0.02988423$

$-0.13571967$

$-0.02988423$

$-0.13571967$

$-0.02988423$

$-0.13571967$

$-0.02988423$

0.04030320

0.14613864

0.04030320

0.14613864

0.04030320

0.14613864

0.04030320

0.14613864

$-0.03195595$

0.07387950

$-0.03195595$

0.07387950

$-0.03195595$

0.07387950

$-0.03195595$

0.07387950

$-0.09333258$

0.01250287

$-0.09333258$

0.01250287

$-0.09333258$

0.01250287
4.39170933

$-4.06051111$

$-4.38794708$

4.01939344

$-4.04485226$

3.77242756

4.06901169

$-3.76612520$

$-1.41218424$

1. 46772993

1.12023675

$-1.16895401$

1.52551913

$-1.59899402$

$-1.17918897$

1.24392033

1.66273105

$-1.53030908$

$-1.38354349$

1.28651130

$-1.69573653$

1.58615768

1.38235295

$-1.30309713$

$-0.65771145$

0.67134356

0.44020900

$-0.46274915$

0.66428405

$-0.68474752$

$-0.45517421$

0.48626766

3.28427029

$-3.10378337$

$-2.91959691$

2.72618937

$-3.15855789$

2.91416264

2. 78190517

$-2.52779984$

0.02724761

$-0.03485684$

0.02700158

$-0.02054774$

0.06462937

$-0.04808721$ 


-2.43289709
-2.43289709
0.74471217
0.74471217
0.74471217
0.74471217
0.85054761
0.85054761
0.85054761
0.85054761
-2.46623826
-2.46623826
-2.46623826
-2.46623826
-2.36040306
-2.36040306
-2.36040306
-2.36040306

64
0.66723585

0.66723585

$-1.31334066$

$-1.31334066$

$-1.20750511$

$-1.20750511$

$-1.31334066$

$-1.31334066$

$-1.20750511$

$-1.20750511$

1.56936514

1.56936514

1.67520046

1.67520046

1.56936514

1.56936514

1.67520046

1.67520046

0 . \# Energy 5->8

$0.1197 \quad 0.1504 \quad-0.0335$

$-0.0277$

2.27520919

2.27520919

2.27520919

2. 27520919

2.38104463

2.38104463

2. 38104463

2.38104463

$-1.40967047$

$-1.40967047$

$-1.40967047$

$-1.40967047$

$-1.30383503$

$-1.30383503$

$-1.30383503$

$-1.30383503$

2. 54510117

2. 54510117

2.54510117

2. 54510117

2.65093660

2.65093660

2.65093660

2.65093660

$-1.43156683$

$-1.43156683$

$-1.43156683$

$-1.43156683$

$-1.32573140$

$-1.32573140$

$-1.32573140$

$-1.32573140$

0.99076527

0.99076527

0.99076527

0.99076527

1.09660077

1.09660077

1.09660077

1.09660077

$-2.53873253$

$-2.53873253$

$-2.53873253$

$-2.53873253$

$-2.43289709$

$-2.43289709$

$$
-0.0125 \quad-0.0047
$$

0.11177704

0.11177704

0.21761249

0.21761249

0.11177704

0.11177704

0.21761249

0.21761249

$-0.18411981$

$-0.18411981$

$-0.07828436$

$-0.07828436$

$-0.18411981$

$-0.18411981$

$-0.07828436$

$-0.07828436$

1.31986856

1.31986856

1. 42570400

1. 42570400

1.31986856

1.31986856

1. 42570400

1. 42570400

$-1.42060065$

$-1.42060065$

$-1.31476510$

$-1.31476510$

$-1.42060065$

$-1.42060065$

$-1.31476510$

$-1.31476510$

$-0.32923394$

$-0.32923394$

$-0.22339852$

$-0.22339852$

$-0.32923394$

$-0.32923394$

$-0.22339852$

$-0.22339852$

0.56140047

0.56140047

0.66723585

0.66723585

0.56140047

0.56140047

$$
\begin{array}{r}
-0.09333258 \\
0.01250287 \\
0.02794744 \\
0.13378289 \\
0.02794744 \\
0.13378289 \\
0.02794744 \\
0.13378289 \\
0.02794744 \\
0.13378289 \\
-0.15553895 \\
-0.04970350 \\
-0.15553895 \\
-0.04970350 \\
-0.15553895 \\
-0.04970350 \\
-0.15553895 \\
-0.04970350
\end{array}
$$

1.000 \# Electr. mom., scale-fac \# Magnetic mom.

$-0.06075061$

0.04508484

$-0.06075061$

0.04508484

$-0.06075061$

0.04508484

$-0.06075061$

0.04508484

$-0.04267391$

0.06316154

$-0.04267391$

0.06316154

$-0.04267391$

0.06316154

$-0.04267391$

0.06316154

$-0.13571967$

$-0.02988423$

$-0.13571967$

$-0.02988423$

$-0.13571967$

$-0.02988423$

$-0.13571967$

$-0.02988423$

0.04030320

0.14613864

0.04030320

0.14613864

0.04030320

0.14613864

0.04030320

0.14613864

$-0.03195595$

0.07387950

$-0.03195595$

0.07387950

$-0.03195595$

0.07387950

$-0.03195595$

0.07387950

$-0.09333258$

0.01250287

$-0.09333258$

0.01250287

$-0.09333258$

0.01250287
4.67749834

$-0.13481151$

0.11931700

0.28974098

$-0.26189795$

$-0.44192263$

0.40042269

$-0.23006862$

0.19025420

0.36360240

$-0.30542216$

$-0.04574530$

0.05191250

0.01707435

$-0.02173857$

0.08702138

$-0.09070923$

$-0.04532433$

0.04813898

$-4.17698193$

$-5.22523403$

4.33303642

$-4.66664839$

4.75343847

5.09384584

$-5.02000141$

1.01111627

2. 57914209

$-1.79050183$

$-2.17513418$

$-0.68299019$

$-2.95064902$

1.47356915

2. 49263334

1.04114830

$-1.72345328$

$-1.11453283$

1. 72098815

$-1.11413181$

1. 70918751

1.16539431

$-1.67131579$

$-0.21525265$

0.66008025

0.21971214

$-0.79714829$

0.34603012

$-0.80128711$

$-0.34538019$

0.93900090

2.57535577

$-1.04168701$

$-3.65965509$

0.83913684

$-3.10151649$

1.13723159

4.22067213

$-0.77544898$

0.49687266

$-0.62537229$

$-0.72980750$

0.69219708

$-0.33918169$

0.60942233 


-2.43289709
-2.43289709
0.74471217
0.74471217
0.74471217
0.74471217
0.85054761
0.85054761
0.85054761
0.85054761
-2.46623826
-2.46623826
-2.46623826
-2.46623826
-2.36040306
-2.36040306
-2.36040306
-2.36040306

64

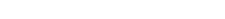

-0.5350
-0.0014

2. 27520919

2.27520919

2.27520919

2.27520919

2.38104463

2. 38104463

2. 38104463

2.38104463

$-1.40967047$

$-1.40967047$

$-1.40967047$

$-1.40967047$

$-1.30383503$

$-1.30383503$

$-1.30383503$

$-1.30383503$

2. 54510117

2. 54510117

2.54510117

2. 54510117

2.65093660

2.65093660

2.65093660

2.65093660

$-1.43156683$

$-1.43156683$

$-1.43156683$

$-1.43156683$

$-1.32573140$

$-1.32573140$

$-1.32573140$

$-1.32573140$

0.99076527

0.99076527

0.99076527

0.99076527

1.09660077

1.09660077

1.09660077

1.09660077

$-2.53873253$

$-2.53873253$

$-2.53873253$

$-2.53873253$

$-2.43289709$

$-2.43289709$
0.66723585

0.66723585

$-1.31334066$

$-1.31334066$

$-1.20750511$

$-1.20750511$

$-1.31334066$

$-1.31334066$

$-1.20750511$

$-1.20750511$

1.56936514

1.56936514

1.67520046

1.67520046

1.56936514

1.56936514

1.67520046

1.67520046

0. \# Energy 5->9

$0.2447 \quad-0.0164$

$0.0005 \quad 0.0295$

0.11177704

0.11177704

0.21761249

0.21761249

0.11177704

0.11177704

0.21761249

0.21761249

$-0.18411981$

$-0.18411981$

$-0.07828436$

$-0.07828436$

$-0.18411981$

$-0.18411981$

$-0.07828436$

$-0.07828436$

1.31986856

1.31986856

1.42570400

1. 42570400

1.31986856

1.31986856

1. 42570400

1. 42570400

$-1.42060065$

$-1.42060065$

$-1.31476510$

$-1.31476510$

$-1.42060065$

$-1.42060065$

$-1.31476510$

$-1.31476510$

$-0.32923394$

$-0.32923394$

$-0.22339852$

$-0.22339852$

$-0.32923394$

$-0.32923394$

$-0.22339852$

$-0.22339852$

0.56140047

0.56140047

0.66723585

0.66723585

0.56140047

0.56140047
-0.09333258
0.01250287
0.02794744
0.13378289
0.02794744
0.13378289
0.02794744
0.13378289
0.02794744
0.13378289
-0.15553895
-0.04970350
-0.15553895
-0.04970350
-0.15553895
-0.04970350
-0.15553895
-0.04970350

0.61997104

$-0.65584904$

$-0.63986617$

0.30549407

1.10340309

$-0.59786034$

0.65259463

$-0.33612067$

$-1.05361748$

0.56457537

0.01441160

0.06529298

0.04232992

$-0.10479548$

0.02673656

$-0.13397759$

$-0.08325808$

0.16713685
1.000 \# Electr. mom., scale-fac
\# Magnetic mom.

$-0.06075061$

0.04508484

$-0.06075061$

0.04508484

$-0.06075061$

0.04508484

$-0.06075061$

0.04508484

$-0.04267391$

0.06316154

$-0.04267391$

0.06316154

$-0.04267391$

0.06316154

$-0.04267391$

0.06316154

$-0.13571967$

$-0.02988423$

$-0.13571967$

$-0.02988423$

$-0.13571967$

$-0.02988423$

$-0.13571967$

$-0.02988423$

0.04030320

0.14613864

0.04030320

0.14613864

0.04030320

0.14613864

0.04030320

0.14613864

$-0.03195595$

0.07387950

$-0.03195595$

0.07387950

$-0.03195595$

0.07387950

$-0.03195595$

0.07387950

$-0.09333258$

0.01250287

$-0.09333258$

0.01250287

$-0.09333258$

0.01250287
$-0.64499152$

$-0.60470122$

1.02520883

0.55435014

0.92944020

$-0.20873019$

$-1.21593022$

0.33660650

$-3.92919946$

$-1.59665024$

4.83525181

1.23484790

3.65770769

1.67245317

$-4.54206324$

$-1.32581544$

0.14926024

$-0.05450534$

$-0.15977734$

0.01558872

$-0.00493623$

$-0.12033805$

0.03278114

0.10911946

0.03859468

$-0.70316726$

0.09662631

0.80528545

$-0.02112093$

0.75369567

$-0.10903199$

$-0.86221343$

1.08799827

$-3.60225797$

$-0.11198123$

3.50892472

$-1.12090325$

3.94893098

0.02557697

$-3.88560200$

0.23158802

2.84234333

$-0.28412589$

$-2.78924608$

$-0.12813628$

$-3.10620117$ 


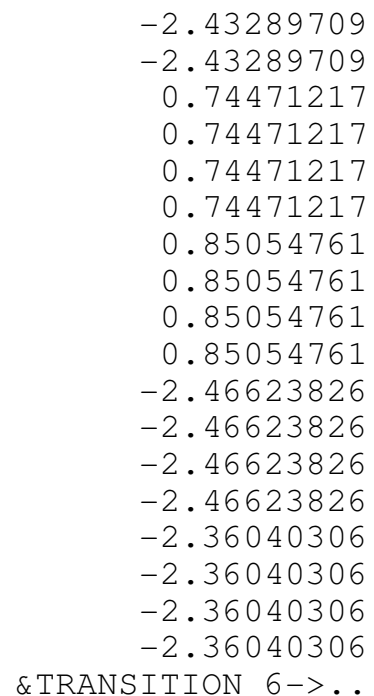
64

$\begin{array}{lll} & 0.0 .0 \text { \#nergy } & 6->7 \\ -0.0241 & -0.0182 & 0.0185 \\ -0.1581 & -0.5710 & 0.0332\end{array}$

$-0.1581$

2.27520919

2. 27520919

2. 27520919

2.27520919

2. 38104463

2. 38104463

2.38104463

2.38104463

$-1.40967047$

$-1.40967047$

$-1.40967047$

$-1.40967047$

$-1.30383503$

$-1.30383503$

$-1.30383503$

$-1.30383503$

2.54510117

2. 54510117

2.54510117

2.54510117

2.65093660

2.65093660

2.65093660

2.65093660

$-1.43156683$

$-1.43156683$

$-1.43156683$

$-1.43156683$

$-1.32573140$

$-1.32573140$

$-1.32573140$

$-1.32573140$

0.99076527

0.99076527

0.99076527

0.99076527

1.09660077

1.09660077

1.09660077

1.09660077

$-2.53873253$

$-2.53873253$

$-2.53873253$

$-2.53873253$

$-2.43289709$
0.66723585

0.66723585

$-1.31334066$

$-1.31334066$

$-1.20750511$

$-1.20750511$

$-1.31334066$

$-1.31334066$

$-1.20750511$

$-1.20750511$

1.56936514

1.56936514

1.67520046

1.67520046

1.56936514

1.56936514

1.67520046

1.67520046

0.11177704

0.11177704

0.21761249

0.21761249

0.11177704

0.11177704

0.21761249

0.21761249

$-0.18411981$

$-0.18411981$

$-0.07828436$

$-0.07828436$

$-0.18411981$

$-0.18411981$

$-0.07828436$

$-0.07828436$

1.31986856

1. 31986856

1. 42570400

1. 42570400

1.31986856

1. 31986856

1. 42570400

1. 42570400

$-1.42060065$

$-1.42060065$

$-1.31476510$

$-1.31476510$

$-1.42060065$

$-1.42060065$

$-1.31476510$

$-1.31476510$

$-0.32923394$

$-0.32923394$

$-0.22339852$

$-0.22339852$

$-0.32923394$

$-0.32923394$

$-0.22339852$

$-0.22339852$

0.56140047

0.56140047

0.66723585

0.66723585

0.56140047

$$
\begin{array}{r}
-0.09333258 \\
0.01250287 \\
0.02794744 \\
0.13378289 \\
0.02794744 \\
0.13378289 \\
0.02794744 \\
0.13378289 \\
0.02794744 \\
0.13378289 \\
-0.15553895 \\
-0.04970350 \\
-0.15553895 \\
-0.04970350 \\
-0.15553895 \\
-0.04970350 \\
-0.15553895 \\
-0.04970350
\end{array}
$$

0.14368524

3. 11662197

0.78225583

$-0.73011321$

$-1.04779100$

0.96407771

$-0.78430825$

0.76309973

1. 05441153

$-1.00163627$

0.18691832

0.61329830

$-0.20334622$

$-0.36733317$

$-0.08296074$

$-0.78227317$

0.12580743

0.48903152
1.000 \# Electr. mom., scale-fac
\# Magnetic mom.

$-0.06075061$

0.04508484

$-0.06075061$

0.04508484

$-0.06075061$

0.04508484

$-0.06075061$

0.04508484

$-0.04267391$

0.06316154

$-0.04267391$

0.06316154

$-0.04267391$

0.06316154

$-0.04267391$

0.06316154

$-0.13571967$

$-0.02988423$

$-0.13571967$

$-0.02988423$

$-0.13571967$

$-0.02988423$

$-0.13571967$

$-0.02988423$

0.04030320

0.14613864

0.04030320

0.14613864

0.04030320

0.14613864

0.04030320

0.14613864

$-0.03195595$

0.07387950

$-0.03195595$

0.07387950

$-0.03195595$

0.07387950

$-0.03195595$

0.07387950

$-0.09333258$

0.01250287

$-0.09333258$

0.01250287

$-0.09333258$
$-6.31314421$

5.70036077

5.58646488

$-4.99749613$

6.48683310

$-5.95236397$

$-5.79156685$

5.27289391

0.96052086

$-0.70784426$

$-1.51988506$

1. 18427157

$-1.19382358$

0.94486636

1. 74775493

$-1.41702211$

$-0.77154350$

0.56510407

$-5.19384193$

5.38950777

2. 47250652

$-2.28282428$

3. 51929951

$-3.70382547$

$-0.12254509$

0.12427911

$-0.00766752$

0.00255413

0.08037070

$-0.08166083$

0.06535968

$-0.06120158$

2.99959612

$-2.51664090$

$-3.10887599$

2. 52743578

$-2.88919139$

2. 54107094

2.87670565

$-2.40814471$

$-0.90611440$

0.83354449

0.90029687

$-0.84376854$

1. 18210316 


-2.43289709
-2.43289709
-2.43289709
0.74471217
0.74471217
0.74471217
0.74471217
0.85054761
0.85054761
0.85054761
0.85054761
-2.46623826
-2.46623826
-2.46623826
-2.46623826
-2.36040306
-2.36040306
-2.36040306
-2.36040306

64

$$
\begin{aligned}
& 0.56140047 \\
& 0.66723585 \\
& 0.66723585 \\
& -1.31334066 \\
& -1.31334066 \\
& -1.20750511 \\
& -1.20750511 \\
& -1.31334066 \\
& -1.31334066 \\
& -1.20750511 \\
& -1.20750511 \\
& 1.56936514 \\
& 1.56936514 \\
& 1.67520046 \\
& 1.67520046 \\
& 1.56936514 \\
& 1.56936514 \\
& 1.67520046 \\
& 1.67520046 \\
& \# \text { Energy } 6->8 \\
& \hline 10
\end{aligned}
$$

$\begin{array}{rrr}0.2034 & 0.0210 & -0.0078 \\ 0.0210 & -0.0514 & 0.0097\end{array}$

2. 27520919

2. 27520919

2.27520919

2. 27520919

2.38104463

2. 38104463

2.38104463

2.38104463

$-1.40967047$

$-1.40967047$

$-1.40967047$

$-1.40967047$

$-1.30383503$

$-1.30383503$

$-1.30383503$

$-1.30383503$

2. 54510117

2. 54510117

2. 54510117

2.54510117

2.65093660

2.65093660

2.65093660

2.65093660

$-1.43156683$

$-1.43156683$

$-1.43156683$

$-1.43156683$

$-1.32573140$

$-1.32573140$

$-1.32573140$

$-1.32573140$

0.99076527

0.99076527

0.99076527

0.99076527

1.09660077

1. 09660077

1.09660077

1.09660077

$-2.53873253$

$-2.53873253$

$-2.53873253$

$-2.53873253$

$-2.43289709$
0.11177704

0.11177704

0.21761249

0.21761249

0.11177704

0.11177704

0.21761249

0.21761249

$-0.18411981$

$-0.18411981$

$-0.07828436$

$-0.07828436$

$-0.18411981$

$-0.18411981$

$-0.07828436$

$-0.07828436$

1. 31986856

1.31986856

1. 42570400

1. 42570400

1.31986856

1. 31986856

1. 42570400

1. 42570400

$-1.42060065$

$-1.42060065$

$-1.31476510$

$-1.31476510$

$-1.42060065$

$-1.42060065$

$-1.31476510$

$-1.31476510$

$-0.32923394$

$-0.32923394$

$-0.22339852$

$-0.22339852$

$-0.32923394$

$-0.32923394$

$-0.22339852$

$-0.22339852$

0.56140047

0.56140047

0.66723585

0.66723585

0.56140047

$\begin{array}{rr}0.01250287 & -1.07534432 \\ -0.09333258 & -1.17069030 \\ 0.01250287 & 1.08416986 \\ 0.02794744 & -0.01027565 \\ 0.13378289 & 0.03931696 \\ 0.02794744 & -0.00535121 \\ 0.13378289 & -0.02304721 \\ 0.02794744 & -0.03908385 \\ 0.13378289 & 0.03841870 \\ 0.02794744 & 0.09667694 \\ 0.13378289 & -0.10899924 \\ -0.15553895 & -0.23243183 \\ -0.04970350 & 0.24763864 \\ -0.15553895 & 0.14870606 \\ -0.04970350 & -0.16100387 \\ -0.15553895 & 0.31642240 \\ -0.04970350 & -0.32824367 \\ -0.15553895 & -0.20825076 \\ -0.04970350 & 0.21866301\end{array}$

1.000 \# Electr. mom., scale-fac -0.06075061 Magnetic mom.

$0.04508484 \quad 0.91769683$

$\begin{array}{ll}-0.06075061 & 0.65654600\end{array}$

$0.04508484 \quad-0.93547451$

$\begin{array}{ll}-0.06075061 & 0.55828655\end{array}$

$0.04508484 \quad-0.80150712$

$-0.06075061 \quad-0.56308407$

$0.04508484 \quad 0.81329888$

$\begin{array}{ll}-0.04267391 & 1.87613237\end{array}$

$0.06316154 \quad-2.30034304$

$-0.04267391$

0.06316154

$-0.04267391$

0.06316154

$-0.04267391$

0.06316154

$-0.13571967$

$-0.02988423$

$-0.13571967$

$-0.02988423$

$-0.13571967$

$-0.02988423$

$-0.13571967$

$-0.02988423$

0.04030320

0.14613864

0.04030320

0.14613864

0.04030320

0.14613864

0.04030320

0.14613864

$-0.03195595$

0.07387950

$-0.03195595$

0.07387950

$-0.03195595$

0.07387950

$-0.03195595$

0.07387950

$-0.09333258$

0.01250287

$-0.09333258$

0.01250287

$-0.09333258$

$-1.71392798$

2.14720488

$-2.04899883$

2. 40640926

1.88348997

$-2.25318980$

$-0.18602733$

0.27987555

0.37058359

$-0.45032728$

0.13800563

$-0.22230311$

$-0.32173204$

0.39479604

0.08131884

$-0.18408374$

$-0.69136006$

0.81693947

$-0.10616239$

0.22436802

0.69442731

$-0.83961922$

$-0.36916238$

0.93291003

0.41335645

$-0.94301969$

0.47838870

$-0.99912417$

$-0.46593189$

0.96809685

$-0.55194640$

0.59058040

0.60688949

$-0.64957553$

0.45865285 


\section{$-2.43289709$ \\ $-2.43289709$ \\ $-2.43289709$ \\ 0.74471217 \\ 0.74471217 \\ 0.74471217 \\ 0.74471217 \\ 0.85054761 \\ 0.85054761 \\ 0.85054761 \\ 0.85054761 \\ $-2.46623826$ \\ $-2.46623826$ \\ $-2.46623826$ \\ $-2.46623826$ \\ $-2.36040306$ \\ $-2.36040306$ \\ $-2.36040306$ \\ $-2.36040306$}

64

0.0000

2. 27520919

2. 27520919

2. 27520919

2. 27520919

2.38104463

2. 38104463

2.38104463

2.38104463

$-1.40967047$

$-1.40967047$

$-1.40967047$

$-1.40967047$

$-1.30383503$

$-1.30383503$

$-1.30383503$

$-1.30383503$

2. 54510117

2. 54510117

2. 54510117

2. 54510117

2.65093660

2.65093660

2.65093660

2.65093660

$-1.43156683$

$-1.43156683$

$-1.43156683$

$-1.43156683$

$-1.32573140$

$-1.32573140$

$-1.32573140$

$-1.32573140$

0.99076527

0.99076527

0.99076527

0.99076527

1.09660077

1. 09660077

1.09660077

1.09660077

$-2.53873253$

$-2.53873253$

$-2.53873253$

$-2.53873253$

$-2.43289709$
0.56140047

0.66723585

0.66723585

$-1.31334066$

$-1.31334066$

$-1.20750511$

$-1.20750511$

$-1.31334066$

$-1.31334066$

$-1.20750511$

$-1.20750511$

1.56936514

1.56936514

1.67520046

1.67520046

1.56936514

1.56936514

1. 67520046

1.67520046

. \# Energy 6->9

$\begin{array}{lr}0.3825 & -0.0235 \\ 0.0016 & 0.0062\end{array}$

0.11177704

0.11177704

0.21761249

0.21761249

0.11177704

0.11177704

0.21761249

0.21761249

$-0.18411981$

$-0.18411981$

$-0.07828436$

$-0.07828436$

$-0.18411981$

$-0.18411981$

$-0.07828436$

$-0.07828436$

1. 31986856

1.31986856

1. 42570400

1. 42570400

1.31986856

1. 31986856

1. 42570400

1. 42570400

$-1.42060065$

$-1.42060065$

$-1.31476510$

$-1.31476510$

$-1.42060065$

$-1.42060065$

$-1.31476510$

$-1.31476510$

$-0.32923394$

$-0.32923394$

$-0.22339852$

$-0.22339852$

$-0.32923394$

$-0.32923394$

$-0.22339852$

$-0.22339852$

0.56140047

0.56140047

0.66723585

0.66723585

0.56140047

$\begin{array}{rr}0.01250287 & -0.47572657 \\ -0.09333258 & -0.51864243 \\ 0.01250287 & 0.53128934 \\ 0.02794744 & 0.13009565 \\ 0.13378289 & -0.05680959 \\ 0.02794744 & -0.30266273 \\ 0.13378289 & 0.20011494 \\ 0.02794744 & -0.11793803 \\ 0.13378289 & 0.06480811 \\ 0.02794744 & 0.25260341 \\ 0.13378289 & -0.17792709 \\ -0.15553895 & -0.10205601 \\ -0.04970350 & 0.13731797 \\ -0.15553895 & 0.05649292 \\ -0.04970350 & -0.08044216 \\ -0.15553895 & 0.06291091 \\ -0.04970350 & -0.08850269 \\ -0.15553895 & -0.02470971 \\ -0.04970350 & 0.04153923\end{array}$

1.000 \# Electr. mom., scale-fac \# Magnetic mom.

$-0.06075061 \quad 1.00565815$

$0.04508484 \quad 1.21155298$

$-0.06075061$

0.04508484

$-0.06075061$

0.04508484

$-0.06075061$

0.04508484

$-0.04267391$

0.06316154

$-0.04267391$

0.06316154

$-0.04267391$

0.06316154

$-0.04267391$

0.06316154

$-0.13571967$

$-0.02988423$

$-0.13571967$

$-0.02988423$

$-0.13571967$

$-0.02988423$

$-0.13571967$

$-0.02988423$

0.04030320

0.14613864

0.04030320

0.14613864

0.04030320

0.14613864

0.04030320

0.14613864

$-0.03195595$

0.07387950

$-0.03195595$

0.07387950

$-0.03195595$

0.07387950

$-0.03195595$

0.07387950

$-0.09333258$

0.01250287

$-0.09333258$

0.01250287

$-0.09333258$
$-0.96616834$

$-1.33168328$

$-0.56880724$

$-1.28581631$

0.50104535

1. 44033587

$-0.92960066$

0.27239022

0.97185904

$-0.40921062$

0.38838241

$-0.04001372$

$-0.45999929$

0.17782746

0.04024242

0.84604239

$-0.03168498$

$-0.65942866$

0.13432629

$-0.86818033$

$-0.11227793$

0.70214683

0.00526755

$-0.33430368$

0.03017726

0.38041544

$-0.05345917$

0.50145191

$-0.00341568$

$-0.57664508$

3. 28402591

0.05157121

$-3.01327133$

0.12577941

$-3.06408644$

$-0.07517102$

2. 83191562

$-0.05389213$

$-1.60616040$

0.99205542

1. 75980079

$-1.15752602$

1. 78412914 


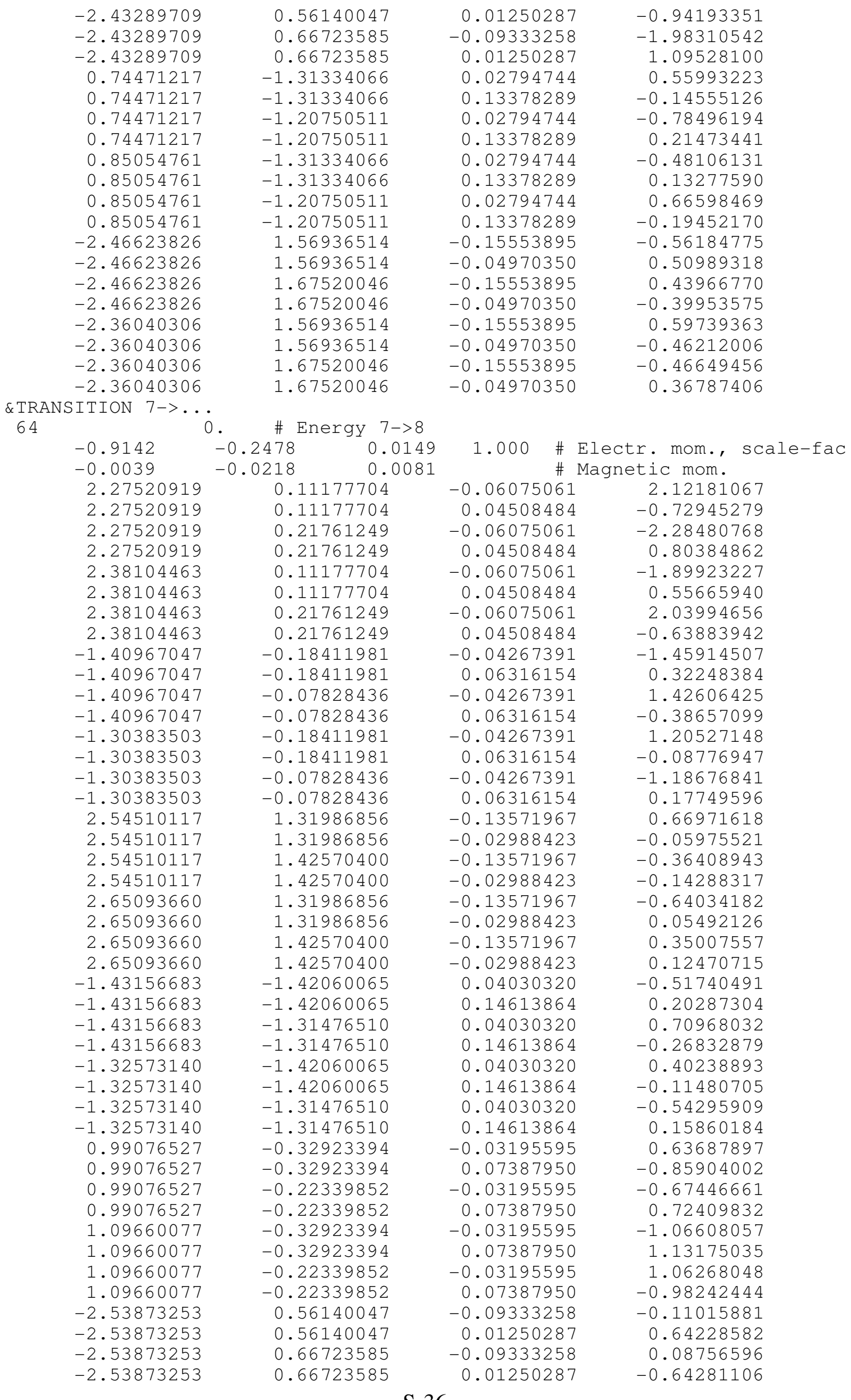




-2.43289709
-2.43289709
-2.43289709
-2.43289709
0.74471217
0.74471217
0.74471217
0.74471217
0.85054761
0.85054761
0.85054761
0.85054761
-2.46623826
-2.46623826
-2.46623826
-2.46623826
-2.36040306
-2.36040306
-2.36040306
-2.36040306

64
0.56140047

0.56140047

0.66723585

0.66723585

$-1.31334066$

$-1.31334066$

$-1.20750511$

$-1.20750511$

$-1.31334066$

$-1.31334066$

$-1.20750511$

$-1.20750511$

1. 56936514

1.56936514

1.67520046

1.67520046

1.56936514

1.56936514

1.67520046

1.67520046

0 .

$-0.0126$

0.0110

2. 27520919

2. 27520919

2. 27520919

2. 27520919

2. 38104463

2.38104463

2.38104463

2. 38104463

$-1.40967047$

$-1.40967047$

$-1.40967047$

$-1.40967047$

$-1.30383503$

$-1.30383503$

$-1.30383503$

$-1.30383503$

2. 54510117

2. 54510117

2.54510117

2. 54510117

2.65093660

2.65093660

2.65093660

2.65093660

$-1.43156683$

$-1.43156683$

$-1.43156683$

$-1.43156683$

$-1.32573140$

$-1.32573140$

$-1.32573140$

$-1.32573140$

0.99076527

0.99076527

0.99076527

0.99076527

1.09660077

1.09660077

1.09660077

1.09660077

$-2.53873253$

$-2.53873253$

$-2.53873253$

$-2.53873253$

$$
\begin{array}{r}
-0.09333258 \\
0.01250287 \\
-0.09333258 \\
0.01250287 \\
0.02794744 \\
0.13378289 \\
0.02794744 \\
0.13378289 \\
0.02794744 \\
0.13378289 \\
0.02794744 \\
0.13378289 \\
-0.15553895 \\
-0.04970350 \\
-0.15553895 \\
-0.04970350 \\
-0.15553895 \\
-0.04970350 \\
-0.15553895 \\
-0.04970350
\end{array}
$$

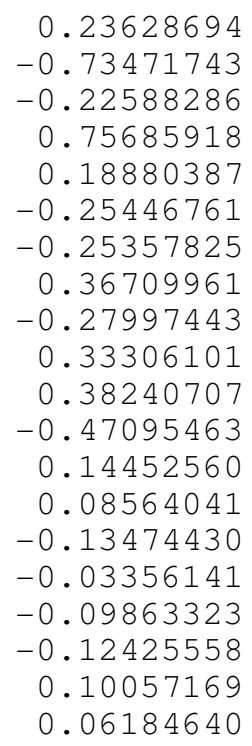
1.000 \# Electr. mom., scale-fac \# Magnetic mom.

-0.06075061
0.04508484

0.76327747

$-0.06075061$

0.04508484

$-0.06075061$

0.04508484

$-0.06075061$

0.04508484

$-0.04267391$

0.06316154

$-0.04267391$

0.06316154

$-0.04267391$

0.06316154

$-0.04267391$

0.06316154

$-0.13571967$

$-0.02988423$

$-0.13571967$

$-0.02988423$

$-0.13571967$

$-0.02988423$

$-0.13571967$

$-0.02988423$

0.04030320

0.14613864

0.04030320

0.14613864

0.04030320

0.14613864

0.04030320

0.14613864

$-0.03195595$

0.07387950

$-0.03195595$

0.07387950

$-0.03195595$

0.07387950

$-0.03195595$

0.07387950

$-0.09333258$

0.01250287

$-0.09333258$

0.01250287
$-0.74294728$

$-0.77803093$

0.75515348

$-0.71433735$

0.69690037

0.73558533

$-0.71771604$

$-0.05999592$

0.01166729

0.08596847

$-0.03067720$

0.08418621

$-0.03759432$

$-0.09279113$

0.03924054

0.23575318

$-0.23523226$

$-0.30657241$

0.30894914

$-0.20608903$

0.21024504

0.27062008

$-0.27644822$

0.00145997

$-0.01275457$

0.21062718

$-0.19337390$

0.00056132

0.00922066

$-0.21689869$

0.20229809

0.64032233

$-0.65581191$

$-0.55180782$

0.55967891

$-0.60628939$

0.61603016

0.51559073

$-0.51779521$

0.30189908

$-0.29123250$

$-0.27608776$

0.26770532 


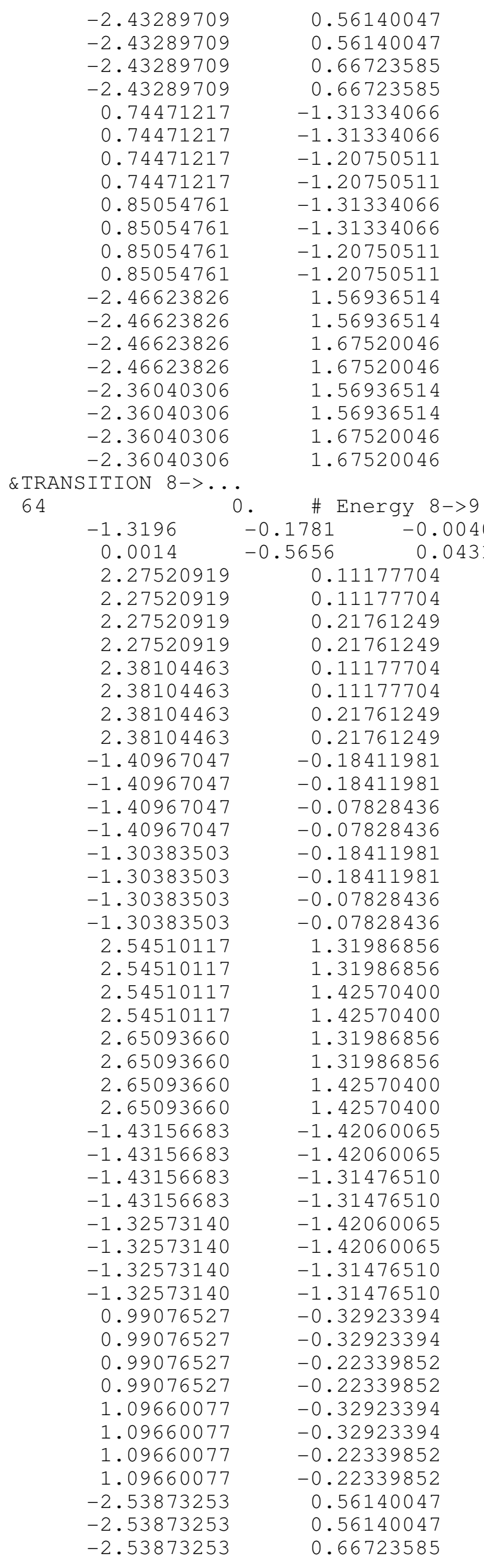

$-2.43289709$

$-2.43289709$

0.74471217

0.74471217

0.74471217

0.74471217

0.85054761

0.85054761

$-2.46623826$

$-2.36040306$

36040306

0.

$\begin{array}{ll}-0.1781 & -0.0040\end{array}$

0.11177704

0.11177704

0.21761249

0.11177704

0.21761249

0.21761249

$-0.18411981$

$-0.07828436$

$-0.18411981$

$-0.18411981$

$-0.07828436$

1.31986856

1.42570400

1.31986856

.31986856

42570400

$-1.42060065$

$-1.31476510$

$-1.42060065$

$-0.32923394$

$-0.22339852$

$-0.22339852$

0.66723585

$$
\begin{array}{r}
-0.09333258 \\
0.01250287 \\
-0.09333258 \\
0.01250287 \\
0.02794744 \\
0.13378289 \\
0.02794744 \\
0.13378289 \\
0.02794744 \\
0.13378289 \\
0.02794744 \\
0.13378289 \\
-0.15553895 \\
-0.04970350 \\
-0.15553895 \\
-0.04970350 \\
-0.15553895 \\
-0.04970350 \\
-0.15553895 \\
-0.04970350
\end{array}
$$

$$
\begin{array}{r}
-0.33289176 \\
0.31703886 \\
0.30032089 \\
-0.28707382 \\
0.07112872 \\
-0.07207227 \\
-0.13701494 \\
0.13844223 \\
-0.06608709 \\
0.06702730 \\
0.12361429 \\
-0.12465358 \\
0.02963115 \\
-0.03122161 \\
-0.02729378 \\
0.02880768 \\
-0.03047278 \\
0.03146352 \\
0.02851234 \\
-0.02966201
\end{array}
$$

$$
\text { \# Magnetic mom }
$$$$
-0.06075061
$$$$
0.04508484
$$$$
-0.06075061
$$$$
0.04508484
$$$$
-0.06075061
$$$$
0.04508484
$$$$
-0.06075061
$$$$
0.04508484
$$$$
-0.04267391
$$$$
0.06316154
$$$$
-0.04267391
$$$$
0.06316154
$$$$
-0.04267391
$$$$
0.06316154
$$$$
-0.04267391
$$$$
0.06316154
$$$$
-0.13571967
$$$$
-0.02988423
$$$$
-0.13571967
$$$$
-0.02988423
$$$$
-0.13571967
$$$$
-0.02988423
$$$$
-0.13571967
$$$$
-0.02988423
$$$$
0.04030320
$$$$
0.14613864
$$$$
0.04030320
$$$$
0.14613864
$$$$
0.04030320
$$$$
0.14613864
$$$$
0.04030320
$$$$
0.14613864
$$$$
-0.03195595
$$$$
0.07387950
$$$$
-0.03195595
$$$$
0.07387950
$$$$
-0.03195595
$$$$
0.07387950
$$$$
-0.03195595
$$$$
0.07387950
$$$$
-0.09333258
$$$$
0.01250287
$$$$
-0.09333258
$$

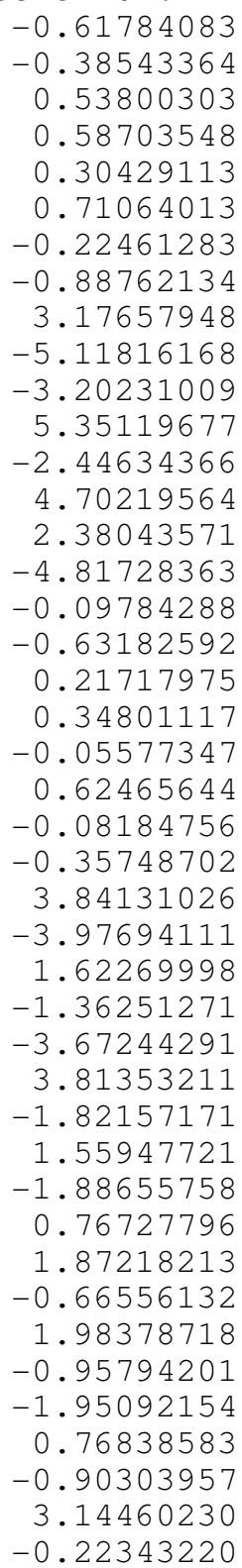


-2.53873253
-2.43289709
-2.43289709
-2.43289709
-2.43289709
0.74471217
0.74471217
0.74471217
0.74471217
0.85054761
0.85054761
0.85054761
0.85054761
-2.46623826
-2.46623826
-2.46623826
-2.46623826
-2.36040306
-2.36040306
-2.36040306
-2.36040306

\&PERMANENT MOMENTS

\begin{tabular}{|c|c|}
\hline & \# Energy 1->1 \\
\hline-3.2564 & -0.1520 \\
\hline 2.27520919 & 0.11177704 \\
\hline 2.27520919 & 0.11177704 \\
\hline 2.27520919 & 0.21761249 \\
\hline 2.27520919 & 0.21761249 \\
\hline 2.38104463 & 0.11177704 \\
\hline 2.38104463 & 0.11177704 \\
\hline 2.38104463 & 0.21761249 \\
\hline 2.38104463 & 0.21761249 \\
\hline-1.40967047 & -0.18411981 \\
\hline-1.40967047 & -0.18411981 \\
\hline-1.40967047 & -0.07828436 \\
\hline-1.40967047 & -0.07828436 \\
\hline-1.30383503 & -0.18411981 \\
\hline-1.30383503 & -0.18411981 \\
\hline-1.30383503 & -0.07828436 \\
\hline-1.30383503 & -0.07828436 \\
\hline 2.54510117 & 1.31986856 \\
\hline 2.54510117 & 1.31986856 \\
\hline 2.54510117 & 1.42570400 \\
\hline 2.54510117 & 1.42570400 \\
\hline 2.65093660 & 1.31986856 \\
\hline 2.65093660 & 1.31986856 \\
\hline 2.65093660 & 1.42570400 \\
\hline 2.65093660 & 1.42570400 \\
\hline-1.43156683 & -1.42060065 \\
\hline-1.43156683 & -1.42060065 \\
\hline-1.43156683 & -1.31476510 \\
\hline-1.43156683 & -1.31476510 \\
\hline-1.32573140 & -1.42060065 \\
\hline-1.32573140 & -1.42060065 \\
\hline-1.32573140 & -1.31476510 \\
\hline-1.32573140 & -1.31476510 \\
\hline 0.99076527 & -0.32923394 \\
\hline 0.99076527 & -0.32923394 \\
\hline 0.99076527 & -0.22339852 \\
\hline 0.99076527 & -0.22339852 \\
\hline 1.09660077 & -0.32923394 \\
\hline 1.09660077 & -0.32923394 \\
\hline 1.09660077 & -0.22339852 \\
\hline 1.09660077 & -0.22339852 \\
\hline-2.53873253 & 0.56140047 \\
\hline-2.53873253 & 0.56140 \\
\hline-2.53873253 & 0.6672 \\
\hline
\end{tabular}

$\begin{array}{rr}0.01250287 & -2.03559422 \\ -0.09333258 & 1.25003552 \\ 0.01250287 & -3.80237198 \\ -0.09333258 & 0.13287368 \\ 0.01250287 & 2.50158906 \\ 0.02794744 & -0.07105391 \\ 0.13378289 & 0.08212353 \\ 0.02794744 & -0.05320212 \\ 0.13378289 & 0.06207341 \\ 0.02794744 & 0.03400822 \\ 0.13378289 & -0.07023038 \\ 0.02794744 & 0.05841102 \\ 0.13378289 & -0.02554031 \\ -0.15553895 & 0.35818273 \\ -0.04970350 & 0.02055832 \\ -0.15553895 & -0.34841731 \\ -0.04970350 & 0.08416093 \\ -0.15553895 & -0.50515079 \\ -0.04970350 & 0.02806995 \\ -0.15553895 & 0.44273236 \\ -0.04970350 & -0.11143014\end{array}$
\# Electr. mom. $\begin{array}{ll}-0.06075061 & 172.33860779\end{array}$ $0.04508484-123.11048889$ $-0.06075061 \quad-166.96473694$ $0.04508484 \quad 122.39292908$ $-0.06075061 \quad-170.91770935$ $0.04508484 \quad 124.29554749$ $-0.06075061 \quad 166.83624268$ $0.04508484 \quad-123.80957794$ $-0.04267391 \quad-53.05001068$ $0.06316154 \quad 3.96958613$ $-0.04267391 \quad 74.01541901$ $0.06316154 \quad-19.04526520$ $-0.04267391 \quad 49.27111816$ $0.06316154 \quad-6.61775017$ $-0.04267391 \quad-67.84795380$ $0.06316154 \quad 20.33152962$ $\begin{array}{ll}-0.13571967 & 28.30279541\end{array}$ $-0.02988423 \quad-22.15081024$ $-0.13571967 \quad-22.94334221$ $-0.02988423 \quad 18.26645088$ $-0.13571967 \quad-30.63180351$ $-0.02988423 \quad 24.99675179$ $-0.13571967 \quad 24.75374603$ $-0.02988423 \quad-21.03478432$ $0.04030320 \quad 7.05732965$ $0.14613864 \quad-16.67008781$ $0.04030320 \quad-6.56781006$ $0.14613864 \quad 18.08987236$ $0.04030320 \quad-7.46918106$ $0.14613864 \quad 15.46862221$ $0.04030320 \quad 6.65001726$ $0.14613864 \quad-16.67440414$ $\begin{array}{ll}-0.03195595 & 3.51498961\end{array}$ $0.07387950 \quad-29.21114540$ $-0.03195595 \quad 7.62966013$ $0.07387950 \quad 21.61389542$ $-0.03195595 \quad-19.82527924$ $0.07387950 \quad 40.64679718$ $-0.03195595 \quad 6.04645109$ $0.07387950 \quad-31.66695023$ $\begin{array}{ll}-0.09333258 & 32.87990570\end{array}$ $0.01250287 \quad-72.18527985$ $-0.09333258 \quad-17.29346275$ 


\begin{tabular}{|c|c|c|c|}
\hline-2.53873253 & 0.66723585 & 0.01250287 & 67.43709564 \\
\hline-2.43289709 & 0.56140047 & -0.09333258 & -41.98970795 \\
\hline-2.43289709 & 0.56140047 & 0.01250287 & 82.33974457 \\
\hline-2.43289709 & 0.66723585 & -0.09333258 & 24.72338867 \\
\hline-2.43289709 & 0.66723585 & 0.01250287 & -77.7186431 \\
\hline 0.74471217 & -1.31334066 & 0.02794744 & 5.5977644 \\
\hline 0.74471217 & -1.31334066 & 0.13378289 & -14.3519783 \\
\hline 0.74471217 & -1.20750511 & 0.02794744 & -7.8452911 \\
\hline 0.74471217 & -1.20750511 & 0.13378289 & 18.1120834 \\
\hline 0.85054761 & -1.31334066 & 0.02794744 & -9.596399 \\
\hline 0.85054761 & -1.31334066 & 0.13378289 & 18.029701 \\
\hline 0.85054761 & -1.20750511 & 0.02794744 & 13.7018985 \\
\hline 0.85054761 & -1.20750511 & 0.13378289 & -22.9429016 \\
\hline-2.46623826 & 1.56936514 & -0.15553895 & -10.8083 \\
\hline-2.46623826 & 1.56936514 & -0.04970350 & -9.924520 \\
\hline-2.46623826 & 1.67520046 & -0.15553895 & 9.6 \\
\hline-2.46623826 & 1.67520046 & -0.049703 & 5.6 \\
\hline-2.36040306 & 1.56936514 & -0.15553 & 9.0 \\
\hline-2.36040306 & 1.56936514 & -0.04970350 & 13.36 \\
\hline-2.36040306 & 1.67520046 & -0.15553895 & -8.29 \\
\hline-2.36040306 & 1.67520046 & -0.04970350 & -7.88 \\
\hline & \# Energy 2->2 & & \\
\hline-2.18 & -0.1824 & & ectr. mom. \\
\hline 2.27 & 0.11177704 & -0.06075061 & 105.81 \\
\hline 0919 & 0.11177704 & 0.04508484 & -142.8495 \\
\hline 2.27520919 & 0.21761249 & -0.06075061 & -94.932914 \\
\hline 20919 & 0.21761249 & 0.04508484 & 138.4 \\
\hline 2.3 & 77704 & -0.06075061 & -117.8 \\
\hline 2.38 & 0.11177704 & 0.04508484 & 146.980 \\
\hline 2.38104463 & 0.21761249 & -0.06075061 & 109.429 \\
\hline $2.3 \xi$ & 0.21 & 0.04508484 & -144.5 \\
\hline-1.4 & -0.18 & -0.04267 & $-71 \cdot 7$ \\
\hline-1.40 & -0.184 & 0.06316154 & .7 \\
\hline-1.4096 & -0.07828436 & -0.04267391 & 96.097 \\
\hline-1.409 & -0.07 & 0.063 & -25.9 \\
\hline$-1 \cdot 30$ & -0.18 & -0.04267391 & 69.6 \\
\hline-1.30383503 & -0.184 & 0.06316154 & $-11 \cdot 1$ \\
\hline-1.30383503 & -0.07828436 & -0.04267391 & -91.272 \\
\hline$-1 \cdot 30$ & -0.07828436 & 0.06316154 & 27.8 \\
\hline 2.54510117 & 1.31986856 & -0.13571967 & 8.522 \\
\hline 2.54510 & 1.31986856 & -0.02988423 & -33.3337 \\
\hline 2.54 & 1.42 & -0.13571 & -6.18 \\
\hline 2. & 1.42 & -0.025 & 26.5 \\
\hline 2.65 & 1.31986856 & -0.13571967 & -14.844 \\
\hline 2.650 & 1.31986856 & -0.02988423 & 39.62 \\
\hline 2. & 1.42 & -0.13 & 11.1 \\
\hline 2.65 & 1.42 & -0.029 & $-31 \cdot 8$ \\
\hline-1.43156 & -1.42 & 0.0403 & .07 \\
\hline-1.43 & -1.42060065 & 0.14613864 & -16.45 \\
\hline$-1 \cdot 43$ & $-1 \cdot 31$ & 0 . & -2.1 \\
\hline-1.431 & $-1 \cdot 31$ & 0.14613864 & 17.4 \\
\hline-1.325 & -1.42060065 & 0.04030320 & $-3 \cdot 7$ \\
\hline$-1 \cdot 32$ & -1.42060065 & 0.14613864 & 14.8 \\
\hline-1.325 & $-1 \cdot 31$ & 0.0 & 2 \\
\hline-1.325731 & -1.31476510 & 0.14613864 & -15.47 \\
\hline 0 & -0.32923394 & -0.03195595 & -89.02 \\
\hline 0 . & -0.3 & 0.0 & -7.5 \\
\hline 0.99 & -0.22 & -0.03195 & 96.1 \\
\hline 0.990 & -0.22339852 & 0.07387950 & -4.086 \\
\hline 1.09 & -0.32 & -0.03 & 78.6 \\
\hline 1.09 & -0 . & 0 . & 17.4 \\
\hline 1.096 & -0.22339852 & -0.03195595 & -88.0921 \\
\hline 1.09 & -0.22 & 0.073879 & -4.2 \\
\hline-2.5387 & 0.56 & -0.09333258 & 37.4 \\
\hline-2.5387 & 0.56 & 0.01250287 & -78.52510 \\
\hline-2.53 & 0.66723 & -0.09333258 & -20.81 \\
\hline-2 . & 0. & 0 & \\
\hline
\end{tabular}




\begin{tabular}{|c|c|c|c|}
\hline-2.43289709 & 0.56140047 & -0.09333258 & -48.32963181 \\
\hline-2.43289709 & 0.56140047 & 0.01250287 & 90.66246033 \\
\hline-2.43289709 & 0.66723585 & -0.09333258 & 29.86554146 \\
\hline-2.43289709 & 0.66723585 & 0.01250287 & -85.40911865 \\
\hline 0.74471217 & -1.31334066 & 0.02794744 & -11.4464483 \\
\hline 0.74471217 & -1.31334066 & 0.13378289 & $-9.07 \mathrm{C}$ \\
\hline 0.74471217 & -1.20750511 & 0.02794744 & 17.313 \\
\hline 0.74471217 & -1.20750511 & 0.13378289 & 9.770961 \\
\hline 0.85054761 & -1.31334066 & 0.02794744 & 7.6 \\
\hline 0.85054761 & -1.31334066 & 0.13378289 & 12 . \\
\hline 0.85054761 & -1.20750511 & 0.02794744 & -11.712 \\
\hline 0.85054761 & -1.20750511 & 0.13378289 & $-14 \cdot 24$ \\
\hline-2.46623826 & 1.56936514 & -0.15553895 & -10.9 \\
\hline 2.46623826 & 1.56936514 & -0.04970 & $-11 \cdot 2$ \\
\hline-2.46623826 & 1.67520046 & -0.155 & 9.8 \\
\hline-2.46623826 & 1.67520046 & -0.04970 & \\
\hline-2.36040306 & 1.56936514 & -0.15553 & 0.0 \\
\hline-2.3604 & 1.56936514 & -0.04970 & 15.2 \\
\hline-2.3604 & 1.67520 & -0 & $-\varepsilon$ \\
\hline 2.3604030 & 1.67520046 & -0.04970350 & -9.2 \\
\hline & \# Energy $3-$ & & \\
\hline-2.6133 & $469 \quad 0.1$ & & ctr. mom. \\
\hline 2.27520919 & 0.11177704 & -0.06075061 & 163.6 \\
\hline 2.27520919 & 0.11177704 & 0.04508484 & -123.5282 \\
\hline & 0.21761249 & -0.06075061 & -157.2 \\
\hline 7520919 & 0.21761249 & 0.04508484 & 122.22 \\
\hline 2.38104463 & 0.11177704 & -0.06075061 & -164.96 \\
\hline 2.3 & 0.11177704 & 0.04508484 & 125 . \\
\hline 2 & 1249 & -0.060 & 160 \\
\hline 04463 & 0.21761249 & 0.0450 & -124.69 \\
\hline 67047 & -0.18411981 & -0.04267391 & -96.85 \\
\hline-1.40 & -0.1 & 0.06 & 26. \\
\hline-1.4 & -0 & -0.042 & 117.4 \\
\hline 1 . & -0 . & 6154 & -46 \\
\hline 3503 & -0.18411981 & -0.042 & 83.86 \\
\hline-1 & -0.184119 & 0.06 & -21.8 \\
\hline-1 & -0.078284 & -0.04267391 & -102.82 \\
\hline-1.30 & -0.07828436 & 0.06316154 & 39.47 \\
\hline 2. & 1.31986856 & -0.135 & 25. \\
\hline & 86856 & -0.0 & $-21 \cdot 2$ \\
\hline 2 . & 1.425704 & -0.13571967 & $-21 \cdot 0$ \\
\hline 2.5 & 1.42570400 & -0.02988423 & $17.5^{\circ}$ \\
\hline 2 . & 1.319868 & -0.13 & -28 \\
\hline 2. & 1 & -0 & 24 \\
\hline 2 . & 1.425704 & -0.13 & 23. \\
\hline 2.6 & 1.425704 & -0.02 & -20 \\
\hline-1.4 & -1.4206 & 0 . & -9 \\
\hline-1 . & -1 & 0 . & -10 \\
\hline-1 . & -1.31 & 0 . & 1. \\
\hline-1 & -1.31476510 & 0.146 & 1 \\
\hline-1 . & -1.42 & 0.04 & 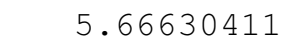 \\
\hline-1 . & -1.42060065 & 0.146 & 1 \\
\hline-1 . & -1.31476510 & 0.040 & -10 \\
\hline-1 & -1.31476510 & 0.14 & -13 \\
\hline & -0 . & -0 . & -38 \\
\hline 0. & -0.32923394 & 0.073 & -20.7 \\
\hline & -0.22339852 & -0.03 & 47 \\
\hline & -0 . & 0 . & 11 \\
\hline & -0.329233 & -0.031 & 21.8 \\
\hline 1. & -0.32923394 & 0.073 & 33 \\
\hline & -0 . & -0 . & -34 \\
\hline & 339852 & 0 . & -22 \\
\hline-2 . & 0.56140047 & -0.09333258 & 17.9 \\
\hline & 0 . & 0 & -59 \\
\hline & 0 . & -0.09 & \\
\hline-2 . & 0 . & 0.01 & 50 \\
\hline-2.43 & 0 & 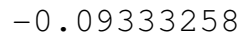 & \\
\hline
\end{tabular}




\begin{tabular}{|c|c|c|c|}
\hline-2.43289709 & 0.56140047 & 0.01250287 & 73.41615295 \\
\hline-2.43289709 & 0.66723585 & -0.09333258 & 4.20598221 \\
\hline-2.43289709 & 0.66723585 & 0.01250287 & -64.38774109 \\
\hline 0.74471217 & -1.31334066 & 0.02794744 & -4.08212805 \\
\hline 0.74471217 & -1.31334066 & 0.13378289 & -12.17274570 \\
\hline 0.74471217 & -1.20750511 & 0.02794744 & 7.24321032 \\
\hline 0.74471217 & -1.20750511 & 0.13378289 & 15.05990982 \\
\hline 0.85054761 & -1.31334066 & 0.02794744 & -0.86836410 \\
\hline 0.85054761 & -1.31334066 & 0.13378289 & 16.56829643 \\
\hline 0.85054761 & -1.20750511 & 0.02794744 & -0.05755687 \\
\hline 0.85054761 & -1.20750511 & 0.13378289 & -20.77493858 \\
\hline-2.46623826 & 1.56936514 & -0.15553895 & -15.64998245 \\
\hline-2.46623826 & 1.56936514 & -0.04970350 & -4.59872818 \\
\hline-2.46623826 & 1.67520046 & -0.15553895 & 13.13133430 \\
\hline-2.46623826 & 1.67520046 & -0.04970350 & 1.92400193 \\
\hline-2.36040306 & 1.56936514 & -0.15553895 & 14.04497528 \\
\hline-2.36040306 & 1.56936514 & -0.04970350 & 9.82288361 \\
\hline-2.36040306 & 1.67520046 & -0.15553895 & -11.88346291 \\
\hline-2.36040306 & 1.67520046 & -0.04970350 & -5.43720388 \\
\hline & \# Energy $4->4$ & & \\
\hline-6.0143 & $-0.1627 \quad-0.0006$ & \# & ectr. mom. \\
\hline 2.27520919 & 0.11177704 & -0.06075061 & 173.56062317 \\
\hline 2.27520919 & 0.11177704 & 0.04508484 & -125.56967163 \\
\hline 2.27520919 & 0.21761249 & -0.06075061 & -168.18128967 \\
\hline 2.27520919 & 0.21761249 & 0.04508484 & 124.79695129 \\
\hline 2.38104463 & 0.11177704 & -0.06075061 & -172.24046326 \\
\hline 2.38104463 & 0.11177704 & 0.04508484 & 126.28600311 \\
\hline 2.38104463 & 0.21761249 & -0.06075061 & 168.19543457 \\
\hline 2.38104463 & 0.21761249 & 0.04508484 & -125.82500458 \\
\hline-1.40967047 & -0.18411981 & -0.04267391 & -40.02493668 \\
\hline-1.40967047 & -0.18411981 & 0.06316154 & -10.34850979 \\
\hline-1.40967047 & -0.07828436 & -0.04267391 & 62.54164886 \\
\hline-1.40967047 & -0.07828436 & 0.06316154 & -4.62865543 \\
\hline-1.30383503 & -0.18411981 & -0.04267391 & 37.47047424 \\
\hline-1.30383503 & -0.18411981 & 0.06316154 & 7.09938908 \\
\hline-1.30383503 & -0.07828436 & -0.04267391 & -57.68953705 \\
\hline-1.30383503 & -0.07828436 & 0.06316154 & 6.24706268 \\
\hline 2.54510117 & 1.31986856 & -0.13571967 & 28.64072990 \\
\hline 2.54510117 & 1.31986856 & -0.02988423 & -22.86768341 \\
\hline 2.54510117 & 1.42570400 & -0.13571967 & -23.20615387 \\
\hline 2.54510117 & 1.42570400 & -0.02988423 & 18.80167580 \\
\hline 2.650 & 1.31986856 & -0.13571967 & -31.00805473 \\
\hline 2.65 & 1.31986856 & -0.02988423 & 25.7216 \\
\hline 2.65093660 & 1.42570400 & -0.13571967 & 25.04117775 \\
\hline 2.65093660 & 1.42570400 & -0.02988423 & -21.59282494 \\
\hline-1.43156683 & -1.42060065 & 0.04030320 & 11.301 \\
\hline-1.43156683 & -1.42060065 & 0.14613864 & -19.59855652 \\
\hline-1.43156683 & -1.31476510 & 0.04030320 & -12.07502556 \\
\hline-1.43156683 & -1.31476510 & 0.14613864 & 21.76894951 \\
\hline-1.32573140 & -1.42060065 & 0.04030320 & -11.55762863 \\
\hline-1.32573140 & -1.42060065 & 0.14613864 & 17.78982544 \\
\hline-1.32573140 & -1.31476510 & 0.04030320 & 11.90369797 \\
\hline-1.32573140 & -1.31476510 & 0.14613864 & -19.67730713 \\
\hline 0.99076527 & -0.32923394 & -0.03195595 & 2.53714895 \\
\hline 0.99076527 & -0.32923394 & 0.07387950 & -30.93278503 \\
\hline 0.99076527 & -0.22339852 & -0.03195595 & 8.68464947 \\
\hline 0.99076527 & -0.22339852 & 0.07387950 & 23.13097382 \\
\hline 1.09660077 & -0.32923394 & -0.03195595 & -18.78204536 \\
\hline 1.09660077 & -0.32923394 & 0.07387950 & 42.68276978 \\
\hline 1.096 & -0.22339852 & -0.03195595 & 4.9566 \\
\hline 1.096 & -0.22339852 & 0.07387950 & -33.38354492 \\
\hline-2.53873253 & 0.56140047 & -0.09333258 & 35.87461090 \\
\hline-2.5387 & 0.56140047 & 0.01250287 & -64.67 \\
\hline-2.53873253 & 0.66723585 & -0.09333258 & -21.15 \\
\hline-2.53873253 & 0.66723585 & 0.01250287 & 59.98513031 \\
\hline-2.43289 & 0.56140047 & -0.09333258 & -45.85377121 \\
\hline-2.432 & 0 . & 0.01250287 & 75. \\
\hline
\end{tabular}


64

$+$

0.66723585

0.66723585

$-1.31334066$

$-1.31334066$

$-1.20750511$

$-1.20750511$

$-1.31334066$

$-1.31334066$

$-1.20750511$

$-1.20750511$

1.56936514

1.56936514

1.67520046

1.67520046

1.56936514

1.56936514

1.67520046

1.67520046

0 .

$-5.7534$

2. 27520919

2. 27520919

2. 27520919

2. 27520919

2.38104463

2.38104463

2.38104463

2. 38104463

$-1.40967047$

$-1.40967047$

$-1.40967047$

$-1.40967047$

$-1.30383503$

$-1.30383503$

$-1.30383503$

$-1.30383503$

2.54510117

2. 54510117

2. 54510117

2.54510117

2.65093660

2.65093660

2.65093660

2.65093660

$-1.43156683$

$-1.43156683$

$-1.43156683$

$-1.43156683$

$-1.32573140$

$-1.32573140$

$-1.32573140$

$-1.32573140$

0.99076527

0.99076527

0.99076527

0.99076527

1.09660077

1.09660077

1.09660077

1.09660077

$-2.53873253$

$-2.53873253$

$-2.53873253$

$-2.53873253$

$-2.43289709$

$-2.43289709$

$-2.43289709$
\# Energy 5->5

0.11177704

0.11177704

0.21761249

0.21761249

0.11177704

0.11177704

0.21761249

0.21761249

$-0.18411981$

$-0.18411981$

$-0.07828436$

$-0.07828436$

$-0.18411981$

$-0.18411981$

$-0.07828436$

$-0.07828436$

1.31986856

1.31986856

1.42570400

1.42570400

1.31986856

1.31986856

1. 42570400

1. 42570400

$-1.42060065$

$-1.42060065$

$-1.31476510$

$-1.31476510$

$-1.42060065$

$-1.42060065$

$-1.31476510$

$-1.31476510$

$-0.32923394$

$-0.32923394$

$-0.22339852$

$-0.22339852$

$-0.32923394$

$-0.32923394$

$-0.22339852$

$-0.22339852$

0.56140047

0.56140047

0.66723585

0.66723585

0.56140047

0.56140047

0.66723585

$$
\begin{array}{rr}
-0.09333258 & 29.73501205 \\
0.01250287 & -70.24802399 \\
0.02794744 & 4.78039837 \\
0.13378289 & -14.45277977 \\
0.02794744 & -6.77504730 \\
0.13378289 & 18.19431496 \\
0.02794744 & -8.84731293 \\
0.13378289 & 18.24086952 \\
0.02794744 & 12.69972420 \\
0.13378289 & -23.17366791 \\
-0.15553895 & -10.38129711 \\
-0.04970350 & -8.82871914 \\
-0.15553895 & 9.23913670 \\
-0.04970350 & 5.09087944 \\
-0.15553895 & 8.18488693 \\
-0.04970350 & 12.32251740 \\
-0.15553895 & -7.53969288 \\
-0.04970350 & -7.39245319
\end{array}
$$

\#
-0.06075061
0.04508484
-0.06075061
0.04508484
-0.06075061
0.04508484
-0.06075061
0.04508484

Electr. mom.

177.66043091

$-135.82839966$

$-172.70074463$

134.90509033

$-177.58418274$

134.44462585

174.16746521

$-134.40460205$

$-47.33258820$

$-0.25141588$

68.53263092

$-14.93526649$

43.98932648

$-2.77184486$

$-62.80541611$

16.51333618

30.85984802

$-26.61978149$

$-25.17505074$

21.92399406

$-33.46125031$

29.78803825

27.12003899

$-25.00492287$

8.30965900

$-17.61978912$

$-8.16953087$

19.28250313

$-8.56845379$

16.21129036

8.05502224

$-17.61820221$

$-5.05085611$

$-35.37920380$

15.52949142

25.98976326

$-10.35760498$

47.82415390

$-2.51780009$

$-36.62639236$

34.82238388

$-72.79528046$

$-19.49899483$

68.17321777

$-44.30916214$

83.24802399

27.38485718

. 01250287

$-0.09333258$

$-0.09333258$

0.01250287

$-0.09333258$ 


\begin{tabular}{|c|c|c|c|}
\hline-2.43289709 & 0.66723585 & 0.01250287 & -78.72726440 \\
\hline 0.74471217 & -1.31334066 & 0.02794744 & 3.34637666 \\
\hline 0.74471217 & -1.31334066 & 0.13378289 & -14.72326469 \\
\hline 0.74471217 & -1.20750511 & 0.02794744 & -4.43723106 \\
\hline 0.74471217 & -1.20750511 & 0.13378289 & 18.43910027 \\
\hline 0.85054761 & -1.31334066 & 0.02794744 & -7.14830256 \\
\hline 0.85054761 & -1.31334066 & 0.13378289 & 18.445 \\
\hline 0.85054761 & -1.20750511 & 0.02794744 & 9.99 \\
\hline 0.85054761 & -1.20750511 & 0.13378289 & $-23 \cdot 3$ \\
\hline 2.46623826 & 1.56936514 & -0.15553895 & -10.1 \\
\hline 623826 & 1.56936514 & -0.049703 & -10.5 \\
\hline-2.46623826 & 1.67520046 & -0.15553895 & 9.1 \\
\hline 623826 & 1.67520046 & -0.04970350 & 6.1 \\
\hline 040306 & 1.56936514 & -0.15553895 & 8.19623 \\
\hline-2.36040306 & 1.56936514 & -0.04970350 & 14.0563926 \\
\hline-2.36040306 & 1.67520046 & -0.15553895 & -7.658880 \\
\hline-2.36040306 & 1.67520046 & -0.04970350 & -8.44 \\
\hline & \# Energy 6->6 & & \\
\hline 9.49 & -0.1766 & & ctr. m \\
\hline 2.27520919 & 0.11177704 & -0.06075061 & 181.7 \\
\hline 20919 & 0.11177704 & 0.04508484 & -113.26 \\
\hline 2.27520919 & 0.21761249 & -0.06075061 & -175.8169708 \\
\hline 2.27520919 & 0.21761249 & 0.04508484 & 110.0456466 \\
\hline 463 & 0.11177704 & -0.06075061 & -177.93 \\
\hline 463 & 0.11177704 & 0.04508484 & 114.66 \\
\hline 2.38104463 & 0.21761249 & -0.06075061 & 173.148 \\
\hline 2.38104463 & 0.21761249 & 0.04508484 & -111.95 \\
\hline 047 & -0.18411981 & -0.04267391 & $-21 \cdot 79$ \\
\hline 0967047 & -0.18411981 & 0.06316154 & -6.4 \\
\hline-1.40967047 & -0.07828436 & -0.04267391 & 42.7 \\
\hline-1.40967047 & -0.07828436 & 0.063 & -8.4 \\
\hline$-1 \cdot 30$ & -0.18 & -0.042 & 17.5 \\
\hline 3503 & -0.1841 & 0.063 & .4 \\
\hline 0383503 & -0.07828436 & -0.04267391 & $-36 \cdot 4$ \\
\hline 503 & -0.07 & 0.06 & 9.5 \\
\hline 17 & 1.31 & -0.1357 & 26.3 \\
\hline 10117 & 1.31986856 & -0.02988423 & -11.17 \\
\hline 10117 & 1.42570400 & -0.13571967 & $-21 \cdot 33$ \\
\hline 2 . & 1.42 & -0.02 & \\
\hline 2.650 & 1.31986856 & -0.13571967 & -27.83 \\
\hline 2.65093660 & 1.31986856 & -0.02988423 & 15.17 \\
\hline 2.65093660 & 1.42570400 & -0.13571967 & 22.36 \\
\hline 2. & 1.42 & -0.029 & -13.2 \\
\hline 3156683 & -1.42 & 0.04030320 & 13 \\
\hline 3156683 & -1.42060065 & 0.14613864 & -20.1 \\
\hline-1 . & $-1 \cdot 31$ & 0.04 & -14.7 \\
\hline-1 & $-1 \cdot 31$ & 0.146 & 22 \\
\hline 73140 & -1.42 & 0.040 & $-13 \cdot 6$ \\
\hline 3140 & -1.42 & 0.146 & 18 \\
\hline-1 . & $-1 \cdot 31$ & 0.04 & 14 \\
\hline$-1 \cdot 32$ & $-1 \cdot 31$ & 0.146 & -20.9 \\
\hline 76527 & -0.329233 & -0.03195595 & 18.8 \\
\hline 0.99 & -0.329233 & 0.07387950 & -24.1 \\
\hline 0 . & -0 . & -0.03 & -6 \\
\hline 0.99 & -0.22 & 0.07387950 & 16. \\
\hline 1.09660077 & -0.329233 & -0.03195595 & -33.5 \\
\hline 1.09 & -0.32 & 0.07 & 34. \\
\hline 1.09 & -0 . & -0.0319 & 19.0 \\
\hline 1.09660077 & -0.223398 & 0.07387950 & $-25 \cdot 3$ \\
\hline-2.53 & 0.56 & -0.09333258 & 29 \\
\hline-2.53 & 0. & 0.0125 & -77.2 \\
\hline-2.53 & 0 . & -0.09333258 & $-12 \cdot 9$ \\
\hline-2.53 & 0 . & 0.01250287 & 71.2 \\
\hline-2.43 & & -0.09333258 & -40.1 \\
\hline 39709 & 0.56 & 0.01250287 & 90.4 \\
\hline-2.43 & 0. & -0.09333258 & 22.06 \\
\hline-2. & & 0.012 & -84 \\
\hline
\end{tabular}




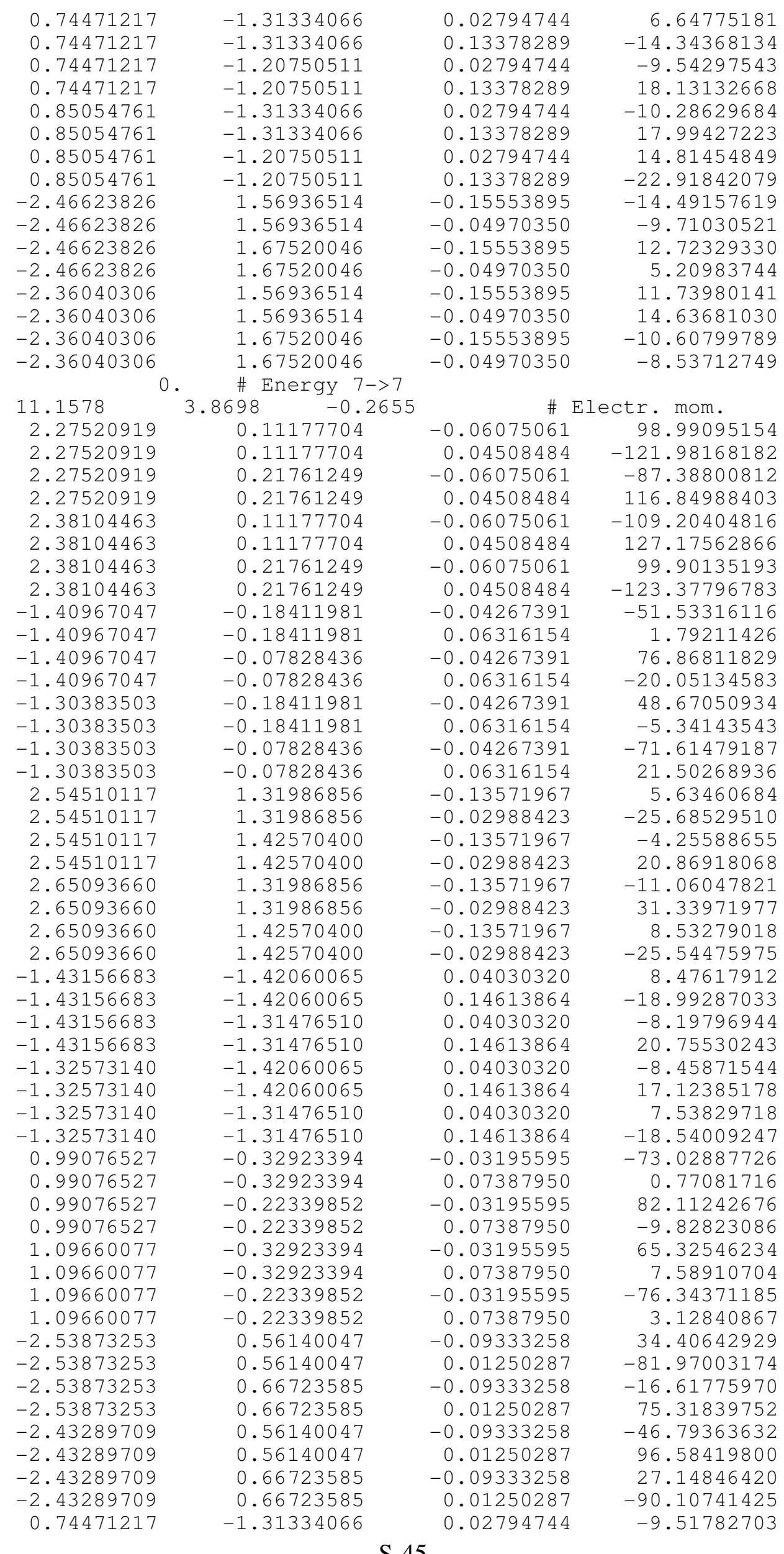




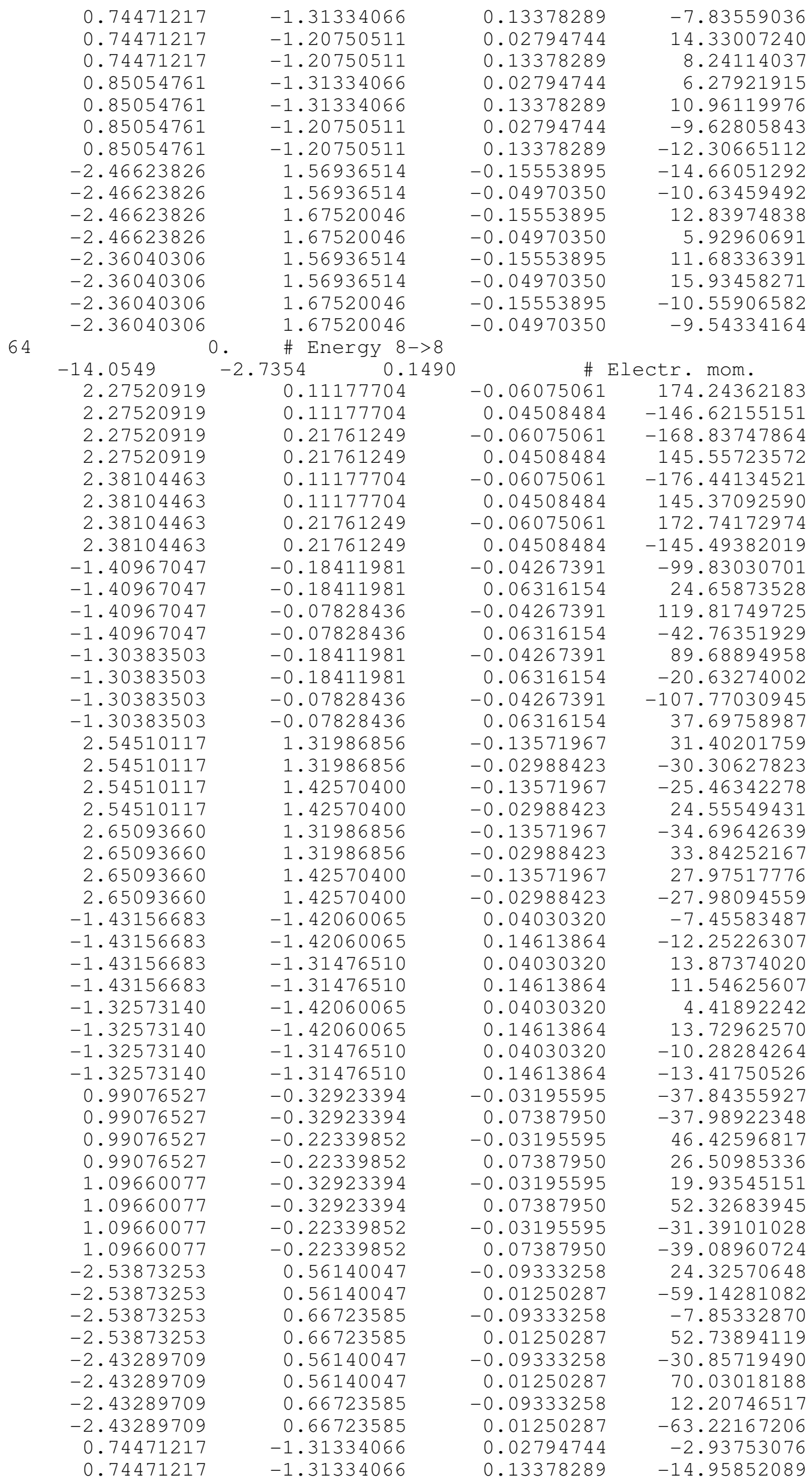




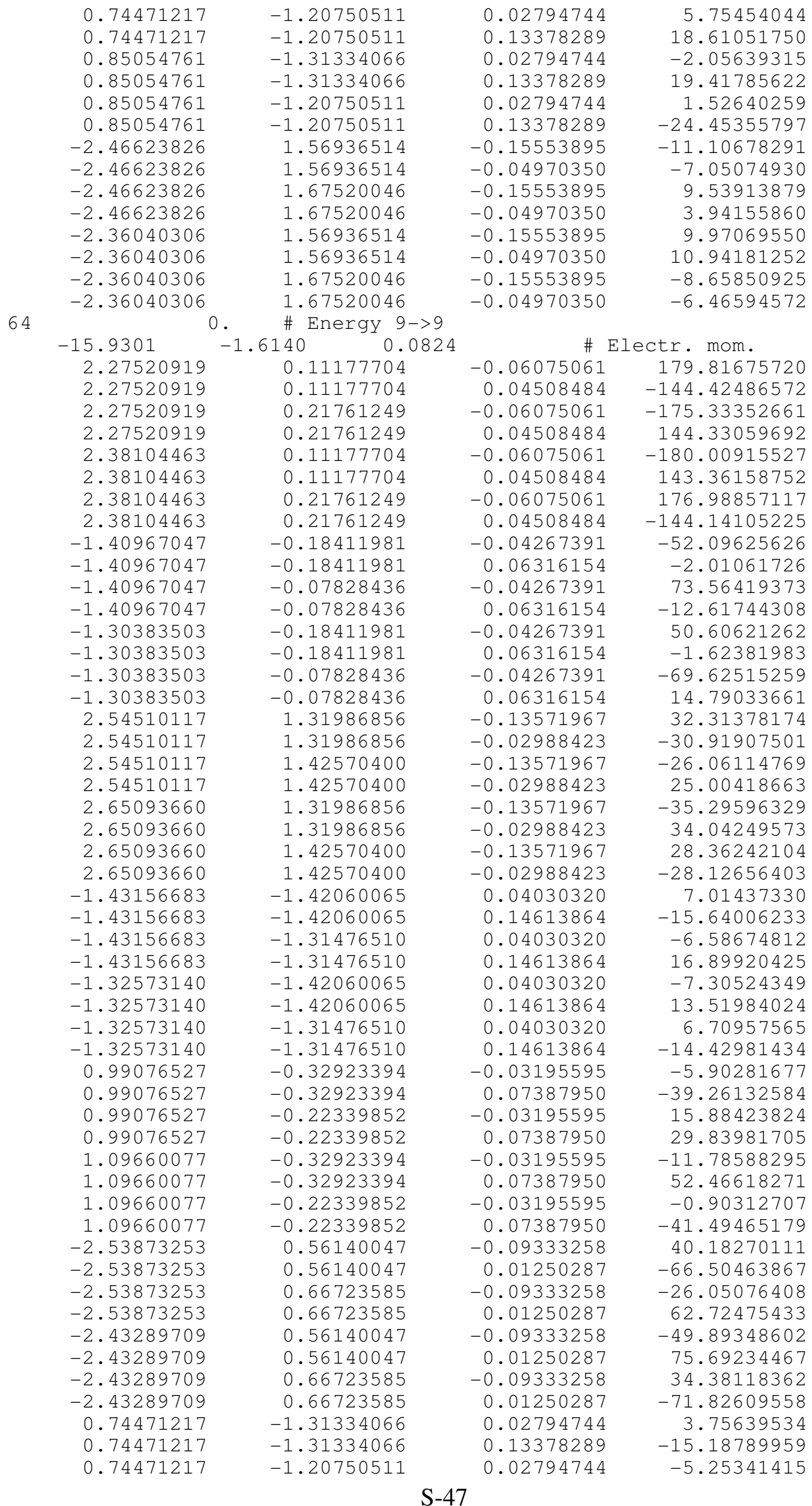




\begin{tabular}{|c|c|c|}
\hline & 0.74471217 & -1.20750511 \\
\hline & 0.85054761 & -1.31334066 \\
\hline & 0.85054761 & -1.31334066 \\
\hline & 0.85054761 & -1.20750511 \\
\hline & 0.85054761 & -1.20750511 \\
\hline & -2.46623826 & 1.56936514 \\
\hline & -2.46623826 & 1.56936514 \\
\hline & -2.46623826 & 1.67520046 \\
\hline & -2.46623826 & 1.67520046 \\
\hline & -2.36040306 & 1.56936514 \\
\hline & -2.36040306 & 1.56936514 \\
\hline & -2.36040306 & 1.67520046 \\
\hline & -2.36040306 & 1.67520046 \\
\hline$-\mathrm{CI}$ & $2009 \mathrm{~B}-$ & \\
\hline \# phi & $=-120, \mathrm{psi}=$ & $180, \operatorname{root} s=9$ \\
\hline & 2.2058 & 0.2180 \\
\hline & 2.3330 & 1.3623 \\
\hline & 1.1131 & -0.1680 \\
\hline & -1.2619 & -0.1414 \\
\hline & -1.1669 & -1.3365 \\
\hline & -2.4381 & -0.1351 \\
\hline$\& \mathrm{TRAN}$ & SITION $1->\ldots$ & \\
\hline 64 & 42611 & \# Energy 1->2 \\
\hline & -0.1902 & -0.0440 \\
\hline & 0.1428 & 0.8573 \\
\hline & 2.15289307 & 0.16506203 \\
\hline & 2.15289307 & 0.16506203 \\
\hline & 2.15289307 & 0.27089748 \\
\hline & 2.15289307 & 0.27089748 \\
\hline & 2.25872827 & 0.16506203 \\
\hline & 2.25872827 & 0.16506203 \\
\hline & 2.25872827 & 0.27089748 \\
\hline & 2.25872827 & 0.27089748 \\
\hline & -1.31485093 & -0.19434193 \\
\hline & -1.31485093 & -0.19434193 \\
\hline & -1.31485093 & -0.08850648 \\
\hline & -1.31485093 & -0.08850648 \\
\hline & -1.20901549 & -0.19434193 \\
\hline & -1.20901549 & -0.19434193 \\
\hline & -1.20901549 & -0.08850648 \\
\hline & -1.20901549 & -0.08850648 \\
\hline & 2.28008008 & 1.30933321 \\
\hline & 2.28008008 & 1.30933321 \\
\hline & 2.28008008 & 1.41516852 \\
\hline & 2.28008008 & 1.41516852 \\
\hline & 2.38591576 & 1.30933321 \\
\hline & 2.38591576 & 1.30933321 \\
\hline & 2.38591576 & 1.41516852 \\
\hline & 2.38591576 & 1.41516852 \\
\hline & -1.21978784 & -1.38936973 \\
\hline & -1.21978784 & -1.38936973 \\
\hline & -1.21978784 & -1.28353417 \\
\hline & -1.21978784 & -1.28353417 \\
\hline & -1.11395252 & -1.38936973 \\
\hline & -1.11395252 & -1.38936973 \\
\hline & -1.11395252 & -1.28353417 \\
\hline & -1.11395252 & -1.28353417 \\
\hline & 1.06017745 & -0.22089551 \\
\hline & 1.06017745 & -0.22089551 \\
\hline & 1.06017745 & -0.11506007 \\
\hline & 1.06017745 & -0.11506007 \\
\hline & 1.16601288 & -0.22089551 \\
\hline & 1.16601288 & -0.22089551 \\
\hline & 1.16601288 & -0.11506007 \\
\hline & 1.16601288 & -0.11506007 \\
\hline & -2.49104571 & 0.48034528 \\
\hline
\end{tabular}

TRANSITION $1->$. .
2.15289307

2.38591576

2.38591576

$-1.21978784$

$-1.21978784$

$-2.49104571$

$$
\begin{array}{r}
0.13378289 \\
0.02794744 \\
0.13378289 \\
0.02794744 \\
0.13378289 \\
-0.15553895 \\
-0.04970350 \\
-0.15553895 \\
-0.04970350 \\
-0.15553895 \\
-0.04970350 \\
-0.15553895 \\
-0.04970350
\end{array}
$$

\begin{tabular}{|c|c|c|c|c|c|}
\hline 2.2058 & 0.2180 & 0.0419 & 1.0000 & $\#$ & $\mathrm{C} 1$ \\
\hline 2.3330 & 1.3623 & 0.4928 & 1.0000 & \# & 01 \\
\hline 1.1131 & -0.1680 & -0.6958 & 1.0000 & \# & N1 \\
\hline-1.2619 & -0.1414 & -0.1731 & 1.0000 & $\#$ & C2 \\
\hline-1.1669 & -1.3365 & 0.1370 & 1.0000 & $\#$ & N2 \\
\hline-2.4381 & 0.5333 & -0.1351 & 1.0000 & $\#$ & 02 \\
\hline
\end{tabular}

19.12154388
-8.14827251
19.18485641
11.65761566
-24.39484024
-7.78814602
-10.48242378
7.18189907
6.42786360
5.90960598
13.44576454
-5.73066950
-8.32592773

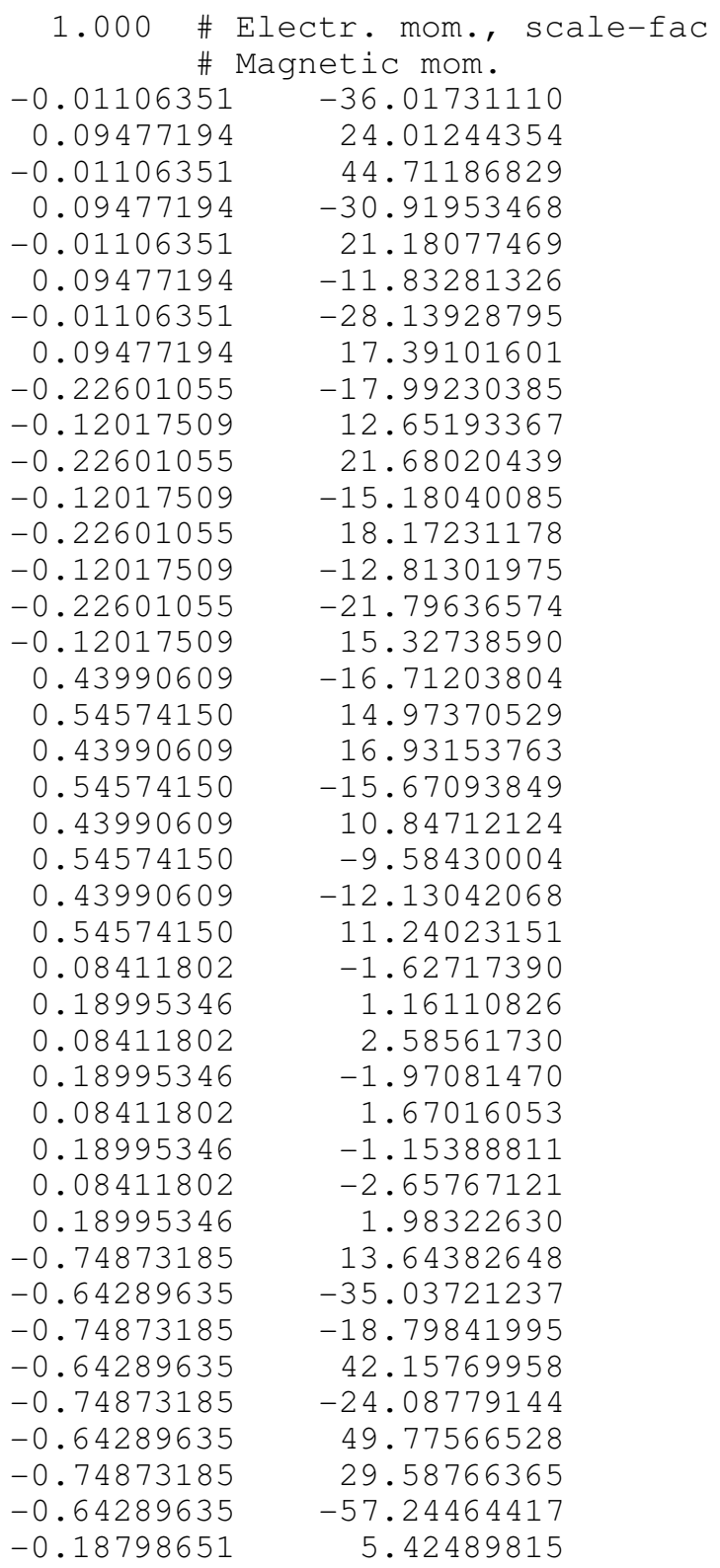




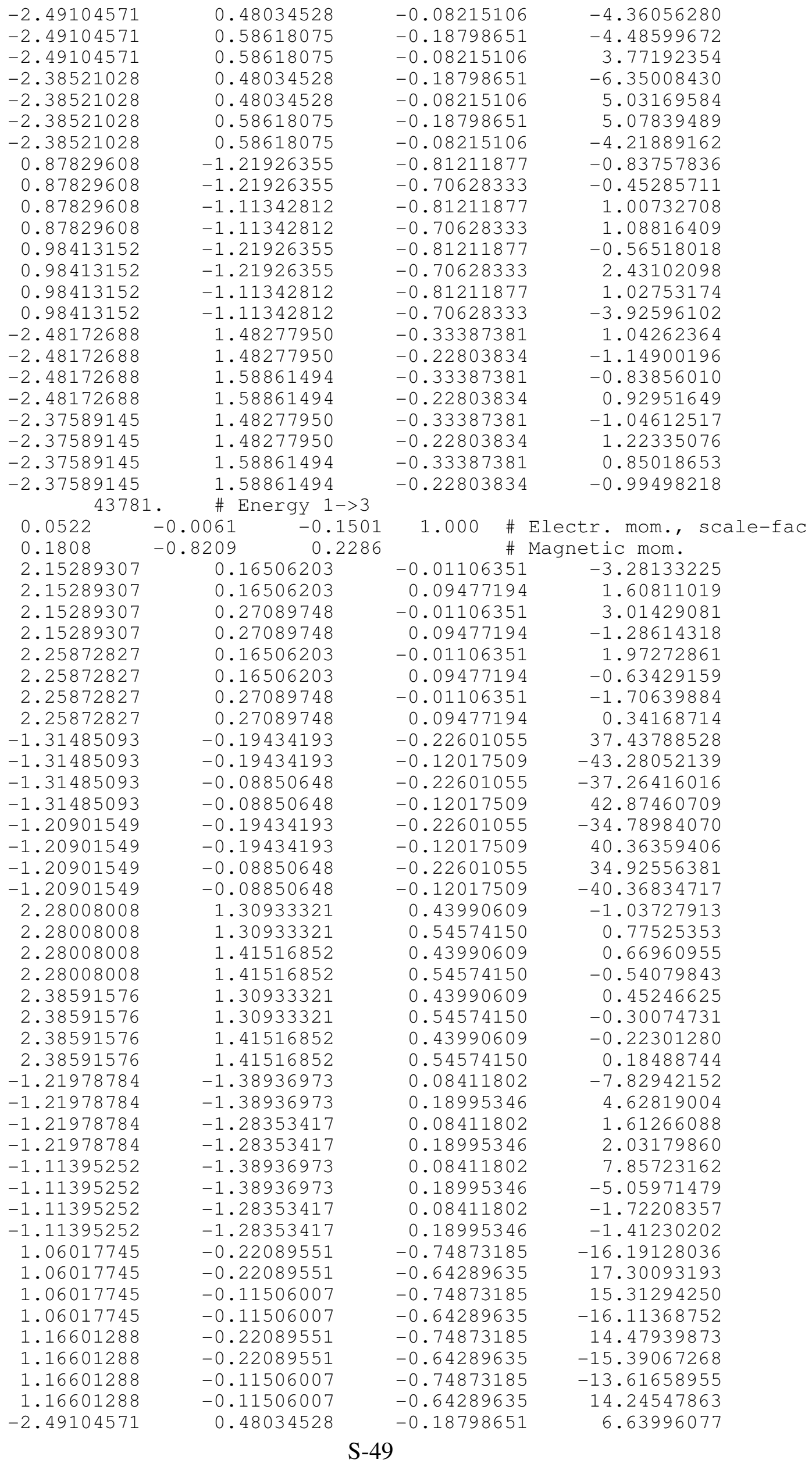




\begin{tabular}{|c|c|c|c|}
\hline-2.49104571 & 0.48034528 & -0.08215106 & -5.76252460 \\
\hline-2.49104571 & 0.58618075 & -0.18798651 & -9.23902035 \\
\hline-2.49104571 & 0.58618075 & -0.08215106 & 8.29741478 \\
\hline-2.38521028 & 0.48034528 & -0.18798651 & -7.02547216 \\
\hline-2.38521028 & 0.48034528 & -0.08215106 & 5.93646336 \\
\hline-2.38521028 & 0.58618075 & -0.18798651 & 9.99017620 \\
\hline-2.38521028 & 0.58618075 & -0.08215106 & -8.80924988 \\
\hline 0.87829608 & -1.21926355 & -0.81211877 & -1.88196731 \\
\hline 0.87829608 & -1.21926355 & -0.70628333 & 3.02270365 \\
\hline 0.87829608 & -1.11342812 & -0.81211877 & 4.18851185 \\
\hline 0.87829608 & -1.11342812 & -0.70628333 & -5.87868738 \\
\hline 0.98413152 & -1.21926355 & -0.81211877 & 1.81435502 \\
\hline 0.98413152 & -1.21926355 & -0.70628333 & -2.85309172 \\
\hline 0.98413152 & -1.11342812 & -0.81211877 & -3.88366604 \\
\hline 0.98413152 & -1.11342812 & -0.70628333 & 5.43080759 \\
\hline-2.48172688 & 1.48277950 & -0.33387381 & 1.80195022 \\
\hline-2.48172688 & 1.48277950 & -0.22803834 & -1.80814791 \\
\hline-2.48172688 & 1.58861494 & -0.33387381 & -1.44581926 \\
\hline-2.48172688 & 1.58861494 & -0.22803834 & 1.51041365 \\
\hline-2.37589145 & 1.48277950 & -0.33387381 & -1.94145024 \\
\hline-2.37589145 & 1.48277950 & -0.22803834 & 1.80419481 \\
\hline-2.37589145 & 1.58861494 & -0.33387381 & 1.50474596 \\
\hline-2.37589145 & 1.58861494 & -0.22803834 & -1.45328856 \\
\hline 52792 & \# Energy 1->4 & & \\
\hline 2.7537 & $\begin{array}{ll}1.1743 & 1.9769\end{array}$ & 1.000 & Electr. mom., scale-fac \\
\hline 0.1368 & -1.1919 & $\#$ & Magnetic mom. \\
\hline 2.15289307 & 0.16506203 & -0.01106351 & -43.58995819 \\
\hline 2.15289307 & 0.16506203 & 0.09477194 & 32.56782150 \\
\hline 2.15289307 & 0.27089748 & -0.01106351 & 44.70822525 \\
\hline 2.15289307 & 0.27089748 & 0.09477194 & -33.13845062 \\
\hline 2.25872827 & 0.16506203 & -0.01106351 & 31.85758781 \\
\hline 2.25872827 & 0.16506203 & 0.09477194 & -22.45444107 \\
\hline 2.25872827 & 0.27089748 & -0.01106351 & -31.94415283 \\
\hline 2.25872827 & 0.27089748 & 0.09477194 & 21.92689133 \\
\hline-1.31485093 & -0.19434193 & -0.22601055 & 6.72975826 \\
\hline-1.31485093 & -0.19434193 & -0.12017509 & -13.72211647 \\
\hline-1.31485093 & -0.08850648 & -0.22601055 & -7.60719585 \\
\hline-1.31485093 & -0.08850648 & -0.12017509 & 15.54795647 \\
\hline-1.20901549 & -0.19434193 & -0.22601055 & -3.81245279 \\
\hline-1.20901549 & -0.19434193 & -0.12017509 & 9.32408905 \\
\hline-1.20901549 & -0.08850648 & -0.22601055 & 4.81962013 \\
\hline-1.20901549 & -0.08850648 & -0.12017509 & -11.26643562 \\
\hline 2.28008008 & 1.30933321 & 0.43990609 & 3.30124545 \\
\hline 2.28008008 & 1.30933321 & 0.54574150 & -2.53284931 \\
\hline 2.28008008 & 1.41516852 & 0.43990609 & -4.27181196 \\
\hline 2.28008008 & 1.41516852 & 0.54574150 & 3.69016337 \\
\hline 2.38591576 & 1.30933321 & 0.43990609 & -4.42687130 \\
\hline 2.38591576 & 1.30933321 & 0.54574150 & 4.48581982 \\
\hline 2.38591576 & 1.41516852 & 0.43990609 & 5.99488926 \\
\hline 2.38591576 & 1.41516852 & 0.54574150 & -5.84747314 \\
\hline-1.21978784 & -1.38936973 & 0.08411802 & 0.81704372 \\
\hline-1.21978784 & -1.38936973 & 0.18995346 & -1.65478909 \\
\hline-1.21978784 & -1.28353417 & 0.08411802 & -0.05401893 \\
\hline-1.21978784 & -1.28353417 & 0.18995346 & 1.23715341 \\
\hline-1.11395252 & -1.38936973 & 0.08411802 & -0.29666656 \\
\hline-1.11395252 & -1.38936973 & 0.18995346 & 0.84957272 \\
\hline-1.11395252 & -1.28353417 & 0.08411802 & -0.35552475 \\
\hline-1.11395252 & -1.28353417 & 0.18995346 & -0.34893277 \\
\hline 1.06017745 & -0.22089551 & -0.74873185 & 39.71094894 \\
\hline 1.06017745 & -0.22089551 & -0.64289635 & -64.89835358 \\
\hline 1.06017745 & -0.11506007 & -0.74873185 & -40.29483414 \\
\hline 1.06017745 & -0.11506007 & -0.64289635 & 64.37893677 \\
\hline 1.16601288 & -0.22089551 & -0.74873185 & -48.00180435 \\
\hline 1.16601288 & -0.22089551 & -0.64289635 & 76.11298370 \\
\hline 1.16601288 & -0.11506007 & -0.74873185 & 48.00011826 \\
\hline 1.16601288 & -0.11506007 & -0.64289635 & -75.10311127 \\
\hline-2.49104571 & 0.48034528 & -0.18798651 & -4.94632196 \\
\hline
\end{tabular}




\begin{tabular}{|c|c|c|c|}
\hline & & & \\
\hline-2.49104571 & 0.48034528 & -0.08215106 & 2.50185895 \\
\hline-2.49104571 & 0.58618075 & -0.18798651 & 4.69213247 \\
\hline-2.49104571 & 0.58618075 & -0.08215106 & -1.87511945 \\
\hline-2.38521028 & 0.48034528 & -0.18798651 & 6.34654140 \\
\hline-2.38521028 & 0.48034528 & -0.08215106 & -3.37563920 \\
\hline-2.38521028 & 0.58618075 & -0.18798651 & -6.26481485 \\
\hline-2.38521028 & 0.58618075 & -0.08215106 & 2.57531238 \\
\hline 0.87829608 & -1.21926355 & -0.81211877 & 1.83888304 \\
\hline 0.87829608 & -1.21926355 & -0.70628333 & -5.23328018 \\
\hline 0.87829608 & -1.11342812 & -0.81211877 & -3.39646745 \\
\hline 0.87829608 & -1.11342812 & -0.70628333 & 8.48740673 \\
\hline 0.98413152 & -1.21926355 & -0.81211877 & -3.00017595 \\
\hline 0.98413152 & -1.21926355 & -0.70628333 & 6.87765408 \\
\hline 0.98413152 & -1.11342812 & -0.81211877 & 5.09066343 \\
\hline 0.98413152 & -1.11342812 & -0.70628333 & -10.83781719 \\
\hline-2.48172688 & 1.48277950 & -0.33387381 & -1.56691265 \\
\hline-2.48172688 & 1.48277950 & -0.22803834 & 1.26804543 \\
\hline-2.48172688 & 1.58861494 & -0.33387381 & 1.26134086 \\
\hline-2.48172688 & 1.58861494 & -0.22803834 & -1.06882215 \\
\hline-2.37589145 & 1.48277950 & -0.33387381 & 1.68687582 \\
\hline-2.37589145 & 1.48277950 & -0.22803834 & -1.12151170 \\
\hline-2.37589145 & 1.58861494 & -0.33387381 & -1.33023775 \\
\hline-2.37589145 & 1.58861494 & -0.22803834 & 0.95182699 \\
\hline 5350 & \# Energy 1->5 & & \\
\hline 1.7283 & $-3.3311 \quad-0.4493$ & 1.000 & Electr. mom., scale-fac \\
\hline 0.0415 & -0.2781 & \# & Magnetic mom. \\
\hline 2.15289307 & 0.16506203 & -0.01106351 & 13.53247929 \\
\hline 2.15289307 & 0.16506203 & 0.09477194 & -5.87827110 \\
\hline 2.15289307 & 0.27089748 & -0.01106351 & -10.84750557 \\
\hline 2.15289307 & 0.27089748 & 0.09477194 & 3.59171939 \\
\hline 2.25872827 & 0.16506203 & -0.01106351 & -8.04648304 \\
\hline 2.25872827 & 0.16506203 & 0.09477194 & 1.64299548 \\
\hline 2.25872827 & 0.27089748 & -0.01106351 & 5.69677162 \\
\hline 2.25872827 & 0.27089748 & 0.09477194 & 0.50188744 \\
\hline-1.31485093 & -0.19434193 & -0.22601055 & 26.35551834 \\
\hline-1.31485093 & -0.19434193 & -0.12017509 & -36.39164734 \\
\hline-1.31485093 & -0.08850648 & -0.22601055 & -24.72663307 \\
\hline-1.31485093 & -0.08850648 & -0.12017509 & 37.07462311 \\
\hline-1.20901549 & -0.19434193 & -0.22601055 & -22.72380066 \\
\hline-1.20901549 & -0.19434193 & -0.12017509 & 28.16882133 \\
\hline-1.20901549 & -0.08850648 & -0.22601055 & 21.58570671 \\
\hline-1.20901549 & -0.08850648 & -0.12017509 & -29.48170662 \\
\hline 2.28008008 & 1.30933321 & 0.43990609 & -2.19702411 \\
\hline 2.28008008 & 1.30933321 & 0.54574150 & 2.35644746 \\
\hline 2.28008008 & 1.41516852 & 0.43990609 & 2.56612039 \\
\hline 2.28008008 & 1.41516852 & 0.54574150 & -2.63760948 \\
\hline 2.38591576 & 1.30933321 & 0.43990609 & 2.47583532 \\
\hline 2.38591576 & 1.30933321 & 0.54574150 & -2.93842196 \\
\hline 2.38591576 & 1.41516852 & 0.43990609 & -3.06603980 \\
\hline 2.38591576 & 1.41516852 & 0.54574150 & 3.30049181 \\
\hline-1.21978784 & -1.38936973 & 0.08411802 & 9.97892475 \\
\hline-1.21978784 & -1.38936973 & 0.18995346 & -10.47235298 \\
\hline-1.21978784 & -1.28353417 & 0.08411802 & -10.97499561 \\
\hline-1.21978784 & -1.28353417 & 0.18995346 & 11.78843880 \\
\hline-1.11395252 & -1.38936973 & 0.08411802 & -10.16969681 \\
\hline-1.11395252 & -1.38936973 & 0.18995346 & 9.40037441 \\
\hline-1.11395252 & -1.28353417 & 0.08411802 & 12.04946423 \\
\hline-1.11395252 & -1.28353417 & 0.18995346 & -10.92565060 \\
\hline 1.06017745 & -0.22089551 & -0.74873185 & -19.57002258 \\
\hline 1.06017745 & -0.22089551 & -0.64289635 & 28.75411606 \\
\hline 1.06017745 & -0.11506007 & -0.74873185 & 14.10875797 \\
\hline 1.06017745 & -0.11506007 & -0.64289635 & -20.85574341 \\
\hline 1.16601288 & -0.22089551 & -0.74873185 & 24.79136848 \\
\hline 1.16601288 & -0.22089551 & -0.64289635 & -35.83337021 \\
\hline 1.16601288 & -0.11506007 & -0.74873185 & -18.62865829 \\
\hline 1.16601288 & -0.11506007 & -0.64289635 & 27.08741188 \\
\hline-2.49104571 & 0.48034528 & -0.18798651 & -6.96047354 \\
\hline
\end{tabular}




\begin{tabular}{|c|c|c|c|}
\hline-2.49104571 & 0.48034528 & -0.08215106 & 1.73073184 \\
\hline-2.49104571 & 0.58618075 & -0.18798651 & 7.54892826 \\
\hline-2.49104571 & 0.58618075 & -0.08215106 & -1.58594334 \\
\hline-2.38521028 & 0.48034528 & -0.18798651 & 8.20784855 \\
\hline-2.38521028 & 0.48034528 & -0.08215106 & -1.73382187 \\
\hline-2.38521028 & 0.58618075 & -0.18798651 & -9.39949989 \\
\hline-2.38521028 & 0.58618075 & -0.08215106 & 1.42954862 \\
\hline 0.87829608 & -1.21926355 & -0.81211877 & -2.06702518 \\
\hline 0.87829608 & -1.21926355 & -0.70628333 & 4.28515148 \\
\hline 0.87829608 & -1.11342812 & -0.81211877 & 4.42766857 \\
\hline 0.87829608 & -1.11342812 & -0.70628333 & -7.80956650 \\
\hline 0.98413152 & -1.21926355 & -0.81211877 & 2.92035675 \\
\hline 0.98413152 & -1.21926355 & -0.70628333 & -5.44129992 \\
\hline 0.98413152 & -1.11342812 & -0.81211877 & -5.56007195 \\
\hline 0.98413152 & -1.11342812 & -0.70628333 & 9.36739922 \\
\hline-2.48172688 & 1.48277950 & -0.33387381 & -4.52623272 \\
\hline-2.48172688 & 1.48277950 & -0.22803834 & 3.98602772 \\
\hline-2.48172688 & 1.58861494 & -0.33387381 & 3.47899485 \\
\hline-2.48172688 & 1.58861494 & -0.22803834 & -3.14743519 \\
\hline-2.37589145 & 1.48277950 & -0.33387381 & 4.51788235 \\
\hline-2.37589145 & 1.48277950 & -0.22803834 & -3.34070063 \\
\hline-2.37589145 & 1.58861494 & -0.33387381 & -3.40916228 \\
\hline-2.37589145 & 1.58861494 & -0.22803834 & 2.63805580 \\
\hline 59891 & \# Energy 1->6 & & \\
\hline-0.1109 & -0.5165 & 1.000 & Electr. mom., scale-fac \\
\hline 0.1093 & 0.0103 & II & Magnetic mom. \\
\hline 2.15289307 & 0.16506203 & -0.01106351 & -5.03410339 \\
\hline 2.15289307 & 0.16506203 & 0.09477194 & 4.91826868 \\
\hline 2.15289307 & 0.27089748 & -0.01106351 & 4.76515722 \\
\hline 2.15289307 & 0.27089748 & 0.09477194 & -4.58457756 \\
\hline 2.25872827 & 0.16506203 & -0.01106351 & 4.03961229 \\
\hline 2.25872827 & 0.16506203 & 0.09477194 & -4.13818645 \\
\hline 2.25872827 & 0.27089748 & -0.01106351 & -3.72974443 \\
\hline 2.25872827 & 0.27089748 & 0.09477194 & 3.82678342 \\
\hline-1.31485093 & -0.19434193 & -0.22601055 & 26.48093605 \\
\hline-1.31485093 & -0.19434193 & -0.12017509 & -27.34802628 \\
\hline-1.31485093 & -0.08850648 & -0.22601055 & -29.84650421 \\
\hline-1.31485093 & -0.08850648 & -0.12017509 & 30.12029648 \\
\hline-1.20901549 & -0.19434193 & -0.22601055 & -28.87833595 \\
\hline-1.20901549 & -0.19434193 & -0.12017509 & 30.55920601 \\
\hline-1.20901549 & -0.08850648 & -0.22601055 & 32.11171722 \\
\hline-1.20901549 & -0.08850648 & -0.12017509 & -33.11984253 \\
\hline 2.28008008 & 1.30933321 & 0.43990609 & 0.06604259 \\
\hline 2.28008008 & 1.30933321 & 0.54574150 & -0.34092042 \\
\hline 2.28008008 & 1.41516852 & 0.43990609 & -0.04190649 \\
\hline 2.28008008 & 1.41516852 & 0.54574150 & 0.29777122 \\
\hline 2.38591576 & 1.30933321 & 0.43990609 & -0.36345175 \\
\hline 2.38591576 & 1.30933321 & 0.54574150 & 0.48543969 \\
\hline 2.38591576 & 1.41516852 & 0.43990609 & 0.21015404 \\
\hline 2.38591576 & 1.41516852 & 0.54574150 & -0.35460275 \\
\hline-1.21978784 & -1.38936973 & 0.08411802 & 0.24174353 \\
\hline-1.21978784 & -1.38936973 & 0.18995346 & -0.32254481 \\
\hline-1.21978784 & -1.28353417 & 0.08411802 & -0.68279546 \\
\hline-1.21978784 & -1.28353417 & 0.18995346 & 0.83765423 \\
\hline-1.11395252 & -1.38936973 & 0.08411802 & -0.92650658 \\
\hline-1.11395252 & -1.38936973 & 0.18995346 & 1.23152590 \\
\hline-1.11395252 & -1.28353417 & 0.08411802 & 1.29187751 \\
\hline-1.11395252 & -1.28353417 & 0.18995346 & -1.81793392 \\
\hline 1.06017745 & -0.22089551 & -0.74873185 & 7.19771385 \\
\hline 1.06017745 & -0.22089551 & -0.64289635 & -10.92832279 \\
\hline 1.06017745 & -0.11506007 & -0.74873185 & -9.63024902 \\
\hline 1.06017745 & -0.11506007 & -0.64289635 & 13.86188698 \\
\hline 1.16601288 & -0.22089551 & -0.74873185 & -7.37547493 \\
\hline 1.16601288 & -0.22089551 & -0.64289635 & 11.23470974 \\
\hline 1.16601288 & -0.11506007 & -0.74873185 & 9.62461281 \\
\hline 1.16601288 & -0.11506007 & -0.64289635 & -13.97888088 \\
\hline-2.49104571 & 0.48034528 & -0.18798651 & -2.54752731 \\
\hline
\end{tabular}




\begin{tabular}{|c|c|c|c|}
\hline-2.49104571 & 0.48034528 & -0.08215106 & 1.86062670 \\
\hline-2.49104571 & 0.58618075 & -0.18798651 & 1.27643383 \\
\hline-2.49104571 & 0.58618075 & -0.08215106 & -0.69061673 \\
\hline-2.38521028 & 0.48034528 & -0.18798651 & 3.00786424 \\
\hline-2.38521028 & 0.48034528 & -0.08215106 & -2.24637699 \\
\hline-2.38521028 & 0.58618075 & -0.18798651 & -1.39865303 \\
\hline-2.38521028 & 0.58618075 & -0.08215106 & 0.78531140 \\
\hline 0.87829608 & -1.21926355 & -0.81211877 & -0.24750675 \\
\hline 0.87829608 & -1.21926355 & -0.70628333 & 0.18153474 \\
\hline 0.87829608 & -1.11342812 & -0.81211877 & 0.19315828 \\
\hline 0.87829608 & -1.11342812 & -0.70628333 & -0.05315339 \\
\hline 0.98413152 & -1.21926355 & -0.81211877 & -0.01090996 \\
\hline 0.98413152 & -1.21926355 & -0.70628333 & 0.09784668 \\
\hline 0.98413152 & -1.11342812 & -0.81211877 & 0.15792055 \\
\hline 0.98413152 & -1.11342812 & -0.70628333 & -0.32562876 \\
\hline-2.48172688 & 1.48277950 & -0.33387381 & 0.29884186 \\
\hline-2.48172688 & 1.48277950 & -0.22803834 & -0.44067797 \\
\hline-2.48172688 & 1.58861494 & -0.33387381 & -0.26428077 \\
\hline-2.48172688 & 1.58861494 & -0.22803834 & 0.34753868 \\
\hline-2.37589145 & 1.48277950 & -0.33387381 & -0.41547003 \\
\hline-2.37589145 & 1.48277950 & -0.22803834 & 0.55226320 \\
\hline-2.37589145 & 1.58861494 & -0.33387381 & 0.36120763 \\
\hline-2.37589145 & 1.58861494 & -0.22803834 & -0.43994540 \\
\hline 65272 & \# Energy 1->7 & & \\
\hline-0.8726 & $\begin{array}{ll}-0.4198 & -0.1449\end{array}$ & 1.000 & Electr. mom., scale-fac \\
\hline 0.0225 & -0.1443 & \# & Magnetic mom. \\
\hline 2.15289307 & 0.16506203 & -0.01106351 & 8.51914597 \\
\hline 2.15289307 & 0.16506203 & 0.09477194 & -4.87403584 \\
\hline 2.15289307 & 0.27089748 & -0.01106351 & -7.44387388 \\
\hline 2.15289307 & 0.27089748 & 0.09477194 & 3.82235217 \\
\hline 2.25872827 & 0.16506203 & -0.01106351 & -6.25197744 \\
\hline 2.25872827 & 0.16506203 & 0.09477194 & 3.15801120 \\
\hline 2.25872827 & 0.27089748 & -0.01106351 & 5.27553892 \\
\hline 2.25872827 & 0.27089748 & 0.09477194 & -2.22565126 \\
\hline-1.31485093 & -0.19434193 & -0.22601055 & -0.06793257 \\
\hline-1.31485093 & -0.19434193 & -0.12017509 & 3.38392687 \\
\hline-1.31485093 & -0.08850648 & -0.22601055 & -0.64397174 \\
\hline-1.31485093 & -0.08850648 & -0.12017509 & -3.08227086 \\
\hline-1.20901549 & -0.19434193 & -0.22601055 & 0.98631340 \\
\hline-1.20901549 & -0.19434193 & -0.12017509 & -4.42994499 \\
\hline-1.20901549 & -0.08850648 & -0.22601055 & -0.06308933 \\
\hline-1.20901549 & -0.08850648 & -0.12017509 & 3.74753785 \\
\hline 2.28008008 & 1.30933321 & 0.43990609 & 1.27856123 \\
\hline 2.28008008 & 1.30933321 & 0.54574150 & -0.84659845 \\
\hline 2.28008008 & 1.41516852 & 0.43990609 & -0.88200068 \\
\hline 2.28008008 & 1.41516852 & 0.54574150 & 0.64415514 \\
\hline 2.38591576 & 1.30933321 & 0.43990609 & -1.00064886 \\
\hline 2.38591576 & 1.30933321 & 0.54574150 & 0.59530294 \\
\hline 2.38591576 & 1.41516852 & 0.43990609 & 0.64214611 \\
\hline 2.38591576 & 1.41516852 & 0.54574150 & -0.43574327 \\
\hline-1.21978784 & -1.38936973 & 0.08411802 & 2.60707831 \\
\hline-1.21978784 & -1.38936973 & 0.18995346 & -2.19812250 \\
\hline-1.21978784 & -1.28353417 & 0.08411802 & -2.71246099 \\
\hline-1.21978784 & -1.28353417 & 0.18995346 & 2.18777633 \\
\hline-1.11395252 & -1.38936973 & 0.08411802 & -2.38277936 \\
\hline-1.11395252 & -1.38936973 & 0.18995346 & 1.80040455 \\
\hline-1.11395252 & -1.28353417 & 0.08411802 & 2.54963994 \\
\hline-1.11395252 & -1.28353417 & 0.18995346 & -1.74766421 \\
\hline 1.06017745 & -0.22089551 & -0.74873185 & -1.99739718 \\
\hline 1.06017745 & -0.22089551 & -0.64289635 & 5.17046213 \\
\hline 1.06017745 & -0.11506007 & -0.74873185 & 1.38151109 \\
\hline 1.06017745 & -0.11506007 & -0.64289635 & -4.16659403 \\
\hline 1.16601288 & -0.22089551 & -0.74873185 & 4.37595129 \\
\hline 1.16601288 & -0.22089551 & -0.64289635 & -8.11864758 \\
\hline 1.1 & -0.11506007 & -0.74873185 & -3.56156278 \\
\hline 1.16601288 & -0.11506007 & -0.64289635 & 6.86931658 \\
\hline-2.49104 & 0.48034528 & -0.18798651 & 0.40 \\
\hline
\end{tabular}




\begin{tabular}{|c|c|c|c|}
\hline-2.49104571 & 0.48034528 & -0.08215106 & 0.64136159 \\
\hline-2.49104571 & 0.58618075 & -0.18798651 & -0.63193744 \\
\hline-2.49104571 & 0.58618075 & -0.08215106 & -0.65892023 \\
\hline-2.38521028 & 0.48034528 & -0.18798651 & -1.02062917 \\
\hline-2.38521028 & 0.48034528 & -0.08215106 & -0.05213632 \\
\hline-2.38521028 & 0.58618075 & -0.18798651 & 1.38359666 \\
\hline-2.38521028 & 0.58618075 & -0.08215106 & 0.05539054 \\
\hline 0.87829608 & -1.21926355 & -0.81211877 & 0.11222284 \\
\hline 0.87829608 & -1.21926355 & -0.70628333 & 0.24034110 \\
\hline 0.87829608 & -1.11342812 & -0.81211877 & -0.00522241 \\
\hline 0.87829608 & -1.11342812 & -0.70628333 & -0.56389332 \\
\hline 0.98413152 & -1.21926355 & -0.81211877 & 0.24595384 \\
\hline 0.98413152 & -1.21926355 & -0.70628333 & -0.70256746 \\
\hline 0.98413152 & -1.11342812 & -0.81211877 & -0.51447511 \\
\hline 0.98413152 & -1.11342812 & -0.70628333 & 1.22872233 \\
\hline-2.48172688 & 1.48277950 & -0.33387381 & 0.17134906 \\
\hline-2.48172688 & 1.48277950 & -0.22803834 & 0.10312744 \\
\hline-2.48172688 & 1.58861494 & -0.33387381 & -0.13840353 \\
\hline-2.48172688 & 1.58861494 & -0.22803834 & -0.04827475 \\
\hline-2.37589145 & 1.48277950 & -0.33387381 & -0.35445860 \\
\hline-2.37589145 & 1.48277950 & -0.22803834 & 0.01484866 \\
\hline-2.37589145 & 1.58861494 & -0.33387381 & 0.27279368 \\
\hline-2.37589145 & 1.58861494 & -0.22803834 & -0.04399500 \\
\hline 7038 & \# Energy 1->8 & & \\
\hline-1.2071 & $0.4822 \quad-0.0554$ & 1.000 & Electr. mom., scale-fac \\
\hline-0.0142 & -0.8110 & & Magnetic mom. \\
\hline 2.15289307 & 0.16506203 & -0.01106351 & -2.64349890 \\
\hline 2.15289307 & 0.16506203 & 0.09477194 & 2.70889258 \\
\hline 2.15289307 & 0.27089748 & -0.01106351 & 11.60466576 \\
\hline 2.15289307 & 0.27089748 & 0.09477194 & -10.62534904 \\
\hline 2.25872827 & 0.16506203 & -0.01106351 & 2.33553433 \\
\hline 2.25872827 & 0.16506203 & 0.09477194 & -2.66674876 \\
\hline 2.25872827 & 0.27089748 & -0.01106351 & -9.97769547 \\
\hline 2.25872827 & 0.27089748 & 0.09477194 & 9.12746716 \\
\hline-1.31485093 & -0.19434193 & -0.22601055 & 6.28119516 \\
\hline-1.31485093 & -0.19434193 & -0.12017509 & -4.69052553 \\
\hline-1.31485093 & -0.08850648 & -0.22601055 & -5.49536037 \\
\hline-1.31485093 & -0.08850648 & -0.12017509 & 3.96849179 \\
\hline-1.20901549 & -0.19434193 & -0.22601055 & -5.65422964 \\
\hline-1.20901549 & -0.19434193 & -0.12017509 & 3.78989291 \\
\hline-1.20901549 & -0.08850648 & -0.22601055 & 5.13075829 \\
\hline-1.20901549 & -0.08850648 & -0.12017509 & -3.70477247 \\
\hline 2.28008008 & 1.30933321 & 0.43990609 & 2.56443810 \\
\hline 2.28008008 & 1.30933321 & 0.54574150 & -1.19674850 \\
\hline 2.28008008 & 1.41516852 & 0.43990609 & -2.79520440 \\
\hline 2.28008008 & 1.41516852 & 0.54574150 & 1.75828946 \\
\hline 2.38591576 & 1.30933321 & 0.43990609 & -1.81843626 \\
\hline 2.38591576 & 1.30933321 & 0.54574150 & 1.25053608 \\
\hline 2.38591576 & 1.41516852 & 0.43990609 & 2.67261744 \\
\hline 2.38591576 & 1.41516852 & 0.54574150 & -2.16232228 \\
\hline-1.21978784 & -1.38936973 & 0.08411802 & 3.83951640 \\
\hline-1.21978784 & -1.38936973 & 0.18995346 & -3.59615564 \\
\hline-1.21978784 & -1.28353417 & 0.08411802 & -4.35906124 \\
\hline-1.21978784 & -1.28353417 & 0.18995346 & 4.02610064 \\
\hline-1.11395252 & -1.38936973 & 0.08411802 & -3.71000862 \\
\hline-1.11395252 & -1.38936973 & 0.18995346 & 3.06096530 \\
\hline-1.11395252 & -1.28353417 & 0.08411802 & 4.45385075 \\
\hline-1.11395252 & -1.28353417 & 0.18995346 & -3.50506592 \\
\hline 1.06017745 & -0.22089551 & -0.74873185 & 4.22658110 \\
\hline 1.06017745 & -0.22089551 & -0.64289635 & -11.41429710 \\
\hline 1.06017745 & -0.11506007 & -0.74873185 & -9.45405293 \\
\hline 1.06017745 & -0.11506007 & -0.64289635 & 18.65522385 \\
\hline 1.16601288 & -0.22089551 & -0.74873185 & -8.01566219 \\
\hline 1.16601288 & -0.22089551 & -0.64289635 & 16.52202034 \\
\hline 1.16601288 & -0.11506007 & -0.74873185 & 13.51072407 \\
\hline 1.16601288 & -0.11506007 & -0.64289635 & -24.08973503 \\
\hline-2.49104571 & 0.48034528 & -0.18798651 & 5.47842264 \\
\hline
\end{tabular}




\begin{tabular}{|c|c|}
\hline & \\
\hline-2.49104571 & 0.48034528 \\
\hline-2.49104571 & 0.58618075 \\
\hline-2.49104571 & 0.58618075 \\
\hline-2.38521028 & 0.48034528 \\
\hline-2.38521028 & 0.48034528 \\
\hline-2.38521028 & 0.58618075 \\
\hline-2.38521028 & 0.58618075 \\
\hline 0.87829608 & -1.21926355 \\
\hline 0.87829608 & -1.21926355 \\
\hline 0.87829608 & -1.11342812 \\
\hline 0.87829608 & -1.11342812 \\
\hline 0.98413152 & -1.21926355 \\
\hline 0.98413152 & -1.21926355 \\
\hline 0.98413152 & -1.11342812 \\
\hline 0.98413152 & -1.11342812 \\
\hline-2.48172688 & 1.48277950 \\
\hline-2.48172688 & 1.48277950 \\
\hline-2.48172688 & 1.58861494 \\
\hline-2.48172688 & 1.58861494 \\
\hline-2.37589145 & 1.48277950 \\
\hline-2.37589145 & 1.48277950 \\
\hline-2.37589145 & 1.58861494 \\
\hline-2.37589145 & 1.58861494 \\
\hline 72207 & \# Energy 1->9 \\
\hline 0.4166 & -1.0012 \\
\hline 0.0360 & 0.0259 \\
\hline 2.15289307 & 0.16506203 \\
\hline 2.15289307 & 0.16506203 \\
\hline 2.15289307 & 0.27089748 \\
\hline 2.15289307 & 0.27089748 \\
\hline 2.25872827 & 0.16506203 \\
\hline 2.25872827 & 0.16506203 \\
\hline 2.25872827 & 0.27089748 \\
\hline 2.25872827 & 0.27089748 \\
\hline-1.31485093 & -0.19434193 \\
\hline-1.31485093 & -0.19434193 \\
\hline-1.31485093 & -0.08850648 \\
\hline$-1 \cdot 31$ & -0.08850648 \\
\hline-1.20901549 & -0.19434193 \\
\hline-1.20901549 & -0.19434193 \\
\hline-1.20901549 & -0.08850648 \\
\hline-1.20901549 & -0.08850648 \\
\hline 2.28008008 & 1.30933321 \\
\hline 2.28008008 & 1.30933321 \\
\hline 2.28008008 & 1.41516852 \\
\hline 2.28008008 & 1.41516852 \\
\hline 2.38591576 & 1.30933321 \\
\hline 2.38591576 & 1.30933321 \\
\hline 2.38591576 & 1.41516852 \\
\hline 2.38591576 & 1.41516852 \\
\hline-1.21978784 & -1.38936973 \\
\hline-1.21978784 & -1.38936973 \\
\hline-1.21978784 & -1.28353417 \\
\hline-1.21978784 & -1.28353417 \\
\hline-1.11395252 & -1.38936973 \\
\hline-1.11395252 & -1.38936973 \\
\hline-1.11395252 & -1.28353417 \\
\hline-1.11395252 & -1.28353417 \\
\hline 1.06017745 & -0.22089551 \\
\hline 1.06017745 & -0.22089551 \\
\hline 1.06017745 & -0.11506007 \\
\hline 1.06017745 & -0.11506007 \\
\hline 1.16601288 & -0.22089551 \\
\hline 1.16601288 & -0.22089551 \\
\hline 1.16601288 & -0.11506007 \\
\hline 01288 & -0.1 \\
\hline 4571 & \\
\hline
\end{tabular}

$$
\begin{array}{lr}
-0.08215106 & -3.11705709 \\
-0.18798651 & -6.20520210 \\
-0.08215106 & 3.59061074 \\
-0.18798651 & -7.47598267 \\
-0.08215106 & 4.92089844 \\
-0.18798651 & 8.41958618 \\
-0.08215106 & -5.45013905 \\
-0.81211877 & -0.16817904 \\
-0.70628333 & 0.26307163 \\
-0.81211877 & 0.39273110 \\
-0.70628333 & -0.47479776 \\
-0.81211877 & -0.40217099 \\
-0.70628333 & 0.39786381 \\
-0.81211877 & 0.38832512 \\
-0.70628333 & -0.43782437 \\
-0.33387381 & 1.75209415 \\
-0.22803834 & -1.52345586 \\
-0.33387381 & -1.33407557 \\
-0.22803834 & 1.20711863 \\
-0.33387381 & -2.28260159 \\
-0.22803834 & 1.97965252 \\
-0.33387381 & 1.71129525 \\
-0.22803834 & -1.54301488
\end{array}
$$

$$
\begin{gathered}
1.000 \text { \# Electr. mom.' scale-fac } \\
\text { \# Magnetic mom. }
\end{gathered}
$$$$
-0.01106351
$$$$
0.09477194
$$$$
-0.01106351
$$$$
0.09477194
$$$$
-0.01106351
$$$$
0.09477194
$$$$
-0.01106351
$$$$
0.09477194
$$$$
-0.22601055
$$$$
-0.12017509
$$$$
-0.22601055
$$$$
-0.12017509
$$$$
-0.22601055
$$$$
-0.12017509
$$$$
-0.22601055
$$$$
-0.12017509
$$$$
0.43990609
$$$$
0.54574150
$$$$
0.43990609
$$$$
0.54574150
$$$$
0.43990609
$$$$
0.54574150
$$$$
0.43990609
$$$$
0.54574150
$$$$
0.08411802
$$$$
0.18995346
$$$$
0.08411802
$$$$
0.18995346
$$$$
0.08411802
$$$$
0.18995346
$$$$
0.08411802
$$$$
0.18995346
$$$$
-0.74873185
$$$$
-0.64289635
$$$$
-0.74873185
$$$$
-0.64289635
$$$$
-0.74873185
$$$$
-0.64289635
$$$$
-0.74873185
$$$$
-0.64289635
$$$$
-0.18798651
$$$$
-0.22504927
$$$$
-6.14117002
$$$$
\text { 5.93179893 }
$$$$
0.18319602
$$$$
0.44412005
$$$$
\text { 5. } 31154346
$$$$
-5.18419552
$$$$
6.00722218
$$$$
-2.36133361
$$$$
-7.48150349
$$$$
2.83455586
$$$$
-6.24346638
$$$$
\text { 2. } 58567595
$$$$
7.90697861
$$$$
-3.53865838
$$$$
-1.29214573
$$$$
0.36384222
$$$$
\text { 1. } 36703813
$$$$
-0.68087941
$$$$
0.90921217
$$$$
-0.50617737
$$$$
-1.38665295
$$$$
1.04865837
$$$$
\text { 1. } 90594423
$$$$
-1.88239717
$$$$
-2.15656638
$$$$
-1.73923302
$$$$
1.48744833
$$$$
\text { 2. } 11949420
$$$$
-1.72383010
$$$$
-6.41464663
$$$$
12.53557968
$$$$
9.18290710
$$$$
-16.58893967
$$$$
8.67910671
$$

$-15.57289600$

$-11.63991547$

19.84346199

$-0.90936613$

$$
-0.20032442
$$$$
\text { 2. } 12311983
$$ 


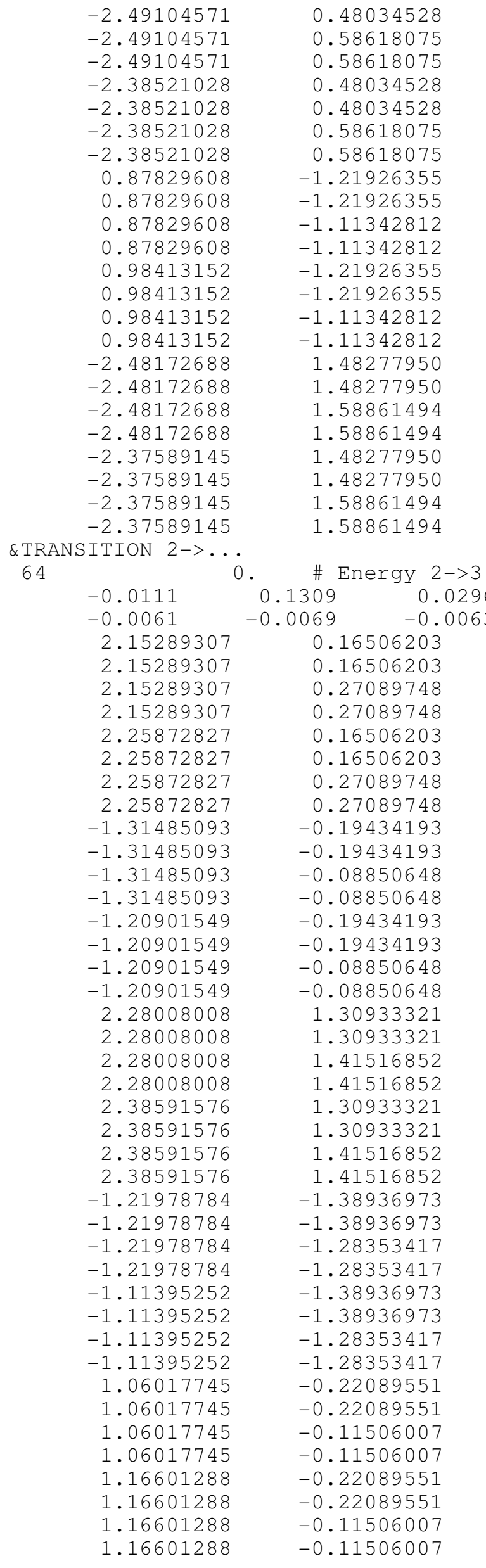

-0.08215106
-0.18798651
-0.08215106
-0.18798651
-0.08215106
-0.18798651
-0.08215106
-0.81211877
-0.70628333
-0.81211877
-0.70628333
-0.81211877
-0.70628333
-0.81211877
-0.70628333
-0.33387381
-0.22803834
-0.33387381
-0.22803834
-0.33387381
-0.22803834
-0.33387381
-0.22803834

0.86456591

0.61192930

$-0.99817497$

0.67273551

$-0.37415910$

$-0.16626452$

0.49120906

$-1.11429429$

1. 41573691

1.64371324

$-2.11455297$

1.34033656

$-1.63543999$

$-1.94827068$

2. 42560387

0.00755699

0.09629467

0.00327780

$-0.06498124$

$-0.19532689$

0.03937770

0.12150633

$-0.02393722$
1.000 \# Electr. mom., scale-fac \# Magnetic mom.

$-0.01106351$ 0.09477194 1.05584157 $-0.99112719$ $-1.00313950$ 0.88385111 $-0.61977428$ 0.64771825 0.50160062 $-0.51176119$ 1. 65846086 $-1.95783508$ $-1.38092148$ 1. 71070170 $-0.97632021$ 1. 27714336 0.71085936 $-1.01983833$

$-1.04717457$

0.77081817

1.23253083

$-0.97403818$ 1. 28639793

$-0.93333822$

$-1.38680744$

1.07305157

0.97413063

$-1.02830315$

$-1.05735362$

1.09787893

$-0.73241311$

0.81085265

0.75764030

$-0.83308512$

$-1.12699020$

1.97602510

1. 51305091

$-2.45383477$

1.69169688

$-2.73436856$

$-2.11042404$

3. 24927711 


-2.49104571
-2.49104571
-2.49104571
-2.49104571
-2.38521028
-2.38521028
-2.38521028
-2.38521028
0.87829608
0.87829608
0.87829608
0.87829608
0.98413152
0.98413152
0.98413152
0.98413152
-2.48172688
-2.48172688
-2.48172688
-2.48172688
-2.37589145
-2.37589145
-2.37589145
-2.37589145

64
0.48034528

0.48034528

0.58618075

0.58618075

0.48034528

0.48034528

0.58618075

0.58618075

$-1.21926355$

$-1.21926355$

$-1.11342812$

$-1.11342812$

$-1.21926355$

$-1.21926355$

$-1.11342812$

$-1.11342812$

1. 48277950

1. 48277950

1.58861494

1. 58861494

1. 48277950

1. 48277950

1.58861494

1. 58861494

0 .

$0.1062=$

$\begin{array}{rrr}0.1062 & 0.0418 & -0.0015 \\ -0.0026 & -0.3282 & -0.1897\end{array}$

2.15289307

2.15289307

2.15289307

2.15289307

2. 25872827

2. 25872827

2. 25872827

2. 25872827

$-1.31485093$

$-1.31485093$

$-1.31485093$

$-1.31485093$

$-1.20901549$

$-1.20901549$

$-1.20901549$

$-1.20901549$

2. 28008008

2. 28008008

2. 28008008

2.28008008

2. 38591576

2. 38591576

2.38591576

2. 38591576

$-1.21978784$

$-1.21978784$

$-1.21978784$

$-1.21978784$

$-1.11395252$

$-1.11395252$

$-1.11395252$

$-1.11395252$

1. 06017745

1.06017745

1. 06017745

1. 06017745

1. 16601288

1. 16601288

1. 16601288

1. 16601288

$-0.18798651$

$-0.08215106$

$-0.18798651$

$-0.08215106$

$-0.18798651$

$-0.08215106$

$-0.18798651$

$-0.08215106$

$-0.81211877$

$-0.70628333$

$-0.81211877$

$-0.70628333$

$-0.81211877$

$-0.70628333$

$-0.81211877$

$-0.70628333$

$-0.33387381$

$-0.22803834$

$-0.33387381$

$-0.22803834$

$-0.33387381$

$-0.22803834$

$-0.33387381$

$-0.22803834$

$$
\begin{gathered}
1.000 \text { \# Electr. mom., scale-fac } \\
\text { \# Magnetic mom. }
\end{gathered}
$$

0.16506203

0.16506203

0.27089748

0.27089748

0.16506203

0.16506203

0.27089748

0.27089748

$-0.19434193$

$-0.19434193$

$-0.08850648$

$-0.08850648$

$-0.19434193$

$-0.19434193$

$-0.08850648$

$-0.08850648$

1. 30933321

1. 30933321

1. 41516852

1. 41516852

1. 30933321

1. 30933321

1. 41516852

1. 41516852

$-1.38936973$

$-1.38936973$

$-1.28353417$

$-1.28353417$

$-1.38936973$

$-1.38936973$

$-1.28353417$

$-1.28353417$

$-0.22089551$

$-0.22089551$

$-0.11506007$

$-0.11506007$

$-0.22089551$

$-0.22089551$

$-0.11506007$

$-0.11506007$
1. 78773260

$-1.51304483$

$-1.90371168$

1.66090178

$-1.94439662$

1.52431750

2. 08538294

$-1.68950629$

$-0.01509069$

0.13055028

0.17839678

$-0.37093115$

0.12384869

$-0.25970611$

$-0.31468076$

0.53430301

0.31198928

$-0.28141335$

0.19595304

$-0.28012338$

0.18767890

$-0.12792452$
$-0.21433330$

0.19312832 $\begin{array}{ll}-0.01106351 & -58.53897095\end{array}$

0.09477194

$-0.01106351$

0.09477194

$-0.01106351$

0.09477194

$-0.01106351$

0.09477194

$-0.22601055$

$-0.12017509$

$-0.22601055$

$-0.12017509$

$-0.22601055$

$-0.12017509$

$-0.22601055$

$-0.12017509$

0.43990609

0.54574150

0.43990609

0.54574150

0.43990609

0.54574150

0.43990609

0.54574150

0.08411802

0.18995346

0.08411802

0.18995346

0.08411802

0.18995346

0.08411802

0.18995346

$-0.74873185$

$-0.64289635$

$-0.74873185$

$-0.64289635$

$-0.74873185$

$-0.64289635$

$-0.74873185$

$-0.64289635$
44.53841782

64.43659210

$-48.67368317$

47.50843811

$-37.26701355$

$-52.05635452$

40.66585541

$-9.62926006$

8.15738678

11.21859360

$-9.22977448$

10.15925694

$-8.71674442$

$-11.72071075$

9.77762699

$-3.58446217$

3.15654993

4.11461067

$-3.87501788$

$-1.47532833$

0.91440803

$-0.23822556$

0.74418062

$-1.26409483$

1.06123614

1.88378942

$-1.63027072$

1. 48973525

$-1.24083328$

$-2.18196201$

1.87748826

17.62521553

$-38.14031982$

$-24.02210045$

46.00568390

$-29.52200890$

54.44388580

36.72174454

$-63.31566238$ 


-2.49104571
-2.49104571
-2.49104571
-2.49104571
-2.38521028
-2.38521028
-2.38521028
-2.38521028
0.87829608
0.87829608
0.87829608
0.87829608
0.98413152
0.98413152
0.98413152
0.98413152
-2.48172688
-2.48172688
-2.48172688
-2.48172688
-2.37589145
-2.37589145
-2.37589145
-2.37589145

64
0.48034528

0.48034528

0.58618075

0.58618075

0.48034528

0.48034528

0.58618075

0.58618075

$-1.21926355$

$-1.21926355$

$-1.11342812$

$-1.11342812$

$-1.21926355$

$-1.21926355$

$-1.11342812$

$-1.11342812$

1. 48277950

1. 48277950

1.58861494

1.58861494

1.48277950

1.48277950

1.58861494

1. 58861494

$0 . \quad \#$ Energy 2->5
$-0.0246 \quad 0.0047$

$-0.0538$

$-0.0003$

2. 15289307

2.15289307

2.15289307

2.15289307

2. 25872827

2. 25872827

2. 25872827

2. 25872827

$-1.31485093$

$-1.31485093$

$-1.31485093$

$-1.31485093$

$-1.20901549$

$-1.20901549$

$-1.20901549$

$-1.20901549$

2. 28008008

2. 28008008

2. 28008008

2.28008008

2. 38591576

2. 38591576

2.38591576

2. 38591576

$-1.21978784$

$-1.21978784$

$-1.21978784$

$-1.21978784$

$-1.11395252$

$-1.11395252$

$-1.11395252$

$-1.11395252$

1.06017745

1.06017745

1. 06017745

1. 06017745

1. 16601288

1. 16601288

1. 16601288

1. 16601288

$$
0.1051 \quad 0.0696
$$

0.16506203

0.16506203

0.27089748

0.27089748

0.16506203

0.16506203

0.27089748

0.27089748

$-0.19434193$

$-0.19434193$

$-0.08850648$

$-0.08850648$

$-0.19434193$

$-0.19434193$

$-0.08850648$

$-0.08850648$

1. 30933321

1. 30933321

1. 41516852

1. 41516852

1.30933321

1.30933321

1. 41516852

1. 41516852

$-1.38936973$

$-1.38936973$

$-1.28353417$

$-1.28353417$

$-1.38936973$

$-1.38936973$

$-1.28353417$

$-1.28353417$

$-0.22089551$

$-0.22089551$

$-0.11506007$

$-0.11506007$

$-0.22089551$

$-0.22089551$

$-0.11506007$

$-0.11506007$
$-0.18798651$

$-0.08215106$

$-0.18798651$

$-0.08215106$

$-0.18798651$

$-0.08215106$

$-0.18798651$

$-0.08215106$

$-0.81211877$

$-0.70628333$

$-0.81211877$

$-0.70628333$

$-0.81211877$

$-0.70628333$

$-0.81211877$

$-0.70628333$

$-0.33387381$

$-0.22803834$

$-0.33387381$

$-0.22803834$

$-0.33387381$

$-0.22803834$

$-0.33387381$

$-0.22803834$

1.000 $-0.01106351$

0.09477194

$-0.01106351$

0.09477194

$-0.01106351$

0.09477194

$-0.01106351$

0.09477194

$-0.22601055$

$-0.12017509$

$-0.22601055$

$-0.12017509$

$-0.22601055$

$-0.12017509$

$-0.22601055$

$-0.12017509$

0.43990609

0.54574150

0.43990609

0.54574150

0.43990609

0.54574150

0.43990609

0.54574150

0.08411802

0.18995346

0.08411802

0.18995346

0.08411802

0.18995346

0.08411802

0.18995346

$-0.74873185$

$-0.64289635$

$-0.74873185$

$-0.64289635$

$-0.74873185$

$-0.64289635$

$-0.74873185$

$-0.64289635$
2. 49187803

$-1.93817019$

$-2.14883828$

1. 71921432

$-2.98723078$

2. 28803587

2. 51546288

$-1.97656393$

$-0.56106883$

$-0.94840890$

0.12235364

2.28987503

$-0.68382078$

2. 51088428

1. 55637372

$-4.42891598$

0.50742960

$-0.54656297$

$-0.36437458$

0.41081676

$-0.53118145$

0.58173466

0.37877318

$-0.43558580$
Electr. mom., scale-fac Magnetic mom.

22.03785706

$-16.93289375$

$-24.38238907$

18.58558464

$-18.01336098$

14.30680084

19.82684135

$-15.66882420$

3.10925317

$-2.64576435$

$-3.65385795$

2. 98617315

$-3.41575408$

2.97205544

3. 94300294

$-3.30190969$

1. 20307422

$-1.06823194$

$-1.42455018$

1. 35110128

0.69307405

$-0.45132110$

$-0.02513989$

$-0.18526702$

0.29709670

$-0.21476486$

$-0.36898822$

0.28252900

$-0.40198094$

0.30753002

0.50737578

$-0.40736493$

$-6.75218248$

14.44885731

9.29888439

$-17.58080292$

11.12279701

$-20.44757080$

$-13.99859905$

23.99809074 


-2.49104571
-2.49104571
-2.49104571
-2.49104571
-2.38521028
-2.38521028
-2.38521028
-2.38521028
0.87829608
0.87829608
0.87829608
0.87829608
0.98413152
0.98413152
0.98413152
0.98413152
-2.48172688
-2.48172688
-2.48172688
-2.48172688
-2.37589145
-2.37589145
-2.37589145
-2.37589145

64
0.48034528

0.48034528

0.58618075

0.58618075

0.48034528

0.48034528

0.58618075

0.58618075

$-1.21926355$

$-1.21926355$

$-1.11342812$

$-1.11342812$

$-1.21926355$

$-1.21926355$

$-1.11342812$

$-1.11342812$

1. 48277950

1. 48277950

1.58861494

1. 58861494

1. 48277950

1. 48277950

1.58861494

1.58861494

$-0.0544$

$-0.0099$

2. 15289307

2.15289307

2.15289307

2.15289307

2. 25872827

2. 25872827

2. 25872827

2. 25872827

$-1.31485093$

$-1.31485093$

$-1.31485093$

$-1.31485093$

$-1.20901549$

$-1.20901549$

$-1.20901549$

$-1.20901549$

2. 28008008

2. 28008008

2. 28008008

2.28008008

2. 38591576

2. 38591576

2.38591576

2. 38591576

$-1.21978784$

$-1.21978784$

$-1.21978784$

$-1.21978784$

$-1.11395252$

$-1.11395252$

$-1.11395252$

$-1.11395252$

1. 06017745

1.06017745

1. 06017745

1. 06017745

1. 16601288

1. 16601288

1. 16601288

1. 16601288

1. 30933321
0 . \# Energy 2->6

$\begin{array}{ll}-0.0210 & 0.0365\end{array}$

$0.0093 \quad-0.0084$

0.16506203

0.16506203

0.27089748

0.27089748

0.16506203

0.16506203

0.27089748

0.27089748

$-0.19434193$

$-0.19434193$

$-0.08850648$

$-0.08850648$

$-0.19434193$

$-0.19434193$

$-0.08850648$

$-0.08850648$

1. 30933321

1. 41516852

1. 41516852

1. 30933321

1.30933321

1. 41516852

1. 41516852

$-1.38936973$

$-1.38936973$

$-1.28353417$

$-1.28353417$

$-1.38936973$

$-1.38936973$

$-1.28353417$

$-1.28353417$

$-0.22089551$

$-0.22089551$

$-0.11506007$

$-0.11506007$

$-0.22089551$

$-0.22089551$

$-0.11506007$

$-0.11506007$ $\begin{array}{lr}-0.18798651 & -1.08262944 \\ -0.08215106 & 0.78537887 \\ -0.18798651 & 0.94380313 \\ -0.08215106 & -0.69446373 \\ -0.18798651 & 1.32422805 \\ -0.08215106 & -0.94619495 \\ -0.18798651 & -1.14076734 \\ -0.08215106 & 0.82306194 \\ -0.81211877 & 0.19137491 \\ -0.70628333 & 0.37362358 \\ -0.81211877 & -0.02863604 \\ -0.70628333 & -0.87044936 \\ -0.81211877 & 0.25829071 \\ -0.70628333 & -0.93090266 \\ -0.81211877 & -0.57210916 \\ -0.70628333 & 1.62776697 \\ -0.33387381 & -0.18881497 \\ -0.22803834 & 0.18376976 \\ -0.33387381 & 0.12799481 \\ -0.22803834 & -0.13107798 \\ -0.33387381 & 0.20304902 \\ -0.22803834 & -0.19454473 \\ -0.33387381 & -0.13903551 \\ -0.22803834 & 0.14082064 \\ -0 & \end{array}$
1.000 \# Electr. mom., scale-fac \# Magnetic mom.

$-0.01106351$

0.09477194

3. 54414105

$-0.01106351$

0.09477194

$-0.01106351$

0.09477194

$-0.01106351$

0.09477194

$-0.22601055$

$-0.12017509$

$-0.22601055$

$-0.12017509$

$-0.22601055$

$-0.12017509$

$-0.22601055$

$-0.12017509$

0.43990609

0.54574150

0.43990609

0.54574150

0.43990609

0.54574150

0.43990609

0.54574150

0.08411802

0.18995346

0.08411802

0.18995346

0.08411802

0.18995346

0.08411802

0.18995346

$-0.74873185$

$-0.64289635$

$-0.74873185$

$-0.64289635$

$-0.74873185$

$-0.64289635$

$-0.74873185$

$-0.64289635$
$-2.85967898$

$-3.96495509$

3.17146921

$-2.93500161$

2. 45636272

3. 24911952

$-2.70384121$

$-1.36589992$

1. 42624819

1. 47659051

$-1.52921009$

1.51924276

$-1.60572088$

$-1.64003754$

1. 71889246

0.12430886

$-0.14582516$

$-0.11933211$

0.14680980

0.18253680

$-0.09778070$

$-0.11360547$

$-0.12113317$

0.09597450

0.08014159

$-0.05808466$

0.14376855

$-0.12877879$

$-0.10000712$

0.09324073

$-1.33157516$

2. 55038929

1. 95377076

$-3.33569503$

1. 95843804

$-3.43779898$

$-2.62264609$

4. 27867126
0.03788780 


-2.49104571
-2.49104571
-2.49104571
-2.49104571
-2.38521028
-2.38521028
-2.38521028
-2.38521028
0.87829608
0.87829608
0.87829608
0.87829608
0.98413152
0.98413152
0.98413152
0.98413152
-2.48172688
-2.48172688
-2.48172688
-2.48172688
-2.37589145
-2.37589145
-2.37589145
-2.37589145

64
0.48034528

0.48034528

0.58618075

0.58618075

0.48034528

0.48034528

0.58618075

0.58618075

$-1.21926355$

$-1.21926355$

$-1.11342812$

$-1.11342812$

$-1.21926355$

$-1.21926355$

$-1.11342812$

$-1.11342812$

1. 48277950

1. 48277950

1.58861494

1. 58861494

1. 48277950

1.48277950

1.58861494

1. 58861494

0. \# Energy 2->7

$\begin{array}{lll}-0.3230 & -0.1153 & 0.1478\end{array}$

-0.0348
2.15289307

2.15289307

2.15289307

2. 15289307

2. 25872827

2. 25872827

2.25872827

2. 25872827

$-1.31485093$

$-1.31485093$

$-1.31485093$

$-1.31485093$

$-1.20901549$

$-1.20901549$

$-1.20901549$

$-1.20901549$

2. 28008008

2. 28008008

2. 28008008

2. 28008008

2.38591576

2. 38591576

2.38591576

2. 38591576

$-1.21978784$

$-1.21978784$

$-1.21978784$

$-1.21978784$

$-1.11395252$

$-1.11395252$

$-1.11395252$

$-1.11395252$

1. 06017745

1.06017745

1.06017745

1. 06017745

1. 16601288

1. 16601288

1. 16601288

1. 16601288

$$
-0.1200 \quad-0.0096
$$

0.16506203

0.16506203

0.27089748

0.27089748

0.16506203

0.16506203

0.27089748

0.27089748

$-0.19434193$

$-0.19434193$

$-0.08850648$

$-0.08850648$

$-0.19434193$

$-0.19434193$

$-0.08850648$

$-0.08850648$

1. 30933321

1.30933321

1. 41516852

1. 41516852

1.30933321

1. 30933321

1. 41516852

1. 41516852

$-1.38936973$

$-1.38936973$

$-1.28353417$

$-1.28353417$

$-1.38936973$

$-1.38936973$

$-1.28353417$

$-1.28353417$

$-0.22089551$

$-0.22089551$

$-0.11506007$

$-0.11506007$

$-0.22089551$

$-0.22089551$

$-0.11506007$

$-0.11506007$
$-0.18798651$

$-0.08215106$

$-0.18798651$

$-0.08215106$

$-0.18798651$

$-0.08215106$

$-0.18798651$

$-0.08215106$

$-0.81211877$

$-0.70628333$

$-0.81211877$

$-0.70628333$

$-0.81211877$

$-0.70628333$

$-0.81211877$

$-0.70628333$

$-0.33387381$

$-0.22803834$

$-0.33387381$

$-0.22803834$

$-0.33387381$

$-0.22803834$

$-0.33387381$

$-0.22803834$

1.000

$-0.01106351$

\# Electr. mom., scale-fac

0.09477194

$-0.01106351$

0.09477194

$-0.01106351$

0.09477194

$-0.01106351$

0.09477194

$-0.22601055$

$-0.12017509$

$-0.22601055$

$-0.12017509$

$-0.22601055$

$-0.12017509$

$-0.22601055$

$-0.12017509$

0.43990609

0.54574150

0.43990609

0.54574150

0.43990609

0.54574150

0.43990609

0.54574150

0.08411802

0.18995346

0.08411802

0.18995346

0.08411802

0.18995346

0.08411802

0.18995346

$-0.74873185$

$-0.64289635$

$-0.74873185$

$-0.64289635$

$-0.74873185$

$-0.64289635$

$-0.74873185$

$-0.64289635$
0.16184527

$-0.12284855$

$-0.11577710$

0.08441568

$-0.14242925$

0.08729154

0.08775638

$-0.03783382$

0.08710953

$-0.04004279$

$-0.07345896$

$-0.01229159$

$-0.01011240$

$-0.05388598$

$-0.02876926$

0.13971010

0.00477621

0.00420487

0.00046734

$-0.00665513$

0.01671797

$-0.03411200$

$-0.01749074$

0.03001571

\section{Magnetic mom.}

$-0.36552048$

$-0.89006335$

$-0.70803320$

1.89057779

$-0.20460810$

1.21990693

1.02960789

$-2.01483059$

$-6.89130640$

6.56218147

8.12824535

$-7.61007547$

7.54361916

$-7.36962557$

$-8.84663868$

8.48125553

0.27802473

$-0.43068984$

$-0.43895394$

0.51239926

$-0.23641542$

0.36384380

0.38105771

$-0.44057801$

$-1.76310802$

1.47429097

1.38606429

$-1.07960820$

1.81460536

$-1.57986283$

$-1.43203926$

1. 20557320

$-4.11919403$

4.45414639

5.62364101

$-6.46353960$

2.93479824

$-3.06179690$

$-4.51507902$

5. 20450211 


-2.49104571
-2.49104571
-2.49104571
-2.49104571
-2.38521028
-2.38521028
-2.38521028
-2.38521028
0.87829608
0.87829608
0.87829608
0.87829608
0.98413152
0.98413152
0.98413152
0.98413152
-2.48172688
-2.48172688
-2.48172688
-2.48172688
-2.37589145
-2.37589145
-2.37589145
-2.37589145

64
0.48034528

0.48034528

0.58618075

0.58618075

0.48034528

0.48034528

0.58618075

0.58618075

$-1.21926355$

$-1.21926355$

$-1.11342812$

$-1.11342812$

$-1.21926355$

$-1.21926355$

$-1.11342812$

$-1.11342812$

1.48277950

1. 48277950

1.58861494

1. 58861494

1.48277950

1.48277950

1.58861494

1.58861494

0 . \# Energy 2->8

$\begin{array}{lll}-0.4114 & -0.0361 & -0.0470\end{array}$

$\begin{array}{lll}-0.0261 & -0.0204 & -0.1041\end{array}$

2. 15289307

2.15289307

2. 15289307

2.15289307

2.25872827

2.25872827

2.25872827

2. 25872827

$-1.31485093$

$-1.31485093$

$-1.31485093$

$-1.31485093$

$-1.20901549$

$-1.20901549$

$-1.20901549$

$-1.20901549$

2. 28008008

2. 28008008

2. 28008008

2. 28008008

2.38591576

2. 38591576

2.38591576

2. 38591576

$-1.21978784$

$-1.21978784$

$-1.21978784$

$-1.21978784$

$-1.11395252$

$-1.11395252$

$-1.11395252$

$-1.11395252$

1. 06017745

1.06017745

1.06017745

1. 06017745

1. 16601288

1. 16601288

1. 16601288

1. 16601288
0.16506203

0.16506203

0.27089748

0.27089748

0.16506203

0.16506203

0.27089748

0.27089748

$-0.19434193$

$-0.19434193$

$-0.08850648$

$-0.08850648$

$-0.19434193$

$-0.19434193$

$-0.08850648$

$-0.08850648$

1. 30933321

1. 30933321

1. 41516852

1. 41516852

1. 30933321

1. 30933321

1. 41516852

1. 41516852

$-1.38936973$

$-1.38936973$

$-1.28353417$

$-1.28353417$

$-1.38936973$

$-1.38936973$

$-1.28353417$

$-1.28353417$

$-0.22089551$

$-0.22089551$

$-0.11506007$

$-0.11506007$

$-0.22089551$

$-0.22089551$

$-0.11506007$

$-0.11506007$
$-0.18798651$

$-0.08215106$

$-0.18798651$

$-0.08215106$

$-0.18798651$

$-0.08215106$

$-0.18798651$

$-0.08215106$

$-0.81211877$

$-0.70628333$

$-0.81211877$

$-0.70628333$

$-0.81211877$

$-0.70628333$

$-0.81211877$

$-0.70628333$

$-0.33387381$

$-0.22803834$

$-0.33387381$

$-0.22803834$

$-0.33387381$

$-0.22803834$

$-0.33387381$

$-0.22803834$

1.000

$-0.01106351$

0.09477194

$-0.01106351$

0.09477194

$-0.01106351$

0.09477194

$-0.01106351$

0.09477194

$-0.22601055$

$-0.12017509$

$-0.22601055$

$-0.12017509$

$-0.22601055$

$-0.12017509$

$-0.22601055$

$-0.12017509$

0.43990609

0.54574150

0.43990609

0.54574150

0.43990609

0.54574150

0.43990609

0.54574150

0.08411802

0.18995346

0.08411802

0.18995346

0.08411802

0.18995346

0.08411802

0.18995346

$-0.74873185$

$-0.64289635$

$-0.74873185$

$-0.64289635$

$-0.74873185$

$-0.64289635$

$-0.74873185$

$-0.64289635$
1. 97897100

$-1.51679802$

$-1.73971295$

1.34972703

$-2.02952504$

1. 41688538

1. 72206998

$-1.17056143$

$-0.04569340$

$-0.11558048$

0.12653415

0.08538869

$-0.09467256$

0.31419209

0.09266256

$-0.38711736$

0.29715258

$-0.26061085$

$-0.23049396$

0.21463555

$-0.18786815$

0.09742708

0.13932773

$-0.08311452$
Electr. mom., scale-fac Magnetic mom.

$-46.97045135$

35.77939224

49.36933899

$-37.18896103$

38.45797348

$-30.30534363$

$-40.20960999$

31.44379425

$-16.19708633$

16.05286217

17.32293129

$-16.88657761$

14.99555874

$-14.47386551$

$-16.20987320$

15.46930408

$-0.63168192$

0.49017137

0.55534220

$-0.59144086$

$-2.60868692$

1.96047246

1. 81928802

$-1.20091283$

0.58372897

0.07602824

1. 53841388

$-2.21185255$

$-0.59575135$

0.17755923

$-1.57307410$

1.93542957

20.28218842

$-34.73980331$

$-23.82801056$

38.95979309

$-29.03531265$

47.13925934

32.77979279

$-51.54694366$ 


-2.49104571
-2.49104571
-2.49104571
-2.49104571
-2.38521028
-2.38521028
-2.38521028
-2.38521028
0.87829608
0.87829608
0.87829608
0.87829608
0.98413152
0.98413152
0.98413152
0.98413152
-2.48172688
-2.48172688
-2.48172688
-2.48172688
-2.37589145
-2.37589145
-2.37589145
-2.37589145

64
0.48034528

0.48034528

0.58618075

0.58618075

0.48034528

0.48034528

0.58618075

0.58618075

$-1.21926355$

$-1.21926355$

$-1.11342812$

$-1.11342812$

$-1.21926355$

$-1.21926355$

$-1.11342812$

$-1.11342812$

1.48277950

1. 48277950

1.58861494

1. 58861494

1. 48277950

1. 48277950

1.58861494

1. 58861494

$-0.1179$

2. 15289307

2. 15289307

2.15289307

2. 15289307

2. 25872827

2. 25872827

2. 25872827

2. 25872827

$-1.31485093$

$-1.31485093$

$-1.31485093$

$-1.31485093$

$-1.20901549$

$-1.20901549$

$-1.20901549$

$-1.20901549$

2. 28008008

2. 28008008

2. 28008008

2. 28008008

2.38591576

2. 38591576

2.38591576

2. 38591576

$-1.21978784$

$-1.21978784$

$-1.21978784$

$-1.21978784$

$-1.11395252$

$-1.11395252$

$-1.11395252$

$-1.11395252$

1. 06017745

1.06017745

1.06017745

1. 06017745

1.16601288

1. 16601288

1. 16601288

1. 16601288
0. \# Energy 2->9

$\begin{array}{ll}-0.1816 & 0.0743\end{array}$

$0.1646 \quad 0.0078$

0.16506203

0.16506203

0.27089748

0.27089748

0.16506203

0.16506203

0.27089748

0.27089748

$-0.19434193$

$-0.19434193$

$-0.08850648$

$-0.08850648$

$-0.19434193$

$-0.19434193$

$-0.08850648$

$-0.08850648$

1. 30933321

1. 30933321

1. 41516852

1. 41516852

1. 30933321

1. 30933321

1. 41516852

1. 41516852

$-1.38936973$

$-1.38936973$

$-1.28353417$

$-1.28353417$

$-1.38936973$

$-1.38936973$

$-1.28353417$

$-1.28353417$

$-0.22089551$

$-0.22089551$

$-0.11506007$

$-0.11506007$

$-0.22089551$

$-0.22089551$

$-0.11506007$

$-0.11506007$
$-0.18798651$

$-0.08215106$

$-0.18798651$

$-0.08215106$

$-0.18798651$

$-0.08215106$

$-0.18798651$

$-0.08215106$

$-0.81211877$

$-0.70628333$

$-0.81211877$

$-0.70628333$

$-0.81211877$

$-0.70628333$

$-0.81211877$

$-0.70628333$

$-0.33387381$

$-0.22803834$

$-0.33387381$

$-0.22803834$

$-0.33387381$

$-0.22803834$

$-0.33387381$

$-0.22803834$

1.000

$-0.01106351$

0.09477194

$-0.01106351$

0.09477194

$-0.01106351$

0.09477194

$-0.01106351$

0.09477194

$-0.22601055$

$-0.12017509$

$-0.22601055$

$-0.12017509$

$-0.22601055$

$-0.12017509$

$-0.22601055$

$-0.12017509$

0.43990609

0.54574150

0.43990609

0.54574150

0.43990609

0.54574150

0.43990609

0.54574150

0.08411802

0.18995346

0.08411802

0.18995346

0.08411802

0.18995346

0.08411802

0.18995346

$-0.74873185$

$-0.64289635$

$-0.74873185$

$-0.64289635$

$-0.74873185$

$-0.64289635$

$-0.74873185$

$-0.64289635$
0.23066950

$-0.16252600$

0.86070818

$-0.82768613$

$-0.41636428$

0.27475178

$-0.87077463$

0.89728630

$-0.20354089$

$-0.98115373$

$-0.68095762$

2.61648035

$-0.82571715$

2.37757397

2. 08987236

$-4.56386042$

$-0.00007332$

$-0.05429914$

$-0.00320128$

0.02780278

0.06841307

0.02161207

$-0.03769348$

$-0.02070156$ Electr. mom., scale-fac
Magnetic mom.

33.35229492

$-25.59484482$

$-34.60023880$

26.32267952

$-27.44235611$

21.57701492

28.32186127

$-22.14915848$

4.51597452

$-3.70081782$

$-4.66473007$

3. 71744037

$-4.54775620$

3. 50899625

4. 70293951

$-3.54651904$

1. 53389204

$-1.20386028$

$-1.56183136$

1. 33108366

0.45699492

$-0.35855156$

0.04328464

$-0.13726686$

0.88876694

$-0.77303332$

$-1.03923488$

0.90936679

$-0.94730669$

0.74435753

1.15473402

$-0.89256364$

$-10.31709385$

19.40505600

12.72572708

$-22.24974442$

15.45591164

$-26.66126633$

$-17.96090317$

29.57904243 


-2.49104571
-2.49104571
-2.49104571
-2.49104571
-2.38521028
-2.38521028
-2.38521028
-2.38521028
0.87829608
0.87829608
0.87829608
0.87829608
0.98413152
0.98413152
0.98413152
0.98413152
-2.48172688
-2.48172688
-2.48172688
-2.48172688
-2.37589145
-2.37589145
-2.37589145
-2.37589145

\&TRANSITION $3->\ldots$ 640 .
0.48034528

0.48034528

0.58618075

0.58618075

0.48034528

0.48034528

0.58618075

0.58618075

$-1.21926355$

$-1.21926355$

$-1.11342812$

$-1.11342812$

$-1.21926355$

$-1.21926355$

$-1.11342812$

$-1.11342812$

1.48277950

1. 48277950

1.58861494

1. 58861494

1. 48277950

1.48277950

1.58861494

1. 58861494

\# Energy $3->4$
$0.0826 \quad-0.0330$

$-0.0229$

$-0.0146$

2. 15289307

2.15289307

2.15289307

2. 15289307

2. 25872827

2. 25872827

2. 25872827

2. 25872827

$-1.31485093$

$-1.31485093$

$-1.31485093$

$-1.31485093$

$-1.20901549$

$-1.20901549$

$-1.20901549$

$-1.20901549$

2. 28008008

2.28008008

2.28008008

2.28008008

2.38591576

2.38591576

2.38591576

2.38591576

$-1.21978784$

$-1.21978784$

$-1.21978784$

$-1.21978784$

$-1.11395252$

$-1.11395252$

$-1.11395252$

$-1.11395252$

1.06017745

1.06017745

1. 06017745

1. 06017745

1. 16601288

1.16601288

1. 16601288
$0.1253 \quad-0.0071$

0.16506203

0.16506203

0.27089748

0.27089748

0.16506203

0.16506203

0.27089748

0.27089748

$-0.19434193$

$-0.19434193$

$-0.08850648$

$-0.08850648$

$-0.19434193$

$-0.19434193$

$-0.08850648$

$-0.08850648$

1. 30933321

1.30933321

1. 41516852

1. 41516852

1. 30933321

1. 30933321

1. 41516852

1. 41516852

$-1.38936973$

$-1.38936973$

$-1.28353417$

$-1.28353417$

$-1.38936973$

$-1.38936973$

$-1.28353417$

$-1.28353417$

$-0.22089551$

$-0.22089551$

$-0.11506007$

$-0.11506007$

$-0.22089551$

$-0.22089551$

$-0.11506007$
$-0.18798651$

$-0.08215106$

$-0.18798651$

$-0.08215106$

$-0.18798651$

$-0.08215106$

$-0.18798651$

$-0.08215106$

$-0.81211877$

$-0.70628333$

$-0.81211877$

$-0.70628333$

$-0.81211877$

$-0.70628333$

$-0.81211877$

$-0.70628333$

$-0.33387381$

$-0.22803834$

$-0.33387381$

$-0.22803834$

$-0.33387381$

$-0.22803834$

$-0.33387381$

$-0.22803834$
$-0.14612690$

$-0.34901077$

$-0.02188481$

0.48439974

0.18895514

0.37489694

0.02356275

$-0.55443442$

0.26181790

0.27947709

$-0.15598075$

$-0.78022832$

0.25843936

$-1.01922727$

$-0.59822267$

1. 85129380

0.03238543

$-0.09359064$

0.01515756

0.02643171

$-0.05267792$

0.11453636

$-0.01065519$

$-0.02765983$
1.000 \# Electr. mom., scale-fac \# Magnetic mom.

$-0.01106351 \quad-1.52043009$

$0.09477194 \quad 1.18174779$

$-0.01106351$

0.09477194

$-0.01106351$

0.09477194

$-0.01106351$

0.09477194

$-0.22601055$

$-0.12017509$

$-0.22601055$

$-0.12017509$

$-0.22601055$

$-0.12017509$

$-0.22601055$

$-0.12017509$

0.43990609

0.54574150

0.43990609

0.54574150

0.43990609

0.54574150

0.43990609

0.54574150

0.08411802

0.18995346

0.08411802

0.18995346

0.08411802

0.18995346

0.08411802

0.18995346

$-0.74873185$

$-0.64289635$

$-0.74873185$

$-0.64289635$

$-0.74873185$

$-0.64289635$

$-0.74873185$
1. 64017224

$-1.25686300$

1.22976029

$-1.06983638$

$-1.33950222$

1.15461135

3. 28281951

$-3.46589756$

$-2.98086786$

3. 36942458

$-1.91017330$

2.11069655

1.70678473

$-2.07727170$

$-0.24838892$

0.15546073

0.24712284

$-0.17872111$

0.05359959

$-0.02279451$

$-0.10943957$

0.08706175

1. 56179142

$-1.78532743$

$-3.36699033$

3. 48611975

$-1.27094340$

1. 46606731

2.98825026

$-3.08260489$

1. 54145694

$-2.21380091$

$-1.87573302$

2. 63740730

$-1.35107028$

2.14312077

1. 74661899 


1.16601288
-2.49104571
-2.49104571
-2.49104571
-2.49104571
-2.38521028
-2.38521028
-2.38521028
-2.38521028
0.87829608
0.87829608
0.87829608
0.87829608
0.98413152
0.98413152
0.98413152
0.98413152
-2.48172688
-2.48172688
-2.48172688
-2.48172688
-2.37589145
-2.37589145
-2.37589145
-2.37589145

64
$-0.11506007$
0.48034528
0.48034528
0.58618075
0.58618075
0.48034528
0.48034528
0.58618075
0.58618075
$-1.21926355$
$-1.21926355$
$-1.11342812$
$-1.11342812$
$-1.21926355$
$-1.21926355$
$-1.11342812$
$-1.11342812$
1.48277950
1.48277950
1.58861494
1.58861494
1. 48277950
1.48277950
1.58861494
1.58861494
$\begin{array}{rr}\text {. } & \text { Energy } 3->5 \\ 0.0953 & 0.0375\end{array}$

$-0.0503$

$-0.0286$

2. 15289307

2.15289307

2. 15289307

2. 15289307

2. 25872827

2. 25872827

2. 25872827

2. 25872827

$-1.31485093$

$-1.31485093$

$-1.31485093$

$-1.31485093$

$-1.20901549$

$-1.20901549$

$-1.20901549$

$-1.20901549$

2. 28008008

2. 28008008

2.28008008

2. 28008008

2. 38591576

2. 38591576

2.38591576

2. 38591576

$-1.21978784$

$-1.21978784$

$-1.21978784$

$-1.21978784$

$-1.11395252$

$-1.11395252$

$-1.11395252$

$-1.11395252$

1.06017745

1.06017745

1. 06017745

1. 06017745

1. 16601288

1.16601288

1. 16601288
$0.3642 \quad-0.0337$

0.16506203

0.16506203

0.27089748

0.16506203

0.16506203

0.27089748

0.27089748

$-0.19434193$

$-0.19434193$

$-0.08850648$

$-0.08850648$

$-0.19434193$

$-0.19434193$

$-0.08850648$

$-0.08850648$

1. 30933321

1.30933321

1. 41516852

1. 41516852

1. 30933321

1. 30933321

1. 41516852

1.41516852

$-1.38936973$

$-1.38936973$

$-1.28353417$

$-1.28353417$

$-1.38936973$

$-1.38936973$

$-1.28353417$

$-1.28353417$

$-0.22089551$

$-0.22089551$

$-0.11506007$

$-0.11506007$

$-0.22089551$

$-0.22089551$

$-0.11506007$
0.27089748

$\begin{array}{rr}-0.64289635 & -2.62690592 \\ -0.18798651 & 3.80747938 \\ -0.08215106 & -2.57494068 \\ -0.18798651 & -3.48341203 \\ -0.08215106 & 2.32434058 \\ -0.18798651 & -4.60550404 \\ -0.08215106 & 2.97421241 \\ -0.18798651 & 4.25861835 \\ -0.08215106 & -2.69353771 \\ -0.81211877 & 0.12669072 \\ -0.70628333 & -0.02043319 \\ -0.81211877 & 0.20253435 \\ -0.70628333 & -0.38333988 \\ -0.81211877 & 0.04524083 \\ -0.70628333 & -0.14504600 \\ -0.81211877 & -0.38699457 \\ -0.70628333 & 0.55078262 \\ -0.33387381 & 0.21435849 \\ -0.22803834 & -0.00357012 \\ -0.33387381 & -0.09808605 \\ -0.22803834 & -0.05055671 \\ -0.33387381 & -0.21943019 \\ -0.22803834 & -0.09816792 \\ -0.33387381 & 0.12263359 \\ -0.22803834 & 0.09959572\end{array}$
1.000 \# Electr. mom., scale-fac \# Magnetic mom.

$-0.01106351 \quad-0.88631946$

$0.09477194 \quad 1.16970789$

$-0.01106351 \quad 0.35926631$

$0.09477194 \quad-0.78430444$

$\begin{array}{ll}-0.01106351 & 0.60827118\end{array}$

$0.09477194 \quad-0.90896863$

$-0.01106351 \quad-0.16692701$

$0.09477194 \quad 0.59316546$

$-0.22601055$

$-0.12017509$

$-0.22601055$

$-0.12017509$

$-0.22601055$

$-0.12017509$

$-0.22601055$

$-0.12017509$

0.43990609

0.54574150

0.43990609

0.54574150

0.43990609

0.54574150

0.43990609

0.54574150

0.08411802

0.18995346

0.08411802

0.18995346

0.08411802

0.18995346

0.08411802

0.18995346

$-0.74873185$

$-0.64289635$

$-0.74873185$

$-0.64289635$

$-0.74873185$

$-0.64289635$

$-0.74873185$

3. 56817222

$-3.45348334$

$-3.75214267$

4. 11269140

$-0.22140124$

0.15599488

0.62399715

$-0.96123761$

0.16086058

$-0.20697267$

$-0.11098265$

0.18670611

$-0.22525521$

0.24093030

0.16432706

$-0.21391518$

3. 11913776

$-3.62583709$

$-8.07696056$

8.28995228

$-2.58180356$

2.96398640

7.37628889

$-7.45255470$

6.58056211

$-7.66357994$

$-6.68546963$

7. 75253296

$-4.40342283$

5.23289776

4. 62927485 


\subsection{8 \\ $-2.49104571$ \\ $-2.49104571$ \\ $-2.49104571$ \\ $-2.49104571$ \\ $-2.38521028$ \\ $-2.38521028$ \\ $-2.38521028$ \\ $-2.38521028$ \\ 0.87829608 \\ 0.87829608 \\ 0.87829608 \\ 0.87829608 \\ 0.98413152 \\ 0.98413152 \\ 0.98413152 \\ 0.98413152 \\ $-2.48172688$ \\ $-2.48172688$ \\ $-2.48172688$ \\ $-2.48172688$ \\ $-2.37589145$ \\ $-2.37589145$ \\ $-2.37589145$ \\ $-2.37589145$}

64

0.0782

2. 15289307

2. 15289307

2. 15289307

2.15289307

2. 25872827

2. 25872827

2. 25872827

2. 25872827

$-1.31485093$

$-1.31485093$

$-1.31485093$

$-1.31485093$

$-1.20901549$

$-1.20901549$

$-1.20901549$

$-1.20901549$

2. 28008008

2.28008008

2. 28008008

2.28008008

2. 38591576

2.38591576

2.38591576

2.38591576

$-1.21978784$

$-1.21978784$

$-1.21978784$

$-1.21978784$

$-1.11395252$

$-1.11395252$

$-1.11395252$

$-1.11395252$

1.06017745

1.06017745

1.06017745

1.06017745

1.16601288

1. 16601288

1. 16601288
$-0.11506007$

0.48034528

0.48034528

0.58618075

0.58618075

0.48034528

0.48034528

0.58618075

0.58618075

$-1.21926355$

$-1.21926355$

$-1.11342812$

$-1.11342812$

$-1.21926355$

$-1.21926355$

$-1.11342812$

$-1.11342812$

1. 48277950

1. 48277950

1.58861494

1. 58861494

1. 48277950

1.48277950

1. 58861494

1. 58861494

\# Energy 3->6

$\begin{array}{lr}0.0916 & -0.0984 \\ 0.0561 & 0.2746\end{array}$

0.16506203

0.16506203

0.27089748

0.27089748

0.16506203

0.16506203

0.27089748

0.27089748

$-0.19434193$

$-0.19434193$

$-0.08850648$

$-0.08850648$

$-0.19434193$

$-0.19434193$

$-0.08850648$

$-0.08850648$

1. 30933321

1.30933321

1. 41516852

1. 41516852

1. 30933321

1.30933321

1. 41516852

1.41516852

$-1.38936973$

$-1.38936973$

$-1.28353417$

$-1.28353417$

$-1.38936973$

$-1.38936973$

$-1.28353417$

$-1.28353417$

$-0.22089551$

$-0.22089551$

$-0.11506007$

$-0.11506007$

$-0.22089551$

$-0.22089551$

$-0.11506007$

$\begin{array}{rr}-0.64289635 & -5.44847727 \\ -0.18798651 & 7.01546860 \\ -0.08215106 & -4.33752966 \\ -0.18798651 & -6.14397860 \\ -0.08215106 & 3.63421130 \\ -0.18798651 & -8.73704338 \\ -0.08215106 & 5.20576286 \\ -0.18798651 & 7.81426144 \\ -0.08215106 & -4.44478989 \\ -0.81211877 & 0.91803259 \\ -0.70628333 & -0.78705060 \\ -0.81211877 & -0.36558983 \\ -0.70628333 & 0.10383948 \\ -0.81211877 & -0.26793748 \\ -0.70628333 & 0.09573049 \\ -0.81211877 & -0.41168913 \\ -0.70628333 & 0.71907312 \\ -0.33387381 & 0.15269090 \\ -0.22803834 & 0.32324234 \\ -0.33387381 & 0.05571745 \\ -0.22803834 & -0.40075606 \\ -0.33387381 & -0.20412934 \\ -0.22803834 & -0.48361522 \\ -0.33387381 & 0.04230233 \\ -0.22803834 & 0.44506827\end{array}$
1.000 \# Electr. mom., scale-fac \# Magnetic mom.

$-0.01106351$

0.09477194

$-0.01106351$

0.09477194

$-0.01106351$

0.09477194

$-0.01106351$

0.09477194

$-0.22601055$

$-0.12017509$

$-0.22601055$

$-0.12017509$

$-0.22601055$

$-0.12017509$

$-0.22601055$

$-0.12017509$

0.43990609

0.54574150

0.43990609

0.54574150

0.43990609

0.54574150

0.43990609

0.54574150

0.08411802

0.18995346

0.08411802

0.18995346

0.08411802

0.18995346

0.08411802

0.18995346

$-0.74873185$

$-0.64289635$

$-0.74873185$

$-0.64289635$

$-0.74873185$

$-0.64289635$

$-0.74873185$
1.85404718

$-0.54512399$

$-1.19311714$

0.00564277

$-1.32271254$

0.24548686

0.79771280

0.19336000

11.48263645

$-8.98150730$

$-12.09545803$

9.46884060

$-12.38889217$

9.56001663

12.95576286

$-10.02925205$

$-0.29317185$

0.46825075

0.22193357

$-0.39777553$

0.17561883

$-0.36174357$

$-0.11936868$

0.30550390

2. 04574156

$-1.15342140$

$-1.34307086$ 0.29382002

$-2.27458119$

1.37759614

1.60051501

$-0.54608780$

$-7.07487917$

7. 49475908

6.51599026

$-6.85089731$

6.94729090

$-7.59245062$

$-6.32998228$ 


1.16601288
-2.49104571
-2.49104571
-2.49104571
-2.49104571
-2.38521028
-2.38521028
-2.38521028
-2.38521028
0.87829608
0.87829608
0.87829608
0.87829608
0.98413152
0.98413152
0.98413152
0.98413152
-2.48172688
-2.48172688
-2.48172688
-2.48172688
-2.37589145
-2.37589145
-2.37589145
-2.37589145

64

0.4823

$-0.0223$

2.15289307

2.15289307

2.15289307

2. 15289307

2. 25872827

2. 25872827

2. 25872827

2. 25872827

$-1.31485093$

$-1.31485093$

$-1.31485093$

$-1.31485093$

$-1.20901549$

$-1.20901549$

$-1.20901549$

$-1.20901549$

2. 28008008

2. 28008008

2. 28008008

2. 28008008

2.38591576

2.38591576

2.38591576

2.38591576

$-1.21978784$

$-1.21978784$

$-1.21978784$

$-1.21978784$

$-1.11395252$

$-1.11395252$

$-1.11395252$

$-1.11395252$

1.06017745

1.06017745

1.06017745

1.06017745

1. 16601288

1.16601288

1. 16601288

$\begin{array}{rr}-0.64289635 & 6.80721092 \\ -0.18798651 & -1.98707628 \\ -0.08215106 & 2.19019818 \\ -0.18798651 & 1.64940798 \\ -0.08215106 & -1.95015085 \\ -0.18798651 & 2.11852360 \\ -0.08215106 & -2.27467775 \\ -0.18798651 & -1.68964875 \\ -0.08215106 & 1.97288620 \\ -0.81211877 & -0.40946016 \\ -0.70628333 & 0.14506292 \\ -0.81211877 & 0.16688821 \\ -0.70628333 & 0.15444803 \\ -0.81211877 & 0.13984936 \\ -0.70628333 & -0.00001267 \\ -0.81211877 & 0.07102415 \\ -0.70628333 & -0.21822792 \\ -0.33387381 & -0.29045752 \\ -0.22803834 & 0.46720120 \\ -0.33387381 & 0.13889280 \\ -0.22803834 & -0.26287255 \\ -0.33387381 & 0.24906369 \\ -0.22803834 & -0.44939989 \\ -0.33387381 & -0.10898901 \\ -0.22803834 & 0.25328359\end{array}$

. \# Energy 3->7

$0.0875 \quad-0.0040$

$\begin{array}{ll}0.1688 & 0.0443\end{array}$

0.16506203

0.16506203

0.27089748

0.27089748

0.16506203

0.16506203

0.27089748

0.27089748

$-0.19434193$

$-0.19434193$

$-0.08850648$

$-0.08850648$

$-0.19434193$

$-0.19434193$

$-0.08850648$

$-0.08850648$

1. 30933321

1.30933321

1. 41516852

1. 41516852

1. 30933321

1. 30933321

1. 41516852

1. 41516852

$-1.38936973$

$-1.38936973$

$-1.28353417$

$-1.28353417$

$-1.38936973$

$-1.38936973$

$-1.28353417$

$-1.28353417$

$-0.22089551$

$-0.22089551$

$-0.11506007$

$-0.11506007$

$-0.22089551$

$-0.22089551$

$-0.11506007$

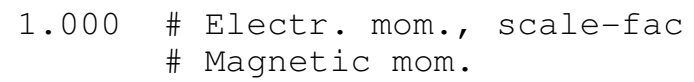




1.16601288
-2.49104571
-2.49104571
-2.49104571
-2.49104571
-2.38521028
-2.38521028
-2.38521028
-2.38521028
0.87829608
0.87829608
0.87829608
0.87829608
0.98413152
0.98413152
0.98413152
0.98413152
-2.48172688
-2.48172688
-2.48172688
-2.48172688
-2.37589145
-2.37589145
-2.37589145
-2.37589145

64
$-0.11506007$
0.48034528
0.48034528
0.58618075
0.58618075
0.48034528
0.48034528
0.58618075
0.58618075
$-1.21926355$
$-1.21926355$
$-1.11342812$
$-1.11342812$
$-1.21926355$
$-1.21926355$
$-1.11342812$
$-1.11342812$
1. 48277950
1. 48277950
1. 58861494
1.58861494
1. 48277950
1. 48277950
1.58861494
1.58861494
-0.64289635
-0.18798651
-0.08215106
-0.18798651
-0.08215106
-0.18798651
-0.08215106
-0.18798651
-0.08215106
-0.81211877
-0.70628333
-0.81211877
-0.70628333
-0.81211877
-0.70628333
-0.81211877
-0.70628333
-0.33387381
-0.22803834
-0.33387381
-0.22803834
-0.33387381
-0.22803834
-0.33387381
-0.22803834

\# Energy 3->8

$\begin{array}{llr}0.1938 & -0.0088 & 0.1329 \\ 0.0050 & -0.1015 & -0.1219\end{array}$

2.15289307

2.15289307

2.15289307

2.15289307

2. 25872827

2.25872827

2. 25872827

2. 25872827

$-1.31485093$

$-1.31485093$

$-1.31485093$

$-1.31485093$

$-1.20901549$

$-1.20901549$

$-1.20901549$

$-1.20901549$

2. 28008008

2. 28008008

2. 28008008

2. 28008008

2. 38591576

2.38591576

2.38591576

2. 38591576

$-1.21978784$

$-1.21978784$

$-1.21978784$

$-1.21978784$

$-1.11395252$

$-1.11395252$

$-1.11395252$

$-1.11395252$

1.06017745

1.06017745

1. 06017745

1.06017745

1. 16601288

1. 16601288

1. 16601288
0.16506203

0.16506203

0.27089748

0.27089748

0.16506203

0.27089748

0.27089748

$-0.19434193$

$-0.19434193$

$-0.08850648$

$-0.08850648$

$-0.19434193$

$-0.19434193$

$-0.08850648$

$-0.08850648$

1. 30933321

1.30933321

1. 41516852

1. 41516852

1. 30933321

1. 30933321

1. 41516852

1. 41516852

$-1.38936973$

$-1.38936973$

$-1.28353417$

$-1.28353417$

$-1.38936973$

$-1.38936973$

$-1.28353417$

$-1.28353417$

$-0.22089551$

$-0.22089551$

$-0.11506007$

$-0.11506007$

$-0.22089551$

$-0.22089551$

$-0.11506007$
0.16506203
1.08092535

4. 26247883

$-4.26704741$

$-4.23282194$

4.35029793

$-4.80872107$

4.55411482

4. 77987576

$-4.64767504$

$-0.54158366$

0.95970529

1.13544881

$-1.78006518$

0.57634383

$-0.89290470$

$-1.10462797$

1.59381497

0.75972247

$-0.96164942$

$-0.52453059$

0.67463100

$-0.80311328$

0.94777137

0.56065536

$-0.67074168$

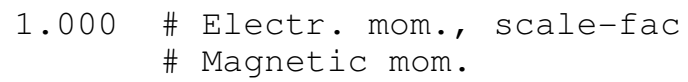




1.16601288
-2.49104571
-2.49104571
-2.49104571
-2.49104571
-2.38521028
-2.38521028
-2.38521028
-2.38521028
0.87829608
0.87829608
0.87829608
0.87829608
0.98413152
0.98413152
0.98413152
0.98413152
-2.48172688
-2.48172688
-2.48172688
-2.48172688
-2.37589145
-2.37589145
-2.37589145
-2.37589145

64
$-0.11506007$
0.48034528
0.48034528
0.58618075
0.58618075
0.48034528
0.48034528
0.58618075
0.58618075
$-1.21926355$
$-1.21926355$
$-1.11342812$
$-1.11342812$
$-1.21926355$
$-1.21926355$
$-1.11342812$
$-1.11342812$
1.48277950
1. 48277950
1.58861494
1.58861494
1. 48277950
1. 48277950
1.58861494
1.58861494
-0.64289635
-0.18798651
-0.08215106
-0.18798651
-0.08215106
-0.18798651
-0.08215106
-0.18798651
-0.08215106
-0.81211877
-0.70628333
-0.81211877
-0.70628333
-0.81211877
-0.70628333
-0.81211877
-0.70628333
-0.33387381
-0.22803834
-0.33387381
-0.22803834
-0.33387381
-0.22803834
-0.33387381
-0.22803834

\# Energy 3->9

$\begin{array}{llr}0.0181 & -0.0242 & 0.0200 \\ 0.0152 & 0.0833 & -0.0162\end{array}$

2.15289307

2.15289307

2.15289307

2.15289307

2. 25872827

2. 25872827

2. 25872827

2. 25872827

$-1.31485093$

$-1.31485093$

$-1.31485093$

$-1.31485093$

$-1.20901549$

$-1.20901549$

$-1.20901549$

$-1.20901549$

2. 28008008

2. 28008008

2.28008008

2. 28008008

2. 38591576

2.38591576

2.38591576

2. 38591576

$-1.21978784$

$-1.21978784$

$-1.21978784$

$-1.21978784$

$-1.11395252$

$-1.11395252$

$-1.11395252$

$-1.11395252$

1.06017745

1. 06017745

1. 06017745

1. 06017745

1. 16601288

1.16601288

1. 16601288
0.16506203

0.16506203

0.27089748

0.27089748

0.16506203

0.16506203

0.27089748

0.27089748

$-0.19434193$

$-0.19434193$

$-0.08850648$

$-0.08850648$

$-0.19434193$

$-0.19434193$

$-0.08850648$

$-0.08850648$

1. 30933321

1.30933321

1. 41516852

1. 41516852

1. 30933321

1. 30933321

1. 41516852

1. 41516852

$-1.38936973$

$-1.38936973$

$-1.28353417$

$-1.28353417$

$-1.38936973$

$-1.38936973$

$-1.28353417$

$-1.28353417$

$-0.22089551$

$-0.22089551$

$-0.11506007$

$-0.11506007$

$-0.22089551$

$-0.22089551$

$-0.11506007$
$-0.12017509$

0.54574150
11.19243431
0.34348026

$-0.86057627$

$-0.83475310$

1.38601959

$-0.21367104$

0.59082848

0.87414533

$-1.26201928$

0.73396605

$-0.69911367$

$-0.86877519$

0.73987448

$-0.30827007$

0.13671491

0.27386048

0.04422877

0.32855472

$-0.53001058$

$-0.16831359$

0.30927882

$-0.36032203$

0.48244783

0.18959972

$-0.27372715$

$$
\begin{gathered}
1.000 \text { \# Electr. mom., scale-fac } \\
\text { \# Magnetic mom. }
\end{gathered}
$$
$-0.01106351$
1.47693896

0.09477194

$-0.01106351$

0.09477194

$-0.01106351$

0.09477194

$-0.01106351$

0.09477194

$-0.22601055$

$-0.12017509$

$-0.22601055$

$-0.12017509$

$-0.22601055$

$-0.12017509$

$-0.22601055$

0.43990609

0.43990609

0.54574150

0.43990609

0.54574150

0.43990609

0.54574150

0.08411802

0.18995346

0.08411802

0.18995346

0.08411802

0.18995346

0.08411802

0.18995346

$-0.74873185$

$-0.64289635$

$-0.74873185$

$-0.64289635$

$-0.74873185$

$-0.64289635$

$-0.74873185$
$-0.79701793$

$-1.96644521$

1. 14720035

$-1.12434256$

0.57962894

1. 50962126

$-0.86064488$

$-0.89670908$

1. 53349197

0.32886794

$-0.91146833$

1. 92940557

$-2.55189800$

$-1.37734556$

1.96247756

0.63671273

$-0.55081600$

$-0.52539825$

0.48555622

$-0.43635440$

0.38650158

0.37682289

$-0.36340809$

1. 45960724

$-1.35451758$

$-2.83552265$

2. 59907484

$-1.28399312$

1.14780664

2. 60936189

$-2.33688402$

1. 70772898

$-1.26596928$

$-1.39998686$

0.81409854

$-0.62608254$

$-0.06860454$

0.31650004 


1.16601288
-2.49104571
-2.49104571
-2.49104571
-2.49104571
-2.38521028
-2.38521028
-2.38521028
-2.38521028
0.87829608
0.87829608
0.87829608
0.87829608
0.98413152
0.98413152
0.98413152
0.98413152
-2.48172688
-2.48172688
-2.48172688
-2.48172688
-2.37589145
-2.37589145
-2.37589145
-2.37589145

64

$-0.4152$

0.0057

2.15289307

2.15289307

2. 15289307

2.15289307

2. 25872827

2. 25872827

2. 25872827

2. 25872827

$-1.31485093$

$-1.31485093$

$-1.31485093$

$-1.31485093$

$-1.20901549$

$-1.20901549$

$-1.20901549$

$-1.20901549$

2. 28008008

2. 28008008

2.28008008

2.28008008

2.38591576

2.38591576

2.38591576

2.38591576

$-1.21978784$

$-1.21978784$

$-1.21978784$

$-1.21978784$

$-1.11395252$

$-1.11395252$

$-1.11395252$

$-1.11395252$

1.06017745

1.06017745

1.06017745

1.06017745

1.16601288

1.16601288

$\begin{aligned}-0.11506007 & -0.64289635 \\ 0.48034528 & -0.18798651 \\ 0.48034528 & -0.08215106 \\ 0.58618075 & -0.18798651 \\ 0.58618075 & -0.08215106 \\ 0.48034528 & -0.18798651 \\ 0.48034528 & -0.08215106 \\ 0.58618075 & -0.18798651 \\ 0.58618075 & -0.08215106 \\ -1.21926355 & -0.81211877 \\ -1.21926355 & -0.70628333 \\ -1.11342812 & -0.81211877 \\ -1.11342812 & -0.70628333 \\ -1.21926355 & -0.81211877 \\ -1.21926355 & -0.70628333 \\ -1.11342812 & -0.81211877 \\ -1.11342812 & -0.70628333 \\ 1.48277950 & -0.33387381 \\ 1.48277950 & -0.22803834 \\ 1.58861494 & -0.33387381 \\ 1.58861494 & -0.22803834 \\ 1.48277950 & -0.33387381 \\ 1.48277950 & -0.22803834 \\ 1.58861494 & -0.33387381 \\ 1.58861494 & -0.22803834\end{aligned}$

0. \# Energy 4->5

$0.5829 \quad 0.5627$

$0.0126 \quad 0.0091$

0.16506203

0.16506203

0.27089748

0.27089748

0.16506203

0.16506203

0.27089748

0.27089748

$-0.19434193$

$-0.19434193$

$-0.08850648$

$-0.08850648$

$-0.19434193$

$-0.19434193$

$-0.08850648$

$-0.08850648$

1.30933321

1.30933321

1.41516852

1.41516852

1.30933321

1.30933321

1.41516852

1.41516852

$-1.38936973$

$-1.38936973$

$-1.28353417$

$-1.28353417$

$-1.38936973$

$-1.38936973$

$-1.28353417$

$-1.28353417$

$-0.22089551$

$-0.22089551$

$-0.11506007$

$-0.11506007$

$-0.22089551$

$-0.22089551$
0.52825415

1.26817572

$-1.41522694$

$-1.57633698$

1.77963257

$-1.43685317$

1.43000674

1.84095788

$-1.88735604$

0.38010353

$-0.34760335$

$-0.33100319$

0.25716689

$-0.16220503$

0.09852427

0.04899898

0.06607091

0.42829883

$-0.56158781$

$-0.27026889$

0.36407587

$-0.49060318$

0.57360876

0.31945279

$-0.37827882$
1.000 \# Electr. mom., scale-fac \# Magnetic mom.

$\begin{array}{rr}-0.01106351 & -13.79150105 \\ 0.09477194 & 10.55551243 \\ -0.01106351 & 11.90519333 \\ 0.09477194 & -8.99508572 \\ -0.01106351 & 11.06784725 \\ 0.09477194 & -7.44684935 \\ -0.01106351 & -9.10742855 \\ 0.09477194 & 5.87283897 \\ -0.22601055 & 6.26790333 \\ -0.12017509 & -7.43925619 \\ -0.22601055 & -8.28031826 \\ -0.12017509 & 9.39132500 \\ -0.22601055 & -5.80727339 \\ -0.12017509 & 7.59502840 \\ -0.22601055 & 7.67366934 \\ -0.12017509 & -9.57928658\end{array}$

$-0.12017509 \quad-9.57928658$

$0.43990609 \quad 1.94309545$

$0.54574150 \quad-1.49205244$

$0.43990609-1.79742801$

$0.54574150 \quad 1.44682407$

$0.43990609 \quad-2.23663497$

$0.54574150 \quad 1.82310867$

$0.43990609 \quad 2.12223983$

$0.54574150 \quad-1.75720167$

$0.08411802 \quad-1.20668352$

$0.18995346 \quad 1.27914667$

$0.08411802 \quad 1.29800892$

$0.18995346 \quad-1.37468708$

$0.08411802 \quad 1.18311894$

0.18995346

0.08411802

0.18995346

$-0.74873185$

$-0.64289635$

$-0.74873185$

$-0.64289635$

$-0.74873185$

$-0.64289635$

$-1.33321714$

$-1.30499220$

1.41997266

5.57740307

$-8.05725956$

$-6.02879286$

8.67773724

$-4.59626245$

6.74005604 
1.16601288
1.16601288
-2.49104571
-2.49104571
-2.49104571
-2.49104571
-2.38521028
-2.38521028
-2.38521028
-2.38521028
0.87829608
0.87829608
0.87829608
0.87829608
0.98413152
0.98413152
0.98413152
0.98413152
-2.48172688
-2.48172688
-2.48172688
-2.48172688
-2.37589145
-2.37589145
-2.37589145
-2.37589145

64

$\begin{array}{llr}1.1204 & 0.2918 & -0.1957 \\ 0.0535 & 0.0830 & 0.0700\end{array}$

2. 15289307

2.15289307

2.15289307

2.15289307

2. 25872827

2. 25872827

2. 25872827

2. 25872827

$-1.31485093$

$-1.31485093$

$-1.31485093$

$-1.31485093$

$-1.20901549$

$-1.20901549$

$-1.20901549$

$-1.20901549$

2. 28008008

2. 28008008

2.28008008

2. 28008008

2.38591576

2.38591576

2. 38591576

2. 38591576

$-1.21978784$

$-1.21978784$

$-1.21978784$

$-1.21978784$

$-1.11395252$

$-1.11395252$

$-1.11395252$

$-1.11395252$

1.06017745

1.06017745

1.06017745

1.06017745

1. 16601288

1.16601288
$-0.11506007$

$-0.11506007$

0.48034528

0.48034528

0.58618075

0.58618075

0.48034528

0.48034528

0.58618075

0.58618075

$-1.21926355$

$-1.21926355$

$-1.11342812$

$-1.11342812$

$-1.21926355$

$-1.21926355$

$-1.11342812$

$-1.11342812$

1.48277950

1. 48277950

1.58861494

1.58861494

1. 48277950

1. 48277950

1. 58861494

1.58861494

\# Energy 4->6

$$
0.0830 \quad 0.0700
$$

0.16506203

0.16506203

0.27089748

0.27089748

0.16506203

0.16506203

0.27089748

0.27089748

$-0.19434193$

$-0.19434193$

$-0.08850648$

$-0.08850648$

$-0.19434193$

$-0.19434193$

$-0.08850648$

$-0.08850648$

1.30933321

1.30933321

1. 41516852

1. 41516852

1. 30933321

1. 30933321

1. 41516852

1. 41516852

$-1.38936973$

$-1.38936973$

$-1.28353417$

$-1.28353417$

$-1.38936973$

$-1.38936973$

$-1.28353417$

$-1.28353417$

$-0.22089551$

$-0.22089551$

$-0.11506007$

$-0.11506007$

$-0.22089551$

$-0.22089551$
$-0.74873185$

$-0.64289635$

$-0.18798651$

$-0.08215106$

$-0.18798651$

$-0.08215106$

$-0.18798651$

$-0.08215106$

$-0.18798651$

$-0.08215106$

$-0.81211877$

$-0.70628333$

$-0.81211877$

$-0.70628333$

$-0.81211877$

$-0.70628333$

$-0.81211877$

$-0.70628333$

$-0.33387381$

$-0.22803834$

$-0.33387381$

$-0.22803834$

$-0.33387381$

$-0.22803834$

$-0.33387381$

$-0.22803834$

$$
\begin{gathered}
1.000 \text { \# Electr. mom., scale-fac } \\
\text { \# Magnetic mom. }
\end{gathered}
$$

$-0.01106351$

0.09477194

$-0.01106351$

0.09477194

$-0.01106351$

0.09477194

$-0.01106351$

0.09477194

$-0.22601055$

$-0.12017509$

$-0.22601055$

$-0.12017509$

$-0.22601055$

$-0.12017509$

$-0.22601055$

$-0.12017509$

0.43990609

0.54574150

0.43990609

0.54574150

0.43990609

0.54574150

0.43990609

0.54574150

0.08411802

0.18995346

0.08411802

0.18995346

0.08411802

0.18995346

0.08411802

0.18995346

$-0.74873185$

$-0.64289635$

$-0.74873185$

$-0.64289635$

$-0.74873185$

$-0.64289635$
5.53916550

$-8.03096962$

$-7.39974737$

10.52285576

5.53243160

$-9.24679756$

7.37429619

$-10.41007710$

$-5.08591080$

8.97743034

0.22893803

$-0.63704729$

$-0.41741386$

0.95431131

$-0.03518542$

0.31357369

0.10880932

$-0.45078307$

$-0.02888640$

0.64162368

$-0.01578263$

$-0.38585377$

$-0.38981315$

$-0.34775925$

0.34160402

0.13916768$$
\text { 2.17049003 }
$$

$-0.75024903$

$-2.47619700$

0.88850915

$-1.10564125$

0.18004981

1. 43933952

$-0.36913228$

11.82656574

$-11.72436523$

$-13.52764225$

13.27364063

$-13.06595707$

13.00227070

14.84571075

$-14.67264271$

0.22167462

$-0.07063234$

$-0.38316053$

0.28660187

0.25636074

$-0.27016890$

0.06573569

$-0.05376560$

0.28270784

$-0.29102838$

$-0.58794630$

$-0.59182733$

0.62313253

0.91034991

$-0.99459577$

2. 52654409

$-1.11975980$

$-3.72322416$

2.66499710

$-0.86770320$

$-0.94428051$
0.62078780 
1.16601288
1.16601288
-2.49104571
-2.49104571
-2.49104571
-2.49104571
-2.38521028
-2.38521028
-2.38521028
-2.38521028
0.87829608
0.87829608
0.87829608
0.87829608
0.98413152
0.98413152
0.98413152
0.98413152
-2.48172688
-2.48172688
-2.48172688
-2.48172688
-2.37589145
-2.37589145
-2.37589145
-2.37589145

64

$\begin{array}{rrr}1.5301 & 0.2979 & 0.2801 \\ -0.0242 & 0.0915 & 0.0683\end{array}$

2. 15289307

2. 15289307

2.15289307

2.15289307

2. 25872827

2. 25872827

2. 25872827

2. 25872827

$-1.31485093$

$-1.31485093$

$-1.31485093$

$-1.31485093$

$-1.20901549$

$-1.20901549$

$-1.20901549$

$-1.20901549$

2. 28008008

2. 28008008

2. 28008008

2. 28008008

2.38591576

2.38591576

2. 38591576

2. 38591576

$-1.21978784$

$-1.21978784$

$-1.21978784$

$-1.21978784$

$-1.11395252$

$-1.11395252$

$-1.11395252$

$-1.11395252$

1.06017745

1.06017745

1.06017745

1. 06017745

1. 16601288

1. 16601288
$-0.11506007$

$-0.11506007$

0.48034528

0.48034528

0.58618075

0.58618075

0.48034528

0.48034528

0.58618075

0.58618075

$-1.21926355$

$-1.11342812$

$-1.11342812$

$-1.21926355$

$-1.21926355$

$-1.11342812$

$-1.11342812$

1.48277950

1. 48277950

1. 58861494

1.58861494

1. 48277950

1. 48277950

1. 58861494

1.58861494

0.0915

0.16506203

0.16506203

0.27089748

0.27089748

0.16506203

0.16506203

0.27089748

0.27089748

$-0.19434193$

$-0.19434193$

$-0.08850648$

$-0.08850648$

$-0.19434193$

$-0.19434193$

$-0.08850648$

$-0.08850648$

1.30933321

1. 30933321

1.41516852

1. 41516852

1.30933321

1. 30933321

1. 41516852

1. 41516852

$-1.38936973$

$-1.38936973$

$-1.28353417$

$-1.28353417$

$-1.38936973$

$-1.38936973$

$-1.28353417$

$-1.28353417$

$-0.22089551$

$-0.22089551$

$-0.11506007$

$-0.11506007$

$-0.22089551$

$-0.22089551$
$-1.21926355$
$-0.74873185$

$-0.64289635$

$-0.18798651$

$-0.08215106$

$-0.18798651$

$-0.08215106$

$-0.18798651$

$-0.08215106$

$-0.18798651$

$-0.08215106$

$-0.81211877$

$-0.70628333$

$-0.81211877$

$-0.70628333$

$-0.81211877$

$-0.70628333$

$-0.81211877$

$-0.70628333$

$-0.33387381$

$-0.22803834$

$-0.33387381$

$-0.22803834$

$-0.33387381$

$-0.22803834$

$-0.33387381$

$-0.22803834$$$
\begin{gathered}
1.000 \text { \# Electr. mom., scale-fac } \\
\text { \# Magnetic mom. }
\end{gathered}
$$

$-0.01106351$

0.09477194

$-0.01106351$

0.09477194

$-0.01106351$

0.09477194

$-0.01106351$

0.09477194

$-0.22601055$

$-0.12017509$

$-0.22601055$

$-0.12017509$

$-0.22601055$

$-0.12017509$

$-0.22601055$

$-0.12017509$

0.43990609

0.54574150

0.43990609

0.54574150

0.43990609

0.54574150

0.43990609

0.54574150

0.08411802

0.18995346

0.08411802

0.18995346

0.08411802

0.18995346

0.08411802

0.18995346

$-0.74873185$

$-0.64289635$

$-0.74873185$

$-0.64289635$

$-0.74873185$

$-0.64289635$
$-2.93192363$

1. 99896681

$-0.50698370$

$-2.40833783$

1.61882329

2.16235709

$-1.39986324$

2. 67512321

$-1.64706838$

$-2.36529088$

1.33487117

$-0.10938546$

0.44046086

0.18137349

$-0.66070276$

0.29126781

$-0.66701680$

$-0.42858291$

0.96944475

$-0.33267963$

0.16261570

0.23409264

$-0.12335396$

0.20793942

0.05060290

$-0.13221444$

$-0.04200731$

$-0.39576176$

1.17165136

1.81890416

2.18336034

1. 24550891

$-0.71801859$

$-2.44396901$

$-1.81695235$

1.17053795

2. 86614776

$-2.14575028$

0.48904189

0.32048360

$-1.69917083$

0.79016888

$-2.62639523$

1.83595049

2.57331848

$-1.94770861$

3. 38281155

$-2.32470155$

$-3.06446743$

2. 25587296

$-1.66584992$

1. 55429292

2. 08317494

$-1.92503142$

1.31910574

$-1.23710477$

$-1.63644636$

1. 48830938

$-5.14278889$

6.99634171

5.92014933

$-7.98472118$

4.76798677

$-6.78547049$ 
1.16601288
1.16601288
-2.49104571
-2.49104571
-2.49104571
-2.49104571
-2.38521028
-2.38521028
-2.38521028
-2.38521028
0.87829608
0.87829608
0.87829608
0.87829608
0.98413152
0.98413152
0.98413152
0.98413152
-2.48172688
-2.48172688
-2.48172688
-2.48172688
-2.37589145
-2.37589145
-2.37589145
-2.37589145

64

$\begin{array}{rr}-0.74873185 & -5.62934160 \\ -0.64289635 & 7.90993643 \\ -0.18798651 & 1.17052627 \\ -0.08215106 & -2.35742521 \\ -0.18798651 & -0.92512274 \\ -0.08215106 & 2.19564891 \\ -0.18798651 & -1.08606374 \\ -0.08215106 & 2.37913609 \\ -0.18798651 & 0.77284777 \\ -0.08215106 & -2.20440865 \\ -0.81211877 & -0.63383472 \\ -0.70628333 & 0.87599218 \\ -0.81211877 & 0.94863743 \\ -0.70628333 & -1.31377888 \\ -0.81211877 & 0.54168487 \\ -0.70628333 & -0.79263419 \\ -0.81211877 & -0.81916338 \\ -0.70628333 & 1.19966471 \\ -0.33387381 & 0.35662949 \\ -0.22803834 & -0.82693434 \\ -0.33387381 & -0.24744809 \\ -0.22803834 & 0.59214032 \\ -0.33387381 & -0.39129543 \\ -0.22803834 & 0.95752805 \\ -0.33387381 & 0.26042953 \\ -0.22803834 & -0.67423660\end{array}$

1.1974

2. 15289307

2.15289307

2.15289307

2.15289307

2. 25872827

2. 25872827

2. 25872827

2. 25872827

$-1.31485093$

$-1.31485093$

$-1.31485093$

$-1.31485093$

$-1.20901549$

$-1.20901549$

$-1.20901549$

$-1.20901549$

2. 28008008

2. 28008008

2.28008008

2. 28008008

2.38591576

2.38591576

2.38591576

2. 38591576

$-1.21978784$

$-1.21978784$

$-1.21978784$

$-1.21978784$

$-1.11395252$

$-1.11395252$

$-1.11395252$

$-1.11395252$

1.06017745

1.06017745

1.06017745

1.06017745

1. 16601288

1. 16601288
$-0.11506007$

$-0.11506007$

0.48034528

0.48034528

0.58618075

0.58618075

0.48034528

0.48034528

0.58618075

0.58618075

$-1.21926355$

$-1.21926355$

$-1.11342812$

$-1.11342812$

$-1.21926355$

$-1.21926355$

$-1.11342812$

$-1.11342812$

1. 48277950

1. 48277950

1.58861494

1.58861494

1.48277950

1.48277950

1.58861494

1.58861494

0 . \# Energy 4->8

$\begin{array}{ll}0.0880 & 0.5208\end{array}$ $0.0898 \quad 0.1023$

0.16506203

0.16506203

0.27089748

0.27089748

0.16506203

0.16506203

0.27089748

0.27089748

$-0.19434193$

$-0.19434193$

$-0.08850648$

$-0.08850648$

$-0.19434193$

$-0.19434193$

$-0.08850648$

$-0.08850648$

1. 30933321

1. 30933321

1. 41516852

1. 41516852

1.30933321

1. 30933321

1. 41516852

1. 41516852

$-1.38936973$

$-1.38936973$

$-1.28353417$

$-1.28353417$

$-1.38936973$

$-1.38936973$

$-1.28353417$

$-1.28353417$

$-0.22089551$

$-0.22089551$

$-0.11506007$

$-0.11506007$

$-0.22089551$

$-0.22089551$

$$
\begin{aligned}
& 1.000 \text { \# Electr. mom., scale-fac } \\
& \text { \# Magnetic mom. }
\end{aligned}
$$

$-3.41161227$

$-4.63756466$

1. 21573615

6.93250179

$-1.40068686$

8.06174088

3. 47712612

$-10.06859016$

$-11.19636345$

9. 42426682

13.20834827

$-11.57555008$

11.62630558

$-9.25431442$

$-13.58547783$

11.43318748

$-5.44060183$

4.10480118

3.95131016

$-2.99376011$

4.80453444

$-3.51335526$

$-3.54252267$

2. 58402276

$-2.47029138$

1. 81717217

2. 93784285

$-2.09843135$

2. 73483634

$-1.97832847$

$-3.29852414$

2. 31082177

4. 38591146

$-9.14651299$

$-2.86025167$

7. 43494558

$-6.25645351$

11.75578880
0.74873185
-0.64289635 


\begin{tabular}{|c|c|c|c|}
\hline 1.16601288 & -0.11506007 & -0.74873185 & 5.26701021 \\
\hline 1.16601288 & -0.11506007 & -0.64289635 & -10.70878696 \\
\hline-2.49104571 & 0.48034528 & -0.18798651 & 5.66342783 \\
\hline-2.49104571 & 0.48034528 & -0.08215106 & -7.07827330 \\
\hline-2.49104571 & 0.58618075 & -0.18798651 & -4.82467508 \\
\hline-2.49104571 & 0.58618075 & -0.08215106 & 6.37152624 \\
\hline-2.38521028 & 0.48034528 & -0.18798651 & -6.18313217 \\
\hline-2.38521028 & 0.48034528 & -0.08215106 & 7.56722403 \\
\hline-2.38521028 & 0.58618075 & -0.18798651 & 5.16933250 \\
\hline-2.38521028 & 0.58618075 & -0.08215106 & -6.74936104 \\
\hline 0.87829608 & -1.21926355 & -0.81211877 & 0.55069304 \\
\hline 0.87829608 & -1.21926355 & -0.70628333 & -1.05983579 \\
\hline 0.87829608 & -1.11342812 & -0.81211877 & -0.69649690 \\
\hline 0.87829608 & -1.11342812 & -0.70628333 & 1.42014849 \\
\hline 0.98413152 & -1.21926355 & -0.81211877 & -0.43491790 \\
\hline 0.98413152 & -1.21926355 & -0.70628333 & 0.89334846 \\
\hline 0.98413152 & -1.11342812 & -0.81211877 & 0.56513298 \\
\hline 0.98413152 & -1.11342812 & -0.70628333 & -1.22445405 \\
\hline-2.48172688 & 1.48277950 & -0.33387381 & 0.78469419 \\
\hline-2.48172688 & 1.48277950 & -0.22803834 & -1.27122188 \\
\hline-2.48172688 & 1.58861494 & -0.33387381 & -0.50336808 \\
\hline-2.48172688 & 1.58861494 & -0.22803834 & 0.85228324 \\
\hline-2.37589145 & 1.48277950 & -0.33387381 & -0.79254216 \\
\hline-2.37589145 & 1.48277950 & -0.22803834 & 1.35488307 \\
\hline-2.37589145 & 1.58861494 & -0.33387381 & 0.49830988 \\
\hline-2.37589145 & 1.58861494 & -0.22803834 & -0.90295571 \\
\hline & \# Energy 4->9 & & \\
\hline-0.1047 & -0.1880 & 1.000 & Electr. mom., scale-fac \\
\hline 0.0289 & 0.0105 & \# & Magnetic mom. \\
\hline 2.15289307 & 0.16506203 & -0.01106351 & 2.58675742 \\
\hline 2.15289307 & 0.16506203 & 0.09477194 & 2.05750012 \\
\hline 2.15289307 & 0.27089748 & -0.01106351 & -2.69775462 \\
\hline 2.15289307 & 0.27089748 & 0.09477194 & -2.31293702 \\
\hline 2.25872827 & 0.16506203 & -0.01106351 & -0.06009739 \\
\hline 2.25872827 & 0.16506203 & 0.09477194 & -3.76806211 \\
\hline 2.25872827 & 0.27089748 & -0.01106351 & -0.05325073 \\
\hline 2.25872827 & 0.27089748 & 0.09477194 & 4.02902079 \\
\hline-1.31485093 & -0.19434193 & -0.22601055 & 5.32450151 \\
\hline-1.31485093 & -0.19434193 & -0.12017509 & -3.36658859 \\
\hline-1.31485093 & -0.08850648 & -0.22601055 & -6.36092997 \\
\hline-1.31485093 & -0.08850648 & -0.12017509 & 4.38548565 \\
\hline-1.20901549 & -0.19434193 & -0.22601055 & -5.92434978 \\
\hline-1.20901549 & -0.19434193 & -0.12017509 & 3.90027404 \\
\hline-1.20901549 & -0.08850648 & -0.22601055 & 6.87975979 \\
\hline-1.20901549 & -0.08850648 & -0.12017509 & -4.82314873 \\
\hline 2.28008008 & 1.30933321 & 0.43990609 & 4.37506390 \\
\hline 2.28008008 & 1.30933321 & 0.54574150 & -3.59806728 \\
\hline 2.28008008 & 1.41516852 & 0.43990609 & -3.37990856 \\
\hline 2.28008008 & 1.41516852 & 0.54574150 & 2.83769631 \\
\hline 2.38591576 & 1.30933321 & 0.43990609 & -3.83014965 \\
\hline 2.38591576 & 1.30933321 & 0.54574150 & 3.14487982 \\
\hline 2.38591576 & 1.41516852 & 0.43990609 & 3.04520965 \\
\hline 2.38591576 & 1.41516852 & 0.54574150 & -2.54321122 \\
\hline-1.21978784 & -1.38936973 & 0.08411802 & 0.89237249 \\
\hline-1.21978784 & -1.38936973 & 0.18995346 & -0.43882442 \\
\hline-1.21978784 & -1.28353417 & 0.08411802 & -1.32629609 \\
\hline-1.21978784 & -1.28353417 & 0.18995346 & 0.72051984 \\
\hline-1.11395252 & -1.38936973 & 0.08411802 & -1.19300354 \\
\hline-1.11395252 & -1.38936973 & 0.18995346 & 0.61754882 \\
\hline-1.11395252 & -1.28353417 & 0.08411802 & 1.75219369 \\
\hline-1.11395252 & -1.28353417 & 0.18995346 & -1.00074899 \\
\hline 1.06017745 & -0.22089551 & -0.74873185 & -5.68747473 \\
\hline 1.06017745 & -0.22089551 & -0.64289635 & 10.26762104 \\
\hline 1.06017745 & -0.11506007 & -0.74873185 & 5.56232166 \\
\hline 1.06017745 & -0.11506007 & -0.64289635 & -10.42983437 \\
\hline 1.16601288 & -0.22089551 & -0.74873185 & 6.03572273 \\
\hline 1.16601288 & -0.22089551 & -0.64289635 & 87636 \\
\hline
\end{tabular}




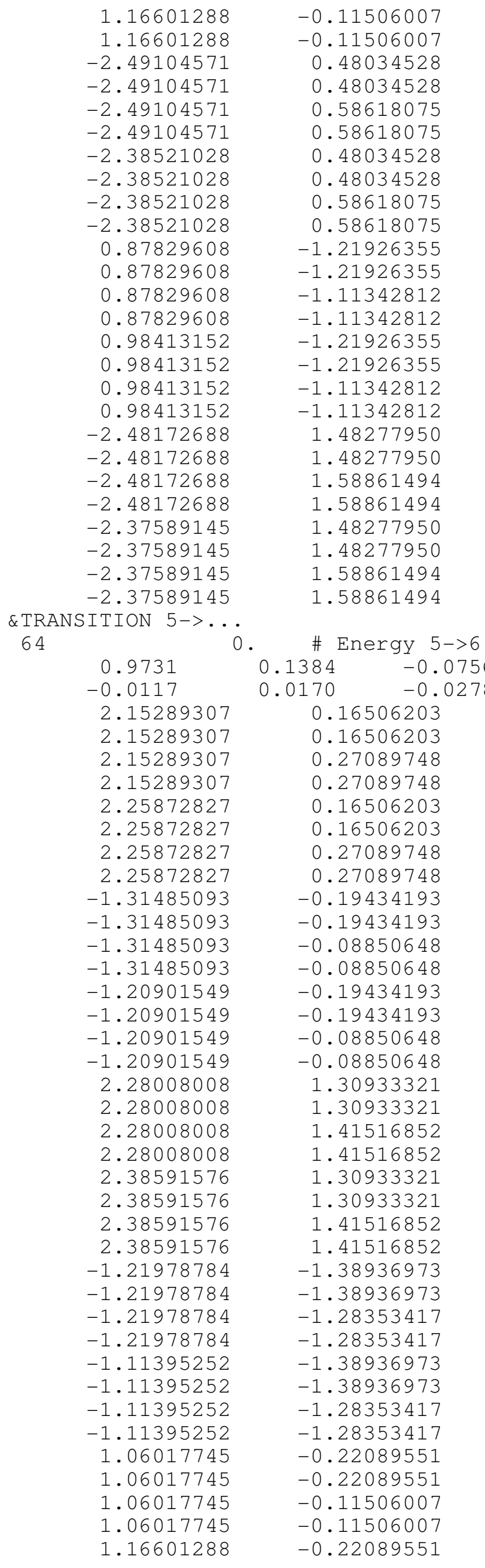

-0.74873185
-0.64289635
-0.18798651
-0.08215106
-0.18798651
-0.08215106
-0.18798651
-0.08215106
-0.18798651
-0.08215106
-0.81211877
-0.70628333
-0.81211877
-0.70628333
-0.81211877
-0.70628333
-0.81211877
-0.70628333
-0.33387381
-0.22803834
-0.33387381
-0.22803834
-0.33387381
-0.22803834
-0.33387381
-0.22803834

$-6.68017530$

11.97935390

0.90919322

$-1.56258523$

$-1.28271604$

1. 98742831

$-0.78070182$

1. 31284630

1. 26108515

$-1.89417052$

$-0.73552430$

1. 27261114

1.18366659

$-1.93011689$

0.49875608

$-0.85415137$

$-0.80321443$

1. 29493153

0.17450275

$-0.34924290$

$-0.12342357$

0.24759798

$-0.20670754$

0.39158869

0.15054077

$-0.28330916$

TRANSITION $5->$..

1.000 \# Electr. mom., scale-fac \# Magnetic mom.

$-0.01106351 \quad-4.52923059$

$0.09477194 \quad 2.96305799$

$-0.01106351 \quad 4.74659920$

$0.09477194 \quad-3.01432538$

$\begin{array}{ll}-0.01106351 & 3.24557877\end{array}$

$0.09477194 \quad-2.06292915$

$-0.01106351 \quad-3.39364743$

$0.09477194 \quad 2.07413530$

$-0.22601055 \quad-6.44614840$

$-0.12017509 \quad 5.93230724$

$\begin{array}{ll}-0.22601055 & 7.26323318\end{array}$

$-0.12017509-6.35701799$

$-0.22601055 \quad 5.94727945$

$-0.12017509 \quad-5.94494057$

$-0.22601055 \quad-6.73515368$

$\begin{array}{lr}-0.12017509 & 6.31040287\end{array}$

$0.43990609 \quad-0.02302814$

$0.54574150 \quad 0.01866239$

$0.43990609-0.14503577$

$0.54574150 \quad 0.12115374$

$0.43990609 \quad-0.14665078$

$0.54574150 \quad 0.16564934$

$\begin{array}{ll}0.43990609 & 0.30907756\end{array}$

$0.54574150 \quad-0.29054385$

$0.08411802 \quad-1.35100222$

$0.18995346 \quad 1.08398008$

$0.08411802 \quad 1.70972478$

$0.18995346-1.38053358$

$0.08411802 \quad 1.21913266$

$0.18995346 \quad-0.91761369$

$0.08411802-1.58813679$

$0.18995346 \quad 1.22259247$

$-0.74873185 \quad 3.92276263$

$-0.64289635 \quad-6.87019825$

$-0.74873185 \quad-4.52349901$

$-0.64289635 \quad 7.51095486$

$-0.74873185 \quad-5.84542084$ 


1.16601288
1.16601288
1.16601288
-2.49104571
-2.49104571
-2.49104571
-2.49104571
-2.38521028
-2.38521028
-2.38521028
-2.38521028
0.87829608
0.87829608
0.87829608
0.87829608
0.98413152
0.98413152
0.98413152
0.98413152
-2.48172688
-2.48172688
-2.48172688
-2.48172688
-2.37589145
-2.37589145
-2.37589145
-2.37589145

64
$-0.22089551$

$-0.11506007$

$-0.11506007$

0.48034528

0.48034528

0.58618075

0.58618075

0.48034528

0.48034528

0.58618075

0.58618075

$-1.21926355$

$-1.21926355$

$-1.11342812$

$-1.11342812$

$-1.21926355$

$-1.21926355$

$-1.11342812$

$-1.11342812$

1.48277950

1.48277950

1.58861494

1.58861494

1. 48277950

1.48277950

1.58861494

1.58861494

$0 . \quad \#$ Energy 5->7
$-0.2722 \quad-0.0802$
-0.0756

$\begin{array}{lll}-0.2826 & -0.2722 & -0.0802\end{array}$

0.0045

2.15289307

2.15289307

2.15289307

2.15289307

2. 25872827

2. 25872827

2. 25872827

2. 25872827

$-1.31485093$

$-1.31485093$

$-1.31485093$

$-1.31485093$

$-1.20901549$

$-1.20901549$

$-1.20901549$

$-1.20901549$

2. 28008008

2.28008008

2.28008008

2. 28008008

2.38591576

2.38591576

2.38591576

2.38591576

$-1.21978784$

$-1.21978784$

$-1.21978784$

$-1.21978784$

$-1.11395252$

$-1.11395252$

$-1.11395252$

$-1.11395252$

1.06017745

1.06017745

1.06017745

1.06017745

1.16601288
0.16506203

0.16506203

0.27089748

0.27089748

0.16506203

0.16506203

0.27089748

0.27089748

$-0.19434193$

$-0.19434193$

$-0.08850648$

$-0.08850648$

$-0.19434193$

$-0.19434193$

$-0.08850648$

$-0.08850648$

1.30933321

1.30933321

1.41516852

1. 41516852

1. 30933321

1.30933321

1. 41516852

1. 41516852

$-1.38936973$

$-1.38936973$

$-1.28353417$

$-1.28353417$

$-1.38936973$

$-1.38936973$

$-1.28353417$

$-1.28353417$

$-0.22089551$

$-0.22089551$

$-0.11506007$

$-0.11506007$

$-0.22089551$
$-0.64289635$

$-0.74873185$

$-0.64289635$

$-0.18798651$

$-0.08215106$

$-0.18798651$

$-0.08215106$

$-0.18798651$

$-0.08215106$

$-0.18798651$

$-0.08215106$

$-0.81211877$

$-0.70628333$

$-0.81211877$

$-0.70628333$

$-0.81211877$

$-0.70628333$

$-0.81211877$

$-0.70628333$

$-0.33387381$

$-0.22803834$

$-0.33387381$

$-0.22803834$

$-0.33387381$

$-0.22803834$

$-0.33387381$

$-0.22803834$

1.000

$-0.01106351$

0.09477194

$-0.01106351$

0.09477194

$-0.01106351$

0.09477194

$-0.01106351$

0.09477194

$-0.22601055$

$-0.12017509$

$-0.22601055$

$-0.12017509$

$-0.22601055$

$-0.12017509$

$-0.22601055$

$-0.12017509$

0.43990609

0.54574150

0.43990609

0.54574150

0.43990609

0.54574150

0.43990609

0.54574150

0.08411802

0.18995346

0.08411802

0.18995346

0.08411802

0.18995346

0.08411802

0.18995346

$-0.74873185$

$-0.64289635$

$-0.74873185$

$-0.64289635$

$-0.74873185$
9.43501472

6.45103884

$-10.00430870$

$-0.24111567$

$-0.13514648$

1.29927421

$-0.68998343$

0.57142913

$-0.06680385$

$-1.88045442$

1.07004285

0.26050362

$-0.66623527$

$-0.63538444$

1.25955081

$-0.48346984$

0.96796995

0.94519353

$-1.68232942$

$-0.43588206$

0.30540916

0.30895638

$-0.22378032$

0.58857363

$-0.38816965$

$-0.42408580$

0.29296434

Electr. mom., scale-fac Magnetic mom.

6.40513515

$-4.35544348$

$-7.52881908$

5.13798571

$-5.06409788$

3.32505226

5.90872622

$-3.93472099$

1.97780073

$-0.34657463$

$-2.19430852$

0.32010174

$-1.89653873$

0.58325636

2.11698127

$-0.47458315$

1.83958316

$-1.51595652$

$-1.71688950$

1.48435533

$-1.38112807$

1.10311258

1.33832872

$-1.14678144$

0.35832831

$-0.20370287$

$-0.35916099$

0.17621021

$-0.33459392$

0.17069185

0.31496403

$-0.12579054$

$-1.40805066$

3.78858924

2.89012218

$-5.81146669$

2. 89529514 


1.16601288
1.16601288
1.16601288
-2.49104571
-2.49104571
-2.49104571
-2.49104571
-2.38521028
-2.38521028
-2.38521028
-2.38521028
0.87829608
0.87829608
0.87829608
0.87829608
0.98413152
0.98413152
0.98413152
0.98413152
-2.48172688
-2.48172688
-2.48172688
-2.48172688
-2.37589145
-2.37589145
-2.37589145
-2.37589145

64
$-0.22089551$

$-0.11506007$

$-0.11506007$

0.48034528

0.48034528

0.58618075

0.58618075

0.48034528

0.48034528

0.58618075

0.58618075

$-1.21926355$

$-1.21926355$

$-1.11342812$

$-1.11342812$

$-1.21926355$

$-1.21926355$

$-1.11342812$

$-1.11342812$

1.48277950

1.48277950

1.58861494

1.58861494

1. 48277950

1. 48277950

1.58861494

1.58861494

0 .

$-0.0714$

0.0071

2. 15289307

2.15289307

2.15289307

2.15289307

2. 25872827

2. 25872827

2. 25872827

2. 25872827

$-1.31485093$

$-1.31485093$

$-1.31485093$

$-1.31485093$

$-1.20901549$

$-1.20901549$

$-1.20901549$

$-1.20901549$

2. 28008008

2. 28008008

2. 28008008

2. 28008008

2.38591576

2. 38591576

2. 38591576

2.38591576

$-1.21978784$

$-1.21978784$

$-1.21978784$

$-1.21978784$

$-1.11395252$

$-1.11395252$

$-1.11395252$

$-1.11395252$

1. 06017745

1. 06017745

1. 06017745

1. 06017745

1. 16601288
\# Energy 5->8

$$
-0.2745 \quad-0.2189
$$$$
-0.0809 \quad-0.0381
$$

0.16506203

0.16506203

0.27089748

0.27089748

0.16506203

0.16506203

0.27089748

0.27089748

$-0.19434193$

$-0.19434193$

$-0.08850648$

$-0.08850648$

$-0.19434193$

$-0.19434193$

$-0.08850648$

$-0.08850648$

1.30933321

1. 30933321

1. 41516852

1. 41516852

1. 30933321

1.30933321

1. 41516852

1. 41516852

$-1.38936973$

$-1.38936973$

$-1.28353417$

$-1.28353417$

$-1.38936973$

$-1.38936973$

$-1.28353417$

$-1.28353417$

$-0.22089551$

$-0.22089551$

$-0.11506007$

$-0.11506007$

$-0.22089551$ $\begin{array}{rr}-0.64289635 & -5.77576494 \\ -0.74873185 & -4.57040739 \\ -0.64289635 & 8.03508663 \\ -0.18798651 & 4.31702185 \\ -0.08215106 & -5.16189098 \\ -0.18798651 & -4.19035625 \\ -0.08215106 & 5.13767147 \\ -0.18798651 & -4.62222815 \\ -0.08215106 & 5.24307394 \\ -0.18798651 & 4.54635143 \\ -0.08215106 & -5.30130529 \\ -0.81211877 & 0.22394820 \\ -0.70628333 & -0.17900468 \\ -0.81211877 & -0.31915212 \\ -0.70628333 & 0.22595905 \\ -0.81211877 & -0.08027004 \\ -0.70628333 & -0.00273613 \\ -0.81211877 & 0.11280955 \\ -0.70628333 & 0.03123677 \\ -0.33387381 & 0.79352123 \\ -0.22803834 & -1.06388772 \\ -0.33387381 & -0.53484994 \\ -0.22803834 & 0.72223032 \\ -0.33387381 & -0.84272546 \\ -0.22803834 & 1.09417748 \\ -0.33387381 & 0.56324548 \\ -0.22803834 & -0.73776633\end{array}$
1.000 \# Electr. mom., scale-fac \# Magnetic mom.

-0.01106351
0.09477194

1. 72622716

0.16716650

$-0.01106351$

0.09477194

$-0.01106351$

0.09477194

$-0.01106351$

0.09477194

$-0.22601055$

$-0.12017509$

$-0.22601055$

$-0.12017509$

$-0.22601055$

$-0.12017509$

$-0.22601055$

$-0.12017509$

0.43990609

0.54574150

0.43990609

0.54574150

0.43990609

0.54574150

0.43990609

0.54574150

0.08411802

0.18995346

0.08411802

0.18995346

0.08411802

0.18995346

0.08411802

0.18995346

$-0.74873185$

$-0.64289635$

$-0.74873185$

$-0.64289635$

$-0.74873185$

$-3.22640491$

0.73588061

$-0.70031220$

$-0.82833278$

1. 75625181

0.15247256

1. 57381690

1. 48994255

$-1.73143101$

$-1.57347751$

$-2.32327795$

0.01993123

2.37343979

0.31526622

2.43621945

$-2.06380129$

$-1.87788832$

1.62754941

$-2.05871177$

1. 75808620

1. 64252067

$-1.43200314$

$-0.18795487$

0.52730268

0.01126708

$-0.45729628$

0.00588074

$-0.35641229$

0.18686418

0.23429307

$-1.86805952$

3. 63320541

3.82325697

$-6.49707651$

2. 75655532 


\begin{tabular}{|c|c|c|c|}
\hline & & & \\
\hline 1.16601288 & -0.22089551 & -0.64289635 & -4.94327164 \\
\hline 1.16601288 & -0.11506007 & -0.74873185 & -5.21178102 \\
\hline 1.16601288 & -0.11506007 & -0.64289635 & 8.47582722 \\
\hline-2.49104571 & 0.48034528 & -0.18798651 & 8.74921322 \\
\hline-2.49104571 & 0.48034528 & -0.08215106 & -9.77093124 \\
\hline-2.49104571 & 0.58618075 & -0.18798651 & -8.14490318 \\
\hline-2.49104571 & 0.58618075 & -0.08215106 & 9.43629646 \\
\hline-2.38521028 & 0.48034528 & -0.18798651 & -9.29732990 \\
\hline-2.38521028 & 0.48034528 & -0.08215106 & 9.80894279 \\
\hline-2.38521028 & 0.58618075 & -0.18798651 & 8.71580410 \\
\hline-2.38521028 & 0.58618075 & -0.08215106 & -9.56199932 \\
\hline 0.87829608 & -1.21926355 & -0.81211877 & 0.10233206 \\
\hline 0.87829608 & -1.21926355 & -0.70628333 & -0.10132370 \\
\hline 0.87829608 & -1.11342812 & -0.81211877 & -0.08466994 \\
\hline 0.87829608 & -1.11342812 & -0.70628333 & 0.09987726 \\
\hline 0.98413152 & -1.21926355 & -0.81211877 & -0.11028305 \\
\hline 0.98413152 & -1.21926355 & -0.70628333 & 0.15471251 \\
\hline 0.98413152 & -1.11342812 & -0.81211877 & 0.12144452 \\
\hline 0.98413152 & -1.11342812 & -0.70628333 & -0.19927692 \\
\hline-2.48172688 & 1.48277950 & -0.33387381 & 1.38874376 \\
\hline-2.48172688 & 1.48277950 & -0.22803834 & -1.91342270 \\
\hline-2.48172688 & 1.58861494 & -0.33387381 & -0.97877914 \\
\hline-2.48172688 & 1.58861494 & -0.22803834 & 1.35283792 \\
\hline-2.37589145 & 1.48277950 & -0.33387381 & -1.44732261 \\
\hline-2.37589145 & 1.48277950 & -0.22803834 & 1.94247270 \\
\hline-2.37589145 & 1.58861494 & -0.33387381 & 1.01715720 \\
\hline-2.37589145 & 1.58861494 & -0.22803834 & -1.37132263 \\
\hline & \# Energy 5->9 & & \\
\hline 0.5614 & -0.0416 & 1.000 & Electr. mom., scale-fac \\
\hline 0.0236 & 0.0503 & \# & Magnetic mom. \\
\hline 2.15289307 & 0.16506203 & -0.01106351 & -4.65571976 \\
\hline 2.15289307 & 0.16506203 & 0.09477194 & 0.41773102 \\
\hline 2.15289307 & 0.27089748 & -0.01106351 & 3.04346108 \\
\hline 2.15289307 & 0.27089748 & 0.09477194 & 1.14303124 \\
\hline 2.25872827 & 0.16506203 & -0.01106351 & 1.63531458 \\
\hline 2.25872827 & 0.16506203 & 0.09477194 & 1.87804031 \\
\hline 2.25872827 & 0.27089748 & -0.01106351 & -0.23829237 \\
\hline 2.25872827 & 0.27089748 & 0.09477194 & -3.20062971 \\
\hline-1.31485093 & -0.19434193 & -0.22601055 & -7.29738522 \\
\hline-1.31485093 & -0.19434193 & -0.12017509 & 8.09469700 \\
\hline-1.31485093 & -0.08850648 & -0.22601055 & 8.98086548 \\
\hline-1.31485093 & -0.08850648 & -0.12017509 & -9.74938393 \\
\hline-1.20901549 & -0.19434193 & -0.22601055 & 7.20803642 \\
\hline-1.20901549 & -0.19434193 & -0.12017509 & -7.37453127 \\
\hline-1.20901549 & -0.08850648 & -0.22601055 & -8.98220158 \\
\hline-1.20901549 & -0.08850648 & -0.12017509 & 9.22937393 \\
\hline 2.28008008 & 1.30933321 & 0.43990609 & -1.93781352 \\
\hline 2.28008008 & 1.30933321 & 0.54574150 & 1.36009228 \\
\hline 2.28008008 & 1.41516852 & 0.43990609 & 1.51792014 \\
\hline 2.28008008 & 1.41516852 & 0.54574150 & -1.11452806 \\
\hline 2.38591576 & 1.30933321 & 0.43990609 & 1.45938981 \\
\hline 2.38591576 & 1.30933321 & 0.54574150 & -0.92159396 \\
\hline 2.38591576 & 1.41516852 & 0.43990609 & -1.14906812 \\
\hline 2.38591576 & 1.41516852 & 0.54574150 & 0.76997787 \\
\hline-1.21978784 & -1.38936973 & 0.08411802 & -1.32608449 \\
\hline-1.21978784 & -1.38936973 & 0.18995346 & 1.24326289 \\
\hline-1.21978784 & -1.28353417 & 0.08411802 & 1.45873773 \\
\hline-1.21978784 & -1.28353417 & 0.18995346 & -1.38809025 \\
\hline-1.11395252 & -1.38936973 & 0.08411802 & 1.31998479 \\
\hline-1.11395252 & -1.38936973 & 0.18995346 & -1.21364725 \\
\hline-1.11395252 & -1.28353417 & 0.08411802 & -1.48722351 \\
\hline-1.11395252 & -1.28353417 & 0.18995346 & 1.35370207 \\
\hline 1.06017745 & -0.22089551 & -0.74873185 & -0.08204957 \\
\hline 1.06017745 & -0.22089551 & -0.64289635 & -3.07693577 \\
\hline 1.06017745 & -0.11506007 & -0.74873185 & 1.74774539 \\
\hline 1.06017745 & -0.11506007 & -0.64289635 & 0.79603404 \\
\hline 1.16601288 & -0.22089551 & -0.74873185 & -1.64791048 \\
\hline
\end{tabular}




$\begin{array}{rrrr}1.16601288 & -0.22089551 & -0.64289635 & 5.26182127 \\ 1.16601288 & -0.11506007 & -0.74873185 & 0.12360353 \\ 1.16601288 & -0.11506007 & -0.64289635 & -3.13655591 \\ -2.49104571 & 0.48034528 & -0.18798651 & 9.13741684 \\ -2.49104571 & 0.48034528 & -0.08215106 & -9.85838413 \\ -2.49104571 & 0.58618075 & -0.18798651 & -8.07112217 \\ -2.49104571 & 0.58618075 & -0.08215106 & 9.07749081 \\ -2.38521028 & 0.48034528 & -0.18798651 & -9.88234329 \\ -2.38521028 & 0.48034528 & -0.08215106 & 10.17095375 \\ -2.38521028 & 0.58618075 & -0.18798651 & 8.68951988 \\ -2.38521028 & 0.58618075 & -0.08215106 & -9.31422329 \\ 0.87829608 & -1.21926355 & -0.81211877 & 0.20283259 \\ 0.87829608 & -1.21926355 & -0.70628333 & -0.80401748 \\ 0.87829608 & -1.11342812 & -0.81211877 & -0.36346081 \\ 0.87829608 & -1.11342812 & -0.70628333 & 1.21237326 \\ 0.98413152 & -1.21926355 & -0.81211877 & -0.36312968 \\ 0.98413152 & -1.21926355 & -0.70628333 & 0.97085100 \\ 0.98413152 & -1.11342812 & -0.81211877 & 0.58226466 \\ 0.98413152 & -1.11342812 & -0.70628333 & -1.44227135 \\ -2.48172688 & 1.48277950 & -0.33387381 & 1.39114904 \\ -2.48172688 & 1.48277950 & -0.22803834 & -1.90277898 \\ -2.48172688 & 1.58861494 & -0.33387381 & -0.97346687 \\ -2.48172688 & 1.58861494 & -0.22803834 & 1.34517860 \\ -2.37589145 & 1.48277950 & -0.33387381 & -1.40187097 \\ -2.37589145 & 1.48277950 & -0.22803834 & 1.89101005 \\ -2.37589145 & 1.58861494 & -0.33387381 & 0.97080243 \\ -2.37589145 & 1.58861494 & -0.22803834 & -1.32795191\end{array}$

\&TRANSITION $6->$. 64 $\begin{array}{cccc}0.5062 & 0.0 .1768 & \text { Energy } & 6->7 \\ 0.1614\end{array}$ $\begin{array}{lll}-0.0256 & -0.4696 & -0.2100\end{array}$

2.15289307

2.15289307

0.16506203

2.15289307

2.15289307

2. 25872827

2. 25872827

2. 25872827

2. 25872827

$-1.31485093$

$-1.31485093$

$-1.31485093$

$-1.31485093$

$-1.20901549$

$-1.20901549$

$-1.20901549$

$-1.20901549$

2.28008008

2. 28008008

2.28008008

2.28008008

2.38591576

2.38591576

2.38591576

2. 38591576

$-1.21978784$

$-1.21978784$

$-1.21978784$

$-1.21978784$

$-1.11395252$

$-1.11395252$

$-1.11395252$

$-1.11395252$

1.06017745

1. 06017745

1.06017745

0.16506203

0.27089748

0.27089748

0.16506203

0.16506203

0.27089748

0.27089748

$-0.19434193$

$-0.19434193$

$-0.08850648$

$-0.08850648$

$-0.19434193$

$-0.19434193$

$-0.08850648$

$-0.08850648$

1. 30933321

1.30933321

1. 41516852

1. 41516852

1. 30933321

1. 30933321

1. 41516852

1. 41516852

$-1.38936973$

$-1.38936973$

$-1.28353417$

$-1.28353417$

$-1.38936973$

$-1.38936973$

$-1.28353417$

$-1.28353417$

$-0.22089551$

$-0.22089551$

$-0.11506007$

1. 06017745

$-0.11506007$
1.000 \# Electr. mom., scale-fac \# Magnetic mom.
$-0.01106351$
60.53982162

0.09477194

$-0.01106351$

0.09477194

$-0.01106351$

0.09477194

$-0.01106351$

0.09477194

$-0.22601055$

$-0.12017509$

$-0.22601055$

$-0.12017509$

$-0.22601055$

$-0.12017509$

$-0.22601055$

$-0.12017509$

0.43990609

0.54574150

0.43990609

0.54574150

0.43990609

0.54574150

0.43990609

0.54574150

0.08411802

0.18995346

0.08411802

0.18995346

0.08411802

0.18995346

0.08411802

0.18995346

$-0.74873185$

$-0.64289635$

$-0.74873185$

$-0.64289635$
$-44.76138687$

$-67.17350006$

49.48270416

$-45.11395264$

33.75631332

50.10674667

$-37.44638824$

9.26754856

$-11.54162502$

7.83427000

$-9.54116917$

6.51646376

11.74917030

$-7.98719454$

4.95839453

$-4.84397411$

$-4.70742416$

4. 86506844

1.63889825

$-0.63965303$

$-0.37628847$

$-0.61314994$

0.97504234

$-0.73556942$

$-1.55925345$

1.23411834

$-1.13822114$

0.86789840

1. 77408111

$-1.41761804$

$-24.21816063$

51.46586609

31.22912788

$-60.17468643$
$-6.33544874$ 


1.16601288
1.16601288
1.16601288
1.16601288
-2.49104571
-2.49104571
-2.49104571
-2.49104571
-2.38521028
-2.38521028
-2.38521028
-2.38521028
0.87829608
0.87829608
0.87829608
0.87829608
0.98413152
0.98413152
0.98413152
0.98413152
-2.48172688
-2.48172688
-2.48172688
-2.48172688
-2.37589145
-2.37589145
-2.37589145
-2.37589145
-

64
0.5837
0.0400

2.15289307

2.15289307

2.15289307

2.15289307

2. 25872827

2. 25872827

2. 25872827

2. 25872827

$-1.31485093$

$-1.31485093$

$-1.31485093$

$-1.31485093$

$-1.20901549$

$-1.20901549$

$-1.20901549$

$-1.20901549$

2. 28008008

2. 28008008

2. 28008008

2.28008008

2.38591576

2. 38591576

2.38591576

2. 38591576

$-1.21978784$

$-1.21978784$

$-1.21978784$

$-1.21978784$

$-1.11395252$

$-1.11395252$

$-1.11395252$

$-1.11395252$

1.06017745

1. 06017745

1. 06017745

1. 06017745
$-0.22089551$

$-0.22089551$

$-0.11506007$

$-0.11506007$

0.48034528

0.48034528

0.58618075

0.58618075

0.48034528

0.48034528

0.58618075

0.58618075

$-1.21926355$

$-1.21926355$

$-1.11342812$

$-1.11342812$

$-1.21926355$

$-1.21926355$

$-1.11342812$

$-1.11342812$

1. 48277950

1. 48277950

1.58861494

1. 58861494

1. 48277950

1. 48277950

1.58861494

1.58861494

\# Energy 6->8

$0.1529 \quad 0.0448$

0.0437

0.0283

0.16506203

0.16506203

0.27089748

0.27089748

0.16506203

0.16506203

0.27089748

0.27089748

$-0.19434193$

$-0.19434193$

$-0.08850648$

$-0.08850648$

$-0.19434193$

$-0.19434193$

$-0.08850648$

$-0.08850648$

1. 30933321

1. 30933321

1. 41516852

1. 41516852

1. 30933321

1. 30933321

1. 41516852

1. 41516852

$-1.38936973$

$-1.38936973$

$-1.28353417$

$-1.28353417$

$-1.38936973$

$-1.38936973$

$-1.28353417$

$-1.28353417$

$-0.22089551$

$-0.22089551$

$-0.11506007$

$-0.11506007$
$-0.74873185$

$-0.64289635$

$-0.74873185$

$-0.64289635$

$-0.18798651$

$-0.08215106$

$-0.18798651$

$-0.08215106$

$-0.18798651$

$-0.08215106$

$-0.18798651$

$-0.08215106$

$-0.81211877$

$-0.70628333$

$-0.81211877$

$-0.70628333$

$-0.81211877$

$-0.70628333$

$-0.81211877$

$-0.70628333$

$-0.33387381$

$-0.22803834$

$-0.33387381$

$-0.22803834$

$-0.33387381$

$-0.22803834$

$-0.33387381$

$-0.22803834$

1.000 \# Electr. mom., scale-fac

$-0.01106351$

0.09477194

$-0.01106351$

0.09477194

$-0.01106351$

0.09477194

$-0.01106351$

0.09477194

$-0.22601055$

$-0.12017509$

$-0.22601055$

$-0.12017509$

$-0.22601055$

$-0.12017509$

$-0.22601055$

$-0.12017509$

0.43990609

0.54574150

0.43990609

0.54574150

0.43990609

0.54574150

0.43990609

0.54574150

0.08411802

0.18995346

0.08411802

0.18995346

0.08411802

0.18995346

0.08411802

0.18995346

$-0.74873185$

$-0.64289635$

$-0.74873185$

$-0.64289635$
39.34477997

$-72.29835510$

$-46.89203644$

81.59844971

$-4.50143623$

3. 48574257

4. 02762556

$-3.22525001$

5. 47939062

$-4.21765566$

$-4.83143282$

3. 84017110

0.38447136

1.57419550

0.10602373

$-3.26734066$

1. 33282936

$-3.88724184$

$-2.54864120$

6.56211376

$-0.87233460$

0.91611856

0.67332375

$-0.72428727$

0.96629292

$-1.03122520$

$-0.74277776$

0.81161642 Magnetic mom.

$-6.98663712$

4.02043533

7.74111223

$-4.47470140$

4. 73886251

$-2.43203592$

$-5.29419804$

2.75796652

$-9.04128075$

7. 99589252

10.19958401

$-8.89888191$

8.84928608

$-8.02648258$

$-10.03095913$

8.93296051

$-1.06088150$

0.88156784

0.73410225

$-0.64613986$

0.39103809

$-0.26771465$

$-0.17929170$

0.14149669

$-1.93795025$

1.65821576

2.59088039

$-2.22450900$

1. 91066968

$-1.57080269$

$-2.60017109$

2. 15856361

3. 23959160

$-6.82579327$

$-3.89309120$

7.66398478 


1.16601288
1.16601288
1.16601288
1.16601288
-2.49104571
-2.49104571
-2.49104571
-2.49104571
-2.38521028
-2.38521028
-2.38521028
-2.38521028
0.87829608
0.87829608
0.87829608
0.87829608
0.98413152
0.98413152
0.98413152
0.98413152
-2.48172688
-2.48172688
-2.48172688
-2.48172688
-2.37589145
-2.37589145
-2.37589145
-2.37589145
-

64
0.1963
0.0096

2. 15289307

2.15289307

2. 15289307

2.15289307

2. 25872827

2. 25872827

2. 25872827

2. 25872827

$-1.31485093$

$-1.31485093$

$-1.31485093$

$-1.31485093$

$-1.20901549$

$-1.20901549$

$-1.20901549$

$-1.20901549$

2. 28008008

2. 28008008

2. 28008008

2.28008008

2.38591576

2.38591576

2.38591576

2. 38591576

$-1.21978784$

$-1.21978784$

$-1.21978784$

$-1.21978784$

$-1.11395252$

$-1.11395252$

$-1.11395252$

$-1.11395252$

1.06017745

1. 06017745

1. 06017745

1. 06017745
$-0.22089551$

$-0.22089551$

$-0.11506007$

$-0.11506007$

0.48034528

0.48034528

0.58618075

0.58618075

0.48034528

0.48034528

0.58618075

0.58618075

$-1.21926355$

$-1.21926355$

$-1.11342812$

$-1.11342812$

$-1.21926355$

$-1.21926355$

$-1.11342812$

$-1.11342812$

1. 48277950

1. 48277950

1. 58861494

1. 58861494

1. 48277950

1. 48277950

1.58861494

1.58861494

\# Energy 6->9

$0.0294^{0.0280} 0.0000$

$0.0280 \quad 0.0171$

0.16506203

0.16506203

0.27089748

0.27089748

0.16506203

0.16506203

0.27089748

0.27089748

$-0.19434193$

$-0.19434193$

$-0.08850648$

$-0.08850648$

$-0.19434193$

$-0.19434193$

$-0.08850648$

$-0.08850648$

1. 30933321

1. 30933321

1. 41516852

1. 41516852

1. 30933321

1. 30933321

1. 41516852

1. 41516852

$-1.38936973$

$-1.38936973$

$-1.28353417$

$-1.28353417$

$-1.38936973$

$-1.38936973$

$-1.28353417$

$-1.28353417$

$-0.22089551$

$-0.22089551$

$-0.11506007$

$-0.11506007$
$-0.74873185$

$-0.64289635$

$-0.74873185$

$-0.64289635$

$-0.18798651$

$-0.08215106$

$-0.18798651$

$-0.08215106$

$-0.18798651$

$-0.08215106$

$-0.18798651$

$-0.08215106$

$-0.81211877$

$-0.70628333$

$-0.81211877$

$-0.70628333$

$-0.81211877$

$-0.70628333$

$-0.81211877$

$-0.70628333$

$-0.33387381$

$-0.22803834$

$-0.33387381$

$-0.22803834$

$-0.33387381$

$-0.22803834$

$-0.33387381$

$-0.22803834$
$-5.86900330$

10.44922733

6.60684872

$-11.33821297$

$-0.55755109$

0.24010120

0.96597743

$-0.58331048$

0.81969637

$-0.41887775$

$-1.36730599$

0.86987633

$-0.03797860$

$-0.31734997$

$-0.24991727$

0.82974833

$-0.28145894$

0.74990535

0.68080163

$-1.42003882$

$-0.10136396$

$-0.00175847$

0.06835100

0.00932378

0.20168667

$-0.05158984$

$-0.14060269$

0.03008702
1.000 \# Electr. mom., scale-fac
\# Magnetic mom.

$-0.01106351$

0.09477194

$-0.01106351$

0.09477194

$-0.01106351$

0.09477194

$-0.01106351$

0.09477194

$-0.22601055$

$-0.12017509$

$-0.22601055$

$-0.12017509$

$-0.22601055$

$-0.12017509$

$-0.22601055$

$-0.12017509$

0.43990609

0.54574150

0.43990609

0.54574150

0.43990609

0.54574150

0.43990609

0.54574150

0.08411802

0.18995346

0.08411802

0.18995346

0.08411802

0.18995346

0.08411802

0.18995346

$-0.74873185$

$-0.64289635$

$-0.74873185$

$-0.64289635$
1.628039

1.62803972

0.65655988

2.18810964

$-1.06425071$

1.03443623

$-0.31058237$

$-1.47215688$

0.63725996

$-3.91872382$

3. 33736634

4.51563406

$-3.87436843$

4.08061314

$-3.60212111$

$-4.64871502$

4.10223150

$-0.74791539$

0.64771962

0.59586054

$-0.53981054$

0.46430841

$-0.39983329$

$-0.37428346$

0.34398150

$-0.60427630$

0.43764439

0.80809283

$-0.59349036$

0.64965987

$-0.45704672$

$-0.88008511$

0.63772345

1. 17098451

$-2.05847263$

$-1.49797893$

2. 54866838 


1.16601288
1.16601288
1.16601288
1.16601288
-2.49104571
-2.49104571
-2.49104571
-2.49104571
-2.38521028
-2.38521028
-2.38521028
-2.38521028
0.87829608
0.87829608
0.87829608
0.87829608
0.98413152
0.98413152
0.98413152
0.98413152
-2.48172688
-2.48172688
-2.48172688
-2.48172688
-2.37589145
-2.37589145
-2.37589145
-2.37589145

\&TRANSITION $7->\ldots$

64
$-0.22089551$

$-0.22089551$

$-0.11506007$

$-0.11506007$

0.48034528

0.48034528

0.58618075

0.58618075

0.48034528

0.48034528

0.58618075

0.58618075

$-1.21926355$

$-1.21926355$

$-1.11342812$

$-1.11342812$

$-1.21926355$

$-1.21926355$

$-1.11342812$

$-1.11342812$

1.48277950

1. 48277950

1.58861494

1. 58861494

1. 48277950

1.48277950

1.58861494

1. 58861494
$-0.74873185$

$-0.64289635$

$-0.74873185$

$-0.64289635$

$-0.18798651$

$-0.08215106$

$-0.18798651$

$-0.08215106$

$-0.18798651$

$-0.08215106$

$-0.18798651$

$-0.08215106$

$-0.81211877$

$-0.70628333$

$-0.81211877$

$-0.70628333$

$-0.81211877$

$-0.70628333$

$-0.81211877$

$-0.70628333$

$-0.33387381$

$-0.22803834$

$-0.33387381$

$-0.22803834$

$-0.33387381$

$-0.22803834$

$-0.33387381$

$-0.22803834$
$-1.92980385$

3. 17349029

2.32445049

$-3.73034620$

$-0.37199670$

0.27635464

0.49217495

$-0.39648348$

0.43825868

$-0.28938425$

$-0.61770004$

0.45566973

$-0.02253143$

0.01075351

$-0.03257072$

0.07792799

$-0.02983800$

0.07605289

0.10486879

$-0.20024332$

$-0.09968495$

0.09679823

0.07212387

$-0.06819417$

0.14120364

$-0.11666492$

$-0.10261454$

0.08322500

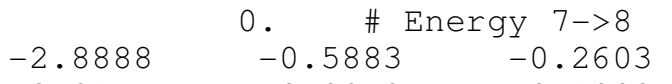

0.0145

2. 15289307

2.15289307

2. 15289307

2.15289307

2. 25872827

2. 25872827

2. 25872827

2. 25872827

$-1.31485093$

$-1.31485093$

$-1.31485093$

$-1.31485093$

$-1.20901549$

$-1.20901549$

$-1.20901549$

$-1.20901549$

2. 28008008

2. 28008008

2. 28008008

2. 28008008

2. 38591576

2. 38591576

2. 38591576

2. 38591576

$-1.21978784$

$-1.21978784$

$-1.21978784$

$-1.21978784$

$-1.11395252$

$-1.11395252$

$-1.11395252$

$-1.11395252$

1. 06017745

1.06017745

1. 06017745
0.16506203

0.16506203

0.27089748

0.27089748

0.16506203

0.16506203

0.27089748

0.27089748

$-0.19434193$

$-0.19434193$

$-0.08850648$

$-0.08850648$

$-0.19434193$

$-0.19434193$

$-0.08850648$

$-0.08850648$

1. 30933321

1. 30933321

1. 41516852

1. 41516852

1. 30933321

1. 30933321

1. 41516852

1. 41516852

$-1.38936973$

$-1.38936973$

$-1.28353417$

$-1.38936973$

$-1.38936973$

$-1.28353417$

$-1.28353417$

$-0.22089551$

$-0.22089551$

$-0.11506007$
$-1.28353417$
1.000 \# Electr. mom., scale-fac
\# Magnetic mom.

$\begin{array}{ll}-0.01106351 & 15.63177299\end{array}$

$0.09477194 \quad-11.06385040$

$-0.01106351 \quad-15.16339493$

0.09477194

$-0.01106351$

0.09477194

$-0.01106351$

0.09477194

$-0.22601055$

$-0.12017509$

$-0.22601055$

$-0.12017509$

$-0.22601055$

$-0.12017509$

$-0.22601055$

$-0.12017509$

0.43990609

0.54574150

0.43990609

0.54574150

0.43990609

0.54574150

0.43990609

0.54574150

0.08411802

0.18995346

0.08411802

0.18995346

0.08411802

0.18995346

0.08411802

0.18995346

$-0.74873185$

$-0.64289635$

$-0.74873185$
10.90200329

$-13.28694725$ 8.64849854

12.88801765

$-8.49304962$

6.02769232

$-5.99178076$

$-4.42173433$

4.19745922

$-5.39275455$

5.69799995

3. 72540951

$-3.73661804$

6.52263069

$-5.15491104$

$-7.57173920$

6.22895050

$-6.86451530$

5.07590628

7.51878500

$-5.90979815$

2. 40464520

$-2.40097094$

$-2.34654593$

2. 33244276

$-1.90376258$

2.01154947

1. 74515808

$-1.85463262$

$-4.73174953$

5.02413082

5. 65912628 
1.06017745
1.16601288
1.16601288
1.16601288
1.16601288
-2.49104571
-2.49104571
-2.49104571
-2.49104571
-2.38521028
-2.38521028
-2.38521028
-2.38521028
0.87829608
0.87829608
0.87829608
0.87829608
0.98413152
0.98413152
0.98413152
0.98413152
-2.48172688
-2.48172688
-2.48172688
-2.48172688
-2.37589145
-2.37589145
-2.37589145
-2.37589145

64
$-0.11506007$

$-0.22089551$

$-0.22089551$

$-0.11506007$

$-0.11506007$

0.48034528

0.48034528

0.58618075

0.58618075

0.48034528

0.48034528

0.58618075

0.58618075

$-1.21926355$

$-1.21926355$

$-1.11342812$

$-1.11342812$

$-1.21926355$

$-1.21926355$

$-1.11342812$

$-1.11342812$

1. 48277950

1.48277950

1.58861494

1. 58861494

1.48277950

1. 48277950

1. 58861494

1.58861494

0 .

$-0.7966$

0.0147

2. 15289307

2.15289307

2. 15289307

2. 15289307

2. 25872827

2. 25872827

2. 25872827

2. 25872827

$-1.31485093$

$-1.31485093$

$-1.31485093$

$-1.31485093$

$-1.20901549$

$-1.20901549$

$-1.20901549$

$-1.20901549$

2. 28008008

2. 28008008

2.28008008

2.28008008

2.38591576

2. 38591576

2. 38591576

2.38591576

$-1.21978784$

$-1.21978784$

$-1.21978784$

$-1.21978784$

$-1.11395252$

$-1.11395252$

$-1.11395252$

$-1.11395252$

1. 06017745

1.06017745

1. 06017745 $\begin{array}{ll}-0.1648 & 0.0620\end{array}$ $\begin{array}{ll}-0.0209 & -0.0185\end{array}$

0.16506203

0.16506203

0.27089748

0.27089748

0.16506203

0.16506203

0.27089748

0.27089748

$-0.19434193$

$-0.19434193$

$-0.08850648$

$-0.08850648$

$-0.19434193$

$-0.19434193$

$-0.08850648$

$-0.08850648$

1. 30933321

1.30933321

1. 41516852

1. 41516852

1. 30933321

1. 30933321

1. 41516852

1. 41516852

$-1.38936973$

$-1.38936973$

$-1.28353417$

$-1.28353417$

$-1.38936973$

$-1.38936973$

$-1.28353417$

$-1.28353417$

$-0.22089551$

$-0.22089551$

$-0.11506007$
$-0.64289635$

$-0.74873185$

$-0.64289635$

$-0.74873185$

$-0.64289635$

$-0.18798651$

$-0.08215106$

$-0.18798651$

$-0.08215106$

$-0.18798651$

$-0.08215106$

$-0.18798651$

$-0.08215106$

$-0.81211877$

$-0.70628333$

$-0.81211877$

$-0.70628333$

$-0.81211877$

$-0.70628333$

$-0.81211877$

$-0.70628333$

$-0.33387381$

$-0.22803834$

$-0.33387381$

$-0.22803834$

$-0.33387381$

$-0.22803834$

$-0.33387381$

$-0.22803834$
$-6.18868113$

3. 84964013

$-4.26630259$

$-4.74407864$

5. 32972670

4.23280001

$-2.16393471$

$-4.51356220$

2.38820386

$-5.01593542$

2. 48499131

5.37620544

$-2.66113257$

0.12891166

$-0.18763350$

$-0.27187777$

0.37770724

$-0.37871408$

0.43984774

0.60247076

$-0.70899588$

0.74181849

$-0.24920809$

$-0.59623873$

0.25598902

$-0.61448729$

$-0.11799262$

0.47777498

0.03926532
1.000 \# Electr. mom., scale-fac \# Magnetic mom.

$\begin{array}{ll}-0.01106351 & 2.49841404\end{array}$

$0.09477194 \quad-1.83500552$

$-0.01106351 \quad-2.61483526$

$0.09477194 \quad 1.96458578$

$-0.01106351 \quad-2.18180346$

$0.09477194 \quad 1.51595592$

$-0.01106351 \quad 2.27732706$

$0.09477194 \quad-1.60730600$

$-0.22601055 \quad-2.14442444$

$-0.12017509 \quad 1.83618808$

$-0.22601055 \quad 2.45711780$

$-0.12017509 \quad-2.12701106$

$-0.22601055 \quad 2.40107989$

$-0.12017509-1.89525425$

$-0.22601055 \quad-2.72747040$

$-0.12017509 \quad 2.22731566$

$0.43990609 \quad 1.02071893$

$0.54574150 \quad-0.81138545$

$0.43990609-1.18088436$

$0.54574150 \quad 0.97567874$

$0.43990609-1.11590600$

$0.54574150 \quad 0.80838752$

$0.43990609 \quad 1.18989336$

$0.54574150 \quad-0.92056227$

$0.08411802 \quad-0.35635716$

$0.18995346 \quad 0.20485137$

$0.08411802 \quad 0.68629235$

$0.18995346 \quad-0.48396519$

$0.08411802 \quad 0.50986481$

$0.18995346 \quad-0.31207180$

$0.08411802 \quad-0.89087683$

$\begin{array}{ll}0.18995346 & 0.63272601\end{array}$

$-0.74873185 \quad 1.37840033$

$-0.64289635-1.76464438$

$-0.74873185 \quad-0.65526098$ 


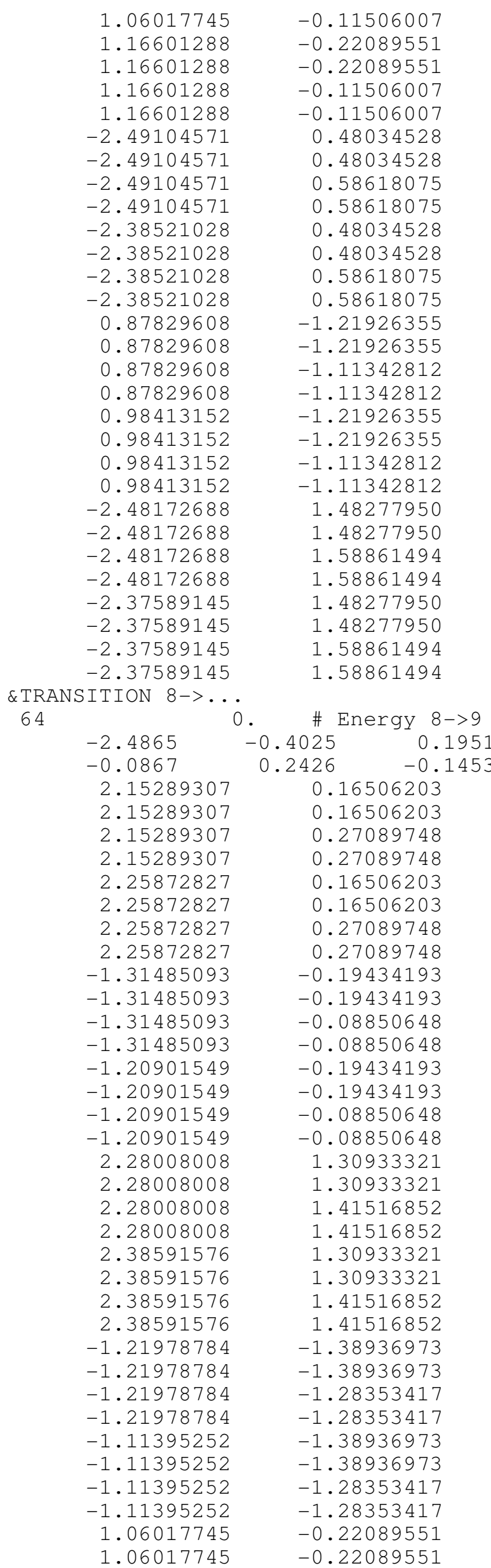

$-0.64289635$

$-0.74873185$

$-0.64289635$

$-0.74873185$

$-0.64289635$

$-0.18798651$

$-0.08215106$

$-0.18798651$

$-0.08215106$

$-0.18798651$

$-0.08215106$

$-0.18798651$

$-0.08215106$

$-0.81211877$

$-0.70628333$

$-0.81211877$

$-0.70628333$

$-0.81211877$

$-0.70628333$

$-0.81211877$

$-0.70628333$

$-0.33387381$

$-0.22803834$

$-0.33387381$

$-0.22803834$

$-0.33387381$

$-0.22803834$

$-0.33387381$

$-0.22803834$
0.88188845

$-0.95910507$

1. 19369388

0.35820693

$-0.47839674$

0.75138271

0.11938015

$-0.61963099$

$-0.26540998$

$-0.94317633$

$-0.05258092$

0.79740614

0.25630182

0.29549164

$-0.45567635$

$-0.52161336$

0.75536406

$-0.22398631$

0.35067087

0.40461192

$-0.58750248$

0.12822236

0.05969543

$-0.08956120$

$-0.04296769$

$-0.10182074$

$-0.14529788$

0.07202056

0.10261630

\footnotetext{
1.000 \# Electr. mom., scale-fac \# Magnetic mom.

$-0.01106351 \quad 17.80643845$

$0.09477194 \quad-8.97967911$

$-0.01106351 \quad-18.50124168$

$0.09477194 \quad 8.95791531$

$-0.01106351 \quad-11.15254974$

$0.09477194 \quad 4.44949484$

$-0.01106351 \quad 11.48426723$

$0.09477194 \quad-4.22952223$

$-0.22601055 \quad 9.41046143$

$-0.12017509-10.11290455$

$-0.22601055 \quad-10.62054920$

$-0.12017509 \quad 11.01130962$

$-0.22601055 \quad-7.36137390$

$-0.12017509 \quad 8.66639423$

$-0.22601055 \quad 8.62083721$

$-0.12017509 \quad-9.62335110$

$0.43990609 \quad 3.13262916$

$0.54574150 \quad-2.43169284$

$0.43990609-1.78572607$

$0.54574150 \quad 1.39275098$

$0.43990609-1.66128063$

$0.54574150 \quad 1.07192421$

$0.43990609 \quad 0.62097603$

$0.54574150 \quad-0.30674282$

$0.08411802 \quad-0.98434746$

$0.18995346 \quad 0.01003593$

$0.08411802 \quad-3.47750664$

$0.18995346 \quad 4.41571712$

$0.08411802 \quad 1.06808269$

$0.18995346 \quad-0.28437835$

$0.08411802 \quad 3.36675072$

$0.18995346-4.05890560$

$-0.74873185 \quad-10.36430264$

$-0.64289635 \quad 20.19251251$
} 


\begin{tabular}{|c|c|c|c|}
\hline & & & \\
\hline 1.06017745 & -0.11506007 & -0.74873185 & 12.18269730 \\
\hline 1.06017745 & -0.11506007 & -0.64289635 & -22.18188095 \\
\hline 1.16601288 & -0.22089551 & -0.74873185 & 18.70262146 \\
\hline 1.16601288 & -0.22089551 & -0.64289635 & -31.47253227 \\
\hline 1.16601288 & -0.11506007 & -0.74873185 & -20.21101761 \\
\hline 1.16601288 & -0.11506007 & -0.64289635 & 32.95469666 \\
\hline-2.49104571 & 0.48034528 & -0.18798651 & 0.812980 \\
\hline-2.49104571 & 0.48034528 & -0.08215106 & 2.3 \\
\hline-2.4910457 & 0.58618075 & -0.18798651 & -1.690079 \\
\hline-2.4910457 & 0.58618075 & -0.08215106 & -1.691592 \\
\hline-2.38521028 & 0.48034528 & -0.18798651 & -1.6132426 \\
\hline-2.38521028 & 0.48034528 & -0.08215106 & -1.9462524 \\
\hline-2.38521028 & 0.58618075 & -0.18798651 & 2.75 \\
\hline-2.38521028 & 0.58618075 & -0.08215106 & 1.24 \\
\hline 0.87829608 & -1.21926355 & -0.81211877 & 0.02 \\
\hline 0.87829608 & -1.21926355 & -0.70628333 & 1.16 \\
\hline 0.87829608 & -1.11342812 & -0.81211877 & 1.05754 \\
\hline 0.87829608 & -1.11342812 & -0.70628333 & -2.9478640 \\
\hline 0.98413152 & -1.21926355 & -0.81211877 & 1.1 \\
\hline 0.98413152 & -1.21926355 & -0.70628333 & -2.73 \\
\hline 0.9841315 & -1.11342812 & -0.81211877 & -2.691 \\
\hline 0.98413152 & -1.11342812 & -0.70628333 & 5.10 \\
\hline-2.48172688 & 1.48277950 & -0.33387381 & 0.36 \\
\hline-2.48172688 & 1.48277950 & -0.22803834 & 0.3 \\
\hline-2.48172688 & 1.58861494 & -0.33387381 & -0.25 \\
\hline-2.48172688 & 1.58861494 & -0.22803834 & -0.21337 \\
\hline-2.37589145 & 1.48277950 & -0.33387381 & -0.47 \\
\hline-2.37589 & 1.4827 & -0.228 & -0.4 \\
\hline-2.375891 & 1.58861494 & -0.33387381 & 0 \\
\hline-2 & 1.58861494 & -0.22803834 & \\
\hline ANENT MOMI & & & \\
\hline & $\# \quad E$ & & \\
\hline-2.6565 & -1.1349 & & ectr. mom. \\
\hline 2.15289307 & 0.16506203 & -0.01106351 & -0.11482 \\
\hline 2.15289307 & 0.16506203 & 0.09477194 & 92.96 \\
\hline 2.15289307 & 0.27089748 & -0.01106351 & 61.299472 \\
\hline 2.15289307 & 0.27089748 & 0.09477194 & -136.91 \\
\hline 2 . & 0.1650 & -0.011 & 63.4 \\
\hline 2.25872827 & 0.16506203 & 0.09477194 & -147.459 \\
\hline 2.25872827 & 0.27089748 & -0.01106351 & -110.663 \\
\hline 2.25872827 & 0.27089748 & 0.09477194 & 181. \\
\hline$-1 \cdot 31$ & -0.1943 & -0.22601055 & -134.27 \\
\hline-1.31 & -0.19434193 & -0.12017509 & 113.4 \\
\hline-1.31485093 & -0.08850648 & -0.22601055 & 160.1 \\
\hline-1 & -0 . & -0.1 & -123.1 \\
\hline-1 & -0.1943 & -0.22601055 & 126.6 \\
\hline-1.209015 & -0.19434193 & -0.12017509 & -109.8 \\
\hline-1.209015 & -0.08850648 & -0.22601055 & -151.46 \\
\hline-1 & -0.08850648 & -0.12017509 & 120.5 \\
\hline 2.280080 & 1.30933321 & 0.43990609 & 32.492 \\
\hline 2.28008008 & 1.30933321 & 0.54574150 & $-16 \cdot 78$ \\
\hline 2.28 & 1.41516852 & 0.43 & -27.7 \\
\hline 2 . & 1. & 0.5 & 15.4 \\
\hline 2.385915 & 1.30933321 & 0.43990609 & -27.54 \\
\hline $2.385915^{\circ}$ & 1.30933321 & 0.545741 & $11.0^{-}$ \\
\hline 2. & 1.41516852 & 0.439 & 22 . \\
\hline 2.38 & 1.41516 & 0.5457 & -10.0 \\
\hline-1.2197878 & -1.38936973 & 0.08411802 & $-13 \cdot 6$ \\
\hline-1.219787 & -1.3893 & 0 & 12 \\
\hline-1 & -1.28353417 & 0 . & 18 \\
\hline-1.219787 & -1.28353417 & 0.18995346 & -17.23 \\
\hline-1.113952 & -1.38936973 & 0.084118 & 12.6 \\
\hline-1.11395252 & -1.38936973 & 0.189 & -11.4 \\
\hline-1.11395252 & -1.28353417 & 0.0841 & -17.758 \\
\hline-1 & $-1 \cdot 2$ & 0.18 & 16.3 \\
\hline & -0.220895 & -0.748 & 45 \\
\hline 1. & -0.22089551 & -0.642 & -47 \\
\hline
\end{tabular}

\section{S-84}




\begin{tabular}{|c|c|c|c|}
\hline 1.06017745 & -0.11506007 & -0.74873185 & -97.32814026 \\
\hline 1.06017745 & -0.11506007 & -0.64289635 & 124.24700928 \\
\hline 1.16601288 & -0.22089551 & -0.74873185 & -22.24190331 \\
\hline 1.16601288 & -0.22089551 & -0.64289635 & 14.7216444 \\
\hline 1.16601288 & -0.11506007 & -0.74873185 & 85.6234436 \\
\hline 1.16601288 & -0.11506007 & -0.64289635 & -108.14171600 \\
\hline-2.49104571 & 0.48034528 & -0.18798651 & 61.9726448 \\
\hline-2.49104571 & 0.48034528 & -0.08215106 & -36.501262 \\
\hline-2.49104571 & 0.58618075 & -0.18798651 & -37.5904846 \\
\hline-2.49104571 & 0.58618075 & -0.08215106 & 23.314090 \\
\hline-2.3852 & 0.48034528 & -0.18798651 & -63.500694 \\
\hline-2.38521028 & 0.48034528 & -0.08215106 & 33.080436 \\
\hline-2.38521028 & 0.58618075 & -0.18798651 & 34.63 \\
\hline-2.38521028 & 0.58618075 & -0.08215106 & -18.12 \\
\hline 0.87829608 & -1.21926355 & -0.81211877 & 3.13 \\
\hline 0.87829608 & -1.21926355 & -0.70628333 & -1.377581 \\
\hline 0.87829608 & -1.11342812 & -0.81211877 & $-2 \cdot 7$ \\
\hline 0.87829608 & -1.11342812 & -0.70628333 & -1.886376 \\
\hline 0.98413152 & -1.21926355 & -0.81211877 & -0.295509 \\
\hline 0.98413152 & -1.21926355 & -0.70628333 & -4.70 \\
\hline 0.984 & -1.11342812 & -0.81211877 & -3.27 \\
\hline 0.98 & -1.11342812 & -0.70628333 & 12.80 \\
\hline-2.48172688 & 1.48277950 & -0.33387381 & -18.285020 \\
\hline-2.48 & 1.48277950 & -0.22803834 & 16.7 \\
\hline-2.4 & 1.58861494 & -0.33387381 & 14.5 \\
\hline-2 & 1.58861494 & -0.22803834 & $-13 \cdot 33$ \\
\hline-2.37589145 & 1.48277950 & -0.33387381 & 19.92 \\
\hline 89145 & 1.48277950 & -0.22803834 & -16.7 \\
\hline-2 & 1.58861494 & -0.33387381 & -15.79 \\
\hline-2.37589145 & 1.58861494 & -0.22803834 & 13.6 \\
\hline & \# Energy 2->2 & \multirow{2}{*}{\multicolumn{2}{|c|}{ \# Electr. mom. }} \\
\hline-2 & -0.1916 & & \\
\hline 2.15289307 & 0.16506203 & -0.01106351 & 66.534767 \\
\hline 2.15289307 & 0.16506203 & 0.09477194 & 43.92845 \\
\hline 2 . & 0.27089748 & -0.01106351 & 1.3 \\
\hline 07 & 0.27089748 & 0.09477194 & -96.0 \\
\hline 2.25872827 & 0.16506203 & -0.01106351 & 21.97716 \\
\hline 2.25872827 & 0.16506203 & 0.09477194 & -117.40647 \\
\hline 327 & 0.27089748 & -0.01106351 & -77.1 \\
\hline 2.25872827 & 0.27089748 & 0.09477194 & 160.1 \\
\hline-1.31485093 & -0.19434193 & -0.22601055 & $-118.7765^{\circ}$ \\
\hline 1485093 & -0.19434193 & -0.12017509 & 100.2 \\
\hline-1 & -0.08850648 & -0.22601055 & 140.6 \\
\hline$-1 \cdot 31$ & -0.08850648 & -0.12017509 & -106.34262 \\
\hline 0901549 & -0.19434193 & -0.22601055 & 110.7 \\
\hline 0901549 & -0.19434193 & -0.12017509 & -96.5 \\
\hline 01549 & -0.08850648 & -0.22601055 & $-131 \cdot 7$ \\
\hline 0901549 & -0.08850648 & -0.12017509 & 103.7 \\
\hline 008008 & 1.30933321 & 0.43990609 & 2.8 \\
\hline 2. & 1.30933 & 0.54 & 6.2 \\
\hline 2. & 1.415 & 0.43 & 8.1 \\
\hline 08008 & 1.41516852 & 0.54574150 & -13.88 \\
\hline 91576 & 1.30933321 & 0.43990609 & 13.1 \\
\hline 91576 & 1.30933 & 0.54 & -19.8 \\
\hline 91576 & 1.41516852 & 0.43990609 & -20.9990 \\
\hline 2.38591576 & 1.41516852 & 0.54574150 & 24.4 \\
\hline 78784 & -1.38936973 & 11802 & -12.83 \\
\hline-1 & -1.38936973 & 0.18995346 & 11.7 \\
\hline 78784 & -1.28353417 & 0.08411802 & 16.8 \\
\hline 1978784 & -1.28353417 & 0.18 & $-16 \cdot 1$ \\
\hline-1 & -1.38936973 & 0.08 & 11.2 \\
\hline-1 & -1.38936973 & 0.18995346 & -10.8 \\
\hline-1.11 & -1.28353417 & 0.08411802 & $-15 \cdot 2$ \\
\hline-1 & -1.28353417 & & 14 \\
\hline 1. & -0.22089551 & -0 & 15 \\
\hline 1. & -0.22089551 & -0.64289635 & 19.2 \\
\hline 1 . & -0.11506007 & -0 . & -58.4 \\
\hline
\end{tabular}

\section{S-85}




\begin{tabular}{|c|c|c|c|}
\hline 1.06017745 & -0.11506007 & -0.64289635 & 47.46169281 \\
\hline 1.16601288 & -0.22089551 & -0.74873185 & 32.19103241 \\
\hline 1.16601288 & -0.22089551 & -0.64289635 & -84.92025757 \\
\hline 1.16601288 & -0.11506007 & -0.74873185 & 21.63122749 \\
\hline 1.16601288 & -0.11506007 & -0.64289635 & 2.38823438 \\
\hline-2.49104571 & 0.48034528 & -0.18798651 & 56.69436264 \\
\hline-2.49104571 & 0.48034528 & -0.08215106 & $-31 \cdot 9$ \\
\hline-2.49104571 & 0.58618075 & -0.18798651 & -33.607131 \\
\hline-2.49104571 & 0.58618075 & -0.08215106 & 19.9940090 \\
\hline-2.38521028 & 0.48034528 & -0.18798651 & -56.45 \\
\hline-2.38521028 & 0.48034528 & -0.08215106 & 26.8 \\
\hline-2.38521028 & 0.58618075 & -0.18798651 & 29.1 \\
\hline-2.38521028 & 0.58618075 & -0.08215106 & -13.480496 \\
\hline 0.87829608 & -1.21926355 & -0.81211877 & 2.70084 \\
\hline 0.87829608 & -1.21926355 & -0.70628333 & 2.4287776 \\
\hline 0.87829608 & -1.11342812 & -0.81211877 & -0.731077 \\
\hline 0.87829608 & -1.11342812 & -0.70628333 & -9.27 \\
\hline 0.98413152 & -1.21926355 & -0.81211877 & 2.79 \\
\hline 0.98413152 & -1.21926355 & -0.70628333 & -12.19 \\
\hline 0.98413152 & -1.11342812 & -0.81211877 & -9.1 \\
\hline 0.98413152 & -1.11342812 & -0.70628333 & 25.5 \\
\hline-2.48172688 & 1.48277950 & -0.33387381 & $-18 \cdot 3$ \\
\hline-2.48172688 & 1.48277950 & -0.22803834 & 16.80 \\
\hline-2.48172688 & 1.58861494 & -0.3338 & 14.6 \\
\hline-2.481726 & 1.58861494 & -0.22 & $-13 \cdot 4$ \\
\hline-2.37589 & 1.48277950 & -0.33 & 20 \\
\hline-2.3758914 & 1.48277950 & -0.22803834 & -16.99402 \\
\hline-2.37 & 1.58861494 & -0.33387381 & -15.9 \\
\hline-2.37 & 1.58861494 & -0.228 & 13.93 \\
\hline & \# Energy 3->3 & & \\
\hline-1.8704 & $-2.0516 \quad-0.6730$ & & ctr. mom. \\
\hline 2.15289307 & 0.16506203 & 5351 & -0.9377 \\
\hline 2.15289307 & 0.16506203 & 0.09477194 & 98.205162 \\
\hline 2.15289307 & 0.27089748 & -0.01106351 & 67.3976898 \\
\hline 2.15289307 & 0.27089748 & 0.09477194 & -146.63 \\
\hline 2.25 & 0.16506203 & -0.01 & 66.22 \\
\hline 2.25872827 & 0.16506203 & 0.09477194 & -153.666275 \\
\hline 2.25872827 & 0.27089748 & -0.01106351 & -117.740547 \\
\hline 2.25 & 0.27089748 & 0.094 & 191.7 \\
\hline-1.31485 & -0.19434193 & -0.22601055 & -199.06532 \\
\hline-1.31485093 & -0.19434193 & -0.12017509 & 188.5668 \\
\hline-1.31485093 & -0.08850648 & -0.22601055 & 215.48898 \\
\hline 35093 & -0.08850648 & -0.12017509 & -189.05 \\
\hline-1.20901549 & -0.19434193 & -0.22601055 & 170.84 \\
\hline-1.20901549 & -0.19434193 & -0.12017509 & -165.44 \\
\hline-1.20901549 & -0.08850648 & -0.22 & -186.9 \\
\hline-1.20 & -0.08850648 & -0.12017509 & 166.9 \\
\hline 2.28008008 & 1.30933321 & 0.43990609 & 32.082 \\
\hline 2.28008008 & 1.30933321 & 0.5457 & -15.1 \\
\hline 2. & 1.41516852 & 0.43 & -26.8 \\
\hline 2.28 & 1.415 & 0.54 & 13.6 \\
\hline $2.385915^{\circ}$ & 1.30933321 & 0.43990 & -26.469 \\
\hline 2.385915 & 1.30933321 & 0.545 & 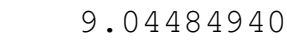 \\
\hline 2 . & 1.4 & 0 & 21.1 \\
\hline 2.38591576 & 1.41516852 & 0.54574 & -8.0 \\
\hline-1.21978784 & -1.38936973 & 0.08411 & -46.98 \\
\hline$-1.21978^{\circ}$ & -1.38936973 & 0.189 & 47.3 \\
\hline-1.21978 & -1.28353417 & 0.08411 & 54.4 \\
\hline-1.21978784 & -1.28353417 & 0.18 & -54.7 \\
\hline-1.113952 & -1.38 & 0.0 & 37.7 \\
\hline-1.11395 & -1.38936973 & $0.1 \varepsilon$ & -39.7 \\
\hline-1.11395 & -1.28353417 & 0.08 & -43.2 \\
\hline-1.113952 & -1.28353417 & 0.18 & 45.1 \\
\hline 1 . & -0.22 & -0.7 & \\
\hline 1. & -0.22089551 & -0.642 & -61 \\
\hline $1.06017^{\circ}$ & -0.11506007 & -0.74873185 & -110.56922 \\
\hline 1.060177 & -0.1150 & -0.6 & 142 \\
\hline
\end{tabular}




\begin{tabular}{|c|c|c|c|}
\hline 1.16601288 & -0.22089551 & -0.74873185 & -34.21302414 \\
\hline 1.16601288 & -0.22089551 & -0.64289635 & 30.85541344 \\
\hline 1.16601288 & -0.11506007 & -0.74873185 & 101.53448486 \\
\hline 1.16601288 & -0.11506007 & -0.64289635 & -129.40505981 \\
\hline-2.49104571 & 0.48034528 & -0.18798651 & 2.80356121 \\
\hline-2.49104571 & 0.48034528 & -0.08215106 & 14.07899094 \\
\hline-2.49104571 & 0.58618075 & -0.18798651 & 25.02890968 \\
\hline-2.49104571 & 0.58618075 & -0.08215106 & -31.4418735 \\
\hline-2.38521028 & 0.48034528 & -0.18798651 & 2.43 \\
\hline-2.38521028 & 0.48034528 & -0.08215106 & -19.74 \\
\hline-2.38521028 & 0.58618075 & -0.18798651 & -35.53 \\
\hline-2.38521028 & 0.58618075 & -0.08215106 & 39.35 \\
\hline 0.87829608 & -1.21926355 & -0.81211877 & 3.41641 \\
\hline 0.87829608 & -1.21926355 & -0.70628333 & -3.79869 \\
\hline 0.87829608 & -1.11342812 & -0.81211877 & -7.18667 \\
\hline 29608 & -1.11342812 & -0.70628333 & 60 \\
\hline 13152 & -1.21926355 & -0.81211877 & $-2.413^{-}$ \\
\hline 0.98413152 & -1.21926355 & -0.70628333 & $-0.554235^{7}$ \\
\hline 0.98413152 & -1.11342812 & -0.81211877 & 3.07 \\
\hline 0.98 & -1.11342812 & -0.70628333 & 3.21 \\
\hline-2.481 & 1.48277950 & -0.33387381 & -27.91 \\
\hline-2.481 & 1.48277950 & -0.22803834 & 25.35 \\
\hline-2.48172688 & 1.58861494 & -0.33387381 & 21.2 \\
\hline-2.48172688 & 1.58861494 & -0.228 & -19.3 \\
\hline$-2 \cdot 3$ & 1.48 & -0.333 & 28 \\
\hline-2.37589 & 1.48277950 & -0.22803834 & -22.97 \\
\hline-2.37589145 & 1.58861494 & -0.33387381 & -21.89 \\
\hline-2.37589145 & 1.58861494 & -0.22803834 & 17.8 \\
\hline & \# Energy 4->4 & & \\
\hline-4.9687 & -2.6169 & $\#$ & ter. mom. \\
\hline 89307 & 0.16506203 & -0.01106351 & 35.5094 \\
\hline 2.15289307 & 0.16506203 & 0.09477194 & 67.020 \\
\hline 2.15289307 & 0.27089748 & -0.01106351 & 33.0065727 \\
\hline 9307 & 0.27089748 & 0.09477194 & -117.081 \\
\hline 2.2 & 0 . & -0.01106351 & 36.1 \\
\hline 2.25872827 & 0.16506203 & 0.09477194 & -130.64039 \\
\hline 2827 & 0.27089748 & -0.01106351 & -90.48206 \\
\hline 2.2 & 89748 & 0.094 & 170.9 \\
\hline$-1 \cdot 31$ & -0.19 & -0.22601055 & -127.39 \\
\hline$-1 \cdot 3$ & -0.19434 & -0.12017509 & 107.72 \\
\hline$-1 \cdot 31$ & -0.08850648 & -0.22601055 & 153.417 \\
\hline$-1 \cdot 3$ & -0.08 & -0.12017509 & -117.0 \\
\hline-1.2 & -0.19 & -0.22601055 & 117.97 \\
\hline-1.20901549 & -0.19434 & -0.12017509 & -102.47 \\
\hline$-1 \cdot 20$ & -0.08850648 & -0.22601055 & -142.91 \\
\hline-1.20 & -0.08 & -0.12017509 & 112.6 \\
\hline 2.28 & 1.30933321 & 0.43990609 & 28.39 \\
\hline 08008 & 1.30933321 & 0.54574150 & -13.182 \\
\hline $2.2 \varepsilon$ & 1.41516852 & 0.43990609 & $-23 \cdot 9$ \\
\hline 2.2 & 1 . & 0.545 & 11.9 \\
\hline 1576 & 1.30933321 & 0.43990609 & -22.197 \\
\hline 2.38 & 1.309333 & 0.54574150 & 6.202 \\
\hline 2.3 & 1.41 & 0.43990609 & 17.3 \\
\hline 2.38 & 1.41 & 0.54574 & -5.45 \\
\hline$-1 \cdot 21$ & -1.389 & 0.08411802 & -11.92 \\
\hline$-1 \cdot 2$ & -1 . & 346 & 10.9 \\
\hline$-1 \cdot 2$ & -1 . & 02 & 16.3 \\
\hline$-1 \cdot 21$ & -1.28353 & 0.18995346 & -15.818 \\
\hline$-1 \cdot 1$ & -1.38936973 & 0.08411802 & 10.17 \\
\hline$-1 \cdot 1$ & -1.38 & 0.18995346 & -9.9 \\
\hline$-1 \cdot 1$ & -1 . & 0.08411802 & -14.50 \\
\hline-1.11 & -1.28353 & 0.18995346 & 14.30 \\
\hline 1.0 & -0.22 & -0.74873185 & 36.25 \\
\hline 1.06 & -0.2208 & -0.64289635 & -31.76 \\
\hline 1.06 & -0.1150 & -0.74873185 & -90.925 \\
\hline 1.0 & -0.11506 & -0.64289635 & 112.342 \\
\hline 1 & -0 . & -0 . & -15.6 \\
\hline
\end{tabular}




\begin{tabular}{|c|c|c|c|}
\hline 1.16601288 & -0.22089551 & -0.64289635 & 2.85842133 \\
\hline 1.16601288 & -0.11506007 & -0.74873185 & 81.01863861 \\
\hline 1.16601288 & -0.11506007 & -0.64289635 & -98.78815460 \\
\hline-2.49104571 & 0.48034528 & -0.18798651 & 62.22805023 \\
\hline-2.4910457 & 0.48034528 & -0.08215106 & -34.5 \\
\hline 2.49104571 & 0.58618075 & -0.18798651 & -38.373023 \\
\hline 2.49104571 & 0.58618075 & -0.08215106 & 21.7426204 \\
\hline-2.38521028 & 0.48034528 & -0.18798651 & -64.2 \\
\hline-2.38521028 & 0.48034528 & -0.08215106 & 31.32 \\
\hline-2.38521028 & 0.58618075 & -0.18798651 & 35 \\
\hline-2.38521028 & 0.58618075 & -0.08215106 & -16.7786636 \\
\hline 0.87829608 & -1.21926355 & -0.81211877 & $1.026886^{\circ}$ \\
\hline 0.87829608 & -1.21926355 & -0.70628333 & 2.316739 \\
\hline 0.87829608 & -1.11342812 & -0.81211877 & 0.2892848 \\
\hline 0.87829608 & -1.11342812 & -0.70628333 & -7.0581798 \\
\hline 0.98413152 & -1.21926355 & -0.81211877 & 1.08 \\
\hline 0.9841315 & -1.21926355 & -0.70628333 & -7.31789 \\
\hline 0.98413152 & -1.11342812 & -0.81211877 & -5.1790285 \\
\hline 0.98413152 & -1.11342812 & -0.70628333 & 16.3 \\
\hline-2.4817268 & 1.48277950 & -0.33387381 & -17.8 \\
\hline-2.48172 & 1.48277950 & -0.228 & 16.6 \\
\hline-2.481726 & 1.58861494 & -0.33387381 & 14.1 \\
\hline-2.48 & 1.58861494 & -0.228038 & -13.1 \\
\hline-2.37 & 1.48277950 & -0.33 & 19.3 \\
\hline-2.37589 & 1.48277950 & -0.22803834 & $-16 \cdot 53$ \\
\hline-2.3758914 & 1.58861494 & -0.33387381 & -15.239 \\
\hline-2.3758914 & 1.58861494 & -0.22803834 & 13.39 \\
\hline & \# Energy 5->5 & & \\
\hline-5.7009 & -1.2613 & & tr. mom. \\
\hline 2 . & 0.16506203 & -0.01106351 & 5.1 \\
\hline 2.15 & 0.165 & 0.094 & 89.71 \\
\hline 2.15 & 0.27089748 & -0.01106351 & 58.67816 \\
\hline 2.15289307 & 0.27089748 & 0.09477194 & -135.78027 \\
\hline 2 . & 0.16506203 & -0.01106351 & 60.1 \\
\hline 2 . & 0.1650 & 0.09477194 & -146.36 \\
\hline 2.25872827 & 0.27089748 & -0.01106351 & -109.73 \\
\hline 2.25872827 & 0.27089748 & 0.094771 & 182.7 \\
\hline$-1 \cdot 31$ & -0.19434 & -0.22601055 & -117.0 \\
\hline-1.31 & -0.19434193 & -0.12017509 & 94.65 \\
\hline-1.31485093 & -0.08850648 & -0.22601055 & 139.8 \\
\hline-1.31 & -0.08850648 & -0.12017509 & $-100 \cdot 7$ \\
\hline-1 & -0.1943 & -0.226 & 109.0 \\
\hline-1 & -0.1943 & -0.120 & -89.4 \\
\hline-1.20 & -0.08850648 & -0.22601055 & $-131 \cdot 0$ \\
\hline-1 & -0.08850648 & -0.120 & 96.3 \\
\hline 2 . & 1.3093 & 0 & 33.3 \\
\hline 2.28 & 1.30933321 & 0.54574150 & -17.09 \\
\hline 2.280 & 1.41516852 & 0.43990609 & $-28 \cdot 5$ \\
\hline 2 . & 1. & 0 & 15 \\
\hline 2.38 & 1.30933321 & 0.43990609 & -27.92 \\
\hline 2.385915 & 1.30933321 & 0.54574150 & 10.9 \\
\hline 2 . & 1.4151 & 0.43 & 22.7 \\
\hline 2 . & 1.4 & 0.54 & -9.9 \\
\hline-1.21978784 & -1.38936973 & 0.08411802 & -14.48 \\
\hline-1 & -1.38936973 & 0.1899 & 13.6 \\
\hline-1 & -1.28353417 & $0.0 \varepsilon$ & 18. \\
\hline-1 & -1.28353417 & 0.1899 & -18.5 \\
\hline-1 & -1.38936973 & 0.0841 & 12.7 \\
\hline-1 & -1.38936973 & 0.1 & $-12 \cdot 8$ \\
\hline-1 & -1.28353417 & 0 & -17.1 \\
\hline-1 & -1.28353417 & 0.18995346 & 17.2 \\
\hline 1 & -0.22089551 & -0.74873185 & 46 \\
\hline 1. & -0.22089551 & -0.64289635 & -46.8 \\
\hline 1. & -0.11506007 & -0.74873185 & -101.062 \\
\hline 1 . & -0.11506007 & -0.64289635 & 127.79919 \\
\hline & -0.2 & -0.748 & -24 \\
\hline & -0 & 661 & 16 \\
\hline
\end{tabular}




\begin{tabular}{|c|c|c|c|}
\hline 1.16601288 & -0.11506007 & -0.74873185 & 90.92971802 \\
\hline 1.16601288 & -0.11506007 & -0.64289635 & 98652649 \\
\hline-2.49104571 & 0.48034528 & -0.18798651 & 50.68270111 \\
\hline-2.49104571 & 0.48034528 & -0.08215106 & -16.68428993 \\
\hline-2.49104571 & 0.58618075 & -0.18798651 & -30.30373955 \\
\hline-2.49104571 & 0.58618075 & -0.08215106 & 6.26830530 \\
\hline-2.38521028 & 0.48034528 & -0.18798651 & -52.963211 \\
\hline-2.38521028 & 0.48034528 & -0.08215106 & 13.6997470 \\
\hline-2.38521028 & 0.58618075 & -0.18798651 & $29.02401^{\circ}$ \\
\hline-2.38521028 & 0.58618075 & -0.08215106 & -1.83 \\
\hline 0.87829608 & -1.21926355 & -0.81211877 & 1.23872 \\
\hline 0.87829608 & -1.21926355 & -0.70628333 & 1.13 \\
\hline 0.87829608 & -1.11342812 & -0.81211877 & -0.1 \\
\hline 0.87829608 & -1.11342812 & -0.70628333 & -5.31 \\
\hline 0.98413152 & -1.21926355 & -0.81211877 & 1.15 \\
\hline 0.98413152 & -1.21926355 & -0.70628333 & -6.693 \\
\hline 0.9841315 & -1.11342812 & -0.81211877 & -5.2 \\
\hline 0.98413152 & -1.11342812 & -0.70628333 & $15.4^{7}$ \\
\hline-2.48172688 & 1.48277950 & -0.33387381 & -17.550760 \\
\hline-2.48 & 1.48277950 & -0.22803834 & 17.40 \\
\hline-2.48 & 1.58861494 & -0.33387381 & 13.88 \\
\hline-2.48 & 1.58861494 & -0.22 & -13.5 \\
\hline-2.37 & 1.48277950 & -0.33387381 & 18.18 \\
\hline-2.375 & 1.48277950 & -0.22803 & -16.7 \\
\hline-2.37 & 1.58861494 & -0.33387381 & -14.32 \\
\hline-2 . & 1.58861494 & -0.22803834 & 13 \\
\hline & \# Energy 6->6 & & \\
\hline 9.43 & -2.6552 & & ctr. mom. \\
\hline 2.15289307 & 0.16506203 & -0.01 & -28.03305 \\
\hline 2.15289307 & 0.16506203 & 0.09477194 & 110.1751632 \\
\hline 39307 & 0.27089748 & -0.01106351 & 86.6 \\
\hline 2. & 0.27089748 & 0.09 & $-151 \cdot 3$ \\
\hline 2.25872827 & 0.16506203 & -0.01106351 & 82.25 \\
\hline 2.25872827 & 0.16506203 & 0.09477194 & -157.498 \\
\hline 72827 & 0.27089748 & -0.01106351 & -126.7 \\
\hline 2.25 & 0.27089748 & 0.0947 & 188.99 \\
\hline 1485093 & -0.19434193 & -0.22601 & -109.732 \\
\hline-1.31485093 & -0.19434193 & -0.12017509 & 95.34 \\
\hline-1 . & -0.08850648 & -0.22601055 & 130.6 \\
\hline-1 . & -0.08850648 & -0.12017509 & -98.33 \\
\hline 0901549 & -0.19434193 & -0.22601055 & 93.97 \\
\hline-1.20901549 & -0.19434193 & -0.12017509 & -84.43 \\
\hline-1 & -0.08850648 & -0.22601055 & -114.02 \\
\hline 0901549 & -0.08850648 & -0.12017509 & 87.9 \\
\hline 8008008 & 1.30933321 & 0.43990609 & 35.63 \\
\hline 08008 & 1.30933321 & 0.54 & -19.4 \\
\hline 2 . & 1.41516 & 0.43 & -32.9 \\
\hline 2.28 & 1.41516852 & 0.54574150 & 20.10 \\
\hline 2.38 & 1.30933321 & 0.43990609 & -30.19 \\
\hline 2 . & 1.30933321 & 0.545 & 14.0 \\
\hline 2 . & 1.41516852 & 0.439 & 27.76 \\
\hline 2.38591576 & 1.41516852 & 0.54574 & -15.35 \\
\hline-1.21978784 & -1.38936973 & 0.08411802 & $-17.2 \varepsilon$ \\
\hline-1 & -1.38936973 & 0 & 17.6 \\
\hline-1 . & -1.28353417 & 08411 & 20.7 \\
\hline-1.21978 & -1.28353417 & 0.1899 & -22.02 \\
\hline-1 . & -1.38936973 & 0.08 & 12.8 \\
\hline-1 . & -1.38936973 & 0.18 & $-14 \cdot 4$ \\
\hline-1 . & -1.28353417 & 0.0841 & -15.85 \\
\hline-1 & -1.28353417 & 0 & 17 \\
\hline 1 . & -0.22089551 & -0.7 & 52.2 \\
\hline 1.060 & -0.22089551 & -0.6428 & $-59.0^{-}$ \\
\hline 1.06017745 & -0.11506007 & -0.74873185 & -116.27977 \\
\hline 1 . & -0.1150 & -0 & 150 . \\
\hline 1 . & -0.22089 & -0 . & -43.6 \\
\hline 1 . & -0.22089551 & -0.64289635 & 47.08741 \\
\hline 1. & -0.1150 & -0 & 117 \\
\hline
\end{tabular}


1.16601288
-2.49104571
-2.49104571
-2.49104571
-2.49104571
-2.38521028
-2.38521028
-2.38521028
-2.38521028
0.87829608
0.87829608
0.87829608
0.87829608
0.98413152
0.98413152
0.98413152
0.98413152
-2.48172688
-2.48172688
-2.48172688
-2.48172688
-2.37589145
-2.37589145
-2.37589145
-2.37589145
-7.7325

7.7325

2. 15289307

2.15289307

2.15289307

2. 15289307

2. 25872827

2. 25872827

2. 25872827

2. 25872827

$-1.31485093$

$-1.31485093$

$-1.31485093$

$-1.31485093$

$-1.20901549$

$-1.20901549$

$-1.20901549$

$-1.20901549$

2. 28008008

2.28008008

2.28008008

2. 28008008

2.38591576

2. 38591576

2.38591576

2.38591576

$-1.21978784$

$-1.21978784$

$-1.21978784$

$-1.21978784$

$-1.11395252$

$-1.11395252$

$-1.11395252$

$-1.11395252$

1. 06017745

1.06017745

1.06017745

1.06017745

1.16601288

1. 16601288

1.16601288

1. 16601288
$-0.11506007$

0.48034528

0.48034528

0.58618075

0.58618075

0.48034528

0.48034528

0.58618075

0.58618075

$-1.21926355$

$-1.21926355$

$-1.11342812$

$-1.11342812$

$-1.21926355$

$-1.21926355$

$-1.11342812$

$-1.11342812$

1. 48277950

1. 48277950

1.58861494

1.58861494

1. 48277950

1.48277950

1.58861494

1.58861494

\# Energy 7->7

$2.8590 \quad-0.7797$

0.16506203

0.16506203

0.27089748

0.27089748

0.16506203

0.16506203

0.27089748

0.27089748

$-0.19434193$

$-0.19434193$

$-0.08850648$

$-0.08850648$

$-0.19434193$

$-0.19434193$

$-0.08850648$

$-0.08850648$

1. 30933321

1. 30933321

1.41516852

1. 41516852

1. 30933321

1.30933321

1. 41516852

1. 41516852

$-1.38936973$

$-1.38936973$

$-1.28353417$

$-1.28353417$

$-1.38936973$

$-1.38936973$

$-1.28353417$

$-1.28353417$

$-0.22089551$

$-0.22089551$

$-0.11506007$

$-0.11506007$

$-0.22089551$

$-0.22089551$

$-0.11506007$

$-0.11506007$
$-0.64289635$

$-0.18798651$

$-0.08215106$

$-0.18798651$

$-0.08215106$

$-0.18798651$

$-0.08215106$

$-0.18798651$

$-0.08215106$

$-0.81211877$

$-0.70628333$

$-0.81211877$

$-0.70628333$

$-0.81211877$

$-0.70628333$

$-0.81211877$

$-0.70628333$

$-0.33387381$

$-0.22803834$

$-0.33387381$

$-0.22803834$

$-0.33387381$

$-0.22803834$

$-0.33387381$

$-0.22803834$$$
\text { \# Electr. mom. }
$$

$\begin{array}{rr}-0.01106351 & 10.25336838 \\ 0.09477194 & 81.83727264 \\ -0.01106351 & 51.04429245 \\ 0.09477194 & -127.51097107\end{array}$

$-0.01106351 \quad 62.86198425$

$0.09477194 \quad-142.17637634$

$-0.01106351$

0.09477194

$-0.22601055$

$-0.12017509$

$-0.22601055$

$-0.12017509$

$-0.22601055$

$-0.12017509$

$-0.22601055$

$-0.12017509$

0.43990609

0.54574150

0.43990609

0.54574150

0.43990609

0.54574150

0.43990609

0.54574150

0.08411802

0.18995346

0.08411802

0.18995346

0.08411802

0.18995346

0.08411802

0.18995346

$-0.74873185$

$-0.64289635$

$-0.74873185$

$-0.64289635$

$-0.74873185$

$-0.64289635$

$-0.74873185$

$-0.64289635$
$-152.64746094$

53.88835907

$-32.04966736$

$-30.18378639$

19.58418655

$-55.03873062$

29.03589249

27.01537323

$-15.26235485$

$-1.04051340$

3. 24503684

2. 21192479

$-7.28158522$

1. 46465313

$-5.87188768$

$-4.67530918$

13.13299370

$-18.75426102$

15.13019466

14.92057800

$-12.18131351$

19.05826187

$-13.14072037$

$-15.06045437$

10.91116047

$-111.30770111$

178.76472473

$-132.55490112$

118.21169281

151.23135376

$-119.94515991$

114.53559875

$-104.80473328$

$-132.54010010$

107.29138947

3.57430172

5.85475302

5.83788157

$-12.16666794$

9.05297089

$-16.60036850$

$-16.00536156$ 20.51058769

$-25.79050064$

25.76061249

30.12389946

$-30.67130470$

20.34319115

$-21.57044792$

$-23.82165909$

25.21033669

20.96379852

4.20971537

$-74.33872223$

75.16240692

13.64086151

$-51.88572311$

50.56290817

$-42.97161484$ 


-2.49104571
-2.49104571
-2.49104571
-2.49104571
-2.38521028
-2.38521028
-2.38521028
-2.38521028
0.87829608
0.87829608
0.87829608
0.87829608
0.98413152
0.98413152
0.98413152
0.98413152
-2.48172688
-2.48172688
-2.48172688
-2.48172688
-2.37589145
-2.37589145
-2.37589145
-2.37589145

64

\begin{abstract}
0.48034528
0.48034528

0.58618075

0.58618075

0.48034528

0.48034528

0.58618075

0.58618075

$-1.21926355$

$-1.21926355$

$-1.11342812$

$-1.11342812$

$-1.21926355$

$-1.21926355$

$-1.11342812$

$-1.11342812$

1. 48277950

1. 48277950

1.58861494

1.58861494

1. 48277950

1. 48277950

1.58861494

1.58861494
\end{abstract}

\section{$-8.9799$ \\ 2.15289307}

2. 15289307

2.15289307

2. 15289307

2. 25872827

2. 25872827

2. 25872827

2. 25872827

$-1.31485093$

$-1.31485093$

$-1.31485093$

$-1.31485093$

$-1.20901549$

$-1.20901549$

$-1.20901549$

$-1.20901549$

2. 28008008

2.28008008

2. 28008008

2. 28008008

2. 38591576

2.38591576

2. 38591576

2. 38591576

$-1.21978784$

$-1.21978784$

$-1.21978784$

$-1.21978784$

$-1.11395252$

$-1.11395252$

$-1.11395252$

$-1.11395252$

1.06017745

1.06017745

1.06017745

1. 06017745

1. 16601288

1.16601288

1. 16601288

1.16601288

$-2.49104571$

$$
\text { \# Energy 8->8 }
$$

0.16506203

0.27089748

0.27089748

0.16506203

0.16506203

0.27089748

0.27089748

$-0.19434193$

$-0.19434193$

$-0.08850648$

$-0.08850648$

$-0.19434193$

$-0.19434193$

$-0.08850648$

$-0.08850648$

1. 30933321

1. 30933321

1. 41516852

1. 41516852

1. 30933321

1. 30933321

1. 41516852

1. 41516852

$-1.38936973$

$-1.38936973$

$-1.28353417$

$-1.28353417$

$-1.38936973$

$-1.38936973$

$-1.28353417$

$-1.28353417$

$-0.22089551$

$-0.22089551$

$-0.11506007$

$-0.11506007$

$-0.22089551$

$-0.22089551$

$-0.11506007$

$-0.11506007$

0.48034528
0.16506203
$-0.18798651$

$-0.08215106$

$-0.18798651$

$-0.08215106$

$-0.18798651$

$-0.08215106$

$-0.18798651$

$-0.08215106$

$-0.81211877$

$-0.70628333$

$-0.81211877$

$-0.70628333$

$-0.81211877$

$-0.70628333$

$-0.81211877$

$-0.70628333$

$-0.33387381$

$-0.22803834$

$-0.33387381$

$-0.22803834$

$-0.33387381$

$-0.22803834$

$-0.33387381$

$-0.22803834$

$$
3
$$

$-0.01106351$

\# Electr. mom.

0.09477194

$-0.01106351$

0.09477194

$-0.01106351$

0.09477194

$-0.01106351$

0.09477194

$-0.22601055$

$-0.12017509$

$-0.22601055$

$-0.12017509$

$-0.22601055$

$-0.12017509$

$-0.22601055$

$-0.12017509$

0.43990609

0.54574150

0.43990609

0.54574150

0.43990609

0.54574150

0.43990609

0.54574150

0.08411802

0.18995346

0.08411802

0.18995346

0.08411802

0.18995346

0.08411802

0.18995346

$-0.74873185$

$-0.64289635$

$-0.74873185$

$-0.64289635$

$-0.74873185$

$-0.64289635$

$-0.74873185$

$-0.64289635$

$-0.18798651$
39.99707794

$-20.11117744$

$-15.77459812$

6.89350557

$-38.47730255$

15.47290707

9.79168129

$-0.76652515$

$-0.77662700$

5.65163326

2.92672110

$-12.35696507$

4.55703068

$-13.06394196$

$-10.37440205$

25.27490807

$-20.70806694$

17.24535942

16.33857727

$-13.75712299$

21.40658951

$-15.41427612$

$-16.78847885$

12.63590050

49.66966248

56.69345856

22.29635811

$-110.26968384$

26.19097328

$-122.98066711$

$-83.70304108$

166.30366516

$-155.78657532$

140.93325806

177.07131958

$-146.44651794$

141.12815857

$-129.90608215$

$-161.63734436$

136.49119568

23.98917961

$-9.19684315$

$-18.48579979$

7.14912605

$-15.67166710$

0.78567743

10.43258667

0.29722583

$-24.94222832$

24.52358627

29.77975082

$-29.57052803$

20.76606941

$-21.43613815$

$-24.79144478$

25.50322533

48.63079834

$-46.44250870$

$-98.78829193$

121.57218933

$-21.33494186$

7. 78872490

82.56708527

$-99.21677399$

41.98486710 


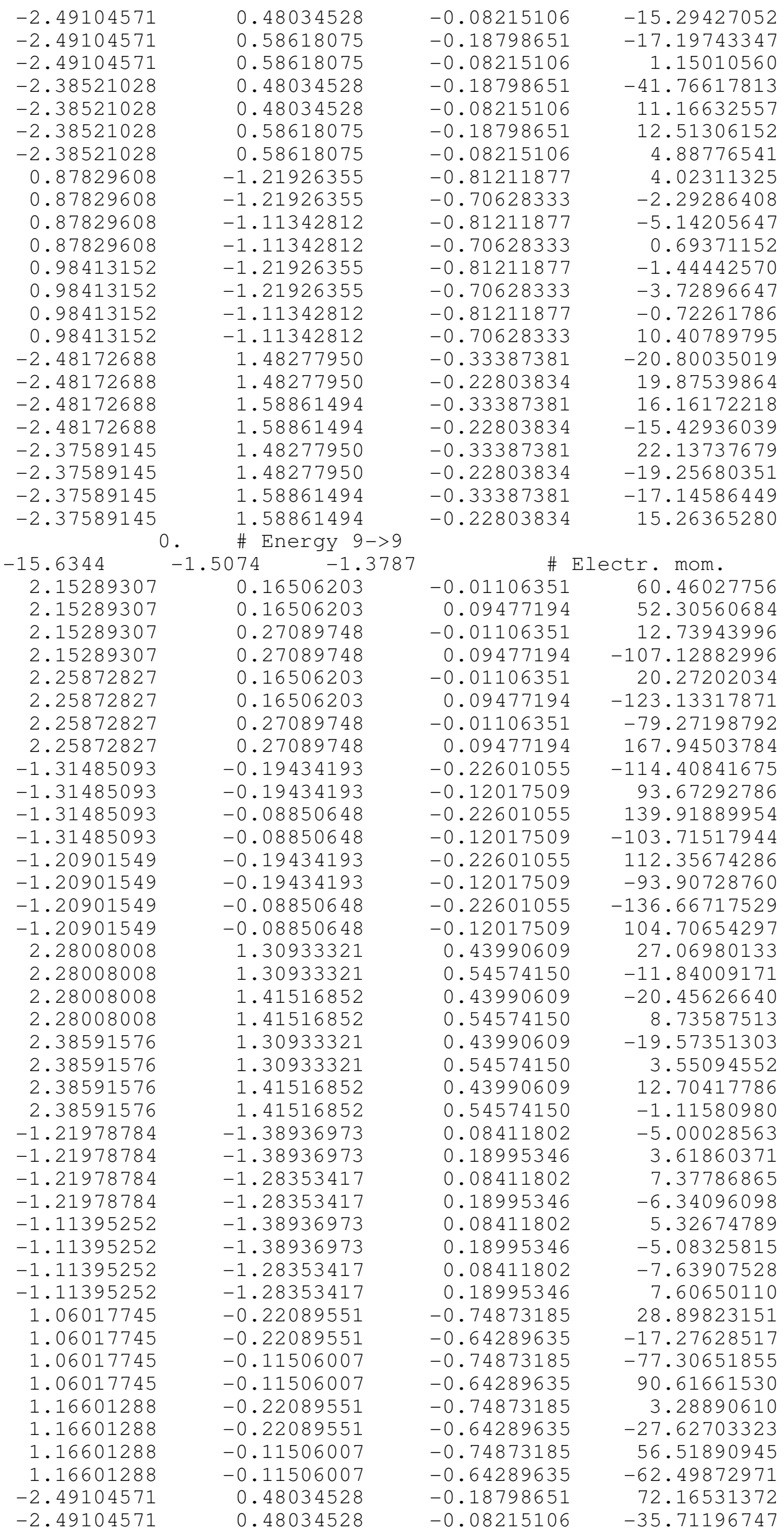




-2.49104571
-2.49104571
-2.38521028
-2.38521028
-2.38521028
-2.38521028
0.87829608
0.87829608
0.87829608
0.87829608
0.98413152
0.98413152
0.98413152
0.98413152
-2.48172688
-2.48172688
-2.48172688
-2.48172688
-2.37589145
-2.37589145
-2.37589145
-2.37589145
-CT03009A-

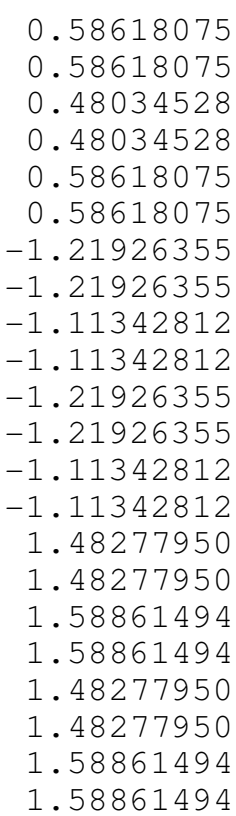

$180, \operatorname{roots}=9$

6

$$
\begin{array}{r}
-1.8828 \\
-1.5089 \\
-1.2684 \\
1.0145 \\
0.6257 \\
2.3163
\end{array}
$$

\&TRANSITION $1->\ldots$

$$
\begin{array}{rr}
0.0653 & -0.2948 \\
-0.2044 & -1.4377 \\
1.0646 & 0.4434 \\
0.1637 & 0.2237 \\
-0.8991 & 0.7158 \\
0.3914 & -0.1081
\end{array}
$$

$-0.18798651$

$-0.08215106$

$-0.18798651$

$-0.08215106$

$-0.18798651$

$-0.08215106$

$-0.81211877$

$-0.70628333$

$-0.81211877$

$-0.70628333$

$-0.81211877$

$-0.81211877$

$-0.70628333$

$-0.33387381$

$-0.22803834$

$-0.33387381$

$-0.22803834$

$-0.33387381$

$-0.22803834$

$-0.33387381$

$-0.22803834$
$-0.70628333$
-51.75628281
25.40567017
-76.98412323
33.89822388
53.18086243
-22.14649963
2.00260687
2.16355515
0.50655735
-8.70877075
1.79265034
-9.47404861
-7.72987318
21.24810791
-14.50567436
15.25579834
11.69502449
-12.04466248
15.55951500
-15.60841465
-12.51919842
12.61484718 $64 \quad 42891$
$-0.1425$
$-0.2383$
$-1.93576097$
$-1.93576097$
$-1.93576097$
$-1.93576097$
$-1.82992566$
$-1.82992566$
$-1.82992566$
$-1.82992566$
0.96153939
0.96153939
0.96153939
0.96153939
1.06737483
1.06737483
1.06737483
1.06737483
$-1.56185186$
$-1.56185186$
$-1.56185186$
$-1.56185186$
$-1.45601630$
$-1.45601630$
$-1.45601630$
$-1.45601630$
0.57276028
0.57276028
0.57276028
0.57276028
0.67859572
0.67859572
0.67859572
0.67859572

\# Energy 1->2
1.0000
1.0000
1.0000
1.0000
1.0000
1.0000

$\begin{array}{ll}\# & \mathrm{C} 1 \\ \# & \mathrm{O} 1 \\ \# & \mathrm{~N} 1 \\ \# & \mathrm{C} 2 \\ \# & \mathrm{~N} 2 \\ \# & \mathrm{O} 2\end{array}$

$$
\begin{aligned}
& 0.0002 \quad-0.1372 \\
& 0.8849 \\
& 1.000 \text { \# Electr. mom., scale-fac } \\
& \text { \# Magnetic mom. }
\end{aligned}
$$

0.01240921

0.01240921

0.11824466

0.11824466

0.01240921

0.01240921

0.11824466

0.11824466

0.11078113

0.11078113

0.21661659

0.21661659

0.11078113

0.11078113

0.21661659

0.21661659

$-0.25733095$

$-0.25733095$

$-0.15149552$

$-0.15149552$

$-0.25733095$

$-0.25733095$

$-0.15149552$

$-0.15149552$

$-0.95197451$

$-0.95197451$

$-0.84613907$

$-0.84613907$

$-0.95197451$

$-0.95197451$

$-0.84613907$

$-0.84613907$ $\begin{array}{ll}-0.34768584 & 21.33906174\end{array}$

$\begin{array}{ll}-0.24185041 & -18.87046432\end{array}$

$-0.34768584 \quad-18.92481232$

$\begin{array}{ll}-0.24185041 & 15.76336670\end{array}$

$-0.34768584$

$-0.24185041$

$-0.34768584$

$-0.24185041$

0.17077343

0.27660885

0.17077343

0.27660885

0.17077343

0.27660885

0.17077343

0.27660885

$-1.49057686$

$-1.38474143$

$-1.49057686$

$-1.38474143$

$-1.49057686$

$-1.38474143$

$-1.49057686$

$-1.38474143$

0.66284108

0.76867646

0.66284108

0.76867646

0.66284108

0.76867646

0.66284108

0.76867646

$-11.85599518$

7.83710670

8.64602947

$-3.40693688$

3.55518603

$-3.94324398$

$-1.62653756$

2.75974107

$-3.02084827$

3.27025390

1.04755628

$-2.09480262$

1.57216704

$-2.58300734$

$-0.45926505$

1.30620337

$-0.16332518$

0.99352294

$-0.70623380$

$-0.02773149$

$-3.13341236$

2.57027602

3.77034426

$-3.15371585$

2. 78923774

$-2.32172298$

$-3.35538864$

2. 85116124 


-1.32129574
-1.32129574
-1.32129574
-1.32129574
-1.21546030
-1.21546030
-1.21546030
-1.21546030
2.26337528
2.26337528
2.26337528
2.26337528
2.36921072
2.36921072
2.36921072
2.36921072
-1.48753464
-1.48753464
-1.48753464
-1.48753464
-1.38169920
-1.38169920
-1.38169920
-1.38169920
2.46758628
2.46758628
2.46758628
2.46758628
2.57342172
2.57342172
2.57342172
2.57342172
-0.10475785
-10

1. 01172543

1. 01172543

1. 11756098

1.11756098

1.01172543

1.01172543

1. 11756098

1. 11756098

0.33846018

0.33846018

0.44429561

0.44429561

0.33846018

0.33846018

0.44429561

0.44429561

0.95463514

0.95463514

1.06047070

1.06047070

0.95463514

0.95463514

1. 06047070

1.06047070

1. 14465213

1. 14465213

1.25048769

1. 25048769

1. 14465213

1.14465213

1.25048769

1. 25048769

64
\# Energy 1->3

$$
\begin{array}{rr}
0.39045236 & -17.66874504 \\
0.49628779 & 13.25396061 \\
0.39045236 & 15.66496944 \\
0.49628779 & -12.86368370 \\
0.39045236 & 9.53880787 \\
0.49628779 & -6.82940006 \\
0.39045236 & -9.44450188 \\
0.49628779 & 7.87249994 \\
-0.16102493 & 2.32115412 \\
-0.05518948 & -1.27885628 \\
-0.16102493 & -2.70861030 \\
-0.05518948 & 1.92137969 \\
-0.16102493 & -2.38930058 \\
-0.05518948 & 1.53196394 \\
-0.16102493 & 2.72627211 \\
-0.05518948 & -2.08039021 \\
1.39116621 & -1.80288720 \\
1.49700165 & 1.33797610 \\
1.39116621 & 2.73170590 \\
1.49700165 & -2.04300523 \\
1.39116621 & 0.63499528 \\
1.49700165 & -0.48120153 \\
1.39116621 & -1.59131002 \\
1.49700165 & 1.20744467 \\
-0.74076766 & -0.02369741 \\
-0.63493228 & 0.25684881 \\
-0.74076766 & -0.12068776 \\
-0.63493228 & -0.01447823 \\
-0.74076766 & -0.25310764 \\
-0.63493228 & 0.10091859 \\
-0.74076766 & 0.31972557 \\
-0.63493228 & -0.25053269
\end{array}
$$

$\begin{array}{rr}0.0504 & 0.1886 \\ -0.7417 & 0.3113\end{array}$

$\begin{array}{lll}-0.3616 & -0.7417 & 0.3113\end{array}$

$-1.93576097$

0.01240921

0.01240921

0.11824466

0.11824466

0.01240921

0.01240921

0.11824466

0.11824466

0.11078113

0.11078113

0.21661659

0.21661659

0.11078113

0.11078113

0.21661659

0.21661659

$-0.25733095$

$-0.25733095$

$-0.15149552$

$-0.15149552$

$-0.25733095$

$-0.25733095$

$-0.15149552$

$-0.15149552$

$-0.95197451$

$-0.95197451$

$-0.84613907$

$-0.84613907$

$-0.95197451$

$-0.95197451$

$-0.84613907$

$-0.84613907$

$-1.49057686$

0.66284108

$$
\begin{gathered}
1.000 \text { \# Electr. mom., scale-fac } \\
\text { \# Magnetic mom. }
\end{gathered}
$$

$-0.34768584$

$-0.24185041$

$-0.34768584$

$-0.24185041$

$-0.34768584$

$-0.24185041$

$-0.34768584$

$-0.24185041$

0.17077343

0.27660885

0.17077343

0.27660885

0.17077343

0.27660885

0.17077343

0.27660885

$-1.49057686$

$-1.38474143$

$-1.38474143$

$-1.49057686$

$-1.38474143$

$-1.49057686$

$-1.38474143$

0.66284108

0.76867646

0.76867646

0.66284108

0.76867646

0.66284108

0.76867646
1. 42160165

$-1.77257228$

2. 04097009

$-3.91388869$

4. 47105646

3.77203345

$-5.01519871$

$-7.24686146$

13.21095371

11.95003891

$-17.42899704$

15.02556801

$-21.87406158$

$-19.51391029$

25.59655952

$-1.25099123$

1. 54862225

0.94169682

$-1.15850925$

0.69591862

$-0.85276932$

$-0.44767407$

0.55321676

$-4.56153822$

7.71263361

$-0.55124819$

$-3.47867465$

7.26939344

$-10.82041740$

$-2.11614251$

6.69230700
$-1.16744101$ 


$\begin{array}{ll}-1.32129574 & 1.01172543 \\ -1.32129574 & 1.01172543 \\ -1.32129574 & 1.11756098 \\ -1.32129574 & 1.11756098 \\ -1.21546030 & 1.01172543 \\ -1.21546030 & 1.01172543 \\ -1.21546030 & 1.11756098 \\ -1.21546030 & 1.11756098 \\ 2.26337528 & 0.33846018 \\ 2.26337528 & 0.33846018 \\ 2.26337528 & 0.44429561 \\ 2.26337528 & 0.44429561 \\ 2.36921072 & 0.33846018 \\ 2.36921072 & 0.33846018 \\ 2.36921072 & 0.44429561 \\ 2.36921072 & 0.44429561 \\ -1.48753464 & 0.95463514 \\ -1.48753464 & 0.95463514 \\ -1.48753464 & 1.06047070 \\ -1.48753464 & 1.06047070 \\ -1.38169920 & 0.95463514 \\ -1.38169920 & 0.95463514 \\ -1.38169920 & 1.06047070 \\ -1.38169920 & 1.06047070 \\ 2.46758628 & 1.14465213 \\ 2.46758628 & 1.14465213 \\ 2.46758628 & 1.25048769 \\ 2.46758628 & 1.25048769 \\ 2.57342172 & 1.14465213 \\ 2.57342172 & 1.14465213 \\ 2.57342172 & 1.25048769 \\ 2.57342172 & 1.25048769 \\ 2.8614 & \# .5 n e r 9 y 1->4 \\ 0.0769 & 874 \\ -1.34101 . & -1.0135\end{array}$

$\begin{array}{lll}2.8614 & 1.8744 & -1.0135\end{array}$ $0.0769-0.3930 \quad-0.1693$

$-1.93576097$

$-1.93576097$

$-1.93576097$

$-1.93576097$

$-1.82992566$

$-1.82992566$

$-1.82992566$

$-1.82992566$

0.96153939

0.96153939

0.96153939

0.96153939

1.06737483

1.06737483

1.06737483

1.06737483

$-1.56185186$

$-1.56185186$

$-1.56185186$

$-1.56185186$

$-1.45601630$

$-1.45601630$

$-1.45601630$

$-1.45601630$

0.57276028

0.57276028

0.57276028

0.57276028

0.67859572

0.67859572

0.67859572

0.67859572
0.01240921

0.01240921

0.11824466

0.11824466

0.01240921

0.01240921

0.11824466

0.11824466

0.11078113

0.11078113

0.21661659

0.21661659

0.11078113

0.11078113

0.21661659

0.21661659

$-0.25733095$

$-0.25733095$

$-0.15149552$

$-0.15149552$

$-0.25733095$

$-0.25733095$

$-0.15149552$

$-0.15149552$

$-0.95197451$

$-0.95197451$

$-0.84613907$

$-0.84613907$

$-0.95197451$

$-0.95197451$

$-0.84613907$

$-0.84613907$
0.39045236

0.49628779

0.39045236

0.49628779

0.39045236

0.49628779

0.39045236

0.49628779

$-0.16102493$

$-0.05518948$

$-0.16102493$

$-0.05518948$

$-0.16102493$

$-0.05518948$

$-0.16102493$

$-0.05518948$

1.39116621

1. 49700165

1. 39116621

1.49700165

1.39116621

1.49700165

1.39116621

1. 49700165

$-0.74076766$

$-0.63493228$

$-0.74076766$

$-0.63493228$

$-0.74076766$

$-0.63493228$

$-0.74076766$

$-0.63493228$
$-0.69315356$

0.82912290

3.56355453

$-4.32603264$

1. 10806322

0.18525089

$-4.82146931$

4.57068157

$-15.20983887$

13.59174633

17.41807747

$-15.06029224$

11.15819359

$-9.88611412$

$-12.67098999$

10.81250572

$-2.01826549$

1. 58905709

3. 30681348

$-2.38093853$

1. 41546679

$-1.18934679$

$-3.46178198$

2.52699494

$-0.11999685$

$-1.11170387$

$-0.12118642$

1. 00005400

0.21473418

0.72806948

0.07854212

$-0.75749171$

$$
\begin{gathered}
1.000 \text { \# Electr. mom., scale-fac } \\
\text { \# Magnetic mom. }
\end{gathered}
$$

$-0.34768584$

$-0.24185041$

$-0.34768584$

$-0.24185041$

$-0.34768584$

$-0.24185041$

$-0.34768584$

$-0.24185041$

0.17077343

0.27660885

0.17077343

0.27660885

0.17077343

0.27660885

0.17077343

0.27660885

$-1.49057686$

$-1.38474143$

$-1.49057686$

$-1.38474143$

$-1.49057686$

$-1.38474143$

$-1.49057686$

$-1.38474143$

0.66284108

0.76867646

0.66284108

0.76867646

0.66284108

0.76867646

0.66284108

0.76867646
$-5.89719391$

4.58129025

4. 20013952

$-2.01539660$

3.79051614

$-1.59329426$

$-1.51993620$

$-1.87389302$

$-20.68127632$

29.80569649

19.49328804

$-29.72284317$

21.60154152

$-32.89077377$

$-19.47872543$

31.49280357

2.94893050

$-3.52278042$

$-3.73752141$

4.51301575

$-3.61495233$

4.28471851

4.26134586

$-5.08675814$

2. 71886897

$-0.91480386$

$-2.94801235$

0.40693000

$-4.71710825$

1. 45075095

6.90978146

$-2.25397635$ 


-1.32129574
-1.32129574
-1.32129574
-1.32129574
-1.21546030
-1.21546030
-1.21546030
-1.21546030
2.26337528
2.26337528
2.26337528
2.26337528
2.36921072
2.36921072
2.36921072
2.36921072
-1.48753464
-1.48753464
-1.48753464
-1.48753464
-1.38169920
-1.38169920
-1.38169920
-1.38169920
2.46758628
2.46758628
2.46758628
2.46758628
2.57342172
2.57342172
2.57342172
2.57342172

64
1. 01172543

1.01172543

1. 11756098

1.11756098

1.01172543

1.01172543

1. 11756098

1.11756098

0.33846018

0.33846018

0.44429561

0.44429561

0.33846018

0.33846018

0.44429561

0.44429561

0.95463514

0.95463514

1.06047070

1.06047070

0.95463514

0.95463514

1.06047070

1.06047070

1.14465213

1.14465213

1. 25048769

1. 25048769

1.14465213

1. 14465213

1. 25048769

1. 25048769

\# Energy 1->5

$\begin{array}{rrr}50881 . & \text { \# Energy } & 1->5 \\ 0.2062 & 1.7057 & 2.9462 \\ -0.0957 & -1.1906 & 0.8536\end{array}$

$-1.93576097$

$-1.93576097$

$-1.93576097$

$-1.93576097$

$-1.82992566$

$-1.82992566$

$-1.82992566$

$-1.82992566$

0.96153939

0.96153939

0.96153939

0.96153939

1.06737483

1. 06737483

1.06737483

1.06737483

$-1.56185186$

$-1.56185186$

$-1.56185186$

$-1.56185186$

$-1.45601630$

$-1.45601630$

$-1.45601630$

$-1.45601630$

0.57276028

0.57276028

0.57276028

0.57276028

0.67859572

0.67859572

0.67859572

0.67859572
0.01240921

0.01240921

0.11824466

0.11824466

0.01240921

0.01240921

0.11824466

0.11824466

0.11078113

0.11078113

0.21661659

0.21661659

0.11078113

0.11078113

0.21661659

0.21661659

$-0.25733095$

$-0.25733095$

$-0.15149552$

$-0.15149552$

$-0.25733095$

$-0.25733095$

$-0.15149552$

$-0.15149552$

$-0.95197451$

$-0.95197451$

$-0.84613907$

$-0.84613907$

$-0.95197451$

$-0.95197451$

$-0.84613907$

$-0.84613907$
0.39045236

0.49628779

0.39045236

0.49628779

0.39045236

0.49628779

0.39045236

0.49628779

$-0.16102493$

$-0.05518948$

$-0.16102493$

$-0.05518948$

$-0.16102493$

$-0.05518948$

$-0.16102493$

$-0.05518948$

1. 39116621

1. 49700165

1.39116621

1. 49700165

1.39116621

1.49700165

1. 39116621

1. 49700165

$-0.74076766$

$-0.63493228$

$-0.74076766$

$-0.63493228$

$-0.74076766$

$-0.63493228$

$-0.74076766$

$-0.63493228$
5.17242813

$-2.78348207$

$-5.54524851$

3.62386417

$-0.48025686$

$-0.73518419$

2. 07063293

$-1.03249514$

18.27050781

$-11.55375862$

$-22.25783157$

15.67896366

$-28.24059677$

20.84459114

33.49391937

$-26.31171227$

1. 51630437

$-1.22030342$

$-1.87239218$

1. 43648720

$-0.80749255$

0.69378203

1.23288369

$-0.96721184$

$-1.52040637$

1. 91745341

1.03143239

$-1.33107126$

0.47898504

$-1.42589247$

$-0.07685765$

0.70958364

$$
\begin{gathered}
1.000 \text { \# Electr. mom., scale-fac } \\
\text { \# Magnetic mom. }
\end{gathered}
$$

$-0.34768584$

$-0.24185041$

$-0.34768584$

$-0.24185041$

$-0.34768584$

$-0.24185041$

$-0.34768584$

$-0.24185041$

0.17077343

0.27660885

0.17077343

0.27660885

0.17077343

0.27660885

0.17077343

0.27660885

$-1.49057686$

$-1.38474143$

$-1.49057686$

$-1.38474143$

$-1.49057686$

$-1.38474143$

$-1.49057686$

$-1.38474143$

0.66284108

0.76867646

0.66284108

0.76867646

0.66284108

0.76867646

0.66284108

0.76867646
8.03673840

$-8.55535316$

$-7.59406567$

9. 57708359

$-16.22356415$

16.62073135

15.46770668

$-17.50369644$

2.35503602

$-1.40582418$

$-2.74823189$

1.70086110

$-2.27076054$

0.69150996

2. 73018074

$-1.11821306$

$-6.67161417$

8.88287735

6.65748692

$-8.93059731$

6.74312449

$-8.83186531$

$-6.65320396$

8.75321770

0.23582087

$-0.09401989$

$-0.12149802$

$-0.10522106$

$-0.81082082$

0.38785714

1. 05815148

$-0.44598985$ 


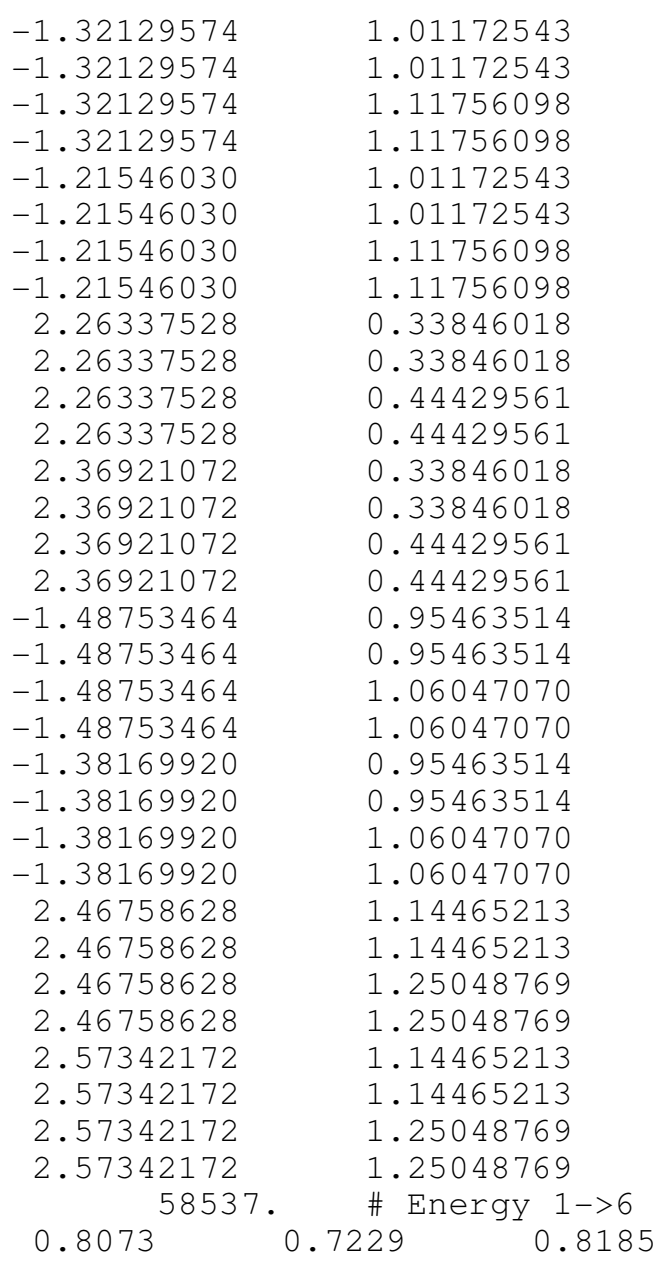

64

$\begin{array}{rr}0.39045236 & -16.78533554 \\ 0.49628779 & 11.21401882 \\ 0.39045236 & 13.57813549 \\ 0.49628779 & -8.40433407 \\ 0.39045236 & 6.92924070 \\ 0.49628779 & -2.32516265 \\ 0.39045236 & -4.10226250 \\ 0.49628779 & 0.14006190 \\ -0.16102493 & 2.22318673 \\ -0.05518948 & -1.32479453 \\ -0.16102493 & -2.85179663 \\ -0.05518948 & 1.89902711 \\ -0.16102493 & -2.28241897 \\ -0.05518948 & 1.66487360 \\ -0.16102493 & 2.80611157 \\ -0.05518948 & -2.18586206 \\ 1.39116621 & 1.56891453 \\ 1.49700165 & -1.11007810 \\ 1.39116621 & -1.32550442 \\ 1.49700165 & 0.90258098 \\ 1.39116621 & -2.30919170 \\ 1.49700165 & 1.61579037 \\ 1.39116621 & 2.01024127 \\ 1.49700165 & -1.38774681 \\ -0.74076766 & -0.06650120 \\ -0.63493228 & 0.37768346 \\ -0.74076766 & -0.12141636 \\ -0.63493228 & -0.06166169 \\ -0.74076766 & -0.19060458 \\ -0.63493228 & -0.00076748 \\ -0.74076766 & 0.27341151 \\ -0.63493228 & -0.18168367\end{array}$

$$
\begin{gathered}
1.000 \text { \# Electr. mom., scale-fac } \\
\text { \# Magnetic mom. }
\end{gathered}
$$

$-0.34768584$

$-0.24185041$

$-0.34768584$

$-0.24185041$

$-0.34768584$

$-0.24185041$

$-0.34768584$

$-0.24185041$

0.17077343

0.27660885

0.17077343

0.27660885

0.17077343

0.27660885

0.17077343

0.27660885

$-1.49057686$

$-1.38474143$

$-1.49057686$

$-1.38474143$

$-1.49057686$

$-1.38474143$

$-1.49057686$

$-1.38474143$

0.66284108

0.76867646

0.66284108

0.76867646

0.66284108

0.76867646

0.66284108

0.76867646
7.40985060

$-7.80047655$

7.46939754

$-11.98037052$

12.29427338

10.77889538

$-11.87453651$

$-13.21070099$

12.50857639

17.83564377

$-15.72912025$

12.46778107

$-10.94878864$

$-17.18016434$

14.20316696

$-4.83845901$

6.24110365

5. 13772106

$-6.66922760$

4.68864679

$-6.01140738$

$-5.01231718$

6.46939087

1.03257596

$-0.42843717$

$-2.24352360$

1. 44088399

$-0.81041628$

0.36321807

1.82266295

$-1.23486662$
$-6.41367197$ 


-1.32129574
-1.32129574
-1.32129574
-1.32129574
-1.21546030
-1.21546030
-1.21546030
-1.21546030
2.26337528
2.26337528
2.26337528
2.26337528
2.36921072
2.36921072
2.36921072
2.36921072
-1.48753464
-1.48753464
-1.48753464
-1.48753464
-1.38169920
-1.38169920
-1.38169920
-1.38169920
2.46758628
2.46758628
2.46758628
2.46758628
2.57342172
2.57342172
2.57342172
2.57342172
-164041.
-0.1760

1. 01172543

1. 01172543

1. 11756098

1. 11756098

1.01172543

1.01172543

1. 11756098

1. 11756098

0.33846018

0.33846018

0.44429561

0.44429561

0.33846018

0.33846018

0.44429561

0.44429561

0.95463514

0.95463514

1.06047070

1.06047070

0.95463514

0.95463514

1.06047070

1.06047070

1. 14465213

1.14465213

1. 25048769

1. 25048769

1. 14465213

1.14465213

1. 25048769

1. 25048769

64
$-0.1760$.

\# Energy 1->7

$$
\begin{array}{rr}
0.39045236 & 3.22670388 \\
0.49628779 & -5.65124607 \\
0.39045236 & -3.63990569 \\
0.49628779 & 6.00745630 \\
0.39045236 & -7.44980240 \\
0.49628779 & 9.61399555 \\
0.39045236 & 7.47278929 \\
0.49628779 & -9.48066616 \\
-0.16102493 & -6.30526114 \\
-0.05518948 & 3.70174074 \\
-0.16102493 & 8.36248302 \\
-0.05518948 & -5.22294760 \\
-0.16102493 & 6.66195869 \\
-0.05518948 & -4.17492056 \\
-0.16102493 & -8.68355942 \\
-0.05518948 & 5.79337025 \\
1.39116621 & 3.33963799 \\
1.49700165 & -2.39465976 \\
1.39116621 & -3.19162393 \\
1.49700165 & 2.28817105 \\
1.39116621 & -3.76006269 \\
1.49700165 & 2.68669534 \\
1.39116621 & 3.57542944 \\
1.49700165 & -2.56320238 \\
-0.74076766 & -0.26441249 \\
-0.63493228 & -0.18235439 \\
-0.74076766 & 0.49310967 \\
-0.63493228 & -0.20879009 \\
-0.74076766 & 0.11032938 \\
-0.63493228 & 0.38046443 \\
-0.74076766 & -0.34035736 \\
-0.63493228 & 0.02213237 \\
-0.035
\end{array}
$$

$\begin{array}{rr}-0.0068 & 0.2985 \\ 0.0351 & -0.0174\end{array}$

$-1.93576097$

$-1.93576097$

$-1.93576097$

$-1.93576097$

$-1.82992566$

$-1.82992566$

$-1.82992566$

$-1.82992566$

0.96153939

0.96153939

0.96153939

0.96153939

1.06737483

1.06737483

1.06737483

1.06737483

$-1.56185186$

$-1.56185186$

$-1.56185186$

$-1.56185186$

$-1.45601630$

$-1.45601630$

$-1.45601630$

$-1.45601630$

0.57276028

0.57276028

0.57276028

0.57276028

0.67859572

0.67859572

0.67859572

0.67859572
0.01240921

0.01240921

0.11824466

0.11824466

0.01240921

0.01240921

0.11824466

0.11824466

0.11078113

0.21661659

0.21661659

0.11078113

0.11078113

0.21661659

0.21661659

$-0.25733095$

$-0.25733095$

$-0.15149552$

$-0.15149552$

$-0.25733095$

$-0.25733095$

$-0.15149552$

$-0.15149552$

$-0.95197451$

$-0.95197451$

$-0.84613907$

$-0.84613907$

$-0.95197451$

$-0.95197451$

$-0.84613907$

$-0.84613907$
0.11078113

9.32908058
1.000 \# Electr. mom., scale-fac \# Magnetic mom.

$-0.3476858$

$-0.24185041$

$-0.34768584$

$-0.24185041$

$-0.34768584$

$-0.24185041$

$-0.34768584$

$-0.24185041$

0.17077343

0.27660885

0.17077343

0.27660885

0.17077343

0.27660885

0.17077343

0.27660885

$-1.49057686$

$-1.38474143$

$-1.49057686$

$-1.38474143$

$-1.49057686$

$-1.38474143$

$-1.49057686$

$-1.38474143$

0.66284108

0.76867646

0.66284108

0.76867646

0.66284108

0.76867646

0.66284108

0.76867646
$-2.66131377$

2. 25391507

2. 57935619

$-2.34171724$

3. 62348008

$-3.07243824$

$-3.33255363$

2.99201393

7.68366575

$-9.30010986$

$-8.42665577$

$-6.14196825$

6.90283394

6.97736835

$-7.04867744$

1.12509799

$-1.32382178$

$-0.83562827$

0.93664050

$-0.94081807$

1.08681953

0.67090374

$-0.73816609$

1. 20989633

$-1.32610416$

$-0.61539197$

0.81134635

$-1.15180182$

1.06622028

0.74934286

$-0.65972179$ 


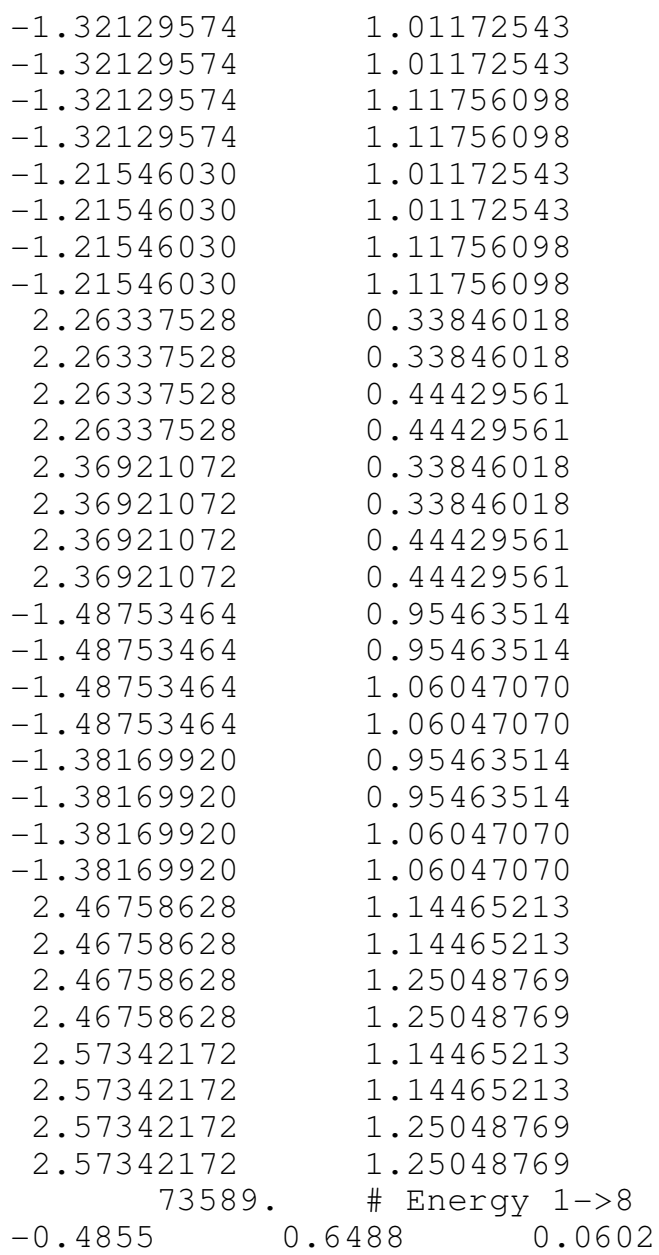

$\begin{array}{rr}0.39045236 & -8.65242386 \\ 0.49628779 & 10.10925579 \\ 0.39045236 & 8.48108768 \\ 0.49628779 & -9.81317806 \\ 0.39045236 & 7.16420174 \\ 0.49628779 & -8.83920574 \\ 0.39045236 & -7.15723419 \\ 0.49628779 & 8.71068192 \\ -0.16102493 & 4.47125435 \\ -0.05518948 & -3.17808151 \\ -0.16102493 & -5.44311905 \\ -0.05518948 & 3.87469578 \\ -0.16102493 & -3.73425126 \\ -0.05518948 & 2.68608093 \\ -0.16102493 & 4.57927275 \\ -0.05518948 & -3.35477042 \\ 1.39116621 & -3.25228500 \\ 1.49700165 & 2.37327957 \\ 1.39116621 & 3.13072634 \\ 1.49700165 & -2.26465774 \\ 1.39116621 & 3.22579002 \\ 1.49700165 & -2.37166953 \\ 1.39116621 & -3.15366054 \\ 1.49700165 & 2.29283452 \\ -0.74076766 & 0.53315848 \\ -0.63493228 & -0.14954285 \\ -0.74076766 & -0.77066612 \\ -0.63493228 & 0.55433768 \\ -0.74076766 & -0.51488692 \\ -0.63493228 & 0.23953117 \\ -0.74076766 & 0.66975343 \\ -0.63493228 & -0.52740163\end{array}$

0.01240921

0.01240921

0.11824466

0.11824466

0.01240921

0.01240921

0.11824466

0.11824466

0.11078113

0.11078113

0.21661659

0.21661659

0.11078113

0.11078113

0.21661659

0.21661659

$-0.25733095$

$-0.25733095$

$-0.15149552$

$-0.15149552$

$-0.25733095$

$-0.25733095$

$-0.15149552$

$-0.15149552$

$-0.95197451$

$-0.95197451$

$-0.84613907$

$-0.84613907$

$-0.95197451$

$-0.95197451$

$-0.84613907$

$-0.84613907$

$$
\begin{array}{r}
0.39045236 \\
0.49628779 \\
0.39045236 \\
0.49628779 \\
0.39045236 \\
0.49628779 \\
0.39045236 \\
0.49628779 \\
-0.16102493 \\
-0.05518948 \\
-0.16102493 \\
-0.05518948 \\
-0.16102493 \\
-0.05518948 \\
-0.16102493 \\
-0.05518948 \\
1.39116621 \\
1.49700165 \\
1.39116621 \\
1.49700165 \\
1.39116621 \\
1.49700165 \\
1.39116621 \\
1.49700165 \\
-0.74076766 \\
-0.63493228 \\
-0.74076766 \\
-0.63493228 \\
-0.74076766 \\
-0.63493228 \\
-0.74076766 \\
-0.63493228
\end{array}
$$

$$
\begin{gathered}
1.000 \text { \# Electr. mom., scale-fac } \\
\text { \# Magnetic mom. }
\end{gathered}
$$

$-0.34768584$

$-0.24185041$

$-0.34768584$

$-0.24185041$

$-0.34768584$

$-0.24185041$

$-0.34768584$

$-0.24185041$

0.17077343

0.27660885

0.17077343

0.27660885

0.17077343

0.27660885

0.17077343

0.27660885

$-1.49057686$

$-1.38474143$

$-1.49057686$

$-1.38474143$

$-1.49057686$

$-1.38474143$

$-1.49057686$

$-1.38474143$

0.66284108

0.76867646

0.66284108

0.76867646

0.66284108

0.76867646

0.66284108

0.76867646
$-3.96008730$

4.50228834

5.17345095

$-5.84609604$

5.13583755

$-5.80288601$

$-6.77429533$

7. 64816189

14.99334908

$-20.89521790$

$-14.67064762$

19.92471123

$-15.38142872$

22.32530212

15.39773273

$-21.06318092$

$-1.05046916$

1. 30857158

$-1.72045267$

1. 65418684

$-2.04736876$

$-1.92698860$

2. 40614486

$-2.13409615$

$-0.08468968$

3. 62162399

$-0.55677265$

3. 73871422

$-6.40052414$

2. 01938677
1.36006379

$-0.55708271$ 


-1.32129574
-1.32129574
-1.32129574
-1.32129574
-1.21546030
-1.21546030
-1.21546030
-1.21546030
2.26337528
2.26337528
2.26337528
2.26337528
2.36921072
2.36921072
2.36921072
2.36921072
-1.48753464
-1.48753464
-1.48753464
-1.48753464
-1.38169920
-1.38169920
-1.38169920
-1.38169920
2.46758628
2.46758628
2.46758628
2.46758628
2.57342172
2.57342172
2.57342172
2.57342172
-1

64
1. 01172543
1. 01172543
1. 11756098
1.11756098
1.01172543
1.01172543
1. 11756098
1.11756098
0.33846018
0.33846018
0.44429561
0.44429561
0.33846018
0.33846018
0.44429561
0.44429561
0.95463514
0.95463514
1. 06047070
1.06047070
0.95463514
0.95463514
1.06047070
1.06047070
1. 14465213
1.14465213
1. 25048769
1. 25048769
1.14465213
1. 14465213
1. 25048769
1. 25048769
\# Energy 1->9

$\begin{array}{lll}-0.0091 & -1.0156 & 0.8063 \\ -0.1533 & -0.2304 & 0.0772\end{array}$

$-1.93576097$

$-1.93576097$

$-1.93576097$

$-1.93576097$

$-1.82992566$

$-1.82992566$

$-1.82992566$

$-1.82992566$

0.96153939

0.96153939

0.96153939

0.96153939

1.06737483

1. 06737483

1.06737483

1.06737483

$-1.56185186$

$-1.56185186$

$-1.56185186$

$-1.56185186$

$-1.45601630$

$-1.45601630$

$-1.45601630$

$-1.45601630$

0.57276028

0.57276028

0.57276028

0.57276028

0.67859572

0.67859572

0.67859572

0.67859572
0.01240921

0.01240921

0.11824466

0.11824466

0.01240921

0.01240921

0.11824466

0.11824466

0.11078113

0.11078113

0.21661659

0.21661659

0.11078113

0.11078113

0.21661659

$-0.25733095$

$-0.25733095$

$-0.15149552$

$-0.15149552$

$-0.25733095$

$-0.25733095$

$-0.15149552$

$-0.15149552$

$-0.95197451$

$-0.95197451$

$-0.84613907$

$-0.84613907$

$-0.95197451$

$-0.95197451$

$-0.84613907$

$-0.84613907$
0.21661659
0.39045236

0.49628779

0.39045236

0.49628779

0.39045236

0.49628779

0.39045236

0.49628779

$-0.16102493$

$-0.05518948$

$-0.16102493$

$-0.05518948$

$-0.16102493$

$-0.05518948$

$-0.16102493$

$-0.05518948$

1. 39116621

1. 49700165

1. 39116621

1.49700165

1.39116621

1.49700165

1. 39116621

1. 49700165

$-0.74076766$

$-0.63493228$

$-0.74076766$

$-0.63493228$

$-0.74076766$

$-0.63493228$

$-0.74076766$

$-0.63493228$
9.03000164

$-6.83057070$

$-5.79222441$

3.82598591

$-8.34230137$

5.85034513

5.00021505

$-2.85804486$

16.14501762

$-15.86676502$

$-17.57800865$

16.57462120

$-17.35004807$

15.86134434

19.56087494

$-17.40064049$

$-1.92499793$

1. 44004464

2.14538932

$-1.56605089$

1. 94834459

$-1.43470573$

$-2.17983198$

1. 57906365

0.02725119

$-0.28069034$

0.18621819

$-0.05727066$

0.53476417

$-0.86005849$

$-0.45698312$

0.73246843

$$
\begin{gathered}
1.000 \text { \# Electr. mom., scale-fac } \\
\text { \# Magnetic mom. }
\end{gathered}
$$

$-0.34768584$

$-0.24185041$

$-0.34768584$

$-0.24185041$

$-0.34768584$

$-0.24185041$

$-0.34768584$

$-0.24185041$

0.17077343

0.27660885

0.17077343

0.27660885

0.17077343

0.27660885

0.17077343

0.27660885

$-1.49057686$

$-1.38474143$

$-1.49057686$

$-1.38474143$

$-1.49057686$

$-1.38474143$

$-1.49057686$

$-1.38474143$

0.66284108

0.76867646

0.66284108

0.76867646

0.66284108

0.76867646

0.66284108

0.76867646
$-10.97903347$

11.65416718

12.00457191

$-11.42684937$

7.56443024

$-7.85532570$

$-9.20110035$

8.04121399

3. 04942942

$-4.45129442$

$-0.69070566$

$-1.60294652$

$-5.21769619$

2. 88926768

3. 46120715

$-0.84204727$

1.32481909

0.99740446

$-1.50991249$

0.58088231

$-1.00699341$

$-0.81395137$

1.29819000

$-0.07618086$

1.06169176

0.02940632

$-1.49367368$

$-1.57749844$

$-0.46166551$

2.93845296

$-0.01651461$
2.03756952 


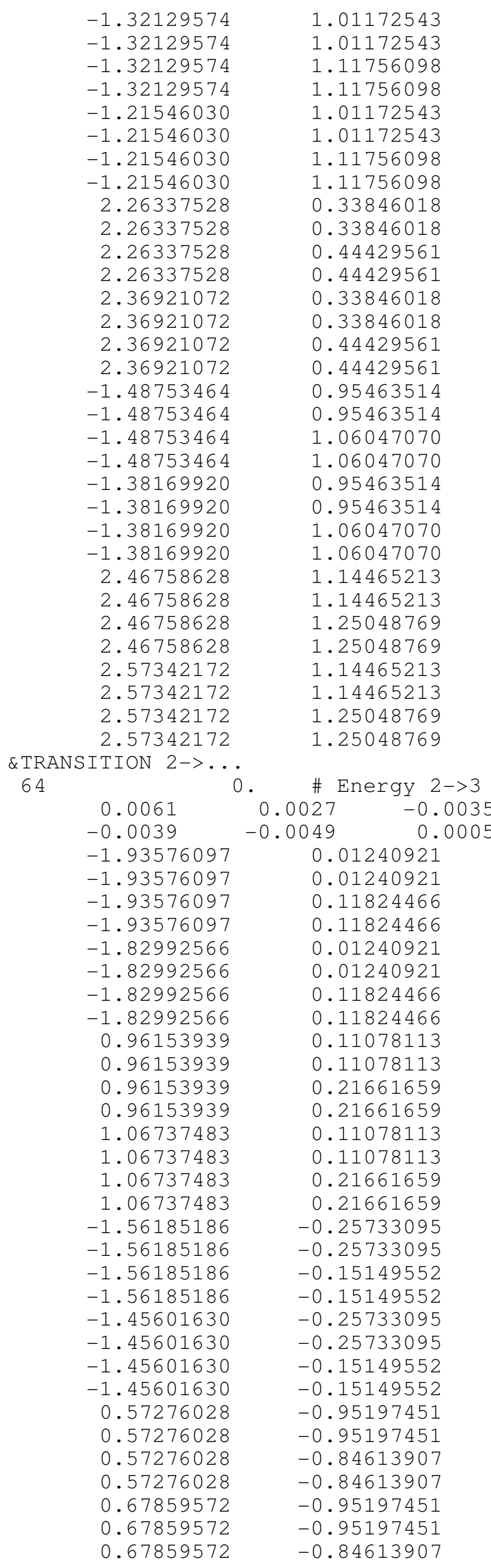

$-1.32129574$

$-1.32129574$

$-1.21546030$

26337528

2.36921072

2.36921072

$-1.48753464$

$-1.48753464$

$-1.38169920$

$-1.38169920$

1.381699

2. 46758628

2.57342172

ITION 2->..

0

\subsection{1}

$-1.93576097$

$-1.93576097$

$-1.82992566$

82992566

0.96153939

0.96153939

1.06737483

1.06737483

1.06737483

56185186

$-1.56185186$

$-1.45601630$

$-1.45601630$

0.57276028

0.67859572
0.39045236

0.49628779

0.39045236

0.49628779

0.39045236

0.49628779

0.39045236

0.49628779

$-0.16102493$

$-0.05518948$

$-0.16102493$

$-0.05518948$

$-0.16102493$

$-0.05518948$

$-0.16102493$

$-0.05518948$

1.39116621

1. 49700165

1. 39116621

1.49700165

1.39116621

1.49700165

1.39116621

1. 49700165

$-0.74076766$

$-0.63493228$

$-0.74076766$

$-0.63493228$

$-0.74076766$

$-0.63493228$

$-0.74076766$

$-0.63493228$
8.46468163

$-4.34946012$

$-5.70758057$

2. 22979355

$-1.70482445$

$-1.29085207$

0.02924649

2. 47938323

$-9.46743870$

11.30633450

9.31354618

$-10.81956959$

11.48446560

$-11.82359791$

$-12.25536633$

12.19823933

$-2.39243484$

1. 65129375

2.06227088

$-1.39772940$

2. 87893891

$-1.99743438$

$-2.58192658$

1. 77934480

0.48389322

0.19022389

$-0.91119999$

0.54412675

$-0.97933012$

0.95397258

1.06838393

$-1.14870751$

$$
\begin{array}{r}
\text { \# Energy } 2->3 \\
0.0027 \quad-0.0035
\end{array}
$$

0.01240921

0.01240921

0.01240921

0.11824466

0.11078113

0.21661659

0.21661659

0.21661659

$-0.25733095$

$-0.25733095$

$-0.25733095$

$-0.15149552$

$-0.95197451$

$-0.84613907$

$-0.84613907$

$-0.95197451$

$-0.84613907$
1.000 \# Electr. mom., scale-fac \# Magnetic mom.

$-0.34768584$

$-0.24185041$

$-0.34768584$

$-0.24185041$

$-0.34768584$

$-0.24185041$

$-0.34768584$

$-0.24185041$

0.17077343

0.27660885

0.17077343

0.27660885

0.17077343

0.27660885

0.17077343

0.27660885

$-1.49057686$

$-1.38474143$

$-1.49057686$

$-1.38474143$

$-1.49057686$

$-1.38474143$

$-1.49057686$

$-1.38474143$

0.66284108

0.76867646

0.66284108

0.76867646

0.66284108

0.76867646

0.66284108
0.03903218

$-0.01440687$

$-0.02160442$

$-0.00183887$

$-0.05139133$

0.02373834

0.03370138

$-0.00749163$

$-0.16294815$

0.30647072

0.21733275

$-0.34972629$

0.24640343

$-0.38671032$

$-0.29704165$

0.42607394

$-0.08062809$

0.10186550

0.08471105

$-0.10800758$

0.07929893

$-0.09957720$

$-0.08498581$

0.10771003

0.03768811

0.01156948

$-0.09767355$

0.03703416

$-0.00773249$

$-0.04191308$

0.06004369 


0.67859572
-1.32129574
-1.32129574
-1.32129574
-1.32129574
-1.21546030
-1.21546030
-1.21546030
-1.21546030
2.26337528
2.26337528
2.26337528
2.26337528
2.36921072
2.36921072
2.36921072
2.36921072
-1.48753464
-1.48753464
-1.48753464
-1.48753464
-1.38169920
-1.38169920
-1.38169920
-1.38169920
2.46758628
2.46758628
2.46758628
2.46758628
2.57342172
2.57342172
2.57342172
2.57342172

64
$-0.84613907$

1.01172543

1. 01172543

1.11756098

1. 11756098

1.01172543

1.01172543

1. 11756098

1. 11756098

0.33846018

0.33846018

0.44429561

0.44429561

0.33846018

0.33846018

0.44429561

0.44429561

0.95463514

0.95463514

1.06047070

1.06047070

0.95463514

0.95463514

1.06047070

1.06047070

1.14465213

1.14465213

1. 25048769

1. 25048769

1. 14465213

1. 14465213

1. 25048769

1. 25048769

\begin{tabular}{|c|c|}
\hline & \# Energy 2->4 \\
\hline-0.0063 & -0.0173 \\
\hline-0.0181 & 0.0321 \\
\hline-1.93576097 & 0.01240921 \\
\hline-1.93576097 & 0.01240921 \\
\hline-1.93576097 & 0.11824466 \\
\hline-1.93576097 & 0.11824466 \\
\hline-1.82992566 & 0.01240921 \\
\hline-1.82992566 & 0.01240921 \\
\hline-1.82992566 & 0.11824466 \\
\hline-1.82992566 & 0.11824466 \\
\hline 0.96153939 & 0.11078113 \\
\hline 0.96153939 & 0.11078113 \\
\hline 0.96153939 & 0.21661659 \\
\hline 0.96153939 & 0.21661659 \\
\hline 1.06737483 & 0.11078113 \\
\hline 1.06737483 & 0.11078113 \\
\hline 1.06737483 & 0.21661659 \\
\hline 1.06737483 & 0.21661659 \\
\hline-1.56185186 & -0.25733095 \\
\hline-1.56185186 & -0.25733095 \\
\hline-1.56185186 & -0.15149552 \\
\hline-1.56185186 & -0.15149552 \\
\hline-1.45601630 & -0.25733095 \\
\hline-1.45601630 & -0.25733095 \\
\hline-1.45601630 & -0.15149552 \\
\hline-1.45601630 & -0.15149552 \\
\hline 0.57276028 & -0.95197451 \\
\hline 0.57276028 & -0.95197451 \\
\hline 0.57276028 & -0.84613907 \\
\hline 0.57276028 & -0.84613907 \\
\hline 0.67859572 & -0.95197451 \\
\hline 0.67859572 & -0.95197451 \\
\hline 0.67859572 & -0.84613907 \\
\hline
\end{tabular}

0.76867646

0.39045236

0.49628779

0.39045236

0.49628779

0.39045236

0.49628779

0.39045236

0.49628779

$-0.16102493$

$-0.05518948$

$-0.16102493$

$-0.05518948$

$-0.16102493$

$-0.05518948$

$-0.16102493$

$-0.05518948$

1. 39116621

1. 49700165

1.39116621

1.49700165

1. 39116621

1. 49700165

1.39116621

1. 49700165

$-0.74076766$

$-0.63493228$

$-0.74076766$

$-0.63493228$

$-0.74076766$

$-0.63493228$

$-0.74076766$

$-0.63493228$
0.00014737

$-0.03628272$

0.01900834

0.04741102

$-0.03602016$

$-0.00894596$

0.03369173

$-0.01147176$

$-0.00539051$

$-0.16819663$

0.15843967

0.19591215

$-0.18370184$

0.14606398

$-0.13589817$

$-0.16914621$

0.15711527

0.00146220

0.00122747

0.00527817

$-0.00482598$

$-0.01702821$

0.00955735

0.00557618

$-0.00279404$

$-0.00887099$

0.00520491

0.00278815

$-0.00010015$

0.00878173

$-0.00538533$

$-0.00345146$

0.00084806

$$
\begin{gathered}
1.000 \text { \# Electr. mom., scale-fac } \\
\text { \# Magnetic mom. }
\end{gathered}
$$

$-0.34768584 \quad-1.12850344$

$\begin{array}{ll}-0.24185041 & 1.50730586\end{array}$

$-0.34768584 \quad 1.88058555$

$-0.24185041$

$-0.34768584$

$-0.24185041$

$-2.29546094$

1.86259186

$-0.34768584$

$-0.24185041$

0.17077343

0.27660885

0.17077343

0.27660885

0.17077343

0.27660885

0.17077343

0.27660885

$-1.49057686$

$-1.38474143$

$-1.49057686$

$-1.38474143$

$-1.49057686$

$-1.38474143$

$-1.49057686$

$-1.38474143$

0.66284108

0.76867646

0.66284108

0.76867646

0.66284108

0.76867646

$-2.30900288$

$-2.82858324$

3. 31309509

$-0.20675123$

0.19048926

0.13366912

$-0.05121300$

0.07465890

$-0.06900816$

$-0.01517885$

$-0.06057361$

0.66484869

$-0.86058104$

$-0.68000937$

0.89306754

$-0.47259778$

0.61090076

0.46408072

$-0.60973990$

$-0.22996385$

0.18760768

0.35143256

$-0.30473065$

0.13165475

$-0.10242141$

0.66284108

$-0.22652793$

\section{S-102}




0.67859572
-1.32129574
-1.32129574
-1.32129574
-1.32129574
-1.21546030
-1.21546030
-1.21546030
-1.21546030
2.26337528
2.26337528
2.26337528
2.26337528
2.36921072
2.36921072
2.36921072
2.36921072
-1.48753464
-1.48753464
-1.48753464
-1.48753464
-1.38169920
-1.38169920
-1.38169920
-1.38169920
2.46758628
2.46758628
2.46758628
2.46758628
2.57342172
2.57342172
2.57342172
2.57342172

64
$-0.84613907$

1.01172543

1. 01172543

1.11756098

1. 11756098

1.01172543

1.01172543

1. 11756098

1. 11756098

0.33846018

0.33846018

0.44429561

0.44429561

0.33846018

0.33846018

0.44429561

0.44429561

0.95463514

0.95463514

1.06047070

1.06047070

0.95463514

0.95463514

1.06047070

1.06047070

1.14465213

1.14465213

1. 25048769

1. 25048769

1.14465213

1. 14465213

1. 25048769

1.25048769

$-0.12850$

$-0.1009$

$-1.93576097$

$-1.93576097$

$-1.93576097$

$-1.93576097$

$-1.82992566$

$-1.82992566$

$-1.82992566$

$-1.82992566$

0.96153939

0.96153939

0.96153939

0.96153939

1. 06737483

1.06737483

1.06737483

1.06737483

$-1.56185186$

$-1.56185186$

$-1.56185186$

$-1.56185186$

$-1.45601630$

$-1.45601630$

$-1.45601630$

$-1.45601630$

0.57276028

0.57276028

0.57276028

0.57276028

0.67859572

0.67859572

0.67859572
. \# Energy 2->5

$$
-0.1565 \quad-0.2840
$$$$
0.0651 \quad 0.2367
$$

0.01240921

0.01240921

0.11824466

0.11824466

0.01240921

0.01240921

0.11824466

0.11824466

0.11078113

0.11078113

0.21661659

0.21661659

0.11078113

0.11078113

0.21661659

0.21661659

$-0.25733095$

$-0.25733095$

$-0.15149552$

$-0.15149552$

$-0.25733095$

$-0.25733095$

$-0.15149552$

$-0.15149552$

$-0.95197451$

$-0.95197451$

$-0.84613907$

$-0.84613907$

$-0.95197451$

$-0.95197451$

$-0.84613907$
0.76867646

0.39045236

0.49628779

0.39045236

0.49628779

0.39045236

0.49628779

0.39045236

0.49628779

$-0.16102493$

$-0.05518948$

$-0.16102493$

$-0.05518948$

$-0.16102493$

$-0.05518948$

$-0.16102493$

$-0.05518948$

1. 39116621

1. 49700165

1.39116621

1. 49700165

1. 39116621

1. 49700165

1.39116621

1.49700165

$-0.74076766$

$-0.63493228$

$-0.74076766$

$-0.63493228$

$-0.74076766$

$-0.63493228$

$-0.74076766$

$-0.63493228$
0.19645773

1.76249456

$-1.72492492$

$-1.37206292$

1.32272005

$-1.52066207$

1.38673043

1. 19333601

$-1.07325244$

0.23264483

$-0.08856036$

$-0.30994606$

0.18725373

$-0.28619248$

0.15285246

0.35762286

$-0.23910122$

0.07895558

$-0.05365054$

$-0.02096237$

0.00831907

$-0.06561230$

0.05069601

0.02766913

$-0.01708072$

$-0.01365260$

0.03162053

0.01244142

$-0.02502037$

0.00093887

$-0.02051042$

$-0.00009454$

0.01339202
1.000 \# Electr. mom., scale-fac \# Magnetic mom.

$-0.34768584$

$-0.24185041$

$-0.34768584$

$-0.24185041$

$-0.34768584$

$-0.24185041$

$-0.34768584$

$-0.24185041$

0.17077343

0.27660885

0.17077343

0.27660885

0.17077343

0.27660885

0.17077343

0.27660885

$-1.49057686$

$-1.38474143$

$-1.49057686$

$-1.38474143$

$-1.49057686$

$-1.38474143$

$-1.49057686$

$-1.38474143$

0.66284108

0.76867646

0.66284108

0.76867646

0.66284108

0.76867646

0.66284108
11.61509228

$-17.32958031$

$-20.30613327$

26.74536324

$-20.31951904$

27.34909630

31.76284790

$-39.61808014$

1.47163475

$-1.48901272$

$-1.78611171$

1. 11853421

$-0.46593741$

0.68062955

0.83474112

$-0.33557227$

$-6.78930092$

8.95085812

6.93921041

$-9.29406929$

4.65006208

$-6.18446255$

$-4.55975485$

6.17984962

3.00669861

$-2.53645563$

$-3.93686962$

3. 40732169

$-2.24069977$

1.87510502

2. 98874092 
64

0.67859572
-1.32129574
-1.32129574
-1.32129574
-1.32129574
-1.21546030
-1.21546030
-1.21546030
-1.21546030
2.26337528
2.26337528
2.26337528
2.26337528
2.36921072
2.36921072
2.36921072
2.36921072
-1.48753464
-1.48753464
-1.48753464
-1.48753464
-1.38169920
-1.38169920
-1.38169920
-1.38169920
2.46758628
2.46758628
2.46758628
2.46758628
2.57342172
2.57342172
2.57342172
2.57342172

$-0.84613907$

1.01172543

1. 01172543

1.11756098

1. 11756098

1.01172543

1.01172543

1. 11756098

1.11756098

0.33846018

0.33846018

0.44429561

0.44429561

0.33846018

0.33846018

0.44429561

0.44429561

0.95463514

0.95463514

1.06047070

1.06047070

0.95463514

0.95463514

1.06047070

1.06047070

1.14465213

1.14465213

1. 25048769

1. 25048769

1. 14465213

1. 14465213

1. 25048769

1. 25048769

$\begin{array}{rcc} & 0 . & \# \text { Energy } 2->6 \\ -0.1348 & -0.0426 & -0.1515 \\ 0.0433 & 0.0941 & 0.0695 \\ -1.93576097 & 0.01240921 \\ -1.93576097 & 0.01240921 \\ -1.93576097 & 0.11824466 \\ -1.93576097 & 0.11824466 \\ -1.82992566 & 0.01240921 \\ -1.82992566 & 0.01240921 \\ -1.82992566 & 0.11824466 \\ -1.82992566 & 0.11824466 \\ 0.96153939 & 0.11078113 \\ 0.96153939 & 0.11078113 \\ 0.96153939 & 0.21661659 \\ 0.96153939 & 0.21661659 \\ 1.06737483 & 0.11078113 \\ 1.06737483 & 0.11078113 \\ 1.06737483 & 0.21661659 \\ 1.06737483 & 0.21661659 \\ -1.56185186 & -0.25733095 \\ -1.56185186 & -0.25733095 \\ -1.56185186 & -0.15149552 \\ -1.56185186 & -0.15149552 \\ -1.45601630 & -0.25733095 \\ -1.45601630 & -0.25733095 \\ -1.45601630 & -0.15149552 \\ -1.45601630 & -0.15149552 \\ 0.57276028 & -0.95197451 \\ 0.57276028 & -0.95197451 \\ 0.57276028 & -0.84613907 \\ 0.57276028 & -0.84613907 \\ 0.67859572 & -0.95197451 \\ 0.67859572 & -0.95197451 \\ 0.67859572 & -0.84613907 \\ & \\ -1.969\end{array}$

0.76867646

0.39045236

0.49628779

0.39045236

0.49628779

0.39045236

0.49628779

0.39045236

0.49628779

$-0.16102493$

$-0.05518948$

$-0.16102493$

$-0.05518948$

$-0.16102493$

$-0.05518948$

$-0.16102493$

$-0.05518948$

1. 39116621

1.49700165

1.39116621

1.49700165

1.39116621

1.49700165

1.39116621

1.49700165

$-0.74076766$

$-0.63493228$

$-0.74076766$

$-0.63493228$

$-0.74076766$

$-0.63493228$

$-0.74076766$

$-0.63493228$
$-2.59094048$

$-18.80316925$

19.22996521

15.19657230

$-15.29096317$

18.33972740

$-17.10846519$

$-15.00338554$

13.78291702

$-2.76793599$

1.10089350

3. 51341891

$-2.01248336$

2. 94672441

$-1.51029336$

$-3.59365153$

2. 27812290

$-0.14211701$

0.02017797

$-0.44825244$

0.41240588

0.19581611

$-0.12703736$

0.23560902

$-0.22151573$

0.16566391

$-0.42211482$

$-0.02302216$

0.16204768

0.04345313

0.16079226

$-0.13661367$

0.03342677
1.000 \# Electr. mom., scale-fac \# Magnetic mom.

$-0.34768584$

$-0.24185041$

$-0.34768584$

$-0.24185041$

$-0.34768584$

$-0.24185041$

$-0.34768584$

$-0.24185041$

0.17077343

0.27660885

0.17077343

0.27660885

0.17077343

0.27660885

0.17077343

0.27660885

$-1.49057686$

$-1.38474143$

$-1.49057686$

$-1.38474143$

$-1.49057686$

$-1.38474143$

$-1.49057686$

$-1.38474143$

0.66284108

0.76867646

0.66284108

0.76867646

0.66284108

0.76867646

0.66284108
3. 14676738

$-5.14322853$

$-6.33607388$

8.53350830

$-5.60857916$

8.04643250

9.73801136

$-12.40437984$

$-2.10260201$

1. 14688802

1.02946389

$-0.20519575$

0.92915094

0.17552622

0.11607431

$-1.07098413$

$-1.23062158$

1. 74621236

1.12346292

$-1.65506804$

0.48072970

$-0.78483200$

$-0.28530091$

0.56697649

1. 79409850

$-1.93343937$

$-1.24218500$

1. 44782019

$-2.12240696$

2. 29050207

1. 64032102 


0.67859572
-1.32129574
-1.32129574
-1.32129574
-1.32129574
-1.21546030
-1.21546030
-1.21546030
-1.21546030
2.26337528
2.26337528
2.26337528
2.26337528
2.36921072
2.36921072
2.36921072
2.36921072
-1.48753464
-1.48753464
-1.48753464
-1.48753464
-1.38169920
-1.38169920
-1.38169920
-1.38169920
2.46758628
2.46758628
2.46758628
2.46758628
2.57342172
2.57342172
2.57342172
2.57342172

64
$-0.84613907$

1.01172543

1. 01172543

1.11756098

1. 11756098

1.01172543

1.01172543

1. 11756098

1.11756098

0.33846018

0.33846018

0.44429561

0.44429561

0.33846018

0.33846018

0.44429561

0.44429561

0.95463514

0.95463514

1.06047070

1.06047070

0.95463514

0.95463514

1.06047070

1.06047070

1.14465213

1.14465213

1. 25048769

1. 25048769

1. 14465213

1. 14465213

1. 25048769

1. 25048769

0

$-0.0419$

0.0555
-1.93576097

$-1.93576097$

$-1.93576097$

$-1.93576097$

$-1.82992566$

$-1.82992566$

$-1.82992566$

$-1.82992566$

0.96153939

0.96153939

0.96153939

0.96153939

1. 06737483

1.06737483

1.06737483

1.06737483

$-1.56185186$

$-1.56185186$

$-1.56185186$

$-1.56185186$

$-1.45601630$

$-1.45601630$

$-1.45601630$

$-1.45601630$

0.57276028

0.57276028

0.57276028

0.57276028

0.67859572

0.67859572

0.67859572
0. \# Energy 2->7

$$
-0.0075 \quad-0.0409
$$$$
0.0841-0.0055
$$

0.01240921

0.01240921

0.11824466

0.11824466

0.01240921

0.01240921

0.11824466

0.11824466

0.11078113

0.11078113

0.21661659

0.21661659

0.11078113

0.11078113

0.21661659

0.21661659

$-0.25733095$

$-0.25733095$

$-0.15149552$

$-0.15149552$

$-0.25733095$

$-0.25733095$

$-0.15149552$

$-0.15149552$

$-0.95197451$

$-0.95197451$

$-0.84613907$

$-0.84613907$

$-0.95197451$

$-0.95197451$

$-0.84613907$
0.76867646

0.39045236

0.49628779

0.39045236

0.49628779

0.39045236

0.49628779

0.39045236

0.49628779

$-0.16102493$

$-0.05518948$

$-0.16102493$

$-0.05518948$

$-0.16102493$

$-0.05518948$

$-0.16102493$

$-0.05518948$

1. 39116621

1. 49700165

1.39116621

1. 49700165

1. 39116621

1. 49700165

1. 39116621

1.49700165

$-0.74076766$

$-0.63493228$

$-0.74076766$

$-0.63493228$

$-0.74076766$

$-0.63493228$

$-0.74076766$

$-0.63493228$

1.000

$-0.34768584$

$-0.24185041$

$-0.34768584$

$-0.24185041$

$-0.34768584$

$-0.24185041$

$-0.34768584$

$-0.24185041$

0.17077343

0.27660885

0.17077343

0.27660885

0.17077343

0.27660885

0.17077343

0.27660885

$-1.49057686$

$-1.38474143$

$-1.49057686$

$-1.38474143$

$-1.49057686$

$-1.38474143$

$-1.49057686$

$-1.38474143$

0.66284108

0.76867646

0.66284108

0.76867646

0.66284108

0.76867646

0.66284108
$-1.88019085$

$-4.66896296$

4. 77296257

3.16423678

$-3.06686044$

5. 32408142

$-5.04430580$

$-3.74001336$

3. 32293129

0.42134815

$-0.76660818$

$-0.27625537$

0.57246733

$-0.49764812$

0.70344782

0.37999052

$-0.53785354$

0.80769527

$-0.67565984$

$-1.12219083$

0.86943555

$-0.50551027$

0.44461241

0.88470739

$-0.69337475$

$-0.08628508$

0.02489209

0.25986579

$-0.25634032$

0.15681256

$-0.15427542$

$-0.28350034$

0.31930038

Electr. mom., scale-fac Magnetic mom.

$-0.34492821$

0.27176797

0.04115097

0.00107645

0.47879225

$-0.39990932$

$-0.12439027$

0.08106389

$-1.06990635$

0.30554849

0.34506780

0.46290934

$-0.14209583$

0.98931456

0.83834153

$-1.71342874$

0.41439265

$-0.47090244$

$-0.48086712$

0.55509245

$-0.46709815$

0.53402185

0.54202157

$-0.63017213$

0.76571417

$-1.05435121$

$-0.06268020$

0.43300053

$-1.22603309$

1.52396274

0.60513991 
0.67859572
-1.32129574
-1.32129574
-1.32129574
-1.32129574
-1.21546030
-1.21546030
-1.21546030
-1.21546030
2.26337528
2.26337528
2.26337528
2.26337528
2.36921072
2.36921072
2.36921072
2.36921072
-1.48753464
-1.48753464
-1.48753464
-1.48753464
-1.38169920
-1.38169920
-1.38169920
-1.38169920
2.46758628
2.46758628
2.46758628
2.46758628
2.57342172
2.57342172
2.57342172
2.57342172

64
$-0.84613907$

1.01172543

1. 01172543

1.11756098

1. 11756098

1.01172543

1.01172543

1. 11756098

1.11756098

0.33846018

0.33846018

0.44429561

0.44429561

0.33846018

0.33846018

0.44429561

0.44429561

0.95463514

0.95463514

1.06047070

1.06047070

0.95463514

0.95463514

1.06047070

1.06047070

1. 14465213

1.14465213

1. 25048769

1. 25048769

1. 14465213

1. 14465213

1. 25048769

1. 25048769

$-0.0745$

0.0253

0 .

\# Energy 2->8

$-1.93576097$

$-1.93576097$

$-1.93576097$

$-1.93576097$

$-1.82992566$

$-1.82992566$

$-1.82992566$

$-1.82992566$

0.96153939

0.96153939

0.96153939

0.96153939

1. 06737483

1.06737483

1.06737483

1.06737483

$-1.56185186$

$-1.56185186$

$-1.56185186$

$-1.56185186$

$-1.45601630$

$-1.45601630$

$-1.45601630$

$-1.45601630$

0.57276028

0.57276028

0.57276028

0.57276028

0.67859572

0.67859572

0.67859572

$$
\begin{array}{r}
0.76867646 \\
0.39045236 \\
0.49628779 \\
0.39045236 \\
0.49628779 \\
0.39045236 \\
0.49628779 \\
0.39045236 \\
0.49628779 \\
-0.16102493 \\
-0.05518948 \\
-0.16102493 \\
-0.05518948 \\
-0.16102493 \\
-0.05518948 \\
-0.16102493 \\
-0.05518948 \\
1.39116621 \\
1.49700165 \\
1.39116621 \\
1.49700165 \\
1.39116621 \\
1.49700165 \\
1.39116621 \\
1.49700165 \\
-0.74076766 \\
-0.63493228 \\
-0.74076766 \\
-0.63493228 \\
-0.74076766 \\
-0.63493228 \\
-0.74076766 \\
-0.63493228
\end{array}
$$

-0.98606503
1.09875655
-1.03091645
-1.39385009
1.42852247
-0.60344589
0.40862033
1.02657568
-0.97425908
1.46996236
-1.27404344
-1.61269486
1.39171028
-1.38640821
1.16920412
1.49855137
-1.25475860
0.62451094
-0.49810657
-0.76040125
0.56989902
-0.42221037
0.35824305
0.65466332
-0.49897280
-0.08069723
0.14535546
0.16896278
-0.23104157
0.07472241
-0.15193585
-0.14738595
0.21731710

1.000 \# Electr. mom., scale-fac \# Magnetic mom.

$-0.34768584$

$-0.24185041$

$-0.34768584$

$-0.24185041$

$-0.34768584$

$-0.24185041$

$-0.34768584$

$-0.24185041$

0.17077343

0.27660885

0.17077343

0.27660885

0.17077343

0.27660885

0.17077343

0.27660885

$-1.49057686$

$-1.38474143$

$-1.49057686$

$-1.38474143$

$-1.49057686$

$-1.38474143$

$-1.49057686$

$-1.38474143$

0.66284108

0.76867646

0.66284108

0.76867646

0.66284108

0.76867646

0.66284108
1. 75452721

$-2.08943272$

$-3.21393824$

3.42644954

$-2.03001213$

2. 29997277

3. 77267480

$-3.86327696$

1. 59585273

$-1.94025135$

$-2.91695905$

2.99050784

$-2.92125869$

3. 35375953

4. 23755455

$-4.35627651$

$-0.28090003$

0.36744264

0.29830855

$-0.40604126$

0.06925300

$-0.08572095$

$-0.01244234$

0.02039701

1.04731679

$-0.23625635$

0.86459053

$-1.52741337$

2. 02545238

0.74994081
$-1.52818906$ 


0.67859572
-1.32129574
-1.32129574
-1.32129574
-1.32129574
-1.21546030
-1.21546030
-1.21546030
-1.21546030
2.26337528
2.26337528
2.26337528
2.26337528
2.36921072
2.36921072
2.36921072
2.36921072
-1.48753464
-1.48753464
-1.48753464
-1.48753464
-1.38169920
-1.38169920
-1.38169920
-1.38169920
2.46758628
2.46758628
2.46758628
2.46758628
2.57342172
2.57342172
2.57342172
2.57342172

64
$-0.84613907$

1. 01172543

1. 01172543

1.11756098

1. 11756098

1.01172543

1.01172543

1. 11756098

1. 11756098

0.33846018

0.33846018

0.44429561

0.44429561

0.33846018

0.33846018

0.44429561

0.44429561

0.95463514

0.95463514

1. 06047070

1.06047070

0.95463514

0.95463514

1.06047070

1.06047070

1.14465213

1.14465213

1. 25048769

1. 25048769

1. 14465213

1. 14465213

1. 25048769

1. 25048769

0 . \# Energy

. \# Energy 2->9

$\begin{array}{lll}-0.0017 & 0.1530 & -0.0479\end{array}$

$-0.0344$

$-1.93576097$

$-1.93576097$

$-1.93576097$

$-1.93576097$

$-1.82992566$

$-1.82992566$

$-1.82992566$

$-1.82992566$

0.96153939

0.96153939

0.96153939

0.96153939

1. 06737483

1.06737483

1.06737483

1.06737483

$-1.56185186$

$-1.56185186$

$-1.56185186$

$-1.56185186$

$-1.45601630$

$-1.45601630$

$-1.45601630$

$-1.45601630$

0.57276028

0.57276028

0.57276028

0.57276028

0.67859572

0.67859572

0.67859572

$$
0.0304 \quad 0.0750
$$

0.01240921

0.01240921

0.11824466

0.11824466

0.01240921

0.01240921

0.11824466

0.11824466

0.11078113

0.11078113

0.21661659

0.21661659

0.11078113

0.11078113

0.21661659

0.21661659

$-0.25733095$

$-0.25733095$

$-0.15149552$

$-0.15149552$

$-0.25733095$

$-0.25733095$

$-0.15149552$

$-0.15149552$

$-0.95197451$

$-0.95197451$

$-0.84613907$

$-0.84613907$

$-0.95197451$

$-0.95197451$

$-0.84613907$
0.76867646

0.39045236

0.49628779

0.39045236

0.49628779

0.39045236

0.49628779

0.39045236

0.49628779

$-0.16102493$

$-0.05518948$

$-0.16102493$

$-0.05518948$

$-0.16102493$

$-0.05518948$

$-0.16102493$

$-0.05518948$

1. 39116621

1. 49700165

1.39116621

1. 49700165

1. 39116621

1. 49700165

1.39116621

1.49700165

$-0.74076766$

$-0.63493228$

$-0.74076766$

$-0.63493228$

$-0.74076766$

$-0.63493228$

$-0.74076766$

$-0.63493228$
1.000 \# Electr. mom., scale-fac \# Magnetic mom.

$-0.34768584$

$-0.24185041$

$-0.34768584$

$-0.24185041$

$-0.34768584$

$-0.24185041$

$-0.34768584$

$-0.24185041$

0.17077343

0.27660885

0.17077343

0.27660885

0.17077343

0.27660885

0.17077343

0.27660885

$-1.49057686$

$-1.38474143$

$-1.49057686$

$-1.38474143$

$-1.49057686$

$-1.38474143$

$-1.49057686$

$-1.38474143$

0.66284108

0.76867646

0.66284108

0.76867646

0.66284108

0.76867646

0.66284108
-1.40423346
-4.63842344
4.20622206
3.08510470
-2.62463665
3.83942461
-3.64748406
-2.33545280
2.06102943
2.80167890
-2.38261652
-3.29841018
2.68779612
-2.22066426
1.87779331
2.59948969
-2.11176181
-0.01082786
-0.03643055
-0.32489341
0.25596410
0.10245111
-0.03296211
0.33432367
-0.26408806
-0.00512545
0.25689825
0.01779633
-0.19475751
-0.01323496
-0.18549527
-0.01451346
0.15440813

5.76543188

$-6.88466930$

$-8.91093636$

10.04207230

$-7.57432842$

8.68764877

11.39991474

$-12.46564674$

4.04630423

$-4.19425297$

$-4.33608389$

4.23255634

$-4.45314884$

4.70650387

4.75563908

$-4.71625376$

$-1.44278121$

1.79460561

1.51710463

$-1.92060125$

0.77058494

$-0.90554804$

$-0.69792408$

0.83071637

0.96662354

$-1.18785238$

$-0.77164418$

1.07916403

$-0.98046368$

1.28361523

0.68929070 


\begin{abstract}
0.67859572
$-1.32129574$

$-1.32129574$

$-1.32129574$

$-1.32129574$

$-1.21546030$

$-1.21546030$

$-1.21546030$

$-1.21546030$

2. 26337528

2. 26337528

2. 26337528

2. 26337528

2. 36921072

2.36921072

2.36921072

2. 36921072

$-1.48753464$

$-1.48753464$

$-1.48753464$

$-1.48753464$

$-1.38169920$

$-1.38169920$

$-1.38169920$

$-1.38169920$

2. 46758628

2.46758628

2. 46758628

2. 46758628

2.57342172

2.57342172

2.57342172

2.57342172
\end{abstract}

\&TRANSITION $3->\ldots$ 64
$-0.84613907$

1. 01172543

1. 01172543

1.11756098

1. 11756098

1.01172543

1.01172543

1. 11756098

1. 11756098

0.33846018

0.33846018

0.44429561

0.44429561

0.33846018

0.33846018

0.44429561

0.44429561

0.95463514

0.95463514

1.06047070

1.06047070

0.95463514

0.95463514

1.06047070

1.06047070

1.14465213

1.14465213

1. 25048769

1. 25048769

1.14465213

1.14465213

1. 25048769

1.25048769

$$
\text { 0. \# Energy 3->4 }
$$

$\begin{array}{ll}-0.0085 & 0.0993\end{array}$ $\begin{array}{ll}-0.3365 & 0.1762\end{array}$

0.01240921

0.01240921

0.11824466

0.11824466

0.01240921

0.01240921

0.11824466

0.11824466

0.11078113

0.11078113

0.21661659

0.21661659

0.11078113

0.11078113

0.21661659

0.21661659

$-0.25733095$

$-0.25733095$

$-0.15149552$

$-0.15149552$

$-0.25733095$

$-0.25733095$

$-0.15149552$

$-0.15149552$

$-0.95197451$

$-0.95197451$

$-0.84613907$

$-0.84613907$

$-0.95197451$

$-0.95197451$
0.76867646

0.39045236

0.49628779

0.39045236

0.49628779

0.39045236

0.49628779

0.39045236

0.49628779

$-0.16102493$

$-0.05518948$

$-0.16102493$

$-0.05518948$

$-0.16102493$

$-0.05518948$

$-0.16102493$

1. 39116621

1. 49700165

1.39116621

1. 49700165

1. 39116621

1.49700165

1.39116621

1.49700165

$-0.74076766$

$-0.63493228$

$-0.74076766$

$-0.63493228$

$-0.74076766$

$-0.74076766$

$-0.63493228$
$-0.05518948$

$-0.63493228$

-1.11517727
-9.44391823
8.57196426
6.94317293
-6.14315939
7.88817310
-7.08656931
-5.69994783
4.98228645
0.42679006
-0.56601542
-0.38405979
0.36031139
0.54152107
-0.28714040
-0.72131181
0.58594382
-0.53411466
0.34887251
0.19981322
-0.11194061
0.56883872
-0.39416096
-0.23299931
0.14772820
0.03744907
0.06481712
-0.04547674
-0.00874659
-0.01959936
-0.00103109
0.00737467
-0.00532868

1.000 \# Electr. mom., scale-fac \# Magnetic mom.

$-0.34768584$

$-0.24185041$ 6.48946047

$-7.80159235$

$-0.34768584$

$-0.24185041$

$-0.34768584$

$-0.24185041$

$-0.34768584$

$-0.24185041$

0.17077343

0.27660885

0.17077343

0.27660885

0.17077343

0.27660885

0.17077343

0.27660885

$-1.49057686$

$-1.38474143$

$-1.49057686$

$-1.38474143$

$-1.49057686$

$-1.38474143$

$-1.49057686$

$-1.38474143$

0.66284108

0.76867646

0.66284108

0.76867646

0.66284108

0.76867646
$-7.40542221$

9.43544292

$-10.66326809$

12.39420795

11.76307583

$-14.40943241$

$-42.72773361$

47.05563354

41.93848419

$-45.69984055$

45.88386536

$-49.35601044$

48.08937454

$-1.05799294$

1. 34360921

0.47166634

$-0.62462306$

0.40736252

$-0.51873291$

0.11589882

$-0.10675859$

0.86209929

1. 71833968

$-4.74534702$

1.37779605

0.80321628

$-3.32135916$
$-45.19725418$ 


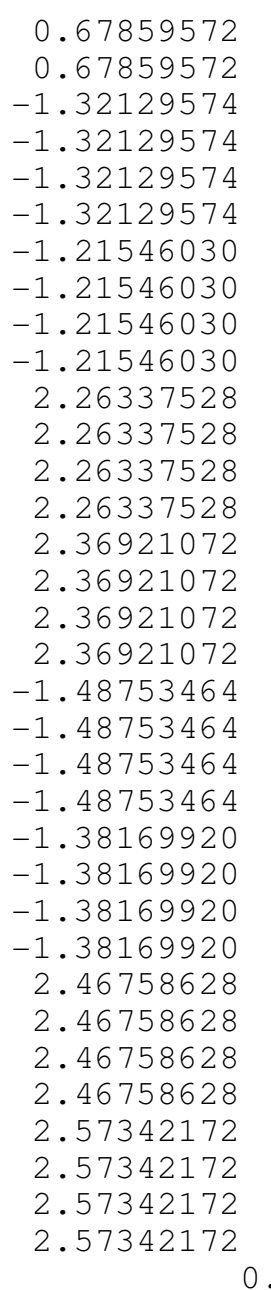

64
$-0.1232$

$-0.0332$

$-1.93576097$

$-1.93576097$

$-1.93576097$

$-1.93576097$

$-1.82992566$

$-1.82992566$

$-1.82992566$

$-1.82992566$

0.96153939

0.96153939

0.96153939

0.96153939

1.06737483

1.06737483

1.06737483

1.06737483

$-1.56185186$

$-1.56185186$

$-1.56185186$

$-1.56185186$

$-1.45601630$

$-1.45601630$

$-1.45601630$

$-1.45601630$

0.57276028

0.57276028

0.57276028

0.57276028

0.67859572

0.67859572

-0.84613907
-0.84613907
1.01172543
1.01172543
1.11756098
1.11756098
1.01172543
1.01172543
1.11756098
1.11756098
0.33846018
0.33846018
0.44429561
0.44429561
0.33846018
0.33846018
0.44429561
0.44429561
0.95463514
0.95463514
1.06047070
1.06047070
0.95463514
0.95463514
1.06047070
1.06047070
1.14465213
1.14465213
1.25048769
1.25048769
1.14465213
1.14465213
1.25048769
1.25048769
$\# 15 n e r 9 y 3->5$
0729
0552

0.66284108

0.76867646

0.39045236

0.49628779

0.39045236

0.49628779

0.39045236

0.49628779

0.39045236

0.49628779

$-0.16102493$

$-0.05518948$

$-0.16102493$

$-0.05518948$

$-0.16102493$

$-0.05518948$

$-0.05518948$

1. 39116621

1.49700165

1.39116621

1.49700165

1. 39116621

1.49700165

1. 39116621

1. 49700165

$-0.74076766$

$-0.63493228$

$-0.74076766$

$-0.63493228$

$-0.74076766$

$-0.63493228$

$-0.74076766$

$-0.63493228$

\# Energy $3->5$
$0.0729 \quad-0.1160$

$0.0552-0.0276$

0.01240921

0.11824466

0.11824466

0.01240921

0.01240921

0.11824466

0.11824466

0.11078113

0.11078113

0.21661659

0.21661659

0.11078113

0.11078113

0.21661659

0.21661659

$-0.25733095$

$-0.25733095$

$-0.15149552$

$-0.15149552$

$-0.25733095$

$-0.25733095$

$-0.15149552$

$-0.15149552$

$-0.95197451$

$-0.95197451$

$-0.84613907$

$-0.84613907$

$-0.95197451$

$-0.95197451$

$-0.24185041$

$-0.34768584$

$-0.24185041$

0.17077343

0.27660885

0.17077343

0.27660885

0.17077343

0.27660885

0.17077343

0.27660885

$-1.49057686$

$-1.38474143$

$-1.49057686$

$-1.38474143$

$-1.49057686$

$-1.38474143$

$-1.49057686$

$-1.38474143$

0.66284108

0.76867646

0.66284108

0.76867646

0.66284108

0.76867646
$-0.16102493$

2.95038438

0.31660560

6.13348055

$-7.94055843$

$-4.93570757$

6.58151245

$-5.11895084$

7.95954132

4. 26037073

$-6.72890377$

$-8.43918419$

6.15937567

11.95576572

$-9.65952396$

6.81085396

$-4.72939301$

$-10.04378700$

7. 94032192

3. 20868349

$-2.28037047$

$-2.83551788$

2. 03713632

$-3.49376082$

2. 47853756

3. 05104232

$-2.19174767$

$-1.03587985$

1. 35157120

0.71877122

$-1.00454414$

0.59797269

$-0.72189039$

$-0.50060076$

0.63422877

1.000 \# Electr. mom., scale-fac \# Magnetic mom.

$-0.34768584 \quad-0.14838959$

$-0.24185041 \quad-0.14982863$

$\begin{array}{ll}-0.34768584 & 0.13521986\end{array}$

$-0.24185041 \quad 0.41034439$

$-0.34768584 \quad-0.14847939$

0.58878416

0.20597382

$-0.95252216$

$-3.66068411$

4.33331776

3. 63232851

$-4.21209478$

3.78029704

$-4.49883652$

$-3.74620891$

4.36212206

0.28963774

$-0.37908509$

$-0.52988368$

0.69724578

$-0.42202047$

0.54137278

0.63968849

$-0.82284558$

0.84586054

$-0.35020289$

$-1.31463706$

0.67902988

$-0.63703072$

0.15549988

\section{S-109}




0.67859572
0.67859572
-1.32129574
-1.32129574
-1.32129574
-1.32129574
-1.21546030
-1.21546030
-1.21546030
-1.21546030
2.26337528
2.26337528
2.26337528
2.26337528
2.36921072
2.36921072
2.36921072
2.36921072
-1.48753464
-1.48753464
-1.48753464
-1.48753464
-1.38169920
-1.38169920
-1.38169920
-1.38169920
2.46758628
2.46758628
2.46758628
2.46758628
2.57342172
2.57342172
2.57342172
2.57342172
-1

64 $\begin{array}{lll}0.3478 & -0.3093 & 0.2307\end{array}$

0.1025

$-1.93576097$

$-1.93576097$

$-1.93576097$

$-1.93576097$

$-1.82992566$

$-1.82992566$

$-1.82992566$

$-1.82992566$

0.96153939

0.96153939

0.96153939

0.96153939

1. 06737483

1.06737483

1.06737483

1.06737483

$-1.56185186$

$-1.56185186$

$-1.56185186$

$-1.56185186$

$-1.45601630$

$-1.45601630$

$-1.45601630$

$-1.45601630$

0.57276028

0.57276028

0.57276028

0.57276028

0.67859572

0.67859572
$-0.84613907$

$-0.84613907$

1.01172543

1.01172543

1. 11756098

1. 11756098

1.01172543

1. 01172543

1.11756098

1. 11756098

0.33846018

0.33846018

0.44429561

0.44429561

0.33846018

0.33846018

0.44429561

0.44429561

0.95463514

0.95463514

1. 06047070

1.06047070

0.95463514

0.95463514

1. 06047070

1.06047070

1.14465213

1. 14465213

1. 25048769

1. 25048769

1. 14465213

1. 14465213

1. 25048769

1. 25048769

\# Energy 3->6

0.66284108

0.76867646

0.39045236

0.49628779

0.39045236

0.49628779

0.39045236

0.49628779

0.39045236

0.49628779

$-0.16102493$

$-0.05518948$

$-0.16102493$

$-0.05518948$

$-0.16102493$

$-0.05518948$

$-0.16102493$

$-0.05518948$

1. 39116621

1.49700165

1.39116621

1. 49700165

1. 39116621

1. 49700165

1. 39116621

1.49700165

$-0.74076766$

$-0.63493228$

$-0.74076766$

$-0.63493228$

$-0.74076766$

$-0.63493228$

$-0.74076766$

$-0.63493228$

0.0277

0.01240921

0.01240921

0.11824466

0.11824466

0.01240921

0.01240921

0.11824466

0.11824466

0.11078113

0.11078113

0.21661659

0.21661659

0.11078113

0.11078113

0.21661659

0.21661659

$-0.25733095$

$-0.25733095$

$-0.15149552$

$-0.15149552$

$-0.25733095$

$-0.25733095$

$-0.15149552$

$-0.15149552$

$-0.95197451$

$-0.95197451$

$-0.84613907$

$-0.84613907$

$-0.95197451$

$-0.95197451$

$-0.34768584$

$-0.24185041$

$-0.34768584$

$-0.24185041$

$-0.34768584$

$-0.24185041$

$-0.34768584$

$-0.24185041$

0.17077343

0.27660885

0.17077343

0.17077343

0.27660885

0.17077343

0.27660885

$-1.49057686$

$-1.38474143$

$-1.49057686$

$-1.38474143$

$-1.49057686$

$-1.38474143$

$-1.49057686$

$-1.38474143$

0.66284108

0.76867646

0.66284108

0.76867646

0.66284108

0.76867646
1.06855381

$-0.45615044$

1. 28196275

$-1.34351265$

$-1.18231177$

1.22971666

$-0.03383471$

0.41201824

0.13410619

$-0.43374228$

$-0.39126441$

0.41002792

0.75409973

$-0.77614743$

0.14735338

$-0.16521800$

$-0.46566895$

0.49067390

0.42477390

$-0.32595071$

$-0.33106908$

0.25677937

$-0.31449041$

0.24622378

0.19234234

$-0.15416291$

$-0.16175479$

0.15618181

0.11345321

$-0.10366297$

0.12619540

$-0.11857076$

$-0.09271307$

0.08179027

1.000 \# Electr. mom., scale-fac \# Magnetic mom.

1.73577893

$-1.45981228$

0.27660885
$-2.45364785$

$-1.70914793$

1.15569651

2. 47897649

$-1.22259521$

$-6.62614584$

5. 24466991

5.69944096

$-4.29744482$

7.01976252

$-5.17595625$

$-6.19511271$

4. 34220409

$-0.25215277$

0.42404377

0.64613920

$-0.96447742$

0.37253356

$-0.56421965$

$-0.70414585$

1. 00448942

$-2.43500161$

1. 62644446

2.92188382

$-1.92994809$

2. 11131406

$-1.34496415$
1. 60741365 


0.67859572
0.67859572
-1.32129574
-1.32129574
-1.32129574
-1.32129574
-1.21546030
-1.21546030
-1.21546030
-1.21546030
2.26337528
2.26337528
2.26337528
2.26337528
2.36921072
2.36921072
2.36921072
2.36921072
-1.48753464
-1.48753464
-1.48753464
-1.48753464
-1.38169920
-1.38169920
-1.38169920
-1.38169920
2.46758628
2.46758628
2.46758628
2.46758628
2.57342172
2.57342172
2.57342172
2.57342172
-1

64 $\begin{array}{lll}-0.3273 & 0.2910 & -0.2384\end{array}$

$\begin{array}{lll}-0.0212 & -0.0920 & -0.3464\end{array}$

$-1.93576097$

$-1.93576097$

$-1.93576097$

$-1.93576097$

$-1.82992566$

$-1.82992566$

$-1.82992566$

$-1.82992566$

0.96153939

0.96153939

0.96153939

0.96153939

1.06737483

1.06737483

1.06737483

1.06737483

$-1.56185186$

$-1.56185186$

$-1.56185186$

$-1.56185186$

$-1.45601630$

$-1.45601630$

$-1.45601630$

$-1.45601630$

0.57276028

0.57276028

0.57276028

0.57276028

0.67859572

0.67859572

0.11824466

-0.84613907
-0.84613907
1.01172543
1.01172543
1.11756098
1.11756098
1.01172543
1.01172543
1.11756098
1.11756098
0.33846018
0.33846018
0.44429561
0.44429561
0.33846018
0.33846018
0.44429561
0.44429561
0.95463514
0.95463514
1.06047070
1.06047070
0.95463514
0.95463514
1.06047070
1.06047070
1.14465213
1.14465213
1.25048769
1.25048769
1.14465213
1.14465213
1.25048769
1.25048769
$\# 15 n e r 9 y 3->7$
2910
0920

0.66284108

0.76867646

0.39045236

0.49628779

0.39045236

0.49628779

0.39045236

0.49628779

0.39045236

0.49628779

$-0.16102493$

$-0.05518948$

$-0.16102493$

$-0.05518948$

$-0.16102493$

$-0.05518948$

$-0.16102493$

$-0.05518948$

1. 39116621

1.49700165

1.39116621

1. 49700165

1. 39116621

1. 49700165

1.39116621

1.49700165

$-0.74076766$

$-0.63493228$

$-0.74076766$

$-0.63493228$

$-0.74076766$

$-0.63493228$

$-0.74076766$

$-0.63493228$

0.01240921

0.01240921

0.11824466

0.11824466

0.01240921

0.01240921

0.11824466

0.11078113

0.11078113

0.21661659

0.21661659

0.11078113

0.11078113

0.21661659

0.21661659

$-0.25733095$

$-0.25733095$

$-0.15149552$

$-0.15149552$

$-0.25733095$

$-0.25733095$

$-0.15149552$

$-0.15149552$

$-0.95197451$

$-0.95197451$

$-0.84613907$

$-0.84613907$

$-0.95197451$

$-0.95197451$

$-1.38474143$
$-2.40541482$

1. 48356569

$-2.16958976$

1. 86088014

2.04045963

$-1.73094940$

$-0.60206443$

0.22666985

0.23211014

$-0.01648435$

$-3.88541436$

3. 12458587

3.53621244

$-2.61478281$

3. 31358862

$-2.77290392$

$-2.97206140$

2. 29950690

$-0.00151498$

0.02963189

$-0.16921476$

0.08874218

$-0.23399624$

0.14307714

0.50892699

$-0.34501147$

0.20462275

$-0.33317530$

$-0.03763432$

0.07522363

$-0.13588266$

0.20729569

0.01714793

$-0.02217077$
\# Magnetic mom.

$-0.34768584$

$-0.24185041$

$-0.34768584$

$-0.24185041$

$-0.34768584$

$-0.24185041$

$-0.34768584$

$-0.24185041$

0.17077343

0.27660885

0.17077343

0.27660885

0.17077343

0.27660885

0.17077343

0.27660885

$-1.49057686$

$-1.38474143$

$-1.49057686$

$-1.49057686$

$-1.38474143$

$-1.49057686$

$-1.38474143$

0.66284108

0.76867646

0.66284108

0.76867646

0.66284108

0.76867646
$-9.85401726$

9.10562229

9.86001492

$-8.48570633$

7.72672033

$-6.36201572$

$-7.53048706$

5.28430843

7.20021105

$-4.13622141$

$-7.20673370$

3.88671017

$-7.89317656$

4.39696503

8.04901695

$-4.29021883$

$-0.59005433$

0.72661954

$-0.02608305$

0.04516585

0.04763874

$-0.12779994$

0.45860642

$-0.48623654$

2. 71876860

$-1.84184778$

$-3.51758242$

2. 43545437

$-2.36039162$

1. 53075707 


0.67859572
0.67859572
-1.32129574
-1.32129574
-1.32129574
-1.32129574
-1.21546030
-1.21546030
-1.21546030
-1.21546030
2.26337528
2.26337528
2.26337528
2.26337528
2.36921072
2.36921072
2.36921072
2.36921072
-1.48753464
-1.48753464
-1.48753464
-1.48753464
-1.38169920
-1.38169920
-1.38169920
-1.38169920
2.46758628
2.46758628
2.46758628
2.46758628
2.57342172
2.57342172
2.57342172
2.57342172
-1

64
$-0.84613907$

$-0.84613907$

1. 01172543

1.01172543

1. 11756098

1. 11756098

1.01172543

1. 01172543

1.11756098

1. 11756098

0.33846018

0.33846018

0.44429561

0.44429561

0.33846018

0.33846018

0.44429561

0.44429561

0.95463514

0.95463514

1. 06047070

1.06047070

0.95463514

0.95463514

1. 06047070

1.06047070

1.14465213

1. 14465213

1. 25048769

1. 25048769

1. 14465213

1.14465213

1. 25048769

1. 25048769

\# Energy 3->8

$\begin{array}{lll}0.0324 & 0.1168 & -0.0537\end{array}$

$-0.0189$

$-1.93576097$

$-1.93576097$

$-1.93576097$

$-1.93576097$

$-1.82992566$

$-1.82992566$

$-1.82992566$

$-1.82992566$

0.96153939

0.96153939

0.96153939

0.96153939

1. 06737483

1.06737483

1.06737483

1.06737483

$-1.56185186$

$-1.56185186$

$-1.56185186$

$-1.56185186$

$-1.45601630$

$-1.45601630$

$-1.45601630$

$-1.45601630$

0.57276028

0.57276028

0.57276028

0.57276028

0.67859572

0.67859572

0.01240921

0.01240921

0.11824466

0.11824466

0.01240921

0.01240921

0.11824466

0.11078113

0.11078113

0.21661659

0.21661659

0.11078113

0.11078113

0.21661659

0.21661659

$-0.25733095$

$-0.25733095$

$-0.15149552$

$-0.15149552$

$-0.25733095$

$-0.25733095$

$-0.15149552$

$-0.15149552$

$-0.95197451$

$-0.95197451$

$-0.84613907$

$-0.84613907$

$-0.95197451$

$-0.95197451$
0.66284108

0.76867646

0.39045236

0.49628779

0.39045236

0.49628779

0.39045236

0.49628779

0.39045236

0.49628779

$-0.16102493$

$-0.05518948$

$-0.16102493$

$-0.05518948$

$-0.16102493$

$-0.05518948$

$-0.16102493$

$-0.05518948$

1. 39116621

1.49700165

1.39116621

1. 49700165

1. 39116621

1. 49700165

1. 39116621

1.49700165

$-0.74076766$

$-0.63493228$

$-0.74076766$

$-0.63493228$

$-0.74076766$

$-0.63493228$

$-0.74076766$

$-0.63493228$

1.000

\# Electr. mom.'

0.11824466
$-0.34768584$

$-0.24185041$

$-0.34768584$

$-0.24185041$

$-0.34768584$

$-0.24185041$

$-0.34768584$

$-0.24185041$

0.17077343

0.27660885

0.17077343

0.27660885

0.17077343

0.27660885

0.17077343

0.27660885

$-1.49057686$

$-1.38474143$

$-1.49057686$

$-1.38474143$

$-1.49057686$

$-1.38474143$

$-1.49057686$

$-1.38474143$

0.66284108

0.76867646

0.66284108

0.76867646

0.66284108

0.76867646

2. 95675159

$-1.95510864$

6.16372442

$-4.44327021$

$-5.80077982$

4.48424339

$-0.94369864$

0.49114120

1.52667379

$-1.17733634$

3.39467406

$-2.45613623$

$-3.32689738$

2. 10521984

$-2.71251273$

2. 07682157

2.57912993

$-1.72455800$

0.17903928

$-0.18468051$

$-0.32265976$

0.30686185

0.31143752

$-0.17709357$

$-0.32582101$

0.17628212

$-0.23839304$

0.43381551

0.02943408

$-0.10268035$

0.18439393

$-0.29312432$

$-0.03076905$

0.05186901

$-8.62385941$

8.84439087

8.44371605

$-8.87197495$

9.13820648

$-9.22464657$

$-8.89021301$

9.15780735

27.70274162

$-29.67404556$

$-27.14006615$

28.41687202

$-29.68971252$

30.75932884

29.25324631

$-29.63237572$

1.00254071

$-1.10434222$

$-1.08620691$

1.20196915

$-1.07260120$

1.14321756

1.14001095

$-1.22297084$

0.96979320

$-1.73816538$

0.76698995

0.37469059

$-1.46587908$

2.13718009 


0.67859572
0.67859572
-1.32129574
-1.32129574
-1.32129574
-1.32129574
-1.21546030
-1.21546030
-1.21546030
-1.21546030
2.26337528
2.26337528
2.26337528
2.26337528
2.36921072
2.36921072
2.36921072
2.36921072
-1.48753464
-1.48753464
-1.48753464
-1.48753464
-1.38169920
-1.38169920
-1.38169920
-1.38169920
2.46758628
2.46758628
2.46758628
2.46758628
2.57342172
2.57342172
2.57342172
2.57342172
-1

64

\begin{abstract}
$-0.84613907$
\end{abstract}
$-0.84613907$

1. 01172543

1.01172543

1. 11756098

1.11756098

1.01172543

1.01172543

1.11756098

1. 11756098

0.33846018

0.33846018

0.44429561

0.44429561

0.33846018

0.33846018

0.44429561

0.44429561

0.95463514

0.95463514

1. 06047070

1.06047070

0.95463514

0.95463514

1. 06047070

1.06047070

1.14465213

1. 14465213

1. 25048769

1. 25048769

1.14465213

1.14465213

1.25048769

1. 25048769

\# Energy 3->9

0.0278

$-1.93576097$

$-1.93576097$

$-1.93576097$

$-1.93576097$

$-1.82992566$

$-1.82992566$

$-1.82992566$

$-1.82992566$

0.96153939

0.96153939

0.96153939

0.96153939

1.06737483

1.06737483

1.06737483

1.06737483

$-1.56185186$

$-1.56185186$

$-1.56185186$

$-1.56185186$

$-1.45601630$

$-1.45601630$

$-1.45601630$

$-1.45601630$

0.57276028

0.57276028

0.57276028

0.57276028

0.67859572

0.67859572
$0.0271 \quad-0.0441$

$0.1094-0.2382$

0.01240921

0.01240921

0.11824466

0.11824466

0.01240921

0.01240921

0.11824466

0.11824466

0.11078113

0.11078113

0.21661659

0.21661659

0.11078113

0.11078113

0.21661659

0.21661659

$-0.25733095$

$-0.25733095$

$-0.15149552$

$-0.15149552$

$-0.25733095$

$-0.25733095$

$-0.15149552$

$-0.15149552$

$-0.95197451$

$-0.95197451$

$-0.84613907$

$-0.84613907$

$-0.95197451$

$-0.95197451$
0.66284108

0.76867646

0.39045236

0.49628779

0.39045236

0.49628779

0.39045236

0.49628779

0.39045236

0.49628779

$-0.16102493$

$-0.05518948$

$-0.16102493$

$-0.05518948$

$-0.16102493$

$-0.05518948$

$-0.16102493$

$-0.05518948$

1. 39116621

1.49700165

1.39116621

1.49700165

1. 39116621

1. 49700165

1. 39116621

1. 49700165

$-0.74076766$

$-0.63493228$

$-0.74076766$

$-0.63493228$

$-0.74076766$

$-0.63493228$

$-0.74076766$

$-0.63493228$

1.000

$-0.34768584$

$-0.24185041$

$-0.34768584$

$-0.24185041$

$-0.34768584$

$-0.24185041$

$-0.34768584$

$-0.24185041$

0.17077343

0.27660885

0.17077343

0.27660885

0.17077343

0.27660885

0.17077343

0.27660885

$-1.49057686$

$-1.38474143$

$-1.49057686$

$-1.38474143$

$-1.49057686$

$-1.38474143$

$-1.49057686$

$-1.38474143$

0.66284108

0.76867646

0.66284108

0.76867646

0.66284108

0.76867646
$-0.20927924$

$-0.79425317$

1.39785016

1.12249196

$-1.05066216$

$-1.09795785$

0.71182209

$-3.22019291$

$-0.94940764$

3.12614679

8.72269440

$-7.90739822$

$-8.82779598$

7.70665932

$-6.80675268$

6.25493526

6.84372854

$-6.03885841$

$-2.15490985$

1. 49889565

1. 91484749

$-1.31834662$

2. 70190835

$-1.89568877$

$-2.53306270$

1. 77273643

0.25893611

$-0.15995304$

$-0.15079384$

0.08821594

$-0.05784157$

$-0.04426492$

$-0.01087996$

0.09079107

Electr. mom., scale-fac Magnetic mom.

$$
\begin{array}{r}
-1.67262125 \\
0.62429935 \\
1.28897619 \\
0.25088918 \\
-0.32128537 \\
1.87291861 \\
0.72481388 \\
-2.95768452 \\
-23.52302742 \\
24.08907700 \\
22.68231010 \\
-22.92938423 \\
24.71834755 \\
-24.98117256 \\
-23.88536644 \\
23.81637192 \\
0.40719178 \\
-0.51243478 \\
-1.04152942 \\
1.30536306 \\
-0.83941519 \\
1.02193975 \\
1.42058802 \\
-1.73411191 \\
-0.30666620 \\
1.02227533 \\
-0.71735197 \\
-0.25553316 \\
0.79589593 \\
-1.48247004
\end{array}
$$

\section{S-113}




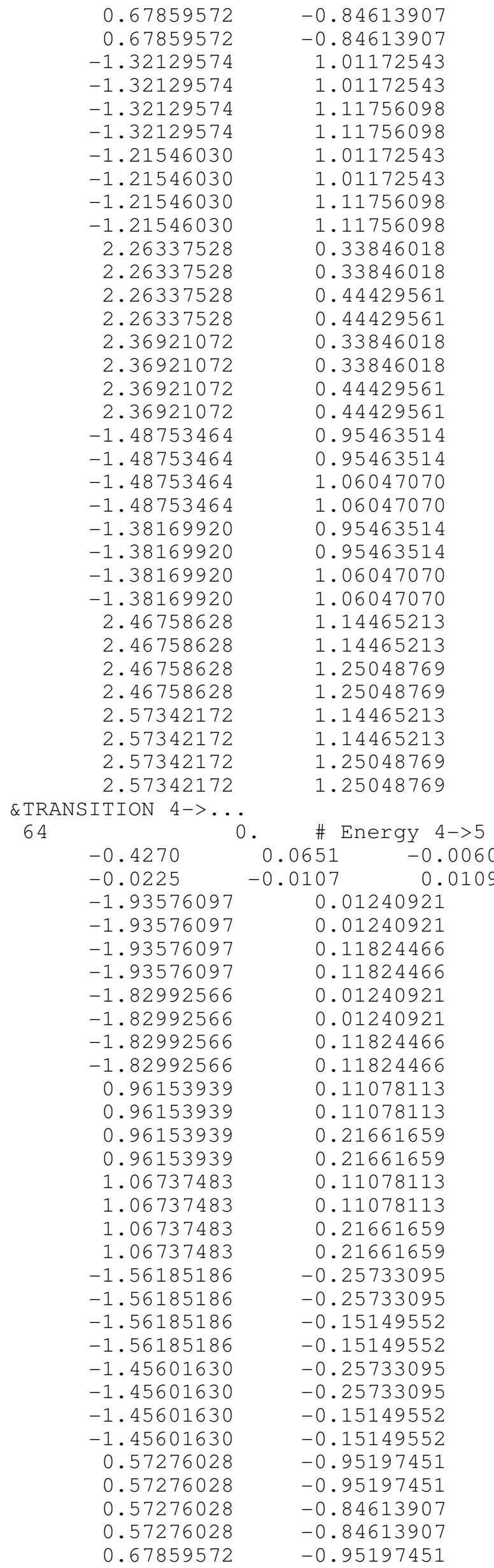

$\begin{array}{rr}0.66284108 & 0.22356401 \\ 0.76867646 & 0.70781112 \\ 0.39045236 & 7.88474083 \\ 0.49628779 & -7.69683361 \\ 0.39045236 & -7.16835117 \\ 0.49628779 & 7.10736179 \\ 0.39045236 & -4.39782381 \\ 0.49628779 & 5.06793022 \\ 0.39045236 & 4.46693993 \\ 0.49628779 & -5.09031010 \\ -0.16102493 & -6.21447563 \\ -0.05518948 & 5.55270338 \\ -0.16102493 & 6.77215672 \\ -0.05518948 & -5.97132206 \\ -0.16102493 & 4.70092726 \\ -0.05518948 & -4.25861979 \\ -0.16102493 & -5.15598917 \\ -0.05518948 & 4.60697746 \\ 1.39116621 & 1.71542275 \\ 1.49700165 & -1.26327443 \\ 1.39116621 & -1.62529647 \\ 1.49700165 & 1.19822311 \\ 1.39116621 & -1.50058329 \\ 1.49700165 & 1.11143208 \\ 1.39116621 & 1.38469231 \\ 1.49700165 & -1.02289200 \\ -0.74076766 & -0.25274035 \\ -0.63493228 & 0.05093415 \\ -0.74076766 & 0.18430176 \\ -0.63493228 & -0.03395324 \\ -0.74076766 & 0.17699133 \\ -0.63493228 & -0.01028671 \\ -0.74076766 & -0.12094712 \\ -0.63493228 & -0.01061536\end{array}$

1.000 \# Electr. mom., scale-fac \# Magnetic mom.

$\begin{array}{ll}-0.34768584 & 0.84509951\end{array}$

$-0.24185041 \quad-0.36321381$

$-0.34768584 \quad-0.92141140$

$-0.24185041 \quad 0.50956982$

$-0.34768584 \quad-1.50473130$

$\begin{array}{ll}-0.24185041 & 0.93591541\end{array}$

$-0.34768584 \quad 1.46662962$

$-0.24185041-1.00333548$

$\begin{array}{ll}0.17077343 & 2.64273286\end{array}$

$0.27660885 \quad-1.02639866$

$0.17077343 \quad-2.45685911$

$0.27660885 \quad 0.87112522$

$0.17077343 \quad-2.31215024$

$0.27660885 \quad 0.86812729$

$0.17077343 \quad 2.10597777$

$0.27660885 \quad-0.69487399$

$-1.49057686 \quad-0.51706368$

$-1.38474143 \quad 0.65615118$

$\begin{array}{lr}-1.49057686 & 0.66487581\end{array}$

$-1.38474143 \quad-0.84635985$

$\begin{array}{ll}-1.49057686 & 0.38749617\end{array}$

$-1.38474143 \quad-0.49395826$

$-1.49057686 \quad-0.54334772$

$-1.38474143 \quad 0.69925523$

$0.66284108 \quad 0.37212828$

$0.76867646 \quad-0.17067696$

$0.66284108 \quad-0.50775361$

$0.76867646 \quad 0.26504895$

$0.66284108 \quad-0.38668847$

\section{S-114}




\begin{abstract}
0.67859572
0.67859572

0.67859572

$-1.32129574$

$-1.32129574$

$-1.32129574$

$-1.32129574$

$-1.21546030$

$-1.21546030$

$-1.21546030$

$-1.21546030$

2. 26337528

2. 26337528

2. 26337528

2. 26337528

2.36921072

2. 36921072

2.36921072

2.36921072

$-1.48753464$

$-1.48753464$

$-1.48753464$

$-1.48753464$

$-1.38169920$

$-1.38169920$

$-1.38169920$

$-1.38169920$

2. 46758628

2.46758628

2. 46758628

2. 46758628

2.57342172

2. 57342172

2.57342172

2. 57342172
\end{abstract}

64
-0.95197451
-0.84613907
-0.84613907
1.01172543
1.01172543
1.11756098
1.11756098
1.01172543
1.01172543
1.11756098
1.11756098
0.33846018
0.33846018
0.44429561
0.44429561
0.33846018
0.33846018
0.44429561
0.44429561
0.95463514
0.95463514
1.06047070
1.06047070
0.95463514
0.95463514
1.06047070
1.06047070
1.14465213
1.14465213
1.25048769
1.25048769
1.14465213
1.14465213
1.25048769
1.25048769
$=1.159599$

0 . \# Energy $4->6$

$\begin{array}{lll}1.4500 & -0.1437 & 0.1159\end{array}$

0.0245

$-1.93576097$

$-1.93576097$

$-1.93576097$

$-1.93576097$

$-1.82992566$

$-1.82992566$

$-1.82992566$

$-1.82992566$

0.96153939

0.96153939

0.96153939

0.96153939

1.06737483

1.06737483

1.06737483

1.06737483

$-1.56185186$

$-1.56185186$

$-1.56185186$

$-1.56185186$

$-1.45601630$

$-1.45601630$

$-1.45601630$

$-1.45601630$

0.57276028

0.57276028

0.57276028

0.57276028

0.67859572 0.0395 $-0.1033$

0.01240921

0.01240921

0.11824466

0.11824466

0.01240921

0.01240921

0.11824466

0.11824466

0.11078113

0.11078113

0.21661659

0.21661659

0.11078113

0.11078113

0.21661659

0.21661659

$-0.25733095$

$-0.25733095$

$-0.15149552$

$-0.15149552$

$-0.25733095$

$-0.25733095$

$-0.15149552$

$-0.15149552$

$-0.95197451$

$-0.95197451$

$-0.84613907$

$-0.84613907$

$-0.95197451$
0.76867646

0.66284108

0.76867646

0.39045236

0.49628779

0.39045236

0.49628779

0.39045236

0.49628779

0.39045236

0.49628779

$-0.16102493$

$-0.05518948$

$-0.16102493$

$-0.05518948$

$-0.16102493$

$-0.05518948$

$-0.16102493$

$-0.05518948$

1.39116621

1.49700165

1. 39116621

1. 49700165

1.39116621

1.49700165

1.39116621

1.49700165

$-0.74076766$

$-0.63493228$

$-0.74076766$

$-0.63493228$

$-0.74076766$

$-0.63493228$

$-0.74076766$

$-0.63493228$
0.16981407

0.49536565

$-0.24554814$

0.83403325

$-0.49051940$

$-0.73027122$

0.43060452

$-0.71094924$

0.42524803

0.61145079

$-0.36599901$

$-1.49812460$

0.94331521

2.00839090

$-1.34723449$

2.10816836

$-1.43684435$

$-2.69650531$

1.93809700

$-0.12228722$

0.09479995

0.05033430

$-0.03620073$

0.10133011

$-0.08203144$

$-0.03184911$

0.02595604

0.26599732

$-0.38526171$

$-0.14609262$

0.22402249

$-0.22951654$

0.38843909

0.11126959

$-0.21271224$$$
1.000 \#
$$

\# Electr. mom., scale-fac \# Magnetic mom.

$-0.34768584$

$-0.24185041$

$-0.34768584$

$-0.24185041$

$-0.34768584$

$-0.24185041$

$-0.34768584$

$-0.24185041$

0.17077343

0.27660885

0.17077343

0.27660885

0.17077343

0.27660885

0.17077343

0.27660885

$-1.49057686$

$-1.38474143$

$-1.49057686$

$-1.38474143$

$-1.49057686$

$-1.38474143$

$-1.49057686$

$-1.38474143$

0.66284108

0.76867646

0.66284108

0.76867646

0.66284108
$-0.52114105$

0.76198667

1.43811166

$-1.80290055$

1.97679543

$-2.30119634$

$-3.08919072$

3.60384941

$-3.76113343$

2. 88680220

3.30645180

$-2.18339348$

3. 23665237

$-2.34600067$

$-2.86571074$

1. 74587095

0.23481262

$-0.35857975$

$-0.39363414$

0.58776653

0.01651514

0.04947116

0.10771138

$-0.22726075$

$-1.28623378$

1.16503179

1.06167138

$-0.98336530$

1. 14748991 


\begin{abstract}
0.67859572
0.67859572

0.67859572

$-1.32129574$

$-1.32129574$

$-1.32129574$

$-1.32129574$

$-1.21546030$

$-1.21546030$

$-1.21546030$

$-1.21546030$

2. 26337528

2. 26337528

2. 26337528

2. 26337528

2.36921072

2. 36921072

2.36921072

2.36921072

$-1.48753464$

$-1.48753464$

$-1.48753464$

$-1.48753464$

$-1.38169920$

$-1.38169920$

$-1.38169920$

$-1.38169920$

2. 46758628

2.46758628

2. 46758628

2. 46758628

2.57342172

2. 57342172

2. 57342172

2. 57342172
\end{abstract}

64

-0.8883
0.0156

$-1.93576097$

$-1.93576097$

$-1.93576097$

$-1.93576097$

$-1.82992566$

$-1.82992566$

$-1.82992566$

$-1.82992566$

0.96153939

0.96153939

0.96153939

0.96153939

1.06737483

1.06737483

1.06737483

1.06737483

$-1.56185186$

$-1.56185186$

$-1.56185186$

$-1.56185186$

$-1.45601630$

$-1.45601630$

$-1.45601630$

$-1.45601630$

0.57276028

0.57276028

0.57276028

0.57276028

0.67859572
$-0.95197451$

$-0.84613907$

$-0.84613907$

1. 01172543

1.01172543

1.11756098

1. 11756098

1. 01172543

1.01172543

1. 11756098

1.11756098

0.33846018

0.33846018

0.44429561

0.44429561

0.33846018

0.33846018

0.44429561

0.44429561

0.95463514

0.95463514

1. 06047070

1. 06047070

0.95463514

0.95463514

1.06047070

1.06047070

1. 14465213

1. 14465213

1.25048769

1. 25048769

1. 14465213

1.14465213

1. 25048769

1. 25048769

0 . \# Energy 4->7

$$
\begin{array}{rr}
0.2411 & -0.0685 \\
-0.0482 & -0.0519
\end{array}
$$

0.01240921

0.01240921

0.11824466

0.11824466

0.01240921

0.01240921

0.11824466

0.11824466

0.11078113

0.11078113

0.21661659

0.21661659

0.11078113

0.11078113

0.21661659

0.21661659

$-0.25733095$

$-0.25733095$

$-0.15149552$

$-0.15149552$

$-0.25733095$

$-0.25733095$

$-0.15149552$

$-0.15149552$

$-0.95197451$

$-0.95197451$

$-0.84613907$

$-0.84613907$

$-0.95197451$
0.76867646

0.66284108

0.76867646

0.39045236

0.49628779

0.39045236

0.49628779

0.39045236

0.49628779

0.39045236

0.49628779

$-0.16102493$

$-0.05518948$

$-0.16102493$

$-0.05518948$

$-0.16102493$

$-0.05518948$

$-0.16102493$

$-0.05518948$

1.39116621

1.49700165

1.39116621

1.49700165

1.39116621

1. 49700165

1.39116621

1. 49700165

$-0.74076766$

$-0.63493228$

$-0.74076766$

$-0.63493228$

$-0.74076766$

$-0.63493228$

$-0.74076766$

$-0.63493228$

$$
\begin{gathered}
1.000 \text { \# Electr. mom., scale-fac } \\
\text { \# Magnetic mom. }
\end{gathered}
$$

$-0.34768584$

$-0.24185041$

$-0.34768584$

$-0.24185041$

$-0.34768584$

$-0.24185041$

$-0.34768584$

$-0.24185041$

0.17077343

0.27660885

0.17077343

0.27660885

0.17077343

0.27660885

0.17077343

0.27660885

$-1.49057686$

$-1.38474143$

$-1.49057686$

$-1.38474143$

$-1.49057686$

$-1.38474143$

$-1.49057686$

$-1.38474143$

0.66284108

0.76867646

0.66284108

0.76867646

0.66284108
$-1.00065899$

$-0.95540333$

0.83520818

$-1.92750001$

0.73554039

1.87148869

$-0.86693460$

1.10162914

$-0.17796713$

$-0.99071676$

0.23588912

5.85719585

$-3.18651175$

$-7.95012331$

4. 94742775

$-7.57062817$

4. 72795010

9.78789234

$-6.68023443$

0.10093137

$-0.05442162$

0.08593896

$-0.08553049$

$-0.24710551$

0.16903712

0.06661465

$-0.02938013$

$-1.20877528$

2.01841831

0.53789532

$-1.04106724$

0.83611310

$-1.61564720$

$-0.26560488$

0.73179156

\section{S-116}




\begin{abstract}
0.67859572
0.67859572

0.67859572

$-1.32129574$

$-1.32129574$

$-1.32129574$

$-1.32129574$

$-1.21546030$

$-1.21546030$

$-1.21546030$

$-1.21546030$

2. 26337528

2. 26337528

2. 26337528

2. 26337528

2.36921072

2. 36921072

2.36921072

2.36921072

$-1.48753464$

$-1.48753464$

$-1.48753464$

$-1.48753464$

$-1.38169920$

$-1.38169920$

$-1.38169920$

$-1.38169920$

2. 46758628

2.46758628

2. 46758628

2. 46758628

2.57342172

2. 57342172

2. 57342172

2. 57342172
\end{abstract}

64

$\begin{array}{lcc} & \text { \# Energy } 4->8 \\ -0.7554 & -0.0362 & 0.2824 \\ 0.0170 & -0.0016 & 0.0001 \\ -1.93576097 & 0.01240921 \\ -1.93576097 & 0.01240921 \\ -1.93576097 & 0.11824466 \\ -1.93576097 & 0.11824466 \\ -1.82992566 & 0.01240921 \\ -1.82992566 & 0.01240921 \\ -1.82992566 & 0.11824466 \\ -1.82992566 & 0.11824466 \\ 0.96153939 & 0.11078113 \\ 0.96153939 & 0.11078113 \\ 0.96153939 & 0.21661659 \\ 0.96153939 & 0.21661659 \\ 1.06737483 & 0.11078113 \\ 1.06737483 & 0.11078113 \\ 1.06737483 & 0.21661659 \\ 1.06737483 & 0.21661659 \\ -1.56185186 & -0.25733095 \\ -1.56185186 & -0.25733095 \\ -1.56185186 & -0.15149552 \\ -1.56185186 & -0.15149552 \\ -1.45601630 & -0.25733095 \\ -1.45601630 & -0.25733095 \\ -1.45601630 & -0.15149552 \\ -1.45601630 & -0.15149552 \\ 0.57276028 & -0.95197451 \\ 0.57276028 & -0.95197451 \\ 0.57276028 & -0.84613907 \\ 0.57276028 & -0.84613907 \\ 0.67859572 & -0.95197451 \\ & \end{array}$

$-0.95197451$

$-0.84613907$

$-0.84613907$

1. 01172543

1.01172543

1. 11756098

1. 11756098

1.01172543

1.01172543

1. 11756098

1.11756098

0.33846018

0.33846018

0.44429561

0.44429561

0.33846018

0.33846018

0.44429561

0.44429561

0.95463514

0.95463514

1. 06047070

1. 06047070

0.95463514

0.95463514

1.06047070

1. 06047070

1. 14465213

1. 14465213

1. 25048769

1. 25048769

1. 14465213

1.14465213

1. 25048769

1.25048769
\# Energy 4->8

-0.7554
0.0170

$-1.93576097$

$-1.93576097$

$-1.93576097$

$-1.93576097$

$-1.82992566$

$-1.82992566$

0.96153939

0.96153939

1.06737483

1.06737483

$-1.56185186$

0.67859572

$$
\begin{array}{r}
0.76867646 \\
0.66284108 \\
0.76867646 \\
0.39045236 \\
0.49628779 \\
0.39045236 \\
0.49628779 \\
0.39045236 \\
0.49628779 \\
0.39045236 \\
0.49628779 \\
-0.16102493 \\
-0.05518948 \\
-0.16102493 \\
-0.05518948 \\
-0.16102493 \\
-0.05518948 \\
-0.16102493 \\
-0.05518948 \\
1.39116621 \\
1.49700165 \\
1.39116621 \\
1.49700165 \\
1.39116621 \\
1.49700165 \\
1.39116621 \\
1.49700165 \\
-0.74076766 \\
-0.63493228 \\
-0.74076766 \\
-0.63493228 \\
-0.74076766 \\
-0.63493228 \\
-0.74076766 \\
-0.63493228
\end{array}
$$

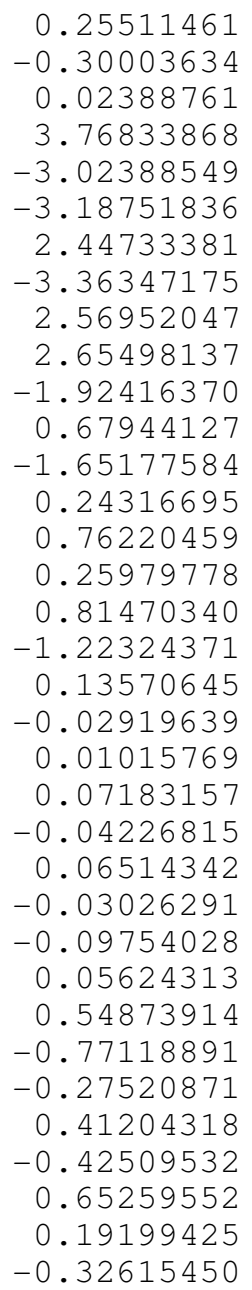
1.000 \# Electr. mom., scale-fac \# Magnetic mom.

$-0.34768584$

$-0.20641597$

$-0.24185041$

$-0.34768584$

$-0.24185041$

$-0.34768584$

$-0.24185041$

$-0.34768584$

$-0.24185041$

0.17077343

0.27660885

0.17077343

0.27660885

0.17077343

0.27660885

0.17077343

0.27660885

$-1.49057686$

$-1.38474143$

$-1.49057686$

$-1.38474143$

$-1.49057686$

$-1.38474143$

$-1.49057686$

$-1.38474143$

0.66284108

0.76867646

0.66284108

0.76867646

0.66284108

0.78941160

$-0.00617721$

$-1.04934633$

2. 14072227

$-3.06043267$

$-1.95580268$

3. 50001240

$-8.29747581$

5. 04602718

7.73624611

$-5.45843267$

7.38413954

$-4.56857300$

$-6.86584663$

4.83116531

0.61816436

$-0.70402509$

$-0.42286929$

0.47994608

$-0.34004599$

0.36211589

0.19782230

$-0.21431129$

0.74284220

$-1.11359584$

$-0.31388229$

0.87802792

$-0.58782303$ 


\begin{abstract}
0.67859572
0.67859572

0.67859572

$-1.32129574$

$-1.32129574$

$-1.32129574$

$-1.32129574$

$-1.21546030$

$-1.21546030$

$-1.21546030$

$-1.21546030$

2. 26337528

2. 26337528

2. 26337528

2. 26337528

2.36921072

2. 36921072

2.36921072

2.36921072

$-1.48753464$

$-1.48753464$

$-1.48753464$

$-1.48753464$

$-1.38169920$

$-1.38169920$

$-1.38169920$

$-1.38169920$

2. 46758628

2.46758628

2. 46758628

2.46758628

2.57342172

2. 57342172

2. 57342172

2. 57342172
\end{abstract}

64

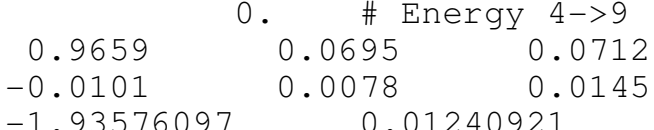

$-1.93576097$

$-1.93576097$

$-1.93576097$

$-1.93576097$

$-1.82992566$

$-1.82992566$

$-1.82992566$

$-1.82992566$

0.96153939

0.96153939

0.96153939

0.96153939

1.06737483

1.06737483

1.06737483

1.06737483

$-1.56185186$

$-1.56185186$

$-1.56185186$

$-1.56185186$

$-1.45601630$

$-1.45601630$

$-1.45601630$

$-1.45601630$

0.57276028

0.57276028

0.57276028

0.57276028

0.67859572
$-0.95197451$

$-0.84613907$

$-0.84613907$

1. 01172543

1.01172543

1.11756098

1. 11756098

1. 01172543

1.01172543

1. 11756098

1.11756098

0.33846018

0.33846018

0.44429561

0.44429561

0.33846018

0.33846018

0.44429561

0.44429561

0.95463514

0.95463514

1. 06047070

1.06047070

0.95463514

0.95463514

1.06047070

1.06047070

1. 14465213

1. 14465213

1.25048769

1. 25048769

1. 14465213

1.14465213

1. 25048769

1. 25048769

$\begin{array}{ll}0.0695 & 0.0712 \\ 0.0078 & 0.0145\end{array}$

0.01240921

0.01240921

0.11824466

0.11824466

0.01240921

0.01240921

0.11824466

0.11824466

0.11078113

0.11078113

0.21661659

0.21661659

0.11078113

0.11078113

0.21661659

0.21661659

$-0.25733095$

$-0.25733095$

$-0.15149552$

$-0.15149552$

$-0.25733095$

$-0.25733095$

$-0.15149552$

$-0.15149552$

$-0.95197451$

$-0.95197451$

$-0.84613907$

$-0.84613907$

$-0.95197451$
0.76867646

0.66284108

0.76867646

0.39045236

0.49628779

0.39045236

0.49628779

0.39045236

0.49628779

0.39045236

0.49628779

$-0.16102493$

$-0.05518948$

$-0.16102493$

$-0.05518948$

$-0.16102493$

$-0.05518948$

$-0.16102493$

$-0.05518948$

1.39116621

1.49700165

1.39116621

1.49700165

1.39116621

1. 49700165

1.39116621

1. 49700165

$-0.74076766$

$-0.63493228$

$-0.74076766$

$-0.63493228$

$-0.74076766$

$-0.63493228$

$-0.74076766$

$-0.63493228$

$$
1.000
$$

Electr. mom., scale-fac

$-0.34768584$

$-0.24185041$

$-0.34768584$

$-0.24185041$

$-0.34768584$

$-0.24185041$

$-0.34768584$

$-0.24185041$

0.17077343

0.27660885

0.17077343

0.27660885

0.17077343

0.27660885

0.17077343

0.27660885

$-1.49057686$

$-1.38474143$

$-1.49057686$

$-1.38474143$

$-1.49057686$

$-1.38474143$

$-1.49057686$

$-1.38474143$

0.66284108

0.76867646

0.66284108

0.76867646

0.66284108

1.00443947

0.22921646

$-0.80372709$

$-2.65580106$

1. 14816320

2.07474613

$-0.93793422$

$-0.13435706$

1. 25080836

0.02853601

$-0.86182600$

$-10.75084019$

4.49778700

14.12242794

$-6.77748680$

12.86775398

$-6.58405066$

$-16.33857155$

9.12378407

$-0.06506793$

0.08470454

0.09109085

$-0.09021488$

$-0.26006204$

0.13966517

0.19518811

$-0.11301612$

3.16379762

$-5.15534639$

$-1.55781531$

2. 79897165

$-2.55070639$

4.36975288

1. 13209462

$-2.22769189$ Magnetic mom.

0.90232050

$-1.25556552$

$-0.40784886$

1. 07352817

$-2.55386782$

3. 12997651

1.98959959

$-2.94631743$

7. 38496494

$-4.21712446$

$-6.73475504$

4.09100246

$-6.66139650$

4.05832386

5.95159101

$-3.74343824$

$-0.82320231$

0.92704648

0.74973601

$-0.83493525$

0.68271428

$-0.76192486$

$-0.68024153$

0.77282429

$-0.12536541$

0.55212426

$-0.48558688$

$-0.10596446$

0.09348092 


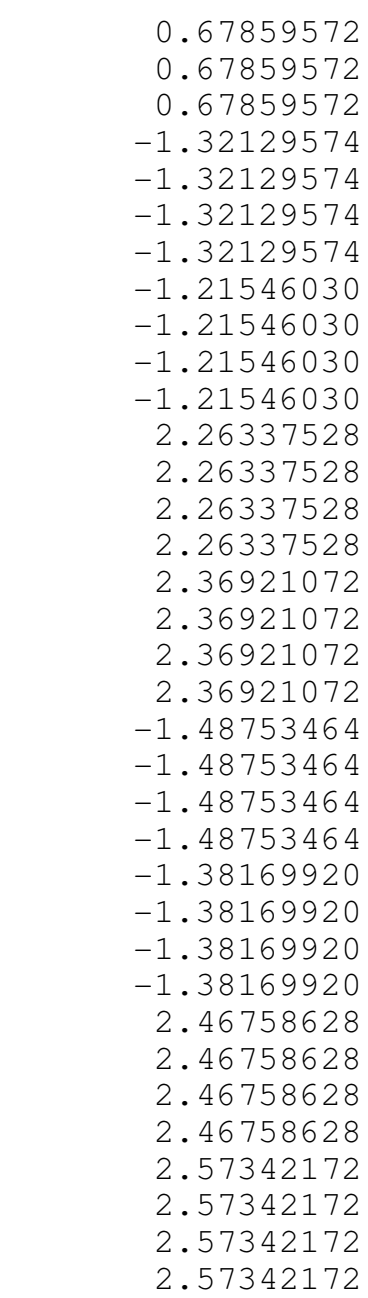

\&TRANSITION 5->...

$\begin{array}{lcc}3.0748 & 0.4933 & 1.3751 \\ -0.0370 & 0.0711 & -0.0477 \\ -1.93576097 & 0.01240921 \\ -1.93576097 & 0.01240921 \\ -1.93576097 & 0.11824466 \\ -1.93576097 & 0.11824466 \\ -1.82992566 & 0.01240921 \\ -1.82992566 & 0.01240921 \\ -1.82992566 & 0.11824466 \\ -1.82992566 & 0.11824466 \\ 0.96153939 & 0.11078113 \\ 0.96153939 & 0.11078113 \\ 0.96153939 & 0.21661659 \\ 0.96153939 & 0.21661659 \\ 1.06737483 & 0.11078113 \\ 1.06737483 & 0.11078113 \\ 1.06737483 & 0.21661659 \\ 1.06737483 & 0.21661659 \\ -1.56185186 & -0.25733095 \\ -1.56185186 & -0.25733095 \\ -1.56185186 & -0.15149552 \\ -1.56185186 & -0.15149552 \\ -1.45601630 & -0.25733095 \\ -1.45601630 & -0.25733095 \\ -1.45601630 & -0.15149552 \\ -1.45601630 & -0.15149552 \\ 0.57276028 & -0.95197451 \\ 0.57276028 & -0.95197451 \\ 0.57276028 & -0.84613907 \\ 0.57276028 & -0.84613907 \\ -1.9635\end{array}$

\section{4}

0 .

-0.95197451
-0.84613907
-0.84613907
1.01172543
1.01172543
1.11756098
1.11756098
1.01172543
1.01172543
1.11756098
1.11756098
0.33846018
0.33846018
0.44429561
0.44429561
0.33846018
0.33846018
0.44429561
0.44429561
0.95463514
0.95463514
1.06047070
1.06047070
0.95463514
0.95463514
1.06047070
1.06047070
1.14465213
1.14465213
1.25048769
1.25048769
1.14465213
1.14465213
1.25048769
1.25048769

$$
\begin{array}{r}
0.76867646 \\
0.66284108 \\
0.76867646 \\
0.39045236 \\
0.49628779 \\
0.39045236 \\
0.49628779 \\
0.39045236 \\
0.49628779 \\
0.39045236 \\
0.49628779 \\
-0.16102493 \\
-0.05518948 \\
-0.16102493 \\
-0.05518948 \\
-0.16102493 \\
-0.05518948 \\
-0.16102493 \\
-0.05518948 \\
1.39116621 \\
1.49700165 \\
1.39116621 \\
1.49700165 \\
1.39116621 \\
1.49700165 \\
1.39116621 \\
1.49700165 \\
-0.74076766 \\
-0.63493228 \\
-0.74076766 \\
-0.63493228 \\
-0.74076766 \\
-0.63493228 \\
-0.74076766 \\
-0.63493228
\end{array}
$$

$-0.51079923$

0.35750449

0.16399156

$-0.46706358$

0.78487974

0.09609696

$-0.32773435$

1. 23153877

$-1.31304598$

$-0.55153245$

0.61614388

10.11487865

$-5.85241461$

$-12.31491375$

7.21399689

$-11.56797504$

7. 21960735

13.90423203

$-8.83651924$

$-0.03201136$

0.02520711

0.00823527

$-0.00607678$

$-0.01023803$

0.00524035

0.00583873

$-0.00053977$

$-2.36509728$

3. 89061356

1.17233419

$-2.12205410$

1. 82164943

$-3.20049429$

$-0.78145796$

1. 60627913

$$
\begin{aligned}
& 1.000 \text { \# Electr. mom., scale-fac } \\
& \text { \# Magnetic mom. }
\end{aligned}
$$

$\begin{array}{ll}-0.34768584 & 12.22823334\end{array}$

$-0.24185041-15.51718903$

$-0.34768584 \quad-12.83815479$

$-0.24185041 \quad 16.82770538$

$-0.34768584 \quad-19.18200493$

$-0.24185041 \quad 23.15062332$

$-0.34768584 \quad 20.71330643$

$-0.24185041 \quad-25.80867577$

$0.17077343 \quad-2.22007036$

$0.27660885 \quad 1.85161054$

$0.17077343 \quad 5.50246954$

$0.27660885 \quad-5.01536560$

$0.17077343 \quad 5.49329805$

$0.27660885 \quad-5.51952887$

$0.17077343 \quad-8.16059875$

$0.27660885 \quad 8.20921421$

$-1.49057686-1.47203135$

$-1.38474143 \quad 1.70406306$

$-1.49057686 \quad 1.15485001$

$-1.38474143 \quad-1.41290998$

$\begin{array}{ll}-1.49057686 & 0.48097977\end{array}$

$-1.38474143 \quad-0.49274379$

$-1.49057686 \quad-0.06736465$

$-1.38474143 \quad 0.06205262$

$0.66284108 \quad 5.20321131$

$0.76867646 \quad-3.75286889$

$0.66284108 \quad-6.09642458$

$0.76867646 \quad 4.41622019$

\section{S-119}




0.67859572
0.67859572
0.67859572
0.67859572
-1.32129574
-1.32129574
-1.32129574
-1.32129574
-1.21546030
-1.21546030
-1.21546030
-1.21546030
2.26337528
2.26337528
2.26337528
2.26337528
2.36921072
2.36921072
2.36921072
2.36921072
-1.48753464
-1.48753464
-1.48753464
-1.48753464
-1.38169920
-1.38169920
-1.38169920
-1.38169920
2.46758628
2.46758628
2.46758628
2.46758628
2.57342172
2.57342172
2.57342172
2.57342172
-

64
$-0.95197451$

$-0.95197451$

$-0.84613907$

$-0.84613907$

1. 01172543

1.01172543

1. 11756098

1. 11756098

1.01172543

1.01172543

1.11756098

1.11756098

0.33846018

0.33846018

0.44429561

0.44429561

0.33846018

0.33846018

0.44429561

0.44429561

0.95463514

0.95463514

1. 06047070

1.06047070

0.95463514

0.95463514

1.06047070

1. 06047070

1. 14465213

1. 14465213

1. 25048769

1. 25048769

1.14465213

1. 14465213

1. 25048769

1. 25048769

0 .
0.66284108

0.76867646

0.66284108

0.76867646

0.39045236

0.49628779

0.39045236

0.49628779

0.39045236

0.49628779

0.39045236

0.49628779

$-0.16102493$

$-0.05518948$

$-0.16102493$

$-0.05518948$

$-0.16102493$

$-0.05518948$

$-0.16102493$

$-0.05518948$

1.39116621

1.49700165

1.39116621

1.49700165

1.39116621

1.49700165

1. 39116621

1. 49700165

$-0.74076766$

$-0.63493228$

$-0.74076766$

$-0.63493228$

$-0.74076766$

$-0.63493228$

$-0.74076766$

$-0.63493228$
$-3.86669946$

2. 41942716

4.58408165

$-2.85656691$

2. 90835357

0.76601475

$-1.35878301$

$-1.18740988$

$-0.98851508$

$-1.43894744$

0.10796842

1. 52137101

$-9.34920502$

7. 40001488

11.65444660

$-9.86209011$

9.97530174

$-8.14731121$

$-12.10363674$

10.44488907

0.23314528

$-0.16464408$

$-0.65720004$

0.49689892

0.34545782

$-0.30026430$

0.06529922

$-0.04545548$

0.26140469

$-1.01654780$

$-0.01965687$

0.49263600

0.42022777

0.15070090

$-0.50080353$

0.17419186

$$
\begin{array}{r}
-0.2565 \\
0.1045
\end{array}
$$

\# Energy 5->7

1.000

\# Electr. mom., scale-fac \# Magnetic mom.

0.01240921

0.01240921

0.11824466

0.11824466

0.01240921

0.01240921

0.11824466

0.11824466

0.11078113

0.11078113

0.21661659

0.21661659

0.11078113

0.11078113

0.21661659

0.21661659

$-0.25733095$

$-0.25733095$

$-0.15149552$

$-0.15149552$

$-0.25733095$

$-0.25733095$

$-0.15149552$

$-0.15149552$

$-0.95197451$

$-0.95197451$

$-0.84613907$

$-0.84613907$

$-0.34768584$

-0.58958209
-0.45415655

$-0.24185041$

$-0.34768584$

$-0.24185041$

$-0.34768584$

$-0.24185041$

$-0.34768584$

$-0.24185041$

0.17077343

0.27660885

0.17077343

0.27660885

0.17077343

0.27660885

0.17077343

0.27660885

$-1.49057686$

$-1.38474143$

$-1.49057686$

$-1.38474143$

$-1.49057686$

$-1.38474143$

$-1.49057686$

$-1.38474143$

0.66284108

0.76867646

0.66284108

0.76867646

0.84266585

$-0.12232975$

2. 96458483

$-2.01340532$

$-3.42416787$

2.84666109

$-6.79410267$

7.03063822

6.75568104

$-6.74674273$

6.20161915

$-6.43046284$

$-6.15870047$

6.11692667

6.28052235

$-8.19834995$

$-6.57079506$

8.58875561

$-5.93870735$

7.70108891

6.28638744

$-8.16711807$

2.05948448

$-1.37021017$

$-1.83047295$

1.08801031

\section{S-120}




\begin{tabular}{|c|c|c|c|}
\hline & & & \\
\hline 0.67859572 & -0.95197451 & 0.66284108 & -2.24149060 \\
\hline 0.67859572 & -0.95197451 & 0.76867646 & 1.49454081 \\
\hline 0.67859572 & -0.84613907 & 0.66284108 & 2.06774688 \\
\hline 0.67859572 & -0.84613907 & 0.76867646 & -1.27279675 \\
\hline-1.32129574 & 1.01172543 & 0.39045236 & -4.09961128 \\
\hline-1.32129574 & 1.01172543 & 0.49628779 & 2.81856799 \\
\hline-1.32129574 & 1.11756098 & 0.39045236 & 3.73662353 \\
\hline-1.32129574 & 1.11756098 & 0.49628779 & -2.54423976 \\
\hline-1.21546030 & 1.01172543 & 0.39045236 & 3.70850134 \\
\hline-1.21546030 & 1.01172543 & 0.49628779 & -2.92025542 \\
\hline-1.21546030 & 1.11756098 & 0.39045236 & -3.22933817 \\
\hline-1.21546030 & 1.11756098 & 0.49628779 & 2.48808837 \\
\hline 2.26337528 & 0.33846018 & -0.16102493 & -1.18021882 \\
\hline 2.26337528 & 0.33846018 & -0.05518948 & 1.03907514 \\
\hline 2.26337528 & 0.44429561 & -0.16102493 & 1.60820591 \\
\hline 2.26337528 & 0.44429561 & -0.05518948 & -1.36404872 \\
\hline 2.36921072 & 0.33846018 & -0.16102493 & 0.25355011 \\
\hline 2.36921072 & 0.33846018 & -0.05518948 & -0.33650354 \\
\hline 2.36921072 & 0.44429561 & -0.16102493 & -0.49665737 \\
\hline 2.36921072 & 0.44429561 & -0.05518948 & 0.53722060 \\
\hline-1.48753464 & 0.95463514 & 1.39116621 & -0.21311820 \\
\hline-1.48753464 & 0.95463514 & 1.49700165 & 0.11758988 \\
\hline-1.48753464 & 1.06047070 & 1.39116621 & 0.14034431 \\
\hline-1.48753464 & 1.06047070 & 1.49700165 & -0.08765326 \\
\hline-1.38169920 & 0.95463514 & 1.39116621 & 0.50355619 \\
\hline-1.38169920 & 0.95463514 & 1.49700165 & -0.32167894 \\
\hline-1.38169920 & 1.06047070 & 1.39116621 & -0.31606525 \\
\hline-1.38169920 & 1.06047070 & 1.49700165 & 0.20744286 \\
\hline 2.46758628 & 1.14465213 & -0.74076766 & -0.29871956 \\
\hline 2.46758628 & 1.14465213 & -0.63493228 & 0.05154308 \\
\hline 2.46758628 & 1.25048769 & -0.74076766 & 0.43107909 \\
\hline 2.46758628 & 1.25048769 & -0.63493228 & -0.29549301 \\
\hline 2.57342172 & 1.14465213 & -0.74076766 & 0.41184965 \\
\hline 2.57342172 & 1.14465213 & -0.63493228 & -0.32866523 \\
\hline 2.57342172 & 1.25048769 & -0.74076766 & -0.46037346 \\
\hline 2.57342172 & 1.25048769 & -0.63493228 & 0.43767881 \\
\hline & \# Energy 5->8 & & \\
\hline 0.3355 & -0.0938 & 1.000 & Electr. mom., \\
\hline 0.0468 & 0.1165 & \# & Magnetic mom. \\
\hline-1.93576097 & 0.01240921 & -0.34768584 & 0.83658952 \\
\hline-1.93576097 & 0.01240921 & -0.24185041 & -1.74752545 \\
\hline-1.93576097 & 0.11824466 & -0.34768584 & -1.76232147 \\
\hline-1.93576097 & 0.11824466 & -0.24185041 & 3.28121614 \\
\hline-1.82992566 & 0.01240921 & -0.34768584 & -4.46332121 \\
\hline-1.82992566 & 0.01240921 & -0.24185041 & 5.71470737 \\
\hline-1.82992566 & 0.11824466 & -0.34768584 & 5.61926889 \\
\hline-1.82992566 & 0.11824466 & -0.24185041 & -7.60182285 \\
\hline 0.96153939 & 0.11078113 & 0.17077343 & -0.41557628 \\
\hline 0.96153939 & 0.11078113 & 0.27660885 & -0.86214697 \\
\hline 0.96153939 & 0.21661659 & 0.17077343 & 0.80461931 \\
\hline 0.96153939 & 0.21661659 & 0.27660885 & 0.23377813 \\
\hline 1.06737483 & 0.11078113 & 0.17077343 & 0.51966387 \\
\hline 1.06737483 & 0.11078113 & 0.27660885 & 0.67859948 \\
\hline 1.06737483 & 0.21661659 & 0.17077343 & -0.79865152 \\
\hline 1.06737483 & 0.21661659 & 0.27660885 & -0.12571527 \\
\hline-1.56185186 & -0.25733095 & -1.49057686 & -1.40698230 \\
\hline-1.56185186 & -0.25733095 & -1.38474143 & 1.82048249 \\
\hline-1.56185186 & -0.15149552 & -1.49057686 & 1.17000270 \\
\hline-1.56185186 & -0.15149552 & -1.38474143 & -1.53071499 \\
\hline-1.45601630 & -0.25733095 & -1.49057686 & 0.93085974 \\
\hline-1.45601630 & -0.25733095 & -1.38474143 & -1.22079420 \\
\hline-1.45601630 & -0.15149552 & -1.49057686 & -0.69469637 \\
\hline-1.45601630 & -0.15149552 & -1.38474143 & 0.93769169 \\
\hline 7276028 & -0.95197451 & 0.66284108 & 0.66952938 \\
\hline 0.57276028 & -0.95197451 & 0.76867646 & -0.78018439 \\
\hline 0.57276028 & -0.84613907 & 0.66284108 & -0.70545435 \\
\hline 0.572 & -0.84613907 & 0.76867646 & 0.84449804 \\
\hline
\end{tabular}

\section{S-121}




\begin{tabular}{|c|c|c|c|}
\hline 0.67859572 & -0.95197451 & 0.66284108 & -0.28728130 \\
\hline 0.67859572 & -0.95197451 & 0.76867646 & 0.48644221 \\
\hline 0.67859572 & -0.84613907 & 0.66284108 & 0.25378144 \\
\hline 0.67859572 & -0.84613907 & 0.76867646 & -0.48448598 \\
\hline-1.32129574 & 1.01172543 & 0.39045236 & 4.29452848 \\
\hline-1.32129574 & 1.01172543 & 0.49628779 & -3.16171741 \\
\hline-1.32129574 & 1.11756098 & 0.39045236 & -4.47339725 \\
\hline-1.32129574 & 1.11756098 & 0.49628779 & 3.51122546 \\
\hline-1.21546030 & 1.01172543 & 0.39045236 & -2.73501611 \\
\hline-1.21546030 & 1.01172543 & 0.49628779 & 2.21342206 \\
\hline-1.21546030 & 1.11756098 & 0.39045236 & 3.01415873 \\
\hline-1.21546030 & 1.11756098 & 0.49628779 & -2.54483271 \\
\hline 2.26337528 & 0.33846018 & -0.16102493 & -0.24016014 \\
\hline 2.26337528 & 0.33846018 & -0.05518948 & -0.74065089 \\
\hline 2.26337528 & 0.44429561 & -0.16102493 & 1.15309834 \\
\hline 2.26337528 & 0.44429561 & -0.05518948 & -0.24884024 \\
\hline 2.36921072 & 0.33846018 & -0.16102493 & 0.63576472 \\
\hline 2.36921072 & 0.33846018 & -0.05518948 & 0.26217121 \\
\hline 2.36921072 & 0.44429561 & -0.16102493 & -1.48171771 \\
\hline 2.36921072 & 0.44429561 & -0.05518948 & 0.65853298 \\
\hline-1.48753464 & 0.95463514 & 1.39116621 & 0.39963466 \\
\hline-1.48753464 & 0.95463514 & 1.49700165 & -0.32506275 \\
\hline-1.48753464 & 1.06047070 & 1.39116621 & -0.47793683 \\
\hline-1.48753464 & 1.06047070 & 1.49700165 & 0.39026672 \\
\hline-1.38169920 & 0.95463514 & 1.39116621 & -0.25577998 \\
\hline-1.38169920 & 0.95463514 & 1.49700165 & 0.21039118 \\
\hline-1.38169920 & 1.06047070 & 1.39116621 & 0.23880276 \\
\hline-1.38169920 & 1.06047070 & 1.49700165 & -0.20578268 \\
\hline 2.46758628 & 1.14465213 & -0.74076766 & 0.18756244 \\
\hline 2.46758628 & 1.14465213 & -0.63493228 & -0.43074220 \\
\hline 2.46758628 & 1.25048769 & -0.74076766 & -0.01926000 \\
\hline 2.46758628 & 1.25048769 & -0.63493228 & 0.17539722 \\
\hline 2.57342172 & 1.14465213 & -0.74076766 & -0.04730450 \\
\hline 2.57342172 & 1.14465213 & -0.63493228 & 0.24986894 \\
\hline 2.57342172 & 1.25048769 & -0.74076766 & -0.07571671 \\
\hline 2.57342172 & 1.25048769 & -0.63493228 & -0.04496409 \\
\hline & \# Energy 5->9 & & \\
\hline 0.0413 & -0.0182 & 000 & Electr. mom., \\
\hline 0.0241 & 0.1039 & \# & Magnetic mom. \\
\hline-1.93576097 & 0.01240921 & -0.34768584 & 2.82263350 \\
\hline-1.93576097 & 0.01240921 & -0.24185041 & -4.79176092 \\
\hline-1.93576097 & 0.11824466 & -0.34768584 & -4.50249624 \\
\hline-1.93576097 & 0.11824466 & -0.24185041 & 7.03528833 \\
\hline-1.82992566 & 0.01240921 & -0.34768584 & -7.04855442 \\
\hline-1.82992566 & 0.01240921 & -0.24185041 & 9.43366528 \\
\hline-1.82992566 & 0.11824466 & -0.34768584 & 9.23450279 \\
\hline-1.82992566 & 0.11824466 & -0.24185041 & -12.15111351 \\
\hline 0.96153939 & 0.11078113 & 0.17077343 & -1.63486898 \\
\hline 0.96153939 & 0.11078113 & 0.27660885 & 0.70323104 \\
\hline 0.96153939 & 0.21661659 & 0.17077343 & 1.57756793 \\
\hline 0.96153939 & 0.21661659 & 0.27660885 & -0.53133458 \\
\hline 1.06737483 & 0.11078113 & 0.17077343 & 1.82298172 \\
\hline 1.06737483 & 0.11078113 & 0.27660885 & -0.44131967 \\
\hline 1.06737483 & 0.21661659 & 0.17077343 & -1.84605110 \\
\hline 1.06737483 & 0.21661659 & 0.27660885 & 0.40462837 \\
\hline-1.56185186 & -0.25733095 & -1.49057686 & -1.95199895 \\
\hline-1.56185186 & -0.25733095 & -1.38474143 & 2.55061722 \\
\hline-1.56185186 & -0.15149552 & -1.49057686 & 1.89878321 \\
\hline-1.56185186 & -0.15149552 & -1.38474143 & -2.50099540 \\
\hline-1.45601630 & -0.25733095 & -1.49057686 & 1.84655893 \\
\hline-1.45601630 & -0.25733095 & -1.38474143 & -2.37891912 \\
\hline-1.45601630 & -0.15149552 & -1.49057686 & -1.76533043 \\
\hline-1.45601630 & -0.15149552 & -1.38474143 & 2.28535628 \\
\hline 0.57276028 & -0.95197451 & 0.66284108 & -0.56544805 \\
\hline 0.57276028 & -0.95197451 & 0.76867646 & 0.18012594 \\
\hline 0.57276028 & -0.84613907 & 0.66284108 & 0.65023226 \\
\hline 0.572 & -0.84613907 & 0.76867646 & -0.19211158 \\
\hline
\end{tabular}

\section{S-122}




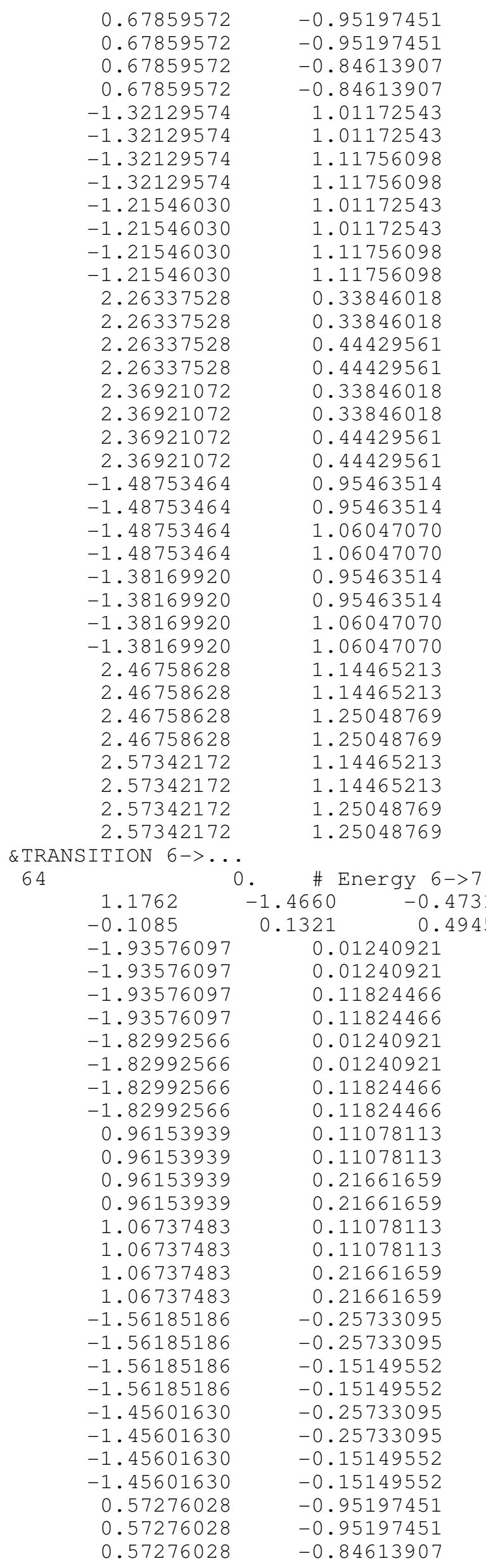

0.67859572

0.67859572

0.67859572

.67859572

$-1.32129574$

$-1.21546030$

2.26337528

. 26337528

2.36921072

2.36921072

2.36921072

$-1.48753464$

$-1.38169920$

2.46758628

2.46758628

2.57342172

2. 57342172

0

$-1.93576097$

$-1.93576097$

$-1.93576097$

$-1.82992566$

$-1.82992566$

$-1.82992566$

1.06737483

1.06737483

1.06737483

06737483

$-1.56185186$

$-1.45601630$

0.57276028
0.66284108

0.76867646

0.66284108

0.76867646

0.39045236

0.49628779

0.39045236

0.49628779

0.39045236

0.49628779

0.39045236

0.49628779

$-0.16102493$

$-0.05518948$

$-0.16102493$

$-0.05518948$

$-0.16102493$

$-0.05518948$

$-0.16102493$

$-0.05518948$

1.39116621

1.49700165

1.39116621

1.49700165

1.39116621

1. 49700165

1.39116621

1.49700165

$-0.74076766$

$-0.63493228$

$-0.74076766$

$-0.63493228$

$-0.74076766$

$-0.63493228$

$-0.74076766$

$-0.63493228$
0.82974845

$-0.26557028$

$-1.11770833$

0.43058953

$-1.30294609$

1. 38500857

$-0.21493335$

0.01411430

0.54936135

$-0.47166780$

0.52082044

$-0.49850032$

$-4.07526541$

3.12547731

4. 24284601

$-3.12969136$

3. 98327470

$-3.24776053$

$-4.12445068$

3.26326513

0.71399891

$-0.53062314$

$-0.70470250$

0.51946789

$-0.90707600$

0.66484290

0.84746450

$-0.62061101$

0.15833101

$-0.45674020$

0.02652471

0.13833487

0.03553891

0.18228905

$-0.15028793$

0.04336625

\section{. \# Energy 6->7}

1.000 \# Electr. mom., scale-fac \# Magnetic mom.

$-0.34768584$

$-0.24185041$

$-0.34768584$

$-0.24185041$

$-0.34768584$

$-0.24185041$

$-0.34768584$

$-0.24185041$

0.17077343

0.27660885

0.17077343

0.27660885

0.17077343

0.27660885

0.17077343

0.27660885

$-1.49057686$

$-1.38474143$

$-1.49057686$

$-1.38474143$

$-1.49057686$

$-1.38474143$

$-1.49057686$

$-1.38474143$

0.66284108

0.76867646

0.66284108
$-0.89922690$

1. 75945723

$-1.59870720$

1.32865000

$-3.61638641$

3. 40304399

7. 32441854

$-7.79112053$

$-0.58001900$

$-4.09766340$

2. 36238885

2. 42841172

0.65964091

4. 14859009

$-2.41769075$

$-2.47961330$

$-13.16281319$

17.74335098

13.72015190

$-18.56833267$

12.07081985

$-16.21471786$

$-12.72459126$

17.14256859

$-3.82537937$

1.80885708

4.57291365 


0.57276028
0.67859572
0.67859572
0.67859572
0.67859572
-1.32129574
-1.32129574
-1.32129574
-1.32129574
-1.21546030
-1.21546030
-1.21546030
-1.21546030
2.26337528
2.26337528
2.26337528
2.26337528
2.36921072
2.36921072
2.36921072
2.36921072
-1.48753464
-1.48753464
-1.48753464
-1.48753464
-1.38169920
-1.38169920
-1.38169920
-1.38169920
2.46758628
2.46758628
2.46758628
2.46758628
2.57342172
2.57342172
2.57342172
2.57342172
-1

64
$-0.84613907$

$-0.95197451$

$-0.95197451$

$-0.84613907$

$-0.84613907$

1.01172543

1.01172543

1. 11756098

1. 11756098

1.01172543

1. 01172543

1. 11756098

1. 11756098

0.33846018

0.33846018

0.44429561

0.44429561

0.33846018

0.33846018

0.44429561

0.44429561

0.95463514

0.95463514

1.06047070

1. 06047070

0.95463514

0.95463514

1. 06047070

1.06047070

1. 14465213

1. 14465213

1. 25048769

1. 25048769

1. 14465213

1.14465213

1. 25048769

1. 25048769

0 .

\# Energy 6->8
0.76867646

0.66284108

0.76867646

0.66284108

0.76867646

0.39045236

0.49628779

0.39045236

0.49628779

0.39045236

0.49628779

0.39045236

0.49628779

$-0.16102493$

$-0.05518948$

$-0.16102493$

$-0.05518948$

$-0.16102493$

$-0.05518948$

$-0.16102493$

$-0.05518948$

1.39116621

1. 49700165

1.39116621

1.49700165

1.39116621

1.49700165

1.39116621

1. 49700165

$-0.74076766$

$-0.63493228$

$-0.74076766$

$-0.63493228$

$-0.74076766$

$-0.63493228$

$-0.74076766$

$-0.63493228$
-2.25200677
3.85175681
-1.82003462
-4.33165550
2.07577848
11.95083809
-7.56875086
-11.67234039
7.76296949
-9.60285187
6.17218447
8.88598728
-5.92671585
-0.29293793
-0.66595119
0.51645368
0.60154688
1.23349905
-0.20614940
-1.62092650
0.41174716
1.60648835
-1.22468472
-1.64803839
1.26067388
-1.61820161
1.22178149
1.52930236
-1.16863465
0.53324676
-0.54739648
-0.42222819
0.42936128
-0.66532850
0.83020073
0.48514435
-0.60112619

1.000 \# Electr. mom., scale-fac \# Magnetic mom.

$-0.34768584$

$-0.24185041$

$-0.34768584$

$-0.24185041$

$-0.34768584$

$-0.24185041$

$-0.34768584$

$-0.24185041$

0.17077343

0.27660885

0.17077343

0.27660885

0.17077343

0.27660885

0.17077343

0.27660885

$-1.49057686$

$-1.38474143$

$-1.49057686$

$-1.38474143$

$-1.49057686$

$-1.38474143$

$-1.49057686$

$-1.38474143$

0.66284108

0.76867646

0.66284108
$-6.64463282$

7. 31360817

10.46114540

$-11.90098000$

13.76271057

$-14.57342529$

$-17.91379356$

19.68478203

2.73568392

$-6.71273232$

$-1.00482321$

5.61582184

$-4.55623770$

8.38974953

2.85494566

$-7.35358143$

2. 44208574

$-3.30138111$

$-1.97390223$

2. 74196553

$-0.93280238$

1.38221216

0.38871157

$-0.73910856$

$-6.02144670$

3. 75920892

7. 20477581 


0.57276028
0.67859572
0.67859572
0.67859572
0.67859572
-1.32129574
-1.32129574
-1.32129574
-1.32129574
-1.21546030
-1.21546030
-1.21546030
-1.21546030
2.26337528
2.26337528
2.26337528
2.26337528
2.36921072
2.36921072
2.36921072
2.36921072
-1.48753464
-1.48753464
-1.48753464
-1.48753464
-1.38169920
-1.38169920
-1.38169920
-1.38169920
2.46758628
2.46758628
2.46758628
2.46758628
2.57342172
2.57342172
2.57342172
2.57342172
-1

64
$-0.84613907$

$-0.95197451$

$-0.95197451$

$-0.84613907$

$-0.84613907$

1.01172543

1.01172543

1. 11756098

1. 11756098

1.01172543

1. 01172543

1. 11756098

1. 11756098

0.33846018

0.33846018

0.44429561

0.44429561

0.33846018

0.33846018

0.44429561

0.44429561

0.95463514

0.95463514

1.06047070

1. 06047070

0.95463514

0.95463514

1. 06047070

1.06047070

1. 14465213

1. 14465213

1. 25048769

1. 25048769

1. 14465213

1.14465213

1.25048769

1.25048769

0 .

\# Energy 6->9

$\begin{array}{lll}0.8468 & -0.8374 & -0.1616\end{array}$

$\begin{array}{lll}0.0592 & -0.0767 & -0.1786\end{array}$

$-1.93576097$

$-1.93576097$

$-1.93576097$

$-1.93576097$

$-1.82992566$

$-1.82992566$

$-1.82992566$

$-1.82992566$

0.96153939

0.96153939

0.96153939

0.96153939

1.06737483

1. 06737483

1.06737483

1.06737483

$-1.56185186$

$-1.56185186$

$-1.56185186$

$-1.56185186$

$-1.45601630$

$-1.45601630$

$-1.45601630$

$-1.45601630$

0.57276028

0.57276028

0.57276028
0.01240921

0.11824466

0.11824466

0.01240921

0.01240921

0.11824466

0.11824466

0.11078113

0.11078113

0.21661659

0.21661659

0.11078113

0.11078113

0.21661659

0.21661659

$-0.25733095$

$-0.25733095$

$-0.15149552$

$-0.15149552$

$-0.25733095$

$-0.25733095$

$-0.15149552$

$-0.15149552$

$-0.95197451$

$-0.95197451$

$-0.84613907$
0.01240921
0.76867646

0.66284108

0.76867646

0.66284108

0.76867646

0.39045236

0.49628779

0.39045236

0.49628779

0.39045236

0.49628779

0.39045236

0.49628779

$-0.16102493$

$-0.05518948$

$-0.16102493$

$-0.05518948$

$-0.16102493$

$-0.05518948$

$-0.16102493$

$-0.05518948$

1.39116621

1. 49700165

1.39116621

1.49700165

1. 39116621

1.49700165

1. 39116621

1. 49700165

$-0.74076766$

$-0.63493228$

$-0.74076766$

$-0.63493228$

$-0.74076766$

$-0.63493228$

$-0.74076766$

$-0.63493228$

1.000

$-0.34768584$

$-0.24185041$

$-0.34768584$

$-0.24185041$

$-0.34768584$

$-0.24185041$

$-0.34768584$

$-0.24185041$

0.17077343

0.27660885

0.17077343

0.27660885

0.17077343

0.27660885

0.17077343

0.27660885

$-1.49057686$

$-1.38474143$

$-1.49057686$

$-1.38474143$

$-1.49057686$

$-1.38474143$

$-1.49057686$

$-1.38474143$

0.66284108

0.76867646

0.66284108
$-4.63257790$

5. 44131517

$-3.20869875$

$-6.28164005$

3. 80551910

9. 14272594

$-8.48898029$

$-8.51269341$

7.60571241

$-8.76552200$

7.42502642

7.85466719

$-6.53620577$

7. 95416498

$-5.88035536$

$-9.84454441$

7.85125637

$-7.78880596$

5.94196558

9.46279621

$-7.71839619$

1.03484547

$-0.73375297$

$-0.59171075$

0.38342625

$-1.11248076$

0.82973415

0.73478782

$-0.51897544$

$-0.12308724$

0.76733691

$-0.15077238$

$-0.23612881$

$-0.36666542$

$-0.09433002$

0.49866581

$-0.25617895$

\section{S-125}




\begin{tabular}{|c|c|}
\hline & \\
\hline $\begin{array}{l}0.57276028 \\
0.67859572\end{array}$ & $\begin{array}{l}-0.84613907 \\
-0.95197451\end{array}$ \\
\hline 0.67859572 & -0.95197451 \\
\hline 0.67859572 & -0.84613907 \\
\hline 0.67859572 & -0.84613907 \\
\hline-1.32129574 & 1.01172543 \\
\hline-1.32129574 & 1.01172543 \\
\hline-1.32129574 & 1.11756098 \\
\hline-1.32129574 & 1.11756098 \\
\hline-1.21546030 & 1.01172543 \\
\hline-1.21546030 & 1.01172543 \\
\hline-1.21546030 & 1.11756098 \\
\hline-1.21546030 & 1.11756098 \\
\hline 2.26337528 & 0.33846018 \\
\hline 2.26337528 & 0.33846018 \\
\hline 2.26337528 & 0.44429561 \\
\hline 2.26337528 & 0.44429561 \\
\hline 2.36921072 & 0.33846018 \\
\hline 2.36921072 & 0.33846018 \\
\hline 2.36921072 & 0.44429561 \\
\hline 2.36921072 & 0.44429561 \\
\hline-1.48753464 & 0.95463514 \\
\hline-1.48753464 & 0.95463514 \\
\hline-1.48753464 & 1.06047070 \\
\hline-1.48753464 & 1.06047070 \\
\hline-1.38169920 & 0.95463514 \\
\hline-1.38169920 & 0.95463514 \\
\hline-1.38169920 & 1.06047070 \\
\hline-1.38169920 & 1.06047070 \\
\hline 2.46758628 & 1.14465213 \\
\hline 2.46758628 & 1.14465213 \\
\hline 2.46758628 & 1.25048769 \\
\hline 2.46758628 & 1.25048769 \\
\hline 2.57342172 & 1.14465213 \\
\hline 2.57342172 & 1.14465213 \\
\hline 2.57342172 & 1.25048769 \\
\hline 2.57342172 & 1.25048769 \\
\hline$\&$ TRANSITION $\quad 7->\ldots$ & \\
\hline 64 & $y$ Y $7->8$ \\
\hline-1.3411 & 0.6072 \\
\hline 0.0371 & -0.0566 \\
\hline-1.93576097 & 0.01240921 \\
\hline-1.93576097 & 0.01240921 \\
\hline-1.93576097 & 0.11824466 \\
\hline-1.93576097 & 0.11824466 \\
\hline-1.82992566 & 0.01240921 \\
\hline-1.82992566 & 0.01240921 \\
\hline-1.82992566 & 0.11824466 \\
\hline-1.82992566 & 0.11824466 \\
\hline 0.96153939 & 0.11078113 \\
\hline 0.96153939 & 0.11078113 \\
\hline 0.96153939 & 0.21661659 \\
\hline 0.96153939 & 0.21661659 \\
\hline 1.06737483 & 0.11078113 \\
\hline 1.06737483 & 0.11078113 \\
\hline 1.06737483 & 0.21661659 \\
\hline 1.06737483 & 0.21661659 \\
\hline-1.56185186 & -0.25733095 \\
\hline-1.56185186 & -0.25733095 \\
\hline-1.56185186 & -0.15149552 \\
\hline-1.56185186 & -0.15149552 \\
\hline-1.45601630 & -0.25733095 \\
\hline-1.45601630 & -0.25733095 \\
\hline-1.45601630 & -0.15149552 \\
\hline-1.45601630 & -0.15149552 \\
\hline 0.57276028 & -0.95197451 \\
\hline 0.57276028 & -0.95197451 \\
\hline
\end{tabular}

$$
\begin{array}{r}
0.76867646 \\
0.66284108 \\
0.76867646 \\
0.66284108 \\
0.76867646 \\
0.39045236 \\
0.49628779 \\
0.39045236 \\
0.49628779 \\
0.39045236 \\
0.49628779 \\
0.39045236 \\
0.49628779 \\
-0.16102493 \\
-0.05518948 \\
-0.16102493 \\
-0.05518948 \\
-0.16102493 \\
-0.05518948 \\
-0.16102493 \\
-0.05518948 \\
1.39116621 \\
1.49700165 \\
1.39116621 \\
1.49700165 \\
1.39116621 \\
1.49700165 \\
1.39116621 \\
1.49700165 \\
-0.74076766 \\
-0.63493228 \\
-0.74076766 \\
-0.63493228 \\
-0.74076766 \\
-0.63493228 \\
-0.74076766 \\
-0.63493228
\end{array}
$$

-2.54552674
1.54321742
-0.82233453
-2.48562098
1.72935057
1.13968873
0.09082932
-1.78330064
0.42756093
-0.52978402
-0.91286314
0.92653972
0.46782950
-2.00505638
2.00947928
1.67283201
-1.42216945
2.99133229
-2.63427401
-2.97607303
2.36824918
0.30087638
-0.22455131
-0.11070838
0.05207794
-0.38871801
0.30517936
0.23798260
-0.16145256
0.78093314
-0.82280523
-0.58688259
0.60622698
-0.77624387
0.93738586
0.54455346
-0.64843351

TRANSITION $7->$. .

1.000 \# Electr. mom., scale-fac \# Magnetic mom.

$-0.34768584$

$-0.24185041$

$-0.34768584$

$-0.24185041$

$-0.34768584$

$-0.24185041$

$-0.34768584$

$-0.24185041$

0.17077343

0.27660885

0.17077343

0.27660885

0.17077343

0.27660885

0.17077343

0.27660885

$-1.49057686$

$-1.38474143$

$-1.49057686$

$-1.38474143$

$-1.49057686$

$-1.38474143$

$-1.49057686$

$-1.38474143$

0.66284108

0.76867646
$-5.58713722$

9.41239357

9.83735085

$-13.54924393$

7. 73364878

$-12.18229103$

$-13.57284069$

17.91892433

$-4.92739201$

6.69406939

6.58159781

$-7.91134930$

5.91954231

$-7.65837765$

$-7.71192360$

8.95461082

$-1.62616634$

2.20729804

1. 96470940

$-2.57825971$

2.18050075

$-2.91049576$

$-2.69742489$

3. 54637289

2.01703835

$-0.60856974$ 


\begin{abstract}
0.57276028
0.57276028

0.67859572

0.67859572

0.67859572

0.67859572

$-1.32129574$

$-1.32129574$

$-1.32129574$

$-1.32129574$

$-1.21546030$

$-1.21546030$

$-1.21546030$

$-1.21546030$

2. 26337528

2. 26337528

2. 26337528

2. 26337528

2.36921072

2.36921072

2.36921072

2.36921072

$-1.48753464$

$-1.48753464$

$-1.48753464$

$-1.48753464$

$-1.38169920$

$-1.38169920$

$-1.38169920$

$-1.38169920$

2. 46758628

2.46758628

2.46758628

2.46758628

2.57342172

2.57342172

2.57342172

2. 57342172
\end{abstract}

64
$-0.84613907$

$-0.84613907$

$-0.95197451$

$-0.95197451$

$-0.84613907$

$-0.84613907$

1.01172543

1.01172543

1.11756098

1.11756098

1.01172543

1.01172543

1.11756098

1.11756098

0.33846018

0.33846018

0.44429561

0.44429561

0.33846018

0.33846018

0.44429561

0.44429561

0.95463514

0.95463514

1.06047070

1.06047070

0.95463514

0.95463514

1.06047070

1.06047070

1.14465213

1.14465213

1.25048769

1.25048769

1. 14465213

1.14465213

1.25048769

1.25048769

0 .

$-0.3890$

0.0468

$-1.93576097$

$-1.93576097$

$-1.93576097$

$-1.93576097$

$-1.82992566$

$-1.82992566$

$-1.82992566$

$-1.82992566$

0.96153939

0.96153939

0.96153939

0.96153939

1.06737483

1.06737483

1.06737483

1.06737483

$-1.56185186$

$-1.56185186$

$-1.56185186$

$-1.56185186$

$-1.45601630$

$-1.45601630$

$-1.45601630$

$-1.45601630$

0.57276028

0.57276028 $\begin{array}{lr}0.3329 & 0.1928 \\ 0.0093 & -0.0535\end{array}$

0.01240921

0.01240921

0.11824466

0.11824466

0.01240921

0.01240921

0.11824466

0.11824466

0.11078113

0.11078113

0.21661659

0.21661659

0.11078113

0.11078113

0.21661659

0.21661659

$-0.25733095$

$-0.25733095$

$-0.15149552$

$-0.15149552$

$-0.25733095$

$-0.25733095$

$-0.15149552$

$-0.15149552$

$-0.95197451$

$-0.95197451$
0.66284108

0.76867646

0.66284108

0.76867646

0.66284108

0.76867646

0.39045236

0.49628779

0.39045236

0.49628779

0.39045236

0.49628779

0.39045236

0.49628779

$-0.16102493$

$-0.05518948$

$-0.16102493$

$-0.05518948$

$-0.16102493$

$-0.05518948$

$-0.16102493$

$-0.05518948$

1.39116621

1. 49700165

1.39116621

1.49700165

1.39116621

1. 49700165

1.39116621

1.49700165

$-0.74076766$

$-0.63493228$

$-0.74076766$

$-0.63493228$

$-0.74076766$

$-0.63493228$

$-0.74076766$

$-0.63493228$
-2.92337561
1.27010906
-1.89971817
0.48092669
2.56065106

$-0.95698446$

6.53417730

$-6.68849277$

$-3.13894773$

3.17067957

$-8.00045967$

7.76374674

4.58670664

$-4.22923374$

1.64232826

$-1.70296884$

$-0.95015562$

1.15978611

$-2.38815069$

2. 32642484

1.94729865

$-1.97814631$

$-1.30756009$

1.02420008

1.69276297

$-1.25729978$

0.75264835

$-0.61428082$

$-1.25324178$

0.94518042

$-0.29561058$

0.30621701

0.15447053

$-0.14853100$

0.13118514

$-0.17957965$

$-0.01732356$

0.03397731
1.000 \# Electr. mom., scale-fac \# Magnetic mom.

$-0.34768584$

$-0.24185041$

$-0.34768584$

$-0.24185041$

$-0.34768584$

$-0.24185041$

$-0.34768584$

$-0.24185041$

0.17077343

0.27660885

0.17077343

0.27660885

0.17077343

0.27660885

0.17077343

0.27660885

$-1.49057686$

$-1.38474143$

$-1.49057686$

$-1.38474143$

$-1.49057686$

$-1.38474143$

$-1.49057686$

$-1.38474143$

0.66284108

0.76867646
$-2.74623346$

5.46712303

6.26909542

$-9.11273861$

5.22935009

$-8.46554470$

$-9.94438076$

13.26855183

$-6.37488174$

4. 58173370

7.91991568

$-5.70320082$

5.79725361

$-3.95671487$

$-7.39846277$

5.12067938

$-1.19405365$

1.54275024

1. 41823041

$-1.78796124$

1.64424002

$-2.11924028$

$-1.95689249$

2.50306821

$-1.58497834$

1. 39375138 


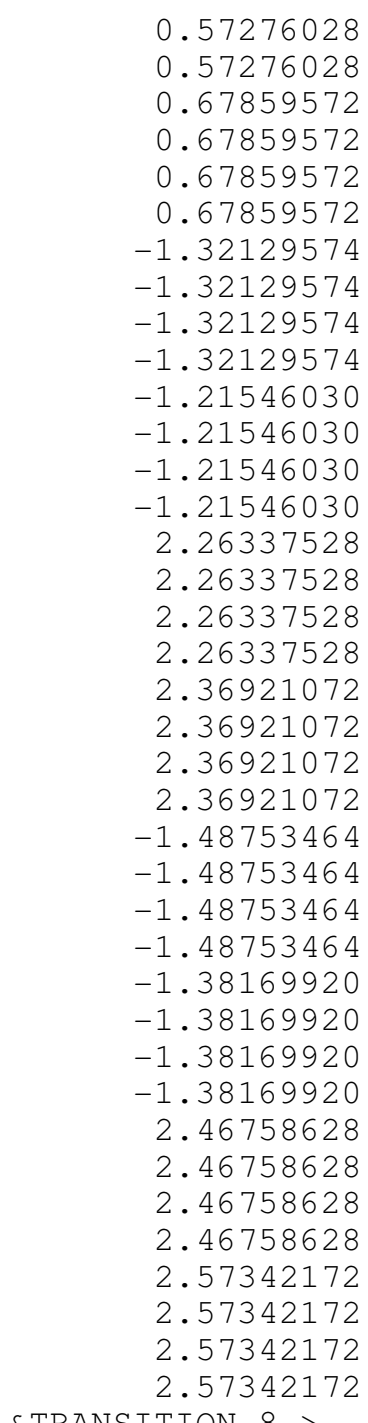

\&TRANSITION $8->\ldots$

64

$\begin{array}{lcc}-1.3872 & 0.0976 & 0.0207 \\ -0.2036 & -0.4293 & 0.2923 \\ -1.93576097 & 0.01240921 \\ -1.93576097 & 0.01240921 \\ -1.93576097 & 0.11824466 \\ -1.93576097 & 0.11824466 \\ -1.82992566 & 0.01240921 \\ -1.82992566 & 0.01240921 \\ -1.82992566 & 0.11824466 \\ -1.82992566 & 0.11824466 \\ 0.96153939 & 0.11078113 \\ 0.96153939 & 0.11078113 \\ 0.96153939 & 0.21661659 \\ 0.96153939 & 0.21661659 \\ 1.06737483 & 0.11078113 \\ 1.06737483 & 0.11078113 \\ 1.06737483 & 0.21661659 \\ 1.06737483 & 0.21661659 \\ -1.56185186 & -0.25733095 \\ -1.56185186 & -0.25733095 \\ -1.56185186 & -0.15149552 \\ -1.56185186 & -0.15149552 \\ -1.45601630 & -0.25733095 \\ -1.45601630 & -0.25733095 \\ -1.45601630 & -0.15149552 \\ -1.45601630 & -0.15149552 \\ 0.57276028 & -0.95197451 \\ & \end{array}$

0.66284108

0.76867646

0.66284108

0.76867646

0.66284108

0.76867646

0.39045236

0.49628779

0.39045236

0.49628779

0.39045236

0.49628779

0.39045236

0.49628779

$-0.16102493$

$-0.05518948$

$-0.16102493$

$-0.05518948$

$-0.16102493$

$-0.05518948$

$-0.16102493$

$-0.05518948$

1.39116621

1. 49700165

1. 39116621

1.49700165

1.39116621

1. 49700165

1.39116621

1.49700165

$-0.74076766$

$-0.63493228$

$-0.74076766$

$-0.63493228$

$-0.74076766$

$-0.63493228$

$-0.74076766$

$-0.63493228$
1. 22044814

$-1.01110744$

1. 75172269

$-1.49861026$

$-1.44610965$

1. 15603185

11.32153320

$-11.05215454$

$-8.01220608$

7. 79777622

$-10.89408588$

10.39855003

7.86266422

$-7.44340992$

2.52069259

$-2.22857642$

$-2.70534229$

2. 51730990

$-3.03435564$

2. 64454579

3. 31634235

$-3.00563788$

$-0.10014029$

0.12066031

0.37190464

$-0.28876346$

$-0.01973046$

$-0.01427344$

$-0.26117098$

0.20340206

$-0.29634050$

0.44448018

0.13938759

$-0.21780330$

0.05493378

$-0.18353511$

0.04903160

0.01147658 


\begin{tabular}{|c|c|c|c|}
\hline \\
\hline \multicolumn{4}{|l|}{0.57276028} \\
\hline 0.57276028 & -0.84613907 & 0.66284108 & 8.94866467 \\
\hline 0.57276028 & -0.84613907 & 0.76867646 & -3.13036203 \\
\hline 0.67859572 & -0.95197451 & 0.66284108 & 3.01385689 \\
\hline 0.67859572 & -0.95197451 & 0.76867646 & 1.87119043 \\
\hline 0.67859572 & -0.84613907 & 0.66284108 & -7.07353354 \\
\hline 0.67859572 & -0.84613907 & 0.76867646 & 1.04379833 \\
\hline-1.32129574 & 1.01172543 & 0.39045236 & -5.75191355 \\
\hline-1.32129574 & 1.01172543 & 0.49628779 & 5.44305182 \\
\hline-1.32129574 & 1.11756098 & 0.39045236 & 4.81565475 \\
\hline-1.32129574 & 1.11756098 & 0.49628779 & -4.55202675 \\
\hline-1.21546030 & 1.01172543 & 0.39045236 & 0.25360677 \\
\hline-1.21546030 & 1.01172543 & 0.49628779 & -2.03059578 \\
\hline-1.21546030 & 1.11756098 & 0.39045236 & -0.15516181 \\
\hline-1.21546030 & 1.11756098 & 0.49628779 & 1.54779649 \\
\hline 2.26337528 & 0.33846018 & -0.16102493 & 22.51969910 \\
\hline 2.26337528 & 0.33846018 & -0.05518948 & -18.70431137 \\
\hline 2.26337528 & 0.44429561 & -0.16102493 & -27.26301765 \\
\hline 2.26337528 & 0.44429561 & -0.05518948 & 22.77962303 \\
\hline 2.36921072 & 0.33846018 & -0.16102493 & -21.47705269 \\
\hline 2.36921072 & 0.33846018 & -0.05518948 & 17.4651 \\
\hline 2.36921072 & 0.44429561 & -0.16102493 & 26.07765770 \\
\hline 2.36921072 & 0.44429561 & -0.05518948 & -21.52573395 \\
\hline-1.48753464 & 0.95463514 & 1.39116621 & -2.39322400 \\
\hline-1.48753464 & 0.95463514 & 1.49700165 & 1.74694312 \\
\hline-1.48753464 & 1.06047070 & 1.39116621 & 2.09989572 \\
\hline-1.48753464 & 1.06047070 & 1.49700165 & -1.57227075 \\
\hline-1.38169920 & 0.95463514 & 1.39116621 & 2.248 \\
\hline-1.38169920 & 0.95463514 & 1.49700165 & -1.62157047 \\
\hline-1.38169920 & 1.06047070 & 1.39116621 & -1.73916817 \\
\hline-1.38169920 & 1.06047070 & 1.49700165 & 1.291 \\
\hline 2.46758628 & 1.14465213 & -0.74076766 & -0.03016488 \\
\hline 2.46758628 & 1.14465213 & -0.63493228 & 1.02997661 \\
\hline 2.46758628 & 1.25048769 & -0.74076766 & -0.20333697 \\
\hline 2.46758628 & 1.25048769 & -0.63493228 & -0.41767594 \\
\hline 2.57342172 & 1.14465213 & -0.74076766 & -0.12348875 \\
\hline 2.57342172 & 1.14465213 & -0.63493228 & -0.90662867 \\
\hline 2.57342172 & 1.25048769 & -0.74076766 & 0.38494948 \\
\hline 2.57342172 & 1.25048769 & -0.63493228 & 0.2 \\
\hline \multicolumn{4}{|l|}{ \&PERMANENT MOMENTS } \\
\hline & \# Energy 1->1 & & \\
\hline 1.85 & 2.5110 & $\#$ & ctr. mom. \\
\hline-1.93576097 & 0.01240921 & -0.34768584 & 94.91687012 \\
\hline-1.93576097 & 0.01240921 & -0.24185041 & -111.66935730 \\
\hline-1.93576097 & 0.11824466 & -0.34768584 & -93.68287659 \\
\hline-1.93576097 & 0.11824466 & -0.24185041 & 110.82323456 \\
\hline-1.82992566 & 0.01240921 & -0.34768584 & -106.33777618 \\
\hline-1.82992566 & 0.01240921 & -0.24185041 & 125.36608124 \\
\hline-1.82992566 & 0.11824466 & -0.34768584 & 109.484 \\
\hline-1.82992566 & 0.11824466 & -0.24185041 & -129.00466 \\
\hline 0.96153939 & 0.11078113 & 0.17077343 & 82.14746857 \\
\hline 0.96153939 & 0.11078113 & 0.27660885 & -36.07565308 \\
\hline 0.96153939 & 0.21661659 & 0.17077343 & -111.707 \\
\hline 0.96153939 & 0.21661659 & 0.27660885 & 62.2831 \\
\hline 1.06737483 & 0.11078113 & 0.17077343 & -106.86974335 \\
\hline 1.06737483 & 0.11078113 & 0.27660885 & 61.6601 \\
\hline 1.0673 & 61659 & 0.17077343 & 138.212 \\
\hline 1.06737483 & 0.21661659 & 0.27660885 & -88.30325317 \\
\hline-1.56185186 & -0.25733095 & -1.49057686 & -26.0456 \\
\hline-1.56185186 & -0.25733095 & -1.38474143 & 31.91 \\
\hline-1.56185186 & -0.15149552 & -1.49057686 & 30.7971763 \\
\hline-1.56185186 & -0.15149552 & -1.38474143 & -37.9357795 \\
\hline-1.45601 & -0.25 & -1.49057686 & 28.806 \\
\hline-1.45601630 & -0.25733095 & -1.38474143 & -35.0964660 \\
\hline-1.45601630 & -0.15149552 & -1.49057686 & -32.7649002 \\
\hline-1.45601630 & -0.15149552 & -1.384741 & 40.0 \\
\hline 0 & -0.9 & 0.66284108 & 33.5 \\
\hline
\end{tabular}

\section{S-129}




\begin{tabular}{|c|c|c|c|}
\hline 0.57276028 & -0.95197451 & 0.76867646 & -20.96156502 \\
\hline 0.57276028 & -0.84613907 & 0.66284108 & -47.73598862 \\
\hline 0.57276028 & -0.84613907 & 0.76867646 & 32.09843445 \\
\hline 0.67859572 & -0.95197451 & 0.66284108 & -41.10173035 \\
\hline 0.67859572 & -0.95197451 & 0.76867646 & 28.05353355 \\
\hline 0.67859572 & -0.84613907 & 0.66284108 & 54.88085175 \\
\hline 0.67859572 & -0.84613907 & 0.76867646 & -39.43695831 \\
\hline-1.32129574 & 1.01172543 & 0.39045236 & 37.95924759 \\
\hline-1.32129574 & 1.01172543 & 0.49628779 & -16.5218181 \\
\hline-1.32129574 & 1.11756098 & 0.39045236 & -33.6604499 \\
\hline-1.32129574 & 1.11756098 & 0.49628779 & 13.4013023 \\
\hline-1.21546030 & 1.01172543 & 0.39045236 & -30.59634209 \\
\hline-1.21546030 & 1.01172543 & 0.49628779 & 13.13179 \\
\hline-1.21546030 & 1.11756098 & 0.39045236 & 25.30 \\
\hline-1.21546030 & 1.11756098 & 0.49628779 & -8.4475936 \\
\hline 6337528 & 0.33846018 & -0.16102493 & 70.64 \\
\hline 2.26337528 & 0.33846018 & -0.05518948 & -54.2607078 \\
\hline 2.26337528 & 0.44429561 & -0.16102493 & -97.82560730 \\
\hline 2.26337528 & 0.44429561 & -0.05518948 & 73.77849579 \\
\hline 2.36921072 & 0.33846018 & -0.161024 & -84.58 \\
\hline 2.36921072 & 0.33846018 & -0.05518948 & 65.972801 \\
\hline 2.36921072 & 0.44429561 & -0.16102493 & 114.672729 \\
\hline 2.36921072 & 0.44429561 & -0.05518948 & -88.75 \\
\hline-1.48753464 & 0.95463514 & 1.391166 & 7.36 \\
\hline-1.48753464 & 0.95463514 & 1.497 & $-4 \cdot 7$ \\
\hline-1.48753464 & 1.06047070 & 1.39116621 & -5.33 \\
\hline-1 & 1.06 & 1.497 & \\
\hline-1 & 0.9546 & 1.3911 & $-5 \cdot 3$ \\
\hline 8169920 & 0.95463514 & 1.49700165 & $1 \S$ \\
\hline-1.38169920 & 1.06047070 & 1.39116621 & 3.5 \\
\hline 69920 & 1.06047070 & 1.497 & $-1 \cdot 8$ \\
\hline 2.46758628 & 1.14465213 & -0.74076766 & 6.87 \\
\hline 2.46758628 & 1.14465213 & -0.63493228 & -4.64729 \\
\hline 58628 & 1.25048769 & -0.7407 & -7.36 \\
\hline 2 . & 1.25048769 & -0.6349 & 6.2 \\
\hline 2.57342172 & 1.14465213 & -0.74076766 & -6.476457 \\
\hline 2.57342172 & 1.14465213 & -0.63493228 & 1.785848 \\
\hline 2.57 & 48769 & -0.7407 & 7 \\
\hline 2.57342 & 1.25048769 & -0.63493228 & -4.19122 \\
\hline & \# Energy 2->2 & \multirow{2}{*}{\multicolumn{2}{|c|}{ \# Electr. mom. }} \\
\hline 2.3170 & -0.6061 & & \\
\hline-1.93576097 & 0.01240921 & -0.34768584 & 96.7410278 \\
\hline 76097 & 0.01240921 & -0.24185041 & -112.85377 \\
\hline 3576097 & 0.11824466 & -0.34768584 & -97.862289 \\
\hline 3576097 & 0.11824466 & -0.24185041 & 113.581314 \\
\hline 92566 & 0.0124 & -0.347 & -106.60136 \\
\hline 2992566 & 0.01240921 & -0.24185041 & 126.03 \\
\hline 2992566 & 0.11824466 & -0.34768584 & 115.38 \\
\hline 92566 & 0.11824466 & -0.241 & -134.4 \\
\hline 3939 & 0.110 & 0.1 & 78.1 \\
\hline 0.96153939 & 0.11078113 & 0.27660885 & -33.01 \\
\hline 0.96153939 & 0.21661659 & 0.17077343 & -103.50 \\
\hline 0 . & 0.21661 & 0 . & 55.7 \\
\hline 1.06737483 & 0.11078113 & 0.17077343 & -102.286010 \\
\hline 1.06737483 & 0.11078113 & 0.27660885 & 58.26114 \\
\hline 1.06737483 & 0.21661659 & 0.17077 & 129.2 \\
\hline 1.06737483 & 0.21661 & 0.27660885 & $-81 \cdot 3$ \\
\hline-1.56185186 & -0.25733095 & -1.49057686 & -47.133087 \\
\hline-1.56185186 & -0.25733095 & -1.38474143 & 60.2 \\
\hline 6185186 & -0.15149552 & -1.49057686 & 52.3 \\
\hline-1.56185186 & -0.15149552 & -1.38474143 & -66.981903 \\
\hline-1.45601630 & -0.25733095 & -1.49057686 & 48.640357 \\
\hline-1.45601630 & -0.25733095 & -1.38474143 & -61.5 \\
\hline-1.45601630 & -0.15149552 & -1.49057686 & -53.2 \\
\hline-1 & -0.15149552 & -1.38474143 & 67.4 \\
\hline 0 . & -0.95197451 & 0.66284108 & 36.25692 \\
\hline 0 . & -0.95197451 & 0.76867646 & -23.28704 \\
\hline
\end{tabular}

\section{S-130}




\begin{abstract}
0.57276028
0.57276028

0.67859572

0.67859572

0.67859572

0.67859572

$-1.32129574$

$-1.32129574$

$-1.32129574$

$-1.32129574$

$-1.21546030$

$-1.21546030$

$-1.21546030$

$-1.21546030$

2. 26337528

2. 26337528

2. 26337528

2. 26337528

2.36921072

2.36921072

2.36921072

2.36921072

$-1.48753464$

$-1.48753464$

$-1.48753464$

$-1.48753464$

$-1.38169920$

$-1.38169920$

$-1.38169920$

$-1.38169920$

2. 46758628

2. 46758628

2. 46758628

2.46758628

2.57342172

2. 57342172

2. 57342172

2. 57342172
\end{abstract}

64

\begin{tabular}{|c|c|}
\hline 0.6905 & 0.6108 \\
\hline-1.93576097 & 0.01240921 \\
\hline-1.93576097 & 0.01240921 \\
\hline-1.93576097 & 0.11824466 \\
\hline-1.93576097 & 0.11824466 \\
\hline-1.82992566 & 0.01240921 \\
\hline-1.82992566 & 0.01240921 \\
\hline-1.82992566 & 0.11824466 \\
\hline-1.82992566 & 0.11824466 \\
\hline 0.96153939 & 0.11078113 \\
\hline 0.96153939 & 0.11078113 \\
\hline 0.96153939 & 0.21661659 \\
\hline 0.96153939 & 0.21661659 \\
\hline 1.06737483 & 0.11078113 \\
\hline 1.06737483 & 0.11078113 \\
\hline 1.06737483 & 0.21661659 \\
\hline 1.06737483 & 0.21661659 \\
\hline-1.56185186 & -0.25733095 \\
\hline-1.56185186 & -0.25733095 \\
\hline-1.56185186 & -0.15149552 \\
\hline-1.56185186 & -0.15149552 \\
\hline-1.45601630 & -0.25733095 \\
\hline-1.45601630 & -0.25733095 \\
\hline-1.45601630 & -0.15149552 \\
\hline-1.45601630 & -0.15149552 \\
\hline 0.57276028 & -0.95197451 \\
\hline 0.57276028 & -0.95197451 \\
\hline 0.57276028 & -0.84613907 \\
\hline
\end{tabular}

$-0.84613907$

$-0.84613907$

$-0.95197451$

$-0.95197451$

$-0.84613907$

$-0.84613907$

1.01172543

1. 01172543

1. 11756098

1. 11756098

1. 01172543

1. 01172543

1.11756098

1.11756098

0.33846018

0.33846018

0.44429561

0.44429561

0.33846018

0.33846018

0.44429561

0.44429561

0.95463514

0.95463514

1. 06047070

1.06047070

0.95463514

0.95463514

1. 06047070

1.06047070

1.14465213

1.14465213

1. 25048769

1. 25048769

1. 14465213

1.14465213

1. 25048769

1. 25048769

\# Energy 3->3

$-1.93576097$

$-1.93576097$

$-1.93576097$

$-1.93576097$

$-1.82992566$

$-1.82992566$

1.06737483

1.06737483

1.06737483

.0673748

$-1.56185186$

$-1.45601630$

$-1.45601630$

$-1.45601630$

$-1.45601630$

0.57276028

0.57276028
0.66284108

0.76867646

0.66284108

0.76867646

0.66284108

0.76867646

0.39045236

0.49628779

0.39045236

0.49628779

0.39045236

0.49628779

0.39045236

0.49628779

$-0.16102493$

$-0.05518948$

$-0.16102493$

$-0.05518948$

$-0.16102493$

$-0.05518948$

$-0.16102493$

$-0.05518948$

1.39116621

1.49700165

1. 39116621

1.49700165

1.39116621

1. 49700165

1.39116621

1.49700165

$-0.74076766$

$-0.63493228$

$-0.74076766$

$-0.63493228$

$-0.74076766$

$-0.63493228$

$-0.74076766$

$-0.63493228$

$-0.34768584$

$-0.24185041$

$-0.34768584$

$-0.24185041$

$-0.34768584$

$-0.24185041$

$-0.34768584$

$-0.24185041$

0.17077343

0.27660885

0.17077343

0.27660885

0.17077343

0.27660885

0.17077343

0.27660885

$-1.49057686$

$-1.38474143$

$-1.49057686$

$-1.38474143$

$-1.49057686$

$-1.38474143$

$-1.49057686$

$-1.38474143$

0.66284108

0.76867646

0.66284108
$-51.37417221$

35.18293381

$-43.52706146$

30.11767006

58.13631821

$-42.20525360$

34.36399078

$-11.17393398$

$-31.17866707$

9.56859493

$-28.26651764$

10.10168552

23.16495132

$-5.88567352$

72.25992584

$-56.43729401$

$-98.44593048$

75.26776886

$-86.29924011$

68.16387177

115.54774475

$-90.39366150$

11.47972107

$-7.75660944$

$-8.99913502$

5.96510983

$-9.95952892$

6.47427893

7.38117456

$-4.71765804$

6.78736496

$-4.60559750$

$-7.40665007$

6.30333328

$-6.73028660$

2.15164781

7.56724596

$-4.59971046$

Electr. mom.

103.56202698

$-120.04143524$

$-98.33913422$

113.35153961

$-107.85207367$

125.66426086

105.99396515

$-121.93646240$

44.55824661

$-23.92446899$

$-79.14422607$

53.90699387

$-74.18637848$

55.52093887

109.28075409

$-85.13499451$

$-27.10730934$

32.54773712

33.40172195

$-40.36659241$

31.28952217

$-37.45471191$

$-36.53675079$

43.88211441

$-7.08743906$

7.48072815

$-4.74154806$ 


\begin{tabular}{|c|c|c|c|}
\hline 0.57276028 & -0.84613907 & 0.76867646 & 2.71411490 \\
\hline 0.67859572 & -0.95197451 & 0.66284108 & -3.26019359 \\
\hline 0.67859572 & -0.95197451 & 0.76867646 & 2.06329250 \\
\hline 0.67859572 & -0.84613907 & 0.66284108 & 16.67 \\
\hline 0.67859572 & -0.84613907 & 0.76867646 & $-14 \cdot 1$ \\
\hline-1.32129574 & 1.01172543 & 0.39045236 & 46.139896 \\
\hline-1.32129574 & 1.01172543 & 0.49628779 & -27.371635 \\
\hline-1.32129574 & 1.11756098 & 0.39045236 & -42.037 \\
\hline-1.32129574 & 1.11756098 & 0.49628779 & 23.184707 \\
\hline-1.21546030 & 1.01172543 & 0.39045236 & -44.7475242 \\
\hline-1.21546030 & 1.01172543 & 0.49628779 & 28.37886047 \\
\hline-1.21546030 & 1.11756098 & 0.39045236 & 38.392890 \\
\hline-1.21546030 & 1.11756098 & 0.49628779 & -21.69636 \\
\hline 2.26337528 & 0.33846018 & -0.16102493 & 93.233428 \\
\hline 2.26337528 & 0.33846018 & -0.05518948 & -77.473838 \\
\hline 2.26337528 & 0.44429561 & -0.16102493 & $-126 \cdot 60$ \\
\hline 2.26337528 & 0.44429561 & -0.05518948 & 103.7 \\
\hline 2.36921072 & 0.33846018 & -0.16102493 & -105.03 \\
\hline 2.36921072 & 0.33846018 & -0.05518948 & 87.79 \\
\hline 2.36921072 & 0.44429561 & -0.16102493 & 139.72 \\
\hline 2.36921072 & 0.44429561 & -0.05518948 & -116.06 \\
\hline-1.48753464 & 0.95463514 & 1.39116621 & 6.96 \\
\hline 753464 & 0.95463514 & 1.4970016 & -4.2 \\
\hline 753464 & 1.06047070 & 1.391166 & -3.89 \\
\hline 753464 & 1.06047070 & 1.49700165 & 2.11 \\
\hline-1.38169920 & 0.95463514 & 1.39116621 & -6.29 \\
\hline-1.38169920 & 0.95463514 & 1.49700165 & 3.73 \\
\hline-1.38169920 & 1.06047070 & 1.391166 & 3.1 \\
\hline-1.38169920 & 1.06047070 & 1.49700165 & -1.49 \\
\hline 58628 & 1.14465213 & -0.7407 & 9.29 \\
\hline 2.46 & 1.1446 & -0.6349 & -4.4 \\
\hline 2.46758628 & 1.25048769 & -0.74076766 & -10.65 \\
\hline 2.46758628 & 1.25048769 & -0.63493228 & 8.027 \\
\hline 72 & 1.1446 & -0.7407 & -11.89 \\
\hline 2.5 & 1.1446 & -0.6349 & 6.1 \\
\hline 2.57342172 & 1.25048769 & -0.74076766 & 12.4866 \\
\hline 2.57342172 & 1.250487 & -0.63493228 & -9.096 \\
\hline & \# Energy $4-$ & & \\
\hline & 0.9984 & $\#$ & Electr. mom. \\
\hline-1.93576097 & 0.01240921 & -0.34768584 & 93.33 \\
\hline-1.93576097 & 0.01240921 & -0.24185041 & -111.32 \\
\hline-1.93 & 0.11824466 & -0.34768584 & -92.8 \\
\hline 76097 & 0.11824466 & -0.24185041 & 111.76 \\
\hline 92566 & 0.01240921 & -0.34768584 & -105.15 \\
\hline 566 & 0.012 & -0.24185041 & 125.7 \\
\hline 66 & 0.11824466 & -0.34768584 & 109.3 \\
\hline-1.8299 & 0.11824466 & -0.24185041 & -130.99 \\
\hline 39 & 0.11078113 & 0.17077343 & 87.08 \\
\hline 39 & 0.11 & 0 . & -25 \\
\hline 0.9 & 0.21661659 & 0.17077343 & -123.25 \\
\hline 53939 & 0.21661659 & 0.27660885 & 58.43 \\
\hline 1.0 & 0.110 & 0.17 & $-113 \cdot 7$ \\
\hline 1.06 & 0.11 & 0.2766 & 55.2 \\
\hline 1.067 & 0.21661659 & 0.17077343 & 150.31 \\
\hline 1.06 & 0.21661659 & 0.27660885 & -87.4 \\
\hline-1.56 & -0.25 & -1.49057686 & -26.3 \\
\hline-1.56 & -0.257330 & -1.38474143 & 32 . \\
\hline-1.561 & -0.15149552 & -1.49057686 & 30.9 \\
\hline-1.56 & -0.15 & -1.38474143 & -38 \\
\hline-1.45 & -0.25 & -1.49057686 & 28. \\
\hline-1.456 & 733095 & -1.38474143 & -35.29 \\
\hline-1.45 & -0 . & -1.49057686 & -32.79 \\
\hline-1.456 & -0 . & -1.38474143 & 40.0 \\
\hline 0.572 & -0 . & 0.66284108 & 30.4 \\
\hline 0.57 & 5197451 & 0.76867646 & -17.21 \\
\hline 0 . & -0 . & 0 . & -45 . \\
\hline 0 . & -0 . & 67646 & 28 \\
\hline
\end{tabular}

\section{S-132}




\begin{tabular}{|c|c|c|c|}
\hline 0.67859572 & -0.95197451 & 0.66284108 & -39.87280273 \\
\hline 0.67859572 & -0.95197451 & 0.76867646 & 25.97546387 \\
\hline 0.67859572 & -0.84613907 & 0.66284108 & 54.53848648 \\
\hline 0.67859572 & -0.84613907 & 0.76867646 & -37.96193695 \\
\hline-1.32129574 & 1.01172543 & 0.39045236 & 11752 \\
\hline-1.32129574 & 1.01172543 & 0.49628779 & -7.17218399 \\
\hline-1.32129574 & 1.11756098 & 0.39045236 & -23.88154602 \\
\hline-1.32129574 & 1.11756098 & 0.49628779 & 6.15209341 \\
\hline-1.21546030 & 1.01172543 & 0.39045236 & -14.36841679 \\
\hline-1.21546030 & 1.01172543 & 0.49628779 & 0.86640537 \\
\hline-1.21546030 & 1.11756098 & 0.39045236 & 12.44696999 \\
\hline-1.21546030 & 1.11756098 & 0.49628779 & 1.16757631 \\
\hline 2.26337528 & 0.33846018 & -0.16102493 & $45.541217 \varepsilon$ \\
\hline 2.26337528 & 0.33846018 & -0.05518948 & -37.43589401 \\
\hline 2.26337528 & 0.44429561 & -0.16102493 & -68.1866607 \\
\hline 2.26337528 & 0.44429561 & -0.05518948 & 54.8196411 \\
\hline 2.36921072 & 0.33846018 & -0.16102493 & -51.93799 \\
\hline 2.36921072 & 0.33846018 & -0.05518948 & 43.336277 \\
\hline 2.36921072 & 0.44429561 & -0.16102493 & 75.839 \\
\hline 2.36921072 & 0.44429561 & -0.05518948 & -62.0 \\
\hline-1.48753464 & 0.95463514 & 1.39116621 & 8.782 \\
\hline-1.48753464 & 0.95463514 & 1.49700165 & -5.7946853 \\
\hline-1.48753464 & 1.06047070 & 1.39116621 & -6.51 \\
\hline-1.48753464 & 1.06047070 & 1.49700165 & 4.1 \\
\hline 169920 & 0.95463514 & 1.39116621 & -6.836 \\
\hline-1.38169920 & 0.95463514 & 1.49700165 & 4.26 \\
\hline$-1 \cdot 38$ & 1.06 & 1.391166 & \\
\hline-1.38169920 & 1.06047070 & 1.49700165 & -2.733 \\
\hline 2.46758628 & 1.14465213 & -0.74076766 & 11.979 \\
\hline 2.46758628 & 1.14465213 & -0.63493228 & -11.707 \\
\hline 58628 & 1.25048 & -0.74076766 & $-10 \cdot 6$ \\
\hline 2.46758628 & 1.25048769 & -0.63493228 & $10.80^{-}$ \\
\hline 2.57342172 & 1.14465213 & -0.74076766 & -11.4663 \\
\hline 2.57342172 & 1.14465213 & -0.63493228 & 9.44 \\
\hline 2.57342172 & 1.25048769 & -0.74076766 & 10.24 \\
\hline 2.57342172 & 1.25048769 & -0.63493228 & -8.97 \\
\hline & \# Energy 5->5 & \multirow{2}{*}{\multicolumn{2}{|c|}{ \# Electr. mom }} \\
\hline 2.930 & 3.1549 & & \\
\hline-1.93576097 & 0.012 & -0.34768584 & 101.54700470 \\
\hline-1.93576097 & 0.01240921 & -0.24185041 & -123.875 \\
\hline-1.93576097 & 0.11824466 & -0.34768584 & -98.268974 \\
\hline-1.93576097 & 4466 & -0.24185041 & 120.5 \\
\hline-1.82992566 & 0.01240921 & -0.34768584 & -111.1885910 \\
\hline-1.82992566 & 0.01240921 & -0.24185041 & $136.80^{\circ}$ \\
\hline-1.82992566 & 0.11824466 & -0.34768584 & 114.1537 \\
\hline 92566 & 0.11 & -0.24 & -140.11 \\
\hline 0.96153939 & 0.11078113 & 0.17077343 & 79.612 \\
\hline 0.96153939 & 0.11078113 & 0.27660885 & -34.565 \\
\hline 53939 & 0.216 & 0.17077343 & -109.48 \\
\hline 0.96 & 0.21 & 0.27660885 & 60.9 \\
\hline 1.06737483 & 0.11078113 & 0.17077343 & -106.003 \\
\hline 1.06737483 & 0.11078113 & 0.27660885 & 60.96 \\
\hline 1.06 & 0.21 & 0.1707 & 137.6 \\
\hline 1.06737483 & 0.216 & 0.27660 & -87.90 \\
\hline-1.56185186 & -0.25733095 & -1.49057686 & -27.472 \\
\hline-1.56185186 & -0.25 & -1.38474143 & 33.47 \\
\hline-1.56185186 & -0.15 & -1.49057686 & 31.05 \\
\hline-1.56185186 & -0.15149552 & -1.38474143 & -38.086 \\
\hline-1.45601630 & -0.257 & -1.49057686 & 30.11 \\
\hline-1.45601630 & -0.25 & -1.38474143 & -36.53 \\
\hline-1.45601630 & -0.151 & -1.49057686 & -32.733 \\
\hline-1.45601630 & -0.15149552 & -1.38474143 & 39.79 \\
\hline 0.57 & -0.95 & 0.6628 & 33.9 \\
\hline 0.57276028 & -0.95 & 0.76867646 & $-21 \cdot 21$ \\
\hline 0.572 & -0.846139 & 0.66284108 & -48.42 \\
\hline 0.572 & -0.84613907 & 0.76867646 & 32.435 \\
\hline 0.67 & -0.95 & 0.6628 & -42.23 \\
\hline
\end{tabular}

\section{S-133}




0.67859572
0.67859572
0.67859572
-1.32129574
-1.32129574
-1.32129574
-1.32129574
-1.21546030
-1.21546030
-1.21546030
-1.21546030
2.26337528
2.26337528
2.26337528
2.26337528
2.36921072
2.36921072
2.36921072
2.36921072
-1.48753464
-1.48753464
-1.48753464
-1.48753464
-1.38169920
-1.38169920
-1.38169920
-1.38169920
2.46758628
2.46758628
2.46758628
2.46758628
2.57342172
2.57342172
2.57342172
2.57342172
-1

64

-0.95197451
-0.84613907
-0.84613907
1.01172543
1.01172543
1.11756098
1.11756098
1.01172543
1.01172543
1.11756098
1.11756098
0.33846018
0.33846018
0.44429561
0.44429561
0.33846018
0.33846018
0.44429561
0.44429561
0.95463514
0.95463514
1.06047070
1.06047070
0.95463514
0.95463514
1.06047070
1.06047070
1.14465213
1.14465213
1.25048769
1.25048769
1.14465213
1.14465213
1.25048769
1.25048769
$\# .5 n e r 9 y 6->6$
8265

-4.7328
-1.93576097

$-1.93576097$

$-1.93576097$

$-1.93576097$

$-1.82992566$

$-1.82992566$

$-1.82992566$

$-1.82992566$

0.96153939

0.96153939

0.96153939

0.96153939

1.06737483

1.06737483

1.06737483

1.06737483

$-1.56185186$

$-1.56185186$

$-1.56185186$

$-1.56185186$

$-1.45601630$

$-1.45601630$

$-1.45601630$

$-1.45601630$

0.57276028

0.57276028

0.57276028

0.57276028

0.67859572

0.67859572

. \# Energy 6->6 3.8265

0.01240921

0.01240921

0.11824466

0.11824466

0.01240921

0.01240921

0.11824466

0.11824466

0.11078113

0.11078113

0.21661659

0.21661659

0.11078113

0.11078113

0.21661659

0.21661659

$-0.25733095$

$-0.25733095$

$-0.15149552$

$-0.15149552$

$-0.25733095$

$-0.25733095$

$-0.15149552$

$-0.15149552$

$-0.95197451$

$-0.95197451$

$-0.84613907$

$-0.84613907$

$-0.95197451$
$-0.95197451$
0.76867646

0.66284108

0.76867646

0.39045236

0.49628779

0.39045236

0.49628779

0.39045236

0.49628779

0.39045236

0.49628779

$-0.16102493$

$-0.05518948$

$-0.16102493$

$-0.05518948$

$-0.16102493$

$-0.05518948$

$-0.16102493$

$-0.05518948$

1.39116621

1. 49700165

1. 39116621

1. 49700165

1.39116621

1.49700165

1.39116621

1.49700165

$-0.74076766$

$-0.63493228$

$-0.74076766$

$-0.63493228$

$-0.74076766$

$-0.63493228$

$-0.74076766$

$-0.63493228$

28.73770905

56.62825012

$-40.49345016$

20.63418579

$-1.89737391$

$-17.27157021$

0.43228209

$-13.13195515$

$-0.78577799$

10.24132061

2.93298697

75.86126709

$-58.28487396$

$-103.70518494$

78.28033447

$-88.85495758$

69.58952332

119.25829315

$-92.62053680$

9.55616951

$-6.38122034$

$-7.30594206$

4.71539068

$-6.91713381$

4. 29868364

4.76507473

$-2.80595732$

6.92015648

$-4.26023817$

$-7.83829880$

6.53735590

$-7.01044273$

2.17783570

8.01734829

$-5.00078726$

\# Electr. mom.

$-0.34768584$

$-0.24185041$

$-0.34768584$

$-0.24185041$

$-0.34768584$

$-0.24185041$

$-0.34768584$

$-0.24185041$

0.17077343

0.27660885

0.17077343

0.27660885

0.17077343

0.27660885

0.17077343

0.27660885

$-1.49057686$

$-1.38474143$

$-1.49057686$

$-1.38474143$

$-1.49057686$

$-1.38474143$

$-1.49057686$

$-1.38474143$

0.66284108

0.76867646

0.66284108

0.76867646

0.66284108

0.76867646
76.59670258

$-89.62718201$

$-69.66665649$

80.87073517

$-70.26455688$

84.54455566

66.94731903

$-78.69046021$

86.70181274

$-42.13638306$

$-119.44223785$

71.50885010

$-119.54069519$

76.35063934

152.61793518

$-105.21691132$

$-29.30552101$

36.03576279

35.07785034

$-43.18844223$

34.56989288

$-42.27711868$

$-39.66571426$

48.49403763

22.50372696

$-13.08730221$

$-36.38013840$

24.15608978

$-33.35145950$

23.30566788 


0.67859572
0.67859572
-1.32129574
-1.32129574
-1.32129574
-1.32129574
-1.21546030
-1.21546030
-1.21546030
-1.21546030
2.26337528
2.26337528
2.26337528
2.26337528
2.36921072
2.36921072
2.36921072
2.36921072
-1.48753464
-1.48753464
-1.48753464
-1.48753464
-1.38169920
-1.38169920
-1.38169920
-1.38169920
2.46758628
2.46758628
2.46758628
2.46758628
2.57342172
2.57342172
2.57342172
2.57342172
-1

64

0

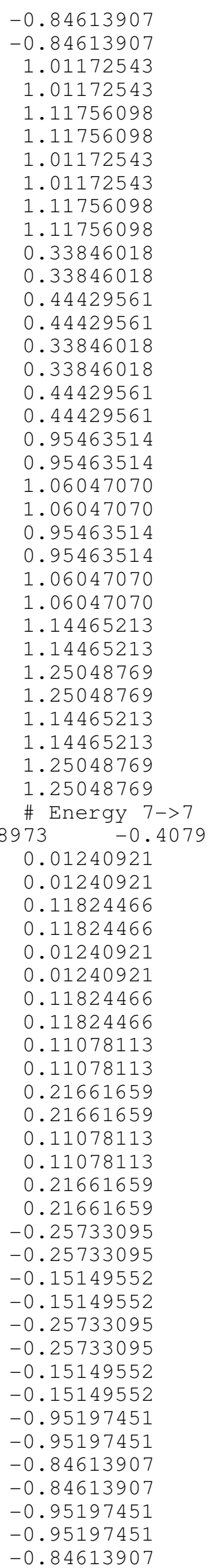

$-0.84613907$

0.84613907

1.01172543

1.11756098

1.11756098

1. 01172543

1.11756098

1.11756098

0.33846018

0.33846018

0.44429561

0.44429561

0. 4442956

0.44429561

0.95463514

1. 06047070

1.06047070

0.95463514

.95463514

1.14465213

1.14465213

1.25048769

.25048769

1.14465213

1. 25048769

$-5.9764$

$-1.93576097$

$-1.93576097$

$-1.93576097$

$-1.93576097$

$-1.82992566$

$-1.82992566$

$-1.82992566$

$-1.82992566$

0.96153939

0.96153939

0.96153939

0.96153939

1.06737483

1.06737483

1.06737483

1.06737483

$-1.56185186$

$-1.56185186$

$-1.56185186$

$-1.56185186$

$-1.45601630$

$-1.45601630$

$-1.45601630$

$-1.45601630$

0.57276028

0.57276028

0.57276028

0.57276028

0.67859572

0.67859572

0.67859572
0.66284108

0.76867646

0.39045236

0.49628779

0.39045236

0.49628779

0.39045236

0.49628779

0.39045236

0.49628779

$-0.16102493$

$-0.05518948$

$-0.16102493$

$-0.05518948$

$-0.16102493$

$-0.05518948$

$-0.16102493$

$-0.05518948$

1. 39116621

1.49700165

1. 39116621

1.49700165

1. 39116621

1. 49700165

1.39116621

1.49700165

$-0.74076766$

$-0.63493228$

$-0.74076766$

$-0.63493228$

$-0.74076766$

$-0.63493228$

$-0.74076766$

$-0.63493228$

\# Electr. mom.

$-0.34768584$

$-0.24185041$

$-0.34768584$

$-0.24185041$

$-0.34768584$

$-0.24185041$

$-0.34768584$

$-0.24185041$

0.17077343

0.27660885

0.17077343

0.27660885

0.17077343

0.27660885

0.17077343

0.27660885

$-1.49057686$

$-1.38474143$

$-1.49057686$

$-1.38474143$

$-1.49057686$

$-1.38474143$

$-1.49057686$

$-1.38474143$

0.66284108

0.76867646

0.66284108

0.76867646

0.66284108

0.76867646

0.66284108
47.21606064

$-35.12998199$

15.34741783

$-4.61112881$

$-13.51356697$

2.18341947

$-17.07808495$

7.83120537

13.53640938

$-3.33874226$

103.57316589

$-81.87544250$

$-137.19639587$

108.11253357

$-116.78516388$

93.82916260

152.45512390

$-122.66130066$

7.35521889

$-4.63154316$

$-4.12734270$

2.28350377

$-6.88957119$

4. 21131182

3. 68693018

$-1.93517208$

6.72876406

$-1.84445167$

$-8.52009392$

5.88255358

$-8.90260220$

2.56260943

10.20088005

$-6.39649820$

83.30580139

$-109.27619171$

$-93.51834106$

118.97896576

$-95.29623413$

125.65296173

114.20551300

$-143.75305176$

82.77605438

$-39.36894608$

$-120.12236023$

71.46434021

$-116.76916504$

75.18061066

154.40298462

$-106.68685913$

$-34.32995987$

42.92473984

39.00818634

$-48.96219635$

35.24807358

$-43.75038910$

$-38.88148117$

48.31760788

28.30554962

$-18.34626007$

$-43.30136108$

30.51001358

$-38.45720291$

28.07310104

53.29616547 


\begin{tabular}{|c|c|c|c|}
\hline 0.67859572 & -0.84613907 & 0.76867646 & -40.90268707 \\
\hline-1.32129574 & 1.01172543 & 0.39045236 & -11.57616234 \\
\hline-1.32129574 & 1.01172543 & 0.49628779 & 23.36064339 \\
\hline-1.32129574 & 1.11756098 & 0.39045236 & 6.63171625 \\
\hline-1.32129574 & 1.11756098 & 0.49628779 & -17.72738457 \\
\hline-1.21546030 & 1.01172543 & 0.39045236 & 14.8469495 \\
\hline-1.21546030 & 1.01172543 & 0.49628779 & -21.6994400 \\
\hline-1.21546030 & 1.11756098 & 0.39045236 & -10.5196571 \\
\hline-1.21546030 & 1.11756098 & 0.49628779 & 17.884460 \\
\hline 2.26337528 & 0.33846018 & -0.16102493 & 101.6654205 \\
\hline 2.26337528 & 0.33846018 & -0.05518948 & -82.3663177 \\
\hline 2.26337528 & 0.44429561 & -0.16102493 & -134.944 \\
\hline 2.26337528 & 0.44429561 & -0.05518948 & 107.7155532 \\
\hline 2.36921072 & 0.33846018 & -0.16102493 & -115.2797546 \\
\hline 2.36921072 & 0.33846018 & -0.05518948 & 94.1278 \\
\hline 2.36921072 & 0.44429561 & -0.16102493 & 150.76 \\
\hline 2.36921072 & 0.44429561 & -0.05518948 & -122.356 \\
\hline-1.48753464 & 0.95463514 & 1.39116621 & 8.470 \\
\hline-1.48753464 & 0.95463514 & 1.49700165 & -5.66 \\
\hline 53464 & 1.06047070 & 1.391166 & $-6 \cdot 9$ \\
\hline-1.48753464 & 1.06047070 & 1.4970 & 53 \\
\hline-1.38169920 & 0.95463514 & 1.39116621 & -6.7 \\
\hline-1.38169920 & 0.95463514 & 1.497001 & 4.2 \\
\hline-1.38169920 & 1.06047070 & 1.39116621 & 5.1 \\
\hline-1.38169920 & 1.06047070 & 1.49700165 & -3.13 \\
\hline 2.46758628 & 1.14465213 & -0.74076766 & 6.63 \\
\hline 58628 & 1.14465213 & -0.63493228 & -2.0 \\
\hline 2.46758628 & 1.25048769 & -0.74076766 & $-8.15 \varepsilon$ \\
\hline 2.46758628 & 1.25048769 & -0.63493228 & 5.667 \\
\hline 2.57342172 & 1.14465213 & -0.740767 & -8.46 \\
\hline 42172 & 1.1446 & -0.634932 & 2.2 \\
\hline 2.57342172 & 1.25048769 & -0.74076766 & 9.61 \\
\hline 7342172 & 1.25048769 & -0.63493228 & -5 \\
\hline & \# Energy 8->8 & \multirow{2}{*}{\multicolumn{2}{|c|}{ \# Electr. mom. }} \\
\hline 5. & 1.2959 & & \\
\hline-1.93576097 & 0.01240921 & -0.34768584 & 114.333183 \\
\hline-1.93576097 & 0.01240921 & -0.24185041 & -134.47 \\
\hline-1.93576097 & 0.11824466 & -0.34768584 & -109.11 \\
\hline-1.93576097 & 0.11824466 & -0.24185041 & 127.462 \\
\hline-1.82992566 & 0.01240921 & -0.34768584 & -117.72 \\
\hline 2992566 & 0.01240921 & -0.24185041 & 140.01 \\
\hline 92566 & 0.11 & -0.34768584 & 118.3 \\
\hline-1.82992566 & 0.11824466 & -0.24185041 & -138.68 \\
\hline 6153939 & 0.11078113 & 0.17077343 & 51.092 \\
\hline 3939 & 0.11 & 0.2766 & -17.82 \\
\hline 3939 & 0.216 & 0.17077343 & -82.03 \\
\hline 0.96153939 & 0.21661659 & 0.27660885 & 45.48 \\
\hline 1.06737483 & 0.11078113 & 0.17077343 & -76.13 \\
\hline 483 & 0.11 & 0 . & 44 \\
\hline 37483 & 0.21661659 & 0.17077343 & 108.12 \\
\hline 1.06737483 & 0.21661659 & 0.27660885 & -72.68 \\
\hline 85186 & -0.25 & -1.49057686 & $-26 \cdot 2$ \\
\hline 35186 & -0.25733095 & -1.38474143 & 31.3 \\
\hline-1.56185186 & -0.15149552 & -1.49057686 & 31.7 \\
\hline 35186 & -0.15 & -1.38474143 & -38.2 \\
\hline 1630 & -0.25 & -1.49057686 & 30. \\
\hline 1630 & -0.25 & -1.38474143 & -36 \\
\hline 1630 & -0.15149552 & -1.49057686 & -34.77 \\
\hline 1630 & -0 . & -1.3847 & 41. \\
\hline 028 & -0 . & $0.662 \varepsilon$ & \\
\hline 6028 & -0.95197451 & 0.76867646 & -0.93 \\
\hline 6028 & -0.84613907 & 0.66284108 & -20.5 \\
\hline 6028 & 13907 & 0.76867646 & 12.3 \\
\hline 9572 & 97451 & 0.66284108 & -15.451 \\
\hline 9572 & 5197451 & 0.76867646 & 9.02703 \\
\hline 9572 & -0 . & 0.66 & 30. \\
\hline 572 & -0 . & 0.76 & -21 \\
\hline
\end{tabular}

\section{S-136}




\begin{tabular}{|c|c|c|c|}
\hline-1.32129574 & 1.01172543 & 0.39045236 & 36.75796890 \\
\hline-1.32129574 & 1.01172543 & 0.49628779 & -17.02089119 \\
\hline-1.32129574 & 1.11756098 & 0.39045236 & -33.59514236 \\
\hline-1.32129574 & 1.11756098 & 0.49628779 & 14.34205055 \\
\hline-1.21546030 & 1.01172543 & 0.39045236 & -33.2976341 \\
\hline-1.21546030 & 1.01172543 & 0.49628779 & 17.1013698 \\
\hline-1.21546030 & 1.11756098 & 0.39045236 & 28.0 \\
\hline-1.21546030 & 1.11756098 & 0.49628779 & $-11.9193^{-}$ \\
\hline 2.26337528 & 0.33846018 & -0.16102493 & 71.31842 \\
\hline 2.26337528 & 0.33846018 & -0.05518948 & -59.4 \\
\hline 2.26337528 & 0.44429561 & -0.16102493 & $-99.119^{-}$ \\
\hline 2.26337528 & 0.44429561 & -0.05518948 & 80.5777 \\
\hline 2.36921072 & 0.33846018 & -0.16102493 & -81.1 \\
\hline 2.36921072 & 0.33846018 & -0.05518948 & 68.2 \\
\hline 2.36921072 & 0.44429561 & -0.16102493 & 110.33 \\
\hline 2.36921072 & 0.44429561 & -0.05518948 & $-91 \cdot 22$ \\
\hline-1.48753464 & 0.95463514 & 1.39116621 & 10 \\
\hline-1.48 & 0.95463514 & 1.497001 & \\
\hline-1. & 1.060470 & 1.3911 & -7.5 \\
\hline-1.48 & 1.06047070 & 1.49700165 & 4.7 \\
\hline$-1 \cdot 38$ & 0.95463514 & 1.391166 & -9.6 \\
\hline-1.3 & 0.95 & 1.49700 & 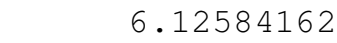 \\
\hline-1. & 1.06047070 & 1.39116 & \\
\hline 69920 & 1.06047070 & 1.49700165 & -3.68 \\
\hline 2. & 1.1446 & -0.74076 & 10 \\
\hline 2. & 1.144652 & -0.63493228 & -7 \\
\hline 2.46758628 & 1.25048769 & -0.74076766 & -10.64 \\
\hline 2.4 & 1.2504 & -0.634932 & 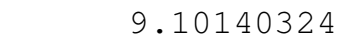 \\
\hline 2.5 & 1.144 & -0.74 & -11.5 \\
\hline 2.573 & 1.14465213 & -0.634932 & 2 \\
\hline 2.573 & 1.25048769 & -0.74076766 & 11.57 \\
\hline 2.573 & 1.25048769 & -0.63493228 & 0 \\
\hline & \# Energy 9->9 & & \\
\hline 7. & 2.4196 & & Electr. \\
\hline 6097 & 0.01240921 & -0.34768584 & 105.7 \\
\hline 6097 & 0.012 & -0.24185041 & -126.0 \\
\hline-1. & 0.11824466 & -0.34768584 & $-101 \cdot 9$ \\
\hline-1.93576097 & 0.11824466 & -0.24185041 & 122.04 \\
\hline 992566 & 0.01240921 & -0.34768584 & -114.61 \\
\hline-1. & 0.01 & -0.24185 & 13 \\
\hline$-1 . \varepsilon$ & 0.11824466 & -0.34768584 & 116.9 \\
\hline 2566 & 0.11824466 & -0.24185041 & -140.42 \\
\hline 0 . & 0.11 & 0.170773 & 94.5 \\
\hline 0. & 0.11 & 0.27 & -34 \\
\hline 0. & 0.21661659 & 0.170773 & $-124 \cdot 9$ \\
\hline 0 . & 0.21661659 & 0.276608 & 62. \\
\hline 1. & 0.11 & 0.17 & -119. \\
\hline 1. & 0.11 & 0.276 & 6 \\
\hline 1. & 0.216616 & 0.17077 & 151. \\
\hline 1 . & 0.21661659 & 0.276608 & -89. \\
\hline-1 & -0.257 & -1.49057 & -25 \\
\hline-1.5 & -0.2573 & -1.38474143 & 3 \\
\hline-1. & -0.1514 & -1.490576 & 30. \\
\hline-1 & -0.15 & -1.384741 & -36 \\
\hline-1 & -0 . & -1.490 & \\
\hline-1. & -0.25733 & -1.384741 & -34 \\
\hline-1 . & -0 . & -1.490 & -3 \\
\hline-1 . & -0 . & -1.384 & 3 \\
\hline 0 & -0 . & 0.662841 & 3 \\
\hline 0. & -0.95197451 & 0.768676 & -19 \\
\hline 0 . & -0.846139 & 0.66284 & -47 . \\
\hline 0 . & -0 . & 0.76867 & 29. \\
\hline 0. & -0.95197451 & 0.66284108 & -43.419 \\
\hline 0 . & -0 . & 0.76867 & \\
\hline & -0 . & 0.662 & \\
\hline 0 . & 1613907 & 0.76867646 & -39.9 \\
\hline$-1 \cdot 3$ & 1 & 0.39045236 & \\
\hline
\end{tabular}

\section{S-137}




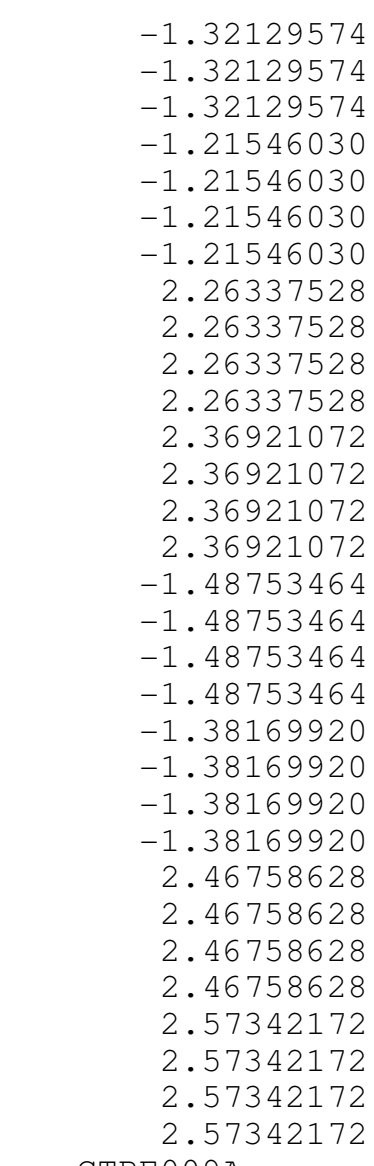

-CTBE009A-

\# phi $=-135$, psi=

6

2.1906
2.3499
1.0719
-1.3259
-1.5072
-2.2385

1.01172543

1. 11756098

1. 11756098

1.01172543

1.01172543

1. 11756098

1.11756098

0.33846018

0.33846018

0.44429561

0.44429561

0.33846018

0.33846018

0.44429561

0.44429561

0.95463514

0.95463514

1.06047070

1.06047070

0.95463514

0.95463514

1. 06047070

1.06047070

1.14465213

1. 14465213

1. 25048769

1. 25048769

1. 14465213

1. 14465213

1.25048769

1. 25048769
0.49628779

0.39045236

0.49628779

0.39045236

0.49628779

0.39045236

0.49628779

$-0.16102493$

$-0.05518948$

$-0.16102493$

$-0.05518948$

$-0.16102493$

$-0.05518948$

$-0.16102493$

$-0.05518948$

1.39116621

1.49700165

1. 39116621

1. 49700165

1.39116621

1. 49700165

1.39116621

1. 49700165

$-0.74076766$

$-0.63493228$

$-0.74076766$

$-0.63493228$

$-0.74076766$

$-0.63493228$

$-0.74076766$

$-0.63493228$
$-6.45081997$

$-26.93885994$

6.86043072

$-16.57240868$

$-0.25246510$

15.53336811

0.41287640

57.70586777

$-42.01352310$

$-83.69207001$

61.48913574

$-66.11968231$

50.43356705

93.34595490

$-71.47920227$

12.04977608

$-8.22724628$

$-9.84797001$

6.53483391

$-9.31318378$

6.10689974

7. 42522669

$-4.74033880$

8.84442616

$-6.49552917$

$-9.11912823$

8.03074551

$-9.13177109$

5.28923225

9.29245377

$-6.94917250$

\&IRANSITION $1->$. . 64 45418 .
$-0.0517$
$-0.0373$
2.13764215
2.13764215
2.13764215
2.13764215
2.24347758
2. 24347758
2.24347758
2.24347758
$-1.37885487$
$-1.37885487$
$-1.37885487$
$-1.37885487$
$-1.27301943$
$-1.27301943$
$-1.27301943$
$-1.27301943$
2.29703021
2.29703021
2.29703021
2. 29703021
2.40286589
2.40286589
2.40286589

135, $\operatorname{root} s=9$

$\begin{array}{rr}0.2133 & 0.1040 \\ 1.4296 & 0.0695 \\ -0.3940 & -0.3763 \\ -0.2279 & -0.3376 \\ -1.4430 & -0.3142 \\ 0.6570 & 0.1190\end{array}$

1.0000

1.0000

1.0000

1.0000

1.0000

1.0000

$\begin{array}{ll}\# & \mathrm{C} 1 \\ \# & \mathrm{O} 1 \\ \# & \mathrm{~N} 1 \\ \# & \mathrm{C} 2 \\ \# & \mathrm{~N} 2 \\ \# & \mathrm{O} 2\end{array}$

\# Energy 1->2

$-0.0384 \quad-0.2022$

$\begin{array}{ll}-0.6871 & 0.0317\end{array}$

0.16034016

0.16034016

0.26617563

0.26617563

0.16034016

0.16034016

0.26617563

0.26617563

$-0.28076980$

$-0.28076980$

$-0.17493436$

$-0.17493436$

$-0.28076980$

$-0.28076980$

$-0.17493436$

$-0.17493436$

1.37668049

1.37668049

1. 48251593

1.48251593

1.37668049

1.37668049

1. 48251593

$\begin{array}{rr}1.000 \text { \# Electr. mom., scal } \\ \text { \# Magnetic mom. } \\ 0.05111535 & -24.85194778 \\ 0.15695079 & 16.62661362 \\ 0.05111535 & 23.11823082 \\ 0.15695079 & -15.01918221 \\ 0.05111535 & 19.55653191 \\ 0.15695079 & -12.97772789 \\ 0.05111535 & -18.05732918 \\ 0.15695079 & 11.59790325 \\ -0.39047247 & 14.40767479 \\ -0.28463703 & -45.51000595 \\ -0.39047247 & -20.64989090 \\ -0.28463703 & 50.85176086 \\ -0.39047247 & -6.21506214 \\ -0.28463703 & 35.10980988 \\ -0.39047247 & 11.30854702 \\ -0.28463703 & -38.95168304 \\ 0.01654314 & -2.78398728 \\ 0.12237859 & 0.79778975 \\ 0.01654314 & 1.43049872 \\ 0.12237859 & 0.10597668 \\ 0.01654314 & 2.05213094 \\ 0.12237859 & -0.37371892 \\ 0.01654314 & -0.89226210\end{array}$

\section{S-138}




2.40286589
-1.56014943
-1.56014943
-1.56014943
-1.56014943
-1.45431399
-1.45431399
-1.45431399
-1.45431399
1.01902342
1.01902342
1.01902342
1.01902342
1.12485886
1.12485886
1.12485886
1.12485886
-2.29137540
-2.29137540
-2.29137540
-2.29137540
-2.18554020
-2.18554020
-2.18554020
-2.18554020
0.88915640
0.88915640
0.88915640
0.88915640
0.99499184
0.99499184
0.99499184
0.99499184
-2.05025959
-2.05025959
-2.05025959
-2.05025959
-1.94442415
-1.94442415
-1.94442415
-1.94442415
-0.07139
-19

1. 48251593

$-1.49588871$

$-1.49588871$

$-1.39005339$

$-1.39005339$

$-1.49588871$

$-1.49588871$

$-1.39005339$

$-1.39005339$

$-0.44691080$

$-0.44691080$

$-0.34107536$

$-0.34107536$

$-0.44691080$

$-0.44691080$

$-0.34107536$

$-0.34107536$

0.60408223

0.60408223

0.70991772

0.70991772

0.60408223

0.60408223

0.70991772

$-1.44335949$

$-1.44335949$

$-1.33752406$

$-1.33752406$

$-1.44335949$

$-1.44335949$

$-1.33752406$

$-1.33752406$

1.58138645

1.58138645

1.68722177

1.68722177

1. 58138645

1. 58138645

1. 68722177

1. 68722177

64
0.70991772 \# Energy 1->3

$$
\begin{array}{rr}
0.12237859 & -0.41411760 \\
-0.36711088 & 3.63081431 \\
-0.26127544 & -10.20055389 \\
-0.36711088 & 1.27853048 \\
-0.26127544 & 7.47345877 \\
-0.36711088 & -4.49179173 \\
-0.26127544 & 11.45122814 \\
-0.36711088 & -0.50640869 \\
-0.26127544 & -8.68550682 \\
-0.42923152 & 23.65463638 \\
-0.32339609 & -38.55130386 \\
-0.42923152 & -26.11114311 \\
-0.32339609 & 39.03166580 \\
-0.42923152 & -24.68494034 \\
-0.32339609 & 40.16084671 \\
-0.42923152 & 27.04380417 \\
-0.32339609 & -40.50550079 \\
0.06612916 & -25.44069099 \\
0.17196462 & 26.03345299 \\
0.06612916 & 25.88723373 \\
0.17196462 & -25.53845215 \\
0.06612916 & 31.06114197 \\
0.17196462 & -30.40737343 \\
0.06612916 & -32.00873184 \\
0.17196462 & 29.94662857 \\
-0.32880956 & -3.37834239 \\
-0.22297411 & 1.02431166 \\
-0.32880956 & 4.01801491 \\
-0.22297411 & -0.25414994 \\
-0.32880956 & 3.21949029 \\
-0.22297411 & -0.88618410 \\
-0.32880956 & -3.82881045 \\
-0.22297411 & 0.09500257 \\
0.09555512 & -1.46933603 \\
0.20139056 & 2.50643849 \\
0.09555512 & 1.30623245 \\
0.20139056 & -1.98617017 \\
0.09555512 & 3.07237482 \\
0.20139056 & -3.34642243 \\
0.09555512 & -2.35645080 \\
0.20139056 & 2.47641230
\end{array}
$$

$\begin{array}{lr}0.0748 & 0.1806 \\ 0.9456 & -0.0675\end{array}$

0.1769

2.13764215

2.13764215

2.13764215

2.13764215

2. 24347758

2. 24347758

2. 24347758

2. 24347758

$-1.37885487$

$-1.37885487$

$-1.37885487$

$-1.37885487$

$-1.27301943$

$-1.27301943$

$-1.27301943$

$-1.27301943$

2. 29703021

2. 29703021

2. 29703021

2. 29703021

2. 40286589

2.40286589

2. 40286589

$$
1.000 \text { \# Electr. mom., scale-fac }
$$$$
\text { \# Magnetic mom. }
$$

0.16034016

0.16034016

0.26617563

0.26617563

0.16034016

0.16034016

0.26617563

0.26617563

$-0.28076980$

$-0.28076980$

$-0.17493436$

$-0.17493436$

$-0.28076980$

$-0.28076980$

$-0.17493436$

$-0.17493436$

1. 37668049

1.37668049

1. 48251593

1.48251593

1.37668049

1.37668049

1.48251593
$-71.91925812$

48.64491653

75.59632874

$-51.70203400$

62.65447998

$-42.15202713$

$-65.38680267$

44.37194824

$-3.81213641$

8.79362869

6.70737553

$-12.58193684$

1.90633774

$-5.79747200$

$-4.42686415$

9.07905579

$-18.86909676$

8.44097424

9.91980648

$-1.71829796$

15.57333183

$-6.40877247$

$-7.47111130$ 


2.40286589
-1.56014943
-1.56014943
-1.56014943
-1.56014943
-1.45431399
-1.45431399
-1.45431399
-1.45431399
1.01902342
1.01902342
1.01902342
1.01902342
1.12485886
1.12485886
1.12485886
1.12485886
-2.29137540
-2.29137540
-2.29137540
-2.29137540
-2.18554020
-2.18554020
-2.18554020
-2.18554020
0.88915640
0.88915640
0.88915640
0.88915640
0.99499184
0.99499184
0.99499184
0.99499184
-2.05025959
-2.05025959
-2.05025959
-2.05025959
-1.94442415
-1.94442415
-1.94442415
-1.94442415
-1
-1553734

1. 48251593

$-1.49588871$

$-1.49588871$

$-1.39005339$

$-1.39005339$

$-1.49588871$

$-1.49588871$

$-1.39005339$

$-1.39005339$

$-0.44691080$

$-0.44691080$

$-0.34107536$

$-0.34107536$

$-0.44691080$

$-0.44691080$

$-0.34107536$

$-0.34107536$

0.60408223

0.60408223

0.70991772

0.70991772

0.60408223

0.60408223

0.70991772

$-1.44335949$

$-1.44335949$

$-1.33752406$

$-1.33752406$

$-1.44335949$

$-1.44335949$

$-1.33752406$

$-1.33752406$

1.58138645

1.58138645

1.68722177

1.68722177

1. 58138645

1. 58138645

1.68722177

1. 68722177

64
0.70991772

0.12237859

$-0.36711088$

$-0.26127544$

$-0.36711088$

$-0.26127544$

$-0.36711088$

$-0.26127544$

$-0.36711088$

$-0.26127544$

$-0.42923152$

$-0.32339609$

$-0.42923152$

$-0.32339609$

$-0.42923152$

$-0.32339609$

$-0.42923152$

$-0.32339609$

0.06612916

0.17196462

0.06612916

0.17196462

0.06612916

0.17196462

0.06612916

0.17196462

$-0.32880956$

$-0.22297411$

$-0.32880956$

$-0.22297411$

$-0.32880956$

$-0.22297411$

$-0.32880956$

$-0.22297411$

0.09555512

0.20139056

0.09555512

0.20139056

0.09555512

0.20139056

0.09555512

0.20139056

\# Energy 1->4

$\begin{array}{lrl}-2.3553 & 2.0740 & 0.4661 \\ -0.2416 & -0.3972 & 1.1739\end{array}$

2.13764215

2. 13764215

2.13764215

2.13764215

2. 24347758

2.24347758

2. 24347758

2.24347758

$-1.37885487$

$-1.37885487$

$-1.37885487$

$-1.37885487$

$-1.27301943$

$-1.27301943$

$-1.27301943$

$-1.27301943$

2. 29703021

2. 29703021

2.29703021

2. 29703021

2. 40286589

2. 40286589

2. 40286589
0.16034016

0.16034016

0.26617563

0.26617563

0.16034016

0.16034016

0.26617563

0.26617563

$-0.28076980$

$-0.28076980$

$-0.17493436$

$-0.17493436$

$-0.28076980$

$-0.28076980$

$-0.17493436$

$-0.17493436$

1. 37668049

1.37668049

1. 48251593

1. 48251593

1.37668049

1.37668049

1. 48251593
0.05111535

0.15695079

0.05111535

0.15695079

0.05111535

0.15695079

0.05111535

0.15695079

$-0.39047247$

$-0.28463703$

$-0.39047247$

$-0.28463703$

$-0.39047247$

$-0.28463703$

$-0.39047247$

$-0.28463703$

0.01654314

0.12237859

0.01654314

0.12237859

0.01654314

0.12237859

0.01654314

0.26114970

$-0.08769230$

0.56056917

$-0.69358492$

0.06083557

0.18188629

$-0.64413375$

0.60882598

$-0.01217819$

$-31.39284515$

31.20100403

33.43367767

$-34.85068893$

35.62724686

$-35.22602844$

$-37.89516449$

39.28773117

1.69351530

$-3.57129622$

$-2.20127344$

3. 70484352

$-1.36843693$

3.81645250

2. 15158057

$-4.07833099$

$-4.19892168$

3. 30388379

6.05988312

$-4.97229958$

5.03185940

$-4.13434076$

$-7.25440931$

6.15432262

$-0.48485449$

0.04024265

0.38594598

$-0.06457074$

0.46608049

$-0.01308419$

$-0.39123234$

0.06146589

$$
1.000 \text { \# Electr. mom., scale-fac }
$$
\# Magnetic mom.

10.02905369

$-5.64962482$

$-8.95302868$

4.92920399

$-6.17560005$

3. 42371917

5.10742044

$-2.76146793$

$-9.71318722$

2.07176781

4.25663042

5.64557552

16.04125023

$-13.28307533$

$-11.33733749$

6.24470854

3. 95077777

$-2.79275417$

$-3.36759067$

2.57989526

$-2.62823582$

2. 20321584

2. 39479303 


2.40286589
-1.56014943
-1.56014943
-1.56014943
-1.56014943
-1.45431399
-1.45431399
-1.45431399
-1.45431399
1.01902342
1.01902342
1.01902342
1.01902342
1.12485886
1.12485886
1.12485886
1.12485886
-2.29137540
-2.29137540
-2.29137540
-2.29137540
-2.18554020
-2.18554020
-2.18554020
-2.18554020
0.88915640
0.88915640
0.88915640
0.88915640
0.99499184
0.99499184
0.99499184
0.99499184
-2.05025959
-2.05025959
-2.05025959
-2.05025959
-1.94442415
-1.94442415
-1.94442415
-1.94442415
1.6612
-19

$-1.39005339$

64

1. 661253948

$-0.0493$

2. 13764215

2.13764215

2.13764215

2.13764215

2. 24347758

2. 24347758

2. 24347758

2. 24347758

$-1.37885487$

$-1.37885487$

$-1.37885487$

$-1.37885487$

$-1.27301943$

$-1.27301943$

$-1.27301943$

$-1.27301943$

2. 29703021

2. 29703021

2. 29703021

2. 29703021

2. 40286589

2.40286589

2. 40286589
1. 48251593

$-1.49588871$

$-1.49588871$

$-1.39005339$

$-1.39005339$

$-1.49588871$

$-1.49588871$

$-1.39005339$

$-0.44691080$

$-0.44691080$

$-0.34107536$

$-0.34107536$

$-0.44691080$

$-0.44691080$

$-0.34107536$

$-0.34107536$

0.60408223

0.60408223

0.70991772

0.70991772

0.60408223

0.60408223

0.70991772

0.70991772

$-1.44335949$

$-1.44335949$

$-1.33752406$

$-1.33752406$

$-1.44335949$

$-1.44335949$

$-1.33752406$

$-1.33752406$

1.58138645

1.58138645

1.68722177

1.68722177

1. 58138645

1. 58138645

1. 68722177

1. 68722177

0.12237859

$-0.36711088$

$-0.26127544$

$-0.36711088$

$-0.26127544$

$-0.36711088$

$-0.26127544$

$-0.36711088$

$-0.26127544$

$-0.42923152$

$-0.32339609$

$-0.42923152$

$-0.32339609$

$-0.42923152$

$-0.32339609$

$-0.42923152$

$-0.32339609$

0.06612916

0.17196462

0.06612916

0.17196462

0.06612916

0.17196462

0.06612916

0.17196462

$-0.32880956$

$-0.22297411$

$-0.32880956$

$-0.22297411$

$-0.32880956$

$-0.22297411$

$-0.32880956$

$-0.22297411$

0.09555512

0.20139056

0.09555512

0.20139056

0.09555512

0.20139056

0.09555512

0.20139056

\# Energy 1->5

$$
\begin{array}{ll}
3.0387 & 0.9708
\end{array}
$$

$0.0130-0.7083$

0.16034016

0.16034016

0.26617563

0.26617563

0.16034016

0.16034016

0.26617563

0.26617563

$-0.28076980$

$-0.28076980$

$-0.17493436$

$-0.17493436$

$-0.28076980$

$-0.28076980$

$-0.17493436$

$-0.17493436$

1. 37668049

1.37668049

1. 48251593

1. 48251593

1.37668049

1.37668049

1. 48251593

0.05111535

0.15695079

0.05111535

0.15695079

0.05111535

0.15695079

0.05111535

0.15695079

$-0.39047247$

$-0.28463703$

$-0.39047247$

$-0.28463703$

$-0.39047247$

$-0.28463703$

$-0.39047247$

$-0.28463703$

0.01654314

0.12237859

0.01654314

0.12237859

0.01654314

0.12237859

0.01654314
-2.09260345
1.64756727

$-0.49853727$

$-0.18267621$

$-1.33455038$

$-1.13167512$

$-1.40959179$

$-0.68013132$

4. 26477289

1. 91108048

0.06049235

$-2.23831224$

0.94252777

$-1.28529119$

$-1.48517191$

1. 73049450

0.15140434

0.15275720

$-6.41996384$

$-2.68152571$

9.35115242

4.55969000

1.85936189

$-3.15141511$

$-4.38233852$

0.56790692

0.08775823

$-0.62774998$

$-0.29553515$

$-0.71166378$

0.02690523

0.81093192

0.17938258

3. 22153187

$-4.12224817$

$-2.45536304$

3.18782115

$-1.59501052$

2.68901300

1.32620370

$-2.16350961$
1.000 \# Electr. mom., scale-fac \# Magnetic mom.

$-16.93791771$

12.34496403

16.57246017

$-11.66054440$

11.00752068

$-8.47784901$

$-10.12110043$

7.59467793

9.44023323

$-12.97263432$

$-11.68400288$

15.90832710

$-8.49205494$

10.34294891

10.47421265

$-13.12288952$

$-7.69897223$

5.65015030

6.79136181

$-5.39122629$

5.26548767

$-4.59172249$

$-4.98150969$ 
2. 40286589

$-1.56014943$

$-1.56014943$

$-1.56014943$

$-1.56014943$

$-1.45431399$

$-1.45431399$

1.01902342

1.01902342

1. 01902342

1.01902342

1.12485886

1. 12485886

1. 12485886

1. 12485886

$-2.29137540$

$-2.29137540$

$-2.29137540$

$-2.29137540$

$-2.18554020$

$-2.18554020$

$-2.18554020$

$-2.18554020$

0.88915640

0.88915640

0.88915640

0.88915640

0.99499184

0.99499184

0.99499184

0.99499184

$-2.05025959$

$-2.05025959$

$-2.05025959$

$-2.05025959$

$-1.94442415$

$-1.94442415$

$-1.94442415$

$-1.94442415$

64
$-1.45431399$

$-1.45431399$

1.48251593

$-1.49588871$

$-1.49588871$

$-1.39005339$

$-1.39005339$

$-1.49588871$

$-1.49588871$

$-1.39005339$

$-1.39005339$

$-0.44691080$

$-0.44691080$

$-0.34107536$

$-0.34107536$

$-0.44691080$

$-0.44691080$

$-0.34107536$

$-0.34107536$

0.60408223

0.60408223

0.70991772

0.70991772

0.60408223

0.60408223

0.70991772

0.70991772

$-1.44335949$

$-1.44335949$

$-1.33752406$

$-1.33752406$

$-1.44335949$

$-1.44335949$

$-1.33752406$

$-1.33752406$

1.58138645

1.58138645

1.68722177

1.68722177

1. 58138645

1. 58138645

1.68722177

1. 68722177

\# Energy 1->6

$\begin{array}{llr}0.5990 & 2.3782 & 0.4625 \\ 0.0972 & 0.2117 & -0.5555\end{array}$

2.13764215

2.13764215

2.13764215

2.13764215

2. 24347758

2. 24347758

2.24347758

2.24347758

$-1.37885487$

$-1.37885487$

$-1.37885487$

$-1.37885487$

$-1.27301943$

$-1.27301943$

$-1.27301943$

$-1.27301943$

2. 29703021

2. 29703021

2. 29703021

2. 29703021

2. 40286589

2.40286589

2. 40286589
0.12237859

$-0.36711088$

$-0.26127544$

$-0.36711088$

$-0.26127544$

$-0.36711088$

$-0.26127544$

$-0.36711088$

$-0.26127544$

$-0.42923152$

$-0.32339609$

$-0.42923152$

$-0.32339609$

$-0.42923152$

$-0.32339609$

$-0.42923152$

$-0.32339609$

0.06612916

0.17196462

0.06612916

0.17196462

0.06612916

0.17196462

0.06612916

0.17196462

$-0.32880956$

$-0.22297411$

$-0.32880956$

$-0.22297411$

$-0.32880956$

$-0.22297411$

$-0.32880956$

$-0.22297411$

0.09555512

0.20139056

0.09555512

0.20139056

0.09555512

0.20139056

0.09555512

0.20139056
1.000 \# Electr. mom., scale-fac \# Magnetic mom.

0.05111535

0.15695079

0.05111535

0.15695079

0.05111535

0.15695079

0.05111535

0.15695079

$-0.39047247$

$-0.28463703$

$-0.39047247$

$-0.28463703$

$-0.39047247$

$-0.28463703$

$-0.39047247$

$-0.28463703$

0.01654314

0.12237859

0.01654314

0.12237859

0.01654314

0.12237859

0.01654314
4. 47662544

2. 70122933

$-2.56158280$

$-2.30188465$

2.15517449

$-2.88930178$

2. 26300716

2. 42925572

$-1.53737593$

$-11.58023167$

9.92393112

7. 17687368

$-3.22362447$

9. 74199867

$-6.15929413$

$-5.34395742$

$-0.26454121$

$-3.80378723$

2.08921409

2. 70530128

$-0.92177540$

5.28766870

$-3.36949110$

$-4.40651035$

2. 22358060

$-2.04499698$

2. 99045682

2. 81308484

$-4.14671326$

2. 16925240

$-2.91795182$

$-2.97884846$

4.00451803

0.82710451

$-0.84533733$

$-0.64597666$

0.67798150

$-0.46768224$

0.58059722

0.41594508

$-0.50185490$

18.63413429

$-11.71308613$

$-18.13947868$

11.17878342

$-13.49692822$

8.67209625

12.78063202

$-8.09935093$

8.29699516

$-7.63603306$

$-6.63802195$

4. 74618149

$-10.57099056$

11.58790207

9.05492687

$-8.88094044$

8.39221478

$-6.33468246$

$-7.07685232$

5.63410187

$-6.22735262$

5. 22972536

5.47662306 


2.40286589
-1.56014943
-1.56014943
-1.56014943
-1.56014943
-1.45431399
-1.45431399
-1.45431399
-1.45431399
1.01902342
1.01902342
1.01902342
1.01902342
1.12485886
1.12485886
1.12485886
1.12485886
-2.29137540
-2.29137540
-2.29137540
-2.29137540
-2.18554020
-2.18554020
-2.18554020
-2.18554020
0.88915640
0.88915640
0.88915640
0.88915640
0.99499184
0.99499184
0.99499184
0.99499184
-2.05025959
-2.05025959
-2.05025959
-2.05025959
-1.94442415
-1.94442415
-1.94442415
-1.94442415
-0.1516
-19495
-1

1. 48251593

$-1.49588871$

$-1.49588871$

$-1.39005339$

$-1.39005339$

$-1.49588871$

$-1.49588871$

$-1.39005339$

$-1.39005339$

$-0.44691080$

$-0.44691080$

$-0.34107536$

$-0.34107536$

$-0.44691080$

$-0.44691080$

$-0.34107536$

$-0.34107536$

0.60408223

0.60408223

0.70991772

0.70991772

0.60408223

0.60408223

0.70991772

0.70991772

$-1.44335949$

$-1.44335949$

$-1.33752406$

$-1.33752406$

$-1.44335949$

$-1.44335949$

$-1.33752406$

$-1.33752406$

1.58138645

1.58138645

1.68722177

1.68722177

1.58138645

1. 58138645

1.68722177

1. 68722177 \# Energy 1->7

$\begin{array}{lll}-0.1516 & -0.0178 & -0.0237\end{array}$

$-0.0143$

2.13764215

2. 13764215

2.13764215

2.13764215

2. 24347758

2.24347758

2. 24347758

2.24347758

$-1.37885487$

$-1.37885487$

$-1.37885487$

$-1.37885487$

$-1.27301943$

$-1.27301943$

$-1.27301943$

$-1.27301943$

2. 29703021

2. 29703021

2. 29703021

2. 29703021

2. 40286589

2.40286589

2. 40286589

0.16034016

0.16034016

0.26617563

0.26617563

0.16034016

0.16034016

0.26617563

0.26617563

$-0.28076980$

$-0.28076980$

$-0.17493436$

$-0.17493436$

$-0.28076980$

$-0.28076980$

$-0.17493436$

$-0.17493436$

1. 37668049

1.37668049

1. 48251593

1. 48251593

1.37668049

1.37668049

1. 48251593
0.12237859

$-0.36711088$

$-0.26127544$

$-0.36711088$

$-0.36711088$

$-0.26127544$

$-0.36711088$

$-0.26127544$

$-0.42923152$

$-0.32339609$

$-0.32339609$

$-0.42923152$

$-0.32339609$

$-0.42923152$

$-0.32339609$

0.06612916

0.06612916

0.17196462

0.06612916

0.17196462

0.06612916

0.17196462

$-0.32880956$

$-0.22297411$

$-0.32880956$

$-0.22297411$

$-0.32880956$

$-0.22297411$

$-0.32880956$

$-0.22297411$

0.09555512

0.20139056

0.09555512

0.20139056

0.09555512

0.20139056

0.09555512

0.20139056 $\begin{array}{rr}-0.0178 & -0.0237 \\ -0.0506 & 0.0874\end{array}$

0.05111535

0.15695079

0.05111535

0.15695079

0.05111535

0.15695079

0.05111535

0.15695079

$-0.39047247$

$-0.28463703$

$-0.39047247$

$-0.28463703$

$-0.39047247$

$-0.28463703$

$-0.39047247$

$-0.28463703$

0.01654314

0.12237859

0.01654314

0.12237859

0.01654314

0.12237859

0.01654314
$-0.26127544$

$-0.42923152$

0.17196462

$-4.72628021$

1.05646753

$-1.93061197$

$-1.45718098$

2.63564205

$-1.64114082$

2. 93382955

2. 20906067

$-3.98771715$

$-11.29231548$

12.39173603

13.47507954

$-14.94754696$

9.60506153

$-12.62962151$

$-12.27917671$

15.38608456

$-4.65996027$

4. 42120504

4. 25209332

$-4.44762039$

5. 21124887

$-4.34745073$

$-4.28264904$

4. 06994152

$-1.05241323$

0.89188814

1. 64849257

$-1.40278602$

0.03052732

$-0.06253377$

$-0.26870748$

0.31730282

$-1.14668214$

1. 55694616

0.92708391

$-1.25839818$

0.90507871

$-1.27108610$

$-0.74093258$

1. 03744435

1.000 \# Electr. mom., scale-fac \# Magnetic mom.

$-5.36657000$

2.73139262

5.34670973

$-2.87205482$

4.11229086

$-1.75177276$

$-4.14999294$

1. 92218065

$-0.88610744$

0.14060140

0.68820316

$-0.11953449$

1. 45983350

$-0.82808346$

$-1.27182019$

0.83411944

$-1.78723776$

0.97420824

1. 12524664

$-0.47024870$

1. 41603255

$-0.67122072$

$-0.86157990$ 


2.40286589
-1.56014943
-1.56014943
-1.56014943
-1.56014943
-1.45431399
-1.45431399
-1.45431399
-1.45431399
1.01902342
1.01902342
1.01902342
1.01902342
1.12485886
1.12485886
1.12485886
1.12485886
-2.29137540
-2.29137540
-2.29137540
-2.29137540
-2.18554020
-2.18554020
-2.18554020
-2.18554020
0.88915640
0.88915640
0.88915640
0.88915640
0.99499184
0.99499184
0.99499184
0.99499184
-2.05025959
-2.05025959
-2.05025959
-2.05025959
-1.94442415
-1.94442415
-1.94442415
-1.94442415
-0.19705

1. 48251593

$-1.49588871$

$-1.49588871$

$-1.39005339$

$-1.39005339$

$-1.49588871$

$-1.49588871$

$-1.39005339$

$-1.39005339$

$-0.44691080$

$-0.44691080$

$-0.34107536$

$-0.34107536$

$-0.44691080$

$-0.44691080$

$-0.34107536$

$-0.34107536$

0.60408223

0.60408223

0.70991772

0.70991772

0.60408223

0.60408223

0.70991772

0.70991772

$-1.44335949$

$-1.44335949$

$-1.33752406$

$-1.33752406$

$-1.44335949$

$-1.44335949$

$-1.33752406$

$-1.33752406$

1.58138645

1.58138645

1.68722177

1.68722177

1.58138645

1. 58138645

1.68722177

1. 68722177

64
0.12237859

$-0.36711088$

$-0.26127544$

$-0.36711088$

$-0.26127544$

$-0.36711088$

$-0.26127544$

$-0.36711088$

$-0.26127544$

$-0.42923152$

$-0.32339609$

$-0.42923152$

$-0.32339609$

$-0.42923152$

$-0.32339609$

$-0.42923152$

$-0.32339609$

0.06612916

0.17196462

0.06612916

0.17196462

0.06612916

0.17196462

0.06612916

0.17196462

$-0.32880956$

$-0.22297411$

$-0.32880956$

$-0.22297411$

$-0.32880956$

$-0.22297411$

$-0.32880956$

$-0.22297411$

0.09555512

0.20139056

0.09555512

0.20139056

0.09555512

0.20139056

0.09555512

0.20139056

$-0.4705-0.7289$

$\begin{array}{lll}-0.0036 & -0.1089 & -0.2540\end{array}$

2.13764215

2.13764215

0.16034016

0.16034016

0.26617563

0.26617563

0.16034016

0.16034016

0.26617563

0.26617563

$-0.28076980$

$-0.28076980$

$-0.17493436$

$-0.17493436$

$-0.28076980$

$-0.28076980$

$-0.17493436$

$-0.17493436$

1. 37668049

1.37668049

1. 48251593

1. 48251593

1.37668049

1.37668049

1. 48251593
1.000 \# Electr. mom., scale-fac \# Magnetic mom.

0.05111535

0.15695079

19.18833542

0.05111535

0.15695079

$-28.89512634$

$-18.70405388$

26.17233658

0.05111535

0.15695079

0.05111535

0.15695079

$-0.39047247$

$-0.28463703$

$-0.39047247$

$-0.28463703$

$-0.39047247$

$-0.28463703$

$-0.39047247$

$-0.28463703$

0.01654314

0.12237859

0.01654314

0.12237859

0.01654314

0.12237859

$-20.22211456$

30.45889091

18.48438072

$-27.20748329$

34.40778732

$-55.69316864$

$-40.48239136$

65.43572235

$-26.44262123$

42.71451187

30.74466705

$-49.90663910$

5.95753431

$-5.08668852$

$-4.67293119$

4.10326481

$-4.52111483$

4.88058138

0.01654314

3. 59910131

\section{S-144}




2.40286589
-1.56014943
-1.56014943
-1.56014943
-1.56014943
-1.45431399
-1.45431399
-1.45431399
-1.45431399
1.01902342
1.01902342
1.01902342
1.01902342
1.12485886
1.12485886
1.12485886
1.12485886
-2.29137540
-2.29137540
-2.29137540
-2.29137540
-2.18554020
-2.18554020
-2.18554020
-2.18554020
0.88915640
0.88915640
0.88915640
0.88915640
0.99499184
0.99499184
0.99499184
0.99499184
-2.05025959
-2.05025959
-2.05025959
-2.05025959
-1.94442415
-1.94442415
-1.94442415
-1.94442415
0.315391352.
-1

1. 48251593

$-1.49588871$

$-1.49588871$

$-1.39005339$

$-1.39005339$

$-1.49588871$

$-1.49588871$

$-1.39005339$

$-1.39005339$

$-0.44691080$

$-0.44691080$

$-0.34107536$

$-0.34107536$

$-0.44691080$

$-0.44691080$

$-0.34107536$

$-0.34107536$

0.60408223

0.60408223

0.70991772

0.70991772

0.60408223

0.60408223

0.70991772

0.70991772

$-1.44335949$

$-1.44335949$

$-1.33752406$

$-1.33752406$

$-1.44335949$

$-1.44335949$

$-1.33752406$

$-1.33752406$

1.58138645

1.58138645

1.68722177

1.68722177

1. 58138645

1. 58138645

1. 68722177

1. 68722177

64 \# Energy 1->9

$\begin{array}{lrl}0.3153 & -0.2234 & 0.0768 \\ 0.0150 & 0.0484 & 0.0013\end{array}$

2.13764215

2.13764215

2.13764215

2.13764215

2. 24347758

2. 24347758

2. 24347758

2.24347758

$-1.37885487$

$-1.37885487$

$-1.37885487$

$-1.37885487$

$-1.27301943$

$-1.27301943$

$-1.27301943$

$-1.27301943$

2. 29703021

2. 29703021

2. 29703021

2.29703021

2. 40286589

2.40286589

2. 40286589

0.16034016

0.16034016

0.26617563

0.26617563

0.16034016

0.16034016

0.26617563

0.26617563

$-0.28076980$

$-0.28076980$

$-0.17493436$

$-0.17493436$

$-0.28076980$

$-0.28076980$

$-0.17493436$

$-0.17493436$

1. 37668049

1.37668049

1. 48251593

1. 48251593

1.37668049

1.37668049

1. 48251593

0.12237859
-0.36711088
-0.26127544
-0.36711088
-0.26127544
-0.36711088
-0.26127544
-0.36711088
-0.26127544
-0.42923152
-0.32339609
-0.42923152
-0.32339609
-0.42923152
-0.32339609
-0.42923152
-0.32339609
0.06612916
0.17196462
0.06612916
0.17196462
0.06612916
0.17196462
0.06612916
0.17196462
-0.32880956
-0.22297411
-0.32880956
-0.22297411
-0.32880956
-0.22297411
-0.32880956
-0.22297411
0.09555512
0.20139056
0.09555512
0.20139056
0.09555512
0.20139056
0.09555512
0.20139056

$-3.89969635$

$-0.04389626$

$-0.50141776$

$-0.38843352$

1.18559611

0.45323813

$-0.14086960$

$-0.19773629$

$-0.30131444$

0.80834258

$-1.22079921$

0.52452165

$-0.19492891$

$-3.81343055$

4. 23354292

1. 36726415

$-1.16818392$

8.84034157

5.38466597

$-7.12637138$

$-3.65559673$

$-15.33594322$

$-2.19470429$

11.89690590

1. 42415476

0.71223617

$-0.53366023$

$-0.84935224$

0.54650980

$-1.21971250$

1. 22069347

1.58867669

$-1.60458279$

3. 82751226

$-3.52573228$

$-3.04165959$

2. 79593182

$-4.33399534$

3.80628943

3.39595079

$-3.03313375$

1.000 \# Electr. mom., scale-fac \# Magnetic mom.

$0.05111535 \quad-1.65339077$

$0.15695079 \quad 3.56739235$

$0.05111535 \quad 1.77376330$

$0.15695079 \quad-3.24714518$

$0.05111535 \quad 1.98349714$

$0.15695079 \quad-3.91534829$

$0.05111535 \quad-1.77855182$

$0.15695079 \quad 3.42688346$

$-0.39047247$

$-0.28463703$

$-0.39047247$

$-0.28463703$

$-0.39047247$

$-0.28463703$

$-0.39047247$

$-0.28463703$

0.01654314

0.12237859

0.01654314

0.12237859

0.01654314

0.12237859

0.01654314

$-0.99186331$

3. 84373546

2.07418299

$-5.76619053$

$-0.43913865$

$-1.60844684$

$-0.39419580$

3.13258862

$-1.28912354$

1.01399755

0.99293578

$-0.79946548$

0.90600812

$-0.90863144$

$-0.71426940$ 
2.40286589
-1.56014943
-1.56014943
-1.56014943
-1.56014943
-1.45431399
-1.45431399
-1.45431399
-1.45431399
1.01902342
1.01902342
1.01902342
1.01902342
1.12485886
1.12485886
1.12485886
1.12485886
-2.29137540
-2.29137540
-2.29137540
-2.29137540
-2.18554020
-2.18554020
-2.18554020
-2.18554020
0.88915640
0.88915640
0.88915640
0.88915640
0.99499184
0.99499184
0.99499184
0.99499184
-2.05025959
-2.05025959
-2.05025959
-2.05025959
-1.94442415
-1.94442415
-1.94442415
-1.94442415

$\&$ TRANSITION $2->\ldots$

64
1.48251593

$-1.49588871$

$-1.49588871$

$-1.39005339$

$-1.39005339$

$-1.49588871$

$-1.49588871$

$-1.39005339$

$-1.39005339$

$-0.44691080$

$-0.44691080$

$-0.34107536$

$-0.34107536$

$-0.44691080$

$-0.44691080$

$-0.34107536$

$-0.34107536$

0.60408223

0.60408223

0.70991772

0.70991772

0.60408223

0.60408223

0.70991772

0.70991772

$-1.44335949$

$-1.44335949$

$-1.33752406$

$-1.33752406$

$-1.44335949$

$-1.44335949$

$-1.33752406$

$-1.33752406$

1.58138645

1.58138645

1.68722177

1.68722177

1. 58138645

1. 58138645

1.68722177

1. 68722177
0.12237859

$-0.36711088$

$-0.26127544$

$-0.36711088$

$-0.26127544$

$-0.36711088$

$-0.26127544$

$-0.36711088$

$-0.26127544$

$-0.42923152$

$-0.32339609$

$-0.42923152$

$-0.32339609$

$-0.42923152$

$-0.32339609$

$-0.42923152$

$-0.32339609$

0.06612916

0.17196462

0.06612916

0.17196462

0.06612916

0.17196462

0.06612916

0.17196462

$-0.32880956$

$-0.22297411$

$-0.32880956$

$-0.22297411$

$-0.32880956$

$-0.22297411$

$-0.32880956$

$-0.22297411$

0.09555512

0.20139056

0.09555512

0.20139056

0.09555512

0.20139056

0.09555512

0.20139056
0.71799248

0.72509664

$-0.63774306$

$-0.72520405$

0.59711540

$-0.81620264$

0.76052248

0.82767630

$-0.73766249$

$-3.55427217$

4.73409081

3. 36347818

$-4.43448639$

4. 22550726

$-5.51224375$

$-3.85997248$

4. 92371082

$-4.82596922$

1. 12722921

3.60540462

$-0.63577259$

7.36229897

$-2.73801398$

$-5.76054049$

1. 98571968

$-0.53907007$

0.60132784

0.56625962

$-0.66753352$

0.55854470

$-0.68365324$

$-0.62777686$

0.82717907

$-0.17662568$

0.08274699

0.19732262

$-0.12361091$

0.51051402

$-0.32651576$

$-0.43839118$

0.31229874

$\begin{array}{ccc}0.1084 & 0.0 .6824 & 0.0310 \\ -0.0009 & -0.0101 & 0.0041 \\ 2.13764215 & 0.16034016 \\ 2.13764215 & 0.16034016 \\ 2.13764215 & 0.26617563 \\ 2.13764215 & 0.26617563 \\ 2.24347758 & 0.16034016 \\ 2.24347758 & 0.16034016 \\ 2.24347758 & 0.26617563 \\ 2.24347758 & 0.26617563 \\ -1.37885487 & -0.28076980 \\ -1.37885487 & -0.28076980 \\ -1.37885487 & -0.17493436 \\ -1.37885487 & -0.17493436 \\ -1.27301943 & -0.28076980 \\ -1.27301943 & -0.28076980 \\ -1.27301943 & -0.17493436 \\ -1.27301943 & -0.17493436 \\ 2.29703021 & 1.37668049 \\ 2.29703021 & 1.37668049 \\ 2.29703021 & 1.48251593 \\ 2.29703021 & 1.48251593 \\ 2.40286589 & 1.37668049 \\ 2.40286589 & 1.37668049 \\ & \end{array}$
1.000 \# Electr. mom., scale-fac \# Magnetic mom.

$0.05111535 \quad-4.38951731$

$0.15695079 \quad-0.87199813$

$0.05111535 \quad 5.53813982$

$0.15695079 \quad-0.59147698$

$0.05111535 \quad 2.38094974$

$0.15695079 \quad 2.37652826$

$0.05111535 \quad-3.57738948$

$0.15695079 \quad-1.04801106$

$-0.39047247 \quad-13.06040096$

$-0.28463703 \quad 18.51740074$

$-0.39047247$

$-0.28463703$

$-0.39047247$

$-0.28463703$

$-0.39047247$

$-0.28463703$

0.01654314

0.12237859

0.01654314

0.12237859

0.01654314

0.12237859

16.06648064

$-21.70321465$

10.42533779

$-15.27054501$

$-13.30496407$

18.38104057

$-3.88833904$

1. 67731512

4.38209009

$-2.59101558$

3.00825930

$-0.66588360$

\section{S-146}




\subsection{9 \\ 2. 40286589 \\ $-1.56014943$ \\ $-1.56014943$ \\ $-1.56014943$ \\ $-1.56014943$ \\ $-1.45431399$ \\ $-1.45431399$ \\ $-1.45431399$ \\ $-1.45431399$ \\ 1. 01902342 \\ 1. 01902342 \\ 1. 01902342 \\ 1. 01902342 \\ 1. 12485886 \\ 1.12485886 \\ 1.12485886 \\ 1.12485886 \\ $-2.29137540$ \\ $-2.29137540$ \\ $-2.29137540$ \\ $-2.29137540$ \\ $-2.18554020$ \\ $-2.18554020$ \\ $-2.18554020$ \\ $-2.18554020$ \\ 0.88915640 \\ 0.88915640 \\ 0.88915640 \\ 0.88915640 \\ 0.99499184 \\ 0.99499184 \\ 0.99499184 \\ 0.99499184 \\ $-2.05025959$ \\ $-2.05025959$ \\ $-2.05025959$ \\ $-2.05025959$ \\ $-1.94442415$ \\ $-1.94442415$ \\ $-1.94442415$ \\ $-1.94442415$}

64

0.08410 .

$-0.0463$

2. 13764215

2. 13764215

2.13764215

2.13764215

2. 24347758

2. 24347758

2. 24347758

2. 24347758

$-1.37885487$

$-1.37885487$

$-1.37885487$

$-1.37885487$

$-1.27301943$

$-1.27301943$

$-1.27301943$

$-1.27301943$

2. 29703021

2. 29703021

2. 29703021

2. 29703021

2. 40286589

2. 40286589
1.48251593

1. 48251593

$-1.49588871$

$-1.49588871$

$-1.39005339$

$-1.39005339$

$-1.49588871$

$-1.49588871$

$-1.39005339$

$-1.39005339$

$-0.44691080$

$-0.44691080$

$-0.34107536$

$-0.34107536$

$-0.44691080$

$-0.44691080$

$-0.34107536$

$-0.34107536$

0.60408223

0.60408223

0.70991772

0.70991772

0.60408223

0.60408223

0.70991772

0.70991772

$-1.44335949$

$-1.44335949$

$-1.33752406$

$-1.33752406$

$-1.44335949$

$-1.44335949$

$-1.33752406$

$-1.33752406$

1.58138645

1.58138645

1.68722177

1. 68722177

1. 58138645

1.58138645

1. 68722177

1.68722177

. \# Energy 2->4

$-0.0422 \quad-0.0856$ $-0.3891-0.0147$

0.16034016

0.16034016

0.26617563

0.26617563

0.16034016

0.16034016

0.26617563

0.26617563

$-0.28076980$

$-0.28076980$

$-0.17493436$

$-0.17493436$

$-0.28076980$

$-0.28076980$

$-0.17493436$

$-0.17493436$

1.37668049

1.37668049

1. 48251593

1.48251593

1.37668049

1. 37668049
0.01654314

0.12237859

$-0.36711088$

$-0.26127544$

$-0.36711088$

$-0.26127544$

$-0.36711088$

$-0.26127544$

$-0.36711088$

$-0.26127544$

$-0.42923152$

$-0.32339609$

$-0.42923152$

$-0.32339609$

$-0.42923152$

$-0.32339609$

$-0.42923152$

$-0.32339609$

0.06612916

0.17196462

0.06612916

0.17196462

0.06612916

0.17196462

0.06612916

0.17196462

$-0.32880956$

$-0.22297411$

$-0.32880956$

$-0.22297411$

$-0.32880956$

$-0.22297411$

$-0.32880956$

$-0.22297411$

0.09555512

0.20139056

0.09555512

0.20139056

0.09555512

0.20139056

0.09555512

0.20139056

$$
\begin{aligned}
& 1.000 \text { \# Electr. mom., scale-fac } \\
& \text { \# Magnetic mom. }
\end{aligned}
$$

0.05111535

0.15695079

0.05111535

0.15695079

0.05111535

0.15695079

0.05111535

0.15695079

$-0.39047247$

$-0.28463703$

$-0.39047247$

$-0.28463703$

$-0.39047247$

$-0.28463703$

$-0.39047247$

$-0.28463703$

0.01654314

0.12237859

0.01654314

0.12237859

0.01654314

0.12237859
$-3.62963605$

1. 75709939

1.24112689

0.20651096

$-1.89985836$

$-1.78971493$

0.54803658

0.48966739

1.00063717

$-11.55373001$

14.79419136

14.02218533

$-18.10252953$

13.32578945

$-17.02057648$

$-16.15688896$

20.81488419

9.54367924

$-8.45014668$

$-8.35306263$

7. 26622343

$-11.55755520$

10.08009434

10.24468136

$-8.69459057$

$-0.49456078$

0.62286413

0.86819154

$-1.19945335$

0.62906832

$-0.74815363$

$-1.07319987$

1. 39102435

0.35793382

$-0.25210801$

$-0.21905524$

0.10673389

$-0.58488595$

0.35968813

0.35719812

$-0.16371422$

$-4.66099739$

1.85096180

3. 49846172

$-0.88402480$

2. 59021688

$-0.50371504$

$-1.60854721$

$-0.31792465$

24.10666656

$-39.78272247$

$-26.10615349$

39.25547791

$-21.31855011$

36.69173050

23.40304184

$-36.51144028$

$-0.01542652$

$-0.55947900$

$-0.16236755$

0.59533787

$-0.21750890$

0.68723762
0.12512282 
2.40286589
2.40286589
-1.56014943
-1.56014943
-1.56014943
-1.56014943
-1.45431399
-1.45431399
-1.45431399
-1.45431399
1.01902342
1.01902342
1.01902342
1.01902342
1.12485886
1.12485886
1.12485886
1.12485886
-2.29137540
-2.29137540
-2.29137540
-2.29137540
-2.18554020
-2.18554020
-2.18554020
-2.18554020
0.88915640
0.88915640
0.88915640
0.88915640
0.99499184
0.99499184
0.99499184
0.99499184
-2.05025959
-2.05025959
-2.05025959
-2.05025959
-1.94442415
-1.94442415
-1.94442415
-1.94442415

64
1. 48251593

1. 48251593

$-1.49588871$

$-1.49588871$

$-1.39005339$

$-1.39005339$

$-1.49588871$

$-1.49588871$

$-1.39005339$

$-1.39005339$

$-0.44691080$

$-0.44691080$

$-0.34107536$

$-0.34107536$

$-0.44691080$

$-0.44691080$

$-0.34107536$

$-0.34107536$

0.60408223

0.60408223

0.70991772

0.70991772

0.60408223

0.60408223

0.70991772

0.70991772

$-1.44335949$

$-1.44335949$

$-1.33752406$

$-1.33752406$

$-1.44335949$

$-1.44335949$

$-1.33752406$

$-1.33752406$

1. 58138645

1.58138645

1.68722177

1.68722177

1. 58138645

1.58138645

1. 68722177

1. 68722177

0 .

$-0.7886$

$-0.0432$

2. 13764215

2.13764215

2.13764215

2.13764215

2. 24347758

2. 24347758

2. 24347758

2. 24347758

$-1.37885487$

$-1.37885487$

$-1.37885487$

$-1.37885487$

$-1.27301943$

$-1.27301943$

$-1.27301943$

$-1.27301943$

2. 29703021

2. 29703021

2. 29703021

2. 29703021

2. 40286589

2. 40286589

0.16034016

0.16034016

0.26617563

0.26617563

0.16034016

0.16034016

0.26617563

0.26617563

$-0.28076980$

$-0.28076980$

$-0.17493436$

$-0.17493436$

$-0.28076980$

$-0.28076980$

$-0.17493436$

$-0.17493436$

1. 37668049

1. 37668049

1. 48251593

1. 48251593

1.37668049

1. 37668049
0.01654314

0.12237859

$-0.36711088$

$-0.26127544$

$-0.36711088$

$-0.26127544$

$-0.36711088$

$-0.26127544$

$-0.36711088$

$-0.26127544$

$-0.42923152$

$-0.32339609$

$-0.42923152$

$-0.32339609$

$-0.42923152$

$-0.32339609$

$-0.42923152$

$-0.32339609$

0.06612916

0.17196462

0.06612916

0.17196462

0.06612916

0.17196462

0.06612916

0.17196462

$-0.32880956$

$-0.22297411$

$-0.32880956$

$-0.22297411$

$-0.32880956$

$-0.22297411$

$-0.32880956$

$-0.22297411$

0.09555512

0.20139056

0.09555512

0.20139056

0.09555512

0.20139056

0.09555512

0.20139056

. \# Energy 2->5

$-0.3899 \quad-0.0321$ $-0.2944 \quad-0.0580$

0.05111535

0.15695079

0.05111535

0.15695079

0.05111535

0.15695079

0.05111535

0.15695079

$-0.39047247$

$-0.28463703$

$-0.39047247$

$-0.28463703$

$-0.39047247$

$-0.28463703$

$-0.39047247$

$-0.28463703$

0.01654314

0.12237859

0.01654314

0.12237859

0.01654314

0.12237859
0.33831352

$-0.68836975$

$-2.10380316$

$-2.46730638$

5.65455103

0.32008466

1.90306473

2.86093855

$-5.57143307$

$-0.58953750$

4.82775211

$-12.94850254$

$-4.27122736$

11.04006100

$-6.37409878$

14.42501163

5.69445992

$-12.36710453$

$-19.68366051$

13.75382805

15.91798973

$-10.91377449$

30.64637184

$-21.80193520$

$-26.05648613$

18.21115494

$-2.32682681$

0.45370802

2.83557081

0.01768554

2.00254893

$-0.23252477$

$-2.37882638$

$-0.32387811$

1. 50764740

$-0.64243406$

$-1.09338582$

0.45189768

0.13982488

$-0.46760106$

$-0.09653695$

0.36651659

$$
\begin{gathered}
1.000 \text { \# Electr. mom., scale-fac } \\
\text { \# Magnetic mom. }
\end{gathered}
$$

$-1.17393470$

1. 26951098

0.88958663

$-0.73033446$

0.75266683

$-1.08759654$

$-0.38987181$

0.57178754

15.10743904

$-25.33593178$

$-16.94705391$

26.37590599

$-12.92108917$

22.68416786

14.65142345

$-23.69002151$

1.93395448

$-1.35471642$

$-1.11765599$

0.61974895

$-1.91228342$

1. 24654245 


\begin{abstract}
2. 40286589
2. 40286589

$-1.56014943$

$-1.56014943$

$-1.56014943$

$-1.56014943$

$-1.45431399$

$-1.45431399$

$-1.45431399$

$-1.45431399$

1.01902342

1. 01902342

1.01902342

1. 01902342

1. 12485886

1. 12485886

1. 12485886

1.12485886

$-2.29137540$

$-2.29137540$

$-2.29137540$

$-2.29137540$

$-2.18554020$

$-2.18554020$

$-2.18554020$

$-2.18554020$

0.88915640

0.88915640

0.88915640

0.88915640

0.99499184

0.99499184

0.99499184

0.99499184

$-2.05025959$

$-2.05025959$

$-2.05025959$

$-2.05025959$

$-1.94442415$

$-1.94442415$

$-1.94442415$

$-1.94442415$
\end{abstract}

64

\subsection{0}

$-0.0025$

2.13764215

2.13764215

2.13764215

2. 13764215

2. 24347758

2. 24347758

2. 24347758

2. 24347758

$-1.37885487$

$-1.37885487$

$-1.37885487$

$-1.37885487$

$-1.27301943$

$-1.27301943$

$-1.27301943$

$-1.27301943$

2. 29703021

2. 29703021

2. 29703021

2. 29703021

2. 40286589

2. 40286589
1. 48251593

1. 48251593

$-1.49588871$

$-1.49588871$

$-1.39005339$

$-1.39005339$

$-1.49588871$

$-1.49588871$

$-1.39005339$

$-1.39005339$

$-0.44691080$

$-0.44691080$

$-0.34107536$

$-0.34107536$

$-0.44691080$

$-0.44691080$

$-0.34107536$

$-0.34107536$

0.60408223

0.60408223

0.70991772

0.70991772

0.60408223

0.60408223

0.70991772

0.70991772

$-1.44335949$

$-1.44335949$

$-1.33752406$

$-1.33752406$

$-1.44335949$

$-1.44335949$

$-1.33752406$

$-1.33752406$

1.58138645

1.58138645

1.68722177

1.68722177

1.58138645

1.58138645

1. 68722177

1. 68722177

0 . \# Energy 2->6

$0.4568 \quad-0.0387$ $-0.0158$ 0.0342

0.16034016

0.16034016

0.26617563

0.26617563

0.16034016

0.16034016

0.26617563

0.26617563

$-0.28076980$

$-0.28076980$

$-0.17493436$

$-0.17493436$

$-0.28076980$

$-0.28076980$

$-0.17493436$

$-0.17493436$

1.37668049

1. 37668049

1. 48251593

1.48251593

1. 37668049

1. 37668049
0.01654314

0.12237859

$-0.36711088$

$-0.26127544$

$-0.36711088$

$-0.26127544$

$-0.36711088$

$-0.26127544$

$-0.36711088$

$-0.26127544$

$-0.42923152$

$-0.32339609$

$-0.42923152$

$-0.32339609$

$-0.42923152$

$-0.32339609$

$-0.42923152$

$-0.32339609$

0.06612916

0.17196462

0.06612916

0.17196462

0.06612916

0.17196462

0.06612916

0.17196462

$-0.32880956$

$-0.22297411$

$-0.32880956$

$-0.22297411$

$-0.32880956$

$-0.22297411$

$-0.32880956$

$-0.22297411$

0.09555512

0.20139056

0.09555512

0.20139056

0.09555512

0.20139056

0.09555512

0.20139056

$$
1.000
$$

0.05111535

0.15695079

0.05111535

0.15695079

0.05111535

0.15695079

0.05111535

0.15695079

$-0.39047247$

$-0.28463703$

$-0.39047247$

$-0.28463703$

$-0.39047247$

$-0.28463703$

$-0.39047247$

$-0.28463703$

0.01654314

0.12237859

0.01654314

0.12237859

0.01654314

0.12237859
1.08872497

$-0.53875452$

$-1.20680177$

$-1.63824427$

1.60801017

2.05287170

1. 41883898

1.41378891

$-1.93016994$

$-1.64960897$

11.33547020

$-17.79844475$

$-12.51484013$

19.02778053

$-13.57186890$

20.49650383

14.96323299

$-22.05043793$

$-12.05857754$

10.10197449

10.58714676

$-8.84236622$

16.47110939

$-13.35802269$

$-14.74963093$

11.80528450

$-0.54838431$

$-0.31335357$

0.72758359

0.67089462

0.38801056

0.46627408

$-0.46205652$

$-0.92155397$

0.01604171

0.42446083

$-0.02603535$

$-0.28556013$

0.67476338

$-0.82819772$

$-0.45990375$

0.57180297$$
\text { mom. }
$$

$-5.84001017$

3. 44885302

5. 94141245

$-3.54560804$

4. 24014759

$-2.27100921$

$-4.30488586$

2.33729935

$-5.45569754$

6.17943335

5. 96402168

$-6.22684097$

5.21016264

$-5.88645458$

$-5.78162670$

6.07728338

$-2.11566567$

1. 25303054

1. 71329594

$-1.03283167$

1.60928142

$-0.91948563$ 


\begin{tabular}{|c|c|c|c|}
\hline 2.40286589 & 1.48251593 & 0.01654314 & -1.33223510 \\
\hline 2.40286589 & 1.48251593 & 0.12237859 & 0.77026492 \\
\hline-1.56014943 & -1.49588871 & -0.36711088 & 0.27773201 \\
\hline-1.56014943 & -1.49588871 & -0.26127544 & -0.30260178 \\
\hline-1.56014943 & -1.39005339 & -0.36711088 & -1.97462642 \\
\hline-1.56014943 & -1.39005339 & -0.26127544 & 1.94649363 \\
\hline-1.45431399 & -1.49588871 & -0.36711088 & 0.00061859 \\
\hline-1.45431399 & -1.49588871 & -0.26127544 & -0.07991269 \\
\hline-1.45431399 & -1.39005339 & -0.36711088 & 1.67805016 \\
\hline-1.45431399 & -1.39005339 & -0.26127544 & -1.48783326 \\
\hline 1.01902342 & -0.44691080 & -0.42923152 & 2.27090240 \\
\hline 1.01902342 & -0.44691080 & -0.32339609 & -2.98735476 \\
\hline 1.01902342 & -0.34107536 & -0.42923152 & -2.06082940 \\
\hline 1.01902342 & -0.34107536 & -0.32339609 & 2.73973465 \\
\hline 1.12485886 & -0.44691080 & -0.42923152 & -1.66808474 \\
\hline 1.12485886 & -0.44691080 & -0.32339609 & 2.51546335 \\
\hline 1.12485886 & -0.34107536 & -0.42923152 & 1.50672138 \\
\hline 1.12485886 & -0.34107536 & -0.32339609 & -2.33135891 \\
\hline-2.29137540 & 0.60408223 & 0.06612916 & 1.43739986 \\
\hline-2.29137540 & 0.60408223 & 0.17196462 & 0.02815307 \\
\hline-2.29137540 & 0.70991772 & 0.06612916 & -0.41968423 \\
\hline-2.29137540 & 0.70991772 & 0.17196462 & -0.75048620 \\
\hline-2.18554020 & 0.60408223 & 0.06612916 & -4.07194328 \\
\hline-2.18554020 & 0.60408223 & 0.17196462 & 2.09423065 \\
\hline-2.18554020 & 0.70991772 & 0.06612916 & 2.84086204 \\
\hline-2.18554020 & 0.70991772 & 0.17196462 & -1.20785582 \\
\hline 0.88915640 & -1.44335949 & -0.32880956 & 0.15478285 \\
\hline 0.88915640 & -1.44335949 & -0.22297411 & -0.11747734 \\
\hline 0.88915640 & -1.33752406 & -0.32880956 & 0.10720931 \\
\hline 0.88915640 & -1.33752406 & -0.22297411 & -0.14193417 \\
\hline 0.99499184 & -1.44335949 & -0.32880956 & 0.12174864 \\
\hline 0.99499184 & -1.44335949 & -0.22297411 & -0.10847558 \\
\hline 0.99499184 & -1.33752406 & -0.32880956 & -0.43186906 \\
\hline 0.99499184 & -1.33752406 & -0.22297411 & 0.40201503 \\
\hline-2.05025959 & 1.58138645 & 0.09555512 & -1.17833054 \\
\hline-2.05025959 & 1.58138645 & 0.20139056 & 1.05751526 \\
\hline-2.05025959 & 1.68722177 & 0.09555512 & 0.85241425 \\
\hline-2.05025959 & 1.68722177 & 0.20139056 & -0.75091350 \\
\hline-1.94442415 & 1.58138645 & 0.09555512 & 0.78359783 \\
\hline-1.94442415 & 1.58138645 & 0.20139056 & -0.69298452 \\
\hline-1.94442415 & 1.68722177 & 0.09555512 & -0.56001949 \\
\hline-1.94442415 & 1.68722177 & 0.20139056 & 0.47679526 \\
\hline & \# Energy 2->7 & & \\
\hline-0.9882 & -0.0734 & 1.000 & Electr. mom., scale-fac \\
\hline 0.0661 & -0.0278 & \# & Magnetic mom. \\
\hline 2.13764215 & 0.16034016 & 0.05111535 & 9.48690510 \\
\hline 2.13764215 & 0.16034016 & 0.15695079 & -4.28124809 \\
\hline 2.13764215 & 0.26617563 & 0.05111535 & -9.45985413 \\
\hline 2.13764215 & 0.26617563 & 0.15695079 & 4.41265011 \\
\hline 2.24347758 & 0.16034016 & 0.05111535 & -7.32298565 \\
\hline 2.24347758 & 0.16034016 & 0.15695079 & 2.73649836 \\
\hline 2.24347758 & 0.26617563 & 0.05111535 & 7.31794500 \\
\hline 2.24347758 & 0.26617563 & 0.15695079 & -2.84816360 \\
\hline-1.37885487 & -0.28076980 & -0.39047247 & 2.11381793 \\
\hline-1.37885487 & -0.28076980 & -0.28463703 & -4.76029110 \\
\hline-1.37885487 & -0.17493436 & -0.39047247 & -2.74159861 \\
\hline-1.37885487 & -0.17493436 & -0.28463703 & 5.51583099 \\
\hline-1.27301943 & -0.28076980 & -0.39047247 & -1.21681142 \\
\hline-1.27301943 & -0.28076980 & -0.28463703 & 3.55800128 \\
\hline-1.27301943 & -0.17493436 & -0.39047247 & 1.72413743 \\
\hline-1.27301943 & -0.17493436 & -0.28463703 & -4.17020655 \\
\hline 2.29703021 & 1.37668049 & 0.01654314 & 3.33045530 \\
\hline 2.29703021 & 1.37668049 & 0.12237859 & -1.25919461 \\
\hline 2.29703021 & 1.48251593 & 0.01654314 & -2.45373917 \\
\hline 2.29703021 & 1.48251593 & 0.12237859 & 0.86821902 \\
\hline 2.40286589 & 1.37668049 & 0.01654314 & -2.60940599 \\
\hline 2.40286589 & 1.37668049 & 0.12237859 & 0.68199807 \\
\hline
\end{tabular}




\begin{tabular}{|c|c|c|c|}
\hline 2.40286589 & 1.48251593 & 0.01654314 & 1.89807773 \\
\hline 2.40286589 & 1.48251593 & 0.12237859 & -0.44088361 \\
\hline-1.56014943 & -1.49588871 & -0.36711088 & -0.23436323 \\
\hline-1.56014943 & -1.49588871 & -0.26127544 & -0.37898880 \\
\hline-1.56014943 & -1.39005339 & -0.36711088 & -0.29263097 \\
\hline-1.56014943 & -1.39005339 & -0.26127544 & 1.02906024 \\
\hline-1.45431399 & -1.49588871 & -0.36711088 & 0.40118656 \\
\hline-1.45431399 & -1.49588871 & -0.26127544 & 0.13468596 \\
\hline-1.45431399 & -1.39005339 & -0.36711088 & 0.08789205 \\
\hline-1.45431399 & -1.39005339 & -0.26127544 & -0.69149393 \\
\hline 1.01902342 & -0.44691080 & -0.42923152 & 2.97228003 \\
\hline 1.01902342 & -0.44691080 & -0.32339609 & -5.45840025 \\
\hline 1.01902342 & -0.34107536 & -0.42923152 & -3.65461326 \\
\hline 1.01902342 & -0.34107536 & -0.32339609 & 6.60110092 \\
\hline 1.12485886 & -0.44691080 & -0.42923152 & -4.54866648 \\
\hline 1.12485886 & -0.44691080 & -0.32339609 & 6.87131596 \\
\hline 1.12485886 & -0.34107536 & -0.42923152 & 5.39418602 \\
\hline 1.12485886 & -0.34107536 & -0.32339609 & -8.31623173 \\
\hline-2.29137540 & 0.60408223 & 0.06612916 & -2.74164152 \\
\hline-2.29137540 & 0.60408223 & 0.17196462 & 3.10905528 \\
\hline-2.29137540 & 0.70991772 & 0.06612916 & 2.67146850 \\
\hline-2.29137540 & 0.70991772 & 0.17196462 & -2.94441867 \\
\hline-2.18554020 & 0.60408223 & 0.06612916 & 2.76297355 \\
\hline-2.18554020 & 0.60408223 & 0.17196462 & -3.15079045 \\
\hline-2.18554020 & 0.70991772 & 0.06612916 & -2.71100044 \\
\hline-2.18554020 & 0.70991772 & 0.17196462 & 2.96447539 \\
\hline 0.88915640 & -1.44335949 & -0.32880956 & 0.06239454 \\
\hline 0.88915640 & -1.44335949 & -0.22297411 & -0.14273229 \\
\hline 0.88915640 & -1.33752406 & -0.32880956 & -0.03936668 \\
\hline 0.88915640 & -1.33752406 & -0.22297411 & 0.27301866 \\
\hline 0.99499184 & -1.44335949 & -0.32880956 & -0.34715393 \\
\hline 0.99499184 & -1.44335949 & -0.22297411 & 0.37225699 \\
\hline 0.99499184 & -1.33752406 & -0.32880956 & 0.45248428 \\
\hline 0.99499184 & -1.33752406 & -0.22297411 & -0.59817994 \\
\hline-2.05025959 & 1.58138645 & 0.09555512 & -0.38398573 \\
\hline-2.05025959 & 1.58138645 & 0.20139056 & 0.50863099 \\
\hline-2.05025959 & 1.68722177 & 0.09555512 & 0.26652059 \\
\hline-2.05025959 & 1.68722177 & 0.20139056 & -0.35551342 \\
\hline-1.94442415 & 1.58138645 & 0.09555512 & 0.35014677 \\
\hline-1.94442415 & 1.58138645 & 0.20139056 & -0.43964455 \\
\hline-1.94442415 & 1.68722177 & 0.09555512 & -0.23699462 \\
\hline-1.94442415 & 1.68722177 & 0.20139056 & 0.30152443 \\
\hline & \# Energy 2->8 & & \\
\hline 0 & -0.0531 & 1.000 & Electr. mom., \\
\hline-0.0669 & $-0.1350 \quad-0.0714$ & $\pi$ & Magnetic mom. \\
\hline 2.13764215 & 0.16034016 & 0.05111535 & 14.62094593 \\
\hline 2.13764215 & 0.16034016 & 0.15695079 & -21.18092728 \\
\hline 2.13764215 & 0.26617563 & 0.05111535 & -16.42048264 \\
\hline 2.13764215 & 0.26617563 & 0.15695079 & 21.69957161 \\
\hline 2.24347758 & 0.16034016 & 0.05111535 & -16.26166916 \\
\hline 2.24347758 & 0.16034016 & 0.15695079 & 22.67014694 \\
\hline 2.24347758 & 0.26617563 & 0.05111535 & 17.36292267 \\
\hline 2.24347758 & 0.26617563 & 0.15695079 & -22.70263863 \\
\hline-1.37885487 & -0.28076980 & -0.39047247 & 6.89063597 \\
\hline-1.37885487 & -0.28076980 & -0.28463703 & -14.39165688 \\
\hline-1.37885487 & -0.17493436 & -0.39047247 & -9.84992409 \\
\hline-1.37885487 & -0.17493436 & -0.28463703 & 19.65060997 \\
\hline-1.27301943 & -0.28076980 & -0.39047247 & -2.69028640 \\
\hline-1.27301943 & -0.28076980 & -0.28463703 & 7.48014593 \\
\hline-1.27301943 & -0.17493436 & -0.39047247 & 4.91394138 \\
\hline-1.27301943 & -0.17493436 & -0.28463703 & -11.60682106 \\
\hline 2.29703021 & 1.37668049 & 0.01654314 & 2.96133494 \\
\hline 2.29703021 & 1.37668049 & 0.12237859 & -2.77687407 \\
\hline 2.29703021 & 1.48251593 & 0.01654314 & -1.69260788 \\
\hline 2.29703021 & 1.48251593 & 0.12237859 & 1.50613165 \\
\hline 2.40286589 & 1.37668049 & 0.01654314 & -2.74264812 \\
\hline 2.40286589 & 1.37668049 & 0.12237859 & 2.70916963 \\
\hline
\end{tabular}

\section{S-151}




\begin{tabular}{|c|c|c|c|}
\hline 2.40286589 & 1.48251593 & 0.01654314 & 1.53500140 \\
\hline 2.40286589 & 1.48251593 & 0.12237859 & -1.48081613 \\
\hline-1.56014943 & -1.49588871 & -0.36711088 & -0.15486300 \\
\hline-1.56014943 & -1.49588871 & -0.26127544 & 0.83793378 \\
\hline-1.56014943 & -1.39005339 & -0.36711088 & -0.42527103 \\
\hline-1.56014943 & -1.39005339 & -0.26127544 & -0.43889120 \\
\hline-1.45431399 & -1.49588871 & -0.36711088 & 0.59976149 \\
\hline-1.45431399 & -1.49588871 & -0.26127544 & -1.56374359 \\
\hline-1.45431399 & -1.39005339 & -0.36711088 & -0.18311349 \\
\hline-1.45431399 & -1.39005339 & -0.26127544 & 1.42659032 \\
\hline 1.01902342 & -0.44691080 & -0.42923152 & 4.56210756 \\
\hline 1.01902342 & -0.44691080 & -0.32339609 & -2.55029178 \\
\hline 1.01902342 & -0.34107536 & -0.42923152 & -2.53611660 \\
\hline 1.01902342 & -0.34107536 & -0.32339609 & -0.08157501 \\
\hline 1.12485886 & -0.44691080 & -0.42923152 & -5.11326551 \\
\hline 1.12485886 & -0.44691080 & -0.32339609 & 3.13481498 \\
\hline 1.12485886 & -0.34107536 & -0.42923152 & 2.60638523 \\
\hline 1.12485886 & -0.34107536 & -0.32339609 & 0.23049584 \\
\hline-2.29137540 & 0.60408223 & 0.06612916 & 9.10815334 \\
\hline-2.29137540 & 0.60408223 & 0.17196462 & 0.73746675 \\
\hline-2.29137540 & 0.70991772 & 0.06612916 & -7.05985832 \\
\hline-2.29137540 & 0.70991772 & 0.17196462 & -0.68846047 \\
\hline-2.18554020 & 0.60408223 & 0.06612916 & -15.65332413 \\
\hline-2.18554020 & 0.60408223 & 0.17196462 & 3.50426245 \\
\hline-2.18554020 & 0.70991772 & 0.06612916 & 12.62937927 \\
\hline-2.18554020 & 0.70991772 & 0.17196462 & -2.94495106 \\
\hline 0.88915640 & -1.44335949 & -0.32880956 & 1.05384755 \\
\hline 0.88915640 & -1.44335949 & -0.22297411 & -0.85210651 \\
\hline 0.88915640 & -1.33752406 & -0.32880956 & -1.54954219 \\
\hline 0.88915640 & -1.33752406 & -0.22297411 & 1.22928131 \\
\hline 0.99499184 & -1.44335949 & -0.32880956 & -1.12486863 \\
\hline 0.99499184 & -1.44335949 & -0.22297411 & 1.07860732 \\
\hline 0.99499184 & -1.33752406 & -0.32880956 & 1.65661919 \\
\hline 0.99499184 & -1.33752406 & -0.22297411 & -1.58441579 \\
\hline-2.05025959 & 1.58138645 & 0.09555512 & 1.26477563 \\
\hline-2.05025959 & 1.58138645 & 0.20139056 & -1.18481851 \\
\hline-2.05025959 & 1.68722177 & 0.09555512 & -1.09170449 \\
\hline-2.05025959 & 1.68722177 & 0.20139056 & 1.03414524 \\
\hline-1.94442415 & 1.58138645 & 0.09555512 & -2.18850446 \\
\hline-1.94442415 & 1.58138645 & 0.20139056 & 1.88980496 \\
\hline-1.94442415 & 1.68722177 & 0.09555512 & 1.74897635 \\
\hline-1.94442415 & 1.68722177 & 0.20139056 & -1.56692696 \\
\hline & \# Energy 2->9 & & \\
\hline-0.1270 & 0.0069 & 1.000 & Electr. mom., \\
\hline 0.0099 & -0.0127 & $\pi$ & Magnetic mom. \\
\hline 2.13764215 & 0.16034016 & 0.05111535 & 1.38380241 \\
\hline 2.13764215 & 0.16034016 & 0.15695079 & 0.22529139 \\
\hline 2.13764215 & 0.26617563 & 0.05111535 & -0.96049190 \\
\hline 2.13764215 & 0.26617563 & 0.15695079 & -0.47433159 \\
\hline 2.24347758 & 0.16034016 & 0.05111535 & -0.75385123 \\
\hline 2.24347758 & 0.16034016 & 0.15695079 & -0.65338916 \\
\hline 2.24347758 & 0.26617563 & 0.05111535 & 0.45443892 \\
\hline 2.24347758 & 0.26617563 & 0.15695079 & 0.81093758 \\
\hline-1.37885487 & -0.28076980 & -0.39047247 & -0.55453569 \\
\hline-1.37885487 & -0.28076980 & -0.28463703 & 1.15592623 \\
\hline-1.37885487 & -0.17493436 & -0.39047247 & 0.77401793 \\
\hline-1.37885487 & -0.17493436 & -0.28463703 & -1.55007780 \\
\hline-1.27301943 & -0.28076980 & -0.39047247 & 0.18934610 \\
\hline-1.27301943 & -0.28076980 & -0.28463703 & -0.56858367 \\
\hline-1.27301943 & -0.17493436 & -0.39047247 & -0.37160704 \\
\hline-1.27301943 & -0.17493436 & -0.28463703 & 0.88619018 \\
\hline 2.29703021 & 1.37668049 & 0.01654314 & 0.15611675 \\
\hline 2.29703021 & 1.37668049 & 0.12237859 & 0.14728595 \\
\hline 2.29703021 & 1.48251593 & 0.01654314 & -0.03764669 \\
\hline 2.29703021 & 1.48251593 & 0.12237859 & -0.18901955 \\
\hline 2.40286589 & 1.37668049 & 0.01654314 & -0.11612642 \\
\hline 2.40286589 & 1.37668049 & 0.12237859 & -0.14435229 \\
\hline
\end{tabular}

\section{S-152}




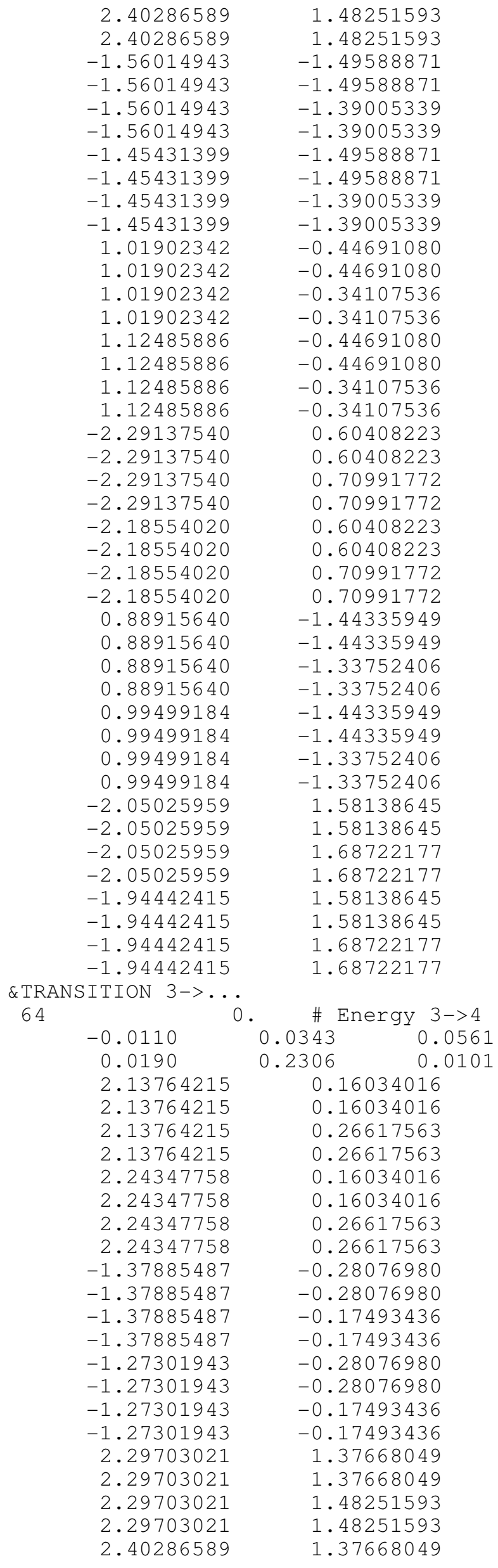

$$
\begin{array}{r}
0.01654314 \\
0.12237859 \\
-0.36711088 \\
-0.26127544 \\
-0.36711088 \\
-0.26127544 \\
-0.36711088 \\
-0.26127544 \\
-0.36711088 \\
-0.26127544 \\
-0.42923152 \\
-0.32339609 \\
-0.42923152 \\
-0.32339609 \\
-0.42923152 \\
-0.32339609 \\
-0.42923152 \\
-0.32339609 \\
0.06612916 \\
0.17196462 \\
0.06612916 \\
0.17196462 \\
0.06612916 \\
0.17196462 \\
0.06612916 \\
0.17196462 \\
-0.32880956 \\
-0.22297411 \\
-0.32880956 \\
-0.22297411 \\
-0.32880956 \\
-0.22297411 \\
-0.32880956 \\
-0.22297411 \\
0.09555512 \\
0.20139056 \\
0.09555512 \\
0.20139056 \\
0.09555512 \\
0.20139056 \\
0.09555512 \\
0.20139056
\end{array}
$$

0.01071129

0.18677324

0.00233846

$-0.10018317$

$-0.02650517$

0.14788616

$-0.04306585$

0.15276881

0.08638632

$-0.22136560$

$-0.17180786$

$-0.02219372$

$-0.34560731$

0.79410654

0.00757878

0.15328625

0.56937736

$-1.03628469$

$-0.98968357$

0.04300038

0.70808429

0.05267106

1. 64220190

$-0.48848674$

$-1.27466512$

0.33353820

$-0.03920335$

0.09585912

0.11283421

$-0.18486443$

0.00677908

$-0.08415292$

$-0.06294434$

0.16694851

$-0.08479459$

0.06630668

0.06844848

$-0.05716002$

0.17910352

$-0.14243963$

$-0.13685217$

0.11593111

TRANSITION $3->$. .
1.000 \# Electr. mom., scale-fac \# Magnetic mom.

0.15695079

0.05111535

0.15695079

0.05111535

0.15695079

0.05111535

0.15695079

$-0.39047247$

$-0.28463703$

$-0.39047247$

$-0.28463703$

$-0.39047247$

$-0.28463703$

$-0.39047247$

$-0.28463703$

0.01654314

0.12237859

0.01654314

0.12237859

0.01654314
$-9.86218548$ 4. 46076727

10.13876057

$-5.18962002$

6.66231871

$-1.85745740$

$-6.97062778$

2.46105409

$-5.67342138$

8.75814342

7.30615425

$-10.58177471$

4.56857824

$-7.17829561$

$-6.08845615$

8.88415241

$-6.99695778$

4.12981319

4.51839542

$-2.18221998$

5.85831451 


\begin{abstract}
2.40286589
2.40286589

2.40286589

$-1.56014943$

$-1.56014943$

$-1.56014943$

$-1.56014943$

$-1.45431399$

$-1.45431399$

$-1.45431399$

$-1.45431399$

1.01902342

1.01902342

1.01902342

1.01902342

1.12485886

1.12485886

1. 12485886

1.12485886

$-2.29137540$

$-2.29137540$

$-2.29137540$

$-2.29137540$

$-2.18554020$

$-2.18554020$

$-2.18554020$

$-2.18554020$

0.88915640

0.88915640

0.88915640

0.88915640

0.99499184

0.99499184

0.99499184

0.99499184

$-2.05025959$

$-2.05025959$

$-2.05025959$

$-2.05025959$

$-1.94442415$

$-1.94442415$

$-1.94442415$

$-1.94442415$
\end{abstract}

64
1.37668049

1. 48251593

1.48251593

$-1.49588871$

$-1.49588871$

$-1.39005339$

$-1.39005339$

$-1.49588871$

$-1.49588871$

$-1.39005339$

$-1.39005339$

$-0.44691080$

$-0.44691080$

$-0.34107536$

$-0.34107536$

$-0.44691080$

$-0.44691080$

$-0.34107536$

$-0.34107536$

0.60408223

0.60408223

0.70991772

0.70991772

0.60408223

0.60408223

0.70991772

0.70991772

$-1.44335949$

$-1.44335949$

$-1.33752406$

$-1.33752406$

$-1.44335949$

$-1.44335949$

$-1.33752406$

$-1.33752406$

1.58138645

1.58138645

1.68722177

1.68722177

1.58138645

1.58138645

1.68722177

1.68722177

0 . \# Energy 3->5

$\begin{array}{lll}0.1789 & -0.0158 & -0.0735\end{array}$

$-0.0252$

2.13764215

2.13764215

2.13764215

2.13764215

2.24347758

2.24347758

2.24347758

2.24347758

$-1.37885487$

$-1.37885487$

$-1.37885487$

$-1.37885487$

$-1.27301943$

$-1.27301943$

$-1.27301943$

$-1.27301943$

2.29703021

2.29703021

2.29703021

2. 29703021

2. 40286589
0.12237859

0.01654314

0.12237859

$-0.36711088$

$-0.26127544$

$-0.36711088$

$-0.26127544$

$-0.36711088$

$-0.26127544$

$-0.36711088$

$-0.26127544$

$-0.42923152$

$-0.32339609$

$-0.42923152$

$-0.32339609$

$-0.42923152$

$-0.32339609$

$-0.42923152$

$-0.32339609$

0.06612916

0.17196462

0.06612916

0.17196462

0.06612916

0.17196462

0.06612916

0.17196462

$-0.32880956$

$-0.22297411$

$-0.32880956$

$-0.22297411$

$-0.32880956$

$-0.22297411$

$-0.32880956$

$-0.22297411$

0.09555512

0.20139056

0.09555512

0.20139056

0.09555512

0.20139056

0.09555512

0.20139056

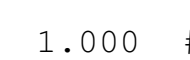

0.05111535

0.15695079

0.05111535

0.15695079

0.05111535

0.15695079

0.05111535

0.15695079

$-0.39047247$

$-0.28463703$

$-0.39047247$

$-0.28463703$

$-0.39047247$

$-0.28463703$

$-0.39047247$

$-0.28463703$

0.01654314

0.12237859

0.01654314

0.12237859

0.01654314
-3.18511915
-3.63384795
1.46363592
0.59975481
-0.16130581
-1.18408751
0.61434388
-0.65110642
0.22047032
1.27162540
-0.72341126
-16.26429367
19.12698936
18.56265259
-22.51782990
19.67277908
-23.43964386
-22.36904335
27.36048317
2.92537618
-2.78229761
-2.52038550
2.37522030
-4.14041376
3.94302011
3.71716714
-3.47524762
-1.39311051
1.26592457
2.17936277
-2.11055207
1.68931043
-1.63229811
-2.58109331
2.61914206
-0.44528672
0.23387043
0.35183167
-0.19095519
0.27988246
-0.09266393
-0.23448464
0.09020173

Electr. mom., scale-fac Magnetic mom.

21.45029259

$-9.42484760$

$-21.93517685$

10.87769604

$-14.06433964$

3.50061965

14.61633396

$-4.68429041$

3.56004739

$-4.56927824$

$-6.52302408$

8.84314823

$-2.11081195$

2. 19237947

4.81930304

$-6.11041546$

14.85041428

$-8.64377117$

$-9.71075439$

4.65791559

$-12.29863834$ 


\subsection{9 \\ 2. 40286589 \\ 2.40286589 \\ $-1.56014943$ \\ $-1.56014943$ \\ $-1.56014943$ \\ $-1.56014943$ \\ $-1.45431399$ \\ $-1.45431399$ \\ $-1.45431399$ \\ $-1.45431399$ \\ 1.01902342 \\ 1.01902342 \\ 1. 01902342 \\ 1.01902342 \\ 1.12485886 \\ 1.12485886 \\ 1.12485886 \\ 1.12485886 \\ $-2.29137540$ \\ $-2.29137540$ \\ $-2.29137540$ \\ $-2.29137540$ \\ $-2.18554020$ \\ $-2.18554020$ \\ $-2.18554020$ \\ $-2.18554020$ \\ 0.88915640 \\ 0.88915640 \\ 0.88915640 \\ 0.88915640 \\ 0.99499184 \\ 0.99499184 \\ 0.99499184 \\ 0.99499184 \\ $-2.05025959$ \\ $-2.05025959$ \\ $-2.05025959$ \\ $-2.05025959$ \\ $-1.94442415$ \\ $-1.94442415$ \\ $-1.94442415$ \\ $-1.94442415$}

64
1.37668049

1. 48251593

1.48251593

$-1.49588871$

$-1.49588871$

$-1.39005339$

$-1.39005339$

$-1.49588871$

$-1.49588871$

$-1.39005339$

$-1.39005339$

$-0.44691080$

$-0.44691080$

$-0.34107536$

$-0.34107536$

$-0.44691080$

$-0.44691080$

$-0.34107536$

$-0.34107536$

0.60408223

0.60408223

0.70991772

0.70991772

0.60408223

0.60408223

0.70991772

0.70991772

$-1.44335949$

$-1.44335949$

$-1.33752406$

$-1.33752406$

$-1.44335949$

$-1.44335949$

$-1.33752406$

$-1.33752406$

1.58138645

1.58138645

1.68722177

1.68722177

1.58138645

1.58138645

1.68722177

1.68722177

$$
\text { 0. \# Energy 3->6 }
$$

$$
-0.1093 \quad-0.0654
$$$$
-0.2441 \quad-0.0101
$$

0.16034016

0.16034016

0.26617563

0.26617563

0.16034016

0.16034016

0.26617563

0.26617563

$-0.28076980$

$-0.28076980$

$-0.17493436$

$-0.17493436$

$-0.28076980$

$-0.28076980$

$-0.17493436$

$-0.17493436$

1.37668049

1.37668049

1.48251593

1.48251593

1.37668049
0.12237859

0.01654314

0.12237859

$-0.36711088$

$-0.26127544$

$-0.36711088$

$-0.26127544$

$-0.36711088$

$-0.26127544$

$-0.36711088$

$-0.26127544$

$-0.42923152$

$-0.32339609$

$-0.42923152$

$-0.32339609$

$-0.42923152$

$-0.32339609$

$-0.42923152$

$-0.32339609$

0.06612916

0.17196462

0.06612916

0.17196462

0.06612916

0.17196462

0.06612916

0.17196462

$-0.32880956$

$-0.22297411$

$-0.32880956$

$-0.22297411$

$-0.32880956$

$-0.22297411$

$-0.32880956$

$-0.22297411$

0.09555512

0.20139056

0.09555512

0.20139056

0.09555512

0.20139056

0.09555512

0.20139056

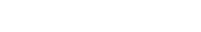
1.000 \# Electr. mom., scale-fac \# Magnetic mom.

0.05111535

0.15695079

0.05111535

0.15695079

0.05111535

0.15695079

0.05111535

0.15695079

$-0.39047247$

$-0.28463703$

$-0.39047247$

$-0.28463703$

$-0.39047247$

$-0.28463703$

$-0.39047247$

$-0.28463703$

0.01654314

0.12237859

0.01654314

0.12237859

0.01654314
6.52748585

7.72420025

$-3.04112601$

$-0.63118452$

1.38218510

0.83739680

$-1.81890774$

0.80456644

$-1.63005483$

$-1.04893160$

2. 12232137

$-36.24262619$

43.86706543

$-39.83014297$

44.72700500

45.66886902

$-53.49679565$

1.10311139

0.65213329

$-0.64132518$

$-0.78875566$

$-2.26858330$

$-0.40587160$

1.55796993

0.67679328

3.83493900

$-2.92900443$

$-5.77366352$

4.64820719

$-4.32571983$

3. 60931063

6.42509699

$-5.58697128$

0.53076488

$-0.40042165$

$-0.44628981$

0.34103408

$-0.73843545$

0.46318135

0.60031229

$-0.39477071$
33.31071854

$-38.26789093$
4.45649147

4.83034658

12.35822392

$-5.50458336$

7.79025269

$-1.06527388$

$-7.84073114$

1.63648570

$-2.93057609$

4.23777437

4.78200436

$-6.92515230$

1.96647382

$-2.65766501$

$-3.62827969$

5.08347559

$-9.37466717$

5.47955799

6.01995516

$-2.84002304$

7.83795691 


\section{40286589 \\ 2.40286589 \\ 2.40286589 \\ $-1.56014943$ \\ $-1.56014943$ \\ $-1.56014943$ \\ $-1.56014943$ \\ $-1.45431399$ \\ $-1.45431399$ \\ $-1.45431399$ \\ $-1.45431399$ \\ 1.01902342 \\ 1.01902342 \\ 1. 01902342 \\ 1.01902342 \\ 1.12485886 \\ 1.12485886 \\ 1.12485886 \\ 1.12485886 \\ $-2.29137540$ \\ $-2.29137540$ \\ $-2.29137540$ \\ $-2.29137540$ \\ $-2.18554020$ \\ $-2.18554020$ \\ $-2.18554020$ \\ $-2.18554020$ \\ 0.88915640 \\ 0.88915640 \\ 0.88915640 \\ 0.88915640 \\ 0.99499184 \\ 0.99499184 \\ 0.99499184 \\ 0.99499184 \\ $-2.05025959$ \\ $-2.05025959$ \\ $-2.05025959$ \\ $-2.05025959$ \\ $-1.94442415$ \\ $-1.94442415$ \\ $-1.94442415$ \\ $-1.94442415$}

64
1.37668049

1. 48251593

1.48251593

$-1.49588871$

$-1.49588871$

$-1.39005339$

$-1.39005339$

$-1.49588871$

$-1.49588871$

$-1.39005339$

$-1.39005339$

$-0.44691080$

$-0.44691080$

$-0.34107536$

$-0.34107536$

$-0.44691080$

$-0.44691080$

$-0.34107536$

$-0.34107536$

0.60408223

0.60408223

0.70991772

0.70991772

0.60408223

0.60408223

0.70991772

0.70991772

$-1.44335949$

$-1.44335949$

$-1.33752406$

$-1.33752406$

$-1.44335949$

$-1.44335949$

$-1.33752406$

$-1.33752406$

1.58138645

1.58138645

1.68722177

1.68722177

1.58138645

1.58138645

1.68722177

1.68722177

0 . \# Energy 3->7

$\begin{array}{lll}-0.7339 & -0.0038 & -0.1589\end{array}$

$\begin{array}{lll}0.0505 & -0.0346 & 0.0258\end{array}$

2.13764215

2.13764215

2.13764215

2.13764215

2.24347758

2.24347758

2.24347758

2.24347758

$-1.37885487$

$-1.37885487$

$-1.37885487$

$-1.37885487$

$-1.27301943$

$-1.27301943$

$-1.27301943$

$-1.27301943$

2.29703021

2.29703021

2.29703021

2. 29703021

2. 40286589
0.16034016

0.16034016

0.26617563

0.26617563

0.16034016

0.16034016

0.26617563

0.26617563

$-0.28076980$

$-0.28076980$

$-0.17493436$

$-0.17493436$

$-0.28076980$

$-0.28076980$

$-0.17493436$

$-0.17493436$

1.37668049

1.37668049

1.48251593

1.48251593

1.37668049
0.12237859

0.01654314

0.12237859

$-0.36711088$

$-0.26127544$

$-0.36711088$

$-0.26127544$

$-0.36711088$

$-0.26127544$

$-0.36711088$

$-0.26127544$

$-0.42923152$

$-0.32339609$

$-0.42923152$

$-0.32339609$

$-0.42923152$

$-0.32339609$

$-0.42923152$

$-0.32339609$

0.06612916

0.17196462

0.06612916

0.17196462

0.06612916

0.17196462

0.06612916

0.17196462

$-0.32880956$

$-0.22297411$

$-0.32880956$

$-0.22297411$

$-0.32880956$

$-0.22297411$

$-0.32880956$

$-0.22297411$

0.09555512

0.20139056

0.09555512

0.20139056

0.09555512

0.20139056

0.09555512

0.20139056

1.000

0.05111535

0.15695079

0.05111535

0.15695079

0.05111535

0.15695079

0.05111535

0.15695079

$-0.39047247$

$-0.28463703$

$-0.39047247$

$-0.28463703$

$-0.39047247$

$-0.28463703$

$-0.39047247$

$-0.28463703$

0.01654314

0.12237859

0.01654314

0.12237859

0.01654314
$-4.18069792$

$-4.81978512$

1.84960783

0.34578249

$-0.61816096$

$-0.24659130$

0.61246562

$-0.49005097$

0.82961124

0.42173842

$-0.88302147$

$-22.34772873$

25.10227394

25.82571411

$-30.44779587$

26.87895775

$-31.00909424$

$-30.97628784$

37.18297195

0.00655711

$-1.23754525$

$-0.33000556$

1.33076942

0.83588189

0.97481948

$-0.34566289$

$-1.15280044$

$-2.39732742$

1. 84204841

3.56042624

$-2.89600158$

2. 71994090

$-2.29312706$

$-3.99550295$

3. 52426291

$-0.15186515$

0.01863406

0.15150453

$-0.04703919$

0.30710143

$-0.09589069$

$-0.26683617$

0.10868405

0.10868405

ctr. mom., scale-fac Magnetic mom.

5.32287931

$-2.19719911$

$-2.71758699$

0.02093888

$-4.19777489$

1.57991242

2.16643119

0.07036017

1.15436816

1.40098369

0.78530264

$-3.70340180$

$-3.01823425$

0.96188146

1.10358620

1.28097105

$-1.80676973$

1.61078274

1.34133315

$-1.15731311$

1. 34051108 


\begin{tabular}{|c|c|c|c|}
\hline 2.40286589 & 1.37668049 & 0.12237859 & -1.15285385 \\
\hline 2.40286589 & 1.48251593 & 0.01654314 & -0.98707241 \\
\hline 2.40286589 & 1.48251593 & 0.12237859 & 0.81147957 \\
\hline-1.56014943 & -1.49588871 & -0.36711088 & 1.26958489 \\
\hline-1.56014943 & -1.49588871 & -0.26127544 & -0.92055792 \\
\hline-1.56014943 & -1.39005339 & -0.36711088 & -1.75797415 \\
\hline-1.56014943 & -1.39005339 & -0.26127544 & 1.34869087 \\
\hline-1.45431399 & -1.49588871 & -0.36711088 & -1.56113422 \\
\hline-1.45431399 & -1.49588871 & -0.26127544 & 1.21782851 \\
\hline-1.45431399 & -1.39005339 & -0.36711088 & 2.12884879 \\
\hline-1.45431399 & -1.39005339 & -0.26127544 & -1.71644104 \\
\hline 1.01902342 & -0.44691080 & -0.42923152 & -11.98518276 \\
\hline 1.01902342 & -0.44691080 & -0.32339609 & 13.47694397 \\
\hline 1.01902342 & -0.34107536 & -0.42923152 & 11.39629650 \\
\hline 1.01902342 & -0.34107536 & -0.32339609 & -12.13324547 \\
\hline 1.12485886 & -0.44691080 & -0.42923152 & 11.96109486 \\
\hline 1.12485886 & -0.44691080 & -0.32339609 & -13.87199116 \\
\hline 1.12485886 & -0.34107536 & -0.42923152 & -11.53452969 \\
\hline 1.12485886 & -0.34107536 & -0.32339609 & 12.57931709 \\
\hline-2.29137540 & 0.60408223 & 0.06612916 & 1.02800274 \\
\hline-2.29137540 & 0.60408223 & 0.17196462 & -1.17496145 \\
\hline-2.29137540 & 0.70991772 & 0.06612916 & -1.26251614 \\
\hline-2.29137540 & 0.70991772 & 0.17196462 & 1.23138535 \\
\hline-2.18554020 & 0.60408223 & 0.06612916 & -1.49943042 \\
\hline-2.18554020 & 0.60408223 & 0.17196462 & 1.75694335 \\
\hline-2.18554020 & 0.70991772 & 0.06612916 & 1.85880947 \\
\hline-2.18554020 & 0.70991772 & 0.17196462 & -1.86834371 \\
\hline 0.88915640 & -1.44335949 & -0.32880956 & -0.81094253 \\
\hline 0.88915640 & -1.44335949 & -0.22297411 & 1.28762209 \\
\hline 0.88915640 & -1.33752406 & -0.32880956 & 1.39082730 \\
\hline 0.88915640 & -1.33752406 & -0.22297411 & -2.11896014 \\
\hline 0.99499184 & -1.44335949 & -0.32880956 & 0.58337539 \\
\hline 0.99499184 & -1.44335949 & -0.22297411 & -1.11572337 \\
\hline 0.99499184 & -1.33752406 & -0.32880956 & -1.10094905 \\
\hline 0.99499184 & -1.33752406 & -0.22297411 & 1.90865088 \\
\hline-2.05025959 & 1.58138645 & 0.09555512 & -0.14799939 \\
\hline-2.05025959 & 1.58138645 & 0.20139056 & 0.29427344 \\
\hline-2.05025959 & 1.68722177 & 0.09555512 & 0.10816537 \\
\hline-2.05025959 & 1.68722177 & 0.20139056 & -0.24028194 \\
\hline-1.94442415 & 1.58138645 & 0.09555512 & -0.02049291 \\
\hline-1.94442415 & 1.58138645 & 0.20139056 & -0.15621318 \\
\hline-1.94442415 & 1.68722177 & 0.09555512 & -0.00024594 \\
\hline-1.94442415 & 1.68722177 & 0.20139056 & 0.15793917 \\
\hline & \# Energy 3->8 & & \\
\hline 3662 & -0.0091 & 1.000 & Electr. mom., scale-fac \\
\hline-0.0330 & 0.0130 & & Magnetic mom. \\
\hline 2.13764215 & 0.16034016 & 0.05111535 & 8.24671936 \\
\hline 2.13764215 & 0.16034016 & 0.15695079 & -16.32663918 \\
\hline 2.13764215 & 0.26617563 & 0.05111535 & -7.94471169 \\
\hline 2.13764215 & 0.26617563 & 0.15695079 & 14.85724258 \\
\hline 2.24347758 & 0.16034016 & 0.05111535 & -11.46659184 \\
\hline 2.24347758 & 0.16034016 & 0.15695079 & 19.29557419 \\
\hline 2.24347758 & 0.26617563 & 0.05111535 & 10.95780277 \\
\hline 2.24347758 & 0.26617563 & 0.15695079 & -17.88922691 \\
\hline-1.37885487 & -0.28076980 & -0.39047247 & 12.04461288 \\
\hline-1.37885487 & -0.28076980 & -0.28463703 & -17.58579636 \\
\hline-1.37885487 & -0.17493436 & -0.39047247 & -12.53917694 \\
\hline-1.37885487 & -0.17493436 & -0.28463703 & 18.82352448 \\
\hline-1.27301943 & -0.28076980 & -0.39047247 & -9.53928089 \\
\hline-1.27301943 & -0.28076980 & -0.28463703 & 13.55237961 \\
\hline-1.27301943 & -0.17493436 & -0.39047247 & 9.64733791 \\
\hline-1.27301943 & -0.17493436 & -0.28463703 & -14.20762062 \\
\hline 2.29703021 & 1.37668049 & 0.01654314 & -5.17457056 \\
\hline 2.29703021 & 1.37668049 & 0.12237859 & 2.51579928 \\
\hline 2.29703021 & 1.48251593 & 0.01654314 & 2.77474093 \\
\hline 2.2970 & 1.48251593 & 0.12237859 & -0.65090150 \\
\hline $2.402 \varepsilon$ & 1.37668049 & 0.01654314 & 3.84809303 \\
\hline
\end{tabular}

\section{S-157}




\begin{tabular}{|c|c|c|c|}
\hline 2.40286589 & 1.37668049 & 0.12237859 & -1.29055035 \\
\hline 2.40286589 & 1.48251593 & 0.01654314 & -1.76788712 \\
\hline 2.40286589 & 1.48251593 & 0.12237859 & -0.25900564 \\
\hline-1.56014943 & -1.49588871 & -0.36711088 & -0.55667996 \\
\hline-1.56014943 & -1.49588871 & -0.26127544 & 0.55052626 \\
\hline-1.56014943 & -1.39005339 & -0.36711088 & -0.32339320 \\
\hline-1.56014943 & -1.39005339 & -0.26127544 & 0.38450089 \\
\hline-1.45431399 & -1.49588871 & -0.36711088 & 0.90265560 \\
\hline-1.45431399 & -1.49588871 & -0.26127544 & -1.06088746 \\
\hline-1.45431399 & -1.39005339 & -0.36711088 & -0.15704055 \\
\hline-1.45431399 & -1.39005339 & -0.26127544 & 0.32176432 \\
\hline 1.01902342 & -0.44691080 & -0.42923152 & -18.40344238 \\
\hline 1.01902342 & -0.44691080 & -0.32339609 & 22.03623009 \\
\hline 1.01902342 & -0.34107536 & -0.42923152 & 21.91264153 \\
\hline 1.01902342 & -0.34107536 & -0.32339609 & -26.94898987 \\
\hline 1.12485886 & -0.44691080 & -0.42923152 & 21.56669617 \\
\hline 1.12485886 & -0.44691080 & -0.32339609 & -26.36902046 \\
\hline 1.12485886 & -0.34107536 & -0.42923152 & -25.73753548 \\
\hline 1.12485886 & -0.34107536 & -0.32339609 & 32.18069839 \\
\hline-2.29137540 & 0.60408223 & 0.06612916 & 4.89782619 \\
\hline-2.29137540 & 0.60408223 & 0.17196462 & 1.35875320 \\
\hline-2.29137540 & 0.70991772 & 0.06612916 & -4.28704405 \\
\hline-2.29137540 & 0.70991772 & 0.17196462 & -0.63723838 \\
\hline-2.18554020 & 0.60408223 & 0.06612916 & -8.12685013 \\
\hline-2.18554020 & 0.60408223 & 0.17196462 & 0.87400258 \\
\hline-2.18554020 & 0.70991772 & 0.06612916 & 7.03157949 \\
\hline-2.18554020 & 0.70991772 & 0.17196462 & -1.29337776 \\
\hline 0.88915640 & -1.44335949 & -0.32880956 & -1.19566643 \\
\hline 0.88915640 & -1.44335949 & -0.22297411 & 0.91197973 \\
\hline 0.88915640 & -1.33752406 & -0.32880956 & 1.82076406 \\
\hline 0.88915640 & -1.33752406 & -0.22297411 & -1.58524704 \\
\hline 0.99499184 & -1.44335949 & -0.32880956 & 1.40623820 \\
\hline 0.99499184 & -1.44335949 & -0.22297411 & -1.16496372 \\
\hline 0.99499184 & -1.33752406 & -0.32880956 & -2.13718009 \\
\hline 0.99499184 & -1.33752406 & -0.22297411 & 1.94542551 \\
\hline-2.05025959 & 1.58138645 & 0.09555512 & 1.37715375 \\
\hline-2.05025959 & 1.58138645 & 0.20139056 & -1.39097011 \\
\hline-2.05025959 & 1.68722177 & 0.09555512 & -1.11771250 \\
\hline-2.05025959 & 1.68722177 & 0.20139056 & 1.13506842 \\
\hline-1.94442415 & 1.58138645 & 0.09555512 & -1.79436564 \\
\hline-1.94442415 & 1.58138645 & 0.20139056 & 1.73657417 \\
\hline-1.94442415 & 1.68722177 & 0.09555512 & 1.39883232 \\
\hline-1.94442415 & 1.68722177 & 0.20139056 & -1.38416898 \\
\hline & \# Energy 3->9 & & \\
\hline-0.2141 & -0.0009 & 1.000 & Electr. mom., scale-fac \\
\hline 0.0124 & 0.0272 & $\#$ & Magnetic mom. \\
\hline 2.13764215 & 0.16034016 & 0.05111535 & -4.58649492 \\
\hline 2.13764215 & 0.16034016 & 0.15695079 & 8.31596661 \\
\hline 2.13764215 & 0.26617563 & 0.05111535 & 4.67966366 \\
\hline 2.13764215 & 0.26617563 & 0.15695079 & -7.88264847 \\
\hline 2.24347758 & 0.16034016 & 0.05111535 & 5.80655336 \\
\hline 2.24347758 & 0.16034016 & 0.15695079 & -9.46109104 \\
\hline 2.24347758 & 0.26617563 & 0.05111535 & -5.76324940 \\
\hline 2.24347758 & 0.26617563 & 0.15695079 & 8.98807144 \\
\hline-1.37885487 & -0.28076980 & -0.39047247 & -8.23268414 \\
\hline-1.37885487 & -0.28076980 & -0.28463703 & 14.42755795 \\
\hline-1.37885487 & -0.17493436 & -0.39047247 & 9.98232746 \\
\hline-1.37885487 & -0.17493436 & -0.28463703 & -16.87399864 \\
\hline-1.27301943 & -0.28076980 & -0.39047247 & 5.91475964 \\
\hline-1.27301943 & -0.28076980 & -0.28463703 & -10.80329895 \\
\hline-1.27301943 & -0.17493436 & -0.39047247 & -7.27630806 \\
\hline-1.27301943 & -0.17493436 & -0.28463703 & 12.68896008 \\
\hline 2.29703021 & 1.37668049 & 0.01654314 & 0.64773732 \\
\hline 2.29703021 & 1.37668049 & 0.12237859 & 0.02893650 \\
\hline 2.29703021 & 1.48251593 & 0.01654314 & -0.15881506 \\
\hline 2.29703021 & 1.48251593 & 0.12237859 & -0.36650881 \\
\hline 2.40286589 & 1.37668049 & 0.01654314 & -0.31824312 \\
\hline
\end{tabular}

\section{S-158}




2.40286589
2.40286589
2.40286589
-1.56014943
-1.56014943
-1.56014943
-1.56014943
-1.45431399
-1.45431399
-1.45431399
-1.45431399
1.01902342
1.01902342
1.01902342
1.01902342
1.12485886
1.12485886
1.12485886
1.12485886
-2.29137540
-2.29137540
-2.29137540
-2.29137540
-2.18554020
-2.18554020
-2.18554020
-2.18554020
0.88915640
0.88915640
0.88915640
0.88915640
0.99499184
0.99499184
0.99499184
0.99499184
-2.05025959
-2.05025959
-2.05025959
-2.05025959
-1.94442415
-1.94442415
-1.94442415
-1.94442415

\&TRANSITION $4->\ldots$

640 .
1. 37668049

1. 48251593

1. 48251593

$-1.49588871$

$-1.49588871$

$-1.39005339$

$-1.39005339$

$-1.49588871$

$-1.49588871$

$-1.39005339$

$-1.39005339$

$-0.44691080$

$-0.44691080$

$-0.34107536$

$-0.34107536$

$-0.44691080$

$-0.44691080$

$-0.34107536$

$-0.34107536$

0.60408223

0.60408223

0.70991772

0.70991772

0.60408223

0.60408223

0.70991772

0.70991772

$-1.44335949$

$-1.44335949$

$-1.33752406$

$-1.33752406$

$-1.44335949$

$-1.44335949$

$-1.33752406$

$-1.33752406$

1.58138645

1. 58138645

1.68722177

1.68722177

1.58138645

1. 58138645

1. 68722177

1.68722177

\# Energy 4->5

0.1431

2. 13764215

2.13764215

2.13764215

2.13764215

2. 24347758

2. 24347758

2. 24347758

2. 24347758

$-1.37885487$

$-1.37885487$

$-1.37885487$

$-1.37885487$

$-1.27301943$

$-1.27301943$

$-1.27301943$

$-1.27301943$

2. 29703021

2. 29703021

2. 29703021

2. 29703021

$0.4271 \quad 0.5326$

$\begin{array}{lr}0.0376 & -0.0268\end{array}$

0.16034016

0.16034016

0.26617563

0.26617563

0.16034016

0.16034016

0.26617563

0.26617563

$-0.28076980$

$-0.28076980$

$-0.17493436$

$-0.17493436$

$-0.28076980$

$-0.28076980$

$-0.17493436$

$-0.17493436$

1.37668049

1.37668049

1. 48251593

1.48251593

$$
\begin{array}{r}
0.12237859 \\
0.01654314 \\
0.12237859 \\
-0.36711088 \\
-0.26127544 \\
-0.36711088 \\
-0.26127544 \\
-0.36711088 \\
-0.26127544 \\
-0.36711088 \\
-0.26127544 \\
-0.42923152 \\
-0.32339609 \\
-0.42923152 \\
-0.32339609 \\
-0.42923152 \\
-0.32339609 \\
-0.42923152 \\
-0.32339609 \\
0.06612916 \\
0.17196462 \\
0.06612916 \\
0.17196462 \\
0.06612916 \\
0.17196462 \\
0.06612916 \\
0.17196462 \\
-0.32880956 \\
-0.22297411 \\
-0.32880956 \\
-0.22297411 \\
-0.32880956 \\
-0.22297411 \\
-0.32880956 \\
-0.22297411 \\
0.09555512 \\
0.20139056 \\
0.09555512 \\
0.20139056 \\
0.09555512 \\
0.20139056 \\
0.09555512 \\
0.20139056
\end{array}
$$

-0.34805629
-0.07997431
0.59384376
0.10305010
0.29181606

$-0.12017807$

$-0.44093278$

$-0.23624884$

$-0.06747972$

0.32635662

0.10234791

2. 28463292

$-2.77019358$

$-3.16715050$

4.12574244

$-2.84733629$

3.57137680

4.02659607

$-5.32683325$

$-1.65269446$

$-2.60391641$

1.05242133

2. 26246929

3.46712446

1.52178442

$-2.46778464$

$-1.39837515$

0.36519927

$-0.23423831$

$-0.53698933$

0.38128543

$-0.38173375$

0.22430968

0.55960596

$-0.35452941$

$-0.65419221$

0.46914858

0.53253770

$-0.39996168$

0.82987112

$-0.62913358$

$-0.66021609$

0.52943587

1.000 \# Electr. mom., scale-fac \# Magnetic mom.

$0.05111535-3.25578260$

$0.15695079 \quad 1.75750208$

$0.05111535 \quad 2.66431284$

0.15695079

0.05111535

0.15695079

0.05111535

0.15695079

$-0.39047247$

$-0.28463703$

$-0.39047247$

$-0.28463703$

$-0.39047247$

$-0.28463703$

$-0.39047247$

$-0.28463703$

0.01654314

0.12237859

0.01654314

$-1.13696611$

3.03190041

$-1.13073373$

$-2.35491300$

0.55430281

2.93089962

$-5.98735857$

$-4.44748688$

7.71522665

$-1.56338632$

4.72653437

3. 08133364

$-6.52792120$

1.62197709

$-1.57269466$

$-1.15651381$

1. 10917056

\section{S-159}


2. 40286589

2. 40286589

2. 40286589

2. 40286589

$-1.56014943$

$-1.56014943$

$-1.56014943$

$-1.56014943$

$-1.45431399$

$-1.45431399$

$-1.45431399$

$-1.45431399$

1.01902342

1.01902342

1. 01902342

1. 01902342

1.12485886

1. 12485886

1. 12485886

1.12485886

$-2.29137540$

$-2.29137540$

$-2.29137540$

$-2.29137540$

$-2.18554020$

$-2.18554020$

$-2.18554020$

$-2.18554020$

0.88915640

0.88915640

0.88915640

0.88915640

0.99499184

0.99499184

0.99499184

0.99499184

$-2.05025959$

$-2.05025959$

$-2.05025959$

$-2.05025959$

$-1.94442415$

$-1.94442415$

$-1.94442415$

64

$-1.94442415$

0.1100
-0.0287

2. 13764215

2.13764215

2. 13764215

2.13764215

2. 24347758

2. 24347758

2. 24347758

2. 24347758

$-1.37885487$

$-1.37885487$

$-1.37885487$

$-1.37885487$

$-1.27301943$

$-1.27301943$

$-1.27301943$

$-1.27301943$

2. 29703021

2. 29703021

2. 29703021

2. 29703021
1. 37668049

1. 37668049

1. 48251593

1.48251593

$-1.49588871$

$-1.49588871$

$-1.39005339$

$-1.39005339$

$-1.49588871$

$-1.49588871$

$-1.39005339$

$-1.39005339$

$-0.44691080$

$-0.44691080$

$-0.34107536$

$-0.34107536$

$-0.44691080$

$-0.44691080$

$-0.34107536$

$-0.34107536$

0.60408223

0.60408223

0.70991772

0.70991772

0.60408223

0.60408223

0.70991772

0.70991772

$-1.44335949$

$-1.44335949$

$-1.33752406$

$-1.33752406$

$-1.44335949$

$-1.44335949$

$-1.33752406$

$-1.33752406$

1. 58138645

1. 58138645

1.68722177

1.68722177

1. 58138645

1.58138645

1.68722177

1.68722177

\# Energy 4->6

$\begin{array}{ll}0.3577 & 0.4092\end{array}$

$-0.0347 \quad 0.0279$

0.16034016

0.16034016

0.26617563

0.26617563

0.16034016

0.16034016

0.26617563

0.26617563

$-0.28076980$

$-0.28076980$

$-0.17493436$

$-0.17493436$

$-0.28076980$

$-0.28076980$

$-0.17493436$

$-0.17493436$

1.37668049

1.37668049

1. 48251593

1. 48251593
0.01654314

0.12237859

0.01654314

0.12237859

$-0.36711088$

$-0.26127544$

$-0.36711088$

$-0.26127544$

$-0.36711088$

$-0.26127544$

$-0.36711088$

$-0.26127544$

$-0.42923152$

$-0.32339609$

$-0.42923152$

$-0.32339609$

$-0.42923152$

$-0.32339609$

$-0.42923152$

$-0.32339609$

0.06612916

0.17196462

0.06612916

0.17196462

0.06612916

0.17196462

0.06612916

0.17196462

$-0.32880956$

$-0.22297411$

$-0.32880956$

$-0.22297411$

$-0.32880956$

$-0.22297411$

$-0.32880956$

$-0.22297411$

0.09555512

0.20139056

0.09555512

0.20139056

0.09555512

0.20139056

0.09555512

0.20139056

$$
1.000 \text { \# Electr. mom., scale-fac }
$$
\# Magnetic mom.

0.05111535

0.15695079

0.05111535

0.15695079

0.05111535

0.15695079

0.05111535

0.15695079

$-0.39047247$

$-0.28463703$

$-0.39047247$

$-0.28463703$

$-0.39047247$

$-0.28463703$

$-0.39047247$

$-0.28463703$

0.01654314

0.12237859

0.01654314

0.12237859
$-1.70531058$

1.56660259

1. 24899304

$-1.09992194$

$-2.81406951$

2.68284845

3.63079238

$-3.47039294$

3.03061938

$-3.05728388$

$-3.91926479$

3. 92928147

1. 11741102

$-0.64339888$

$-1.83530712$

1.62221980

$-0.25358441$

$-0.67403436$

1. 38328373

$-0.94297844$

$-2.06595540$

2. 78631663

1.63684690

$-2.46106791$

2.47061300

$-3.10935116$

$-1.81082880$

2.64021516

0.20124558

$-0.15038070$

$-0.15469941$

0.04003063

$-0.09179361$

$-0.13069156$

0.00050026

0.35999328

0.45958143

$-0.67118269$

$-0.45287177$

0.63006270

$-0.50131243$

0.66228205

0.48507860

$-0.62853944$

$$
\begin{array}{r}
4.78327608 \\
-2.17743993 \\
-3.89178801 \\
1.44122612 \\
-4.02015495 \\
1.28392982 \\
3.20812154 \\
-0.67979181 \\
-1.21472371 \\
0.04963336 \\
1.65864849 \\
-0.37458482 \\
0.65016788 \\
0.34469146 \\
-1.25471532 \\
0.26554513 \\
-1.04530883 \\
1.37350607 \\
0.88383526 \\
-1.13282669
\end{array}
$$




2.40286589
2.40286589
2.40286589
2.40286589
-1.56014943
-1.56014943
-1.56014943
-1.56014943
-1.45431399
-1.45431399
-1.45431399
-1.45431399
1.01902342
1.01902342
1.01902342
1.01902342
1.12485886
1.12485886
1.12485886
1.12485886
-2.29137540
-2.29137540
-2.29137540
-2.29137540
-2.18554020
-2.18554020
-2.18554020
-2.18554020
0.88915640
0.88915640
0.88915640
0.88915640
0.99499184
0.99499184
0.99499184
0.99499184
-2.05025959
-2.05025959
-2.05025959
-2.05025959
-1.94442415
-1.94442415
-1.94442415
-1.94442415
-

64
1.37668049

1.37668049

1. 48251593

1.48251593

$-1.49588871$

$-1.49588871$

$-1.39005339$

$-1.39005339$

$-1.49588871$

$-1.49588871$

$-1.39005339$

$-1.39005339$

$-0.44691080$

$-0.44691080$

$-0.34107536$

$-0.34107536$

$-0.44691080$

$-0.44691080$

$-0.34107536$

$-0.34107536$

0.60408223

0.60408223

0.70991772

0.70991772

0.60408223

0.60408223

0.70991772

0.70991772

$-1.44335949$

$-1.44335949$

$-1.33752406$

$-1.33752406$

$-1.44335949$

$-1.44335949$

$-1.33752406$

$-1.33752406$

1.58138645

1.58138645

1.68722177

1.68722177

1.58138645

1.58138645

1.68722177

1.68722177

$-0.0001$

2.13764215

2.13764215

2.13764215

2.13764215

2.24347758

2.24347758

2.24347758

2.24347758

$-1.37885487$

$-1.37885487$

$-1.37885487$

$-1.37885487$

$-1.27301943$

$-1.27301943$

$-1.27301943$

$-1.27301943$

2. 29703021

2.29703021

2. 29703021

2. 29703021
0. \# Energy $4->7$
$-0.0636 \quad-0.0054$

$0.0029 \quad 0.0402$

0.16034016

0.16034016

0.26617563

0.26617563

0.16034016

0.16034016

0.26617563

0.26617563

$-0.28076980$

$-0.28076980$

$-0.17493436$

$-0.17493436$

$-0.28076980$

$-0.28076980$

$-0.17493436$

$-0.17493436$

1.37668049

1.37668049

1. 48251593

1. 48251593
0.01654314

0.12237859

0.01654314

0.12237859

$-0.36711088$

$-0.26127544$

$-0.36711088$

$-0.26127544$

$-0.36711088$

$-0.26127544$

$-0.36711088$

$-0.26127544$

$-0.42923152$

$-0.32339609$

$-0.42923152$

$-0.32339609$

$-0.42923152$

$-0.32339609$

$-0.42923152$

$-0.32339609$

0.06612916

0.17196462

0.06612916

0.17196462

0.06612916

0.17196462

0.06612916

0.17196462

$-0.32880956$

$-0.22297411$

$-0.32880956$

$-0.22297411$

$-0.32880956$

$-0.22297411$

$-0.32880956$

$-0.22297411$

0.09555512

0.20139056

0.09555512

0.20139056

0.09555512

0.20139056

0.09555512

0.20139056

$$
\begin{gathered}
1.000 \text { \# Electr. mom., scale-fac } \\
\text { \# Magnetic mom. }
\end{gathered}
$$

0.05111535

0.15695079

0.05111535

0.15695079

0.05111535

0.15695079

0.05111535

0.15695079

$-0.39047247$

$-0.28463703$

$-0.39047247$

$-0.28463703$

$-0.39047247$

$-0.28463703$

$-0.39047247$

$-0.28463703$

0.01654314

0.12237859

0.01654314

0.12237859
0.65540916

1.11051083

$-1.33783507$

$-0.96241409$

1.11206830

1.35264850

$-1.99600017$

$-1.49927258$

2.35351443

$-1.71426666$

2.50657845

1. 93242574

$-2.94312000$

3.42118883

$-5.45721674$

$-4.10655642$

6.27778864

$-4.74397230$

7.19747353

5.14849901

$-7.61248922$

2. 91343784

$-1.77379632$

1.78646302

$-3.42967916$

2.70676160

1.87330329

$-1.65792847$

$-0.12285382$

0.09946364

0.18866898

$-0.07343425$

$-0.04501186$

0.21165587

0.05129426

$-0.36866644$

$-0.95048100$

1.14585078

0.74486381

$-0.91643602$

1.16118646

$-1.30096662$

$-0.91733509$

1.05029154

0.27648890

$-0.67530781$

$-0.18146066$

$-0.27560285$

$-0.63143218$

0.32453033

0.52030027

1.55795288

$-3.52304959$

$-1.46527255$

3.33501840

$-1.11155665$

2. 92319345

0.97764337

$-2.70018697$

0.43104652

$-0.24737220$

$-0.63295710$

0.47862297
$-2.56745243$ 


2.40286589
2.40286589
2.40286589
2.40286589
-1.56014943
-1.56014943
-1.56014943
-1.56014943
-1.45431399
-1.45431399
-1.45431399
-1.45431399
1.01902342
1.01902342
1.01902342
1.01902342
1.12485886
1.12485886
1.12485886
1.12485886
-2.29137540
-2.29137540
-2.29137540
-2.29137540
-2.18554020
-2.18554020
-2.18554020
-2.18554020
0.88915640
0.88915640
0.88915640
0.88915640
0.99499184
0.99499184
0.99499184
0.99499184
-2.05025959
-2.05025959
-2.05025959
-2.05025959
-1.94442415
-1.94442415
-1.94442415
-1.94442415
-1

64
1. 37668049

1. 37668049

1. 48251593

1. 48251593

$-1.49588871$

$-1.49588871$

$-1.39005339$

$-1.39005339$

$-1.49588871$

$-1.49588871$

$-1.39005339$

$-1.39005339$

$-0.44691080$

$-0.44691080$

$-0.34107536$

$-0.34107536$

$-0.44691080$

$-0.44691080$

$-0.34107536$

$-0.34107536$

0.60408223

0.60408223

0.70991772

0.70991772

0.60408223

0.60408223

0.70991772

0.70991772

$-1.44335949$

$-1.44335949$

$-1.33752406$

$-1.33752406$

$-1.44335949$

$-1.44335949$

$-1.33752406$

$-1.33752406$

1. 58138645

1. 58138645

1.68722177

1.68722177

1.58138645

1.58138645

1.68722177

1.68722177

\# Energy 4->8

$\begin{array}{lll}0.1798 & -0.2455 & -0.2062\end{array}$

$-0.0019$

2.13764215

2.13764215

2.13764215

2.13764215

2. 24347758

2. 24347758

2. 24347758

2. 24347758

$-1.37885487$

$-1.37885487$

$-1.37885487$

$-1.37885487$

$-1.27301943$

$-1.27301943$

$-1.27301943$

$-1.27301943$

2. 29703021

2. 29703021

2. 29703021

2. 29703021
0.01654314

0.12237859

0.01654314

0.12237859

$-0.36711088$

$-0.26127544$

$-0.36711088$

$-0.26127544$

$-0.36711088$

$-0.26127544$

$-0.36711088$

$-0.26127544$

$-0.42923152$

$-0.32339609$

$-0.42923152$

$-0.32339609$

$-0.42923152$

$-0.32339609$

$-0.42923152$

$-0.32339609$

0.06612916

0.17196462

0.06612916

0.17196462

0.06612916

0.17196462

0.06612916

0.17196462

$-0.32880956$

$-0.22297411$

$-0.32880956$

$-0.22297411$

$-0.32880956$

$-0.22297411$

$-0.32880956$

$-0.22297411$

0.09555512

0.20139056

0.09555512

0.20139056

0.09555512

0.20139056

0.09555512

0.20139056
1.000 \# Electr. mom., scale-fac \# Magnetic mom.

0.05111535

0.15695079

0.05111535

0.15695079

0.05111535

0.15695079

0.05111535

0.15695079

$-0.39047247$

$-0.28463703$

$-0.39047247$

$-0.28463703$

$-0.39047247$

$-0.28463703$

$-0.39047247$

$-0.28463703$

0.01654314

0.12237859

0.01654314

0.12237859
$-0.29132420$

0.07654995

0.52015609

$-0.34996170$

$-0.09075232$

$-0.25450671$

0.38299453

0.07416512

0.08851077

0.24710578

$-0.40232506$

$-0.03274346$

$-0.50984198$

$-0.17462951$

0.56051081

0.14999443

0.02676384

0.54343671

$-0.04272150$

$-0.58140820$

$-1.71927404$

1.60600674

$-1.74593377$

2.06297565

$-2.05954337$

$-1.91640210$

1. 91898680

$-0.12079068$

0.04429109

0.13118400

$-0.00293468$

0.02786860

0.01655908

$-0.00393742$

$-0.07952230$

0.16042674

$-0.04528511$

$-0.13643210$

0.05740223

$-0.12109348$

0.04257505

0.10565690

$-0.05373991$
1. 85497522
36.28311157

$-54.21933365$

$-36.32888794$

52.11949539

$-39.66654968$

57.94307709

39.27584457

$-55.61934662$

52.68994522

$-91.26396179$

$-64.26911163$

108.91082001

$-35.47538757$

63.97315598

44.40859604

$-77.95228577$

5.05386019

$-5.74133873$

$-3.37144589$

3. 84956074 


\begin{tabular}{|c|c|c|c|}
\hline & & & \\
\hline 2.40286589 & 1.37668049 & 0.01654314 & -5.29780483 \\
\hline 2.40286589 & 1.37668049 & 0.12237859 & 6.38805819 \\
\hline 2.40286589 & 1.48251593 & 0.01654314 & 3.55357122 \\
\hline 2.40286589 & 1.48251593 & 0.12237859 & -4.34898567 \\
\hline-1.56014943 & -1.49588871 & -0.36711088 & -0.41020760 \\
\hline-1.56014943 & -1.49588871 & -0.26127544 & -0.32996014 \\
\hline-1.56014943 & -1.39005339 & -0.36711088 & 0.01320578 \\
\hline-1.56014943 & -1.39005339 & -0.26127544 & 1.11417294 \\
\hline-1.45431399 & -1.49588871 & -0.36711088 & 1.57569563 \\
\hline-1.45431399 & -1.49588871 & -0.26127544 & -1.74659896 \\
\hline-1.45431399 & -1.39005339 & -0.36711088 & -1.74934328 \\
\hline-1.45431399 & -1.39005339 & -0.26127544 & 1.89623666 \\
\hline 1.01902342 & -0.44691080 & -0.42923152 & 3.39806414 \\
\hline 1.01902342 & -0.44691080 & -0.32339609 & -3.09352231 \\
\hline 1.01902342 & -0.34107536 & -0.42923152 & -1.57955432 \\
\hline 1.01902342 & -0.34107536 & -0.32339609 & 1.06088591 \\
\hline 1.12485886 & -0.44691080 & -0.42923152 & -6.82193089 \\
\hline 1.12485886 & -0.44691080 & -0.32339609 & 6.55214596 \\
\hline 1.12485886 & -0.34107536 & -0.42923152 & 3.93663263 \\
\hline 1.12485886 & -0.34107536 & -0.32339609 & -3.13322306 \\
\hline-2.29137540 & 0.60408223 & 0.06612916 & 13.79901123 \\
\hline-2.29137540 & 0.60408223 & 0.17196462 & 14.01774883 \\
\hline-2.29137540 & 0.70991772 & 0.06612916 & -10.15302372 \\
\hline-2.29137540 & 0.70991772 & 0.17196462 & -11.53748417 \\
\hline-2.18554020 & 0.60408223 & 0.06612916 & -26.70896721 \\
\hline-2.18554020 & 0.60408223 & 0.17196462 & -6.45559692 \\
\hline-2.18554020 & 0.70991772 & 0.06612916 & 20.29785156 \\
\hline-2.18554020 & 0.70991772 & 0.17196462 & 5.51022959 \\
\hline 0.88915640 & -1.44335949 & -0.32880956 & 0.15851708 \\
\hline 0.88915640 & -1.44335949 & -0.22297411 & -0.12390502 \\
\hline 0.88915640 & -1.33752406 & -0.32880956 & -0.59763086 \\
\hline 0.88915640 & -1.33752406 & -0.22297411 & 0.49676633 \\
\hline 0.99499184 & -1.44335949 & -0.32880956 & -0.89804202 \\
\hline 0.99499184 & -1.44335949 & -0.22297411 & 1.07449865 \\
\hline 0.99499184 & -1.33752406 & -0.32880956 & 1.54834294 \\
\hline 0.99499184 & -1.33752406 & -0.22297411 & -1.83718097 \\
\hline-2.05025959 & 1.58138645 & 0.09555512 & 6.11999416 \\
\hline-2.05025959 & 1.58138645 & 0.20139056 & -5.08372164 \\
\hline-2.05025959 & 1.68722177 & 0.09555512 & -4.95031738 \\
\hline-2.05025959 & 1.68722177 & 0.20139056 & 4.20698071 \\
\hline-1.94442415 & 1.58138645 & 0.09555512 & -7.53057241 \\
\hline-1.94442415 & 1.58138645 & 0.20139056 & 6.18492603 \\
\hline-1.94442415 & 1.68722177 & 0.09555512 & 5.94883394 \\
\hline-1.94442415 & 1.68722177 & 0.20139056 & -5.06461859 \\
\hline & \# Energy 4->9 & & \\
\hline-0.3275 & $-0.0927 \quad 0.0342$ & 1.000 & Electr. mom., scale-fac \\
\hline 0.0314 & -0.0399 & \# & Magnetic mom. \\
\hline 2.13764215 & 0.16034016 & 0.05111535 & -21.40760231 \\
\hline 2.13764215 & 0.16034016 & 0.15695079 & 36.74084473 \\
\hline 2.13764215 & 0.26617563 & 0.05111535 & 23.03059578 \\
\hline 2.13764215 & 0.26617563 & 0.15695079 & -36.29881287 \\
\hline 2.24347758 & 0.16034016 & 0.05111535 & 24.64215469 \\
\hline 2.24347758 & 0.16034016 & 0.15695079 & -39.82510757 \\
\hline 2.24347758 & 0.26617563 & 0.05111535 & -25.41232681 \\
\hline 2.24347758 & 0.26617563 & 0.15695079 & 38.84090042 \\
\hline-1.37885487 & -0.28076980 & -0.39047247 & -36.22263718 \\
\hline-1.37885487 & -0.28076980 & -0.28463703 & 60.95485306 \\
\hline-1.37885487 & -0.17493436 & -0.39047247 & 44.13092804 \\
\hline-1.37885487 & -0.17493436 & -0.28463703 & -72.70591736 \\
\hline-1.27301943 & -0.28076980 & -0.39047247 & 25.09313011 \\
\hline-1.27301943 & -0.28076980 & -0.28463703 & -43.30861282 \\
\hline-1.27301943 & -0.17493436 & -0.39047247 & -31.27967262 \\
\hline-1.27301943 & -0.17493436 & -0.28463703 & 52.61740112 \\
\hline 2.29703021 & 1.37668049 & 0.01654314 & -4.61232758 \\
\hline 2.29703021 & 1.37668049 & 0.12237859 & 5.47283506 \\
\hline 2.29703021 & 1.48251593 & 0.01654314 & 3.24281430 \\
\hline 2.29703021 & 1.48251593 & 0.12237859 & -3.86278582 \\
\hline
\end{tabular}

\section{S-163}


2. 40286589

2. 40286589

2. 40286589

2. 40286589

$-1.56014943$

$-1.56014943$

$-1.56014943$

$-1.45431399$

$-1.45431399$

$-1.45431399$

1.01902342

1.01902342

1. 01902342

1. 01902342

1.12485886

1. 12485886

1. 12485886

1.12485886

$-2.29137540$

$-2.29137540$

$-2.29137540$

$-2.29137540$

$-2.18554020$

$-2.18554020$

$-2.18554020$

$-2.18554020$

0.88915640

0.88915640

0.88915640

0.88915640

0.99499184

0.99499184

0.99499184

0.99499184

$-2.05025959$

$-2.05025959$

$-2.05025959$

$-2.05025959$

$-1.94442415$

$-1.94442415$

$-1.94442415$

$-1.94442415$

\&TRANSITION 5->... 64
$-1.56014943$

$-1.45431399$

1. 37668049

1. 37668049

1. 48251593

1.48251593

$-1.49588871$

$-1.49588871$

$-1.39005339$

$-1.39005339$

$-1.49588871$

$-1.49588871$

$-1.39005339$

$-1.39005339$

$-0.44691080$

$-0.44691080$

$-0.34107536$

$-0.34107536$

$-0.44691080$

$-0.44691080$

$-0.34107536$

$-0.34107536$

0.60408223

0.60408223

0.70991772

0.70991772

0.60408223

0.60408223

0.70991772

0.70991772

$-1.44335949$

$-1.44335949$

$-1.33752406$

$-1.33752406$

$-1.44335949$

$-1.44335949$

$-1.33752406$

$-1.33752406$

1. 58138645

1.58138645

1.68722177

1.68722177

1. 58138645

1. 58138645

1.68722177

1. 68722177

\# Energy 5->6

$\begin{array}{lll}-7.3358 & -1.3336 & -0.3672\end{array}$

0.0258

2.13764215

2.13764215

2.13764215

2.13764215

2.24347758

2. 24347758

2. 24347758

2.24347758

$-1.37885487$

$-1.37885487$

$-1.37885487$

$-1.37885487$

$-1.27301943$

$-1.27301943$

$-1.27301943$

$-1.27301943$

2. 29703021

2. 29703021

2. 29703021 $\begin{array}{lr}-1.3336 & -0.3672 \\ -0.0485 & 0.0699\end{array}$

0.16034016

0.16034016

0.26617563

0.26617563

0.16034016

0.16034016

0.26617563

0.26617563

$-0.28076980$

$-0.28076980$

$-0.17493436$

$-0.17493436$

$-0.28076980$

$-0.28076980$

$-0.17493436$

$-0.17493436$

1. 37668049

1. 37668049

1. 48251593
0.01654314

0.12237859

0.01654314

0.12237859

$-0.36711088$

$-0.26127544$

$-0.36711088$

$-0.26127544$

$-0.36711088$

$-0.26127544$

$-0.36711088$

$-0.26127544$

$-0.42923152$

$-0.32339609$

$-0.42923152$

$-0.32339609$

$-0.42923152$

$-0.32339609$

$-0.42923152$

$-0.32339609$

0.06612916

0.17196462

0.06612916

0.17196462

0.06612916

0.17196462

0.06612916

0.17196462

$-0.32880956$

$-0.22297411$

$-0.32880956$

$-0.22297411$

$-0.32880956$

$-0.22297411$

$-0.32880956$

$-0.22297411$

0.09555512

0.20139056

0.09555512

0.20139056

0.09555512

0.20139056

0.09555512

0.20139056
4. 52374363

$-5.63707781$

$-3.18065834$

4.00170994

0.44886407

0.00567830

$-0.49031842$

$-0.18888427$

$-1.32474387$

1. 46541917

1. 77878308

$-1.92356372$

$-2.93863249$

1. 52618992

$-0.17371435$

2. 77688122

4. 76134109

$-3.29132938$

$-0.65268123$

$-2.40942693$

$-8.33185482$

$-10.26391315$

5.94776154

8.45529556

16.66336632

5.50670385

$-12.42839241$

$-4.72093201$

$-0.12483734$

0.35980380

0.59881908

$-0.89219111$

0.55350244

$-0.99735171$

$-1.15946281$

1.81189716

$-4.01531839$

3. 42733884

3. 22464204

$-2.80917120$

4.92660332

$-4.14926195$

$-3.87945366$

3. 38817239
1.000 \# Electr. mom., scale-fac \# Magnetic mom.

0.05111535

0.15695079

0.05111535

0.15695079

0.05111535

0.15695079

0.05111535

0.15695079

$-0.39047247$

$-0.28463703$

$-0.39047247$

$-0.28463703$

$-0.39047247$

$-0.28463703$

$-0.39047247$

$-0.28463703$

0.01654314

0.12237859

0.01654314
5.47005701

$-4.20218706$

$-8.79481983$

6.96080399

$-3.36623001$

3. 43771648

6.11504507

$-5.60039949$

15.69149208

$-14.63543224$

$-22.04579544$

21.02320099

$-13.25417805$

12.42350483

19.82220268

$-19.37173843$

7.56513882

$-5.33593941$

$-6.11521530$ 
2. 29703021

2. 40286589

2. 40286589

2. 40286589

2.40286589

$-1.56014943$

$-1.56014943$

$-1.56014943$

$-1.56014943$

$-1.45431399$

$-1.45431399$

$-1.45431399$

$-1.45431399$

1.01902342

1. 01902342

1.01902342

1. 01902342

1. 12485886

1. 12485886

1.12485886

1. 12485886

$-2.29137540$

$-2.29137540$

$-2.29137540$

$-2.29137540$

$-2.18554020$

$-2.18554020$

$-2.18554020$

$-2.18554020$

0.88915640

0.88915640

0.88915640

0.88915640

0.99499184

0.99499184

0.99499184

0.99499184

$-2.05025959$

$-2.05025959$

$-2.05025959$

$-2.05025959$

$-1.94442415$

$-1.94442415$

$-1.94442415$

64

$-1.94442415$

0.11390 .

$-0.0435$

2.13764215

2. 13764215

2.13764215

2. 13764215

2. 24347758

2. 24347758

2. 24347758

2. 24347758

$-1.37885487$

$-1.37885487$

$-1.37885487$

$-1.37885487$

$-1.27301943$

$-1.27301943$

$-1.27301943$

$-1.27301943$

2. 29703021

2. 29703021

2. 29703021
1. 48251593

1. 37668049

1. 37668049

1. 48251593

1. 48251593

$-1.49588871$

$-1.49588871$

$-1.39005339$

$-1.39005339$

$-1.49588871$

$-1.49588871$

$-1.39005339$

$-1.39005339$

$-0.44691080$

$-0.44691080$

$-0.34107536$

$-0.34107536$

$-0.44691080$

$-0.44691080$

$-0.34107536$

$-0.34107536$

0.60408223

0.60408223

0.70991772

0.70991772

0.60408223

0.60408223

0.70991772

0.70991772

$-1.44335949$

$-1.44335949$

$-1.33752406$

$-1.33752406$

$-1.44335949$

$-1.44335949$

$-1.33752406$

$-1.33752406$

1. 58138645

1. 58138645

1.68722177

1. 68722177

1.58138645

1.58138645

1.68722177

1.68722177

. \# Energy 5->7

$\begin{array}{lr}-0.0084 & 0.0339 \\ -0.3393 & -0.0140\end{array}$
$-0.0140$

0.16034016

0.16034016

0.26617563

0.26617563

0.16034016

0.16034016

0.26617563

0.26617563

$-0.28076980$

$-0.28076980$

$-0.17493436$

$-0.17493436$

$-0.28076980$

$-0.28076980$

$-0.17493436$

$-0.17493436$

1. 37668049

1.37668049

1. 48251593
0.12237859

0.01654314

0.12237859

0.01654314

0.12237859

$-0.36711088$

$-0.26127544$

$-0.36711088$

$-0.26127544$

$-0.36711088$

$-0.26127544$

$-0.36711088$

$-0.26127544$

$-0.42923152$

$-0.32339609$

$-0.42923152$

$-0.32339609$

$-0.42923152$

$-0.32339609$

$-0.42923152$

$-0.32339609$

0.06612916

0.17196462

0.06612916

0.17196462

0.06612916

0.17196462

0.06612916

0.17196462

$-0.32880956$

$-0.22297411$

$-0.32880956$

$-0.22297411$

$-0.32880956$

$-0.22297411$

$-0.32880956$

$-0.22297411$

0.09555512

0.20139056

0.09555512

0.20139056

0.09555512

0.20139056

0.09555512

0.20139056

1.000

0.05111535 \#

0.15695079

0.05111535

0.15695079

0.05111535

0.15695079

0.05111535

0.15695079

$-0.39047247$

$-0.28463703$

$-0.39047247$

$-0.28463703$

$-0.39047247$

$-0.28463703$

$-0.39047247$

$-0.28463703$

0.01654314

0.12237859

0.01654314
4. 44434452

$-5.85316515$

4.30194998

4.85490322

$-3.59112906$

$-3.02463794$

2. 64711261

2.85147643

$-2.49788189$

3. 44105983

$-2.91329527$

$-3.31502271$

2.66392565

6.39418077

$-2.14323044$

$-10.10277271$

6.53936100

$-6.54829168$

2. 51736188

10.35312843

$-6.84864569$

$-10.87404823$

3.81877518

7.06926298

$-0.76757932$

18.58806610

$-10.32571983$

$-14.28612900$

6.78904629

0.60922539

$-0.47697425$

$-1.21293938$

0.95311207

$-0.62000293$

0.45239538

1.25205612

$-0.96129853$

1. 48711765

$-2.84371233$

$-1.05718195$

2.09481072

$-0.19538733$

1. 62446260

0.17286018

$-1.24817610$ 
2. 29703021

2. 40286589

2. 40286589

2. 40286589

2.40286589

$-1.56014943$

$-1.56014943$

$-1.56014943$

$-1.56014943$

$-1.45431399$

$-1.45431399$

$-1.45431399$

$-1.45431399$

1.01902342

1. 01902342

1.01902342

1. 01902342

1. 12485886

1. 12485886

1.12485886

1. 12485886

$-2.29137540$

$-2.29137540$

$-2.29137540$

$-2.29137540$

$-2.18554020$

$-2.18554020$

$-2.18554020$

$-2.18554020$

0.88915640

0.88915640

0.88915640

0.99499184

0.99499184

0.99499184

0.99499184

$-2.05025959$

$-2.05025959$

$-2.05025959$

$-2.05025959$

$-1.94442415$

$-1.94442415$

$-1.94442415$

64

$-1.94442415$
0.88915640

1. 48251593

1. 37668049

1. 37668049

1. 48251593

1. 48251593

$-1.49588871$

$-1.49588871$

$-1.39005339$

$-1.39005339$

$-1.49588871$

$-1.49588871$

$-1.39005339$

$-1.39005339$

$-0.44691080$

$-0.44691080$

$-0.34107536$

$-0.34107536$

$-0.44691080$

$-0.44691080$

$-0.34107536$

$-0.34107536$

0.60408223

0.60408223

0.70991772

0.70991772

0.60408223

0.60408223

0.70991772

0.70991772

$-1.44335949$

$-1.44335949$

$-1.33752406$

$-1.33752406$

$-1.44335949$

$-1.44335949$

$-1.33752406$

$-1.33752406$

1.58138645

1. 58138645

1.68722177

1.68722177

1.58138645

1. 58138645

1. 68722177

1.68722177

$\begin{array}{lll}0.1086 & 0.0 .2 \text { Energy } 5->8 \\ -0.0202 & 0.2228 & 0.2703\end{array}$

$-0.0202$

2. 13764215

2.13764215

2.13764215

2.13764215

2. 24347758

2. 24347758

2. 24347758

2. 24347758

$-1.37885487$

$-1.37885487$

$-1.37885487$

$-1.37885487$

$-1.27301943$

$-1.27301943$

$-1.27301943$

$-1.27301943$

2. 29703021

2. 29703021

2. 29703021

$$
\begin{array}{rr}
0.2228 & 0.2703 \\
-0.0730 & -0.0330
\end{array}
$$

0.16034016

0.16034016

0.26617563

0.26617563

0.16034016

0.16034016

0.26617563

0.26617563

$-0.28076980$

$-0.28076980$

$-0.17493436$

$-0.17493436$

$-0.28076980$

$-0.28076980$

$-0.17493436$

$-0.17493436$

1. 37668049

1.37668049

1. 48251593
0.12237859

0.01654314

0.12237859

0.01654314

0.12237859

$-0.36711088$

$-0.26127544$

$-0.36711088$

$-0.26127544$

$-0.36711088$

$-0.26127544$

$-0.36711088$

$-0.26127544$

$-0.42923152$

$-0.32339609$

$-0.42923152$

$-0.32339609$

$-0.42923152$

$-0.32339609$

$-0.42923152$

$-0.32339609$

0.06612916

0.17196462

0.06612916

0.17196462

0.06612916

0.17196462

0.06612916

0.17196462

$-0.32880956$

$-0.22297411$

$-0.32880956$

$-0.22297411$

$-0.32880956$

$-0.22297411$

$-0.32880956$

$-0.22297411$

0.09555512

0.20139056

0.09555512

0.20139056

0.09555512

0.20139056

0.09555512

0.20139056
$-2.64382696$

10.00257587

$-5.32477665$

$-5.45366192$

1. 69253027

0.13549680

$-0.41916639$

$-0.25924793$

0.60541642

$-0.20177419$

0.52516484

0.33021688

$-0.73385519$

$-18.27892494$

19.60914612

20.57657242

$-23.51130486$

22.77803230

$-24.81202507$

$-25.53072357$

29.33948326

$-0.29165125$

$-1.00127280$

$-0.02151283$

1. 11033213

0.99584514

0.85043287

$-0.51829171$

$-1.05010653$

$-2.53193593$

1.91606700

3. 66932178

$-2.88664794$

3. 02562428

$-2.46512699$

$-4.35964966$

3. 66417527

$-0.30570722$

0.14034136

0.27295890

$-0.14910537$

0.39783353

$-0.16184834$

$-0.33964860$

0.16658218
1.000 \# Electr. mom., scale-fac \# Magnetic mom.

0.05111535

0.15695079

0.05111535

0.15695079

0.05111535

0.15695079

0.05111535

0.15695079

$-0.39047247$

$-0.28463703$

$-0.39047247$

$-0.28463703$

$-0.39047247$

$-0.28463703$

$-0.39047247$

$-0.28463703$

0.01654314

0.12237859

0.01654314
$-47.95782471$

79.04728699

$-76.29985046$

54.76366806

$-85.69020844$

$-54.55619431$

82.50851440

$-74.48988342$

124.10848999

90.29718781

$-148.46388245$ 51.83040619

$-88.29077911$

$-63.87124252$

107.35926819

$-5.69542456$

7.39694691

3. 81056976
48.44678116 
2. 29703021

2. 40286589

2. 40286589

2. 40286589

2.40286589

$-1.56014943$

$-1.56014943$

$-1.56014943$

$-1.56014943$

$-1.45431399$

$-1.45431399$

$-1.45431399$

$-1.45431399$

1.01902342

1. 01902342

1.01902342

1. 01902342

1. 12485886

1. 12485886

1.12485886

1. 12485886

$-2.29137540$

$-2.29137540$

$-2.29137540$

$-2.29137540$

$-2.18554020$

$-2.18554020$

$-2.18554020$

$-2.18554020$

0.88915640

0.88915640

0.88915640

0.88915640

0.99499184

0.99499184

0.99499184

0.99499184

$-2.05025959$

$-2.05025959$

$-2.05025959$

$-2.05025959$

$-1.94442415$

$-1.94442415$

$-1.94442415$

64

$-1.94442415$
1. 48251593

1. 37668049

1. 37668049

1.48251593

1. 48251593

$-1.49588871$

$-1.49588871$

$-1.39005339$

$-1.49588871$

$-1.49588871$

$-1.39005339$

$-1.39005339$

$-0.44691080$

$-0.44691080$

$-0.34107536$

$-0.34107536$

$-0.44691080$

$-0.44691080$

$-0.34107536$

$-0.34107536$

0.60408223

0.60408223

0.70991772

0.70991772

0.60408223

0.60408223

0.70991772

0.70991772

$-1.44335949$

$-1.44335949$

$-1.33752406$

$-1.33752406$

$-1.44335949$

$-1.33752406$

$-1.33752406$

1.58138645

1. 58138645

1. 68722177

1. 68722177

1. 58138645

1.58138645

1.68722177

1.68722177

0.

0.0095

2.13764215

2.13764215

2.13764215

2. 13764215

2. 24347758

2. 24347758

2. 24347758

2. 24347758

$-1.37885487$

$-1.37885487$

$-1.37885487$

$-1.37885487$

$-1.27301943$

$-1.27301943$

$-1.27301943$

$-1.27301943$

2. 29703021

2. 29703021

2. 29703021

$-1.39005339$

$-1.44335949$

0.12237859

0.01654314

0.12237859

0.01654314

0.12237859

$-0.36711088$

$-0.26127544$

$-0.36711088$

$-0.26127544$

$-0.36711088$

$-0.26127544$

$-0.36711088$

$-0.26127544$

$-0.42923152$

$-0.32339609$

$-0.42923152$

$-0.32339609$

$-0.42923152$

$-0.32339609$

$-0.42923152$

$-0.32339609$

0.06612916

0.17196462

0.06612916

0.17196462

0.06612916

0.17196462

0.06612916

0.17196462

$-0.32880956$

$-0.22297411$

$-0.32880956$

$-0.22297411$

$-0.32880956$

$-0.22297411$

$-0.32880956$

$-0.22297411$

0.09555512

0.20139056

0.09555512

0.20139056

0.09555512

0.20139056

0.09555512

0.20139056

\# Energy 5->9

$\begin{array}{rr}-0.2403 & -0.0342 \\ 0.0305 & 0.0645\end{array}$

0.16034016

0.16034016

0.26617563

0.26617563

0.16034016

0.16034016

0.26617563

0.26617563

$-0.28076980$

$-0.28076980$

$-0.17493436$

$-0.17493436$

$-0.28076980$

$-0.28076980$

$-0.17493436$

$-0.17493436$

1. 37668049

1.37668049

1. 48251593
$-5.02693844$

6.14000654

$-8.42054844$

$-4.13475323$

5.82109165

1. 51041925

$-1.02507913$

$-0.89654589$

0.06005336

$-3.21634603$

4.02678251

3.39736557

$-4.33971643$

$-7.42843676$

8.06844425

4.14519310

$-4.48687983$

13.28131104

$-14.47861099$

$-8.58692551$

9.04415321

$-19.65264893$

$-19.82699013$

14.55710793

16.08011627

38.39268112

9.08759212

$-29.34744835$

$-7.55161667$

$-0.56520087$

0.53588855

1. 19572413

$-1.07753158$

1.53254557

$-1.85160637$

$-2.46924043$

2.98472309

$-7.72237206$

6.77684546

6.29466105

$-5.61409140$

9.75621223

$-8.30395126$

$-7.72776222$

6.80849028

$$
\begin{gathered}
1.000 \text { \# Electr. mom., scale-fac } \\
\text { \# Magnetic mom. }
\end{gathered}
$$

0.05111535

0.15695079

0.05111535

0.15695079

0.05111535

0.15695079

0.05111535

0.15695079

$-0.39047247$

$-0.28463703$

$-0.39047247$

$-0.28463703$

$-0.39047247$

$-0.28463703$

$-0.39047247$

$-0.28463703$

0.01654314

0.12237859

0.01654314
$-42.06179428$

67.70555115

43.17560577

$-65.15435791$

46.38624191

$-72.50447845$

$-46.23777390$

69.29107666

$-58.13715363$

103.11510468

73.10147858

$-125.80686951$

37.72225571

$-70.59621429$

$-49.35350037$

88.55650330

$-7.63576221$

9. 28168011

5.49297380 


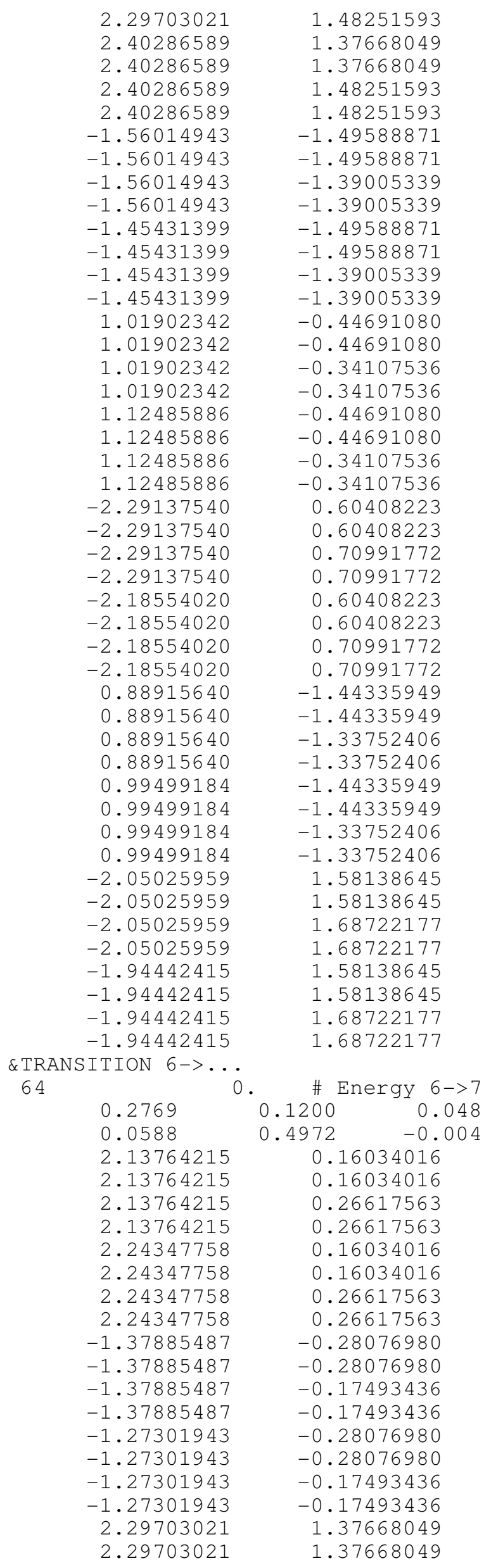

2. 29703021

2. 40286589

2. 40286589

$-1.56014943$

$-1.56014943$

$-1.56014943$

$-1.56014943$

$-1.45431399$

1.01902342

1.12485886

1.12485886

1.12485886

. 12485886

$-2.29137540$

$-2.29137540$

0.889156

0.99499184

0.99499184

0.99499184

$-2.05025959$

$-1.94442415$

2.13764215

2.13764215

2.24347758

2. 24347758

2.24347758

$-1.378854$

$-1.37885487$

$-1.27301943$

2. 29703021
0.12237859

0.01654314

0.12237859

0.01654314

0.12237859

$-0.36711088$

$-0.26127544$

$-0.36711088$

$-0.26127544$

$-0.36711088$

$-0.26127544$

$-0.36711088$

$-0.26127544$

$-0.42923152$

$-0.32339609$

$-0.42923152$

$-0.32339609$

$-0.42923152$

$-0.32339609$

$-0.42923152$

$-0.32339609$

0.06612916

0.17196462

0.06612916

0.17196462

0.06612916

0.17196462

0.06612916

0.17196462

$-0.32880956$

$-0.22297411$

$-0.32880956$

$-0.22297411$

$-0.32880956$

$-0.22297411$

$-0.32880956$

$-0.22297411$

0.09555512

0.20139056

0.09555512

0.20139056

0.09555512

0.20139056

0.09555512

0.20139056
$-6.73884964$

7.70559359

$-9.86509037$

$-5.57090139$

7.19942999

1.65506077

$-1.05759609$

$-2.01240230$

1.05154967

$-3.04114485$

3. 61876416

4. 07673216

$-4.73917866$

0.34551534

$-4.98503017$

$-4.87126112$

11.22410870

1. 83783233

3. 44053268

4. 72840261

$-12.45753956$

$-21.42895889$

$-14.84226894$

15.80449295

12.33741760

38.88158798

4.69793272

$-29.50959396$

$-4.34088564$

0.00846612

$-0.11518668$

0.23030363

0.03828474

0.65503561

$-0.94701123$

$-1.13740611$

1.54480541

$-7.04722071$

6.05697918

5.73663187

$-5.02414083$

8.83991528

$-7.41384459$

$-7.00691652$

6.09648752

$$
0.1200 \quad 0.0488
$$

0.16034016

0.16034016

0.16034016

0.16034016

0.26617563

0.26617563

$-0.28076980$

$-0.17493436$

$-0.17493436$

$-0.28076980$

$-0.17493436$

1.37668049

1.37668049
1.000 \# Electr. mom., scale-fac \# Magnetic mom.

$0.05111535 \quad-34.02855682$

$\begin{array}{ll}0.15695079 & 22.06467438\end{array}$

$0.05111535 \quad 35.83983231$

$0.15695079 \quad-24.09525681$

0.05111535

0.15695079

0.05111535

0.15695079

$-0.39047247$

$-0.28463703$

$-0.39047247$

$-0.28463703$

$-0.39047247$

$-0.28463703$

$-0.39047247$

$-0.28463703$

0.01654314

0.12237859
27.38224411

$-16.92369270$

$-28.87583923$

18.47693634

$-3.57925010$

4.38313007

5.95016718

$-7.66717720$

2.16896296

$-2.18574762$

$-4.37378502$

5.24254560

$-15.90637398$

9. 04622459 


\begin{tabular}{|c|c|c|c|}
\hline 2.29703021 & 1.48251593 & 0.01654314 & 8.68311119 \\
\hline 2.29703021 & 1.48251593 & 0.12237859 & -3.16532493 \\
\hline 2.40286589 & 1.37668049 & 0.01654314 & 13.57617664 \\
\hline 2.40286589 & 1.37668049 & 0.12237859 & -7.41012478 \\
\hline 2.40286589 & 1.48251593 & 0.01654314 & -6.93459463 \\
\hline 2.40286589 & 1.48251593 & 0.12237859 & 1.97318423 \\
\hline-1.56014943 & -1.49588871 & -0.36711088 & 0.67141092 \\
\hline-1.56014943 & -1.49588871 & -0.26127544 & -1.12790895 \\
\hline-1.56014943 & -1.39005339 & -0.36711088 & -0.89702320 \\
\hline-1.56014943 & -1.39005339 & -0.26127544 & 1.50560117 \\
\hline-1.45431399 & -1.49588871 & -0.36711088 & -0.80296928 \\
\hline-1.45431399 & -1.49588871 & -0.26127544 & 1.32209229 \\
\hline-1.45431399 & -1.39005339 & -0.36711088 & 1.07284939 \\
\hline-1.45431399 & -1.39005339 & -0.26127544 & -1.76530612 \\
\hline 1.01902342 & -0.44691080 & -0.42923152 & -34.48151779 \\
\hline 1.01902342 & -0.44691080 & -0.32339609 & 37.03147125 \\
\hline 1.01902342 & -0.34107536 & -0.42923152 & 37.99669647 \\
\hline 1.01902342 & -0.34107536 & -0.32339609 & -42.51202393 \\
\hline 1.12485886 & -0.44691080 & -0.42923152 & 40.16238022 \\
\hline 1.12485886 & -0.44691080 & -0.32339609 & -44.19154358 \\
\hline 1.12485886 & -0.34107536 & -0.42923152 & -44.29381561 \\
\hline 1.12485886 & -0.34107536 & -0.32339609 & 50.48623276 \\
\hline-2.29137540 & 0.60408223 & 0.06612916 & -0.06986186 \\
\hline-2.29137540 & 0.60408223 & 0.17196462 & -0.94425946 \\
\hline-2.29137540 & 0.70991772 & 0.06612916 & -0.04581374 \\
\hline-2.29137540 & 0.70991772 & 0.17196462 & 0.86722136 \\
\hline-2.18554020 & 0.60408223 & 0.06612916 & 0.48431990 \\
\hline-2.18554020 & 0.60408223 & 0.17196462 & 1.08615935 \\
\hline-2.18554020 & 0.70991772 & 0.06612916 & -0.23904218 \\
\hline-2.18554020 & 0.70991772 & 0.17196462 & -1.06091130 \\
\hline 0.88915640 & -1.44335949 & -0.32880956 & -4.01567554 \\
\hline 0.88915640 & -1.44335949 & -0.22297411 & 3.34364820 \\
\hline 0.88915640 & -1.33752406 & -0.32880956 & 5.97720814 \\
\hline 0.88915640 & -1.33752406 & -0.22297411 & -5.19042492 \\
\hline 0.99499184 & -1.44335949 & -0.32880956 & 4.54531574 \\
\hline 0.99499184 & -1.44335949 & -0.22297411 & -4.04116917 \\
\hline 0.99499184 & -1.33752406 & -0.32880956 & -6.69248009 \\
\hline 0.99499184 & -1.33752406 & -0.22297411 & 6.15177965 \\
\hline-2.05025959 & 1.58138645 & 0.09555512 & -0.61903816 \\
\hline-2.05025959 & 1.58138645 & 0.20139056 & 0.45940918 \\
\hline-2.05025959 & 1.68722177 & 0.09555512 & 0.50347143 \\
\hline-2.05025959 & 1.68722177 & 0.20139056 & -0.37781909 \\
\hline-1.94442415 & 1.58138645 & 0.09555512 & 0.76325530 \\
\hline-1.94442415 & 1.58138645 & 0.20139056 & -0.50902814 \\
\hline-1.94442415 & 1.68722177 & 0.09555512 & -0.61610848 \\
\hline-1.94442415 & 1.68722177 & 0.20139056 & 0.42175439 \\
\hline & \# Energy 6->8 & & \\
\hline 0 & 0.0980 & 1.000 & Electr. \\
\hline-0.0029 & $.0052 \quad 0.0062$ & $\# 1$ & Magnetic mom. \\
\hline 2.13764215 & 0.16034016 & 0.05111535 & 21.89165306 \\
\hline 2.13764215 & 0.16034016 & 0.15695079 & -37.22931290 \\
\hline 2.13764215 & 0.26617563 & 0.05111535 & -22.66840172 \\
\hline 2.13764215 & 0.26617563 & 0.15695079 & 36.39520645 \\
\hline 2.24347758 & 0.16034016 & 0.05111535 & -25.24409294 \\
\hline 2.24347758 & 0.16034016 & 0.15695079 & 40.47691727 \\
\hline 2.24347758 & 0.26617563 & 0.05111535 & 25.62394333 \\
\hline 2.24347758 & 0.26617563 & 0.15695079 & -39.35977936 \\
\hline-1.37885487 & -0.28076980 & -0.39047247 & 34.36778641 \\
\hline-1.37885487 & -0.28076980 & -0.28463703 & -57.88094711 \\
\hline-1.37885487 & -0.17493436 & -0.39047247 & -42.02970123 \\
\hline-1.37885487 & -0.17493436 & -0.28463703 & 69.47608185 \\
\hline-1.27301943 & -0.28076980 & -0.39047247 & -23.89787292 \\
\hline-1.27301943 & -0.28076980 & -0.28463703 & 41.26491547 \\
\hline-1.27301943 & -0.17493436 & -0.39047247 & 29.77203369 \\
\hline-1.27301943 & -0.17493436 & -0.28463703 & -50.33035278 \\
\hline 2 & 1.37668049 & 0.01654314 & 2.92826486 \\
\hline 2 . & 1.37668049 & 0.12237859 & -4.00517511 \\
\hline
\end{tabular}




\begin{tabular}{|c|c|c|c|}
\hline 2.29703021 & 1.48251593 & 0.01654314 & -1.90249288 \\
\hline 2.29703021 & 1.48251593 & 0.12237859 & 2.66998863 \\
\hline 2.40286589 & 1.37668049 & 0.01654314 & -3.28196096 \\
\hline 2.40286589 & 1.37668049 & 0.12237859 & 4.47851944 \\
\hline 2.40286589 & 1.48251593 & 0.01654314 & 2.17802525 \\
\hline 2.40286589 & 1.48251593 & 0.12237859 & -3.05644369 \\
\hline-1.56014943 & -1.49588871 & -0.36711088 & -0.90907395 \\
\hline-1.56014943 & -1.49588871 & -0.26127544 & 0.59474522 \\
\hline-1.56014943 & -1.39005339 & -0.36711088 & 0.75797212 \\
\hline-1.56014943 & -1.39005339 & -0.26127544 & -0.25096601 \\
\hline-1.45431399 & -1.49588871 & -0.36711088 & 1.68444967 \\
\hline-1.45431399 & -1.49588871 & -0.26127544 & -1.93495595 \\
\hline-1.45431399 & -1.39005339 & -0.36711088 & -1.89374995 \\
\hline-1.45431399 & -1.39005339 & -0.26127544 & 2.15948629 \\
\hline 1.01902342 & -0.44691080 & -0.42923152 & 0.23872079 \\
\hline 1.01902342 & -0.44691080 & -0.32339609 & 0.37090942 \\
\hline 1.01902342 & -0.34107536 & -0.42923152 & 1.67802060 \\
\hline 1.01902342 & -0.34107536 & -0.32339609 & -2.61683536 \\
\hline 1.12485886 & -0.44691080 & -0.42923152 & -2.47322917 \\
\hline 1.12485886 & -0.44691080 & -0.32339609 & 1.96646464 \\
\hline 1.12485886 & -0.34107536 & -0.42923152 & -0.15374549 \\
\hline 1.12485886 & -0.34107536 & -0.32339609 & 1.22954345 \\
\hline-2.29137540 & 0.60408223 & 0.06612916 & 9.46327782 \\
\hline-2.29137540 & 0.60408223 & 0.17196462 & 9.27175140 \\
\hline-2.29137540 & 0.70991772 & 0.06612916 & -6.89144659 \\
\hline-2.29137540 & 0.70991772 & 0.17196462 & -7.60824919 \\
\hline-2.18554020 & 0.60408223 & 0.06612916 & -18.18185043 \\
\hline-2.18554020 & 0.60408223 & 0.17196462 & -4.32045984 \\
\hline-2.18554020 & 0.70991772 & 0.06612916 & 13.71151352 \\
\hline-2.18554020 & 0.70991772 & 0.17196462 & 3.72708678 \\
\hline 0.88915640 & -1.44335949 & -0.32880956 & -0.17767927 \\
\hline 0.88915640 & -1.44335949 & -0.22297411 & 0.17727078 \\
\hline 0.88915640 & -1.33752406 & -0.32880956 & 0.05357708 \\
\hline 0.88915640 & -1.33752406 & -0.22297411 & -0.10644053 \\
\hline 0.99499184 & -1.44335949 & -0.32880956 & -0.21053456 \\
\hline 0.99499184 & -1.44335949 & -0.22297411 & 0.37214935 \\
\hline 0.99499184 & -1.33752406 & -0.32880956 & 0.47161031 \\
\hline 0.99499184 & -1.33752406 & -0.22297411 & -0.71259123 \\
\hline-2.05025959 & 1.58138645 & 0.09555512 & 3.63735390 \\
\hline-2.05025959 & 1.58138645 & 0.20139056 & -3.19638038 \\
\hline-2.05025959 & 1.68722177 & 0.09555512 & -2.96057415 \\
\hline-2.05025959 & 1.68722177 & 0.20139056 & 2.64252901 \\
\hline-1.94442415 & 1.58138645 & 0.09555512 & -4.53520679 \\
\hline-1.94442415 & 1.58138645 & 0.20139056 & 3.87254548 \\
\hline-1.94442415 & 1.68722177 & 0.09555512 & 3.59378099 \\
\hline-1.94442415 & 1.68722177 & 0.20139056 & -3.17758703 \\
\hline & \# Energy 6->9 & & \\
\hline 0.2006 & -0.0316 & 1.000 & Electr. mom., \\
\hline 0.0074 & -0.0108 & & Magnetic mom. \\
\hline 2.13764215 & 0.16034016 & 0.05111535 & -40.57488632 \\
\hline 2.13764215 & 0.16034016 & 0.15695079 & 64.70013428 \\
\hline 2.13764215 & 0.26617563 & 0.05111535 & 41.08550262 \\
\hline 2.13764215 & 0.26617563 & 0.15695079 & -62.08843994 \\
\hline 2.24347758 & 0.16034016 & 0.05111535 & 44.91519165 \\
\hline 2.24347758 & 0.16034016 & 0.15695079 & -69.37031555 \\
\hline 2.24347758 & 0.26617563 & 0.05111535 & -44.53009415 \\
\hline 2.24347758 & 0.26617563 & 0.15695079 & 66.29834747 \\
\hline-1.37885487 & -0.28076980 & -0.39047247 & -58.29314041 \\
\hline-1.37885487 & -0.28076980 & -0.28463703 & 99.36479187 \\
\hline-1.37885487 & -0.17493436 & -0.39047247 & 71.64964294 \\
\hline-1.37885487 & -0.17493436 & -0.28463703 & -119.52584839 \\
\hline-1.27301943 & -0.28076980 & -0.39047247 & 39.54606247 \\
\hline-1.27301943 & -0.28076980 & -0.28463703 & -69.62626648 \\
\hline-1.27301943 & -0.17493436 & -0.39047247 & -49.83963776 \\
\hline-1.27301943 & -0.17493436 & -0.28463703 & 85.48799133 \\
\hline 2.29703021 & 1.37668049 & 0.01654314 & -6.30569220 \\
\hline 2.29703021 & 1.37668049 & 0.12237859 & 7.71503592 \\
\hline \multicolumn{4}{|c|}{17} \\
\hline
\end{tabular}




2.29703021
2.29703021
2.40286589
2.40286589
2.40286589
2.40286589
-1.56014943
-1.56014943
-1.56014943
-1.56014943
-1.45431399
-1.45431399
-1.45431399
-1.45431399
1.01902342
1.01902342
1.01902342
1.01902342
1.12485886
1.12485886
1.12485886
1.12485886
-2.29137540
-2.29137540
-2.29137540
-2.29137540
-2.18554020
-2.18554020
-2.18554020
-2.18554020
0.88915640
0.88915640
0.88915640
0.88915640
0.99499184
0.99499184
0.99499184
0.99499184
-2.05025959
-2.05025959
-2.05025959
-2.05025959
-1.94442415
-1.94442415
-1.94442415
-1.94442415

\&TRANSITION $7->\ldots$ 64
1. 48251593

1. 48251593

1. 37668049

1. 37668049

1. 48251593

1.48251593

$-1.49588871$

$-1.49588871$

$-1.39005339$

$-1.39005339$

$-1.49588871$

$-1.49588871$

$-1.39005339$

$-1.39005339$

$-0.44691080$

$-0.44691080$

$-0.34107536$

$-0.34107536$

$-0.44691080$

$-0.44691080$

$-0.34107536$

$-0.34107536$

0.60408223

0.60408223

0.70991772

0.70991772

0.60408223

0.60408223

0.70991772

0.70991772

$-1.44335949$

$-1.44335949$

$-1.33752406$

$-1.33752406$

$-1.44335949$

$-1.44335949$

$-1.33752406$

$-1.33752406$

1. 58138645

1.58138645

1.68722177

1. 68722177

1.58138645

1. 58138645

1.68722177

1.68722177

0.

0.2049

0.0151

2. 13764215

2.13764215

2.13764215

2. 13764215

2. 24347758

2. 24347758

2. 24347758

2. 24347758

$-1.37885487$

$-1.37885487$

$-1.37885487$

$-1.37885487$

$-1.27301943$

$-1.27301943$

$-1.27301943$

$-1.27301943$

2. 29703021

0.16034016

0.16034016

0.26617563

0.26617563

0.16034016

0.16034016

0.26617563

0.26617563

$-0.28076980$

$-0.28076980$

$-0.17493436$

$-0.17493436$

$-0.28076980$

$-0.28076980$

$-0.17493436$

$-0.17493436$

1. 37668049
0.01654314

0.12237859

0.01654314

0.12237859

0.01654314

0.12237859

$-0.36711088$

$-0.26127544$

$-0.36711088$

$-0.26127544$

$-0.36711088$

$-0.26127544$

$-0.36711088$

$-0.26127544$

$-0.42923152$

$-0.32339609$

$-0.42923152$

$-0.32339609$

$-0.42923152$

$-0.32339609$

$-0.42923152$

$-0.32339609$

0.06612916

0.17196462

0.06612916

0.17196462

0.06612916

0.17196462

0.06612916

0.17196462

$-0.32880956$

$-0.22297411$

$-0.32880956$

$-0.22297411$

$-0.32880956$

$-0.22297411$

$-0.32880956$

$-0.22297411$

0.09555512

0.20139056

0.09555512

0.20139056

0.09555512

0.20139056

0.09555512

0.20139056
4. 47857523

$-5.52571678$

6.52809477

$-8.43607712$

$-4.66468287$

6.09407043

1.23737514

$-0.67611611$

$-1.31005430$

0.39603746

$-2.58283234$

3. 07808280

3. 28734684

$-3.82951236$

$-0.09363120$

$-2.56127548$

$-3.51879001$

7.25657225

3.16743326

$-0.25304312$

2.13497591

$-6.69979477$

$-16.17808723$

$-16.32171249$

12.10015392

13.13575077

31.18421936

7.69140148

$-23.88663483$

$-6.29866886$

0.06017483

$-0.08726179$

0.18082912

$-0.04773260$

0.66838694

$-0.96998316$

$-1.16341162$

1.60571837

$-6.81489801$

5.88292551

5.50936413

$-4.83712339$

8. 43604374

$-7.12458229$

$-6.66211987$

5.82183743

\subsection{Energy $7->8$ \\ $0.1400 \quad-0.0007$
1.000 \# Electr. mom., scale-fac \# Magnetic mom.

0.05111535

0.15695079

0.05111535

0.15695079

0.05111535

0.15695079

0.05111535

0.15695079

$-0.39047247$

$-0.28463703$

$-0.39047247$

$-0.28463703$

$-0.39047247$

$-0.28463703$

$-0.39047247$

$-0.28463703$

0.01654314
34.04035187

$-43.95824814$

$-35.06733322$

43.16350937

$-33.99990845$

44.70650101

34.37123871

$-43.48421097$

38.83107376

$-64.69480133$

$-47.72642899$

77.95104980

$-27.19355011$

46.16149139

34.16212845

$-56.71682739$

9.41737843 


\begin{tabular}{|c|c|c|c|}
\hline 2.29703021 & 1.37668049 & 0.12237859 & -8.11288452 \\
\hline 2.29703021 & 1.48251593 & 0.01654314 & -6.31339216 \\
\hline 2.29703021 & 1.48251593 & 0.12237859 & 5.20737886 \\
\hline 2.40286589 & 1.37668049 & 0.01654314 & -8.61371231 \\
\hline 2.40286589 & 1.37668049 & 0.12237859 & 7.76268291 \\
\hline 2.40286589 & 1.48251593 & 0.01654314 & 5.70493984 \\
\hline 2.40286589 & 1.48251593 & 0.12237859 & -4.95519876 \\
\hline-1.56014943 & -1.49588871 & -0.36711088 & -0.71186411 \\
\hline-1.56014943 & -1.49588871 & -0.26127544 & 0.38125041 \\
\hline-1.56014943 & -1.39005339 & -0.36711088 & 0.65828794 \\
\hline-1.56014943 & -1.39005339 & -0.26127544 & -0.12701134 \\
\hline-1.45431399 & -1.49588871 & -0.36711088 & 1.57279289 \\
\hline-1.45431399 & -1.49588871 & -0.26127544 & -1.87533092 \\
\hline-1.45431399 & -1.39005339 & -0.36711088 & -1.91657090 \\
\hline-1.45431399 & -1.39005339 & -0.26127544 & 2.25746250 \\
\hline 1.01902342 & -0.44691080 & -0.42923152 & 8.32923031 \\
\hline 1.01902342 & -0.44691080 & -0.32339609 & -6.26560688 \\
\hline 1.01902342 & -0.34107536 & -0.42923152 & -7.04231405 \\
\hline 1.01902342 & -0.34107536 & -0.32339609 & 4.83415127 \\
\hline 1.12485886 & -0.44691080 & -0.42923152 & -11.84327030 \\
\hline 1.12485886 & -0.44691080 & -0.32339609 & 9.84407043 \\
\hline 1.12485886 & -0.34107536 & -0.42923152 & 9.63428497 \\
\hline 1.12485886 & -0.34107536 & -0.32339609 & -7.18391562 \\
\hline-2.29137540 & 0.60408223 & 0.06612916 & 9.24219704 \\
\hline-2.29137540 & 0.60408223 & 0.17196462 & 10.69502163 \\
\hline-2.29137540 & 0.70991772 & 0.06612916 & -6.71335030 \\
\hline-2.29137540 & 0.70991772 & 0.17196462 & -8.78532028 \\
\hline-2.18554020 & 0.60408223 & 0.06612916 & -18.25171661 \\
\hline-2.18554020 & 0.60408223 & 0.17196462 & -5.68249369 \\
\hline-2.18554020 & 0.70991772 & 0.06612916 & 13.73272038 \\
\hline-2.18554020 & 0.70991772 & 0.17196462 & 4.87307453 \\
\hline 0.88915640 & -1.44335949 & -0.32880956 & 0.97312266 \\
\hline 0.88915640 & -1.44335949 & -0.22297411 & -0.66006058 \\
\hline 0.88915640 & -1.33752406 & -0.32880956 & -1.72599328 \\
\hline 0.88915640 & -1.33752406 & -0.22297411 & 1.26568282 \\
\hline 0.99499184 & -1.44335949 & -0.32880956 & -1.65121627 \\
\hline 0.99499184 & -1.44335949 & -0.22297411 & 1.57089365 \\
\hline 0.99499184 & -1.33752406 & -0.32880956 & 2.64400935 \\
\hline 0.99499184 & -1.33752406 & -0.22297411 & -2.58219004 \\
\hline-2.05025959 & 1.58138645 & 0.09555512 & 4.33253574 \\
\hline-2.05025959 & 1.58138645 & 0.20139056 & -3.66398454 \\
\hline-2.05025959 & 1.68722177 & 0.09555512 & -3.50421500 \\
\hline-2.05025959 & 1.68722177 & 0.20139056 & 3.01854634 \\
\hline-1.94442415 & 1.58138645 & 0.09555512 & -5.30805779 \\
\hline-1.94442415 & 1.58138645 & 0.20139056 & 4.38898849 \\
\hline-1.94442415 & 1.68722177 & 0.09555512 & 4.19552040 \\
\hline-1.94442415 & 1.68722177 & 0.20139056 & -3.59258175 \\
\hline & \# Energy 7->9 & & \\
\hline 0.0029 & 0.0061 & 1.000 & Electr. mom., scale-fac \\
\hline 0.0044 & 0.0282 & $\pi$ & Magnetic mom. \\
\hline 2.13764215 & 0.16034016 & 0.05111535 & 2.48845768 \\
\hline 2.13764215 & 0.16034016 & 0.15695079 & -1.96886015 \\
\hline 2.13764215 & 0.26617563 & 0.05111535 & -2.57010865 \\
\hline 2.13764215 & 0.26617563 & 0.15695079 & 2.03297448 \\
\hline 2.24347758 & 0.16034016 & 0.05111535 & -1.99299014 \\
\hline 2.24347758 & 0.16034016 & 0.15695079 & 1.60330188 \\
\hline 2.24347758 & 0.26617563 & 0.05111535 & 2.05104589 \\
\hline 2.24347758 & 0.26617563 & 0.15695079 & -1.63802075 \\
\hline-1.37885487 & -0.28076980 & -0.39047247 & 1.64996827 \\
\hline-1.37885487 & -0.28076980 & -0.28463703 & -2.71044374 \\
\hline-1.37885487 & -0.17493436 & -0.39047247 & -2.15299678 \\
\hline-1.37885487 & -0.17493436 & -0.28463703 & 3.41222787 \\
\hline-1.27301943 & -0.28076980 & -0.39047247 & -1.17806387 \\
\hline-1.27301943 & -0.28076980 & -0.28463703 & 1.95639253 \\
\hline-1.27301943 & -0.17493436 & -0.39047247 & 1.59962308 \\
\hline-1.27301943 & -0.17493436 & -0.28463703 & -2.54800868 \\
\hline 2.29 & 1.37668049 & 0.016543 & 1.26789582 \\
\hline
\end{tabular}


2. 29703021

2. 29703021

2. 29703021

2. 40286589

2. 40286589

2. 40286589

2. 40286589

$-1.56014943$

$-1.56014943$

$-1.56014943$

$-1.56014943$

$-1.45431399$

$-1.45431399$

$-1.45431399$

$-1.45431399$

1.01902342

1. 01902342

1.01902342

1. 01902342

1.12485886

1. 12485886

1. 12485886

1.12485886

$-2.29137540$

$-2.29137540$

$-2.29137540$

$-2.29137540$

$-2.18554020$

$-2.18554020$

$-2.18554020$

$-2.18554020$

0.88915640

0.88915640

0.88915640

0.88915640

0.99499184

0.99499184

0.99499184

0.99499184

$-2.05025959$

$-2.05025959$

$-2.05025959$

$-2.05025959$

$-1.94442415$

$-1.94442415$

$-1.94442415$

$-1.94442415$

\&TRANSITION $8->$. . 64
$-0.4659$
0.0640
2.13764215
2.13764215
2.13764215
2.13764215
2. 24347758
2. 24347758
2. 24347758
2. 24347758
$-1.37885487$
$-1.37885487$
$-1.37885487$
$-1.37885487$
$-1.27301943$
$-1.27301943$
$-1.27301943$
$-1.27301943$

1. 37668049

1. 48251593

1. 48251593

1.37668049

1. 37668049

1. 48251593

1. 48251593

$-1.49588871$

$-1.49588871$

$-1.39005339$

$-1.39005339$

$-1.49588871$

$-1.49588871$

$-1.39005339$

$-1.39005339$

$-0.44691080$

$-0.44691080$

$-0.34107536$

$-0.34107536$

$-0.44691080$

$-0.44691080$

$-0.34107536$

$-0.34107536$

0.60408223

0.60408223

0.70991772

0.70991772

0.60408223

0.60408223

0.70991772

0.70991772

$-1.44335949$

$-1.44335949$

$-1.33752406$

$-1.33752406$

$-1.44335949$

$-1.44335949$

$-1.33752406$

$-1.33752406$

1.58138645

1. 58138645

1. 68722177

1.68722177

1. 58138645

1. 58138645

1.68722177

1. 68722177

$\begin{array}{cc}0 . & \# \text { Energy } \\ 0.4->9 \\ 0.4949 & 0.2341 \\ 0.5474 & 0.0032\end{array}$

0.16034016

0.16034016

0.26617563

0.26617563

0.16034016

0.16034016

0.26617563

0.26617563

$-0.28076980$

$-0.28076980$

$-0.17493436$

$-0.17493436$

$-0.28076980$

$-0.28076980$

$-0.17493436$

$-0.17493436$
0.12237859

0.01654314

0.12237859

0.01654314

0.12237859

0.01654314

0.12237859

$-0.36711088$

$-0.26127544$

$-0.36711088$

$-0.26127544$

$-0.36711088$

$-0.26127544$

$-0.36711088$

$-0.26127544$

$-0.42923152$

$-0.32339609$

$-0.42923152$

$-0.32339609$

$-0.42923152$

$-0.32339609$

$-0.42923152$

$-0.32339609$

0.06612916

0.17196462

0.06612916

0.17196462

0.06612916

0.17196462

0.06612916

0.17196462

$-0.32880956$

$-0.22297411$

$-0.32880956$

$-0.22297411$

$-0.32880956$

$-0.22297411$

$-0.32880956$

$-0.22297411$

0.09555512

0.20139056

0.09555512

0.20139056

0.09555512

0.20139056

0.09555512

0.20139056
-0.81787032
-0.84225285
0.47903579
-1.05881989
0.66103780
0.68071812

$-0.35890725$

$-0.07846908$

0.05247194

0.11440832

$-0.07748794$

0.10613432

$-0.10715878$

$-0.15581416$

0.15685101

2. 65601659

$-2.71547365$

$-2.93807864$

3. 14210296

$-3.14116955$

3. 30702424

3. 41440630

$-3.72600913$

0.18070111

0.51520526

$-0.09450622$

$-0.44587159$

$-0.44467655$

$-0.40957007$

0.28251725

0.37895346

0.26459780

$-0.19247039$

$-0.39576229$

0.30533779

$-0.32318053$

0.27460665

0.47846678

$-0.42439348$

0.17020380

$-0.12410251$

$-0.13918003$

0.10425112

$-0.19581908$

0.13910358

0.15777645

$-0.11727888$

$$
\begin{aligned}
& 1.000 \text { \# Electr. mom., scale-fac } \\
& \text { \# Magnetic mom. }
\end{aligned}
$$

0.05111535

0.15695079

0.05111535

0.15695079

0.05111535

0.15695079

0.05111535

0.15695079

$-0.39047247$

$-0.28463703$

$-0.39047247$

$-0.28463703$

$-0.39047247$

$-0.28463703$

$-0.39047247$

$-0.28463703$
6.85240030

$-3.47530341$

$-4.97425032$

1. 63896692

$-4.00113821$

1. 74975908

2.33220792

$-0.15046787$

$-38.88592148$

66.92168427

43.84907150

$-70.20355988$

31.71842384

$-58.16925049$

$-36.04469681$

60.70881271 


2.29703021
2.29703021
2.29703021
2.29703021
2.40286589
2.40286589
2.40286589
2.40286589
-1.56014943
-1.56014943
-1.56014943
-1.56014943
-1.45431399
-1.45431399
-1.45431399
-1.45431399
1.01902342
1.01902342
1.01902342
1.01902342
1.12485886
1.12485886
1.12485886
1.12485886
-2.29137540
-2.29137540
-2.29137540
-2.29137540
-2.18554020
-2.18554020
-2.18554020
-2.18554020
0.88915640
0.88915640
0.88915640
0.88915640
0.99499184
0.99499184
0.99499184
0.99499184
-1.05025959
-1.905025959
-1.9405025959
-2.05025959
-1.94442415
-1.94442415
-1.9442415
-1015

\&PERMANENT MOMENTS

64
1.37668049

1.37668049

1. 48251593

1. 48251593

1. 37668049

1. 37668049

1. 48251593

1. 48251593

$-1.49588871$

$-1.49588871$

$-1.39005339$

$-1.39005339$

$-1.49588871$

$-1.49588871$

$-1.39005339$

$-1.39005339$

$-0.44691080$

$-0.44691080$

$-0.34107536$

$-0.34107536$

$-0.44691080$

$-0.44691080$

$-0.34107536$

$-0.34107536$

0.60408223

0.60408223

0.70991772

0.70991772

0.60408223

0.60408223

0.70991772

0.70991772

$-1.44335949$

$-1.44335949$

$-1.33752406$

$-1.33752406$

$-1.44335949$

$-1.44335949$

$-1.33752406$

$-1.33752406$

1. 58138645

1.58138645

1.68722177

1.68722177

1. 58138645

1.58138645

1. 68722177

1.68722177
0.01654314

0.12237859

0.01654314

0.12237859

0.01654314

0.12237859

0.01654314

0.12237859

$-0.36711088$

$-0.26127544$

$-0.36711088$

$-0.26127544$

$-0.36711088$

$-0.26127544$

$-0.36711088$

$-0.26127544$

$-0.42923152$

$-0.32339609$

$-0.42923152$

$-0.32339609$

$-0.42923152$

$-0.32339609$

$-0.42923152$

$-0.32339609$

0.06612916

0.17196462

0.06612916

0.17196462

0.06612916

0.17196462

0.06612916

0.17196462

$-0.32880956$

$-0.22297411$

$-0.32880956$

$-0.22297411$

$-0.32880956$

$-0.22297411$

$-0.32880956$

$-0.22297411$

0.09555512

0.20139056

0.09555512

0.20139056

0.09555512

0.20139056

0.09555512

0.20139056
$-1.64401364$

1. 85481739

1.17742980

$-1.29199874$

1.80574203

$-1.84537196$

$-1.29403746$

1. 28948355

$-0.01603648$

7.64595270

$-0.11943321$

$-9.78178406$

$-0.20615928$

$-7.84751368$

0.56162995

9.77048111

$-20.81637573$

36.53628540

21.96985626

$-36.09008408$

24.44004631

$-40.70641708$

$-25.49982452$

40.14601517

29.48974228

$-24.70972061$

$-26.68034363$

21.84390640

$-39.64080048$

32.46424103

36.73753738

$-29.14755630$

2.65832829

0.11790171

$-3.09338355$

$-1.30654001$

$-2.12865949$

$-0.61216772$

2.33566809

2.00418210

0.88263720

$-1.70396066$

$-0.73988348$

1. 29217684

$-2.77982521$

2. 80373883

2.05147147

$-2.04411817$

$$
0 \text {. \# Energy 1->1 }
$$$$
0.1560
$$
0.5003

0.16034016

0.16034016

0.26617563

0.26617563

0.16034016

0.16034016

0.26617563

0.26617563

$-0.28076980$

$-0.28076980$

$-0.17493436$

$-0.17493436$

$-0.28076980$

$-0.28076980$

$-0.17493436$

$-0.17493436$
\# Electr. mom.

$0.05111535 \quad-300.80075073$

$0.15695079 \quad 250.16873169$

$0.05111535 \quad 281.22213745$

$0.15695079-225.91487122$

$0.05111535 \quad 275.52645874$

$0.15695079 \quad-234.82000732$

$0.05111535-255.65911865$

$0.15695079 \quad 211.69210815$

$-0.39047247$

$-0.28463703$

$-0.39047247$

$-0.28463703$

$-0.39047247$

$-0.28463703$

$-0.39047247$

$-0.28463703$
211.69210815
35.18000031

$-67.69459534$

$-66.17034912$

108.55467224

$-16.64756393$

42.74257278

43.54051971

$-77.87226105$ 


\begin{tabular}{|c|c|c|c|}
\hline 2.29703021 & 1.37668049 & 0.01654314 & -16.19223404 \\
\hline 2.29703021 & 1.37668049 & 0.12237859 & 7.84216118 \\
\hline 2.29703021 & 1.48251593 & 0.01654314 & 7.84719038 \\
\hline 2.29703021 & 1.48251593 & 0.12237859 & -1.45162368 \\
\hline 2.40286589 & 1.37668049 & 0.01654314 & 29297 \\
\hline 2.40286589 & 1.37668049 & 0.12237859 & -8.18280792 \\
\hline 2.40286589 & 1.48251593 & 0.01654314 & -7.75834084 \\
\hline 2.40286589 & 1.48251593 & 0.12237859 & 1.65950072 \\
\hline-1.56014943 & -1.49588871 & -0.36711088 & 6.06 \\
\hline 1.56014943 & -1.49588871 & -0.26127544 & -7.78133726 \\
\hline-1.56014943 & -1.39005339 & -0.36711088 & -3.0222816 \\
\hline-1.56014943 & -1.39005339 & -0.26127544 & 4.79109669 \\
\hline-1.45431399 & -1.49588871 & -0.36711088 & -4.66 \\
\hline-1.454 & -1.49588871 & -0.26127544 & 5.29 \\
\hline-1.45431399 & -1.39005339 & -0.36711088 & 1.3299807 \\
\hline-1.45431399 & -1.39005339 & -0.26127544 & $-2 \cdot 32$ \\
\hline 1.01902342 & -0.44691080 & -0.42923152 & $305.5^{\prime}$ \\
\hline 1.01902342 & -0.44691080 & -0.32339609 & -409.22552490 \\
\hline 1.01902342 & -0.34107536 & -0.42923152 & -327.05 \\
\hline 2342 & -0.34107536 & -0.32339609 & 432.4 \\
\hline 1.12485886 & -0.44691080 & -0.42923152 & -329.68 \\
\hline 1.12485886 & -0.44691080 & -0.32339609 & 446.57 \\
\hline 5886 & -0.34107536 & -0.42923152 & 354.5 \\
\hline 1.12 & -0.34107536 & -0.3233 & -473.4 \\
\hline 7540 & 0.60408223 & 0.06612916 & 21.8 \\
\hline 7540 & 0.60408223 & 0.17196462 & -40.21 \\
\hline-2 & 0.7099 & 0.06612 & -19.2 \\
\hline-2 . & 0.70991772 & 0.1719 & 44.6 \\
\hline 4020 & 0.60408223 & 0.06612916 & 1.58966 \\
\hline 8554020 & 0.60408223 & 0.17196462 & 10.49 \\
\hline 4020 & 0.70991772 & 0.06 & -8.1 \\
\hline-2 & 0.70991772 & 0.17196462 & $-12 \cdot 3$ \\
\hline 8915640 & -1.44335949 & -0.32880956 & 28.98362 \\
\hline 15640 & -1.44335949 & -0.22297411 & -41.1 \\
\hline 40 & $-1 \cdot 33$ & -0.32 & -35.8 \\
\hline 0.88915640 & -1.33752406 & -0.22297411 & 53.6 \\
\hline 9499184 & -1.44335949 & -0.32880956 & -29.93 \\
\hline 84 & 35949 & -0.22 & 43.7 \\
\hline 84 & -1.33752406 & -0.32 & 36.5 \\
\hline 0.99499184 & -1.33752406 & -0.22297411 & -56.1 \\
\hline 5025959 & 1.58138645 & 0.09555512 & -0.6 \\
\hline-2 . & 138645 & 0.2 & -10.9 \\
\hline-2 & 1.68722177 & 0 . & 0.8 \\
\hline-2 . & 1.68722177 & 0.20 & 7.4 \\
\hline-1 & 1.58138645 & 0.0 & 10.0 \\
\hline-1 & 1.58 & 0 & 0.9 \\
\hline-1.94442415 & 1.68722177 & 0.0 & -7.2 \\
\hline-1.94442415 & 1.68722177 & 39056 & -0.2 \\
\hline & \# Energy 2->2 & & \\
\hline-2 . & -2.5074 & $\#$ & tr. mom. \\
\hline 3764215 & 0.16034016 & 0.05 & $-328 \cdot 25$ \\
\hline 3764215 & 0.16034016 & 0.15695079 & 267.7 \\
\hline 54215 & 0.26617563 & 0 . & 307 . \\
\hline 764215 & 0.26617563 & 95079 & -241.9 \\
\hline 347758 & 0.16034016 & 0.05111535 & 295.3 \\
\hline 347758 & 0.16034016 & 95079 & -247 \\
\hline 347758 & 0.2661 & 0.0 & -274.2 \\
\hline 2.24347758 & 0.26617563 & 0.15695079 & 222.5 \\
\hline 885487 & -0.28076980 & -0.39047247 & 125.9 \\
\hline 885487 & -0.28076980 & -0.28463703 & -196.3 \\
\hline 885487 & -0.17493436 & -0.39047247 & -172.1 \\
\hline 7885487 & -0.17493436 & -0.28463703 & 251.0 \\
\hline-1 & -0.280 & -0.39 & -90.1 \\
\hline 301943 & -0.28076980 & -0.28463703 & 151.7 \\
\hline 01943 & -0.17493436 & -0.39047247 & 131.9 \\
\hline-1 & -0.174 & -0.28463703 & -200 \\
\hline 2 & 1. & 0.01 & -19.2 \\
\hline
\end{tabular}

\section{S-175}




\begin{tabular}{|c|c|c|c|}
\hline 2.29703021 & 1.37668049 & 0.12237859 & 8.94422054 \\
\hline 2.29703021 & 1.48251593 & 0.01654314 & 10.33623219 \\
\hline 2.29703021 & 1.48251593 & 0.12237859 & -2.47244430 \\
\hline 2.40286589 & 1.37668049 & 0.01654314 & 17.2 \\
\hline 2.40286589 & 1.37668049 & 0.12237859 & -8.42917347 \\
\hline 2.40286589 & 1.48251593 & 0.01654314 & -9.3518981 \\
\hline 2.40286589 & 1.48251593 & 0.12237859 & 2.002 \\
\hline 1.56014943 & -1.49588871 & -0.36711088 & -0.63 \\
\hline 1.56014943 & -1.49588871 & -0.26127544 & -12.372619 \\
\hline 1.56014943 & -1.39005339 & -0.36711088 & -7.626 \\
\hline-1.560 & -1.39005339 & -0.26127544 & 23.49 \\
\hline-1.45431399 & -1.49588871 & -0.36711088 & 5.8 \\
\hline-1.45431399 & -1.49588871 & -0.26127544 & 5.2 \\
\hline-1.45431399 & -1.39005339 & -0.36711088 & 1.05 \\
\hline-1.45431399 & -1.39005339 & -0.26127544 & $-14 \cdot 8$ \\
\hline 1.01902342 & -0.44691080 & -0.42923152 & 336.43 \\
\hline 1.01902342 & -0.44691080 & -0.32339609 & -470.9468383 \\
\hline 1.01902342 & -0.34107536 & -0.42923152 & -359.0 \\
\hline 1.01902342 & -0.34107536 & -0.32339609 & 492.6 \\
\hline 1.12485886 & -0.44691080 & -0.42923152 & -364.98 \\
\hline 1.12485886 & -0.44691080 & -0.32339609 & 513.92 \\
\hline 5886 & -0.34107536 & -0.42923152 & 390.8 \\
\hline 1.1 & -0.34 & -0.3233 & -539.2 \\
\hline-2.291 & 0.60408 & 0.0661 & -51.0 \\
\hline-2.2 & 0.60408223 & 0.17196462 & 20.1 \\
\hline-2.2 & 0.70991 & 0.06612916 & 43.8 \\
\hline-2.2 & 0.70 & 0.1719 & -6.6 \\
\hline-2 & 0.60 & 0.0661 & 91.6 \\
\hline-2.18554020 & 0.60408223 & 0.17196462 & -62.2 \\
\hline-2 & 0.70 & 0.06 & -87 \\
\hline-2.1 & 0.70 & 0.1719 & 49.5 \\
\hline 5640 & -1.44335949 & -0.32880956 & 25.6 \\
\hline 15640 & -1.44335949 & -0.22297411 & $-43 \cdot 3$ \\
\hline 0. & $-1 \cdot 33$ & -0.32880 & -31.5 \\
\hline 0. & -1.33752406 & -0.22297411 & 57.9 \\
\hline 9499184 & -1.44335949 & -0.32880956 & -27.01 \\
\hline 0 . & -1.44 & -0.22 & 46.2 \\
\hline 0 . & $-1 \cdot 33$ & -0.32 & 32.8 \\
\hline 0. & -1.33752406 & -0.22297411 & -60.99 \\
\hline-2 & 1.58138645 & 0.09555512 & $-4.7 \varepsilon$ \\
\hline-2 . & 1.58 & 0.2013 & $-8 \cdot 3$ \\
\hline-2 . & 1.68 & 0 . & 3.6 \\
\hline-2 . & 1.68 & 0 . & 6.0 \\
\hline-1 . & 1.58 & 0 . & 16.2 \\
\hline-1 . & $1.5 \varepsilon$ & 0 . & -2 \\
\hline-1. & 1.68 & 0 . & $-11 \cdot 3$ \\
\hline-1 . & 1.68722177 & 0.20 & 1.6 \\
\hline & \# Energy 3- & \multirow{2}{*}{\multicolumn{2}{|c|}{ \# Electr. mom. }} \\
\hline-1 . & 2.4539 & & \\
\hline 2 . & 0.16 & 0.0 & -350.1525573 \\
\hline 3764215 & 34016 & 0.15695079 & 256.83 \\
\hline 2 . & 0 . & 0 . & 339. \\
\hline 2 . & 0.26 & 0.15695079 & -242.74 \\
\hline 4347758 & 0.16 & 0.05111535 & 305.20 \\
\hline 2. & 0.16 & 0.1 & $-226 \cdot 9$ \\
\hline 2. & 0.26 & 0 . & -293.8 \\
\hline$\$ 347758$ & 0.26617563 & 0.15695079 & 212.86 \\
\hline 5487 & -0.28076 & -0.39047247 & 32.05 \\
\hline 5487 & -0.28 & $-0.2 \varepsilon$ & -62.25 \\
\hline 487 & -0.17 & -0.39047247 & $-56 \cdot 2$ \\
\hline-1.37 & -0.174934 & -0.28463703 & 93.7 \\
\hline-1.27 & -0.280769 & -0.39047247 & -15.8 \\
\hline-1.27 & -0.28076980 & -0.28463703 & 41.6 \\
\hline 301943 & -0.17493436 & -0.39047247 & 36.4 \\
\hline-1.2 & -0 . & -0 & -68.0 \\
\hline 03021 & 1.37 & 0.01654314 & -45.11 \\
\hline 2.2 & 1.37668049 & 0.12237859 & 20.45 \\
\hline
\end{tabular}




\begin{tabular}{|c|c|c|c|}
\hline 2.29703021 & 1.48251593 & 0.01654314 & 40.85416794 \\
\hline 2.29703021 & 1.48251593 & 0.12237859 & -21.24513054 \\
\hline 2.40286589 & 1.37668049 & 0.01654314 & 37.18571472 \\
\hline 2.40286589 & 1.37668049 & 0.12237859 & -12.79539871 \\
\hline 2.40286589 & 1.48251593 & 0.01654314 & -34.68117523 \\
\hline 2.40286589 & 1.48251593 & 0.12237859 & 14.85539532 \\
\hline-1.56014943 & -1.49588871 & -0.36711088 & 8.80775070 \\
\hline 1.56014943 & -1.49588871 & -0.26127544 & -11.6966133 \\
\hline-1.56014943 & -1.39005339 & -0.36711088 & -6.731846 \\
\hline 1.56014943 & -1.39005339 & -0.26127544 & 10.04 \\
\hline-1.45431399 & -1.49588871 & -0.36711088 & -7.685743 \\
\hline-1.45431399 & -1.49588871 & -0.26127544 & 9.5 \\
\hline-1.45431399 & -1.39005339 & -0.36711088 & 5.36 \\
\hline-1.454 & -1.39005339 & -0.26127544 & -8.0 \\
\hline 1.01902342 & -0.44691080 & -0.42923152 & 250.3 \\
\hline 1.01902342 & -0.44691080 & -0.32339609 & -361.5 \\
\hline 1.01902342 & -0.34107536 & -0.42923152 & -256.54568 \\
\hline 1.01902342 & -0.34107536 & -0.32339609 & 361.761199 \\
\hline 1.12485886 & -0.44691080 & -0.42923152 & -267.42 \\
\hline 1.12 & -0.44691080 & -0.32339609 & 389.7 \\
\hline 85886 & -0.34107536 & -0.42923152 & $274.3^{\prime}$ \\
\hline 1.12485886 & -0.34107536 & -0.32339609 & -390.227355 \\
\hline 37540 & 0.60408223 & 0.06612916 & 18.14 \\
\hline-2 . & 0.60408223 & 0.17 & -41.0 \\
\hline-2 . & 0.70991 & 0.0661 & $-16 \cdot 8$ \\
\hline-2.29 & 0.70991772 & 0.17196462 & 46.04 \\
\hline 4020 & 0.60408223 & 0.06612916 & 7.7 \\
\hline-2 . & 0.60408223 & 0.1719 & 10.7 \\
\hline 54020 & 0.70991772 & 0.06612916 & -12.4 \\
\hline 8554020 & 0.70991772 & 0.17196462 & -13.5 \\
\hline 15640 & -1.4433 & -0.32880 & 22.0 \\
\hline 0.88915 & -1.44335949 & -0.22297411 & -38.41 \\
\hline 0.88915640 & -1.33752406 & -0.32880956 & -25.13 \\
\hline 40 & -1.33752406 & -0.22297411 & 48. \\
\hline 0 . & -1.44 & -0.3288 & -22 . \\
\hline 84 & -1.44335949 & -0.22297411 & 40.67 \\
\hline 949 & -1.33752406 & -0.32880956 & 25.3 \\
\hline 0 . & -1.33 & -0 . & -50 . \\
\hline-2 & 1.58 & 0 & -2 \\
\hline-2 & 1.581 & 0.2 & -10 \\
\hline-2 . & 1.68722177 & 0.09555512 & 2.0 \\
\hline-2 & 1.6872 & 0 & 6 \\
\hline-1 & 1.5813 & 512 & 11 \\
\hline-1 & 1.58138645 & 0.20 & 0.0 \\
\hline-1.94 & 1.68722177 & 55512 & -8.7 \\
\hline-1.94 & 1.68722177 & 0.20139056 & 0 \\
\hline & \# Energy 4->4 & \multirow{2}{*}{\multicolumn{2}{|c|}{ \# Electr. mom }} \\
\hline-4 . & 1.2815 & & \\
\hline 2 & 34016 & 535 & -311.65426 \\
\hline 2 & 0.16 & 079 & 258.6 \\
\hline 764215 & 0.26617563 & 0.05111535 & 292.8 \\
\hline 764215 & 0.26617563 & 0.15695079 & -235.3 \\
\hline 2. & 0 . & 35 & 284 \\
\hline 2 . & 0.16034016 & 95079 & -241.9 \\
\hline 17758 & 0.26617563 & 0.05111535 & -265.1 \\
\hline 7758 & 0.26617563 & 95079 & 219. \\
\hline 85487 & -0.28076980 & -0.39047247 & 51.6 \\
\hline 885487 & -0.28076980 & -0.28463703 & -90.9 \\
\hline 885487 & -0.17493436 & -0.39047247 & -87.8 \\
\hline-1 & -0.17493436 & -0.28463703 & 137.7 \\
\hline 01943 & -0.28076980 & -0.39047247 & -28.4 \\
\hline 01943 & -0.28076980 & -0.28463703 & 60.8 \\
\hline-1 & -0.17493436 & -0.39047247 & 60 \\
\hline-1 & -0.17493436 & -0.28463703 & -102.5 \\
\hline 2 . & 1.37668049 & 0.01654314 & -20.56 \\
\hline & 1.3766 & 0.12237859 & 11 \\
\hline 2 . & 1.48251593 & 54314 & 11 \\
\hline
\end{tabular}


2. 29703021

2. 40286589

2. 40286589

2. 40286589

2.40286589

$-1.56014943$

$-1.56014943$

$-1.56014943$

$-1.56014943$

$-1.45431399$

$-1.45431399$

$-1.45431399$

1.01902342

1. 01902342

1.01902342

1.01902342

1. 12485886

1. 12485886

1.12485886

1.12485886

$-2.29137540$

$-2.29137540$

$-2.29137540$

$-2.29137540$

$-2.18554020$

$-2.18554020$

$-2.18554020$

$-2.18554020$

0.88915640

0.88915640

0.88915640

0.99499184

0.99499184

0.99499184

0.99499184

$-2.05025959$

$-2.05025959$

$-2.05025959$

$-2.05025959$

$-1.94442415$

$-1.94442415$

$-1.94442415$

64
$-1.45431399$

0.88915640

1. 48251593

1. 37668049

1. 37668049

1. 48251593

1. 48251593

$-1.49588871$

$-1.49588871$

$-1.39005339$

$-1.39005339$

$-1.49588871$

$-1.49588871$

$-1.39005339$

$-1.39005339$

$-0.44691080$

$-0.44691080$

$-0.34107536$

$-0.34107536$

$-0.44691080$

$-0.44691080$

$-0.34107536$

$-0.34107536$

0.60408223

0.60408223

0.70991772

0.70991772

0.60408223

0.60408223

0.70991772

0.70991772

$-1.44335949$

$-1.44335949$

$-1.33752406$

$-1.33752406$

$-1.44335949$

$-1.44335949$

$-1.33752406$

$-1.33752406$

1.58138645

1. 58138645

1. 68722177

1. 68722177

1. 58138645

1. 58138645

1.68722177

1.68722177

.94442415

0.7007

2.13764215

2.13764215

2. 13764215

2.13764215

2. 24347758

2. 24347758

2. 24347758

2. 24347758

$-1.37885487$

$-1.37885487$

$-1.37885487$

$-1.37885487$

$-1.27301943$

$-1.27301943$

$-1.27301943$

$-1.27301943$

2. 29703021

2. 29703021

2. 29703021

2. 29703021

0.16034016

0.16034016

0.26617563

0.26617563

0.16034016

0.16034016

0.26617563

0.26617563

$-0.28076980$

$-0.28076980$

$-0.17493436$

$-0.17493436$

$-0.28076980$

$-0.28076980$

$-0.17493436$

$-0.17493436$

1.37668049

1. 37668049

1. 48251593

1. 48251593
$-0.42923152$

. \# Energy 5->5

$0.3302^{0.0387}$
0.12237859

0.01654314

0.12237859

0.01654314

0.12237859

$-0.36711088$

$-0.26127544$

$-0.36711088$

$-0.26127544$

$-0.36711088$

$-0.26127544$

$-0.36711088$

$-0.26127544$

$-0.42923152$

$-0.32339609$

$-0.42923152$

$-0.32339609$

$-0.32339609$

$-0.42923152$

$-0.32339609$

0.06612916

0.17196462

0.06612916

0.17196462

0.06612916

0.17196462

0.06612916

0.17196462

$-0.32880956$

$-0.22297411$

$-0.32880956$

$-0.22297411$

$-0.32880956$

$-0.22297411$

$-0.32880956$

$-0.22297411$

0.09555512

0.20139056

0.09555512

0.20139056

0.09555512

0.20139056

0.09555512

0.20139056

$-4.49214745$

19.26997185

$-11.53486824$

$-10.84063244$

4. 34097528

$-1.02482998$

0.19885898

5.53557205

$-4.94974661$

3. 48385501

$-4.35216045$

$-8.57491779$

9.42964745

311.58297729

$-416.31985474$

$-332.07876587$

437.60369873

$-333.77230835$

452.03018188

357.38360596

$-476.64825439$

14.06660938

$-28.87180138$

$-13.65143490$

34.81342697

9.41615486

$-0.51402211$

$-12.70160580$

$-3.63928437$

30.14666939

$-42.92896652$

$-37.42206573$

55.97607422

$-30.72969246$

45.13352966

37.51520920

$-57.95261383$

2.61541271

$-14.47897625$

$-1.96050048$

10.52025032

5.90798712

5.07050705

$-3.80707598$

$-3.77760410$

Electr. mom.

0.05111535

0.15695079

$-300.49145508$

0.05111535

0.15695079

0.05111535

0.15695079

0.05111535

0.15695079

$-0.39047247$

$-0.28463703$

$-0.39047247$

$-0.28463703$

$-0.39047247$

$-0.28463703$

$-0.39047247$

$-0.28463703$

0.01654314

0.12237859

0.01654314

0.12237859
251.69401550

279.76370239

$-227.03533936$

274.91864014

$-237.20956421$

$-254.77658081$

214.11924744

53.90521240

$-88.63038635$

$-92.24853516$

137.45202637

$-32.01917267$

59.92830658

66.41540527

$-103.41461945$

$-18.49895096$

11.24570656

9.33814144
$-3.75819325$ 
2.40286589

2. 40286589

2. 40286589

2. 40286589

$-1.56014943$

$-1.56014943$

$-1.56014943$

$-1.45431399$

$-1.45431399$

$-1.45431399$

$-1.45431399$

1.01902342

1.01902342

1. 01902342

1.01902342

1.12485886

1. 12485886

1.12485886

$-2.29137540$

$-2.29137540$

$-2.29137540$

$-2.29137540$

$-2.18554020$

$-2.18554020$

$-2.18554020$

$-2.18554020$

0.88915640

0.88915640

0.88915640

0.88915640

0.99499184

0.99499184

0.99499184

0.99499184

$-2.05025959$

$-2.05025959$

$-2.05025959$

$-2.05025959$

$-1.94442415$

$-1.94442415$

$-1.94442415$

64
$-1.56014943$

1. 12485886

1. 37668049

1. 37668049

1. 48251593

1.48251593

$-1.49588871$

$-1.49588871$

$-1.39005339$

$-1.39005339$

$-1.49588871$

$-1.49588871$

$-1.39005339$

$-1.39005339$

$-0.44691080$

$-0.44691080$

$-0.34107536$

$-0.34107536$

$-0.44691080$

$-0.44691080$

$-0.34107536$

$-0.34107536$

0.60408223

0.60408223

0.70991772

0.70991772

0.60408223

0.60408223

0.70991772

0.70991772

$-1.44335949$

$-1.44335949$

$-1.33752406$

$-1.33752406$

$-1.44335949$

$-1.44335949$

$-1.33752406$

$-1.33752406$

1. 58138645

1.58138645

1.68722177

1.68722177

1. 58138645

1. 58138645

1.68722177

1.68722177

\# Energy 6->6

4.8340

2.13764215

2.13764215

2.13764215

2.13764215

2. 24347758

2. 24347758

2. 24347758

2. 24347758

$-1.37885487$

$-1.37885487$

$-1.37885487$

$-1.37885487$

$-1.27301943$

$-1.27301943$

$-1.27301943$

$-1.27301943$

2. 29703021

2. 29703021

2. 29703021

2. 29703021

2. 40286589

$$
\begin{array}{ll}
1.3373 & 0.0419
\end{array}
$$

0.16034016

0.16034016

0.26617563

0.26617563

0.16034016

0.16034016

0.26617563

0.26617563

$-0.28076980$

$-0.28076980$

$-0.17493436$

$-0.17493436$

$-0.28076980$

$-0.28076980$

$-0.17493436$

$-0.17493436$

1.37668049

1.37668049

1.48251593

1.48251593

1.37668049
0.01654314

0.12237859

0.01654314

0.12237859

$-0.36711088$

$-0.26127544$

$-0.36711088$

$-0.26127544$

$-0.36711088$

$-0.26127544$

$-0.36711088$

$-0.26127544$

$-0.42923152$

$-0.32339609$

$-0.42923152$

$-0.32339609$

$-0.42923152$

$-0.32339609$

$-0.42923152$

$-0.32339609$

0.06612916

0.17196462

0.06612916

0.17196462

0.06612916

0.17196462

0.06612916

0.17196462

$-0.32880956$

$-0.22297411$

$-0.32880956$

$-0.22297411$

$-0.32880956$

$-0.22297411$

$-0.32880956$

$-0.22297411$

0.09555512

0.20139056

0.09555512

0.20139056

0.09555512

0.20139056

0.09555512

0.20139056

\# Electr. mom.

0.05111535

0.15695079

0.05111535

0.15695079

0.05111535

0.15695079

0.05111535

0.15695079

$-0.39047247$

$-0.28463703$

$-0.39047247$

$-0.28463703$

$-0.39047247$

$-0.28463703$

$-0.39047247$

$-0.28463703$

0.01654314

0.12237859

0.01654314

0.12237859

0.01654314
18.94461632

$-12.10274792$

$-10.24060726$

4.39149332

2. 20980835

$-4.23758841$

1.27429032

0.77679586

$-0.20213605$

1. 10190701

$-3.71354675$

2.39747834

325.74990845

$-431.15701294$

$-347.71316528$

453.39230347

$-350.83959961$

471.33343506

374.96676636

$-495.20803833$

12.99316025

$-35.26407623$

$-13.05452728$

41.86231995

15.94321632

0.56642467

$-19.54171753$

$-5.02474022$

30.54776955

$-43.60941315$

$-38.52983856$

57.63393784

$-31.33878517$

46.51924133

38.97480011

$-60.68000793$

1.05906975

$-13.74445629$

$-0.50302792$

9.63321209

9.20770741

2.85225463

$-6.52000523$

$-1.80449629$

$-283.35559082$

242.66757202

264.54452515

$-219.44180298$

262.14901733

$-230.56413269$

$-243.30735779$

208.46269226

70.03755951

$-98.71911621$

$-109.83022308$

148.36529541

$-49.50629807$

72.34730530

85.54427338

$-117.17703247$

$-15.31070709$

10.31078720

6.86864710

$-2.95744300$

16.29580688 
2.40286589

2. 40286589

2. 40286589

$-1.56014943$

$-1.56014943$

$-1.56014943$

$-1.56014943$

$-1.45431399$

$-1.45431399$

$-1.45431399$

1.01902342

1.01902342

1.01902342

1. 01902342

1. 12485886

1. 12485886

1. 12485886

1. 12485886

$-2.29137540$

$-2.29137540$

$-2.29137540$

$-2.29137540$

$-2.18554020$

$-2.18554020$

$-2.18554020$

$-2.18554020$

0.88915640

0.88915640

0.88915640

0.88915640

0.99499184

0.99499184

0.99499184

0.99499184

$-2.05025959$

$-2.05025959$

$-2.05025959$

$-2.05025959$

$-1.94442415$

$-1.94442415$

$-1.94442415$

$-1.94442415$

64
$-1.45431399$

0.12237859

0.01654314

$-0.26127544$

0.68722177

\# Energy 7->7

12.2423

2.13764215

2.13764215

2.13764215

2. 13764215

2. 24347758

2. 24347758

2. 24347758

2. 24347758

$-1.37885487$

$-1.37885487$

$-1.37885487$

$-1.37885487$

$-1.27301943$

$-1.27301943$

$-1.27301943$

$-1.27301943$

2. 29703021

2. 29703021

2. 29703021

2. 29703021

2.40286589

2. 40286589
4.0682 1.1866

0.16034016

0.16034016

0.26617563

0.26617563

0.16034016

0.16034016

0.26617563

0.26617563

$-0.28076980$

$-0.28076980$

$-0.17493436$

$-0.17493436$

$-0.28076980$

$-0.28076980$

$-0.17493436$

$-0.17493436$

1.37668049

1.37668049

1. 48251593

1. 48251593

1. 37668049

1. 37668049
0.12237859

$-0.36711088$

$-0.36711088$

$-0.26127544$

$-0.36711088$

$-0.26127544$

$-0.36711088$

$-0.26127544$

$-0.42923152$

$-0.32339609$

$-0.42923152$

$-0.32339609$

$-0.42923152$

$-0.32339609$

$-0.42923152$

$-0.32339609$

0.06612916

0.17196462

0.06612916

0.17196462

0.06612916

0.17196462

0.06612916

0.17196462

$-0.32880956$

$-0.22297411$

$-0.32880956$

$-0.22297411$

$-0.32880956$

$-0.22297411$

$-0.32880956$

$-0.22297411$

0.09555512

0.20139056

0.09555512

0.20139056

0.09555512

0.20139056

0.09555512

0.20139056

$-11.19087029$

$-8.17162418$

3.66768885

3.01697278

$-4.40580177$

$-0.93672866$

2.13181019

$-1.11190426$

1. 49813557

$-1.30659842$

0.61903721

312.91400146

$-413.39080811$

$-339.43035889$

442.47592163

$-339.07019043$

454.42330933

367.69314575

$-485.22833252$

4.41194725

$-32.47837448$

$-8.29829502$

41.92218399

29.49295425

$-6.00133276$

$-28.54770660$

$-1.86712444$

29.92410278

$-41.96452332$

$-37.62033463$

55.16539001

$-31.22580147$

45.26476669

38.72242737

$-58.71574402$

2.17026997

$-15.59072208$

$-1.28217673$

10.94425678

8.61936092

4.16349888

$-6.09123898$

$-2.73839235$

0.05111535

\# Electr. mom.

0.15695079

0.05111535

0.15695079

0.05111535

0.15695079

0.05111535

0.15695079

$-0.39047247$

$-0.28463703$

$-0.39047247$

$-0.28463703$

$-0.39047247$

$-0.28463703$

$-0.39047247$

$-0.28463703$

0.01654314

0.12237859

0.01654314

0.12237859

0.01654314

0.12237859
$-363.64691162$

265.61178589

346.90582275

$-246.29328918$

316.12023926

$-232.56843567$

$-299.28143311$

213.81166077

67.64842224

$-92.86249542$

$-103.77649689$

136.11842346

$-47.70039368$

69.53993988

80.81584930

$-44.59814835$

20.04830742

39.40836716

$-19.84101105$

36.67095947

$-12.43279743$
$-109.18279266$ 


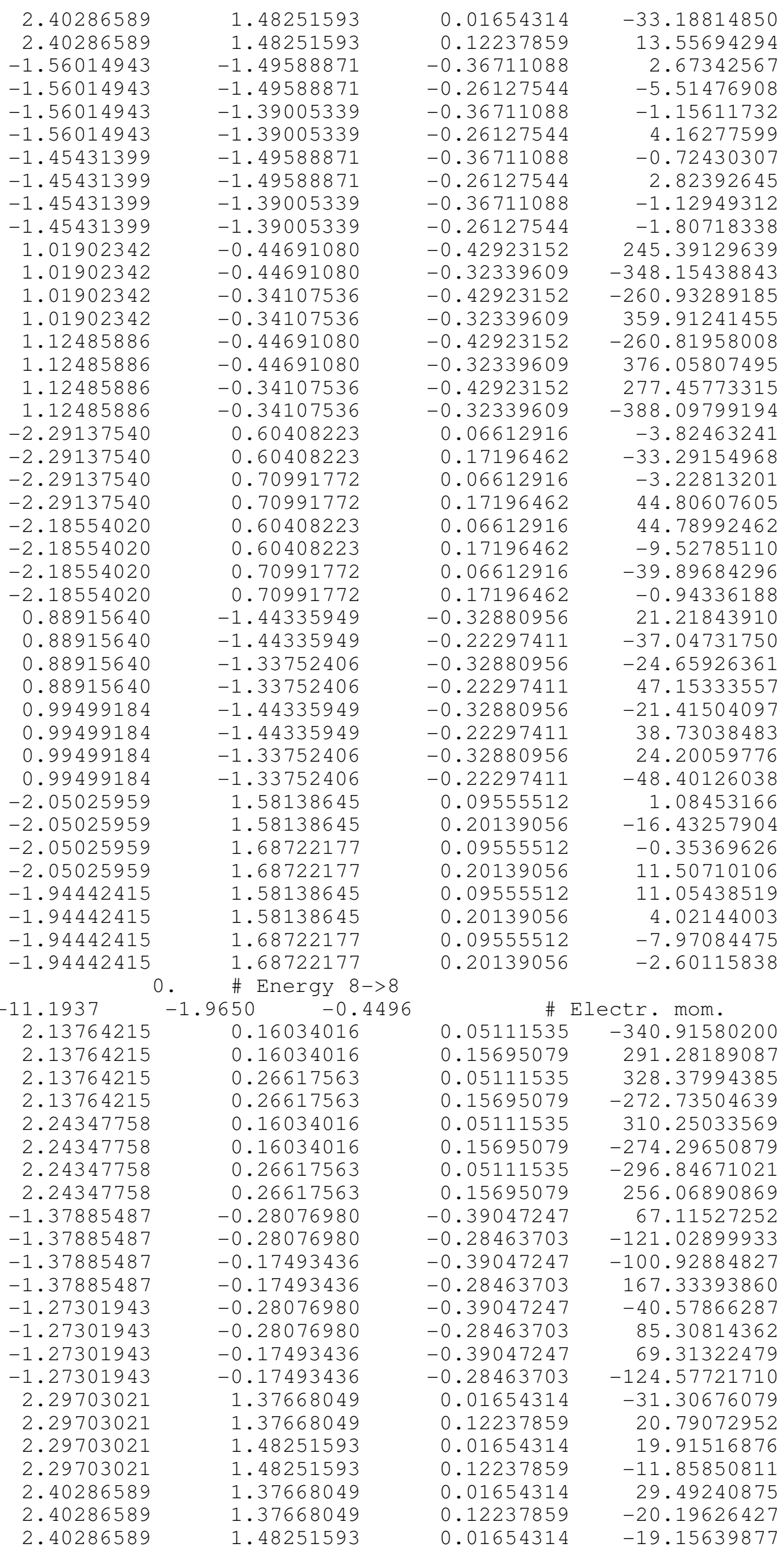

\section{S-181}




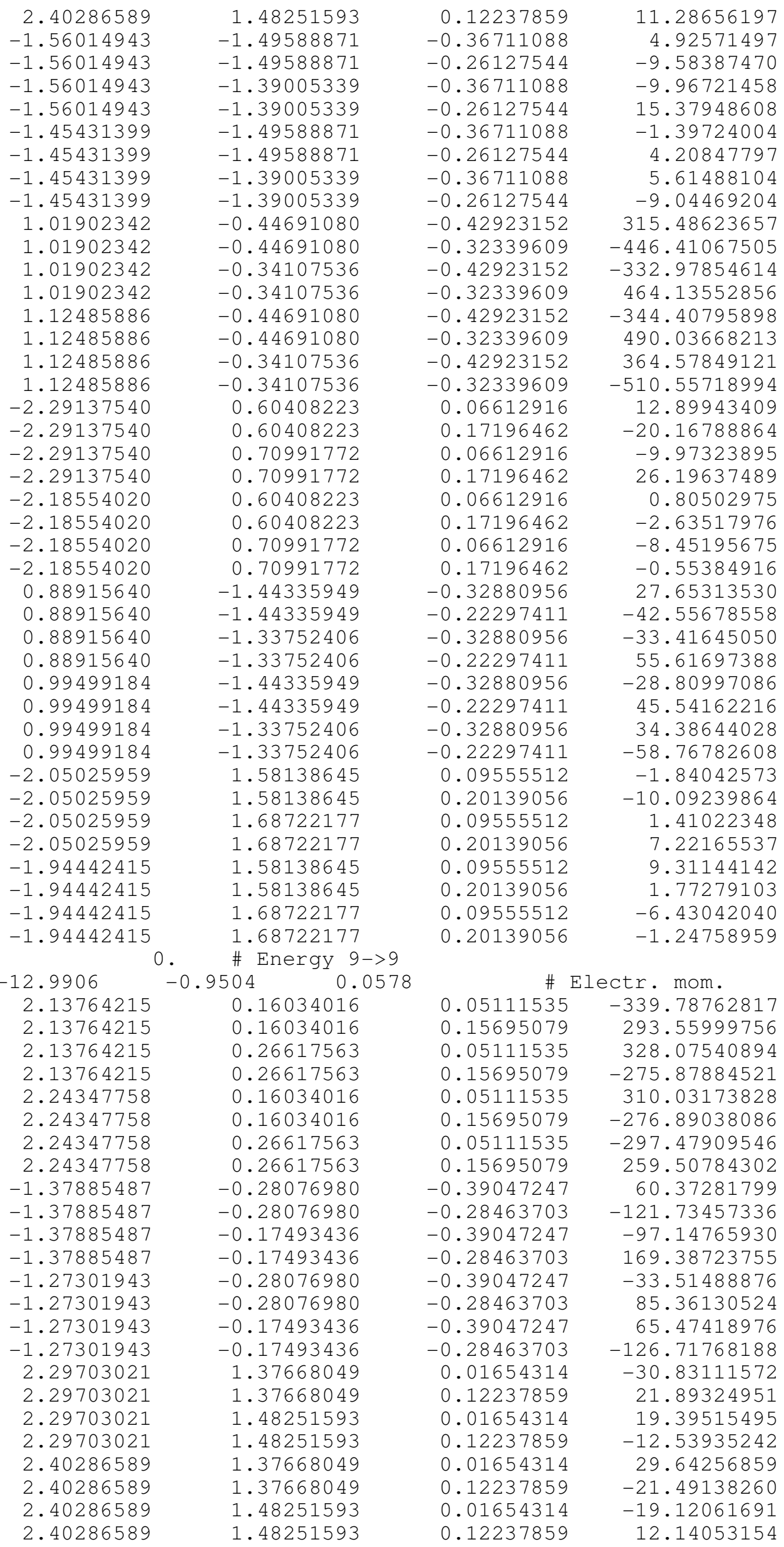

\section{S-182}




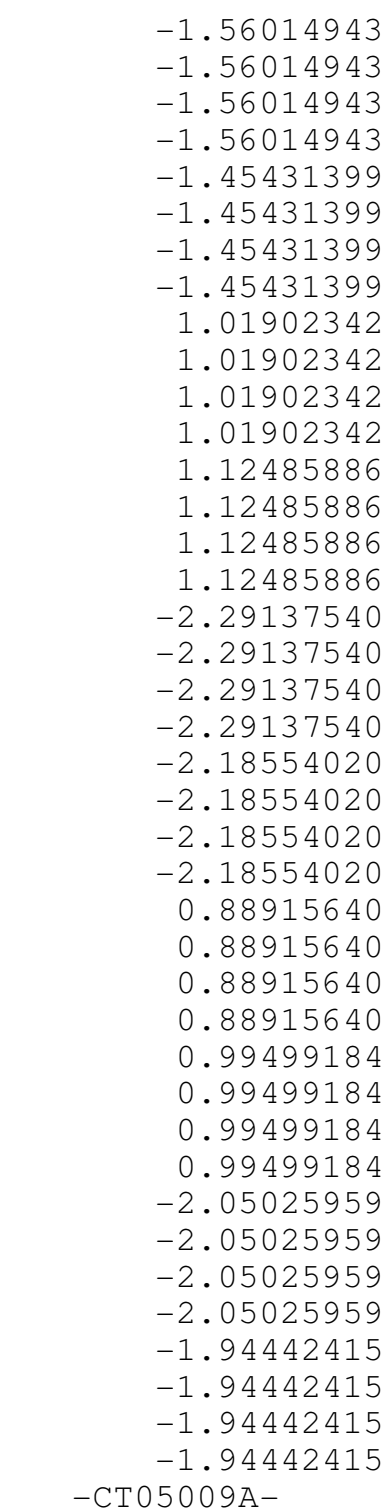

\# phi $=-120$, psi= 6

2.0839
2.1109
1.0789
-1.3231
-1.6312
-2.0739

$$
\begin{array}{r}
-0.36711088 \\
-0.26127544 \\
-0.36711088 \\
-0.26127544 \\
-0.36711088 \\
-0.26127544 \\
-0.36711088 \\
-0.26127544 \\
-0.42923152 \\
-0.32339609 \\
-0.42923152 \\
-0.32339609 \\
-0.42923152 \\
-0.32339609 \\
-0.42923152 \\
-0.32339609 \\
0.06612916 \\
0.17196462 \\
0.06612916 \\
0.17196462 \\
0.06612916 \\
0.17196462 \\
0.06612916 \\
0.17196462 \\
-0.32880956 \\
-0.22297411 \\
-0.32880956 \\
-0.22297411 \\
-0.32880956 \\
-0.22297411 \\
-0.32880956 \\
-0.22297411 \\
0.09555512 \\
0.20139056 \\
0.09555512 \\
0.20139056 \\
0.09555512 \\
0.20139056 \\
0.09555512 \\
0.20139056
\end{array}
$$

1.58138645

1.68722177

58138645

1.58138645

1.68722177

1.68722177

$120, \operatorname{root} s=9$

$$
\begin{array}{rr}
0.2464 & 0.0312 \\
1.4258 & -0.3453 \\
-0.6125 & -0.3111 \\
-0.4073 & -0.3157 \\
-1.5361 & 0.0936 \\
0.6953 & -0.0756
\end{array}
$$

\&TRANSITION $1->\ldots$

64 41505.

$$
\begin{gathered}
-0.1610 \quad-0.2079 \\
-0.6546 \quad 0.2523 \\
0.19347408 \\
0.19347408 \\
0.29930952 \\
0.29930952 \\
0.19347408 \\
0.19347408 \\
0.29930952 \\
0.29930952 \\
-0.46025667 \\
-0.46025667 \\
-0.35442120 \\
-0.35442120 \\
-0.46025667 \\
-0.46025667
\end{gathered}
$$

1.96355677

$-5.75835514$

6.15477800

$-1.52882016$

0.04927015

1.38860846

$-8.94629860$

6.93704557

314.85507202

$-445.02172852$

$-333.79595947$

464.84600830

$-343.60879517$

488.11553955

365.48138428

$-511.20965576$

6.99602938

$-13.63387966$

$-5.47077131$

20.12629318

12.79353809

$-13.49336529$

$-18.05018806$

9.25930882

27.11037064

$-41.48453903$

$-32.81468582$

54.14785004

$-28.44173241$

44.43641663

33.93244553

$-57.17068100$

1.88867891

$-12.49428940$

$-1.37710011$

9.00711060

6.31569386

3. 54407001

$-4.18483973$

$-2.57220745$

$\begin{array}{ll}1.0000 & \# \text { C1 } \\ 1.0000 & \# \text { O1 } \\ 1.0000 & \# \text { N1 } \\ 1.0000 & \# \text { C2 } \\ 1.0000 & \# \text { N2 } \\ 1.0000 & \# \text { O2 }\end{array}$

$\begin{array}{rr}1.000 \# \text { Electr. mom., scale-fac } \\ \# \text { Magnetic mom. } \\ -0.02169468 & 2.19170237 \\ 0.08414077 & -0.77578312 \\ -0.02169468 & -3.88646984 \\ 0.08414077 & 2.07817245 \\ -0.02169468 & -2.63382983 \\ 0.08414077 & 1.52719903 \\ -0.02169468 & 4.20396376 \\ 0.08414077 & -2.77430201 \\ -0.36857194 & 5.32560158 \\ -0.26273650 & -29.37474823 \\ -0.36857194 & 0.35494158 \\ -0.26273650 & 22.88758087 \\ -0.36857194 & -7.22967434 \\ -0.26273650 & 29.49219513\end{array}$




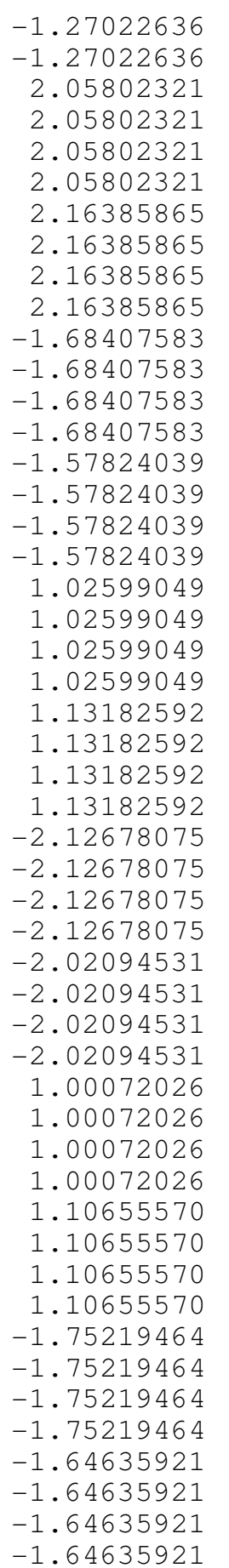

64
$-0.35442120$

$-0.35442120$

1. 37286413

1.37286413

1. 47869956

1. 47869956

1. 37286413

1. 37286413

1. 47869956

1.47869956

$-1.58903348$

$-1.58903348$

$-1.48319805$

$-1.48319805$

$-1.58903348$

$-1.58903348$

$-1.48319805$

$-1.48319805$

$-0.66542661$

$-0.66542661$

$-0.55959117$

$-0.55959117$

$-0.66542661$

$-0.66542661$

$-0.55959117$

$-0.55959117$

0.64235818

0.64235818

0.74819362

0.74819362

0.64235818

0.64235818

0.74819362

0.74819362

$-1.60289264$

$-1.60289264$

$-1.49705720$

$-1.49705720$

$-1.60289264$

$-1.60289264$

$-1.49705720$

$-1.49705720$

1. 54989612

1.54989612

1.65573156

1.65573156

1. 54989612

1.54989612

1.65573156

1.65573156 43802 .
\# Energy 1->3

$$
0.0734 \quad 0.1505
$$

$0.9553-0.2889$

0.19347408

0.19347408

0.29930952

0.29930952

0.19347408

0.19347408

0.29930952

0.29930952

$-0.46025667$

$-0.46025667$

$-0.35442120$

$-0.35442120$

$-0.46025667$

$-0.46025667$
$-0.36857194$

$-0.26273650$

$-0.39818472$

$-0.29234925$

$-0.39818472$

$-0.29234925$

$-0.39818472$

$-0.29234925$

$-0.39818472$

$-0.29234925$

0.04071225

0.14654770

0.04071225

0.14654770

0.04071225

0.14654770

0.04071225

0.14654770

$-0.36399299$

$-0.25815752$

$-0.36399299$

$-0.25815752$

$-0.36399299$

$-0.25815752$

$-0.36399299$

$-0.25815752$

$-0.12852763$

$-0.02269218$

$-0.12852763$

$-0.02269218$

$-0.12852763$

$-0.02269218$

$-0.12852763$

$-0.02269218$

0.02148618

0.12732163

0.02148618

0.12732163

0.02148618

0.12732163

0.02148618

0.12732163

$-0.37628788$

$-0.27045241$

$-0.37628788$

$-0.27045241$

$-0.37628788$

$-0.27045241$

$-0.37628788$

$-0.27045241$

1.000

$-0.02169468$

0.08414077

$-0.02169468$

0.08414077

$-0.02169468$

0.08414077

$-0.02169468$

0.08414077

$-0.36857194$

$-0.26273650$

$-0.36857194$

$-0.26273650$

$-0.36857194$

$-0.26273650$
0.76190400

$-21.78552246$

1. 47269750

$-1.26033342$

$-1.79248309$

1.67721367

$-2.09621811$

2.02369928

2. 28000355

$-2.27310324$

$-4.24585867$

$-0.98853505$

9. 70781803

$-2.84537435$

0.28041837

5. 03981018

$-5.21091986$

$-1.75582945$

5.51130295

$-13.94660091$

$-4.16129017$

11.07197380

$-3.54299378$

9.94897270

2.65700603

$-7.76112938$

7.61333036

$-0.67510426$

$-6.32952356$

0.30227238

$-16.35893822$

6.60653114

14.61545181

$-6.01587057$

$-4.92277384$

3.20692754

6.78383207

$-4.09669781$

4.32917070

$-3.10418463$

$-5.79998302$

3. 82114482

$-0.73899871$

$-0.26305616$

0.36682913

0.34666184

$-0.17155942$

0.92838430

0.37521055

$-0.97223455$ 


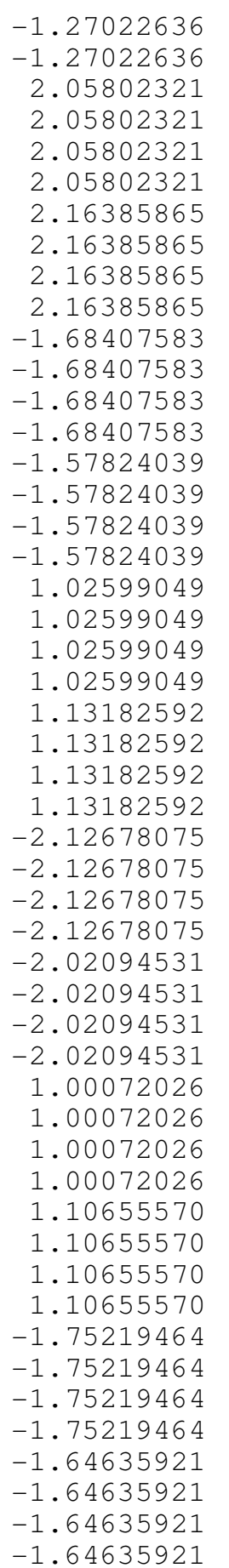

64
$-0.35442120$

$-0.35442120$

1. 37286413

1.37286413

1. 47869956

1. 47869956

1. 37286413

1. 37286413

1. 47869956

1.47869956

$-1.58903348$

$-1.58903348$

$-1.48319805$

$-1.48319805$

$-1.58903348$

$-1.58903348$

$-1.48319805$

$-1.48319805$

$-0.66542661$

$-0.66542661$

$-0.55959117$

$-0.55959117$

$-0.66542661$

$-0.66542661$

$-0.55959117$

$-0.55959117$

0.64235818

0.64235818

0.74819362

0.74819362

0.64235818

0.64235818

0.74819362

0.74819362

$-1.60289264$

$-1.60289264$

$-1.49705720$

$-1.49705720$

$-1.60289264$

$-1.60289264$

$-1.49705720$

$-1.49705720$

1. 54989612

1. 54989612

1.65573156

1.65573156

1. 54989612

1.54989612

1.65573156

1.65573156 51848 .
-0.36857194
-0.26273650
-0.39818472
-0.29234925
-0.39818472
-0.29234925
-0.39818472
-0.29234925
-0.39818472
-0.29234925
0.04071225
0.14654770
0.04071225
0.14654770
0.04071225
0.14654770
0.04071225
0.14654770
-0.36399299
-0.25815752
-0.36399299
-0.25815752
-0.36399299
-0.25815752
-0.36399299
-0.25815752
-0.12852763
-0.02269218
-0.12852763
-0.02269218
-0.12852763
-0.02269218
-0.12852763
-0.02269218
0.02148618
0.12732163
0.02148618
0.12732163
0.02148618
0.12732163
0.02148618
0.12732163
-0.37628788
-0.27045241
-0.37628788
-0.27045241
-0.37628788
-0.27045241
-0.37628788
-0.27045241 \# Energy 1->4
5.68681717

$-12.27623367$

$-8.67630482$

13.62764740

9.57833672

$-13.44088268$

15.18676376

$-20.02011299$

$-14.97248936$

18.79304695

$-0.59628797$

$-0.21692704$

1.39871597

$-0.33356890$

$-0.03967761$

0.75929701

$-0.61570036$

$-0.30726311$

5. 43716574

$-10.41644669$

$-3.01851082$

8.56089878

$-4.86572075$

9. 75265408

2.49455738

$-8.08188438$

0.97421610

2. 35468507

$-0.91751140$

$-2.16868424$

$-3.55747700$

$-1.23910475$

3. 44689870

1. 04202890

$-3.63181639$

3. 64681697

4.85507154

$-4.77306128$

4.07310390

$-4.20723295$

$-5.34781504$

5. 43306541

0.19326240

$-0.74872732$

$-0.23135944$

0.66324520

$-0.24916668$

0.51781696

0.31821504

$-0.55596119$
$\begin{array}{lll}-2.2141 & 1.2538 & -0.1416\end{array}$
$-0.2911$ .1416
1.4480
2.03096962
2.03096962
2.03096962
2. 03096962
2.13680482
2.13680482
2.13680482
2.13680482
$-1.37606192$
$-1.37606192$
$-1.37606192$
$-1.37606192$
$-1.27022636$
$-1.27022636$

$\begin{array}{cc}1.2538 & -0.1416 \\ -0.0573 & 1.4480 \\ 0.19347408 \\ 0.19347408 \\ 0.29930952 \\ 0.29930952 \\ 0.19347408 \\ 0.19347408 \\ 0.29930952 \\ 0.29930952 \\ -0.46025667 \\ -0.46025667 \\ -0.35442120 \\ -0.35442120 \\ -0.46025667 \\ -0.46025667\end{array}$
1.000
$-0.02169468$
0.08414077
$-0.02169468$
0.08414077
$-0.02169468$
0.08414077
$-0.02169468$
0.08414077
$-0.36857194$
$-0.26273650$
$-0.36857194$
$-0.26273650$
$-0.36857194$
$-0.26273650$
\# Electr. mom., scale-fac Magnetic mom.
15.05834866
$-10.94272423$
$-13.62886143$
9.73705769
$-9.20760441$
6.85253000
7.58173800
$-5.57169199$
$-24.77965927$
26.86650467
25.64413834
$-26.75302696$
26.03034019
$-31.73385620$ 


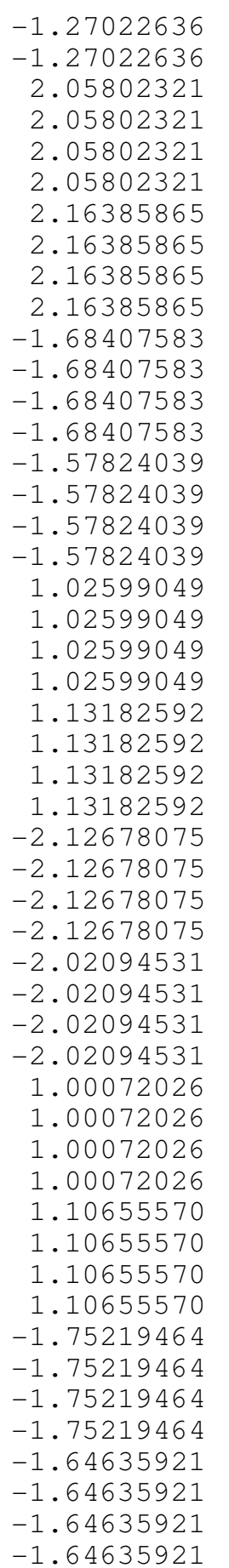

64
$-0.35442120$

$-0.35442120$

1. 37286413

1.37286413

1. 47869956

1. 47869956

1. 37286413

1. 37286413

1. 47869956

1.47869956

$-1.58903348$

$-1.58903348$

$-1.48319805$

$-1.48319805$

$-1.58903348$

$-1.58903348$

$-1.48319805$

$-1.48319805$

$-0.66542661$

$-0.66542661$

$-0.55959117$

$-0.55959117$

$-0.66542661$

$-0.66542661$

$-0.55959117$

$-0.55959117$

0.64235818

0.64235818

0.74819362

0.74819362

0.64235818

0.64235818

0.74819362

0.74819362

$-1.60289264$

$-1.60289264$

$-1.49705720$

$-1.49705720$

$-1.60289264$

$-1.60289264$

$-1.49705720$

$-1.49705720$

1. 54989612

1. 54989612

1.65573156

1.65573156

1. 54989612

1. 54989612

1.65573156

1.65573156 53886 .
-0.36857194
-0.26273650
-0.39818472
-0.29234925
-0.39818472
-0.29234925
-0.39818472
-0.29234925
-0.39818472
-0.29234925
0.04071225
0.14654770
0.04071225
0.14654770
0.04071225
0.14654770
0.04071225
0.14654770
-0.36399299
-0.25815752
-0.36399299
-0.25815752
-0.36399299
-0.25815752
-0.36399299
-0.25815752
-0.12852763
-0.02269218
-0.12852763
-0.02269218
-0.12852763
-0.02269218
-0.12852763
-0.02269218
0.02148618
0.12732163
0.02148618
0.12732163
0.02148618
0.12732163
0.02148618
0.12732163
-0.37628788
-0.27045241
-0.37628788
-0.27045241
-0 \# Energy 1->5 $\begin{array}{rr}-4.0927 & 0.0184 \\ 0.0559 & 0.2903\end{array}$

0.19347408

0.19347408

0.29930952

0.29930952

0.19347408

0.19347408

0.29930952

0.29930952

$-0.46025667$

$-0.46025667$

$-0.35442120$

$-0.35442120$

$-0.46025667$

$-0.46025667$
$-26.13591003$

30.68104172

2.97833276

$-2.26749921$

$-3.82817054$

3. 41796660

$-2.34154367$

2. 50195146

2.99684978

$-3.11900878$

6.53076982

$-4.26421595$

$-6.31749868$

3.37011576

$-7.79200315$

4.41637850

8.54643631

$-3.90245485$

$-4.39799738$

5.67113638

6.47734547

$-7.82682705$

4.83291435

$-6.85145664$

$-7.00713015$

8.87359047

25.17000961

$-27.07855034$

$-25.40481377$

27.13998413

$-28.40723038$

30.43153954

27.91647911

$-30.31254768$

$-0.90122402$

0.62394524

1. 46752560

$-1.11968744$

0.34398177

$-0.14823975$

$-0.69932520$

0.47923139

$-1.29850543$

2. 04928684

0.71149629

$-1.14699984$

0.41254863

$-0.79513073$

$-0.12705480$

0.29693305
1.000 \# Electr. mom., scale-fac
\# Magnetic mom.

$-0.02169468$

0.08414077

$-0.02169468$

0.08414077

$-0.02169468$

0.08414077

$-0.02169468$

0.08414077

$-0.36857194$

$-0.26273650$

$-0.36857194$

$-0.26273650$

$-0.36857194$

$-0.26273650$
$-11.72586918$

7. 92906189

11.22579288

$-6.99822426$

6.10446024

$-3.72929597$

$-5.07651567$

$-7.50267458$

4.00483084

6.29673910

$-2.27578664$

10.58145809

$-9.95132637$
2. 53304148 


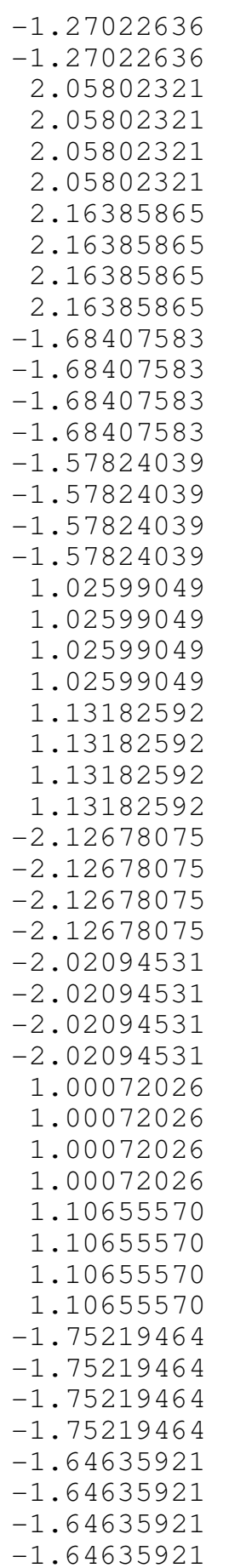

64
$-0.35442120$

$-0.35442120$

1. 37286413

1. 37286413

1. 47869956

1. 47869956

1. 37286413

1. 37286413

1. 47869956

1.47869956

$-1.58903348$

$-1.58903348$

$-1.48319805$

$-1.48319805$

$-1.58903348$

$-1.58903348$

$-1.48319805$

$-1.48319805$

$-0.66542661$

$-0.66542661$

$-0.55959117$

$-0.55959117$

$-0.66542661$

$-0.66542661$

$-0.55959117$

$-0.55959117$

0.64235818

0.64235818

0.74819362

0.74819362

0.64235818

0.64235818

0.74819362

0.74819362

$-1.60289264$

$-1.60289264$

$-1.49705720$

$-1.49705720$

$-1.60289264$

$-1.60289264$

$-1.49705720$

$-1.49705720$

1. 54989612

1.54989612

1.65573156

1.65573156

1. 54989612

1. 54989612

1.65573156

1.65573156 57619.
-0.36857194
-0.26273650
-0.39818472
-0.29234925
-0.39818472
-0.29234925
-0.39818472
-0.29234925
-0.39818472
-0.29234925
0.04071225
0.14654770
0.04071225
0.14654770
0.04071225
0.14654770
0.04071225
0.14654770
-0.36399299
-0.25815752
-0.36399299
-0.25815752
-0.36399299
-0.25815752
-0.36399299
-0.25815752
-0.12852763
-0.02269218
-0.12852763
-0.02269218
-0.12852763
-0.02269218
-0.12852763
-0.02269218
0.02148618
0.12732163
0.02148618
0.12732163
0.02148618
0.12732163
0.02148618
0.12732163
-0.37678788
-0.27045241
-0.37628788
-0.27045241
-0

\# Energy 1->6

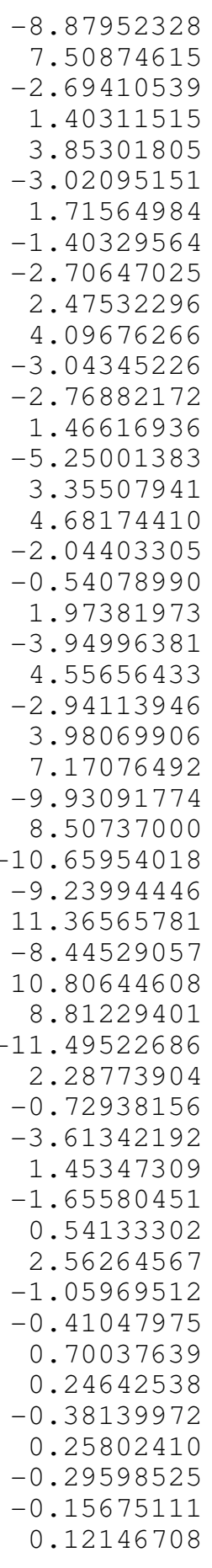

1.000 $-0.02169468$ 0.08414077 $-0.02169468$ 0.08414077 $-0.02169468$ 0.08414077 $-0.02169468$ 0.08414077 $-0.36857194$ $-0.26273650$ $-0.36857194$ $-0.26273650$ $-0.36857194$ $-0.26273650$
$-0.0847$
066
0.1212
2.03096962
.19347408
0.29930952
0.29930952
0.19347408
0.29930952
0.29930952
$-0.46025667$
$-0.46025667$
$-0.35442120$
$-0.35442120$
$-0.46025667$
$-0.46025667$
\# Electr. mom., scale-fac Magnetic mom.
$-18.84490967$
12.37860298
18.47711563
$-11.66364002$
12.07347584
$-7.54371929$
$-11.19876003$
6.58159685
$-0.03515843$
$-0.62896812$
$-1.87680197$
3. 34682918
2. 92968416
$-4.08914328$
-1.27022636
-1.27022636 


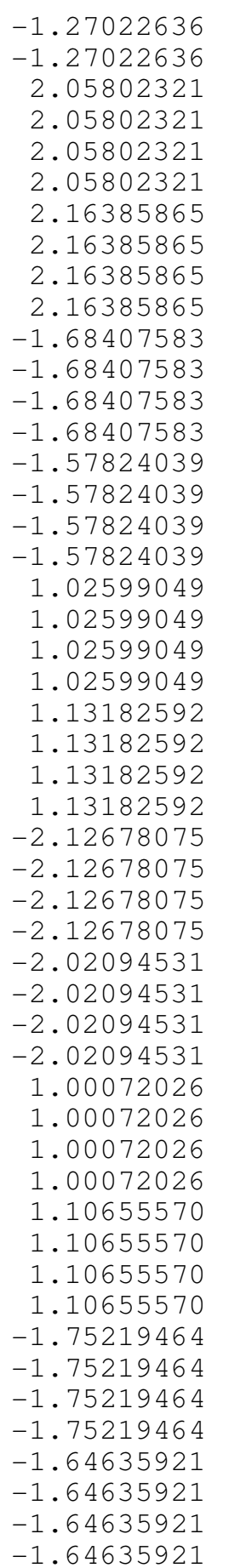

64
$-0.35442120$

$-0.35442120$

1. 37286413

1. 37286413

1. 47869956

1. 47869956

1. 37286413

1. 37286413

1. 47869956

1.47869956

$-1.58903348$

$-1.58903348$

$-1.48319805$

$-1.48319805$

$-1.58903348$

$-1.58903348$

$-1.48319805$

$-1.48319805$

$-0.66542661$

$-0.66542661$

$-0.55959117$

$-0.55959117$

$-0.66542661$

$-0.66542661$

$-0.55959117$

$-0.55959117$

0.64235818

0.64235818

0.74819362

0.74819362

0.64235818

0.64235818

0.74819362

0.74819362

$-1.60289264$

$-1.60289264$

$-1.49705720$

$-1.49705720$

$-1.60289264$

$-1.60289264$

$-1.49705720$

$-1.49705720$

1. 54989612

1.54989612

1.65573156

1.65573156

1. 54989612

1. 54989612

1.65573156

1.65573156 64213.
0.4114

0.0243

2. 03096962

2.03096962

2.03096962

2.03096962

2.13680482

2.13680482

2.13680482

2.13680482

$-1.37606192$

$-1.37606192$

$-1.37606192$

$-1.37606192$

$-1.27022636$

$-1.27022636$
-0.36857194
-0.26273650
-0.39818472
-0.29234925
-0.39818472
-0.29234925
-0.39818472
-0.29234925
-0.39818472
-0.29234925
0.04071225
0.14654770
0.04071225
0.14654770
0.04071225
0.14654770
0.04071225
0.14654770
-0.36399299
-0.25815752
-0.36399299
-0.25815752
-0.36399299
-0.25815752
-0.36399299
-0.25815752
-0.12852763
-0.02269218
-0.12852763
-0.02269218
-0.12852763
-0.02269218
-0.12852763
-0.02269218
0.02148618
0.12732163
0.02148618
0.12732163
0.02148618
0.12732163
0.02148618
0.12732163
-0.37628788
-0.27045241
-0.37628788
-0.27045241
-0.37628788
-0.27045241
-0.37628788
-0.27045241 \# Energy 1->7

$0.3605 \quad-0.0151$

$0.0365-0.1362$

0.19347408

0.19347408

0.29930952

0.29930952

0.19347408

0.19347408

0.29930952

0.29930952

$-0.46025667$

$-0.46025667$

$-0.35442120$

$-0.35442120$

$-0.46025667$

$-0.46025667$
$-0.84723848$

1. 15988231

$-4.55943918$

3.20719099

5. 38630486

$-4.54470921$

3.36699796

$-3.02631021$

$-4.07300091$

3.84525490

0.56068850

0.35454407

$-0.71604848$

$-0.54871809$

$-0.60799837$

$-0.69851786$

0.99983394

0.83498925

5.50105619

$-11.04113102$

$-9.33935738$

15.43406200

$-8.18163586$

16.27512550

12.18780518

$-20.50152206$

4.13525343

$-4.27137280$

$-4.09530973$

4. 40025902

$-4.17786598$

3. 92136216

4.02058077

$-4.10644245$

$-0.37150812$

$-0.06392397$

0.22934894

0.37073928

1. 01842725

$-0.25164965$

$-1.21959424$

0.12398645

0.47598633

$-0.69803095$

$-0.41813198$

0.60690534

$-0.50561374$

0.73677140

0.43673390

$-0.63122171$
1.000 \# Electr. mom., scale-fac
\# Magnetic mom.

$-0.02169468$

0.08414077

$-0.02169468$

0.08414077

$-0.02169468$

0.08414077

$-0.02169468$

0.08414077

$-0.36857194$

$-0.26273650$

$-0.36857194$

$-0.26273650$

$-0.36857194$

$-0.26273650$
5.65824699

$-5.62018251$

$-5.35599184$

4.83648586

$-4.68160629$

4.91352463

4.20978165

$-4.05499220$

$-4.27421904$

5.69660282

5.17913008

$-6.65615797$

3. 33925247

$-4.41870070$ 


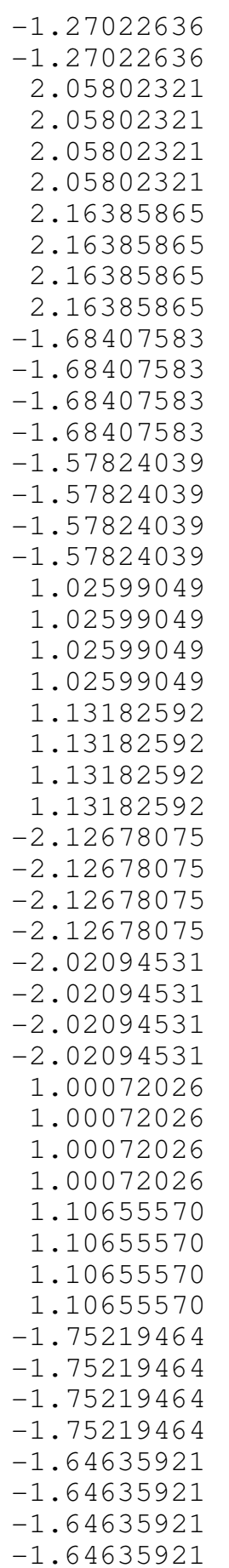

64
$-0.35442120$

$-0.35442120$

1. 37286413

1.37286413

1. 47869956

1. 47869956

1. 37286413

1. 37286413

1. 47869956

1.47869956

$-1.58903348$

$-1.58903348$

$-1.48319805$

$-1.48319805$

$-1.58903348$

$-1.58903348$

$-1.48319805$

$-1.48319805$

$-0.66542661$

$-0.66542661$

$-0.55959117$

$-0.55959117$

$-0.66542661$

$-0.66542661$

$-0.55959117$

$-0.55959117$

0.64235818

0.64235818

0.74819362

0.74819362

0.64235818

0.64235818

0.74819362

0.74819362

$-1.60289264$

$-1.60289264$

$-1.49705720$

$-1.49705720$

$-1.60289264$

$-1.60289264$

$-1.49705720$

$-1.49705720$

1. 54989612

1. 54989612

1.65573156

1.65573156

1. 54989612

1. 54989612

1.65573156

1.65573156 79090.
$-0.36857194$

$-0.26273650$

$-0.39818472$

$-0.29234925$

$-0.39818472$

$-0.29234925$

$-0.39818472$

$-0.29234925$

$-0.39818472$

$-0.29234925$

0.04071225

0.14654770

0.04071225

0.14654770

0.04071225

0.14654770

0.04071225

0.14654770

$-0.36399299$

$-0.25815752$

$-0.36399299$

$-0.25815752$

$-0.36399299$

$-0.25815752$

$-0.36399299$

$-0.25815752$

$-0.12852763$

$-0.02269218$

$-0.12852763$

$-0.02269218$

$-0.12852763$

$-0.02269218$

$-0.12852763$

$-0.02269218$

0.02148618

0.12732163

0.02148618

0.12732163

0.02148618

0.12732163

0.02148618

0.12732163

$-0.37628788$

$-0.27045241$

$-0.37628788$

$-0.27045241$

$-0.37628788$

$-0.27045241$

$-0.37628788$

$-0.27045241$ \# Energy 1->8 $\begin{array}{lll}0.1769 & 0.0621 & -0.4183\end{array}$

$-0.0033$

2.03096962

2.03096962

2.03096962

2.03096962

2.13680482

2.13680482

2.13680482

2.13680482

$-1.37606192$

$-1.37606192$

$-1.37606192$

$-1.37606192$

$-1.27022636$

$-1.27022636$
$-0.0139-0.1134$

0.19347408

0.19347408

0.29930952

0.29930952

0.19347408

0.19347408

0.29930952

0.29930952

$-0.46025667$

$-0.46025667$

$-0.35442120$

$-0.35442120$

$-0.46025667$

$-0.46025667$
-4.27359295
5.42344809
0.04076965
0.11121136
-0.17477129

0.11016044

$-0.11721218$

0.15129068

0.18409707

$-0.23366654$

0.07341027

$-0.20439667$

$-0.20713612$

0.33655667

$-0.24137306$

0.43233615

0.34635532

$-0.57517326$

$-3.90601969$

5.70375538

4.62720680

$-6.77422428$

4.79368925

$-7.42843533$

$-5.63218260$

8.59453487

2. 52889991

$-2.55144548$

$-2.36481881$

2. 34250975

$-3.20072818$

3. 27856064

3.01254296

$-3.03559446$

$-0.10200376$

0.14699872

0.07652995

$-0.15743023$

$-0.18327777$

0.02214463

0.33738202

$-0.08620078$

$-0.34549433$

0.44632399

0.25517452

$-0.34764978$

0.21410640

$-0.29300016$

$-0.16797604$

0.24263532

$$
1.000 \text { \# Electr. mom., scale-fac }
$$$$
-0.02169468
$$$$
0.08414077
$$$$
-0.02169468
$$$$
0.08414077
$$$$
-0.02169468
$$$$
0.08414077
$$$$
-0.02169468
$$$$
0.08414077
$$$$
-0.36857194
$$$$
-0.26273650
$$$$
-0.36857194
$$$$
-0.26273650
$$$$
-0.36857194
$$$$
-0.26273650
$$

$$
\begin{array}{r}
4.47618246 \\
-6.27692795 \\
-4.23939753 \\
5.76518488 \\
-4.28949308 \\
6.03259659 \\
4.13580608 \\
-5.53631592 \\
3.49810600 \\
-3.03688502 \\
-5.80546570 \\
5.25512028 \\
-0.67827553 \\
-0.52902621
\end{array}
$$

\section{S-189}




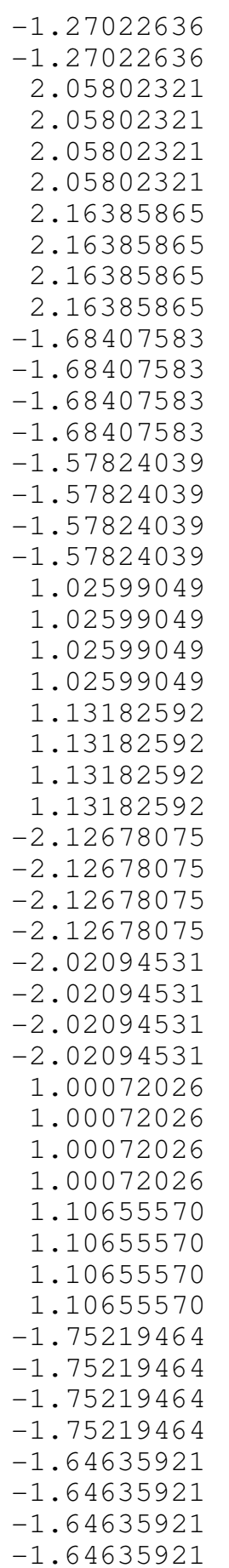

64
$-0.35442120$

$-0.35442120$

1. 37286413

1.37286413

1. 47869956

1. 47869956

1. 37286413

1. 37286413

1. 47869956

1.47869956

$-1.58903348$

$-1.58903348$

$-1.48319805$

$-1.48319805$

$-1.58903348$

$-1.58903348$

$-1.48319805$

$-1.48319805$

$-0.66542661$

$-0.66542661$

$-0.55959117$

$-0.55959117$

$-0.66542661$

$-0.66542661$

$-0.55959117$

$-0.55959117$

0.64235818

0.64235818

0.74819362

0.74819362

0.64235818

0.74819362

0.74819362

$-1.60289264$

$-1.60289264$

$-1.49705720$

$-1.49705720$

$-1.60289264$

$-1.60289264$

$-1.49705720$

$-1.49705720$

1. 54989612

1. 54989612

1.65573156

1.65573156

1. 54989612

1. 54989612

1.65573156

1.65573156 73988.
0.64235818

-0.36857194
-0.26273650
-0.39818472
-0.29234925
-0.39818472
-0.29234925
-0.39818472
-0.29234925
-0.39818472
-0.29234925
0.04071225
0.14654770
0.04071225
0.14654770
0.04071225
0.14654770
0.04071225
0.14654770
-0.36399299
-0.25815752
-0.36399299
-0.25815752
-0.36399299
-0.25815752
-0.36399299
-0.25815752
-0.12852763
-0.02269218
-0.12852763
-0.02269218
-0.12852763
-0.02269218
-0.12852763
-0.02269218
0.02148618
0.12732163
0.02148618
0.12732163
0.02148618
0.12732163
0.02148618
0.12732163
-0.37628788
-0.27045241
-0.37628788
-0.27045241
-0.37628788
-0.37045241
-0.27045241 \# Energy 1->9

$\begin{array}{lll}-0.6471 & 0.8074 & -1.4521\end{array}$

$\begin{array}{lll}-0.0170 & -0.2803 & -0.2342\end{array}$

2.03096962

2.03096962

0.19347408

0.19347408

0.29930952

2.03096962

2.03096962

2.13680482

2.13680482

2.13680482

2.13680482

$-1.37606192$

$-1.37606192$

$-1.37606192$

$-1.37606192$

$-1.27022636$

$-1.27022636$

0.29930952

0.19347408

0.19347408

0.29930952

0.29930952

$-0.46025667$

$-0.46025667$

$-0.35442120$

$-0.35442120$

$-0.46025667$

$-0.46025667$

3. 09434175

$-2.01852942$

$-0.00932577$

$-0.11275176$

0.31964430

$-0.25850326$

$-0.01137085$

$-0.05381013$

$-0.24183719$

0.30313268

0.14264756

0.43053475

$-0.03562028$

$-0.70046604$

$-0.20856647$

$-0.65008843$

0.31274533

0.85008371

$-4.83588791$

6.30934858

5.19773769

$-6.89820671$

5.46826839

$-7.40059900$

$-5.99236345$

8.14520264

$-10.11735058$

10.42764473

9. 28971958

$-9.31577682$

11.95486832

$-12.00620174$

$-10.93162251$

10.77897358

$-1.00202370$

0.89844644

1. 40382814

$-1.30665505$

0.79736990

$-0.71355939$

$-1.09857082$

1.02916014

0.54646397

$-0.64523667$

$-0.36075664$

0.42593005

$-0.30702615$

0.38754401

0.20393881

$-0.25607699$

$$
\begin{gathered}
1.000 \text { \# Electr. mom., scale-fac } \\
\text { \# Magnetic mom. }
\end{gathered}
$$

$-0.02169468$

0.08414077

$-0.02169468$

0.08414077

$-0.02169468$

0.08414077

$-0.02169468$

0.08414077

$-0.36857194$

$-0.26273650$

$-0.36857194$

$-0.26273650$

$-0.36857194$

$-0.26273650$
$-6.72769308$
7. 92218351
4. 32254314
$-4.01029539$
6.82693195
$-7.82005024$
$-3.33645821$
3. 57535791
$-19.81744385$
30.21428680
18.53649902
$-29.67834282$
14.90250874
$-23.58959579$ 


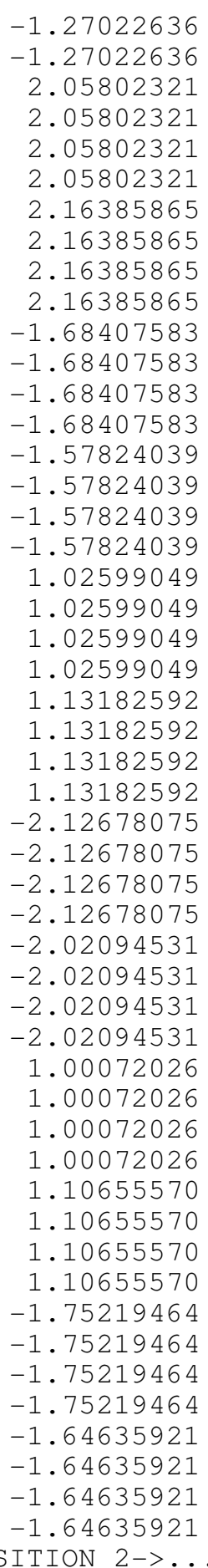

\&TRANSITION 2->...
$-0.35442120$

$-0.35442120$

1. 37286413

1.37286413

1. 47869956

1.47869956

1. 37286413

1. 37286413

1. 47869956

1.47869956

$-1.58903348$

$-1.58903348$

$-1.48319805$

$-1.48319805$

$-1.58903348$

$-1.58903348$

$-1.48319805$

$-1.48319805$

$-0.66542661$

$-0.66542661$

$-0.55959117$

$-0.55959117$

$-0.66542661$

$-0.66542661$

$-0.55959117$

$-0.55959117$

0.64235818

0.64235818

0.74819362

0.74819362

0.64235818

0.64235818

0.74819362

0.74819362

$-1.60289264$

$-1.60289264$

$-1.49705720$

$-1.49705720$

$-1.60289264$

$-1.60289264$

$-1.49705720$

$-1.49705720$

1. 54989612

1. 54989612

1.65573156

1.65573156

1. 54989612

1.54989612

1.65573156

1.65573156
$-0.36857194$

$-0.26273650$

$-0.39818472$

$-0.29234925$

$-0.39818472$

$-0.29234925$

$-0.39818472$

$-0.29234925$

$-0.39818472$

$-0.29234925$

0.04071225

0.14654770

0.04071225

0.14654770

0.04071225

0.14654770

0.04071225

0.14654770

$-0.36399299$

$-0.25815752$

$-0.36399299$

$-0.25815752$

$-0.36399299$

$-0.25815752$

$-0.36399299$

$-0.25815752$

$-0.12852763$

$-0.02269218$

$-0.12852763$

$-0.02269218$

$-0.12852763$

$-0.02269218$

$-0.12852763$

$-0.02269218$

0.02148618

0.12732163

0.02148618

0.12732163

0.02148618

0.12732163

0.02148618

0.12732163

$-0.37628788$

$-0.27045241$

$-0.37628788$

$-0.27045241$

$-0.37628788$

$-0.27045241$

$-0.37628788$

$-0.27045241$

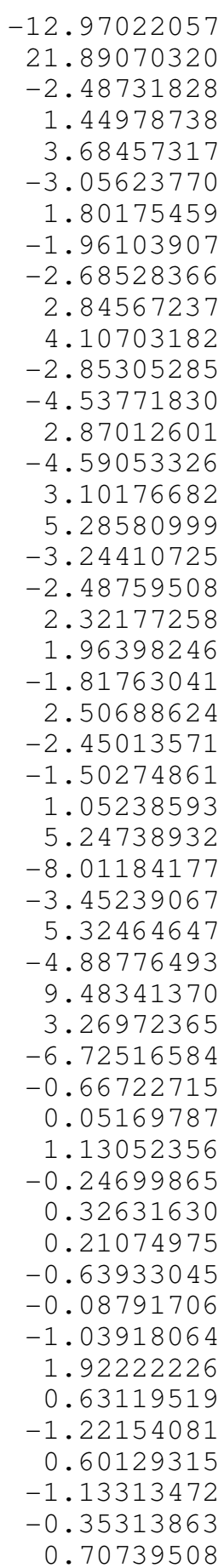

\# Energy 2->3

$\begin{array}{lll}0.1395 & 0.7292 & -0.1930\end{array}$

$-0.0026$ 0.0094

2. 03096962

2.03096962

$-0.0141$

1.000

Electr. mom., scale-fac

0.19347408

0.19347408

0.29930952

0.29930952

2.03096962

0.19347408

2.13680482

2.13680482

2.13680482

2. 13680482

$-1.37606192$

$-1.37606192$

$-1.37606192$

$-1.37606192$

$-1.27022636$

0.19347408

0.29930952

0.29930952

$-0.46025667$

$-0.46025667$

$-0.35442120$

$-0.35442120$

$-0.46025667$

$-0.02169468$

0.08414077

$-0.02169468$

0.08414077

$-0.02169468$

0.08414077

$-0.02169468$

0.08414077

$-0.36857194$

$-0.26273650$

$-0.36857194$

$-0.26273650$

$-0.36857194$
Magnetic mom.
6.14078236
$-4.28932762$
$-7.86522293$
5.86931610
$-3.05762863$
1.47593367
4.59703588
$-2.80341053$
$-1.93876755$
0.26751775
1.93232203
$-0.39653841$
0.90391731 


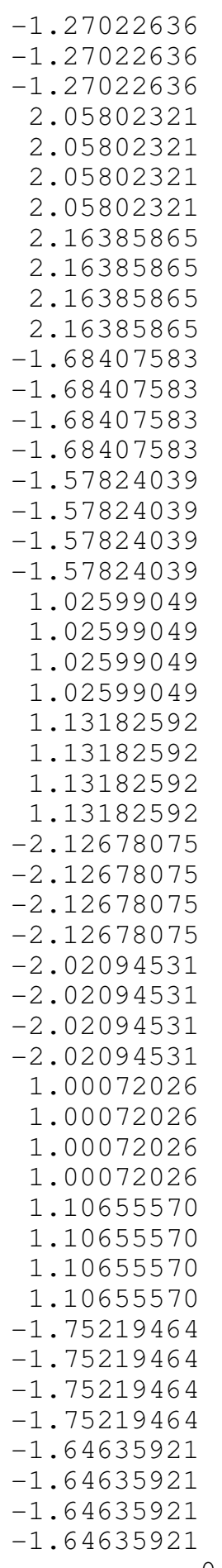

64
$-0.46025667$

$-0.35442120$

$-0.35442120$

1. 37286413

1. 37286413

1. 47869956

1. 47869956

1. 37286413

1. 37286413

1. 47869956

1.47869956

$-1.58903348$

$-1.58903348$

$-1.48319805$

$-1.48319805$

$-1.58903348$

$-1.58903348$

$-1.48319805$

$-1.48319805$

$-0.66542661$

$-0.66542661$

$-0.55959117$

$-0.55959117$

$-0.66542661$

$-0.66542661$

$-0.55959117$

$-0.55959117$

0.64235818

0.64235818

0.74819362

0.74819362

0.64235818

0.64235818

0.74819362

0.74819362

$-1.60289264$

$-1.60289264$

$-1.49705720$

$-1.49705720$

$-1.60289264$

$-1.60289264$

$-1.49705720$

$-1.49705720$

1. 54989612

1.54989612

1.65573156

1.65573156

1. 54989612

1.54989612

1.65573156

1.65573156

\# Energy 2->4

$0.3723 \quad 0.0598 \quad-0.1303$

$-0.0417$

2.03096962

2. 03096962

2. 03096962

2.03096962

2.13680482

2.13680482

2. 13680482

2.13680482

$-1.37606192$

$-1.37606192$

$-1.37606192$

$-1.37606192$

$-1.27022636$

$$
\begin{array}{rr}
0.0221 & 0.0762
\end{array}
$$

0.19347408

0.19347408

0.29930952

0.29930952

0.19347408

0.19347408

0.29930952

0.29930952

$-0.46025667$

$-0.46025667$

$-0.35442120$

$-0.35442120$

$-0.46025667$

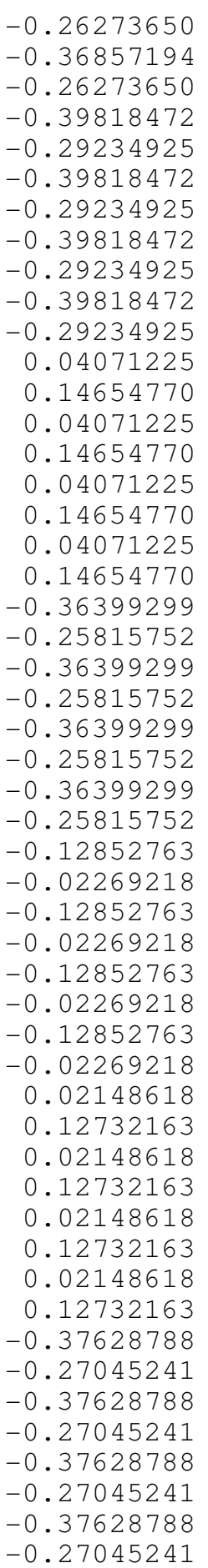

0.91268682

$-0.92545557$

$-0.82586884$

2.33810568

$-1.94240928$

$-3.41694784$

3.18315887

$-0.64480555$

$-0.00818252$

2.08459663

$-1.65825403$

$-3.96550775$

2. 53133655

2. 94726515

$-1.32734084$

3. 88297009

$-2.53214717$

$-2.68691301$

1.21706522

$-1.61453187$

0.93541491

2. 05557775

$-1.26409614$

1. 39908504

$-0.86710829$

$-1.73511577$

1.06652462

0.83532888

$-0.47447664$

$-1.24616528$

0.81765801

$-1.53143311$

1.28367162

1. 94179809

$-1.63337100$

$-1.20143867$

1. 04093111

1. 45335364

$-1.20258141$

1.32279062

$-1.20974600$

$-1.56797493$

1.38569605

0.04706921

$-0.17869981$

$-0.17358813$

0.32327569

$-0.31587678$

0.50199461

0.36967638

$-0.56292492$
1.000 \# Electr. mom., scale-fac
\# Magnetic mom.

$-0.02169468$

14.02121925

$-10.35600853$

$-14.02814960$

10.26471424

$-12.23049259$

9.20215511

12.32784939

$-9.24189854$

$-5.86981392$

$-5.50254440$

9.26420116

1.79581428

2.01938367

273650 
$-1.27022636$

$-1.27022636$

$-1.27022636$

2. 05802321

2. 05802321

2. 05802321

2. 05802321

2.16385865

2.16385865

2.16385865

2.16385865

$-1.68407583$

$-1.68407583$

$-1.68407583$

$-1.68407583$

$-1.57824039$

$-1.57824039$

$-1.57824039$

$-1.57824039$

1.02599049

1.02599049

1. 02599049

1.02599049

1. 13182592

1. 13182592

1.13182592

1.13182592

$-2.12678075$

$-2.12678075$

$-2.12678075$

$-2.12678075$

$-2.02094531$

$-2.02094531$

$-2.02094531$

$-2.02094531$

1. 00072026

1.00072026

1.00072026

1.00072026

1.10655570

1. 10655570

1. 10655570

1.10655570

$-1.75219464$

$-1.75219464$

$-1.75219464$

$-1.75219464$

$-1.64635921$

$-1.64635921$

$-1.64635921$

64

$-1.64635921$

0.86470

0.0635

2. 03096962

2.03096962

2.03096962

2.03096962

2.13680482

2.13680482

2.13680482

2. 13680482

$-1.37606192$

$-1.37606192$

$-1.37606192$

$-1.37606192$

$-1.27022636$
$-0.46025667$

$-0.35442120$

$-0.35442120$

1. 37286413

1.37286413

1. 47869956

1.47869956

1. 37286413

1. 37286413

1. 47869956

1.47869956

$-1.58903348$

$-1.58903348$

$-1.48319805$

$-1.48319805$

$-1.58903348$

$-1.58903348$

$-1.48319805$

$-1.48319805$

$-0.66542661$

$-0.66542661$

$-0.55959117$

$-0.55959117$

$-0.66542661$

$-0.66542661$

$-0.55959117$

$-0.55959117$

0.64235818

0.64235818

0.74819362

0.74819362

0.64235818

0.64235818

0.74819362

0.74819362

$-1.60289264$

$-1.60289264$

$-1.49705720$

$-1.49705720$

$-1.60289264$

$-1.60289264$

$-1.49705720$

$-1.49705720$

1. 54989612

1.54989612

1.65573156

1.65573156

1.54989612

1.54989612

1.65573156

1.65573156

\# Energy 2->5

$\begin{array}{ll}0.4531 & -0.1090 \\ 0.3884 & -0.0679\end{array}$

0.19347408

0.19347408

0.29930952

0.29930952

0.19347408

0.19347408

0.29930952

0.29930952

$-0.46025667$

$-0.46025667$

$-0.35442120$

$-0.35442120$

$-0.46025667$
$-0.26273650$

$-0.36857194$

$-0.26273650$

$-0.39818472$

$-0.29234925$

$-0.39818472$

$-0.29234925$

$-0.39818472$

$-0.29234925$

$-0.39818472$

$-0.29234925$

0.04071225

0.14654770

0.04071225

0.14654770

0.04071225

0.14654770

0.04071225

0.14654770

$-0.36399299$

$-0.25815752$

$-0.36399299$

$-0.25815752$

$-0.36399299$

$-0.25815752$

$-0.36399299$

$-0.25815752$

$-0.12852763$

$-0.02269218$

$-0.12852763$

$-0.02269218$

$-0.12852763$

$-0.02269218$

$-0.12852763$

$-0.02269218$

0.02148618

0.12732163

0.02148618

0.12732163

0.02148618

0.12732163

0.02148618

0.12732163

$-0.37628788$

$-0.27045241$

$-0.37628788$

$-0.27045241$

$-0.37628788$

$-0.27045241$

$-0.37628788$

$-0.27045241$
8.64347935

$-5.32386684$

$-4.92624521$

0.46931097

$-0.03120632$

$-0.68143761$

0.40873003

$-0.89638191$

0.62974375

1. 01593673

$-0.86323237$

$-5.23789120$

2.17875600

8.88209629

$-4.86861706$

2. 70552158

0.33862373

$-6.03104019$

2. 03222322

$-6.41916895$

2. 22569609

9.70348454

$-6.05637646$

7.86146402

$-4.80368662$

$-11.02705193$

8.42246723

14.29325676

$-9.76613331$

$-13.66894245$

9.37709332

$-18.66092300$

12.87433720

17.78741646

$-12.33029175$

$-2.76010275$

1.38609278

3. 80953693

$-1.80574834$

2. 35450888

$-1.24256229$

$-3.17122149$

1. 55367839

0.14277750

$-0.18158430$

$-0.33150887$

0.32471609

$-0.75290018$

0.72302216

0.74746472

$-0.71974403$
1.000 \# Electr. mom., scale-fac
\# Magnetic mom.

$-0.02169468$

0.08414077

$-0.02169468$

0.08414077

$-0.02169468$

0.08414077

$-0.02169468$

0.08414077

$-0.36857194$

$-0.26273650$

$-0.36857194$

$-0.26273650$

$-0.36857194$
6.09799147

$-3.14867759$

$-5.87623692$

2.85796189

$-3.49166155$

0.98225260

3. 23333502

$-0.62517905$

$-4.04952526$

$-8.20729351$

8.37389851

3.61033511

$-0.41965058$ 


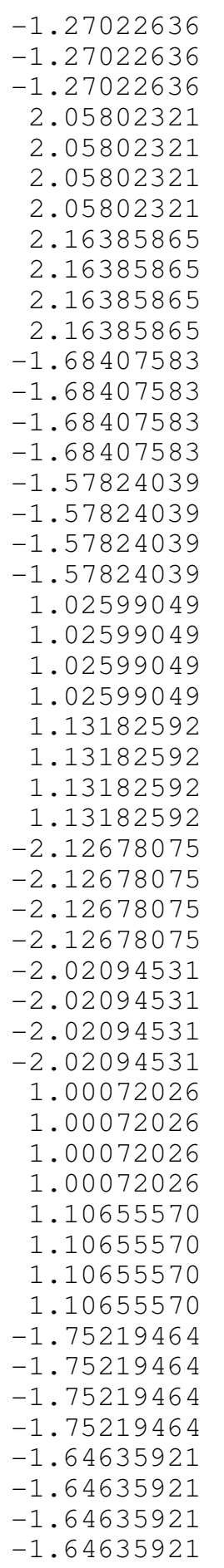

64
$-0.46025667$

$-0.35442120$

$-0.35442120$

1. 37286413

1. 37286413

1. 47869956

1.47869956

1. 37286413

1. 37286413

1. 47869956

1.47869956

$-1.58903348$

$-1.58903348$

$-1.48319805$

$-1.48319805$

$-1.58903348$

$-1.58903348$

$-1.48319805$

$-1.48319805$

$-0.66542661$

$-0.66542661$

$-0.55959117$

$-0.55959117$

$-0.66542661$

$-0.66542661$

$-0.55959117$

$-0.55959117$

0.64235818

0.64235818

0.74819362

0.74819362

0.64235818

0.64235818

0.74819362

0.74819362

$-1.60289264$

$-1.60289264$

$-1.49705720$

$-1.49705720$

$-1.60289264$

$-1.60289264$

$-1.49705720$

$-1.49705720$

1. 54989612

1.54989612

1.65573156

1.65573156

1.54989612

1.54989612

1.65573156

1.65573156

\# Energy 2->6
0 $\begin{array}{ll}-0.4719 & 0.1868\end{array}$ $-0.0385-0.0026$

0.19347408

0.19347408

0.29930952

0.29930952

0.19347408

0.19347408

0.29930952

0.29930952

$-0.46025667$

$-0.46025667$

$-0.35442120$

$-0.35442120$

$-0.46025667$
$-0.26273650$

$-0.36857194$

$-0.26273650$

$-0.39818472$

$-0.29234925$

$-0.39818472$

$-0.29234925$

$-0.39818472$

$-0.29234925$

$-0.39818472$

$-0.29234925$

0.04071225

0.14654770

0.04071225

0.14654770

0.04071225

0.14654770

0.04071225

0.14654770

$-0.36399299$

$-0.25815752$

$-0.36399299$

$-0.25815752$

$-0.36399299$

$-0.25815752$

$-0.36399299$

$-0.25815752$

$-0.12852763$

$-0.02269218$

$-0.12852763$

$-0.02269218$

$-0.12852763$

$-0.02269218$

$-0.12852763$

$-0.02269218$

0.02148618

0.12732163

0.02148618

0.12732163

0.02148618

0.12732163

0.02148618

0.12732163

$-0.37628788$

$-0.27045241$

$-0.37628788$

$-0.27045241$

$-0.37628788$

$-0.27045241$

$-0.37628788$

$-0.27045241$
12.05247784

$-3.90761542$

$-7.32738876$

$-1.21951818$

2.03493071

1. 26367843

$-1.89677358$

1. 95983970

$-2.78704405$

$-1.86473858$

2.50823545

$-7.31242800$

3.12420368

9.87674427

$-4.56837559$

5.31825304

$-1.16691327$

$-7.47311831$

2. 27810645

1. 04795074

$-5.39533424$

1. 26651525

2. 77155209

$-0.89276624$

4. 46334076

$-1.06818545$

$-2.37247086$

12.99213505

$-7.46017075$

$-12.29131126$

7.05282164

$-18.80462837$

11.80724430

17.78126335

$-11.17548656$

$-2.88618660$

1.83059287

3. 99203539

$-2.39483762$

2. 64960194

$-1.82158899$

$-3.58044434$

2. 31637788

$-0.29726452$

0.19420186

$-0.09851809$

0.16237149

$-0.45883936$

0.47809207

0.62809837

$-0.66626960$
1.000 \# Electr. mom., scale-fac \# Magnetic mom.

$-0.02169468$

0.08414077

$-0.02169468$

0.08414077

$-0.02169468$

0.08414077

$-0.02169468$

0.08414077

$-0.36857194$

$-0.26273650$

$-0.36857194$

$-0.26273650$

$-0.36857194$
7.57273102

$-5.58860588$

$-7.36328650$

$-4.72229910$

3. 17496610

4.47393322

$-2.82527232$

$-2.94425130$

2. 32045293

2. 84427166

$-2.25953650$

2.54790378
5.25098705 


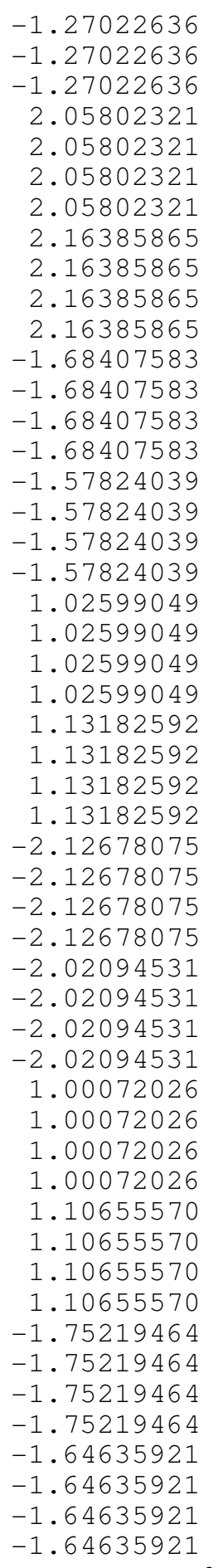

64
$-0.46025667$

$-0.35442120$

$-0.35442120$

1. 37286413

1. 37286413

1. 47869956

1. 47869956

1. 37286413

1. 37286413

1. 47869956

1.47869956

$-1.58903348$

$-1.58903348$

$-1.48319805$

$-1.48319805$

$-1.58903348$

$-1.58903348$

$-1.48319805$

$-1.48319805$

$-0.66542661$

$-0.66542661$

$-0.55959117$

$-0.55959117$

$-0.66542661$

$-0.66542661$

$-0.55959117$

$-0.55959117$

0.64235818

0.64235818

0.74819362

0.74819362

0.64235818

0.64235818

0.74819362

0.74819362

$-1.60289264$

$-1.60289264$

$-1.49705720$

$-1.49705720$

$-1.60289264$

$-1.60289264$

$-1.49705720$

$-1.49705720$

1. 54989612

1.54989612

1.65573156

1.65573156

1.54989612

1.54989612

1.65573156

1.65573156
$-0.26273650$

$-0.36857194$

$-0.26273650$

$-0.39818472$

$-0.29234925$

$-0.39818472$

$-0.29234925$

$-0.39818472$

$-0.29234925$

$-0.39818472$

$-0.29234925$

0.04071225

0.14654770

0.04071225

0.14654770

0.04071225

0.14654770

0.04071225

0.14654770

$-0.36399299$

$-0.25815752$

$-0.36399299$

$-0.25815752$

$-0.36399299$

$-0.25815752$

$-0.36399299$

$-0.25815752$

$-0.12852763$

$-0.02269218$

$-0.12852763$

$-0.02269218$

$-0.12852763$

$-0.02269218$

$-0.12852763$

$-0.02269218$

0.02148618

0.12732163

0.02148618

0.12732163

0.02148618

0.12732163

0.02148618

0.12732163

$-0.37628788$

$-0.27045241$

$-0.37628788$

$-0.27045241$

$-0.37628788$

$-0.27045241$

$-0.37628788$

$-0.27045241$

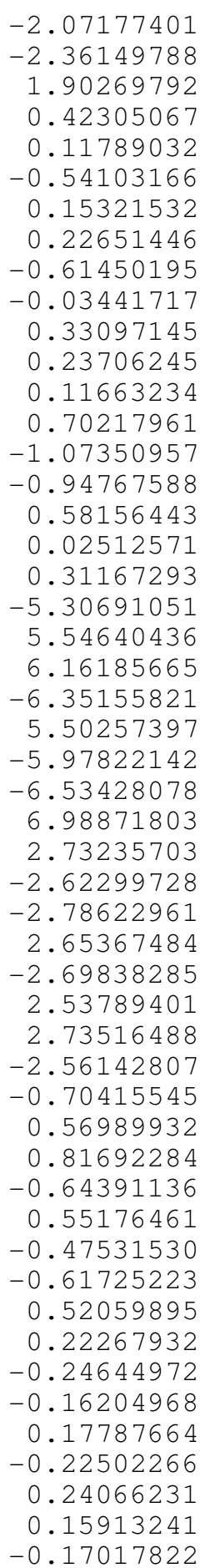

\footnotetext{
0.6346

$-0.0615$

2.03096962

2.03096962

2.03096962

2.03096962

2.13680482

2.13680482

2.13680482

2.13680482

$-1.37606192$

$-1.37606192$

$-1.37606192$

$-1.37606192$

$-1.27022636$
}
1.14751685 0.74654216
$-1.16388392$
$-0.60368389$
0.51119596
$-2.28345180$
$-0.58389157$
2.25716758
0.03430895
$-0.61377728$
0.14970200
0.54030704
0.11498668 


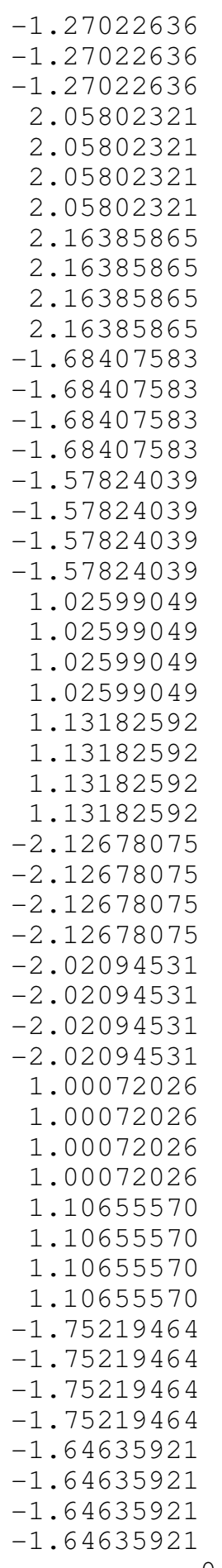

64
$-0.46025667$

$-0.35442120$

$-0.35442120$

1. 37286413

1.37286413

1. 47869956

1.47869956

1. 37286413

1. 37286413

1. 47869956

1.47869956

$-1.58903348$

$-1.58903348$

$-1.48319805$

$-1.48319805$

$-1.58903348$

$-1.58903348$

$-1.48319805$

$-1.48319805$

$-0.66542661$

$-0.66542661$

$-0.55959117$

$-0.55959117$

$-0.66542661$

$-0.66542661$

$-0.55959117$

$-0.55959117$

0.64235818

0.64235818

0.74819362

0.74819362

0.64235818

0.64235818

0.74819362

0.74819362

$-1.60289264$

$-1.60289264$

$-1.49705720$

$-1.49705720$

$-1.60289264$

$-1.60289264$

$-1.49705720$

$-1.49705720$

1. 54989612

1.54989612

1.65573156

1.65573156

1.54989612

1.54989612

1.65573156

1.65573156

0 .

\# Energy 2->8

$\begin{array}{lll}0.0842 & 0.0340 & -0.0442\end{array}$

$-0.0198$

2. 03096962

2.03096962

2.03096962

2.03096962

2.13680482

2.13680482

2.13680482

2.13680482

$-1.37606192$

$-1.37606192$

$-1.37606192$

$-1.37606192$

$-1.27022636$

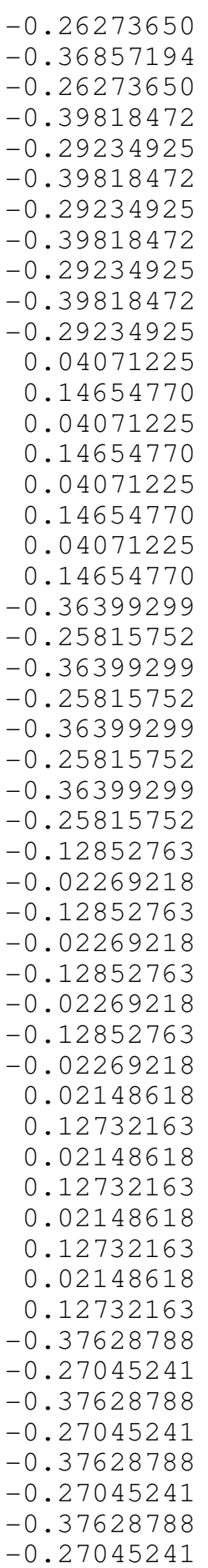

0.40562928

$-0.33993739$

$-0.26952106$

$-0.96368372$

1. 68981886

0.87244552

$-1.40555692$

1.97565794

$-2.74411058$

$-1.67685151$

2. 24083877

0.00295511

$-0.04467073$

$-0.32399336$

0.32824904

0.16683029

$-0.13323560$

0.19200210

$-0.16091470$

2.06181955

$-3.43173337$

$-2.03454804$

3. 59892869

$-2.83362103$

4. 45296621

2.85402131

$-4.70660114$

$-0.12050755$

0.50076151

0.10837753

$-0.47584674$

$-0.14287375$

$-0.46811837$

0.16653274

0.43719435

$-0.50375867$

0.53722507

0.80638629

$-0.79453546$

0.59860098

$-0.60696793$

$-0.93017781$

0.88080001

$-0.07195288$

$-0.04038931$

0.04533747

0.03491435

0.12835486

$-0.07733582$

$-0.08179308$

0.04355009
1.000 \# Electr. mom., scale-fac \# Magnetic mom.

0.19347408

0.19347408

0.29930952

0.29930952

0.19347408

0.19347408

0.29930952

0.29930952

$-0.46025667$

$-0.46025667$

$-0.35442120$

$-0.35442120$

$-0.46025667$
$-7.16966009$

10.21602631

6.17059708

$-8.51103210$

7.32241297

$-10.06496906$

$-6.20595598$

8.34254646

$-8.62027454$

8.80639172

9.61618805

$-9.71489906$

5. 01287127 
$-1.27022636$

$-1.27022636$

$-1.27022636$

2. 05802321

2. 05802321

2. 05802321

2. 05802321

2.16385865

2.16385865

2.16385865

2.16385865

$-1.68407583$

$-1.68407583$

$-1.68407583$

$-1.68407583$

$-1.57824039$

$-1.57824039$

$-1.57824039$

$-1.57824039$

1.02599049

1. 02599049

1.02599049

1.02599049

1.13182592

1. 13182592

1.13182592

1.13182592

$-2.12678075$

$-2.12678075$

$-2.12678075$

$-2.12678075$

$-2.02094531$

$-2.02094531$

$-2.02094531$

$-2.02094531$

1. 00072026

1. 00072026

1.00072026

1.00072026

1.10655570

1. 10655570

1.10655570

1.10655570

$-1.75219464$

$-1.75219464$

$-1.75219464$

$-1.75219464$

$-1.64635921$

$-1.64635921$

$-1.64635921$

64

$-1.64635921$

0.4962

$-0.0554$

2. 03096962

2.03096962

2.03096962

2.03096962

2.13680482

2.13680482

2.13680482

2. 13680482

$-1.37606192$

$-1.37606192$

$-1.37606192$

$-1.37606192$

$-1.27022636$
$-0.46025667$

$-0.35442120$

$-0.35442120$

1. 37286413

1. 37286413

1. 47869956

1.47869956

1. 37286413

1. 37286413

1. 47869956

1.47869956

$-1.58903348$

$-1.58903348$

$-1.48319805$

$-1.48319805$

$-1.58903348$

$-1.58903348$

$-1.48319805$

$-1.48319805$

$-0.66542661$

$-0.66542661$

$-0.55959117$

$-0.55959117$

$-0.66542661$

$-0.66542661$

$-0.55959117$

$-0.55959117$

0.64235818

0.64235818

0.74819362

0.74819362

0.64235818

0.64235818

0.74819362

0.74819362

$-1.60289264$

$-1.60289264$

$-1.49705720$

$-1.49705720$

$-1.60289264$

$-1.60289264$

$-1.49705720$

$-1.49705720$

1. 54989612

1.54989612

1.65573156

1.65573156

1.54989612

1.54989612

1.65573156

1.65573156

0 . \# Energy 2->9

$\begin{array}{rr}0.2219 & -0.1009 \\ -0.1398 & -0.0468\end{array}$

0.19347408

0.19347408

0.29930952

0.29930952

0.19347408

0.19347408

0.29930952

0.29930952

$-0.46025667$

$-0.46025667$

$-0.35442120$

$-0.35442120$

$-0.46025667$
$-0.26273650$

$-0.36857194$

$-0.26273650$

$-0.39818472$

$-0.29234925$

$-0.39818472$

$-0.29234925$

$-0.39818472$

$-0.29234925$

$-0.39818472$

$-0.29234925$

0.04071225

0.14654770

0.04071225

0.14654770

0.04071225

0.14654770

0.04071225

0.14654770

$-0.36399299$

$-0.25815752$

$-0.36399299$

$-0.25815752$

$-0.36399299$

$-0.25815752$

$-0.36399299$

$-0.25815752$

$-0.12852763$

$-0.02269218$

$-0.12852763$

$-0.02269218$

$-0.12852763$

$-0.02269218$

$-0.12852763$

$-0.02269218$

0.02148618

0.12732163

0.02148618

0.12732163

0.02148618

0.12732163

0.02148618

0.12732163

$-0.37628788$

$-0.27045241$

$-0.37628788$

$-0.27045241$

$-0.37628788$

$-0.27045241$

$-0.37628788$

$-0.27045241$
$-4.59549189$

$-5.80252838$

5.30294657

$-0.43302637$

0.50803500

0.24237381

$-0.29071689$

0.36781561

$-0.43953061$

$-0.17111149$

0.21872292

$-0.54409975$

0.01434543

1. 10757649

$-0.41847044$

$-0.07902976$

0.68171251

$-0.43691123$

$-0.36611557$

5.01791143

$-9.28975582$

$-5.52395964$

10.06158447

$-6.36500645$

11.24628544

7.19273233

$-12.42776966$

11.42525482

$-10.86161613$

$-9.58849144$

8.62440491

$-14.14503288$

13.30577660

12.21283531

$-10.95790863$

$-0.72651142$

0.20928441

1. 17186916

$-0.37245473$

0.81320167

$-0.28266409$

$-1.29522300$

0.48635253

$-0.96836996$

1.19004202

0.62318766

$-0.77906227$

0.56892097

$-0.77044785$

$-0.33997047$

0.47786114
1.000 \# Electr. mom., scale-fac \# Magnetic mom.

$-0.02169468$

0.08414077

$-0.02169468$

0.08414077

$-0.02169468$

0.08414077

$-0.02169468$

0.08414077

$-0.36857194$

$-0.26273650$

$-0.36857194$

$-0.26273650$

$-0.36857194$
$-9.01244164$

15.51448059

8.07027340

$-13.39560413$

11.41748333

$-16.93278694$

$-10.20724583$

14.71760941

$-16.10581017$

15.94341755

18.48295784

$-18.23524475$

9. 23783112 


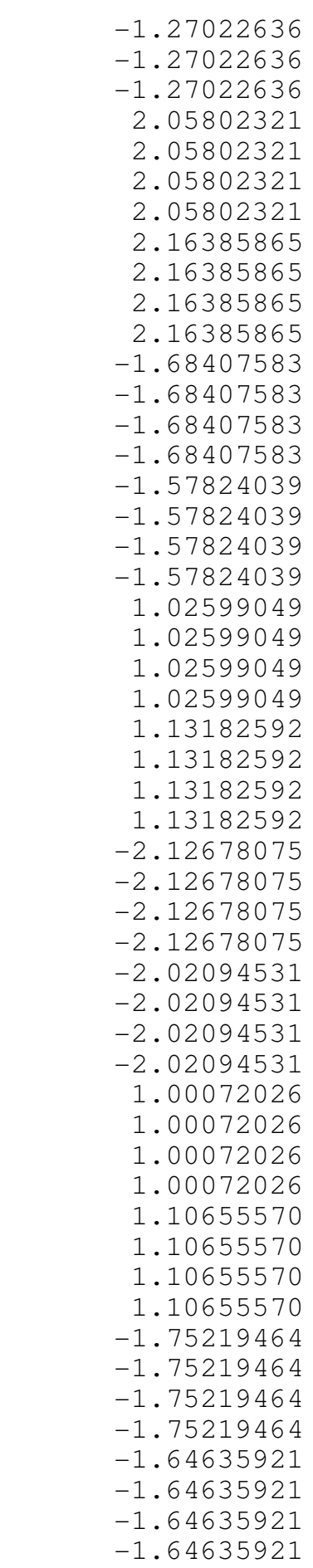

\&TRANSITION 3->...

64
$-0.46025667$

$-0.35442120$

$-0.35442120$

1.37286413

1.37286413

1. 47869956

1.47869956

1.37286413

1. 37286413

1. 47869956

1.47869956

$-1.58903348$

$-1.58903348$

$-1.48319805$

$-1.48319805$

$-1.58903348$

$-1.58903348$

$-1.48319805$

$-1.48319805$

$-0.66542661$

$-0.66542661$

$-0.55959117$

$-0.55959117$

$-0.66542661$

$-0.66542661$

$-0.55959117$

$-0.55959117$

0.64235818

0.64235818

0.74819362

0.74819362

0.64235818

0.64235818

0.74819362

0.74819362

$-1.60289264$

$-1.60289264$

$-1.49705720$

$-1.49705720$

$-1.60289264$

$-1.60289264$

$-1.49705720$

$-1.49705720$

1. 54989612

1.54989612

1.65573156

1.65573156

1.54989612

1.54989612

1.65573156

1.65573156
$-0.26273650$

$-0.36857194$

$-0.26273650$

$-0.39818472$

$-0.29234925$

$-0.39818472$

$-0.29234925$

$-0.39818472$

$-0.29234925$

$-0.39818472$

$-0.29234925$

0.04071225

0.14654770

0.04071225

0.14654770

0.04071225

0.14654770

0.04071225

0.14654770

$-0.36399299$

$-0.25815752$

$-0.36399299$

$-0.25815752$

$-0.36399299$

$-0.25815752$

$-0.36399299$

$-0.25815752$

$-0.12852763$

$-0.02269218$

$-0.12852763$

$-0.02269218$

$-0.12852763$

$-0.02269218$

$-0.12852763$

$-0.02269218$

0.02148618

0.12732163

0.02148618

0.12732163

0.02148618

0.12732163

0.02148618

0.12732163

$-0.37628788$

$-0.27045241$

$-0.37628788$

$-0.27045241$

$-0.37628788$

$-0.27045241$

$-0.37628788$

$-0.27045241$
$-8.01021862$

$-11.29461861$

9.99478912

$-0.66232437$

1. 11271548

0.19135778

$-0.51115710$

0.86211509

$-1.18252218$

$-0.36954892$

0.61321402

$-1.35988569$

0.18265578

2. 47563171

$-0.93991750$

0.15262920

1.15243793

$-1.14235210$

$-0.58866674$

6.93460798

$-13.36574459$

$-7.78983974$

14.85986423

$-9.30213547$

16.60171127

10.64685154

$-18.78281975$

20.77376938

$-19.44221878$

$-17.88205910$

15.88464069

$-25.92301178$

24.09161377

22.80765152

$-20.30226517$

$-1.35261846$

0.63948548

2. 14683247

$-1.02508676$

1. 43778992

$-0.73107213$

$-2.26875710$

1. 16865122

$-1.74918866$

2.14544129

1.08641815

$-1.36000812$

0.99188113

$-1.34493589$

$-0.55730772$

0.79060823

\section{0. \# Energy 3->4}

$\begin{array}{ll}-0.0047 & 0.0648\end{array}$ $0.2786-0.0799$

0.19347408

0.19347408

0.29930952

0.29930952

0.19347408

0.19347408

0.29930952

0.29930952

$-0.46025667$

$-0.46025667$

$-0.35442120$

$-0.35442120$
1.000 \# Electr. mom., scale-fac \# Magnetic mom.

$-0.02169468$

$-1.21133947$

0.08414077

$-0.02169468$

0.08414077

$-0.02169468$

0.08414077

$-0.02169468$

0.08414077

$-0.36857194$

$-0.26273650$

$-0.36857194$

$-0.26273650$
2. 48020411

1.31298172

$-2.96901965$

7.73168993

$-8.93335056$

$-8.36595345$

9.96925354

$-0.60728520$

$-1.70378792$

1. 46482110

1. 01220524 
$-1.27022636$

$-1.27022636$

$-1.27022636$

$-1.27022636$

2. 05802321

2. 05802321

2. 05802321

2. 05802321

2.16385865

2.16385865

2.16385865

2.16385865

$-1.68407583$

$-1.68407583$

$-1.68407583$

$-1.68407583$

$-1.57824039$

$-1.57824039$

$-1.57824039$

$-1.57824039$

1. 02599049

1.02599049

1.02599049

1. 13182592

1.13182592

1.13182592

1. 13182592

$-2.12678075$

$-2.12678075$

$-2.12678075$

$-2.12678075$

$-2.02094531$

$-2.02094531$

$-2.02094531$

$-2.02094531$

1.00072026

1.00072026

1.00072026

1.00072026

1. 10655570

1. 10655570

1.10655570

1. 10655570

$-1.75219464$

$-1.75219464$

$-1.75219464$

$-1.75219464$

$-1.64635921$

$-1.64635921$

$-1.64635921$

$-1.64635921$

64
1. 02599049

$-0.46025667$

$-0.46025667$

$-0.35442120$

$-0.35442120$

1. 37286413

1. 37286413

1.47869956

1.47869956

1. 37286413

1. 37286413

1.47869956

$-1.58903348$

$-1.58903348$

$-1.48319805$

$-1.48319805$

$-1.58903348$

$-1.58903348$

$-1.48319805$

$-1.48319805$

$-0.66542661$

$-0.66542661$

$-0.55959117$

$-0.55959117$

$-0.66542661$

$-0.66542661$

$-0.55959117$

$-0.55959117$

0.64235818

0.64235818

0.74819362

0.74819362

0.64235818

0.64235818

0.74819362

0.74819362

$-1.60289264$

$-1.60289264$

$-1.49705720$

$-1.49705720$

$-1.60289264$

$-1.60289264$

$-1.49705720$

1. 54989612

1.54989612

1.65573156

1.65573156

1. 54989612

1.54989612

1.65573156

1.65573156

0 .

\# Energy

$\begin{array}{lll}-0.1515 & -0.0560 & 0.0682\end{array}$

$-0.0165$

2.03096962

2.03096962

2.03096962

2.03096962

2.13680482

2.13680482

2.13680482

2.13680482

$-1.37606192$

$-1.37606192$

$-1.37606192$

$-1.37606192$

$0.2119-0.0723$

0.19347408

0.19347408

0.29930952

0.29930952

0.19347408

0.19347408

0.29930952

0.29930952

$-0.46025667$

$-0.46025667$

$-0.35442120$

$-0.35442120$
1. 47869956

$-1.49705720$

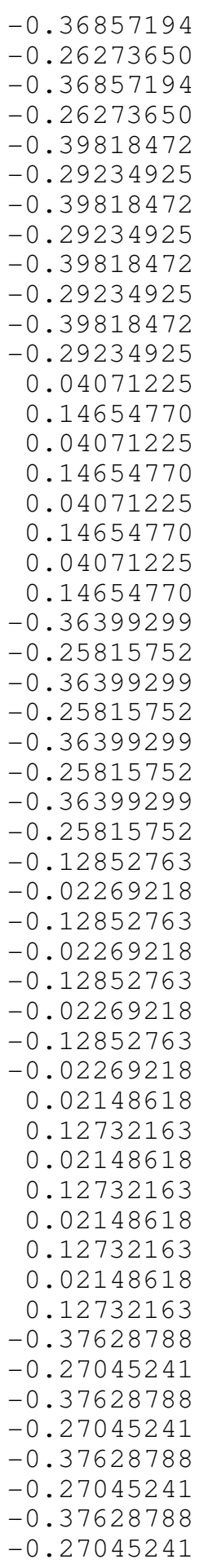

0.04845473

1.80442512

$-0.89991391$

$-1.08729029$

$-2.06990647$

3.65093279

2. 58712006

$-3.90193558$

5.79661131

$-7.44746637$

$-5.67884827$

7.06640768

$-0.54480761$

0.21585719

1. 00303054

$-0.58127946$

0.06164848

0.19989327

$-0.42229024$

0.08665098

5.77453232

$-5.87990141$

$-5.71902132$

6.05942822

$-7.70748711$

8.15444183

7.64747429

$-8.30333900$

2. 64809871

$-0.93634754$

$-2.48392510$

0.86622053

$-4.07351255$

1. 76884770

3.83036017

$-1.64735043$

$-1.96212971$

2. 20216250

2. 20725989

$-2.50884581$

2. 04698896

$-2.26941872$

$-2.34746742$

2.60429049

0.03611222

$-0.18244062$

$-0.11216945$

0.22848657

$-0.07830094$

0.12264934

0.14647266

$-0.19988164$

$$
\begin{aligned}
& 1.000 \text { \# Electr. mom., scale-fac } \\
& \text { \# Magnetic mom. }
\end{aligned}
$$

$-0.02169468$

0.08414077

$-0.02169468$

0.08414077

$-0.02169468$

0.08414077

$-0.02169468$

0.08414077

$-0.36857194$

$-0.26273650$

$-0.36857194$

$-0.26273650$
4.81131983

$-5.05499554$

$-4.84963036$

5.52858639

$-11.46435070$

11.82398510

12.12155342

$-12.93246269$

$-0.97515047$

$-0.03587152$

1. 22835493

$-0.49103254$ 
$-1.27022636$

$-1.27022636$

$-1.27022636$

$-1.27022636$

2. 05802321

2. 05802321

2. 05802321

2. 05802321

2.16385865

2.16385865

2.16385865

2.16385865

$-1.68407583$

$-1.68407583$

$-1.68407583$

$-1.68407583$

$-1.57824039$

$-1.57824039$

$-1.57824039$

$-1.57824039$

1. 02599049

1. 02599049

1.02599049

1.02599049

1. 13182592

1.13182592

1.13182592

1. 13182592

$-2.12678075$

$-2.12678075$

$-2.12678075$

$-2.12678075$

$-2.02094531$

$-2.02094531$

$-2.02094531$

$-2.02094531$

1.00072026

1.00072026

1.00072026

1.00072026

1. 10655570

1. 10655570

1.10655570

1. 10655570

$-1.75219464$

$-1.75219464$

$-1.75219464$

$-1.75219464$

$-1.64635921$

$-1.64635921$

$-1.64635921$

64

$-1.64635921$

\subsection{1}

$-0.0096$

2.03096962

2.03096962

2.03096962

2.03096962

2.13680482

2.13680482

2. 13680482

2.13680482

$-1.37606192$

$-1.37606192$

$-1.37606192$

$-1.37606192$
$-0.46025667$

$-0.46025667$

$-0.35442120$

$-0.35442120$

1. 37286413

1. 37286413

1.47869956

1.47869956

1. 37286413

1. 37286413

1.47869956

1.47869956

$-1.58903348$

$-1.58903348$

$-1.48319805$

$-1.48319805$

$-1.58903348$

$-1.58903348$

$-1.48319805$

$-1.48319805$

$-0.66542661$

$-0.66542661$

$-0.55959117$

$-0.55959117$

$-0.66542661$

$-0.66542661$

$-0.55959117$

$-0.55959117$

0.64235818

0.64235818

0.74819362

0.74819362

0.64235818

0.64235818

0.74819362

0.74819362

$-1.60289264$

$-1.60289264$

$-1.49705720$

$-1.49705720$

$-1.60289264$

$-1.60289264$

$-1.49705720$

$-1.49705720$

1. 54989612

1.54989612

1.65573156

1.65573156

1.54989612

1.54989612

1.65573156

1.65573156

0 . \# Energy 3->6

$0.0796 \quad 0.0333$

$$
0.2505 \quad-0.0747
$$

0.19347408

0.19347408

0.29930952

0.29930952

0.19347408

0.19347408

0.29930952

0.29930952

$-0.46025667$

$-0.46025667$

$-0.35442120$

$-0.35442120$
$-0.36857194$

$-0.26273650$

$-0.36857194$

$-0.26273650$

$-0.39818472$

$-0.29234925$

$-0.39818472$

$-0.29234925$

$-0.39818472$

$-0.29234925$

$-0.39818472$

$-0.29234925$

0.04071225

0.14654770

0.04071225

0.14654770

0.04071225

0.14654770

0.04071225

0.14654770

$-0.36399299$

$-0.25815752$

$-0.36399299$

$-0.25815752$

$-0.36399299$

$-0.25815752$

$-0.36399299$

$-0.25815752$

$-0.12852763$

$-0.02269218$

$-0.12852763$

$-0.02269218$

$-0.12852763$

$-0.02269218$

$-0.12852763$

$-0.02269218$

0.02148618

0.12732163

0.02148618

0.12732163

0.02148618

0.12732163

0.02148618

0.12732163

$-0.37628788$

$-0.27045241$

$-0.37628788$

$-0.27045241$

$-0.37628788$

$-0.27045241$

$-0.37628788$

$-0.27045241$

1.000
1.000 \# Electr. mom., scale-fac \# Magnetic mom.

$-0.02169468$

5.48829651

$-0.02169468$

0.08414077

$-0.02169468$

0.08414077

$-0.02169468$

0.08414077

$-0.36857194$

$-0.26273650$

$-0.36857194$

$-0.26273650$

0.34395483

0.98094457

$-0.58866012$

$-0.46511790$

2.19383025

$-3.77801371$

$-2.78530264$

4. 12611532

$-6.37747145$

8.07208633

6.25559807

$-7.70047665$

$-1.25992215$

0.53079450

1.69741273

$-0.75875109$

1.10294580

$-0.30620852$

$-1.54032266$

0.52458858

$-7.47353220$

6.15187883

8.29699135

$-7.35261583$

9. 89242363

$-9.24189854$

$-10.63491726$

10.28656006

1. 17113698

$-1.55285680$

$-1.16516602$

1. 52275991

$-1.12224889$

1. 75979805

1.11591065

$-1.72685456$

1.33654201

$-1.99749565$

$-1.26504421$

2.18336272

$-1.53214514$

2.09694290

1.58608651

$-2.34193611$

$-0.05574640$

0.19178088

0.04871027

$-0.16438049$

$-0.10595134$

0.06194132

0.05763317

$-0.00599994$

\section{S-200}

$-5.73119783$

$-5.63378859$

6.23371649

$-11.48412800$

11.80374432

12.16114521

$-12.86739540$

$-0.59954268$

1.05893254

0.19162399

$-0.86777174$ 
$-1.27022636$

$-1.27022636$

$-1.27022636$

$-1.27022636$

2. 05802321

2. 05802321

2. 05802321

2. 05802321

2.16385865

2.16385865

2.16385865

2.16385865

$-1.68407583$

$-1.68407583$

$-1.68407583$

$-1.68407583$

$-1.57824039$

$-1.57824039$

$-1.57824039$

$-1.57824039$

1. 02599049

1. 02599049

1.02599049

1.02599049

1. 13182592

1.13182592

1.13182592

1. 13182592

$-2.12678075$

$-2.12678075$

$-2.12678075$

$-2.12678075$

$-2.02094531$

$-2.02094531$

$-2.02094531$

$-2.02094531$

1.00072026

1.00072026

1.00072026

1.00072026

1. 10655570

1. 10655570

1.10655570

1. 10655570

$-1.75219464$

$-1.75219464$

$-1.75219464$

$-1.75219464$

$-1.64635921$

$-1.64635921$

$-1.64635921$

64

$-1.64635921$

0.9804

$-0.0428$

2.03096962

2.03096962

2.03096962

2.03096962

2.13680482

2.13680482

2.13680482

2.13680482

$-1.37606192$

$-1.37606192$

$-1.37606192$

$-1.37606192$
$-0.46025667$

$-0.46025667$

$-0.35442120$

$-0.35442120$

1. 37286413

1. 37286413

1.47869956

1.47869956

1. 37286413

1. 37286413

1.47869956

1.47869956

$-1.58903348$

$-1.58903348$

$-1.48319805$

$-1.48319805$

$-1.58903348$

$-1.58903348$

$-1.48319805$

$-1.48319805$

$-0.66542661$

$-0.66542661$

$-0.55959117$

$-0.55959117$

$-0.66542661$

$-0.66542661$

$-0.55959117$

$-0.55959117$

0.64235818

0.64235818

0.74819362

0.74819362

0.64235818

0.64235818

0.74819362

0.74819362

$-1.60289264$

$-1.60289264$

$-1.49705720$

$-1.49705720$

$-1.60289264$

$-1.60289264$

$-1.49705720$

$-1.49705720$

1. 54989612

1.54989612

1.65573156

1.65573156

1.54989612

1.54989612

1.65573156

1.65573156

0 .

$0.1012 \quad 0.1192$ $0.0723-0.0478$

0.19347408

0.19347408

0.29930952

0.29930952

0.19347408

0.19347408

0.29930952

0.29930952

$-0.46025667$

$-0.46025667$

$-0.35442120$

$-0.35442120$
$-0.36857194$

$-0.26273650$

$-0.36857194$

$-0.26273650$

$-0.39818472$

$-0.29234925$

$-0.39818472$

$-0.29234925$

$-0.39818472$

$-0.29234925$

$-0.39818472$

$-0.29234925$

0.04071225

0.14654770

0.04071225

0.14654770

0.04071225

0.14654770

0.04071225

0.14654770

$-0.36399299$

$-0.25815752$

$-0.36399299$

$-0.25815752$

$-0.36399299$

$-0.25815752$

$-0.36399299$

$-0.25815752$

$-0.12852763$

$-0.02269218$

$-0.12852763$

$-0.02269218$

$-0.12852763$

$-0.02269218$

$-0.12852763$

$-0.02269218$

0.02148618

0.12732163

0.02148618

0.12732163

0.02148618

0.12732163

0.02148618

0.12732163

$-0.37628788$

$-0.27045241$

$-0.37628788$

$-0.27045241$

$-0.37628788$

$-0.27045241$

$-0.37628788$

$-0.27045241$

$$
\begin{aligned}
& 1.000 \text { \# Electr. mom., scale-fac } \\
& \text { \# Magnetic mom. }
\end{aligned}
$$

$\begin{array}{rr}-0.02169468 & -2.54821277 \\ 0.08414077 & -0.33400226 \\ -0.02169468 & 0.70906061 \\ 0.08414077 & 1.64374459 \\ -0.02169468 & 2.18536639 \\ 0.08414077 & 0.14669681 \\ -0.02169468 & -0.81364185 \\ 0.08414077 & -1.03289783 \\ -0.36857194 & -1.81610584 \\ -0.26273650 & 0.85383886 \\ -0.36857194 & 1.37641835 \\ -0.26273650 & -0.17662315\end{array}$

$-0.73931056$

$-0.17614456$

0.50610137

2.24450707

4.02313328

$-6.00458384$

7.55399036

5.91828632

$-7.24185324$

$-0.24575813$

0.15010332

0.45360434

$-0.31148201$

0.24053286

0.08336817

$-7.98467588$

7. 31804180

8.72073460

$-8.41045284$

.41070747

11.14935398

11.41452312

$-0.24429095$

$-0.79591101$

0.14729238

0.83193904

1.03395581

0.41486281

8960801

1. 41835105

$-1.94752812$

$-1.46214676$

2.17693830

$-1.58605850$

2.03954983

1.73444796

$-2.31883144$

0.04833409

0.07152041

0.01130989

$-0.10997771$

$-0.11017945$

0.08367905

0.02720397

0.01062866 


\begin{tabular}{|c|c|c|c|}
\hline & & & \\
\hline-1.27022636 & -0.46025667 & -0.36857194 & 1.96412694 \\
\hline-1.27022636 & -0.46025667 & -0.26273650 & -1.45735753 \\
\hline-1.27022636 & -0.35442120 & -0.36857194 & -1.51181304 \\
\hline-1.27022636 & -0.35442120 & -0.26273650 & 0.76673794 \\
\hline 2.05802321 & 1.37286413 & -0.39818472 & 0.99748993 \\
\hline 2.05802321 & 1.37286413 & -0.29234925 & -0.90893972 \\
\hline 2.05802321 & 1.47869956 & -0.39818472 & -0.64688492 \\
\hline 2.05802321 & 1.47869956 & -0.29234925 & 0.56217247 \\
\hline 2.16385865 & 1.37286413 & -0.39818472 & -0.01321720 \\
\hline 2.16385865 & 1.37286413 & -0.29234925 & -0.15879817 \\
\hline 2.16385865 & 1.47869956 & -0.39818472 & -0.15375435 \\
\hline 2.16385865 & 1.47869956 & -0.29234925 & 0.30807501 \\
\hline-1.68407583 & -1.58903348 & 0.04071225 & -0.92690080 \\
\hline-1.68407583 & -1.58903348 & 0.14654770 & 0.69158316 \\
\hline-1.68407583 & -1.48319805 & 0.04071225 & 1.02455807 \\
\hline-1.68407583 & -1.48319805 & 0.14654770 & -0.82827055 \\
\hline-1.57824039 & -1.58903348 & 0.04071225 & 0.49427295 \\
\hline-1.57824039 & -1.58903348 & 0.14654770 & -0.33050862 \\
\hline-1.57824039 & -1.48319805 & 0.04071225 & -0.52785331 \\
\hline-1.57824039 & -1.48319805 & 0.14654770 & 0.40104708 \\
\hline 1.02599049 & -0.66542661 & -0.36399299 & 1.43246114 \\
\hline 1.02599049 & -0.66542661 & -0.25815752 & -2.20898438 \\
\hline 1.02599049 & -0.55959117 & -0.36399299 & -0.60425401 \\
\hline 1.02599049 & -0.55959117 & -0.25815752 & 0.66444528 \\
\hline 1.13182592 & -0.66542661 & -0.36399299 & -0.67840976 \\
\hline 1.13182592 & -0.66542661 & -0.25815752 & 1.06156409 \\
\hline 1.13182592 & -0.55959117 & -0.36399299 & -0.02213119 \\
\hline 1.13182592 & -0.55959117 & -0.25815752 & 0.43395096 \\
\hline-2.12678075 & 0.64235818 & -0.12852763 & 2.34934664 \\
\hline-2.12678075 & 0.64235818 & -0.02269218 & -1.66934049 \\
\hline-2.12678075 & 0.74819362 & -0.12852763 & -1.98769772 \\
\hline-2.12678075 & 0.74819362 & -0.02269218 & 1.40546775 \\
\hline-2.02094531 & 0.64235818 & -0.12852763 & -3.55363345 \\
\hline-2.02094531 & 0.64235818 & -0.02269218 & 2.51936126 \\
\hline-2.02094531 & 0.74819362 & -0.12852763 & 3.14735413 \\
\hline-2.02094531 & 0.74819362 & -0.02269218 & -2.24871039 \\
\hline 1.00072026 & -1.60289264 & 0.02148618 & -1.63549328 \\
\hline 1.00072026 & -1.60289264 & 0.12732163 & 1.02008057 \\
\hline 1.00072026 & -1.49705720 & 0.02148618 & 1.92016304 \\
\hline 1.00072026 & -1.49705720 & 0.12732163 & -1.07156324 \\
\hline 1.10655570 & -1.60289264 & 0.02148618 & 1.56562483 \\
\hline 1.10655570 & -1.60289264 & 0.12732163 & -1.02624500 \\
\hline 1.10655570 & -1.49705720 & 0.02148618 & -1.76827741 \\
\hline 1.10655570 & -1.49705720 & 0.12732163 & 1.03379190 \\
\hline-1.75219464 & 1.54989612 & -0.37628788 & 0.14737855 \\
\hline-1.75219464 & 1.54989612 & -0.27045241 & -0.40050495 \\
\hline-1.75219464 & 1.65573156 & -0.37628788 & -0.16723759 \\
\hline-1.75219464 & 1.65573156 & -0.27045241 & 0.36763853 \\
\hline-1.64635921 & 1.54989612 & -0.37628788 & -0.35114920 \\
\hline-1.64635921 & 1.54989612 & -0.27045241 & 0.57276517 \\
\hline-1.64635921 & 1.65573156 & -0.37628788 & 0.33669889 \\
\hline-1.64635921 & 1.65573156 & -0.27045241 & -0.52386779 \\
\hline & \# Energy 3->8 & & \\
\hline-0 . & -0.0046 & 1.000 & Electr. mom., scale-fac \\
\hline 0.0105 & -0.0336 & & Magnetic mom. \\
\hline 2.03096962 & 0.19347408 & -0.02169468 & -7.07600832 \\
\hline 2.03096962 & 0.19347408 & 0.08414077 & 8.54114342 \\
\hline 2.03096962 & 0.29930952 & -0.02169468 & 6.43183231 \\
\hline 2.03096962 & 0.29930952 & 0.08414077 & -7.19734097 \\
\hline 2.13680482 & 0.19347408 & -0.02169468 & 5.19488907 \\
\hline 2.13680482 & 0.19347408 & 0.08414077 & -6.56922579 \\
\hline 2.13680482 & 0.29930952 & -0.02169468 & -4.36357307 \\
\hline 2.13680482 & 0.29930952 & 0.08414077 & 5.12538290 \\
\hline-1.37606192 & -0.46025667 & -0.36857194 & -5.19560432 \\
\hline-1.37606192 & -0.46025667 & -0.26273650 & 8.00287819 \\
\hline-1.37606192 & -0.35442120 & -0.36857194 & 5.45398283 \\
\hline-1.37606192 & -0.35442120 & -0.26273650 & -8.25753880 \\
\hline
\end{tabular}


$-0.46025667$

$-0.46025667$

$-0.35442120$

$-0.35442120$

1. 37286413

1. 37286413

1. 47869956

1.47869956

1. 37286413

1. 37286413

1.47869956

1.47869956

$-1.58903348$

$-1.58903348$

$-1.48319805$

$-1.48319805$

$-1.58903348$

$-1.58903348$

$-1.48319805$

$-1.48319805$

$-0.66542661$

$-0.66542661$

$-0.55959117$

$-0.55959117$

$-0.66542661$

$-0.66542661$

$-0.55959117$

$-0.55959117$

0.64235818

0.64235818

0.74819362

0.74819362

0.64235818

0.64235818

0.74819362

0.74819362

$-1.60289264$

$-1.60289264$

$-1.49705720$

$-1.49705720$

$-1.60289264$

$-1.60289264$

$-1.49705720$

$-1.49705720$

1. 54989612

1.54989612

1.65573156

1.65573156

1.54989612

1.54989612

1.65573156

1.65573156

\# Energy 3->9
$-0.36857194$

$-0.26273650$

$-0.36857194$

$-0.26273650$

$-0.39818472$

$-0.29234925$

$-0.39818472$

$-0.29234925$

$-0.39818472$

$-0.29234925$

$-0.39818472$

$-0.29234925$

0.04071225

0.14654770

0.04071225

0.14654770

0.04071225

0.14654770

0.04071225

0.14654770

$-0.36399299$

$-0.25815752$

$-0.36399299$

$-0.25815752$

$-0.36399299$

$-0.25815752$

$-0.36399299$

$-0.25815752$

$-0.12852763$

$-0.02269218$

$-0.12852763$

$-0.02269218$

$-0.12852763$

$-0.02269218$

$-0.12852763$

$-0.02269218$

0.02148618

0.12732163

0.02148618

0.12732163

0.02148618

0.12732163

0.02148618

0.12732163

$-0.37628788$

$-0.27045241$

$-0.37628788$

$-0.27045241$

$-0.37628788$

$-0.27045241$

$-0.37628788$

$-0.27045241$
3. 05572581

$-4.89051199$

$-3.22104597$

5.03146601

$-0.26347023$

$-0.13232982$

0.09410996

0.23127583

$-0.66368729$

1.06858957

0.68982130

$-1.02698350$

0.98709440

$-0.83346170$

$-1.37813938$

1.16993594

$-0.89386898$

0.85688573

1.19073892

$-1.15834308$

4. 52270174

$-8.23875237$

$-5.35361004$

9.25112629

$-5.46060658$

9.79893780

6.60058880

$-11.19312382$

5.21451616

$-6.07645369$

$-4.22248697$

4.69570351

$-5.90986824$

7. 03808069

4.94344091

$-5.62940311$

0.22212538

$-0.67275441$

0.02722856

0.65352184

$-0.11913245$

0.60116118

$-0.18702137$

$-0.53180796$

$-0.61422861$

0.77424073

0.38848719

$-0.48728147$

0.41123918

$-0.51176023$

$-0.24647257$

0.30704460

64

$\begin{array}{lrr}0.0759 & 0.0477 & -0.0450 \\ 0.0034 & -0.2009 & 0.0967\end{array}$

2.03096962

0.19347408

2.03096962

0.19347408

1.000 \#

0.29930952

0.29930952

2.03096962

2.13680482

2.13680482

2.13680482

2.13680482

$-1.37606192$

$-1.37606192$

$-1.37606192$

$-1.37606192$

0.19347408

0.19347408

0.29930952

0.29930952

$-0.46025667$

$-0.46025667$

$-0.35442120$

$-0.35442120$
$-0.02169468$
0.08414077
$-0.02169468$
0.08414077
$-0.02169468$
0.08414077
$-0.02169468$
0.08414077
$-0.36857194$
$-0.26273650$
$-0.36857194$
$-0.26273650$

\# Electr. mom., scale-fac Magnetic mom.

9.97988701

$-10.68542767$

$-8.27889824$

9. 51755619

$-16.75395012$

17.52758598

15.74760914

$-17.06277657$

1.66014993

$-2.87671018$

$-1.85464036$

2. 70153236 


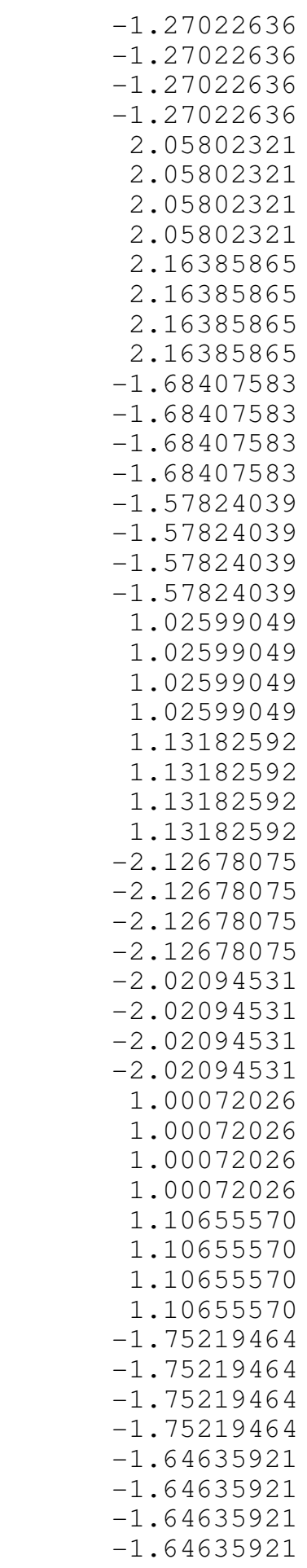

\&TRANSITION $4->\ldots$ 64
$-0.46025667$

$-0.46025667$

$-0.35442120$

$-0.35442120$

1. 37286413

1. 37286413

1.47869956

1.47869956

1. 37286413

1.37286413

1.47869956

1. 47869956

$-1.58903348$

$-1.58903348$

$-1.48319805$

$-1.48319805$

$-1.58903348$

$-1.58903348$

$-1.48319805$

$-1.48319805$

$-0.66542661$

$-0.66542661$

$-0.55959117$

$-0.55959117$

$-0.66542661$

$-0.66542661$

$-0.55959117$

$-0.55959117$

0.64235818

0.64235818

0.74819362

0.74819362

0.64235818

0.64235818

0.74819362

0.74819362

$-1.60289264$

$-1.60289264$

$-1.49705720$

$-1.49705720$

$-1.60289264$

$-1.60289264$

$-1.49705720$

$-1.49705720$

1. 54989612

1.54989612

1.65573156

1.65573156

1. 54989612

1. 54989612

1.65573156

1.65573156
$-0.36857194$

$-0.26273650$

$-0.36857194$

$-0.26273650$

$-0.39818472$

$-0.29234925$

$-0.39818472$

$-0.29234925$

$-0.39818472$

$-0.29234925$

$-0.39818472$

$-0.29234925$

0.04071225

0.14654770

0.04071225

0.14654770

0.04071225

0.14654770

0.04071225

0.14654770

$-0.36399299$

$-0.25815752$

$-0.36399299$

$-0.25815752$

$-0.36399299$

$-0.25815752$

$-0.36399299$

$-0.25815752$

$-0.12852763$

$-0.02269218$

$-0.12852763$

$-0.02269218$

$-0.12852763$

$-0.02269218$

$-0.12852763$

$-0.02269218$

0.02148618

0.12732163

0.02148618

0.12732163

0.02148618

0.12732163

0.02148618

0.12732163

$-0.37628788$

$-0.27045241$

$-0.37628788$

$-0.27045241$

$-0.37628788$

$-0.27045241$

$-0.37628788$

$-0.27045241$
-0.59804195
1.83051181
0.77985781

$-1.66732788$

$-0.47600263$

$-1.15081346$

$-0.55796802$

1. 93326628

$-4.20179415$

5.93916321

4.40727806

$-5.88505745$

$-1.37038147$

0.95125329

1. 58811033

$-1.02921987$

1. 42220473

$-1.01775205$

$-1.64345086$

1.11578202

$-9.09669018$

6.79715204

10.56925011

$-8.75194931$

11.51209450

$-9.66611385$

$-12.88309383$

11.44522190

$-4.15649557$

3. 64464903

3.20977902

$-2.54878640$

5.35093594

$-4.37493944$

$-4.37554836$

3. 24824977

1.88173234

$-2.91101789$

$-1.69732845$

3. 09359574

$-2.14579749$

3. 08950114

2.03754258

$-3.29910278$

0.32879046

$-0.30492151$

$-0.19033837$

0.17146377

$-0.36534446$

0.44886175

0.20808651

$-0.26097178$

$$
\text { 0. \# Energy 4->5 }
$$

$$
\begin{aligned}
& -0.8731 \quad-0.6728 \\
& 0.0398 \\
& 0.19347408 \\
& 0.19347408 \\
& 0.29930952 \\
& 0.29930952 \\
& 0.19347408 \\
& 0.19347408 \\
& 0.29930952 \\
& 0.29930952 \\
& -0.46025667 \\
& -0.46025667 \\
& -0.35442120 \\
& 1.000 \text { \# Electr. mom., scale-fac } \\
& \text { \# Magnetic mom. } \\
& \begin{array}{r}
-0.02169468 \\
0.08414077 \\
-0.02169468 \\
0.08414077 \\
-0.02169468 \\
0.08414077 \\
-0.02169468 \\
0.08414077 \\
-0.36857194 \\
-0.26273650 \\
-0.36857194
\end{array}
\end{aligned}
$$




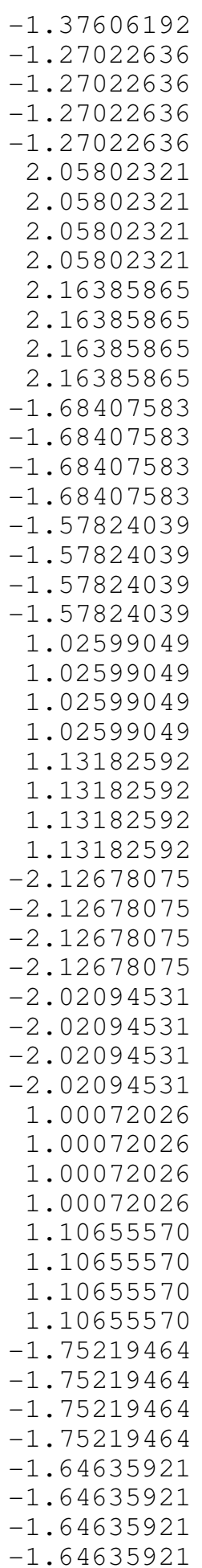

64
$-0.35442120$

$-0.46025667$

$-0.46025667$

$-0.35442120$

$-0.35442120$

1. 37286413

1.37286413

1.47869956

1.47869956

1.37286413

1.37286413

1. 47869956

1.47869956

$-1.58903348$

$-1.58903348$

$-1.48319805$

$-1.48319805$

$-1.58903348$

$-1.58903348$

$-1.48319805$

$-1.48319805$

$-0.66542661$

$-0.66542661$

$-0.55959117$

$-0.55959117$

$-0.66542661$

$-0.66542661$

$-0.55959117$

$-0.55959117$

0.64235818

0.64235818

0.74819362

0.74819362

0.64235818

0.64235818

0.74819362

0.74819362

$-1.60289264$

$-1.60289264$

$-1.49705720$

$-1.49705720$

$-1.60289264$

$-1.60289264$

$-1.49705720$

$-1.49705720$

1. 54989612

1. 54989612

1.65573156

1.65573156

1. 54989612

1. 54989612

1.65573156

1.65573156

0. \# Energy 4->6

$\begin{array}{lll}-2.7063 & -1.1120 & -0.2758\end{array}$

0.0369

$-0.0306$

0.0019

0.19347408

0.19347408

0.29930952

0.29930952

0.19347408

0.19347408

0.29930952

0.29930952

$-0.46025667$

$-0.46025667$

$-0.35442120$

$-1.37606192$
$-0.26273650$

$-0.36857194$

$-0.26273650$

$-0.36857194$

$-0.26273650$

$-0.39818472$

$-0.29234925$

$-0.39818472$

$-0.29234925$

$-0.39818472$

$-0.29234925$

$-0.39818472$

$-0.29234925$

0.04071225

0.14654770

0.04071225

0.14654770

0.04071225

0.14654770

0.04071225

0.14654770

$-0.36399299$

$-0.25815752$

$-0.36399299$

$-0.25815752$

$-0.36399299$

$-0.25815752$

$-0.36399299$

$-0.25815752$

$-0.12852763$

$-0.02269218$

$-0.12852763$

$-0.02269218$

$-0.12852763$

$-0.02269218$

$-0.12852763$

$-0.02269218$

0.02148618

0.12732163

0.02148618

0.12732163

0.02148618

0.12732163

0.02148618

0.12732163

$-0.37628788$

$-0.27045241$

$-0.37628788$

$-0.27045241$

$-0.37628788$

$-0.27045241$

$-0.37628788$

$-0.27045241$$$
.27045241-1.52946925
$$

. 90128803

0.68954730

1.40135169

$-0.83335865$

060056

3.03370762

2.46588016

$-2.63540578$

3.37318778

1.38141537

$-0.46069333$

$-1.30962706$

$-2.21938300$

$-0.18804635$

14.67669582

13.18309116

23.03842926

$-17.85977364$

6. 13982201

0.36161530

0.60685116

0.54733270

0.60385901

0.71185327

0.59882855

1.89501822

1.68760753

1.92930567

$-1.52946925$

1.000 \# Electr. mom., scale-fac
\# Magnetic mom.

$-0.02169468$

$-0.85036123$

0.08414077

$-2.19934368$

$-0.26614544$

2. 63124275

1.11551225

2.04694104

$-0.32557321$

$-2.15478587$

$-2.94720602$

9. 04898834

1. 75321174
$-0.26273650$

0.36857194
$-0.02169468$

$-0.02169468$

0.08414077

$-0.36857194$ 


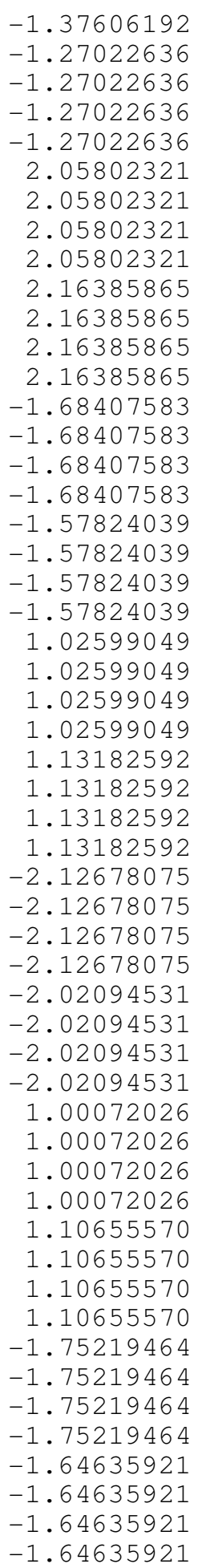

64
$-0.35442120$

$-0.46025667$

$-0.46025667$

$-0.35442120$

1. 37286413

1.37286413

1.47869956

1.47869956

1.37286413

1.37286413

1. 47869956

1.47869956

$-1.58903348$

$-1.58903348$

$-1.48319805$

$-1.48319805$

$-1.58903348$

$-1.48319805$

$-1.48319805$

$-0.66542661$

$-0.66542661$

$-0.55959117$

$-0.55959117$

$-0.66542661$

$-0.66542661$

$-0.55959117$

$-0.55959117$

0.64235818

0.64235818

0.74819362

0.74819362

0.64235818

0.64235818

0.74819362

0.74819362

$-1.60289264$

$-1.60289264$

$-1.49705720$

$-1.49705720$

$-1.60289264$

$-1.60289264$

$-1.49705720$

$-1.49705720$

1. 54989612

1. 54989612

1.65573156

1.65573156

1.54989612

1. 54989612

1.65573156

1.65573156

0 .
$-0.35442120$

$-1.58903348$

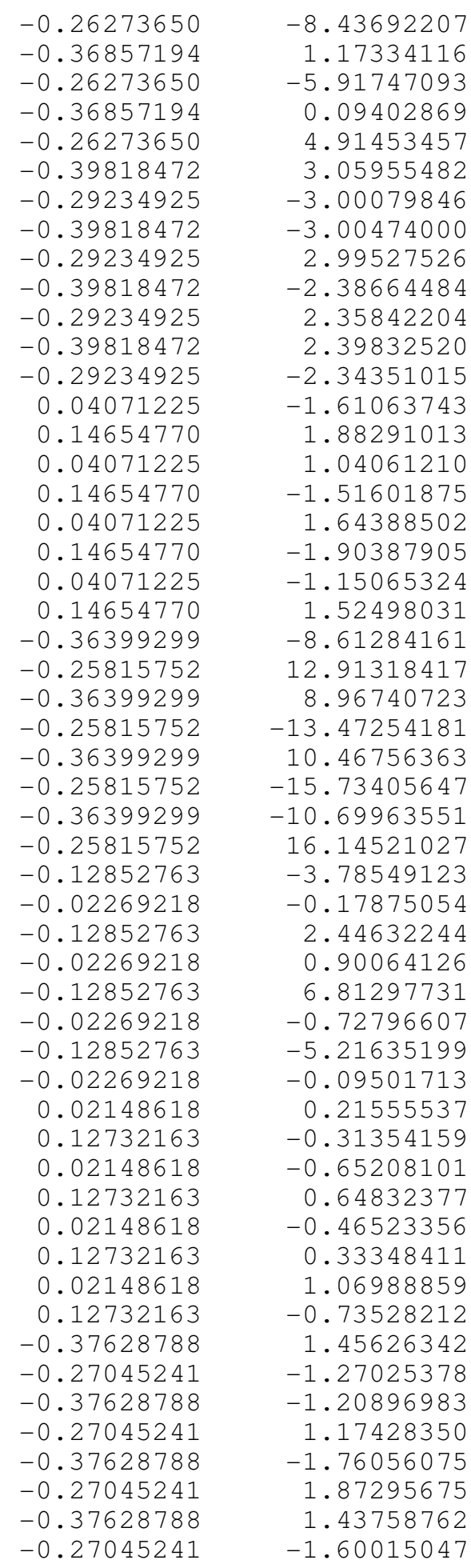

\# Energy 4->7

$\begin{array}{lrr}0.6551 & 0.1235 & 0.0161\end{array}$

$0.0066-0.0985 \quad-0.0180$

2.03096962

2.03096962

2.03096962

2.03096962

2.13680482

2.13680482

2.13680482

2.13680482

$-1.37606192$

$-1.37606192$

$-1.37606192$
0.19347408

0.19347408

0.29930952

0.29930952

0.19347408

0.19347408

0.29930952

0.29930952

$-0.46025667$

$-0.46025667$

$-0.35442120$
1.000 \# Electr. mom., scale-fac \# Magnetic mom.

$\begin{array}{rr}-0.02169468 & 0.89078033 \\ 0.08414077 & 0.88914078 \\ -0.02169468 & -1.09412527 \\ 0.08414077 & -0.59432399 \\ -0.02169468 & 1.88581252 \\ 0.08414077 & -3.67289376 \\ -0.02169468 & -1.87189519 \\ 0.08414077 & 3.58635855 \\ -0.36857194 & 2.76849508 \\ -0.26273650 & -5.76316166 \\ -0.36857194 & -2.30462956\end{array}$




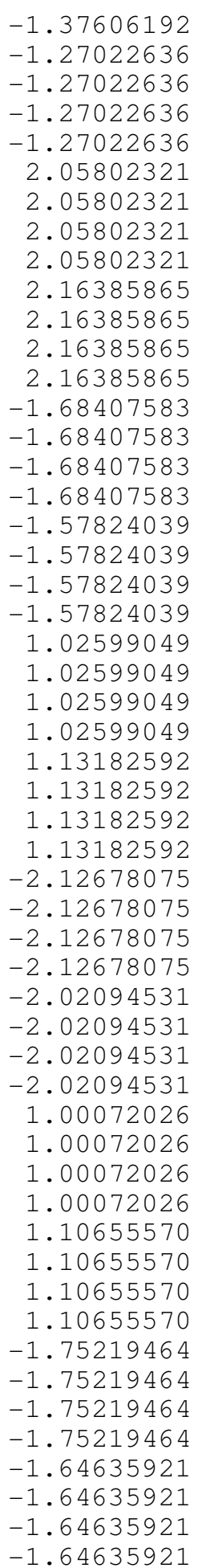

64
$-0.35442120$

$-0.46025667$

$-0.46025667$

$-0.35442120$

$-0.35442120$

1. 37286413

1.37286413

1.47869956

1.47869956

1.37286413

1.37286413

1. 47869956

1.47869956

$-1.58903348$

$-1.58903348$

$-1.48319805$

$-1.48319805$

$-1.58903348$

$-1.58903348$

$-1.48319805$

$-1.48319805$

$-0.66542661$

$-0.66542661$

$-0.55959117$

$-0.55959117$

$-0.66542661$

$-0.66542661$

$-0.55959117$

$-0.55959117$

0.64235818

0.64235818

0.74819362

0.74819362

0.64235818

0.64235818

0.74819362

0.74819362

$-1.60289264$

$-1.60289264$

$-1.49705720$

$-1.49705720$

$-1.60289264$

$-1.60289264$

$-1.49705720$

$-1.49705720$

1. 54989612

1. 54989612

1.65573156

1.65573156

1.54989612

1. 54989612

1.65573156

1.65573156

0 . \# Energy 4->8

0.0258

2.03096962

2.03096962

2.03096962

2.03096962

2.13680482

2.13680482

2.13680482

2.13680482

$-1.37606192$

$-1.37606192$

$-1.37606192$

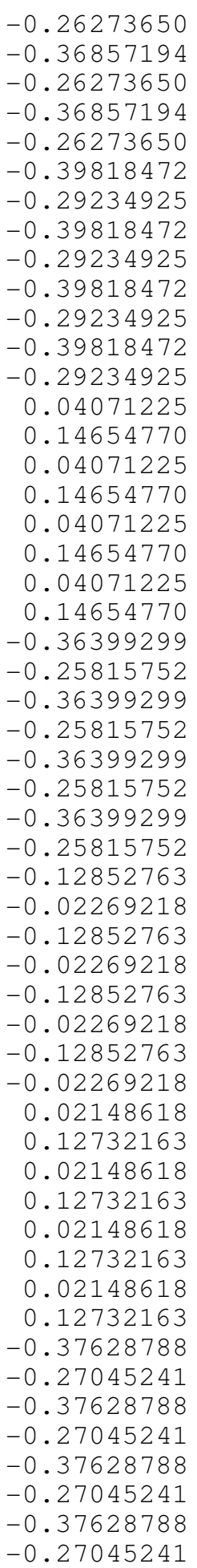

5.41473913

$-1.64680922$

4.00878811

1.15497339

$-3.57732010$

$-0.61833942$

1. 27201974

0.73478293

$-1.27235436$

2. 28798914

$-3.02183461$

$-2.09365296$

2. 68676734

0.18106651

$-0.33068451$

0.08790760

0.13098897

$-0.26451418$

0.36844105

0.02856376

$-0.16960354$

4.57589960

$-6.10209608$

$-4.45498848$

6.09430361

$-5.57279730$

7. 43127871

5.48094559

$-7.45487881$

$-1.61182344$

2. 79087567

1.58164990

$-2.60206771$

1. 07942057

$-2.70602965$

$-1.10724962$

2. 54921794

$-0.99292344$

0.99459022

1. 26607537

$-1.21790969$

1. 11656880

$-1.08219504$

$-1.44798338$

1.33967471

0.18006235

$-0.35053000$

$-0.17644246$

0.31668749

$-0.08649287$

0.16888572

0.12258868

$-0.20378980$ $\begin{array}{rrrrr}-0.0728 & -0.3183 & -0.1106 & 1.000 & \# \text { Electr. mom., scale-fac } \\ 0.0258 & -0.0626 & 0.0143 & & \# \text { Magnetic mom. }\end{array}$

0.19347408

0.19347408

0.29930952

0.29930952

0.19347408

0.19347408

0.29930952

0.29930952

$-0.46025667$

$-0.46025667$

$-0.35442120$
$-36.41294861$

42.75169373

31.92390251

$-36.08328247$

33.05049133

$-39.18096924$

$-28.51558304$

32.71701431

$-19.50854301$

30.49623489

20.83874130 


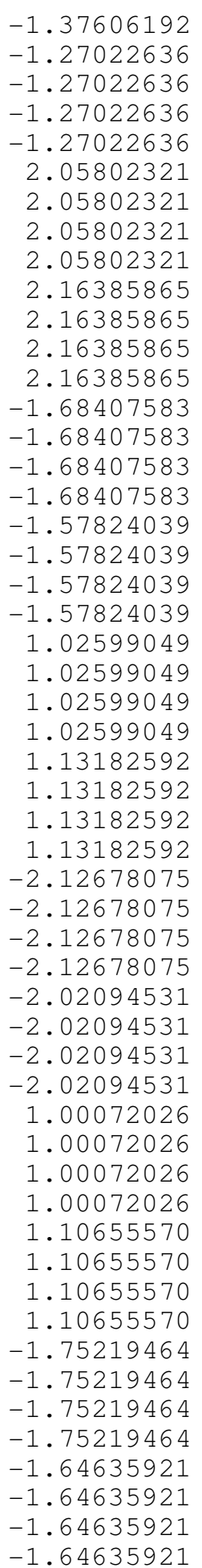

64
$-0.35442120$

$-0.46025667$

$-0.46025667$

$-0.35442120$

$-0.35442120$

1. 37286413

1.37286413

1.47869956

1.47869956

1.37286413

1.37286413

1. 47869956

1.47869956

$-1.58903348$

$-1.58903348$

$-1.48319805$

$-1.48319805$

$-1.58903348$

$-1.58903348$

$-1.48319805$

$-1.48319805$

$-0.66542661$

$-0.66542661$

$-0.55959117$

$-0.55959117$

$-0.66542661$

$-0.66542661$

$-0.55959117$

$-0.55959117$

0.64235818

0.64235818

0.74819362

0.74819362

0.64235818

0.64235818

0.74819362

0.74819362

$-1.60289264$

$-1.60289264$

$-1.49705720$

$-1.49705720$

$-1.60289264$

$-1.60289264$

$-1.49705720$

$-1.49705720$

1. 54989612

1. 54989612

1.65573156

1.65573156

1.54989612

1. 54989612

1.65573156

1.65573156

0 . \# Energy 4->9

$\begin{array}{lll}0.0789 & -0.7066 & -0.2928\end{array}$

0.0172

2.03096962

2.03096962

2.03096962

2.03096962

2.13680482

2.13680482

2.13680482

2.13680482

$-1.37606192$

$-1.37606192$

$-1.37606192$
$0.1036-0.0046$

0.19347408

0.19347408

0.29930952

0.29930952

0.19347408

0.19347408

0.29930952

0.29930952

$-0.46025667$

$-0.46025667$

$-0.35442120$
$-0.26273650$

$-0.36857194$

$-0.26273650$

$-0.36857194$

$-0.26273650$

$-0.39818472$

$-0.29234925$

$-0.39818472$

$-0.29234925$

$-0.39818472$

$-0.29234925$

$-0.39818472$

$-0.29234925$

0.04071225

0.14654770

0.04071225

0.14654770

0.04071225

0.14654770

0.04071225

0.14654770

$-0.36399299$

$-0.25815752$

$-0.36399299$

$-0.25815752$

$-0.36399299$

$-0.25815752$

$-0.36399299$

$-0.25815752$

$-0.12852763$

$-0.02269218$

$-0.12852763$

$-0.02269218$

$-0.12852763$

$-0.02269218$

$-0.12852763$

$-0.02269218$

0.02148618

0.12732163

0.02148618

0.12732163

0.02148618

0.12732163

0.02148618

0.12732163

$-0.37628788$

$-0.27045241$

$-0.37628788$

$-0.27045241$

$-0.37628788$

$-0.27045241$

$-0.37628788$

$-0.27045241$
$-31.86042404$

7.53753185

$-14.11917400$

$-8.54859543$

15.20277691

$-0.41306031$

$-0.05196548$

0.18627560

0.16906703

$-0.04637776$

0.40156287

0.27726358

$-0.55871987$

3. 85299492

$-3.89633536$

$-4.73734093$

4.82528639

$-3.74824357$

4.35407448

4.28930569

$-5.20706797$

25.44684219

$-37.73417282$

$-28.81647301$

41.51777267

$-29.14555168$

43.53619003

33.64699173

$-48.64433670$

30.77217102

$-33.66976166$

$-25.15103531$

26.23882675

$-35.32725906$

38.59823608

29.83222771

$-31.12575531$

$-0.00035542$

$-1.09160614$

0.57639843

1. 19913185

0.60207719

0.62675959

$-1.41006756$

$-0.52167660$

$-3.05316448$

4. 03658390

2.07968998

$-2.78139758$

2.04541254

$-2.85117078$

$-1.37698209$

1. 95986819
1.000 \# Electr. mom., scale-fac

\# Magnetic mom.

$-0.02169468$

0.08414077

$-0.02169468$

0.08414077

$-0.02169468$

0.08414077

$-0.02169468$

0.08414077

$-0.36857194$

$-0.26273650$

$-0.36857194$
$-139.54222107$

169.84033203

125.39134979

$-146.32620239$

131.01329041

$-158.94401550$

$-115.97768402$ 135.70031738

$-78.79502869$

119.76528168

82.79708862 
$-1.37606192$

$-1.27022636$

$-1.27022636$

$-1.27022636$

$-1.27022636$

2. 05802321

2. 05802321

2. 05802321

2. 05802321

2.16385865

2.16385865

2.16385865

2.16385865

$-1.68407583$

$-1.68407583$

$-1.68407583$

$-1.68407583$

$-1.57824039$

$-1.57824039$

$-1.57824039$

$-1.57824039$

1.02599049

1.02599049

1.02599049

1. 02599049

1.13182592

1.13182592

1. 13182592

1. 13182592

$-2.12678075$

$-2.12678075$

$-2.12678075$

$-2.12678075$

$-2.02094531$

$-2.02094531$

$-2.02094531$

$-2.02094531$

1. 00072026

1.00072026

1.00072026

1. 00072026

1.10655570

1.10655570

1.10655570

1.10655570

$-1.75219464$

$-1.75219464$

$-1.75219464$

$-1.75219464$

$-1.64635921$

$-1.64635921$

$-1.64635921$

$-1.64635921$

$\&$ TRANSITION $5->\ldots$ 64
$-0.35442120$

$-0.46025667$

$-0.46025667$

$-0.35442120$

$-0.35442120$

1. 37286413

1.37286413

1.47869956

1. 47869956

1.37286413

1.37286413

1. 47869956

1.47869956

$-1.58903348$

$-1.58903348$

$-1.48319805$

$-1.48319805$

$-1.58903348$

$-1.58903348$

$-1.48319805$

$-1.48319805$

$-0.66542661$

$-0.66542661$

$-0.55959117$

$-0.55959117$

$-0.66542661$

$-0.66542661$

$-0.55959117$

$-0.55959117$

0.64235818

0.64235818

0.74819362

0.74819362

0.64235818

0.64235818

0.74819362

0.74819362

$-1.60289264$

$-1.60289264$

$-1.49705720$

$-1.49705720$

$-1.60289264$

$-1.60289264$

$-1.49705720$

$-1.49705720$

1. 54989612

1. 54989612

1.65573156

1.65573156

1. 54989612

1. 54989612

1.65573156

1.65573156
$-0.26273650$

$-0.36857194$

$-0.26273650$

$-0.36857194$

$-0.26273650$

$-0.39818472$

$-0.29234925$

$-0.39818472$

$-0.29234925$

$-0.39818472$

$-0.29234925$

$-0.39818472$

$-0.29234925$

0.04071225

0.14654770

0.04071225

0.14654770

0.04071225

0.14654770

0.04071225

0.14654770

$-0.36399299$

$-0.25815752$

$-0.36399299$

$-0.25815752$

$-0.36399299$

$-0.25815752$

$-0.36399299$

$-0.25815752$

$-0.12852763$

$-0.02269218$

$-0.12852763$

$-0.02269218$

$-0.12852763$

$-0.02269218$

$-0.12852763$

$-0.02269218$

0.02148618

0.12732163

0.02148618

0.12732163

0.02148618

0.12732163

0.02148618

0.12732163

$-0.37628788$

$-0.27045241$

$-0.37628788$

$-0.27045241$

$-0.37628788$

$-0.27045241$

$-0.37628788$

$-0.27045241$
$-123.30023193$

36.99531555

$-62.83613968$

$-39.68704987$

64.99835205

$-3.93868804$

2. 76213002

2. 24919629

$-1.32934606$

1.86046743

$-0.89648348$

$-0.27943128$

$-0.47836331$

15.67431355

$-14.68769836$

$-19.24810982$

18.21759033

$-15.43050194$

16.22565842

18.08171272

$-19.67395401$

97.20241547

$-145.83033752$

$-114.22280884$

166.22840881

$-114.11652374$

171.98640442

135.36662292

$-197.51882935$

113.87495422

$-125.22935486$

$-93.55202484$

97.89516449

$-130.01893616$

143.12538147

110.21903229

$-115.67333984$

$-0.66040319$

$-2.71311665$

3.88135672

1.78423560

3.11109900

0.94492966

$-7.33733416$

0.82474530

$-12.09718609$

15.98806381

8.23633194

$-11.00224304$

8.52015877

$-11.77771187$

$-5.77678108$

8.13636398

$-6.6745 \quad 0 . \quad$ \# Energy 5->6

$\begin{array}{ll}-1.3295 & 0.0753\end{array}$

$-0.1068$

0.1132

2. 03096962

0.19347408

0.19347408

0.29930952

0.29930952

0.19347408

0.19347408

0.29930952

0.29930952

$-0.46025667$

$-0.46025667$
1.000 \# Electr. mom., scale-fac \# Magnetic mom.

$-0.02169468$

0.08414077

$-0.02169468$

0.08414077

$-0.02169468$

0.08414077

$-0.02169468$

0.08414077

$-0.36857194$

$-0.26273650$
$-11.04430676$

11.39112186

12.62782764

$-11.82980061$ 6.82596827

$-7.81458664$

$-7.78907490$

7.73232079

18.92863464

$-26.11862373$ 
$-1.37606192$

$-1.37606192$

$-1.27022636$

$-1.27022636$

$-1.27022636$

$-1.27022636$

2. 05802321

2. 05802321

2. 05802321

2. 05802321

2.16385865

2.16385865

2.16385865

2.16385865

$-1.68407583$

$-1.68407583$

$-1.68407583$

$-1.68407583$

$-1.57824039$

$-1.57824039$

$-1.57824039$

1.02599049

1.02599049

1. 02599049

1.02599049

1. 13182592

1. 13182592

1. 13182592

1.13182592

$-2.12678075$

$-2.12678075$

$-2.12678075$

$-2.12678075$

$-2.02094531$

$-2.02094531$

$-2.02094531$

$-2.02094531$

1.00072026

1.00072026

1. 00072026

1.00072026

1.10655570

1.10655570

1.10655570

1.10655570

$-1.75219464$

$-1.75219464$

$-1.75219464$

$-1.75219464$

$-1.64635921$

$-1.64635921$

$-1.64635921$

64

$-1.64635921$
$-1.57824039$

$-0.35442120$

$-0.35442120$

$-0.46025667$

$-0.46025667$

$-0.35442120$

$-0.35442120$

1. 37286413

1.37286413

1.47869956

1.47869956

1. 37286413

1. 37286413

1.47869956

1.47869956

$-1.58903348$

$-1.58903348$

$-1.48319805$

$-1.48319805$

$-1.58903348$

$-1.58903348$

$-1.48319805$

$-1.48319805$

$-0.66542661$

$-0.66542661$

$-0.55959117$

$-0.55959117$

$-0.66542661$

$-0.66542661$

$-0.55959117$

$-0.55959117$

0.64235818

0.64235818

0.74819362

0.74819362

0.64235818

0.64235818

0.74819362

0.74819362

$-1.60289264$

$-1.60289264$

$-1.49705720$

$-1.49705720$

$-1.60289264$

$-1.60289264$

$-1.49705720$

$-1.49705720$

1. 54989612

1.54989612

1.65573156

1.65573156

1.54989612

1.54989612

1.65573156

1.65573156

\# Energy 5->7

$0.7498 \quad 0.2246 \quad 0.0239$

0.0014

2.03096962

2.03096962

2.03096962

2.03096962

2.13680482

2.13680482

2.13680482

2.13680482

$-1.37606192$

$-1.37606192$

$\begin{array}{rr}0.3017 & 0.0967\end{array}$

0.19347408

0.19347408

0.29930952

0.29930952

0.19347408

0.19347408

0.29930952

0.29930952

$-0.46025667$

$-0.46025667$
$-0.36857194$

$-0.26273650$

$-0.36857194$

$-0.26273650$

$-0.36857194$

$-0.26273650$

$-0.39818472$

$-0.29234925$

$-0.39818472$

$-0.29234925$

$-0.39818472$

$-0.29234925$

$-0.39818472$

$-0.29234925$

0.04071225

0.14654770

0.04071225

0.14654770

0.04071225

0.14654770

0.04071225

0.14654770

$-0.36399299$

$-0.25815752$

$-0.36399299$

$-0.25815752$

$-0.36399299$

$-0.25815752$

$-0.36399299$

$-0.25815752$

$-0.12852763$

$-0.02269218$

$-0.12852763$

$-0.02269218$

$-0.12852763$

$-0.02269218$

$-0.12852763$

$-0.02269218$

0.02148618

0.12732163

0.02148618

0.12732163

0.02148618

0.12732163

0.02148618

0.12732163

$-0.37628788$

$-0.27045241$

$-0.37628788$

$-0.27045241$

$-0.37628788$

$-0.27045241$

$-0.37628788$

$-0.27045241$
$-16.89123535$

23.93656540

$-13.71837044$

19.46166801

11.48999310

$-16.82199478$

$-3.53954768$

2. 43408251

4.04190969

$-3.30745959$

1. 82680786

$-1.18247926$

$-2.43147802$

1. 92091644

2. 93272066

$-2.68464470$

$-1.90790749$

1.83853292

$-2.17634797$

1. 80976689

1. 15344870

$-0.78772175$

14.52456093

$-20.55348396$

$-13.68633366$

19.47597694

$-16.57361794$

23.55807877

16.02590370

$-22.98324585$

$-12.51365185$

14.32814693

12.76333141

$-14.04994774$

14.06820774

$-16.64583778$

$-14.26904774$

16.34242439

0.71325845

$-0.99083275$

$-0.23400138$

0.81565022

$-0.55807239$

1.00659394

$-0.05052717$

$-0.76870573$

0.60656953

$-0.53112847$

$-0.26370960$

0.12710407

0.16188762

$-0.44149199$

$-0.28117004$

0.57040662
1.000 \# Electr. mom., scale-fac

$-0.02169468$

0.08414077

$-0.02169468$

0.08414077

$-0.02169468$

0.08414077

$-0.02169468$

0.08414077

$-0.36857194$

$-0.26273650$
Magnetic mom.

$$
\begin{array}{r}
-0.25705266 \\
-2.73289871 \\
0.12012993 \\
3.06697726 \\
-6.84088993 \\
9.95595551 \\
7.60532761 \\
-10.92614269 \\
-5.99479246 \\
9.19608688
\end{array}
$$


$-1.37606192$

$-1.37606192$

$-1.27022636$

$-1.27022636$

$-1.27022636$

$-1.27022636$

2. 05802321

2. 05802321

2. 05802321

2. 05802321

2.16385865

2.16385865

2.16385865

2.16385865

$-1.68407583$

$-1.68407583$

$-1.68407583$

$-1.68407583$

$-1.57824039$

$-1.57824039$

$-1.57824039$

$-1.57824039$

1.02599049

1.02599049

1. 02599049

1.02599049

1. 13182592

1. 13182592

1. 13182592

1.13182592

$-2.12678075$

$-2.12678075$

$-2.12678075$

$-2.12678075$

$-2.02094531$

$-2.02094531$

$-2.02094531$

$-2.02094531$

1.00072026

1.00072026

1. 00072026

1.00072026

1.10655570

1.10655570

1. 10655570

1. 10655570

$-1.75219464$

$-1.75219464$

$-1.75219464$

$-1.75219464$

$-1.64635921$

$-1.64635921$

$-1.64635921$

64

$-1.64635921$

0.6401

$-0.0140$

2. 03096962

2.03096962

2. 03096962

2.03096962

2. 13680482

2.13680482

2.13680482

2.13680482

$-1.37606192$

$-1.37606192$
$-0.35442120$

$-0.35442120$

$-0.46025667$

$-0.46025667$

$-0.35442120$

$-0.35442120$

1. 37286413

1.37286413

1. 47869956

1.47869956

1.37286413

1. 37286413

1. 47869956

1.47869956

$-1.58903348$

$-1.58903348$

$-1.48319805$

$-1.48319805$

$-1.58903348$

$-1.58903348$

$-1.48319805$

$-1.48319805$

$-0.66542661$

$-0.66542661$

$-0.55959117$

$-0.55959117$

$-0.66542661$

$-0.66542661$

$-0.55959117$

$-0.55959117$

0.64235818

0.64235818

0.74819362

0.74819362

0.64235818

0.64235818

0.74819362

0.74819362

$-1.60289264$

$-1.60289264$

$-1.49705720$

$-1.49705720$

$-1.60289264$

$-1.60289264$

$-1.49705720$

$-1.49705720$

1. 54989612

1. 54989612

1.65573156

1.65573156

1.54989612

1. 54989612

1.65573156

1.65573156

0 .

\# Energy 5->8

$0.2137 \quad-0.1161$

$0.0147 \quad-0.0687$

0.19347408

0.19347408

0.29930952

0.29930952

0.19347408

0.19347408

0.29930952

0.29930952

$-0.46025667$

$-0.46025667$
$-0.36857194$

$-0.26273650$

$-0.36857194$

$-0.26273650$

$-0.36857194$

$-0.26273650$

$-0.39818472$

$-0.29234925$

$-0.39818472$

$-0.29234925$

$-0.39818472$

$-0.29234925$

$-0.39818472$

$-0.29234925$

0.04071225

0.14654770

0.04071225

0.14654770

0.04071225

0.14654770

0.04071225

0.14654770

$-0.36399299$

$-0.25815752$

$-0.36399299$

$-0.25815752$

$-0.36399299$

$-0.25815752$

$-0.36399299$

$-0.25815752$

$-0.12852763$

$-0.02269218$

$-0.12852763$

$-0.02269218$

$-0.12852763$

$-0.02269218$

$-0.12852763$

$-0.02269218$

0.02148618

0.12732163

0.02148618

0.12732163

0.02148618

0.12732163

0.02148618

0.12732163

$-0.37628788$

$-0.27045241$

$-0.37628788$

$-0.27045241$

$-0.37628788$

$-0.27045241$

$-0.37628788$

$-0.27045241$
5.54471540

$-8.96708775$

4.93968678

$-7.42786503$

$-4.40453768$

7.01250744

3.38803029

$-5.49626923$

$-3.84263992$

5.57923841

$-7.82952547$

10.08508778

7.50340939

$-9.35898113$

$-0.38890299$

0.40949765

0.10824756

$-0.19633675$

0.38702333

$-0.34301785$

$-0.15313976$

0.13841198

$-9.27588654$

10.39648724

8.96656895

$-10.25081348$

10.95364094

$-12.57240963$

$-10.63399982$

12.40144539

1.13233149

$-2.86025810$

$-1.21624613$

2. 77365065

$-0.20223381$

2. 72399211

0.32796967

$-2.63896108$

1.87344360

$-2.21745658$

$-2.21611691$

2.63285017

$-2.15623450$

2. 44239020

2.61876893

$-2.93626881$

$-0.11241501$

0.32588395

0.09793732

$-0.26550278$

$-0.02109329$

$-0.02796498$

$-0.02103113$

0.07727873
1.000 \# Electr. mom., scale-fac
\# Magnetic mom.

$-0.02169468$

0.08414077

$-0.02169468$

0.08414077

$-0.02169468$

0.08414077

$-0.02169468$

0.08414077

$-0.36857194$

$-0.26273650$
141.22866821

$-173.60673523$

$-126.48014832$

148.66648865

$-132.81228638$

162.93397522

116.72531891

$-138.08827209$

91.05835724

$-129.37199402$ 
$-1.37606192$

$-1.37606192$

$-1.27022636$

$-1.27022636$

$-1.27022636$

$-1.27022636$

2. 05802321

2. 05802321

2. 05802321

2. 05802321

2.16385865

2.16385865

2.16385865

2.16385865

$-1.68407583$

$-1.68407583$

$-1.68407583$

$-1.68407583$

$-1.57824039$

$-1.57824039$

$-1.57824039$

$-1.57824039$

1.02599049

1.02599049

1. 02599049

1.02599049

1. 13182592

1. 13182592

1. 13182592

1.13182592

$-2.12678075$

$-2.12678075$

$-2.12678075$

$-2.12678075$

$-2.02094531$

$-2.02094531$

$-2.02094531$

$-2.02094531$

1.00072026

1.00072026

1. 00072026

1.00072026

1.10655570

1.10655570

1. 10655570

1. 10655570

$-1.75219464$

$-1.75219464$

$-1.75219464$

$-1.75219464$

$-1.64635921$

$-1.64635921$

$-1.64635921$

64

$-1.64635921$

$-0.7289$

0.0462

2.03096962

2. 03096962

2. 03096962

2. 03096962

2. 13680482

2.13680482

2.13680482

2.13680482

$-1.37606192$

$-1.37606192$
$-0.35442120$

$-0.35442120$

$-0.46025667$

$-0.46025667$

$-0.35442120$

$-0.35442120$

1. 37286413

1.37286413

1.47869956

1.47869956

1.37286413

1. 37286413

1. 47869956

1. 47869956

$-1.58903348$

$-1.58903348$

$-1.48319805$

$-1.48319805$

$-1.58903348$

$-1.58903348$

$-1.48319805$

$-1.48319805$

$-0.66542661$

$-0.66542661$

$-0.55959117$

$-0.55959117$

$-0.66542661$

$-0.66542661$

$-0.55959117$

$-0.55959117$

0.64235818

0.64235818

0.74819362

0.74819362

0.64235818

0.64235818

0.74819362

0.74819362

$-1.60289264$

$-1.60289264$

$-1.49705720$

$-1.49705720$

$-1.60289264$

$-1.60289264$

$-1.49705720$

$-1.49705720$

1. 54989612

1.54989612

1.65573156

1.65573156

1.54989612

1. 54989612

1.65573156

1.65573156

0 . \# Energy 5->9

$\begin{array}{rr}-0.4558 & -0.2205 \\ 0.0100 & 0.0104\end{array}$

0.19347408

0.19347408

0.29930952

0.29930952

0.19347408

0.19347408

0.29930952

0.29930952

$-0.46025667$

$-0.46025667$
$-0.36857194$

$-0.26273650$

$-0.36857194$

$-0.26273650$

$-0.36857194$

$-0.26273650$

$-0.39818472$

$-0.29234925$

$-0.39818472$

$-0.29234925$

$-0.39818472$

$-0.29234925$

$-0.39818472$

$-0.29234925$

0.04071225

0.14654770

0.04071225

0.14654770

0.04071225

0.14654770

0.04071225

0.14654770

$-0.36399299$

$-0.25815752$

$-0.36399299$

$-0.25815752$

$-0.36399299$

$-0.25815752$

$-0.36399299$

$-0.25815752$

$-0.12852763$

$-0.02269218$

$-0.12852763$

$-0.02269218$

$-0.12852763$

$-0.02269218$

$-0.12852763$

$-0.02269218$

0.02148618

0.12732163

0.02148618

0.12732163

0.02148618

0.12732163

0.02148618

0.12732163

$-0.37628788$

$-0.27045241$

$-0.37628788$

$-0.27045241$

$-0.37628788$

$-0.27045241$

$-0.37628788$

$-0.27045241$
$-95.71196747$

133.55776978

$-48.87798309$

73.12397766

51.82625580

$-75.34253693$

6.26174498

$-5.56121635$

$-4.13686991$

3.59937167

$-4.16679955$

3. 69237661

2.15117121

$-1.76944792$

$-15.29388523$

14.48497868

19.38768196

$-18.48189545$

15.46929836

$-16.39072418$

$-18.76679039$

20.39269066

$-99.70523071$

152.87960815

118.00893402

$-175.61540222$

120.06726837

$-184.03529358$

$-142.96647644$

212.43397522

$-116.77475739$

128.97155762

96.16091156

$-100.96481323$

132.77214050

$-147.53324890$

$-112.57363129$

119.26136017

1. 96916234

1.72341311

$-5.69358826$

$-0.47136486$

$-4.53309679$

0.10082393

9.37235737

$-2.26829457$

11.39126587

$-15.69106483$

$-7.58847332$

10.59912682

$-7.66840410$

11.18382072

5.03181791

$-7.54495668$
1.000 \# Electr. mom., scale-fac
\# Magnetic mom.

$-0.02169468$

0.08414077

$-0.02169468$

0.08414077

$-0.02169468$

0.08414077

$-0.02169468$

0.08414077

$-0.36857194$

$-0.26273650$
$-24.30535316$

25.54737854

21.05988312

$-20.59039688$

20.40571594

$-22.40224457$

$-16.60684776$

17.40279388

$-10.33403301$

21.28404236 
$-1.37606192$

$-1.37606192$

$-1.27022636$

$-1.27022636$

$-1.27022636$

$-1.27022636$

2. 05802321

2. 05802321

2. 05802321

2. 05802321

2.16385865

2.16385865

2.16385865

2.16385865

$-1.68407583$

$-1.68407583$

$-1.68407583$

$-1.68407583$

$-1.57824039$

$-1.57824039$

$-1.57824039$

$-1.57824039$

1.02599049

1.02599049

1. 02599049

1.02599049

1. 13182592

1. 13182592

1. 13182592

1.13182592

$-2.12678075$

$-2.12678075$

$-2.12678075$

$-2.12678075$

$-2.02094531$

$-2.02094531$

$-2.02094531$

$-2.02094531$

1.00072026

1.00072026

1. 00072026

1.00072026

1.10655570

1.10655570

1.10655570

1.10655570

$-1.75219464$

$-1.75219464$

$-1.75219464$

$-1.75219464$

$-1.64635921$

$-1.64635921$

$-1.64635921$

$-1.64635921$

\&TRANSITION $6->\ldots$

64

0.8404

0.0183

2.03096962

2. 03096962

2.03096962

2.03096962

2.13680482

2.13680482

2.13680482

2. 13680482

$-1.37606192$
$-0.35442120$

$-0.35442120$

$-0.46025667$

$-0.46025667$

$-0.35442120$

$-0.35442120$

1. 37286413

1. 37286413

1. 47869956

1.47869956

1.37286413

1.37286413

1.47869956

1. 47869956

$-1.58903348$

$-1.58903348$

$-1.48319805$

$-1.48319805$

$-1.58903348$

$-1.58903348$

$-1.48319805$

$-1.48319805$

$-0.66542661$

$-0.66542661$

$-0.55959117$

$-0.55959117$

$-0.66542661$

$-0.66542661$

$-0.55959117$

$-0.55959117$

0.64235818

0.64235818

0.74819362

0.74819362

0.64235818

0.64235818

0.74819362

0.74819362

$-1.60289264$

$-1.60289264$

$-1.49705720$

$-1.49705720$

$-1.60289264$

$-1.60289264$

$-1.49705720$

$-1.49705720$

1. 54989612

1.54989612

1.65573156

1.65573156

1.54989612

1.54989612

1.65573156

1. 65573156
$-0.36857194$

$-0.26273650$

$-0.36857194$

$-0.26273650$

$-0.36857194$

$-0.26273650$

$-0.39818472$

$-0.29234925$

$-0.39818472$

$-0.29234925$

$-0.39818472$

$-0.29234925$

$-0.39818472$

$-0.29234925$

0.04071225

0.14654770

0.04071225

0.14654770

0.04071225

0.14654770

0.04071225

0.14654770

$-0.36399299$

$-0.25815752$

$-0.36399299$

$-0.25815752$

$-0.36399299$

$-0.25815752$

$-0.36399299$

$-0.25815752$

$-0.12852763$

$-0.02269218$

$-0.12852763$

$-0.02269218$

$-0.12852763$

$-0.02269218$

$-0.12852763$

$-0.02269218$

0.02148618

0.12732163

0.02148618

0.12732163

0.02148618

0.12732163

0.02148618

0.12732163

$-0.37628788$

$-0.27045241$

$-0.37628788$

$-0.27045241$

$-0.37628788$

$-0.27045241$

$-0.37628788$

$-0.27045241$
11.51090717

$-22.47142220$

1.19535446

$-8.51470470$

$-2.22907090$

9.54447269

$-3.31523991$

3. 40084743

2. 51959229

$-2.63403416$

2. 93122673

$-3.18609977$

$-2.15809298$

2.35641599

2. 13870931

$-1.79956043$

$-3.23209476$

2. 81106853

$-1.89043844$

1.89347279

2.76742840

$-2.87843251$

22.62404633

$-32.87052536$

$-26.18856049$

37.71815491

$-28.55906296$

41.85180664

33.28418732

$-48.19937134$

20.77494621

$-23.26159096$

$-17.20303917$

18.30810928

$-23.36788940$

26.38467979

19.96664429

$-21.44327927$

$-0.99925518$

0.52944654

1. 70440936

$-0.85379022$

1.81453109

$-1.12623715$

$-2.87265563$

1. 71664941

$-2.24991250$

3. 03448153

1. 52304614

$-2.09149861$

1. 56723619

$-2.21354675$

$-1.06382191$

1. 54041398

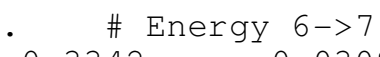

0.19347408

0.19347408

0.29930952

0.29930952

0.19347408

0.19347408

0.29930952

0.29930952

$-0.46025667$
1.000 \# Electr. mom., scale-fac \# Magnetic mom.
$-0.02169468$
0.08414077
$-0.02169468$
0.08414077
$-0.02169468$
0.08414077
$-0.02169468$
0.08414077
$-0.36857194$

1.01806819
1.32731760

0.55202436

$-3.61378407$

8.52907372

$-10.87257957$

$-10.99879074$

14.05173016

$-1.95744789$ 
$-1.37606192$

$-1.37606192$

$-1.37606192$

$-1.27022636$

$-1.27022636$

$-1.27022636$

$-1.27022636$

2. 05802321

2. 05802321

2. 05802321

2. 05802321

2.16385865

2.16385865

2.16385865

2.16385865

$-1.68407583$

$-1.68407583$

$-1.68407583$

$-1.68407583$

$-1.57824039$

$-1.57824039$

$-1.57824039$

$-1.57824039$

1.02599049

1. 02599049

1.02599049

1.02599049

1. 13182592

1. 13182592

1.13182592

1. 13182592

$-2.12678075$

$-2.12678075$

$-2.12678075$

$-2.12678075$

$-2.02094531$

$-2.02094531$

$-2.02094531$

$-2.02094531$

1.00072026

1. 00072026

1.00072026

1.00072026

1.10655570

1. 10655570

1.10655570

1. 10655570

$-1.75219464$

$-1.75219464$

$-1.75219464$

$-1.75219464$

$-1.64635921$

$-1.64635921$

$-1.64635921$

64

$-1.64635921$

$-0.4623 \quad 0$.

0.0020

2.03096962

2. 03096962

2.03096962

2. 03096962

2.13680482

2.13680482

2.13680482

2. 13680482

$-1.37606192$
$-0.46025667$

$-0.35442120$

$-0.35442120$

$-0.46025667$

$-0.46025667$

$-0.35442120$

$-0.35442120$

1.37286413

1. 37286413

1. 47869956

1.47869956

1.37286413

1.37286413

1.47869956

1. 47869956

$-1.58903348$

$-1.58903348$

$-1.48319805$

$-1.48319805$

$-1.58903348$

$-1.58903348$

$-1.48319805$

$-1.48319805$

$-0.66542661$

$-0.66542661$

$-0.55959117$

$-0.55959117$

$-0.66542661$

$-0.66542661$

$-0.55959117$

$-0.55959117$

0.64235818

0.64235818

0.74819362

0.74819362

0.64235818

0.64235818

0.74819362

0.74819362

$-1.60289264$

$-1.60289264$

$-1.49705720$

$-1.49705720$

$-1.60289264$

$-1.60289264$

$-1.49705720$

$-1.49705720$

1.54989612

1.54989612

1.65573156

1.65573156

1.54989612

1.54989612

1.65573156

1.65573156

\# Energy 6->8

$\begin{array}{cc}\text { \# Energy } & 6->8 \\ -0.0009 & 0.0026\end{array}$ $-0.0048 \quad 0.0069$

0.19347408

0.19347408

0.29930952

0.29930952

0.19347408

0.19347408

0.29930952

0.29930952

$-0.46025667$
$-0.26273650$

$-0.36857194$

$-0.26273650$

$-0.36857194$

$-0.26273650$

$-0.36857194$

$-0.26273650$

$-0.39818472$

$-0.29234925$

$-0.39818472$

$-0.29234925$

$-0.39818472$

$-0.29234925$

$-0.39818472$

$-0.29234925$

0.04071225

0.14654770

0.04071225

0.14654770

0.04071225

0.14654770

0.04071225

0.14654770

$-0.36399299$

$-0.25815752$

$-0.36399299$

$-0.25815752$

$-0.36399299$

$-0.25815752$

$-0.36399299$

$-0.25815752$

$-0.12852763$

$-0.02269218$

$-0.12852763$

$-0.02269218$

$-0.12852763$

$-0.02269218$

$-0.12852763$

$-0.02269218$

0.02148618

0.02148618

0.12732163

0.02148618

0.12732163

0.02148618

0.12732163

$-0.37628788$

$-0.27045241$

$-0.37628788$

$-0.27045241$

$-0.37628788$

$-0.27045241$

$-0.37628788$

$-0.27045241$
0.12732163

2.84005189

2.30017662

$-2.87559295$

1. 27141380

$-2.36069393$

$-1.57098615$

2. 29876995

$-6.07734489$

9.11430073

7. 74506140

$-10.26399040$

11.71422863

$-14.75537682$

$-12.44603634$

15.01876068

0.02058165

0.23797050

$-0.45609680$

0.04144493

$-0.29514584$

$-0.07864985$

0.81524128

$-0.26835233$

4. 85894537

$-4.03934240$

$-4.27270746$

3. 86701322

$-6.58249426$

6.02532864

5. 91528082

$-5.74231339$

2. 22331762

$-1.23901343$

$-2.20396709$

1. 17888522

$-3.11590290$

1.83870077

3. 08957314

$-1.76254618$

$-2.34239340$

2.83936644

2. 59466171

$-3.29587054$

2. 44453859

$-2.96316385$

$-2.75533342$

3. 46352983

0.10266044

$-0.27455279$

$-0.17563155$

0.33030093

$-0.15913036$

0.24280223

0.22519375

$-0.32108560$

$$
\begin{aligned}
& 1.000 \text { \# Electr. mom., scale-fac } \\
& \text { \# Magnetic mom. }
\end{aligned}
$$

$-88.18706512$

105.60028839

78.46437836

$-89.82788086$

81.61135864

$-98.18314362$

$-71.14067078$

82.57772064

$-53.15103149$ 
$-1.37606192$

$-1.37606192$

$-1.37606192$

$-1.27022636$

$-1.27022636$

$-1.27022636$

$-1.27022636$

2. 05802321

2. 05802321

2. 05802321

2. 05802321

2.16385865

2.16385865

2.16385865

2. 16385865

$-1.68407583$

$-1.68407583$

$-1.68407583$

$-1.68407583$

$-1.57824039$

$-1.57824039$

$-1.57824039$

$-1.57824039$

1.02599049

1. 02599049

1.02599049

1.02599049

1. 13182592

1. 13182592

1.13182592

1. 13182592

$-2.12678075$

$-2.12678075$

$-2.12678075$

$-2.12678075$

$-2.02094531$

$-2.02094531$

$-2.02094531$

$-2.02094531$

1.00072026

1. 00072026

1.00072026

1.00072026

1.10655570

1.10655570

1.10655570

1. 10655570

$-1.75219464$

$-1.75219464$

$-1.75219464$

$-1.75219464$

$-1.64635921$

$-1.64635921$

$-1.64635921$

64

$-1.64635921$

$-0.3880$

0.0171

2.03096962

2. 03096962

2.03096962

2. 03096962

2.13680482

2.13680482

2.13680482

2. 13680482

$-1.37606192$
$-0.46025667$

$-0.35442120$

$-0.35442120$

$-0.46025667$

$-0.46025667$

$-0.35442120$

$-0.35442120$

1.37286413

1. 37286413

1. 47869956

1. 47869956

1. 37286413

1.37286413

1.47869956

1. 47869956

$-1.58903348$

$-1.58903348$

$-1.48319805$

$-1.48319805$

$-1.58903348$

$-1.58903348$

$-1.48319805$

$-1.48319805$

$-0.66542661$

$-0.66542661$

$-0.55959117$

$-0.55959117$

$-0.66542661$

$-0.66542661$

$-0.55959117$

$-0.55959117$

0.64235818

0.64235818

0.74819362

0.74819362

0.64235818

0.64235818

0.74819362

0.74819362

$-1.60289264$

$-1.60289264$

$-1.49705720$

$-1.49705720$

$-1.60289264$

$-1.60289264$

$-1.49705720$

$-1.49705720$

1.54989612

1.54989612

1.65573156

1.65573156

1. 54989612

1.54989612

1.65573156

1.65573156

0. \# Energy 6->9

$-0.2043-0.1449$ $-0.0231 \quad-0.0024$

0.19347408

0.19347408

0.29930952

0.29930952

0.19347408

0.19347408

0.29930952

0.29930952

$-0.46025667$
$-0.26273650$

$-0.36857194$

$-0.26273650$

$-0.36857194$

$-0.26273650$

$-0.36857194$

$-0.26273650$

$-0.39818472$

$-0.39818472$

$-0.29234925$

$-0.39818472$

$-0.29234925$

$-0.39818472$

$-0.29234925$

0.04071225

0.14654770

0.04071225

0.14654770

0.04071225

0.14654770

0.04071225

0.14654770

$-0.36399299$

$-0.25815752$

$-0.36399299$

$-0.25815752$

$-0.36399299$

$-0.25815752$

$-0.36399299$

$-0.25815752$

$-0.12852763$

$-0.02269218$

$-0.12852763$

$-0.02269218$

$-0.12852763$

$-0.02269218$

$-0.12852763$

$-0.02269218$

0.02148618

0.12732163

0.02148618

0.12732163

0.02148618

0.12732163

0.02148618

0.12732163

$-0.37628788$

$-0.27045241$

$-0.37628788$

$-0.27045241$

$-0.37628788$

$-0.27045241$

$-0.37628788$

$-0.27045241$
$-0.29234925$

77.48235321

55.70092010

$-79.59296417$

27.33839417

$-42.88640976$

$-28.91425896$

43.92092133

$-3.91556406$

3. 30792260

2. 80060410

$-2.35683727$

2. 75905037

$-2.41059756$

$-1.66789567$

1. 39815140

9. 48809624

$-8.84506226$

$-12.11339474$

11.34155655

$-9.52188492$

9.92638111

11.62539005

$-12.39771843$

63.23436737

$-95.03980255$

$-74.53061676$

108.97888947

$-75.72068024$

114.24314117

89.92581940

$-131.73779297$

72.17034912

$-79.15754700$

$-59.15292358$

61.72642899

$-82.55101776$

90.69127655

69.78290558

$-73.11317444$

$-1.10947287$

$-1.00697911$

3.19407797

0.37288126

2.79073358

$-0.19605353$

$-5.59810877$

1. 42132473

$-7.55963564$

10.03834248

5.11051273

$-6.86190176$

5.31172943

$-7.38018227$

$-3.56654239$

5.05854273

$$
\begin{aligned}
& 1.000 \text { \# Electr. mom., scale-fac } \\
& \text { \# Magnetic mom. }
\end{aligned}
$$

$-0.02169468$

69.75109100

$-0.02169468$

0.08414077

$-0.02169468$

0.08414077

$-0.02169468$

0.08414077

$-0.36857194$

$-87.38925171$

$-63.14738083$

75.87659454

$-66.14298248$

82.01975250

59.09888458

$-70.55279541$

44.48581314 


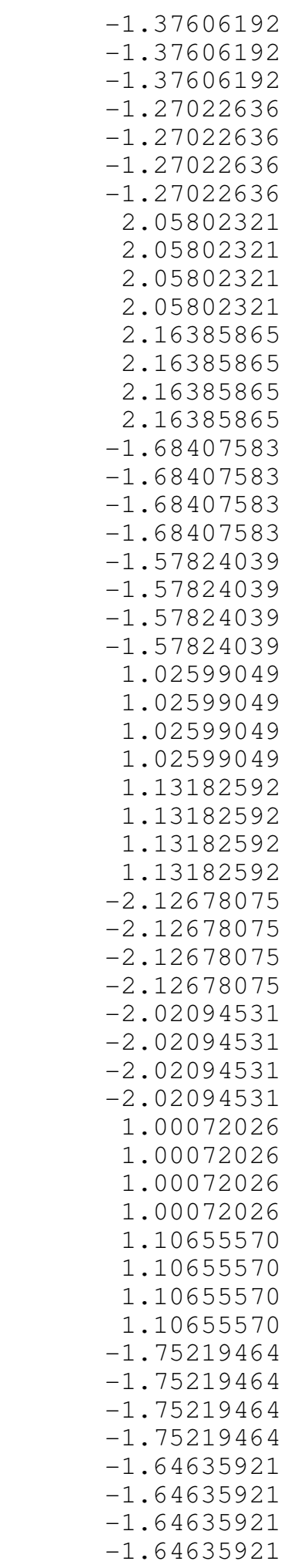

\&TRANSITION $7->\ldots$

64
$-0.46025667$

$-0.35442120$

$-0.35442120$

$-0.46025667$

$-0.46025667$

$-0.35442120$

$-0.35442120$

1. 37286413

1. 37286413

1. 47869956

1. 47869956

1.37286413

1. 37286413

1.47869956

1. 47869956

$-1.58903348$

$-1.58903348$

$-1.48319805$

$-1.48319805$

$-1.58903348$

$-1.58903348$

$-1.48319805$

$-1.48319805$

$-0.66542661$

$-0.66542661$

$-0.55959117$

$-0.55959117$

$-0.66542661$

$-0.66542661$

$-0.55959117$

$-0.55959117$

0.64235818

0.64235818

0.74819362

0.74819362

0.64235818

0.64235818

0.74819362

0.74819362

$-1.60289264$

$-1.60289264$

$-1.49705720$

$-1.49705720$

$-1.60289264$

$-1.60289264$

$-1.49705720$

$-1.49705720$

1. 54989612

1.54989612

1.65573156

1.65573156

1.54989612

1. 54989612

1.65573156

1.65573156
$-0.26273650$

$-0.36857194$

$-0.26273650$

$-0.36857194$

$-0.26273650$

$-0.36857194$

$-0.26273650$

$-0.39818472$

$-0.29234925$

$-0.39818472$

$-0.29234925$

$-0.39818472$

$-0.29234925$

$-0.39818472$

$-0.29234925$

0.04071225

0.14654770

0.04071225

0.14654770

0.04071225

0.14654770

0.04071225

0.14654770

$-0.36399299$

$-0.25815752$

$-0.36399299$

$-0.25815752$

$-0.36399299$

$-0.25815752$

$-0.36399299$

$-0.25815752$

$-0.12852763$

$-0.02269218$

$-0.12852763$

$-0.02269218$

$-0.12852763$

$-0.02269218$

$-0.12852763$

$-0.02269218$

0.02148618

0.12732163

0.02148618

0.12732163

0.02148618

0.12732163

0.02148618

0.12732163

$-0.37628788$

$-0.27045241$

$-0.37628788$

$-0.27045241$

$-0.37628788$

$-0.27045241$

$-0.37628788$

$-0.27045241$
$-62.07586288$

$-46.59658813$

63.86707687

$-24.92953682$

35.99034500

26.20922279

$-36.83638000$

1.15185773

$-0.42594159$

$-0.18518591$

$-0.42162716$

0.29185542

$-1.04898667$

$-1.10386133$

1. 71203029

$-7.57634830$

7.20559406

9.06591129

$-8.67439365$

7.85463285

$-8.26125717$

$-9.01309586$

9.75223064

$-46.90620422$

72.58790588

55.38465500

$-82.56610107$

54.88401031

$-84.84497833$

$-65.38852692$

97.29240417

$-56.54549408$

62.02352905

46.47221756

$-48.43858719$

64.57553101

$-71.10215759$

$-54.70794296$

57.40240097

0.66932070

1. 41035807

$-2.63635564$

$-0.75823987$

$-1.64887512$

$-0.71493858$

4.02355146

$-0.29197103$

5.61330795

$-7.63897038$

$-3.76781988$

5.19127893

$-3.80900931$

5.48930550

2. 52298760

$-3.72801805$

$\begin{array}{ccc}-0.5031 & -0.1385 & 0.0359 \\ 0.0015 & -0.0500 & 0.0276 \\ 2.03096962 & 0.19347408 \\ 2.03096962 & 0.19347408 \\ 2.03096962 & 0.29930952 \\ 2.03096962 & 0.29930952 \\ 2.13680482 & 0.19347408 \\ 2.13680482 & 0.19347408 \\ 2.13680482 & 0.29930952 \\ 2.13680482 & 0.29930952\end{array}$

1.000

$-0.02169468$

0.08414077

$-0.02169468$

0.08414077

$-0.02169468$

0.08414077

$-0.02169468$

0.08414077
\# Electr. mom., scale-fac Magnetic mom.

$-134.01412964$ 160.22793579 120.06195831

$-136.98388672$ 122.24735260

$-147.04333496$

$-106.98432922$ 123.82389069 
$-1.37606192$

$-1.37606192$

$-1.37606192$

$-1.37606192$

$-1.27022636$

$-1.27022636$

$-1.27022636$

$-1.27022636$

2. 05802321

2. 05802321

2. 05802321

2.05802321

2. 16385865

2.16385865

2.16385865

2.16385865

$-1.68407583$

$-1.68407583$

$-1.68407583$

$-1.68407583$

$-1.57824039$

$-1.57824039$

$-1.57824039$

1.02599049

1.02599049

1.02599049

1. 02599049

1. 13182592

1.13182592

1. 13182592

1. 13182592

$-2.12678075$

$-2.12678075$

$-2.12678075$

$-2.12678075$

$-2.02094531$

$-2.02094531$

$-2.02094531$

$-2.02094531$

1. 00072026

1.00072026

1.00072026

1.00072026

1.10655570

1.10655570

1.10655570

1.10655570

$-1.75219464$

$-1.75219464$

$-1.75219464$

$-1.75219464$

$-1.64635921$

$-1.64635921$

$-1.64635921$

$-1.64635921$

64
$-1.57824039$

$-0.46025667$

$-0.46025667$

$-0.35442120$

$-0.35442120$

$-0.46025667$

$-0.46025667$

$-0.35442120$

$-0.35442120$

1. 37286413

1. 37286413

1.47869956

1. 47869956

1.37286413

1.37286413

1. 47869956

1. 47869956

$-1.58903348$

$-1.58903348$

$-1.48319805$

$-1.48319805$

$-1.58903348$

$-1.58903348$

$-1.48319805$

$-1.48319805$

$-0.66542661$

$-0.66542661$

$-0.55959117$

$-0.55959117$

$-0.66542661$

$-0.66542661$

$-0.55959117$

$-0.55959117$

0.64235818

0.64235818

0.74819362

0.74819362

0.64235818

0.64235818

0.74819362

0.74819362

$-1.60289264$

$-1.60289264$

$-1.49705720$

$-1.49705720$

$-1.60289264$

$-1.60289264$

$-1.49705720$

$-1.49705720$

1.54989612

1.54989612

1.65573156

1.65573156

1. 54989612

1.54989612

1.65573156

1.65573156

0 . \# Energy 7->9

$\begin{array}{lll}-0.5443 & -0.1243 & 0.0485\end{array}$

$\begin{array}{lll}-0.0058 & -0.0735 & 0.0231\end{array}$

2.03096962

2.03096962

2. 03096962

2.03096962

2.13680482

2.13680482

2.13680482

2. 13680482
0.19347408

0.19347408

0.29930952

0.29930952

0.19347408

0.19347408

0.29930952

0.29930952
$-0.36857194$

$-0.26273650$

$-0.36857194$

$-0.26273650$

$-0.36857194$

$-0.26273650$

$-0.36857194$

$-0.26273650$

$-0.39818472$

$-0.29234925$

$-0.39818472$

$-0.29234925$

$-0.39818472$

$-0.29234925$

$-0.39818472$

$-0.29234925$

0.04071225

0.14654770

0.04071225

0.14654770

0.04071225

0.14654770

0.04071225

0.14654770

$-0.36399299$

$-0.25815752$

$-0.36399299$

$-0.25815752$

$-0.36399299$

$-0.25815752$

$-0.36399299$

$-0.25815752$

$-0.12852763$

$-0.02269218$

$-0.12852763$

$-0.02269218$

$-0.12852763$

$-0.02269218$

$-0.12852763$

$-0.02269218$

0.02148618

0.12732163

0.02148618

0.12732163

0.02148618

0.12732163

0.02148618

0.12732163

$-0.37628788$

$-0.27045241$

$-0.37628788$

$-0.27045241$

$-0.37628788$

$-0.27045241$

$-0.37628788$

$-0.27045241$
$-80.98577881$

117.55757141

84.82145691

$-120.87716675$

42.31849670

$-65.70957947$

$-44.63111877$

67.29375458

$-5.92529678$

4.63774633

4.07715225

$-3.09808493$

2. 75684381

$-1.69792211$

$-1.22669959$

0.38885900

14.25984764

$-13.34344959$

$-17.97496986$

16.92158318

$-14.39338684$

15.03857517

17.37387848

$-18.61248589$

93.90464783

$-143.45973206$

$-110.80887604$

164.21438599

$-112.65515900$

172.29393005

133.92550659

$-198.40286255$

107.63969421

$-118.91564178$

$-88.41268158$

92.94348907

$-122.44398499$

135.89706421

103.64834595

$-109.73464203$

$-1.66651857$

$-1.92462111$

5.14555359

0.79921603

4.05441284

0.24149553

$-8.57065868$

1. 72965121

$-10.83873272$

14.73315716

7.29510021

$-10.04596043$

7.41540289

$-10.61511803$

$-4.94095135$

7. 24979782
1.000

$-0.02169468$

0.08414077

$-0.02169468$

0.08414077

$-0.02169468$

0.08414077

$-0.02169468$

0.08414077
\# Electr. mom., scale-fac

Magnetic mom.

$-139.07356262$

166.13378906

124.85591125

$-142.33255005$

126.96919250

$-152.46057129$

$-111.41551208$

128.67965698 
$-1.37606192$

$-1.37606192$

$-1.37606192$

$-1.37606192$

$-1.27022636$

$-1.27022636$

$-1.27022636$

$-1.27022636$

2. 05802321

2. 05802321

2. 05802321

2.05802321

2. 16385865

2.16385865

2.16385865

2.16385865

$-1.68407583$

$-1.68407583$

$-1.68407583$

$-1.68407583$

$-1.57824039$

$-1.57824039$

$-1.57824039$

$-1.57824039$

1. 02599049

1.02599049

1.02599049

1. 02599049

1. 13182592

1.13182592

1. 13182592

1. 13182592

$-2.12678075$

$-2.12678075$

$-2.12678075$

$-2.12678075$

$-2.02094531$

$-2.02094531$

$-2.02094531$

$-2.02094531$

1. 00072026

1.00072026

1.00072026

1.00072026

1.10655570

1.10655570

1.10655570

1.10655570

$-1.75219464$

$-1.75219464$

$-1.75219464$

$-1.75219464$

$-1.64635921$

$-1.64635921$

$-1.64635921$

$-1.64635921$

$\&$ TRANSITION $8->\ldots$

640 .
$-0.46025667$

$-0.46025667$

$-0.35442120$

$-0.35442120$

$-0.46025667$

$-0.46025667$

$-0.35442120$

$-0.35442120$

1. 37286413

1. 37286413

1.47869956

1. 47869956

1.37286413

1.37286413

1. 47869956

1.47869956

$-1.58903348$

$-1.58903348$

$-1.48319805$

$-1.48319805$

$-1.58903348$

$-1.58903348$

$-1.48319805$

$-1.48319805$

$-0.66542661$

$-0.66542661$

$-0.55959117$

$-0.55959117$

$-0.66542661$

$-0.66542661$

$-0.55959117$

$-0.55959117$

0.64235818

0.64235818

0.74819362

0.74819362

0.64235818

0.64235818

0.74819362

0.74819362

$-1.60289264$

$-1.60289264$

$-1.49705720$

$-1.49705720$

$-1.60289264$

$-1.60289264$

$-1.49705720$

$-1.49705720$

1.54989612

1.54989612

1.65573156

1.65573156

1.54989612

1. 54989612

1.65573156

1. 65573156
$-0.36857194$

$-0.26273650$

$-0.36857194$

$-0.26273650$

$-0.36857194$

$-0.26273650$

$-0.36857194$

$-0.26273650$

$-0.39818472$

$-0.29234925$

$-0.39818472$

$-0.29234925$

$-0.39818472$

$-0.29234925$

$-0.39818472$

$-0.29234925$

0.04071225

0.14654770

0.04071225

0.14654770

0.04071225

0.14654770

0.04071225

0.14654770

$-0.36399299$

$-0.25815752$

$-0.36399299$

$-0.25815752$

$-0.36399299$

$-0.25815752$

$-0.36399299$

$-0.25815752$

$-0.12852763$

$-0.02269218$

$-0.12852763$

$-0.02269218$

$-0.12852763$

$-0.02269218$

$-0.12852763$

$-0.02269218$

0.02148618

0.12732163

0.02148618

0.12732163

0.02148618

0.12732163

0.02148618

0.12732163

$-0.37628788$

$-0.27045241$

$-0.37628788$

$-0.27045241$

$-0.37628788$

$-0.27045241$

$-0.37628788$

$-0.27045241$
$-84.38874054$

123.14339447

88.04106140

$-126.17947388$

44.58233643

$-69.70725250$

$-46.64149857$

70.90516663

$-5.83794498$

4.42395163

3.99229193

$-2.91401100$

2. 48350382

$-1.27449882$

$-0.98885822$

0.03834480

15.11717319

$-13.91042137$

$-19.01169968$

17.59054375

$-15.23006916$

15.60240746

18.37913132

$-19.28033447$

96.51477814

$-147.18811035$

$-114.16620636$

168.85978699

$-115.79437256$

176.87750244

137.89503479

$-203.99032593$

111.20547485

$-123.02867126$

$-91.34253693$

96.18054962

$-126.28829193$

140.39810181

106.89604950

$-113.37255096$

$-1.53457999$

$-2.09679699$

5.08444500

0.93036103

4.00257015

0.38169226

$-8.62749958$

1.65189159

$-11.29127312$

15.32377625

7.62427139

$-10.47885036$

7.78545904

$-11.10383701$

$-5.21737337$

7.61865664

$1.8553 \quad 0 . \quad$ \# Energy 8->9

$\begin{array}{lll}1.8553 & 0.3181 & -0.0937\end{array}$

$-0.0746$

2.03096962

0.1154

2. 03096962

$-0.2965$

0.19347408

0.19347408

0.29930952

2.03096962

0.29930952

0.19347408

0.19347408

2.13680482

2.13680482

2.13680482

0.29930952

$1.000 \#$
-0.02169468
0.08414077
-0.02169468
0.08414077
-0.02169468
0.08414077
-0.02169468

Electr. mom., scale-fac \# Magnetic mom.

$$
\begin{array}{r}
-4.11020088 \\
0.64975864 \\
3.57623577 \\
-0.28359094 \\
2.97601938 \\
0.10136325 \\
-2.53599215
\end{array}
$$


2.13680482

$-1.37606192$

$-1.37606192$

$-1.37606192$

$-1.37606192$

$-1.27022636$

$-1.27022636$

$-1.27022636$

$-1.27022636$

2. 05802321

2. 05802321

2. 05802321

2. 05802321

2.16385865

2.16385865

2.16385865

2.16385865

$-1.68407583$

$-1.68407583$

$-1.68407583$

$-1.68407583$

$-1.57824039$

$-1.57824039$

$-1.57824039$

$-1.57824039$

1.02599049

1.02599049

1. 02599049

1.02599049

1.13182592

1. 13182592

1. 13182592

1.13182592

$-2.12678075$

$-2.12678075$

$-2.12678075$

$-2.12678075$

$-2.02094531$

$-2.02094531$

$-2.02094531$

$-2.02094531$

1. 00072026

1.00072026

1.00072026

1.00072026

1.10655570

1.10655570

1.10655570

1. 10655570

$-1.75219464$

$-1.75219464$

$-1.75219464$

$-1.75219464$

$-1.64635921$

$-1.64635921$

$-1.64635921$

$-1.64635921$
0.29930952

$-0.46025667$

$-0.46025667$

$-0.35442120$

$-0.35442120$

$-0.46025667$

$-0.46025667$

$-0.35442120$

$-0.35442120$

1. 37286413

1. 37286413

1.47869956

1.47869956

1.37286413

1. 37286413

1. 47869956

1.47869956

$-1.58903348$

$-1.58903348$

$-1.48319805$

$-1.48319805$

$-1.58903348$

$-1.58903348$

$-1.48319805$

$-1.48319805$

$-0.66542661$

$-0.66542661$

$-0.55959117$

$-0.55959117$

$-0.66542661$

$-0.66542661$

$-0.55959117$

$-0.55959117$

0.64235818

0.64235818

0.74819362

0.74819362

0.64235818

0.64235818

0.74819362

0.74819362

$-1.60289264$

$-1.60289264$

$-1.49705720$

$-1.49705720$

$-1.60289264$

$-1.60289264$

$-1.49705720$

$-1.49705720$

1.54989612

1. 54989612

1.65573156

1.65573156

1.54989612

1.54989612

1.65573156

1. 65573156
0.08414077

$-0.36857194$

$-0.26273650$

$-0.36857194$

$-0.26273650$

$-0.36857194$

$-0.26273650$

$-0.36857194$

$-0.26273650$

$-0.39818472$

$-0.29234925$

$-0.39818472$

$-0.29234925$

$-0.39818472$

$-0.29234925$

$-0.39818472$

$-0.29234925$

0.04071225

0.14654770

0.04071225

0.14654770

0.04071225

0.14654770

0.04071225

0.14654770

$-0.36399299$

$-0.25815752$

$-0.36399299$

$-0.25815752$

$-0.36399299$

$-0.25815752$

$-0.36399299$

$-0.25815752$

$-0.12852763$

$-0.02269218$

$-0.12852763$

$-0.02269218$

$-0.12852763$

$-0.02269218$

$-0.12852763$

$-0.02269218$

0.02148618

0.12732163

0.02148618

0.12732163

0.02148618

0.12732163

0.02148618

0.12732163

$-0.37628788$

$-0.27045241$

$-0.37628788$

$-0.27045241$

$-0.37628788$

$-0.27045241$

$-0.37628788$

$-0.27045241$
$-0.37404662$

$-5.02624607$

18.96770859

1.25977325

$-14.72178364$

6.64801121

$-19.47568130$

$-2.76121569$

14.97805882

1.13867712

$-1.67456424$

$-0.95430583$

1.35604119

$-1.17797613$

1.66101134

0.98799759

$-1.34259224$

4.65074873

$-1.47858322$

$-8.22432327$

4.05550385

$-2.45457363$

$-0.55009139$

5.59845781

$-1.71715653$

$-3.78889728$

10.13988876

1.67329037

$-7.63899374$

3.96203303

$-9.76019764$

$-2.19397616$

7.82295418

$-5.62559032$

0.69483417

5.06602764

$-0.54231083$

9. 86244488

$-3.50932574$

$-9.10806274$

3. 22907758

2.71665883

$-1.60936856$

$-3.95534325$

2. 22843909

$-2.50761652$

1.60996449

3.60676074

$-2.18223619$

0.05005132

0.16652983

0.15456834

$-0.28577375$

0.40672642

$-0.48685572$

$-0.48635811$

0.54821563

\&PERMANENT MOMENTS

64
0 . \# Energy $1->1$

$0.2997 \quad 0.3653$

0.19347408

0.19347408

0.29930952

0.29930952

0.19347408

0.19347408

0.29930952
\# Electr. mom.

$-0.02169468$

0.08414077

$-0.02169468$

0.08414077

$-0.02169468$

0.08414077

$-0.02169468$
$-247.18289185$

218.22950745

231.08070374

$-196.59223938$

203.60493469

$-178.89437866$

$-187.28442383$ 
2.13680482

$-1.37606192$

$-1.37606192$

$-1.37606192$

$-1.37606192$

$-1.27022636$

$-1.27022636$

$-1.27022636$

$-1.27022636$

2. 05802321

2. 05802321

2. 05802321

2. 05802321

2.16385865

2.16385865

2.16385865

2.16385865

$-1.68407583$

$-1.68407583$

$-1.68407583$

$-1.68407583$

$-1.57824039$

$-1.57824039$

$-1.57824039$

$-1.57824039$

1.02599049

1.02599049

1. 02599049

1.02599049

1. 13182592

1. 13182592

1. 13182592

1.13182592

$-2.12678075$

$-2.12678075$

$-2.12678075$

$-2.12678075$

$-2.02094531$

$-2.02094531$

$-2.02094531$

$-2.02094531$

1. 00072026

1.00072026

1.00072026

1.00072026

1.10655570

1. 10655570

1.10655570

1. 10655570

$-1.75219464$

$-1.75219464$

$-1.75219464$

$-1.75219464$

$-1.64635921$

$-1.64635921$

$-1.64635921$

$-1.64635921$

64
0.29930952

$-0.46025667$

$-0.46025667$

$-0.35442120$

$-0.35442120$

$-0.46025667$

$-0.46025667$

$-0.35442120$

$-0.35442120$

1. 37286413

1. 37286413

1.47869956

1.47869956

1.37286413

1. 37286413

1. 47869956

1.47869956

$-1.58903348$

$-1.58903348$

$-1.48319805$

$-1.48319805$

$-1.58903348$

$-1.58903348$

$-1.48319805$

$-1.48319805$

$-0.66542661$

$-0.66542661$

$-0.55959117$

$-0.55959117$

$-0.66542661$

$-0.66542661$

$-0.55959117$

$-0.55959117$

0.64235818

0.64235818

0.74819362

0.74819362

0.64235818

0.64235818

0.74819362

0.74819362

$-1.60289264$

$-1.60289264$

$-1.49705720$

$-1.49705720$

$-1.60289264$

$-1.60289264$

$-1.49705720$

$-1.49705720$

1. 54989612

1.54989612

1.65573156

1.65573156

1.54989612

1.54989612

1.65573156

1.65573156 \# Energy 2->2

$\begin{array}{lll}-1.5387 & -2.2330 & 1.1473\end{array}$

2.03096962

2.03096962

2. 03096962

2.03096962

2.13680482

2.13680482

2. 13680482

2.13680482
0.19347408

0.19347408

0.29930952

0.29930952

0.19347408

0.19347408

0.29930952

0.29930952
0.08414077

$-0.36857194$

$-0.26273650$

$-0.36857194$

$-0.26273650$

$-0.36857194$

$-0.26273650$

$-0.36857194$

$-0.26273650$

$-0.39818472$

$-0.29234925$

$-0.29234925$

$-0.39818472$

$-0.29234925$

$-0.39818472$

$-0.29234925$

0.04071225

0.14654770

0.04071225

0.14654770

0.04071225

0.14654770

0.04071225

0.14654770

$-0.36399299$

$-0.25815752$

$-0.36399299$

$-0.25815752$

$-0.36399299$

$-0.25815752$

$-0.36399299$

$-0.25815752$

$-0.12852763$

$-0.02269218$

$-0.12852763$

$-0.02269218$

$-0.12852763$

$-0.02269218$

$-0.12852763$

$-0.02269218$

0.02148618

0.12732163

0.02148618

0.12732163

0.02148618

0.12732163

0.02148618

0.12732163

$-0.37628788$

$-0.27045241$

$-0.37628788$

$-0.27045241$

$-0.27045241$

$-0.37628788$

$-0.27045241$
$-0.39818472$

$-0.37628788$

158.63183594

$-73.45983887$

30.39963913

57.93566513

$-0.15014566$

81.07949829

$-56.89385605$

$-66.54796600$

30.45522499

$-10.08775806$

3.58515334

6.76976013

$-2.29047585$

2. 99579287

2. 44766402

$-0.52059823$

$-3.74549174$

0.06835068

5.51812649

3. 07255769

$-10.81432629$

$-8.97149277$

0.73055297

7.96899271

2.63019419

158.08155823

$-232.20315552$

$-179.75773621$

255.88562012

$-199.63920593$

282.64239502

225.13917542

$-310.89440918$

126.09044647

$-76.46729279$

$-113.94143677$

74.03568268

$-178.22019958$

102.41210938

163.59416199

$-99.61539459$

$-11.06995869$

$-0.52754897$

23.82871056

$-7.19095135$

6.23233175

5.21203995

$-17.86141777$

1. 43276191

$-21.98195267$

9.63283157

15.66120434

$-6.93636036$

21.04287338

$-12.00396347$

$-15.17324638$

8.79207134

\# Electr. mom.

$-0.02169468$

0.08414077

$-0.02169468$

0.08414077

$-0.02169468$

0.08414077

$-0.02169468$

0.08414077
$-235.69869995$

211.99876404

220.53392029

$-191.32627869$

194.18850708

$-173.94012451$

$-178.54020691$

154.34678650 
$-1.37606192$

$-1.37606192$

$-1.37606192$

$-1.37606192$

$-1.27022636$

$-1.27022636$

$-1.27022636$

$-1.27022636$

2. 05802321

2. 05802321

2. 05802321

2.05802321

2.16385865

2.16385865

2.16385865

2.16385865

$-1.68407583$

$-1.68407583$

$-1.68407583$

$-1.68407583$

$-1.57824039$

$-1.57824039$

$-1.57824039$

$-1.57824039$

1. 02599049

1.02599049

1.02599049

1. 02599049

1. 13182592

1.13182592

1. 13182592

1. 13182592

$-2.12678075$

$-2.12678075$

$-2.12678075$

$-2.12678075$

$-2.02094531$

$-2.02094531$

$-2.02094531$

$-2.02094531$

1. 00072026

1. 00072026

1.00072026

1.00072026

1.10655570

1.10655570

1.10655570

1.10655570

$-1.75219464$

$-1.75219464$

$-1.75219464$

$-1.75219464$

$-1.64635921$

$-1.64635921$

$-1.64635921$

64

$-1.64635921$

$-0.7175$

2.03096962

2. 03096962

2.03096962

2. 03096962

2.13680482

2.13680482

2.13680482

2. 13680482

$-1.37606192$
$-0.46025667$

$-0.46025667$

$-0.35442120$

$-0.35442120$

$-0.46025667$

$-0.46025667$

$-0.35442120$

$-0.35442120$

1. 37286413

1. 37286413

1.47869956

1. 47869956

1.37286413

1. 37286413

1. 47869956

1. 47869956

$-1.58903348$

$-1.58903348$

$-1.48319805$

$-1.48319805$

$-1.58903348$

$-1.58903348$

$-1.48319805$

$-1.48319805$

$-0.66542661$

$-0.66542661$

$-0.55959117$

$-0.55959117$

$-0.66542661$

$-0.66542661$

$-0.55959117$

$-0.55959117$

0.64235818

0.64235818

0.74819362

0.74819362

0.64235818

0.64235818

0.74819362

0.74819362

$-1.60289264$

$-1.60289264$

$-1.49705720$

$-1.49705720$

$-1.60289264$

$-1.60289264$

$-1.49705720$

$-1.49705720$

1.54989612

1.54989612

1.65573156

1.65573156

1.54989612

1. 54989612

1.65573156

1.65573156

0 . \# Energy 3->3

$2.6319-0.1845$

0.19347408

0.19347408

0.29930952

0.29930952

0.19347408

0.19347408

0.29930952

0.29930952

$-0.46025667$
$-0.36857194$

$-0.26273650$

$-0.36857194$

$-0.26273650$

$-0.36857194$

$-0.26273650$

$-0.36857194$

$-0.26273650$

$-0.39818472$

$-0.29234925$

$-0.39818472$

$-0.29234925$

$-0.39818472$

$-0.29234925$

$-0.39818472$

$-0.29234925$

0.04071225

0.14654770

0.04071225

0.14654770

0.04071225

0.14654770

0.04071225

0.14654770

$-0.36399299$

$-0.25815752$

$-0.36399299$

$-0.25815752$

$-0.36399299$

$-0.25815752$

$-0.36399299$

$-0.25815752$

$-0.12852763$

$-0.02269218$

$-0.12852763$

$-0.02269218$

$-0.12852763$

$-0.02269218$

$-0.12852763$

$-0.02269218$

0.02148618

0.12732163

0.02148618

0.12732163

0.02148618

0.12732163

0.02148618

0.12732163

$-0.37628788$

$-0.27045241$

$-0.37628788$

$-0.27045241$

$-0.37628788$

$-0.27045241$

$-0.37628788$

$-0.27045241$
$-79.85681152$

25.02271271

63.19936752

4.69933653

80.31347656

$-42.86151886$

$-64.83056641$

16.58326340

$-11.68924904$

6.27027130

8.13548851

$-4.46417618$

4.02264690

0.58005762

$-1.44832635$

$-2.19430542$

$-27.45221710$

22.70588875

23.74155045

$-19.71132851$

17.73501968

$-16.20328903$

$-10.67619991$

10.50170898

150.41201782

$-235.02778625$

$-166.26348877$

251.71176147

$-190.60061646$

282.08642578

210.69953918

$-304.03570557$

129.91659546

$-81.24338531$

$-120.65491486$

81.16497040

$-184.38471985$

111.67771912

172.70919800

$-111.37759399$

$-15.30340195$

0.93066943

30.32101631

$-9.55426311$

9.83515263

3.86719370

$-23.27218437$

3. 47027206

$-21.16046715$

8.43200207

14.09886360

$-4.77537441$

18.01210785

$-7.83048296$

$-12.01438522$

4.46782112 $\begin{array}{rr}\text { \# } \text { Electr. mom. } \\ -0.02169468 & -276.27133179 \\ 0.08414077 & 241.93983459 \\ -0.02169468 & 273.12591553 \\ 0.08414077 & -232.29072571 \\ -0.02169468 & 216.97349548 \\ 0.08414077 & -187.69670105 \\ -0.02169468 & -212.20968628 \\ 0.08414077 & 177.56877136 \\ -0.36857194 & -67.69657135\end{array}$ 
$-1.37606192$

$-1.37606192$

$-1.37606192$

$-1.27022636$

$-1.27022636$

$-1.27022636$

$-1.27022636$

2. 05802321

2. 05802321

2. 05802321

2. 05802321

2.16385865

2.16385865

2.16385865

2. 16385865

$-1.68407583$

$-1.68407583$

$-1.68407583$

$-1.68407583$

$-1.57824039$

$-1.57824039$

$-1.57824039$

$-1.57824039$

1.02599049

1. 02599049

1.02599049

1.02599049

1. 13182592

1. 13182592

1.13182592

1. 13182592

$-2.12678075$

$-2.12678075$

$-2.12678075$

$-2.12678075$

$-2.02094531$

$-2.02094531$

$-2.02094531$

$-2.02094531$

1.00072026

1. 00072026

1.00072026

1.00072026

1.10655570

1.10655570

1.10655570

1.10655570

$-1.75219464$

$-1.75219464$

$-1.75219464$

$-1.75219464$

$-1.64635921$

$-1.64635921$

$-1.64635921$

64

$-1.64635921$

$-2.8076$

2.03096962

2.03096962

2. 03096962

2.03096962

2. 13680482

2.13680482

2.13680482

2.13680482

$-1.37606192$

$-1.37606192$
$-0.46025667$

$-0.35442120$

$-0.35442120$

$-0.46025667$

$-0.46025667$

$-0.35442120$

$-0.35442120$

1.37286413

1. 37286413

1. 47869956

1.47869956

1. 37286413

1.37286413

1.47869956

1. 47869956

$-1.58903348$

$-1.58903348$

$-1.48319805$

$-1.48319805$

$-1.58903348$

$-1.58903348$

$-1.48319805$

$-1.48319805$

$-0.66542661$

$-0.66542661$

$-0.55959117$

$-0.55959117$

$-0.66542661$

$-0.66542661$

$-0.55959117$

$-0.55959117$

0.64235818

0.64235818

0.74819362

0.74819362

0.64235818

0.64235818

0.74819362

0.74819362

$-1.60289264$

$-1.60289264$

$-1.49705720$

$-1.49705720$

$-1.60289264$

$-1.60289264$

$-1.49705720$

$-1.49705720$

1.54989612

1.54989612

1.65573156

1.65573156

1. 54989612

1.54989612

1.65573156

1.65573156

\# Energy 4->4

$\begin{array}{ll}1.2611 & 0.6683\end{array}$

0.19347408

0.19347408

0.29930952

0.29930952

0.19347408

0.19347408

0.29930952

0.29930952

$-0.46025667$

$-0.46025667$
$-0.26273650$

$-0.36857194$

$-0.26273650$

$-0.36857194$

$-0.26273650$

$-0.36857194$

$-0.26273650$

$-0.39818472$

$-0.29234925$

$-0.39818472$

$-0.29234925$

$-0.39818472$

$-0.29234925$

$-0.29234925$

0.04071225

0.14654770

0.04071225

0.14654770

0.04071225

0.14654770

0.04071225

0.14654770

$-0.25815752$

$-0.36399299$

$-0.25815752$

$-0.36399299$

$-0.25815752$

$-0.36399299$

$-0.25815752$

$-0.12852763$

$-0.02269218$

$-0.12852763$

$-0.02269218$

$-0.12852763$

$-0.02269218$

$-0.12852763$

$-0.02269218$

0.02148618

0.12732163

0.02148618

0.12732163

0.02148618

0.12732163

0.02148618

0.12732163

$-0.37628788$

$-0.27045241$

$-0.37628788$

$-0.27045241$

$-0.37628788$

$-0.27045241$

$-0.27045241$
$-0.39818472$

$-0.36399299$

$-0.37628788$

24.05865288

51.35595322

6.34962368

74.79444885

$-49.49169540$

$-59.46181107$

22.84260368

$-28.83177567$

21.68145370

32.57346344

$-28.05088234$

11.22765827

$-3.16105604$

$-18.11883163$

12.33601665

$-0.63910604$

5.62381935

3.65435886

$-10.64484501$

$-8.49235916$

0.86096287

7. 65585041

2.17815852

162.96295166

$-243.47512817$

$-182.08763123$

263.00378418

$-202.83976746$

291.52554321

225.48786926

$-315.45446777$

125.07933044

$-78.47837830$

$-113.07016754$

76.08982849

$-175.21063232$

103.62590790

160.78512573

$-100.95922089$

$-7.78273296$

$-5.31128073$

21.30231094

$-2.46510673$

1.55843389

11.20804310

$-13.58041286$

$-4.79616117$

$-21.55818939$

9.62417030

15.31237984

$-6.92865753$

20.26085854

$-11.29637814$

$-14.57664871$ 8.30081749

Electr. mom.

$-0.02169468$

0.08414077

$-0.02169468$

0.08414077

$-0.02169468$

0.08414077

$-0.02169468$

0.08414077

$-0.36857194$

$-0.26273650$
$-245.95677185$

217.71755981

229.78945923

$-196.20129395$

201.50679016

$-178.35360718$

$-185.16558838$

158.06970215

$-46.11359787$

3. 72287250 
$-1.37606192$

$-1.37606192$

$-1.27022636$

$-1.27022636$

$-1.27022636$

$-1.27022636$

2. 05802321

2. 05802321

2. 05802321

2. 05802321

2.16385865

2.16385865

2.16385865

2.16385865

$-1.68407583$

$-1.68407583$

$-1.68407583$

$-1.68407583$

$-1.57824039$

$-1.57824039$

$-1.57824039$

$-1.57824039$

1.02599049

1.02599049

1. 02599049

1.02599049

1. 13182592

1. 13182592

1. 13182592

1.13182592

$-2.12678075$

$-2.12678075$

$-2.12678075$

$-2.12678075$

$-2.02094531$

$-2.02094531$

$-2.02094531$

$-2.02094531$

1.00072026

1.00072026

1. 00072026

1.00072026

1.10655570

1.10655570

1.10655570

1. 10655570

$-1.75219464$

$-1.75219464$

$-1.75219464$

$-1.75219464$

$-1.64635921$

$-1.64635921$

$-1.64635921$

64

$-1.64635921$

1.9563

2. 03096962

2.03096962

2.03096962

2. 03096962

2.13680482

2. 13680482

2.13680482

2.13680482

$-1.37606192$

$-1.37606192$

$-1.37606192$
$-0.35442120$

$-0.35442120$

$-0.46025667$

$-0.46025667$

$-0.35442120$

$-0.35442120$

1. 37286413

1.37286413

1.47869956

1.47869956

1.37286413

1. 37286413

1. 47869956

1. 47869956

$-1.58903348$

$-1.58903348$

$-1.48319805$

$-1.48319805$

$-1.58903348$

$-1.58903348$

$-1.48319805$

$-1.48319805$

$-0.66542661$

$-0.66542661$

$-0.55959117$

$-0.55959117$

$-0.66542661$

$-0.66542661$

$-0.55959117$

$-0.55959117$

0.64235818

0.64235818

0.74819362

0.74819362

0.64235818

0.64235818

0.74819362

0.74819362

$-1.60289264$

$-1.60289264$

$-1.49705720$

$-1.49705720$

$-1.60289264$

$-1.60289264$

$-1.49705720$

$-1.49705720$

1. 54989612

1.54989612

1.65573156

1.65573156

1.54989612

1. 54989612

1.65573156

1.65573156

0 .

0.6610

\# Energy 5->5

0.19347408

0.19347408

0.29930952

0.29930952

0.19347408

0.19347408

0.29930952

0.29930952

$-0.46025667$

$-0.46025667$

$-0.35442120$
$-0.36857194$

$-0.26273650$

$-0.36857194$

$-0.26273650$

$-0.36857194$

$-0.26273650$

$-0.39818472$

$-0.29234925$

$-0.39818472$

$-0.29234925$

$-0.39818472$

$-0.29234925$

$-0.39818472$

$-0.29234925$

0.04071225

0.14654770

0.04071225

0.14654770

0.04071225

0.14654770

0.04071225

0.14654770

$-0.36399299$

$-0.25815752$

$-0.36399299$

$-0.25815752$

$-0.36399299$

$-0.25815752$

$-0.36399299$

$-0.25815752$

$-0.12852763$

$-0.02269218$

$-0.12852763$

$-0.02269218$

$-0.12852763$

$-0.02269218$

$-0.12852763$

$-0.02269218$

0.02148618

0.12732163

0.02148618

0.12732163

0.02148618

0.12732163

0.02148618

0.12732163

$-0.37628788$

$-0.27045241$

$-0.37628788$

$-0.27045241$

$-0.37628788$

$-0.27045241$

$-0.37628788$

$-0.27045241$

$$
5
$$

$-0.02169468$

0.08414077

$-0.02169468$

0.08414077

$-0.02169468$

0.08414077

$-0.02169468$

0.08414077

$-0.36857194$

$-0.26273650$

$-0.36857194$
26.10936165

29.83163452

59.42140579

$-35.20169067$

$-40.53909683$

4.87431288

$-13.01878929$

6.76154137

9.19765854

$-4.90977335$

5.56204414

$-0.24359351$

$-2.65633512$

$-1.55818808$

$-6.06156158$

11.99479580

9.65136623

$-17.99456787$

$-2.59258270$

$-6.44120169$

1. 15705979

10.53599930

159.98344421

$-236.02255249$

$-180.77308655$

257.65515137

$-202.26766968$

287.24914551

226.37960815

$-312.71237183$

92.78849792

$-50.68195724$

$-84.84839630$

51.54985809

$-132.93006897$

69.02113342

123.57773590

$-70.08766937$

$-11.74870014$

$-0.87943560$

24.98584747

$-6.90953398$

6.60015583

5.84404182

$-18.63400269$

0.77713919

$-16.36538887$ 3.86929679

11.36193466

$-2.34049940$

15.85371113

$-6.31047583$

$-11.12137604$

4. 24114132

Electr. mom.

$-234.43948364$

208.59217834

216.71348572

$-185.99363708$

192.96847534

$-171.85963440$

$-175.49264526$

150.78150940

$-67.15034485$

26.09436417

46.86204529 


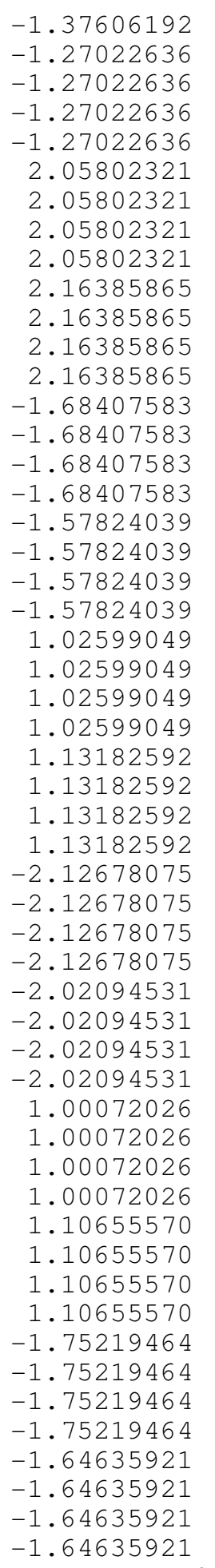

64
$-0.35442120$

$-0.46025667$

$-0.46025667$

$-0.35442120$

$-0.35442120$

1. 37286413

1.37286413

1.47869956

1. 47869956

1.37286413

1.37286413

1. 47869956

1.47869956

$-1.58903348$

$-1.58903348$

$-1.48319805$

$-1.48319805$

$-1.58903348$

$-1.58903348$

$-1.48319805$

$-1.48319805$

$-0.66542661$

$-0.66542661$

$-0.55959117$

$-0.55959117$

$-0.66542661$

$-0.66542661$

$-0.55959117$

$-0.55959117$

0.64235818

0.64235818

0.74819362

0.74819362

0.64235818

0.64235818

0.74819362

0.74819362

$-1.60289264$

$-1.60289264$

$-1.49705720$

$-1.49705720$

$-1.60289264$

$-1.60289264$

$-1.49705720$

$-1.49705720$

1. 54989612

1. 54989612

1.65573156

1.65573156

1. 54989612

1. 54989612

1.65573156

1.65573156

0 . \# Energy 6->6

4.9504
2.03096962

2. 03096962

2.03096962

2.03096962

2.13680482

2.13680482

2.13680482

2.13680482

$-1.37606192$

$-1.37606192$

$-1.37606192$

$-1.37606192$

0.19347408

0.19347408

0.29930952

0.29930952

0.19347408

0.19347408

0.29930952

0.29930952

$-0.46025667$

$-0.46025667$

$-0.35442120$

$-0.35442120$ $0.9610 \quad-0.4398$
$-0.26273650$

$-0.36857194$

$-0.26273650$

$-0.36857194$

$-0.26273650$

$-0.39818472$

$-0.29234925$

$-0.39818472$

$-0.29234925$

$-0.39818472$

$-0.29234925$

$-0.39818472$

$-0.29234925$

0.04071225

0.14654770

0.04071225

0.14654770

0.04071225

0.14654770

0.04071225

0.14654770

$-0.36399299$

$-0.25815752$

$-0.36399299$

$-0.25815752$

$-0.36399299$

$-0.25815752$

$-0.36399299$

$-0.25815752$

$-0.12852763$

$-0.02269218$

$-0.12852763$

$-0.02269218$

$-0.12852763$

$-0.02269218$

$-0.12852763$

$-0.02269218$

0.02148618

0.12732163

0.02148618

0.12732163

0.02148618

0.12732163

0.02148618

0.12732163

$-0.37628788$

$-0.27045241$

$-0.37628788$

$-0.27045241$

$-0.37628788$

$-0.27045241$

$-0.37628788$

$-0.27045241$

8.99165058

74.96607971

$-51.94801712$

$-55.70579147$

20.25336838

$-11.97620392$

6.51276016

7.54425240

$-3.76141834$

5.38490105

$-0.39802858$

$-1.86686027$

$-2.14071822$

$-6.88147211$

12.06510162

10.34341908

$-17.85568619$

$-2.93409896$

$-5.20077133$

1.73962247

8.87513256

156.69030762

$-232.15356445$

$-178.46716309$

254.88337708

$-198.49838257$

282.96145630

223.20028687

$-308.79873657$

122.20439911

$-76.17362976$

$-111.82778168$

74.87538910

$-170.65116882$

100.06785583

158.29501343

$-98.76426697$

$-13.02483654$

0.49686790

26.24338531

$-8.33969402$

8.06559086

4.39245415

$-20.14407349$

2. 29452062

$-19.90995216$

6.96271944

13.94170761

$-4.64919424$

18.63273048

$-8.83464336$

$-13.17397499$

6.11944389

Electr. mom.

$-0.02169468$

$-217.62454224$

198.96850586

201.90017700

$-178.22171021$

183.81895447

$-168.75914001$

$-168.23605347$

149.41137695

$-82.70021057$

47.16651535

61.67767334

$-11.27306461$
$-0.26273650$
$-0.36857194$ 


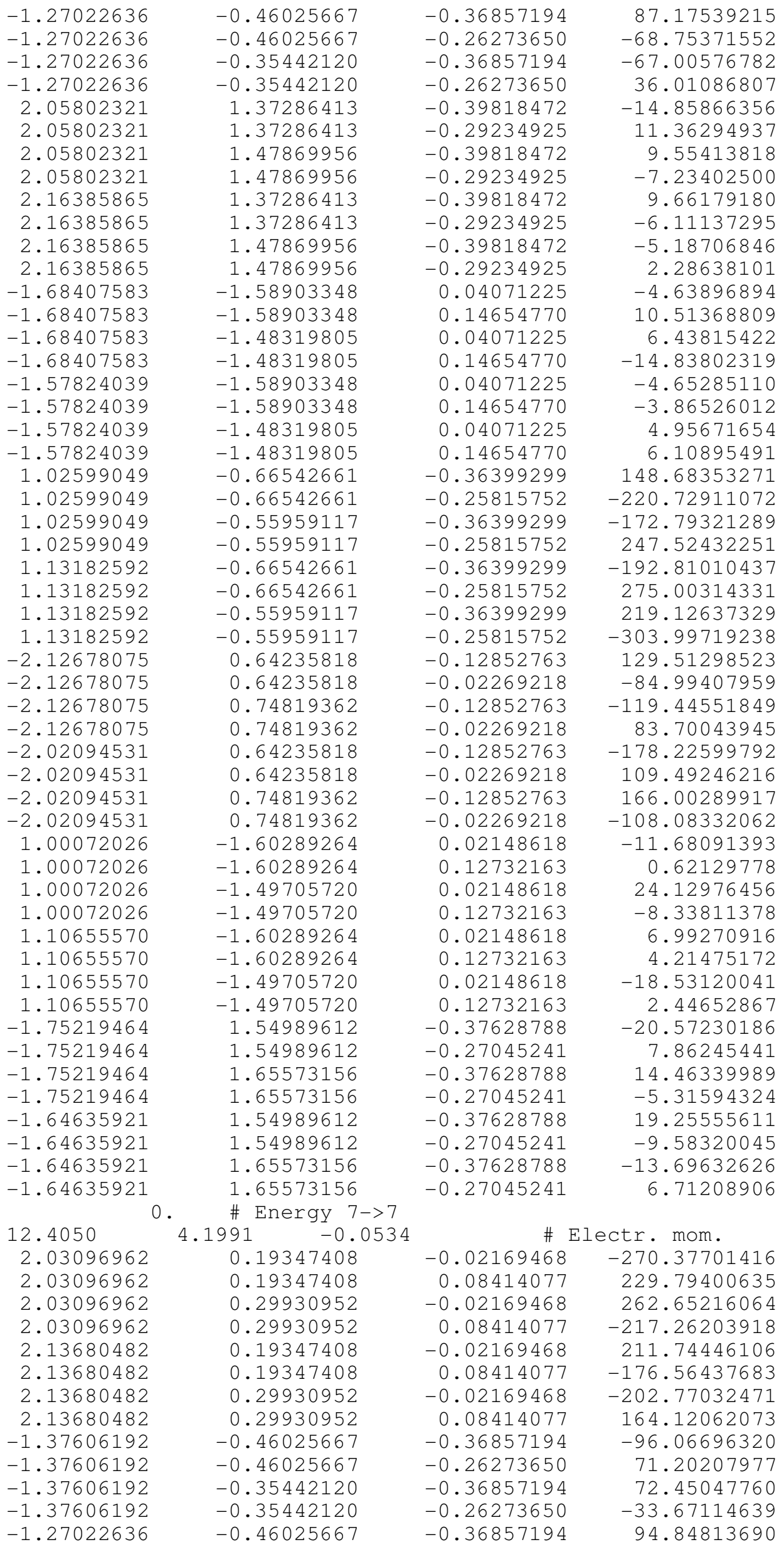

\section{S-225}




\begin{tabular}{|c|c|c|c|}
\hline-1.27022636 & -0.46025667 & -0.26273650 & -84.56722260 \\
\hline-1.27022636 & -0.35442120 & -0.36857194 & -71.86264038 \\
\hline-1.27022636 & -0.35442120 & -0.26273650 & 49.58371735 \\
\hline 2.05802321 & 1.37286413 & -0.39818472 & -25.14019966 \\
\hline 2.05802321 & 1.37286413 & -0.29234925 & 18.49551773 \\
\hline 2.05802321 & 1.47869956 & -0.39818472 & 28.70871735 \\
\hline 2.0580232 & 1.47869956 & -0.29234925 & -24.4943714 \\
\hline 2.1638586 & 1.37286413 & -0.39818472 & 8.6701736 \\
\hline 2.1638586 & 1.37286413 & -0.29234925 & -1.2564448 \\
\hline 2.16 & 1.47869956 & -0.39818472 & -15.2 \\
\hline 2.1638586 & 1.47869956 & -0.29234925 & 9.96 \\
\hline-1.68407583 & -1.58903348 & 0.04071225 & -7.106771 \\
\hline 1.6840758 & -1.58903348 & 0.14654770 & $12.7^{7}$ \\
\hline 1.6840758 & -1.48319805 & 0.04071225 & 7.62 \\
\hline-1.684075 & -1.48319805 & 0.14654770 & -16.02 \\
\hline-1.578240 & -1.58903348 & 0.04071225 & -2.95 \\
\hline-1.57 & -1.58903348 & 0.1465 & -5.2 \\
\hline-1.57 & -1.48319805 & 0.0407 & \\
\hline-1 & -1.483 & 0.146 & 6.2 \\
\hline 1. & -0.66542661 & -0.36399299 & 132.0 \\
\hline 1. & -0.66542661 & -0.25815752 & -200.9 \\
\hline 1. & -0.55959117 & -0.36399299 & -156.1 \\
\hline 1.02 & -0.55959117 & -0.25815752 & 226.8 \\
\hline 1.13 & -0.66542661 & -0.36399299 & -169.9 \\
\hline 1. & -0.66542661 & -0.25815752 & 246.9 \\
\hline 1 . & -0.55959117 & -0.36399299 & 197.0 \\
\hline 1.13 & -0.55959117 & -0.25815752 & -276.08 \\
\hline-2 . & 0.64235818 & -0.128 & 137.4 \\
\hline-2 . & 0.64 & -0.02 & -99.5 \\
\hline-2 . & 0.74 & -0.12 & -127.5 \\
\hline-2 . & 0.74 & -0.02 & 97.8 \\
\hline-2 . & 0.64 & -0.1 & -184.1 \\
\hline-2 . & 0.6 & -0.02 & 125.0 \\
\hline-2 . & 0.74 & -0.1 & 172.3 \\
\hline-2 . & 0.74819 & -0.022 & $-123 \cdot 2$ \\
\hline 1. & -1.602 & 0.02 & -7.2 \\
\hline 1 . & -1.60289264 & 0.12 & -5.1 \\
\hline 1.00072 & -1.49705720 & 0.02 & 18.9 \\
\hline 1.00 & -1.49705720 & 0.12 & $-1 \cdot 63$ \\
\hline 1. & -1.60 & 0 . & 1 \\
\hline 1 . & -1.60289264 & 0.12732 & 10.8 \\
\hline 1.10655 & -1.49705720 & 0.021 & $-11 \cdot 2$ \\
\hline 1 . & -1.49705720 & 0.12 & -5.4 \\
\hline-1 & 1.54 & -0.37 & -20.6 \\
\hline-1 & 1.549 & -0.27 & 8.4 \\
\hline-1 . & 1.6557 & -0.376 & 14.3 \\
\hline-1 & 1.6557 & -0.27 & -5.5 \\
\hline-1 & 1.54 & -0 & 18.2 \\
\hline-1 . & 1.549 & -0 . & -8.4 \\
\hline-1 . & 1.65573156 & -0.37 & -12.7 \\
\hline-1 . & 1.65573156 & -0.27045241 & 5.6 \\
\hline & \# Energy 8->8 & & \\
\hline 11 & 0.4870 & & ectr. m \\
\hline 2 & 0.193 & -0.02 & -289.5 \\
\hline 2 & 0.19 & 0 . & 270.1 \\
\hline 2. & 0.29930952 & -0.02169468 & 274.7 \\
\hline & 0.29930952 & 0.08414077 & -246.7 \\
\hline 2 . & 0. & -0 & 241.0 \\
\hline 2 . & 0.19347 & 0 & -227.1 \\
\hline 2 . & 0.29930952 & -0.02169468 & -225.5 \\
\hline 2. & 0.2993 & 4077 & 204.7 \\
\hline & -0.46025 & $\$ 857194$ & 9.0 \\
\hline & -0.46025667 & -0.26273650 & -69.1 \\
\hline-1 & -0 . & -0 . & $-31 \cdot 1$ \\
\hline-1 & -0 & -0 . & 105 \\
\hline & -0.4 & -0.36 & 17. \\
\hline & 0 & -0.2 & \\
\hline
\end{tabular}




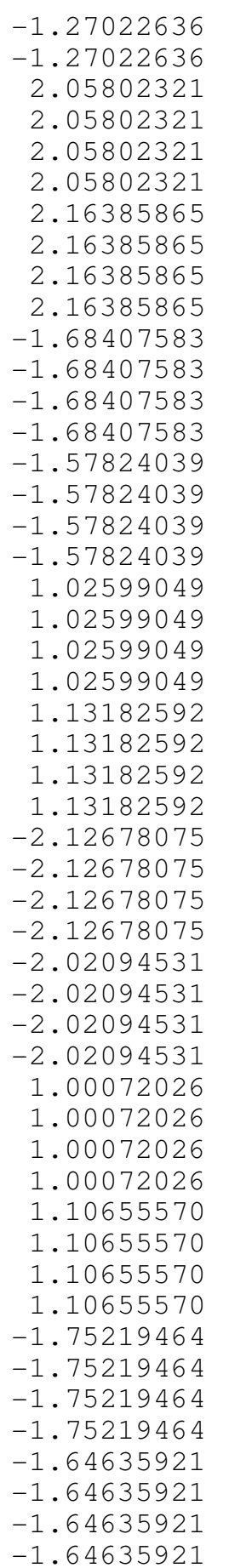

64
$-0.35442120$

$-0.35442120$

1. 37286413

1.37286413

1. 47869956

1. 47869956

1. 37286413

1. 37286413

1.47869956

1.47869956

$-1.58903348$

$-1.58903348$

$-1.48319805$

$-1.48319805$

$-1.58903348$

$-1.58903348$

$-1.48319805$

$-1.48319805$

$-0.66542661$

$-0.66542661$

$-0.55959117$

$-0.55959117$

$-0.66542661$

$-0.66542661$

$-0.55959117$

$-0.55959117$

0.64235818

0.64235818

0.74819362

0.74819362

0.64235818

0.64235818

0.74819362

0.74819362

$-1.60289264$

$-1.60289264$

$-1.49705720$

$-1.49705720$

$-1.60289264$

$-1.60289264$

$-1.49705720$

$-1.49705720$

1. 54989612

1. 54989612

1.65573156

1.65573156

1. 54989612

1.54989612

1.65573156

1.65573156

0 . \# Energy 9->9

$\begin{array}{lll}-6.6064 & -1.2839 & -0.1162\end{array}$

2.03096962

2.03096962

2.03096962

2. 03096962

2. 13680482

2.13680482

2.13680482

2.13680482

$-1.37606192$

$-1.37606192$

$-1.37606192$

$-1.37606192$

$-1.27022636$

$-1.27022636$

$-1.27022636$$$
-1.2839-0 \text {. }
$$

0.19347408

0.29930952

0.29930952

0.19347408

0.19347408

0.29930952

0.29930952

$-0.46025667$

$-0.46025667$

$-0.35442120$

$-0.35442120$

$-0.46025667$

$-0.46025667$

$-0.35442120$
$-0.36857194$

$-0.26273650$

$-0.39818472$

$-0.29234925$

$-0.39818472$

$-0.29234925$

$-0.39818472$

$-0.29234925$

$-0.39818472$

$-0.29234925$

0.04071225

0.14654770

0.04071225

0.14654770

0.04071225

0.14654770

0.04071225

0.14654770

$-0.36399299$

$-0.25815752$

$-0.36399299$

$-0.25815752$

$-0.36399299$

$-0.25815752$

$-0.36399299$

$-0.25815752$

$-0.12852763$

$-0.02269218$

$-0.12852763$

$-0.02269218$

$-0.12852763$

$-0.02269218$

$-0.12852763$

$-0.02269218$

0.02148618

0.12732163

0.02148618

0.12732163

0.02148618

0.12732163

0.02148618

0.12732163

$-0.37628788$

$-0.27045241$

$-0.37628788$

$-0.27045241$

$-0.37628788$

$-0.27045241$

$-0.37628788$

$-0.27045241$
2. 72598958

$-52.61500931$

$-20.36290550$

14.20491028

15.91625881

$-11.83127117$

12.55760288

$-6.99900198$

$-9.19444942$

4.80157614

$-2.15531707$

9.30265808

9.57736492

$-19.06561089$

$-6.42008305$

$-4.91925764$

1.79396832

12.83643818

215.39778137

$-315.76412964$

$-238.09918213$

340.88159180

$-270.73645020$

385.35549927

297.70431519

$-415.50012207$

64.67737579

$-21.04703331$

$-59.23271561$

25.38598824

$-98.71134949$

30.81990433

92.35348511

$-35.93129730$

$-13.13530827$

$-0.16784295$

28.48300171

$-8.84920883$

9.08173275

4.73325634

$-23.94876862$ 3.45336509

$-13.26154518$

$-0.41573185$

9.69707680

0.03756093

14.52915192

$-4.53568125$

$-10.65702724$

3. 62117124
$-0.02169468$
0.08414077
$-0.02169468$
0.08414077
$-0.02169468$
0.08414077
$-0.02169468$
0.08414077
$-0.36857194$
$-0.26273650$
$-0.36857194$
$-0.26273650$
$-0.36857194$
$-0.26273650$
$-0.36857194$

Electr. mom.

$-265.07736206$

247.73905945

249.35453796

$-223.89955139$

219.01873779

$-206.81600952$

$-202.48583984$

183.71069336

$-26.85108757$

$-41.89929199$

8.29756832

75.74657440

45.87664413

0.04500496

$-29.16689873$ 


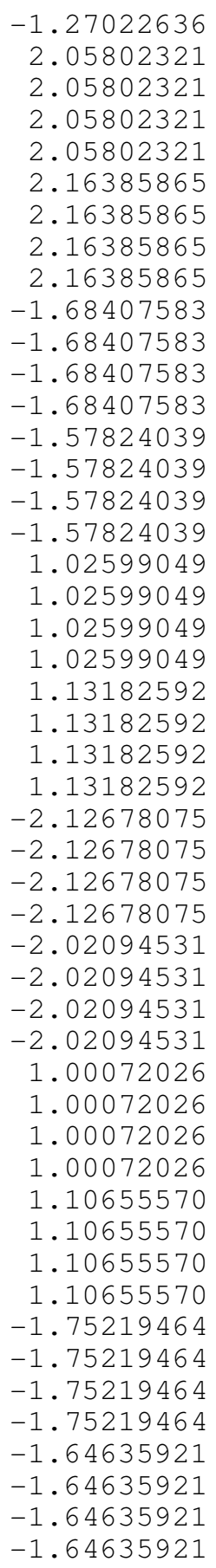
$-\mathrm{CT} 08009 \mathrm{~A}-$

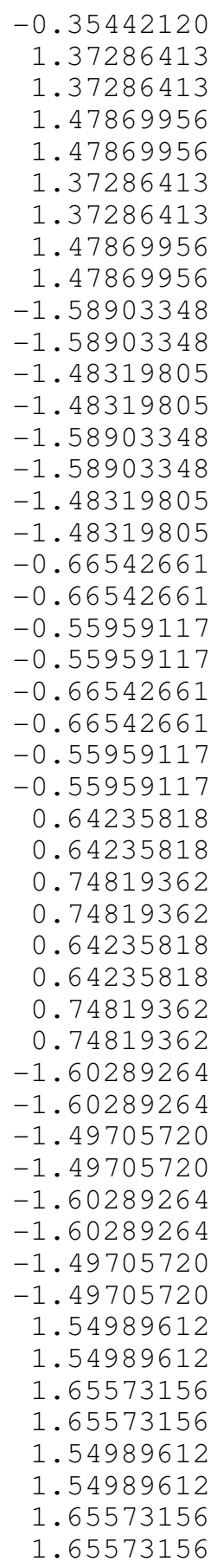

$60, \operatorname{root} s=9$

\begin{tabular}{|c|c|}
\hline & \\
\hline-0.26273650 & -29.25893402 \\
\hline-0.39818472 & -1 \\
\hline-0.29234925 & 12.051 \\
\hline-0.39818472 & 13.3 \\
\hline-0.29234925 & -9.4 \\
\hline-0.39818472 & \\
\hline-0.29234925 & -3.9678 \\
\hline-0.39818472 & -6.07 \\
\hline-0.29234925 & 1.64 \\
\hline 0.04071225 & -11.810952 \\
\hline 0.14654770 & 13.46 \\
\hline 0.04071225 & $16.3^{\circ}$ \\
\hline 0.14654770 & -19.37997 \\
\hline 0.04071225 & 2.40331 \\
\hline 0.14654770 & -7.4 \\
\hline 0.04071225 & -4.2 \\
\hline 54770 & 11 \\
\hline-0.36399299 & 202 \\
\hline 5752 & -301 \\
\hline-0.36 & -223 \\
\hline-0.25 & 324 \\
\hline 9299 & -253 \\
\hline 5752 & 365 . \\
\hline 299 & 278 \\
\hline-0.25815752 & -392 \\
\hline-0.12852763 & 99.4 \\
\hline 9218 & -48.1 \\
\hline 52763 & -91 \\
\hline 59218 & 50 \\
\hline 2763 & -146. \\
\hline-0 & 67. \\
\hline 52763 & 136 \\
\hline 59218 & -70 \\
\hline 0. & -15 \\
\hline 0 & 0 . \\
\hline 8618 & 30.6 \\
\hline 163 & -9.9 \\
\hline 0 . & 10 \\
\hline 2163 & 3.74 \\
\hline 0.02 & -25.52 \\
\hline 0. & 4. \\
\hline-0.37 & -16.8 \\
\hline-0.27 & 2.5 \\
\hline 8788 & 12. \\
\hline-0 & -2.0 \\
\hline-0 . & 16.8 \\
\hline-0 . & -6 \\
\hline-0 . & 12. \\
\hline-0 . & 4 . \\
\hline
\end{tabular}

1.0000

1.0000

1.0000

1.0000

1.0000

1.0000

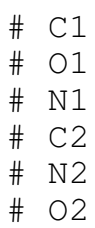

\&TRANSITION $1->\ldots$

64
44207 .

\footnotetext{
1.000 \# Electr. mom., scale-fac \# Magnetic mom.

$-0.13595992$

$-0.03012447$

$-0.13595992$

$-0.03012447$

$-0.13595992$

17.16557121

$-21.51730919$

$-18.91749382$

24.93519592

$-12.52886581$
} 
$-1.94716847$

$-1.94716847$

$-1.94716847$

1. 40458930

1. 40458930

1. 40458930

1.40458930

1.51042473

1. 51042473

1.51042473

1.51042473

$-2.05135775$

$-2.05135775$

$-2.05135775$

$-2.05135775$

$-1.94552219$

$-1.94552219$

$-1.94552219$

$-1.94552219$

2. 33443308

2. 33443308

2. 33443308

2. 33443308

2. 44026875

2. 44026875

2. 44026875

2. 44026875

$-1.00705183$

$-1.00705183$

$-1.00705183$

$-1.00705183$

$-0.90121639$

$-0.90121639$

$-0.90121639$

$-0.90121639$

1. 51851332

1. 51851332

1. 51851332

1. 51851332

1.62434876

1. 62434876

1. 62434876

1.62434876

$-1.10039973$

$-1.10039973$

$-1.10039973$

$-1.10039973$

$-0.99456429$

$-0.99456429$

$-0.99456429$

$-0.99456429$

0.70601028

0.70601028

0.70601028

0.70601028

0.81184572

0.81184572

0.81184572

0.81184572 43589
$-0.19663274$

$-0.09079729$

$-0.09079729$

0.53337198

0.53337198

0.63920748

0.63920748

0.53337198

0.53337198

0.63920748

0.63920748

$-0.61681372$

$-0.61681372$

$-0.51097828$

$-0.51097828$

$-0.61681372$

$-0.61681372$

$-0.51097828$

$-0.51097828$

1. 26356936

1. 26356936

1. 36940491

1. 36940491

1. 26356936

1. 26356936

1. 36940491

1.36940491

0.52339011

0.52339011

0.62922561

0.62922561

0.52339011

0.52339011

0.62922561

0.62922561

$-0.81257546$

$-0.81257546$

$-0.70673996$

$-0.70673996$

$-0.81257546$

$-0.81257546$

$-0.70673996$

$-0.70673996$

0.90373951

0.90373951

1.00957489

1.00957489

0.90373951

0.90373951

1. 00957489

1.00957489

$-1.34548187$

$-1.34548187$

$-1.23964632$

$-1.23964632$

$-1.34548187$

$-1.34548187$

$-1.23964632$

$-1.23964632$

\# Energy 1->3

$$
\begin{aligned}
& -0.03012447 \\
& -0.13595992 \\
& -0.03012447 \\
& -0.10552958 \\
& 0.00030586 \\
& -0.10552958 \\
& 0.00030586 \\
& -0.10552958 \\
& 0.00030586 \\
& -0.10552958 \\
& 0.00030586 \\
& -1.29993546 \\
& -1.19410002 \\
& -1.29993546 \\
& -1.19410002 \\
& -1.29993546 \\
& -1.19410002 \\
& -1.29993546 \\
& -1.19410002 \\
& 0.26371813 \\
& 0.36955357 \\
& 0.26371813 \\
& 0.36955357 \\
& 0.26371813 \\
& 0.36955357 \\
& 0.26371813 \\
& 0.36955357 \\
& 0.37719119 \\
& 0.48302662 \\
& 0.37719119 \\
& 0.48302662 \\
& 0.37719119 \\
& 0.48302662 \\
& 0.37719119 \\
& 0.48302662 \\
& -0.22067484 \\
& -0.11483940 \\
& -0.22067484 \\
& -0.11483940 \\
& -0.22067484 \\
& -0.11483940 \\
& -0.22067484 \\
& -0.11483940 \\
& \text { 1. } 31258702 \\
& \text { 1. } 41842246 \\
& \text { 1. } 31258702 \\
& \text { 1. } 41842246 \\
& \text { 1. } 31258702 \\
& \text { 1. } 41842246 \\
& \text { 1. } 31258702 \\
& \text { 1. } 41842246 \\
& -0.51015860 \\
& -0.40432316 \\
& -0.51015860 \\
& -0.40432316 \\
& -0.51015860 \\
& -0.40432316 \\
& -0.51015860 \\
& -0.40432316 \\
& 17.66453743 \\
& 14.08520126 \\
& -21.15651703 \\
& -6.25289249 \\
& 7.11170292 \\
& \text { 7. } 45439291 \\
& -8.45404339 \\
& 6.97736311 \\
& -8.01693344 \\
& -8.16744614 \\
& \text { 9.34064007 } \\
& 6.14022732 \\
& -7.81611729 \\
& -5.61653519 \\
& 7.27287149 \\
& -3.46094561 \\
& 4.56156445 \\
& 2.55664134
\end{aligned}
$$
$\begin{array}{lll}-0.1637 & -0.1089 & 0.1007\end{array}$
$-0.6005 \quad-0.5013 \quad-0.2811$
$-2.05300403$
$-2.05300403$
$-2.05300403$
$-2.05300403$
$-0.19663274$
$-0.19663274$
$-0.09079729$
$-0.09079729$
$-0.19663274$

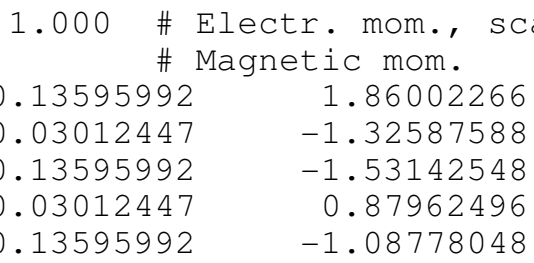


$-1.94716847$

$-1.94716847$

$-1.94716847$

1. 40458930

1. 40458930

1. 40458930

1.40458930

1. 51042473

1. 51042473

1.51042473

1.51042473

$-2.05135775$

$-2.05135775$

$-2.05135775$

$-2.05135775$

$-1.94552219$

$-1.94552219$

$-1.94552219$

$-1.94552219$

2. 33443308

2. 33443308

2. 33443308

2. 33443308

2. 44026875

2. 44026875

2. 44026875

$-1.00705183$

$-1.00705183$

$-1.00705183$

$-1.00705183$

$-0.90121639$

$-0.90121639$

$-0.90121639$

$-0.90121639$

1. 51851332

1. 51851332

1. 51851332

1. 51851332

1.62434876

1. 62434876

1. 62434876

1.62434876

$-1.10039973$

$-1.10039973$

$-1.10039973$

$-1.10039973$

$-0.99456429$

$-0.99456429$

$-0.99456429$

$-0.99456429$

0.70601028

0.70601028

0.70601028

0.70601028

0.81184572

0.81184572

0.81184572

0.81184572

64
2. 44026875

$-0.19663274$

$-0.09079729$

$-0.09079729$

0.53337198

0.53337198

0.63920748

0.63920748

0.53337198

0.53337198

0.63920748

0.63920748

$-0.61681372$

$-0.61681372$

$-0.51097828$

$-0.51097828$

$-0.61681372$

$-0.61681372$

$-0.51097828$

$-0.51097828$

1. 26356936

1. 26356936

1. 36940491

1. 36940491

1.26356936

1. 26356936

1. 36940491

1.36940491

0.52339011

0.52339011

0.62922561

0.62922561

0.52339011

0.52339011

0.62922561

0.62922561

$-0.81257546$

$-0.81257546$

$-0.70673996$

$-0.70673996$

$-0.81257546$

$-0.81257546$

$-0.70673996$

$-0.70673996$

0.90373951

0.90373951

1.00957489

1.00957489

0.90373951

0.90373951

1.00957489

1.00957489

$-1.34548187$

$-1.34548187$

$-1.23964632$

$-1.23964632$

$-1.34548187$

$-1.34548187$

$-1.23964632$

$-1.23964632$

\# Energy 1->4

$\begin{array}{lll}0.6042 & 3.2266 & 0.3436\end{array}$

$-0.1785$

$-2.05300403$

$-2.05300403$

$-2.05300403$

$-2.05300403$

$-1.94716847$

$$
0.3786-0.7865
$$

$-0.19663274$

$-0.19663274$

$-0.09079729$

$-0.09079729$

$-0.19663274$
$-0.03012447$

$-0.13595992$

$-0.03012447$

$-0.10552958$

0.00030586

$-0.10552958$

0.00030586

$-0.10552958$

0.00030586

$-0.10552958$

0.00030586

$-1.29993546$

$-1.19410002$

$-1.29993546$

$-1.19410002$

$-1.29993546$

$-1.19410002$

$-1.29993546$

$-1.19410002$

0.26371813

0.36955357

0.26371813

0.36955357

0.26371813

0.36955357

0.26371813

0.36955357

0.37719119

0.48302662

0.37719119

0.48302662

0.37719119

0.48302662

0.37719119

0.48302662

$-0.22067484$

$-0.11483940$

$-0.22067484$

$-0.11483940$

$-0.22067484$

$-0.11483940$

$-0.22067484$

$-0.11483940$

1.31258702

1. 41842246

1. 31258702

1. 41842246

1.31258702

1. 41842246

1.31258702

1. 41842246

$-0.51015860$

$-0.40432316$

$-0.51015860$

$-0.40432316$

$-0.51015860$

$-0.40432316$

$-0.51015860$

$-0.40432316$
0.45091310

0.67161310

0.12177196

$-12.10792828$

5.86220074

13.28503418

$-9.02734470$

8.02767181

$-3.38209105$

$-10.01179314$

7.48305559

0.25523338

$-0.32329580$

$-0.11923231$

0.14091372

$-0.16340593$

0.19926724

$-0.01542019$

0.03113722

$-9.33791447$

5.16360331

4. 19051790

$-1.01335084$

12.36306858

$-9.15705109$

$-7.51840687$

5.08857346

$-13.39725780$

13.86221886

16.87489891

$-18.06035423$

15.23284531

$-16.01665688$

$-19.49590683$

21.06550026

$-10.44475365$

3.17680097

10.02371883

$-1.79997993$

8. 25527287

$-1.72034967$

$-7.61180353$

0.18448034

$-1.48951781$

1. 37790394

2. 44778824

$-1.94434679$

1. 41752553

$-1.38577282$

$-2.64697742$

2.13199306

$-1.52559900$

0.98906219

1. 40595877

$-0.88106763$

1. 61368728

$-0.88520688$

$-1.39322722$

0.68721670

1.000 \# Electr. mom., scale-fac

\# Magnetic mom.

$-0.13595992$

$-0.03012447$

$-1.19714522$

$-0.13595992$

0.46959031

$-0.03012447$

0.43732592

0.60556346

$-0.13595992$

0.37575305

\section{S-230}


$-1.94716847$

$-1.94716847$

$-1.94716847$

1. 40458930

1. 40458930

1. 40458930

1.40458930

1.51042473

1. 51042473

1.51042473

1.51042473

$-2.05135775$

$-2.05135775$

$-2.05135775$

$-2.05135775$

$-1.94552219$

$-1.94552219$

$-1.94552219$

$-1.94552219$

2. 33443308

2. 33443308

2. 33443308

2. 33443308

2. 44026875

2. 44026875

2. 44026875

2. 44026875

$-1.00705183$

$-1.00705183$

$-1.00705183$

$-1.00705183$

$-0.90121639$

$-0.90121639$

$-0.90121639$

$-0.90121639$

1. 51851332

1. 51851332

1. 51851332

1. 51851332

1.62434876

1. 62434876

1. 62434876

1.62434876

$-1.10039973$

$-1.10039973$

$-1.10039973$

$-1.10039973$

$-0.99456429$

$-0.99456429$

$-0.99456429$

$-0.99456429$

0.70601028

0.70601028

0.70601028

0.70601028

0.81184572

0.81184572

0.81184572

0.81184572

64 49738 .
$-0.19663274$

$-0.09079729$

$-0.09079729$

0.53337198

0.53337198

0.63920748

0.63920748

0.53337198

0.53337198

0.63920748

0.63920748

$-0.61681372$

$-0.61681372$

$-0.51097828$

$-0.51097828$

$-0.61681372$

$-0.61681372$

$-0.51097828$

$-0.51097828$

1. 26356936

1. 26356936

1. 36940491

1. 36940491

1. 26356936

1. 26356936

1. 36940491

1.36940491

0.52339011

0.52339011

0.62922561

0.62922561

0.52339011

0.52339011

0.62922561

0.62922561

$-0.81257546$

$-0.81257546$

$-0.70673996$

$-0.70673996$

$-0.81257546$

$-0.81257546$

$-0.70673996$

$-0.70673996$

0.90373951

0.90373951

1.00957489

1.00957489

0.90373951

0.90373951

1. 00957489

1.00957489

$-1.34548187$

$-1.34548187$

$-1.23964632$

$-1.23964632$

$-1.34548187$

$-1.34548187$

$-1.23964632$

$-1.23964632$

\# Energy 1->5
$-0.03012447$

$-0.13595992$

$-0.03012447$

$-0.10552958$

0.00030586

$-0.10552958$

0.00030586

$-0.10552958$

0.00030586

$-0.10552958$

0.00030586

$-1.29993546$

$-1.19410002$

$-1.29993546$

$-1.19410002$

$-1.29993546$

$-1.19410002$

$-1.29993546$

$-1.19410002$

0.26371813

0.36955357

0.26371813

0.36955357

0.26371813

0.36955357

0.26371813

0.36955357

0.37719119

0.48302662

0.37719119

0.48302662

0.37719119

0.48302662

0.37719119

0.48302662

$-0.22067484$

$-0.11483940$

$-0.22067484$

$-0.11483940$

$-0.22067484$

$-0.11483940$

$-0.22067484$

$-0.11483940$

1. 31258702

1. 41842246

1. 31258702

1. 41842246

1.31258702

1. 41842246

1.31258702

1. 41842246

$-0.51015860$

$-0.40432316$

$-0.51015860$

$-0.40432316$

$-0.51015860$

$-0.40432316$

$-0.51015860$

$-0.40432316$
0.73431003

0.77590197

$-2.31396365$

5. 42438221

$-16.99555206$

$-9.99468899$

25.28907013

$-9.89391518$

26.07810974

15.46274471

$-35.91887283$

$-0.04571056$

$-0.09472079$

$-0.08275040$

0.29454315

$-0.32233334$

0.54517967

0.41183853

$-0.68776554$

15.84440708

$-6.87692356$

$-11.34976864$

5.96516466

$-11.41993904$

5.44797134

9.03161812

$-5.52517557$

18.56605911

$-16.05920792$

$-16.08575630$

15.38194370

$-18.57919312$

16.66337013

16.38777351

$-16.27268600$

9.64897251

$-14.50765419$

$-9.17308140$

14.77567387

$-10.12307453$

14.70442963

10.07283688

$-15.84346581$

3. 15418577

$-2.53970337$

$-3.45623279$

2.68599153

$-3.51776648$

2. 83759761

3. 88317394

$-3.02321672$

1.19157946

$-3.31258559$

$-0.94191259$

3. 44880581

$-1.56410396$

4.20881796

1. 22917986

$-4.31500101$
$\begin{array}{lll}1.7263 & 2.1603 & 2.5046\end{array}$

$-2.05300403$

$-2.05300403$

$-2.05300403$

$-2.05300403$

$-1.94716847$

$\begin{array}{cc}2.1603 & 2.5046 \\ -1.0666 & 0.5518 \\ -0.19663274 \\ -0.19663274 \\ -0.09079729 \\ -0.09079729 \\ -0.19663274\end{array}$

1.000 \# \#

$-0.13595992$

$-0.03012447$

$-0.13595992$

$-0.03012447$

$-0.13595992$
\# Electr. mom., scale-fac Magnetic mom. 
$-1.94716847$

$-1.94716847$

$-1.94716847$

1. 40458930

1. 40458930

1.40458930

1.51042473

1. 51042473

1.51042473

1.51042473

$-2.05135775$

$-2.05135775$

$-2.05135775$

$-2.05135775$

$-1.94552219$

$-1.94552219$

$-1.94552219$

$-1.94552219$

2. 33443308

2. 33443308

2. 33443308

2. 33443308

2. 44026875

2. 44026875

2. 44026875

2. 44026875

$-1.00705183$

$-1.00705183$

$-1.00705183$

$-1.00705183$

$-0.90121639$

$-0.90121639$

$-0.90121639$

$-0.90121639$

1. 51851332

1. 51851332

1. 51851332

1. 51851332

1.62434876

1. 62434876

1. 62434876

1.62434876

$-1.10039973$

$-1.10039973$

$-1.10039973$

$-1.10039973$

$-0.99456429$

$-0.99456429$

$-0.99456429$

$-0.99456429$

0.70601028

0.70601028

0.70601028

0.70601028

0.81184572

0.81184572

0.81184572

0.81184572

64
1. 40458930

$-0.19663274$

$-0.09079729$

$-0.09079729$

0.53337198

0.53337198

0.63920748

0.63920748

0.53337198

0.53337198

0.63920748

0.63920748

$-0.61681372$

$-0.61681372$

$-0.51097828$

$-0.51097828$

$-0.61681372$

$-0.61681372$

$-0.51097828$

$-0.51097828$

1. 26356936

1. 26356936

1. 36940491

1. 36940491

1. 26356936

1. 26356936

1. 36940491

1.36940491

0.52339011

0.52339011

0.62922561

0.62922561

0.52339011

0.52339011

0.62922561

0.62922561

$-0.81257546$

$-0.81257546$

$-0.70673996$

$-0.70673996$

$-0.81257546$

$-0.81257546$

$-0.70673996$

$-0.70673996$

0.90373951

0.90373951

1.00957489

1.00957489

0.90373951

0.90373951

1. 00957489

1.00957489

$-1.34548187$

$-1.34548187$

$-1.23964632$

$-1.23964632$

$-1.34548187$

$-1.34548187$

$-1.23964632$

$-1.23964632$

\# Energy 1->6

$\begin{array}{lll}-1.2571 & -0.5491 & 0.1961\end{array}$

$0.0373-0.1309 \quad-0.0088$

$-2.05300403$

$-2.05300403$

$-2.05300403$

$-2.05300403$

$-1.94716847$
$-0.19663274$

$-0.19663274$

$-0.09079729$

$-0.09079729$

$-0.19663274$
$-0.03012447$

$-0.13595992$

$-0.03012447$

$-0.10552958$

0.00030586

$-0.10552958$

0.00030586

$-0.10552958$

0.00030586

$-0.10552958$

0.00030586

$-1.29993546$

$-1.19410002$

$-1.29993546$

$-1.19410002$

$-1.29993546$

$-1.19410002$

$-1.29993546$

$-1.19410002$

0.26371813

0.36955357

0.26371813

0.36955357

0.26371813

0.36955357

0.26371813

0.36955357

0.37719119

0.48302662

0.37719119

0.48302662

0.37719119

0.48302662

0.37719119

0.48302662

$-0.22067484$

$-0.11483940$

$-0.22067484$

$-0.11483940$

$-0.22067484$

$-0.11483940$

$-0.22067484$

$-0.11483940$

1. 31258702

1. 41842246

1. 31258702

1. 41842246

1. 31258702

1. 41842246

1. 31258702

1. 41842246

$-0.51015860$

$-0.40432316$

$-0.51015860$

$-0.40432316$

$-0.51015860$

$-0.40432316$

$-0.51015860$

$-0.40432316$
5.01011276

1. 80674136

$-9.26656628$

$-21.23712730$

13.77935219

19.78784180

$-12.42273521$

18.32553101

$-10.67314339$

$-16.85549927$

8.97991371

$-4.68775034$

6.34642696

5.14938116

$-7.02447701$

6.66336489

$-8.73841190$

$-7.09787560$

9.33271503

4.60744715

$-2.30404115$

$-3.34918904$

1.98451257

$-3.06581688$

1. 49801469

2. 41474533

$-1.51345432$

$-4.81000853$

4.53305674

4. 53957415

$-3.95323491$

4.02353668

$-3.22302842$

$-2.87246966$

2. 31346774

$-7.07119370$

3. 01262927

8.55906010

$-3.53738213$

5.75156450

$-2.10492206$

$-6.96072721$

2. 42538166

$-2.16332674$

1. 46271920

2. 24695921

$-1.50850713$

1. 55163431

$-1.02763391$

$-1.90650654$

1. 26972806

0.24572590

$-1.34119880$

$-0.44824398$

1.88595307

$-0.43606889$

1.86393499

0.72737402

$-2.57387567$
1.000 \# Electr. mom., scale-fac \# Magnetic mom.

$-0.13595992$

$-0.03012447$

$-0.13595992$

$-0.03012447$

$-0.13595992$
3. 61542392

$-4.11865091$

$-3.98419476$

4.78073263

$-3.95448303$ 
$-1.94716847$

$-1.94716847$

$-1.94716847$

1. 40458930

1. 40458930

1.40458930

1.51042473

1. 51042473

1. 51042473

1.51042473

$-2.05135775$

$-2.05135775$

$-2.05135775$

$-2.05135775$

$-1.94552219$

$-1.94552219$

$-1.94552219$

2. 33443308

2. 33443308

2. 33443308

2. 33443308

2. 44026875

2. 44026875

2. 44026875

$-1.00705183$

$-1.00705183$

$-1.00705183$

$-1.00705183$

$-0.90121639$

$-0.90121639$

$-0.90121639$

$-0.90121639$

1. 51851332

1. 51851332

1. 51851332

1. 51851332

1.62434876

1. 62434876

1. 62434876

1.62434876

$-1.10039973$

$-1.10039973$

$-1.10039973$

$-1.10039973$

$-0.99456429$

$-0.99456429$

$-0.99456429$

$-0.99456429$

0.70601028

0.70601028

0.70601028

0.70601028

0.81184572

0.81184572

0.81184572

0.81184572

64

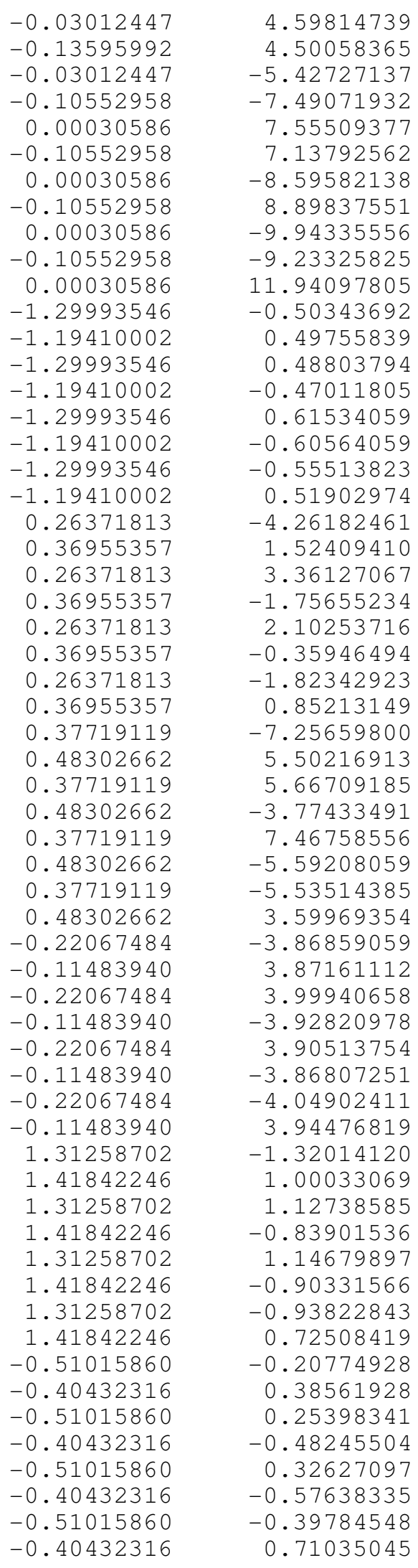

1. 40458930

$-1.94552219$

2. 44026875

$-0.19663274$

$-0.09079729$

$-0.09079729$

0.53337198

0.53337198

0.63920748

0.63920748

0.53337198

0.53337198

0.63920748

0.63920748

$-0.61681372$

$-0.61681372$

$-0.51097828$

$-0.51097828$

$-0.61681372$

$-0.61681372$

$-0.51097828$

$-0.51097828$

1. 26356936

1.26356936

1. 36940491

1. 36940491

1.26356936

1. 26356936

1. 36940491

1.36940491

0.52339011

0.52339011

0.62922561

0.62922561

0.52339011

0.52339011

0.62922561

0.62922561

$-0.81257546$

$-0.81257546$

$-0.70673996$

$-0.70673996$

$-0.81257546$

$-0.81257546$

$-0.70673996$

$-0.70673996$

0.90373951

0.90373951

1.00957489

1.00957489

0.90373951

0.90373951

1.00957489

1.00957489

$-1.34548187$

$-1.34548187$

$-1.23964632$

$-1.23964632$

$-1.34548187$

$-1.34548187$

$-1.23964632$

$-1.23964632$

\# Energy 1->7

$\begin{array}{lll}-2.0520 & -1.0554 & -1.5184\end{array}$

$\begin{array}{lll}0.0293 & 0.5170 & -0.5676\end{array}$

$-2.05300403$

$-2.05300403$

$-2.05300403$

$-2.05300403$

$-1.94716847$
$-0.19663274$

$-0.19663274$

$-0.09079729$

$-0.09079729$

$-0.19663274$
1.000 \# Electr. mom., scale-fac \# Magnetic mom.

$-0.13595992$

$-0.03012447$

$-0.13595992$

$-0.03012447$

$-0.13595992$
2.05262685

0.06151667

$-1.58513355$

$-1.31719863$

0.10822861 
$-1.94716847$

$-1.94716847$

$-1.94716847$

1. 40458930

1. 40458930

1. 40458930

1.40458930

1.51042473

1. 51042473

1.51042473

1.51042473

$-2.05135775$

$-2.05135775$

$-2.05135775$

$-2.05135775$

$-1.94552219$

$-1.94552219$

$-1.94552219$

$-1.94552219$

2. 33443308

2. 33443308

2. 33443308

2. 33443308

2. 44026875

2. 44026875

2. 44026875

2. 44026875

$-1.00705183$

$-1.00705183$

$-1.00705183$

$-1.00705183$

$-0.90121639$

$-0.90121639$

$-0.90121639$

$-0.90121639$

1. 51851332

1. 51851332

1. 51851332

1. 51851332

1.62434876

1. 62434876

1. 62434876

1.62434876

$-1.10039973$

$-1.10039973$

$-1.10039973$

$-1.10039973$

$-0.99456429$

$-0.99456429$

$-0.99456429$

$-0.99456429$

0.70601028

0.70601028

0.70601028

0.70601028

0.81184572

0.81184572

0.81184572

0.81184572

64
$-0.19663274$

$-0.09079729$

$-0.09079729$

0.53337198

0.53337198

0.63920748

0.63920748

0.53337198

0.53337198

0.63920748

0.63920748

$-0.61681372$

$-0.61681372$

$-0.51097828$

$-0.51097828$

$-0.61681372$

$-0.61681372$

$-0.51097828$

$-0.51097828$

1. 26356936

1. 26356936

1. 36940491

1. 36940491

1. 26356936

1. 26356936

1. 36940491

1.36940491

0.52339011

0.52339011

0.62922561

0.62922561

0.52339011

0.52339011

0.62922561

0.62922561

$-0.81257546$

$-0.81257546$

$-0.70673996$

$-0.70673996$

$-0.81257546$

$-0.81257546$

$-0.70673996$

$-0.70673996$

0.90373951

0.90373951

1.00957489

1.00957489

0.90373951

0.90373951

1.00957489

1.00957489

$-1.34548187$

$-1.34548187$

$-1.23964632$

$-1.23964632$

$-1.34548187$

$-1.34548187$

$-1.23964632$

$-1.23964632$

\# Energy 1->8

$\begin{array}{lll}0.7172 & 0.0260 & -0.2723\end{array}$

$\begin{array}{lll}-0.0406 & 0.2086 & 0.0511\end{array}$

$-2.05300403$

$-2.05300403$

$-2.05300403$

$-2.05300403$

$-1.94716847$
$-0.19663274$

$-0.19663274$

$-0.09079729$

$-0.09079729$

$-0.19663274$

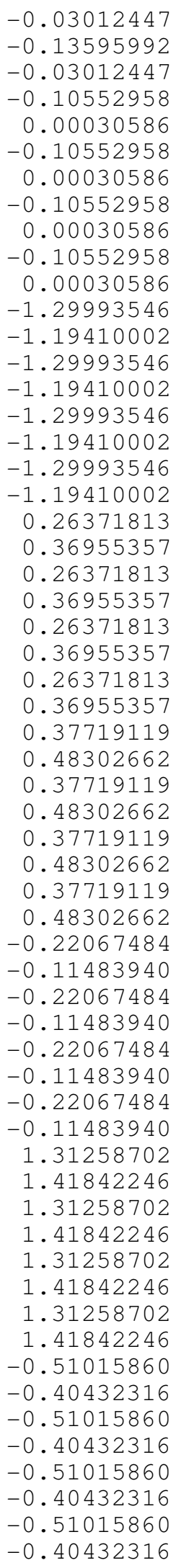

$-2.37478733$

$-0.09982540$

3.39585304

9.71506882

$-3.72073174$

$-7.53749752$

0.77959400

$-7.58695316$

1. 59601188

5.28537750

1.80747414

1.61897397

$-2.34036589$

$-1.58437681$

2.36358404

$-2.62810564$

3. 59819198

2. 59232664

$-3.59095716$

$-3.59181857$

0.92969817

2.61862922

$-0.86674654$

2. 57927227

$-0.74112052$

$-2.10798955$

0.91099602

2. 77164078

$-2.07554269$

$-2.60977459$

1.79667687

$-2.89168501$

1. 99882209

2. 24329495

$-1.49952447$

6.99798107

$-5.20455360$

$-7.53596878$

5.53201532

$-6.06554365$

4.62368155

6.56435871

$-5.00668335$

1.07082295

$-0.80882758$

$-1.14505363$

0.86811769

$-0.83698201$

0.65353721

0.99566060

$-0.77179396$

$-0.24788889$

0.45770854

0.38906202

$-0.62013203$

0.24495313

$-0.48267347$

$-0.42382509$

0.67830241
1.000 \# Electr. mom., scale-fac \# Magnetic mom.

$-0.13595992$

$-0.03012447$

$-0.13595992$

$-0.03012447$

$-0.13595992$

\author{
$-0.40446284$ \\ 1. 54566038 \\ 0.79111499 \\ $-2.51474333$ \\ 1. 57461023
}


$-1.94716847$

$-1.94716847$

$-1.94716847$

1. 40458930

1. 40458930

1. 40458930

1.40458930

1.51042473

1. 51042473

1.51042473

1.51042473

$-2.05135775$

$-2.05135775$

$-2.05135775$

$-2.05135775$

$-1.94552219$

$-1.94552219$

$-1.94552219$

$-1.94552219$

2. 33443308

2. 33443308

2. 33443308

2. 33443308

2. 44026875

2. 44026875

2. 44026875

$-1.00705183$

$-1.00705183$

$-1.00705183$

$-1.00705183$

$-0.90121639$

$-0.90121639$

$-0.90121639$

$-0.90121639$

1. 51851332

1. 51851332

1. 51851332

1. 51851332

1.62434876

1. 62434876

1. 62434876

1.62434876

$-1.10039973$

$-1.10039973$

$-1.10039973$

$-1.10039973$

$-0.99456429$

$-0.99456429$

$-0.99456429$

$-0.99456429$

0.70601028

0.70601028

0.70601028

0.70601028

0.81184572

0.81184572

0.81184572

0.81184572

64
2. 44026875

$-0.19663274$

$-0.09079729$

$-0.09079729$

0.53337198

0.53337198

0.63920748

0.63920748

0.53337198

0.53337198

0.63920748

0.63920748

$-0.61681372$

$-0.61681372$

$-0.51097828$

$-0.51097828$

$-0.61681372$

$-0.61681372$

$-0.51097828$

$-0.51097828$

1. 26356936

1. 26356936

1. 36940491

1. 36940491

1.26356936

1. 26356936

1. 36940491

1.36940491

0.52339011

0.52339011

0.62922561

0.62922561

0.52339011

0.52339011

0.62922561

0.62922561

$-0.81257546$

$-0.81257546$

$-0.70673996$

$-0.70673996$

$-0.81257546$

$-0.81257546$

$-0.70673996$

$-0.70673996$

0.90373951

0.90373951

1.00957489

1.00957489

0.90373951

0.90373951

1. 00957489

1.00957489

$-1.34548187$

$-1.34548187$

$-1.23964632$

$-1.23964632$

$-1.34548187$

$-1.34548187$

$-1.23964632$

$-1.23964632$

\# Energy 1->9

0.2579

$-0.0370$ 73973.

$-2.05300403$

$-2.05300403$

$-2.05300403$

$-2.05300403$

$-1.94716847$

$-0.19663274$

$-0.19663274$

$-0.09079729$

$-0.09079729$

$-0.19663274$

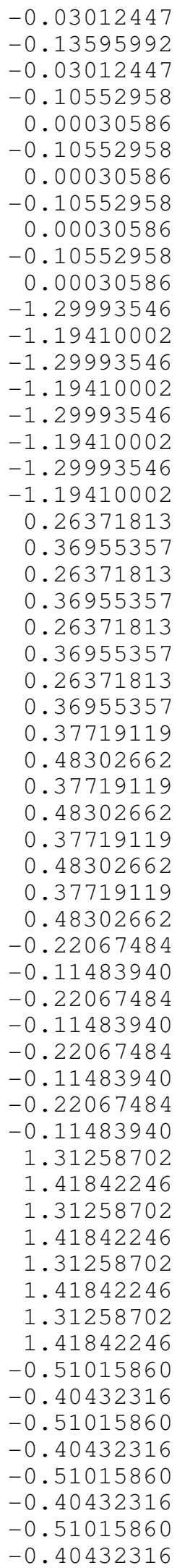

$$
0.0205 \quad-0.2564
$$

$0.1327-0.0386$
$-2.89395428$

$-1.83235598$

3. 78459382

$-1.13717031$

$-0.67096430$

0.53371251

1. 63165879

0.28977051

1. 54083967

0.41800848

$-2.72857332$

1. 27757537

$-1.69715905$

$-1.53094637$

2.02635479

$-1.54219842$

1. 99635231

1. 74662197

$-2.26107025$

0.31776452

0.29531181

$-0.10268153$

$-0.18755350$

$-0.01291669$

$-0.33303586$

$-0.01695032$

0.15140226

$-3.54504347$

1. 76726425

1. 55974448

$-0.34982175$

2. 61507082

$-1.09414315$

$-0.97424757$

$-0.08193302$

$-1.92266798$

1. 24904788

2. 00588298

$-1.34929812$

1. 57407546

$-1.06612837$

$-1.66934860$

1.19769228

0.96315765

$-0.70778686$

$-0.79380435$

0.56149340

$-0.84849960$

0.62150878

0.73107743

$-0.51492804$

$-0.08124261$

0.15000945

0.09137647

$-0.23316173$

0.12086717

$-0.20701165$

$-0.14149144$

0.31767371
\# Magnetic mom.

$-0.13595992$

$-0.03012447$

$-0.13595992$

$-0.03012447$

$-0.13595992$
$-0.98721975$

1. 73258567

1. 43528783

$-2.49264383$

1. 72588766 


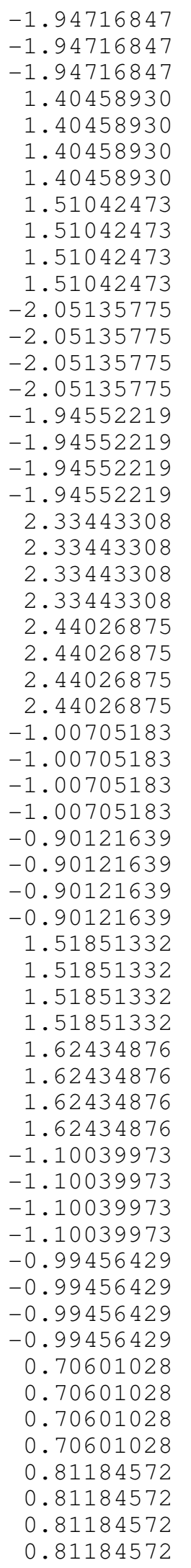

$\&$ TRANSITION $2->\ldots$
$-0.19663274$

$-0.09079729$

$-0.09079729$

0.53337198

0.53337198

0.63920748

0.63920748

0.53337198

0.53337198

0.63920748

0.63920748

$-0.61681372$

$-0.61681372$

$-0.51097828$

$-0.51097828$

$-0.61681372$

$-0.61681372$

$-0.51097828$

$-0.51097828$

1.26356936

1. 26356936

1. 36940491

1. 36940491

1. 26356936

1. 26356936

1. 36940491

1.36940491

0.52339011

0.52339011

0.62922561

0.62922561

0.52339011

0.52339011

0.62922561

0.62922561

$-0.81257546$

$-0.81257546$

$-0.70673996$

$-0.70673996$

$-0.81257546$

$-0.81257546$

$-0.70673996$

$-0.70673996$

0.90373951

0.90373951

1.00957489

1.00957489

0.90373951

0.90373951

1. 00957489

1.00957489

$-1.34548187$

$-1.34548187$

$-1.23964632$

$-1.23964632$

$-1.34548187$

$-1.34548187$

$-1.23964632$

$-1.23964632$

$$
\begin{aligned}
& -0.03012447 \\
& -0.13595992 \\
& -0.03012447 \\
& -0.10552958 \\
& 0.00030586 \\
& -0.10552958 \\
& 0.00030586 \\
& -0.10552958 \\
& 0.00030586 \\
& -0.10552958 \\
& 0.00030586 \\
& -1.29993546 \\
& -1.19410002 \\
& -1.29993546 \\
& -1.19410002 \\
& -1.29993546 \\
& -1.19410002 \\
& -1.29993546 \\
& -1.19410002 \\
& 0.26371813 \\
& 0.36955357 \\
& 0.26371813 \\
& 0.36955357 \\
& 0.26371813 \\
& 0.36955357 \\
& 0.26371813 \\
& 0.36955357 \\
& 0.37719119 \\
& 0.48302662 \\
& 0.37719119 \\
& 0.48302662 \\
& 0.37719119 \\
& 0.48302662 \\
& 0.37719119 \\
& 0.48302662 \\
& -0.22067484 \\
& -0.11483940 \\
& -0.22067484 \\
& -0.11483940 \\
& -0.22067484 \\
& -0.11483940 \\
& -0.22067484 \\
& -0.11483940 \\
& \text { 1. } 31258702 \\
& \text { 1. } 41842246 \\
& \text { 1. } 31258702 \\
& \text { 1. } 41842246 \\
& \text { 1. } 31258702 \\
& \text { 1. } 41842246 \\
& \text { 1. } 31258702 \\
& \text { 1. } 41842246 \\
& -0.51015860 \\
& -0.40432316 \\
& -0.51015860 \\
& -0.40432316 \\
& -0.51015860 \\
& -0.40432316 \\
& -0.51015860 \\
& -0.40432316
\end{aligned}
$$

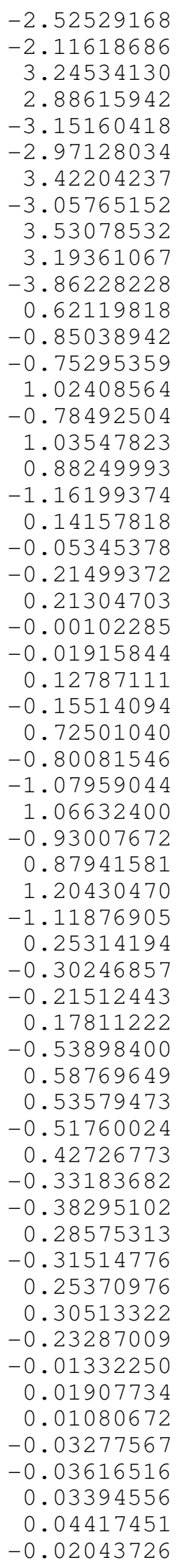
64
$-0.0126$
$-0.0085$
$-2.05300403$
$-2.05300403$
$-2.05300403$
$-2.05300403$

\# Energy 2->3 $\begin{array}{ll}-0.0123 & -0.0125\end{array}$ $-0.0068 \quad-0.0215$

$-0.19663274$

$-0.19663274$

$-0.09079729$

$-0.09079729$ 1.000 \# Electr. mom., scale-fac
\# Magnetic mom.
$-0.13595992$
$-0.28197625$
$-0.03012447$
0.32496122
0.52478540
$-0.13595992$
$-0.55843592$ 
$-1.94716847$

$-1.94716847$

$-1.94716847$

$-1.94716847$

1. 40458930

1. 40458930

1. 40458930

1. 40458930

1. 51042473

1.51042473

1.51042473

1. 51042473

$-2.05135775$

$-2.05135775$

$-2.05135775$

$-2.05135775$

$-1.94552219$

$-1.94552219$

$-1.94552219$

$-1.94552219$

2. 33443308

2. 33443308

2. 33443308

2. 33443308

2. 44026875

2. 44026875

2. 44026875

2. 44026875

$-1.00705183$

$-1.00705183$

$-1.00705183$

$-1.00705183$

$-0.90121639$

$-0.90121639$

$-0.90121639$

$-0.90121639$

1. 51851332

1. 51851332

1. 51851332

1. 51851332

1. 62434876

1. 62434876

1.62434876

1. 62434876

$-1.10039973$

$-1.10039973$

$-1.10039973$

$-1.10039973$

$-0.99456429$

$-0.99456429$

$-0.99456429$

$-0.99456429$

0.70601028

0.70601028

0.70601028

0.70601028

0.81184572

0.81184572

0.81184572

0.81184572

64
$-0.19663274$

$-0.19663274$

$-0.09079729$

$-0.09079729$

0.53337198

0.53337198

0.63920748

0.63920748

0.53337198

0.63920748

0.63920748

$-0.61681372$

$-0.61681372$

$-0.51097828$

$-0.51097828$

$-0.61681372$

$-0.61681372$

$-0.51097828$

$-0.51097828$

1. 26356936

1.26356936

1.36940491

1. 36940491

1. 26356936

1.26356936

1. 36940491

1. 36940491

0.52339011

0.52339011

0.62922561

0.62922561

0.52339011

0.52339011

0.62922561

0.62922561

$-0.81257546$

$-0.81257546$

$-0.70673996$

$-0.70673996$

$-0.81257546$

$-0.81257546$

$-0.70673996$

$-0.70673996$

0.90373951

0.90373951

1.00957489

1.00957489

0.90373951

0.90373951

1.00957489

1.00957489

$-1.34548187$

$-1.34548187$

$-1.23964632$

$-1.23964632$

$-1.34548187$

$-1.34548187$

$-1.23964632$

$-1.23964632$

$$
\text { 0. \# Energy 2->4 }
$$

$\begin{array}{lll}-0.0230 & 0.0051 & 0.0045\end{array}$

$\begin{array}{lll}-0.0080 & -0.0150 & -0.0609\end{array}$

$-2.05300403$

$-2.05300403$

$-2.05300403$

$-2.05300403$

19663274

$-0.09079729$

$-0.09079729$
$-0.13595992$

$-0.03012447$

$-0.13595992$

$-0.03012447$

0.00030586

$-0.10552958$

0.00030586

$-0.10552958$

0.00030586

$-0.10552958$

0.00030586

$-1.29993546$

$-1.19410002$

$-1.29993546$

$-1.19410002$

$-1.29993546$

$-1.19410002$

$-1.29993546$

$-1.19410002$

0.26371813

0.36955357

0.26371813

0.36955357

0.26371813

0.36955357

0.26371813

0.36955357

0.37719119

0.37719119

0.48302662

0.37719119

0.48302662

0.37719119

0.48302662

$-0.22067484$

$-0.11483940$

$-0.22067484$

$-0.11483940$

$-0.22067484$

$-0.11483940$

$-0.22067484$

$-0.11483940$

1. 31258702

1. 41842246

1. 31258702

1. 41842246

1. 31258702

1. 41842246

1. 31258702

1.41842246

$-0.51015860$

$-0.40432316$

$-0.51015860$

$-0.40432316$

$-0.51015860$

$-0.40432316$

$-0.51015860$

$-0.40432316$

1.000 \# Electr. mom., scale-fac
\# Magnetic mom.

$-0.13595992$

$-0.03012447$

$-0.13595992$

$-0.03012447$

$-0.69424319$

$-0.97088897$

0.98107851

$-0.42347708$

0.43847415

0.46053112

$-0.47587061$

0.43813649

$-0.46236035$

$-0.47692251$

0.33035401

$-0.38653675$

$-0.33294713$

0.38855624

$-0.19764088$

0.22092126

0.17654349

$-0.19369601$

0.12927911

$-0.07170970$

$-0.15630600$

0.10012239

$-0.04405709$

$-0.00567156$

0.07490296

$-0.02754233$

0.32259557

$-0.02577962$

0.03648654

$-0.30617312$

$-0.25959226$

$-0.01005199$

$-0.04515190$

0.04461001

$-0.03113946$

$-0.03012247$

0.03924041

$-0.01680736$

0.01589058

0.00795198

$-0.02531053$

$-0.22535986$

0.17097642

0.20803440

$-0.15283334$

0.18540001

$-0.13881743$

$-0.16878322$

0.12231608

0.00286390

$-0.02113037$

$-0.00534860$

0.04040685

$-0.00584984$

0.03079661

0.01246233

$-0.05791905$

-1.22889769
1.22314024
1.76084447
-1.69034278

0.53337198

$-0.10552958$

0.48302662

0.66706949

0.50170225

0.28900304

\section{S-237}


$-1.94716847$

$-1.94716847$

$-1.94716847$

$-1.94716847$

1. 40458930

1. 40458930

1. 40458930

1. 40458930

1. 51042473

1.51042473

1.51042473

1. 51042473

$-2.05135775$

$-2.05135775$

$-2.05135775$

$-2.05135775$

$-1.94552219$

$-1.94552219$

$-1.94552219$

$-1.94552219$

2. 33443308

2. 33443308

2. 33443308

2. 33443308

2. 44026875

2. 44026875

2. 44026875

2. 44026875

$-1.00705183$

$-1.00705183$

$-1.00705183$

$-1.00705183$

$-0.90121639$

$-0.90121639$

$-0.90121639$

$-0.90121639$

1. 51851332

1. 51851332

1. 51851332

1. 51851332

1. 62434876

1. 62434876

1.62434876

1. 62434876

$-1.10039973$

$-1.10039973$

$-1.10039973$

$-1.10039973$

$-0.99456429$

$-0.99456429$

$-0.99456429$

$-0.99456429$

0.70601028

0.70601028

0.70601028

0.70601028

0.81184572

0.81184572

0.81184572

0.81184572

64
$-0.19663274$

$-0.19663274$

$-0.09079729$

$-0.09079729$

0.53337198

0.53337198

0.63920748

0.63920748

0.53337198

0.53337198

0.63920748

0.63920748

$-0.61681372$

$-0.61681372$

$-0.51097828$

$-0.51097828$

$-0.61681372$

$-0.61681372$

$-0.51097828$

$-0.51097828$

1. 26356936

1.26356936

1. 36940491

1. 36940491

1. 26356936

1.26356936

1. 36940491

1. 36940491

0.52339011

0.52339011

0.62922561

0.62922561

0.52339011

0.52339011

0.62922561

0.62922561

$-0.81257546$

$-0.81257546$

$-0.70673996$

$-0.70673996$

$-0.81257546$

$-0.81257546$

$-0.70673996$

$-0.70673996$

0.90373951

0.90373951

1.00957489

0.90373951

0.90373951

1. 00957489

1.00957489

$-1.34548187$

$-1.34548187$

$-1.23964632$

$-1.23964632$

$-1.34548187$

$-1.34548187$

$-1.23964632$

$-1.23964632$

0 .

0.3498

0.0558

$-2.05300403$

$-2.05300403$

$-2.05300403$

$-2.05300403$

$-0.19663274$

$-0.19663274$

$-0.09079729$

$-0.09079729$
1.00957489

$-0.13595992$

$-0.03012447$

$-0.13595992$

$-0.03012447$

$-0.10552958$

0.00030586

$-0.10552958$

0.00030586

$-0.10552958$

0.00030586

$-0.10552958$

0.00030586

$-1.29993546$

$-1.19410002$

$-1.29993546$

$-1.19410002$

$-1.29993546$

$-1.19410002$

$-1.29993546$

$-1.19410002$

0.26371813

0.36955357

0.26371813

0.36955357

0.26371813

0.36955357

0.26371813

0.36955357

0.37719119

0.48302662

0.37719119

0.48302662

0.37719119

0.48302662

0.37719119

0.48302662

$-0.22067484$

$-0.11483940$

$-0.22067484$

$-0.11483940$

$-0.22067484$

$-0.11483940$

$-0.22067484$

$-0.11483940$

1. 31258702

1. 41842246

1. 31258702

1.41842246

1.31258702

1. 41842246

1. 31258702

1.41842246

$-0.51015860$

$-0.40432316$

$-0.51015860$

$-0.40432316$

$-0.51015860$

$-0.40432316$

$-0.51015860$

$-0.40432316$

H Energy 2->5

$\begin{array}{lr}0.0567 & -0.0096 \\ 0.0704 & 0.3590\end{array}$
2. 20051384

$-2.13491201$

$-2.94669533$

2.77269673

$-1.83918715$

1. 91799045

2. 09825110

$-2.25873232$

1. 91512442

$-2.05037498$

$-2.19347262$

2. 40979719

1.56706059

$-1.88456309$

$-1.61606300$

1. 94780993

$-1.15298223$

1. 36635208

1. 12854850

$-1.33701539$

0.29884550

$-0.25407004$

$-0.25137159$

0.20598571

$-0.20550296$

0.16399194

0.17828907

$-0.13835111$

1. 25130939

$-0.04578358$

$-0.12034638$

$-0.90584058$

$-0.88277078$

$-0.18609187$

$-0.06948726$

0.98710376

0.35795015

$-0.37537935$

$-0.25338760$

0.35386786

$-0.22348580$

0.25578293

0.13790137

$-0.23978589$

$-0.88799131$

0.65866995

0.78695697

$-0.56583023$

0.70489621

$-0.52017736$

$-0.63162357$

0.45120174

0.00729953

$-0.07970298$

$-0.00280634$

0.12653197

$-0.01364265$

0.10910774

0.01318026

$-0.17033385$
1.000 \# Electr. mom., scale-fac
\# Magnetic mom.

$-0.13595992$

$-0.03012447$

$-0.13595992$

$-0.03012447$
8.50540447

$-8.49579525$

$-11.88030434$

11.47970581 
$-1.94716847$

$-1.94716847$

$-1.94716847$

$-1.94716847$

1. 40458930

1. 40458930

1. 40458930

1. 40458930

1. 51042473

1. 51042473

1.51042473

1. 51042473

$-2.05135775$

$-2.05135775$

$-2.05135775$

$-2.05135775$

$-1.94552219$

$-1.94552219$

$-1.94552219$

$-1.94552219$

2. 33443308

2. 33443308

2. 33443308

2. 33443308

2. 44026875

2. 44026875

2. 44026875

2. 44026875

$-1.00705183$

$-1.00705183$

$-1.00705183$

$-1.00705183$

$-0.90121639$

$-0.90121639$

$-0.90121639$

$-0.90121639$

1. 51851332

1. 51851332

1. 51851332

1. 51851332

1. 62434876

1. 62434876

1.62434876

1. 62434876

$-1.10039973$

$-1.10039973$

$-1.10039973$

$-1.10039973$

$-0.99456429$

$-0.99456429$

$-0.99456429$

$-0.99456429$

0.70601028

0.70601028

0.70601028

0.70601028

0.81184572

0.81184572

0.81184572

0.81184572

64
$-0.19663274$

$-0.19663274$

$-0.09079729$

$-0.09079729$

0.53337198

0.53337198

0.63920748

0.63920748

0.53337198

0.53337198

0.63920748

0.63920748

$-0.61681372$

$-0.61681372$

$-0.51097828$

$-0.51097828$

$-0.61681372$

$-0.61681372$

$-0.51097828$

$-0.51097828$

1. 26356936

1. 26356936

1.36940491

1.36940491

1. 26356936

1.26356936

1.36940491

1. 36940491

0.52339011

0.52339011

0.62922561

0.62922561

0.52339011

0.52339011

0.62922561

0.62922561

$-0.81257546$

$-0.81257546$

$-0.70673996$

$-0.70673996$

$-0.81257546$

$-0.81257546$

$-0.70673996$

$-0.70673996$

0.90373951

0.90373951

1.00957489

1.00957489

0.90373951

0.90373951

1.00957489

1.00957489

$-1.34548187$

$-1.34548187$

$-1.23964632$

$-1.23964632$

$-1.34548187$

$-1.34548187$

$-1.23964632$

$-1.23964632$

$$
0 \text {. \# Energy 2->6 }
$$

$\begin{array}{lll}0.1797 & -0.0169 & -0.0359\end{array}$

0.0394

$-2.05300403$

$-2.05300403$

$-2.05300403$

$-2.05300403$ $0.0039 \quad 0.0489$

$-0.19663274$

$-0.19663274$

$-0.09079729$

$-0.09079729$
$-0.13595992$

$-0.03012447$

$-0.13595992$

$-0.03012447$

$-0.10552958$

0.00030586

$-0.10552958$

0.00030586

$-0.10552958$

0.00030586

$-0.10552958$

0.00030586

$-1.29993546$

$-1.19410002$

$-1.29993546$

$-1.19410002$

$-1.29993546$

$-1.19410002$

$-1.29993546$

$-1.19410002$

0.26371813

0.36955357

0.26371813

0.36955357

0.26371813

0.36955357

0.26371813

0.36955357

0.37719119

0.48302662

0.37719119

0.48302662

0.37719119

0.48302662

0.37719119

0.48302662

$-0.22067484$

$-0.11483940$

$-0.22067484$

$-0.11483940$

$-0.22067484$

$-0.11483940$

$-0.22067484$

$-0.11483940$

1. 31258702

1. 41842246

1. 31258702

1. 41842246

1. 31258702

1. 41842246

1. 31258702

1. 41842246

$-0.51015860$

$-0.40432316$

$-0.51015860$

$-0.40432316$

$-0.51015860$

$-0.40432316$

$-0.51015860$

$-0.40432316$
$-14.55735397$

14.15376759

19.24991608

$-18.19447899$

12.28159046

$-12.01045990$

$-14.01128864$

14.40766239

$-12.84919071$

13.05046558

14.75419712

$-15.63885689$

$-8.86547279$

10.57500076

9.14155483

$-10.91321182$

6.34280062

$-7.41025352$

$-6.15837240$

7.16823339

$-1.17571652$

1. 35852230

1.12687087

$-1.15729117$

0.67267066

$-0.74158931$

$-0.67749387$

0.64769071

$-6.53246260$

$-0.83949333$

$-0.36548960$

6.67634058

4.19824457

2. 33575845

1. 60232079

$-7.25656700$

$-1.77977645$

2. 36139154

1. 26853740

$-2.36675000$

1. 01348817

$-1.62853324$

$-0.59636474$

1. 64928377

5.57989550

$-4.15722513$

$-5.00187588$

3.61391401

$-4.42208481$

3.29007554

4. 02554703

$-2.90050435$

$-0.04397615$

0.47869122

$-0.01512992$

$-0.70364296$

0.08361963

$-0.64427996$

$-0.04437534$

0.94250154

1.000

* Magnect mom.,

$-0.13595992$

$-0.03012447$

ic mom.

$-0.13595992$

$-0.03012447$

1.17011654

1. 02623153

$-1.59223330$

\section{S-239}


$-1.94716847$

$-1.94716847$

$-1.94716847$

$-1.94716847$

1. 40458930

1. 40458930

1. 40458930

1. 40458930

1. 51042473

1. 51042473

1.51042473

1. 51042473

$-2.05135775$

$-2.05135775$

$-2.05135775$

$-2.05135775$

$-1.94552219$

$-1.94552219$

$-1.94552219$

$-1.94552219$

2. 33443308

2. 33443308

2. 33443308

2. 33443308

2. 44026875

2. 44026875

2. 44026875

2. 44026875

$-1.00705183$

$-1.00705183$

$-1.00705183$

$-1.00705183$

$-0.90121639$

$-0.90121639$

$-0.90121639$

$-0.90121639$

1. 51851332

1. 51851332

1. 51851332

1. 51851332

1. 62434876

1. 62434876

1.62434876

1. 62434876

$-1.10039973$

$-1.10039973$

$-1.10039973$

$-1.10039973$

$-0.99456429$

$-0.99456429$

$-0.99456429$

$-0.99456429$

0.70601028

0.70601028

0.70601028

0.70601028

0.81184572

0.81184572

0.81184572

0.81184572

64
$-0.19663274$

$-0.19663274$

$-0.09079729$

$-0.09079729$

0.53337198

0.53337198

0.63920748

0.63920748

0.53337198

0.53337198

0.63920748

0.63920748

$-0.61681372$

$-0.61681372$

$-0.51097828$

$-0.51097828$

$-0.61681372$

$-0.61681372$

$-0.51097828$

$-0.51097828$

1. 26356936

1.26356936

1. 36940491

1. 36940491

1. 26356936

1.26356936

1. 36940491

1. 36940491

0.52339011

0.52339011

0.62922561

0.52339011

0.52339011

0.62922561

0.62922561

$-0.81257546$

$-0.81257546$

$-0.70673996$

$-0.70673996$

$-0.81257546$

$-0.81257546$

$-0.70673996$

$-0.70673996$

0.90373951

0.90373951

1.00957489

1.00957489

0.90373951

0.90373951

1. 00957489

1.00957489

$-1.34548187$

$-1.34548187$

$-1.23964632$

$-1.23964632$

$-1.34548187$

$-1.34548187$

$-1.23964632$

$-1.23964632$ 0 . \# Energy 2->7

$\begin{array}{lll}0.6538 & -0.0682 & -0.1343\end{array}$

$0.1053 \quad-0.0073 \quad-0.0231$

$-2.05300403$

$-2.05300403$

$-2.05300403$

$-2.05300403$

$-0.19663274$

$-0.19663274$

$-0.09079729$

$-0.09079729$
0.62922561
1.000 \# Electr. mom., scale-fac
\# Magnetic mom.

$-0.13595992$

$-0.03012447$

$-0.13595992$

$-0.03012447$

$-0.10552958$

0.00030586

$-0.10552958$

0.00030586

$-0.10552958$

0.00030586

$-0.10552958$

0.00030586

$-1.29993546$

$-1.19410002$

$-1.29993546$

$-1.19410002$

$-1.29993546$

$-1.19410002$

$-1.29993546$

$-1.19410002$

0.26371813

0.36955357

0.26371813

0.36955357

0.26371813

0.36955357

0.26371813

0.36955357

0.37719119

0.48302662

0.37719119

0.48302662

0.37719119

0.48302662

0.37719119

0.48302662

$-0.22067484$

$-0.11483940$

$-0.22067484$

$-0.11483940$

$-0.22067484$

$-0.11483940$

$-0.22067484$

$-0.11483940$

1. 31258702

1. 41842246

1. 31258702

1. 41842246

1. 31258702

1. 41842246

1. 31258702

1.41842246

$-0.51015860$

$-0.40432316$

$-0.51015860$

$-0.40432316$

$-0.51015860$

$-0.40432316$

$-0.51015860$

$-0.40432316$

0.66056955

$-1.18113041$

$-0.92806387$

1.63091564

2. 37371802

$-1.50867069$

$-2.53986478$

1. 87031674

$-2.27093911$

1. 53389680

2. 50134921

$-1.95638156$

$-0.59457475$

0.74044049

0.62985820

$-0.78857976$

0.45475337

$-0.56925631$

$-0.46239176$

0.58297139

0.11194668

0.00543182

0.02080797

$-0.09975812$

$-0.25504482$

0.17480248

0.11727640

$-0.06431719$

1. 67605937

$-2.38729358$

$-1.78872883$

2. 34564662

$-1.74691200$

2.34115982

1.80795336

$-2.29763126$

0.38271183

0.22583091

$-0.41070512$

$-0.32992572$

$-0.36821109$

$-0.17723018$

0.38156563

0.28859317

0.75871426

$-0.70049727$

0.49969202

$-0.57163590$

0.43254152

0.55888575

$-0.40993589$

$-0.02397948$

0.13122943

0.02650340

$-0.16045558$

0.02797857

$-0.16346900$

$-0.03267894$

0.19866070

$-0.13595992$

$-9.59777355$

$-0.03012447$

11.73359680

13.57783031

$-0.03012447$

$-16.18636513$
$-0.55570656$ 


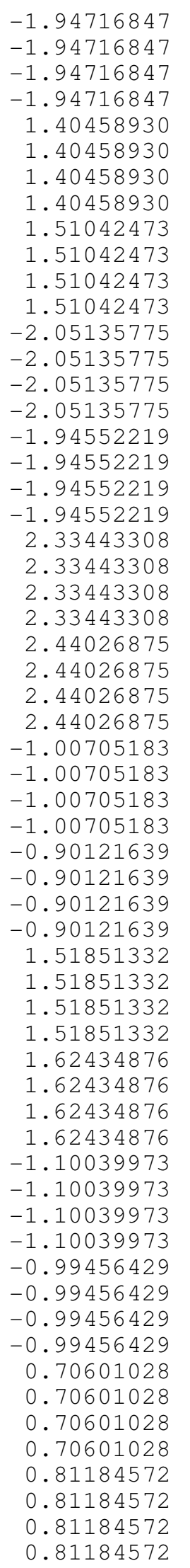

64
$-0.19663274$

$-0.19663274$

$-0.09079729$

$-0.09079729$

0.53337198

0.53337198

0.63920748

0.63920748

0.53337198

0.53337198

0.63920748

0.63920748

$-0.61681372$

$-0.61681372$

$-0.51097828$

$-0.51097828$

$-0.61681372$

$-0.61681372$

$-0.51097828$

$-0.51097828$

1. 26356936

1.26356936

1. 36940491

1. 36940491

1. 26356936

1.26356936

1. 36940491

1. 36940491

0.52339011

0.52339011

0.62922561

0.62922561

0.52339011

0.52339011

0.62922561

0.62922561

$-0.81257546$

$-0.81257546$

$-0.70673996$

$-0.70673996$

$-0.81257546$

$-0.81257546$

$-0.70673996$

$-0.70673996$

0.90373951

0.90373951

1.00957489

1.00957489

0.90373951

0.90373951

1. 00957489

1.00957489

$-1.34548187$

$-1.34548187$

$-1.23964632$

$-1.23964632$

$-1.34548187$

$-1.34548187$

$-1.23964632$

$-1.23964632$

0 . \# Energy 2->8

$-0.2467$

0.0812

$-2.05300403$

$-2.05300403$

$-2.05300403$

$-2.05300403$

$0.0667 \quad 0.0315$

$0.0597 \quad-0.0807$

$-0.19663274$

$-0.19663274$

$-0.09079729$

$-0.09079729$
$-0.13595992$

$-0.03012447$

$-0.13595992$

$-0.03012447$

$-0.10552958$

0.00030586

$-0.10552958$

0.00030586

$-0.10552958$

0.00030586

$-0.10552958$

0.00030586

$-1.29993546$

$-1.19410002$

$-1.29993546$

$-1.19410002$

$-1.29993546$

$-1.19410002$

$-1.29993546$

$-1.19410002$

0.26371813

0.36955357

0.26371813

0.36955357

0.26371813

0.36955357

0.26371813

0.36955357

0.37719119

0.48302662

0.37719119

0.48302662

0.37719119

0.48302662

0.37719119

0.48302662

$-0.22067484$

$-0.11483940$

$-0.22067484$

$-0.11483940$

$-0.22067484$

$-0.11483940$

$-0.22067484$

$-0.11483940$

1. 31258702

1. 41842246

1. 31258702

1.41842246

1. 31258702

1. 41842246

1. 31258702

1.41842246

$-0.51015860$

$-0.40432316$

$-0.51015860$

$-0.40432316$

$-0.51015860$

$-0.40432316$

$-0.51015860$

$-0.40432316$
13.46323681

$-15.85128403$

$-18.41463089$

21.25097466

2. 10112739

1. 40852678

$-1.74753499$

$-1.26879263$

$-1.42198884$

$-1.79669511$

1. 25325716

1. 48968124

3.72316670

$-4.27896070$

$-3.78151011$

4.32577515

$-2.58482170$

2.83218503

2.44983840

$-2.63331175$

1. 44814539

$-0.87020236$

$-0.89082730$

0.44002724

$-1.50851154$

1.08052421

1.00361729

$-0.67010134$

13.58955669

$-11.52825642$

$-9.20541763$

7. 18354607

$-12.20753479$

10.18447781

8.36509132

$-6.49444199$

2. 82856607

$-0.56242377$

$-2.60467887$

0.13323480

$-2.27219439$

0.32568371

2. 05814004

0.11557891

$-0.54042226$

0.44918787

0.34418115

$-0.28258878$

0.56456560

$-0.38747251$

$-0.28522593$

0.17488049

$-0.06016357$

0.20807840

0.07398072

$-0.12458947$

0.04040903

$-0.21219356$

$-0.03948826$

0.08973480

1.000

\# Electr. mom., scale-fac

\# Magnetic mom.

$-0.13595992$

$-3.45139790$

$-0.03012447$

2. 70138717

3. 33083773

-0.13595992
-0.03012447

$-2.25956893$

\section{S-241}




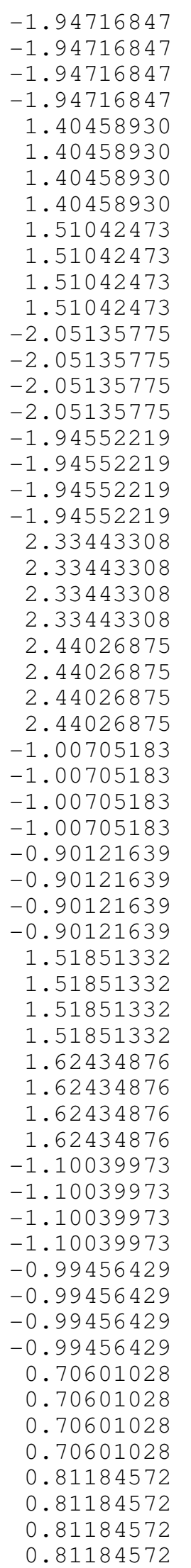

64
$-0.19663274$

$-0.19663274$

$-0.09079729$

$-0.09079729$

0.53337198

0.53337198

0.63920748

0.63920748

0.53337198

0.53337198

0.63920748

0.63920748

$-0.61681372$

$-0.61681372$

$-0.51097828$

$-0.51097828$

$-0.61681372$

$-0.61681372$

$-0.51097828$

$-0.51097828$

1. 26356936

1.26356936

1. 36940491

1. 36940491

1.26356936

1.26356936

1. 36940491

1. 36940491

0.52339011

0.52339011

0.62922561

0.62922561

0.52339011

0.52339011

0.62922561

0.62922561

$-0.81257546$

$-0.81257546$

$-0.70673996$

$-0.70673996$

$-0.81257546$

$-0.81257546$

$-0.70673996$

$-0.70673996$

0.90373951

0.90373951

1.00957489

1. 00957489

0.90373951

0.90373951

1.00957489

1.00957489

$-1.34548187$

$-1.34548187$

$-1.23964632$

$-1.23964632$

$-1.34548187$

$-1.34548187$

$-1.23964632$

$-1.23964632$

$$
0 \text {. \# Energy 2->9 }
$$

$\begin{array}{rrr}-0.2360 & -0.2755 & -0.0695 \\ 0.0157 & -0.0164 & -0.0182\end{array}$

$-2.05300403$

$-2.05300403$

$-2.05300403$

$-2.05300403$
$-0.19663274$

$-0.19663274$

$-0.09079729$

$-0.09079729$
$-0.13595992$

$-0.03012447$

$-0.13595992$

$-0.03012447$

$-0.10552958$

0.00030586

$-0.10552958$

0.00030586

$-0.10552958$

0.00030586

$-0.10552958$

0.00030586

$-1.29993546$

$-1.19410002$

$-1.29993546$

$-1.19410002$

$-1.29993546$

$-1.19410002$

$-1.29993546$

$-1.19410002$

0.26371813

0.36955357

0.26371813

0.36955357

0.26371813

0.36955357

0.26371813

0.36955357

0.37719119

0.48302662

0.37719119

0.48302662

0.37719119

0.48302662

0.37719119

0.48302662

$-0.22067484$

$-0.11483940$

$-0.22067484$

$-0.11483940$

$-0.22067484$

$-0.11483940$

$-0.22067484$

$-0.11483940$

1. 31258702

1. 41842246

1. 31258702

1. 41842246

1. 31258702

1. 41842246

1. 31258702

1. 41842246

$-0.51015860$

$-0.40432316$

$-0.51015860$

$-0.40432316$

$-0.51015860$

$-0.40432316$

$-0.51015860$

$-0.40432316$
4. 41315556

$-3.39239144$

$-4.52595139$

3.05808496

$-0.89797550$

1. 41032624

1. 17178631

$-1.55308652$

1.60217404

$-1.91866720$

$-1.80436039$

1. 95841527

1.98175037

$-2.37260652$

$-2.09212470$

2. 51372981

$-1.41641843$

1.65806270

1. 41230464

$-1.65223825$

2.42388654

$-1.52749979$

$-1.42239559$

0.72645020

$-2.75777006$

2.04205203

1.85797262

$-1.30349839$

3. 27441001

$-0.57005197$

$-1.42058146$

$-0.55350608$

$-2.42490220$

0.18212278

1.01485884

0.61019593

2.39061522

$-1.69226635$

$-2.21298313$

1.57353020

$-1.85259926$

1. 24398351

1.68189657

$-1.12846684$

$-1.35219550$

0.94896281

1.08190644

$-0.73584318$

0.94359183

$-0.65308332$

$-0.73918039$

0.49466959

0.25493315

$-0.39552450$

$-0.23295024$

0.46986875

$-0.28533337$

0.45316774

0.24939142

$-0.53306210$
1.000 \# Electr. mom., scale-fac \# Magnetic mom.

$-0.13595992$

$-0.03012447$

$-0.13595992$

$-0.03012447$
-2.20987129
2.24653172
2.88658524
-2.89046288 
$-1.94716847$

$-1.94716847$

$-1.94716847$

$-1.94716847$

1. 40458930

1. 40458930

1. 40458930

1. 40458930

1. 51042473

1. 51042473

1.51042473

1. 51042473

$-2.05135775$

$-2.05135775$

$-2.05135775$

$-2.05135775$

$-1.94552219$

$-1.94552219$

$-1.94552219$

$-1.94552219$

2. 33443308

2. 33443308

2. 33443308

2. 33443308

2. 44026875

2. 44026875

2. 44026875

2. 44026875

$-1.00705183$

$-1.00705183$

$-1.00705183$

$-1.00705183$

$-0.90121639$

$-0.90121639$

$-0.90121639$

$-0.90121639$

1. 51851332

1. 51851332

1. 51851332

1. 51851332

1. 62434876

1. 62434876

1.62434876

1. 62434876

$-1.10039973$

$-1.10039973$

$-1.10039973$

$-1.10039973$

$-0.99456429$

$-0.99456429$

$-0.99456429$

$-0.99456429$

0.70601028

0.70601028

0.70601028

0.70601028

0.81184572

0.81184572

0.81184572

0.81184572

\&TRANSITION $3->\ldots$ 64

-0.3422
0.3002
-2.05300403
-2.05300403
-2.05300403

$-0.19663274$

$-0.19663274$

$-0.09079729$

$-0.09079729$

0.53337198

0.53337198

0.63920748

0.63920748

0.53337198

0.53337198

0.63920748

0.63920748

$-0.61681372$

$-0.61681372$

$-0.51097828$

$-0.51097828$

$-0.61681372$

$-0.61681372$

$-0.51097828$

$-0.51097828$

1. 26356936

1.26356936

1. 36940491

1. 36940491

1.26356936

1.26356936

1. 36940491

1. 36940491

0.52339011

0.52339011

0.62922561

0.62922561

0.52339011

0.52339011

0.62922561

0.62922561

$-0.81257546$

$-0.81257546$

$-0.70673996$

$-0.70673996$

$-0.81257546$

$-0.81257546$

$-0.70673996$

$-0.70673996$

0.90373951

0.90373951

1.00957489

1.00957489

0.90373951

0.90373951

1.00957489

1.00957489

$-1.34548187$

$-1.34548187$

$-1.23964632$

$-1.23964632$

$-1.34548187$

$-1.34548187$

$-1.23964632$

$-1.23964632$
$-0.13595992$

$-0.03012447$

$-0.13595992$

$-0.03012447$

$-0.10552958$

0.00030586

$-0.10552958$

0.00030586

$-0.10552958$

0.00030586

$-0.10552958$

0.00030586

$-1.29993546$

$-1.19410002$

$-1.29993546$

$-1.19410002$

$-1.29993546$

$-1.19410002$

$-1.29993546$

$-1.19410002$

0.26371813

0.36955357

0.26371813

0.36955357

0.26371813

0.36955357

0.26371813

0.36955357

0.37719119

0.48302662

0.37719119

0.48302662

0.37719119

0.48302662

0.37719119

0.48302662

$-0.22067484$

$-0.11483940$

$-0.22067484$

$-0.11483940$

$-0.22067484$

$-0.11483940$

$-0.22067484$

$-0.11483940$

1. 31258702

1. 41842246

1. 31258702

1.41842246

1. 31258702

1. 41842246

1. 31258702

1.41842246

$-0.51015860$

$-0.40432316$

$-0.51015860$

$-0.40432316$

$-0.51015860$

$-0.40432316$

$-0.51015860$

$-0.40432316$
3. 38452911

$-3.36820865$

$-4.31442022$

4.23235083

$-1.76838350$

2. 44507647

2. 44049716

$-3.49392653$

2. 34636331

$-3.41014671$

$-3.11919498$

4.61595488

1.11007750

$-1.27966571$

$-1.11639011$

1.27609932

$-0.70064628$

0.75947398

0.62324625

$-0.65010488$

$-1.05576611$

0.24085344

0.82064372

$-0.33147687$

0.64380586

$-0.10832565$

$-0.57774186$

0.26648206

$-0.22331475$

1. 36161280

1. 54519284

$-2.61158824$

0.78950149

$-1.81313980$

$-1.93955982$

2. 91881227

0.13510945

0.02447836

$-0.22799914$

0.05020643

0.10221873

$-0.25958270$

$-0.05141039$

0.25642633

$-1.04399693$

0.80769795

1.01214731

$-0.75743765$

0.89392895

$-0.69785112$

$-0.89269978$

0.67281103

$-0.04215360$

0.13168322

0.08878442

$-0.18674310$

0.06036867

$-0.18626858$

$-0.10970207$

0.24862903

$\begin{array}{rr}-0.0827 & -0.0926 \\ 0.2440 & 0.1013\end{array}$

1.000 \# Electr. mom., scale-fac \# Magnetic mom.

$-0.19663274$

$-0.19663274$

$-0.13595992$

0.08722487

$-0.03012447$

0.20129479

$-0.09079729$

$-0.13595992$

0.32838920 


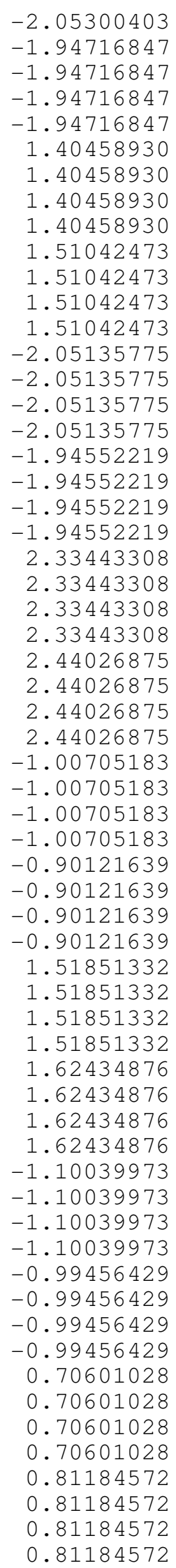

64
$-0.09079729$

$-0.19663274$

$-0.19663274$

$-0.09079729$

$-0.09079729$

0.53337198

0.53337198

0.63920748

0.63920748

0.53337198

0.53337198

0.63920748

0.63920748

$-0.61681372$

$-0.61681372$

$-0.51097828$

$-0.51097828$

$-0.61681372$

$-0.61681372$

$-0.51097828$

$-0.51097828$

1. 26356936

1.26356936

1. 36940491

1. 36940491

1. 26356936

1.26356936

1. 36940491

1. 36940491

0.52339011

0.52339011

0.62922561

0.62922561

0.52339011

0.52339011

0.62922561

0.62922561

$-0.81257546$

$-0.81257546$

$-0.70673996$

$-0.70673996$

$-0.81257546$

$-0.81257546$

$-0.70673996$

$-0.70673996$

0.90373951

0.90373951

1.00957489

1.00957489

0.90373951

0.90373951

1.00957489

1.00957489

$-1.34548187$

$-1.34548187$

$-1.23964632$

$-1.23964632$

$-1.34548187$

$-1.34548187$

$-1.23964632$

$-1.23964632$

\# Energy 3->5
$-0.03012447$

$-0.13595992$

$-0.03012447$

$-0.13595992$

$-0.03012447$

$-0.10552958$

0.00030586

$-0.10552958$

0.00030586

$-0.10552958$

0.00030586

$-0.10552958$

0.00030586

$-1.29993546$

$-1.19410002$

$-1.29993546$

$-1.19410002$

$-1.29993546$

$-1.19410002$

$-1.29993546$

$-1.19410002$

0.26371813

0.36955357

0.26371813

0.36955357

0.26371813

0.36955357

0.26371813

0.36955357

0.37719119

0.48302662

0.37719119

0.48302662

0.37719119

0.48302662

0.37719119

0.48302662

$-0.22067484$

$-0.11483940$

$-0.22067484$

$-0.11483940$

$-0.22067484$

$-0.11483940$

$-0.22067484$

$-0.11483940$

1. 31258702

1. 41842246

1. 31258702

1.41842246

1. 31258702

1. 41842246

1. 31258702

1.41842246

$-0.51015860$

$-0.40432316$

$-0.51015860$

$-0.40432316$

$-0.51015860$

$-0.40432316$

$-0.51015860$

$-0.40432316$
$-0.79537135$

0.19981138

$-0.59734845$

$-0.66123545$

1. 29189444

$-0.70560944$

$-1.80349672$

1.81252694

$-0.01264220$

4.36202335

$-1.99787867$

$-5.72284174$

4.17716789

0.19875322

$-0.20951478$

$-0.18922175$

0.19489974

$-0.20187795$

0.21317212

0.19625065

$-0.20159784$

1. 92017806

$-3.30968976$

$-4.44394255$

5. 26483297

1. 52615035

$-0.38758275$

0.92825681

$-1.61476994$

0.08366603

$-1.74116874$

$-2.05531168$

3. 44331098

$-1.00623119$

2. 59159184

3. 04689026

$-4.40480900$

4.90762091

$-5.83674335$

$-4.85527754$

6.14798594

$-5.35367823$

6.55394793

5. 42469168

$-7.00075722$

0.13562281

$-0.16817383$

$-0.33430424$

0.28674734

$-0.19981751$

0.23233999

0.44434449

$-0.37750852$

$-0.02380992$

0.30220452

0.09320512

$-0.34791756$

$-0.18431228$

$-0.09178453$

0.06842740

0.17080249

$$
\begin{array}{lll}
0.3152 & 0.0606 & -0.0230 \\
0.0557 & 0.0888 & -0.0359
\end{array}
$$

1.000

\# Electr. mom., scale-fac

$-0.19663274$

$-0.13595992$

\# Magnetic mom.

$-0.19663274$

$-0.03012447$

$-0.35926726$

$-0.09079729$

$-0.13595992$

0.66393864

0.00759056

\section{S-244}




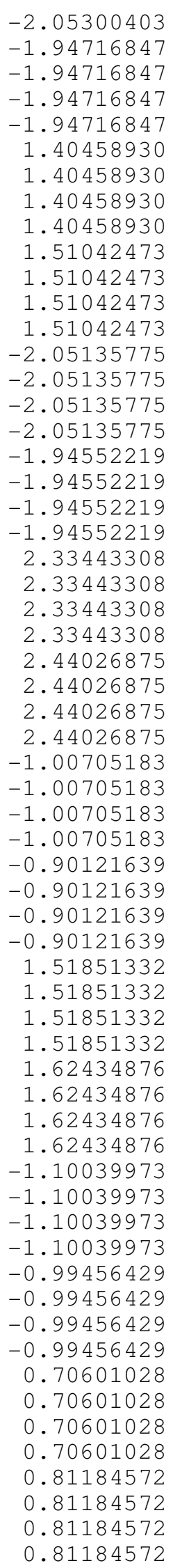

64
$-0.09079729$

$-0.19663274$

$-0.19663274$

$-0.09079729$

$-0.09079729$

0.53337198

0.63920748

0.63920748

0.53337198

0.53337198

0.63920748

0.63920748

$-0.61681372$

$-0.61681372$

$-0.51097828$

$-0.51097828$

$-0.61681372$

$-0.61681372$

$-0.51097828$

$-0.51097828$

1. 26356936

1.26356936

1. 36940491

1. 36940491

1. 26356936

1.26356936

1. 36940491

1. 36940491

0.52339011

0.52339011

0.62922561

0.62922561

0.52339011

0.52339011

0.62922561

0.62922561

$-0.81257546$

$-0.81257546$

$-0.70673996$

$-0.70673996$

$-0.81257546$

$-0.81257546$

$-0.70673996$

$-0.70673996$

0.90373951

0.90373951

1.00957489

1.00957489

0.90373951

0.90373951

1.00957489

1. 00957489

$-1.34548187$

$-1.34548187$

$-1.23964632$

$-1.23964632$

$-1.34548187$

$-1.34548187$

$-1.23964632$

$-1.23964632$
0.53337198

0 . \# Energy 3->6 $-0.0879 \quad-0.1774$

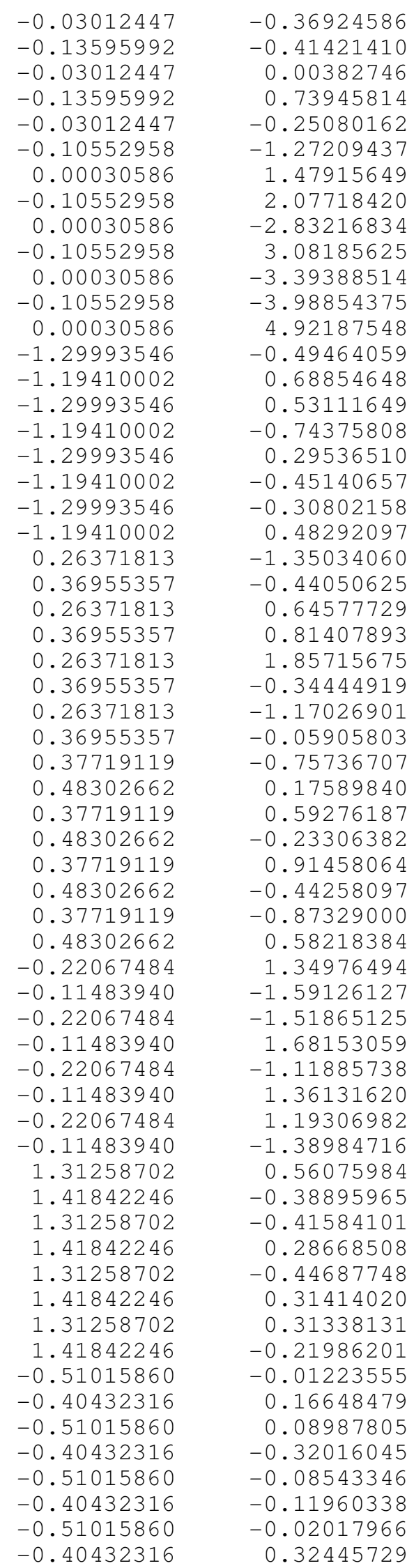

$$
\begin{aligned}
& -0.03012447 \\
& 0.13595992 \\
& -0.13595992 \\
& -0.03012447 \\
& -0.10552958 \\
& -0.10552958 \\
& 0.00030586 \\
& -0.10552958 \\
& \text { 0.00030586 } \\
& 0.00030586 \\
& -1.29993546 \\
& -1.19410002 \\
& -1.29993546 \\
& -1.29993546 \\
& -1.19410002 \\
& -1.29993546 \\
& -1.19410002 \\
& 0.26371813 \\
& 0.36955357 \\
& 0.26371813 \\
& 0.48302662 \\
& 0.37719119 \\
& 0.37719119 \\
& 0.48302662 \\
& -0.22067484 \\
& -0.11483940 \\
& -0.22067484 \\
& -0.2206748 \\
& -0.11483940 \\
& \text { 1. } 41842246 \\
& 1.31258702 \\
& \text { 1. } 41842246 \\
& \text { 1.31258702 } \\
& \text { 1. } 41842246 \\
& -0.51015860 \\
& -0.40432316 \\
& -0.51015860 \\
& -0.51015860 \\
& -0.51015860 \\
& -0.40432316
\end{aligned}
$$

$-0.19663274$

$-0.19663274$

$-0.09079729$

$\begin{array}{lcc}-1.7826 & -0.4302 & -0.1803 \\ -0.1657 & -0.0879 & -0.177 \\ -2.05300403 & -0.19663274 \\ -2.05300403 & -0.19663274 \\ -2.05300403 & -0.09079729\end{array}$

$$
\begin{aligned}
& 1.000 \text { \# Electr. mom., scale-fac } \\
& \text { \# Magnetic mom. } \\
& -0.13595992 \\
& -0.03012447 \\
& -0.13595992
\end{aligned}
$$


$-2.05300403$

$-1.94716847$

$-1.94716847$

$-1.94716847$

$-1.94716847$

1. 40458930

1. 40458930

1. 40458930

1. 40458930

1.51042473

1.51042473

1. 51042473

1. 51042473

$-2.05135775$

$-2.05135775$

$-2.05135775$

$-2.05135775$

$-1.94552219$

$-1.94552219$

$-1.94552219$

$-1.94552219$

2. 33443308

2. 33443308

2. 33443308

2. 33443308

2. 44026875

2. 44026875

2. 44026875

2. 44026875

$-1.00705183$

$-1.00705183$

$-1.00705183$

$-1.00705183$

$-0.90121639$

$-0.90121639$

$-0.90121639$

$-0.90121639$

1. 51851332

1. 51851332

1. 51851332

1. 51851332

1.62434876

1.62434876

1. 62434876

1. 62434876

$-1.10039973$

$-1.10039973$

$-1.10039973$

$-1.10039973$

$-0.99456429$

$-0.99456429$

$-0.99456429$

$-0.99456429$

0.70601028

0.70601028

0.70601028

0.70601028

0.81184572

0.81184572

0.81184572

0.81184572

64
$-0.09079729$

$-0.19663274$

$-0.19663274$

$-0.09079729$

$-0.09079729$

0.53337198

0.53337198

0.63920748

0.63920748

0.53337198

0.53337198

0.63920748

0.63920748

$-0.61681372$

$-0.61681372$

$-0.51097828$

$-0.51097828$

$-0.61681372$

$-0.61681372$

$-0.51097828$

$-0.51097828$

1. 26356936

1.26356936

1. 36940491

1. 36940491

1. 26356936

1.26356936

1. 36940491

1. 36940491

0.52339011

0.52339011

0.62922561

0.62922561

0.52339011

0.52339011

0.62922561

0.62922561

$-0.81257546$

$-0.81257546$

$-0.70673996$

$-0.70673996$

$-0.81257546$

$-0.81257546$

$-0.70673996$

$-0.70673996$

0.90373951

0.90373951

1.00957489

1.00957489

0.90373951

0.90373951

1.00957489

1. 00957489

$-1.34548187$

$-1.34548187$

$-1.23964632$

$-1.23964632$

$-1.34548187$

$-1.34548187$

$-1.23964632$

$-1.23964632$

0 .

\# Energy 3->7

$\begin{array}{lll}0.4556 & 0.1027 & 0.0969\end{array}$

$-0.0965$

$-0.0612$ $-0.1001$

$-0.19663274$

$-0.19663274$

$-0.09079729$

$-2.05300403$

$-2.05300403$
$-0.03012447$

$-0.13595992$

$-0.03012447$

$-0.13595992$

$-0.03012447$

$-0.10552958$

0.00030586

$-0.10552958$

0.00030586

$-0.10552958$

0.00030586

$-0.10552958$

0.00030586

$-1.29993546$

$-1.19410002$

$-1.29993546$

$-1.19410002$

$-1.29993546$

$-1.19410002$

$-1.29993546$

$-1.19410002$

0.26371813

0.36955357

0.26371813

0.36955357

0.26371813

0.36955357

0.26371813

0.36955357

0.37719119

0.48302662

0.37719119

0.48302662

0.37719119

0.48302662

0.37719119

0.48302662

$-0.22067484$

$-0.11483940$

$-0.22067484$

$-0.11483940$

$-0.22067484$

$-0.11483940$

$-0.22067484$

$-0.11483940$

1. 31258702

1. 41842246

1. 31258702

1. 41842246

1. 31258702

1. 41842246

1. 31258702

1. 41842246

$-0.51015860$

$-0.40432316$

$-0.51015860$

$-0.40432316$

$-0.51015860$

$-0.40432316$

$-0.51015860$

$-0.40432316$
$-2.79329395$

2.26936126

$-2.57884169$

$-2.36467934$

2.86617255

3. 74116325

$-4.75494099$

$-4.34235334$

6.10262537

$-6.23887157$

7. 43233681

6.92804670

$-9.00034809$

0.03333110

$-0.18021408$

$-0.10679254$

0.28836867

$-0.38399535$

0.61456376

0.48611420

$-0.75130200$

3.10589767

$-0.27599728$

$-1.88276172$

$-0.50156844$

$-3.56694126$

1. 11920130

2. 34268022

$-0.30399111$

4.88733292

$-5.56759024$

$-6.35950470$

7.31726503

$-4.64308786$

5.40447903

5.99603653

$-6.96966076$

$-0.16508196$

$-0.41441947$

0.40113044

0.42325312

$-0.64668185$

1. 15449846

0.62439299

$-1.33673286$

0.07316776

$-0.20665082$

$-0.52415538$

0.49305323

$-0.12455521$

0.22503565

0.50387639

$-0.46165097$

$-0.29734984$

0.09867825

0.22736306

0.05469122

0.38395002

$-0.07822187$

$-0.28865322$

$-0.13015728$

$$
1.000
$$

$-0.13595992$

$-0.03012447$

$-0.13595992$
Electr. mom., scale-fac Magnetic mom.

$-2.76146960$

2.90526342

2. 31284046 


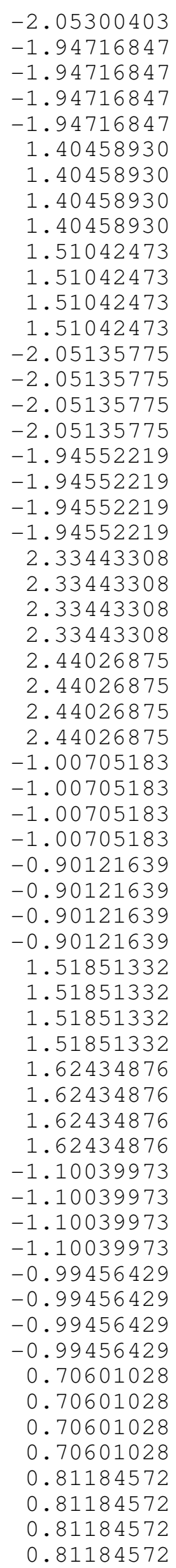

64
$-0.09079729$

$-0.19663274$

$-0.19663274$

$-0.09079729$

$-0.09079729$

0.53337198

0.53337198

0.63920748

0.63920748

0.53337198

0.53337198

0.63920748

0.63920748

$-0.61681372$

$-0.61681372$

$-0.51097828$

$-0.51097828$

$-0.61681372$

$-0.61681372$

$-0.51097828$

$-0.51097828$

1. 26356936

1.26356936

1. 36940491

1. 36940491

1. 26356936

1.26356936

1. 36940491

1. 36940491

0.52339011

0.52339011

0.62922561

0.62922561

0.52339011

0.52339011

0.62922561

0.62922561

$-0.81257546$

$-0.81257546$

$-0.70673996$

$-0.70673996$

$-0.81257546$

$-0.81257546$

$-0.70673996$

$-0.70673996$

0.90373951

0.90373951

1.00957489

1.00957489

0.90373951

0.90373951

1.00957489

1.00957489

$-1.34548187$

$-1.34548187$

$-1.23964632$

$-1.23964632$

$-1.34548187$

$-1.34548187$

$-1.23964632$

$-1.23964632$

\# Energy 3->8

$$
\begin{aligned}
& -0.03012447 \\
& -0.13595992 \\
& -0.03012447 \\
& -0.13595992 \\
& -0.03012447 \\
& -0.10552958 \\
& 0.00030586 \\
& -0.10552958 \\
& 0.00030586 \\
& -0.10552958 \\
& 0.00030586 \\
& -0.10552958 \\
& 0.00030586 \\
& -1.29993546 \\
& -1.19410002 \\
& -1.29993546 \\
& -1.19410002 \\
& -1.29993546 \\
& -1.19410002 \\
& -1.29993546 \\
& -1.19410002 \\
& 0.26371813 \\
& 0.36955357 \\
& 0.26371813 \\
& 0.36955357 \\
& 0.26371813 \\
& 0.36955357 \\
& 0.26371813 \\
& 0.36955357 \\
& 0.37719119 \\
& 0.48302662 \\
& 0.37719119 \\
& 0.48302662 \\
& 0.37719119 \\
& 0.48302662 \\
& 0.37719119 \\
& 0.48302662 \\
& -0.22067484 \\
& -0.11483940 \\
& -0.22067484 \\
& -0.11483940 \\
& -0.22067484 \\
& -0.11483940 \\
& -0.22067484 \\
& -0.11483940 \\
& \text { 1. } 31258702 \\
& \text { 1. } 41842246
\end{aligned}
$$

$-2.54990292$

1.64303803

$-1.83153951$

$-1.13541830$

1. 40239525

4.50150394

$-3.60819697$

$-4.77044439$

4.08908653

$-5.58919191$

4.76874685

5.89367390

$-5.28901863$

$-1.06662548$

1. 34359062

0.98714930

$-1.26167905$

0.71850675

$-0.92743045$

$-0.58695978$

0.78221714

$-0.67890441$

0.77799761

0.93819153

$-0.94276673$

$-0.19989578$

0.11502478

$-0.09792830$

0.11439901

1. 23390234

$-1.15031528$

$-1.15878606$

1.17029071

$-0.73836458$

0.62901837

0.54289317

$-0.51587057$

0.30528668

0.44853380

$-0.08756987$

$-0.82379830$

$-0.53287733$

$-0.22055410$

0.39462778

0.51192659

0.67911822

$-0.47409201$

$-0.53818029$

0.36298412

$-0.60391045$

0.40550724

0.43481669

$-0.27824426$

$-0.09647153$

0.19814153

0.12588415

$-0.28163081$

0.22200376

$-0.36432666$

$-0.29251069$

0.49631470

$$
\begin{array}{ccc}
-0.0802 & -0.0724 & -0.0643 \\
0.0017 & 0.0125 & -0.1254 \\
-2.05300403 & -0.19663274 \\
-2.05300403 & -0.19663274 \\
-2.05300403 & -0.09079729
\end{array}
$$

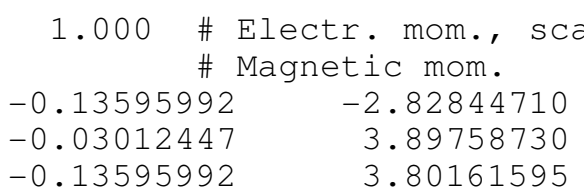


$-2.05300403$

$-1.94716847$

$-1.94716847$

$-1.94716847$

$-1.94716847$

1. 40458930

1. 40458930

1. 40458930

1. 40458930

1.51042473

1.51042473

1. 51042473

1. 51042473

$-2.05135775$

$-2.05135775$

$-2.05135775$

$-2.05135775$

$-1.94552219$

$-1.94552219$

$-1.94552219$

$-1.94552219$

2. 33443308

2. 33443308

2. 33443308

2. 33443308

2. 44026875

2. 44026875

2. 44026875

2. 44026875

$-1.00705183$

$-1.00705183$

$-1.00705183$

$-1.00705183$

$-0.90121639$

$-0.90121639$

$-0.90121639$

$-0.90121639$

1. 51851332

1. 51851332

1. 51851332

1. 51851332

1.62434876

1.62434876

1. 62434876

1. 62434876

$-1.10039973$

$-1.10039973$

$-1.10039973$

$-1.10039973$

$-0.99456429$

$-0.99456429$

$-0.99456429$

$-0.99456429$

0.70601028

0.70601028

0.70601028

0.70601028

0.81184572

0.81184572

0.81184572

64
0.81184572

$-0.09079729$

$-0.19663274$

$-0.19663274$

$-0.09079729$

$-0.09079729$

0.53337198

0.53337198

0.63920748

0.63920748

0.53337198

0.53337198

0.63920748

0.63920748

$-0.61681372$

$-0.61681372$

$-0.51097828$

$-0.51097828$

$-0.61681372$

$-0.61681372$

$-0.51097828$

$-0.51097828$

1. 26356936

1.26356936

1. 36940491

1. 36940491

1. 26356936

1.26356936

1. 36940491

1. 36940491

0.52339011

0.52339011

0.62922561

0.62922561

0.52339011

0.52339011

0.62922561

0.62922561

$-0.81257546$

$-0.81257546$

$-0.70673996$

$-0.70673996$

$-0.81257546$

$-0.81257546$

$-0.70673996$

$-0.70673996$

0.90373951

0.90373951

1.00957489

1.00957489

0.90373951

0.90373951

1.00957489

1. 00957489

$-1.34548187$

$-1.34548187$

$-1.23964632$

$-1.23964632$

$-1.34548187$

$-1.34548187$

$-1.23964632$

$-1.23964632$

0 .

0.0235

0.0038

$-2.05300403$

$-2.05300403$

$-2.05300403$

$-0.19663274$

$-0.19663274$

$-0.09079729$

$$
\begin{aligned}
& -0.03012447 \\
& -0.13595992 \\
& -0.03012447 \\
& -0.13595992 \\
& -0.03012447 \\
& -0.10552958 \\
& 0.00030586 \\
& -0.10552958 \\
& 0.00030586 \\
& -0.10552958 \\
& 0.00030586 \\
& -0.10552958 \\
& 0.00030586 \\
& -1.29993546 \\
& -1.19410002 \\
& -1.29993546 \\
& -1.19410002 \\
& -1.29993546 \\
& -1.19410002 \\
& -1.29993546 \\
& -1.19410002 \\
& 0.26371813 \\
& 0.36955357 \\
& 0.26371813 \\
& 0.36955357 \\
& 0.26371813 \\
& 0.36955357
\end{aligned}
$$

\# Energy 3->9

$$
\begin{array}{ll}
0.0006 & -0.0045 \\
0.0068 & -0.0012
\end{array}
$$

$$
1.000
$$

\# Electr. mom., scale-fac

$-0.13595992$

$-0.03012447$

$-0.13595992$
$-0.16511814$
0.24844129
0.30919671

\section{S-248}




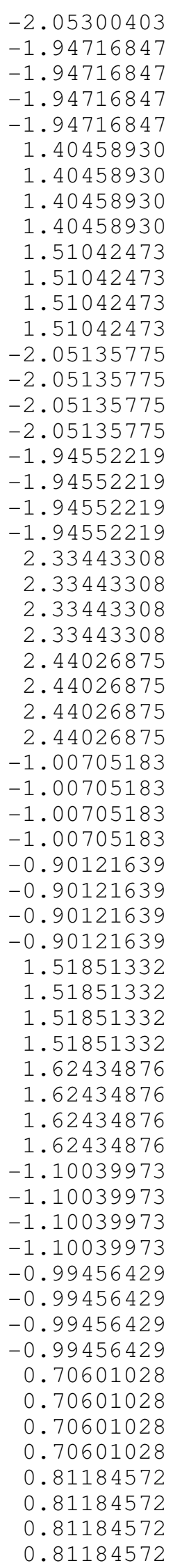

\&TRANSITION $4->$. .
$-0.09079729$
$-0.19663274$
$-0.19663274$
$-0.09079729$
$-0.09079729$
0.53337198
0.53337198
0.63920748
0.63920748
0.53337198
0.53337198
0.63920748
0.63920748
$-0.61681372$
$-0.61681372$
$-0.51097828$
$-0.51097828$
$-0.61681372$
$-0.61681372$
$-0.51097828$
$-0.51097828$
1. 26356936
1.26356936
1. 36940491
1. 36940491
1.26356936
1.26356936
1. 36940491
1. 36940491
0.52339011
0.52339011
0.62922561
0.62922561
0.52339011
0.52339011
0.62922561
0.62922561
$-0.81257546$
$-0.81257546$
$-0.70673996$
$-0.70673996$
$-0.81257546$
$-0.81257546$
$-0.70673996$
$-0.70673996$
0.90373951
0.90373951
1.00957489
1.00957489
0.90373951
0.90373951
1.00957489
1.00957489
$-1.34548187$
$-1.34548187$
$-1.23964632$
$-1.23964632$
$-1.34548187$
$-1.34548187$
$-1.23964632$
$-1.23964632$

$$
\begin{aligned}
& -0.03012447 \\
& -0.13595992 \\
& -0.03012447 \\
& -0.13595992 \\
& -0.03012447 \\
& -0.10552958 \\
& 0.00030586 \\
& -0.10552958 \\
& 0.00030586 \\
& -0.10552958 \\
& 0.00030586 \\
& -0.10552958 \\
& 0.00030586 \\
& -1.29993546 \\
& -1.19410002 \\
& -1.29993546 \\
& -1.19410002 \\
& -1.29993546 \\
& -1.19410002 \\
& -1.29993546 \\
& -1.19410002 \\
& 0.26371813 \\
& 0.36955357 \\
& 0.26371813 \\
& 0.36955357 \\
& 0.26371813 \\
& 0.36955357 \\
& 0.26371813 \\
& 0.36955357 \\
& 0.37719119 \\
& 0.48302662 \\
& 0.37719119 \\
& 0.48302662 \\
& 0.37719119 \\
& 0.48302662 \\
& 0.37719119 \\
& 0.48302662 \\
& -0.22067484 \\
& -0.11483940 \\
& -0.22067484 \\
& -0.11483940 \\
& -0.22067484 \\
& -0.11483940 \\
& -0.22067484 \\
& -0.11483940 \\
& \text { 1. } 31258702 \\
& \text { 1. } 41842246
\end{aligned}
$$

-0.42493460
0.19839846
-0.30055565
-0.36459044
0.50757784
0.36919039

$-0.21395397$

$-0.34512904$

0.18486035

$-0.17926873$

0.04554684

0.15347359

$-0.00805503$

0.03369129

$-0.03663382$

$-0.03248050$

0.03494889

$-0.03000366$

0.03148911

0.02964776

$-0.03079260$

$-0.01492694$

$-0.06763126$

$-0.01114590$

0.07807320

0.05968332

0.01326922

$-0.03331508$

$-0.02604020$

0.43204299

$-0.56295931$

$-0.35027575$

0.43348631

$-0.45552877$

0.55259627

0.38301417

$-0.44601515$

0.27711672

$-0.17147020$

$-0.28893542$

0.15763514

$-0.24566843$

0.16026735

0.25232455

$-0.14632235$

0.10777328

$-0.07094798$

$-0.09040839$

0.05801991

$-0.07069286$

0.04909552

0.06751566

$-0.04611993$

0.00081653

0.02839507

0.00783964

$-0.04198717$

$-0.01002441$

$-0.02724567$

$-0.00129294$

0.04504297

$$
\begin{aligned}
& 0.4010 \quad-0.5076 \quad \text { Energy } 4->5 \\
& \begin{array}{lll}
-0.0533 & -0.0221 & -0.0930
\end{array} \\
& -2.05300403 \\
& -0.19663274 \\
& -0.19663274
\end{aligned}
$$$$
\begin{gathered}
1.000 \text { \# Electr. mom., scale-fac } \\
\text { \# Magnetic mom. }
\end{gathered}
$$ 


\begin{tabular}{|c|c|c|c|}
\hline-205300403 & -0 09079729 & 13595090 & -288129139 \\
\hline-2.05300403 & -0.09079729 & -0.03012447 & 4. 49715042 \\
\hline-1.94716847 & -0.19663274 & -0.13595992 & -2.94279623 \\
\hline-1.94716847 & -0.19663274 & -0.03012447 & 4.06484747 \\
\hline-1.94716847 & -0.09079729 & -0.13595992 & 4.85529089 \\
\hline-1.94716847 & -0.09079729 & -0.03012447 & -6.77076626 \\
\hline 1.40458930 & 0.53337198 & -0.10552958 & 3.19715357 \\
\hline 1.40458930 & 0.53337198 & 0.00030586 & 1.09514928 \\
\hline 1.40458930 & 0.63920748 & -0.10552958 & -2.64069843 \\
\hline 1.40458930 & 0.63920748 & 0.00030586 & -1.98172975 \\
\hline 1.51042473 & 0.53337198 & -0.10552958 & -1.92879486 \\
\hline 1.51042473 & 0.53337198 & 0.00030586 & -2.87732029 \\
\hline 1.51042473 & 0.63920748 & -0.10552958 & 1.29905224 \\
\hline 1.51042473 & 0.63920748 & 0.00030586 & 3.83798814 \\
\hline-2.05135775 & -0.61681372 & -1.29993546 & -1.03799272 \\
\hline-2.05135775 & -0.61681372 & -1.19410002 & 1.31832278 \\
\hline-2.05135775 & -0.51097828 & -1.29993546 & 1.18696547 \\
\hline-2.05135775 & -0.51097828 & -1.19410002 & -1.49289000 \\
\hline-1.94552219 & -0.61681372 & -1.29993546 & 0.85364068 \\
\hline-1.94552219 & -0.61681372 & -1.19410002 & -1.09590173 \\
\hline-1.94552219 & -0.51097828 & -1.29993546 & -1.01348054 \\
\hline-1.94552219 & -0.51097828 & -1.19410002 & 1.29936159 \\
\hline 2.33443308 & 1.26356936 & 0.26371813 & 0.39783141 \\
\hline 2.33443308 & 1.26356936 & 0.36955357 & -0.29931003 \\
\hline 2.33443308 & 1.36940491 & 0.26371813 & -0.47372255 \\
\hline 2.33443308 & 1.36940491 & 0.36955357 & 0.31260934 \\
\hline 2.44026875 & 1.26356936 & 0.26371813 & -0.09958963 \\
\hline 2.44026875 & 1.26356936 & 0.36955357 & 0.00160385 \\
\hline 2.44026875 & 1.36940491 & 0.26371813 & 0.14409247 \\
\hline 2.44026875 & 1.36940491 & 0.36955357 & -0.02219939 \\
\hline-1.00705183 & 0.52339011 & 0.37719119 & 5.09995890 \\
\hline-1.00705183 & 0.52339011 & 0.48302662 & -2.27901888 \\
\hline-1.00705183 & 0.62922561 & 0.37719119 & -3.07291007 \\
\hline-1.00705183 & 0.62922561 & 0.48302662 & 0.98765093 \\
\hline-0.90121639 & 0.52339011 & 0.37719119 & -3.43981051 \\
\hline-0.90121639 & 0.52339011 & 0.48302662 & 0.99705946 \\
\hline-0.90121639 & 0.62922561 & 0.37719119 & 1.41432559 \\
\hline-0.90121639 & 0.62922561 & 0.48302662 & 0.35472128 \\
\hline 1.51851332 & -0.81257546 & -0.22067484 & 2.60377812 \\
\hline 1.51851332 & -0.81257546 & -0.11483940 & -2.60022974 \\
\hline 1.51851332 & -0.70673996 & -0.22067484 & -2.90992737 \\
\hline 1.51851332 & -0.70673996 & -0.11483940 & 2.79082274 \\
\hline 1.62434876 & -0.81257546 & -0.22067484 & -2.25498295 \\
\hline 1.62434876 & -0.81257546 & -0.11483940 & 1.96933591 \\
\hline 1.62434876 & -0.70673996 & -0.22067484 & 2.43987179 \\
\hline 1.62434876 & -0.70673996 & -0.11483940 & -1.95460927 \\
\hline-1.10039973 & 0.90373951 & 1.31258702 & 0.14426915 \\
\hline-1.10039973 & 0.90373951 & 1.41842246 & -0.11377243 \\
\hline-1.10039973 & 1.00957489 & 1.31258702 & -0.20454113 \\
\hline-1.10039973 & 1.00957489 & 1.41842246 & 0.17659736 \\
\hline-0.99456429 & 0.90373951 & 1.31258702 & -0.12461401 \\
\hline-0.99456429 & 0.90373951 & 1.41842246 & 0.09334640 \\
\hline-0.99456429 & 1.00957489 & 1.31258702 & 0.14505331 \\
\hline-0.99456429 & 1.00957489 & 1.41842246 & -0.12864868 \\
\hline 0.70601028 & -1.34548187 & -0.51015860 & 0.23632726 \\
\hline 0.70601028 & -1.34548187 & -0.40432316 & -0.07048801 \\
\hline 0.70601028 & -1.23964632 & -0.51015860 & -0.17204235 \\
\hline 0.70601028 & -1.23964632 & -0.40432316 & -0.06993313 \\
\hline 0.81184572 & -1.34548187 & -0.51015860 & -0.21114990 \\
\hline 0.81184572 & -1.34548187 & -0.40432316 & -0.00779465 \\
\hline 0.81184572 & -1.23964632 & -0.51015860 & 0.10251423 \\
\hline 0.81184572 & -1.23964632 & -0.40432316 & 0.21422365 \\
\hline & \# Energy 4->6 & & \\
\hline & -0.0958 & 00 & scale-fac \\
\hline 0.0672 & 0.0624 & & Magnetic \\
\hline-2.05300403 & -0.19663274 & -0.13595992 & 0.52852738 \\
\hline-2.05300403 & -0.19663274 & 3012447 & -0.81024081 \\
\hline & & & \\
\hline
\end{tabular}




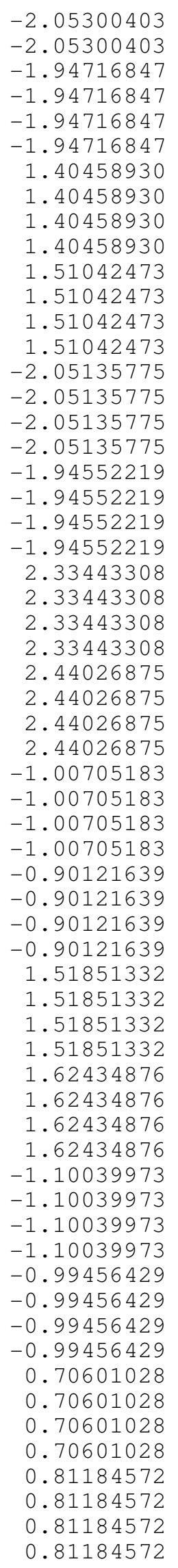

64
$-0.09079729$

$-0.09079729$

$-0.19663274$

$-0.19663274$

$-0.09079729$

$-0.09079729$

0.53337198

0.53337198

0.63920748

0.63920748

0.53337198

0.53337198

0.63920748

0.63920748

$-0.61681372$

$-0.61681372$

$-0.51097828$

$-0.51097828$

$-0.61681372$

$-0.61681372$

$-0.51097828$

$-0.51097828$

1. 26356936

1.26356936

1. 36940491

1. 36940491

1.26356936

1. 26356936

1. 36940491

1.36940491

0.52339011

0.52339011

0.62922561

0.62922561

0.52339011

0.52339011

0.62922561

0.62922561

$-0.81257546$

$-0.81257546$

$-0.70673996$

$-0.70673996$

$-0.81257546$

$-0.81257546$

$-0.70673996$

$-0.70673996$

0.90373951

0.90373951

1.00957489

1.00957489

0.90373951

0.90373951

1.00957489

1.00957489

$-1.34548187$

$-1.34548187$

$-1.23964632$

$-1.23964632$

$-1.34548187$

$-1.34548187$

$-1.23964632$

$-1.23964632$

\# Energy 4->7
$-0.13595992$

$-0.03012447$

$-0.13595992$

$-0.03012447$

$-0.13595992$

$-0.03012447$

$-0.10552958$

0.00030586

$-0.10552958$

0.00030586

$-0.10552958$

0.00030586

$-0.10552958$

0.00030586

$-1.29993546$

$-1.19410002$

$-1.29993546$

$-1.19410002$

$-1.29993546$

$-1.19410002$

$-1.29993546$

$-1.19410002$

0.26371813

0.36955357

0.26371813

0.36955357

0.26371813

0.36955357

0.26371813

0.36955357

0.37719119

0.48302662

0.37719119

0.48302662

0.37719119

0.48302662

0.37719119

0.48302662

$-0.22067484$

$-0.11483940$

$-0.22067484$

$-0.11483940$

$-0.22067484$

$-0.11483940$

$-0.22067484$

$-0.11483940$

1. 31258702

1.41842246

1. 31258702

1. 41842246

1. 31258702

1.41842246

1. 31258702

1. 41842246

$-0.51015860$

$-0.40432316$

$-0.51015860$

$-0.40432316$

$-0.51015860$

$-0.40432316$

$-0.51015860$

$-0.40432316$
$-1.12403238$

1. 13785887

$-0.23129018$

0.58475268

1. 25397694

$-1.22712362$

$-5.81860399$

$-0.01277721$

6.49255848

$-1.08413541$

4.86079836

1.09726977

$-5.68363237$

0.06611198

0.73768985

$-1.18702781$

$-0.94650555$

1. 49046814

$-1.17791367$

1. 75274551

1. 45898736

$-2.14619589$

$-0.63524687$

$-0.23780623$

1. 03530931

$-0.32919821$

0.10133705

0.50727034

$-0.51681024$

0.02871924

$-7.61019945$

6.05991220

5.31604099

$-4.10877037$

6.28536797

$-5.05431175$

$-4.21816635$

3. 43483949

1.13839900

$-3.73362279$

$-0.69516981$

3. 56740832

$-1.82620549$

4. 14787769

1. 51500034

$-4.15428209$

$-0.81388474$

0.51857889

0.83369762

$-0.53891838$

0.68933845

$-0.46268627$

$-0.84293091$

0.57694322

$-0.39642128$

0.15276667

0.58868349

$-0.36655596$

0.31013370

0.00630932

$-0.52955556$

0.24454270

$$
\begin{array}{rrr}
1.1778 & -0.1246 & 0.1611 \\
-0.0062 & -0.0478 & -0.0288
\end{array}
$$

$-2.05300403$

$-0.19663274$

1.000

\# Electr. mom., scale-fac

\# Magnetic mom.

$-2.05300403$

$-0.19663274$

$-0.13595992$

$-3.42827845$

$-0.03012447 \quad 3.67926836$ 


\begin{tabular}{|c|c|c|c|}
\hline-205300403 & -0 09079729 & 13595090 & 394914842 \\
\hline-2.05300403 & -0.09079729 & -0.03012447 & -4.46376228 \\
\hline-1.94716847 & -0.19663274 & -0.13595992 & 2.51394939 \\
\hline-1.94716847 & -0.19663274 & -0.03012447 & -2.92175651 \\
\hline-1.94716847 & -0.09079729 & -0.13595992 & -3.04636192 \\
\hline-1.94716847 & -0.09079729 & -0.03012447 & 3.70692849 \\
\hline 1.40458930 & 0.53337198 & -0.10552958 & -1.23374188 \\
\hline 1.40458930 & 0.53337198 & 0.00030586 & -0.04758259 \\
\hline 1.40458930 & 0.63920748 & -0.10552958 & -0.10279158 \\
\hline 1.40458930 & 0.63920748 & 0.00030586 & 2.07509685 \\
\hline 1.51042473 & 0.53337198 & -0.10552958 & 0.72350872 \\
\hline 1.51042473 & 0.53337198 & 0.00030586 & 0.85360444 \\
\hline 1.51042473 & 0.63920748 & -0.10552958 & 0.76583320 \\
\hline 1.51042473 & 0.63920748 & 0.00030586 & -3.10359192 \\
\hline-2.05135775 & -0.61681372 & -1.29993546 & -1.56208563 \\
\hline-2.05135775 & -0.61681372 & -1.19410002 & 1.88276207 \\
\hline-2.05135775 & -0.51097828 & -1.29993546 & 1.52010882 \\
\hline-2.05135775 & -0.51097828 & -1.19410002 & -1.84596229 \\
\hline-1.94552219 & -0.61681372 & -1.29993546 & 1.15614879 \\
\hline-1.94552219 & -0.61681372 & -1.19410002 & -1.39667308 \\
\hline-1.94552219 & -0.51097828 & -1.29993546 & -1.03166115 \\
\hline-1.94552219 & -0.51097828 & -1.19410002 & 1.25108576 \\
\hline 2.33443308 & 1.26356936 & 0.26371813 & -0.17348053 \\
\hline 2.33443308 & 1.26356936 & 0.36955357 & 0.46911061 \\
\hline 2.33443308 & 1.36940491 & 0.26371813 & 0.03071491 \\
\hline 2.33443308 & 1.36940491 & 0.36955357 & -0.19510463 \\
\hline 2.44026875 & 1.26356936 & 0.26371813 & 0.16961765 \\
\hline 2.44026875 & 1.26356936 & 0.36955357 & -0.33982006 \\
\hline 2.44026875 & 1.36940491 & 0.26371813 & -0.00742543 \\
\hline 2.44026875 & 1.36940491 & 0.36955357 & 0.10595122 \\
\hline-1.00705183 & 0.52339011 & 0.37719119 & 7.05816126 \\
\hline-1.00705183 & 0.52339011 & 0.48302662 & -7.07309198 \\
\hline-1.00705183 & 0.62922561 & 0.37719119 & -6.54085159 \\
\hline-1.00705183 & 0.62922561 & 0.48302662 & 6.70630121 \\
\hline-0.90121639 & 0.52339011 & 0.37719119 & -6.78486538 \\
\hline-0.90121639 & 0.52339011 & 0.48302662 & 6.74782753 \\
\hline-0.90121639 & 0.62922561 & 0.37719119 & 6.26665926 \\
\hline-0.90121639 & 0.62922561 & 0.48302662 & -6.42418623 \\
\hline 1.51851332 & -0.81257546 & -0.22067484 & -2.65419126 \\
\hline 1.51851332 & -0.81257546 & -0.11483940 & 1.46345246 \\
\hline 1.51851332 & -0.70673996 & -0.22067484 & 2.59259486 \\
\hline 1.51851332 & -0.70673996 & -0.11483940 & -1.65368557 \\
\hline 1.62434876 & -0.81257546 & -0.22067484 & 2.14496684 \\
\hline 1.62434876 & -0.81257546 & -0.11483940 & -1.28908932 \\
\hline 1.62434876 & -0.70673996 & -0.22067484 & -2.19583488 \\
\hline 1.62434876 & -0.70673996 & -0.11483940 & 1.62134218 \\
\hline-1.10039973 & 0.90373951 & 1.31258702 & 1.50033033 \\
\hline-1.10039973 & 0.90373951 & 1.41842246 & -1.04243100 \\
\hline-1.10039973 & 1.00957489 & 1.31258702 & -1.35515296 \\
\hline-1.10039973 & 1.00957489 & 1.41842246 & 0.90926564 \\
\hline-0.99456429 & 0.90373951 & 1.31258702 & -1.25849175 \\
\hline-0.99456429 & 0.90373951 & 1.41842246 & 0.86856115 \\
\hline-0.99456429 & 1.00957489 & 1.31258702 & 1.18078840 \\
\hline-0.99456429 & 1.00957489 & 1.41842246 & -0.78847355 \\
\hline 0.70601028 & -1.34548187 & -0.51015860 & 0.21925411 \\
\hline 0.70601028 & -1.34548187 & -0.40432316 & 0.04846172 \\
\hline 0.70601028 & -1.23964632 & -0.51015860 & -0.24904317 \\
\hline 0.70601028 & -1.23964632 & -0.40432316 & -0.20169717 \\
\hline 0.81184572 & -1.34548187 & -0.51015860 & -0.05961392 \\
\hline 0.81184572 & -1.34548187 & -0.40432316 & -0.30187571 \\
\hline 0.81184572 & -1.23964632 & -0.51015860 & 0.03345365 \\
\hline 0.81184572 & -1.23964632 & -0.40432316 & 0.55839705 \\
\hline & \# Energy 4->8 & & \\
\hline-0 . & 0.0579 & 000 & Electr. mom., \\
\hline 0.0087 & 0.0112 & & Magnetic \\
\hline-2.05300403 & -0.19663274 & 3595992 & 1.74681807 \\
\hline-2.05300403 & -0.196 & 3012447 & -2.39361262 \\
\hline & & & \\
\hline
\end{tabular}


$-2.05300403$

$-2.05300403$

$-1.94716847$

$-1.94716847$

$-1.94716847$

$-1.94716847$

1. 40458930

1. 40458930

1. 40458930

1. 40458930

1.51042473

1. 51042473

1. 51042473

1.51042473

$-2.05135775$

$-2.05135775$

$-2.05135775$

$-2.05135775$

$-1.94552219$

$-1.94552219$

$-1.94552219$

$-1.94552219$

2. 33443308

2. 33443308

2. 33443308

2. 33443308

2. 44026875

2. 44026875

2. 44026875

2. 44026875

$-1.00705183$

$-1.00705183$

$-1.00705183$

$-1.00705183$

$-0.90121639$

$-0.90121639$

$-0.90121639$

$-0.90121639$

1. 51851332

1. 51851332

1. 51851332

1. 51851332

1.62434876

1. 62434876

1.62434876

1.62434876

$-1.10039973$

$-1.10039973$

$-1.10039973$

$-1.10039973$

$-0.99456429$

$-0.99456429$

$-0.99456429$

$-0.99456429$

0.70601028

0.70601028

0.70601028

0.70601028

0.81184572

0.81184572

0.81184572

0.81184572
$-0.09079729$

$-0.09079729$

$-0.19663274$

$-0.19663274$

$-0.09079729$

$-0.09079729$

0.53337198

0.53337198

0.63920748

0.63920748

0.53337198

0.53337198

0.63920748

0.63920748

$-0.61681372$

$-0.61681372$

$-0.51097828$

$-0.51097828$

$-0.61681372$

$-0.61681372$

$-0.51097828$

$-0.51097828$

1. 26356936

1.26356936

1. 36940491

1. 36940491

1.26356936

1. 26356936

1. 36940491

1.36940491

0.52339011

0.52339011

0.62922561

0.62922561

0.52339011

0.52339011

0.62922561

0.62922561

$-0.81257546$

$-0.81257546$

$-0.70673996$

$-0.70673996$

$-0.81257546$

$-0.81257546$

$-0.70673996$

$-0.70673996$

0.90373951

0.90373951

1.00957489

1.00957489

0.90373951

0.90373951

1.00957489

1.00957489

$-1.34548187$

$-1.34548187$

$-1.23964632$

$-1.23964632$

$-1.34548187$

$-1.34548187$

$-1.23964632$

$-1.23964632$

\# Energy 4->9

$\begin{array}{lll}-0.3530 & -0.1050 & -0.0475\end{array}$

$-0.0444$

$-2.05300403$

$-2.05300403$

$-0.19663274$

$-0.19663274$
$-0.13595992$

$-0.03012447$

$-0.13595992$

$-0.03012447$

$-0.13595992$

$-0.03012447$

$-0.10552958$

0.00030586

$-0.10552958$

0.00030586

$-0.10552958$

0.00030586

$-0.10552958$

0.00030586

$-1.29993546$

$-1.19410002$

$-1.29993546$

$-1.19410002$

$-1.29993546$

$-1.19410002$

$-1.29993546$

$-1.19410002$

0.26371813

0.36955357

0.26371813

0.36955357

0.26371813

0.36955357

0.26371813

0.36955357

0.37719119

0.48302662

0.37719119

0.48302662

0.37719119

0.48302662

0.37719119

0.48302662

$-0.22067484$

$-0.11483940$

$-0.22067484$

$-0.11483940$

$-0.22067484$

$-0.11483940$

$-0.22067484$

$-0.11483940$

1. 31258702

1. 41842246

1. 31258702

1. 41842246

1. 31258702

1. 41842246

1. 31258702

1. 41842246

$-0.51015860$

$-0.40432316$

$-0.51015860$

$-0.40432316$

$-0.51015860$

$-0.40432316$

$-0.51015860$

$-0.40432316$
$-2.27585673$

3. 12227106

$-1.76563609$

2. 57226801

2.37832880

$-3.40134764$

3.14100266

$-1.98090005$

$-2.99391747$

1. 83811355

$-3.02108359$

1. 84695435

2.88915920

$-1.70064390$

0.76300681

$-0.98811644$

$-0.78604001$

1.03004718

$-0.62715238$

0.82735217

0.62971646

$-0.84610987$

1.02819693

$-0.36257815$

$-0.96863037$

0.38053185

$-0.81174260$

0.24458729

0.75425303

$-0.26552081$

$-1.97403526$

2. 34730554

2.07308459

$-2.35026860$

1. 70636618

$-2.01818442$

$-1.72825801$

1. 97024500

0.80058873

0.21013109

$-0.97436112$

0.00004097

$-0.53087997$

$-0.27734053$

0.72620565

0.03789367

$-0.47098884$

0.35313669

0.45048237

$-0.33068994$

0.39864460

$-0.30288666$

$-0.37695152$

0.28018424

0.08197038

$-0.15012681$

$-0.15777384$

0.30432367

$-0.12162681$

0.21409863

0.23489782

$-0.42894563$
1.000 \# Electr. mom., scale-fac \# Magnetic mom.
$\begin{array}{ll}-0.13595992 & -1.61372209\end{array}$
$\begin{array}{rr}-0.13595992 & -1.61372209 \\ -0.03012447 & 2.09147787\end{array}$




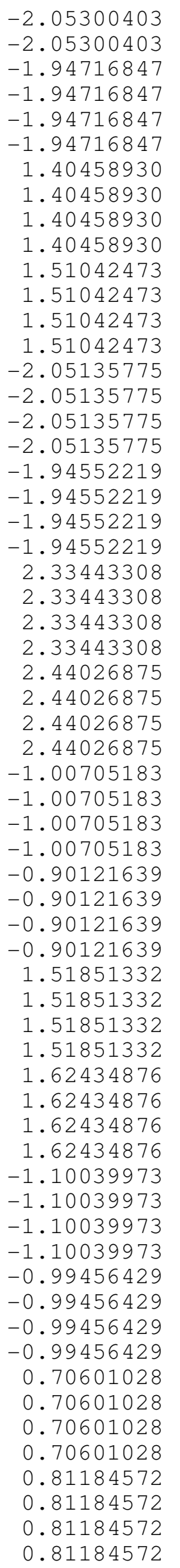

\&TRANSITION 5->...
$-0.09079729$

$-0.09079729$

$-0.19663274$

$-0.19663274$

$-0.09079729$

$-0.09079729$

0.53337198

0.53337198

0.63920748

0.63920748

0.53337198

0.53337198

0.63920748

0.63920748

$-0.61681372$

$-0.61681372$

$-0.51097828$

$-0.51097828$

$-0.61681372$

$-0.61681372$

$-0.51097828$

$-0.51097828$

1. 26356936

1. 26356936

1.36940491

1. 36940491

1.26356936

1. 26356936

1. 36940491

1.36940491

0.52339011

0.52339011

0.62922561

0.62922561

0.52339011

0.52339011

0.62922561

0.62922561

$-0.81257546$

$-0.81257546$

$-0.70673996$

$-0.70673996$

$-0.81257546$

$-0.81257546$

$-0.70673996$

$-0.70673996$

0.90373951

0.90373951

1.00957489

1.00957489

0.90373951

0.90373951

1.00957489

1.00957489

$-1.34548187$

$-1.34548187$

$-1.23964632$

$-1.23964632$

$-1.34548187$

$-1.34548187$

$-1.23964632$

$-1.23964632$
$-0.13595992$

$-0.03012447$

$-0.13595992$

$-0.03012447$

$-0.13595992$

$-0.03012447$

$-0.10552958$

0.00030586

$-0.10552958$

0.00030586

$-0.10552958$

0.00030586

$-0.10552958$

0.00030586

$-1.29993546$

$-1.19410002$

$-1.29993546$

$-1.19410002$

$-1.29993546$

$-1.19410002$

$-1.29993546$

$-1.19410002$

0.26371813

0.36955357

0.26371813

0.36955357

0.26371813

0.36955357

0.26371813

0.36955357

0.37719119

0.48302662

0.37719119

0.48302662

0.37719119

0.48302662

0.37719119

0.48302662

$-0.22067484$

$-0.11483940$

$-0.22067484$

$-0.11483940$

$-0.22067484$

$-0.11483940$

$-0.22067484$

$-0.11483940$

1. 31258702

1. 41842246

1. 31258702

1. 41842246

1. 31258702

1.41842246

1. 31258702

1. 41842246

$-0.51015860$

$-0.40432316$

$-0.51015860$

$-0.40432316$

$-0.51015860$

$-0.40432316$

$-0.51015860$

$-0.40432316$
2.12898636

$-2.77144742$

1. 44960868

$-2.05392790$

$-2.03828955$

2. 85923409

1.93096197

$-0.94844937$

$-1.78304684$

0.83965874

$-1.68782449$

0.80261427

1.54056835

$-0.68118536$

0.03030296

$-0.03774517$

$-0.00957485$

0.01647880

$-0.17197575$

0.20985883

0.17704724

$-0.21652141$

0.01704167

$-0.01222596$

0.22740123

$-0.23958707$

$-0.11003043$

0.08979496

$-0.14710966$

0.16217370

3.31544137

$-4.16843939$

$-2.78123927$

3. 43776345

$-3.03827691$

3.73606896

2.44669461

$-2.99358821$

1. 07677543

$-0.82909763$

$-1.16461217$

0.83390123

$-1.03400064$

0.81884503

1. 11315382

$-0.83474141$

0.76822829

$-0.54249102$

$-0.70533878$

0.49280602

$-0.54987752$

0.40425733

0.51955003

$-0.37827554$

$-0.12182662$

0.19361003

0.15080854

$-0.24373206$

0.10339015

$-0.19191983$

$-0.13866265$

0.25427932

64

$$
\begin{array}{cccc} 
& 0.04 & \text { Energy } & 5->6 \\
0.6834 & -0.0796 & -0.1826 \\
0.1105 & 0.0348 & 0.3394 \\
-2.05300403 & -0.19663274
\end{array}
$$

1.000 \# Electr. mom., scale-fac \# Magnetic mom. $-0.13595992 \quad-5.32080317$ 


\begin{tabular}{|c|c|c|c|}
\hline & & 7 & \\
\hline-2.05300403 & -0.19663274 & -0.03012447 & 7.94619226 \\
\hline-2.05300403 & -0.09079729 & -0.13595992 & 7.28658915 \\
\hline-2.05300403 & -0.09079729 & -0.03012447 & -10.62573624 \\
\hline-1.94716847 & -0.19663274 & -0.13595992 & 5.18821621 \\
\hline-1.94716847 & -0.19663274 & -0.03012447 & -8.16316128 \\
\hline-1.94716847 & -0.09079729 & -0.13595992 & -6.96958065 \\
\hline-1.94716847 & -0.09079729 & -0.03012447 & 10.88090038 \\
\hline 1.40458930 & 0.53337198 & -0.10552958 & 7.02008533 \\
\hline 1.40458930 & 0.53337198 & 0.00030586 & -5.16986561 \\
\hline 1.40458930 & 0.63920748 & -0.10552958 & -7.79036522 \\
\hline 1.40458930 & 0.63920748 & 0.00030586 & 6.56097031 \\
\hline 1.51042473 & 0.53337198 & -0.10552958 & -7.64366102 \\
\hline 1.51042473 & 0.53337198 & 0.00030586 & 6.22168350 \\
\hline 1.51042473 & 0.63920748 & -0.10552958 & 8.57978725 \\
\hline 1.51042473 & 0.63920748 & 0.00030586 & -7.73673916 \\
\hline-2.05135775 & -0.61681372 & -1.29993546 & -6.79875231 \\
\hline-2.05135775 & -0.61681372 & -1.19410002 & 8.78350544 \\
\hline-2.05135775 & -0.51097828 & -1.29993546 & 7.04420328 \\
\hline-2.05135775 & -0.51097828 & -1.19410002 & -9.19435692 \\
\hline-1.94552219 & -0.61681372 & -1.29993546 & 6.14255953 \\
\hline-1.94552219 & -0.61681372 & -1.19410002 & -7.99518299 \\
\hline-1.94552219 & -0.51097828 & -1.29993546 & -6.26780748 \\
\hline-1.94552219 & -0.51097828 & -1.19410002 & 8.24969196 \\
\hline 2.33443308 & 1.26356936 & 0.26371813 & -1.60763395 \\
\hline 2.33443308 & 1.26356936 & 0.36955357 & 1.14276445 \\
\hline 2.33443308 & 1.36940491 & 0.26371813 & 1.38730979 \\
\hline 2.33443308 & 1.36940491 & 0.36955357 & -0.96596682 \\
\hline 2.44026875 & 1.26356936 & 0.26371813 & 0.86202902 \\
\hline 2.44026875 & 1.26356936 & 0.36955357 & -0.45421585 \\
\hline 2.44026875 & 1.36940491 & 0.26371813 & -0.73669726 \\
\hline 2.44026875 & 1.36940491 & 0.36955357 & 0.38689736 \\
\hline-1.00705183 & 0.52339011 & 0.37719119 & 4.84178448 \\
\hline-1.00705183 & 0.52339011 & 0.48302662 & -8.69803047 \\
\hline-1.00705183 & 0.62922561 & 0.37719119 & -6.57148790 \\
\hline-1.00705183 & 0.62922561 & 0.48302662 & 9.47604942 \\
\hline-0.90121639 & 0.52339011 & 0.37719119 & -5.17420673 \\
\hline-0.90121639 & 0.52339011 & 0.48302662 & 8.37649155 \\
\hline-0.90121639 & 0.62922561 & 0.37719119 & 6.53619623 \\
\hline-0.90121639 & 0.62922561 & 0.48302662 & -9.04824162 \\
\hline 1.51851332 & -0.81257546 & -0.22067484 & -0.82392412 \\
\hline 1.51851332 & -0.81257546 & -0.11483940 & 2.40392852 \\
\hline 1.51851332 & -0.70673996 & -0.22067484 & 0.90834266 \\
\hline 1.51851332 & -0.70673996 & -0.11483940 & -2.91979241 \\
\hline 1.62434876 & -0.81257546 & -0.22067484 & 0.37156898 \\
\hline 1.62434876 & -0.81257546 & -0.11483940 & -1.78310943 \\
\hline 1.62434876 & -0.70673996 & -0.22067484 & -0.44615686 \\
\hline 1.62434876 & -0.70673996 & -0.11483940 & 2.24944472 \\
\hline-1.10039973 & 0.90373951 & 1.31258702 & 3.53997922 \\
\hline-1.10039973 & 0.90373951 & 1.41842246 & -2.57752776 \\
\hline-1.10039973 & 1.00957489 & 1.31258702 & -3.16588140 \\
\hline-1.10039973 & 1.00957489 & 1.41842246 & 2.24554110 \\
\hline-0.99456429 & 0.90373951 & 1.31258702 & -2.71573973 \\
\hline-0.99456429 & 0.90373951 & 1.41842246 & 2.01917243 \\
\hline-0.99456429 & 1.00957489 & 1.31258702 & 2.54505587 \\
\hline-0.99456429 & 1.00957489 & 1.41842246 & -1.84293747 \\
\hline 0.70601028 & -1.34548187 & -0.51015860 & -0.30016983 \\
\hline 0.70601028 & -1.34548187 & -0.40432316 & 0.60648710 \\
\hline 0.70601028 & -1.23964632 & -0.51015860 & 0.31330773 \\
\hline 0.70601028 & -1.23964632 & -0.40432316 & -0.72783804 \\
\hline 0.81184572 & -1.34548187 & -0.51015860 & 0.34361342 \\
\hline 0.81184572 & -1.34548187 & -0.40432316 & -0.72343224 \\
\hline 0.81184572 & -1.23964632 & -0.51015860 & -0.35338879 \\
\hline 0.81184572 & -1.23964632 & -0.40432316 & 0.85204273 \\
\hline & \# Energy 5->7 & & \\
\hline-6.5238 & -1.2076 & .000 & scale-fac \\
\hline 0.0267 & -0.0518 & & Magnetic \\
\hline-2.0530 & -0.1 & -0 & 4.0 \\
\hline & & & \\
\hline
\end{tabular}




\begin{tabular}{|c|c|c|c|}
\hline & & & \\
\hline-2.05300403 & -0.19663274 & -0.03012447 & -5.61539745 \\
\hline-2.05300403 & -0.09079729 & -0.13595992 & -8.02417946 \\
\hline-2.05300403 & -0.09079729 & -0.03012447 & 11.58897686 \\
\hline-1.94716847 & -0.19663274 & -0.13595992 & -0.10330988 \\
\hline-1.94716847 & -0.19663274 & -0.03012447 & 2.92488503 \\
\hline-1.94716847 & -0.09079729 & -0.13595992 & 4.31038284 \\
\hline-1.94716847 & -0.09079729 & -0.03012447 & -9.27264118 \\
\hline 1.40458930 & 0.53337198 & -0.10552958 & -23.10019684 \\
\hline 1.40458930 & 0.53337198 & 0.00030586 & 22.84970856 \\
\hline 1.40458930 & 0.63920748 & -0.10552958 & 30.63722992 \\
\hline 1.40458930 & 0.63920748 & 0.00030586 & -34.08528900 \\
\hline 1.51042473 & 0.53337198 & -0.10552958 & 27.40182686 \\
\hline 1.51042473 & 0.53337198 & 0.00030586 & -29.00415421 \\
\hline 1.51042473 & 0.63920748 & -0.10552958 & -35.81069183 \\
\hline 1.51042473 & 0.63920748 & 0.00030586 & 41.12103271 \\
\hline-2.05135775 & -0.61681372 & -1.29993546 & -3.88725972 \\
\hline-2.05135775 & -0.61681372 & -1.19410002 & 5.86072063 \\
\hline-2.05135775 & -0.51097828 & -1.29993546 & 4.52525616 \\
\hline-2.05135775 & -0.51097828 & -1.19410002 & -6.75580549 \\
\hline-1.94552219 & -0.61681372 & -1.29993546 & 5.65889597 \\
\hline-1.94552219 & -0.61681372 & -1.19410002 & -8.05593395 \\
\hline-1.94552219 & -0.51097828 & -1.29993546 & -6.82280350 \\
\hline-1.94552219 & -0.51097828 & -1.19410002 & 9.64478970 \\
\hline 2.33443308 & 1.26356936 & 0.26371813 & -0.52939880 \\
\hline 2.33443308 & 1.26356936 & 0.36955357 & -3.32797909 \\
\hline 2.33443308 & 1.36940491 & 0.26371813 & 1.50312614 \\
\hline 2.33443308 & 1.36940491 & 0.36955357 & 1.13975322 \\
\hline 2.44026875 & 1.26356936 & 0.26371813 & 0.32679856 \\
\hline 2.44026875 & 1.26356936 & 0.36955357 & 2.33213878 \\
\hline 2.44026875 & 1.36940491 & 0.26371813 & -1.49970734 \\
\hline 2.44026875 & 1.36940491 & 0.36955357 & -0.35731402 \\
\hline-1.00705183 & 0.52339011 & 0.37719119 & -23.02889061 \\
\hline-1.00705183 & 0.52339011 & 0.48302662 & 28.30766487 \\
\hline-1.00705183 & 0.62922561 & 0.37719119 & 26.21015549 \\
\hline-1.00705183 & 0.62922561 & 0.48302662 & -31.34921455 \\
\hline-0.90121639 & 0.52339011 & 0.37719119 & 23.66664124 \\
\hline-0.90121639 & 0.52339011 & 0.48302662 & -28.16421700 \\
\hline-0.90121639 & 0.62922561 & 0.37719119 & -26.90079689 \\
\hline-0.90121639 & 0.62922561 & 0.48302662 & 31.66449165 \\
\hline 1.51851332 & -0.81257546 & -0.22067484 & 10.91659451 \\
\hline 1.51851332 & -0.81257546 & -0.11483940 & -15.10989857 \\
\hline 1.51851332 & -0.70673996 & -0.22067484 & -10.26993465 \\
\hline 1.51851332 & -0.70673996 & -0.11483940 & 15.65053463 \\
\hline 1.62434876 & -0.81257546 & -0.22067484 & -9.14246559 \\
\hline 1.62434876 & -0.81257546 & -0.11483940 & 12.69636726 \\
\hline 1.62434876 & -0.70673996 & -0.22067484 & 8.56268597 \\
\hline 1.62434876 & -0.70673996 & -0.11483940 & -13.20821190 \\
\hline-1.10039973 & 0.90373951 & 1.31258702 & -6.36006260 \\
\hline-1.10039973 & 0.90373951 & 1.41842246 & 4.59489012 \\
\hline-1.10039973 & 1.00957489 & 1.31258702 & 6.45895910 \\
\hline-1.10039973 & 1.00957489 & 1.41842246 & -4.46392679 \\
\hline-0.99456429 & 0.90373951 & 1.31258702 & 4.75038004 \\
\hline-0.99456429 & 0.90373951 & 1.41842246 & -3.48032856 \\
\hline-0.99456429 & 1.00957489 & 1.31258702 & -5.39519310 \\
\hline-0.99456429 & 1.00957489 & 1.41842246 & 3.76136589 \\
\hline 0.70601028 & -1.34548187 & -0.51015860 & -0.35316846 \\
\hline 0.70601028 & -1.34548187 & -0.40432316 & -0.49020338 \\
\hline 0.70601028 & -1.23964632 & -0.51015860 & 1.11583579 \\
\hline 0.70601028 & -1.23964632 & -0.40432316 & -0.00445046 \\
\hline 0.81184572 & -1.34548187 & -0.51015860 & 0.06083896 \\
\hline 0.81184572 & -1.34548187 & -0.40432316 & 1.00909138 \\
\hline 0.81184572 & -1.23964632 & -0.51015860 & -0.93050104 \\
\hline 0.81184572 & -1.23964632 & -0.40432316 & -0.44664776 \\
\hline & \# Energy 5->8 & & \\
\hline 86 & 0.7115 & 1.000 & Electr. \\
\hline 0.0188 & 0.0193 & $\#$ & Magnetic mom. \\
\hline 300403 & -0.19663274 & -0.13595992 & 0.24296248 \\
\hline & & & \\
\hline
\end{tabular}




\begin{tabular}{|c|c|c|c|}
\hline & & ค100010 & \\
\hline-2.05300403 & -0.19663274 & -0.03012447 & 2.80113411 \\
\hline-2.05300403 & -0.09079729 & -0.13595992 & 2.06487894 \\
\hline-2.05300403 & -0.09079729 & -0.03012447 & -5.91081095 \\
\hline-1.94716847 & -0.19663274 & -0.13595992 & 0.61160618 \\
\hline-1.94716847 & -0.19663274 & -0.03012447 & -4.44503403 \\
\hline-1.94716847 & -0.09079729 & -0.13595992 & -3.44718313 \\
\hline-1.94716847 & -0.09079729 & -0.03012447 & 8.11497498 \\
\hline 1.40458930 & 0.53337198 & -0.10552958 & -3.76273131 \\
\hline 1.40458930 & 0.53337198 & 0.00030586 & 3.09861445 \\
\hline 1.40458930 & 0.63920748 & -0.10552958 & 3.05138826 \\
\hline 1.40458930 & 0.63920748 & 0.00030586 & -2.50296330 \\
\hline 1.51042473 & 0.53337198 & -0.10552958 & 3.78541970 \\
\hline 1.51042473 & 0.53337198 & 0.00030586 & -3.25574708 \\
\hline 1.51042473 & 0.63920748 & -0.10552958 & -3.08129144 \\
\hline 1.51042473 & 0.63920748 & 0.00030586 & 2.76484299 \\
\hline-2.05135775 & -0.61681372 & -1.29993546 & -0.07741001 \\
\hline-2.05135775 & -0.61681372 & -1.19410002 & 0.23848797 \\
\hline-2.05135775 & -0.51097828 & -1.29993546 & 0.13609765 \\
\hline-2.05135775 & -0.51097828 & -1.19410002 & -0.34855244 \\
\hline-1.94552219 & -0.61681372 & -1.29993546 & -0.08038070 \\
\hline-1.94552219 & -0.61681372 & -1.19410002 & -0.05892859 \\
\hline-1.94552219 & -0.51097828 & -1.29993546 & 0.06369780 \\
\hline-1.94552219 & -0.51097828 & -1.19410002 & 0.12094927 \\
\hline 2.33443308 & 1.26356936 & 0.26371813 & -5.33813810 \\
\hline 2.33443308 & 1.26356936 & 0.36955357 & 2.48582244 \\
\hline 2.33443308 & 1.36940491 & 0.26371813 & 4.92193556 \\
\hline 2.33443308 & 1.36940491 & 0.36955357 & -2.35211706 \\
\hline 2.44026875 & 1.26356936 & 0.26371813 & 3.84631467 \\
\hline 2.44026875 & 1.26356936 & 0.36955357 & -1.31527698 \\
\hline 2.44026875 & 1.36940491 & 0.26371813 & -3.45002508 \\
\hline 2.44026875 & 1.36940491 & 0.36955357 & 1.23518121 \\
\hline-1.00705183 & 0.52339011 & 0.37719119 & 1.52097440 \\
\hline-1.00705183 & 0.52339011 & 0.48302662 & -3.60360408 \\
\hline-1.00705183 & 0.62922561 & 0.37719119 & -4.14706039 \\
\hline-1.00705183 & 0.62922561 & 0.48302662 & 5.63293695 \\
\hline-0.90121639 & 0.52339011 & 0.37719119 & -1.23777866 \\
\hline-0.90121639 & 0.52339011 & 0.48302662 & 3.16773939 \\
\hline-0.90121639 & 0.62922561 & 0.37719119 & 3.74355960 \\
\hline-0.90121639 & 0.62922561 & 0.48302662 & -5.27001286 \\
\hline 1.51851332 & -0.81257546 & -0.22067484 & -3.24499702 \\
\hline 1.51851332 & -0.81257546 & -0.11483940 & 3.60973382 \\
\hline 1.51851332 & -0.70673996 & -0.22067484 & 3.66694260 \\
\hline 1.51851332 & -0.70673996 & -0.11483940 & -4.32273531 \\
\hline 1.62434876 & -0.81257546 & -0.22067484 & 3.36332750 \\
\hline 1.62434876 & -0.81257546 & -0.11483940 & -3.75579047 \\
\hline 1.62434876 & -0.70673996 & -0.22067484 & -3.86529636 \\
\hline 1.62434876 & -0.70673996 & -0.11483940 & 4.51217699 \\
\hline-1.10039973 & 0.90373951 & 1.31258702 & 0.79019612 \\
\hline-1.10039973 & 0.90373951 & 1.41842246 & -0.62386948 \\
\hline-1.10039973 & 1.00957489 & 1.31258702 & -1.03470385 \\
\hline-1.10039973 & 1.00957489 & 1.41842246 & 0.76454437 \\
\hline-0.99456429 & 0.90373951 & 1.31258702 & -0.59462494 \\
\hline-0.99456429 & 0.90373951 & 1.41842246 & 0.49590811 \\
\hline-0.99456429 & 1.00957489 & 1.31258702 & 0.94497502 \\
\hline-0.99456429 & 1.00957489 & 1.41842246 & -0.71123660 \\
\hline 0.70601028 & -1.34548187 & -0.51015860 & -0.01057638 \\
\hline 0.70601028 & -1.34548187 & -0.40432316 & 0.21722414 \\
\hline 0.70601028 & -1.23964632 & -0.51015860 & 0.12468043 \\
\hline 0.70601028 & -1.23964632 & -0.40432316 & -0.46507016 \\
\hline 0.81184572 & -1.34548187 & -0.51015860 & 0.10886162 \\
\hline 0.81184572 & -1.34548187 & -0.40432316 & -0.41397196 \\
\hline 0.81184572 & -1.23964632 & -0.51015860 & -0.27961138 \\
\hline 0.81184572 & -1.23964632 & -0.40432316 & 0.75944024 \\
\hline & \# Energy 5->9 & & \\
\hline $6<$ & -0.1774 & 00 & Electr. mom., \\
\hline 0.0273 & -0.0139 & & Magnetic mom. \\
\hline-2.05300403 & -0.19 & -0.135 & -2.33754587 \\
\hline & & & \\
\hline
\end{tabular}


$-2.05300403$

$-2.05300403$

$-2.05300403$

$-1.94716847$

$-1.94716847$

$-1.94716847$

$-1.94716847$

1. 40458930

1. 40458930

1. 40458930

1. 40458930

1. 51042473

1. 51042473

1.51042473

1. 51042473

$-2.05135775$

$-2.05135775$

$-2.05135775$

$-2.05135775$

$-1.94552219$

$-1.94552219$

$-1.94552219$

$-1.94552219$

2. 33443308

2. 33443308

2. 33443308

2. 33443308

2. 44026875

2. 44026875

2. 44026875

2. 44026875

$-1.00705183$

$-1.00705183$

$-1.00705183$

$-1.00705183$

$-0.90121639$

$-0.90121639$

$-0.90121639$

$-0.90121639$

1. 51851332

1. 51851332

1. 51851332

1.51851332

1. 62434876

1.62434876

1.62434876

1.62434876

$-1.10039973$

$-1.10039973$

$-1.10039973$

$-1.10039973$

$-0.99456429$

$-0.99456429$

$-0.99456429$

$-0.99456429$

0.70601028

0.70601028

0.70601028

0.70601028

0.81184572

0.81184572

0.81184572

0.81184572

\&TRANSITION $6->$. .

64

-0.3444
0.0612

0. \# Energy 6->7

$-0.1465-0.1854$

$-0.2251-0.4900$

$-0.19663274$

$-0.09079729$

$-0.09079729$

$-0.19663274$

$-0.19663274$

$-0.09079729$

$-0.09079729$

0.53337198

0.53337198

0.63920748

0.63920748

0.53337198

0.53337198

0.63920748

0.63920748

$-0.61681372$

$-0.61681372$

$-0.51097828$

$-0.51097828$

$-0.61681372$

$-0.61681372$

$-0.51097828$

$-0.51097828$

1. 26356936

1. 26356936

1. 36940491

1.36940491

1. 26356936

1.26356936

1.36940491

1. 36940491

0.52339011

0.52339011

0.62922561

0.62922561

0.52339011

0.52339011

0.62922561

0.62922561

$-0.81257546$

$-0.81257546$

$-0.70673996$

$-0.70673996$

$-0.81257546$

$-0.81257546$

$-0.70673996$

$-0.70673996$

0.90373951

0.90373951

1.00957489

1. 00957489

0.90373951

0.90373951

1.00957489

1.00957489

$-1.34548187$

$-1.34548187$

$-1.23964632$

$-1.23964632$

$-1.34548187$

$-1.34548187$

$-1.23964632$

$-1.23964632$
$-0.03012447$

$-0.13595992$

$-0.03012447$

$-0.13595992$

$-0.03012447$

$-0.13595992$

$-0.03012447$

$-0.10552958$

0.00030586

$-0.10552958$

0.00030586

$-0.10552958$

0.00030586

$-0.10552958$

0.00030586

$-1.29993546$

$-1.19410002$

$-1.29993546$

$-1.19410002$

$-1.29993546$

$-1.19410002$

$-1.29993546$

$-1.19410002$

0.26371813

0.36955357

0.26371813

0.36955357

0.26371813

0.36955357

0.26371813

0.36955357

0.37719119

0.48302662

0.37719119

0.48302662

0.37719119

0.48302662

0.37719119

0.48302662

$-0.22067484$

$-0.11483940$

$-0.22067484$

$-0.11483940$

$-0.22067484$

$-0.11483940$

$-0.22067484$

$-0.11483940$

1. 31258702

1. 41842246

1. 31258702

1. 41842246

1. 31258702

1. 41842246

1. 31258702

1. 41842246

$-0.51015860$

$-0.40432316$

$-0.51015860$

$-0.40432316$

$-0.51015860$

$-0.40432316$

$-0.51015860$

$-0.40432316$
3.62750769

3. 23916650

$-4.74549770$

2. 96089268

$-4.48860502$

$-4.10833597$

5.81773949

0.08933717

0.51464456

0.29246587

$-1.30244315$

0.47499421

$-1.48083472$

$-0.98611736$

2. 45817113

$-0.62571812$

0.89627916

0.65657783

$-0.95530587$

0.63589996

$-0.92096448$

$-0.67028630$

0.98946404

$-2.10432053$

0.40697476

0.81353396

0.45026228

2. 29013205

$-0.93166113$

$-1.14351988$

0.13925628

3. 04682469

$-1.96880877$

$-2.48989034$

1. 50782621

$-2.07300234$

0.99069345

1. 55573690

$-0.59542030$

0.33006072

$-0.51306158$

$-0.06375359$

0.17793787

$-0.75505757$

0.84275299

0.50974971

$-0.46180978$

$-0.20376363$

0.11279327

0.11912664

$-0.05130068$

0.28952363

$-0.17148715$

$-0.21793514$

0.12013469

$-0.20351890$

0.41650778

0.23556043

$-0.50080955$

0.18055487

$-0.42697102$

$-0.22241107$

0.53107458

1.000 \# Electr. mom., scale-fac \# Magnetic mom. 


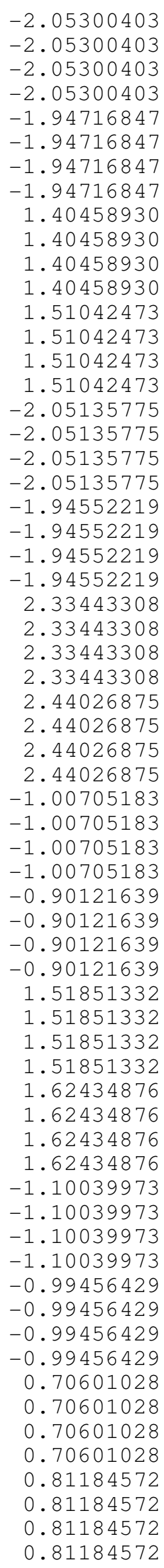

64
$-0.19663274$

$-0.19663274$

$-0.09079729$

$-0.09079729$

$-0.19663274$

$-0.19663274$

$-0.09079729$

$-0.09079729$

0.53337198

0.53337198

0.63920748

0.63920748

0.53337198

0.53337198

0.63920748

0.63920748

$-0.61681372$

$-0.61681372$

$-0.51097828$

$-0.51097828$

$-0.61681372$

$-0.61681372$

$-0.51097828$

$-0.51097828$

1. 26356936

1.26356936

1. 36940491

1. 36940491

1. 26356936

1.26356936

1. 36940491

1. 36940491

0.52339011

0.52339011

0.62922561

0.62922561

0.52339011

0.52339011

0.62922561

0.62922561

$-0.81257546$

$-0.81257546$

$-0.70673996$

$-0.70673996$

$-0.81257546$

$-0.81257546$

$-0.70673996$

$-0.70673996$

0.90373951

0.90373951

1. 00957489

1.00957489

0.90373951

0.90373951

1.00957489

1.00957489

$-1.34548187$

$-1.34548187$

$-1.23964632$

$-1.23964632$

$-1.34548187$

$-1.34548187$

$-1.23964632$

$-1.23964632$

\# Energy 6->8
$-0.13595992$

$-0.03012447$

$-0.13595992$

$-0.03012447$

$-0.13595992$

$-0.03012447$

$-0.13595992$

$-0.03012447$

$-0.10552958$

0.00030586

$-0.10552958$

0.00030586

$-0.10552958$

0.00030586

$-0.10552958$

0.00030586

$-1.29993546$

$-1.19410002$

$-1.29993546$

$-1.19410002$

$-1.29993546$

$-1.19410002$

$-1.29993546$

$-1.19410002$

0.26371813

0.36955357

0.26371813

0.36955357

0.26371813

0.36955357

0.26371813

0.36955357

0.37719119

0.48302662

0.37719119

0.48302662

0.37719119

0.48302662

0.37719119

0.48302662

$-0.22067484$

$-0.11483940$

$-0.22067484$

$-0.11483940$

$-0.22067484$

$-0.11483940$

$-0.22067484$

$-0.11483940$

1. 31258702

1. 41842246

1. 31258702

1. 41842246

1.31258702

1. 41842246

1.31258702

1.41842246

$-0.51015860$

$-0.40432316$

$-0.51015860$

$-0.40432316$

$-0.51015860$

$-0.40432316$

$-0.51015860$

$-0.40432316$
2. 42867064

$-1.25286651$

$-4.93846703$

3.98215199

$-6.17919588$

4.95891380

9.46611309

$-8.35702515$

2.78653407

$-3.17837214$

$-3.18636370$

3. 34848475

$-2.62190795$

2. 90093899

2. 94981194

$-2.98001099$

$-12.06066799$

15.51458549

12.47777843

$-16.18378067$

10.62292767

$-13.73627281$

$-10.85379696$

14.18036461

$-1.44210315$

0.88503331

1.08647776

$-0.62461168$

1. 24333394

$-0.78223962$

$-0.93964154$

0.55773550

$-6.80690193$

4. 46146202

2.38955951

$-0.42932716$

6.56705475

$-4.40961409$

$-2.80856395$

0.99151117

$-1.40842402$

1. 27028918

1.13580275

$-1.06719077$

1. 17412972

$-1.09474516$

$-0.95942372$

0.92692143

2.07008433

$-1.59074855$

$-1.83422661$

1.37235320

$-1.83210766$

1. 36771417

1. 58389699

$-1.15250421$

0.12412392

$-0.16909970$

$-0.23701099$

0.22495674

$-0.20271780$

0.25468585

0.34498468

$-0.32945776$

$\begin{array}{lll}1.7158 & 0.4207 & 0.0460 \\ 0.0207 & 0.0438 & 0.1798\end{array}$

1.000

\# Electr. mom., scale-fac

\# Magnetic mom. 


\begin{abstract}
$-2.05300403$
$-2.05300403$

$-2.05300403$

$-2.05300403$

$-1.94716847$

$-1.94716847$

$-1.94716847$

$-1.94716847$

1. 40458930

1. 40458930

1. 40458930

1. 40458930

1. 51042473

1. 51042473

1. 51042473

1.51042473

$-2.05135775$

$-2.05135775$

$-2.05135775$

$-2.05135775$

$-1.94552219$

$-1.94552219$

$-1.94552219$

$-1.94552219$

2. 33443308

2. 33443308

2.33443308

2. 33443308

2. 44026875

2. 44026875

2. 44026875

2. 44026875

$-1.00705183$

$-1.00705183$

$-1.00705183$

$-1.00705183$

$-0.90121639$

$-0.90121639$

$-0.90121639$

$-0.90121639$

1. 51851332

1. 51851332

1. 51851332

1. 51851332

1.62434876

1.62434876

1.62434876

1.62434876

$-1.10039973$

$-1.10039973$

$-1.10039973$

$-1.10039973$

$-0.99456429$

$-0.99456429$

$-0.99456429$

$-0.99456429$

0.70601028

0.70601028

0.70601028

0.70601028

0.81184572

0.81184572

0.81184572
\end{abstract}

64
$-0.19663274$

$-0.19663274$

$-0.09079729$

$-0.09079729$

$-0.19663274$

$-0.19663274$

$-0.09079729$

$-0.09079729$

0.53337198

0.53337198

0.63920748

0.63920748

0.53337198

0.53337198

0.63920748

0.63920748

$-0.61681372$

$-0.61681372$

$-0.51097828$

$-0.51097828$

$-0.61681372$

$-0.61681372$

$-0.51097828$

$-0.51097828$

1. 26356936

1.26356936

1. 36940491

1. 36940491

1. 26356936

1.26356936

1. 36940491

1. 36940491

0.52339011

0.52339011

0.62922561

0.62922561

0.52339011

0.52339011

0.62922561

0.62922561

$-0.81257546$

$-0.81257546$

$-0.70673996$

$-0.70673996$

$-0.81257546$

$-0.81257546$

$-0.70673996$

$-0.70673996$

0.90373951

0.90373951

1. 00957489

1.00957489

0.90373951

0.90373951

1.00957489

1.00957489

$-1.34548187$

$-1.34548187$

$-1.23964632$

$-1.23964632$

$-1.34548187$

$-1.34548187$

$-1.23964632$

$-1.23964632$

\# Energy 6->9
$-0.13595992$

$-0.03012447$

$-0.13595992$

$-0.03012447$

$-0.13595992$

$-0.03012447$

$-0.13595992$

$-0.03012447$

$-0.10552958$

0.00030586

$-0.10552958$

0.00030586

$-0.10552958$

0.00030586

$-0.10552958$

0.00030586

$-1.29993546$

$-1.19410002$

$-1.29993546$

$-1.19410002$

$-1.29993546$

$-1.19410002$

$-1.29993546$

$-1.19410002$

0.26371813

0.36955357

0.26371813

0.36955357

0.26371813

0.36955357

0.26371813

0.36955357

0.37719119

0.48302662

0.37719119

0.48302662

0.37719119

0.48302662

0.37719119

0.48302662

$-0.22067484$

$-0.11483940$

$-0.22067484$

$-0.11483940$

$-0.22067484$

$-0.11483940$

$-0.22067484$

$-0.11483940$

1. 31258702

1. 41842246

1. 31258702

1.41842246

1. 31258702

1. 41842246

1. 31258702

1.41842246

$-0.51015860$

$-0.40432316$

$-0.51015860$

$-0.40432316$

$-0.51015860$

$-0.40432316$

$-0.51015860$

$-0.40432316$
0.74673182

0.15837464

0.87395239

$-2.23691678$

1. 29610407

$-2.40456557$

$-3.16921115$

4. 78375340

$-5.49211645$

4.84731722

5.39617920

$-4.90836859$

5.46249771

$-4.93911648$

$-5.38427544$

5.08476734

2. 21328068

$-2.64334059$

$-2.26370215$

2.69174504

$-1.65216577$

1.93910336

1.62513661

$-1.90210402$

$-3.96752429$

1. 67458677

3.68091059

$-1.63837075$

2. 81306887

$-0.81897777$

$-2.53873467$

0.80641592

$-3.19174314$

1. 96123290

2. 31331825

$-1.64309645$

2.64667773

$-1.58197582$

$-1.83299220$

1.15824974

$-1.58604908$

1. 76581299

2. 13630080

$-2.46226025$

1. 89101458

$-2.11390209$

$-2.46481466$

2. 81317711

0.01152920

0.04493348

0.01410151

$-0.05657634$

0.03247351

$-0.05282034$

0.05950265

$-0.01873109$

$-0.19174130$

0.34588021

0.42363894

$-0.66207570$

0.32162401

$-0.54683572$

$-0.62704730$

0.95875901

$\begin{array}{lrr}0.8814 & -0.0493 & -0.0310 \\ 0.0534 & 0.0318 & 0.0768\end{array}$

1.000

\# Electr. mom., scale-fac

\# Magnetic mom.

\section{S-260}




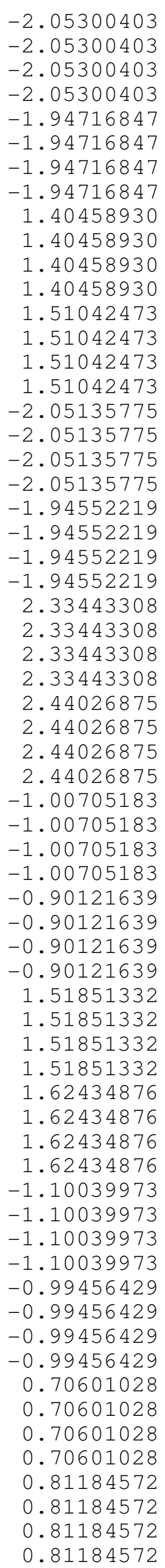

\&TRANSITION $7->\ldots$
$-0.19663274$

$-0.19663274$

$-0.09079729$

$-0.09079729$

$-0.19663274$

$-0.19663274$

$-0.09079729$

$-0.09079729$

0.53337198

0.53337198

0.63920748

0.63920748

0.53337198

0.53337198

0.63920748

0.63920748

$-0.61681372$

$-0.61681372$

$-0.51097828$

$-0.51097828$

$-0.61681372$

$-0.61681372$

$-0.51097828$

$-0.51097828$

1. 26356936

1.26356936

1. 36940491

1. 36940491

1.26356936

1.26356936

1. 36940491

1. 36940491

0.52339011

0.52339011

0.62922561

0.62922561

0.52339011

0.52339011

0.62922561

0.62922561

$-0.81257546$

$-0.81257546$

$-0.70673996$

$-0.70673996$

$-0.81257546$

$-0.81257546$

$-0.70673996$

$-0.70673996$

0.90373951

0.90373951

1. 00957489

1.00957489

0.90373951

0.90373951

1. 00957489

1.00957489

$-1.34548187$

$-1.34548187$

$-1.23964632$

$-1.23964632$

$-1.34548187$

$-1.34548187$

$-1.23964632$

$-1.23964632$
$-0.13595992$

$-0.03012447$

$-0.13595992$

$-0.03012447$

$-0.13595992$

$-0.03012447$

$-0.13595992$

$-0.03012447$

$-0.10552958$

0.00030586

$-0.10552958$

0.00030586

$-0.10552958$

0.00030586

$-0.10552958$

0.00030586

$-1.29993546$

$-1.19410002$

$-1.29993546$

$-1.19410002$

$-1.29993546$

$-1.19410002$

$-1.29993546$

$-1.19410002$

0.26371813

0.36955357

0.26371813

0.36955357

0.26371813

0.36955357

0.26371813

0.36955357

0.37719119

0.48302662

0.37719119

0.48302662

0.37719119

0.48302662

0.37719119

0.48302662

$-0.22067484$

$-0.11483940$

$-0.22067484$

$-0.11483940$

$-0.22067484$

$-0.11483940$

$-0.22067484$

$-0.11483940$

1. 31258702

1. 41842246

1. 31258702

1. 41842246

1. 31258702

1. 41842246

1. 31258702

1.41842246

$-0.51015860$

$-0.40432316$

$-0.51015860$

$-0.40432316$

$-0.51015860$

$-0.40432316$

$-0.51015860$

$-0.40432316$
$-0.90797764$

1. 23266518

1. 55600178

$-1.98720384$

1. 71679151

$-2.05428267$

$-2.47870827$

2. 90447569

$-0.32526720$

0.53972828

0.49574363

$-0.90908754$

0.69321722

$-1.13568413$

$-0.93446106$

1. 62020254

0.29442921

$-0.28407949$

$-0.31597227$

0.29535097

$-0.03113582$

$-0.05055878$

0.01260609

0.08460895

$-1.34770870$

0.28924420

0.38797766

0.41123226

1. 58825994

$-0.72424251$

$-0.70708603$

0.06252369

0.30575049

0.37588003

0.02223998

$-0.68105894$

$-0.10800169$

$-0.58746576$

$-0.15754388$

0.79022807

$-0.56183702$

0.57288057

0.73210990

$-0.79319859$

0.35248959

$-0.38184297$

$-0.50956714$

0.62538630

$-0.31142583$

0.24039194

0.30453992

$-0.22898228$

0.31940043

$-0.24103355$

$-0.28481767$

0.20993704

$-0.12093560$

0.26900858

0.13306224

$-0.30891865$

0.12587348

$-0.30138621$

$-0.14248484$

0.34971899

64

3.4309

0. \# Energy 7->8

$0.8637 \quad 0.1524$

1.000

\# Electr. mom., scale-fac

\section{S-261}


0.0153

$-2.05300403$

$-2.05300403$

$-2.05300403$

$-2.05300403$

$-1.94716847$

$-1.94716847$

$-1.94716847$

$-1.94716847$

1. 40458930

1. 40458930

1. 40458930

1. 40458930

1.51042473

1. 51042473

1. 51042473

1. 51042473

$-2.05135775$

$-2.05135775$

$-2.05135775$

$-2.05135775$

$-1.94552219$

$-1.94552219$

$-1.94552219$

$-1.94552219$

2. 33443308

2. 33443308

2. 33443308

2. 33443308

2. 44026875

2. 44026875

2. 44026875

2. 44026875

$-1.00705183$

$-1.00705183$

$-1.00705183$

$-1.00705183$

$-0.90121639$

$-0.90121639$

$-0.90121639$

$-0.90121639$

1. 51851332

1. 51851332

1. 51851332

1. 51851332

1.62434876

1.62434876

1.62434876

1. 62434876

$-1.10039973$

$-1.10039973$

$-1.10039973$

$-1.10039973$

$-0.99456429$

$-0.99456429$

$-0.99456429$

$-0.99456429$

0.70601028

0.70601028

0.70601028

0.70601028

0.81184572

0.81184572

0.81184572

64
0.0190

$-0.19663274$

$-0.19663274$

$-0.09079729$

$-0.09079729$

$-0.19663274$

$-0.19663274$

$-0.09079729$

$-0.09079729$

0.53337198

0.53337198

0.63920748

0.63920748

0.53337198

0.53337198

0.63920748

0.63920748

$-0.61681372$

$-0.61681372$

$-0.51097828$

$-0.51097828$

$-0.61681372$

$-0.61681372$

$-0.51097828$

$-0.51097828$

1. 26356936

1.26356936

1. 36940491

1. 36940491

1.26356936

1. 26356936

1. 36940491

1.36940491

0.52339011

0.52339011

0.62922561

0.62922561

0.52339011

0.52339011

0.62922561

0.62922561

$-0.81257546$

$-0.81257546$

$-0.70673996$

$-0.70673996$

$-0.81257546$

$-0.81257546$

$-0.70673996$

$-0.70673996$

0.90373951

0.90373951

1.00957489

1. 00957489

0.90373951

0.90373951

1.00957489

1.00957489

$-1.34548187$

$-1.34548187$

$-1.23964632$

$-1.23964632$

$-1.34548187$

$-1.34548187$

$-1.23964632$

$-1.23964632$

\# Energy 7->9
\# Magnetic mom.

$\begin{array}{ll}-0.13595992 & -0.36702833\end{array}$

$\begin{array}{ll}-0.03012447 & 3.10254622\end{array}$

$\begin{array}{ll}-0.13595992 & 3.04496336\end{array}$

$-0.03012447 \quad-7.11822510$

$\begin{array}{ll}-0.13595992 & 1.37766159\end{array}$

$-0.03012447$

$-0.13595992$

$-0.03012447$

$-0.10552958$

0.00030586

$-0.10552958$

0.00030586

$-0.10552958$

0.00030586

$-0.10552958$

0.00030586

$-1.29993546$

$-1.19410002$

$-1.29993546$

$-1.19410002$

$-1.29993546$

$-1.19410002$

$-1.29993546$

$-1.19410002$

0.26371813

0.36955357

0.26371813

0.36955357

0.26371813

0.36955357

0.26371813

0.36955357

0.37719119

0.48302662

0.37719119

0.48302662

0.37719119

0.48302662

0.37719119

0.48302662

$-0.22067484$

$-0.11483940$

$-0.22067484$

$-0.11483940$

$-0.22067484$

$-0.11483940$

$-0.22067484$

$-0.11483940$

1. 31258702

1.41842246

1. 31258702

1. 41842246

1. 31258702

1. 41842246

1.31258702

1. 41842246

$-0.51015860$

$-0.40432316$

$-0.51015860$

$-0.40432316$

$-0.51015860$

$-0.40432316$

$-0.51015860$

$-0.40432316$

$-4.82082510$

$-4.14497232$

9.15127659

$-1.65413857$

1.06992793

0.56115752

0.02011793

1. 44693136

$-0.79722339$

$-0.29731205$

$-0.24470231$

$-0.53118312$

0.75756878

0.48402509

$-0.74613637$

0.18908820

$-0.34482133$

$-0.03272044$

0.17840081

$-7.47388554$

3. 61593366

6.95077181

$-3.38405776$

5. 40219212

$-1.95352376$

$-4.87555599$

1.78794765

$-1.91486144$

$-2.93118906$

$-2.03129148$

5.49005556

0.47284657

3.63333726

3. 00463033

$-6.13346672$

$-3.10924435$

3.83349133

3. 68244147

$-4.95682907$

3.32863069

$-4.13956213$

$-4.01657867$

5.29508066

2. 91005850

$-2.05387354$

$-2.66411495$

1. 82630193

$-2.28051066$

1. 63548195

2. 32316351

$-1.62275732$

$-0.30402529$

0.74851024

0.59869558

$-1.31634521$

0.50692397

$-1.13219547$

$-0.92563003$

1. 88862658

$\begin{array}{lll}0.7294 & 0.0455 & -0.0163\end{array}$

1.000

\# Electr. mom., scale-fac

\section{S-262}




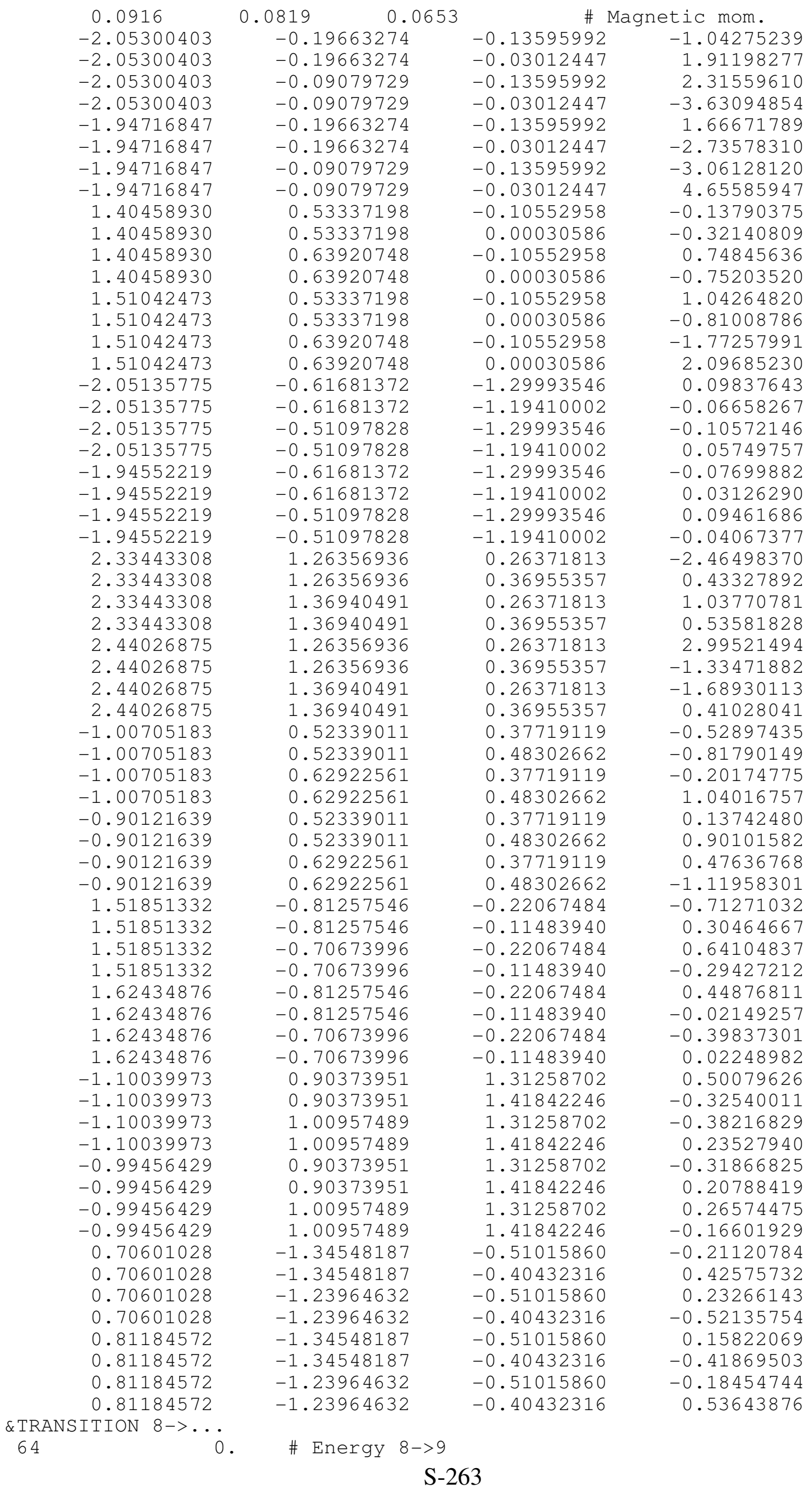




\begin{tabular}{|c|c|c|c|}
\hline-0.7561 & -0.0113 & 00 & mom., \\
\hline 0.4994 & 4165 & \# & ic mom. \\
\hline-2.05300403 & -0.19663274 & -0.13595992 & 5.9799571 \\
\hline 2.05300403 & -0.19663274 & -0.03012447 & -5.037226 \\
\hline 2.05300403 & -0.09079729 & -0.13595992 & -5.620533 \\
\hline-2.05300403 & -0.09079729 & -0.03012447 & 4.191113 \\
\hline-1.94716847 & -0.19663274 & -0.13595992 & -5.46 \\
\hline-1.94716847 & -0.19 & -0.03012447 & 4.15 \\
\hline-1.94716847 & -0.09079729 & -0.13595992 & 5.02 \\
\hline-1.94716847 & -0.09079729 & -0.03012447 & -3.00 \\
\hline 1.40458930 & 0.53337198 & -0.10552958 & -18.045700 \\
\hline 1.40458930 & 0.53337198 & 0.00030586 & 11.5284671 \\
\hline 1.40458930 & 0.63920748 & -0.10552958 & 22.11 \\
\hline 1.40458930 & 0.63920748 & 0.00030586 & -18.6 \\
\hline 1.51042473 & 0.53337198 & -0.10552958 & 22.21 \\
\hline 1.51042473 & 0.53337198 & 0.00030586 & -16.64 \\
\hline 1.51042473 & 0.63920748 & -0.10552958 & -27.06 \\
\hline 1.51042473 & 0.63920748 & 0.00030586 & 25.02 \\
\hline-2.05135775 & -0.61681372 & -1.29993546 & 0.88 \\
\hline-2.05135775 & 81372 & -1.19410002 & -0.99 \\
\hline 35775 & -0.51097828 & -1.29993546 & -0.7 \\
\hline 35775 & -0.51097828 & -1.19410002 & 0.8 \\
\hline 52219 & 81372 & -1.29993546 & -0.9 \\
\hline-1.94552219 & -0.61681372 & -1.19410002 & 1.05 \\
\hline 52219 & -0.51 & -1.29993546 & 0.8 \\
\hline 52219 & 7828 & -1.19410002 & -0.8 \\
\hline 2.33443308 & 1.26356936 & 0.26371813 & -9.36 \\
\hline 43308 & 1.26356936 & 0.36955357 & 0.6 \\
\hline 3308 & 0491 & 371813 & 4.4 \\
\hline 2.33443308 & 1.36940491 & 0.36955357 & 2.29 \\
\hline 2.44026875 & 1.26356936 & 0.26371813 & 12.0429544 \\
\hline 26875 & 1.26356936 & 0.36955357 & -5.0 \\
\hline 6875 & 40491 & 0.26371813 & -7.5 \\
\hline 26875 & 1.36940491 & 0.36955357 & 2.14 \\
\hline 05183 & 0.52339011 & 0.37719119 & -19.08 \\
\hline 05183 & 0.52339011 & 0.48302662 & 14.25 \\
\hline-1 . & 22561 & 0.37719119 & 15.8 \\
\hline 05183 & 0.62922561 & 0.48302662 & -12.95 \\
\hline 21639 & 0.52339011 & 0.37719119 & 17.31 \\
\hline 21639 & 339011 & 302662 & -13.1 \\
\hline 21639 & 0.62922561 & 0.37719119 & -14.69 \\
\hline 21639 & 0.62922561 & 0.48302662 & 12.35 \\
\hline 1.51851332 & -0.81257546 & -0.22067484 & 0.08 \\
\hline 51332 & -0.8 & -0.11483940 & -4.3 \\
\hline 351332 & -0.70673996 & -0.22067484 & -0.53 \\
\hline 1.51851332 & -0.70673996 & -0.11483940 & 5.38 \\
\hline 4876 & -0.81257546 & -0.22067484 & -0.22 \\
\hline 4876 & -0.81257546 & -0.11483940 & 4.5 \\
\hline 34876 & -0.70673996 & -0.22067484 & 0.61 \\
\hline 3876 & -0.70673996 & -0.11483940 & -5.74 \\
\hline 9973 & 0.90373951 & 1.31258702 & -0.94 \\
\hline-1 & 0.90373951 & 1.41842246 & 0.8 \\
\hline 9973 & 1.00957489 & 1.31258702 & 1.4779 \\
\hline 9973 & 1.00957489 & 1.41842246 & -1.17 \\
\hline 29 & 0.90373951 & 1.31258702 & 0.9 \\
\hline 56429 & 0.90373951 & 1.41842246 & -0.845320 \\
\hline 56429 & 1.00957489 & 1.31258702 & -1.51 \\
\hline 6429 & 1.00957489 & 1.41842246 & 1.20 \\
\hline 01028 & -1.34548187 & -0.51015860 & -0.49 \\
\hline 0.70601028 & -1.34548187 & -0.40432316 & 0.464119 \\
\hline 1028 & -1.23964632 & -0.51015860 & 0.73 \\
\hline 01028 & -1.23964632 & -0.40432316 & -0.78 \\
\hline 84572 & -1.34548187 & -0.51015860 & 0.1423435 \\
\hline 84572 & -1.34548187 & -0.40432316 & -0.1048976 \\
\hline & -1.23964632 & -0.51015860 & -0.41 \\
\hline 84572 & -1.23964632 & -0.40432316 & 0.491495 \\
\hline
\end{tabular}


64 $\begin{array}{llll} & 0 . & \# \text { Energy } 1->1 \\ -1.9803 & -1.4055 & 2.1610 \quad \# \text { Electr. mom. }\end{array}$

$-2.05300403$

$-2.05300403$

$-2.05300403$

$-2.05300403$

$-1.94716847$

$-1.94716847$

$-1.94716847$

$-1.94716847$

1. 40458930

1.40458930

1. 40458930

1. 40458930

1.51042473

1.51042473

1. 51042473

1. 51042473

$-2.05135775$

$-2.05135775$

$-2.05135775$

$-2.05135775$

$-1.94552219$

$-1.94552219$

$-1.94552219$

$-1.94552219$

2. 33443308

2. 33443308

2. 33443308

2. 33443308

2. 44026875

2. 44026875

2. 44026875

2. 44026875

$-1.00705183$

$-1.00705183$

$-1.00705183$

$-1.00705183$

$-0.90121639$

$-0.90121639$

$-0.90121639$

$-0.90121639$

1. 51851332

1. 51851332

1. 51851332

1. 51851332

1.62434876

1.62434876

1. 62434876

1.62434876

$-1.10039973$

$-1.10039973$

$-1.10039973$

$-1.10039973$

$-0.99456429$

$-0.99456429$

$-0.99456429$

$-0.99456429$

0.70601028

0.70601028

0.70601028

0.70601028

0.81184572

0.81184572

0.81184572

0.81184572
64
$-0.19663274$

$-0.19663274$

$-0.09079729$

$-0.09079729$

$-0.19663274$

$-0.19663274$

$-0.09079729$

$-0.09079729$

0.53337198

0.53337198

0.63920748

0.63920748

0.53337198

0.53337198

0.63920748

0.63920748

$-0.61681372$

$-0.61681372$

$-0.51097828$

$-0.51097828$

$-0.61681372$

$-0.61681372$

$-0.51097828$

$-0.51097828$

1. 26356936

1. 26356936

1. 36940491

1.36940491

1.26356936

1.26356936

1. 36940491

1. 36940491

0.52339011

0.52339011

0.62922561

0.62922561

0.52339011

0.52339011

0.62922561

0.62922561

$-0.81257546$

$-0.81257546$

$-0.70673996$

$-0.70673996$

$-0.81257546$

$-0.81257546$

$-0.70673996$

$-0.70673996$

0.90373951

0.90373951

1.00957489

1.00957489

0.90373951

0.90373951

1.00957489

1.00957489

$-1.34548187$

$-1.34548187$

$-1.23964632$

$-1.23964632$

$-1.34548187$

$-1.34548187$

$-1.23964632$

$-1.23964632$

\# Energy 2->2
$-0.13595992 \quad 136.30987549$

$-126.74493408$

$-122.22817993$

125.27703857

$-136.34790039$

132.30108643

131.51036072

$-141.23381042$

$-70.93521881$

32.39118958

82.82276154

$-48.08309174$

70.37551880

$-29.61118126$

$-83.34574127$

46.74179077

0.89493102

$-0.20313041$

10.81495857

$-14.90692616$

0.76757926

$-1.29294300$

$-10.33940887$

13.62089729

23.50335312

$-14.00042534$

$-13.03879166$

5.60743284

$-12.27943230$

4.59411383

3. 77845168

1. 68952787

44.60737228

25.87374496

$-18.58879852$

$-39.58811951$

$-5.06750965$

$-61.12213898$

$-24.15002632$

81.01173401

$-68.79624939$

8.87730026

46.37445068

12.41852570

61.54727554

$-4.85271931$

$-40.85295105$

$-16.03833580$

$-22.38517761$

15.65679646

21.52016449

$-14.45031261$

21.68944740

$-15.97817993$

$-24.42258644$

17.23515892

10.18363190

$-18.11796570$

$-12.48626137$

20.11540604

$-14.83237934$

23.60020828

17.20092583

$-24.59219551$ 


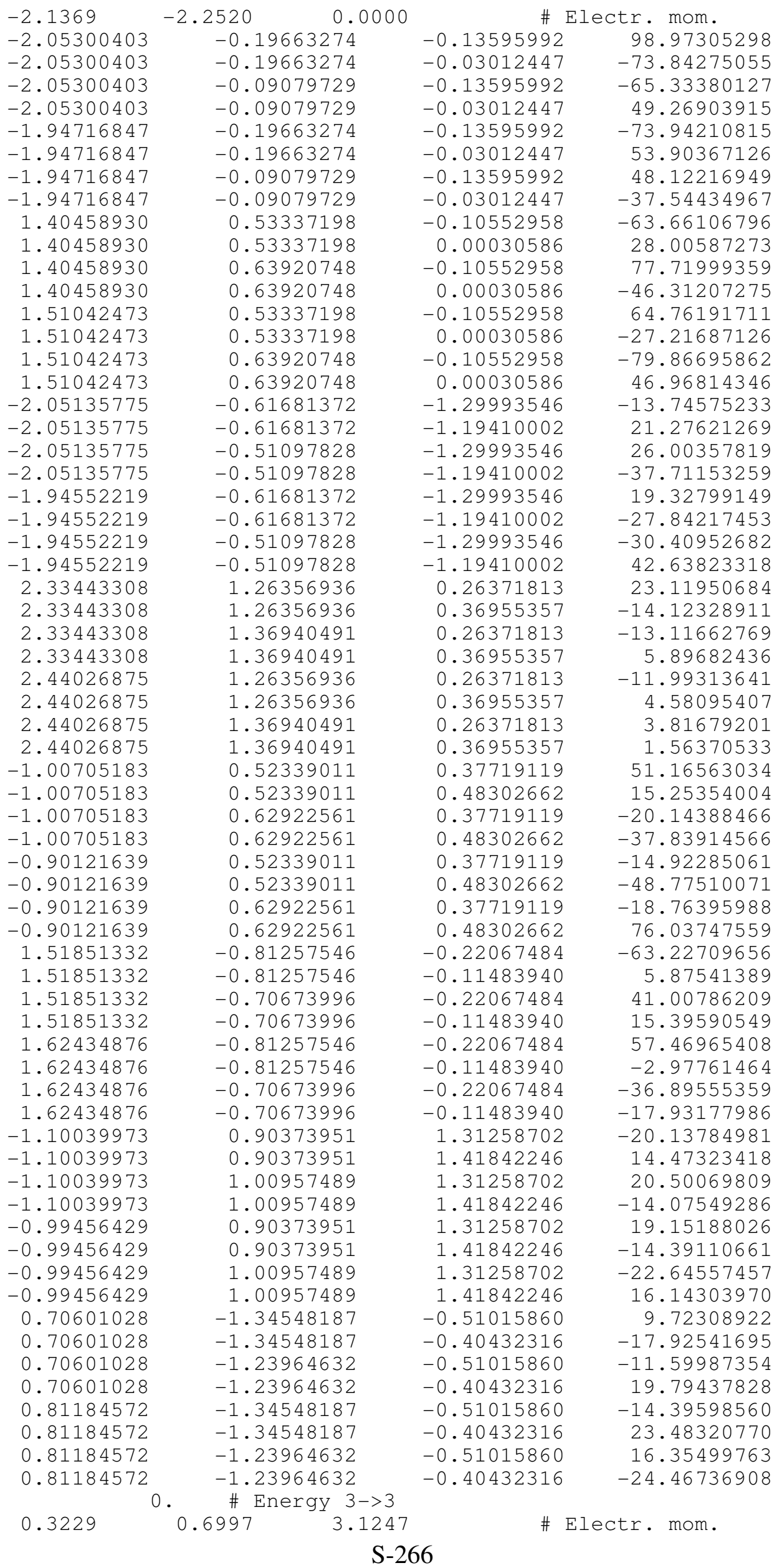




\begin{tabular}{|c|c|c|c|}
\hline & & & \\
\hline-2.05300403 & -0.19663274 & -0.13595992 & 148.8 \\
\hline-2.05300403 & -0.19663274 & -0.03012447 & -135.0 \\
\hline-2.05300403 & -0.09079729 & -0.13595992 & -132.65849304 \\
\hline-2.05300403 & -0.09079729 & -0.03012447 & 129.30224609 \\
\hline-1.94716847 & -0.19663274 & -0.13595992 & -145.75866699 \\
\hline-1.94716847 & -0.19663274 & -0.03012447 & 136.02682495 \\
\hline-1.94716847 & -0.09079729 & -0.13595992 & 138.59576416 \\
\hline-1.94716847 & -0.09079729 & -0.03012447 & -139.7431945 \\
\hline 1.40458930 & 0.53337198 & -0.10552958 & -121.1340332 \\
\hline 1.40458930 & 0.53337198 & 0.00030586 & 71.113769 \\
\hline 1.40458930 & 0.63920748 & -0.10552958 & 143.1213531 \\
\hline 1.40458930 & 0.63920748 & 0.00030586 & -106.19271088 \\
\hline 1.51042473 & 0.53337198 & -0.10552958 & 128.5 \\
\hline 1.51042473 & 0.53337198 & 0.00030586 & -80.8973 \\
\hline 1.51042473 & 0.63920748 & -0.10552958 & -153.63200378 \\
\hline 1.51042473 & 0.63920748 & 0.00030586 & 119.7 \\
\hline-2.05135775 & -0.61681372 & -1.29993546 & 4.4 \\
\hline-2.05135775 & -0.61681372 & -1.19410002 & 1.33 \\
\hline-2.05135775 & -0.51097828 & -1.29993546 & 7.3702678 \\
\hline-2.05135775 & -0.51097828 & -1.19410002 & -10.98 \\
\hline-1.94552219 & -0.61681372 & -1.29993546 & -2.76 \\
\hline$-1 \cdot 94$ & -0.61681372 & -1.1941 & 8 \\
\hline-1.94552219 & -0.51097828 & -1.29993546 & -6.7 \\
\hline-1.94552219 & -0.51097828 & -1.19410002 & 9.3 \\
\hline 2.33443308 & 1.26356936 & 0.26371813 & -11.2963 \\
\hline 2.33443308 & 1.26356936 & 0.36955357 & -4.67509079 \\
\hline 2.33443308 & 1.36940491 & 0.26371813 & 20.364 \\
\hline 2.33 & 1.36940491 & 0.369 & $-6 \cdot 3$ \\
\hline 2.44 & 1.26356936 & 0.26371813 & 14.7725076 \\
\hline 2.44026875 & 1.26356936 & 0.36955357 & -1.33779871 \\
\hline 2.44 & 1.36940491 & 0.2637 & $-22 \cdot 3$ \\
\hline 2.4 & 1.36940491 & 0.36 & 10.0 \\
\hline$-1.00^{\prime}$ & 0.523390 & 0.377 & $-0.7 \xi$ \\
\hline-1.00705183 & 0.52339011 & 0.48302662 & 56.060 \\
\hline-1.00 & 0.62922561 & 0.377 & 10.3 \\
\hline-1.00 & 0.62922561 & 0.483 & -58.98 \\
\hline-0.90121639 & 0.52339011 & 0.377 & 2 \\
\hline-0.90121639 & 0.52339011 & 0.48302662 & -86.48 \\
\hline-0.90 & 0.62922561 & 0.377 & -47.7 \\
\hline-0.901 & 0.62922561 & 0.4830 & 96.8 \\
\hline 1.51851332 & -0.81257546 & -0.22067484 & -62.45833588 \\
\hline 1.51851332 & -0.81257546 & -0.11483940 & -9.7 \\
\hline 1.51 & -0.70673996 & -0.2206 & 42.26 \\
\hline 1.51851332 & -0.70673996 & -0.11483940 & 29.0937862 \\
\hline 1.62434876 & -0.81257546 & -0.22067484 & 57.969 \\
\hline 1.62 & -0.81257546 & -0.11483940 & 8.3 \\
\hline 1.62 & -0.70673996 & -0.22067484 & -40.1 \\
\hline 1.62434876 & -0.70673996 & -0.11483940 & -26.8 \\
\hline-1.10039973 & 0.90373951 & 1.31258702 & -23.22 \\
\hline-1.10 & 3951 & 1.41 & 16. \\
\hline$-1 \cdot 10$ & 1.00957489 & 1.31258702 & 22.87 \\
\hline-1.10039973 & 1.00957489 & 1.41842246 & -15.47 \\
\hline-0.99456429 & 0.90373951 & 1.31258702 & 22.3 \\
\hline 6429 & 0.90373951 & 1.41842246 & -16.59 \\
\hline-0.99456429 & 1.00957489 & 1.31258702 & -25.6 \\
\hline-0.99456429 & 1.00957489 & 1.41842246 & 18.26 \\
\hline 0.70601028 & -1.34548187 & -0.5101 & 8.8 \\
\hline 0.70601028 & -1.34548187 & -0.40432316 & -16.49 \\
\hline 0.70601028 & -1.23964632 & -0.51015860 & -8.597076 \\
\hline 0.70601028 & -1.23964632 & -0.40432316 & 14.8 \\
\hline 0.81184572 & -1.34548187 & -0.5101 & -13.4 \\
\hline 0.81184572 & -1.34548187 & -0.40432316 & 21.4638481 \\
\hline 0.81184572 & -1.23964632 & -0.51015860 & 12.4 \\
\hline 0.81184572 & -1.23964632 & -0.40432316 & -17.6698856 \\
\hline & \# Energy 4->4 & & \\
\hline & 2.3396 & H & ctr \\
\hline 0403 & -0.19663274 & -0 & 123 \\
\hline & & & \\
\hline
\end{tabular}




\begin{tabular}{|c|c|c|c|}
\hline \\
\hline \multicolumn{4}{|l|}{$\begin{array}{l}-2.05300403 \\
-2.05300403\end{array}$} \\
\hline \multicolumn{4}{|l|}{$\begin{array}{l}-2.05300403 \\
-2.05300403\end{array}$} \\
\hline-1.94716847 & & 992 & 870 \\
\hline \multicolumn{4}{|l|}{-1.94716847} \\
\hline-1.94716847 & & & \\
\hline & -0 . & & \\
\hline 1.40458930 & 0.53337198 & -0.10552958 & -82.0299758 \\
\hline 1.40458930 & 0.53337198 & 0.00030586 & 49.6396942 \\
\hline 1.40458930 & 0.63920748 & -0.10552958 & 97.1340103 \\
\hline 1.40458930 & 0.63920748 & 0.00030586 & -72.7194824 \\
\hline 1.51042473 & 0.53337198 & -0.10552958 & 85.5947647 \\
\hline 1.51042473 & 0.53337198 & 0.00030586 & -55.21 \\
\hline 1.51042473 & 0.63920748 & -0.10552958 & -102.852264 \\
\hline 1.51042473 & 0.63920748 & 0.00030586 & 80.3110580 \\
\hline-2.05135775 & -0.61681372 & -1.29993546 & -0.0653392 \\
\hline-2.05135775 & -0.61681372 & -1.19410002 & 1.094698 \\
\hline-2.05135775 & -0.51097828 & -1.29993546 & 11.644231 \\
\hline-2.05135775 & -0.51097828 & -1.19410002 & -16.01 \\
\hline-1.94552219 & -0.61681372 & -1.29993546 & 2.235406 \\
\hline-1.9455221 & -0.61681372 & -1.19410002 & -3.202 \\
\hline-1.9455221 & -0.51097828 & -1.29993546 & -11.6 \\
\hline-1.945 & -0.51097828 & -1.19410002 & 15.3377676 \\
\hline 2.334433 & 1.26356936 & 0.26371813 & 22.6622467 \\
\hline 2.334 & 1.26356936 & 0.36955357 & -14.740366 \\
\hline 2.33443308 & 1.36940491 & 0.26371813 & -11.4820013 \\
\hline 2.33443308 & 1.36940491 & 0.36955357 & 4.953433 \\
\hline 2.44 & 1.26356936 & 0.26371813 & $-11 \cdot 71$ \\
\hline 2.440 & 1.26356936 & 0.36955357 & 4.997441 \\
\hline 2.44026875 & 1.36940491 & 0.26371813 & 2.3212966 \\
\hline 2.44026875 & 1.36940491 & 0.36955357 & 2.51 \\
\hline-1.007 & 0.52339011 & 0.37719119 & 67.9606 \\
\hline-1.00705183 & 0.52339011 & 0.48302662 & 7.8730516 \\
\hline-1.00705183 & 0.62922561 & 0.37719119 & -39.704647 \\
\hline-1.00705183 & 0.62922561 & 0.48302662 & -22.62 \\
\hline-0.901 & 0.52339011 & 0.37719119 & -28.13 \\
\hline-0.901 & 0.52339011 & 0.48302662 & -43.0 \\
\hline-0.90121639 & 0.62922561 & 0.37719119 & -3.117877 \\
\hline-0.90121639 & 0.62922561 & 0.48302662 & 64.0 \\
\hline 1.51851332 & -0.81257546 & -0.22067484 & -58.119056 \\
\hline 1.51851332 & -0.81257546 & -0.11483940 & -13.5390358 \\
\hline 1.51851332 & -0.70673996 & -0.22067484 & 36.721633 \\
\hline 1.51 & -0.70673996 & -0.11483940 & 34.6 \\
\hline 1.62434876 & -0.81257546 & -0.22067484 & 50.50 \\
\hline 1.62434876 & -0.81257546 & -0.11483940 & 14.329661 \\
\hline 1.62 & -0.70673996 & -0.22067484 & $-31 \cdot 21$ \\
\hline 1.624 & -0.70673996 & -0.11483940 & $-33 \cdot 9$ \\
\hline-1.10039973 & 0.90373951 & 1.31258702 & $-21 \cdot 51$ \\
\hline-1.100 & 0.90373951 & 1.41842246 & 14.93 \\
\hline-1.10 & 1.00957489 & 1.31258702 & 20.2 \\
\hline-1.10 & 1.00957489 & 1.4184 & -13.40 \\
\hline-0.994 & 0.90373951 & 1.31258702 & 20.08 \\
\hline-0.9945642 & 0.90373951 & 1.41842246 & -14.75730 \\
\hline 9456429 & 1.00957489 & 1.31258702 & -22.7 \\
\hline-0.994 & 1.00957489 & 1.41842246 & 15.9 \\
\hline 0.706010 & -1.34548187 & -0.51015860 & 10.3 \\
\hline 0.7060102 & -1.34548187 & -0.40432316 & -17.7 \\
\hline 0.706010 & -1.23964632 & -0.51015860 & -11.5 \\
\hline 0.706010 & -1.23964632 & -0.40432316 & 18.1 \\
\hline 0.811845 & -1.34548187 & -0.51015860 & -14.4 \\
\hline 0 . & -1.34548187 & -0.4043 & 22 . \\
\hline 0.811 & -1.23964632 & -0.51015860 & 15.2 \\
\hline 0.811 & -1.23964632 & -0.40432316 & -21.3326988 \\
\hline & ergy 5-> & & \\
\hline & 2.8835 & & \\
\hline-2 & -0.1 & -0.135 & 114 \\
\hline & -0 . & -0 & -9 \\
\hline
\end{tabular}

\section{S-268}




\begin{tabular}{|c|c|c|c|}
\hline-2.05300403 & -0.09079729 & -0.13595992 & -86.92144775 \\
\hline-2.05300403 & -0.09079729 & -0.03012447 & 77.84149170 \\
\hline-1.94716847 & -0.19663274 & -0.13595992 & -102.45237732 \\
\hline-1.94716847 & -0.19663274 & -0.03012447 & 89.49040222 \\
\hline-1.94716847 & -0.09079729 & -0.13595992 & 83.7565918 \\
\hline-1.94716847 & -0.09079729 & -0.03012447 & -79.27653503 \\
\hline 1.40458930 & 0.53337198 & -0.10552958 & -75.50883484 \\
\hline 1.40458930 & 0.53337198 & 0.00030586 & 26.9197254 \\
\hline 1.40458930 & 0.63920748 & -0.10552958 & 87.6542587 \\
\hline 1.40458930 & 0.63920748 & 0.00030586 & -45.2161598 \\
\hline 1.51042473 & 0.53337198 & -0.10552958 & 74.620246 \\
\hline 1.51042473 & 0.53337198 & 0.00030586 & -25.170562 \\
\hline 1.51042473 & 0.63920748 & -0.10552958 & -88.44241 \\
\hline 1.51042473 & 0.63920748 & 0.00030586 & 45.3 \\
\hline-2.05135775 & -0.61681372 & -1.29993546 & -0.194 \\
\hline-2.05135775 & -0.61681372 & -1.19410002 & 151 \\
\hline-2.0513 & -0.51097828 & -1.29993546 & 11.4 \\
\hline-2.0513 & -0.51097828 & -1.19410002 & -16.2 \\
\hline-1.945 & -0.61 & -1.2999 & 7 \\
\hline 219 & -0.61 & -1.19410002 & -4.0 \\
\hline-1.945 & -0.51 & -1.29993 & $-11 \cdot 7$ \\
\hline-1.94 & -0.51097828 & -1.19410002 & 15.7 \\
\hline 2.33443308 & 1.26356936 & 0.26371813 & 20.182 \\
\hline 2.33443308 & 1.26356936 & 0.36955357 & -12.80 \\
\hline 08 & 1.36940491 & 0.26371813 & -9.69 \\
\hline 08 & 1.36940491 & 0.36955357 & 3.93 \\
\hline 6875 & 1.26356936 & 0.26371813 & -9.609657 \\
\hline 2.4 & 1.26356936 & 0.3695 & 49 \\
\hline 75 & 1.36 & 0.2637 & $.92-2 \cdot-1$ \\
\hline 875 & 1.36940491 & 0.369553 & 3.2 \\
\hline-1.00705183 & 0.523390 & 0.37719119 & 44.2 \\
\hline-1.00 & 0.52 & 0.4830 & \\
\hline-1 & 0 . & 0.377 & -30 \\
\hline-1 . & 0.62 & 0.4830 & -14 \\
\hline 1639 & 0.523390 & 0.37719119 & $-14 \cdot 7 \mathrm{C}$ \\
\hline-0. & 0 . & 0.4830 & -36.4 \\
\hline-0 . & 2922561 & 0.37719 & -2 \\
\hline-0.90 & 0.62922561 & 0.48302662 & 48.07 \\
\hline 1332 & -0.81257546 & -0.22067484 & -70.72200 \\
\hline 1. & -0.81 & -0.1148 & 1 \\
\hline $1.51 \varepsilon$ & -0.70673996 & -0.22067484 & 48.93 \\
\hline 1332 & -0.70673996 & -0.11483940 & 17.337 \\
\hline 376 & -0.81257546 & -0.22067484 & 62. \\
\hline 76 & -0.81 & -0.1148 & 0 \\
\hline 1.62 & -0.70673996 & -0.22067484 & $-41 \cdot 93$ \\
\hline 876 & -0.70673996 & -0.11483940 & $-21 \cdot 4$ \\
\hline-1 . & 0.90 & 1.312 & -17 . \\
\hline-1 . & 0 . & 1.4 & 12 . \\
\hline-1. & 1.00 & 1.312 & 16. \\
\hline 9973 & 1.00957489 & 1.41842246 & $-11 \cdot 2$ \\
\hline-0 . & 0 . & 1.31 & 17 \\
\hline-0 . & 0 . & 1.41842246 & -12 \\
\hline-0 . & 1 . & 1.312 & -19 \\
\hline-0 . & 1.00 & 1.4 & 13. \\
\hline 0 & -1 . & -0.5 & \\
\hline 0.70 & -1.3454818 & -0.40432316 & -17.2 \\
\hline 0.7 & -1.23 & -0.51015 & -10 \\
\hline 0.7 & -1 & -0.404 & 18 \\
\hline 0.8 & -1.345481 & -0.51 & -13.75 \\
\hline 0.8 & -1.34548187 & -0.404323 & 22.64 \\
\hline 4572 & -1.23964632 & -0.51015860 & 15. \\
\hline 0.81 & -1.23964632 & -0.40432316 & -22.718023 \\
\hline & \# Energy 6-> & & \\
\hline & 1.9257 & & ctr. \\
\hline-2 . & -0.1 & -0 & 140 \\
\hline-2 . & -0.1 & -0 . & -128 \\
\hline 然 & -0 & -0.13595992 & -126.3 \\
\hline
\end{tabular}

\section{S-269}


$-2.05300403$

$-1.94716847$

$-1.94716847$

$-1.94716847$

$-1.94716847$

1. 40458930

1. 40458930

1. 40458930

1. 40458930

1. 51042473

1.51042473

1. 51042473

1. 51042473

$-2.05135775$

$-2.05135775$

$-2.05135775$

$-2.05135775$

$-1.94552219$

$-1.94552219$

$-1.94552219$

$-1.94552219$

2. 33443308

2. 33443308

2. 33443308

2. 33443308

2. 44026875

2. 44026875

2. 44026875

2. 44026875

$-1.00705183$

$-1.00705183$

$-1.00705183$

$-1.00705183$

$-0.90121639$

$-0.90121639$

$-0.90121639$

$-0.90121639$

1. 51851332

1. 51851332

1. 51851332

1. 51851332

1.62434876

1.62434876

1.62434876

1. 62434876

$-1.10039973$

$-1.10039973$

$-1.10039973$

$-1.10039973$

$-0.99456429$

$-0.99456429$

$-0.99456429$

$-0.99456429$

0.70601028

0.70601028

0.70601028

0.70601028

0.81184572

0.81184572

0.81184572

0.81184572

64

-10.0498
-2.05300403
-2.05300403
-2.05300403
-2.05300403

$-0.09079729$

$-0.19663274$

$-0.19663274$

$-0.09079729$

$-0.09079729$

0.53337198

0.53337198

0.63920748

0.63920748

0.53337198

0.53337198

0.63920748

0.63920748

$-0.61681372$

$-0.61681372$

$-0.51097828$

$-0.51097828$

$-0.61681372$

$-0.61681372$

$-0.51097828$

$-0.51097828$

1. 26356936

1.26356936

1. 36940491

1. 36940491

1. 26356936

1.26356936

1. 36940491

1. 36940491

0.52339011

0.52339011

0.62922561

0.62922561

0.52339011

0.52339011

0.62922561

0.62922561

$-0.81257546$

$-0.81257546$

$-0.70673996$

$-0.70673996$

$-0.81257546$

$-0.81257546$

$-0.70673996$

$-0.70673996$

0.90373951

0.90373951

1.00957489

1.00957489

0.90373951

0.90373951

1.00957489

1. 00957489

$-1.34548187$

$-1.34548187$

$-1.23964632$

$-1.23964632$

$-1.34548187$

$-1.34548187$

$-1.23964632$

$-1.23964632$

\# Energy 7->7

$-2.4746 \quad 1.3868$

$-0.19663274$

$-0.19663274$

$-0.09079729$

$-0.09079729$
$-0.03012447$

$-0.13595992$

$-0.03012447$

$-0.13595992$

$-0.03012447$

$-0.10552958$

0.00030586

$-0.10552958$

0.00030586

$-0.10552958$

0.00030586

$-0.10552958$

0.00030586

$-1.29993546$

$-1.19410002$

$-1.29993546$

$-1.19410002$

$-1.29993546$

$-1.19410002$

$-1.29993546$

$-1.19410002$

0.26371813

0.36955357

0.26371813

0.36955357

0.26371813

0.36955357

0.26371813

0.36955357

0.37719119

0.48302662

0.37719119

0.48302662

0.37719119

0.48302662

0.37719119

0.48302662

$-0.22067484$

$-0.11483940$

$-0.22067484$

$-0.11483940$

$-0.22067484$

$-0.11483940$

$-0.22067484$

$-0.11483940$

1. 31258702

1. 41842246

1. 31258702

1. 41842246

1. 31258702

1.41842246

1. 31258702

1.41842246

$-0.51015860$

$-0.40432316$

$-0.51015860$

$-0.51015860$

$-0.40432316$

$-0.51015860$

$-0.40432316$
$-0.40432316$

124.29208374

$-138.74391174$

132.22732544

136.89411926

$-139.86952209$

$-91.37918091$

34.35710907

110.55445099

$-64.40979004$

94.48234558

$-38.59092712$

$-116.52406311$

71.45940399

$-1.02150762$

0.97150761

12.04366112

$-15.07880306$

$-0.55408859$

1.67271709

$-7.75843287$

8.91609001

19.71990585

$-15.90789032$

$-7.37620831$

4. 15629244

$-8.63189983$

4.92999411

$-2.01058054$

4.37934542

4.87365294

40.24427032

$-4.93123007$

$-33.62727356$

19.76464653

$-63.76620483$

$-25.14649200$

65.56422424

$-60.30043411$

$-17.11408234$

37.58935165

40.82046509

51.49868774

20.06036568

$-30.52646065$

$-43.41549301$

$-17.79142570$

11.99787426

17.38280678

$-11.24410343$

16.86227989

$-12.27275181$

$-20.39599037$

14.20313549

8.93979263

$-17.55513000$

$-10.02270412$

17.80593491

$-13.99993706$

23.63549805

14.76584816

$-22.36047363$

\# Electr. mom.

$-0.13595992$

$-0.03012447$

$-0.13595992$

$-0.03012447$
114.27309418

$-97.51228333$

$-88.07203674$

82.16475677 
$-1.94716847$

$-1.94716847$

$-1.94716847$

$-1.94716847$

1. 40458930

1. 40458930

1. 40458930

1. 40458930

1. 51042473

1. 51042473

1.51042473

1. 51042473

$-2.05135775$

$-2.05135775$

$-2.05135775$

$-2.05135775$

$-1.94552219$

$-1.94552219$

$-1.94552219$

$-1.94552219$

2. 33443308

2. 33443308

2. 33443308

2. 33443308

2. 44026875

2. 44026875

2. 44026875

2. 44026875

$-1.00705183$

$-1.00705183$

$-1.00705183$

$-1.00705183$

$-0.90121639$

$-0.90121639$

$-0.90121639$

$-0.90121639$

1. 51851332

1. 51851332

1. 51851332

1.51851332

1. 62434876

1. 62434876

1.62434876

1.62434876

$-1.10039973$

$-1.10039973$

$-1.10039973$

$-1.10039973$

$-0.99456429$

$-0.99456429$

$-0.99456429$

$-0.99456429$

0.70601028

0.70601028

0.70601028

0.70601028

0.81184572

0.81184572

0.81184572

0.81184572

64
$-0.19663274$

$-0.19663274$

$-0.09079729$

$-0.09079729$

0.53337198

0.53337198

0.63920748

0.63920748

0.53337198

0.53337198

0.63920748

0.63920748

$-0.61681372$

$-0.61681372$

$-0.51097828$

$-0.51097828$

$-0.61681372$

$-0.61681372$

$-0.51097828$

$-0.51097828$

1. 26356936

1.26356936

1.36940491

1. 36940491

1. 26356936

1.26356936

1.36940491

1. 36940491

0.52339011

0.52339011

0.62922561

0.62922561

0.52339011

0.52339011

0.62922561

0.62922561

$-0.81257546$

$-0.81257546$

$-0.70673996$

$-0.70673996$

$-0.81257546$

$-0.81257546$

$-0.70673996$

$-0.70673996$

0.90373951

1.00957489

1.00957489

0.90373951

0.90373951

1.00957489

$-1.34548187$

$-1.34548187$

$-1.23964632$

$-1.23964632$

$-1.34548187$

$-1.34548187$

$-1.23964632$

$-1.23964632$

\# Energy 8->8

$\begin{array}{lll}1.7829 & -0.0687 & 1.5840\end{array}$

$-2.05300403$

$-2.05300403$

$-2.05300403$

$-2.05300403$

$-1.94716847$

$-0.19663274$

$-0.19663274$

$-0.09079729$

$-0.09079729$

$-0.19663274$
0.90373951

1. 00957489
$-0.13595992$

$-0.03012447$

$-0.13595992$

$-0.03012447$

$-0.10552958$

0.00030586

$-0.10552958$

0.00030586

$-0.10552958$

0.00030586

$-0.10552958$

0.00030586

$-1.29993546$

$-1.19410002$

$-1.29993546$

$-1.19410002$

$-1.29993546$

$-1.19410002$

$-1.29993546$

$-1.19410002$

0.26371813

0.36955357

0.26371813

0.36955357

0.26371813

0.36955357

0.26371813

0.36955357

0.37719119

0.48302662

0.37719119

0.48302662

0.37719119

0.48302662

0.37719119

0.48302662

$-0.22067484$

$-0.11483940$

$-0.22067484$

$-0.11483940$

$-0.22067484$

$-0.11483940$

$-0.22067484$

$-0.11483940$

1. 31258702

1. 41842246

1. 31258702

1.41842246

1.31258702

1. 41842246

1. 31258702

1.41842246

$-0.51015860$

$-0.40432316$

$-0.51015860$

$-0.40432316$

$-0.51015860$

$-0.40432316$

$-0.51015860$

$-0.40432316$

\# Electr. mom.

$-94.20732880$

83.28791809

76.36600494

$-76.75263214$

$-102.71356201$

52.94316101

124.18388367

$-84.27401733$

106.66394043

$-57.92101669$

$-130.58921814$

91.77999878

$-3.91936898$

8.01393795

15.98422813

$-23.92611313$

9.81353664

$-14.81332493$

$-20.53919411$

28.89455605

21.77222824

$-17.56887817$

$-9.42718887$

5.84488726

$-10.86212540$

6.90711260

0.34723216

2.30032945

20.37934113

41.78338242

2. 72902727

$-56.94298172$

12.28462315

$-71.58547974$

$-38.41462708$

92.68716431

$-55.78052521$

$-15.45320511$

34.49342346

37.81927109

49.58181000

16.74193001

$-29.84930229$

$-38.74253082$

$-26.70608330$

18.91815186

26.32113457

$-17.88470459$

24.09035492

$-17.79381561$

$-27.69277191$

19.53133392

8.94982338

$-18.14275742$

$-9.77315331$

18.85942650

$-14.01240540$

24.35001183

14.64758587

$-23.83595657$

$-0.13595992$

$-0.03012447$

$-0.13595992$

$-0.03012447$

$-0.13595992$
116.71530914

$-90.44838715$

$-87.06589508$

67.93778229

$-98.83926392$ 
$-1.94716847$

$-1.94716847$

$-1.94716847$

1. 40458930

1. 40458930

1. 40458930

1.40458930

1.51042473

1. 51042473

1.51042473

1.51042473

$-2.05135775$

$-2.05135775$

$-2.05135775$

$-2.05135775$

$-1.94552219$

$-1.94552219$

$-1.94552219$

$-1.94552219$

2. 33443308

2. 33443308

2. 33443308

2. 33443308

2. 44026875

2. 44026875

2. 44026875

$-1.00705183$

$-1.00705183$

$-1.00705183$

$-1.00705183$

$-0.90121639$

$-0.90121639$

$-0.90121639$

$-0.90121639$

1. 51851332

1. 51851332

1. 51851332

1. 51851332

1.62434876

1. 62434876

1. 62434876

1.62434876

$-1.10039973$

$-1.10039973$

$-1.10039973$

$-1.10039973$

$-0.99456429$

$-0.99456429$

$-0.99456429$

$-0.99456429$

0.70601028

0.70601028

0.70601028

0.70601028

0.81184572

0.81184572

0.81184572

0.81184572

64
2. 44026875

$-0.19663274$

$-0.09079729$

$-0.09079729$

0.53337198

0.53337198

0.63920748

0.63920748

0.53337198

0.53337198

0.63920748

0.63920748

$-0.61681372$

$-0.61681372$

$-0.51097828$

$-0.51097828$

$-0.61681372$

$-0.61681372$

$-0.51097828$

$-0.51097828$

1. 26356936

1. 26356936

1. 36940491

1. 36940491

1.26356936

1. 26356936

1. 36940491

1.36940491

0.52339011

0.52339011

0.62922561

0.62922561

0.52339011

0.52339011

0.62922561

0.62922561

$-0.81257546$

$-0.81257546$

$-0.70673996$

$-0.70673996$

$-0.81257546$

$-0.81257546$

$-0.70673996$

$-0.70673996$

0.90373951

0.90373951

1.00957489

1.00957489

0.90373951

0.90373951

1.00957489

1.00957489

$-1.34548187$

$-1.34548187$

$-1.23964632$

$-1.23964632$

$-1.34548187$

$-1.34548187$

$-1.23964632$

$-1.23964632$

\# Energy 9->9

$\begin{array}{lll}3.8656 & -2.3021 & 1.4776\end{array}$

$-0.19663274$

$-0.19663274$

$-0.09079729$

$-0.09079729$

$-0.19663274$

$-0.19663274$
$-0.03012447$

$-0.13595992$

$-0.03012447$

$-0.10552958$

0.00030586

$-0.10552958$

0.00030586

$-0.10552958$

$-0.10552958$

0.00030586

$-1.29993546$

$-1.19410002$

$-1.29993546$

$-1.19410002$

$-1.29993546$

$-1.19410002$

$-1.29993546$

$-1.19410002$

0.26371813

0.36955357

0.26371813

0.36955357

0.26371813

0.36955357

0.26371813

0.36955357

0.37719119

0.48302662

0.37719119

0.48302662

0.37719119

0.48302662

0.37719119

0.48302662

$-0.22067484$

$-0.11483940$

$-0.11483940$

$-0.22067484$

$-0.11483940$

$-0.22067484$

$-0.11483940$

1.31258702

1. 41842246

1. 31258702

1. 41842246

1. 31258702

1. 41842246

1.31258702

1. 41842246

$-0.51015860$

$-0.40432316$

$-0.51015860$

$-0.40432316$

$-0.51015860$

$-0.40432316$

$-0.51015860$

$-0.40432316$
0.00030586

$-0.22067484$

76.16495514

78.16240692

$-62.41367340$

$-97.19223785$

53.88446426

116.35029602

$-84.22614288$

103.02502441

$-61.23677063$

$-124.73915100$

94.72635651

$-12.41875553$

18.58895493

24.48091888

$-34.62474442$

15.06171799

$-21.37153244$

$-25.11029053$

34.70129013

$-7.63884401$

$-3.80957007$

17.58910942

$-7.23710155$

10.12660599

$-0.43887648$

$-18.58159637$

9.35379696

17.10724258

32.23110580

$-7.45580292$

$-37.85496140$

11.52540207

$-60.40190125$

$-25.72370148$

72.24797058

$-64.29920197$

$-2.35310459$

43.88888550

21.27806282

60.02098083

1. 25644267

$-41.92187500$

$-19.34005928$

$-16.32810211$

11.65209293

17.08106422

$-11.59532833$

16.17954254

$-12.25228405$

$-20.02576637$

14.28606606

8.95826340

$-16.35217094$

$-8.94396782$

15.09650707

$-13.30262089$

21.04004478

12.65445709

$-17.82944107$

\# Electr. mom.

$\begin{array}{ll}-0.13595992 & 97.65152740\end{array}$

$-0.03012447$

$-0.13595992$

$-0.03012447$

$-0.13595992$

$-0.03012447$
$-74.54786682$

$-68.81169128$

54.33218384

$-81.52006531$

63.08601761 


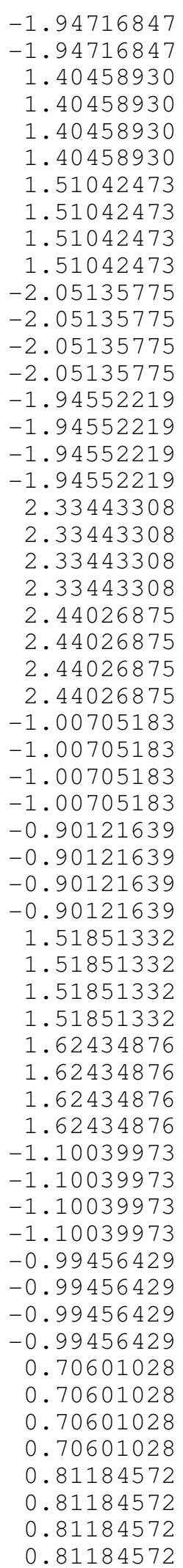
-CT31009A-

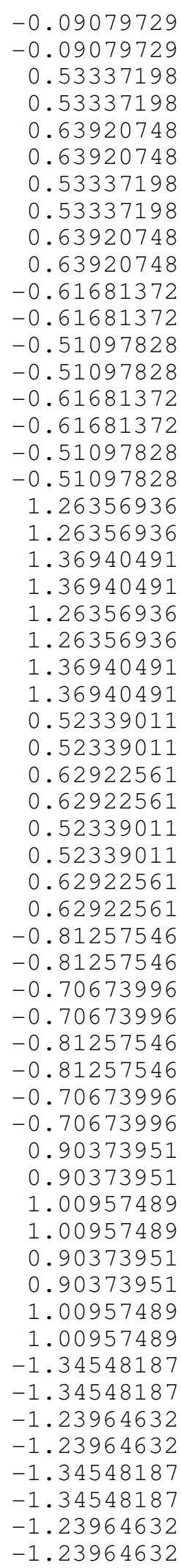

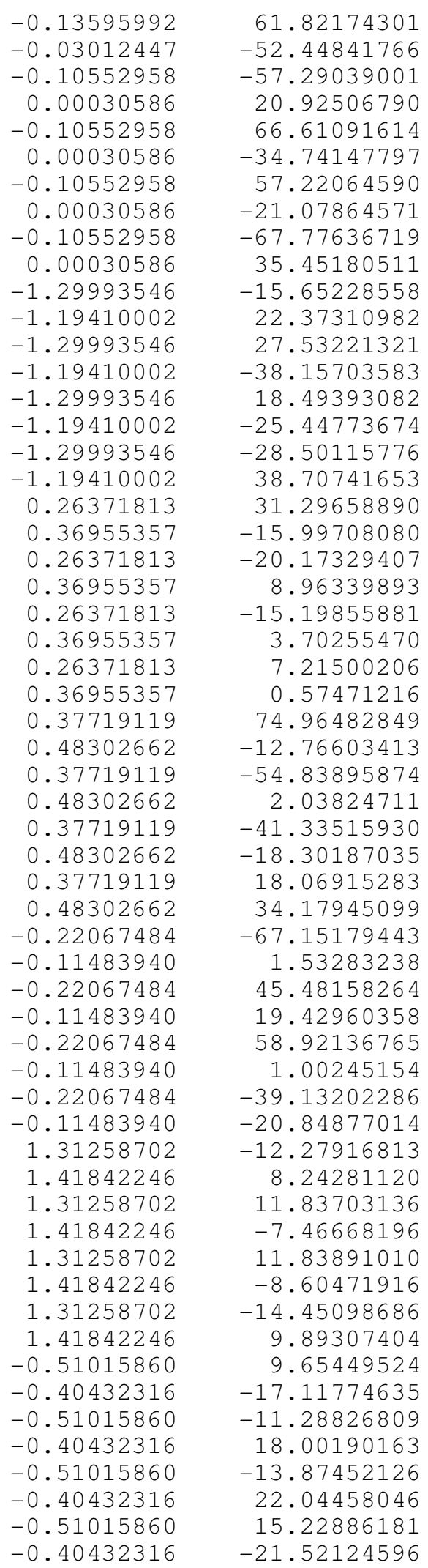
\# $p h i=-74$, psi= 6

$\begin{array}{rrr}-1.8022 & -0.0904 & -0.2109 \\ -1.3992 & -0.3708 & -1.3340 \\ -1.1017 & 0.7461 & 0.6090 \\ 1.3607 & 0.5714 & 0.0211 \\ 2.3915 & 1.0953 & -0.3847 \\ 1.2847 & -0.7220 & 0.4086\end{array}$

1.0000

1.0000

1.0000

1.0000

1.0000

1.0000

\# C1

\# $\mathrm{O} 1$

\# N1

\# C2

\# $\mathrm{N} 2$

\# 02 
$\&$ TRANSITION $1->\ldots$

6

$\begin{array}{llr}0.0830 & -0.1005 & 0.0666 \\ 0.2887 & -0.3053 & -0.8870\end{array}$

$-1.85509956$

$-0.14332448$

$-1.85509956$

$-0.14332448$

$-1.85509956$

$-0.03748903$

$-1.85509956$

$-0.03748903$

$-1.74926412$

$-0.14332448$

$-1.74926412$

$-0.14332448$

$-1.74926412$

$-0.03748903$

$-1.74926412$

$-0.03748903$

1.30779219

0.51848310

1.30779219

0.51848310

1. 30779219

0.62431854

1.30779219

0.62431854

1. 41362774

0.51848310

1. 41362774

1. 41362774

1. 41362774

$-1.45213747$

$-1.45213747$

$-1.45213747$

$-1.45213747$

$-1.34630203$

$-1.34630203$

$-1.34630203$

$-1.34630203$

2. 33856606

2.33856606

2.33856606

2. 33856606

2.44440126

2.44440126

2. 44440126

2. 44440126

$-1.15465784$

$-1.15465784$

$-1.15465784$

$-1.15465784$

$-1.04882240$

$-1.04882240$

$-1.04882240$

$-1.04882240$

1. 23173511

1. 23173511

1. 23173511

1. 23173511

1. 33757067

1.33757067

1. 33757067

1.33757067

$-1.51449943$

$-1.51449943$

$-1.51449943$

$-1.51449943$

$-1.40866399$

$-1.40866399$

$-1.40866399$

$-1.40866399$

0.31038415

0.31038415

0.31038415

0.31038415

0.41621959

0.41621959

0.51848310

0.62431854

0.62431854

$-0.42367253$

$-0.42367253$

$-0.31783706$

$-0.31783706$

$-0.42367253$

$-0.42367253$

$-0.31783706$

$-0.31783706$

1. 04233944

1.04233944

1. 14817488

1. 14817488

1.04233944

1.04233944

1. 14817488

1. 14817488

0.69317716

0.69317716

0.79901266

0.79901266

0.69317716

0.69317716

0.79901266

0.79901266

$-0.77488583$

$-0.77488583$

$-0.66905046$

$-0.66905046$

$-0.77488583$

$-0.77488583$

$-0.66905046$

$-0.66905046$

0.88849652

0.88849652

0.99433196

0.99433196

0.88849652

0.88849652

0.99433196

0.99433196

$-1.17169046$

$-1.17169046$

$-1.06585503$

$-1.06585503$

$-1.17169046$

$-1.17169046$

0.41621959

$-1.06585503$

-0.26382661
-0.15799116
-0.26382661

-0.26382661
-0.15799116

$-0.26382661$

$-0.15799116$

$-0.26382661$

$-0.15799116$

$-0.03186282$

0.07397263

$-0.03186282$

0.07397263

$-0.03186282$

0.07397263

$-0.03186282$

0.07397263

$-1.38695288$

$-1.28111744$

$-1.38695288$

$-1.28111744$

$-1.38695288$

$-1.28111744$

$-1.38695288$

$-1.28111744$

$-0.43766770$

$-0.33183220$

$-0.43766770$

$-0.33183220$

$-0.43766770$

$-0.33183220$

$-0.43766770$

$-0.33183220$

0.55604303

0.66187847

0.55604303

0.66187847

0.55604303

0.66187847

0.55604303

0.66187847

0.35565418

0.46148965

0.35565418

0.46148965

0.35565418

0.46148965

0.35565418

0.46148965

1. 47651505

1. 58235061

1. 47651505

1. 58235061

1. 47651505

1. 58235061

1.47651505

1. 58235061

0.44945931

0.55529481

0.44945931

0.55529481

0.44945931

0.55529481

0.44945931
Electr. mom., scale-fac Magnetic mom. 27.07287025

$-27.69585609$

$-26.01611519$

27.64042664

$-25.72789192$

26.34858704

24.86979294

$-26.29009438$

3. 21773791

$-2.04256868$

$-3.56848931$

2. 43802977

$-3.37986040$

2. 20600986

3. 74331260

$-2.62430763$

1. 73291993

$-2.50299668$

0.83706897

$-0.52677268$

$-1.21172976$

2. 03223705

$-1.12427580$

0.71820456

$-0.35778955$

0.51460421

0.20064148

$-0.33860880$

0.25079256

$-0.36285824$

$-0.13403316$

0.22329389

$-28.80267525$

24.93418121

24.90095901

$-22.28715706$

22.85928917

$-19.53720856$

$-20.07682419$

17.87113571

$-2.59820175$

2. 24989247

2. 45507264

$-2.20509553$

2. 22401857

$-1.95495272$

$-2.06763887$

1. 88262582

$-0.34924379$

0.36269379

1. 00703764

$-0.85194021$

$-0.31305727$

0.06320686

$-0.39975530$

0.46409944

$-2.70591092$

2. 02784920

3.55236506

$-2.71947885$

2.36945939

$-1.80185235$

$-3.06797981$ 
$-1.06585503$ 45272 .
$-0.0485 \quad-0.2072$

0.7483

$-1.85509956$

$-1.85509956$

$-1.85509956$

$-1.85509956$

$-1.74926412$

$-1.74926412$

$-1.74926412$

$-1.74926412$

1.30779219

1.30779219

1. 30779219

1.30779219

1. 41362774

1. 41362774

1. 41362774

1. 41362774

$-1.45213747$

$-1.45213747$

$-1.45213747$

$-1.45213747$

$-1.34630203$

$-1.34630203$

$-1.34630203$

$-1.34630203$

2.33856606

2.33856606

2.33856606

2.33856606

2. 44440126

2. 44440126

2. 44440126

2. 44440126

$-1.15465784$

$-1.15465784$

$-1.15465784$

$-1.15465784$

$-1.04882240$

$-1.04882240$

$-1.04882240$

$-1.04882240$

1. 23173511

1. 23173511

1. 23173511

1. 23173511

1.33757067

1.33757067

1.33757067

1.33757067

$-1.51449943$

$-1.51449943$

$-1.51449943$

$-1.51449943$

$-1.40866399$

$-1.40866399$

$-1.40866399$

$-1.40866399$

0.31038415

0.31038415

0.31038415

0.31038415

0.41621959

0.41621959

0.41621959

$$
\begin{array}{rr}
-0.2072 & -0.0875 \\
0.3841 & -0.2839
\end{array}
$$

$-0.14332448$

$-0.14332448$

$-0.03748903$

$-0.03748903$

$-0.14332448$

$-0.14332448$

$-0.03748903$

$-0.03748903$

0.51848310

0.51848310

0.62431854

0.62431854

0.51848310

0.51848310

0.62431854

0.62431854

$-0.42367253$

$-0.42367253$

$-0.31783706$

$-0.31783706$

$-0.42367253$

$-0.42367253$

$-0.31783706$

$-0.31783706$

1. 04233944

1.04233944

1. 14817488

1. 14817488

1.04233944

1. 04233944

1. 14817488

1. 14817488

0.69317716

0.69317716

0.79901266

0.79901266

0.69317716

0.69317716

0.79901266

0.79901266

$-0.77488583$

$-0.77488583$

$-0.66905046$

$-0.66905046$

$-0.77488583$

$-0.77488583$

$-0.66905046$

$-0.66905046$

0.88849652

0.88849652

0.99433196

0.99433196

0.88849652

0.88849652

0.99433196

0.99433196

$-1.17169046$

$-1.17169046$

$-1.06585503$

$-1.06585503$

$-1.17169046$

$-1.17169046$

$-1.06585503$

$0.55529481 \quad 2.37280226$

1.000 \# Electr. mom., scale-fac \# Magnetic mom.

$-0.26382661$

$-0.15799116$

$-0.26382661$

$-0.15799116$

$-0.26382661$

$-0.15799116$

$-0.26382661$

$-0.15799116$

$-0.03186282$

0.07397263

$-0.03186282$

0.07397263

$-0.03186282$

0.07397263

$-0.03186282$

0.07397263

$-1.38695288$

$-1.28111744$

$-1.38695288$

$-1.28111744$

$-1.38695288$

$-1.28111744$

$-1.38695288$

$-1.28111744$

$-0.43766770$

$-0.33183220$

$-0.43766770$

$-0.33183220$

$-0.43766770$

$-0.33183220$

$-0.43766770$

$-0.33183220$

0.55604303

0.66187847

0.55604303

0.66187847

0.55604303

0.66187847

0.55604303

0.66187847

0.35565418

0.46148965

0.35565418

0.46148965

0.35565418

0.46148965

0.35565418

0.46148965

1. 47651505

1.58235061

1. 47651505

1. 58235061

1. 47651505

1. 58235061

1.47651505

1. 58235061

0.44945931

0.55529481

0.44945931

0.55529481

0.44945931

0.55529481

0.44945931
4.80466604

$-5.11268044$

$-5.67223787$

5.88559628

$-5.68679285$

6.10531092

6.97522020

$-7.28104210$

14.97285461

$-11.83507442$

$-17.60617638$

15.32226372

$-16.88181877$

14.31011868

19.28170586

$-17.49824333$

0.57677823

$-0.62836069$

$-0.53126884$

0.53995961

$-0.63740349$

0.73782390

0.66022664

$-0.74166656$

$-6.69239092$

7. 98919153

2. 33936024

$-3.41344476$

10.59854031

$-11.76092243$

$-6.50990629$

7.47987700

2. 21780491

0.30938575

$-0.92122257$

$-1.04287267$

$-4.46061039$

1.83667159

2.37223458

$-0.26648322$

0.58230758

1.92564905

$-7.00464058$

3. 07136345

$-1.34841359$

$-1.19337130$

7. 21169233

$-3.45260620$

0.36567205

$-0.15257703$

$-0.11742162$

0.03122644

$-1.22580111$

0.75498635

0.69283795

$-0.42625111$

1. 93688262

$-1.39742339$

$-4.05005550$

3. 10923076

$-2.96838021$

2. 04984450

5.86701107 
$-1.06585503$ 55399.

$\begin{array}{lll}1.3103 & 2.8264 & -1.5717\end{array}$

0.1771

- 85509956

$-1.85509956$

$-1.85509956$

$-1.85509956$

$-1.74926412$

$-1.74926412$

$-1.74926412$

$-1.74926412$

1.30779219

1.30779219

1.30779219

1.30779219

1. 41362774

1. 41362774

1. 41362774

1. 41362774

$-1.45213747$

$-1.45213747$

$-1.45213747$

$-1.45213747$

$-1.34630203$

$-1.34630203$

$-1.34630203$

$-1.34630203$

2.33856606

2.33856606

2.33856606

2. 33856606

2. 44440126

2. 44440126

2. 44440126

2. 44440126

$-1.15465784$

$-1.15465784$

$-1.15465784$

$-1.15465784$

$-1.04882240$

$-1.04882240$

$-1.04882240$

$-1.04882240$

1. 23173511

1. 23173511

1. 23173511

1. 23173511

1. 33757067

1.33757067

1. 33757067

1.33757067

$-1.51449943$

$-1.51449943$

$-1.51449943$

$-1.51449943$

$-1.40866399$

$-1.40866399$

$-1.40866399$

$-1.40866399$

0.31038415

0.31038415

0.31038415

0.31038415

0.41621959

0.41621959

0.41621959

$-0.5971$

$-0.14332448$

$-0.14332448$

$-0.03748903$

$-0.03748903$

$-0.14332448$

$-0.14332448$

$-0.03748903$

$-0.03748903$

0.51848310

0.51848310

0.62431854

0.62431854

0.51848310

0.51848310

0.62431854

0.62431854

$-0.42367253$

$-0.42367253$

$-0.31783706$

$-0.31783706$

$-0.42367253$

$-0.42367253$

$-0.31783706$

$-0.31783706$

1. 04233944

1.04233944

1. 14817488

1. 14817488

1.04233944

1.04233944

1. 14817488

1. 14817488

0.69317716

0.69317716

0.79901266

0.79901266

0.69317716

0.69317716

0.79901266

0.79901266

$-0.77488583$

$-0.77488583$

$-0.66905046$

$-0.66905046$

$-0.77488583$

$-0.77488583$

$-0.66905046$

$-0.66905046$

0.88849652

0.88849652

0.99433196

0.99433196

0.88849652

0.88849652

0.99433196

0.99433196

$-1.17169046$

$-1.17169046$

$-1.06585503$

$-1.06585503$

$-1.17169046$

$-1.17169046$

$-1.06585503$
0.55529481

$-4.39673567$

1.000 \# Electr. mom., scale-fac \# Magnetic mom.
$-0.26382661$

$-0.15799116$

$-0.26382661$

$-0.15799116$

$-0.26382661$

$-0.15799116$

$-0.26382661$

$-0.15799116$

$-0.03186282$

0.07397263

$-0.03186282$

0.07397263

$-0.03186282$

0.07397263

$-0.03186282$

0.07397263

$-1.38695288$

$-1.28111744$

$-1.38695288$

$-1.28111744$

$-1.38695288$

$-1.28111744$

$-1.38695288$

$-1.28111744$

$-0.43766770$

$-0.33183220$

$-0.43766770$

$-0.33183220$

$-0.43766770$

$-0.33183220$

$-0.43766770$

$-0.33183220$

0.55604303

0.66187847

0.55604303

0.66187847

0.55604303

0.66187847

0.55604303

0.66187847

0.35565418

0.46148965

0.35565418

0.46148965

0.35565418

0.46148965

0.35565418

0.46148965

1. 47651505

1. 58235061

1. 47651505

1. 58235061

1. 47651505

1. 58235061

1. 47651505

1. 58235061

0.44945931

0.55529481

0.44945931

0.55529481

0.44945931

0.55529481

0.44945931
5.20000315

$-5.92444134$

$-5.28609467$

6.09216881

$-5.54885006$

6.35412598

5.56694221

$-6.46216393$

$-2.89333797$

6.66250706

1. 11452007

$-6.78889704$

2.35978675

$-7.15477896$

0.72847396

6.40922260

1.19351399

$-1.58253706$

$-1.39647353$

1.83172333

$-1.22339618$

1.63828301

1. 47812092

$-1.95355296$

6.30427647

$-11.30410385$

$-4.79484606$

7. 37442160

$-4.37076855$

7.63066149

3.98981786

$-5.60713434$

$-8.89111233$

6.75317669

6.63225317

$-5.09510803$

9.90420246

$-7.67858982$

$-7.32221699$

5.76724386

5.77779531

3. 60645247

$-15.64153862$

4.54954004

$-4.93763733$

$-2.95641899$

13.63594532

$-3.95033669$

0.10591084

$-0.18422434$

$-0.04422049$

0.13237680

$-0.05034403$

0.16865535

$-0.00590205$

$-0.11798524$

9.88928318

$-4.63906240$

$-14.88882637$

8.37250042

$-12.99576283$

6.39411068

19.30072403 
$-1.06585503$ 51052 .

$\begin{array}{lrl}1.1506 & 2.1051 & 2.7248 \\ 0.1147 & -1.2842 & 0.7327\end{array}$

$-1.85509956$

$-1.85509956$

$-1.85509956$

$-1.85509956$

$-1.74926412$

$-1.74926412$

$-1.74926412$

$-1.74926412$

1.30779219

1.30779219

1.30779219

1.30779219

1. 41362774

1. 41362774

1. 41362774

1. 41362774

$-1.45213747$

$-1.45213747$

$-1.45213747$

$-1.45213747$

$-1.34630203$

$-1.34630203$

$-1.34630203$

$-1.34630203$

2. 33856606

2.33856606

2.33856606

2. 33856606

2. 44440126

2.44440126

2. 44440126

2. 44440126

$-1.15465784$

$-1.15465784$

$-1.15465784$

$-1.15465784$

$-1.04882240$

$-1.04882240$

$-1.04882240$

$-1.04882240$

1. 23173511

1. 23173511

1. 23173511

1. 23173511

1. 33757067

1.33757067

1. 33757067

1.33757067

$-1.51449943$

$-1.51449943$

$-1.51449943$

$-1.51449943$

$-1.40866399$

$-1.40866399$

$-1.40866399$

$-1.40866399$

0.31038415

0.31038415

0.31038415

0.31038415

0.41621959

0.41621959

0.41621959
$-0.14332448$

$-0.14332448$

$-0.03748903$

$-0.03748903$

$-0.14332448$

$-0.14332448$

$-0.03748903$

$-0.03748903$

0.51848310

0.51848310

0.62431854

0.62431854

0.51848310

0.51848310

0.62431854

0.62431854

$-0.42367253$

$-0.42367253$

$-0.31783706$

$-0.31783706$

$-0.42367253$

$-0.42367253$

$-0.31783706$

$-0.31783706$

1. 04233944

1.04233944

1. 14817488

1. 14817488

1.04233944

1.04233944

1. 14817488

1. 14817488

0.69317716

0.69317716

0.79901266

0.79901266

0.69317716

0.69317716

0.79901266

0.79901266

$-0.77488583$

$-0.77488583$

$-0.66905046$

$-0.66905046$

$-0.77488583$

$-0.77488583$

$-0.66905046$

$-0.66905046$

0.88849652

0.88849652

0.99433196

0.99433196

0.88849652

0.88849652

0.99433196

0.99433196

$-1.17169046$

$-1.17169046$

$-1.06585503$

$-1.06585503$

$-1.17169046$

$-1.17169046$

$-1.06585503$
$0.55529481 \quad-11.22807884$

1.000 \# Electr. mom., scale-fac \# Magnetic mom.

$-0.26382661 \quad-11.37316036$

$-0.15799116 \quad 11.57299805$

$-0.26382661 \quad 11.41541290$

$-0.15799116$

$-0.26382661$

$-0.15799116$

$-0.26382661$

$-0.15799116$

$-0.03186282$

0.07397263

$-0.03186282$

0.07397263

$-0.03186282$

0.07397263

$-0.03186282$

0.07397263

$-1.38695288$

$-1.28111744$

$-1.38695288$

$-1.28111744$

$-1.38695288$

$-1.28111744$

$-1.38695288$

$-1.28111744$

$-0.43766770$

$-0.33183220$

$-0.43766770$

$-0.33183220$

$-0.43766770$

$-0.33183220$

$-0.43766770$

$-0.33183220$

0.55604303

0.66187847

0.55604303

0.66187847

0.55604303

0.66187847

0.55604303

0.66187847

0.35565418

0.46148965

0.35565418

0.46148965

0.35565418

0.46148965

0.35565418

0.46148965

1. 47651505

1. 58235061

1. 47651505

1.58235061

1. 47651505

1. 58235061

1.47651505

1. 58235061

0.44945931

0.55529481

0.44945931

0.55529481

0.44945931

0.55529481

0.44945931
$-14.12333965$

19.42205238

$-20.38177299$

$-19.78597069$

23.62923241

3. 49837112

4. 51752424

$-4.76332235$

$-3.16300774$

$-3.68309927$

$-3.71756363$

5.18559837

2. 36582017

5.24801493

$-6.84210396$

$-6.18089581$

8. 08153248

$-5.40827084$

6.86952019

6.09671640

$-7.77197170$

0.81443495

$-1.90146101$

$-0.37232631$

0.88009369

$-0.54540318$

1. 26871872

0.35603565

$-0.67963231$

11.09067822

$-7.25069237$

$-9.80989075$

6.43523312

$-8.41900921$

4. 34477615

5.65210295

$-2.44538832$

12.75909710

$-7.31742573$

$-17.17100334$

10.37841702

$-11.29195786$

6.59976292

15.05053520

$-9.20176506$

0.06238356

$-0.02069325$

$-0.42404386$

0.34622598

$-0.33073762$

0.22560714

0.55864453

$-0.45779085$

4.37310743

$-2.76509452$

$-5.94486237$

3.83190131

$-5.49708891$

3.40420747

7.43466759 


$$
0.41621959
$$
72363.

$\begin{array}{lrl} & 72363 & \# \text { Energy } 1->6 \\ 0.3323 & 0.3828 & 0.6283 \\ 0.0451 & -0.2275 & 0.1454\end{array}$

$-1.85509956$

$-1.85509956$

$-1.85509956$

$-1.85509956$

$-1.74926412$

$-1.74926412$

$-1.74926412$

$-1.74926412$

1.30779219

1.30779219

1.30779219

1.30779219

1. 41362774

1. 41362774

1. 41362774

1. 41362774

$-1.45213747$

$-1.45213747$

$-1.45213747$

$-1.45213747$

$-1.34630203$

$-1.34630203$

$-1.34630203$

$-1.34630203$

2.33856606

2.33856606

2.33856606

2. 33856606

2. 44440126

2. 44440126

2. 44440126

2. 44440126

$-1.15465784$

$-1.15465784$

$-1.15465784$

$-1.15465784$

$-1.04882240$

$-1.04882240$

$-1.04882240$

$-1.04882240$

1. 23173511

1. 23173511

1. 23173511

1. 23173511

1. 33757067

1.33757067

1. 33757067

1.33757067

$-1.51449943$

$-1.51449943$

$-1.51449943$

$-1.51449943$

$-1.40866399$

$-1.40866399$

$-1.40866399$

$-1.40866399$

0.31038415

0.31038415

0.31038415

0.31038415

0.41621959

0.41621959

0.41621959

$-0.14332448$

$-0.03748903$

$-0.14332448$

$-0.14332448$

$-0.03748903$

$-0.03748903$

0.51848310

0.51848310

0.62431854

0.62431854

0.51848310

0.51848310

0.62431854

0.62431854

$-0.42367253$

$-0.42367253$

$-0.31783706$

$-0.31783706$

$-0.42367253$

$-0.42367253$

$-0.31783706$

$-0.31783706$

1. 04233944

1.04233944

1. 14817488

1. 14817488

1.04233944

1.04233944

1. 14817488

1. 14817488

0.69317716

0.69317716

0.79901266

0.79901266

0.69317716

0.69317716

0.79901266

0.79901266

$-0.77488583$

$-0.77488583$

$-0.66905046$

$-0.66905046$

$-0.77488583$

$-0.77488583$

$-0.66905046$

$-0.66905046$

0.88849652

0.88849652

0.99433196

0.99433196

0.88849652

0.88849652

0.99433196

0.99433196

$-1.17169046$

$-1.17169046$

$-1.06585503$

$-1.06585503$

$-1.17169046$

$-1.17169046$

$-1.06585503$
$0.55529481 \quad-4.72867966$

1.000 \# Electr. mom., scale-fac \# Magnetic mom.

$-0.26382661$

$-0.47213241$

$\begin{array}{ll}-0.15799116 & 0.87347680\end{array}$

$-0.26382661$

$-0.15799116$

0.39056951
$-0.26382661$

$-0.15799116$

$-0.26382661$

$-0.15799116$

$-0.03186282$

0.07397263

$-0.03186282$

0.07397263

$-0.03186282$

0.07397263

$-0.03186282$

0.07397263

$-1.38695288$

$-1.28111744$

$-1.38695288$

$-1.28111744$

$-1.38695288$

$-1.28111744$

$-1.38695288$

$-1.28111744$

$-0.43766770$

$-0.43766770$

$-0.33183220$

$-0.43766770$

$-0.33183220$

$-0.43766770$

$-0.33183220$

0.55604303

0.66187847

0.55604303

0.66187847

0.55604303

0.66187847

0.55604303

0.66187847

0.35565418

0.46148965

0.35565418

0.46148965

0.35565418

0.46148965

0.35565418

0.46148965

1. 47651505

1. 58235061

1. 47651505

1.58235061

1. 47651505

1. 58235061

1. 47651505

1. 58235061

0.44945931

0.55529481

0.44945931

0.55529481

0.44945931

0.55529481

0.44945931
$-0.33183220$
$-1.57148647$

$-3.36556578$

$-2.69385076$

4.25055647

0.68005729

$-0.45253140$

$-1.66461718$

1.08347273

$-0.69418067$

0.67674321

1. 76912820

$-1.22880006$

2. 12936354

$-2.81514215$

$-2.45337272$

3. 24601364

$-2.15255332$

2. 81153584

2.39958882

$-3.14199257$

0.93808067

$-1.99099219$

$-0.66108066$

1. 30718136

$-0.60265118$

1. 32274318

0.49472326

$-0.92550665$

2. 05713940

$-1.15592694$

$-1.80708683$

0.97582370

$-2.73084331$

1. 58272612

1.89995968

$-0.94618970$

2. 77425027

$-2.25268173$

$-3.07089305$

2.40474486

$-2.35666704$

2. 04179335

2. 50751400

$-2.13037777$

$-0.38129097$

0.35956031

0.34860113

$-0.31558657$

0.24672948

$-0.26887050$

$-0.25734335$

0.25472587

0.93595594

$-0.66444504$

$-1.23119330$

0.85423374

$-1.30147147$

0.90821451

1. 69680583
2. 70109010 
$-1.06585503$ 65324 . $\begin{array}{lll}-0.0761 & -0.1706 & -0.1910\end{array}$

$\begin{array}{lll}-0.0675 & -0.0087 & -0.0757\end{array}$

$-1.85509956$

$-1.85509956$

$-1.85509956$

$-1.85509956$

$-1.74926412$

$-1.74926412$

$-1.74926412$

$-1.74926412$

1.30779219

1.30779219

1.30779219

1.30779219

1. 41362774

1. 41362774

1. 41362774

1. 41362774

$-1.45213747$

$-1.45213747$

$-1.45213747$

$-1.45213747$

$-1.34630203$

$-1.34630203$

$-1.34630203$

$-1.34630203$

2.33856606

2.33856606

2. 33856606

2.33856606

2. 44440126

2. 44440126

2. 44440126

2. 44440126

$-1.15465784$

$-1.15465784$

$-1.15465784$

$-1.15465784$

$-1.04882240$

$-1.04882240$

$-1.04882240$

$-1.04882240$

1. 23173511

1. 23173511

1. 23173511

1. 23173511

1.33757067

1.33757067

1. 33757067

1.33757067

$-1.51449943$

$-1.51449943$

$-1.51449943$

$-1.51449943$

$-1.40866399$

$-1.40866399$

$-1.40866399$

$-1.40866399$

0.31038415

0.31038415

0.31038415

0.31038415

0.41621959

0.41621959

0.41621959
$-0.14332448$

$-0.14332448$

$-0.03748903$

$-0.03748903$

$-0.14332448$

$-0.14332448$

$-0.03748903$

$-0.03748903$

0.51848310

0.51848310

0.62431854

0.62431854

0.51848310

0.51848310

0.62431854

0.62431854

$-0.42367253$

$-0.42367253$

$-0.31783706$

$-0.31783706$

$-0.42367253$

$-0.42367253$

$-0.31783706$

$-0.31783706$

1. 04233944

1.04233944

1. 14817488

1. 14817488

1.04233944

1. 04233944

1. 14817488

1. 14817488

0.69317716

0.69317716

0.79901266

0.79901266

0.69317716

0.69317716

0.79901266

0.79901266

$-0.77488583$

$-0.77488583$

$-0.66905046$

$-0.66905046$

$-0.77488583$

$-0.77488583$

$-0.66905046$

$-0.66905046$

0.88849652

0.88849652

0.99433196

0.99433196

0.88849652

0.88849652

0.99433196

0.99433196

$-1.17169046$

$-1.17169046$

$-1.06585503$

$-1.06585503$

$-1.17169046$

$-1.17169046$

$-1.06585503$
$0.55529481 \quad-1.16577947$

1.000 \# Electr. mom., scale-fac \# Magnetic mom.

$\begin{array}{ll}-0.26382661 & -1.70971811\end{array}$

$-0.15799116 \quad 1.49275398$

$-0.26382661 \quad 1.35065806$

$-0.15799116 \quad-1.26419413$

$-0.26382661 \quad 2.28341126$

$-0.15799116 \quad-2.06002116$

$-0.26382661 \quad-1.93242931$

$-0.15799116 \quad 1.82560909$

$-0.03186282$

0.07397263

$-0.03186282$

0.07397263

$-0.03186282$

0.07397263

$-0.03186282$

0.07397263

$-1.38695288$

$-1.28111744$

$-1.38695288$

$-1.28111744$

$-1.38695288$

$-1.28111744$

$-1.38695288$

$-1.28111744$

$-0.43766770$

$-0.33183220$

$-0.43766770$

$-0.33183220$

$-0.43766770$

$-0.33183220$

$-0.43766770$

$-0.33183220$

0.55604303

0.66187847

0.55604303

0.66187847

0.55604303

0.66187847

0.55604303

0.66187847

0.35565418

0.46148965

0.35565418

0.46148965

0.35565418

0.46148965

0.35565418

0.46148965

1. 47651505

1.58235061

1. 47651505

1. 58235061

1.47651505

1. 58235061

1.47651505

1. 58235061

0.44945931

0.55529481

0.44945931

0.55529481

0.44945931

0.55529481

0.44945931
$-1.07965231$

$-0.39465481$

0.48741716

0.87110633

0.83446354

0.63783932

$-0.25269502$

$-1.11695516$

$-0.22833368$

0.24961369

$-0.16188379$

0.25568724

0.36030057

$-0.42643207$

0.02509163

$-0.07306834$

$-0.22279806$

0.27745131

0.17005114

$-0.19091861$

0.07952035

$-0.11301402$

$-0.08265346$

0.09514551

$-1.81167448$

0.41928929

1. 18457985

$-0.08251146$

1. 51880801

$-0.21389531$

$-0.75921839$

$-0.24448334$

$-3.00897026$

1.38801587

3. 40182710

$-1.61790454$

3. 12128258

$-1.62230921$

$-3.56780100$

1.90372562

0.33840650

$-0.27462074$

$-0.21924148$

0.17032596

$-0.13621110$

0.13294263

0.07533817

$-0.06995436$

$-0.93079895$

0.71374214

1. 27818334

$-1.04430902$

1. 47204971

$-1.11381638$

$-1.93419516$ 
$-1.06585503$ 68541 .
$0.55529481 \quad 1.54670036$ $\begin{array}{lll}-0.1270 & -0.1681 & 0.5098\end{array}$ $\begin{array}{lll}-0.0757 & -0.0147 & 0.0449\end{array}$

$-1.85509956$

$-0.14332448$

$-1.85509956$

$-1.85509956$

$-1.85509956$

$-1.74926412$

$-1.74926412$

$-1.74926412$

$-1.74926412$

1.30779219

1.30779219

1.30779219

1.30779219

1. 41362774

1. 41362774

1. 41362774

1.41362774

$-1.45213747$

$-1.45213747$

$-1.45213747$

$-1.45213747$

$-1.34630203$

$-1.34630203$

$-1.34630203$

$-1.34630203$

2. 33856606

2.33856606

2.33856606

2. 33856606

2. 44440126

2.44440126

2. 44440126

2. 44440126

$-1.15465784$

$-1.15465784$

$-1.15465784$

$-1.15465784$

$-1.04882240$

$-1.04882240$

$-1.04882240$

$-1.04882240$

1. 23173511

1. 23173511

1. 23173511

1. 23173511

1. 33757067

1.33757067

1.33757067

1.33757067

$-1.51449943$

$-1.51449943$

$-1.51449943$

$-1.51449943$

$-1.40866399$

$-1.40866399$

$-1.40866399$

$-1.40866399$

0.31038415

0.31038415

0.31038415

0.31038415

0.41621959

0.41621959

0.41621959
$-0.14332448$

$-0.03748903$

$-0.03748903$

$-0.14332448$

$-0.03748903$

$-0.03748903$

0.51848310

0.51848310

0.62431854

0.62431854

0.51848310

0.51848310

0.62431854

0.62431854

$-0.42367253$

$-0.42367253$

$-0.31783706$

$-0.31783706$

$-0.42367253$

$-0.42367253$

$-0.31783706$

$-0.31783706$

1. 04233944

1.04233944

1. 14817488

1. 14817488

1.04233944

1. 04233944

1. 14817488

1. 14817488

0.69317716

0.69317716

0.79901266

0.79901266

0.69317716

0.69317716

0.79901266

0.79901266

$-0.77488583$

$-0.77488583$

$-0.66905046$

$-0.66905046$

$-0.77488583$

$-0.77488583$

$-0.66905046$

$-0.66905046$

0.88849652

0.88849652

0.99433196

0.99433196

0.88849652

0.88849652

0.99433196

0.99433196

$-1.17169046$

$-1.17169046$

$-1.06585503$

$-1.06585503$

$-1.17169046$

$-1.17169046$

$-1.06585503$
$-0.14332448$

$-14.08492470$

$-1.82042038$
1.000 \# Electr. mom., scale-fac \# Magnetic mom.

$-0.26382661$

$-0.15799116$

$-0.26382661$

$-0.15799116$

$-0.26382661$

$-0.15799116$

$-0.26382661$

$-0.15799116$

$-0.03186282$

0.07397263

$-0.03186282$

0.07397263

$-0.03186282$

0.07397263

$-0.03186282$

0.07397263

$-1.38695288$

$-1.28111744$

$-1.38695288$

$-1.28111744$

$-1.38695288$

$-1.28111744$

$-1.38695288$

$-1.28111744$

$-0.43766770$

$-0.33183220$

$-0.43766770$

$-0.33183220$

$-0.43766770$

$-0.33183220$

$-0.43766770$

$-0.33183220$

0.55604303

0.66187847

0.55604303

0.66187847

0.55604303

0.66187847

0.55604303

0.66187847

0.35565418

0.46148965

0.35565418

0.46148965

0.35565418

0.46148965

0.35565418

0.46148965

1. 47651505

1. 58235061

1. 47651505

1.58235061

1. 47651505

1. 58235061

1. 47651505

1. 58235061

0.44945931

0.55529481

0.44945931

0.55529481

0.44945931

0.55529481

0.44945931
$-2.34431815$

2. 81690717

$-2.29602790$

1. 98741817

$-1.49861717$

$-2.57282162$

1. 63928354

$-16.17925644$

19.42118454

16.84967422

$-19.25609779$

12.48268604

$-15.11846924$

$-13.24232292$

14.94378567

$-0.08674767$

$-0.05580184$

$-0.30745754$

0.59347177

0.08956992

0.00309299

0.20903997

$-0.40502486$

$-0.92510676$

1. 68052745

0.46593297

$-0.85599554$

1.24378169

$-1.73395860$

$-0.98886091$

1.23558319

15.69179440

$-14.00109863$

12.69635963

$-11.20316505$

10.13133430

10.79938793

$-10.01545620$

$-0.13114591$

1.69364369

0.61280859

$-2.34058547$

0.20904806

1. 61692715

0.17080052

$-1.66713476$

1. 20886803

0.93525440

$-0.72854447$

2.78175569

$-1.97023714$

$-1.68622398$

1. 22804451

$-1.03282630$

0.97756052

1. 12282586

$-1.02262902$

1.35657024

$-1.21069276$

$-1.56880879$
2. 11222506 
$-1.06585503$ 61378 . $\begin{array}{lll}1.0971 & -0.1612 & -1.3886\end{array}$

$-0.0093$

$-1.85509956$

$-1.85509956$

$-1.85509956$

$-1.85509956$

$-1.74926412$

$-1.74926412$

$-1.74926412$

$-1.74926412$

1.30779219

1.30779219

1.30779219

1.30779219

1. 41362774

1. 41362774

1. 41362774

1. 41362774

$-1.45213747$

$-1.45213747$

$-1.45213747$

$-1.45213747$

$-1.34630203$

$-1.34630203$

$-1.34630203$

$-1.34630203$

2.33856606

2.33856606

2. 33856606

2.33856606

2. 44440126

2.44440126

2.44440126

2. 44440126

$-1.15465784$

$-1.15465784$

$-1.15465784$

$-1.15465784$

$-1.04882240$

$-1.04882240$

$-1.04882240$

$-1.04882240$

1. 23173511

1. 23173511

1. 23173511

1. 23173511

1.33757067

1.33757067

1.33757067

1.33757067

$-1.51449943$

$-1.51449943$

$-1.51449943$

$-1.51449943$

$-1.40866399$

$-1.40866399$

$-1.40866399$

$-1.40866399$

0.31038415

0.31038415

0.31038415

0.31038415

0.41621959

0.41621959

0.41621959

$$
0.5700 \quad-0.0279
$$

$-0.14332448$

$-0.14332448$

$-0.03748903$

$-0.03748903$

$-0.14332448$

$-0.14332448$

$-0.03748903$

$-0.03748903$

0.51848310

0.51848310

0.62431854

0.62431854

0.51848310

0.51848310

0.62431854

0.62431854

$-0.42367253$

$-0.42367253$

$-0.31783706$

$-0.31783706$

$-0.42367253$

$-0.42367253$

$-0.31783706$

$-0.31783706$

1. 04233944

1.04233944

1. 14817488

1. 14817488

1.04233944

1. 04233944

1. 14817488

1. 14817488

0.69317716

0.69317716

0.79901266

0.79901266

0.69317716

0.69317716

0.79901266

0.79901266

$-0.77488583$

$-0.77488583$

$-0.66905046$

$-0.66905046$

$-0.77488583$

$-0.77488583$

$-0.66905046$

$-0.66905046$

0.88849652

0.88849652

0.99433196

0.99433196

0.88849652

0.88849652

0.99433196

0.99433196

$-1.17169046$

$-1.17169046$

$-1.06585503$

$-1.06585503$

$-1.17169046$

$-1.17169046$

$-1.06585503$
$0.55529481 \quad 1.34363174$

1.000 \# Electr. mom., scale-fac \# Magnetic mom.

$-0.26382661$ 16.22741318

$-0.15799116 \quad-16.85372162$

$-0.26382661 \quad-17.80922699$

$-0.15799116 \quad 21.14413452$

$-0.26382661 \quad-27.17255402$

$-0.15799116 \quad 28.74585915$

$-0.26382661$

$-0.15799116$

$-0.03186282$

0.07397263

$-0.03186282$

0.07397263

$-0.03186282$

0.07397263

$-0.03186282$

0.07397263

$-1.38695288$

$-1.28111744$

$-1.38695288$

$-1.28111744$

$-1.38695288$

$-1.28111744$

$-1.38695288$

$-1.28111744$

$-0.43766770$

$-0.33183220$

$-0.43766770$

$-0.33183220$

$-0.43766770$

$-0.33183220$

$-0.43766770$

$-0.33183220$

0.55604303

0.66187847

0.55604303

0.66187847

0.55604303

0.66187847

0.55604303

0.66187847

0.35565418

0.46148965

0.35565418

0.46148965

0.35565418

0.46148965

0.35565418

0.46148965

1.47651505

1.58235061

1. 47651505

1.58235061

1. 47651505

1. 58235061

1.47651505

1.58235061

0.44945931

0.55529481

0.44945931

0.55529481

0.44945931

0.55529481

0.44945931
29.68818474

$-34.23368073$

$-2.89547229$

6.02265453

1. 93552852

$-4.99686337$

1. 45924294

$-4.46434975$

$-0.53732026$

3. 53899741

$-4.83595181$

6.69423342

6.21654081

$-8.50081921$

4.20470619

$-5.69048119$

$-5.24194288$

7.06074047

$-0.07036173$

0.51843470

0.08681665

$-0.66288537$

0.09277026

$-0.54607642$

$-0.03931850$

0.55373389

$-1.57236552$

4. 20148230

4.20799732

$-5.83048773$

3. 26511431

$-4.80367851$

$-4.91583204$

5.85436964

2. 59485817

$-0.49994442$

$-3.11357093$

1.00469315

$-2.93921232$

1.08579814

3. 51388168

$-1.61299300$

$-0.73621565$

0.46484408

0.64217675

$-0.36294138$

0.18670692

$-0.07483477$

$-0.28275099$

0.12000653

$-2.46969318$

1. 72572386

3. 28050923

$-2.15269160$

2.46339107

$-1.74768519$

$-3.28957653$ 


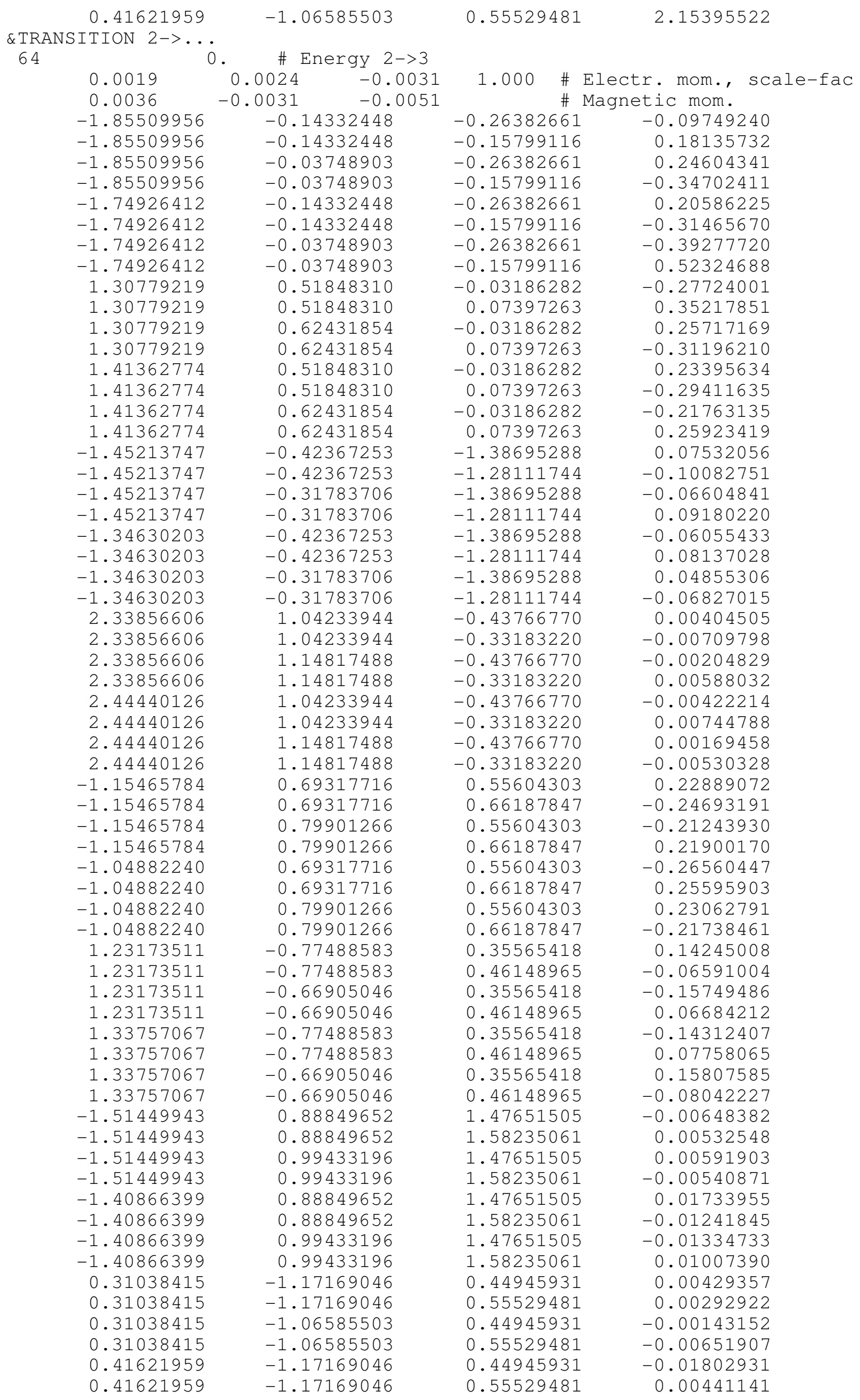

\section{S-282}


64

$$
\begin{array}{ccc}
0.41621959 & -1.06585503 \\
0.41621959 & -1.06585503 \\
& 0.0 . \# \text { Energy } 2->4 \\
0.0083 & 0.0032 & 0.0018 \\
-0.0019 & 0.0059 & 0.0215
\end{array}
$$

$-1.85509956$

$-1.85509956$

$-1.85509956$

$-1.85509956$

$-1.74926412$

$-1.74926412$

$-1.74926412$

$-1.74926412$

1.30779219

1.30779219

1.30779219

1.30779219

1. 41362774

1. 41362774

1. 41362774

1. 41362774

$-1.45213747$

$-1.45213747$

$-1.45213747$

$-1.45213747$

$-1.34630203$

$-1.34630203$

$-1.34630203$

$-1.34630203$

2. 33856606

2.33856606

2.33856606

2.33856606

2. 44440126

2. 44440126

2. 44440126

2. 44440126

$-1.15465784$

$-1.15465784$

$-1.15465784$

$-1.15465784$

$-1.04882240$

$-1.04882240$

$-1.04882240$

$-1.04882240$

1. 23173511

1. 23173511

1. 23173511

1. 23173511

1.33757067

1. 33757067

1.33757067

1.33757067

$-1.51449943$

$-1.51449943$

$-1.51449943$

$-1.51449943$

$-1.40866399$

$-1.40866399$

$-1.40866399$

$-1.40866399$

0.31038415

0.31038415

0.31038415

0.31038415

0.41621959

0.41621959

$\begin{array}{ll}0.0032 & 0.0018 \\ 0.0059 & 0.0215\end{array}$

$-0.14332448$

$-0.14332448$

$-0.03748903$

$-0.03748903$

$-0.14332448$

$-0.14332448$

$-0.03748903$

$-0.03748903$

0.51848310

0.51848310

0.62431854

0.62431854

0.51848310

0.51848310

0.62431854

0.62431854

$-0.42367253$

$-0.42367253$

$-0.31783706$

$-0.31783706$

$-0.42367253$

$-0.42367253$

$-0.31783706$

$-0.31783706$

1.04233944

1.04233944

1.14817488

1.14817488

1. 04233944

1.04233944

1. 14817488

1. 14817488

0.69317716

0.69317716

0.79901266

0.79901266

0.69317716

0.69317716

0.79901266

0.79901266

$-0.77488583$

$-0.77488583$

$-0.66905046$

$-0.66905046$

$-0.77488583$

$-0.77488583$

$-0.66905046$

$-0.66905046$

0.88849652

0.88849652

0.99433196

0.99433196

0.88849652

0.88849652

0.99433196

0.99433196

$-1.17169046$

$-1.17169046$

$-1.06585503$

$-1.06585503$

$-1.17169046$

$-1.17169046$ $\begin{array}{lr}0.44945931 & 0.01762786 \\ 0.55529481 & -0.00229055\end{array}$

1.000 \# Electr. mom., scale-fac \# Magnetic mom.

$-0.26382661 \quad 1.01099133$

$-0.15799116-1.57820344$

$-0.26382661-1.98886490$

$\begin{array}{ll}-0.15799116 & 2.68448067\end{array}$

$-0.26382661 \quad-1.69624734$

$-0.15799116$

$-0.26382661$

$-0.15799116$

$-0.03186282$

0.07397263

$-0.03186282$

0.07397263

$-0.03186282$

0.07397263

$-0.03186282$

0.07397263

$-1.38695288$

$-1.28111744$

$-1.38695288$

$-1.28111744$

$-1.38695288$

$-1.28111744$

$-1.38695288$

$-1.28111744$

$-0.43766770$

$-0.33183220$

$-0.43766770$

$-0.33183220$

$-0.43766770$

$-0.33183220$

$-0.43766770$

$-0.33183220$

0.55604303

0.66187847

0.55604303

0.66187847

0.55604303

0.66187847

0.55604303

0.66187847

0.35565418

0.46148965

0.35565418

0.46148965

0.35565418

0.46148965

0.35565418

0.46148965

1. 47651505

1. 58235061

1. 47651505

1. 58235061

1. 47651505

1. 58235061

1. 47651505

1.58235061

0.44945931

0.55529481

0.44945931

0.55529481

0.44945931

0.55529481
2.43488836

2.94865966

$-3.84590483$

$-0.42063636$

0.69136512

0.37290972

$-0.64625281$

0.32295561

$-0.57400161$

$-0.27132571$

0.52930957

0.30806592

0.18098201

$-0.29090965$

0.07443158

$-0.12899239$

$-0.02023934$

0.07571712

0.09030383

$-0.16277577$

$-0.05630941$

0.10701109

$-0.06728193$

0.12359483

0.04172706

$-0.08107989$

$-1.24041593$

1.44404638

1.29949689

$-1.41282380$

1.70480907

$-1.69457591$

$-1.63527977$

1.58366168

0.31375277

$-0.10572705$

$-0.45404249$

0.20968892

$-0.33755252$

0.14888468

0.46756443

$-0.24604753$

$-0.03104107$

0.01181376

0.00978619

0.00463785

$-0.01195570$

0.01793126

0.02120323

$-0.02611906$

0.19165345

$-0.11179194$

$-0.29846466$

0.19607098

$-0.22444548$

0.13452348
$-0.21026866$ 
64

$$
\begin{array}{ccc}
0.41621959 & -1.06585503 \\
0.41621959 & -1.06585503 \\
& 0 . & \# \text { Energy } 2->5 \\
0.0131 & -0.0597 & 0.0306
\end{array}
$$

0.1065

$-1.85509956$

$-1.85509956$

$-1.85509956$

$-1.85509956$

$-1.74926412$

$-1.74926412$

$-1.74926412$

$-1.74926412$

1.30779219

1.30779219

1.30779219

1.30779219

1. 41362774

1. 41362774

1. 41362774

1. 41362774

$-1.45213747$

$-1.45213747$

$-1.45213747$

$-1.45213747$

$-1.34630203$

$-1.34630203$

$-1.34630203$

$-1.34630203$

2. 33856606

2.33856606

2.33856606

2. 33856606

2. 44440126

2. 44440126

2. 44440126

2. 44440126

$-1.15465784$

$-1.15465784$

$-1.15465784$

$-1.15465784$

$-1.04882240$

$-1.04882240$

$-1.04882240$

$-1.04882240$

1. 23173511

1. 23173511

1. 23173511

1. 23173511

1.33757067

1.33757067

1.33757067

1. 33757067

$-1.51449943$

$-1.51449943$

$-1.51449943$

$-1.51449943$

$-1.40866399$

$-1.40866399$

$-1.40866399$

$-1.40866399$

0.31038415

0.31038415

0.31038415

0.31038415

0.41621959

0.41621959 $\begin{array}{rr}-0.0597 & 0.0306 \\ -0.0498 & -0.2974\end{array}$

$-0.14332448$

$-0.14332448$

$-0.03748903$

$-0.03748903$

$-0.14332448$

$-0.14332448$

$-0.03748903$

$-0.03748903$

0.51848310

0.51848310

0.62431854

0.62431854

0.51848310

0.51848310

0.62431854

0.62431854

$-0.42367253$

$-0.42367253$

$-0.31783706$

$-0.31783706$

$-0.42367253$

$-0.42367253$

$-0.31783706$

$-0.31783706$

1. 04233944

1.04233944

1. 14817488

1.14817488

1. 04233944

1.04233944

1.14817488

1. 14817488

0.69317716

0.69317716

0.79901266

0.79901266

0.69317716

0.69317716

0.79901266

0.79901266

$-0.77488583$

$-0.77488583$

$-0.66905046$

$-0.66905046$

$-0.77488583$

$-0.77488583$

$-0.66905046$

$-0.66905046$

0.88849652

0.88849652

0.99433196

0.99433196

0.88849652

0.88849652

0.99433196

0.99433196

$-1.17169046$

$-1.17169046$

$-1.06585503$

$-1.06585503$

$-1.17169046$

$-1.17169046$ $\begin{array}{lr}0.44945931 & 0.34187579 \\ 0.55529481 & -0.22921759\end{array}$

1.000 \# Electr. mom., scale-fac \# Magnetic mom.

$-0.26382661$

$-13.24185753$

$-0.15799116 \quad 20.38767624$

$-0.26382661$

$-0.15799116$

$-0.26382661$

$-0.15799116$

$-0.26382661$

$-0.15799116$

$-0.03186282$

0.07397263

$-0.03186282$

0.07397263

$-0.03186282$

0.07397263

$-0.03186282$

0.07397263

$-1.38695288$

$-1.28111744$

$-1.38695288$

$-1.28111744$

$-1.38695288$

$-1.28111744$

$-1.38695288$

$-1.28111744$

$-0.43766770$

$-0.33183220$

$-0.43766770$

$-0.33183220$

$-0.43766770$

$-0.33183220$

$-0.43766770$

$-0.33183220$

0.55604303

0.66187847

0.55604303

0.66187847

0.55604303

0.66187847

0.55604303

0.66187847

0.35565418

0.46148965

0.35565418

0.46148965

0.35565418

0.46148965

0.35565418

0.46148965

1. 47651505

1. 58235061

1.47651505

1.58235061

1. 47651505

1. 58235061

1.47651505

1.58235061

0.44945931

0.55529481

0.44945931

0.55529481

0.44945931

0.55529481
25.44814110

$-34.06230164$

21.27238083

$-30.49529648$

$-36.86938095$

47.89053345

$-1.55137789$

$-0.05321678$

1. 77355194

0.06746554

1. 56247163

0.04102425

$-1.86531591$

$-0.00137522$

3.38014579

$-4.80228519$

$-2.97718096$

4. 51656628

$-1.83260560$

2. 74322581

1. 12648642

$-2.01845145$

$-0.34295243$

0.73081195

0.27652907

$-0.50684035$

0.06447968

$-0.34517667$

$-0.05822669$

0.22224522

17.29482269

$-19.50765228$

$-17.18301010$

18.36868668

$-21.29352379$

21.14093399

19.87909698

$-19.28475189$

$-1.54858923$

0.53602821

2.92458439

$-1.68150926$

1. 58035338

$-2.76752424$

1. 62261403

0.19190598

$-0.06662768$

$-0.09200283$

$-0.03257266$

0.56611741

$-0.44445285$

$-0.44853246$

0.39120826

$-1.95325208$

1. 24952245

3. 13634706

$-2.18241477$

2. 07378435

$-1.35118961$
$-0.62781471$ 
64

$$
\begin{array}{ccc}
0.41621959 & \multicolumn{1}{c}{-1.06585503} \\
0.41621959 & -1.06585503 \\
& 0.0 .04 \text { Energy 2->6 } \\
0.0292 & 0.0492 & 0.0776 \\
-0.1346 & -0.0674 & -0.0316
\end{array}
$$

$-1.85509956$

$-1.85509956$

$-1.85509956$

$-1.85509956$

$-1.74926412$

$-1.74926412$

$-1.74926412$

$-1.74926412$

1.30779219

1.30779219

1.30779219

1.30779219

1. 41362774

1. 41362774

1. 41362774

1. 41362774

$-1.45213747$

$-1.45213747$

$-1.45213747$

$-1.45213747$

$-1.34630203$

$-1.34630203$

$-1.34630203$

$-1.34630203$

2. 33856606

2.33856606

2.33856606

2. 33856606

2. 44440126

2. 44440126

2. 44440126

2. 44440126

$-1.15465784$

$-1.15465784$

$-1.15465784$

$-1.15465784$

$-1.04882240$

$-1.04882240$

$-1.04882240$

$-1.04882240$

1. 23173511

1. 23173511

1. 23173511

1. 23173511

1.33757067

1. 33757067

1.33757067

1.33757067

$-1.51449943$

$-1.51449943$

$-1.51449943$

$-1.51449943$

$-1.40866399$

$-1.40866399$

$-1.40866399$

$-1.40866399$

0.31038415

0.31038415

0.31038415

0.31038415

0.41621959

0.41621959 $-0.14332448$

$-0.14332448$

$-0.03748903$

$-0.03748903$

$-0.14332448$

$-0.14332448$

$-0.03748903$

$-0.03748903$

0.51848310

0.51848310

0.62431854

0.62431854

0.51848310

0.51848310

0.62431854

0.62431854

$-0.42367253$

$-0.42367253$

$-0.31783706$

$-0.31783706$

$-0.42367253$

$-0.42367253$

$-0.31783706$

$-0.31783706$

1. 04233944

1. 04233944

1. 14817488

1.14817488

1. 04233944

1.04233944

1. 14817488

1. 14817488

0.69317716

0.69317716

0.79901266

0.79901266

0.69317716

0.69317716

0.79901266

0.79901266

$-0.77488583$

$-0.77488583$

$-0.66905046$

$-0.66905046$

$-0.77488583$

$-0.77488583$

$-0.66905046$

$-0.66905046$

0.88849652

0.88849652

0.99433196

0.99433196

0.88849652

0.88849652

0.99433196

0.99433196

$-1.17169046$

$-1.17169046$

$-1.06585503$

$-1.06585503$

$-1.17169046$

$-1.17169046$ $\begin{array}{lr}0.44945931 & -3.34480524 \\ 0.55529481 & 2.37432551\end{array}$

1.000 \# Electr. mom., scale-fac \# Magnetic mom.

$-0.26382661 \quad-1.79546797$

$-0.15799116 \quad 3.46956229$

$-0.26382661$

$-0.15799116$

$-0.26382661$

$-0.15799116$

$-0.26382661$

$-0.15799116$

$-0.03186282$

0.07397263

$-0.03186282$

0.07397263

$-0.03186282$

0.07397263

$-0.03186282$

0.07397263

$-1.38695288$

$-1.28111744$

$-1.38695288$

$-1.28111744$

$-1.38695288$

$-1.28111744$

$-1.38695288$

$-1.28111744$

$-0.43766770$

$-0.33183220$

$-0.43766770$

$-0.33183220$

$-0.43766770$

$-0.33183220$

$-0.43766770$

$-0.33183220$

0.55604303

0.66187847

0.55604303

0.66187847

0.55604303

0.66187847

0.55604303

0.66187847

0.35565418

0.46148965

0.35565418

0.46148965

0.35565418

0.46148965

0.35565418

0.46148965

1. 47651505

1. 58235061

1. 47651505

1. 58235061

1. 47651505

1.58235061

1. 47651505

1.58235061

0.44945931

0.55529481

0.44945931

0.55529481

0.44945931

0.55529481
4.47813797

3. 44738150

$-5.60447741$

$-6.85459805$

9.44208813

7.53586245

$-8.91549778$

$-7.69263315$

8.95733166

$-6.90845490$

8.14826584

7.06409025

$-8.20403862$

$-0.70928037$

$-0.17854096$

0.40863755

$-0.02195189$

0.14802338

$-0.30456227$

0.22635502

$-1.18920815$

1. 42921054

0.32873124

$-0.49516085$

1. 79891038

$-2.00241804$

$-1.00362980$

1. 14892375

1. 24080491

$-1.83667445$

$-1.61399543$

1. 95674443

$-3.38229275$

3.33454967

3. 21363354

$-3.03642869$

$-2.97972131$

1. 77706957

2. 45824027

$-1.23657644$

2.90383554

$-1.90672719$

$-2.45309138$

1. 42648005

0.45505440

$-0.29037574$

$-0.27675295$

0.16832840

$-0.56515580$

0.36377841

0.34903613

$-0.21348283$

$-0.58543640$

0.24210703

0.61698133

$-0.24429880$

0.55525124

$-0.20527036$
$-6.48817444$

0.45577884 
64

$$
\begin{array}{ccc}
0.41621959 & -1.06585503 \\
0.41621959 & -1.06585503 \\
& 0.0 . \# \text { Energy 2->7 } \\
0.2220 & -0.1214 & 0.1691 \\
-0.0601 & 0.0130 & 0.0064
\end{array}
$$

$-1.85509956$

$-1.85509956$

$-1.85509956$

$-1.85509956$

$-1.74926412$

$-1.74926412$

$-1.74926412$

$-1.74926412$

1.30779219

1.30779219

1.30779219

1.30779219

1. 41362774

1. 41362774

1. 41362774

1. 41362774

$-1.45213747$

$-1.45213747$

$-1.45213747$

$-1.45213747$

$-1.34630203$

$-1.34630203$

$-1.34630203$

$-1.34630203$

2. 33856606

2.33856606

2.33856606

2. 33856606

2. 44440126

2. 44440126

2. 44440126

2. 44440126

$-1.15465784$

$-1.15465784$

$-1.15465784$

$-1.15465784$

$-1.04882240$

$-1.04882240$

$-1.04882240$

$-1.04882240$

1. 23173511

1. 23173511

1. 23173511

1. 23173511

1.33757067

1. 33757067

1.33757067

1.33757067

$-1.51449943$

$-1.51449943$

$-1.51449943$

$-1.51449943$

$-1.40866399$

$-1.40866399$

$-1.40866399$

$-1.40866399$

0.31038415

0.31038415

0.31038415

0.31038415

0.41621959

0.41621959

$\begin{array}{rr}-0.1214 & 0.1691 \\ 0.0130 & 0.0064\end{array}$

$-0.14332448$

$-0.14332448$

$-0.03748903$

$-0.03748903$

$-0.14332448$

$-0.14332448$

$-0.03748903$

$-0.03748903$

0.51848310

0.51848310

0.62431854

0.62431854

0.51848310

0.51848310

0.62431854

0.62431854

$-0.42367253$

$-0.42367253$

$-0.31783706$

$-0.31783706$

$-0.42367253$

$-0.42367253$

$-0.31783706$

$-0.31783706$

1. 04233944

1.04233944

1. 14817488

1.14817488

1. 04233944

1.04233944

1. 14817488

1. 14817488

0.69317716

0.69317716

0.79901266

0.79901266

0.69317716

0.69317716

0.79901266

0.79901266

$-0.77488583$

$-0.77488583$

$-0.66905046$

$-0.66905046$

$-0.77488583$

$-0.77488583$

$-0.66905046$

$-0.66905046$

0.88849652

0.88849652

0.99433196

0.99433196

0.88849652

0.88849652

0.99433196

0.99433196

$-1.17169046$

$-1.17169046$

$-1.06585503$

$-1.06585503$

$-1.17169046$

$-1.17169046$ $\begin{array}{lr}0.44945931 & -0.46434376 \\ 0.55529481 & 0.10953333\end{array}$

1.000 \# Electr. mom., scale-fac \# Magnetic mom.

$-0.26382661$

0.50699532

$-0.15799116 \quad-0.79412794$

$-0.26382661$

$-0.15799116$

$-0.26382661$

$-0.15799116$

$-0.26382661$

$-0.15799116$

$-0.03186282$

0.07397263

$-0.03186282$

0.07397263

$-0.03186282$

0.07397263

$-0.03186282$

0.07397263

$-1.38695288$

$-1.28111744$

$-1.38695288$

$-1.28111744$

$-1.38695288$

$-1.28111744$

$-1.38695288$

$-1.28111744$

$-0.43766770$

$-0.33183220$

$-0.43766770$

$-0.33183220$

$-0.43766770$

$-0.33183220$

$-0.43766770$

$-0.33183220$

0.55604303

0.66187847

0.55604303

0.66187847

0.55604303

0.66187847

0.55604303

0.66187847

0.35565418

0.46148965

0.35565418

0.46148965

0.35565418

0.46148965

0.35565418

0.46148965

1. 47651505

1. 58235061

1. 47651505

1. 58235061

1. 47651505

1.58235061

1. 47651505

1.58235061

0.44945931

0.55529481

0.44945931

0.55529481

0.44945931

0.55529481
$-0.93475878$

1. 29178393

$-1.11044073$

1. 44809878

1. 59123790

$-2.03805614$

1. 41282034

$-1.16627002$

$-1.60398960$

1.30568469

$-1.24241972$

0.95394439

1. 47079742

$-1.11393201$

0.38772193

$-0.48482695$

$-0.66762626$

0.83567870

$-0.43019158$

0.53422576

0.72363585

$-0.90221632$

0.23551401

$-0.45846829$

$-0.29203275$

0.41356185

0.09566234

0.04931434

0.00823516

$-0.08001499$

$-0.49838021$

0.68405497

0.62248719

$-0.74843192$

0.94961959

$-0.98826176$

$-0.98342317$

0.99953777

1.12653339

$-0.62528133$

$-2.05829072$

1.41071689

$-0.78060168$

0.32434544

1. 64126849

$-1.01742256$

0.14371093

$-0.12684418$

$-0.14525226$

0.13264188

$-0.19941878$

0.16970730

0.17724438

$-0.15839939$

0.45942616

$-0.30213881$

$-0.60374123$

0.42644760

$-0.52436018$

0.33680314 
64

$$
\begin{array}{ccc}
0.41621959 & & -1.06585503 \\
0.41621959 & & -1.06585503 \\
& 0 . & \# \text { Energy } 2->8 \\
-0.0114 & 0.0059 & -0.0098 \\
0.0148 & -0.0019 & -0.0095
\end{array}
$$$$
-1.85509956
$$$$
-1.85509956
$$$$
-1.85509956
$$$$
-1.85509956
$$$$
-1.74926412
$$$$
-1.74926412
$$$$
-1.74926412
$$$$
-1.74926412
$$$$
1.30779219
$$$$
\text { 1.30779219 }
$$$$
\text { 1.30779219 }
$$$$
1.30779219
$$$$
\text { 1. } 41362774
$$$$
\text { 1. } 41362774
$$$$
\text { 1. } 41362774
$$$$
\text { 1. } 41362774
$$$$
-1.45213747
$$$$
-1.45213747
$$$$
-1.45213747
$$$$
-1.45213747
$$$$
-1.34630203
$$$$
-1.34630203
$$$$
-1.34630203
$$$$
-1.34630203
$$$$
\text { 2. } 33856606
$$$$
2.33856606
$$$$
2.33856606
$$$$
\text { 2. } 33856606
$$$$
\text { 2. } 44440126
$$$$
\text { 2. } 44440126
$$$$
2.44440126
$$$$
\text { 2. } 44440126
$$$$
-1.15465784
$$$$
-1.15465784
$$$$
-1.15465784
$$$$
-1.15465784
$$$$
-1.04882240
$$$$
-1.04882240
$$$$
-1.04882240
$$$$
-1.04882240
$$$$
\text { 1. } 23173511
$$$$
\text { 1. } 23173511
$$$$
\text { 1. } 23173511
$$$$
\text { 1. } 23173511
$$$$
1.33757067
$$$$
\text { 1.33757067 }
$$$$
\text { 1.33757067 }
$$$$
\text { 1. } 33757067
$$

$-1.51449943$

$-1.51449943$

$-1.51449943$

$-1.51449943$

$-1.40866399$

$-1.40866399$

$-1.40866399$

$-1.40866399$

0.31038415

0.31038415

0.31038415

0.31038415

0.41621959

0.41621959

$-0.14332448$

$-0.14332448$

$-0.03748903$

$-0.03748903$

$-0.14332448$

$-0.14332448$

$-0.03748903$

$-0.03748903$

0.51848310

0.51848310

0.62431854

0.62431854

0.51848310

0.62431854

0.62431854

$-0.42367253$

$-0.42367253$

$-0.31783706$

$-0.31783706$

$-0.42367253$

$-0.42367253$

$-0.31783706$

$-0.31783706$

1. 04233944

1. 04233944

1. 14817488

1. 14817488

1. 04233944

1.14817488

1. 14817488

0.69317716

0.69317716

0.79901266

0.79901266

0.69317716

0.69317716

0.79901266

0.79901266

$-0.77488583$

$-0.77488583$

$-0.66905046$

$-0.66905046$

$-0.77488583$

$-0.77488583$

$-0.66905046$

$-0.66905046$

0.88849652

0.88849652

0.99433196

0.99433196

0.88849652

0.88849652

0.99433196

0.99433196

$-1.17169046$

$-1.17169046$

$-1.06585503$

$-1.06585503$

$-1.17169046$

$-1.17169046$

$$
\begin{array}{lr}
0.44945931 & 0.69980443 \\
0.55529481 & -0.48964214
\end{array}
$$

0.51848310

1.04233944

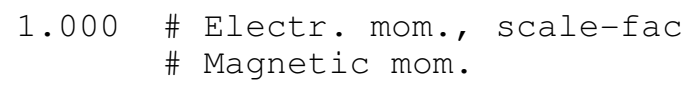


64

$$
\begin{aligned}
& 0.41621959 \\
& 0.41621959
\end{aligned}
$$

$-0.1981$

$-0.0541$

$-1.85509956$

$-1.85509956$

$-1.85509956$

$-1.85509956$

$-1.74926412$

$-1.74926412$

$-1.74926412$

$-1.74926412$

1.30779219

1.30779219

1.30779219

1.30779219

1. 41362774

1. 41362774

1. 41362774

1. 41362774

$-1.45213747$

$-1.45213747$

$-1.45213747$

$-1.45213747$

$-1.34630203$

$-1.34630203$

$-1.34630203$

$-1.34630203$

2. 33856606

2.33856606

2.33856606

2. 33856606

2. 44440126

2. 44440126

2. 44440126

2. 44440126

$-1.15465784$

$-1.15465784$

$-1.15465784$

$-1.15465784$

$-1.04882240$

$-1.04882240$

$-1.04882240$

$-1.04882240$

1. 23173511

1. 23173511

1. 23173511

1. 23173511

1.33757067

1. 33757067

1.33757067

1. 33757067

$-1.51449943$

$-1.51449943$

$-1.51449943$

$-1.51449943$

$-1.40866399$

$-1.40866399$

$-1.40866399$

$-1.40866399$

0.31038415

0.31038415

0.31038415

0.31038415

0.41621959

0.41621959
$-1.06585503$

$-1.06585503$

\# Energy 2->9

$0.0429^{0.0597}-0.0621$

$0.0597 \quad 0.1403$

$-0.14332448$

$-0.14332448$

$-0.03748903$

$-0.03748903$

$-0.14332448$

$-0.14332448$

$-0.03748903$

$-0.03748903$

0.51848310

0.51848310

0.62431854

0.62431854

0.51848310

0.51848310

0.62431854

0.62431854

$-0.42367253$

$-0.42367253$

$-0.31783706$

$-0.31783706$

$-0.42367253$

$-0.42367253$

$-0.31783706$

$-0.31783706$

1.04233944

1.04233944

1.14817488

1.14817488

1. 04233944

1.04233944

1. 14817488

1. 14817488

0.69317716

0.69317716

0.79901266

0.79901266

0.69317716

0.69317716

0.79901266

0.79901266

$-0.77488583$

$-0.77488583$

$-0.66905046$

$-0.66905046$

$-0.77488583$

$-0.77488583$

$-0.66905046$

$-0.66905046$

0.88849652

0.88849652

0.99433196

0.99433196

0.88849652

0.88849652

0.99433196

0.99433196

$-1.17169046$

$-1.17169046$

$-1.06585503$

$-1.06585503$

$-1.17169046$

$-1.17169046$ $\begin{array}{lr}0.44945931 & -0.21015154 \\ 0.55529481 & 0.16028707\end{array}$

1.000 \# Electr. mom., scale-fac \# Magnetic mom.

.26382661

12.92445660

$-18.68206215$

$-22.21750069$

29.10497284

$-0.26382661$

$-0.15799116$

$-0.26382661$

$-0.15799116$

$-0.26382661$

$-0.15799116$

$-0.03186282$

0.07397263

$-0.03186282$

0.07397263

$-0.03186282$

0.07397263

$-0.03186282$

0.07397263

$-1.38695288$

$-1.28111744$

$-1.38695288$

$-1.28111744$

$-1.38695288$

$-1.28111744$

$-1.38695288$

$-1.28111744$

$-0.43766770$

$-0.33183220$

$-0.43766770$

$-0.33183220$

$-0.43766770$

$-0.33183220$

$-0.43766770$

$-0.33183220$

0.55604303

0.66187847

0.55604303

0.66187847

0.55604303

0.66187847

0.55604303

0.66187847

0.35565418

0.46148965

0.35565418

0.46148965

0.35565418

0.46148965

0.35565418

0.46148965

1. 47651505

1. 58235061

1. 47651505

1. 58235061

1. 47651505

1. 58235061

1. 47651505

1.58235061

0.44945931

0.55529481

0.44945931

0.55529481

0.44945931

0.55529481
$-18.88364792$

26.21315193

30.74439240

$-39.42110062$

2. 51925421

$-3.61466908$

$-2.14027405$

2.96786904

$-1.82634759$

2. 71388435

1. 46170032

$-2.12415886$

$-2.91519833$

4.10030031

2.60716391

$-3.84712958$

1. 79250979

$-2.58169818$

$-1.25683093$

2.00168061

$-0.16286938$

0.40018153

0.26965284

$-0.45465475$

$-0.05193742$

$-0.14469793$

$-0.05453786$

0.20431115

$-14.47832394$

15.77832699

13.71336079

$-14.30899429$

16.03091240

$-15.78415394$

$-14.47300148$

13.93570137

$-1.50360918$

0.33387393

1. 93128633

$-0.52527136$

1. 56239629

$-0.56591856$

$-1.99235523$

0.77923310

$-0.63737208$

0.48850492

0.60776699

$-0.43943855$

0.00021222

$-0.07237354$

$-0.14051856$

0.14313643

1. 01581371

$-0.79372829$

$-1.53754151$

1.20732749

$-0.70089561$

0.63323486 


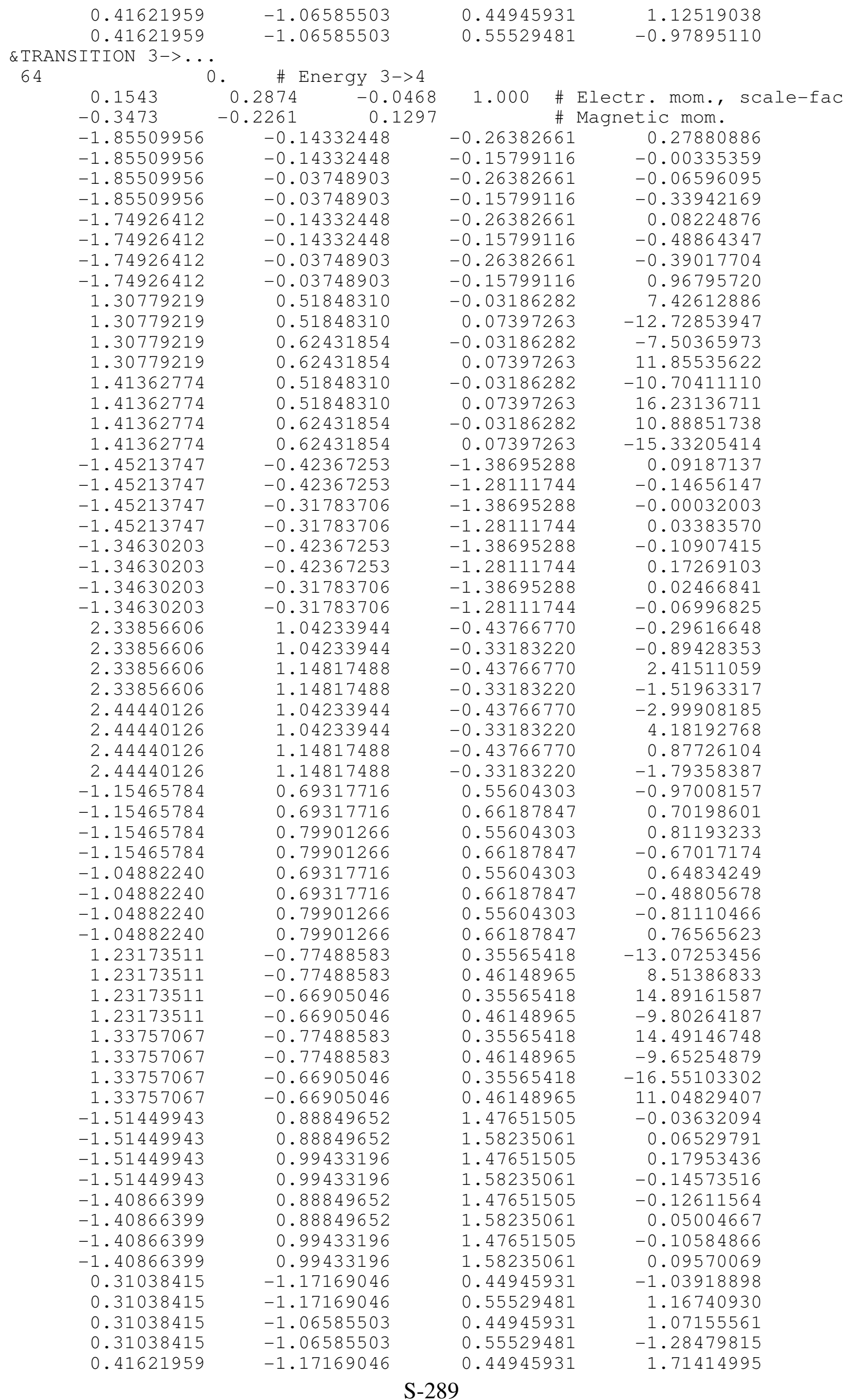




$$
\begin{aligned}
& 0.41621959 \\
& 0.41621959 \\
& 0.41621959
\end{aligned}
$$

$-0.0024$

$-0.0067$

$-1.85509956$

$-1.85509956$

$-1.85509956$

$-1.85509956$

$-1.74926412$

$-1.74926412$

$-1.74926412$

$-1.74926412$

1. 30779219

1.30779219

1.30779219

1.30779219

1. 41362774

1. 41362774

1. 41362774

1. 41362774

$-1.45213747$

$-1.45213747$

$-1.45213747$

$-1.45213747$

$-1.34630203$

$-1.34630203$

$-1.34630203$

$-1.34630203$

2. 33856606

2.33856606

2. 33856606

2.33856606

2. 44440126

2. 44440126

2. 44440126

2. 44440126

$-1.15465784$

$-1.15465784$

$-1.15465784$

$-1.15465784$

$-1.04882240$

$-1.04882240$

$-1.04882240$

$-1.04882240$

1. 23173511

1. 23173511

1. 23173511

1. 23173511

1. 33757067

1.33757067

1.33757067

1. 33757067

$-1.51449943$

$-1.51449943$

$-1.51449943$

$-1.51449943$

$-1.40866399$

$-1.40866399$

$-1.40866399$

$-1.40866399$

0.31038415

0.31038415

0.31038415

0.31038415

0.41621959
$-1.17169046$

$-1.06585503$

$-1.06585503$

. \# Energy 3->5

$\begin{array}{ll}-0.0019 & 0.0776 \\ -0.0534 & 0.0028\end{array}$

$-0.14332448$

$-0.14332448$

$-0.03748903$

$-0.03748903$

$-0.14332448$

$-0.14332448$

$-0.03748903$

$-0.03748903$

0.51848310

0.51848310

0.62431854

0.62431854

0.51848310

0.51848310

0.62431854

0.62431854

$-0.42367253$

$-0.42367253$

$-0.31783706$

$-0.31783706$

$-0.42367253$

$-0.42367253$

$-0.31783706$

$-0.31783706$

1. 04233944

1. 04233944

1. 14817488

1. 14817488

1.04233944

1.04233944

1. 14817488

1. 14817488

0.69317716

0.69317716

0.79901266

0.79901266

0.69317716

0.69317716

0.79901266

0.79901266

$-0.77488583$

$-0.77488583$

$-0.66905046$

$-0.66905046$

$-0.77488583$

$-0.77488583$

$-0.66905046$

$-0.66905046$

0.88849652

0.88849652

0.99433196

0.99433196

0.88849652

0.88849652

0.99433196

0.99433196

$-1.17169046$

$-1.17169046$

$-1.06585503$

$-1.06585503$

$-1.17169046$

$$
\begin{array}{lr}
0.55529481 & -1.77761996 \\
0.44945931 & -1.70853937 \\
0.55529481 & 1.88232481
\end{array}
$$
1.000 \# Electr. mom., scale-fac \# Magnetic mom.

$\begin{array}{rr}-0.26382661 & -0.31313565 \\ -0.15799116 & 0.17358164\end{array}$

$-0.26382661 \quad 0.13124879$

$-0.15799116 \quad 0.01110530$

$-0.26382661 \quad 0.13531575$

$-0.15799116 \quad 0.03984968$

$\begin{array}{ll}-0.26382661 & 0.09340374\end{array}$

$-0.15799116$

$-0.03186282$

0.07397263

$-0.03186282$

0.07397263

$-0.03186282$

0.07397263

$-0.03186282$

0.07397263

$-1.38695288$

$-1.28111744$

$-1.38695288$

$-1.28111744$

$-1.38695288$

$-1.28111744$

$-1.38695288$

$-1.28111744$

$-0.43766770$

$-0.33183220$

$-0.43766770$

$-0.33183220$

$-0.43766770$

$-0.33183220$

$-0.43766770$

$-0.33183220$

0.55604303

0.66187847

0.55604303

0.66187847

0.55604303

0.66187847

0.55604303

0.66187847

0.35565418

0.46148965

0.35565418

0.46148965

0.35565418

0.46148965

0.35565418

0.46148965

1. 47651505

1. 58235061

1. 47651505

1. 58235061

1.47651505

1. 58235061

1.47651505

1. 58235061

0.44945931

0.55529481

0.44945931

0.55529481

0.44945931

$-0.28174475$

4.31991529

$-5.79140759$

$-4.03236294$

5.20330095

$-4.21703386$

5.52320671

3.96910620

$-4.97957945$

$-0.55049026$

0.68074656

0.52190757

$-0.64773327$

0.58989978

$-0.73166251$

$-0.56110018$

0.69699770

$-0.51271904$

0.71012944

0.75772589

$-0.97550160$

$-0.00612711$

$-0.13832425$

$-0.23664717$

0.39608532

$-0.17636208$

0.35996664

0.18146306

$-0.33773482$

$-0.07444621$

$-0.08399364$

0.07812838

0.08030491

$-3.91351295$

2. 26561999

4.59877634

$-2.66896439$

4.16112661

$-2.60116148$

$-4.84582186$

3. 02058911

0.06994801

$-0.03016898$

$-0.00834977$

$-0.00570277$

$-0.18111902$

0.10540757

0.07930677

$-0.03998973$

$-0.53781670$

0.36583957

0.69896328

$-0.48631677$

0.82799989 
64

0.0127

$-0.1167$

$-1.85509956$

$-1.85509956$

$-1.85509956$

$-1.85509956$

$-1.74926412$

$-1.74926412$

$-1.74926412$

$-1.74926412$

1.30779219

1.30779219

1.30779219

1.30779219

1.41362774

1. 41362774

1. 41362774

1. 41362774

$-1.45213747$

$-1.45213747$

$-1.45213747$

$-1.45213747$

$-1.34630203$

$-1.34630203$

$-1.34630203$

$-1.34630203$

2.33856606

2.33856606

2.33856606

2.33856606

2.44440126

2.44440126

2. 44440126

2. 44440126

$-1.15465784$

$-1.15465784$

$-1.15465784$

$-1.15465784$

$-1.04882240$

$-1.04882240$

$-1.04882240$

$-1.04882240$

1.23173511

1.23173511

1.23173511

1.23173511

1.33757067

1.33757067

1.33757067

1.33757067

$-1.51449943$

$-1.51449943$

$-1.51449943$

$-1.51449943$

$-1.40866399$

$-1.40866399$

$-1.40866399$

$-1.40866399$

0.31038415

0.31038415

0.31038415

0.31038415

0.41621959
$-1.17169046$

$-1.06585503$

$-1.06585503$

. \# Energy 3->6

$0.0330 \quad-0.0109$

$0.0599 \quad 0.1637$

$-0.14332448$

$-0.14332448$

$-0.03748903$

$-0.03748903$

$-0.14332448$

$-0.14332448$

$-0.03748903$

$-0.03748903$

0.51848310

0.51848310

0.62431854

0.62431854

0.51848310

0.51848310

0.62431854

0.62431854

$-0.42367253$

$-0.42367253$

$-0.31783706$

$-0.31783706$

$-0.42367253$

$-0.42367253$

$-0.31783706$

$-0.31783706$

1. 04233944

1.04233944

1. 14817488

1. 14817488

1.04233944

1.04233944

1. 14817488

1. 14817488

0.69317716

0.69317716

0.79901266

0.79901266

0.69317716

0.69317716

0.79901266

0.79901266

$-0.77488583$

$-0.77488583$

$-0.66905046$

$-0.66905046$

$-0.77488583$

$-0.77488583$

$-0.66905046$

$-0.66905046$

0.88849652

0.88849652

0.99433196

0.99433196

0.88849652

0.88849652

0.99433196

0.99433196

$-1.17169046$

$-1.17169046$

$-1.06585503$

$-1.06585503$

$-1.17169046$

$$
\begin{array}{lr}
0.55529481 & -0.56904590 \\
0.44945931 & -1.03628743 \\
0.55529481 & 0.72539788
\end{array}
$$
1.000 \# Electr. mom., scale-fac \# Magnetic mom.

$\begin{array}{ll}-0.26382661 & 3.62730670\end{array}$

$-0.15799116 \quad-3.51076865$

$-0.26382661 \quad-2.82980180$

$\begin{array}{ll}-0.15799116 & 2.74424338\end{array}$

$-0.26382661 \quad-2.73991084$

$\begin{array}{ll}-0.15799116 & 2.50347900\end{array}$

$\begin{array}{ll}-0.26382661 & 1.78722489\end{array}$

$-0.15799116$

$-0.03186282$

0.07397263

$-0.03186282$

0.07397263

$-0.03186282$

0.07397263

$-0.03186282$

0.07397263

$-1.38695288$

$-1.28111744$

$-1.38695288$

$-1.28111744$

$-1.38695288$

$-1.28111744$

$-1.38695288$

$-1.28111744$

$-0.43766770$

$-0.33183220$

$-0.43766770$

$-0.33183220$

$-0.43766770$

$-0.33183220$

$-0.43766770$

$-0.33183220$

0.55604303

0.66187847

0.55604303

0.66187847

0.55604303

0.66187847

0.55604303

0.66187847

0.35565418

0.46148965

0.35565418

0.46148965

0.35565418

0.46148965

0.35565418

0.46148965

1.47651505

1.58235061

1. 47651505

1.58235061

1.47651505

1.58235061

1.47651505

1.58235061

0.44945931

0.55529481

0.44945931

0.55529481

0.44945931

$-1.53600419$

0.87419009

$-1.31601620$

$-0.84570569$

1.29066968

$-1.49485636$

2.00062323

1. 42533875

$-1.92257369$

0.76423466

$-1.05954325$

$-0.39154059$

0.63785201

$-0.61779028$

0.89151132

0.25938922

$-0.48323363$

$-0.37445238$

0.48686439

0.58819282

$-0.69975567$

$-0.06514302$

$-0.00644610$

$-0.16112255$

0.23229772

$-2.36858058$

2. 05640054

2. 18138981

$-2.05519152$

1. 40590310

$-1.28365064$

$-1.48255122$

1. 49188685

$-0.64867330$

0.15408029

1.29190385

$-0.68215382$

0.70550537

$-0.17449485$

$-1.27146018$

0.62401569

$-0.40874785$

0.33821172

0.50268823

$-0.40311190$

0.34408936

$-0.29563609$

$-0.44271240$

0.36443585

$-0.59401220$

0.47524464

0.92306155

$-0.75524437$

0.64992487 
64

0.1186

$-0.0165$

$-1.85509956$

$-1.85509956$

$-1.85509956$

$-1.85509956$

$-1.74926412$

$-1.74926412$

$-1.74926412$

$-1.74926412$

1.30779219

1.30779219

1.30779219

1.30779219

1.41362774

1. 41362774

1. 41362774

1. 41362774

$-1.45213747$

$-1.45213747$

$-1.45213747$

$-1.45213747$

$-1.34630203$

$-1.34630203$

$-1.34630203$

$-1.34630203$

2.33856606

2.33856606

2.33856606

2.33856606

2.44440126

2.44440126

2. 44440126

2. 44440126

$-1.15465784$

$-1.15465784$

$-1.15465784$

$-1.15465784$

$-1.04882240$

$-1.04882240$

$-1.04882240$

$-1.04882240$

1.23173511

1.23173511

1.23173511

1.23173511

1.33757067

1.33757067

1.33757067

1.33757067

$-1.51449943$

$-1.51449943$

$-1.51449943$

$-1.51449943$

$-1.40866399$

$-1.40866399$

$-1.40866399$

$-1.40866399$

0.31038415

0.31038415

0.31038415

0.31038415

0.41621959
$-1.17169046$

$-1.06585503$

$-1.06585503$

. \# Energy 3->7

$0.0154 \quad-0.0167$

$0.0151 \quad 0.0620$

$-0.14332448$

$-0.14332448$

$-0.03748903$

$-0.03748903$

$-0.14332448$

$-0.14332448$

$-0.03748903$

$-0.03748903$

0.51848310

0.51848310

0.62431854

0.62431854

0.51848310

0.51848310

0.62431854

0.62431854

$-0.42367253$

$-0.42367253$

$-0.31783706$

$-0.31783706$

$-0.42367253$

$-0.42367253$

$-0.31783706$

$-0.31783706$

1. 04233944

1.04233944

1. 14817488

1. 14817488

1.04233944

1.04233944

1. 14817488

1. 14817488

0.69317716

0.69317716

0.79901266

0.79901266

0.69317716

0.69317716

0.79901266

0.79901266

$-0.77488583$

$-0.77488583$

$-0.66905046$

$-0.66905046$

$-0.77488583$

$-0.77488583$

$-0.66905046$

$-0.66905046$

0.88849652

0.88849652

0.99433196

0.99433196

0.88849652

0.88849652

0.99433196

0.99433196

$-1.17169046$

$-1.17169046$

$-1.06585503$

$-1.06585503$

$-1.17169046$

$$
\begin{array}{lr}
0.55529481 & -0.52935129 \\
0.44945931 & -1.01618290 \\
0.55529481 & 0.84426028
\end{array}
$$

1.000 \# Electr. mom., scale-fac
\# Magnetic mom.

$-0.26382661$

1.91712546

$-0.15799116$

$-0.26382661$

$-0.15799116$

$-0.26382661$

$-0.15799116$

$-0.26382661$

$-0.15799116$

$-0.03186282$

0.07397263

$-0.03186282$

0.07397263

$-0.03186282$

0.07397263

$-0.03186282$

0.07397263

$-1.38695288$

$-1.28111744$

$-1.38695288$

$-1.28111744$

$-1.38695288$

$-1.28111744$

$-1.38695288$

$-1.28111744$

$-0.43766770$

$-0.33183220$

$-0.43766770$

$-0.33183220$

$-0.43766770$

$-0.33183220$

$-0.43766770$

$-0.33183220$

0.55604303

0.66187847

0.55604303

0.66187847

0.55604303

0.66187847

0.55604303

0.66187847

0.35565418

0.46148965

0.35565418

0.46148965

0.35565418

0.46148965

0.35565418

0.46148965

1. 47651505

1.58235061

1.47651505

1.58235061

1.47651505

1.58235061

1.47651505

1.58235061

0.44945931

0.55529481

0.44945931

0.55529481

0.44945931
$-1.89539695$

1. 78946018

$-1.86874473$

1. 81177557

1.69461215

$-1.66115487$

0.12487783

0.02360879

$-0.16861777$

0.04448909

$-0.19926578$

0.09708791

0.24700138

$-0.16278206$

0.20714353

$-0.27383867$

$-0.06998489$

0.11391604

$-0.17526558$

0.24557617

0.05125713

$-0.10126110$

0.23774345

$-0.41822284$

$-0.27893460$

0.45943257

$-0.16591142$

0.31830609

0.20038138

$-0.35016057$

$-2.28189707$

1. 83188808

1.90425110

$-1.56787550$

1.87894416

$-1.49222529$

$-1.61226213$

1.31479371

0.56398928

$-0.89183575$

0.53453219

$-0.63070607$

0.37834188

0.93215054

$-0.60415798$

0.07215768

$-0.05117575$

$-0.02810684$

0.01609756

$-0.09319504$

0.06371684

0.05615411

$-0.03416419$

0.06966897

$-0.01205870$

$-0.14746808$

0.06446860

$-0.18204860$
$-1.77014244$

$-0.29261088$ 


$\begin{array}{lllr}0.41621959 & -1.17169046 & 0.55529481 & 0.08779280 \\ 0.41621959 & -1.06585503 & 0.44945931 & 0.30268770 \\ 0.41621959 & -1.06585503 & 0.55529481 & -0.17395802\end{array}$

$\begin{array}{ccc}1.4915 & 0.0 .14 \text { Energy } 3->8 \\ 0.0822 & 0.1482 & -0.0737\end{array}$

$\begin{array}{lll}0.0822 & -0.1125 & 0.1389\end{array}$

$-1.85509956$

$-1.85509956$

$-1.85509956$

$-1.85509956$

$-1.74926412$

$-1.74926412$

$-1.74926412$

$-1.74926412$

1. 30779219

1.30779219

1.30779219

1.30779219

1. 41362774

1. 41362774

1. 41362774

1. 41362774

$-1.45213747$

$-1.45213747$

$-1.45213747$

$-1.45213747$

$-1.34630203$

$-1.34630203$

$-1.34630203$

$-1.34630203$

2. 33856606

2.33856606

2. 33856606

2.33856606

2. 44440126

2. 44440126

2. 44440126

2. 44440126

$-1.15465784$

$-1.15465784$

$-1.15465784$

$-1.15465784$

$-1.04882240$

$-1.04882240$

$-1.04882240$

$-1.04882240$

1. 23173511

1. 23173511

1. 23173511

1. 23173511

1. 33757067

1.33757067

1.33757067

1. 33757067

$-1.51449943$

$-1.51449943$

$-1.51449943$

$-1.51449943$

$-1.40866399$

$-1.40866399$

$-1.40866399$

$-1.40866399$

0.31038415

0.31038415

0.31038415

0.31038415

0.41621959
$-0.14332448$

$-0.14332448$

$-0.03748903$

$-0.03748903$

$-0.14332448$

$-0.14332448$

$-0.03748903$

$-0.03748903$

0.51848310

0.51848310

0.62431854

0.62431854

0.51848310

0.51848310

0.62431854

0.62431854

$-0.42367253$

$-0.42367253$

$-0.31783706$

$-0.31783706$

$-0.42367253$

$-0.42367253$

$-0.31783706$

$-0.31783706$

1.04233944

1.04233944

1.14817488

1. 14817488

1.04233944

1.04233944

1.14817488

1. 14817488

0.69317716

0.69317716

0.79901266

0.79901266

0.69317716

0.69317716

0.79901266

0.79901266

$-0.77488583$

$-0.77488583$

$-0.66905046$

$-0.66905046$

$-0.77488583$

$-0.77488583$

$-0.66905046$

$-0.66905046$

0.88849652

0.88849652

0.99433196

0.99433196

0.88849652

0.88849652

0.99433196

0.99433196

$-1.17169046$

$-1.17169046$

$-1.06585503$

$-1.06585503$

$-1.17169046$

$$
\begin{gathered}
1.000 \text { \# Electr. mom., scale-fac } \\
\text { \# Magnetic mom. }
\end{gathered}
$$

$\begin{array}{ll}-0.26382661 & 7.15199566\end{array}$

$-0.15799116 \quad-6.79833937$

$-0.26382661 \quad-7.91680956$

$-0.15799116 \quad 7.73245811$

$-0.26382661$

$-0.15799116$

$-0.26382661$

$-0.15799116$

$-0.03186282$

0.07397263

$-0.03186282$

0.07397263

$-0.03186282$

0.07397263

$-0.03186282$

0.07397263

$-1.38695288$

$-1.28111744$

$-1.38695288$

$-1.28111744$

$-1.38695288$

$-1.28111744$

$-1.38695288$

$-1.28111744$

$-0.43766770$

$-0.33183220$

$-0.43766770$

$-0.33183220$

$-0.43766770$

$-0.33183220$

$-0.43766770$

$-0.33183220$

0.55604303

0.66187847

0.55604303

0.66187847

0.55604303

0.66187847

0.55604303

0.66187847

0.35565418

0.46148965

0.35565418

0.46148965

0.35565418

0.46148965

0.35565418

0.46148965

1. 47651505

1. 58235061

1. 47651505

1. 58235061

1.47651505

1. 58235061

1.47651505

1. 58235061

0.44945931

0.55529481

0.44945931

0.55529481

0.44945931
$-9.38514614$

8.89636612

10.24640369

$-9.88129616$

5.12854433

$-3.69678545$

$-5.47316504$

3.91675782

$-5.16395950$

4.15658998

5.62210751

$-4.40259027$

$-0.21970460$

0.47168565

0.33550021

$-0.64500087$

0.09545734

$-0.25217101$

$-0.13371699$

0.32081184

3.23867297

$-5.49428654$

$-3.48861194$

5.67457438

$-2.41877341$

4.29309034

2. 62800407

$-4.41453218$

$-15.29073429$

12.02325535

12.48435593

$-9.76601124$

13.62336540

$-10.53509903$

$-11.39120102$

8.75627613

8.16310120

$-5.12727833$

$-12.21538925$

8.30844784

$-8.67177677$

5.80473661

12.35454750

$-8.72094917$

2.26228833

$-1.74398923$

$-1.96265066$

1. 49993849

$-2.46056724$

1.87302995

2.17726421

$-1.64661586$

1. 78022087

$-0.98977542$

$-3.06465292$

1. 95003200

$-2.76036572$ 


\begin{abstract}
0.41621959
0.41621959

0.41621959
\end{abstract}

\subsection{4}

0.0374

$-1.85509956$

$-1.85509956$

$-1.85509956$

$-1.85509956$

$-1.74926412$

$-1.74926412$

$-1.74926412$

$-1.74926412$

1.30779219

1.30779219

1.30779219

1.30779219

1.41362774

1.41362774

1.41362774

1.41362774

$-1.45213747$

$-1.45213747$

$-1.45213747$

$-1.45213747$

$-1.34630203$

$-1.34630203$

$-1.34630203$

$-1.34630203$

2. 33856606

2.33856606

2.33856606

2.33856606

2.44440126

2.44440126

2.44440126

2. 44440126

$-1.15465784$

$-1.15465784$

$-1.15465784$

$-1.15465784$

$-1.04882240$

$-1.04882240$

$-1.04882240$

$-1.04882240$

1.23173511

1.23173511

1.23173511

1.23173511

1.33757067

1.33757067

1.33757067

1.33757067

$-1.51449943$

$-1.51449943$

$-1.51449943$

$-1.51449943$

$-1.40866399$

$-1.40866399$

$-1.40866399$

$-1.40866399$

0.31038415

0.31038415

0.31038415

0.31038415

0.41621959
$-1.17169046$

$-1.06585503$

$-1.06585503$

0. \# Energy 3->9

$\begin{array}{ll}-0.0413 & 0.0383 \\ -0.0408 & 0.0290\end{array}$

$-0.14332448$

$-0.14332448$

$-0.03748903$

$-0.03748903$

$-0.14332448$

$-0.14332448$

$-0.03748903$

$-0.03748903$

0.51848310

0.51848310

0.62431854

0.62431854

0.51848310

0.51848310

0.62431854

0.62431854

$-0.42367253$

$-0.42367253$

$-0.31783706$

$-0.31783706$

$-0.42367253$

$-0.42367253$

$-0.31783706$

$-0.31783706$

1. 04233944

1.04233944

1.14817488

1.14817488

1.04233944

1.04233944

1.14817488

1.14817488

0.69317716

0.69317716

0.79901266

0.79901266

0.69317716

0.69317716

0.79901266

0.79901266

$-0.77488583$

$-0.77488583$

$-0.66905046$

$-0.66905046$

$-0.77488583$

$-0.77488583$

$-0.66905046$

$-0.66905046$

0.88849652

0.88849652

0.99433196

0.99433196

0.88849652

0.88849652

0.99433196

0.99433196

$-1.17169046$

$-1.17169046$

$-1.06585503$

$-1.06585503$

$-1.17169046$

$$
\begin{array}{lr}
0.55529481 & 1.66389668 \\
0.44945931 & 4.43478298 \\
0.55529481 & -2.93661499
\end{array}
$$
1.000 \# Electr. mom., scale-fac \# Magnetic mom.

$-0.26382661 \quad 1.04095614$

$-0.15799116 \quad-1.21709073$

$-0.26382661 \quad-1.16970634$

$-0.15799116 \quad 1.37587273$

$-0.26382661 \quad-1.39089155$

$-0.15799116 \quad 1.54647815$

$-0.26382661 \quad 1.47127926$

$-0.15799116$

$-0.03186282$

0.07397263

$-0.03186282$

0.07397263

$-0.03186282$

0.07397263

$-0.03186282$

0.07397263

$-1.38695288$

$-1.28111744$

$-1.38695288$

$-1.28111744$

$-1.38695288$

$-1.28111744$

$-1.38695288$

$-1.28111744$

$-0.43766770$

$-0.33183220$

$-0.43766770$

$-0.33183220$

$-0.43766770$

$-0.33183220$

$-0.43766770$

$-0.33183220$

0.55604303

0.66187847

0.55604303

0.66187847

0.55604303

0.66187847

0.55604303

0.66187847

0.35565418

0.46148965

0.35565418

0.46148965

0.35565418

0.46148965

0.35565418

0.46148965

1. 47651505

1.58235061

1.47651505

1.58235061

1.47651505

1.58235061

1.47651505

1.58235061

0.44945931

0.55529481

0.44945931

0.55529481

0.44945931

$-1.65806401$

7.40071869

$-9.89626408$

$-6.95354700$

8.98492813

$-6.73811769$

9.00581932

6.39809608

$-8.21782017$

$-0.70207995$

0.85935122

0.69889754

$-0.84169489$

0.79968059

$-0.98124701$

$-0.79561877$

0.96434605

0.20247594

$-0.55317301$

$-0.23568334$

0.56663316

$-0.31252131$

0.61376023

0.32705036

$-0.60744810$

$-3.30491877$

2. 54763460

2.45775723

$-1.84661925$

2. 87451148

$-2.14494181$

$-2.06125593$

1.47373319

$-3.87640524$

1.88452685

3.93514919

$-1.69303632$

3.89260817

$-2.12411761$

$-4.00531197$

2.00873804

0.31790444

$-0.22823323$

$-0.23546576$

0.16943449

$-0.36745384$

0.25457162

0.27638605

$-0.18985555$

$-0.38288587$

0.22613375

0.35589984

$-0.20776623$

0.61395097 


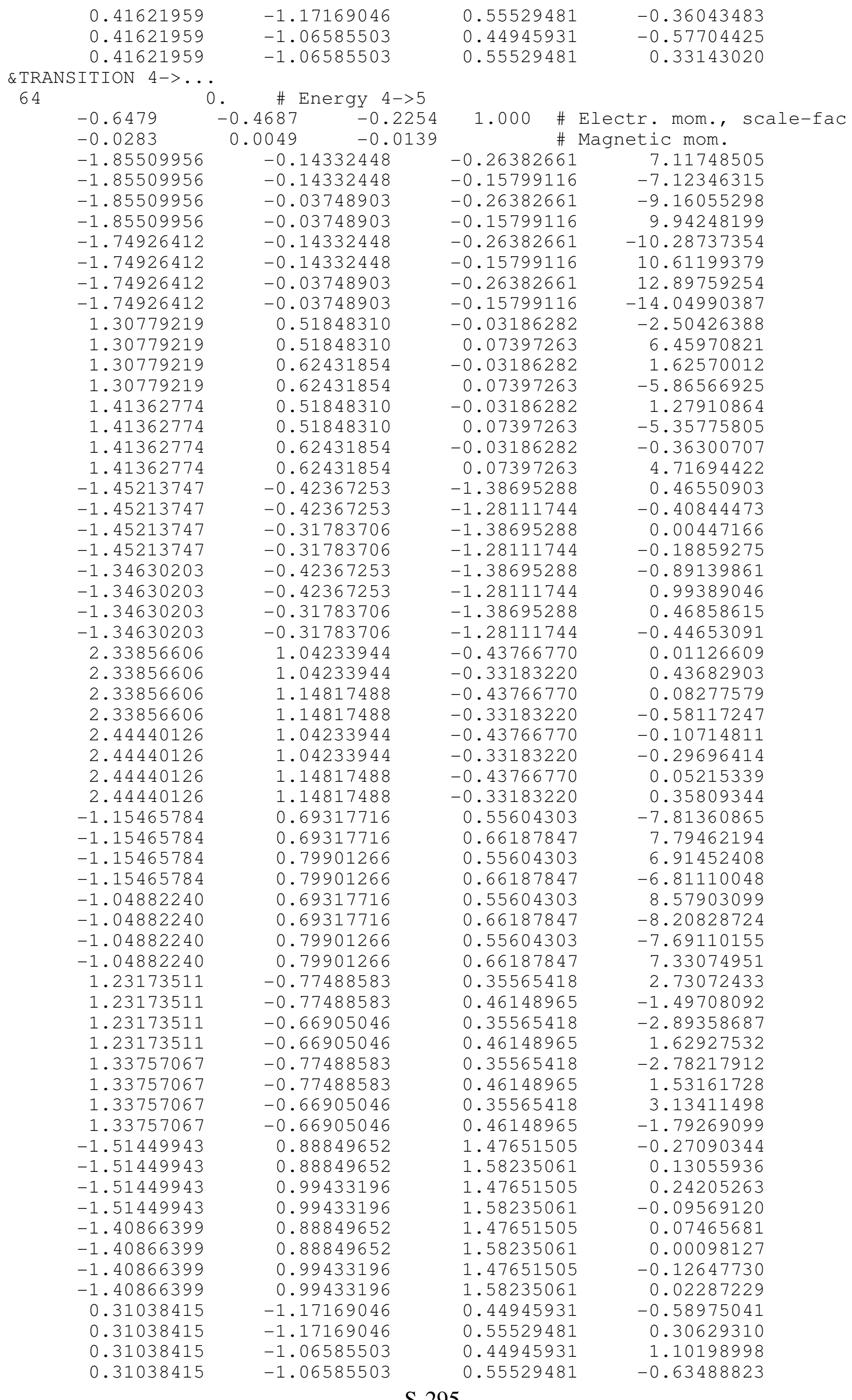


64

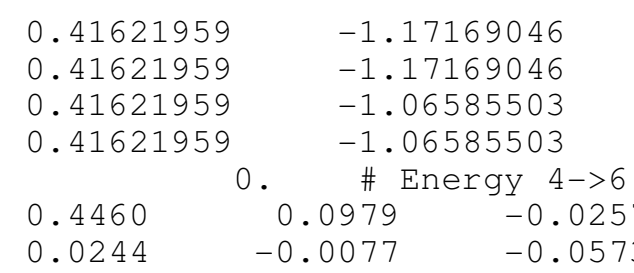

$-1.85509956$

$-1.85509956$

$-1.85509956$

$-1.85509956$

$-1.74926412$

$-1.74926412$

$-1.74926412$

$-1.74926412$

1.30779219

1.30779219

1.30779219

1. 30779219

1. 41362774

1. 41362774

1. 41362774

1. 41362774

$-1.45213747$

$-1.45213747$

$-1.45213747$

$-1.45213747$

$-1.34630203$

$-1.34630203$

$-1.34630203$

$-1.34630203$

2. 33856606

2.33856606

2.33856606

2. 33856606

2. 44440126

2. 44440126

2. 44440126

2. 44440126

$-1.15465784$

$-1.15465784$

$-1.15465784$

$-1.15465784$

$-1.04882240$

$-1.04882240$

$-1.04882240$

$-1.04882240$

1. 23173511

1. 23173511

1. 23173511

1. 23173511

1.33757067

1.33757067

1.33757067

1.33757067

$-1.51449943$

$-1.51449943$

$-1.51449943$

$-1.51449943$

$-1.40866399$

$-1.40866399$

$-1.40866399$

$-1.40866399$

0.31038415

0.31038415

0.31038415

0.31038415

$\begin{array}{rr}0.0979 & -0.0257 \\ -0.0077 & -0.0573\end{array}$

$-0.14332448$

$-0.14332448$

$-0.03748903$

$-0.03748903$

$-0.14332448$

$-0.14332448$

$-0.03748903$

$-0.03748903$

0.51848310

0.51848310

0.62431854

0.62431854

0.51848310

0.51848310

0.62431854

0.62431854

$-0.42367253$

$-0.42367253$

$-0.31783706$

$-0.31783706$

$-0.42367253$

$-0.42367253$

$-0.31783706$

$-0.31783706$

1. 04233944

1.04233944

1. 14817488

1. 14817488

1.04233944

1. 04233944

1. 14817488

1. 14817488

0.69317716

0.69317716

0.79901266

0.79901266

0.69317716

0.69317716

0.79901266

0.79901266

$-0.77488583$

$-0.77488583$

$-0.66905046$

$-0.66905046$

$-0.77488583$

$-0.77488583$

$-0.66905046$

$-0.66905046$

0.88849652

0.88849652

0.99433196

0.99433196

0.88849652

0.88849652

0.99433196

0.99433196

$-1.17169046$

$-1.17169046$

$-1.06585503$

$-1.06585503$

$$
\begin{array}{lr}
0.44945931 & 0.58808351 \\
0.55529481 & -0.28508398 \\
0.44945931 & -1.16143692 \\
0.55529481 & 0.64937454
\end{array}
$$
1.000 \# Electr. mom., scale-fac \# Magnetic mom.

$\begin{array}{ll}-0.26382661 & 1.66162443\end{array}$

$-0.15799116 \quad-1.99762905$

$-0.26382661 \quad-2.31684947$

$-0.15799116$

$-0.26382661$

$-0.15799116$

$-0.26382661$

$-0.15799116$

$-0.03186282$

0.07397263

$-0.03186282$

0.07397263

$-0.03186282$

0.07397263

$-0.03186282$

0.07397263

$-1.38695288$

$-1.28111744$

$-1.38695288$

$-1.28111744$

$-1.38695288$

$-1.28111744$

$-1.38695288$

$-1.28111744$

$-0.43766770$

$-0.33183220$

$-0.43766770$

$-0.33183220$

$-0.43766770$

$-0.33183220$

$-0.43766770$

$-0.33183220$

0.55604303

0.66187847

0.55604303

0.66187847

0.55604303

0.66187847

0.55604303

0.66187847

0.35565418

0.46148965

0.35565418

0.46148965

0.35565418

0.46148965

0.35565418

0.46148965

1. 47651505

1. 58235061

1. 47651505

1.58235061

1. 47651505

1.58235061

1. 47651505

1. 58235061

0.44945931

0.55529481

0.44945931

0.55529481

2.72697520

$-2.44847322$

2.86905503

3. 28385854

$-3.81671858$

0.03570602

0.19952813

0.29944208

$-0.69691640$

0.27147540

$-0.57059175$

$-0.54498690$

1. 04525042

$-0.15139741$

0.19264433

0.15198660

$-0.21331806$

0.05522571

$-0.06627242$

$-0.03337895$

0.05506469

0.57003772

$-0.63398337$

$-0.46733239$

0.54514366

$-0.45557269$

0.52335137

0.36619291

$-0.44393319$

0.19986123

0.40293503

$-0.00810796$

$-0.45783192$

0.17653146

$-0.61471272$

$-0.36655340$

0.70004547

0.88670743

$-0.66052014$

$-1.11960876$

0.80383748

$-0.77552605$

0.62298584

0.89669281

$-0.69382399$

$-0.06337773$

0.06870700

0.08002851

$-0.06901862$

$-0.04685040$

0.00782661

$-0.01274059$

0.02362111

0.27168000

$-0.06124275$

$-0.50113207$

0.19134149 
64

0.41621959
0.41621959
0.41621959
0.41621959

$-0.0401$

$-0.0827$

$-1.85509956$

$-1.85509956$

$-1.85509956$

$-1.85509956$

$-1.74926412$

$-1.74926412$

$-1.74926412$

$-1.74926412$

1.30779219

1.30779219

1.30779219

1. 30779219

1. 41362774

1. 41362774

1. 41362774

1. 41362774

$-1.45213747$

$-1.45213747$

$-1.45213747$

$-1.45213747$

$-1.34630203$

$-1.34630203$

$-1.34630203$

$-1.34630203$

2. 33856606

2.33856606

2.33856606

2. 33856606

2. 44440126

2. 44440126

2. 44440126

2. 44440126

$-1.15465784$

$-1.15465784$

$-1.15465784$

$-1.15465784$

$-1.04882240$

$-1.04882240$

$-1.04882240$

$-1.04882240$

1. 23173511

1. 23173511

1. 23173511

1. 23173511

1.33757067

1.33757067

1. 33757067

1.33757067

$-1.51449943$

$-1.51449943$

$-1.51449943$

$-1.51449943$

$-1.40866399$

$-1.40866399$

$-1.40866399$

$-1.40866399$

0.31038415

0.31038415

0.31038415

0.31038415
$-1.17169046$

$-1.17169046$

$-1.06585503$

$-1.06585503$

. \# Energy 4->7

$$
\begin{array}{lr}
0.0943 & -0.0402 \\
0.0075 & 0.0050
\end{array}
$$

$-0.14332448$

$-0.14332448$

$-0.03748903$

$-0.03748903$

$-0.14332448$

$-0.14332448$

$-0.03748903$

$-0.03748903$

0.51848310

0.51848310

0.62431854

0.62431854

0.51848310

0.51848310

0.62431854

0.62431854

$-0.42367253$

$-0.42367253$

$-0.31783706$

$-0.31783706$

$-0.42367253$

$-0.42367253$

$-0.31783706$

$-0.31783706$

1. 04233944

1.04233944

1. 14817488

1. 14817488

1.04233944

1. 04233944

1. 14817488

1. 14817488

0.69317716

0.69317716

0.79901266

0.79901266

0.69317716

0.69317716

0.79901266

0.79901266

$-0.77488583$

$-0.77488583$

$-0.66905046$

$-0.66905046$

$-0.77488583$

$-0.77488583$

$-0.66905046$

$-0.66905046$

0.88849652

0.88849652

0.99433196

0.99433196

0.88849652

0.88849652

0.99433196

0.99433196

$-1.17169046$

$-1.17169046$

$-1.06585503$

$-1.06585503$
0.44945931
0.55529481
0.44945931
-0.43371591
0.15229145
0.73850507
-0.33404338
0.55529481
1.000 \# Electr. mom., scale-fac \# Magnetic mom.

$-0.26382661-1.63803697$

$-0.15799116 \quad 2.05131960$

$\begin{array}{ll}-0.26382661 & 2.30958867\end{array}$

$-0.15799116$

$-0.26382661$

$-0.15799116$

$-0.26382661$

$-0.15799116$

$-0.03186282$

0.07397263

$-0.03186282$

0.07397263

$-0.03186282$

0.07397263

$-0.03186282$

0.07397263

$-1.38695288$

$-1.28111744$

$-1.38695288$

$-1.28111744$

$-1.38695288$

$-1.28111744$

$-1.38695288$

$-1.28111744$

$-0.43766770$

$-0.33183220$

$-0.43766770$

$-0.33183220$

$-0.43766770$

$-0.33183220$

$-0.43766770$

$-0.33183220$

0.55604303

0.66187847

0.55604303

0.66187847

0.55604303

0.66187847

0.55604303

0.66187847

0.35565418

0.46148965

0.35565418

0.46148965

0.35565418

0.46148965

0.35565418

0.46148965

1. 47651505

1. 58235061

1. 47651505

1.58235061

1. 47651505

1.58235061

1. 47651505

1. 58235061

0.44945931

0.55529481

0.44945931

0.55529481

$-2.92364025$

2. 49897718

$-3.03449130$

$-3.32156014$

4.08341360

$-5.39084864$

5.85145569

5.00057030

$-5.21986866$

4.37454128

$-4.54739571$

$-4.06764841$

4.04037666

0.86377794

$-1.09986055$

$-0.88232183$

1.11764729

$-0.90965295$

1. 14664626

0.91340554

$-1.14701092$

0.17404647

$-0.45990789$

0.23208800

$-0.00560429$

$-0.50065935$

0.74823040

0.11007036

$-0.30420607$

3. 92705059

$-3.45249200$

$-3.07412243$

2. 67189121

$-3.67172718$

3.10109830

2. 74064732

$-2.28234768$

1. 75367153

$-0.81450832$

$-1.51750863$

0.44732925

$-1.71651876$

0.99540907

1. 51068413

$-0.70128685$

$-0.35454455$

0.26870036

0.28153908

$-0.21699472$

0.45677367

$-0.33102283$

$-0.35881904$

0.26174486

0.54138124

$-0.25771016$

$-0.67654854$

0.33447555 
64

$$
\begin{aligned}
& 0.41621959 \\
& 0.41621959 \\
& 0.41621959 \\
& 0.41621959
\end{aligned}
$$

$-0.6301$

0.0916

$-1.85509956$

$-1.85509956$

$-1.85509956$

$-1.85509956$

$-1.74926412$

$-1.74926412$

$-1.74926412$

$-1.74926412$

1.30779219

1.30779219

1.30779219

1. 30779219

1. 41362774

1. 41362774

1. 41362774

1. 41362774

$-1.45213747$

$-1.45213747$

$-1.45213747$

$-1.45213747$

$-1.34630203$

$-1.34630203$

$-1.34630203$

$-1.34630203$

2. 33856606

2. 33856606

2.33856606

2. 33856606

2. 44440126

2. 44440126

2. 44440126

2. 44440126

$-1.15465784$

$-1.15465784$

$-1.15465784$

$-1.15465784$

$-1.04882240$

$-1.04882240$

$-1.04882240$

$-1.04882240$

1. 23173511

1. 23173511

1. 23173511

1. 23173511

1.33757067

1.33757067

1. 33757067

1.33757067

$-1.51449943$

$-1.51449943$

$-1.51449943$

$-1.51449943$

$-1.40866399$

$-1.40866399$

$-1.40866399$

$-1.40866399$

0.31038415

0.31038415

0.31038415

0.31038415
$-1.17169046$

$-1.17169046$

$-1.06585503$

$-1.06585503$

. \# Energy 4->8

$$
\begin{array}{lr}
0.1210 & -0.1248 \\
0.0181 & 0.0192
\end{array}
$$

$-0.14332448$

$-0.14332448$

$-0.03748903$

$-0.03748903$

$-0.14332448$

$-0.14332448$

$-0.03748903$

$-0.03748903$

0.51848310

0.51848310

0.62431854

0.62431854

0.51848310

0.51848310

0.62431854

0.62431854

$-0.42367253$

$-0.42367253$

$-0.31783706$

$-0.31783706$

$-0.42367253$

$-0.42367253$

$-0.31783706$

$-0.31783706$

1. 04233944

1.04233944

1. 14817488

1. 14817488

1.04233944

1. 04233944

1. 14817488

1. 14817488

0.69317716

0.69317716

0.79901266

0.79901266

0.69317716

0.69317716

0.79901266

0.79901266

$-0.77488583$

$-0.77488583$

$-0.66905046$

$-0.66905046$

$-0.77488583$

$-0.77488583$

$-0.66905046$

$-0.66905046$

0.88849652

0.88849652

0.99433196

0.99433196

0.88849652

0.88849652

0.99433196

0.99433196

$-1.17169046$

$-1.17169046$

$-1.06585503$

$-1.06585503$

$$
\begin{array}{lr}
0.44945931 & -0.73032922 \\
0.55529481 & 0.35178208 \\
0.44945931 & 0.88392293 \\
0.55529481 & -0.43506062
\end{array}
$$
1.000 \# Electr. mom., scale-fac \# Magnetic mom.

$-0.26382661 \quad-2.67601633$

$\begin{array}{ll}-0.15799116 & 2.37439346\end{array}$

$\begin{array}{ll}-0.26382661 & 2.70792127\end{array}$

$-0.15799116$

$-0.26382661$

$-0.15799116$

$-0.26382661$

$-0.15799116$

$-0.03186282$

0.07397263

$-0.03186282$

0.07397263

$-0.03186282$

0.07397263

$-0.03186282$

0.07397263

$-1.38695288$

$-1.28111744$

$-1.38695288$

$-1.28111744$

$-1.38695288$

$-1.28111744$

$-1.38695288$

$-1.28111744$

$-0.43766770$

$-0.33183220$

$-0.43766770$

$-0.33183220$

$-0.43766770$

$-0.33183220$

$-0.43766770$

$-0.33183220$

0.55604303

0.66187847

0.55604303

0.66187847

0.55604303

0.66187847

0.55604303

0.66187847

0.35565418

0.46148965

0.35565418

0.46148965

0.35565418

0.46148965

0.35565418

0.46148965

1. 47651505

1. 58235061

1.47651505

1.58235061

1. 47651505

1.58235061

1. 47651505

1. 58235061

0.44945931

0.55529481

0.44945931

0.55529481

$-2.39897251$

3. 24531198

$-2.86745620$

$-3.26845264$

2. 89404964

0.11620612

$-2.95379543$

$-0.70568961$

3. 29219937

$-1.20786071$

4.13375044

1. 76444304

$-4.45683718$

$-0.10339565$

0.04056367

$-0.01258653$

0.13297969

0.20749360

$-0.19006267$

$-0.10462275$

0.03616957

$-0.62536407$

0.15913595

0.33842477

0.08363948

0.21074593

0.17573728

0.04859292

$-0.39980525$

$-1.82516980$

0.97324824

1. 02066255

$-0.31130433$

2. 20914769

$-1.34078956$

$-1.04913199$

0.32970154

$-3.16313624$

1. 77952349

4. 91404152

$-2.94830346$

2.32921839

$-1.01680219$

$-3.89763093$

2. 04898739

$-0.27442366$

0.17633028

0.13362010

$-0.09598877$

0.56573462

$-0.38760105$

$-0.32575998$

0.23502861

$-1.68475902$

1.06123042

2. 34801316

$-1.47647882$ 
64

$$
\begin{aligned}
& 0.41621959 \\
& 0.41621959 \\
& 0.41621959 \\
& 0.41621959
\end{aligned}
$$

$-0.8565$

0.0160

$-1.85509956$

$-1.85509956$

$-1.85509956$

$-1.85509956$

$-1.74926412$

$-1.74926412$

$-1.74926412$

$-1.74926412$

1.30779219

1.30779219

1.30779219

1. 30779219

1. 41362774

1. 41362774

1. 41362774

1. 41362774

$-1.45213747$

$-1.45213747$

$-1.45213747$

$-1.45213747$

$-1.34630203$

$-1.34630203$

$-1.34630203$

$-1.34630203$

2. 33856606

2.33856606

2.33856606

2. 33856606

2. 44440126

2. 44440126

2. 44440126

2. 44440126

$-1.15465784$

$-1.15465784$

$-1.15465784$

$-1.15465784$

$-1.04882240$

$-1.04882240$

$-1.04882240$

$-1.04882240$

1. 23173511

1. 23173511

1. 23173511

1. 23173511

1.33757067

1. 33757067

1. 33757067

1.33757067

$-1.51449943$

$-1.51449943$

$-1.51449943$

$-1.51449943$

$-1.40866399$

$-1.40866399$

$-1.40866399$

$-1.40866399$

0.31038415

0.31038415

0.31038415

0.31038415
$-1.17169046$

$-1.17169046$

$-1.06585503$

$-1.06585503$

. \# Energy 4->9

$\begin{array}{ll}-0.1849 & -0.0788\end{array}$

$0.0587-0.1688$

$-0.14332448$

$-0.14332448$

$-0.03748903$

$-0.03748903$

$-0.14332448$

$-0.14332448$

$-0.03748903$

$-0.03748903$

0.51848310

0.51848310

0.62431854

0.62431854

0.51848310

0.51848310

0.62431854

0.62431854

$-0.42367253$

$-0.42367253$

$-0.31783706$

$-0.31783706$

$-0.42367253$

$-0.42367253$

$-0.31783706$

$-0.31783706$

1. 04233944

1. 04233944

1. 14817488

1. 14817488

1.04233944

1. 04233944

1. 14817488

1. 14817488

0.69317716

0.69317716

0.79901266

0.79901266

0.69317716

0.69317716

0.79901266

0.79901266

$-0.77488583$

$-0.77488583$

$-0.66905046$

$-0.66905046$

$-0.77488583$

$-0.77488583$

$-0.66905046$

$-0.66905046$

0.88849652

0.88849652

0.99433196

0.99433196

0.88849652

0.88849652

0.99433196

0.99433196

$-1.17169046$

$-1.17169046$

$-1.06585503$

$-1.06585503$
0.44945931
0.55529481
0.44945931
0.55529481
2. 21942878
$-1.35645771$
$-3.09686494$
1. 91584444
1.000 \# Electr. mom., scale-fac \# Magnetic mom.

$\begin{array}{ll}-0.26382661 & 1.98550689\end{array}$

$-0.15799116 \quad-2.63773966$

$-0.26382661 \quad-3.79647207$

$-0.15799116$

$-0.26382661$

$-0.15799116$

$-0.26382661$

$-0.15799116$

$-0.03186282$

0.07397263

$-0.03186282$

0.07397263

$-0.03186282$

0.07397263

$-0.03186282$

0.07397263

$-1.38695288$

$-1.28111744$

$-1.38695288$

$-1.28111744$

$-1.38695288$

$-1.28111744$

$-1.38695288$

$-1.28111744$

$-0.43766770$

$-0.33183220$

$-0.43766770$

$-0.33183220$

$-0.43766770$

$-0.33183220$

$-0.43766770$

$-0.33183220$

0.55604303

0.66187847

0.55604303

0.66187847

0.55604303

0.66187847

0.55604303

0.66187847

0.35565418

0.46148965

0.35565418

0.46148965

0.35565418

0.46148965

0.35565418

0.46148965

1. 47651505

1. 58235061

1. 47651505

1. 58235061

1. 47651505

1.58235061

1. 47651505

1. 58235061

0.44945931

0.55529481

0.44945931

0.55529481
4.85579681

$-3.83538389$

4.85790873

6.21933222

$-7.69625235$

$-2.41513038$

6.59310246

1.83510506

$-5.92684507$

1. 55182362

$-5.76870680$

$-1.01755965$

5.13991833

0.35214475

$-0.37841740$

$-0.21246523$

0.18158528

$-0.60162616$

0.70073330

0.49264020

$-0.54402310$

$-1.52100611$

3. 01434374

1. 51057005

$-2.91651535$

1.06143379

$-2.31914425$

$-1.04693127$

2.19745755

$-2.02393627$

3. 17652249

2. 54974794

$-3.39155555$

2. 31534147

$-3.15775037$

$-2.66170001$

3. 31358004

2. 24160743

$-0.91795313$

$-1.84104502$

$-2.01001620$

0.80120564

1. 72113669

$-0.48237693$

$-0.29810342$

0.20362189

0.28404689

$-0.17672189$

0.06014831

$-0.03953106$

$-0.12891355$

0.07391334

$-0.64467663$

0.33753711

1. 24621356

$-0.75174910$
0.50499332 


0.41621959
0.41621959
0.41621959
0.41621959
\&TRANSITION 5->.
64

$-0.0242$

$-1.85509956$

$-1.85509956$

$-1.85509956$

$-1.85509956$

$-1.74926412$

$-1.74926412$

$-1.74926412$

$-1.74926412$

1.30779219

1.30779219

1.30779219

1.30779219

1.41362774

1.41362774

1.41362774

1.41362774

$-1.45213747$

$-1.45213747$

$-1.45213747$

$-1.45213747$

$-1.34630203$

$-1.34630203$

$-1.34630203$

$-1.34630203$

2.33856606

2. 33856606

2.33856606

2.33856606

2.44440126

2. 44440126

2.44440126

2. 44440126

$-1.15465784$

$-1.15465784$

$-1.15465784$

$-1.15465784$

$-1.04882240$

$-1.04882240$

$-1.04882240$

$-1.04882240$

1.23173511

1.23173511

1.23173511

1.23173511

1.33757067

1.33757067

1.33757067

1.33757067

$-1.51449943$

$-1.51449943$

$-1.51449943$

$-1.51449943$

$-1.40866399$

$-1.40866399$

$-1.40866399$

$-1.40866399$

0.31038415

0.31038415

0.31038415
$-1.17169046$

$-1.17169046$

$-1.06585503$

$-1.06585503$

. \# Energy 5->6

$\begin{array}{lr}0.2388 & 0.4765 \\ 0.0701 & -0.0315\end{array}$

$-0.14332448$

$-0.14332448$

$-0.03748903$

$-0.03748903$

$-0.14332448$

$-0.14332448$

$-0.03748903$

$-0.03748903$

0.51848310

0.51848310

0.62431854

0.62431854

0.51848310

0.51848310

0.62431854

0.62431854

$-0.42367253$

$-0.42367253$

$-0.31783706$

$-0.31783706$

$-0.42367253$

$-0.42367253$

$-0.31783706$

$-0.31783706$

1.04233944

1.04233944

1.14817488

1.14817488

1.04233944

1.04233944

1.14817488

1.14817488

0.69317716

0.69317716

0.79901266

0.79901266

0.69317716

0.69317716

0.79901266

0.79901266

$-0.77488583$

$-0.77488583$

$-0.66905046$

$-0.66905046$

$-0.77488583$

$-0.77488583$

$-0.66905046$

$-0.66905046$

0.88849652

0.88849652

0.99433196

0.99433196

0.88849652

0.88849652

0.99433196

0.99433196

$-1.17169046$

$-1.17169046$

$-1.06585503$
0.44945931
0.55529481
0.44945931
0.80267227
0.55529481
$-0.47305208$
$-1.52175593$
0.97336483

1.000 \# Electr. mom., scale-fac \# Magnetic mom.

$\begin{array}{ll}-0.26382661 & -3.77222061\end{array}$

$-0.15799116 \quad 1.73763072$

$\begin{array}{ll}-0.26382661 & 3.50832129\end{array}$

$-0.15799116 \quad-1.40322435$

$\begin{array}{ll}-0.26382661 & 3.11543012\end{array}$

$-0.15799116 \quad-0.72079754$

$\begin{array}{ll}-0.26382661 & -2.60389018\end{array}$

$-0.15799116 \quad 0.03321234$

$-0.03186282$

0.07397263

$-0.03186282$

0.07397263

$-0.03186282$

0.07397263

$-0.03186282$

0.07397263

$-1.38695288$

$-1.28111744$

$-1.38695288$

$-1.28111744$

$-1.38695288$

$-1.28111744$

$-1.38695288$

$-1.28111744$

$-0.43766770$

$-0.33183220$

$-0.43766770$

$-0.33183220$

$-0.43766770$

$-0.33183220$

$-0.43766770$

$-0.33183220$

0.55604303

0.66187847

0.55604303

0.66187847

0.55604303

0.66187847

0.55604303

0.66187847

0.35565418

0.46148965

0.35565418

0.46148965

0.35565418

0.46148965

0.35565418

0.46148965

1.47651505

1.58235061

1.47651505

1.58235061

1.47651505

1.58235061

1.47651505

1.58235061

0.44945931

0.55529481

0.44945931

$-0.66897565$

3.55924416

2.24551606

$-4.44701719$

1.99440730

$-4.89832687$

$-3.60979724$

5.80329800

0.87300241

$-1.26937735$

$-1.24810755$

1.70628059

$-0.80167305$

1.11500692

1.19844890

$-1.58861899$

$-2.70993209$

5.29339218

3.00180745

$-5.28314400$

1. 86428165

$-3.97228718$

$-2.16442251$

4.01153564

6.99829054

$-4.65869617$

$-4.69781351$

2.92538071

$-6.80362701$

4.81526327

5.02463961

$-3.46324420$

0.86940414

$-0.33487558$

$-0.04457606$

$-0.54160482$

0.57924241

$-0.86603016$

$-1.48271251$

1.81249988

$-0.09140647$

0.11645984

0.03839309

$-0.06524804$

$-0.13828896$

0.05544634

0.15456994

$-0.07742855$

$-0.02866667$

0.00253447

0.55700690 


\begin{tabular}{|c|c|c|c|}
\hline $\begin{array}{l}0.31038415 \\
0.41621959 \\
0.41621959 \\
0.41621959 \\
0.41621959\end{array}$ & $\begin{array}{l}-1.06585503 \\
-1.17169046 \\
-1.17169046 \\
-1.06585503 \\
-1.06585503\end{array}$ & $\begin{array}{l}0.55529481 \\
0.44945931 \\
0.55529481 \\
0.44945931 \\
0.55529481\end{array}$ & $\begin{array}{r}-0.44034556 \\
0.37244481 \\
-0.31441781 \\
-1.09622347 \\
0.92462635\end{array}$ \\
\hline 11744 & 0. \# Energy 5->7 & & \\
\hline $\begin{array}{l}1.1744 \\
0.0859\end{array}$ & $\begin{array}{rr}-0.3119 & 0.0350 \\
0.0966 & -0.0901\end{array}$ & 1.000 & $\begin{array}{l}\text { Electr. mom., scale-fac } \\
\text { Magnetic mom. }\end{array}$ \\
\hline-1.85509956 & -0.14332448 & -0.26382661 & 4.11723900 \\
\hline-1.85509956 & -0.14332448 & -0.15799116 & -4.90850353 \\
\hline-1.85509956 & -0.03748903 & -0.26382661 & -5.22842503 \\
\hline-1.85509956 & -0.03748903 & -0.15799116 & 6.48014545 \\
\hline-1.74926412 & -0.14332448 & -0.26382661 & -6.43733501 \\
\hline-1.74926412 & -0.14332448 & -0.15799116 & 7.63069105 \\
\hline-1.74926412 & -0.03748903 & -0.26382661 & 8.05638504 \\
\hline-1.74926412 & -0.03748903 & -0.15799116 & -9.84651566 \\
\hline 1.30779219 & 0.51848310 & -0.03186282 & -6.81700087 \\
\hline 1.30779219 & 0.51848310 & 0.07397263 & 13.15369225 \\
\hline 1.30779219 & 0.62431854 & -0.03186282 & 7.23054075 \\
\hline 1.30779219 & 0.62431854 & 0.07397263 & -12.75932884 \\
\hline 1.41362774 & 0.51848310 & -0.03186282 & 6.40978527 \\
\hline 1.41362774 & 0.51848310 & 0.07397263 & -12.67595959 \\
\hline 1.41362774 & 0.62431854 & -0.03186282 & -6.96499538 \\
\hline 1.41362774 & 0.62431854 & 0.07397263 & 12.37378597 \\
\hline-1.45213747 & -0.42367253 & -1.38695288 & 0.35663307 \\
\hline-1.45213747 & -0.42367253 & -1.28111744 & -0.32547218 \\
\hline-1.45213747 & -0.31783706 & -1.38695288 & -0.51488024 \\
\hline-1.45213747 & -0.31783706 & -1.28111744 & 0.49057442 \\
\hline-1.34630203 & -0.42367253 & -1.38695288 & -0.44667596 \\
\hline-1.34630203 & -0.42367253 & -1.28111744 & 0.45740306 \\
\hline-1.34630203 & -0.31783706 & -1.38695288 & 0.62722880 \\
\hline-1.34630203 & -0.31783706 & -1.28111744 & -0.65556955 \\
\hline 2.33856606 & 1.04233944 & -0.43766770 & -1.06370246 \\
\hline 2.33856606 & 1.04233944 & -0.33183220 & 2.45063853 \\
\hline 2.33856606 & 1.14817488 & -0.43766770 & 0.31972322 \\
\hline 2.33856606 & 1.14817488 & -0.33183220 & -1.41834152 \\
\hline 2.44440126 & 1.04233944 & -0.43766770 & 2.09430575 \\
\hline 2.44440126 & 1.04233944 & -0.33183220 & -3.24288988 \\
\hline 2.44440126 & 1.14817488 & -0.43766770 & -1.35555267 \\
\hline 2.44440126 & 1.14817488 & -0.33183220 & 2.27337837 \\
\hline-1.15465784 & 0.69317716 & 0.55604303 & 2.14750838 \\
\hline-1.15465784 & 0.69317716 & 0.66187847 & 0.14340921 \\
\hline-1.15465784 & 0.79901266 & 0.55604303 & -0.99886757 \\
\hline-1.15465784 & 0.79901266 & 0.66187847 & -0.82140571 \\
\hline-1.04882240 & 0.69317716 & 0.55604303 & -0.88293779 \\
\hline-1.04882240 & 0.69317716 & 0.66187847 & -0.98287350 \\
\hline-1.04882240 & 0.79901266 & 0.55604303 & -0.02085804 \\
\hline-1.04882240 & 0.79901266 & 0.66187847 & 1.46895444 \\
\hline 1.23173511 & -0.77488583 & 0.35565418 & 4.86701155 \\
\hline 1.23173511 & -0.77488583 & 0.46148965 & -1.55453527 \\
\hline 1.23173511 & -0.66905046 & 0.35565418 & -6.60453176 \\
\hline 1.23173511 & -0.66905046 & 0.46148965 & 2.87548852 \\
\hline 1.33757067 & -0.77488583 & 0.35565418 & -5.70885897 \\
\hline 1.33757067 & -0.77488583 & 0.46148965 & 2.46530414 \\
\hline 1.33757067 & -0.66905046 & 0.35565418 & 7.58347273 \\
\hline 1.33757067 & -0.66905046 & 0.46148965 & -3.80975914 \\
\hline-1.51449943 & 0.88849652 & 1.47651505 & -0.13893545 \\
\hline-1.51449943 & 0.88849652 & 1.58235061 & 0.11842920 \\
\hline-1.51449943 & 0.99433196 & 1.47651505 & 0.07030469 \\
\hline-1.51449943 & 0.99433196 & 1.58235061 & -0.06671247 \\
\hline-1.40866399 & 0.88849652 & 1.47651505 & 0.04783895 \\
\hline-1.40866399 & 0.88849652 & 1.58235061 & -0.04721204 \\
\hline-1.40866399 & 0.99433196 & 1.47651505 & 0.03275331 \\
\hline-1.40866399 & 0.99433196 & 1.58235061 & -0.01369465 \\
\hline 0.31038415 & -1.17169046 & 0.44945931 & 0.93839926 \\
\hline 0.31038415 & -1.17169046 & 0.55529481 & -0.69180799 \\
\hline 0.31038415 & -1.06585503 & 0.44945931 & -1.12505448 \\
\hline
\end{tabular}

\section{S-301}




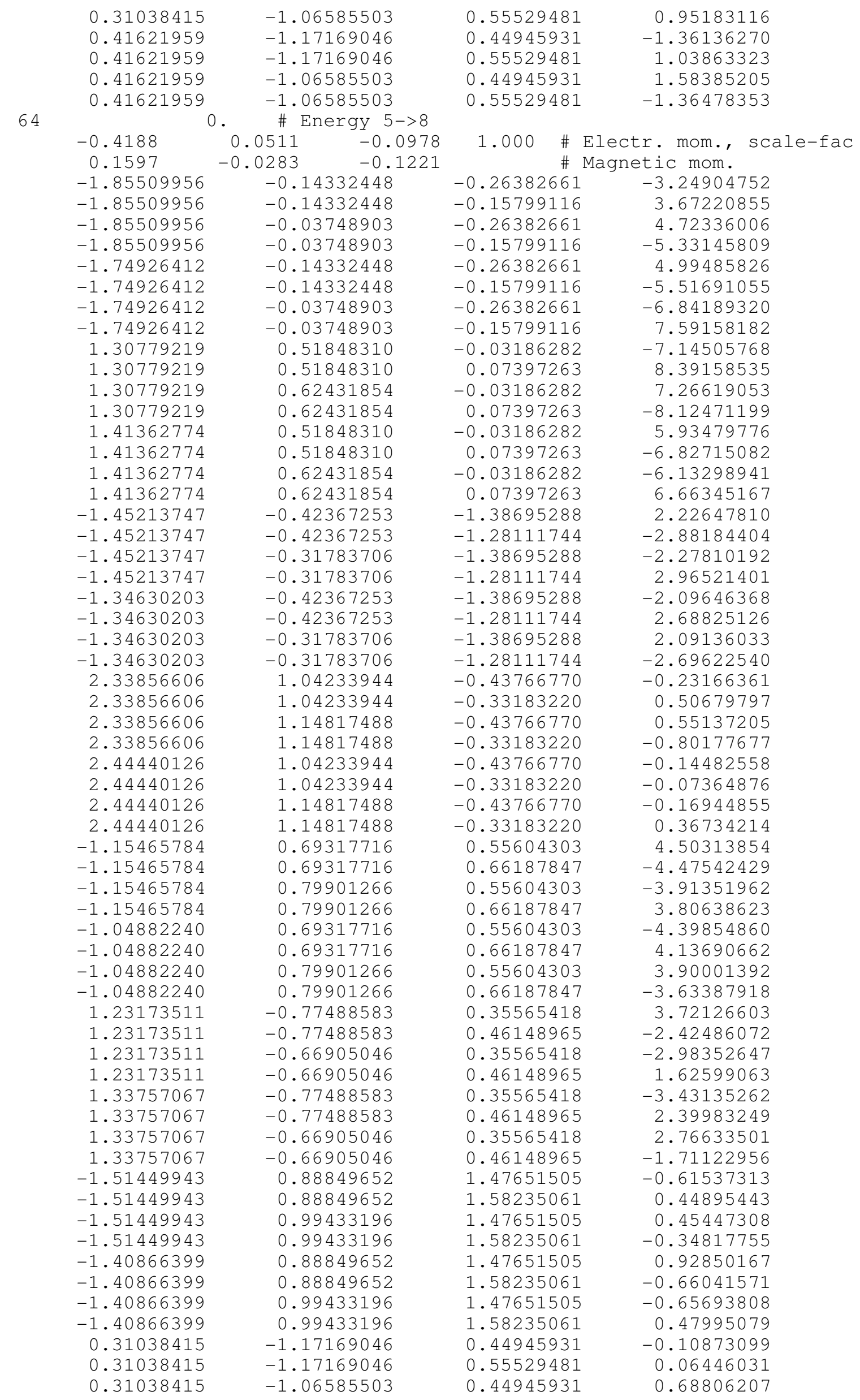

\section{S-302}




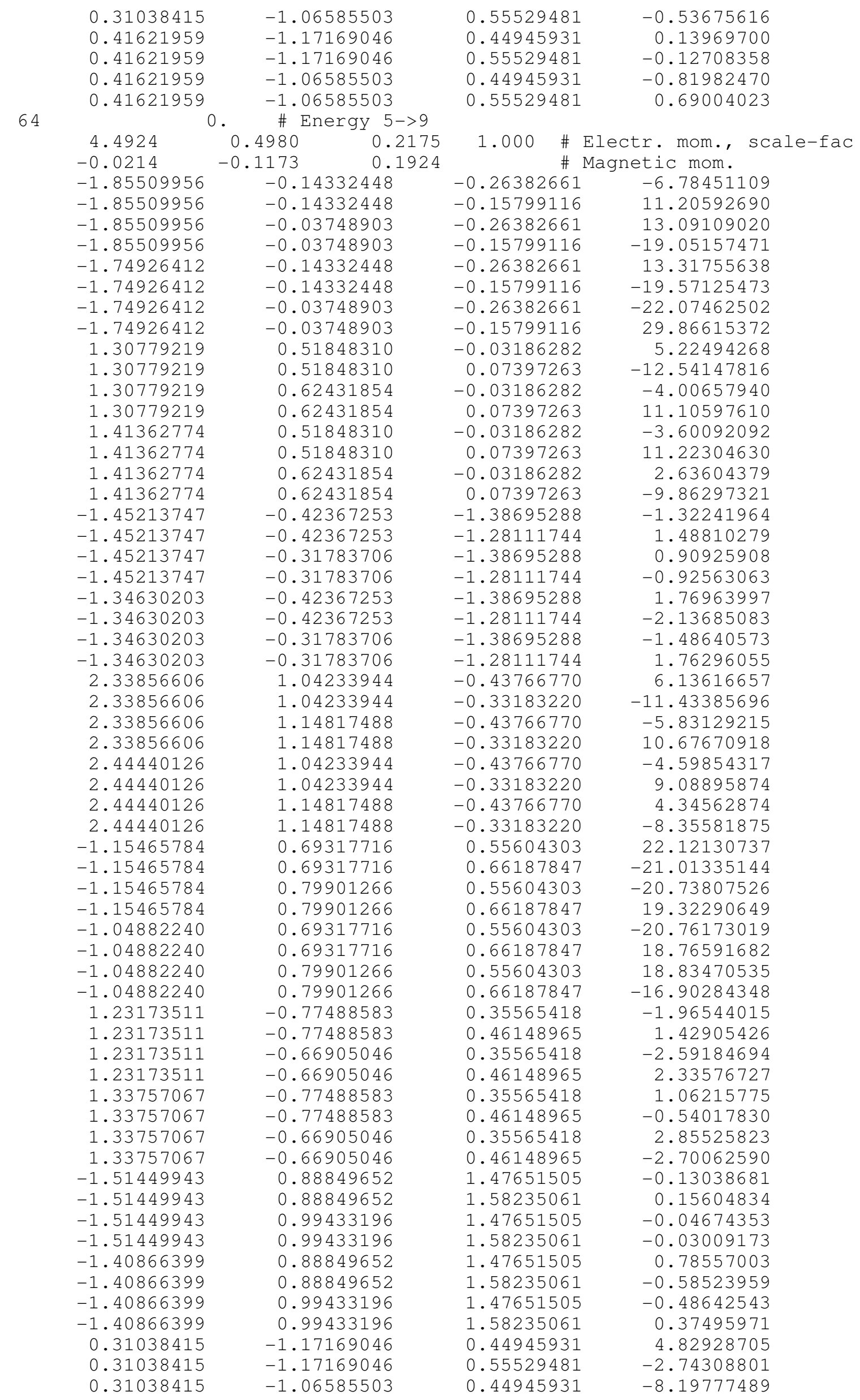

\section{S-303}




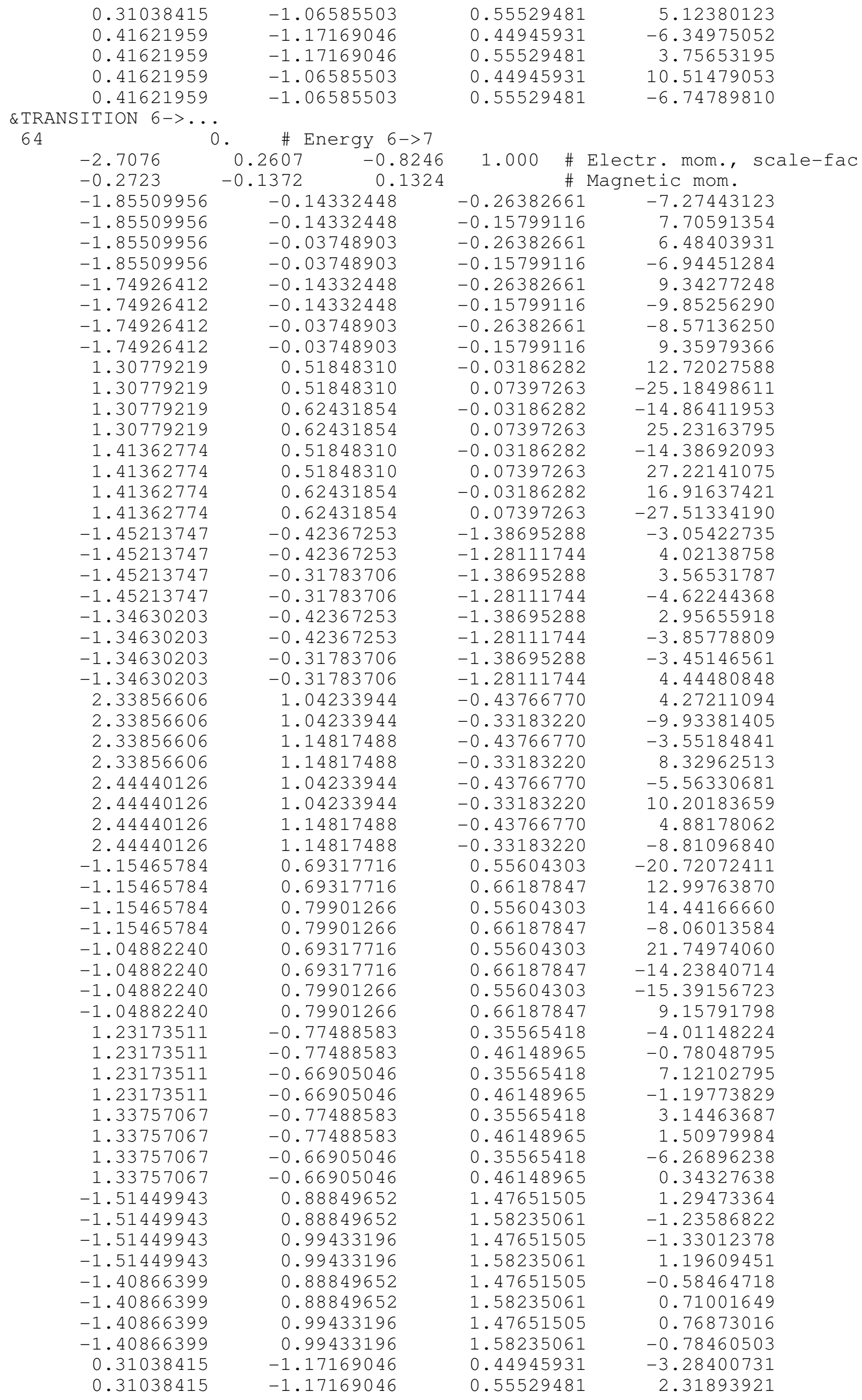


64

$$
\begin{aligned}
& 0.31038415 \\
& 0.31038415 \\
& 0.41621959 \\
& 0.41621959 \\
& 0.41621959 \\
& 0.41621959
\end{aligned}
$$

1609

$-0.1113$

$-1.85509956$

$-1.85509956$

$-1.85509956$

$-1.85509956$

$-1.74926412$

$-1.74926412$

$-1.74926412$

$-1.74926412$

1.30779219

1. 30779219

1.30779219

1.30779219

1. 41362774

1. 41362774

1. 41362774

1. 41362774

$-1.45213747$

$-1.45213747$

$-1.45213747$

$-1.45213747$

$-1.34630203$

$-1.34630203$

$-1.34630203$

$-1.34630203$

2. 33856606

2. 33856606

2.33856606

2.33856606

2. 44440126

2. 44440126

2. 44440126

2. 44440126

$-1.15465784$

$-1.15465784$

$-1.15465784$

$-1.15465784$

$-1.04882240$

$-1.04882240$

$-1.04882240$

$-1.04882240$

1. 23173511

1. 23173511

1. 23173511

1. 23173511

1.33757067

1.33757067

1.33757067

1.33757067

$-1.51449943$

$-1.51449943$

$-1.51449943$

$-1.51449943$

$-1.40866399$

$-1.40866399$

$-1.40866399$

$-1.40866399$

0.31038415

0.31038415
$-1.06585503$

$-1.06585503$

$-1.17169046$

$-1.17169046$

$-1.06585503$

$-1.06585503$

. \# Energy 6->8

$\begin{array}{lr}0.0824 & -0.1596 \\ 0.1453 & 0.5105\end{array}$

$-0.14332448$

$-0.14332448$

$-0.03748903$

$-0.03748903$

$-0.14332448$

$-0.14332448$

$-0.03748903$

$-0.03748903$

0.51848310

0.51848310

0.62431854

0.62431854

0.51848310

0.51848310

0.62431854

0.62431854

$-0.42367253$

$-0.42367253$

$-0.31783706$

$-0.31783706$

$-0.42367253$

$-0.42367253$

$-0.31783706$

$-0.31783706$

1. 04233944

1.04233944

1.14817488

1. 14817488

1. 04233944

1. 04233944

1. 14817488

1. 14817488

0.69317716

0.69317716

0.79901266

0.79901266

0.69317716

0.69317716

0.79901266

0.79901266

$-0.77488583$

$-0.77488583$

$-0.66905046$

$-0.66905046$

$-0.77488583$

$-0.77488583$

$-0.66905046$

$-0.66905046$

0.88849652

0.88849652

0.99433196

0.99433196

0.88849652

0.88849652

0.99433196

0.99433196

$-1.17169046$

$-1.17169046$

$\begin{array}{lr}0.44945931 & 4.01836681 \\ 0.55529481 & -2.89573121 \\ 0.44945931 & 4.25469780 \\ 0.55529481 & -2.89226985 \\ 0.44945931 & -5.23597193 \\ 0.55529481 & 3.66790104\end{array}$

1.000 \# Electr. mom., scale-fac \# Magnetic mom.

$-0.26382661$

$-0.15799116$

$-0.26382661$

$-0.15799116$

$-0.26382661$

$-0.15799116$

$-0.26382661$

$-0.15799116$

$-0.03186282$

0.07397263

$-0.03186282$

0.07397263

$-0.03186282$

0.07397263

$-0.03186282$

0.07397263

$-1.38695288$

$-1.28111744$

$-1.38695288$

$-1.28111744$

$-1.38695288$

$-1.28111744$

$-1.38695288$

$-1.28111744$

$-0.43766770$

$-0.33183220$

$-0.43766770$

$-0.33183220$

$-0.43766770$

$-0.33183220$

$-0.43766770$

$-0.33183220$

0.55604303

0.66187847

0.55604303

0.66187847

0.55604303

0.66187847

0.55604303

0.66187847

0.35565418

0.46148965

0.35565418

0.46148965

0.35565418

0.46148965

0.35565418

0.46148965

1. 47651505

1.58235061

1. 47651505

1. 58235061

1.47651505

1. 58235061

1.47651505

1. 58235061

0.44945931

0.55529481
3.11318755

$-8.92531967$

$-13.59232044$

20.05884171

$-9.50901413$

16.99580765

22.75416183

$-31.22005081$

$-1.74668336$

2. 05039120

$-3.29595685$

1. 40667915

$-2.43523979$

$-1.66955066$

2.68082690

$-4.40939808$

6.25241041

3.00168800

$-4.74676800$

2. 96812701

$-4.37783337$

$-1.38497710$

2. 58950377

1. 70732951

$-2.94790316$

$-1.50774550$

2.62786913

$-1.12757301$

2.16459155

0.97053564

$-1.88357401$

$-2.52746320$

5.84005070

3. 84917927

$-5.91053581$

8.04583073

$-9.27656937$

$-8.10376740$

8.53554058

4.02060890

$-2.00621104$

$-6.54841280$

3. 99347758

$-4.32744646$

2.47927809

6.55980301

$-4.24176359$

0.21823174

$-0.22720791$

$-0.48587751$

0.44083816

$-0.54925174$

0.45851418

0.69614595

$-0.59054983$

4.12697411

$-2.60237741$
3. 06934738 


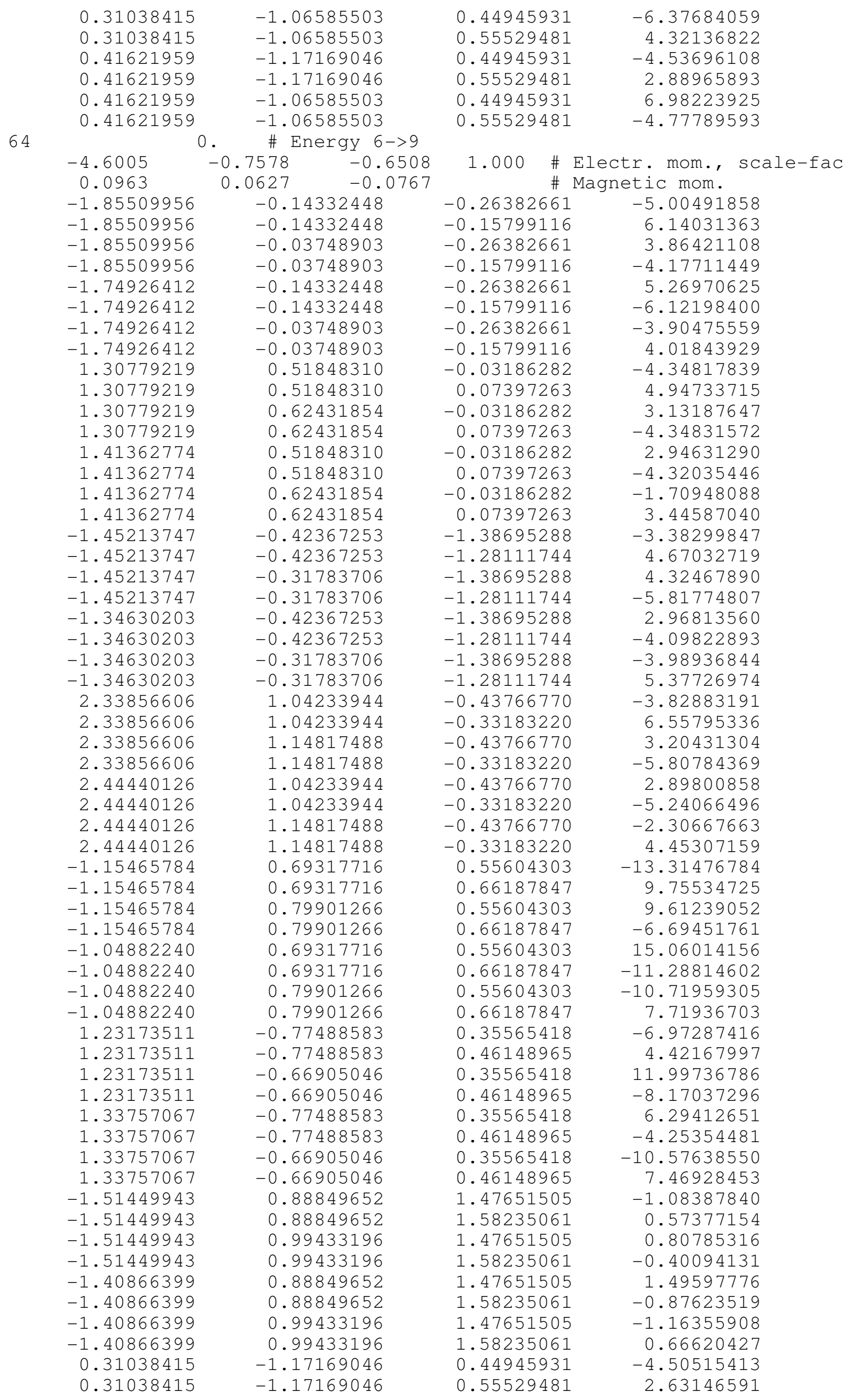

\section{S-306}




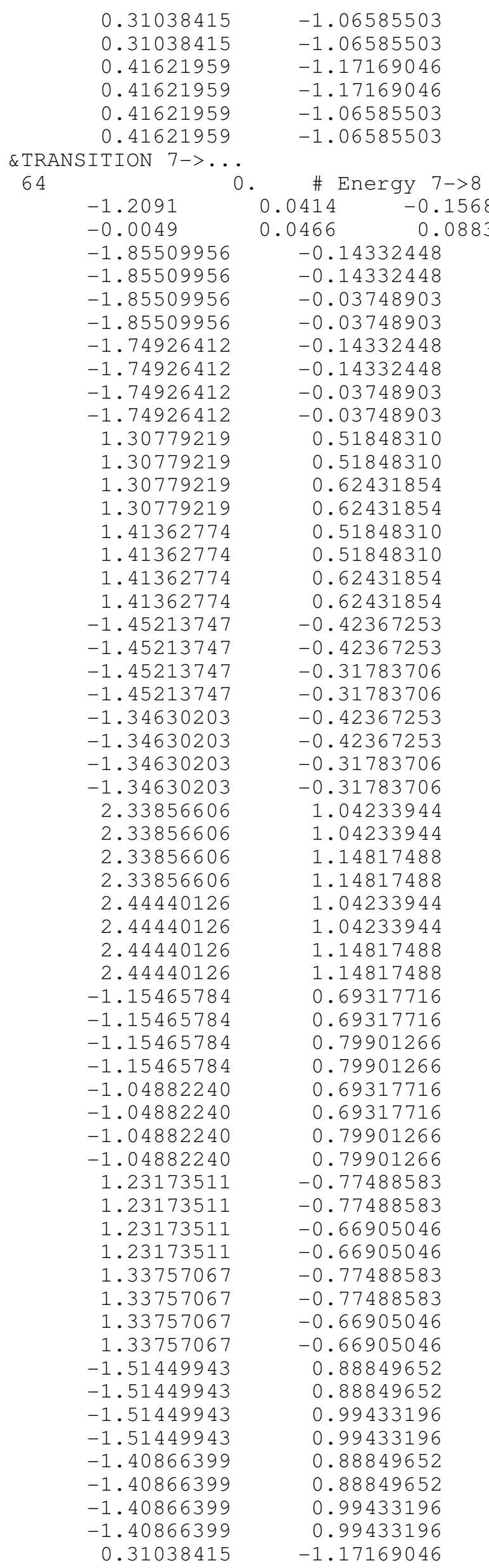

0.44945931

0.55529481

0.44945931

0.55529481

0.44945931

0.55529481
6.74055672

$-4.12372684$

6.00860357

$-3.53269386$

$-8.89573765$

5.50762844
1.000 \# Electr. mom., scale-fac \# Magnetic mom.

$\begin{array}{ll}-0.26382661 & 0.13119762\end{array}$

$-0.15799116 \quad-1.84966767$

$-0.26382661 \quad-3.13343358$

$-0.15799116 \quad 5.17648458$

$-0.26382661 \quad-1.69655132$

$\begin{array}{ll}-0.15799116 & 3.90043640\end{array}$

$-0.26382661 \quad 5.44367361$

$-0.15799116 \quad-7.99618292$

$\begin{array}{ll}-0.03186282 & 3.25771713\end{array}$

$0.07397263-6.18059111$

$-0.03186282 \quad-3.95311975$

$0.07397263 \quad 6.29397154$

$-0.03186282 \quad-3.48685098$

$0.07397263 \quad 6.38253021$

$\begin{array}{ll}-0.03186282 & 4.27522373\end{array}$

$0.07397263-6.58306170$

$-1.38695288$

$-1.28111744$

$-1.38695288$

$-1.28111744$

$-1.38695288$

$-1.28111744$

$-1.38695288$

$-1.28111744$

$-0.43766770$

$-0.33183220$

$-0.43766770$

$-0.33183220$

$-0.43766770$

$-0.33183220$

$-0.43766770$

$-0.33183220$

0.55604303

0.66187847

0.55604303

0.66187847

0.55604303

0.66187847

0.55604303

0.66187847

0.35565418

0.46148965

0.35565418

0.46148965

0.35565418

0.46148965

0.35565418

0.46148965

1. 47651505

1. 58235061

1.47651505

1. 58235061

1. 47651505

1. 58235061

1. 47651505

1. 58235061

0.44945931
0.73880619

0.44506359

$-0.66197014$

0.13883273

$-0.23778006$

0.00704299

0.07854174

0.51867294

$-1.52414978$

$-0.67090946$

1. 47937322

$-0.59192252$

1.36970162

0.75672549

$-1.39305091$

$-7.70310974$

6.56126976

6.33740664

$-5.18158627$

8.67297459

$-7.05909491$

$-6.86041355$

5. 38018227

$-0.15998788$

$-0.89917874$

1. 07554579

0.32711464

$-0.02904242$

0.99938071

$-0.84666193$

$-0.48432136$

$-0.06021281$

$-0.03308244$

$-0.06621462$

0.11875085

0.08190783

0.00330972

0.03366137

$-0.08308429$

$-0.62749022$
$-0.53166521$ 
64

\begin{abstract}
0.31038415
0.31038415

0.31038415

0.41621959

0.41621959

0.41621959

0.41621959
\end{abstract}

$-0.6606 \quad 0$

-0.3649
-1.85509956

$-1.85509956$

$-1.85509956$

$-1.85509956$

$-1.74926412$

$-1.74926412$

$-1.74926412$

$-1.74926412$

1.30779219

1.30779219

1.30779219

1. 30779219

1. 41362774

1. 41362774

1. 41362774

1. 41362774

$-1.45213747$

$-1.45213747$

$-1.45213747$

$-1.45213747$

$-1.34630203$

$-1.34630203$

$-1.34630203$

$-1.34630203$

2. 33856606

2. 33856606

2. 33856606

2. 33856606

2. 44440126

2. 44440126

2. 44440126

2. 44440126

$-1.15465784$

$-1.15465784$

$-1.15465784$

$-1.15465784$

$-1.04882240$

$-1.04882240$

$-1.04882240$

$-1.04882240$

1. 23173511

1. 23173511

1. 23173511

1. 23173511

1.33757067

1.33757067

1. 33757067

1.33757067

$-1.51449943$

$-1.51449943$

$-1.51449943$

$-1.51449943$

$-1.40866399$

$-1.40866399$

$-1.40866399$

$-1.40866399$

0.31038415
$-1.17169046$

$-1.06585503$

$-1.06585503$

$-1.17169046$

$-1.17169046$

$-1.06585503$

$-1.06585503$

. \# Energy 7->9

$-0.2359 \quad 0.1413$

$-0.2114 \quad 0.0638$

$-0.14332448$

$-0.14332448$

$-0.03748903$

$-0.03748903$

$-0.14332448$

$-0.14332448$

$-0.03748903$

$-0.03748903$

0.51848310

0.51848310

0.62431854

0.62431854

0.51848310

0.51848310

0.62431854

0.62431854

$-0.42367253$

$-0.42367253$

$-0.31783706$

$-0.31783706$

$-0.42367253$

$-0.42367253$

$-0.31783706$

$-0.31783706$

1. 04233944

1.04233944

1. 14817488

1. 14817488

1.04233944

1.04233944

1. 14817488

1. 14817488

0.69317716

0.69317716

0.79901266

0.79901266

0.69317716

0.69317716

0.79901266

0.79901266

$-0.77488583$

$-0.77488583$

$-0.66905046$

$-0.66905046$

$-0.77488583$

$-0.77488583$

$-0.66905046$

$-0.66905046$

0.88849652

0.88849652

0.99433196

0.99433196

0.88849652

0.88849652

0.99433196

0.99433196

$-1.17169046$ $\begin{array}{lr}0.55529481 & 0.30884418 \\ 0.44945931 & 0.86380684 \\ 0.55529481 & -0.46524709 \\ 0.44945931 & 0.98187423 \\ 0.55529481 & -0.50932473 \\ 0.44945931 & -1.33731043 \\ 0.55529481 & 0.75624490\end{array}$
1.000 \# Electr. mom., scale-fac \# Magnetic mom.

$\begin{array}{ll}-0.26382661 & 2.07616878\end{array}$

$-0.15799116 \quad-2.65357161$

$-0.26382661 \quad-3.07447171$

$\begin{array}{ll}-0.15799116 & 3.98832583\end{array}$

$-0.26382661 \quad-3.41350412$

$-0.15799116 \quad 4.27446604$

$-0.26382661 \quad 4.72827911$

$-0.15799116 \quad-5.98458624$

$-0.03186282-1.23464942$

$0.07397263 \quad 6.11798477$

$-0.03186282$

0.07397263

$-0.03186282$

0.07397263

$-0.03186282$

0.07397263

$-1.38695288$

$-1.28111744$

$-1.38695288$

$-1.28111744$

$-1.38695288$

$-1.28111744$

$-1.38695288$

$-1.28111744$

$-0.43766770$

$-0.33183220$

$-0.43766770$

$-0.33183220$

$-0.43766770$

$-0.33183220$

$-0.43766770$

$-0.33183220$

0.55604303

0.66187847

0.55604303

0.66187847

0.55604303

0.66187847

0.55604303

0.66187847

0.35565418

0.46148965

0.35565418

0.46148965

0.35565418

0.46148965

0.35565418

0.46148965

1.47651505

1. 58235061

1.47651505

1. 58235061

1. 47651505

1. 58235061

1. 47651505

1. 58235061

0.44945931
0.89572525

$-4.80600309$

2.34662795

$-7.19482183$

$-2.12578416$

5.91176081

0.26952806

$-0.24848272$

$-0.22174615$

0.16607372

$-0.40723675$

0.42884907

0.37231514

$-0.36019856$

$-2.23252964$

3.85247111

$-0.40033749$

$-0.93091899$

5.41453075

$-6.94332933$

$-2.85432172$

4.11471033

$-0.69694537$

1. 30959392

1.00263023

$-1.36066580$

0.30505645

$-0.73915547$

$-0.43207210$

0.68638301

8.35557270

$-4.88766766$

$-11.26205254$

7.07032299

$-9.43916512$

5.75868320

12.36670876

$-7.93039227$

$-0.05042058$

0.02024047

$-0.02550254$

0.03126245

$-0.08675795$

0.07474359

0.15686047

$-0.12083061$

1. 14409578 


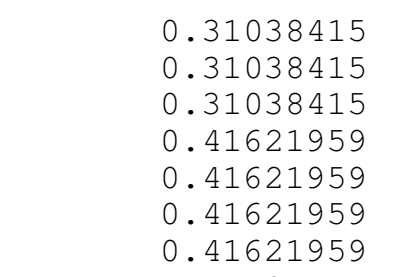

\&TRANSITION $8->$. 64

\begin{tabular}{|c|c|}
\hline $\begin{array}{r}-1.1850 \\
0.1325\end{array}$ & $\begin{array}{l}-0.1284 \\
-0.0432\end{array}$ \\
\hline-1.85509956 & -0.14332448 \\
\hline-1.85509956 & -0.14332448 \\
\hline-1.85509956 & -0.03748903 \\
\hline-1.85509956 & -0.03748903 \\
\hline-1.74926412 & -0.14332448 \\
\hline-1.74926412 & -0.14332448 \\
\hline-1.74926412 & -0.03748903 \\
\hline-1.74926412 & -0.03748903 \\
\hline 1.30779219 & 0.51848310 \\
\hline 1.30779219 & 0.51848310 \\
\hline 1.30779219 & 0.62431854 \\
\hline 1.30779219 & 0.62431854 \\
\hline 1.41362774 & 0.51848310 \\
\hline 1.41362774 & 0.51848310 \\
\hline 1.41362774 & 0.62431854 \\
\hline 1.41362774 & 0.62431854 \\
\hline-1.45213747 & -0.42367253 \\
\hline-1.45213747 & -0.42367253 \\
\hline-1.45213747 & -0.31783706 \\
\hline-1.45213747 & -0.31783706 \\
\hline-1.34630203 & -0.42367253 \\
\hline-1.34630203 & -0.42367253 \\
\hline-1.34630203 & -0.31783706 \\
\hline-1.34630203 & -0.31783706 \\
\hline 2.33856606 & 1.04233944 \\
\hline 2.33856606 & 1.04233944 \\
\hline 2.33856606 & 1.14817488 \\
\hline 2.33856606 & 1.14817488 \\
\hline 2.44440126 & 1.04233944 \\
\hline 2.44440126 & 1.04233944 \\
\hline 2.44440126 & 1.14817488 \\
\hline 2.44440126 & 1.14817488 \\
\hline-1.15465784 & 0.69317716 \\
\hline-1.15465784 & 0.69317716 \\
\hline-1.15465784 & 0.79901266 \\
\hline-1.15465784 & 0.79901266 \\
\hline-1.04882240 & 0.69317716 \\
\hline-1.04882240 & 0.69317716 \\
\hline-1.04882240 & 0.79901266 \\
\hline-1.04882240 & 0.79901266 \\
\hline 1.23173511 & -0.77488583 \\
\hline 1.23173511 & -0.77488583 \\
\hline 1.23173511 & -0.66905046 \\
\hline 1.23173511 & -0.66905046 \\
\hline 1.33757067 & -0.77488583 \\
\hline 1.33757067 & -0.77488583 \\
\hline 1.33757067 & -0.66905046 \\
\hline 1.33757067 & -0.66905046 \\
\hline-1.51449943 & 0.88849652 \\
\hline-1.51449943 & 0.88849652 \\
\hline-1.51449943 & 0.99433196 \\
\hline-1.51449943 & 0.99433196 \\
\hline-1.40866399 & 0.88849652 \\
\hline-1.40866399 & 0.88849652 \\
\hline-1.40866399 & 0.99433196 \\
\hline-1.40866399 & 0.99433196 \\
\hline
\end{tabular}

$-1.17169046$

$-1.06585503$

$-1.06585503$

$-1.17169046$

$-1.17169046$

$-1.06585503$

$-1.06585503$

0.

$\begin{array}{cc}\text { \# Energy } & 8->9 \\ -0.1284 & -0.0271 \\ -0.0432 & -0.1968\end{array}$

8

-0.26382661
-0.15799116

$-0.26382661$

$-0.15799116$

$-0.26382661$

$-0.15799116$

$-0.26382661$

$-0.15799116$

$-0.03186282$

0.07397263

$-0.03186282$

0.07397263

$-0.03186282$

0.07397263

$-0.03186282$

0.07397263

$-1.38695288$

$-1.28111744$

$-1.38695288$

$-1.28111744$

$-1.38695288$

$-1.28111744$

$-1.38695288$

$-1.28111744$

$-0.43766770$

$-0.33183220$

$-0.43766770$

$-0.33183220$

$-0.43766770$

$-0.33183220$

$-0.43766770$

$-0.33183220$

0.55604303

0.66187847

0.55604303

0.66187847

0.55604303

0.66187847

0.55604303

0.66187847

0.35565418

0.46148965

0.35565418

0.46148965

0.35565418

0.46148965

0.35565418

0.46148965

1. 47651505

1.58235061

1.47651505

1. 58235061

1.47651505

1. 58235061

1.47651505

1. 58235061
-1.14237809
-1.56545353
1.54888332
-1.72267389
1.60838747
2.30736780
-2.15598202
Electr. mom., scale-fac Magnetic mom.

$$
\begin{aligned}
& \text { 1. } 37683392 \\
& -4.13363600 \\
& -6.40357637 \\
& 9.67612743 \\
& -4.39168310 \\
& \text { 7. } 96813011 \\
& 10.69232178 \\
& -14.88852596 \\
& 4.43370199 \\
& -6.21127272 \\
& -4.60700083 \\
& 5.84770250 \\
& -3.77456522 \\
& \text { 5. } 13993740 \\
& 3.99001503 \\
& -4.88818741 \\
& -1.18283761 \\
& \text { 1. } 70624232 \\
& 0.93586713 \\
& -1.48426461 \\
& 0.50615078 \\
& -0.83953416 \\
& -0.17922182 \\
& 0.49951512 \\
& -0.98415631 \\
& \text { 1. } 52239716 \\
& 0.84400713 \\
& -1.39440894 \\
& 0.70061326 \\
& -1.18558705 \\
& -0.54833114 \\
& \text { 1. } 01560390 \\
& -8.28721237 \\
& 8.60127544 \\
& 7.68567753 \\
& -7.62471819 \\
& 9.58477306 \\
& -9.02857971 \\
& -8.36957073 \\
& 7.70862818 \\
& -2.74164772 \\
& 0.94357103 \\
& \text { 3. } 98606730 \\
& -1.71149814 \\
& \text { 3. } 05525279 \\
& -1.49432814 \\
& -4.20185518 \\
& \text { 2. } 21235466 \\
& -0.32436553 \\
& 0.18850288 \\
& 0.22168809 \\
& -0.09596645 \\
& 0.01952745 \\
& 0.00918696 \\
& -0.03061105 \\
& -0.02017656
\end{aligned}
$$




\begin{tabular}{|c|c|c|c|}
\hline 0.31038415 & -1.17169046 & 0.44945931 & -0.19906788 \\
\hline 0.31038415 & -1.17169046 & 0.55529481 & -0.14853027 \\
\hline 0.31038415 & -1.06585503 & 0.44945931 & 0.46000826 \\
\hline 0.31038415 & -1.06585503 & 0.55529481 & 0.00038813 \\
\hline 0.41621959 & -1.17169046 & 0.44945931 & 0.72964436 \\
\hline 0.41621959 & -1.17169046 & 0.55529481 & -0.16197141 \\
\hline 0.41621959 & -1.06585503 & 0.44945931 & -1.1615486 \\
\hline 0.41621959 & -1.06585503 & 0.55529481 & 0.436723 \\
\hline PERMANENT MOMENTS & & & \\
\hline 64 & \# Energy 1->1 & & \\
\hline-3.6450 & -1.2119 & $\#$ & ectr. mom. \\
\hline-1.85509956 & -0.14332448 & -0.26382661 & 160.84364 \\
\hline-1.85509956 & -0.14332448 & -0.15799116 & -168.2941284 \\
\hline-1.85509956 & -0.03748903 & -0.26382661 & -159.2243652 \\
\hline-1.85509956 & -0.03748903 & -0.15799116 & 176.534545 \\
\hline-1.74926412 & -0.14332448 & -0.26382661 & -204.93580627 \\
\hline-1.74926412 & -0.14332448 & -0.15799116 & 222.97695923 \\
\hline-1.74926412 & -0.03748903 & -0.26382661 & 222.466415 \\
\hline-1.74926412 & -0.03748903 & -0.15799116 & -252.5768432 \\
\hline 1.30779219 & 0.51848310 & -0.03186282 & 72.01787567 \\
\hline 1.30779219 & 0.51848310 & 0.07397263 & -56.94678116 \\
\hline 1.30779215 & 0.62431854 & -0.03186282 & -88.500411 \\
\hline 1.30779219 & 0.62431854 & 0.07397263 & 73.52066803 \\
\hline 1.41362774 & 0.51848310 & -0.03186282 & -83.68054962 \\
\hline 1.41362774 & 0.51848310 & 0.07397263 & 70.393348 \\
\hline 1.41362774 & 0.62431854 & -0.03186282 & 101.27171326 \\
\hline 1.41362774 & 0.62431854 & 0.07397263 & -87.21294403 \\
\hline-1.45213747 & -0.42367253 & -1.38695288 & -15.61686 \\
\hline-1.45213747 & -0.42367253 & -1.28111744 & 23.0292 \\
\hline-1.45213747 & -0.31783706 & -1.38695288 & 27.897792 \\
\hline-1.45213747 & -0.31783706 & -1.28111744 & -39.4161262 \\
\hline-1.34630203 & -0.42367253 & -1.38695288 & 10.972 \\
\hline-1.34630203 & -0.42367253 & -1.28111744 & -16.403 \\
\hline-1.34630203 & -0.31783706 & -1.38695288 & -20.24537 \\
\hline-1.34630203 & -0.31783706 & -1.28111744 & 28.94361496 \\
\hline 3856606 & 1.04233944 & -0.43766770 & $-18 \cdot 12$ \\
\hline 2.33856606 & 1.04233944 & -0.33183220 & 28.94365692 \\
\hline 2.33856606 & 1.14817488 & -0.43766770 & 8.43911743 \\
\hline 2.33856606 & 1.14817488 & -0.33183220 & -18.02234268 \\
\hline 2.44440126 & 1.04233944 & -0.43766770 & 11.1990 \\
\hline 2.44440126 & 1.04233944 & -0.33183220 & -19.90995598 \\
\hline 2.44440126 & 1.14817488 & -0.43766770 & -3.41879344 \\
\hline 2.44440126 & 1.14817488 & -0.33183220 & 10.5378656 \\
\hline-1.15465784 & 0.69317716 & 0.55604303 & 90.90381 \\
\hline-1.15465784 & 0.69317716 & 0.66187847 & -43.53651 \\
\hline-1.15465784 & 0.79901266 & 0.55604303 & -65.6538467 \\
\hline-1.15465784 & 0.799 & 0.661 & 33.44 \\
\hline-1.04882240 & 0.69317716 & 0.55604303 & -41.60279846 \\
\hline-1.04882240 & 0.69317716 & 0.66187847 & 7.70203 \\
\hline-1.04882240 & 0.79901266 & 0.55604303 & 24.7134761 \\
\hline-1.04882240 & 0.79901266 & 0.66187847 & -2.59911370 \\
\hline 1.23173511 & -0.77488583 & 0.35565418 & 75.9410018 \\
\hline 1.23173511 & -0.77488583 & 0.46148965 & -95.5984878 \\
\hline 1.23173511 & -0.66905046 & 0.35565418 & -65.4411 \\
\hline 1.23173511 & -0.66905046 & 0.46148965 & 80.8044128 \\
\hline 1.33757067 & -0.77488583 & 0.35565418 & -72.8914566 \\
\hline 1.33757067 & -0.77488583 & 0.46148965 & 92.58990 \\
\hline 1.33757067 & -0.66905046 & 0.35565418 & 62.3 \\
\hline 1.33757067 & -0.66905046 & 0.46148965 & -79.1831359 \\
\hline-1.51449943 & 0.88849652 & 1.47651505 & -0.769273 \\
\hline-1.51449943 & 0.88849652 & 1.582 & 0.4 \\
\hline-1.51449943 & 0.99433196 & 1.47651505 & -1.2773753 \\
\hline-1.51449943 & 0.99433196 & 1.58235061 & 1.2223783 \\
\hline-1.40866399 & 0.88849652 & 1.47651505 & 1.74282 \\
\hline-1.4086 & 49652 & 1.58 & $-1 \cdot 4$ \\
\hline-1.40866399 & 0.99433196 & 1.47651505 & $-0.52309^{\circ}$ \\
\hline-1 & 196 & 1. & \\
\hline
\end{tabular}




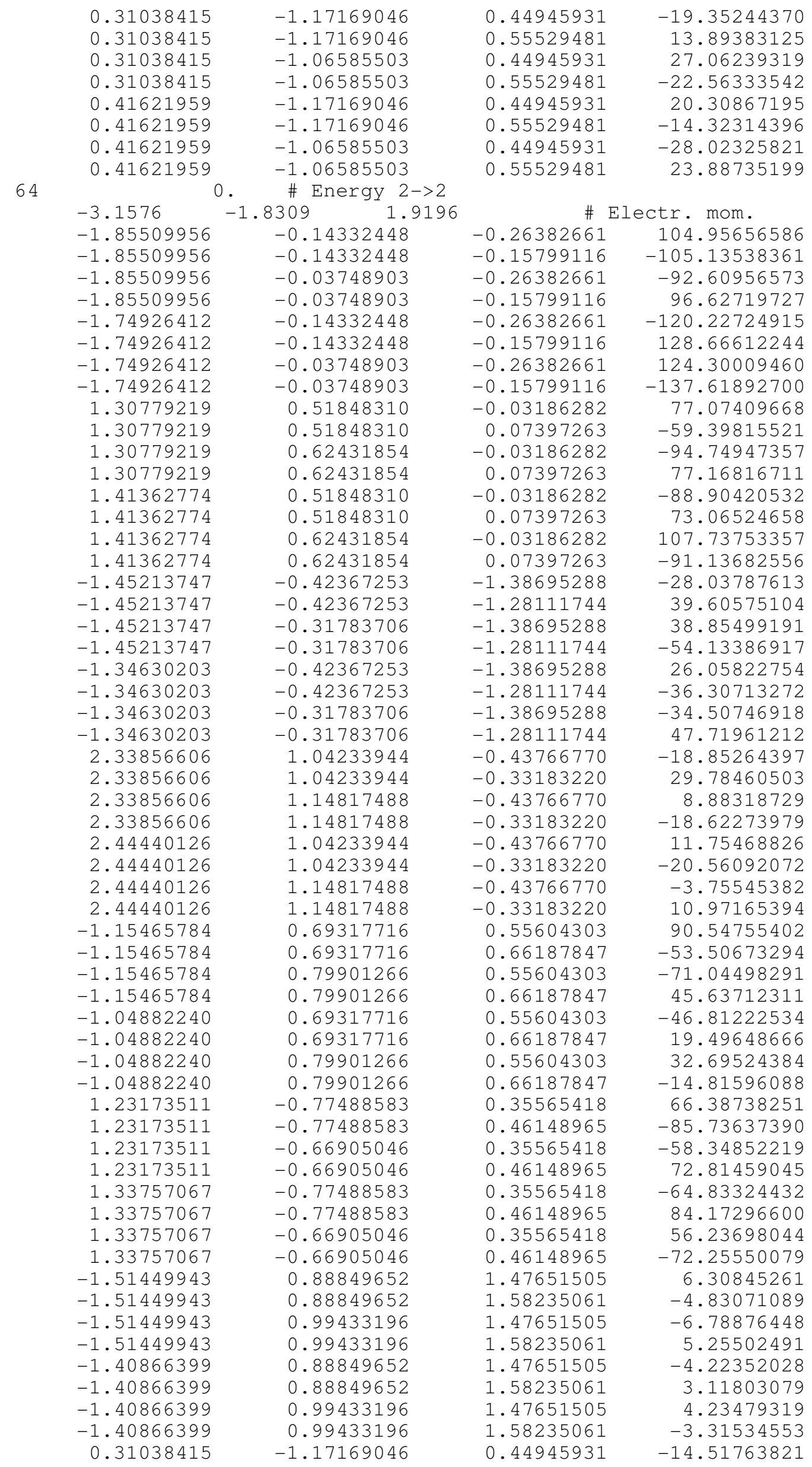

\section{S-311}


64

1

-1.17169046
-1.06585503
-1.06585503
-1.17169046
-1.17169046
-1.06585503
-1.06585503

$-2.1233$

$-1.85509956$

$-1.85509956$

$-1.85509956$

$-1.85509956$

$-1.74926412$

$-1.74926412$

$-1.74926412$

$-1.74926412$

1. 30779219

1.30779219

1.30779219

1.30779219

1. 41362774

1. 41362774

1. 41362774

1. 41362774

$-1.45213747$

$-1.45213747$

$-1.45213747$

$-1.45213747$

$-1.34630203$

$-1.34630203$

$-1.34630203$

$-1.34630203$

2.33856606

2.33856606

2.33856606

2.33856606

2. 44440126

2. 44440126

2. 44440126

2. 44440126

$-1.15465784$

$-1.15465784$

$-1.15465784$

$-1.15465784$

$-1.04882240$

$-1.04882240$

$-1.04882240$

$-1.04882240$

1. 23173511

1. 23173511

1. 23173511

1. 23173511

1.33757067

1.33757067

1.33757067

1.33757067

$-1.51449943$

$-1.51449943$

$-1.51449943$

$-1.51449943$

$-1.40866399$

$-1.40866399$

$-1.40866399$

$-1.40866399$

0.31038415

0.31038415

$$
0.1331 \quad 3.2732
$$

$-0.14332448$

$-0.14332448$

$-0.03748903$

$-0.03748903$

$-0.14332448$

$-0.14332448$

$-0.03748903$

$-0.03748903$

0.51848310

0.51848310

0.62431854

0.62431854

0.51848310

0.51848310

0.62431854

0.62431854

$-0.42367253$

$-0.42367253$

$-0.31783706$

$-0.31783706$

$-0.42367253$

$-0.42367253$

$-0.31783706$

$-0.31783706$

1. 04233944

1.04233944

1.14817488

1. 14817488

1.04233944

1. 04233944

1. 14817488

1. 14817488

0.69317716

0.69317716

0.79901266

0.79901266

0.69317716

0.69317716

0.79901266

0.79901266

$-0.77488583$

$-0.77488583$

$-0.66905046$

$-0.66905046$

$-0.77488583$

$-0.77488583$

$-0.66905046$

$-0.66905046$

0.88849652

0.88849652

0.99433196

0.99433196

0.88849652

0.88849652

0.99433196

0.99433196

$-1.17169046$

$-1.17169046$ $\begin{array}{lr}0.55529481 & 11.26555634 \\ 0.44945931 & 18.23761559 \\ 0.55529481 & -16.90135956 \\ 0.44945931 & 14.94835949 \\ 0.55529481 & -11.39985943 \\ 0.44945931 & -18.23044205 \\ 0.55529481 & 17.53517532\end{array}$

\# Electr. mom.

$-0.26382661 \quad 152.07150269$

$-0.15799116-158.45024109$

$-0.26382661 \quad-147.20625305$

$-0.15799116 \quad 161.65609741$

$-0.26382661 \quad-188.35513306$

$\begin{array}{ll}-0.15799116 & 203.70878601\end{array}$

$-0.26382661 \quad 200.65821838$

$-0.15799116 \quad-225.64584351$

$-0.03186282 \quad 107.10971832$

$0.07397263-131.60455322$

$-0.03186282 \quad-130.57769775$

$0.07397263 \quad 148.51300049$

$-0.03186282-123.41156769$

$0.07397263 \quad 149.66267395$

$-0.03186282 \quad 149.09980774$

$0.07397263-167.92807007$

$-1.38695288 \quad-17.52225113$

$-1.28111744 \quad 24.91777802$

$-1.38695288 \quad 30.49414635$

$-1.28111744 \quad-41.98378372$

$-1.38695288 \quad 13.57512760$

$-1.28111744 \quad-19.18569946$

$-1.38695288 \quad-23.38283920$

$-1.28111744 \quad 32.19227219$

$-0.43766770 \quad 5.57978916$

$-0.33183220 \quad-17.93866539$

$-0.43766770 \quad-17.05248070$

$-0.33183220 \quad 28.68403435$

$-0.43766770 \quad-9.31104755$

$-0.33183220 \quad 19.95580292$

$-0.43766770 \quad 18.49256706$

$-0.33183220 \quad-28.95050621$

$0.55604303 \quad 48.84165192$

$0.66187847 \quad-22.43647003$

$0.55604303 \quad-38.22436523$

$0.66187847 \quad 23.14417458$

$0.55604303 \quad 1.31845653$

$0.66187847 \quad-16.01372528$

$0.55604303 \quad-4.25315905$

$0.66187847 \quad 10.41694736$

$0.35565418 \quad 63.32297134$

$0.46148965 \quad-94.94593048$

$0.35565418 \quad-58.10400391$

$0.46148965 \quad 86.23593140$

$0.35565418 \quad-68.81716156$

$0.46148965 \quad 98.07678223$

$0.35565418 \quad 63.47424316$

$0.46148965 \quad-90.84993744$

$\begin{array}{ll}1.47651505 & 3.59649158\end{array}$

$1.58235061 \quad-3.33467889$

$1.47651505 \quad-5.77186775$

$1.58235061 \quad 4.94568157$

$\begin{array}{ll}1.47651505 & 0.03571178\end{array}$

$\begin{array}{ll}1.58235061 & 0.43432769\end{array}$

$1.47651505 \quad 1.96748245$

$1.58235061 \quad-2.04133296$

$0.44945931 \quad-14.61949730$

$0.55529481 \quad 10.80191135$ 


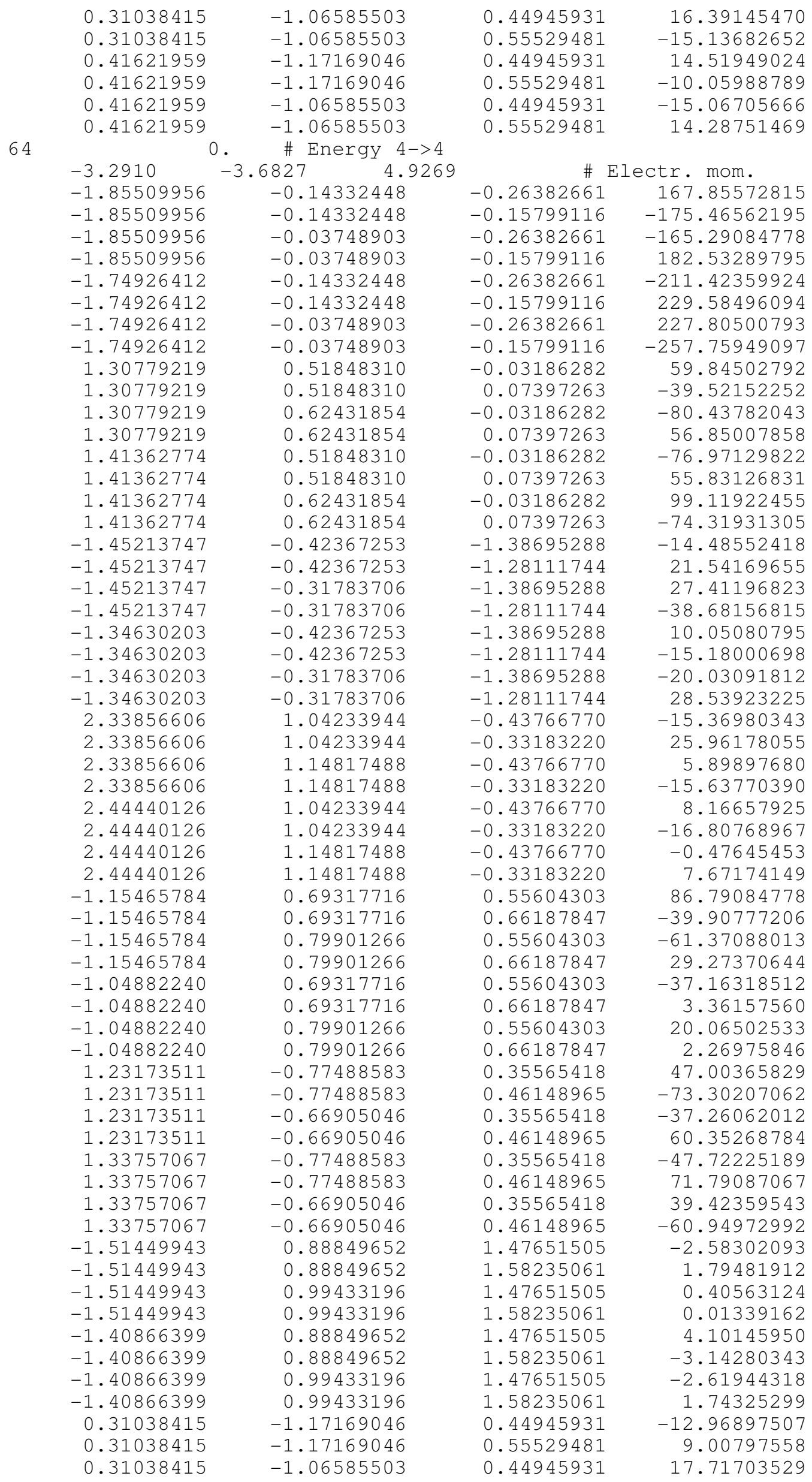

\section{S-313}




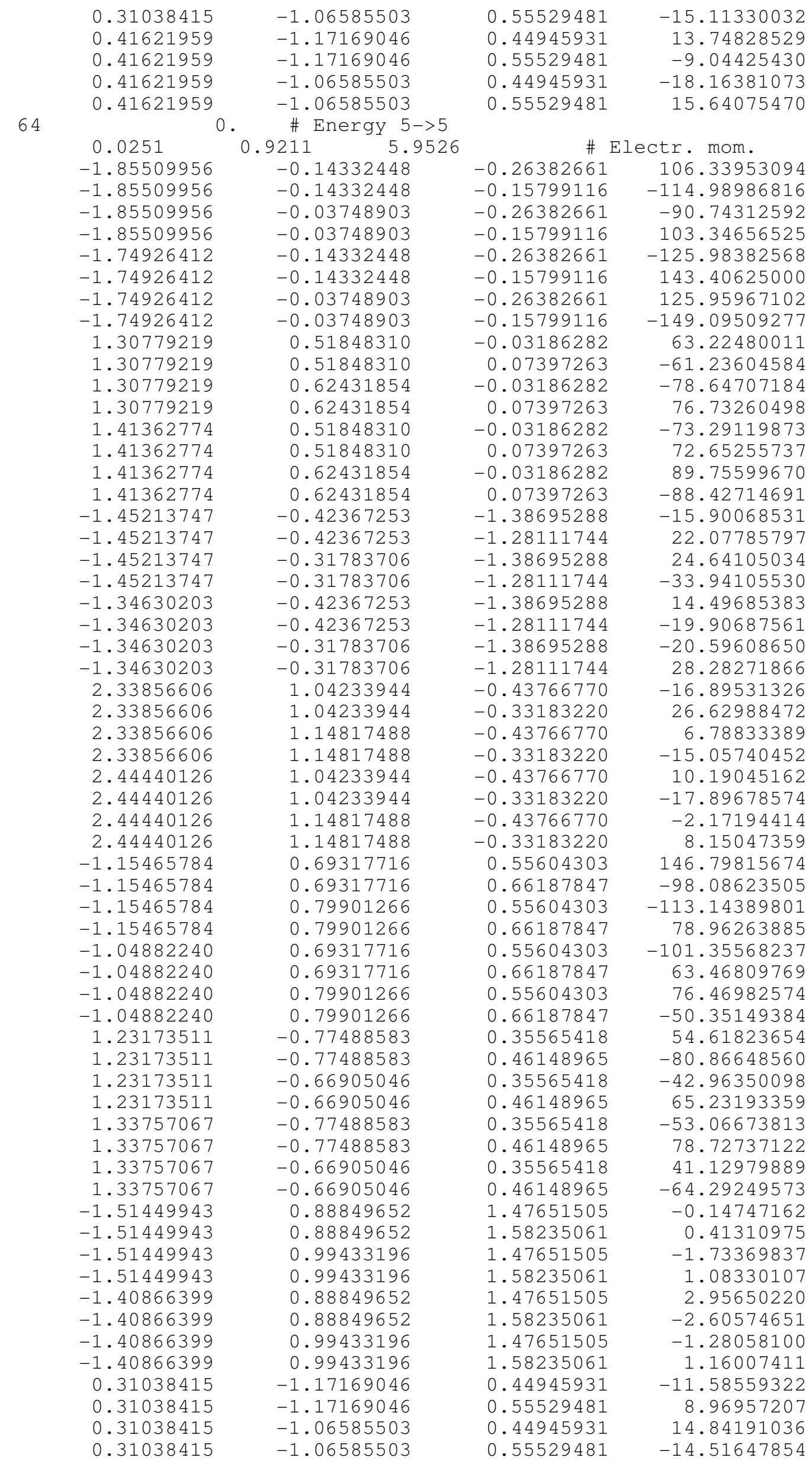

\section{S-314}


64

$$
\begin{aligned}
0.41621959 & -1.17169046 \\
0.41621959 & -1.17169046 \\
0.41621959 & -1.06585503 \\
0.41621959 & -1.06585503 \\
0 . & \# \text { Energy 6->6 }
\end{aligned}
$$

$$
-5.0957
$$

$-1.85509956$

$-1.85509956$

$-1.85509956$

$-1.85509956$

$-1.74926412$

$-1.74926412$

$-1.74926412$

$-1.74926412$

1. 30779219

1.30779219

1.30779219

1.30779219

1. 41362774

1. 41362774

1. 41362774

1. 41362774

$-1.45213747$

$-1.45213747$

$-1.45213747$

$-1.45213747$

$-1.34630203$

$-1.34630203$

$-1.34630203$

$-1.34630203$

2. 33856606

2.33856606

2.33856606

2.33856606

2. 44440126

2. 44440126

2. 44440126

2. 44440126

$-1.15465784$

$-1.15465784$

$-1.15465784$

$-1.15465784$

$-1.04882240$

$-1.04882240$

$-1.04882240$

$-1.04882240$

1. 23173511

1. 23173511

1. 23173511

1. 23173511

1. 33757067

1.33757067

1.33757067

1. 33757067

$-1.51449943$

$-1.51449943$

$-1.51449943$

$-1.51449943$

$-1.40866399$

$-1.40866399$

$-1.40866399$

$-1.40866399$

0.31038415

0.31038415

0.31038415

0.31038415

0.41621959
$-1.1750$

2.0091

$-0.14332448$

$-0.14332448$

$-0.03748903$

$-0.03748903$

$-0.14332448$

$-0.14332448$

$-0.03748903$

$-0.03748903$

0.51848310

0.51848310

0.62431854

0.62431854

0.51848310

0.51848310

0.62431854

0.62431854

$-0.42367253$

$-0.42367253$

$-0.31783706$

$-0.31783706$

$-0.42367253$

$-0.42367253$

$-0.31783706$

$-0.31783706$

1. 04233944

1.04233944

1.14817488

1.14817488

1.04233944

1.04233944

1. 14817488

1. 14817488

0.69317716

0.69317716

0.79901266

0.79901266

0.69317716

0.69317716

0.79901266

0.79901266

$-0.77488583$

$-0.77488583$

$-0.66905046$

$-0.66905046$

$-0.77488583$

$-0.77488583$

$-0.66905046$

$-0.66905046$

0.88849652

0.88849652

0.99433196

0.99433196

0.88849652

0.88849652

0.99433196

0.99433196

$-1.17169046$

$-1.17169046$

$-1.06585503$

$-1.06585503$

$-1.17169046$ $\begin{array}{lr}0.44945931 & 12.53202820 \\ 0.55529481 & -9.51059341 \\ 0.44945931 & -15.44739437 \\ 0.55529481 & 15.69548607\end{array}$

\# Electr. mom.

$-0.26382661 \quad 109.25251770$

$-0.15799116-109.28842926$

$-0.26382661 \quad-95.67638397$

$-0.15799116 \quad 99.81940460$

$-0.26382661-123.83026123$

$-0.15799116 \quad 131.51997375$

$-0.26382661 \quad 125.58142090$

$-0.15799116-138.12532043$

$-0.03186282 \quad 91.76084900$

$0.07397263-104.02803040$

$-0.03186282-115.50778961$

$0.07397263 \quad 122.82188416$

$-0.03186282 \quad-107.79447937$

$0.07397263 \quad 120.81900787$

$-0.03186282 \quad 133.33219910$

$0.07397263-140.87510681$

$-1.38695288 \quad-27.27281761$

$-1.28111744 \quad 38.05303574$

$-1.38695288 \quad 39.44480896$

$-1.28111744 \quad-54.08366776$

$-1.38695288 \quad 25.38932800$

$-1.28111744 \quad-34.87009811$

$-1.38695288 \quad-35.03345871$

$-1.28111744 \quad 47.60154343$

$-0.43766770 \quad-5.16764927$

$-0.33183220 \quad 2.21217918$

$-0.43766770 \quad-7.90224552$

$-0.33183220 \quad 10.96936798$

$-0.43766770 \quad 1.60220778$

$-0.33183220 \quad 1.06804776$

$-0.43766770$

$-0.33183220$

0.55604303

0.66187847

0.55604303

0.66187847

0.55604303

0.66187847

0.55604303

0.66187847

0.35565418

0.46148965

0.35565418

0.46148965

0.35565418

0.46148965

0.35565418

0.46148965

1. 47651505

1. 58235061

1. 47651505

1. 58235061

1. 47651505

1. 58235061

1.47651505

1. 58235061

0.44945931

0.55529481

0.44945931

0.55529481

0.44945931
9. 14283657

$-12.45542812$

55.70956421

$-47.00049210$

34.33148193

$-11.28440189$

$-1.56342447$

8.48197269

$-2.68425560$

50.19915390

$-80.79962158$

$-41.97298431$

69.36552429

$-56.27951431$

84.31715393

48.33492661

$-74.57602692$ 6.19336748

$-5.16491890$

$-7.26611376$

5.89337921

$-1.98003459$

1.89009976

3.11260939

$-2.79128480$

$-14.38145733$

10.67182827

15.75812912

$-14.47359943$

14.92835999
$-33.93177032$ 


$$
\begin{array}{ll}
0.41621959 & -1.17169046 \\
0.41621959 & -1.06585503 \\
0.41621959 & -1.06585503 \\
8.6516 \quad 0 . & \text { \# Energy 7->7 }
\end{array}
$$

8.6516

$-1.85509956$

$-1.85509956$

$-1.85509956$

$-1.85509956$

$-1.74926412$

$-1.74926412$

$-1.74926412$

$-1.74926412$

1.30779219

1.30779219

1.30779219

1.30779219

1. 41362774

1. 41362774

1. 41362774

1. 41362774

$-1.45213747$

$-1.45213747$

$-1.45213747$

$-1.45213747$

$-1.34630203$

$-1.34630203$

$-1.34630203$

$-1.34630203$

2. 33856606

2. 33856606

2.33856606

2.33856606

2. 44440126

2. 44440126

2. 44440126

2. 44440126

$-1.15465784$

$-1.15465784$

$-1.15465784$

$-1.15465784$

$-1.04882240$

$-1.04882240$

$-1.04882240$

$-1.04882240$

1. 23173511

1. 23173511

1. 23173511

1. 23173511

1.33757067

1. 33757067

1.33757067

1.33757067

$-1.51449943$

$-1.51449943$

$-1.51449943$

$-1.51449943$

$-1.40866399$

$-1.40866399$

$-1.40866399$

$-1.40866399$

0.31038415

0.31038415

0.31038415

0.31038415

0.41621959

0.41621959
5.7092

$-0.14332448$

$-0.14332448$

$-0.03748903$

$-0.03748903$

$-0.14332448$

$-0.14332448$

$-0.03748903$

$-0.03748903$

0.51848310

0.51848310

0.62431854

0.62431854

0.51848310

0.51848310

0.62431854

0.62431854

$-0.42367253$

$-0.42367253$

$-0.31783706$

$-0.31783706$

$-0.42367253$

$-0.42367253$

$-0.31783706$

$-0.31783706$

1. 04233944

1. 04233944

1. 14817488

1.14817488

1. 04233944

1.04233944

1. 14817488

1. 14817488

0.69317716

0.69317716

0.79901266

0.79901266

0.69317716

0.69317716

0.79901266

0.79901266

$-0.77488583$

$-0.77488583$

$-0.66905046$

$-0.66905046$

$-0.77488583$

$-0.77488583$

$-0.66905046$

$-0.66905046$

0.88849652

0.88849652

0.99433196

0.99433196

0.88849652

0.88849652

0.99433196

0.99433196

$-1.17169046$

$-1.17169046$

$-1.06585503$

$-1.06585503$

$-1.17169046$

$-1.17169046$ $\begin{array}{lr}0.55529481 & -10.40010834 \\ 0.44945931 & -15.39217186 \\ 0.55529481 & 14.27519226\end{array}$

\# Electr. mom.

$-0.26382661 \quad 135.10565186$

$-0.15799116-138.25282288$

$-0.26382661 \quad-119.35251617$

$-0.15799116 \quad 126.60315704$

$-0.26382661 \quad-158.68931580$

$-0.15799116 \quad 169.78698730$

$-0.26382661 \quad 158.41091919$

$-0.15799116 \quad-175.48901367$

$-0.03186282$

0.07397263

$-0.03186282$

0.07397263

$-0.03186282$

0.07397263

$-0.03186282$

0.07397263

$-1.38695288$

$-1.28111744$

$-1.38695288$

$-1.28111744$

$-1.38695288$

$-1.28111744$

$-1.38695288$

$-1.28111744$

$-0.43766770$

$-0.33183220$

$-0.43766770$

$-0.33183220$

$-0.43766770$

$-0.33183220$

$-0.43766770$

$-0.33183220$

0.55604303

0.66187847

0.55604303

0.66187847

0.55604303

0.66187847

0.55604303

0.66187847

0.35565418

0.46148965

0.35565418

0.46148965

0.35565418

0.46148965

0.35565418

0.46148965

1. 47651505

1. 58235061

1. 47651505

1. 58235061

1. 47651505

1.58235061

1. 47651505

1.58235061

0.44945931

0.55529481

0.44945931

0.55529481

0.44945931

0.55529481

56.08536148

$-35.05222321$

$-68.56636810$

48.18336487

$-66.82474518$

45.45455170

80.05702209

$-58.96001434$

$-17.14418411$

24.56890488

26.74460030

$-37.60278702$

15.51749611

$-21.90225410$

$-22.59028244$

31.59265518

$-16.07312012$

30.03890038

8.99914646

$-20.50834274$

7.64499426

$-18.30031967$

$-2.70119810$

10.95056915

140.34626770

$-87.75212860$

$-106.17971039$

68.04608917

$-97.05860138$

54.76099014

68.66426086

$-38.45729065$

41.52233887

$-65.72276306$

$-37.34719467$

56.17467499

$-39.80985641$

62.63734055

35.73452377

$-54.13662338$

1. 71889853

$-0.74362266$

$-2.44618893$

1. 49837589

$-0.28391045$

$-0.45960453$

0.42586133

0.02638541

$-3.57284856$

3. 99374247

3. 30821157

$-6.81828260$

2.38456964

$-3.09074855$ 
64

$$
\begin{array}{rr}
0.41621959 & -1.06585503 \\
0.41621959 & -1.06585503 \\
0 . & \# \text { Energy 8->8 }
\end{array}
$$$$
\begin{array}{lll}
-12.2505 & -1.0010 & 3.6135
\end{array}
$$

$-1.85509956$

$-1.85509956$

$-1.85509956$

$-1.85509956$

$-1.74926412$

$-1.74926412$

$-1.74926412$

$-1.74926412$

1.30779219

1.30779219

1. 30779219

1.30779219

1.41362774

1.41362774

1. 41362774

1. 41362774

$-1.45213747$

$-1.45213747$

$-1.45213747$

$-1.45213747$

$-1.34630203$

$-1.34630203$

$-1.34630203$

$-1.34630203$

2.33856606

2.33856606

2. 33856606

2. 33856606

2. 44440126

2. 44440126

2. 44440126

2. 44440126

$-1.15465784$

$-1.15465784$

$-1.15465784$

$-1.15465784$

$-1.04882240$

$-1.04882240$

$-1.04882240$

$-1.04882240$

1. 23173511

1. 23173511

1. 23173511

1. 23173511

1.33757067

1.33757067

1. 33757067

1.33757067

$-1.51449943$

$-1.51449943$

$-1.51449943$

$-1.51449943$

$-1.40866399$

$-1.40866399$

$-1.40866399$

$-1.40866399$

0.31038415

0.31038415

0.31038415

0.31038415

0.41621959

0.41621959

0.41621959
$-0.14332448$

$-0.14332448$

$-0.03748903$

$-0.03748903$

$-0.14332448$

$-0.14332448$

$-0.03748903$

$-0.03748903$

0.51848310

0.51848310

0.62431854

0.62431854

0.51848310

0.51848310

0.62431854

0.62431854

$-0.42367253$

$-0.42367253$

$-0.31783706$

$-0.31783706$

$-0.42367253$

$-0.42367253$

$-0.31783706$

$-0.31783706$

1. 04233944

1.04233944

1. 14817488

1. 14817488

1.04233944

1.04233944

1. 14817488

1. 14817488

0.69317716

0.69317716

0.79901266

0.79901266

0.69317716

0.69317716

0.79901266

0.79901266

$-0.77488583$

$-0.77488583$

$-0.66905046$

$-0.66905046$

$-0.77488583$

$-0.77488583$

$-0.66905046$

$-0.66905046$

0.88849652

0.88849652

0.99433196

0.99433196

0.88849652

0.88849652

0.99433196

0.99433196

$-1.17169046$

$-1.17169046$

$-1.06585503$

$-1.06585503$

$-1.17169046$

$-1.17169046$

$-1.06585503$
$0.44945931 \quad-0.83270508$

$\begin{array}{ll}0.55529481 & 5.73476887\end{array}$

\# Electr. mom.

$-0.26382661 \quad 126.21659851$

$-0.15799116-138.30921936$

$-0.26382661 \quad-124.51422119$

$-0.15799116 \quad 143.55256653$

$-0.26382661 \quad-155.00735474$

$-0.15799116 \quad 177.72297668$

$\begin{array}{ll}-0.26382661 & 171.98104858\end{array}$

$-0.15799116 \quad-203.15153503$

$-0.03186282 \quad 68.91506195$

$0.07397263 \quad-78.76000977$

$-0.03186282 \quad-93.53532410$

$0.07397263 \quad 98.44851685$

$-0.03186282 \quad-86.67588806$

$0.07397263 \quad 96.27837372$

$-0.03186282 \quad 112.84927368$

$0.07397263-117.27094269$

$-1.38695288 \quad-10.51720715$

$-1.28111744 \quad 15.21984100$

$-1.38695288 \quad 21.78538704$

$-1.28111744 \quad-30.09140587$

$-1.38695288 \quad 6.93253565$

$-1.28111744 \quad-10.21447468$

$-1.38695288 \quad-15.39565754$

$-1.28111744 \quad 21.45731163$

$-0.43766770 \quad-15.01325798$

$-0.33183220 \quad 20.41291618$

$\begin{array}{ll}-0.43766770 & 2.11053538\end{array}$

$-0.33183220 \quad-6.94809961$

$-0.43766770 \quad 8.36279392$

$-0.33183220 \quad-12.88599491$

$-0.43766770 \quad 2.28796291$

$-0.33183220 \quad 1.02602279$

$\begin{array}{lr}0.55604303 & 63.77802277\end{array}$

$0.66187847 \quad-32.48299789$

$0.55604303 \quad-47.76947784$

$0.66187847 \quad 28.65594482$

$0.55604303 \quad-17.25727844$

$0.66187847 \quad-2.13435912$

$0.55604303 \quad 11.14108181$

$0.66187847 \quad-0.65633219$

$0.35565418 \quad 48.91116333$

$0.46148965 \quad-82.54073334$

$0.35565418 \quad-29.33485794$

$0.46148965 \quad 61.85882950$

$0.35565418 \quad-51.88478851$

$0.46148965 \quad 82.93778229$

$0.35565418 \quad 33.87955475$

$0.46148965 \quad-64.95168304$

$1.47651505 \quad-2.02008390$

$1.58235061 \quad 1.10017133$

$1.47651505 \quad-0.66310924$

$1.58235061 \quad 0.88824970$

$1.47651505 \quad 5.74003458$

$1.58235061-4.06377602$

$1.47651505-3.09794188$

$1.58235061 \quad 1.99462128$

$0.44945931 \quad-20.61737251$

$0.55529481 \quad 13.75713634$

$0.44945931 \quad 28.30182266$

$0.55529481 \quad-22.33551598$

$0.44945931 \quad 24.22516632$

$0.55529481 \quad-15.65934181$

$0.44945931 \quad-32.56872559$ 
64
$0.55529481 \quad 25.59965706$

\# Electr. mom.

$\begin{array}{ll}-0.26382661 & 127.76020813\end{array}$

$-0.15799116 \quad-135.02131653$

$-0.26382661-116.61727905$

$-0.15799116 \quad 128.47059631$

$-0.26382661 \quad-152.89552307$

$-0.15799116 \quad 168.78213501$

$-0.26382661 \quad 158.29759216$

$-0.15799116-180.35865784$

$-0.03186282 \quad 91.03588867$

$0.07397263 \quad-94.18930054$

$-0.03186282-108.01981354$

$0.07397263 \quad 109.16867828$

$-0.03186282-102.08438873$

$0.07397263 \quad 107.65865326$

$-0.03186282 \quad 120.89880371$

$0.07397263-123.29237366$

$-1.38695288 \quad-18.34646034$

$-1.28111744 \quad 25.85430145$

$-1.38695288 \quad 28.12793159$

$-1.28111744 \quad-39.10501862$

$-1.38695288 \quad 16.46855545$

$-1.28111744 \quad-22.83778954$

$-1.38695288 \quad-23.36089706$

$-1.28111744 \quad 32.26393127$

$-0.43766770 \quad-1.42757916$

$-0.33183220 \quad-2.11982512$

$-0.43766770 \quad-8.04810524$

$-0.33183220 \quad 11.59508419$

$-0.43766770 \quad-2.60325718$

$-0.33183220 \quad 5.93656921$

$-0.43766770 \quad 10.13993645$

$-0.33183220 \quad-13.88217449$

$0.55604303 \quad 101.68840027$

$0.66187847 \quad-63.09520340$

$0.55604303 \quad-78.97127533$

$0.66187847 \quad 53.02009583$

$0.55604303 \quad-56.37948608$

$0.66187847 \quad 28.38240051$

$0.55604303 \quad 40.35636139$

$0.66187847 \quad-22.55753326$

$\begin{array}{ll}0.35565418 & 70.87943268\end{array}$

$0.46148965 \quad-91.81511688$

$0.35565418 \quad-71.81623077$

$0.46148965 \quad 86.71395111$

$0.35565418 \quad-71.46767426$

$0.46148965 \quad 92.12908936$

$\begin{array}{ll}0.35565418 & 70.96248627\end{array}$

$0.46148965 \quad-87.33547974$

$1.47651505 \quad 6.28422832$

$1.58235061 \quad-4.67871571$

$\begin{array}{ll}1.47651505 & -7.22973394\end{array}$

$1.58235061 \quad 5.43482542$

$1.47651505-3.65792298$

$1.58235061 \quad 2.57526922$

$1.47651505 \quad 4.33472204$

$1.58235061 \quad-3.26040053$

$0.44945931 \quad-5.30111980$

$0.55529481 \quad 5.73942423$

$0.44945931 \quad 3.77658653$

$0.55529481 \quad-7.77429914$

$0.44945931 \quad 3.07589626$

$0.55529481 \quad-4.05776691$

$0.44945931 \quad 0.27805147$

$0.55529481 \quad 5.51761198$ 
-CTBT009B-

\# phi= -62, psi= $-41, \operatorname{roots}=9$

6

$\begin{array}{rrrrr}-1.7247 & -0.1243 & -0.2242 & 1.0000 & \# \text { C1 } \\ -1.1937 & -0.2450 & -1.3192 & 1.0000 & \text { \# O1 } \\ -1.1252 & 0.5921 & 0.7783 & 1.0000 & \text { \# N1 } \\ 1.2384 & 0.5876 & -0.0127 & 1.0000 & \text { \# C2 } \\ 2.0133 & 1.0468 & -0.8384 & 1.0000 & \text { \# N2 } \\ 1.4433 & -0.5911 & 0.6333 & 1.0000 & \text { \# O2 }\end{array}$

\&TRANSITION $1->\ldots$

64

\begin{tabular}{|c|c|}
\hline \multirow{2}{*}{\multicolumn{2}{|c|}{0 . }} \\
\hline & \\
\hline $\begin{array}{l}-0.3562 \\
-1.77768791\end{array}$ & $0.1411 \quad 0.899$ \\
\hline-1.77768791 & -0.17730296 \\
\hline-1.77768791 & -0.07146750 \\
\hline-1.77768791 & -0.07146750 \\
\hline-1.67185235 & -0.17730296 \\
\hline-1.67185235 & -0.17730296 \\
\hline-1.67185235 & -0.0714675 \\
\hline-1.67185235 & -0.07146750 \\
\hline-1.24671459 & -0.29797390 \\
\hline-1.24671459 & -0.29797390 \\
\hline-1.24671459 & -0.19213843 \\
\hline-1.24671459 & -0.19213843 \\
\hline-1.14087915 & -0.29797390 \\
\hline-1.14087915 & -0.29797390 \\
\hline-1.14087915 & -0.19213843 \\
\hline-1.14087915 & -0.19213843 \\
\hline-1.17812049 & 0.53925329 \\
\hline-1.17812049 & 0.53925329 \\
\hline-1.17812049 & 0.64508873 \\
\hline-1.17812049 & 0.64508873 \\
\hline-1.07228506 & 0.53925329 \\
\hline-1.07228506 & 0.53925329 \\
\hline-1.07228506 & 0.64508873 \\
\hline-1.07228506 & 0.64508873 \\
\hline-1.71410346 & 0.74141330 \\
\hline-1.71410346 & 0.74141330 \\
\hline-1.71410346 & 0.84724873 \\
\hline-1.71410346 & 0.84724873 \\
\hline-1.60826802 & 0.74141330 \\
\hline-1.60826802 & 0.74141330 \\
\hline-1.60826802 & 0.84724873 \\
\hline-1.60826802 & 0.84724873 \\
\hline 1.18551624 & 0.53476322 \\
\hline 1.18551624 & 0.53476322 \\
\hline 1.18551624 & 0.64059871 \\
\hline 1.18551624 & 0.64059871 \\
\hline 1.29135168 & 0.53476322 \\
\hline 1.29135168 & 0.53476322 \\
\hline 1.29135168 & 0.64059871 \\
\hline 1.29135168 & 0.64059871 \\
\hline 1.96047521 & 0.99393350 \\
\hline 1.96047521 & 0.99393350 \\
\hline 1.96047521 & 1.09976900 \\
\hline 1.96047521 & 1.09976900 \\
\hline 2.06631064 & 0.99393350 \\
\hline 2.06631064 & 0.99393350 \\
\hline 2.06631064 & 1.09976900 \\
\hline 2.06631064 & 1.09976900 \\
\hline 1.39046717 & -0.64405900 \\
\hline 1.39046717 & -0.64405900 \\
\hline 1.39046717 & -0.53822351 \\
\hline 1.39046717 & -0.53822351 \\
\hline 1.49630260 & -0.64405900 \\
\hline 1.49630260 & -0.64405900 \\
\hline
\end{tabular}

1.000 \# Electr. mom., scale-fac \# Magnetic mom.

$\begin{array}{ll}-0.27713856 & 35.98752213\end{array}$

$-0.17130314 \quad-38.47553253$

$-0.27713856 \quad-32.19065475$

$-0.17130314 \quad 36.13224792$

$-0.27713856 \quad-34.90025330$

$\begin{array}{ll}-0.17130314 & 39.10438538\end{array}$

$-0.27713856 \quad 35.41856766$

$-0.17130314 \quad-41.21416092$

$-1.37213480 \quad 5.98633385$

$-1.26629949 \quad-10.51654053$

$-1.37213480 \quad 2.90012360$

$-1.26629949 \quad-0.24986145$

$-1.37213480 \quad-5.06453991$

$\begin{array}{ll}-1.26629949 & 9.42467403\end{array}$

$-1.37213480 \quad-2.41055441$

$-1.26629949 \quad-0.41086146$

$0.72545010 \quad 22.89628220$

$0.83128560-13.11017513$

$0.72545010 \quad-13.24318504$

$0.83128560 \quad 5.20048523$

$0.72545010 \quad-20.54600143$

$0.83128560 \quad 12.49708176$

$0.72545010 \quad 13.06535721$

$0.83128560 \quad-6.36593676$

$\begin{array}{ll}1.55540633 & 2.98037529\end{array}$

$1.66124189 \quad-2.33930182$

$1.55540633 \quad-2.57017851$

$1.66124189 \quad 1.94192755$

$1.55540633 \quad-3.69869637$

$\begin{array}{ll}1.66124189 & 2.91586399\end{array}$

$\begin{array}{ll}1.55540633 & 3.33561373\end{array}$

$1.66124189 \quad-2.52318406$

$-0.06571164 \quad 18.10423660$

$0.04012381 \quad-13.36912251$

$-0.06571164 \quad-15.41787148$

$0.04012381 \quad 11.53984356$

$-0.06571164 \quad-16.96733856$

$0.04012381 \quad 12.85474586$

$-0.06571164 \quad 13.91791916$

$0.04012381 \quad-10.69101334$

$-0.89135671$

$-0.78552121$

$-0.89135671$

$-0.78552121$

$-0.89135671$

$-0.78552121$

$-0.89135671$

$-0.78552121$

0.58045453

0.68628997

0.58045453

0.68628997

0.58045453

0.68628997

$-0.56197613$

1.19255996

$-0.48016053$

0.27507108

$-0.17988759$

$-0.07100125$

0.86370951

$-0.92697448$

$-0.80083889$

1. 23319566

$-0.61787617$

$-0.56924486$

1.68748522

$-1.95913398$

\section{S-319}




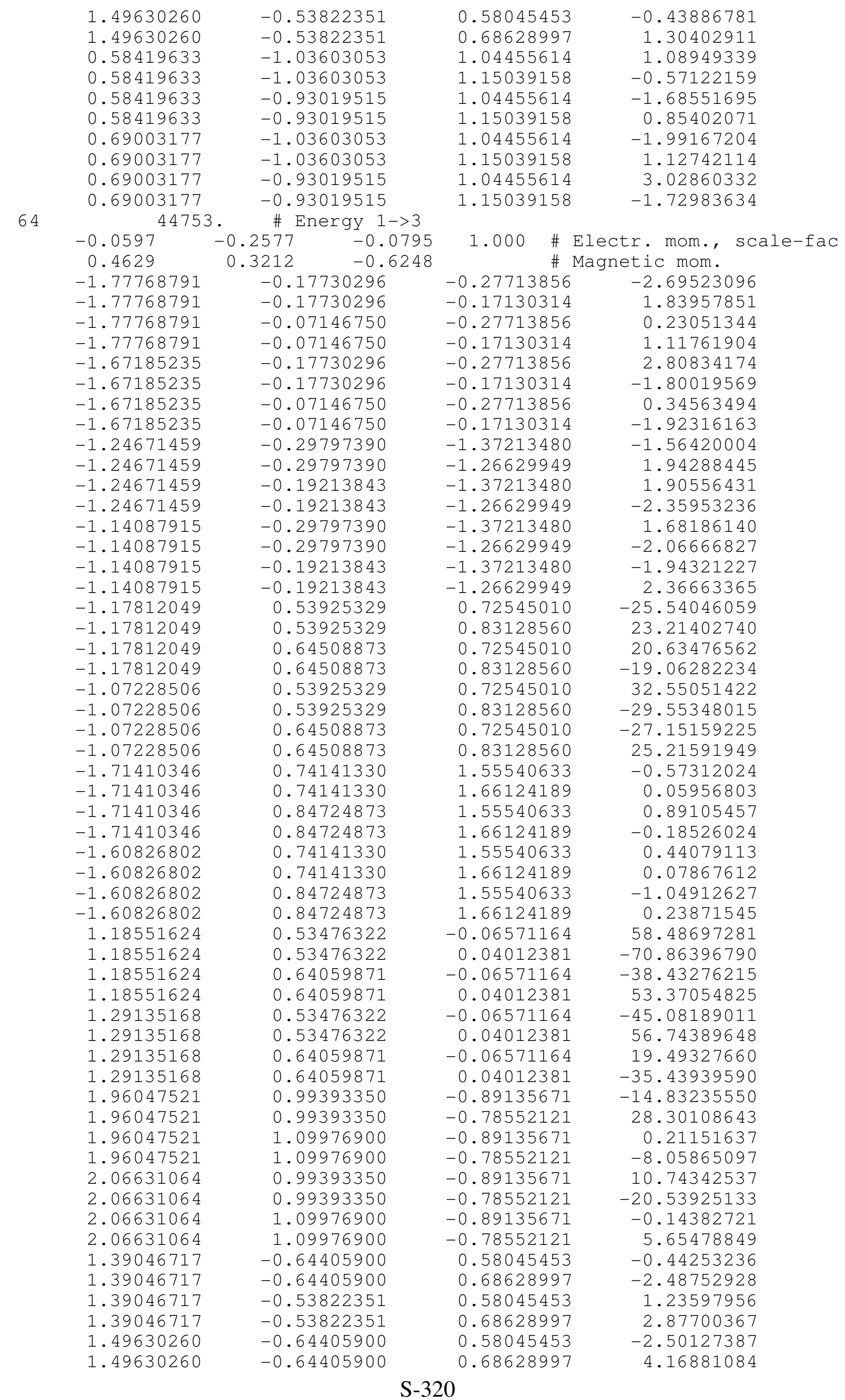




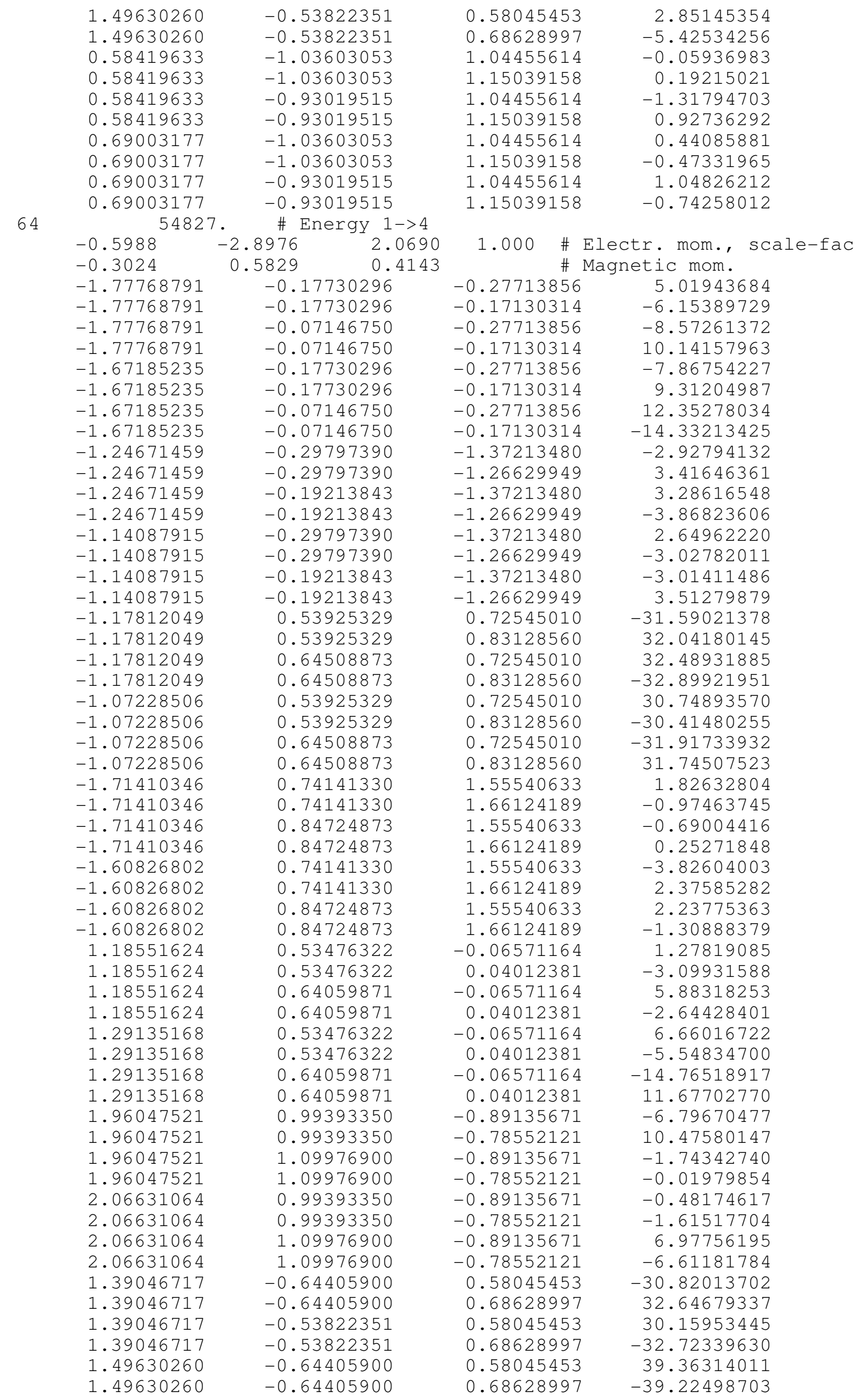




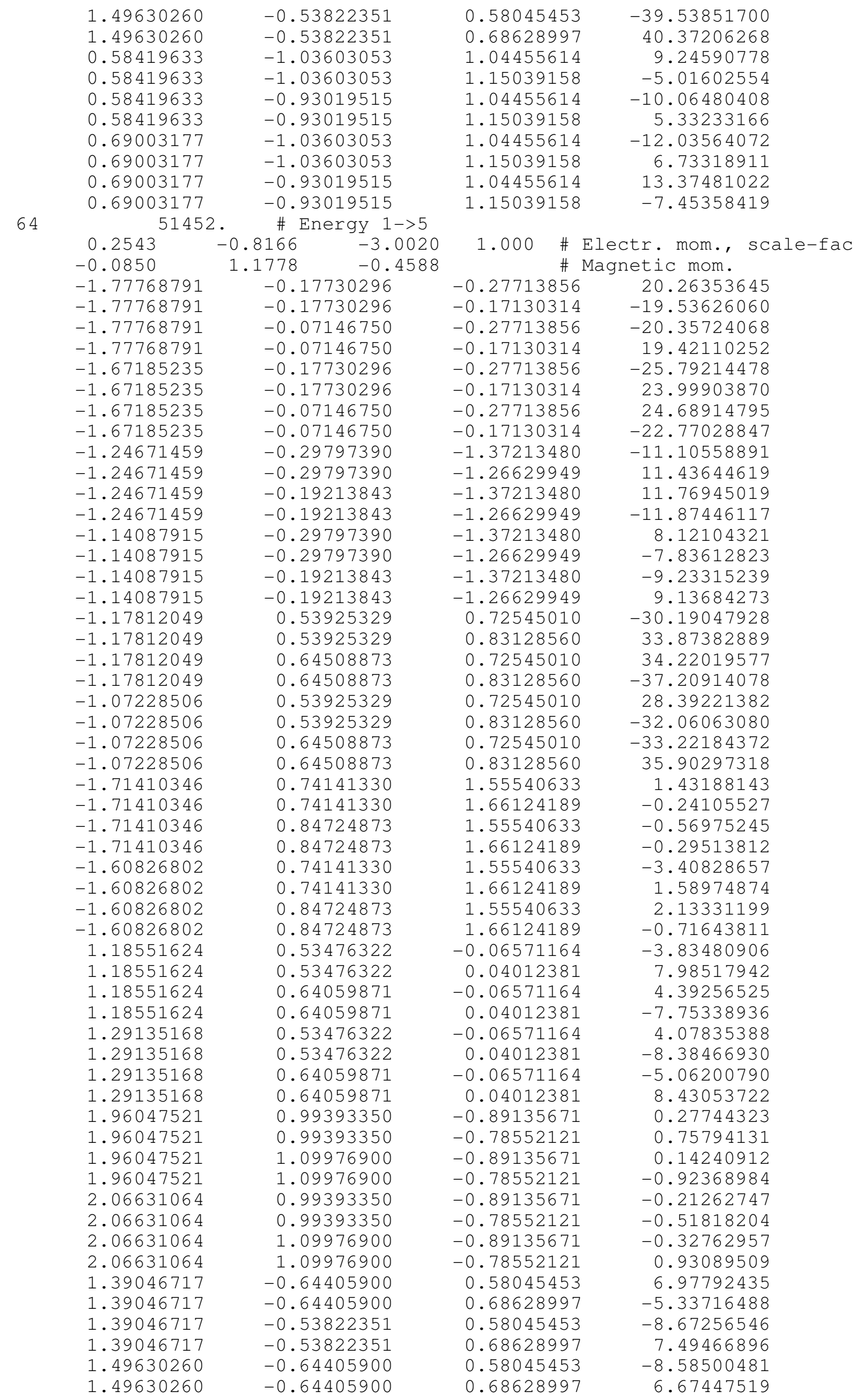

\section{S-322}




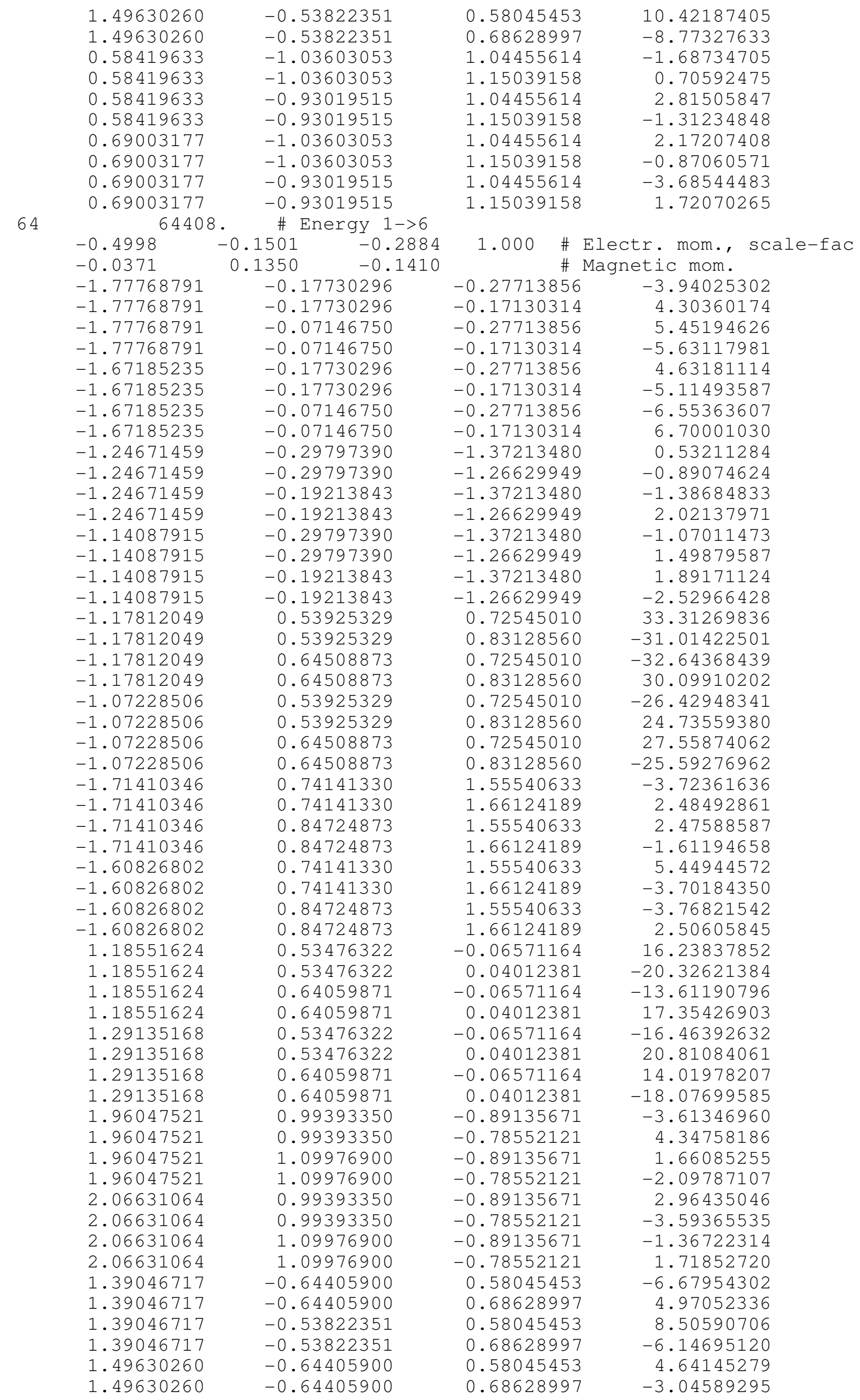

\section{S-323}




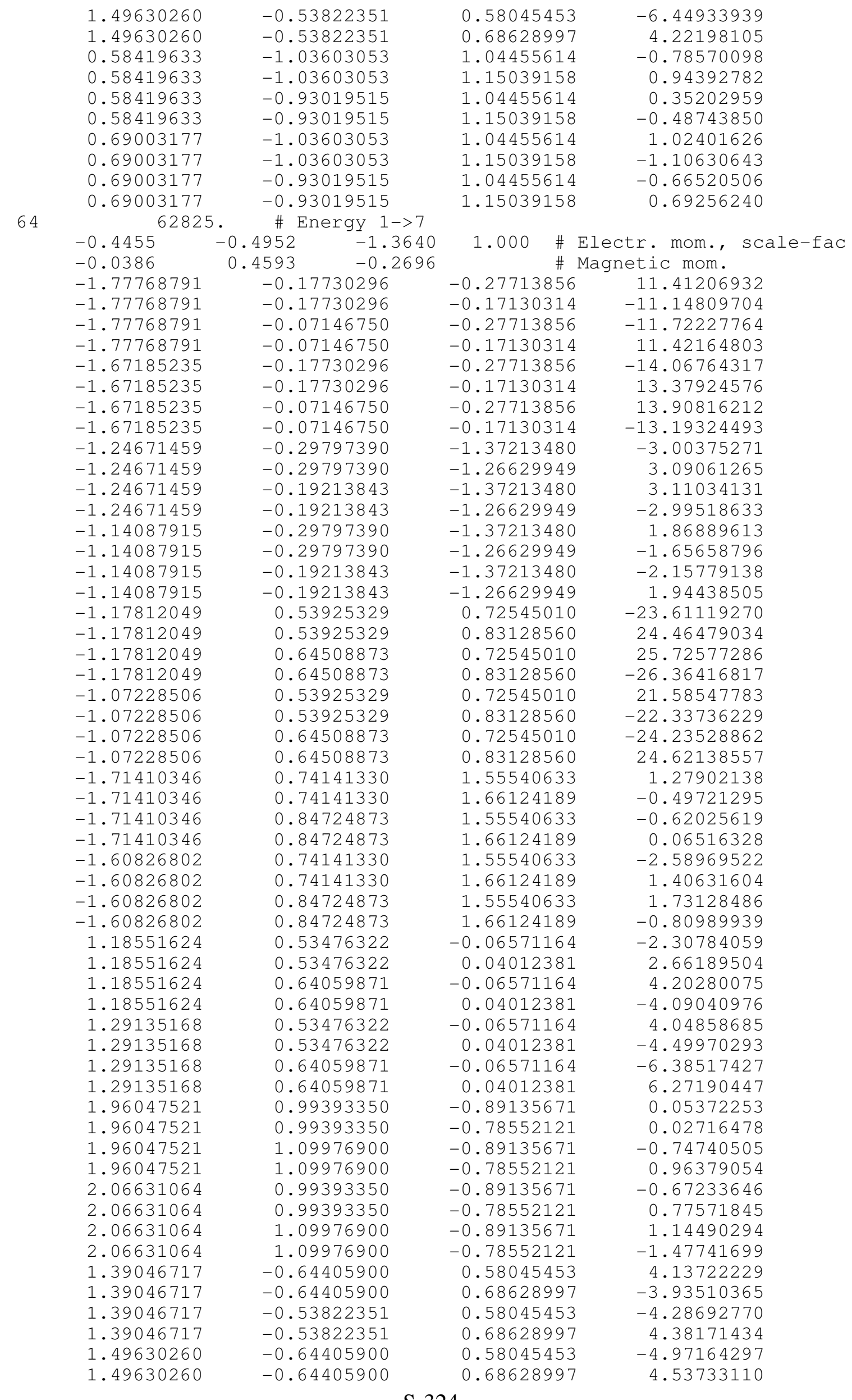

\section{S-324}




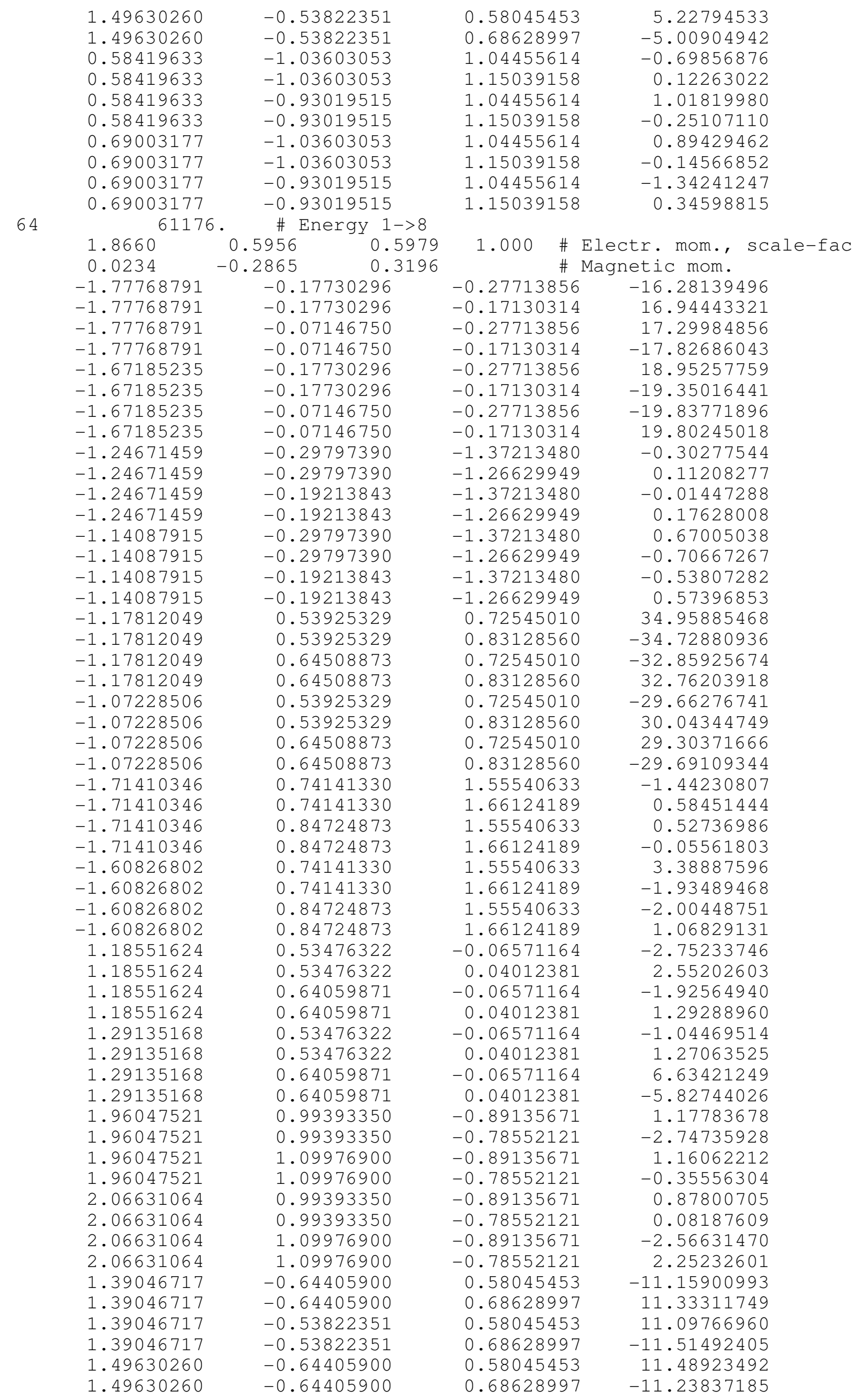

\section{S-325}




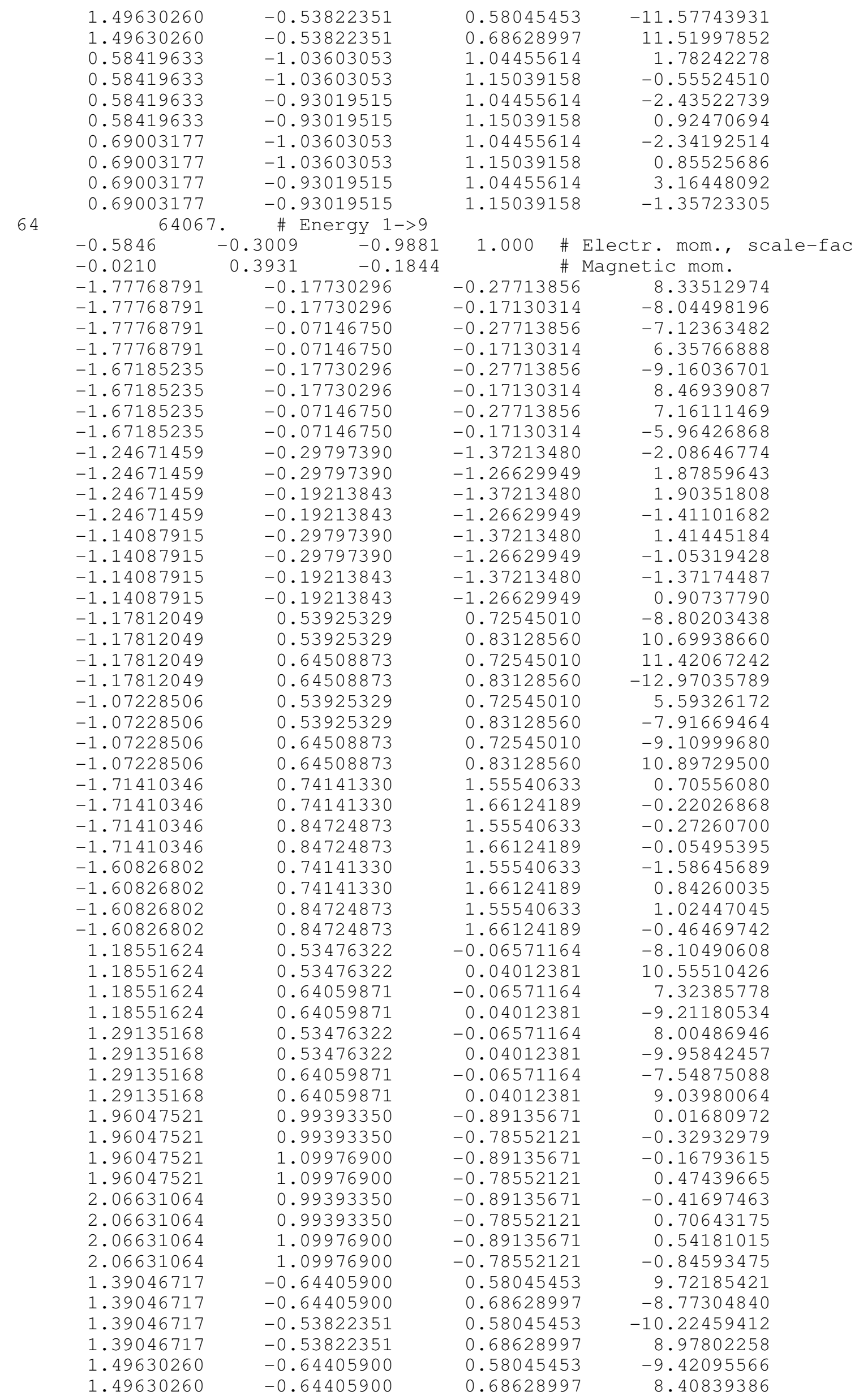




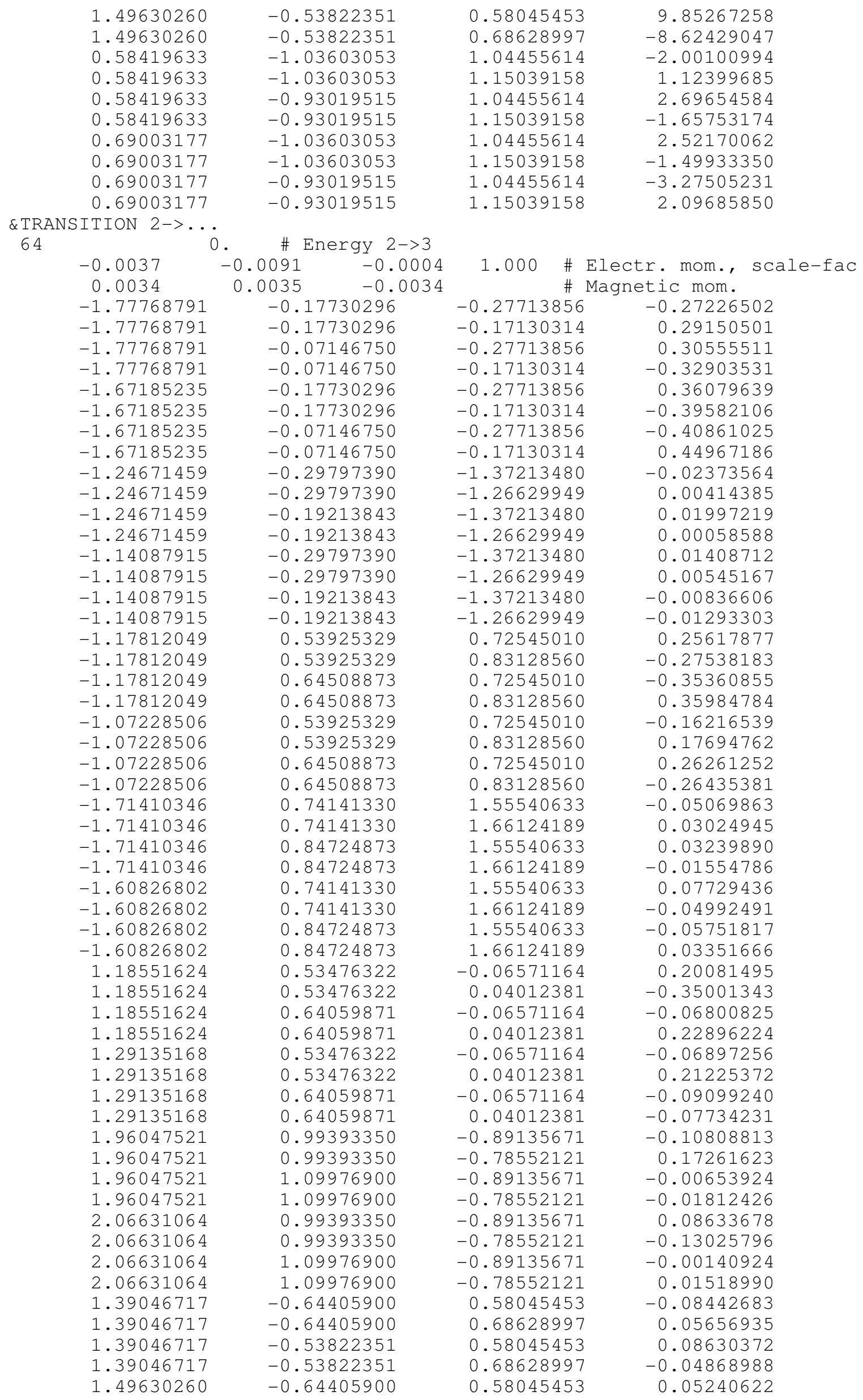

\section{S-327}




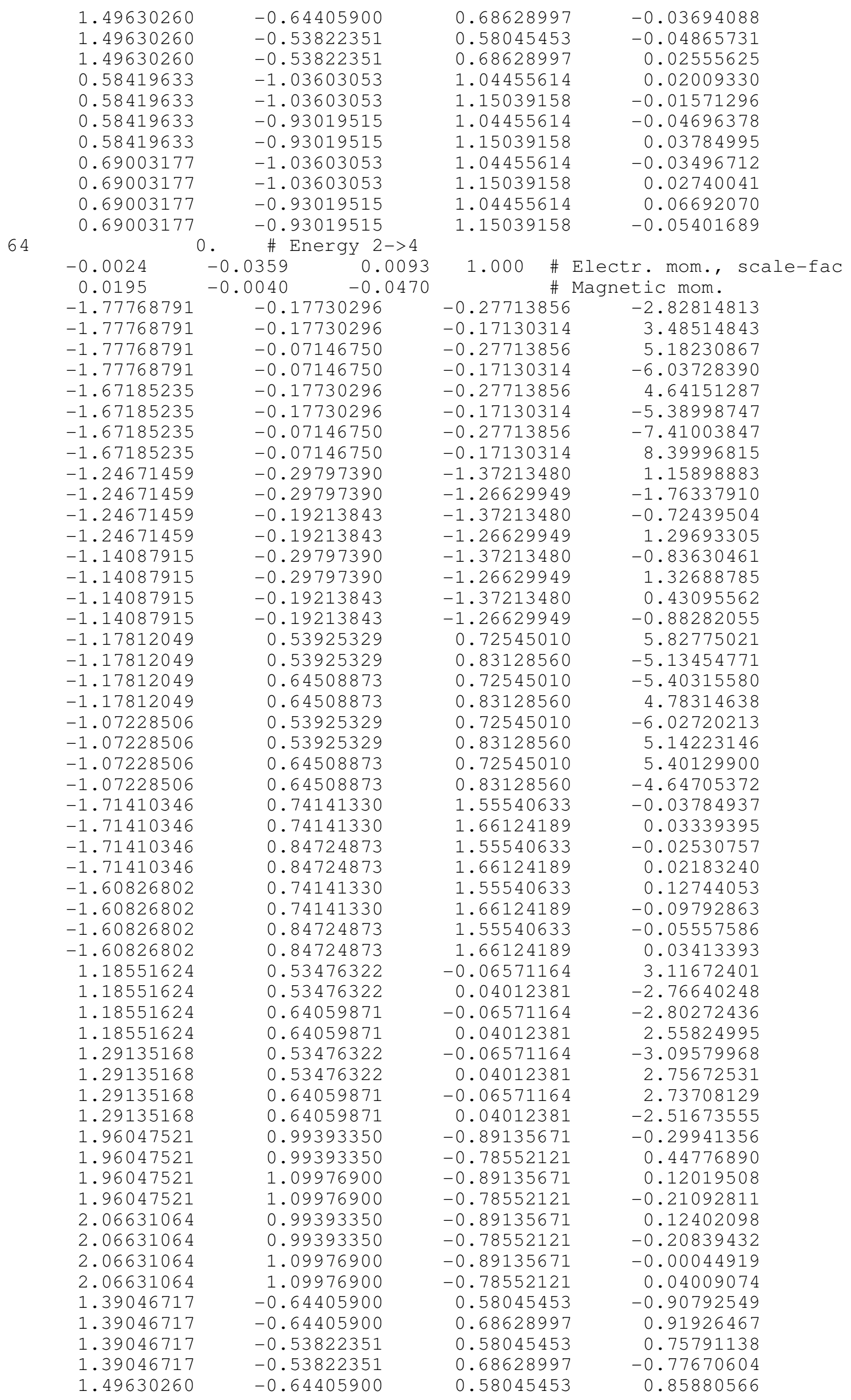

\section{S-328}




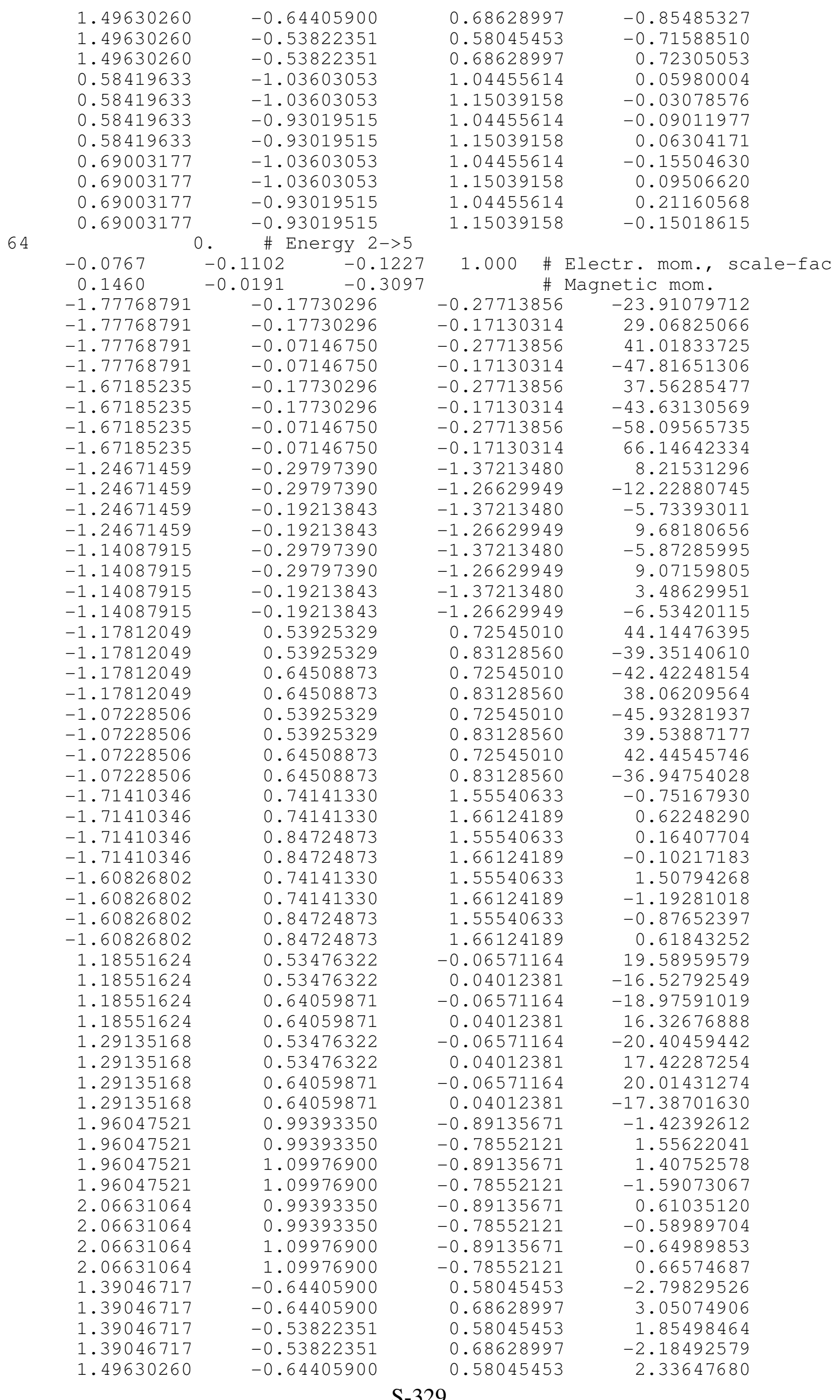




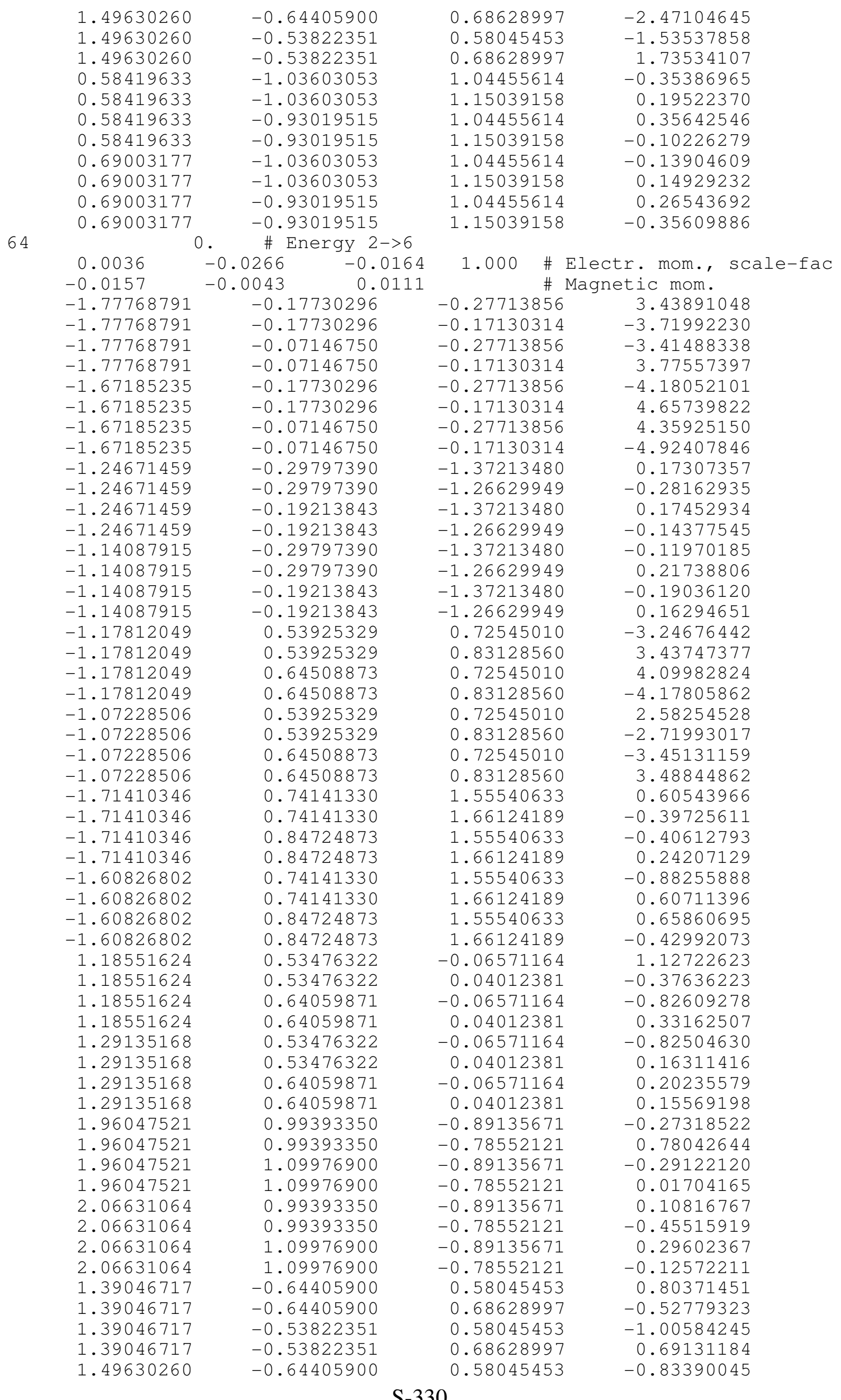




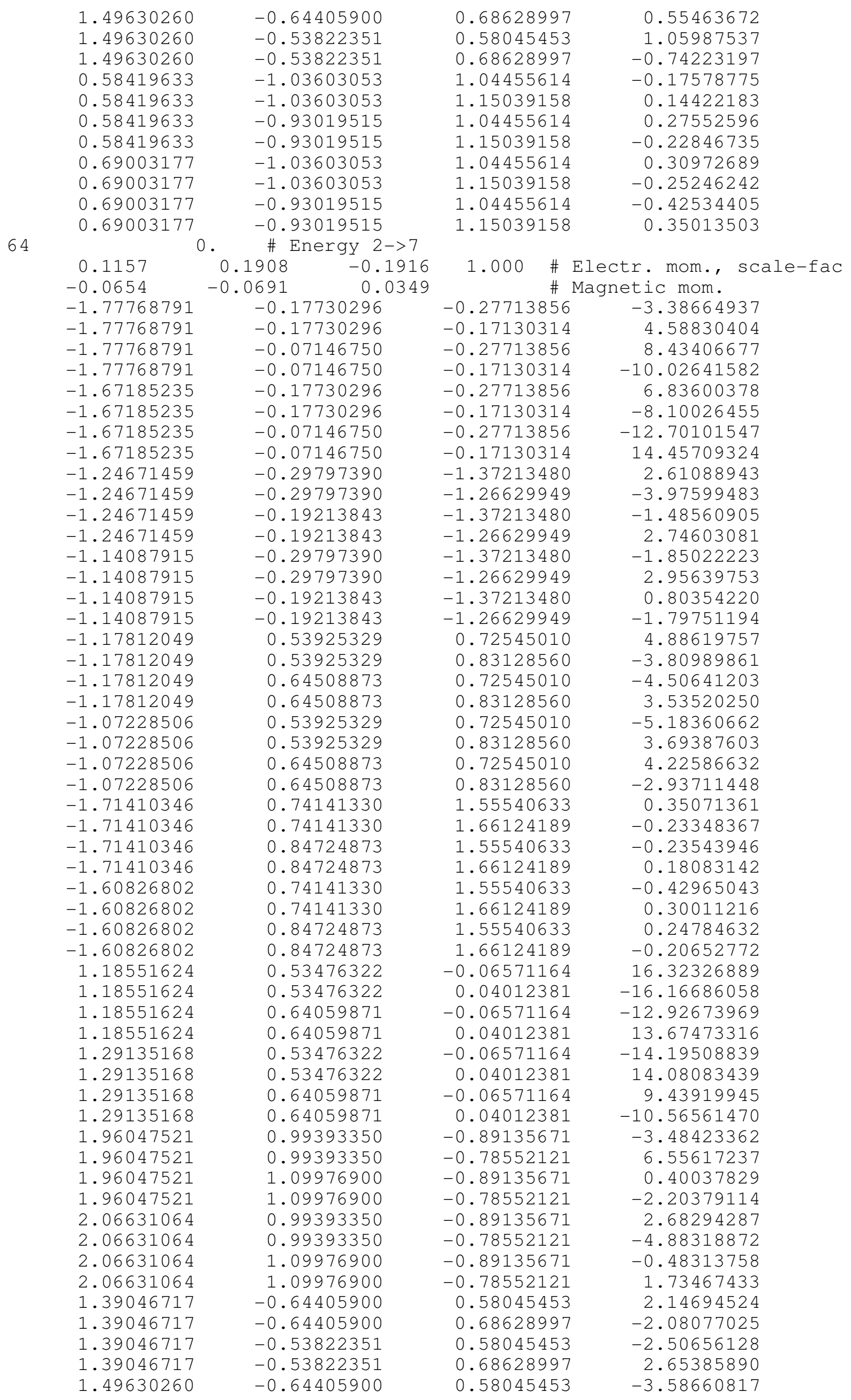

\section{S-331}




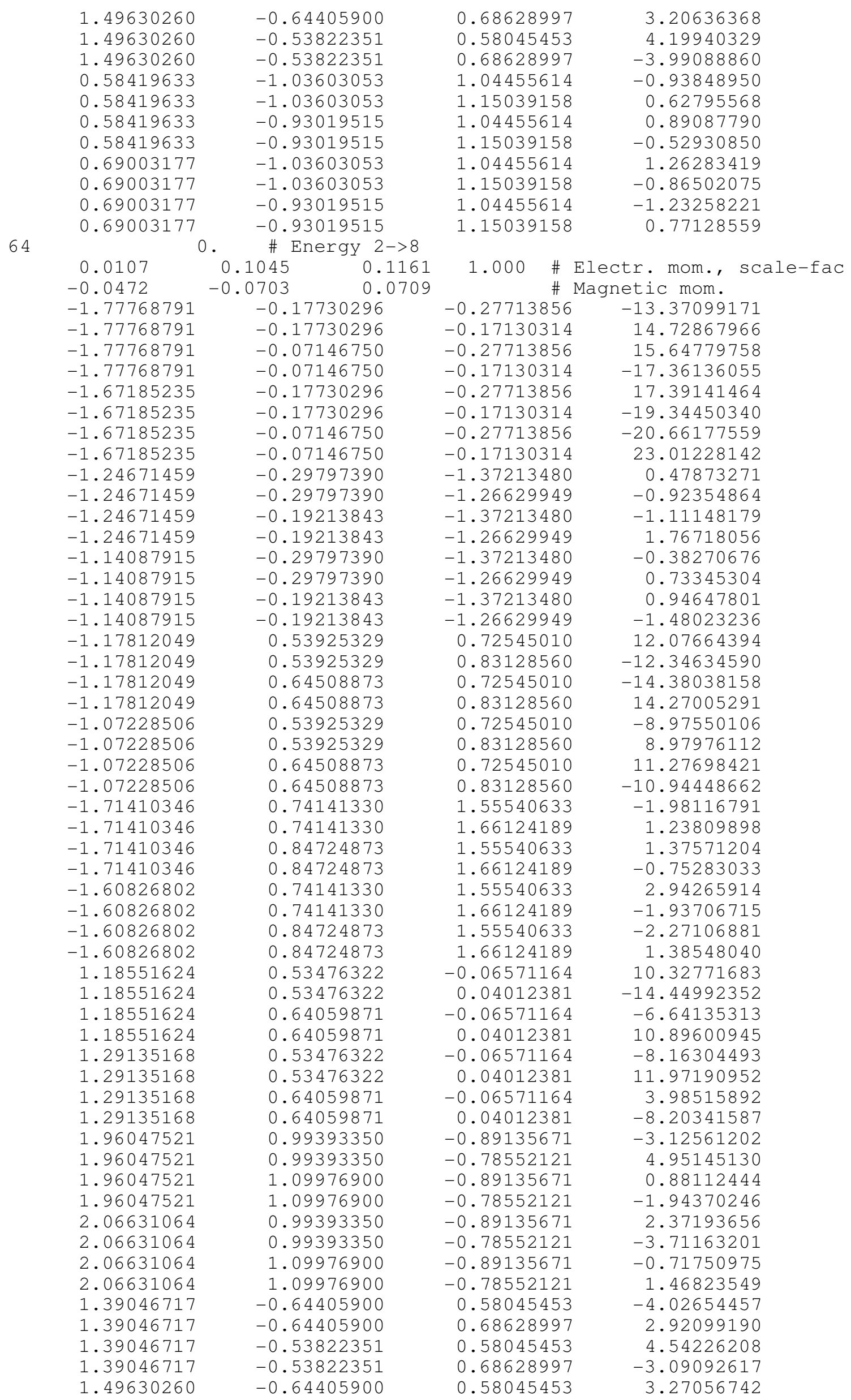

\section{S-332}




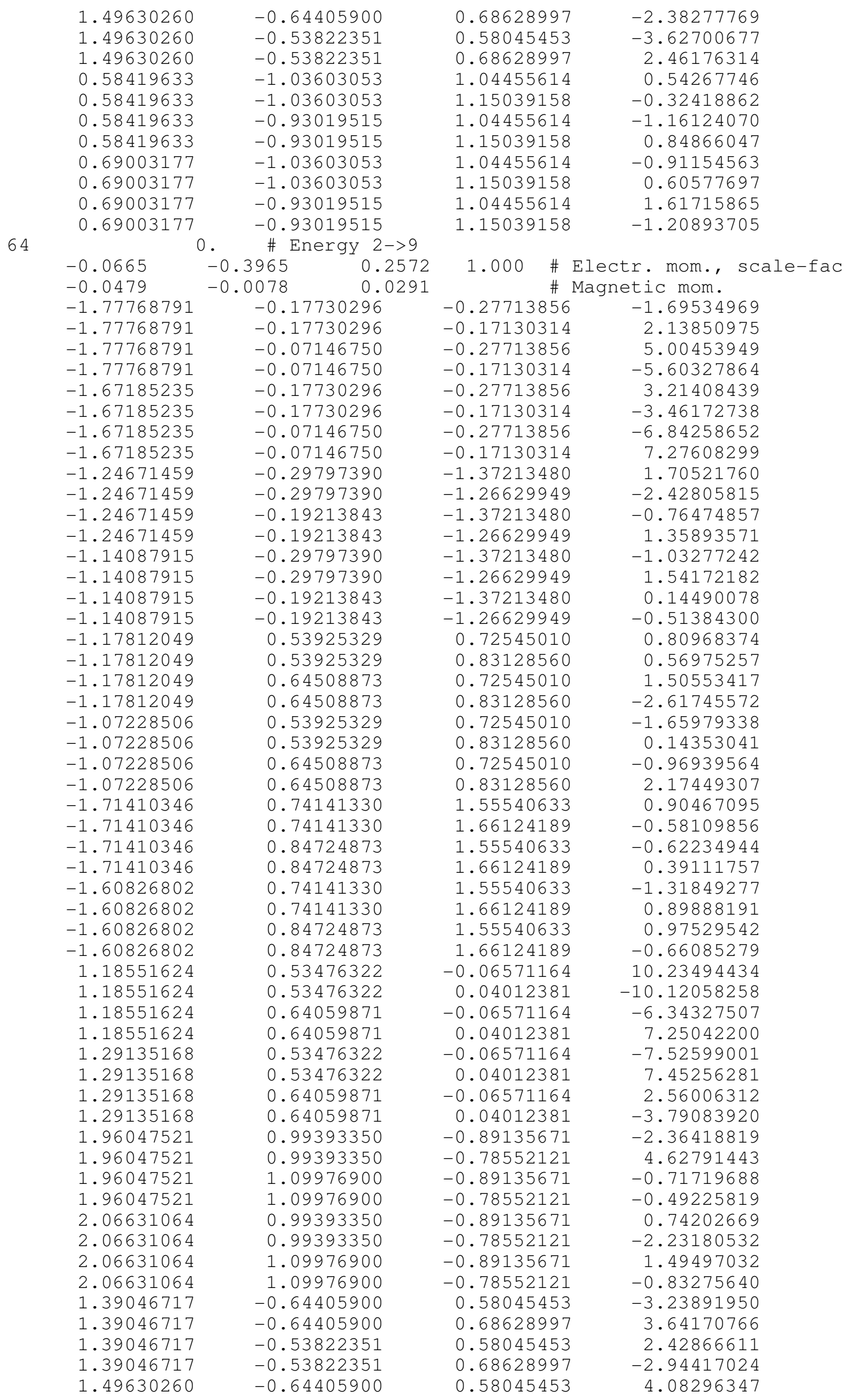

\section{S-333}




\begin{tabular}{|c|c|c|c|}
\hline & & & \\
\hline 1.49630260 & -0.64405900 & 0.68628997 & -4.34503889 \\
\hline 1.49630260 & -0.53822351 & 0.58045453 & -3.28297591 \\
\hline 1.49630260 & -0.53822351 & 0.68628997 & 3.70904160 \\
\hline 0.58419633 & -1.03603053 & 1.04455614 & 0.81771541 \\
\hline 0.58419633 & -1.03603053 & 1.15039158 & -0.40567937 \\
\hline 0.58419633 & -0.93019515 & 1.04455614 & -0.86229646 \\
\hline 0.58419633 & -0.93019515 & 1.15039158 & 0.41092530 \\
\hline 0.69003177 & -1.03603053 & 1.04455614 & -1.11520708 \\
\hline 0.69003177 & -1.03603053 & 1.15039158 & 0.56988961 \\
\hline 0.69003177 & -0.93019515 & 1.04455614 & 1.25661933 \\
\hline 0.69003177 & -0.93019515 & 1.15039158 & -0.65240341 \\
\hline$\&$ TRANSITION $3->\ldots$ & & & \\
\hline 64 & \# Energy 3->4 & & \\
\hline-0.0221 & 0.1127 & 1.000 & Electr. mom., scale-fac \\
\hline 0.2640 & -0.2528 & $\#$ & Magnetic mom. \\
\hline-1.77768791 & -0.17730296 & -0.27713856 & $5 \quad 7.64867449$ \\
\hline-1.77768791 & -0.17730296 & -0.17130314 & -7.83062887 \\
\hline-1.77768791 & -0.07146750 & -0.27713856 & -8.61569881 \\
\hline-1.77768791 & -0.07146750 & -0.17130314 & 8.64830875 \\
\hline-1.67185235 & -0.17730296 & -0.27713856 & -8.87133026 \\
\hline-1.67185235 & -0.17730296 & -0.17130314 & 8.95734882 \\
\hline-1.67185235 & -0.07146750 & -0.27713856 & 9.81497765 \\
\hline-1.67185235 & -0.07146750 & -0.17130314 & -9.66776371 \\
\hline-1.24671459 & -0.29797390 & -1.37213480 & 1.54697824 \\
\hline-1.24671459 & -0.29797390 & -1.26629949 & -1.85390580 \\
\hline-1.24671459 & -0.19213843 & -1.37213480 & -2.02130771 \\
\hline-1.24671459 & -0.19213843 & -1.26629949 & 2.42159986 \\
\hline-1.14087915 & -0.29797390 & -1.37213480 & -1.83272755 \\
\hline-1.14087915 & -0.29797390 & -1.26629949 & 2.24081087 \\
\hline-1.14087915 & -0.19213843 & -1.37213480 & 2.36422658 \\
\hline-1.14087915 & -0.19213843 & -1.26629949 & -2.88112164 \\
\hline-1.17812049 & 0.53925329 & 0.72545010 & -13.52228832 \\
\hline-1.17812049 & 0.53925329 & 0.83128560 & 14.67171383 \\
\hline-1.17812049 & 0.64508873 & 0.72545010 & 12.85163689 \\
\hline-1.17812049 & 0.64508873 & 0.83128560 & -13.78882122 \\
\hline-1.07228506 & 0.53925329 & 0.72545010 & 9.50542831 \\
\hline-1.07228506 & 0.53925329 & 0.83128560 & -11.09432030 \\
\hline-1.07228506 & 0.64508873 & 0.72545010 & -10.11682606 \\
\hline-1.07228506 & 0.64508873 & 0.83128560 & 11.47371101 \\
\hline-1.71410346 & 0.74141330 & 1.55540633 & 0.29291075 \\
\hline-1.71410346 & 0.74141330 & 1.66124189 & 0.08317867 \\
\hline-1.71410346 & 0.84724873 & 1.55540633 & 0.24636260 \\
\hline-1.71410346 & 0.84724873 & 1.66124189 & -0.38169667 \\
\hline-1.60826802 & 0.74141330 & 1.55540633 & -1.41690028 \\
\hline-1.60826802 & 0.74141330 & 1.66124189 & 0.66330087 \\
\hline-1.60826802 & 0.84724873 & 1.55540633 & 0.55727136 \\
\hline-1.60826802 & 0.84724873 & 1.66124189 & -0.13599361 \\
\hline 1.18551624 & 0.53476322 & -0.06571164 & -4.26668119 \\
\hline 1.18551624 & 0.53476322 & 0.04012381 & 7.01009655 \\
\hline 1.18551624 & 0.64059871 & -0.06571164 & 3.94153810 \\
\hline 1.18551624 & 0.64059871 & 0.04012381 & -6.55720901 \\
\hline 1.29135168 & 0.53476322 & -0.06571164 & -1.96508050 \\
\hline 1.29135168 & 0.53476322 & 0.04012381 & -0.96700108 \\
\hline 1.29135168 & 0.64059871 & -0.06571164 & 3.26142383 \\
\hline 1.29135168 & 0.64059871 & 0.04012381 & -0.02104377 \\
\hline 1.96047521 & 0.99393350 & -0.89135671 & 0.10039198 \\
\hline 1.96047521 & 0.99393350 & -0.78552121 & -3.08699965 \\
\hline 1.96047521 & 1.09976900 & -0.89135671 & 5.37739086 \\
\hline 1.96047521 & 1.09976900 & -0.78552121 & -3.91829467 \\
\hline 2.06631064 & 0.99393350 & -0.89135671 & -1.91189957 \\
\hline 2.06631064 & 0.99393350 & -0.78552121 & 4.60296202 \\
\hline 2.06631064 & 1.09976900 & -0.89135671 & -2.63434815 \\
\hline 2.06631064 & 1.09976900 & -0.78552121 & 1.21280146 \\
\hline 1.39046717 & -0.64405900 & 0.58045453 & 0.60791159 \\
\hline 1.39046717 & -0.64405900 & 0.68628997 & 0.79386878 \\
\hline 1.39046717 & -0.53822351 & 0.58045453 & -1.82336116 \\
\hline 1.39046717 & -0.53822351 & 0.68628997 & 0.04781737 \\
\hline
\end{tabular}

\section{S-334}




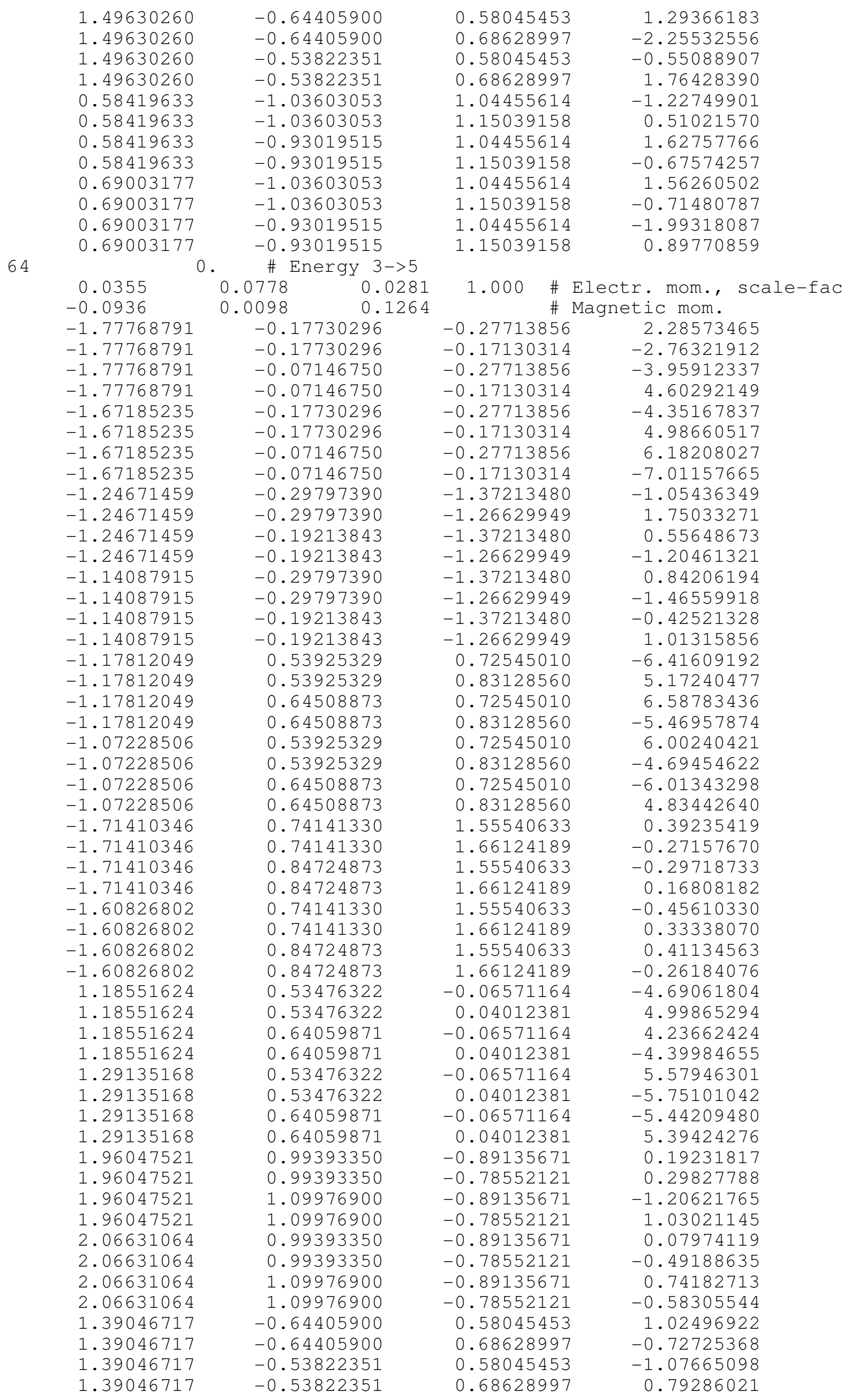

\section{S-335}




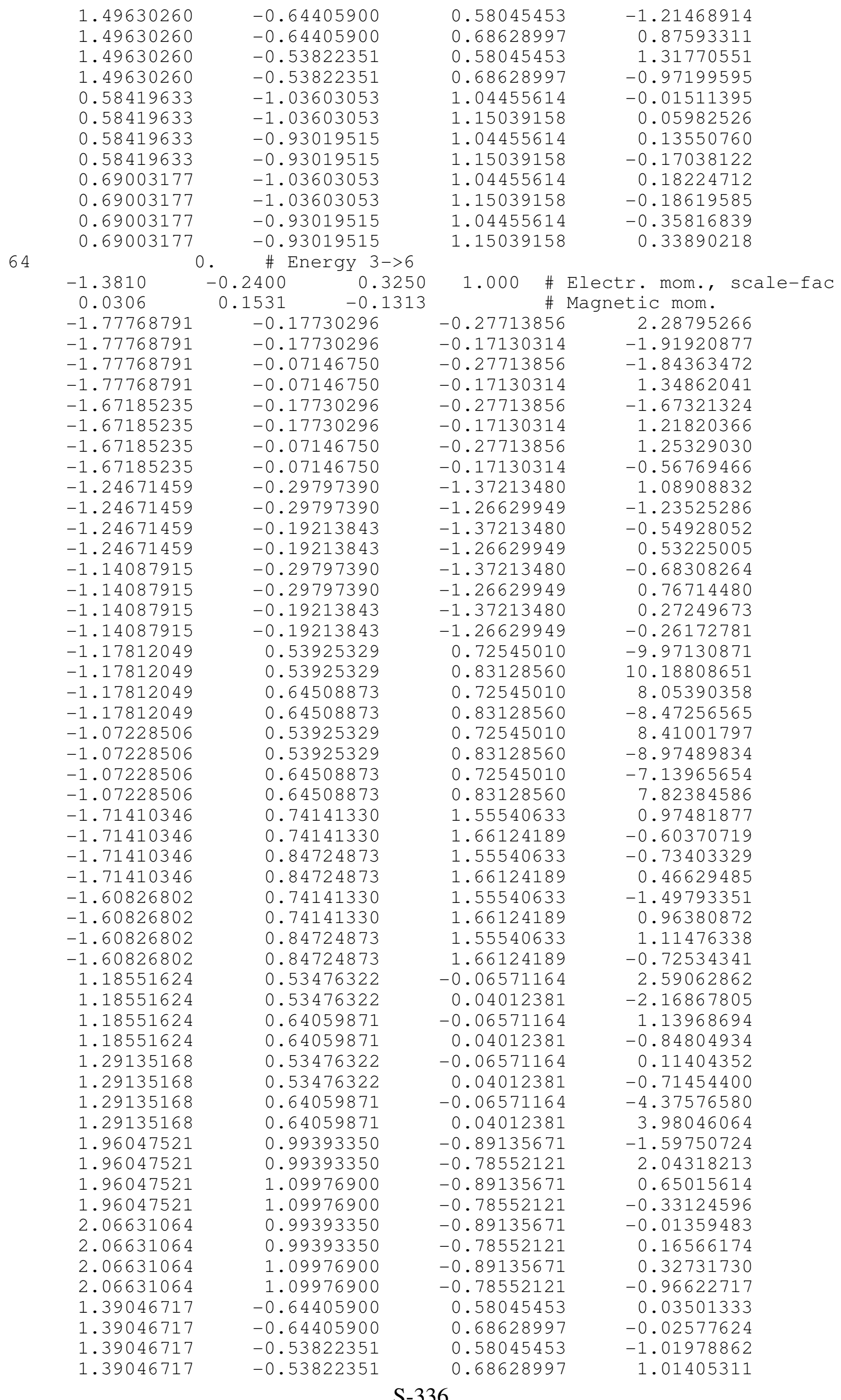




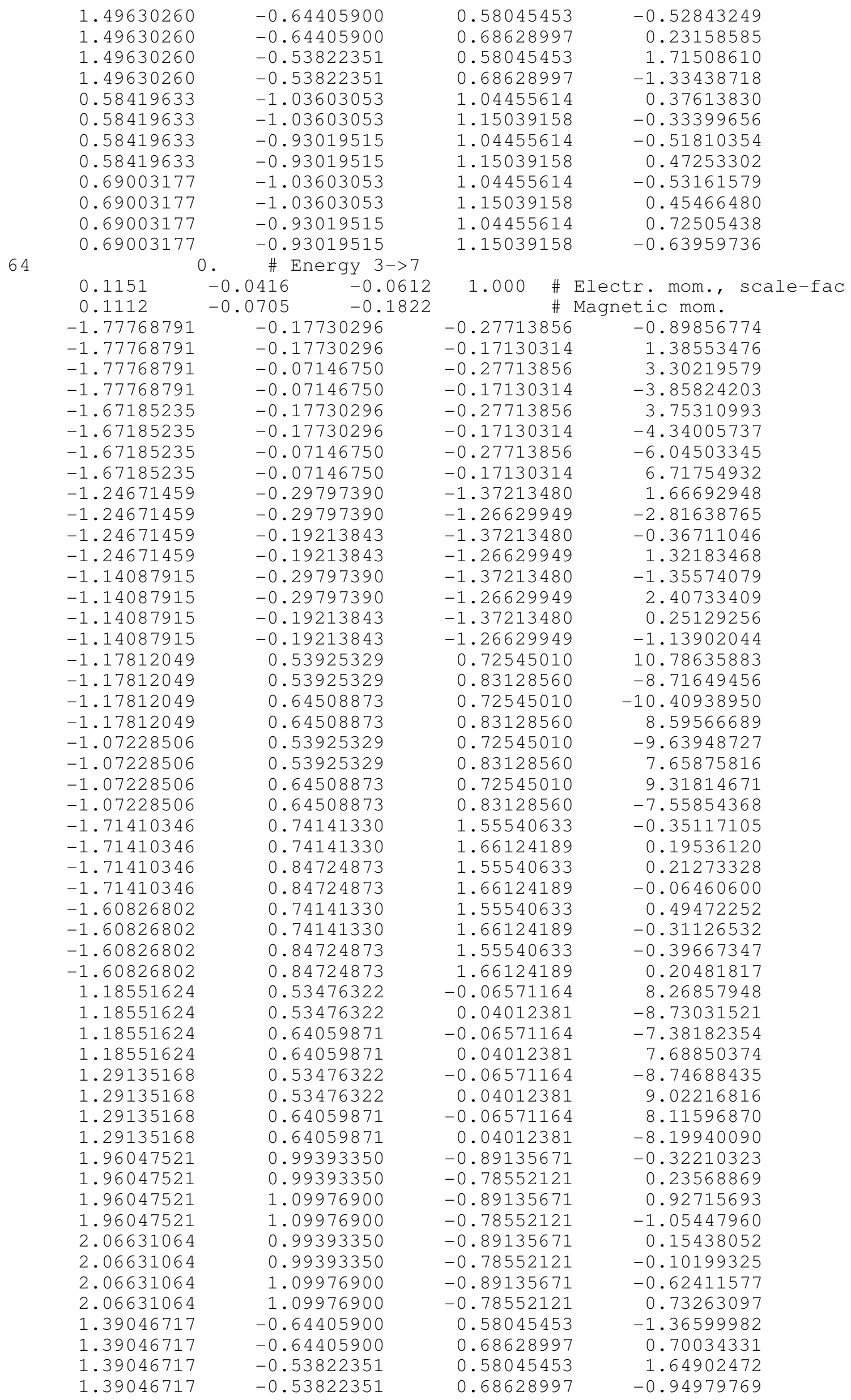

\section{S-337}




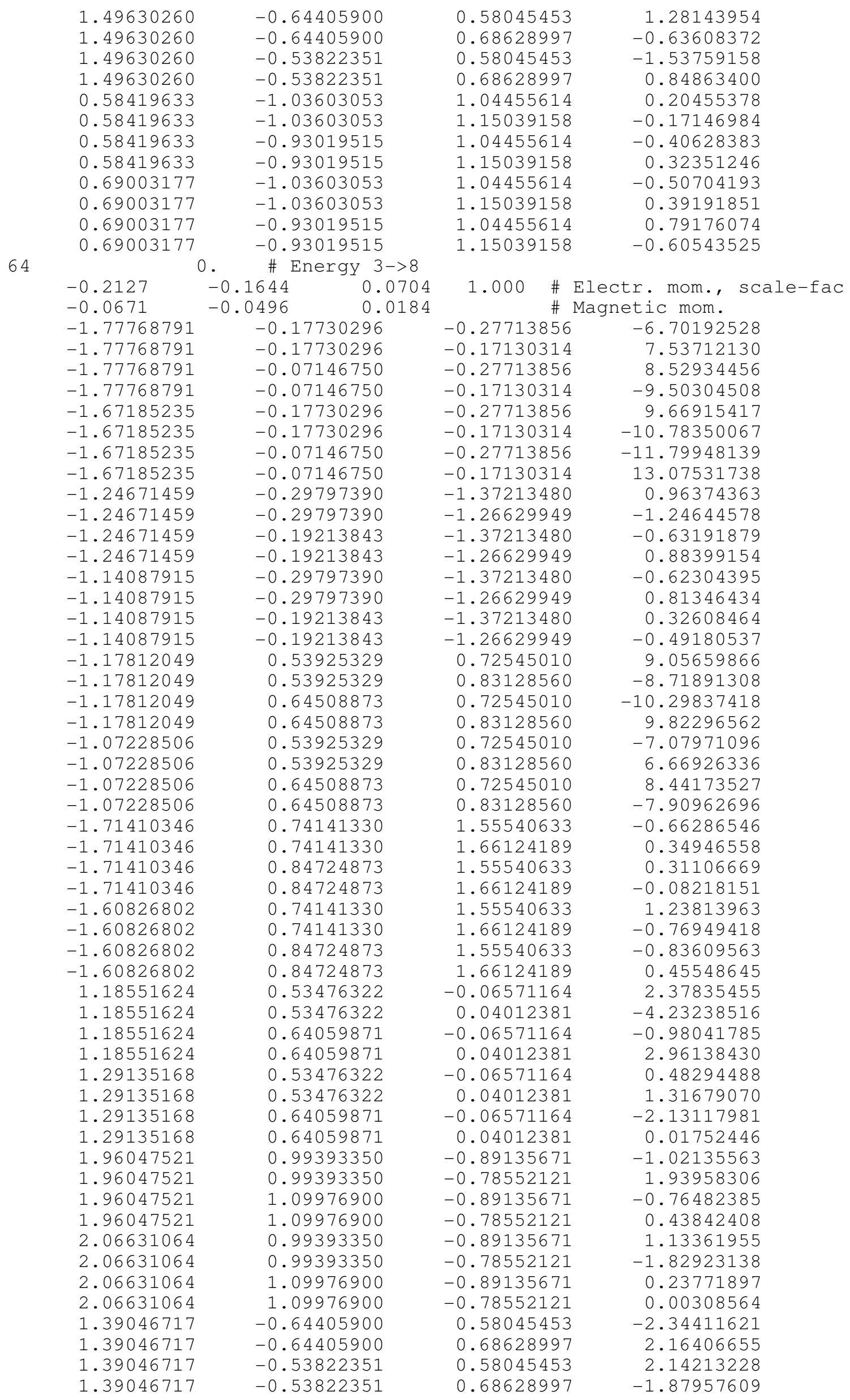

\section{S-338}




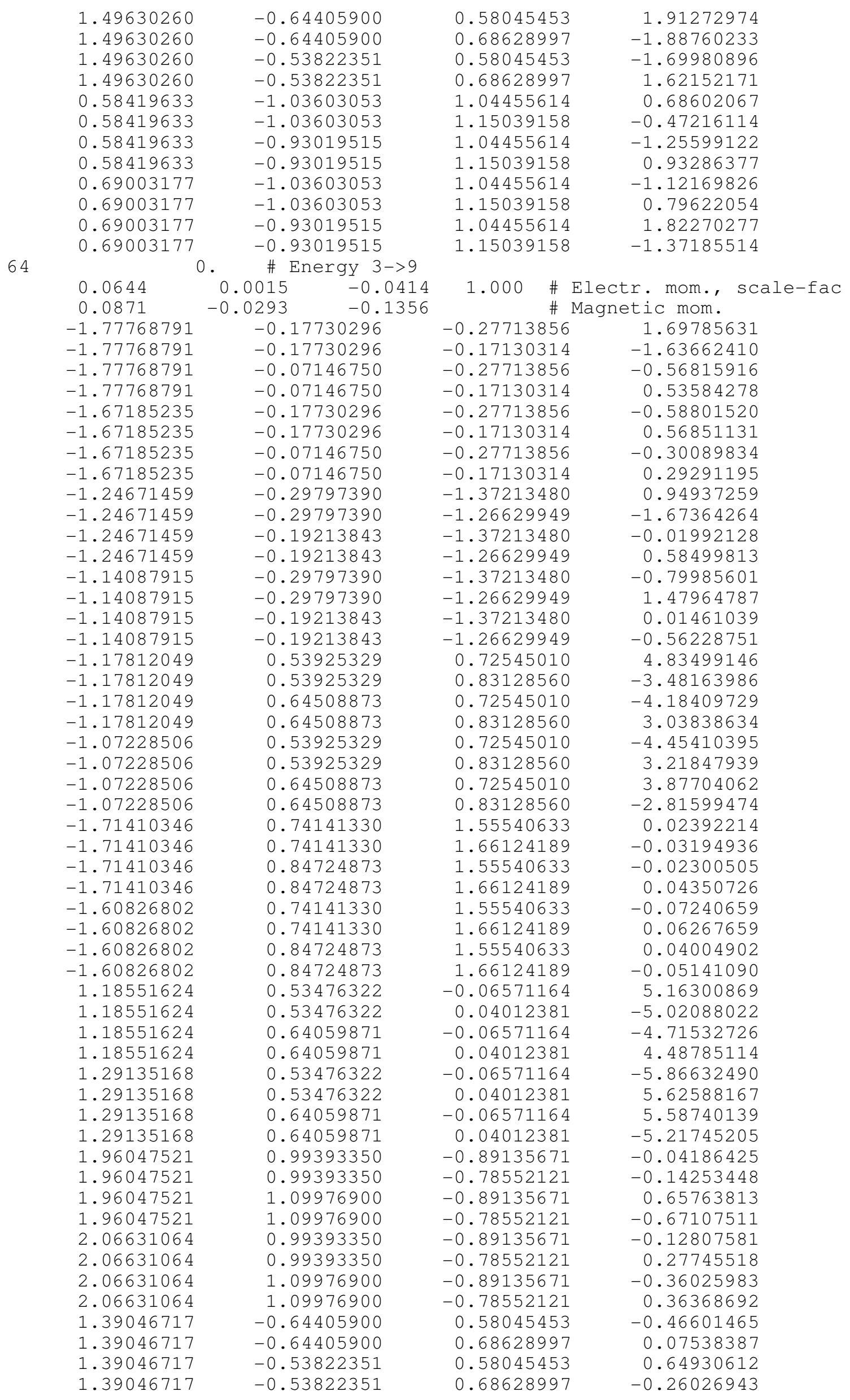

\section{S-339}




\begin{tabular}{|c|c|c|c|}
\hline & & & \\
\hline 1.49630260 & -0.64405900 & 0.58045453 & 0.46395966 \\
\hline 1.49630260 & -0.64405900 & 0.68628997 & -0.06800146 \\
\hline 1.49630260 & -0.53822351 & 0.58045453 & -0.62037009 \\
\hline 1.49630260 & -0.53822351 & 0.68628997 & 0.21576796 \\
\hline 0.58419633 & -1.03603053 & 1.04455614 & 0.04680593 \\
\hline 0.58419633 & -1.03603053 & 1.15039158 & -0.04076711 \\
\hline 0.58419633 & -0.93019515 & 1.04455614 & -0.08349936 \\
\hline 0.58419633 & -0.93019515 & 1.15039158 & 0.05763949 \\
\hline 0.69003177 & -1.03603053 & 1.04455614 & -0.16570181 \\
\hline 0.69003177 & -1.03603053 & 1.15039158 & 0.12389209 \\
\hline 0.69003177 & -0.93019515 & 1.04455614 & 0.23697817 \\
\hline 0.69003177 & -0.93019515 & 1.15039158 & -0.16303031 \\
\hline$\&$ TRANSITION $4->\ldots$ & & & \\
\hline 64 & \# Energy 4->5 & & \\
\hline-0.2801 & 0.1537 & 1.000 & Electr. mom., scale-fac \\
\hline-0.0073 & 0.0231 & 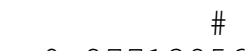 & Magnetic mom. \\
\hline-1.77768791 & -0.17730296 & -0.27713856 & -7.12291050 \\
\hline-1.77768791 & -0.17730296 & -0.17130314 & 7.24155951 \\
\hline-1.77768791 & -0.07146750 & -0.27713856 & 8.38854122 \\
\hline-1.77768791 & -0.07146750 & -0.17130314 & -8.74365711 \\
\hline-1.67185235 & -0.17730296 & -0.27713856 & 9.25311947 \\
\hline-1.67185235 & -0.17730296 & -0.17130314 & -9.31139660 \\
\hline-1.67185235 & -0.07146750 & -0.27713856 & -10.51319122 \\
\hline-1.67185235 & -0.07146750 & -0.17130314 & 10.74519634 \\
\hline-1.24671459 & -0.29797390 & -1.37213480 & 1.21697879 \\
\hline-1.24671459 & -0.29797390 & -1.26629949 & -1.27661657 \\
\hline-1.24671459 & -0.19213843 & -1.37213480 & -1.39875674 \\
\hline-1.24671459 & -0.19213843 & -1.26629949 & 1.55823541 \\
\hline-1.14087915 & -0.29797390 & -1.37213480 & -0.65082181 \\
\hline-1.14087915 & -0.29797390 & -1.26629949 & 0.55396026 \\
\hline-1.14087915 & -0.19213843 & -1.37213480 & 0.77010262 \\
\hline-1.14087915 & -0.19213843 & -1.26629949 & -0.76346725 \\
\hline-1.17812049 & 0.53925329 & 0.72545010 & 8.00079536 \\
\hline-1.17812049 & 0.53925329 & 0.83128560 & -6.52530861 \\
\hline-1.17812049 & 0.64508873 & 0.72545010 & -7.07006359 \\
\hline-1.17812049 & 0.64508873 & 0.83128560 & 5.93877506 \\
\hline-1.07228506 & 0.53925329 & 0.72545010 & -6.03090096 \\
\hline-1.07228506 & 0.53925329 & 0.83128560 & 4.74535227 \\
\hline-1.07228506 & 0.64508873 & 0.72545010 & 5.33131123 \\
\hline-1.07228506 & 0.64508873 & 0.83128560 & -4.28400707 \\
\hline-1.71410346 & 0.74141330 & 1.55540633 & -0.44384888 \\
\hline-1.71410346 & 0.74141330 & 1.66124189 & 0.24678835 \\
\hline-1.71410346 & 0.84724873 & 1.55540633 & 0.42871064 \\
\hline-1.71410346 & 0.84724873 & 1.66124189 & -0.22217363 \\
\hline-1.60826802 & 0.74141330 & 1.55540633 & 0.57121456 \\
\hline-1.60826802 & 0.74141330 & 1.66124189 & -0.32216275 \\
\hline-1.60826802 & 0.84724873 & 1.55540633 & -0.63728452 \\
\hline-1.60826802 & 0.84724873 & 1.66124189 & 0.35501808 \\
\hline 1.18551624 & 0.53476322 & -0.06571164 & 8.85262871 \\
\hline 1.18551624 & 0.53476322 & 0.04012381 & -10.98092270 \\
\hline 1.18551624 & 0.64059871 & -0.06571164 & -7.76364422 \\
\hline 1.18551624 & 0.64059871 & 0.04012381 & 10.00780487 \\
\hline 1.29135168 & 0.53476322 & -0.06571164 & -7.67643356 \\
\hline 1.29135168 & 0.53476322 & 0.04012381 & 9.73497200 \\
\hline 1.29135168 & 0.64059871 & -0.06571164 & 6.42206621 \\
\hline 1.29135168 & 0.64059871 & 0.04012381 & -8.61504555 \\
\hline 1.96047521 & 0.99393350 & -0.89135671 & -1.23835099 \\
\hline 1.96047521 & 0.99393350 & -0.78552121 & 1.46743488 \\
\hline 1.96047521 & 1.09976900 & -0.89135671 & 1.09168506 \\
\hline 1.96047521 & 1.09976900 & -0.78552121 & -1.28617942 \\
\hline 2.06631064 & 0.99393350 & -0.89135671 & 1.05779946 \\
\hline 2.06631064 & 0.99393350 & -0.78552121 & -1.23739040 \\
\hline 2.06631064 & 1.09976900 & -0.89135671 & -0.94577688 \\
\hline 2.06631064 & 1.09976900 & -0.78552121 & 1.10805786 \\
\hline 1.39046717 & -0.64405900 & 0.58045453 & 0.73286456 \\
\hline 1.39046717 & -0.64405900 & 0.68628997 & -0.87582493 \\
\hline 1.39046717 & -0.53822351 & 0.58045453 & -0.40050986 \\
\hline
\end{tabular}




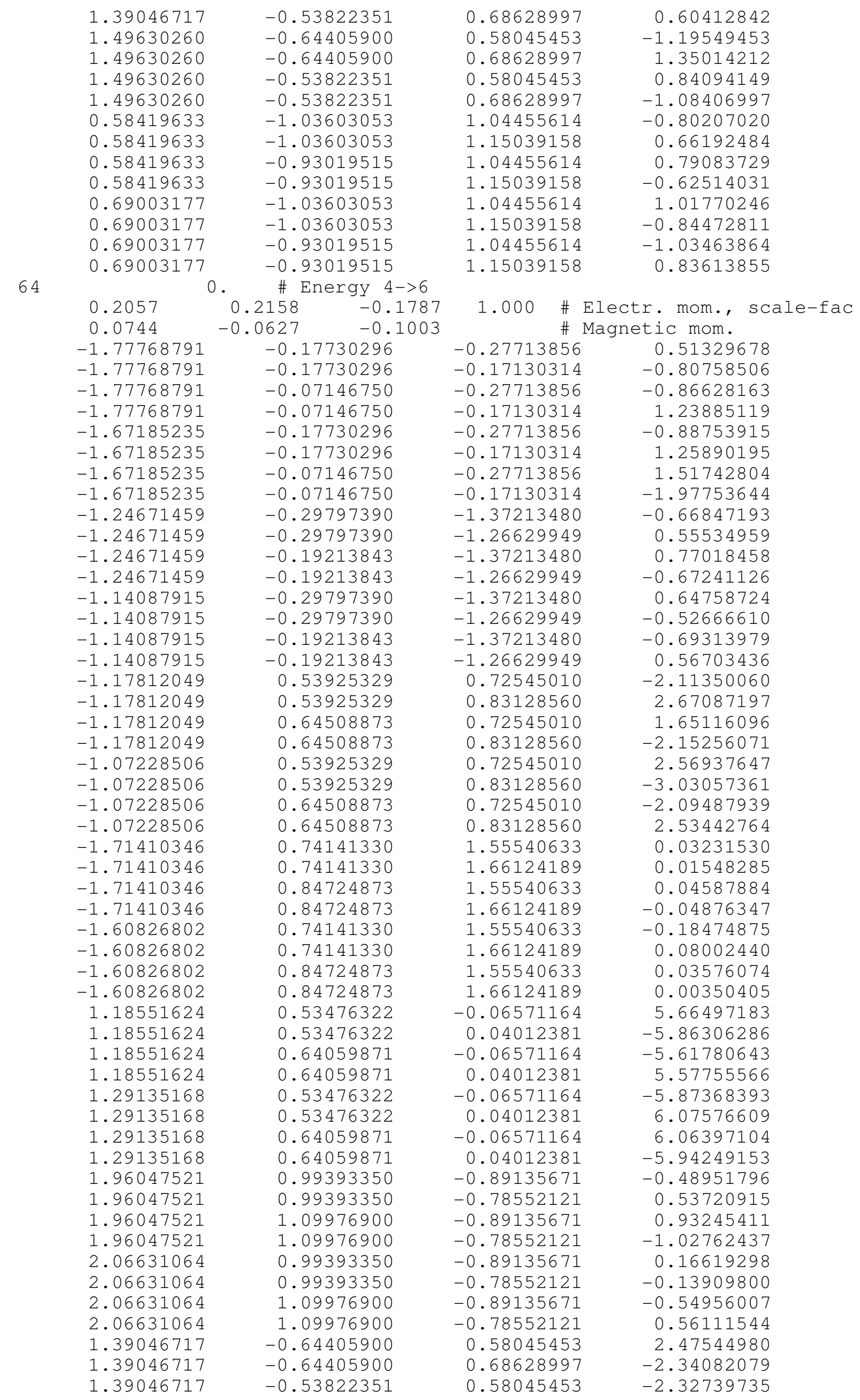




\begin{tabular}{|c|c|c|c|}
\hline $\begin{array}{l}1.39046717 \\
1.49630260 \\
1.49630260 \\
1.49630260 \\
1.49630260 \\
0.58419633 \\
0.58419633 \\
0.58419633 \\
0.58419633 \\
0.69003177 \\
0.69003177 \\
0.69003177 \\
0.69003177\end{array}$ & $\begin{array}{l}-0.53822351 \\
-0.64405900 \\
-0.64405900 \\
-0.53822351 \\
-0.53822351 \\
-1.03603053 \\
-1.03603053 \\
-0.93019515 \\
-0.93019515 \\
-1.03603053 \\
-1.03603053 \\
-0.93019515 \\
-0.93019515 \\
\#-F n e r 9 y \quad 4->7\end{array}$ & $\begin{array}{l}0.68628997 \\
0.58045453 \\
0.68628997 \\
0.58045453 \\
0.68628997 \\
1.04455614 \\
1.15039158 \\
1.04455614 \\
1.15039158 \\
1.04455614 \\
1.15039158 \\
1.04455614 \\
1.15039158\end{array}$ & $\begin{array}{r}2.39949751 \\
-2.31682134 \\
2.42196918 \\
1.81811452 \\
-2.14662313 \\
-0.66672051 \\
0.30386177 \\
1.13378918 \\
-0.55823475 \\
0.81369364 \\
-0.34534466 \\
-1.41066337 \\
0.68707991\end{array}$ \\
\hline 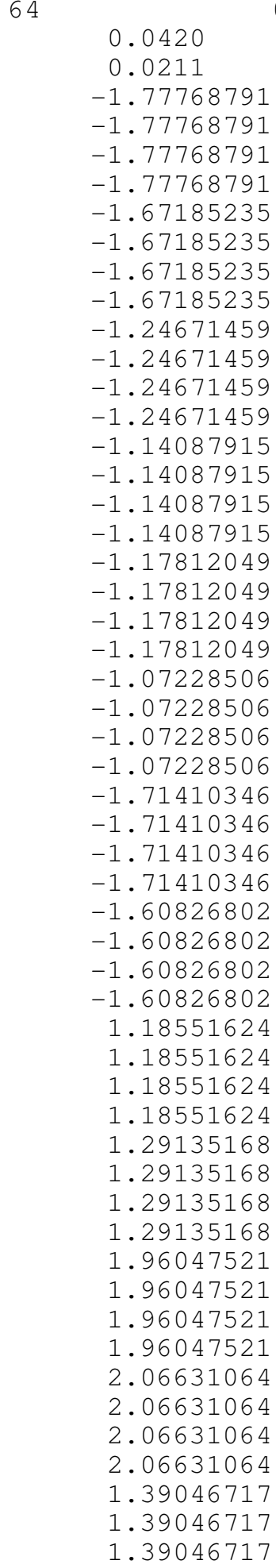 & 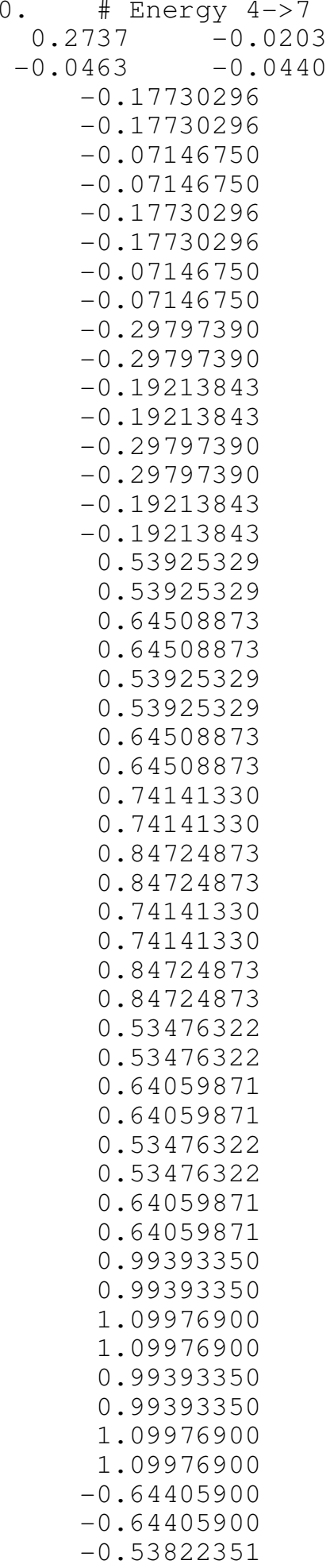 & 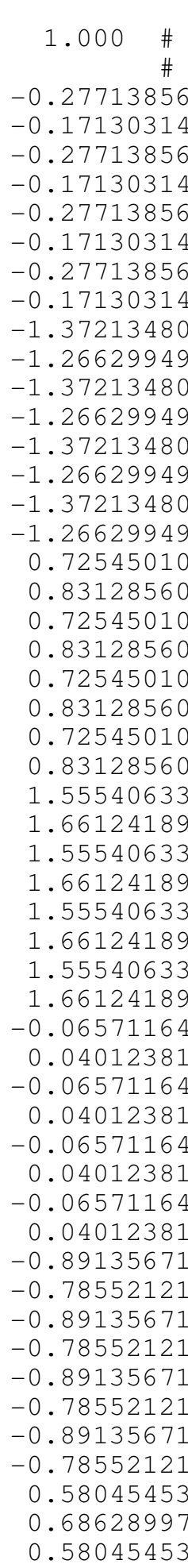 & 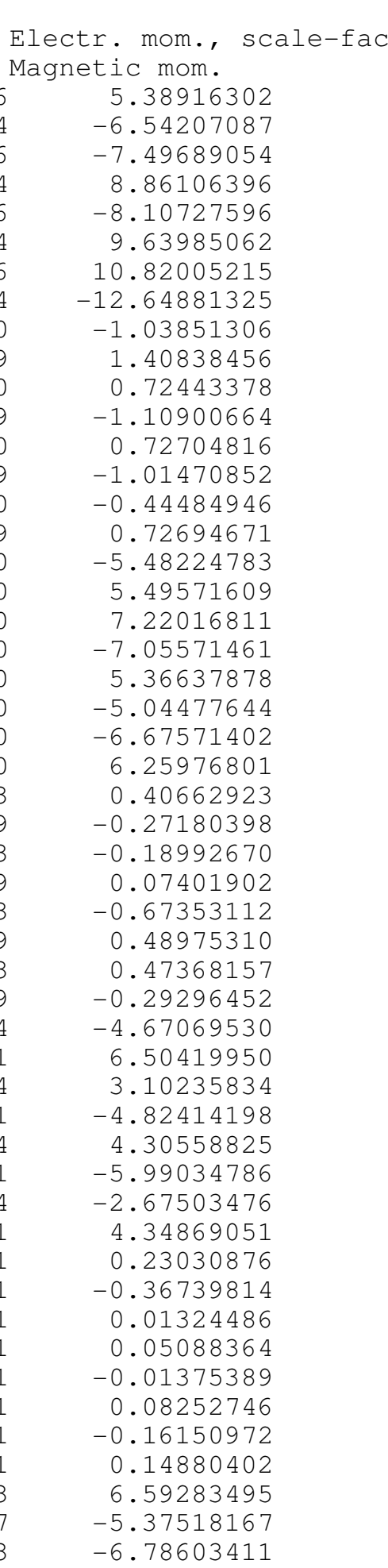 \\
\hline
\end{tabular}

\section{S-342}




\begin{tabular}{|c|c|c|c|}
\hline $\begin{array}{l}1.39046717 \\
1.49630260 \\
1.49630260 \\
1.49630260 \\
1.49630260 \\
0.58419633 \\
0.58419633 \\
0.58419633 \\
0.58419633 \\
0.69003177 \\
0.69003177 \\
0.69003177 \\
0.69003177\end{array}$ & $\begin{array}{l}-0.53822351 \\
-0.64405900 \\
-0.64405900 \\
-0.53822351 \\
-0.53822351 \\
-1.03603053 \\
-1.03603053 \\
-0.93019515 \\
-0.93019515 \\
-1.03603053 \\
-1.03603053 \\
-0.93019515 \\
-0.93019515\end{array}$ & $\begin{array}{l}0.68628997 \\
0.58045453 \\
0.68628997 \\
0.58045453 \\
0.68628997 \\
1.04455614 \\
1.15039158 \\
1.04455614 \\
1.15039158 \\
1.04455614 \\
1.15039158 \\
1.04455614 \\
1.15039158\end{array}$ & $\begin{array}{r}5.31803703 \\
-6.54618502 \\
5.41587877 \\
6.63724422 \\
-5.33146906 \\
-1.03281927 \\
0.79602331 \\
1.28941536 \\
-1.01885986 \\
1.55720890 \\
-1.20575356 \\
-1.89023066 \\
1.50191951\end{array}$ \\
\hline 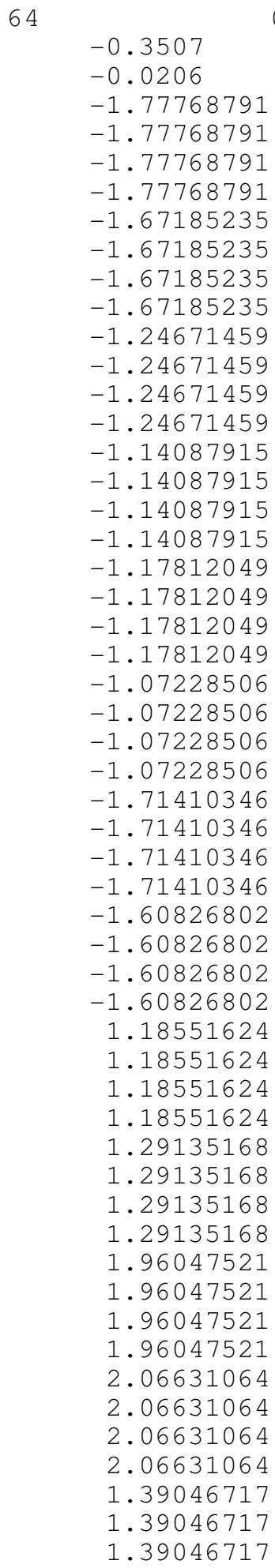 & 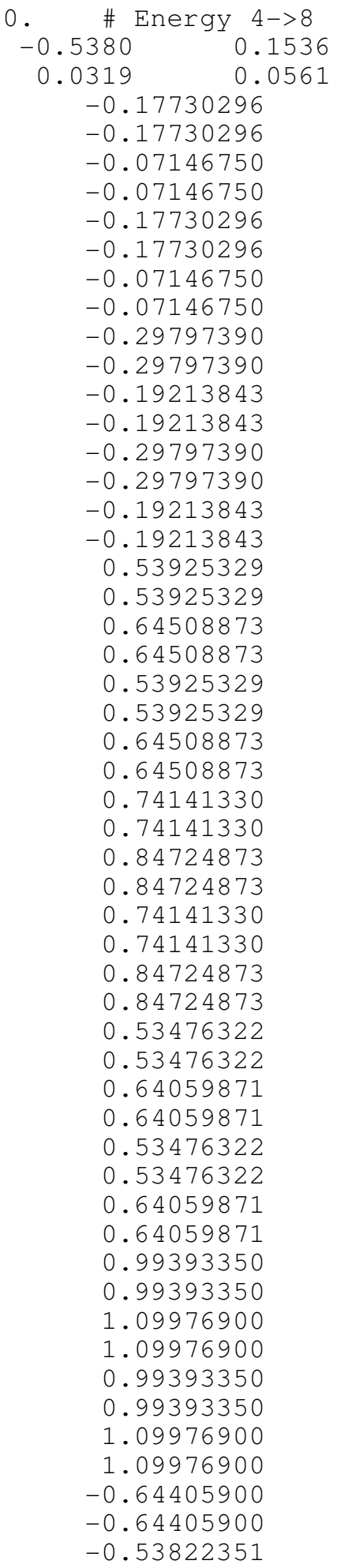 & 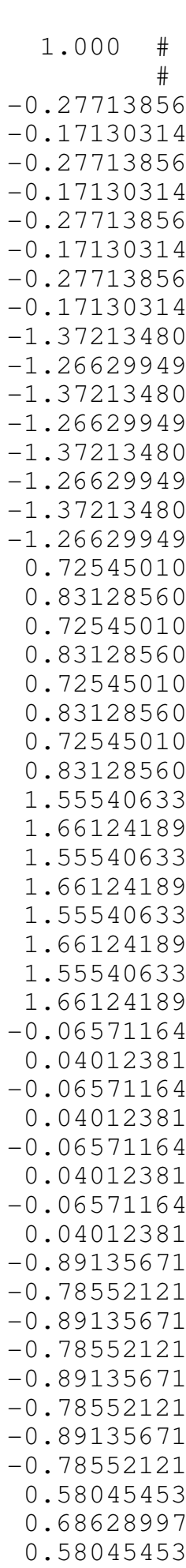 & 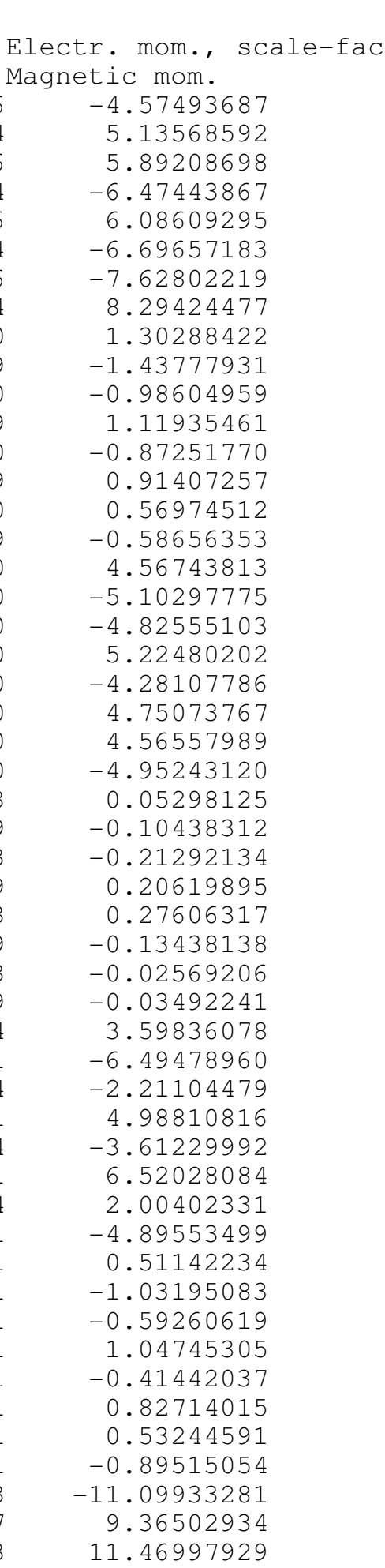 \\
\hline
\end{tabular}

\section{S-343}




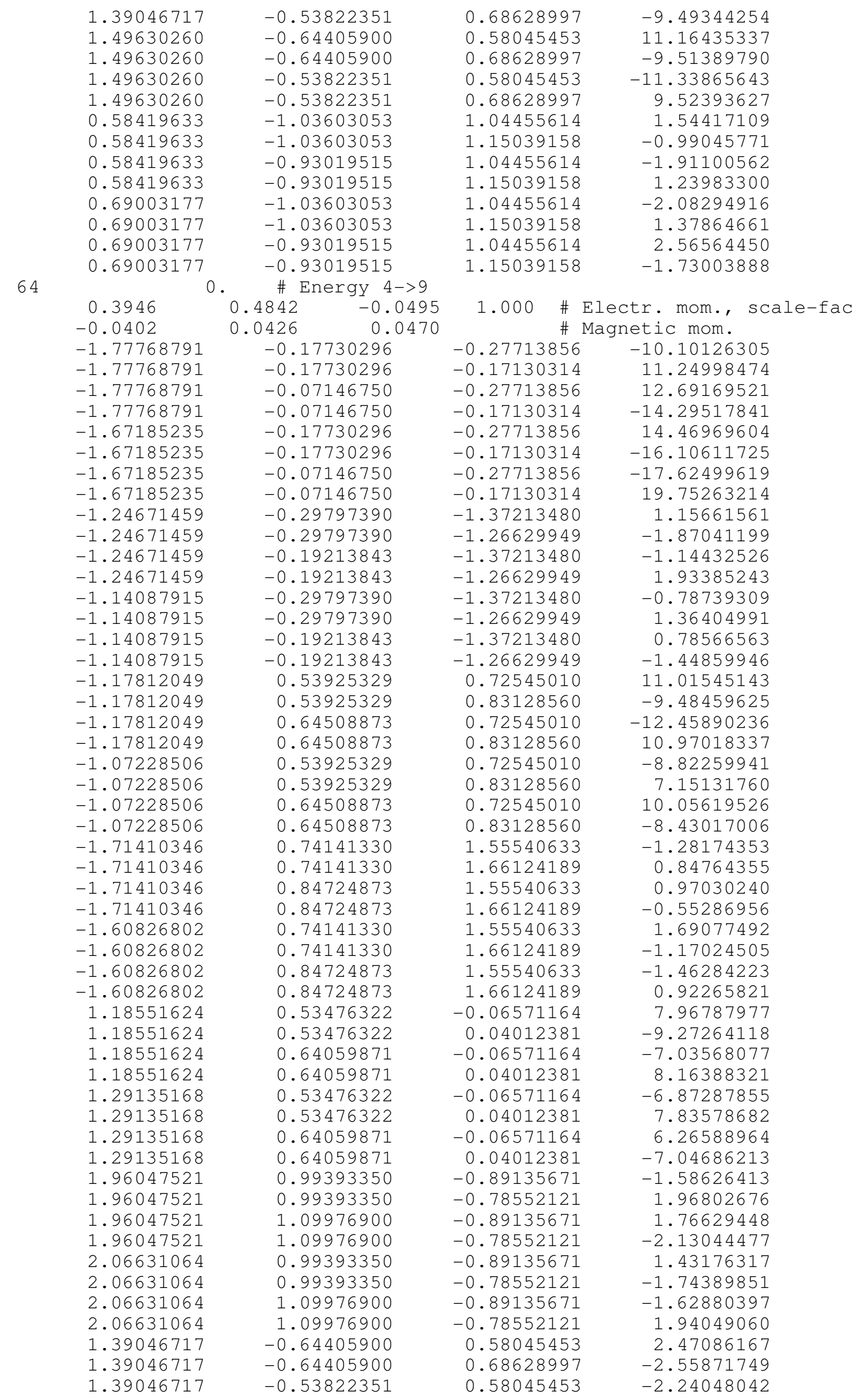

\section{S-344}




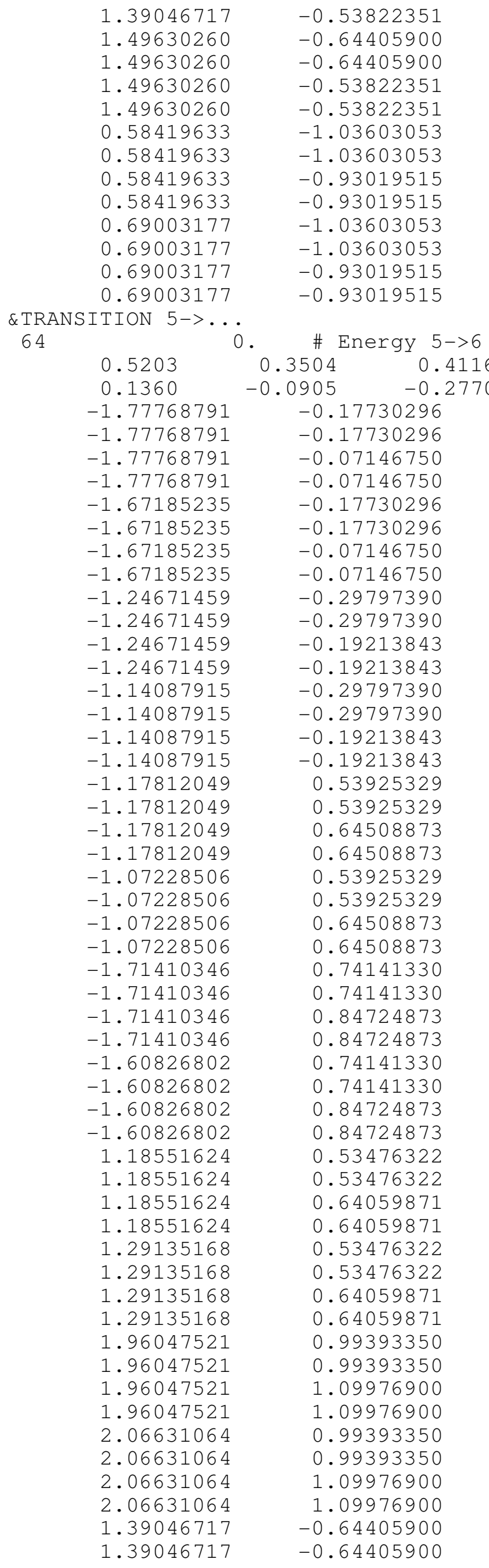

0.68628997
0.58045453
0.68628997
0.58045453
0.68628997
1.04455614
1.15039158
1.04455614
1.15039158
1.04455614
1.15039158
1.04455614
1.15039158

2.39813495

$-2.94065046$

2. 97212481

2. 64283276

$-2.76210785$

$-0.41488209$

0.22018106

0.14474769

0.04596018

0.27719927

$-0.10345363$

0.03681206

$-0.19756980$
1.000 \# Electr. mom., scale-fac $-0.27713856$

$-0.17130314$

$-0.27713856$

$-0.17130314$

$-0.27713856$

$-0.17130314$

$-0.27713856$

$-0.17130314$

$-1.37213480$

$-1.26629949$

$-1.37213480$

$-1.26629949$

$-1.37213480$

$-1.26629949$

$-1.37213480$

$-1.26629949$

0.72545010

0.83128560

0.72545010

0.83128560

0.72545010

0.83128560

0.72545010

0.83128560

1.55540633

1.66124189

1.55540633

1.66124189

1.55540633

1.66124189

1.55540633

1.66124189

$-0.06571164$

0.04012381

$-0.06571164$

0.04012381

$-0.06571164$

0.04012381

$-0.06571164$

0.04012381

$-0.89135671$

$-0.78552121$

$-0.89135671$

$-0.78552121$

$-0.89135671$

$-0.78552121$

$-0.89135671$

$-0.78552121$

0.58045453

0.68628997
$-17.3196945$

19.01471329

26.41875839

$-29.13257790$

26.26166534

$-28.46916199$

$-36.57557678$

39.86987686

1.83461905

$-0.30351338$

3. 71763897

$-1.31659806$

4.43048286

$-0.03992995$

$-2.98942685$

38.16905212

$-32.62601471$

$-36.58641052$

31.79534721

$-35.25296402$

29.39844322

33.49005127

$-28.42249107$

$-1.90161586$

1.29060256

1. 24422634

$-0.73232180$

2. 65512753

$-1.85254431$

$-2.05772877$

1.32050931

16.44960213

$-17.67097664$

$-14.71503162$

15.96530342

$-16.05295372$

16.92296982

14.56054688

$-15.46913338$

$-1.66084647$

2. 26160145

1. 91328120

$-2.56654382$

1.39434993

$-1.89127171$

$-1.64496636$

2.19515610

$-4.73050785$

4. 17643261
$-5.37157059$ 


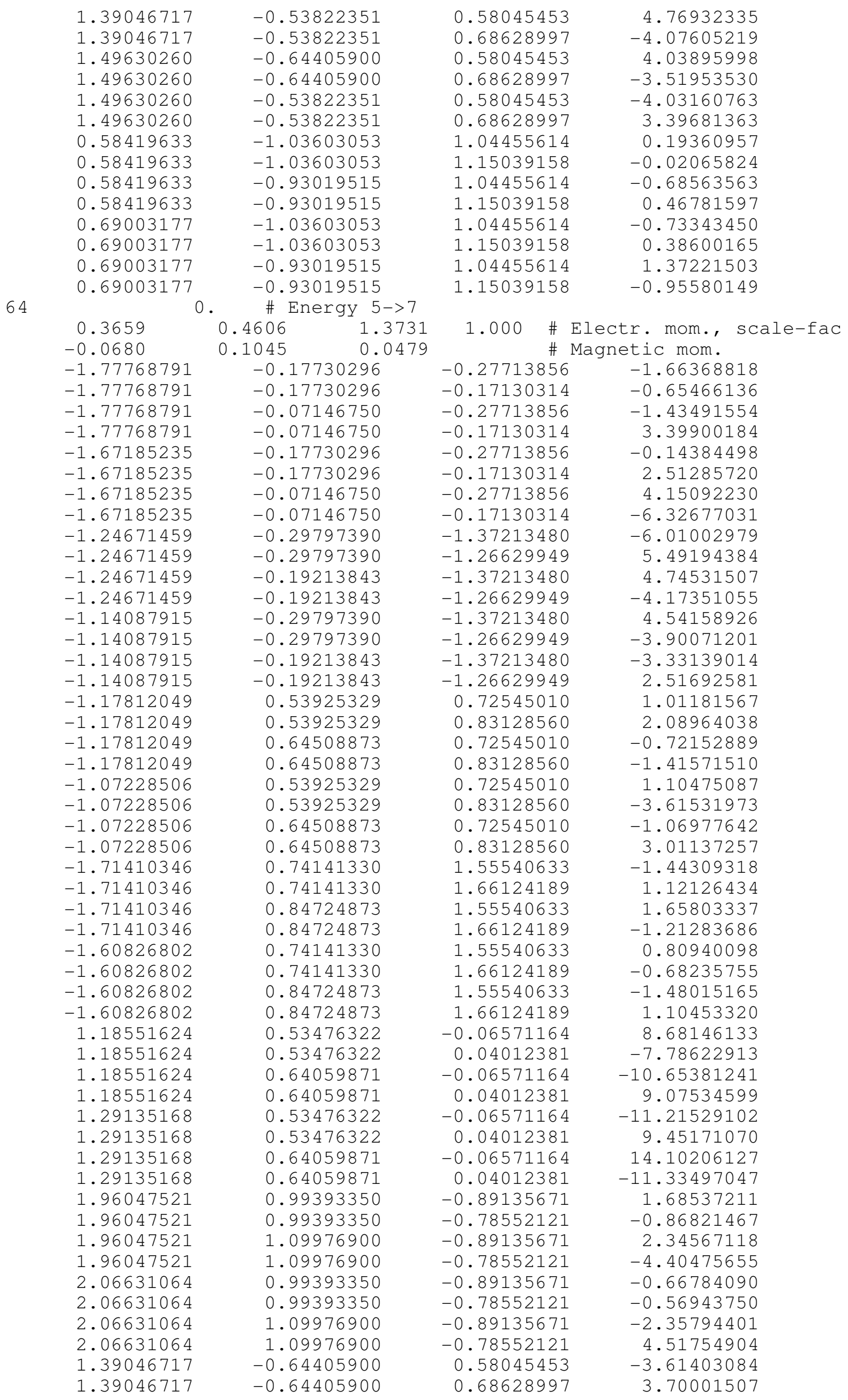

\section{S-346}




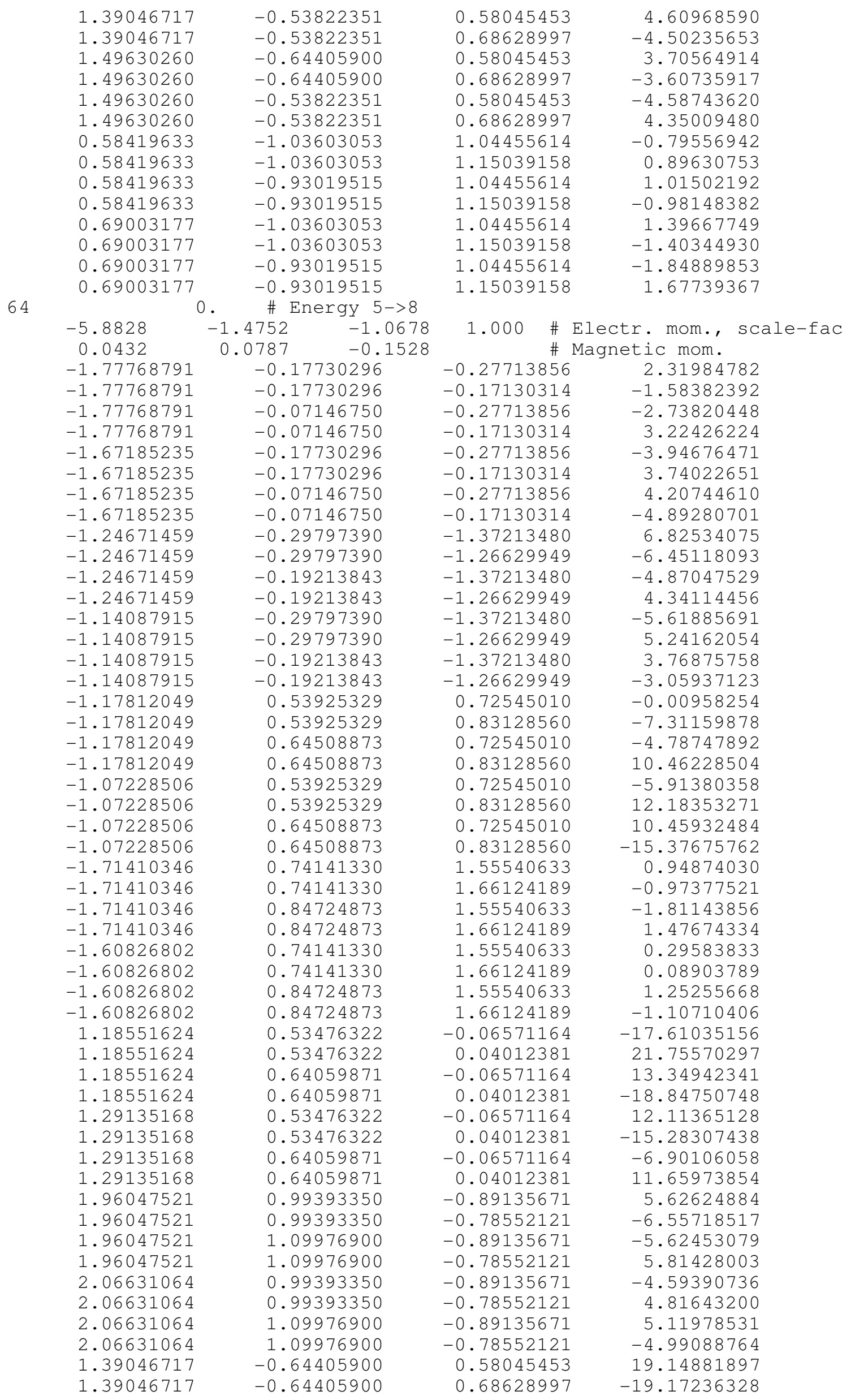

\section{S-347}




\begin{tabular}{|c|c|c|c|}
\hline 1.39046717 & -0.53822351 & 0.58045453 & -19.41924667 \\
\hline 1.39046717 & -0.53822351 & 0.68628997 & 18.90200615 \\
\hline 1.49630260 & -0.64405900 & 0.58045453 & -16.69096184 \\
\hline 1.49630260 & -0.64405900 & 0.68628997 & 16.79937553 \\
\hline 1.49630260 & -0.53822351 & 0.58045453 & 16.53262711 \\
\hline 1.49630260 & -0.53822351 & 0.68628997 & -16.36319160 \\
\hline 0.58419633 & -1.03603053 & 1.04455614 & -0.70688647 \\
\hline 0.58419633 & -1.03603053 & 1.15039158 & -0.48783830 \\
\hline 0.58419633 & -0.93019515 & 1.04455614 & 1.44599867 \\
\hline 0.58419633 & -0.93019515 & 1.15039158 & -0.15870368 \\
\hline 0.69003177 & -1.03603053 & 1.04455614 & 0.70891285 \\
\hline 0.69003177 & -1.03603053 & 1.15039158 & 0.63884169 \\
\hline 0.69003177 & -0.93019515 & 1.04455614 & -1.45895922 \\
\hline 0.69003177 & -0.93019515 & 1.15039158 & 0.05113225 \\
\hline 4 & \# Energy 5->9 & & \\
\hline 1.6103 & $0.4186 \quad 0.9266$ & $1.000 \quad \#$ & Electr. mom., \\
\hline 0.0427 & -0.0460 & - & Magnetic mom. \\
\hline-1.77768791 & -0.17730296 & -0.27713856 & -2.90798044 \\
\hline-1.77768791 & -0.17730296 & -0.17130314 & 0.63127792 \\
\hline-1.77768791 & -0.07146750 & -0.27713856 & -0.41547665 \\
\hline-1.77768791 & -0.07146750 & -0.17130314 & 2.64916730 \\
\hline-1.67185235 & -0.17730296 & -0.27713856 & 0.98707902 \\
\hline-1.67185235 & -0.17730296 & -0.17130314 & 1.54053390 \\
\hline-1.67185235 & -0.07146750 & -0.27713856 & 3.67192173 \\
\hline-1.67185235 & -0.07146750 & -0.17130314 & -6.39175224 \\
\hline-1.24671459 & -0.29797390 & -1.37213480 & -6.40484571 \\
\hline-1.24671459 & -0.29797390 & -1.26629949 & 6.37511492 \\
\hline-1.24671459 & -0.19213843 & -1.37213480 & 5.59010887 \\
\hline-1.24671459 & -0.19213843 & -1.26629949 & -5.60593081 \\
\hline-1.14087915 & -0.29797390 & -1.37213480 & 5.34293890 \\
\hline-1.14087915 & -0.29797390 & -1.26629949 & -5.23979473 \\
\hline-1.14087915 & -0.19213843 & -1.37213480 & -4.54057980 \\
\hline-1.14087915 & -0.19213843 & -1.26629949 & 4.34673452 \\
\hline-1.17812049 & 0.53925329 & 0.72545010 & -2.20666981 \\
\hline-1.17812049 & 0.53925329 & 0.83128560 & 4.79352045 \\
\hline-1.17812049 & 0.64508873 & 0.72545010 & 2.74390960 \\
\hline-1.17812049 & 0.64508873 & 0.83128560 & -4.58803177 \\
\hline-1.07228506 & 0.53925329 & 0.72545010 & 7.68222618 \\
\hline-1.07228506 & 0.53925329 & 0.83128560 & -9.16055870 \\
\hline-1.07228506 & 0.64508873 & 0.72545010 & -7.13645840 \\
\hline-1.07228506 & 0.64508873 & 0.83128560 & 8.26373577 \\
\hline-1.71410346 & 0.74141330 & 1.55540633 & -0.80802542 \\
\hline-1.71410346 & 0.74141330 & 1.66124189 & 0.57664168 \\
\hline-1.71410346 & 0.84724873 & 1.55540633 & 1.06073046 \\
\hline-1.71410346 & 0.84724873 & 1.66124189 & -0.72395790 \\
\hline-1.60826802 & 0.74141330 & 1.55540633 & 0.41794223 \\
\hline-1.60826802 & 0.74141330 & 1.66124189 & -0.28757620 \\
\hline-1.60826802 & 0.84724873 & 1.55540633 & -1.01837862 \\
\hline-1.60826802 & 0.84724873 & 1.66124189 & 0.69338208 \\
\hline 1.18551624 & 0.53476322 & -0.06571164 & 21.33677101 \\
\hline 1.18551624 & 0.53476322 & 0.04012381 & -24.66602135 \\
\hline 1.18551624 & 0.64059871 & -0.06571164 & -19.22014809 \\
\hline 1.18551624 & 0.64059871 & 0.04012381 & 22.29529190 \\
\hline 1.29135168 & 0.53476322 & -0.06571164 & -18.45838737 \\
\hline 1.29135168 & 0.53476322 & 0.04012381 & 21.01025963 \\
\hline 1.29135168 & 0.64059871 & -0.06571164 & 15.96043015 \\
\hline 1.29135168 & 0.64059871 & 0.04012381 & -18.42817497 \\
\hline 1.96047521 & 0.99393350 & -0.89135671 & -1.24571753 \\
\hline 1.96047521 & 0.99393350 & -0.78552121 & 4.36124182 \\
\hline 1.96047521 & 1.09976900 & -0.89135671 & 0.61936706 \\
\hline 1.96047521 & 1.09976900 & -0.78552121 & -3.24794149 \\
\hline 2.06631064 & 0.99393350 & -0.89135671 & 1.86621571 \\
\hline 2.06631064 & 0.99393350 & -0.78552121 & -4.57279968 \\
\hline 2.06631064 & 1.09976900 & -0.89135671 & -1.41037607 \\
\hline 2.06631064 & 1.09976900 & -0.78552121 & 3.74040866 \\
\hline 1.39046717 & -0.64405900 & 0.58045453 & -9.20236206 \\
\hline 1.39046717 & -0.64405900 & 0.68628997 & 8.07068062 \\
\hline
\end{tabular}

\section{S-348}




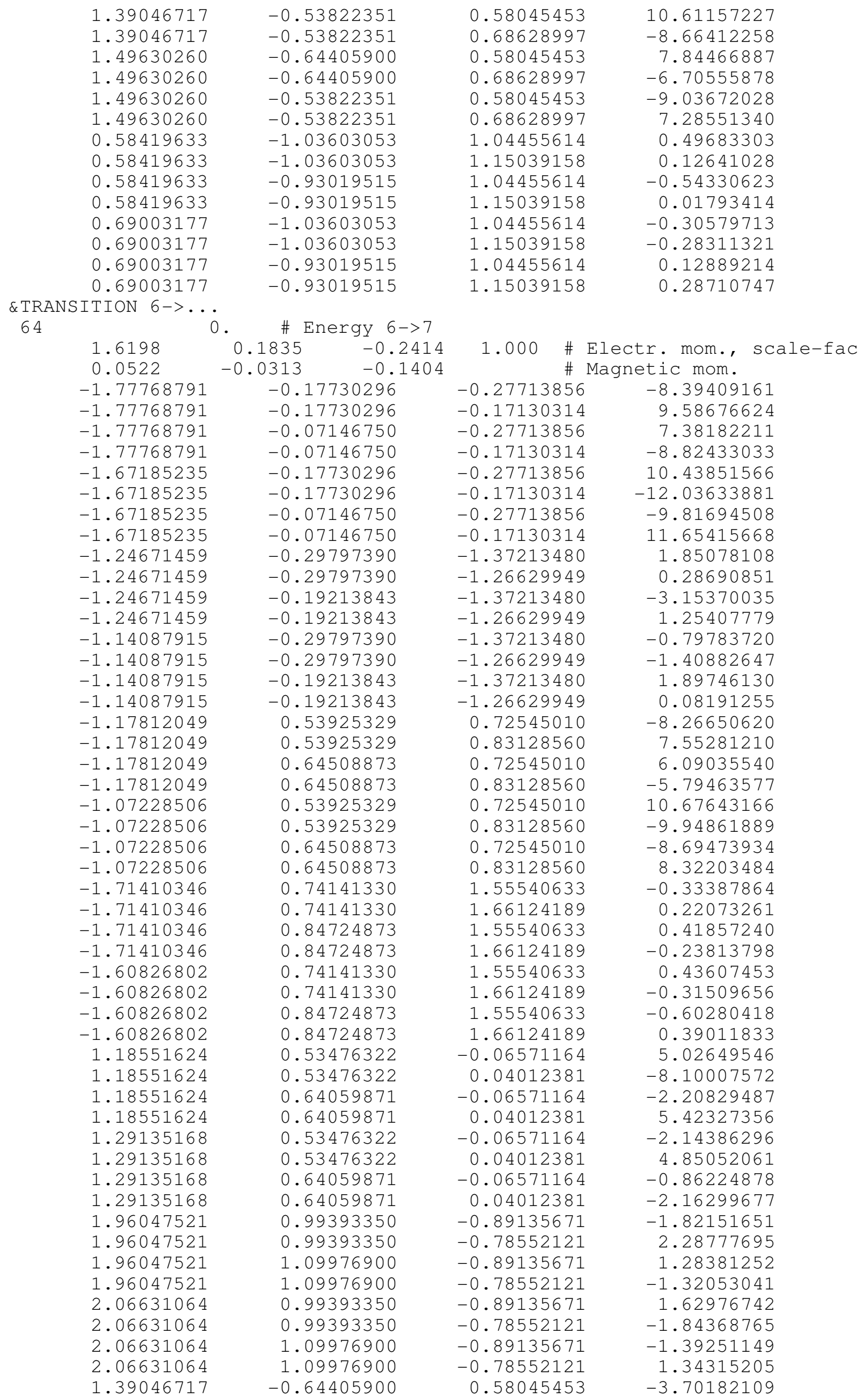

\section{S-349}




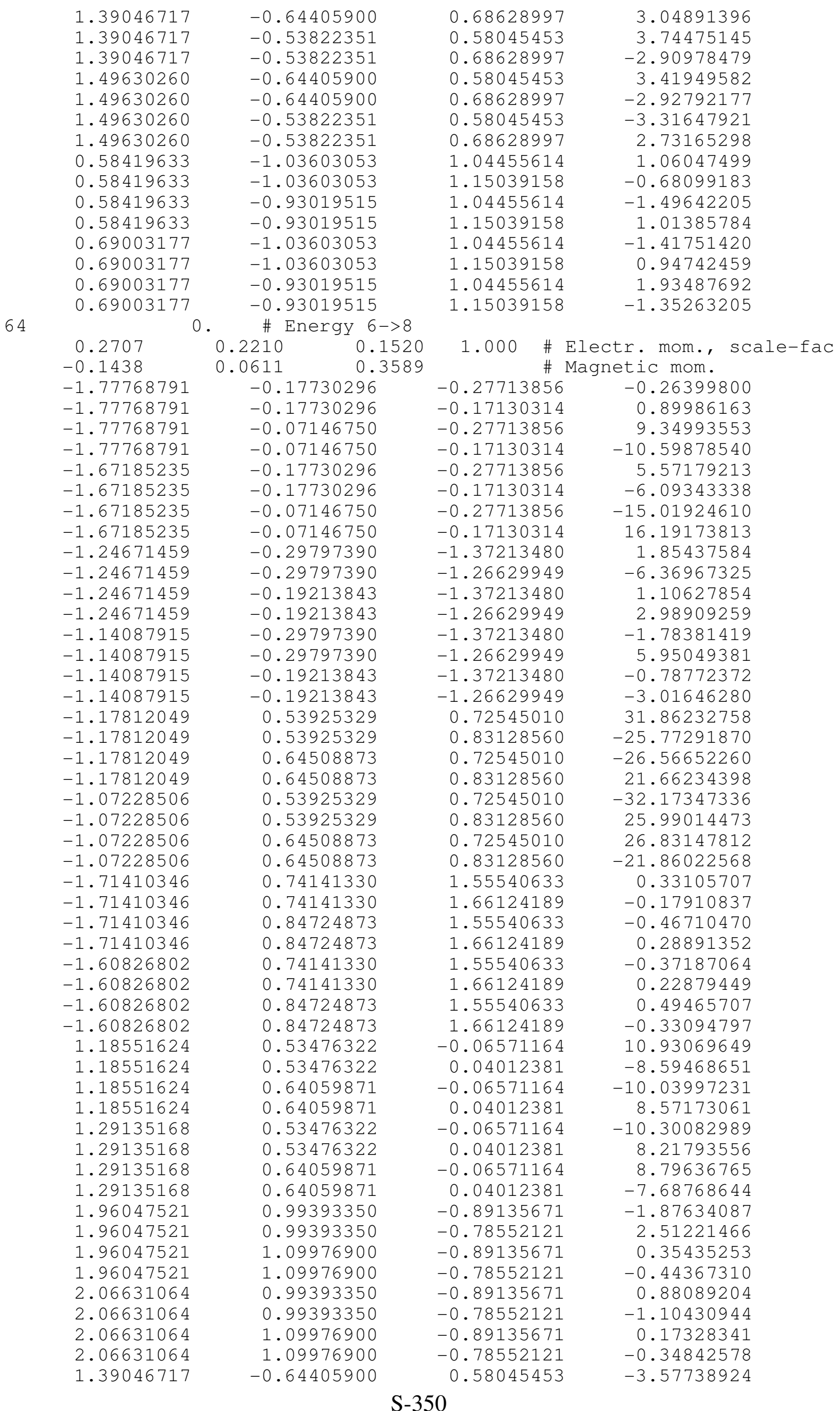




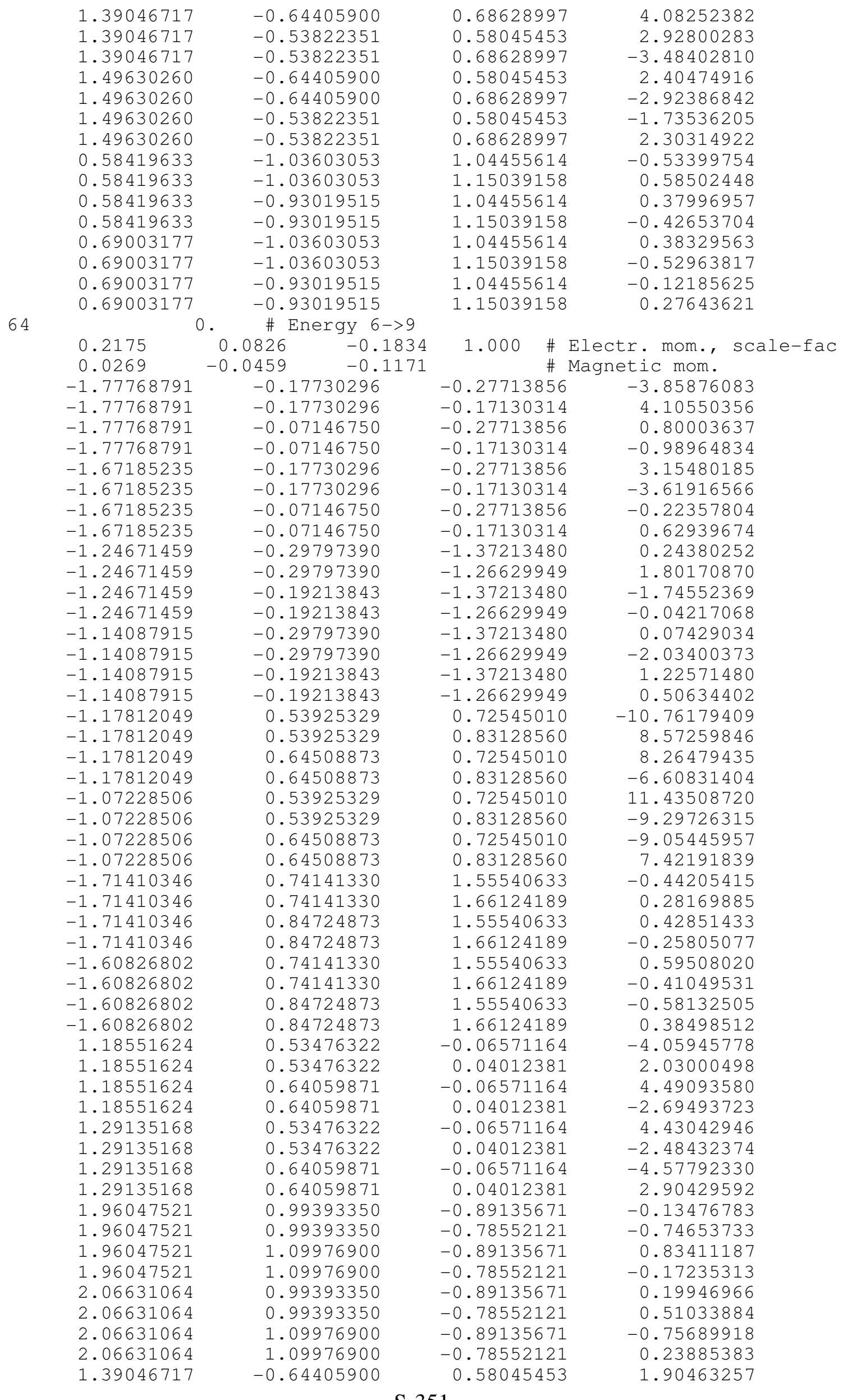

\section{S-351}




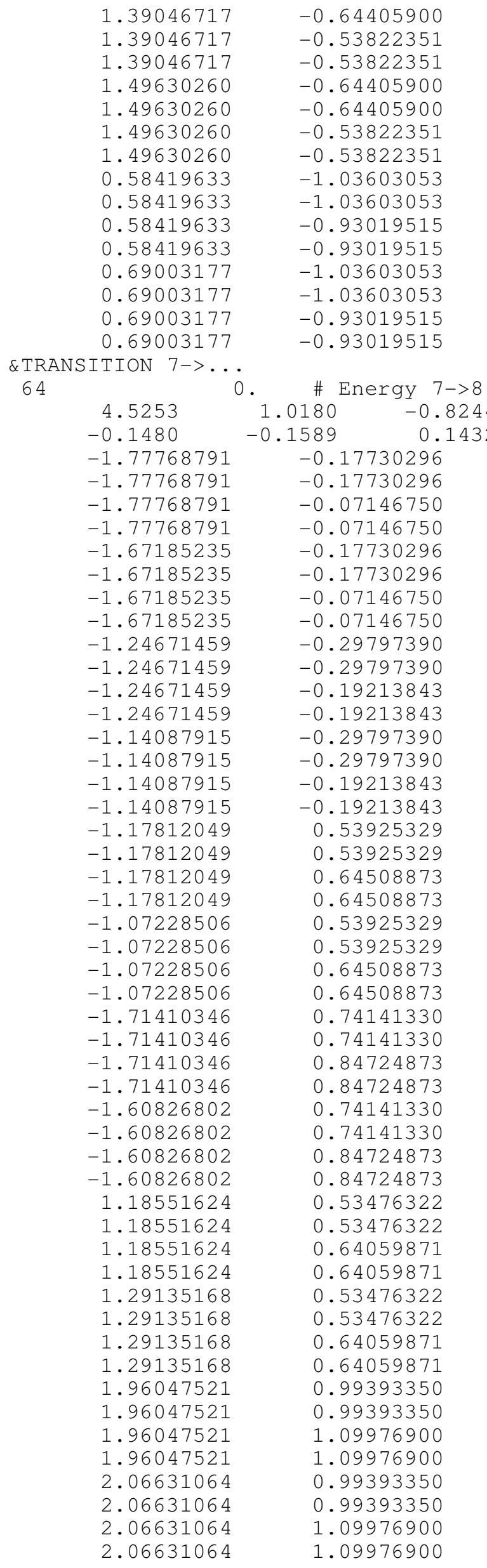

$\begin{array}{lr}0.68628997 & -2.17432117 \\ 0.58045453 & -1.73415637 \\ 0.68628997 & 1.98212254 \\ 0.58045453 & -1.49820518 \\ 0.68628997 & 1.74187315 \\ 0.58045453 & 1.28937972 \\ 0.68628997 & -1.52988899 \\ 1.04455614 & 0.08394631 \\ 1.15039158 & -0.17321748 \\ 1.04455614 & -0.12370262 \\ 1.15039158 & 0.18995994 \\ 1.04455614 & -0.08784288 \\ 1.15039158 & 0.19718859 \\ 1.04455614 & 0.13350341 \\ 1.15039158 & -0.21218127\end{array}$

1. 15039158
1.000 \# Electr. mom., scale-fac \# Magnetic mom.

$-0.27713856$

$-0.17130314$

$-0.27713856$

$-0.17130314$

$-0.27713856$

$-0.17130314$

$-0.27713856$

$-0.17130314$

$-1.37213480$

$-1.26629949$

$-1.37213480$

$-1.26629949$

$-1.37213480$

$-1.26629949$

$-1.37213480$

$-1.26629949$

0.72545010

0.83128560

0.72545010

0.83128560

0.72545010

0.83128560

0.72545010

0.83128560

1.55540633

1.66124189

1. 55540633

1.66124189

1.55540633

1.66124189

1.55540633

1.66124189

$-0.06571164$

0.04012381

$-0.06571164$

0.04012381

$-0.06571164$

0.04012381

$-0.06571164$

0.04012381

$-0.89135671$

$-0.78552121$

$-0.89135671$

$-0.78552121$

$-0.89135671$

$-0.78552121$

$-0.89135671$

$-0.78552121$
$-3.2417740$

3. 94550753

3. 40125370

$-5.15653324$

6.10947180

$-7.36294842$

$-6.63340521$

8. 94732094

0.49761051

0.04554666

$-2.14102769$

1. 90524483

$-0.00977563$

$-0.64110285$

1.56772947

$-1.28699601$

$-15.48873615$

17.97608566

14.81813526

$-17.15285110$

16.78645325

$-19.47281075$

$-17.17597198$

19.59049416

1.00010860

$-0.50185359$

$-0.27223429$

0.09464314

$-2.02987504$

1.23406529

0.89202142

$-0.53309590$

2. 41309571

$-7.81413937$

6.98408508

1. 01795697

8.95288181

$-3.58118081$

$-20.91809654$

12.07973862

$-8.66887093$

9.93499279

2. 48721361

$-0.89519489$

4.90412426

$-4.48201370$

$-0.99540079$

$-1.35284042$ 


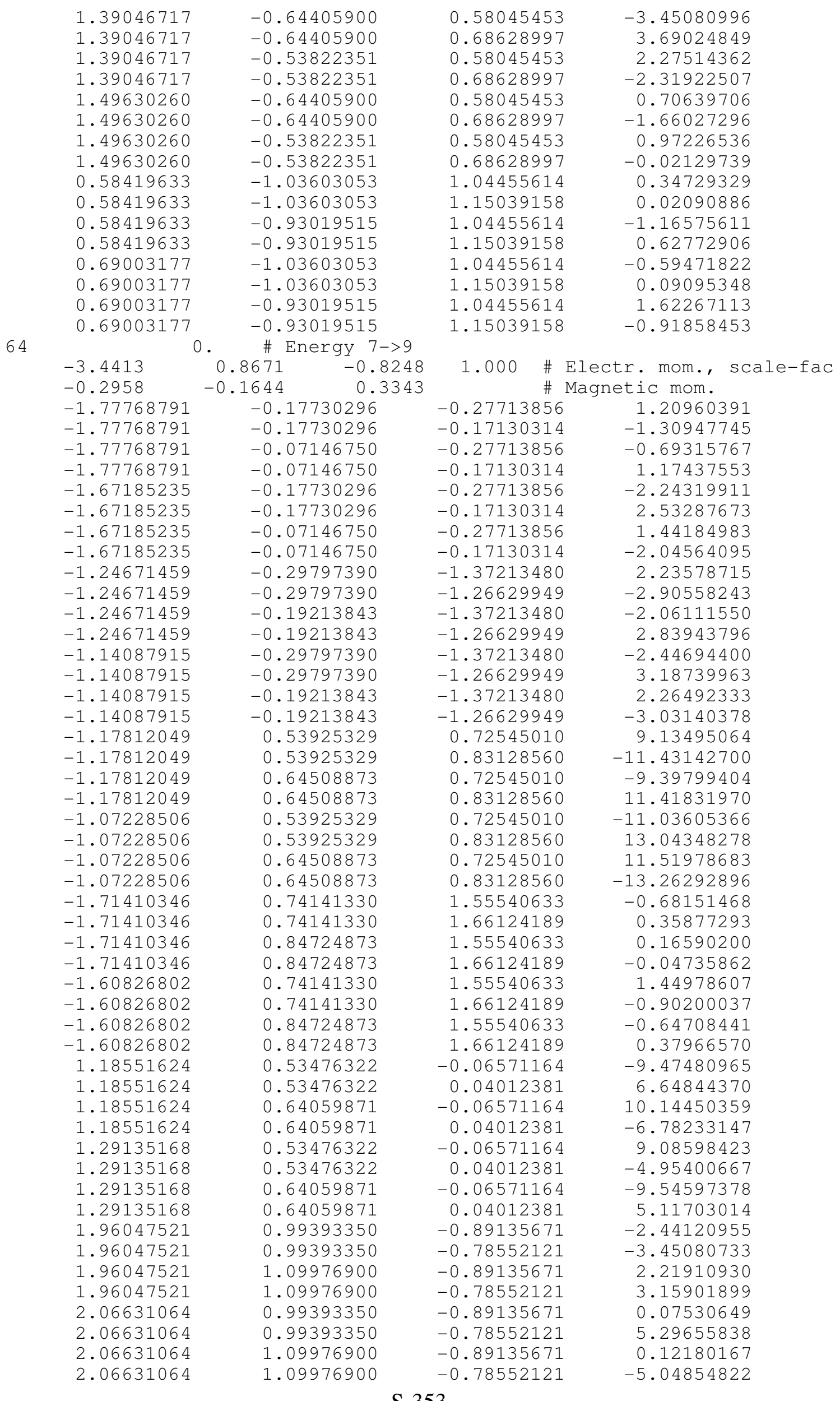




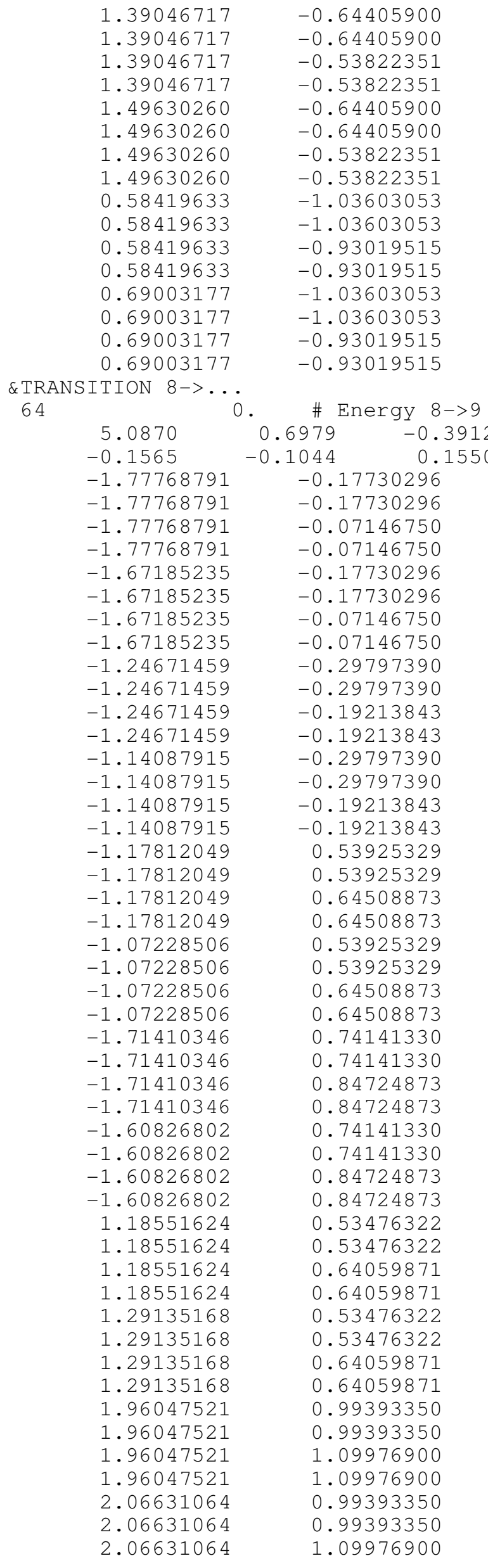
0.58045453
0.68628997
0.58045453
0.68628997
0.58045453
0.68628997
0.58045453
0.68628997
1. 04455614
1.15039158
1.04455614
1. 15039158
1.04455614
1.15039158
1.04455614
1. 15039158

22.13530731
-21.25754356
-22.29848862
20.91720009
-23.30455208
22.27478027
22.96154594
-21.83283615
-5.17474461
3.00047350
5.73480272
-3.36412239
6.46131706
-3.86885548
-7.08962440
4.32123375

1.000 \# Electr. mom., scale-fac \# Magnetic mom.

$\begin{array}{ll}-0.27713856 & 2.88384581\end{array}$

$-0.17130314 \quad-2.22662735$

$-0.27713856 \quad-2.61995006$

$\begin{array}{ll}-0.17130314 & 0.80611432\end{array}$

$-0.27713856 \quad-0.97724944$

$-0.17130314 \quad-0.24004976$

$\begin{array}{ll}-0.27713856 & 0.56170541\end{array}$

$-0.17130314 \quad 1.79376125$

$-1.37213480 \quad 1.05817425$

$-1.26629949-1.00431538$

$-1.37213480 \quad-2.70148301$

$\begin{array}{ll}-1.26629949 & 2.91866612\end{array}$

$-1.37213480 \quad-0.93288147$

$-1.26629949 \quad 0.84063137$

$-1.37213480 \quad 2.51513338$

$-1.26629949$

0.72545010

0.83128560

0.72545010

0.83128560

0.72545010

0.83128560

0.72545010

0.83128560

1.55540633

1. 66124189

1.55540633

1.66124189

1.55540633

1.66124189

1.55540633

1.66124189

$-0.06571164$

0.04012381

$-0.06571164$

0.04012381

$-0.06571164$

0.04012381

$-0.06571164$

0.04012381

$-0.89135671$

$-0.78552121$

$-0.89135671$

$-0.78552121$

$-0.89135671$

$-0.78552121$

$-0.89135671$

$-2.77834344$

$-15.42404461$

20.75781631

17.01429558

$-21.44947243$

13.58618069

$-19.08793831$

$-16.76065063$

21.25543976

1. 42616713

$-0.46649319$

$-0.32389015$

$-0.18339899$

$-3.37176871$

1.80225515

1.65479326

$-0.70120752$

$-6.44385910$

8.60825157

7.69070482

$-8.94556713$

6.42278147

$-9.44964600$

$-7.29003716$

9.47066975

$-0.93879080$

$-1.61888099$

5.28313780

$-3.21654582$

$-0.84260225$

3.61188436

$-3.22426486$ 


2.06631064
1.39046717
1.39046717
1.39046717
1.39046717
1.49630260
1.49630260
1.49630260
1.49630260
0.58419633
0.58419633
0.58419633
0.58419633
0.69003177
0.69003177
0.69003177
0.69003177
\&PERMANENT MOMENTS

64
1.09976900

$-0.64405900$

$-0.64405900$

$-0.53822351$

$-0.53822351$

$-0.64405900$

$-0.64405900$

$-0.53822351$

$-0.53822351$

$-1.03603053$

$-1.03603053$

$-0.93019515$

$-0.93019515$

$-1.03603053$

$-1.03603053$

$-0.93019515$

$-0.93019515$
$-0.78552121$
0.58045453
0.68628997
0.58045453
0.68628997
0.58045453
0.68628997
0.58045453
0.68628997
1.04455614
1.15039158
1.04455614
1.15039158
1.04455614
1.15039158
1.04455614
1.15039158

1.08559251

$-14.18343735$

15.42554569

12.58507442

$-13.77403927$

13.87138557

$-15.02431488$

$-12.22847939$

13.53146839

1.48512590

$-0.87709856$

$-1.80783749$

1.26670229

$-2.02940369$

1. 26360238

2.43272042

$-1.76505899$

$-3.5947$

0 . \# Energy 1->1

$-1.77768791$

$-1.4217$ 5.0345

$-1.77768791$

$-1.77768791$

$-1.77768791$

$-1.67185235$

$-1.67185235$

$-1.67185235$

$-1.67185235$

$-1.24671459$

$-1.24671459$

$-1.24671459$

$-1.24671459$

$-1.14087915$

$-1.14087915$

$-1.14087915$

$-1.14087915$

$-1.17812049$

$-1.17812049$

$-1.17812049$

$-1.17812049$

$-1.07228506$

$-1.07228506$

$-1.07228506$

$-1.07228506$

$-1.71410346$

$-1.71410346$

$-1.71410346$

$-1.71410346$

$-1.60826802$

$-1.60826802$

$-1.60826802$

$-1.60826802$

1.18551624

1.18551624

1.18551624

1.18551624

1. 29135168

1.29135168

1.29135168

1.29135168

1.96047521

1.96047521

1.96047521

1.96047521

2.06631064

2.06631064

$-0.17730296$

$-0.17730296$

$-0.07146750$

$-0.07146750$

$-0.17730296$

$-0.17730296$

$-0.07146750$

$-0.07146750$

$-0.29797390$

$-0.29797390$

$-0.19213843$

$-0.19213843$

$-0.29797390$

$-0.29797390$

$-0.19213843$

$-0.19213843$

0.53925329

0.53925329

0.64508873

0.64508873

0.53925329

0.53925329

0.64508873

0.64508873

0.74141330

0.74141330

0.84724873

0.84724873

0.74141330

0.74141330

0.84724873

0.84724873

0.53476322

0.53476322

0.64059871

0.64059871

0.53476322

0.53476322

0.64059871

0.64059871

0.99393350

0.99393350

1.09976900

1.09976900

0.99393350

0.99393350

2.06631064

1.09976900

-0.27713856
-0.17130314

$-0.27713856$

$-0.17130314$

$-0.27713856$

$-0.17130314$

$-0.27713856$

$-0.17130314$

$-1.37213480$

$-1.26629949$

$-1.37213480$

$-1.26629949$

$-1.37213480$

$-1.26629949$

$-1.37213480$

$-1.26629949$

0.72545010

0.83128560

0.72545010

0.83128560

0.72545010

0.83128560

0.72545010

0.83128560

1.55540633

1.66124189

1.55540633

1.66124189

1.55540633

1.66124189

1.55540633

1.66124189

$-0.06571164$

0.04012381

$-0.06571164$

0.04012381

$-0.06571164$

0.04012381

$-0.06571164$

0.04012381

$-0.89135671$

$-0.78552121$

$-0.89135671$

$-0.78552121$

$-0.89135671$

$-0.78552121$

$-0.89135671$
Electr. mom.

27.82242393

$-21.65666199$

$-42.11869812$

39.46121979

$-28.14096069$

23.61341286

48.45865631

$-47.30563354$

$-10.04841137$

5.74285698

10.97851181

$-8.03430271$

6.73815203

$-2.21204066$

$-6.93590498$

2.89102507

$-72.08042145$

70.61484528

66.13167572

$-68.91027832$

101.21786499

$-95.75193024$

$-86.59014130$

85.47227478

$-6.68150806$

5.81809759

4.00643539

$-3.73834443$

9.32914352

$-7.41957855$

$-5.22639608$

4.66180706

29.70226669

$-52.66197205$

$-28.79569817$

48.22182083

$-32.33048630$

52.40977097

33.66021347

$-49.25653076$

15.75187683

$-16.97171211$

$-9.01048756$

7.35928011

$-11.86632061$

11.15091133

6.99556208 


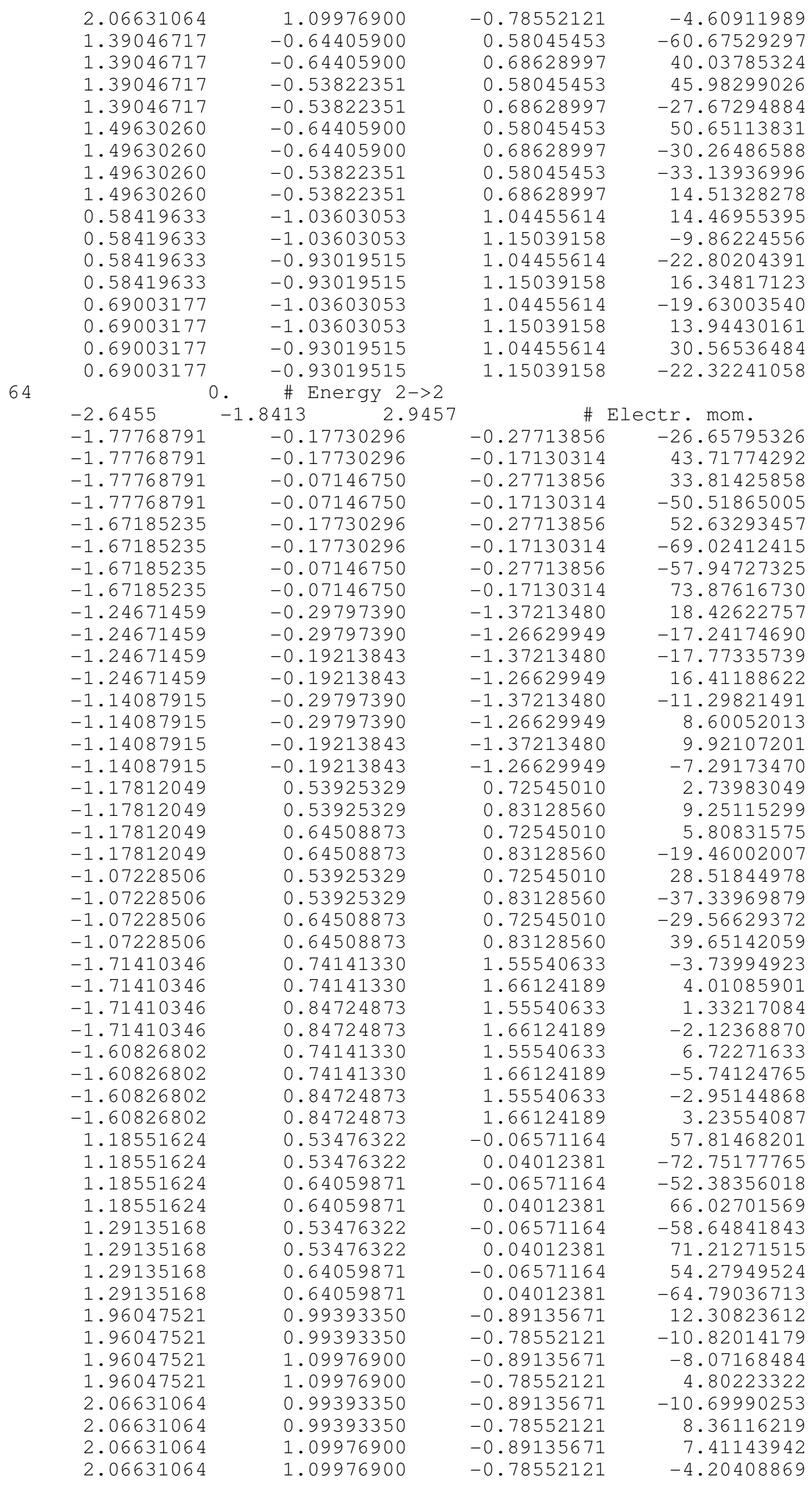

\section{S-356}




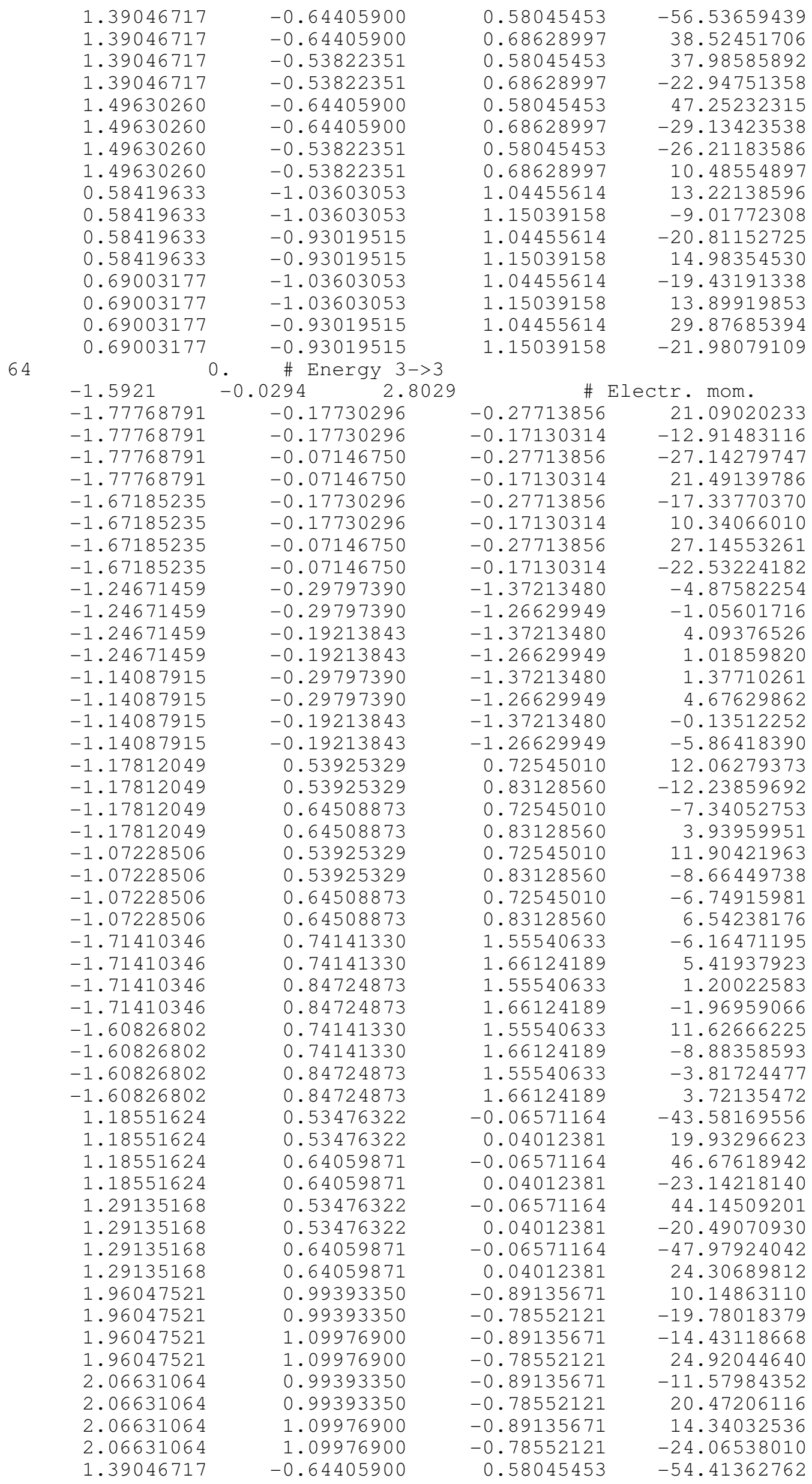

\section{S-357}




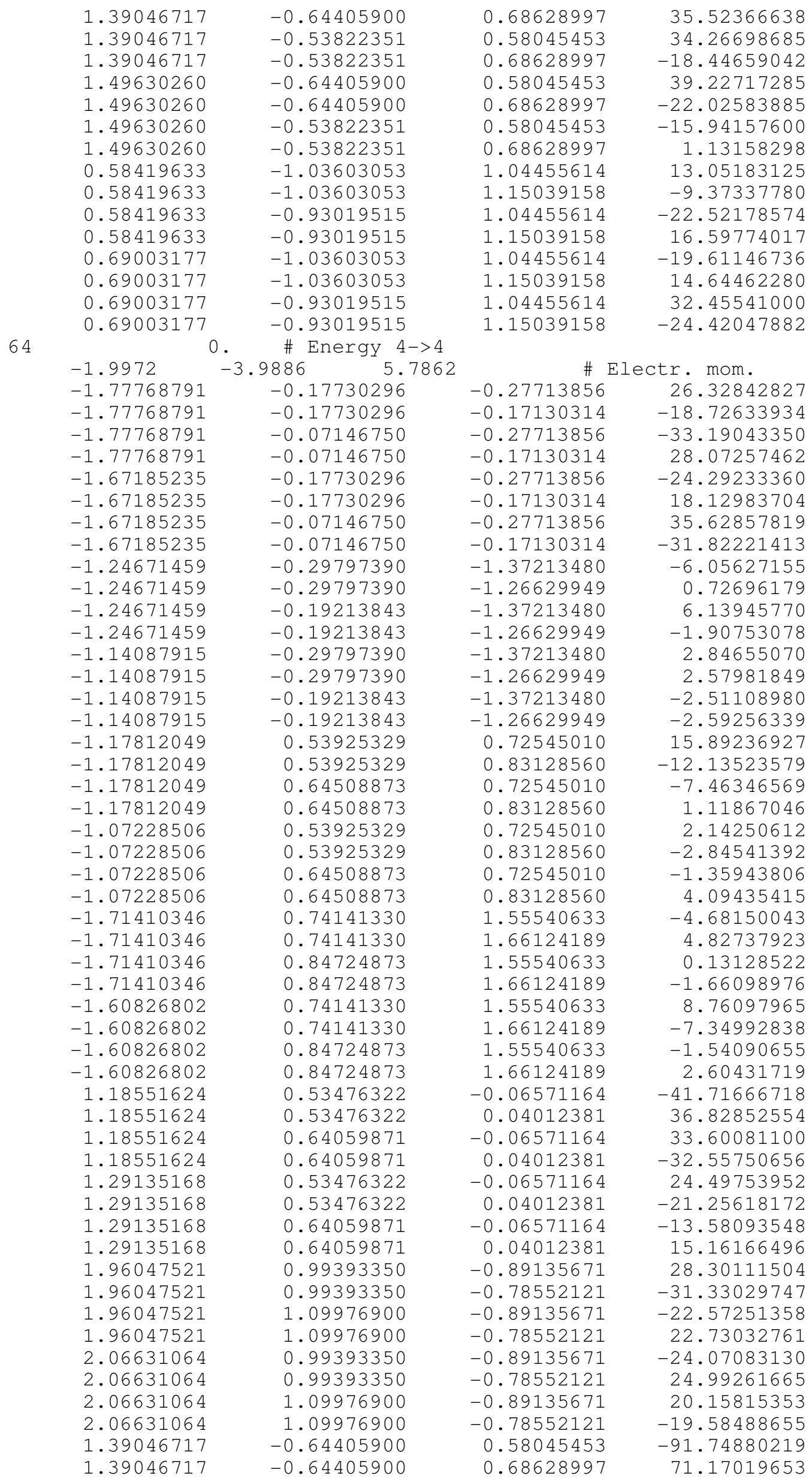

\section{S-358}




\begin{tabular}{|c|c|c|c|}
\hline 1.39046717 & -0.53822351 & 058045453 & 72.59873199 \\
\hline 1.39046717 & -0.53822351 & 0.68628997 & -54.86151123 \\
\hline 1.49630260 & -0.64405900 & 0.58045453 & 86.03157806 \\
\hline 1.49630260 & -0.64405900 & 0.68628997 & -65.36965179 \\
\hline 1.49630260 & -0.53822351 & 0.58045453 & -63.95581055 \\
\hline 1.49630260 & -0.53822351 & 0.68628997 & 45.88867188 \\
\hline 0.58419633 & -1.03603053 & 1.04455614 & 22.17017174 \\
\hline 0.58419633 & -1.03603053 & 1.15039158 & -15.44123650 \\
\hline 0.58419633 & -0.93019515 & 1.04455614 & -30.60635185 \\
\hline 0.58419633 & -0.93019515 & 1.15039158 & 21.75527573 \\
\hline 0.69003177 & -1.03603053 & 1.04455614 & -31.24298859 \\
\hline 0.69003177 & -1.03603053 & 1.15039158 & 22.56247520 \\
\hline 0.69003177 & -0.93019515 & 1.04455614 & 42.9 \\
\hline 0.69003177 & -0.93019515 & 1.15039158 & -31.39183807 \\
\hline 64 & \# Energy 5->5 & & \\
\hline-4.0404 & -0.3337 & $\pi$ & ctr. mom \\
\hline-1.77768791 & -0.17730296 & -0.27713856 & -30.80095863 \\
\hline-1.77768791 & -0.17730296 & -0.17130314 & 40.88220215 \\
\hline-1.77768791 & -0.07146750 & -0.27713856 & 36.43330765 \\
\hline-1.77768791 & -0.07146750 & -0.17130314 & -46.41468430 \\
\hline-1.67185235 & -0.17730296 & -0.27713856 & 53.21351242 \\
\hline-1.67185235 & -0.17730296 & -0.17130314 & -61.94202423 \\
\hline-1.67185235 & -0.07146750 & -0.27713856 & -55.38321304 \\
\hline-1.67185235 & -0.07146750 & -0.17130314 & 63.62846756 \\
\hline-1.24671459 & -0.29797390 & -1.37213480 & 5.98927689 \\
\hline-1.24671459 & -0.29797390 & -1.26629949 & -12.00328827 \\
\hline-1.24671459 & -0.19213843 & -1.37213480 & -7.86659193 \\
\hline-1.24671459 & -0.19213843 & -1.26629949 & 13.81844234 \\
\hline-1.14087915 & -0.29797390 & -1.37213480 & -4.41001797 \\
\hline-1.14087915 & -0.29797390 & -1.26629949 & 9.278706 \\
\hline-1.14087915 & -0.19213843 & -1.37213480 & 6.08067560 \\
\hline-1.14087915 & -0.19213843 & -1.26629949 & -11.54650688 \\
\hline-1.17812049 & 0.53925329 & 0.72545010 & 85.37867737 \\
\hline-1.17812049 & 0.53925329 & 0.83128560 & -69.80171204 \\
\hline-1.17812049 & 0.64508873 & 0.72545010 & -70.73264313 \\
\hline-1.17812049 & 0.64508873 & 0.83128560 & 55.18522644 \\
\hline-1.07228506 & 0.53925329 & 0.72545010 & -49.49596024 \\
\hline-1.07228506 & 0.53925329 & 0.83128560 & 38.47682953 \\
\hline-1.07228506 & 0.64508873 & 0.72545010 & 45.90423584 \\
\hline-1.07228506 & 0.64508873 & 0.83128560 & -34.63884354 \\
\hline-1.71410346 & 0.74141330 & 1.55540633 & -9.87174034 \\
\hline-1.71410346 & 0.74141330 & 1.66124189 & 7.92632818 \\
\hline-1.71410346 & 0.84724873 & 1.55540633 & 5.09769106 \\
\hline-1.71410346 & 0.84724873 & 1.66124189 & -4.47655344 \\
\hline-1.60826802 & 0.74141330 & 1.55540633 & 15.44094944 \\
\hline-1.60826802 & 0.74141330 & 1.66124189 & -11.39682865 \\
\hline-1.60826802 & 0.84724873 & 1.55540633 & -8.66404533 \\
\hline-1.60826802 & 0.84724873 & 1.66124189 & 6.83651876 \\
\hline 1.18551624 & 0.53476322 & -0.06571164 & 31.6764 \\
\hline 1.18551624 & 0.53476322 & 0.04012381 & -52.12873459 \\
\hline 1.18551624 & 0.64059871 & -0.06571164 & -35.00327682 \\
\hline 1.18551624 & 0.64059871 & 0.04012381 & 52.35182190 \\
\hline 1.29135168 & 0.53476322 & -0.06571164 & -40.08549500 \\
\hline 1.29135168 & 0.53476322 & 0.04012381 & 58.50317001 \\
\hline 1.29135168 & 0.64059871 & -0.06571164 & 45.32774734 \\
\hline 1.29135168 & 0.64059871 & 0.04012381 & -59.55976486 \\
\hline 1.96047521 & 0.99393350 & -0.89135671 & 14.46808720 \\
\hline 1.96047521 & 0.99393350 & -0.78552121 & -16.67277718 \\
\hline 1.96047521 & 1.09976900 & -0.89135671 & -9.64781380 \\
\hline 1.96047521 & 1.09976900 & -0.78552121 & 9.09448624 \\
\hline 2.06631064 & 0.99393350 & -0.89135671 & -11.34386349 \\
\hline 2.06631064 & 0.99393350 & -0.78552121 & 11.60969448 \\
\hline 2.06631064 & 1.09976900 & -0.89135671 & 8.16319370 \\
\hline 2.06631064 & 1.09976900 & -0.78552121 & -6.91117716 \\
\hline 1.39046717 & -0.64405900 & 0.58045453 & -66.26797485 \\
\hline 1.39046717 & -0.64405900 & 0.68628997 & 46.41582870 \\
\hline 1.39046717 & -0.53822351 & 0.58045453 & 50.96761703 \\
\hline
\end{tabular}

\section{S-359}




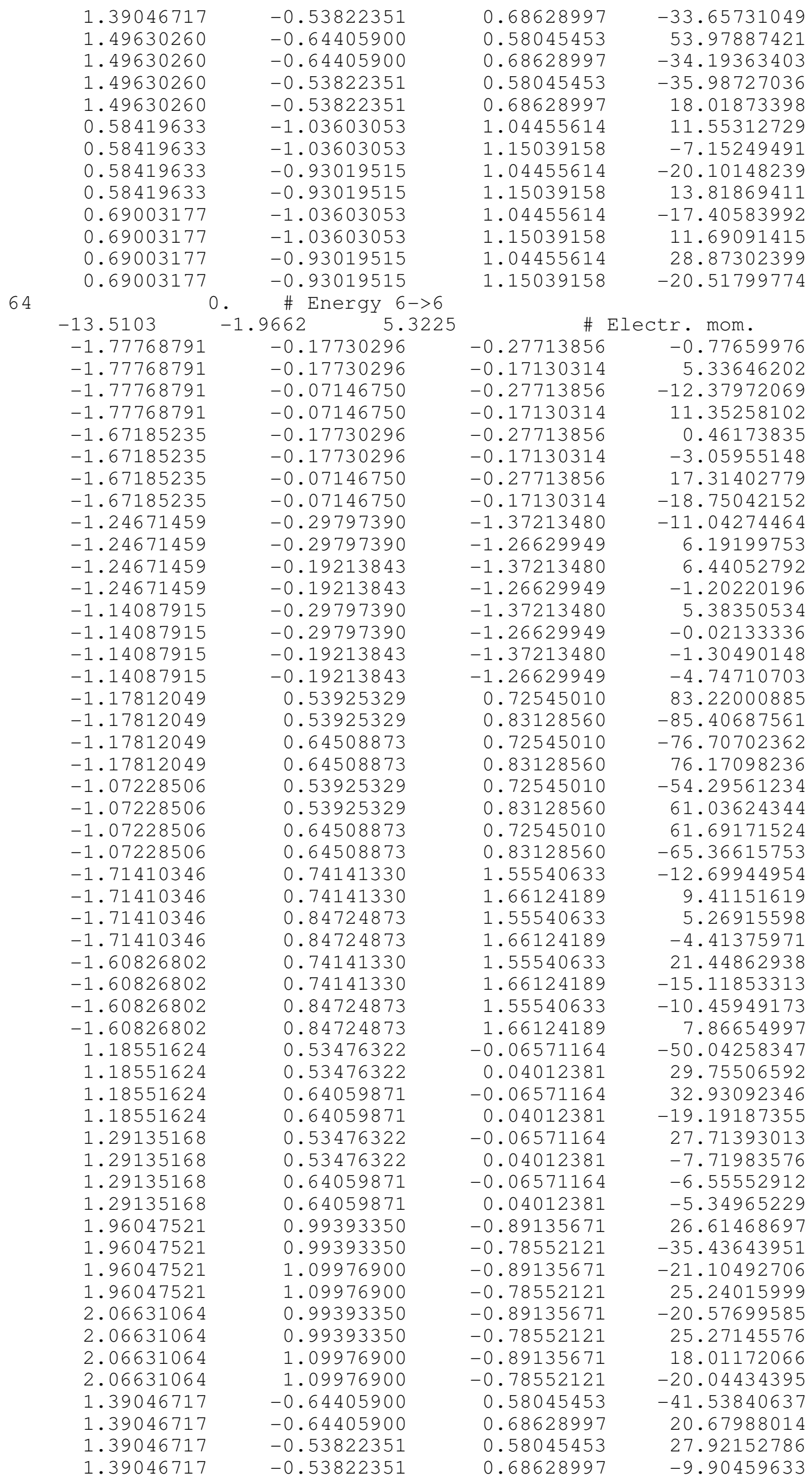

\section{S-360}




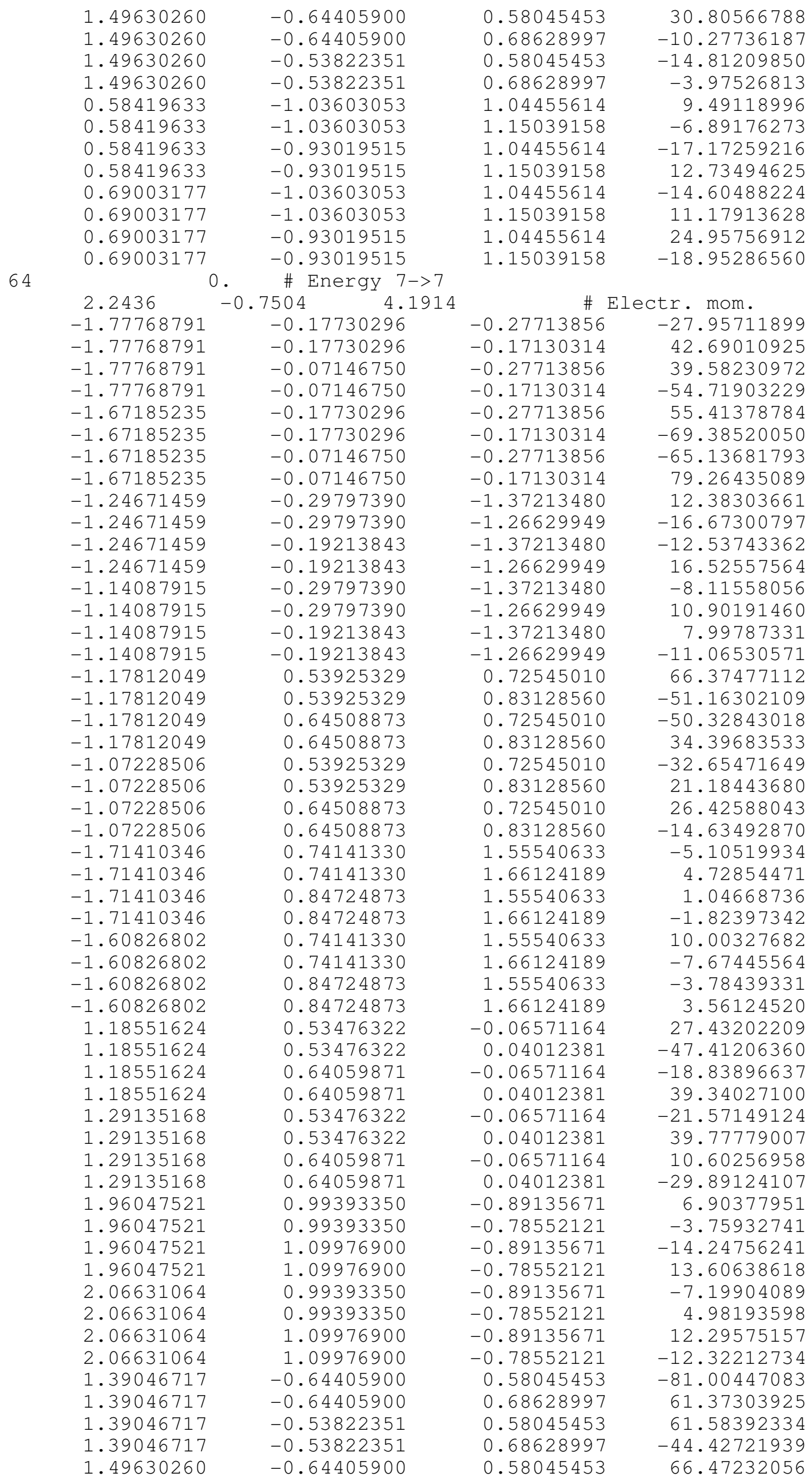

\section{S-361}




\begin{tabular}{|c|c|c|c|}
\hline 1.49630260 & -0.64405900 & 0.68628997 & -47.99791336 \\
\hline 1.49630260 & -0.53822351 & 0.58045453 & -43.32922745 \\
\hline 1.49630260 & -0.53822351 & 0.68628997 & 27.08380890 \\
\hline 0.58419633 & -1.03603053 & 1.04455614 & 17.07597351 \\
\hline 0.58419633 & -1.03603053 & 1.15039158 & -10.70132256 \\
\hline 0.58419633 & -0.93019515 & 1.04455614 & -27.14374161 \\
\hline 0.58419633 & -0.93019515 & 1.15039158 & 18.35638809 \\
\hline 0.69003177 & -1.03603053 & 1.04455614 & -24.97459221 \\
\hline 0.69003177 & -1.03603053 & 1.15039158 & 16.70985031 \\
\hline 0.69003177 & -0.93019515 & 1.04455614 & 38.54327774 \\
\hline 0.69003177 & -0.93019515 & 1.15039158 & -27.04080009 \\
\hline 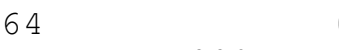 & \# Energy 8->8 & & \\
\hline-5.4228 & -1.5401 & 开 & ctr. mom. \\
\hline-1.77768791 & -0.17730296 & -0.27713856 & -8.82981586 \\
\hline-1.77768791 & -0.17730296 & -0.17130314 & 23.12895966 \\
\hline-1.77768791 & -0.07146750 & -0.27713856 & 17.26880074 \\
\hline-1.77768791 & -0.07146750 & -0.17130314 & -30.43634033 \\
\hline-1.67185235 & -0.17730296 & -0.27713856 & 29.45422554 \\
\hline-1.67185235 & -0.17730296 & -0.17130314 & -42.6357841 \\
\hline-1.67185235 & -0.07146750 & -0.27713856 & -36.14198685 \\
\hline-1.67185235 & -0.07146750 & -0.17130314 & 48.33953094 \\
\hline-1.24671459 & -0.29797390 & -1.37213480 & 14.65937328 \\
\hline-1.24671459 & -0.29797390 & -1.26629949 & -18.99596405 \\
\hline-1.24671459 & -0.19213843 & -1.37213480 & -13.20928001 \\
\hline-1.24671459 & -0.19213843 & -1.26629949 & 16.98000908 \\
\hline-1.14087915 & -0.29797390 & -1.37213480 & -11.24437523 \\
\hline-1.14087915 & -0.29797390 & -1.26629949 & 14.48354721 \\
\hline-1.14087915 & -0.19213843 & -1.37213480 & 9.80025101 \\
\hline-1.14087915 & -0.19213843 & -1.26629949 & -12.94639683 \\
\hline-1.17812049 & 0.53925329 & 0.72545010 & 55.07211685 \\
\hline-1.17812049 & 0.53925329 & 0.83128560 & -47.43030167 \\
\hline-1.17812049 & 0.64508873 & 0.72545010 & -45.88732529 \\
\hline-1.17812049 & 0.64508873 & 0.83128560 & 36.18087387 \\
\hline-1.07228506 & 0.53925329 & 0.72545010 & -31.84730530 \\
\hline-1.07228506 & 0.53925329 & 0.83128560 & 26.50434685 \\
\hline-1.07228506 & 0.64508873 & 0.72545010 & 31.17395973 \\
\hline-1.07228506 & 0.64508873 & 0.83128560 & -24.28413200 \\
\hline-1.71410346 & 0.74141330 & 1.55540633 & -5.43543673 \\
\hline-1.71410346 & 0.74141330 & 1.66124189 & 4.95977879 \\
\hline-1.71410346 & 0.84724873 & 1.55540633 & 0.87686497 \\
\hline-1.71410346 & 0.84724873 & 1.66124189 & -1.72699201 \\
\hline-1.60826802 & 0.74141330 & 1.55540633 & 10.64686108 \\
\hline-1.60826802 & 0.74141330 & 1.66124189 & -8.20977497 \\
\hline-1.60826802 & 0.84724873 & 1.55540633 & -3.65577769 \\
\hline-1.60826802 & 0.84724873 & 1.66124189 & 3.55108237 \\
\hline 1.18551624 & 0.53476322 & -0.06571 & 0.06662474 \\
\hline 1.18551624 & 0.53476322 & 0.04012381 & -12.93669224 \\
\hline 1.18551624 & 0.64059871 & -0.06571164 & -2.08385491 \\
\hline 1.18551624 & 0.64059871 & 0.04012381 & 13.06844616 \\
\hline 1.29135168 & 0.53476322 & -0.06571164 & -8.65847683 \\
\hline 1.29135168 & 0.53476322 & 0.04012381 & 20.82038498 \\
\hline 1.29135168 & 0.64059871 & -0.06571164 & 12.10132694 \\
\hline 1.29135168 & 0.64059871 & 0.04012381 & -21.57293320 \\
\hline 1.96047521 & 0.99393350 & -0.89135671 & 16.04126930 \\
\hline 1.96047521 & 0.99393350 & -0.78552121 & -20.98002434 \\
\hline 1.96047521 & 1.09976900 & -0.89135671 & -14.02134228 \\
\hline 1.96047521 & 1.09976900 & -0.78552121 & 17.13805389 \\
\hline 2.06631064 & 0.99393350 & -0.89135671 & -15.57741833 \\
\hline 2.06631064 & 0.99393350 & -0.78552121 & 19.04079819 \\
\hline 2.06631064 & 1.09976900 & -0.89135671 & 14.21301937 \\
\hline 2.06631064 & 1.09976900 & -0.78552121 & -16.81348991 \\
\hline 1.39046717 & -0.64405900 & 0.58045453 & -53.87173462 \\
\hline 1.39046717 & -0.64405900 & 0.68628997 & 35.82720184 \\
\hline 1.39046717 & -0.53822351 & 0.58045453 & 34.46444321 \\
\hline 1.39046717 & -0.53822351 & 0.68628997 & -19.89678955 \\
\hline 1.49630260 & -0.64405900 & 0.58045453 & 43.87206268 \\
\hline 1.49630260 & -0.64405900 & 0.68628997 & -26.03819466 \\
\hline
\end{tabular}

\section{S-362}




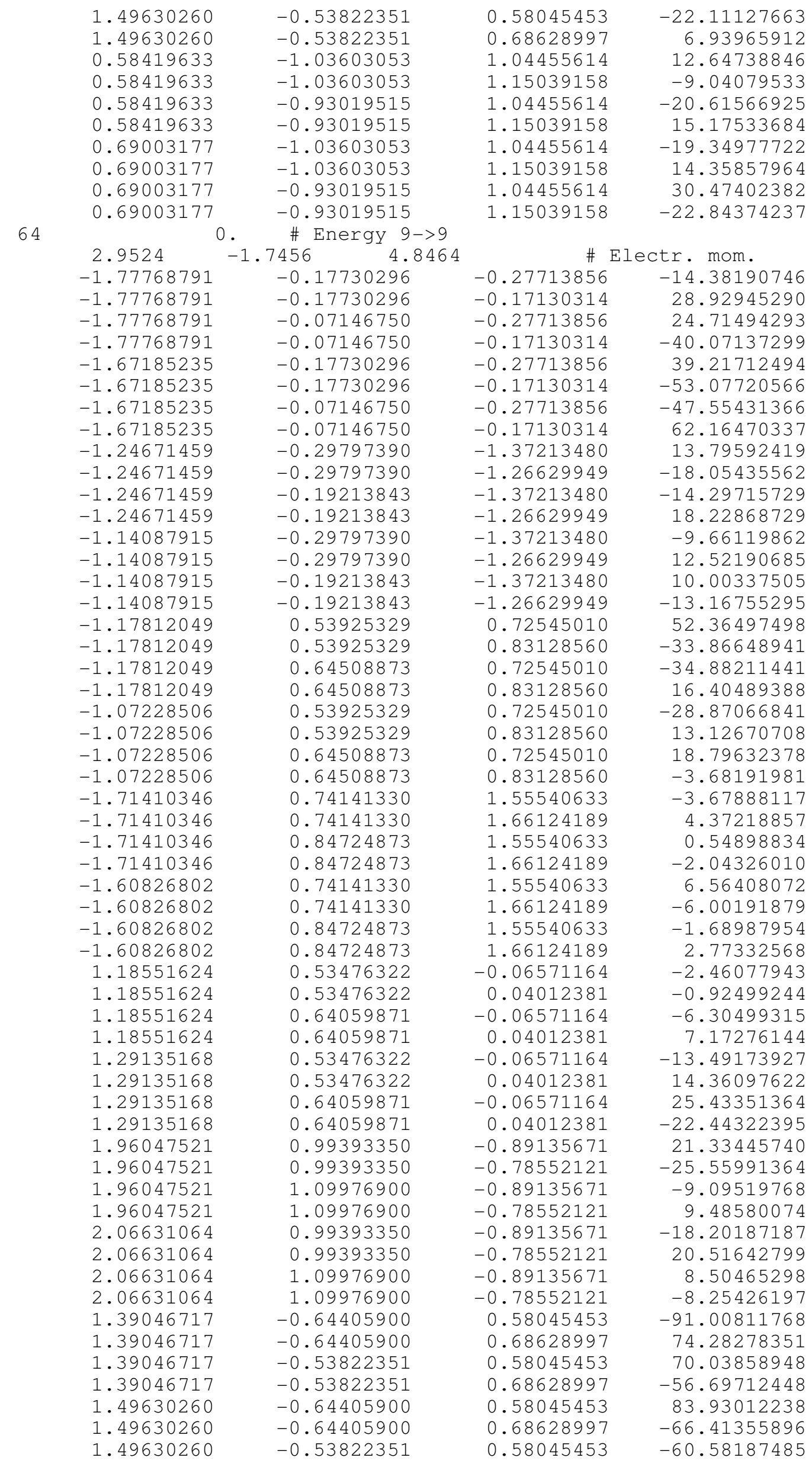

\section{S-363}




1.49630260
0.58419633
0.58419633
0.58419633
0.58419633
0.69003177
0.69003177
0.69003177
0.69003177
-CTAL009A-

$-0.53822351$

$-1.03603053$

$-1.03603053$

$-0.93019515$

$-0.93019515$

$-1.03603053$

$-1.03603053$

$-0.93019515$

$-0.93019515$

\subsection{7 \\ 1.04455614 \\ 1.15039158 \\ 1.04455614 \\ 1.15039158 \\ 1.04455614 \\ 1.15039158 \\ 1.04455614 \\ 1.15039158}

46.07367325

18.67245674

$-12.27451420$

$-26.75722313$

18.51725769

$-27.02256393$

18.66997719

38.36414337

$-27.41305542$
\# $\mathrm{ph} i=-48, \mathrm{ps} i=-57$, roots $=9$

6

$$
\begin{array}{r}
-1.6541 \\
-1.0407 \\
-1.1509 \\
1.2015 \\
1.8338 \\
1.5737
\end{array}
$$

-0.1714
-1.2045
0.7935
-0.0278
-0.9898
0.7164
1.0000
1.0000
1.0000
1.0000
1.0000
1.0000

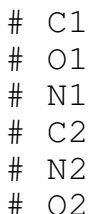

\# $\mathrm{C} 1$

\# $\mathrm{N} 1$

\# $\mathrm{C} 2$

\# $\mathrm{O} 2$

\&TRANSITION $1->.$.

6443983.

\subsection{8 \\ 0.3778}

$-1.70699394$

$-1.70699394$

$-1.70699394$

$-1.70699394$

$-1.60115862$

$-1.60115862$

$-1.60115862$

$-1.60115862$

1.14857543

1.14857543

1.14857543

1.14857543

1.25441086

1.25441086

1.25441086

1.25441086

$-1.09362578$

$-1.09362578$

$-1.09362578$

$-1.09362578$

$-0.98779023$

$-0.98779023$

$-0.98779023$

$-0.98779023$

1.78089249

1.78089249

1.78089249

1.78089249

1.88672793

1.88672793

1.88672793

1.88672793

$-1.20384598$

$-1.20384598$

$-1.20384598$

$-1.20384598$

$-1.09801054$

$-1.09801054$

$-1.09801054$

$-1.09801054$

1.52079928

1.52079928

1.52079928

1.52079928

1.62663472

$\begin{array}{cc}\quad \# \text { Energy } & 1->2 \\ 0.2585 & 0.0612 \\ 0.3176 & -0.7391\end{array}$

$-0.23974958$

$-0.23974958$

$-0.13391411$

$-0.13391411$

$-0.23974958$

$-0.23974958$

$-0.13391411$

$-0.13391411$

0.57671642

0.57671642

0.68255186

0.68255186

0.57671642

0.57671642

0.68255186

0.68255186

$-0.46797100$

$-0.46797100$

$-0.36213556$

$-0.36213556$

$-0.46797100$

$-0.46797100$

$-0.36213556$

$-0.36213556$

0.98020822

0.98020822

1.08604372

1.08604372

0.98020822

0.98020822

1.08604372

1.08604372

0.58981353

0.58981353

0.69564903

0.69564903

0.58981353

0.58981353

0.69564903

0.69564903

$-0.50571668$

$-0.50571668$

$-0.39988124$

$-0.39988124$

$-0.50571668$

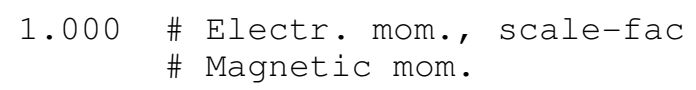

$-0.22430076$

$-0.11846532$

$-0.22430076$

$-0.11846532$

$-0.22430076$

$-0.11846532$

$-0.22430076$

$-0.11846532$

$-0.08068259$

0.02515285

$-0.08068259$

0.02515285

$-0.08068259$

0.02515285

$-0.08068259$

0.02515285

$-1.25737166$

$-1.15153623$

$-1.25737166$

$-1.15153623$

$-1.25737166$

$-1.15153623$

$-1.25737166$

$-1.15153623$

$-1.04271245$

$-0.93687707$

$-1.04271245$

$-0.93687707$

$-1.04271245$

$-0.93687707$

$-1.04271245$

$-0.93687707$

0.74054700

0.84638250

0.74054700

0.84638250

0.74054700

0.84638250

0.74054700

0.84638250

0.66345012

0.76928562

0.66345012

0.76928562

0.66345012

0.09120998

$-1.56865883$

$-1.19162643$

3.30569100

$-2.41467977$

4.69490480

4.73867512

$-7.86968279$

$-9.18325806$

14.22533131

6.98312902

$-10.75526524$

18.32760048

$-25.11934471$

$-17.27565384$

21.91418648

$-1.41459191$

2.54687285

2.51259637

$-4.01184464$

1.38067281

$-2.50555778$

$-2.46329451$

3. 94885945

$-10.10013199$

13.93560028

5.04222584

$-7.40495014$

9.00409508

$-11.72022152$

$-5.18518305$

6.82020092

7.89961672

$-3.90618587$

$-7.11914635$

3. 65652132

$-3.35089898$

0.17902157

3.63924575

$-0.66990912$

$-14.29949093$

18.04890633

9.85871124

$-10.50313091$

5.86387682 


1.62663472
1.62663472
1.62663472
-1.79626679
-1.79626679
-1.79626679
-1.79626679
-1.69043136
-1.69043136
-1.69043136
-1.69043136
0.79423416
0.79423416
0.79423416
0.79423416
0.90006965
0.90006965
0.90006965
0.90006965

$-0.50571668$

$-0.39988124$

$-0.39988124$

0.82610810

0.82610810

0.93194360

0.93194360

0.82610810

0.82610810

0.93194360

0.93194360

$-0.89343792$

$-0.89343792$

$-0.78760254$

$-0.78760254$

$-0.89343792$

$-0.89343792$

$-0.78760254$

$-0.78760254$

64 44666 .

\begin{tabular}{|c|c|c|c|}
\hline-0.0181 & -0.1516 & 1.000 & Electr. mom., scale-fac \\
\hline-0.3664 & 0.1823 & $\#$ & Magnetic mom. \\
\hline-1.70699394 & -0.23974958 & -0.22430076 & -23.08307076 \\
\hline-1.70699394 & -0.23974958 & -0.11846532 & 24.58387756 \\
\hline-1.70699394 & -0.13391411 & -0.22430076 & 24.07382965 \\
\hline-1.70699394 & -0.13391411 & -0.11846532 & -25.25660324 \\
\hline-1.60115862 & -0.23974958 & -0.22430076 & 17.38698196 \\
\hline-1.60115862 & -0.23974958 & -0.11846532 & -18.41958427 \\
\hline-1.60115862 & -0.13391411 & -0.22430076 & -17.76334190 \\
\hline-1.60115862 & -0.13391411 & -0.11846532 & 17.97506142 \\
\hline 1.14857543 & 0.57671642 & -0.08068259 & -18.79381180 \\
\hline 1.14857543 & 0.57671642 & 0.02515285 & 15.75342751 \\
\hline 1.14857543 & 0.68255186 & -0.08068259 & 17.65857315 \\
\hline 1.14857543 & 0.68255186 & 0.02515285 & -14.94701290 \\
\hline 1.25441086 & 0.57671642 & -0.08068259 & 19.85055923 \\
\hline 1.25441086 & 0.57671642 & 0.02515285 & -16.16602898 \\
\hline 1.25441086 & 0.68255186 & -0.08068259 & -18.60967636 \\
\hline 1.25441086 & 0.68255186 & 0.02515285 & 15.44754601 \\
\hline-1.09362578 & -0.46797100 & -1.25737166 & -4.84528160 \\
\hline-1.09362578 & -0.46797100 & -1.15153623 & 6.72172499 \\
\hline-1.09362578 & -0.36213556 & -1.25737166 & 3.64978981 \\
\hline-1.09362578 & -0.36213556 & -1.15153623 & -5.38768291 \\
\hline-0.98779023 & -0.46797100 & -1.25737166 & 4.53218746 \\
\hline-0.98779023 & -0.46797100 & -1.15153623 & -6.49637651 \\
\hline-0.98779023 & -0.36213556 & -1.25737166 & -3.88406110 \\
\hline-0.98779023 & -0.36213556 & -1.15153623 & 5.83418655 \\
\hline 1.78089249 & 0.98020822 & -1.04271245 & 1.11753201 \\
\hline 1.78089249 & 0.98020822 & -0.93687707 & -1.61844838 \\
\hline 1.78089249 & 1.08604372 & -1.04271245 & -0.52190685 \\
\hline 1.78089249 & 1.08604372 & -0.93687707 & 0.80887562 \\
\hline 1.88672793 & 0.98020822 & -1.04271245 & -0.22144540 \\
\hline 1.88672793 & 0.98020822 & -0.93687707 & 0.38992801 \\
\hline 1.88672793 & 1.08604372 & -1.04271245 & -0.15014087 \\
\hline 1.88672793 & 1.08604372 & -0.93687707 & 0.14661337 \\
\hline-1.20384598 & 0.58981353 & 0.74054700 & 31.85647774 \\
\hline-1.20384598 & 0.58981353 & 0.84638250 & -28.10309410 \\
\hline-1.20384598 & 0.69564903 & 0.74054700 & -25.43373489 \\
\hline-1.20384598 & 0.69564903 & 0.84638250 & 23.11967278 \\
\hline-1.09801054 & 0.58981353 & 0.74054700 & -21.93818665 \\
\hline-1.09801054 & 0.58981353 & 0.84638250 & 19.55144119 \\
\hline-1.09801054 & 0.69564903 & 0.74054700 & 18.00775146 \\
\hline-1.09801054 & 0.69564903 & 0.84638250 & -16.77087593 \\
\hline 1.52079928 & -0.50571668 & 0.66345012 & 14.85828686 \\
\hline 1.52079928 & -0.50571668 & 0.76928562 & -12.97862053 \\
\hline 1.52079928 & -0.39988124 & 0.66345012 & -17.20733643 \\
\hline 1.52079928 & -0.39988124 & 0.76928562 & 14.53580475 \\
\hline 1.62663472 & -0.50571668 & 0.66345012 & -10.25165367 \\
\hline
\end{tabular}
0.76928562
0.66345012
0.76928562
1.52134740
1.62718296
1.52134740
1. 62718296
1.52134740
1.62718296
1.52134740
1.62718296
1. 24928117
1. 35511649
1. 24928117
1. 35511649
1. 24928117
1. 35511649
1. 24928117
1. 35511649

$$
\begin{array}{r}
-10.26552105 \\
-0.94237393 \\
3.18412209 \\
0.12957361 \\
-0.32773161 \\
-0.15911941 \\
0.41039249 \\
-0.50591475 \\
0.65254915 \\
0.35990083 \\
-0.60667670 \\
1.09859324 \\
-1.30193233 \\
0.53263974 \\
0.74801779 \\
0.43163162 \\
0.48414516 \\
-3.49212790 \\
0.98478675
\end{array}
$$

$-0.11846532$

$-0.22430076$

$-0.11846532$

$-0.22430076$

1184653

0.08068259

0.02515285

$-1.04271245$

$-0.93687707$

$-1.04271245$

$-0.93687707$

0.84638250

0.74054700

.84638250

.6634501
24.58387756

18.79381180

15.75342751

.65857315

14.94701290

15.44754601

4.5321874

$-6.49637651$

1.11753201

$-1.61844838$

.52190685

.80887562

0.14661337

23.11967278

.93818665

19.55144119

18.00775146

14.85828686

$-12.97862053$

14.53580475

$-10.25165367$ 


1.62663472
1.62663472
1.62663472
-1.79626679
-1.79626679
-1.79626679
-1.79626679
-1.69043136
-1.69043136
-1.69043136
-1.69043136
0.79423416
0.79423416
0.79423416
0.79423416
0.90006965
0.90006965
0.90006965
0.90006965

64

51716.
$-0.50571668$

$-0.39988124$

$-0.39988124$

0.82610810

0.82610810

0.93194360

0.93194360

0.82610810

0.82610810

0.93194360

0.93194360

$-0.89343792$

$-0.89343792$

$-0.78760254$

$-0.78760254$

$-0.89343792$

$-0.89343792$

$-0.78760254$

$-0.78760254$

$-0.0090$

$-0.4146$

$-1.70699394$

$-1.70699394$

$-1.70699394$

$-1.70699394$

$-1.60115862$

$-1.60115862$

$-1.60115862$

$-1.60115862$

1. 14857543

1.14857543

1. 14857543

1. 14857543

1. 25441086

1. 25441086

1. 25441086

1. 25441086

$-1.09362578$

$-1.09362578$

$-1.09362578$

$-1.09362578$

$-0.98779023$

$-0.98779023$

$-0.98779023$

$-0.98779023$

1.78089249

1. 78089249

1. 78089249

1. 78089249

1.88672793

1.88672793

1.88672793

1.88672793

$-1.20384598$

$-1.20384598$

$-1.20384598$

$-1.20384598$

$-1.09801054$

$-1.09801054$

$-1.09801054$

$-1.09801054$

1. 52079928

1. 52079928

1. 52079928

1. 52079928

1. 62663472

0.76928562

0.66345012

0.76928562

1.52134740

1.62718296

1.52134740

1.62718296

1.52134740

1.62718296

1.52134740

1. 62718296

1. 24928117

1. 35511649

1. 24928117

1. 35511649

1. 24928117

1. 35511649

1. 24928117

1. 35511649
9.08004665

11.72930908

$-9.92192841$

$-0.98211414$

0.13719743

$-0.12565926$

0.60427189

2.23197484

$-0.89079928$

$-0.79927880$

$-0.08515480$

$-0.28048453$

$-0.03454646$

0.69404066

$-0.27996212$

0.43578789

0.01931831

$-0.92312056$

0.37895390 $\begin{array}{lr}2.9320 & 0.0254 \\ 1.2041 & -0.0396\end{array}$

$-0.23974958$

$-0.23974958$

$-0.13391411$

$-0.13391411$

$-0.23974958$

$-0.23974958$

$-0.13391411$

$-0.13391411$

0.57671642

0.57671642

0.68255186

0.68255186

0.57671642

0.57671642

0.68255186

0.68255186

$-0.46797100$

$-0.46797100$

$-0.36213556$

$-0.36213556$

$-0.46797100$

$-0.46797100$

$-0.36213556$

$-0.36213556$

0.98020822

0.98020822

1. 08604372

1. 08604372

0.98020822

0.98020822

1. 08604372

1. 08604372

0.58981353

0.58981353

0.69564903

0.69564903

0.58981353

0.58981353

0.69564903

0.69564903

$-0.50571668$

$-0.50571668$

$-0.39988124$

$-0.39988124$

$-0.50571668$
1.000 \# Electr. mom., scale-fac
\# Magnetic mom.

$-0.22430076$

$-0.11846532$

$-0.22430076$

$-0.11846532$

$-0.22430076$

$-0.11846532$

$-0.22430076$

$-0.11846532$

$-0.08068259$

0.02515285

$-0.08068259$

0.02515285

$-0.08068259$

0.02515285

$-0.08068259$

0.02515285

$-1.25737166$

$-1.15153623$

$-1.25737166$

$-1.15153623$

$-1.25737166$

$-1.15153623$

$-1.25737166$

$-1.15153623$

$-1.04271245$

$-0.93687707$

$-1.04271245$

$-0.93687707$

$-1.04271245$

$-0.93687707$

$-1.04271245$

$-0.93687707$

0.74054700

0.84638250

0.74054700

0.84638250

0.74054700

0.84638250

0.74054700

0.84638250

0.66345012

0.76928562

0.66345012

0.76928562

0.66345012
10.58334827

$-11.08779240$

$-9.74146748$

11.41168785

$-16.87105179$

18.17154312

16.77941513

$-19.49696159$

10.01785755

$-10.20660019$

$-15.45608425$

14.37543201

$-16.52916718$

17.13525772

22.83328629

$-21.74629021$

$-3.28521204$

3. 87817335

3. 09860730

$-3.80904174$

3. 16870093

$-3.59087729$

$-2.66407466$

3.09693074

$-4.41273594$

4. 31152487

5.90049028

$-6.27828932$

5.30945778

$-5.51718521$

$-6.54314947$

7. 15650225

16.99426460

$-15.46242905$

$-14.76228428$

13.35709763

$-18.29964447$

16.66806602

17.76306534

$-16.04943275$

$-1.90808141$

$-1.16978800$

2. 44095159

1. 31329310

$-0.76972985$ 


1.62663472
1.62663472
1.62663472
-1.79626679
-1.79626679
-1.79626679
-1.79626679
-1.69043136
-1.69043136
-1.69043136
-1.69043136
0.79423416
0.79423416
0.79423416
0.79423416
0.90006965
0.90006965
0.90006965
0.90006965

64
-0.50571668
-0.39988124
-0.39988124
0.82610810
0.82610810
0.93194360
0.93194360
0.82610810
0.82610810
0.93194360
0.93194360
-0.89343792
-0.89343792
-0.78760254
-0.78760254
-0.89343792
-0.89343792
-0.78760254
-0.78760254
$\#$ Energy $1->5$
$4233 \quad-3.9196$

0.144751220
0.76928562

0.66345012

0.76928562

1.52134740

1.62718296

1. 52134740

1.62718296

1. 52134740

1.62718296

1.52134740

1.62718296

1. 24928117

1. 35511649

1. 24928117

1. 35511649

1. 24928117

1. 35511649

1. 24928117

1. 35511649
3. 47123528

$-0.66530210$

$-2.89329576$

$-2.57521892$

1. 56969035

2.02442265

$-1.32484210$

3. 99926281

$-2.55293965$

$-3.07394052$

2.04799247

$-6.32819986$

3. 70232606

8.66849422

$-5.27508593$

8.18475437

$-4.90203381$

$-11.05322361$

6.86831856

$\begin{array}{lll}-0.1196 & -0.5136 & 0.7542\end{array}$

$-1.70699394$

$-0.23974958$

1.000

\# Electr. mom., scale-fac \# Magnetic mom.

$-1.70699394$

$-0.23974958$

$-0.22430076$

$-7.99734497$

$-0.13391411$

$-1.70699394$

$-0.11846532$

$-0.13391411$

$-0.23974958$

$-1.60115862$

$-1.60115862$

$-1.60115862$

$-1.60115862$

1.14857543

1.14857543

1.14857543

1. 14857543

1. 25441086

1. 25441086

1. 25441086

1. 25441086

$-1.09362578$

$-1.09362578$

$-1.09362578$

$-1.09362578$

$-0.98779023$

$-0.98779023$

$-0.98779023$

$-0.98779023$

1. 78089249

1. 78089249

1. 78089249

1. 78089249

1.88672793

1.88672793

1.88672793

1.88672793

$-1.20384598$

$-1.20384598$

$-1.20384598$

$-1.20384598$

$-1.09801054$

$-1.09801054$

$-1.09801054$

$-1.09801054$

1. 52079928

1.52079928

1. 52079928

1. 52079928

1. 62663472

$-0.23974958$

$-0.13391411$

$-0.13391411$

0.57671642

0.57671642

0.68255186

0.68255186

0.57671642

0.57671642

0.68255186

0.68255186

$-0.46797100$

$-0.46797100$

$-0.36213556$

$-0.36213556$

$-0.46797100$

$-0.46797100$

$-0.36213556$

$-0.36213556$

0.98020822

0.98020822

1.08604372

1. 08604372

0.98020822

0.98020822

1.08604372

1. 08604372

0.58981353

0.58981353

0.69564903

0.69564903

0.58981353

0.58981353

0.69564903

0.69564903

$-0.50571668$

$-0.50571668$

$-0.39988124$

$-0.39988124$

$-0.22430076$

$-0.11846532$

$-0.22430076$

$-0.11846532$

$-0.22430076$

$-0.11846532$

$-0.08068259$

0.02515285

$-0.08068259$

0.02515285

$-0.08068259$

0.02515285

$-0.08068259$

0.02515285

$-1.25737166$

$-1.15153623$

$-1.25737166$

$-1.15153623$

$-1.25737166$

$-1.15153623$

$-1.25737166$

$-1.15153623$

$-1.04271245$

$-0.93687707$

$-1.04271245$

$-0.93687707$

$-1.04271245$

$-0.93687707$

$-1.04271245$

$-0.93687707$

0.74054700

0.84638250

0.74054700

0.84638250

0.74054700

0.84638250

0.74054700

0.84638250

0.66345012

0.76928562

0.66345012

0.76928562

$-0.50571668$

0.66345012

9.49739456

9. 00202942

$-12.20000362$

14.97308636

$-17.74888039$

$-17.31629181$

22.18823242

$-12.32237434$

21.05413055

4.56112480

$-13.18983841$

6.55747652

$-14.47228909$

1.15148973

6.93970776

2. 21813011

$-2.83437347$

$-2.10914588$

2. 90203881

$-2.21996045$

2. 71618962

1. 85071564

$-2.42312789$

$-5.98959923$

7.02347803

6.61093807

$-7.64869690$

5.77526665

$-6.66134834$

$-6.57773590$

7.53590536

2.09874630

$-0.94883001$

$-4.09371042$

3. 54364085

$-3.16439700$

1. 07860208

2. 49370933

$-1.38133693$

13.77559471

$-10.66114330$

$-18.80114746$

16.47517395

$-15.54280281$ 


1.62663472
1.62663472
1.62663472
-1.79626679
-1.79626679
-1.79626679
-1.79626679
-1.69043136
-1.69043136
-1.69043136
-1.69043136
0.79423416
0.79423416
0.79423416
0.79423416
0.90006965
0.90006965
0.90006965
0.90006965

64
$-0.50571668$

$-0.39988124$

$-0.39988124$

0.82610810

0.82610810

0.93194360

0.93194360

0.82610810

0.82610810

0.93194360

0.93194360

$-0.89343792$

$-0.89343792$

$-0.78760254$

$-0.78760254$

$-0.89343792$

$-0.89343792$

$-0.78760254$

$-0.78760254$

61659 .
0.76928562

0.66345012

0.76928562

1.52134740

1.62718296

1.52134740

1.62718296

1.52134740

1.62718296

1.52134740

1.62718296

1.24928117

1.35511649

1.24928117

1.35511649

1.24928117

1.35511649

1.24928117

1.35511649
12.83156586

18.98121071

$-17.00637627$

0.88940823

$-0.28992924$

$-0.56659836$

0.22433776

$-1.91419995$

0.96385610

1.15054369

$-0.60266042$

$-1.20033514$

$-0.24532226$

2.99226069

$-0.58263016$

3.54532862

$-1.09617686$

$-6.65648460$

2.86378264

$\begin{array}{rrr}-0.2395 & -0.2698 & -0.5718 \\ 0.0412 & -0.1777 & 0.2122\end{array}$

$-1.70699394$

$-1.70699394$

$-1.70699394$

$-1.70699394$

$-1.60115862$

$-1.60115862$

$-1.60115862$

$-1.60115862$

1.14857543

1.14857543

1.14857543

1.14857543

1.25441086

1.25441086

1.25441086

1.25441086

$-1.09362578$

$-1.09362578$

$-1.09362578$

$-1.09362578$

$-0.98779023$

$-0.98779023$

$-0.98779023$

$-0.98779023$

1.78089249

1.78089249

1.78089249

1.78089249

1.88672793

1.88672793

1.88672793

1.88672793

$-1.20384598$

$-1.20384598$

$-1.20384598$

$-1.20384598$

$-1.09801054$

$-1.09801054$

$-1.09801054$

$-1.09801054$

1.52079928

1.52079928

1.52079928

1.52079928

1.62663472

$$
-0.1777 \quad 0.2122
$$

$-0.23974958$

$-0.23974958$

$-0.13391411$

$-0.13391411$

$-0.23974958$

$-0.23974958$

$-0.13391411$

$-0.13391411$

0.57671642

0.57671642

0.68255186

0.68255186

0.57671642

0.57671642

0.68255186

0.68255186

$-0.46797100$

$-0.46797100$

$-0.36213556$

$-0.36213556$

$-0.46797100$

$-0.46797100$

$-0.36213556$

$-0.36213556$

0.98020822

0.98020822

1.08604372

1.08604372

0.98020822

0.98020822

1.08604372

1.08604372

0.58981353

0.58981353

0.69564903

0.69564903

0.58981353

0.58981353

0.69564903

0.69564903

$-0.50571668$

$-0.50571668$

$-0.39988124$

$-0.39988124$

$-0.50571668$
1.000 \# Electr. mom., scale-fac
\# Magnetic mom.

$-0.22430076$

$-0.11846532$

$-0.22430076$

$-0.11846532$

$-0.22430076$

$-0.11846532$

$-0.22430076$

$-0.11846532$

$-0.08068259$

0.02515285

$-0.08068259$

0.02515285

$-0.08068259$

0.02515285

$-0.08068259$

0.02515285

$-1.25737166$

$-1.15153623$

$-1.25737166$

$-1.15153623$

$-1.25737166$

$-1.15153623$

$-1.25737166$

$-1.15153623$

$-1.04271245$

$-0.93687707$

$-1.04271245$

$-0.93687707$

$-1.04271245$

$-0.93687707$

$-1.04271245$

$-0.93687707$

0.74054700

0.84638250

0.74054700

0.84638250

0.74054700

0.84638250

0.74054700

0.84638250

0.66345012

0.76928562

0.66345012

0.76928562

0.66345012
$-5.27773285$

6.50228596

6.16829395

$-8.05206776$

8.78010654

$-10.56664371$

$-10.40878010$

13.01790237

1.96244359

$-2.46461773$

$-0.54972219$

1. 34015417

$-0.96064216$

1.58612871

$-0.63826817$

$-0.30990970$

1.65680492

$-2.23604822$

$-1.80992317$

2.51414680

$-1.45770013$

1.92892337

1.47857487

$-2.02888799$

1.78705370

$-2.26288795$

$-1.99346840$

2.53263450

$-1.64104450$

2.07385612

1.79806161

$-2.27543879$

$-2.23536468$

0.74000007

1.10649693

0.31886938

2.00516033

$-0.88589966$

$-1.86011791$

0.63998532

1.39060259

$-1.60582435$

$-0.58849514$

1.04048669

$-2.20648456$ 


1.62663472
1.62663472
1.62663472
-1.79626679
-1.79626679
-1.79626679
-1.79626679
-1.69043136
-1.69043136
-1.69043136
-1.69043136
0.79423416
0.79423416
0.79423416
0.79423416
0.90006965
0.90006965
0.90006965
0.90006965

64

60768 .
$-0.50571668$

$-0.39988124$

$-0.39988124$

0.82610810

0.82610810

0.93194360

0.93194360

0.82610810

0.82610810

0.93194360

0.93194360

$-0.89343792$

$-0.89343792$

$-0.78760254$

$-0.78760254$

$-0.89343792$

$-0.89343792$

$-0.78760254$

$-0.78760254$ $\begin{array}{rr}0.6382 & 0 \\ -0.0518 & 0\end{array}$

$-1.70699394$

$-1.70699394$

$-1.70699394$

$-1.70699394$

$-1.60115862$

$-1.60115862$

$-1.60115862$

$-1.60115862$

1. 14857543

1.14857543

1. 14857543

1. 14857543

1. 25441086

1. 25441086

1. 25441086

1. 25441086

$-1.09362578$

$-1.09362578$

$-1.09362578$

$-1.09362578$

$-0.98779023$

$-0.98779023$

$-0.98779023$

$-0.98779023$

1. 78089249

1. 78089249

1. 78089249

1. 78089249

1.88672793

1.88672793

1.88672793

1.88672793

$-1.20384598$

$-1.20384598$

$-1.20384598$

$-1.20384598$

$-1.09801054$

$-1.09801054$

$-1.09801054$

$-1.09801054$

1. 52079928

1. 52079928

1. 52079928

1. 52079928

1. 62663472
$0.1525 \quad-0.2825$

$$
0.0956-0.1395
$$

$-0.23974958$

$-0.23974958$

$-0.13391411$

$-0.13391411$

$-0.23974958$

$-0.23974958$

$-0.13391411$

$-0.13391411$

0.57671642

0.57671642

0.68255186

0.68255186

0.57671642

0.57671642

0.68255186

0.68255186

$-0.46797100$

$-0.46797100$

$-0.36213556$

$-0.36213556$

$-0.46797100$

$-0.46797100$

$-0.36213556$

$-0.36213556$

0.98020822

0.98020822

1. 08604372

1. 08604372

0.98020822

0.98020822

1. 08604372

1.08604372

0.58981353

0.58981353

0.69564903

0.69564903

0.58981353

0.58981353

0.69564903

0.69564903

$-0.50571668$

$-0.50571668$

$-0.39988124$

$-0.39988124$

$-0.50571668$
0.76928562

0.66345012

0.76928562

1. 52134740

1.62718296

1. 52134740

1.62718296

1.52134740

1.62718296

1.52134740

1. 62718296

1. 24928117

1.35511649

1. 24928117

1.35511649

1. 24928117

1.35511649

1. 24928117

1. 35511649
2. 27050519

1. 48238707

$-1.76024806$

$-0.25974962$

0.31511179

0.31464422

$-0.31290030$

0.22925007

$-0.31769648$

$-0.35284540$

0.35496405

0.24500206

$-0.17990600$

$-0.41042304$

0.33132944

$-0.11160247$

0.11023417

0.21361163

$-0.21467058$
1.000 \# Electr. mom., scale-fac
\# Magnetic mom.

$-0.22430076$

$-0.11846532$

$-0.22430076$

$-0.11846532$

$-0.22430076$

$-0.11846532$

$-0.22430076$

$-0.11846532$

$-0.08068259$

0.02515285

$-0.08068259$

0.02515285

$-0.08068259$

0.02515285

$-0.08068259$

0.02515285

$-1.25737166$

$-1.15153623$

$-1.25737166$

$-1.15153623$

$-1.25737166$

$-1.15153623$

$-1.25737166$

$-1.15153623$

$-1.04271245$

$-0.93687707$

$-1.04271245$

$-0.93687707$

$-1.04271245$

$-0.93687707$

$-1.04271245$

$-0.93687707$

0.74054700

0.84638250

0.74054700

0.84638250

0.74054700

0.84638250

0.74054700

0.84638250

0.66345012

0.76928562

0.66345012

0.76928562

0.66345012
$-5.62583733$

5.40189791

5.88446999

$-6.07423639$

8.16723442

$-8.09834576$

$-8.81202793$

9.24577999

5.53945160

$-7.13358307$

$-5.87194157$

6.98998117

$-6.80429029$

7. 95006466

7. 16262865

$-7.79319334$

2. 77551222

$-3.55960250$

$-2.78410459$

3. 54806733

$-2.77279139$

3. 50121784

2. 76010132

$-3.48156404$

$-0.72949266$

0.88532585

1.27307343

$-1.52697921$

0.66812247

$-0.81357902$

$-1.16040397$

1. 39310265

$-1.23651695$

1.19723809

0.27154833

$-0.66953373$

$-0.84820855$

0.42645586

1.63340318

$-0.88416666$

$-0.56502134$

0.32436681

1.65749526

$-1.72256601$

2. 10470128 


1.62663472
1.62663472
1.62663472
-1.79626679
-1.79626679
-1.79626679
-1.79626679
-1.69043136
-1.69043136
-1.69043136
-1.69043136
0.79423416
0.79423416
0.79423416
0.79423416
0.90006965
0.90006965
0.90006965
0.90006965

$-0.50571668$

$-0.39988124$

$-0.39988124$

0.82610810

0.82610810

0.93194360

0.93194360

0.82610810

0.82610810

0.93194360

0.93194360

$-0.89343792$

$-0.89343792$

$-0.78760254$

$-0.78760254$

$-0.89343792$

$-0.89343792$

$-0.78760254$

$-0.78760254$

64 $\begin{array}{ccc}65540 . & \# \text { Energy } 1->8 \\ -0.3532 & -0.0634 & 0.5979\end{array}$

$-0.0176$

$-1.70699394$

$-1.70699394$

$-1.70699394$

$-1.70699394$

$-1.60115862$

$-1.60115862$

$-1.60115862$

$-1.60115862$

1.14857543

1.14857543

1. 14857543

1. 14857543

1.25441086

1. 25441086

1. 25441086

1. 25441086

$-1.09362578$

$-1.09362578$

$-1.09362578$

$-1.09362578$

$-0.98779023$

$-0.98779023$

$-0.98779023$

$-0.98779023$

1. 78089249

1. 78089249

1. 78089249

1. 78089249

1.88672793

1.88672793

1.88672793

1.88672793

$-1.20384598$

$-1.20384598$

$-1.20384598$

$-1.20384598$

$-1.09801054$

$-1.09801054$

$-1.09801054$

$-1.09801054$

1. 52079928

1.52079928

1. 52079928

1.52079928

1. 62663472

$$
0.1072 \quad-0.0011
$$

$-0.23974958$

$-0.23974958$

$-0.13391411$

$-0.13391411$

$-0.23974958$

$-0.23974958$

$-0.13391411$

$-0.13391411$

0.57671642

0.57671642

0.68255186

0.68255186

0.57671642

0.57671642

0.68255186

0.68255186

$-0.46797100$

$-0.46797100$

$-0.36213556$

$-0.36213556$

$-0.46797100$

$-0.36213556$

$-0.36213556$

0.98020822

0.98020822

1.08604372

1.08604372

0.98020822

0.98020822

1.08604372

1. 08604372

0.58981353

0.58981353

0.69564903

0.69564903

0.58981353

0.58981353

0.69564903

0.69564903

$-0.50571668$

$-0.50571668$

$-0.39988124$

$-0.39988124$

$-0.50571668$
$-0.46797100$
0.76928562
0.66345012
0.76928562
1.52134740
1.62718296
1.52134740
1. 62718296
1.52134740
1.62718296
1.52134740
1.62718296
1. 24928117
1. 35511649
1. 24928117
1. 35511649
1. 24928117
1.35511649
1. 24928117
1. 35511649
-1.72836423
-2.92885780
2.75579667
-0.19794424
0.13259096
0.23155758
-0.18576415
0.40097633
-0.28744918
-0.37768608
0.30042595
0.76316559
-0.39650854
-1.24813199
0.61371583
-1.35016441
0.74471831
2.14168859
-1.17702007

1.000 \# Electr. mom., scale-fac

$-0.22430076$ \# Magnetic mom.

$-0.11846532$

$-0.22430076$

$-0.11846532$

$-0.22430076$

$-0.11846532$

$-0.22430076$

$-0.11846532$

$-0.08068259$

0.02515285

$-0.08068259$

0.02515285

$-0.08068259$

0.02515285

$-0.08068259$

0.02515285

$-1.25737166$

$-1.15153623$

$-1.25737166$

$-1.15153623$

$-1.25737166$

$-1.15153623$

$-1.25737166$

$-1.15153623$

$-1.04271245$

$-0.93687707$

$-1.04271245$

$-0.93687707$

$-1.04271245$

$-0.93687707$

$-1.04271245$

$-0.93687707$

0.74054700

0.84638250

0.74054700

0.84638250

0.74054700

0.84638250

0.74054700

0.84638250

0.66345012

0.76928562

0.66345012

0.76928562

0.66345012
6.27219629

$-6.61299801$

$-6.96091223$

7.99533844

$-9.65310669$

10.23237896

10.85496426

$-12.15122795$

0.85593528

$-0.90859282$

0.34658784

$-0.18415567$

0.05183414

$-0.48127773$

$-1.17590427$

1. 33225691

$-1.57536805$

2. 24855995

2. 27873111

$-3.24073839$

1.32651949

$-1.85645676$

$-1.88115144$

2. 64428592

$-0.35038945$

0.77263737

$-0.02406133$

$-0.26661351$

0.05557414

$-0.33275190$

0.22630905

$-0.05338536$

$-5.11649179$

5.05576563

4. 50922203

$-4.36099291$

5.59309721

$-5.19624567$

$-4.79720402$

4.44046879

$-0.90173531$

0.48636988

1.97341919

$-0.98613161$

$-0.32774350$ 


1.62663472
1.62663472
1.62663472
-1.79626679
-1.79626679
-1.79626679
-1.79626679
-1.69043136
-1.69043136
-1.69043136
-1.69043136
0.79423416
0.79423416
0.79423416
0.79423416
0.90006965
0.90006965
0.90006965
0.90006965

64
$-0.50571668$

$-0.39988124$

$-0.39988124$

0.82610810

0.82610810

0.93194360

0.93194360

0.82610810

0.82610810

0.93194360

0.93194360

$-0.89343792$

$-0.89343792$

$-0.78760254$

$-0.78760254$

$-0.89343792$

$-0.89343792$

$-0.78760254$

$-0.78760254$

\# Energy 1->9

$\begin{array}{rrr}-1.8704 & -0.5892 & -0.5135 \\ 0.0236 & -0.2433 & 0.3397\end{array}$

$-1.70699394$

$-1.70699394$

$-1.70699394$

$-1.70699394$

$-1.60115862$

$-1.60115862$

$-1.60115862$

$-1.60115862$

1.14857543

1.14857543

1. 14857543

1. 14857543

1. 25441086

1. 25441086

1. 25441086

1. 25441086

$-1.09362578$

$-1.09362578$

$-1.09362578$

$-1.09362578$

$-0.98779023$

$-0.98779023$

$-0.98779023$

$-0.98779023$

1. 78089249

1. 78089249

1. 78089249

1. 78089249

1.88672793

1.88672793

1.88672793

1.88672793

$-1.20384598$

$-1.20384598$

$-1.20384598$

$-1.20384598$

$-1.09801054$

$-1.09801054$

$-1.09801054$

$-1.09801054$

1. 52079928

1.52079928

1. 52079928

1. 52079928

1. 62663472
$-0.23974958$

$-0.23974958$

$-0.13391411$

$-0.13391411$

$-0.23974958$

$-0.23974958$

$-0.13391411$

$-0.13391411$

0.57671642

0.57671642

0.68255186

0.68255186

0.57671642

0.57671642

0.68255186

0.68255186

$-0.46797100$

$-0.46797100$

$-0.36213556$

$-0.36213556$

$-0.46797100$

$-0.46797100$

$-0.36213556$

$-0.36213556$

0.98020822

0.98020822

1.08604372

1. 08604372

0.98020822

0.98020822

1. 08604372

1. 08604372

0.58981353

0.58981353

0.69564903

0.69564903

0.58981353

0.58981353

0.69564903

0.69564903

$-0.50571668$

$-0.50571668$

$-0.39988124$

$-0.39988124$

$-0.50571668$
0.76928562

0.66345012

0.76928562

1.52134740

1.62718296

1. 52134740

1. 62718296

1.52134740

1.62718296

1.52134740

1.62718296

1. 24928117

1. 35511649

1. 24928117

1. 35511649

1. 24928117

1. 35511649

1. 24928117

1. 35511649
0.45439380

$-0.36979768$

$-0.14972384$

0.57218009

$-0.39065972$

$-0.42477530$

0.31477126

$-1.04958320$

0.72300351

0.77418298

$-0.55498546$

$-0.18667494$

0.08899210

0.53827292

$-0.30437306$

0.36128145

$-0.17606997$

$-0.89325982$

0.51600975
1.000 \# Electr. mom., scale-fac
\# Magnetic mom.

$-0.22430076$

$-0.11846532$

$-0.22430076$

$-0.11846532$

$-0.22430076$

$-0.11846532$

$-0.22430076$

$-0.11846532$

$-0.08068259$

0.02515285

$-0.08068259$

0.02515285

$-0.08068259$

0.02515285

$-0.08068259$

0.02515285

$-1.25737166$

$-1.15153623$

$-1.25737166$

$-1.15153623$

$-1.25737166$

$-1.15153623$

$-1.25737166$

$-1.15153623$

$-1.04271245$

$-0.93687707$

$-1.04271245$

$-0.93687707$

$-1.04271245$

$-0.93687707$

$-1.04271245$

$-0.93687707$

0.74054700

0.84638250

0.74054700

0.84638250

0.74054700

0.84638250

0.74054700

0.84638250

0.66345012

0.76928562

0.66345012

0.76928562

0.66345012
$-0.01750143$

1. 28178012

0.58019143

$-2.66640806$

3. 90580058

$-6.01587629$

$-5.60414314$

8. 96484661

0.00736093

$-0.30682474$

5.10543585

$-3.70160270$

5.97302103

$-5.67931890$

$-12.15855598$

10.57788372

2.58702779

$-3.37384462$

$-2.78541923$

3.69398355

$-2.66314173$

3. 47467065

2. 86252761

$-3.78207254$

3. 36552739

$-3.62850285$

$-4.33645010$

4. 98453093

$-3.92800617$

4.39410210

4.65178204

$-5.41176701$

$-14.09340191$

11.96032715

10.90756226

$-9.29543114$

9.32343864

$-8.33732796$

$-8.05012608$

7.28661776

5.20427084

$-4.89289951$

$-5.50195312$

5.73654270

$-6.73367691$ 


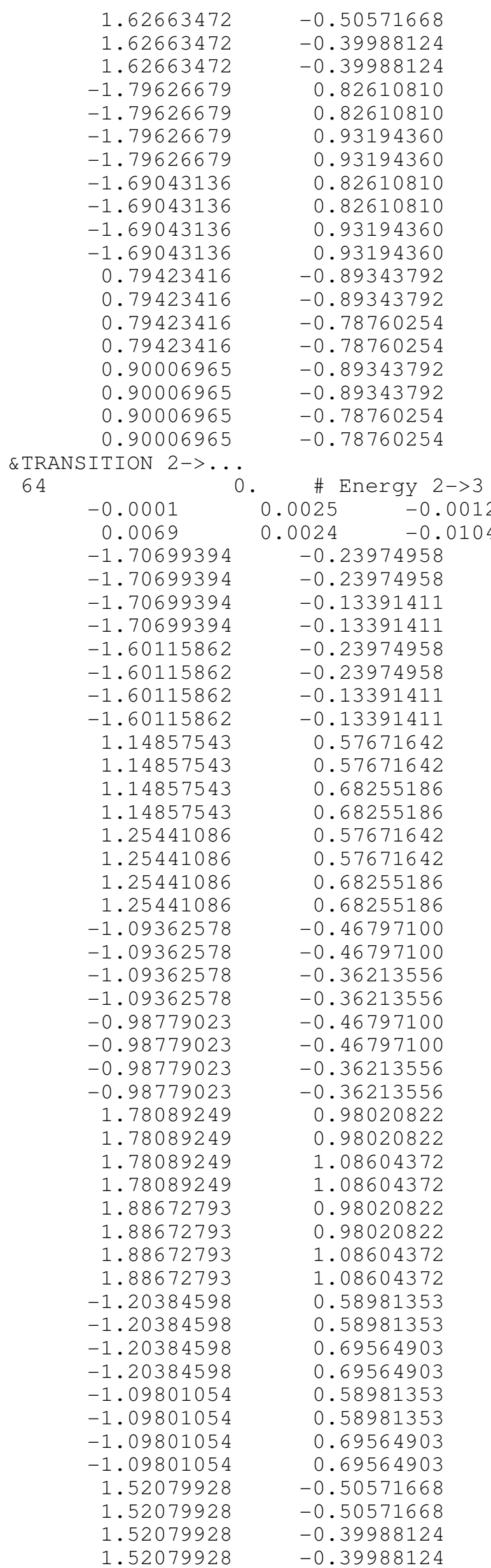

$-0.50571668$

$-0.39988124$

0.82610810

0.82610810

0.93194360

0.82610810

0.82610810

0.93194360

0.93194360

$-0.89343792$

$-0.78760254$

$-0.78760254$

$-0.78760254$

\# Energy 2->3
0.76928562

0.66345012

0.76928562

1.52134740

1.62718296

1.52134740

1.62718296

1.52134740

1.62718296

1.52134740

1.62718296

1. 24928117

1. 35511649

1. 24928117

1.35511649

1. 24928117

1. 35511649

1. 24928117

1. 35511649
6.12758064

7.02700996

$-6.88384867$

0.78796220

$-0.32400322$

$-0.36915073$

0.10225401

$-1.45298529$

0.74629200

0.81588507

$-0.37239504$

$-1.05148304$

0.49563041

1.59994769

$-0.78849036$

1. 49841297

$-0.75744796$

$-2.25648451$

1. 19033313
1.000 \# Electr. mom., scale-fac \# Magnetic mom.

$-0.22430076$

$-0.11846532$

$-0.22430076$

$-0.11846532$

$-0.22430076$

$-0.11846532$

$-0.22430076$

$-0.11846532$

$-0.08068259$

0.02515285

$-0.08068259$

0.02515285

$-0.08068259$

0.02515285

$-0.08068259$

0.02515285

$-1.25737166$

$-1.15153623$

$-1.25737166$

$-1.15153623$

$-1.25737166$

$-1.15153623$

$-1.25737166$

$-1.15153623$

$-1.04271245$

$-0.93687707$

$-1.04271245$

$-0.93687707$

$-1.04271245$

$-0.93687707$

$-1.04271245$

$-0.93687707$

0.74054700

0.84638250

0.74054700

0.84638250

0.74054700

0.84638250

0.74054700

0.84638250

0.66345012

0.76928562

0.66345012

0.76928562

\begin{abstract}
$-0.01597248$
\end{abstract}
0.07488509

0.12006063

$-0.19210254$

0.11056730

$-0.20002639$

$-0.27054197$

0.38026825

0.63004351

$-0.84491003$

$-0.53400528$

0.71314621

$-0.83968163$

1.10375047

0.74214154

$-0.95505595$

0.15289445

$-0.22352971$

$-0.17232211$

0.25237900

$-0.13674308$

0.19965692

0.15662861

$-0.22820550$

0.22974543

$-0.31990379$

$-0.15286419$

0.22077706

$-0.22235063$

0.29963136

0.15828320

$-0.21733689$

$-0.15873346$

0.06415883

0.08912803

$-0.00821403$

0.02335409

0.03139892

0.00094686

$-0.05150264$

0.09053354

$-0.14844927$

$-0.00836102$

0.00977258 


\begin{abstract}
1.62663472
1.62663472

1.62663472

1.62663472

$-1.79626679$

$-1.79626679$

$-1.79626679$

$-1.79626679$

$-1.69043136$

$-1.69043136$

$-1.69043136$

$-1.69043136$

0.79423416

0.79423416

0.79423416

0.79423416

0.90006965

0.90006965

0.90006965

0.90006965
\end{abstract}

64
$-0.50571668$

$-0.50571668$

$-0.39988124$

$-0.39988124$

0.82610810

0.82610810

0.93194360

0.93194360

0.82610810

0.82610810

0.93194360

0.93194360

$-0.89343792$

$-0.89343792$

$-0.78760254$

$-0.78760254$

$-0.89343792$

$-0.89343792$

$-0.78760254$

$-0.78760254$

0 . \# Energy 2->4

$\begin{array}{lll}0.0082 & -0.2018 & 0.1546\end{array}$

$-0.1334$

$-1.70699394$

$-1.70699394$

$-1.70699394$

$-1.70699394$

$-1.60115862$

$-1.60115862$

$-1.60115862$

$-1.60115862$

1.14857543

1.14857543

1.14857543

1.14857543

1. 25441086

1. 25441086

1. 25441086

1. 25441086

$-1.09362578$

$-1.09362578$

$-1.09362578$

$-1.09362578$

$-0.98779023$

$-0.98779023$

$-0.98779023$

$-0.98779023$

1. 78089249

1.78089249

1.78089249

1. 78089249

1.88672793

1.88672793

1.88672793

1.88672793

$-1.20384598$

$-1.20384598$

$-1.20384598$

$-1.20384598$

$-1.09801054$

$-1.09801054$

$-1.09801054$

$-1.09801054$

1.52079928

1. 52079928

1.52079928

1. 52079928

$$
-0.1438 \quad 0.1843
$$

$-0.23974958$

$-0.23974958$

$-0.13391411$

$-0.13391411$

$-0.23974958$

$-0.23974958$

$-0.13391411$

$-0.13391411$

0.57671642

0.57671642

0.68255186

0.68255186

0.57671642

0.57671642

0.68255186

0.68255186

$-0.46797100$

$-0.46797100$

$-0.36213556$

$-0.36213556$

$-0.46797100$

$-0.46797100$

$-0.36213556$

$-0.36213556$

0.98020822

0.98020822

1. 08604372

1. 08604372

0.98020822

0.98020822

1. 08604372

1.08604372

0.58981353

0.58981353

0.69564903

0.69564903

0.58981353

0.58981353

0.69564903

0.69564903

$-0.50571668$

$-0.50571668$

$-0.39988124$

$-0.39988124$
0.66345012

0.76928562

0.66345012

0.76928562

1. 52134740

1. 62718296

1.52134740

1.62718296

1.52134740

1.62718296

1.52134740

1.62718296

1. 24928117

1. 35511649

1. 24928117

1. 35511649

1. 24928117

1. 35511649

1. 24928117

1.35511649

$$
\begin{array}{r}
0.03299030 \\
0.04224579 \\
-0.12195530 \\
0.08241877 \\
-0.02893182 \\
0.02568885 \\
0.03236351 \\
-0.02773094 \\
0.03690831 \\
-0.03270305 \\
-0.04030886 \\
0.03446808 \\
-0.02543583 \\
0.03777610 \\
-0.01659565 \\
-0.01962039 \\
-0.01635077 \\
-0.01532588 \\
0.09219368 \\
-0.02543410
\end{array}
$$

$-0.22430076$

$-0.11846532$

$-0.22430076$

$-0.11846532$

$-0.22430076$

$-0.11846532$

$-0.22430076$

$-0.11846532$

$-0.08068259$

0.02515285

$-0.08068259$

0.02515285

$-0.08068259$

0.02515285

$-0.08068259$

0.02515285

$-1.25737166$

$-1.15153623$

$-1.25737166$

$-1.15153623$

$-1.25737166$

$-1.15153623$

$-1.25737166$

$-1.15153623$

$-1.04271245$

$-0.93687707$

$-1.04271245$

$-0.93687707$

$-1.04271245$

$-0.93687707$

$-1.04271245$

$-0.93687707$

0.74054700

0.84638250

0.74054700

0.84638250

0.74054700

0.84638250

0.74054700

0.84638250

0.66345012

0.76928562

0.66345012

0.76928562
Electr. mom., scale-fac Magnetic mom. $-2.19204354$

2. 14611101

2. 51768017

$-2.35980606$

2.17747116

$-1.99216604$

$-2.39724827$

2.03430271

$-8.37252522$

13.22980881

4.30672169

$-8.79220581$

15.11578369

$-22.06049919$

$-10.08671379$

16.21225357

$-1.14435923$

1. 58228934

1.54353416

$-2.07922840$

1. 32359374

$-1.82450449$

$-1.79617584$

2.40695119

$-1.07108760$

1.73846459

$-0.65803587$

0.33628052

2.15352535

$-2.96322107$

$-0.54225230$

1. 02335548

7.69380856

$-8.13626575$

$-7.09477901$

7.44170141

$-5.89408922$

6.54268265

5. 91983843

$-6.45926714$

$-6.82171059$

6.43679905

5.86356068

$-3.72893119$ 
1.62663472

1. 62663472

1. 62663472

1.62663472

$-1.79626679$

$-1.79626679$

$-1.79626679$

$-1.79626679$

$-1.69043136$

$-1.69043136$

$-1.69043136$

$-1.69043136$

0.79423416

0.79423416

0.79423416

0.79423416

0.90006965

0.90006965

0.90006965

64 $\begin{array}{lr}0.66345012 & 0.66331732 \\ 0.76928562 & -1.57937145 \\ 0.66345012 & 1.19872808 \\ 0.76928562 & -1.34235466 \\ 1.52134740 & -0.84343225 \\ 1.62718296 & 0.47548911 \\ 1.52134740 & 0.48605701 \\ 1.62718296 & -0.26486754 \\ 1.52134740 & 1.59059429 \\ 1.62718296 & -0.98906344 \\ 1.52134740 & -1.03983939 \\ 1.62718296 & 0.63240856 \\ 1.24928117 & 1.01156259 \\ 1.35511649 & -1.06313467 \\ 1.24928117 & -0.60906839 \\ 1.35511649 & 1.05754852 \\ 1.24928117 & -0.07918506 \\ 1.35511649 & 0.55933672 \\ 1.24928117 & -1.09238970 \\ 1.35511649 & -0.05173632\end{array}$ 0 . \# Energy 2->5

$-0.50571668$

$-0.39988124$

$-0.39988124$

0.82610810

0.82610810

0.93194360

0.93194360

0.82610810

0.82610810

0.93194360

0.93194360

$-0.89343792$

$-0.89343792$

$-0.78760254$

$-0.78760254$

$-0.89343792$

$-0.89343792$

$-0.78760254$

$-0.78760254$
0.90006965

$-0.50571668$

$\begin{array}{rrr}0.0156 & -0.2094 & 0.0578 \\ -0.1567 & -0.1090 & 0.2274\end{array}$

$-1.70699394$

$-1.70699394$

$-1.70699394$

$-1.70699394$

$-1.60115862$

$-1.60115862$

$-1.60115862$

$-1.60115862$

1.14857543

1.14857543

1. 14857543

1.14857543

1. 25441086

1. 25441086

1. 25441086

1. 25441086

$-1.09362578$

$-1.09362578$

$-1.09362578$

$-1.09362578$

$-0.98779023$

$-0.98779023$

$-0.98779023$

$-0.98779023$

1. 78089249

1. 78089249

1. 78089249

1. 78089249

1.88672793

1.88672793

1.88672793

1.88672793

$-1.20384598$

$-1.20384598$

$-1.20384598$

$-1.20384598$

$-1.09801054$

$-1.09801054$

$-1.09801054$

$-1.09801054$

1.52079928

1. 52079928

1.52079928

1. 52079928
$-0.23974958$

$-0.23974958$

$-0.13391411$

$-0.13391411$

$-0.23974958$

$-0.23974958$

$-0.13391411$

$-0.13391411$

0.57671642

0.57671642

0.68255186

0.68255186

0.57671642

0.57671642

0.68255186

0.68255186

$-0.46797100$

$-0.46797100$

$-0.36213556$

$-0.36213556$

$-0.46797100$

$-0.46797100$

$-0.36213556$

$-0.36213556$

0.98020822

0.98020822

1. 08604372

1. 08604372

0.98020822

0.98020822

1. 08604372

1.08604372

0.58981353

0.58981353

0.69564903

0.69564903

0.58981353

0.58981353

0.69564903

0.69564903

$-0.50571668$

$-0.50571668$

$-0.39988124$

$-0.39988124$

1.000 \# Electr. mom., scale-fac \# Magnetic mom.

$-0.22430076$

$-0.11846532$

$-0.22430076$

$-0.11846532$

$-0.22430076$

$-0.11846532$

$-0.22430076$

$-0.11846532$

$-0.08068259$

0.02515285

$-0.08068259$

0.02515285

$-0.08068259$

0.02515285

$-0.08068259$

0.02515285

$-1.25737166$

$-1.15153623$

$-1.25737166$

$-1.15153623$

$-1.25737166$

$-1.15153623$

$-1.25737166$

$-1.15153623$

$-1.04271245$

$-0.93687707$

$-1.04271245$

$-0.93687707$

$-1.04271245$

$-0.93687707$

$-1.04271245$

$-0.93687707$

0.74054700

0.84638250

0.74054700

0.84638250

0.74054700

0.84638250

0.74054700

0.84638250

0.66345012

0.76928562

0.66345012

0.76928562
0.09781991

$-0.23946904$

$-0.25306630$

0.71802121

$-1.53611517$

1. 90066481

1. 93968415

$-2.72923970$

$-10.67006874$

16.15043068

6.39889097

$-11.42361164$

17.86037445

$-25.42859840$

$-12.63503075$

19.28880501

$-1.84871495$

2. 54653049

2. 21228123

$-3.01129794$

1. 88845503

$-2.60120034$

$-2.32623672$

3.15443611

$-0.41362038$

0.82910150

$-1.30633128$

1. 24914277

1. 48050904

$-2.04100108$

0.08850898

0.14373808

5.50640583

$-5.94170570$

$-4.73336077$

5.02277803

$-3.60427952$

4.37201262

3.63459802

$-4.21807384$

$-6.54870939$

6.54838371

4.92234087

$-3.07602453$ 


\begin{abstract}
1.62663472
1.62663472

1.62663472

1.62663472

$-1.79626679$

$-1.79626679$

$-1.79626679$

$-1.79626679$

$-1.69043136$

$-1.69043136$

$-1.69043136$

$-1.69043136$

0.79423416

0.79423416

0.79423416

0.79423416

0.90006965

0.90006965

0.90006965

0.90006965
\end{abstract}

64 $\begin{array}{rr}0.66345012 & 0.22018926 \\ 0.76928562 & -1.48176825 \\ 0.66345012 & 2.20720053 \\ 0.76928562 & -2.06866384 \\ 1.52134740 & -0.61868382 \\ 1.62718296 & 0.31104353 \\ 1.52134740 & 0.28219649 \\ 1.62718296 & -0.12595744 \\ 1.52134740 & 1.30802369 \\ 1.62718296 & -0.78115231 \\ 1.52134740 & -0.76092184 \\ 1.62718296 & 0.44217724 \\ 1.24928117 & 1.05882907 \\ 1.35511649 & -1.14214408 \\ 1.24928117 & -0.54067653 \\ 1.35511649 & 1.09766221 \\ 1.24928117 & 0.02282310 \\ 1.35511649 & 0.55588686 \\ 1.24928117 & -1.41643608 \\ 1.35511649 & 0.06221401\end{array}$

1.000 \# Electr. mom., scale-fac \# Magnetic mom. $\begin{array}{lll}0.3598 & 0.1399 & -0.0205\end{array}$

$\begin{array}{lll}0.0885 & -0.0128 & -0.1895\end{array}$

$-1.70699394$

$-1.70699394$

$-1.70699394$

$-1.70699394$

$-1.60115862$

$-1.60115862$

$-1.60115862$

$-1.60115862$

1.14857543

1.14857543

1.14857543

1.14857543

1.25441086

1. 25441086

1. 25441086

1. 25441086

$-1.09362578$

$-1.09362578$

$-1.09362578$

$-1.09362578$

$-0.98779023$

$-0.98779023$

$-0.98779023$

$-0.98779023$

1. 78089249

1.78089249

1.78089249

1. 78089249

1.88672793

1.88672793

1.88672793

1.88672793

$-1.20384598$

$-1.20384598$

$-1.20384598$

$-1.20384598$

$-1.09801054$

$-1.09801054$

$-1.09801054$

$-1.09801054$

1.52079928

1. 52079928

1.52079928

1. 52079928
$-0.50571668$

$-0.50571668$

0.82610810

0.82610810

0.93194360

0.93194360

0.82610810

0.82610810

0.93194360

0.93194360

$-0.89343792$

$-0.89343792$

$-0.78760254$

$-0.78760254$

$-0.89343792$

$-0.89343792$

$-0.78760254$

$-0.78760254$

$-0.23974958$

$-0.23974958$

$-0.13391411$

$-0.13391411$

$-0.23974958$

$-0.23974958$

$-0.13391411$

$-0.13391411$

0.57671642

0.57671642

0.68255186

0.68255186

0.57671642

0.57671642

0.68255186

0.68255186

$-0.46797100$

$-0.46797100$

$-0.36213556$

$-0.36213556$

$-0.46797100$

$-0.46797100$

$-0.36213556$

$-0.36213556$

0.98020822

0.98020822

1.08604372

1. 08604372

0.98020822

0.98020822

1. 08604372

1.08604372

0.58981353

0.58981353

0.69564903

0.69564903

0.58981353

0.58981353

0.69564903

0.69564903

$-0.50571668$

$-0.50571668$

$-0.39988124$

$-0.39988124$
$-0.22430076$

$-0.11846532$

$-0.22430076$

$-0.11846532$

$-0.22430076$

$-0.11846532$

$-0.22430076$

$-0.11846532$

$-0.08068259$

0.02515285

$-0.08068259$

0.02515285

$-0.08068259$

0.02515285

$-0.08068259$

0.02515285

$-1.25737166$

$-1.15153623$

$-1.25737166$

$-1.15153623$

$-1.25737166$

$-1.15153623$

$-1.25737166$

$-1.15153623$

$-1.04271245$

$-0.93687707$

$-1.04271245$

$-0.93687707$

$-1.04271245$

$-0.93687707$

$-1.04271245$

$-0.93687707$

0.74054700

0.84638250

0.74054700

0.84638250

0.74054700

0.84638250

0.74054700

0.84638250

0.66345012

0.76928562

0.66345012

0.76928562
$-0.91442925$

1.19929624

1.38374996

$-2.03151536$

3. 76512766

$-4.32743120$

$-4.54311800$

5.60745573

4.90735102

$-5.11722946$

$-4.04994297$

4.13725615

$-6.42749977$

6.79252529

5.54105473

$-5.74419403$

1.60460365

$-2.14121532$

$-1.37542737$

1.87688851

$-1.38189423$

1.87154114

1. 22530007

$-1.70371950$

0.19735134

$-0.24845102$

0.03033975

$-0.01168301$

$-0.54407251$

0.67838335

0.28372929

$-0.37990648$

$-6.09063101$

5. 43803263

3.96117520

$-3.48894143$

4.14955950

$-3.90500212$

$-2.94160938$

2. 78989339

1.45348895

$-1.48737097$

$-0.86418152$

0.71994841 
1.62663472

1.62663472

1. 62663472

1.62663472

$-1.79626679$

$-1.79626679$

$-1.79626679$

$-1.79626679$

$-1.69043136$

$-1.69043136$

$-1.69043136$

$-1.69043136$

0.79423416

0.79423416

0.79423416

0.79423416

0.90006965

0.90006965

0.90006965

0.90006965

64
$-0.50571668$

$-0.50571668$

$-0.39988124$

$-0.39988124$

0.82610810

0.82610810

0.93194360

0.93194360

0.82610810

0.82610810

0.93194360

0.93194360

$-0.89343792$

$-0.89343792$

$-0.78760254$

$-0.78760254$

$-0.89343792$

$-0.89343792$

$-0.78760254$

$-0.78760254$

0 . \# Energy 2->7

$\begin{array}{lll}-0.6292 & -0.1018 & 0.1559\end{array}$

$-0.0318$

$-1.70699394$

$-1.70699394$

$-1.70699394$

$-1.70699394$

$-1.60115862$

$-1.60115862$

$-1.60115862$

$-1.60115862$

1.14857543

1. 14857543

1. 14857543

1. 14857543

1. 25441086

1. 25441086

1. 25441086

1. 25441086

$-1.09362578$

$-1.09362578$

$-1.09362578$

$-1.09362578$

$-0.98779023$

$-0.98779023$

$-0.98779023$

$-0.98779023$

1.78089249

1. 78089249

1.78089249

1. 78089249

1.88672793

1.88672793

1.88672793

1. 88672793

$-1.20384598$

$-1.20384598$

$-1.20384598$

$-1.20384598$

$-1.09801054$

$-1.09801054$

$-1.09801054$

$-1.09801054$

1. 52079928

1. 52079928

1.52079928

1. 52079928
0.66345012

0.76928562

0.66345012

0.76928562

1. 52134740

1. 62718296

1.52134740

1. 62718296

1.52134740

1.62718296

1.52134740

1. 62718296

1. 24928117

1. 35511649

1. 24928117

1. 35511649

1. 24928117

1. 35511649

1. 24928117

1.35511649
-1.10879850
1.19872057
0.60460633
-0.57421970
0.66134113
-0.34355658
-0.38224673
0.19533880
-1.13629675
0.64886004
0.69959700
-0.39601013
0.13577759
-0.06626900
-0.26079226
0.10999424
-0.35174903
0.18914714
0.58943659
-0.30746618

1.000 \# Electr. mom., scale-fac \# Magnetic mom.

$-0.22430076$

$-0.11846532$

$-0.22430076$

$-0.11846532$

$-0.22430076$

$-0.11846532$

$-0.22430076$

$-0.11846532$

$-0.08068259$

0.02515285

$-0.08068259$

0.02515285

$-0.08068259$

0.02515285

$-0.08068259$

0.02515285

$-1.25737166$

$-1.15153623$

$-1.25737166$

$-1.15153623$

$-1.25737166$

$-1.15153623$

$-1.25737166$

$-1.15153623$

$-1.04271245$

$-0.93687707$

$-1.04271245$

$-0.93687707$

$-1.04271245$

$-0.93687707$

$-1.04271245$

$-0.93687707$

0.74054700

0.84638250

0.74054700

0.84638250

0.74054700

0.84638250

0.74054700

0.84638250

0.66345012

0.76928562

0.66345012

0.76928562
$-3.36442900$

3. 27230144

4.07027864

$-4.25254107$

4.90871286

$-4.83591175$

$-5.73412275$

5.95398998

1. 44197595

$-3.08624744$

$-1.35926950$

3. 10369563

$-0.23438105$

1.38907397

0.15425614

$-1.53145981$

0.92419082

$-1.24000633$

$-0.92424142$

1. 25091791

$-0.88927227$

1. 15756190

0.86202478

$-1.12849200$

$-2.56036758$

3. 46975565

2.00584221

$-2.80567384$

2.77561736

$-3.67404652$

$-2.19306898$

2.97390962

10.96091938

$-10.05012798$

$-9.65748310$

9.06637573

$-10.11109161$

9.08995247

8.90224361

$-8.18472385$

$-4.66613007$

2. 95464563

6.57794809

$-4.61058378$ 
1.62663472

1. 62663472

$-1.79626679$

$-1.79626679$

$-1.79626679$

$-1.69043136$

$-1.69043136$

$-1.69043136$

$-1.69043136$

0.79423416

0.79423416

0.79423416

0.79423416

0.90006965

0.90006965

0.90006965

0.90006965

64
1. 62663472

1.62663472

$-1.79626679$

$\begin{array}{rr}0.66345012 & 3.87358546 \\ 0.76928562 & -2.57260418 \\ 0.66345012 & -5.39812374 \\ 0.76928562 & 3.88246822 \\ 1.52134740 & -1.30845714 \\ 1.62718296 & 0.88623440 \\ 1.52134740 & 1.08785760 \\ 1.62718296 & -0.72556514 \\ 1.52134740 & 1.71868289 \\ 1.62718296 & -1.16575420 \\ 1.52134740 & -1.48030269 \\ 1.62718296 & 0.98884559 \\ 1.24928117 & -0.91511148 \\ 1.35511649 & 0.69875968 \\ 1.24928117 & 0.91822511 \\ 1.35511649 & -0.76481819 \\ 1.24928117 & 0.82970715 \\ 1.35511649 & -0.66386282 \\ 1.24928117 & -0.69613940 \\ 1.35511649 & 0.63385612\end{array}$ 0 . \# Energy 2->8

$\begin{array}{lll}0.0126 & -0.0368 & -0.0453\end{array}$

$\begin{array}{lll}-0.0371 & 0.0621 & 0.0779\end{array}$

$-1.70699394$

$-1.70699394$

$-1.70699394$

$-1.70699394$

$-1.60115862$

$-1.60115862$

$-1.60115862$

$-1.60115862$

1.14857543

1.14857543

1. 14857543

1.14857543

1. 25441086

1. 25441086

1. 25441086

1. 25441086

$-1.09362578$

$-1.09362578$

$-1.09362578$

$-1.09362578$

$-0.98779023$

$-0.98779023$

$-0.98779023$

$-0.98779023$

1. 78089249

1.78089249

1.78089249

1.78089249

1.88672793

1.88672793

1.88672793

1.88672793

$-1.20384598$

$-1.20384598$

$-1.20384598$

$-1.20384598$

$-1.09801054$

$-1.09801054$

$-1.09801054$

$-1.09801054$

1.52079928

1. 52079928

1. 52079928

1. 52079928
$-0.50571668$

$-0.50571668$

$-0.39988124$

$-0.39988124$

0.82610810

0.82610810

0.93194360

0.93194360

0.82610810

0.82610810

0.93194360

0.93194360

$-0.89343792$

$-0.89343792$

$-0.78760254$

$-0.78760254$

$-0.89343792$

$-0.89343792$

$-0.78760254$

$-0.78760254$$$
-0.23974958
$$

$-0.23974958$

$-0.13391411$

$-0.13391411$

$-0.23974958$

$-0.23974958$

$-0.13391411$

$-0.13391411$

0.57671642

0.57671642

0.68255186

0.68255186

0.57671642

0.57671642

0.68255186

0.68255186

$-0.46797100$

$-0.46797100$

$-0.36213556$

$-0.36213556$

$-0.46797100$

$-0.46797100$

$-0.36213556$

$-0.36213556$

0.98020822

0.98020822

1.08604372

1.08604372

0.98020822

0.98020822

1. 08604372

1.08604372

0.58981353

0.58981353

0.69564903

0.69564903

0.58981353

0.58981353

0.69564903

0.69564903

$-0.50571668$

$-0.39988124$

$-0.39988124$
$-0.50571668$
1.000 \# Electr. mom., scale-fac \# Magnetic mom.

$-0.22430076$

$-0.11846532$

$-0.22430076$

$-0.11846532$

$-0.11846532$

$-0.22430076$

$-0.11846532$

$-0.08068259$

0.02515285

$-0.08068259$

$-0.08068259$

0.02515285

$-0.08068259$

0.02515285

$-1.25737166$

$-1.15153623$

$-1.25737166$

$-1.15153623$

$-1.25737166$

$-1.15153623$

$-1.15153623$

$-1.04271245$

$-0.93687707$

$-1.04271245$

$-0.93687707$

$-1.04271245$

$-0.93687707$

$-1.04271245$

$-0.93687707$

0.74054700

0.84638250

0.74054700

0.84638250

0.74054700

0.84638250

0.74054700

0.84638250

0.66345012

0.76928562

0.66345012

0.76928562
1. 24358010

$-1.34304214$

$-1.73163402$

2.11768413

$-3.40070891$

3.66392207

4. 10854721

$-4.74634504$

$-2.97823143$

3. 34377575

2. 66940689

$-3.03132725$

3.02781606

$-3.18167472$

$-2.76293421$

2.96091986

$-1.20299530$

1. 60849094

1. 04123425

$-1.42496920$

1.06128800

$-1.42546773$

$-0.93467587$

1. 28704190

0.71436721

$-0.99822724$

$-0.56295562$

0.80900639

$-0.64671504$

0.88841879

0.51242667

$-0.71820271$

0.02317541

0.16201854

0.80264044

$-0.95259482$

0.86967695

$-1.21181488$

1.13573289

1.38037896

$-0.72674108$

$-2.34285736$

1. 59480727
$-0.22430076$

0.02515285

$-1.25737166$

$-0.78050488$ 


\subsection{2 \\ 1.62663472 \\ 1.62663472 \\ 1.62663472 \\ $-1.79626679$ \\ $-1.79626679$ \\ $-1.79626679$ \\ $-1.79626679$ \\ $-1.69043136$ \\ $-1.69043136$ \\ $-1.69043136$ \\ $-1.69043136$ \\ 0.79423416 \\ 0.79423416 \\ 0.79423416 \\ 0.79423416 \\ 0.90006965 \\ 0.90006965 \\ 0.90006965 \\ 0.90006965}

64
0.66345012
-0.90026397
0.43972117
0.76928562
1.61973369
0.76928562
1.52134740
$-1.08645844$
0.10296078
$-0.13302188$
$-0.18327968$
0.16402605
$-0.00164748$
0.07666303
0.15472908
$-0.15392230$
0.16311871
$-0.13538194$
$-0.09853864$
0.12084861
$-0.05808723$
0.07964819
$-0.07760980$
$-0.01497565$
1.35511649
$-0.2906$
0.1192

$$
\begin{array}{ll}
-0.1724 & 0.1205
\end{array}
$$$$
0.0071-0.1225
$$

$-1.70699394$

$-1.70699394$

$-1.70699394$

$-1.70699394$

$-1.60115862$

$-1.60115862$

$-1.60115862$

$-1.60115862$

1.14857543

1.14857543

1.14857543

1. 14857543

1.25441086

1.25441086

1.25441086

1.25441086

$-1.09362578$

$-1.09362578$

$-1.09362578$

$-1.09362578$

$-0.98779023$

$-0.98779023$

$-0.98779023$

$-0.98779023$

1.78089249

1.78089249

1.78089249

1.78089249

1.88672793

1.88672793

1.88672793

1. 88672793

$-1.20384598$

$-1.20384598$

$-1.20384598$

$-1.20384598$

$-1.09801054$

$-1.09801054$

$-1.09801054$

$-1.09801054$

1. 52079928

1. 52079928

1. 52079928

1. 52079928
0. \# Energy 2->9

$-0.23974958$

$-0.23974958$

$-0.13391411$

$-0.13391411$

$-0.23974958$

$-0.23974958$

$-0.13391411$

$-0.13391411$

0.57671642

0.57671642

0.68255186

0.68255186

0.57671642

0.57671642

0.68255186

0.68255186

$-0.46797100$

$-0.46797100$

$-0.36213556$

$-0.36213556$

$-0.46797100$

$-0.46797100$

$-0.36213556$

$-0.36213556$

0.98020822

0.98020822

1.08604372

1.08604372

0.98020822

0.98020822

1. 08604372

1.08604372

0.58981353

0.58981353

0.69564903

0.69564903

0.58981353

0.58981353

0.69564903

0.69564903

$-0.50571668$

$-0.50571668$

$-0.39988124$

$-0.39988124$

$$
\begin{gathered}
1.000 \text { \# Electr. mom., scale-fac } \\
\text { \# Magnetic mom. }
\end{gathered}
$$

$-0.22430076$

$-0.11846532$

$-0.22430076$

$-0.11846532$

$-0.22430076$

$-0.11846532$

$-0.22430076$

$-0.11846532$

$-0.08068259$

0.02515285

$-0.08068259$

0.02515285

$-0.08068259$

0.02515285

$-0.08068259$

0.02515285

$-1.25737166$

$-1.15153623$

$-1.25737166$

$-1.15153623$

$-1.25737166$

$-1.15153623$

$-1.25737166$

$-1.15153623$

$-1.04271245$

$-0.93687707$

$-1.04271245$

$-0.93687707$

$-1.04271245$

$-0.93687707$

$-1.04271245$

$-0.93687707$

0.74054700

0.84638250

0.74054700

0.84638250

0.74054700

0.84638250

0.74054700

0.84638250

0.66345012

0.76928562

0.66345012

0.76928562
0.93848175

$-0.85998851$

$-0.96790951$

0.76802504

$-0.20785563$

0.00250170

0.00117487

0.37710851

16.18959427

$-21.00964355$

$-13.00389862$

17.12520599

$-22.33811760$

28.72435188

18.50740242

$-23.82909775$

1.39191604

$-2.00765944$

$-1.76322007$

2.51034665

$-1.39066315$

2.00569010

1. 80128205

$-2.55150247$

$-0.15151732$

0.07122021

1.59378004

$-1.90394938$

$-0.24615654$

0.31913856

$-0.88982463$

1.11831319

$-1.51908839$

1.26835442

1.25443244

$-0.98129559$

0.62703949

$-0.56788200$

$-0.67244953$

0.55910599

1.16975045

$-2.26275373$

0.94319069

$-1.23894286$ 


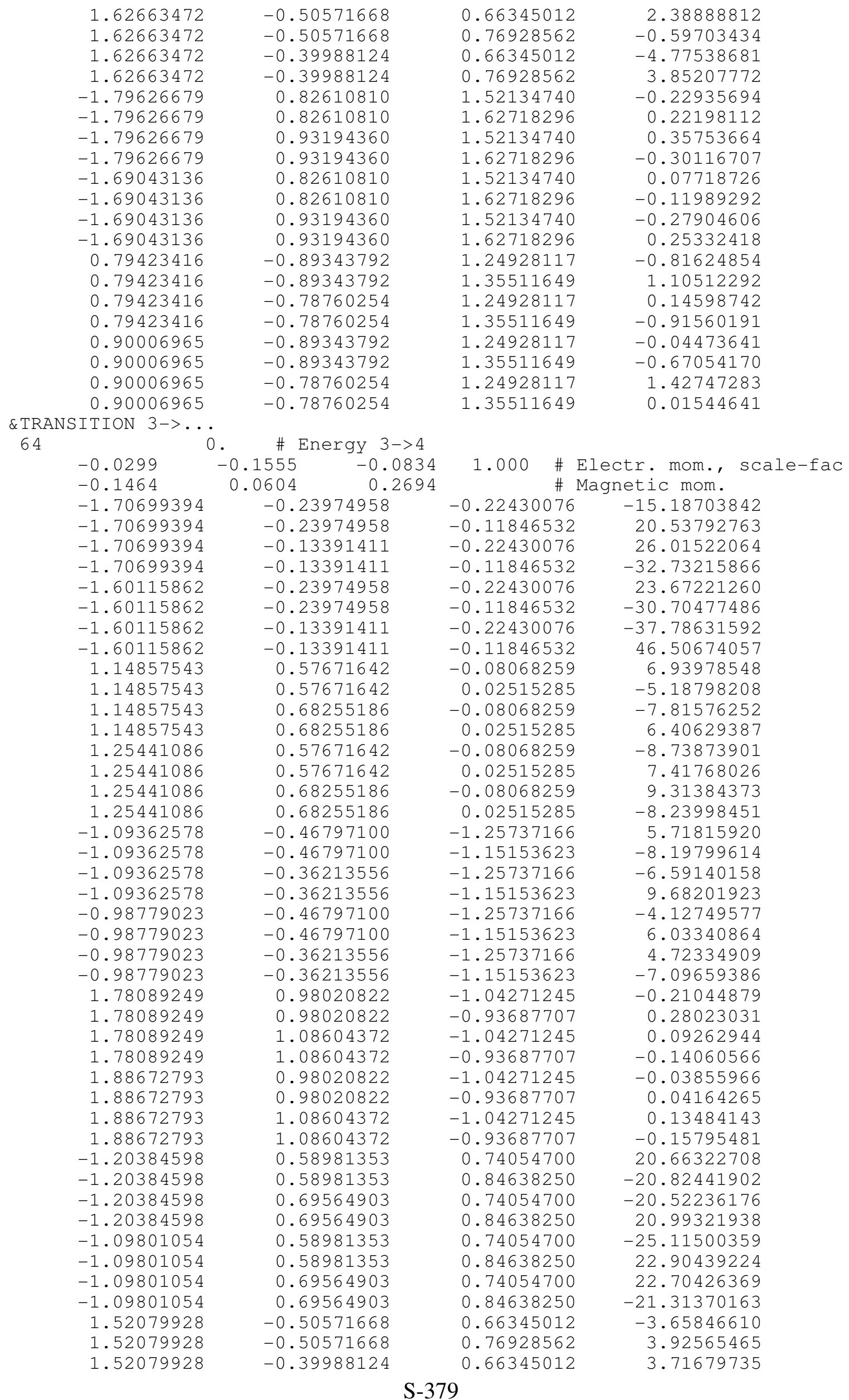


1.52079928

1.62663472

1. 62663472

1. 62663472

1. 62663472

$-1.79626679$

$-1.79626679$

$-1.79626679$

$-1.79626679$

$-1.69043136$

$-1.69043136$

$-1.69043136$

$-1.69043136$

0.79423416

0.79423416

0.79423416

0.79423416

0.90006965

0.90006965

0.90006965

0.90006965

64

$$
\begin{array}{lrr} 
& 0 . & \# \text { Energy } 3->5 \\
0.0344 & 0.1244 & 0.1279 \\
0.1464 & -0.0524 & -0.2706
\end{array}
$$

$-1.70699394$

$-1.70699394$

$-1.70699394$

$-1.70699394$

$-1.60115862$

$-1.60115862$

$-1.60115862$

$-1.60115862$

1.14857543

1.14857543

1. 14857543

1. 14857543

1.25441086

1. 25441086

1. 25441086

1. 25441086

$-1.09362578$

$-1.09362578$

$-1.09362578$

$-1.09362578$

$-0.98779023$

$-0.98779023$

$-0.98779023$

$-0.98779023$

1. 78089249

1.78089249

1. 78089249

1.78089249

1.88672793

1. 88672793

1.88672793

1.88672793

$-1.20384598$

$-1.20384598$

$-1.20384598$

$-1.20384598$

$-1.09801054$

$-1.09801054$

$-1.09801054$

$-1.09801054$

1. 52079928

1.52079928

1. 52079928
$-0.39988124$

$-0.50571668$

$-0.50571668$

$-0.39988124$

$-0.39988124$

0.82610810

0.82610810

0.93194360

0.93194360

0.82610810

0.82610810

0.93194360

0.93194360

$-0.89343792$

$-0.89343792$

$-0.78760254$

$-0.78760254$

$-0.89343792$

$-0.89343792$

$-0.78760254$

$-0.78760254$

$-0.23974958$

$-0.23974958$

$-0.13391411$

$-0.13391411$

$-0.23974958$

$-0.23974958$

$-0.13391411$

$-0.13391411$

0.57671642

0.57671642

0.68255186

0.68255186

0.57671642

0.57671642

0.68255186

0.68255186

$-0.46797100$

$-0.46797100$

$-0.36213556$

$-0.36213556$

$-0.46797100$

$-0.46797100$

$-0.36213556$

$-0.36213556$

0.98020822

0.98020822

1. 08604372

1.08604372

0.98020822

0.98020822

1. 08604372

1.08604372

0.58981353

0.58981353

0.69564903

0.69564903

0.58981353

0.58981353

0.69564903

0.69564903

$-0.50571668$

$-0.50571668$

$-0.39988124$
0.76928562

0.66345012

0.76928562

0.66345012

0.76928562

1. 52134740

1. 62718296

1.52134740

1.62718296

1.52134740

1.62718296

1.52134740

1. 62718296

1. 24928117

1. 35511649

1. 24928117

1. 35511649

1. 24928117

1. 35511649

1. 24928117

1. 35511649

1.000 \# Electr. mom., scale-fac \# Magnetic mom.

$-0.22430076$

$-0.11846532$

$-0.22430076$

$-0.11846532$

$-0.22430076$

$-0.11846532$

$-0.22430076$

$-0.11846532$

$-0.08068259$

0.02515285

$-0.08068259$

0.02515285

$-0.08068259$

0.02515285

$-0.08068259$

0.02515285

$-1.25737166$

$-1.15153623$

$-1.25737166$

$-1.15153623$

$-1.25737166$

$-1.15153623$

$-1.25737166$

$-1.15153623$

$-1.04271245$

$-0.93687707$

$-1.04271245$

$-0.93687707$

$-1.04271245$

$-0.93687707$

$-1.04271245$

$-0.93687707$

0.74054700

0.84638250

0.74054700

0.84638250

0.74054700

0.84638250

0.74054700

0.84638250

0.66345012

0.76928562

0.66345012
$-4.24907017$

3.31569266

$-3.42308426$

$-3.40246153$

3.69936991

$-1.46318173$

1.14556313

1. 25038469

$-0.93246716$

2.32924891

$-1.77509999$

$-2.09136677$

1. 51850033

$-0.41761228$

0.45006213

0.47834039

$-0.54072309$

0.07935138

$-0.25574020$

0.01485151

0.23367080

15.81772804

$-21.30335426$

$-26.83477402$

33.74128342

$-24.55449677$

31.75742722

38.92290115

$-47.87770081$

$-6.80514050$

4.97899675

7.80872059

$-6.28274345$

9.01907253

$-7.70910358$

$-9.75609684$

8.61034298

$-5.86442852$

8.41320419

6.77402878

$-9.94667530$

4.23822689

$-6.19811726$

$-4.86180449$

7. 29965496

0.13297492

$-0.14116751$

$-0.16659589$

0.19590604

0.11183682

$-0.15675575$

$-0.08569934$

0.11760674

$-21.62384987$

21.76617622

21.52769470

$-22.02135849$

26.16970062

$-23.86596298$

$-23.72680283$

2.31898308

$-2.64270377$

$-2.42007399$
22.29815292 


1.52079928
1.62663472
1.62663472
1.62663472
1.62663472
-1.79626679
-1.79626679
-1.79626679
-1.79626679
-1.69043136
-1.69043136
-1.69043136
-1.69043136
0.79423416
0.79423416
0.79423416
0.79423416
0.90006965
0.90006965
0.90006965
0.90006965

64

$-0.1283$

$-1.70699394$

$-1.70699394$

$-1.70699394$

$-1.70699394$

$-1.60115862$

$-1.60115862$

$-1.60115862$

$-1.60115862$

1.14857543

1.14857543

1.14857543

1.14857543

1.25441086

1.25441086

1.25441086

1.25441086

$-1.09362578$

$-1.09362578$

$-1.09362578$

$-1.09362578$

$-0.98779023$

$-0.98779023$

$-0.98779023$

$-0.98779023$

1.78089249

1.78089249

1.78089249

1.78089249

1.88672793

1.88672793

1.88672793

1.88672793

$-1.20384598$

$-1.20384598$

$-1.20384598$

$-1.20384598$

$-1.09801054$

$-1.09801054$

$-1.09801054$

$-1.09801054$

1.52079928

1.52079928

1.52079928
$-0.39988124$

$-0.50571668$

$-0.50571668$

$-0.39988124$

$-0.39988124$

0.82610810

0.82610810

0.93194360

0.93194360

0.82610810

0.82610810

0.93194360

0.93194360

$-0.89343792$

$-0.89343792$

$-0.78760254$

$-0.78760254$

$-0.89343792$

$-0.89343792$

$-0.78760254$

$-0.78760254$

. \# Energy 3->6

$\begin{array}{ll}-0.1885 & 0.1389\end{array}$

$-0.0600 \quad 0.1184$

$-0.23974958$

$-0.23974958$

$-0.13391411$

$-0.13391411$

$-0.23974958$

$-0.23974958$

$-0.13391411$

$-0.13391411$

0.57671642

0.57671642

0.68255186

0.68255186

0.57671642

0.57671642

0.68255186

0.68255186

$-0.46797100$

$-0.46797100$

$-0.36213556$

$-0.36213556$

$-0.46797100$

$-0.46797100$

$-0.36213556$

$-0.36213556$

0.98020822

0.98020822

1.08604372

1.08604372

0.98020822

0.98020822

1.08604372

1.08604372

0.58981353

0.58981353

0.69564903

0.69564903

0.58981353

0.58981353

0.69564903

0.69564903

$-0.50571668$

$-0.50571668$

$-0.39988124$
0.76928562

0.66345012

0.76928562

0.66345012

0.76928562

1.52134740

1.62718296

1.52134740

1.62718296

1.52134740

1.62718296

1.52134740

1.62718296

1.24928117

1. 35511649

1. 24928117

1.35511649

1.24928117

1.35511649

1.24928117

1.35511649

1.000 \# Electr. mom., scale-fac \# Magnetic mom.

$-0.22430076$

$-0.11846532$

$-0.22430076$

$-0.11846532$

$-0.22430076$

$-0.11846532$

$-0.22430076$

$-0.11846532$

$-0.08068259$

0.02515285

$-0.08068259$

0.02515285

$-0.08068259$

0.02515285

$-0.08068259$

0.02515285

$-1.25737166$

$-1.15153623$

$-1.25737166$

$-1.15153623$

$-1.25737166$

$-1.15153623$

$-1.25737166$

$-1.15153623$

$-1.04271245$

$-0.93687707$

$-1.04271245$

$-0.93687707$

$-1.04271245$

$-0.93687707$

$-1.04271245$

$-0.93687707$

0.74054700

0.84638250

0.74054700

0.84638250

0.74054700

0.84638250

0.74054700

0.84638250

0.66345012

0.76928562

0.66345012
3.12580276

$-2.31817198$

2. 42924547

2. 51292658

$-2.89016938$

1.55704153

$-1.21843362$

$-1.33224928$

0.99299634

$-2.45195913$

1.87018430

2.20667863

$-1.60293424$

0.47113821

$-0.48118266$

$-0.51835448$

0.57768679

$-0.11357011$

0.27758992

$-0.02865725$

$-0.24082278$

3.04029012

$-4.33831167$

$-6.36144209$

8.00652504

$-4.84208536$

6.50292683

8.93897152

$-11.00349712$

3.37505198

$-7.34375095$

$-1.02906132$

4.44811869

$-5.12285471$

9.66718769

2.99430323

$-6.78706646$

$-1.35736108$

1.90226126

1.37818623

$-2.00906396$

0.91740662

$-1.28839242$

$-0.87325078$

1.29408860

3.28608751

$-4.39480400$

$-1.99049675$

2. 71274328

$-2.94405913$

3.74879503

1.97600269

$-2.49408245$

$-10.96848011$

9.37197399

10.18826771

$-8.89237881$

11.89978218

$-9.62319469$

$-10.62059689$

8.73912048

4.54271173

$-5.68847466$

$-3.29227662$ 

1.52079928
1.62663472
1.62663472
1.62663472
1. 62663472
$-1.79626679$
$-1.79626679$
$-1.79626679$
$-1.79626679$
$-1.69043136$
$-1.69043136$
$-1.69043136$
$-1.69043136$
0.79423416
0.79423416
0.79423416
0.79423416
0.90006965
0.90006965
0.90006965
0.90006965

64
$-0.39988124$

$-0.50571668$

$-0.50571668$

$-0.39988124$

$-0.39988124$

0.82610810

0.82610810

0.93194360

0.93194360

0.82610810

0.82610810

0.93194360

0.93194360

$-0.89343792$

$-0.89343792$

$-0.78760254$

$-0.78760254$

$-0.89343792$

$-0.89343792$

$-0.78760254$

$-0.78760254$

$$
\text { 0. \# Energy 3->7 }
$$

$\begin{array}{lll}-0.0511 & -0.1483 & 0.0718 \\ -0.0177 & -0.0038 & 0.0038\end{array}$

$-1.70699394$

$-1.70699394$

$-1.70699394$

$-1.70699394$

$-1.60115862$

$-1.60115862$

$-1.60115862$

$-1.60115862$

1.14857543

1. 14857543

1. 14857543

1. 14857543

1. 25441086

1. 25441086

1. 25441086

1.25441086

$-1.09362578$

$-1.09362578$

$-1.09362578$

$-1.09362578$

$-0.98779023$

$-0.98779023$

$-0.98779023$

$-0.98779023$

1. 78089249

1.78089249

1. 78089249

1.78089249

1.88672793

1.88672793

1.88672793

1.88672793

$-1.20384598$

$-1.20384598$

$-1.20384598$

$-1.20384598$

$-1.09801054$

$-1.09801054$

$-1.09801054$

$-1.09801054$

1. 52079928

1.52079928

1. 52079928

$-0.23974958$

$-0.23974958$

$-0.13391411$

$-0.13391411$

$-0.23974958$

$-0.23974958$

$-0.13391411$

$-0.13391411$

0.57671642

0.57671642

0.68255186

0.68255186

0.57671642

0.57671642

0.68255186

0.68255186

$-0.46797100$

$-0.46797100$

$-0.36213556$

$-0.36213556$

$-0.46797100$

$-0.46797100$

$-0.36213556$

$-0.36213556$

0.98020822

0.98020822

1. 08604372

1.08604372

0.98020822

0.98020822

1.08604372

1.08604372

0.58981353

0.58981353

0.69564903

0.69564903

0.58981353

0.58981353

0.69564903

0.69564903

$-0.50571668$

$-0.50571668$

$-0.39988124$
1.000 \# Electr. mom., scale-fac
0.76928562

0.66345012

0.76928562

0.66345012

0.76928562

1. 52134740

1. 62718296

1.52134740

1.62718296

1.52134740

1.62718296

1. 52134740

1.62718296

1. 24928117

1. 35511649

1. 24928117

1. 35511649

1. 24928117

1. 35511649

1. 24928117

1. 35511649 \# Magnetic mom.

3. 42325377

$-1.36224973$

2. 73627710

0.00514670

$-0.66578525$

0.63839287

$-0.46857342$

$-0.55212951$

0.37813535

$-0.85304773$

0.61600596

0.79941028

$-0.54240370$

0.26898596

0.00836764

$-1.12518179$

0.40684956

$-0.90354198$

0.34784213

2. 28175378

$-1.10332787$

$-0.22430076$

$-0.11846532$

2.84106660

$-3.38685060$

$-0.22430076$

$-0.11846532$

$-0.22430076$

$-0.11846532$

$-0.22430076$

$-0.11846532$

$-0.08068259$

0.02515285

$-0.08068259$

0.02515285

$-0.08068259$

0.02515285

$-0.08068259$

0.02515285

$-1.25737166$

$-1.15153623$

$-1.25737166$

$-1.15153623$

$-1.25737166$

$-1.15153623$

$-1.25737166$

$-1.15153623$

$-1.04271245$

$-0.93687707$

$-1.04271245$

$-0.93687707$

$-1.04271245$

$-0.93687707$

$-1.04271245$

$-0.93687707$

0.74054700

0.84638250

0.74054700

0.84638250

0.74054700

0.84638250

0.74054700

0.84638250

0.66345012

0.76928562

0.66345012
$-3.91296697$

4. 71475172

$-4.23468351$

4.99271441

5.61774635

$-6.68933344$

1. 35423970

$-2.34656715$

$-0.54002875$

1. 49798203

$-1.09924650$

2. 22635674

0.21185933

$-1.28325248$

$-0.68485349$

0.94169241

0.61830240

$-0.86329848$

0.53558600

$-0.72675723$

$-0.45680913$

0.63348842

1.10767484

$-1.41729474$

$-0.94197214$

1.19977939

$-1.00921893$

1. 25560534

0.90176034

$-1.11090219$

$-3.15527368$

2. 85184622

3. 49019241

$-3.30357599$

3. 54750848

$-2.95735478$

$-3.47397327$

3. 04342818

$-0.84503204$

0.63990563

0.87166882 


1.52079928
1.62663472
1.62663472
1.62663472
1.62663472
-1.79626679
-1.79626679
-1.79626679
-1.79626679
-1.69043136
-1.69043136
-1.69043136
-1.69043136
0.79423416
0.79423416
0.79423416
0.79423416
0.90006965
0.90006965
0.90006965
0.90006965

64
$-0.39988124$

$-0.50571668$

$-0.50571668$

$-0.39988124$

$-0.39988124$

0.82610810

0.82610810

0.93194360

0.93194360

0.82610810

0.82610810

0.93194360

0.93194360

$-0.89343792$

$-0.89343792$

$-0.78760254$

$-0.78760254$

$-0.89343792$

$-0.89343792$

$-0.78760254$

$-0.78760254$

-0.2156
0.0019

$-1.70699394$

$-1.70699394$

$-1.70699394$

$-1.70699394$

$-1.60115862$

$-1.60115862$

$-1.60115862$

$-1.60115862$

1.14857543

1.14857543

1.14857543

1.14857543

1.25441086

1. 25441086

1.25441086

1.25441086

$-1.09362578$

$-1.09362578$

$-1.09362578$

$-1.09362578$

$-0.98779023$

$-0.98779023$

$-0.98779023$

$-0.98779023$

1.78089249

1.78089249

1.78089249

1.78089249

1.88672793

1.88672793

1.88672793

1.88672793

$-1.20384598$

$-1.20384598$

$-1.20384598$

$-1.20384598$

$-1.09801054$

$-1.09801054$

$-1.09801054$

$-1.09801054$

1.52079928

1.52079928

1.52079928

$-0.46797100$

$$
0 \text {. \# Energy 3->8 }
$$

$\begin{array}{ll}-0.2703 & 0.2145\end{array}$

$$
0.1014 \quad-0.0238
$$

$-0.23974958$

$-0.23974958$

$-0.13391411$

$-0.13391411$

$-0.23974958$

$-0.23974958$

$-0.13391411$

$-0.13391411$

0.57671642

0.57671642

0.68255186

0.68255186

0.57671642

0.57671642

0.68255186

0.68255186

$-0.46797100$

$-0.36213556$

$-0.36213556$

$-0.46797100$

$-0.46797100$

$-0.36213556$

$-0.36213556$

0.98020822

0.98020822

1.08604372

1.08604372

0.98020822

0.98020822

1.08604372

1.08604372

0.58981353

0.58981353

0.69564903

0.69564903

0.58981353

0.58981353

0.69564903

0.69564903

$-0.50571668$

$-0.50571668$

$-0.39988124$
0.76928562

0.66345012

0.76928562

0.66345012

0.76928562

1.52134740

1.62718296

1.52134740

1.62718296

1.52134740

1.62718296

1.52134740

1.62718296

1.24928117

1. 35511649

1. 24928117

1.35511649

1.24928117

1.35511649

1.24928117

1.35511649

1.000 \# Electr. mom., scale-fac \# Magnetic mom.

$-0.22430076$

$-0.11846532$

$-0.22430076$

$-0.11846532$

$-0.22430076$

$-0.11846532$

$-0.22430076$

$-0.11846532$

$-0.08068259$

0.02515285

$-0.08068259$

0.02515285

$-0.08068259$

0.02515285

$-0.08068259$

0.02515285

$-1.25737166$

$-1.15153623$

$-1.25737166$

$-1.15153623$

$-1.25737166$

$-1.15153623$

$-1.25737166$

$-1.15153623$

$-1.04271245$

$-0.93687707$

$-1.04271245$

$-0.93687707$

$-1.04271245$

$-0.93687707$

$-1.04271245$

$-0.93687707$

0.74054700

0.84638250

0.74054700

0.84638250

0.74054700

0.84638250

0.74054700

0.84638250

0.66345012

0.76928562

0.66345012
$-0.86635810$

1.36233687

$-1.09819365$

$-1.39710140$

1.28154802

$-0.14624259$

$-0.16511589$

0.11951884

$-0.12782741$

0.12879024

0.19604841

$-0.14128695$

0.19410084

$-0.02807889$

$-0.46787450$

0.18918654

$-0.36469686$

0.12165356

0.75757235

$-0.36501828$
0.16112803
$-5.66749668$

6.91592932

8.67668533

$-10.04054260$

6.86574459

$-8.35394478$

$-10.49182796$

12.08811474

$-0.85912895$

0.86023581

1.63376570

$-1.09690833$

3.66688681

$-3.88101363$

$-4.84959793$

4.31524563

0.83693457

$-1.04512548$

$-0.99459559$

1.35467863

$-0.46507847$

0.53507793

0.49414432

$-0.65524429$

$-0.66641068$

$-0.53199279$

0.35217223

0.48409256

$-0.80688572$

0.47751632

$-0.40720186$

6.52046633

$-5.82250690$

$-5.50094795$

4.96363640

$-6.02820063$

5.08387136

4.92521095

$-4.18647146$

$-5.37268543$

5.97599745

4.24805689
1.17671156 


1.52079928
1.62663472
1.62663472
1.62663472
1.62663472
-1.79626679
-1.79626679
-1.79626679
-1.79626679
-1.69043136
-1.69043136
-1.69043136
-1.69043136
0.79423416
0.79423416
0.79423416
0.79423416
0.90006965
0.90006965
0.90006965
0.90006965

64

$\begin{array}{ccc}-0.1199 & 0.1356 & 0.0595 \\ 0.0246 & 0.0178 & -0.0030 \\ -1.70699394 & -0.2397495 \\ -1.70699394 & -0.23974958 \\ -1.70699394 & -0.13391411 \\ -1.70699394 & -0.13391411 \\ -1.60115862 & -0.23974958 \\ -1.60115862 & -0.23974958 \\ -1.60115862 & -0.13391411 \\ -1.60115862 & -0.13391411 \\ 1.14857543 & 0.57671642 \\ 1.14857543 & 0.57671642 \\ 1.14857543 & 0.68255186 \\ 1.14857543 & 0.68255186 \\ 1.25441086 & 0.57671642 \\ 1.25441086 & 0.57671642 \\ 1.25441086 & 0.68255186 \\ 1.25441086 & 0.68255186 \\ -1.09362578 & -0.46797100 \\ -1.09362578 & -0.46797100 \\ -1.09362578 & -0.36213556 \\ -1.09362578 & -0.36213556 \\ -0.98779023 & -0.46797100 \\ -0.98779023 & -0.46797100 \\ -0.98779023 & -0.36213556 \\ -0.98779023 & -0.36213556 \\ 1.78089249 & 0.98020822 \\ 1.78089249 & 0.98020822 \\ 1.78089249 & 1.08604372 \\ 1.78089249 & 1.08604372 \\ 1.88672793 & 0.98020822 \\ 1.88672793 & 0.98020822 \\ 1.88672793 & 1.08604372 \\ 1.88672793 & 1.08604372 \\ -1.20384598 & 0.58981353 \\ -1.20384598 & 0.58981353 \\ -1.20384598 & 0.69564903 \\ -1.20384598 & 0.69564903 \\ -1.09801054 & 0.58981353 \\ -1.09801054 & 0.58981353 \\ -1.09801054 & 0.69564903 \\ -1.09801054 & 0.69564903 \\ 1.52079928 & -0.50571668 \\ 1.52079928 & -0.50571668 \\ 1.52079928 & -0.39988124 \\ - & \end{array}$

$-0.39988124$

$-0.50571668$

$-0.50571668$

$-0.39988124$

$-0.39988124$

0.82610810

0.82610810

0.93194360

0.93194360

0.82610810

0.82610810

0.93194360

0.93194360

$-0.89343792$

$-0.89343792$

$-0.78760254$

$-0.78760254$

$-0.89343792$

$-0.89343792$

$-0.78760254$

$-0.78760254$

0.

\# Energy $3->9$
0.1356

$\begin{array}{lr}0.1356 & 0.0595 \\ 0.0178 & -0.0030\end{array}$

0.2243007

$-0.11846532$

$-0.22430076$

$-0.11846532$

$-0.22430076$

$-0.11846532$

$-0.22430076$

$-0.11846532$

$-0.08068259$

0.02515285

$-0.08068259$

0.02515285

$-0.08068259$

0.02515285

$-0.08068259$

0.02515285

$-1.25737166$

$-1.15153623$

$-1.25737166$

$-1.15153623$

$-1.25737166$

$-1.15153623$

$-1.25737166$

$-1.15153623$

$-1.04271245$

$-0.93687707$

$-1.04271245$

$-0.93687707$

$-1.04271245$

$-0.93687707$

$-1.04271245$

$-0.93687707$

0.74054700

0.84638250

0.74054700

0.84638250

0.74054700

0.84638250

0.74054700

0.84638250

0.66345012

0.76928562

0.66345012
$-4.45293713$

4.11205196

$-4.80974293$

$-2.84732437$

3. 32373285

$-0.03092192$

$-0.06007257$

$-0.09797040$

0.16339707

0.24154040

$-0.06597212$

$-0.07473089$

$-0.06592669$

0.18209501

$-0.11147095$

$-0.04395896$

0.09629233

$-0.21166745$

0.15807222

$-0.06529576$

$-0.06655705$

\section{S-384}




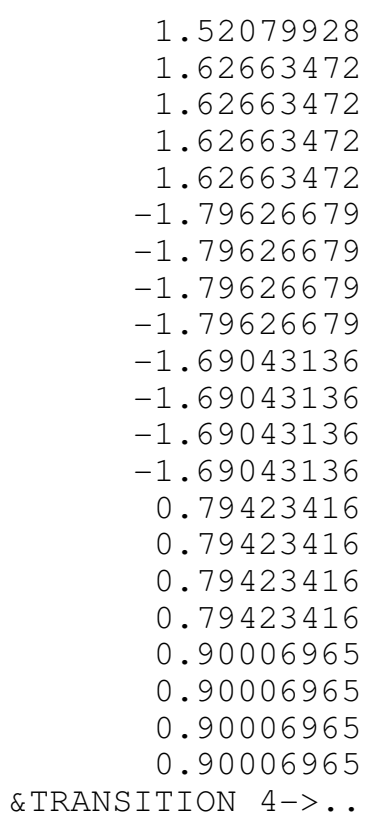

0.2197

$-0.0078$

$-1.70699394$

$-1.70699394$

$-1.70699394$

$-1.70699394$

$-1.60115862$

$-1.60115862$

$-1.60115862$

$-1.60115862$

1.14857543

1.14857543

1.14857543

1.14857543

1.25441086

1.25441086

1.25441086

1.25441086

$-1.09362578$

$-1.09362578$

$-1.09362578$

$-1.09362578$

$-0.98779023$

$-0.98779023$

$-0.98779023$

$-0.98779023$

1.78089249

1.78089249

1.78089249

1.78089249

1.88672793

1.88672793

1.88672793

1.88672793

$-1.20384598$

$-1.20384598$

$-1.20384598$

$-1.20384598$

$-1.09801054$

$-1.09801054$

$-1.09801054$

$-1.09801054$

1.52079928

1.52079928
$-0.39988124$

$-0.50571668$

$-0.50571668$

$-0.39988124$

$-0.39988124$

0.82610810

0.82610810

0.93194360

0.93194360

0.82610810

0.82610810

0.93194360

0.93194360

$-0.89343792$

$-0.89343792$

$-0.78760254$

$-0.78760254$

$-0.89343792$

$-0.89343792$

$-0.78760254$

$-0.78760254$

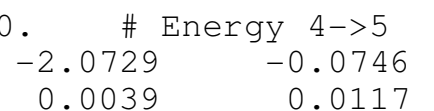

$-0.23974958$

$-0.23974958$

$-0.13391411$

$-0.13391411$

$-0.23974958$

$-0.23974958$

$-0.13391411$

$-0.13391411$

0.57671642

0.57671642

0.68255186

0.68255186

0.57671642

0.57671642

0.68255186

0.68255186

$-0.46797100$

$-0.46797100$

$-0.36213556$

$-0.36213556$

$-0.46797100$

$-0.46797100$

$-0.36213556$

$-0.36213556$

0.98020822

0.98020822

1.08604372

1.08604372

0.98020822

0.98020822

1.08604372

1.08604372

0.58981353

0.58981353

0.69564903

0.69564903

0.58981353

0.58981353

0.69564903

0.69564903

$-0.50571668$

$-0.50571668$
0.76928562

0.66345012

0.76928562

0.66345012

0.76928562

1.52134740

1.62718296

1.52134740

1.62718296

1.52134740

1.62718296

1.52134740

1.62718296

1.24928117

1. 35511649

1. 24928117

1. 35511649

1. 24928117

1.35511649

1. 24928117

1.35511649
2.01250410

$-2.10030437$

2. 21212530

1.77957833

$-1.99744821$

0.25448290

$-0.15325949$

$-0.17590626$

0.13812071

$-0.55866891$

0.36111391

0.36123300

$-0.26688990$

$-0.24634986$

0.03179715

0.40734607

$-0.13819526$

0.32442790

$-0.06368862$

$-0.53561121$

0.20826758
1.000 \# Electr. mom., scale-fac \# Magnetic mom.

$-0.22430076$

$-0.11846532$

$-0.22430076$

$-0.11846532$

$-0.22430076$

$-0.11846532$

$-0.22430076$

$-0.11846532$

$-0.08068259$

0.02515285

$-0.08068259$

0.02515285

$-0.08068259$

0.02515285

$-0.08068259$

0.02515285

$-1.25737166$

$-1.15153623$

$-1.25737166$

$-1.15153623$

$-1.25737166$

$-1.15153623$

$-1.25737166$

$-1.15153623$

$-1.04271245$

$-0.93687707$

$-1.04271245$

$-0.93687707$

$-1.04271245$

$-0.93687707$

$-1.04271245$

$-0.93687707$

0.74054700

0.84638250

0.74054700

0.84638250

0.74054700

0.84638250

0.74054700

0.84638250

0.66345012

0.76928562
19.77939796

$-18.03742218$

$-25.54547501$

25.48263741

$-22.72091675$

20.40228844

28.08109856

$-27.18497276$

2.63628387

1.12698090

2.26541257

$-6.56934166$

$-0.70095479$

$-4.83802366$

$-3.64047885$

9.15766335

0.50887638

$-0.26476151$

0.69031876

$-1.28308773$

$-1.12023067$

1.14569843

0.05291832

0.24814251

0.02908882

1.05658257

$-0.64133942$

$-0.08719080$

$-0.79294622$

0.05874791

1.17658758

$-0.72971505$

$-57.15514755$

46.01284790

45.00876236

$-36.40247345$

54.08411789

$-43.16481018$

$-43.87387466$

35.34171295

$-17.22352791$
14.41907978 


\begin{abstract}
1.52079928
1. 52079928

1.62663472

1.62663472

1.62663472

1.62663472

$-1.79626679$

$-1.79626679$

$-1.79626679$

$-1.79626679$

$-1.69043136$

$-1.69043136$

$-1.69043136$

$-1.69043136$

0.79423416

0.79423416

0.79423416

0.79423416

0.90006965

0.90006965

0.90006965

0.90006965
\end{abstract}

64
$-0.39988124$

$-0.39988124$

$-0.50571668$

$-0.50571668$

$-0.39988124$

$-0.39988124$

0.82610810

0.82610810

0.93194360

0.93194360

0.82610810

0.82610810

0.93194360

0.93194360

$-0.89343792$

$-0.89343792$

$-0.78760254$

$-0.78760254$

$-0.89343792$

$-0.89343792$

$-0.78760254$

$-0.78760254$

0 . \# Energy 4->6

$\begin{array}{lll}0.2956 & -0.2773 & -0.1774\end{array}$

0.0226

$-1.70699394$

$-1.70699394$

$-1.70699394$

$-1.70699394$

$-1.60115862$

$-1.60115862$

$-1.60115862$

$-1.60115862$

1.14857543

1.14857543

1.14857543

1.14857543

1. 25441086

1. 25441086

1. 25441086

1. 25441086

$-1.09362578$

$-1.09362578$

$-1.09362578$

$-1.09362578$

$-0.98779023$

$-0.98779023$

$-0.98779023$

$-0.98779023$

1. 78089249

1. 78089249

1.78089249

1. 78089249

1.88672793

1.88672793

1.88672793

1.88672793

$-1.20384598$

$-1.20384598$

$-1.20384598$

$-1.20384598$

$-1.09801054$

$-1.09801054$

$-1.09801054$

$-1.09801054$

1.52079928

1. 52079928

$$
-0.0314 \quad-0.0833
$$

$-0.23974958$

$-0.23974958$

$-0.13391411$

$-0.13391411$

$-0.23974958$

$-0.23974958$

$-0.13391411$

$-0.13391411$

0.57671642

0.57671642

0.68255186

0.68255186

0.57671642

0.57671642

0.68255186

0.68255186

$-0.46797100$

$-0.46797100$

$-0.36213556$

$-0.36213556$

$-0.46797100$

$-0.46797100$

$-0.36213556$

$-0.36213556$

0.98020822

0.98020822

1.08604372

1.08604372

0.98020822

0.98020822

1.08604372

1.08604372

0.58981353

0.58981353

0.69564903

0.69564903

0.58981353

0.58981353

0.69564903

0.69564903

$-0.50571668$

$-0.50571668$
0.66345012

0.76928562

0.66345012

0.76928562

0.66345012

0.76928562

1.52134740

1.62718296

1.52134740

1.62718296

1.52134740

1.62718296

1.52134740

1.62718296

1. 24928117

1. 35511649

1. 24928117

1. 35511649

1. 24928117

1. 35511649

1. 24928117

1. 35511649
18.19704819

$-13.46472645$

14.70337486

$-12.78034496$

$-13.93903160$

10.75331402

2. 01433563

$-1.03908253$

$-0.92911696$

0.36718017

$-3.37571192$

1.89561617

1.95268512

$-0.98692012$

8.27193546

$-6.00941372$

$-10.12954998$

7.54773235

$-8.31180096$

6.11664200

9. 72452354

$-7.36724615$
1.000 \# Electr. mom., scale-fac \# Magnetic mom.

$-0.22430076$

$-0.11846532$

$-0.22430076$

$-0.11846532$

$-0.22430076$

$-0.11846532$

$-0.22430076$

$-0.11846532$

$-0.08068259$

0.02515285

$-0.08068259$

0.02515285

$-0.08068259$

0.02515285

$-0.08068259$

0.02515285

$-1.25737166$

$-1.15153623$

$-1.25737166$

$-1.15153623$

$-1.25737166$

$-1.15153623$

$-1.25737166$

$-1.15153623$

$-1.04271245$

$-0.93687707$

$-1.04271245$

$-0.93687707$

$-1.04271245$

$-0.93687707$

$-1.04271245$

$-0.93687707$

0.74054700

0.84638250

0.74054700

0.84638250

0.74054700

0.84638250

0.74054700

0.84638250

0.66345012

0.76928562
0.94337535

$-0.12733020$

$-1.68990850$

1.05841351

$-0.65920413$

$-0.26399106$

1.39734757

$-0.63714254$

5.81174850

$-7.49259090$

$-4.58570290$

6.16427565

$-4.09447145$

5.28193521

2. 89041352

$-4.16281986$

$-0.94837254$

1. 44191206

1.09035707

$-1.61167634$

0.88243616

$-1.30353010$

$-1.03075910$

1.48499799

1.80726302

$-2.40882111$

$-2.16834259$

2.88050699

$-1.66229689$

2. 25222135

1.89419734

$-2.54932880$

$-5.15906191$

3. 72959018

3. 57974005

$-2.36258388$

5.82834578

$-4.35915136$

$-4.53662109$

3. 27191806

$-6.21539354$

4. 79569054 


\begin{abstract}
1.52079928
1. 52079928

1. 62663472

1.62663472

1. 62663472

1. 62663472

$-1.79626679$

$-1.79626679$

$-1.79626679$

$-1.79626679$

$-1.69043136$

$-1.69043136$

$-1.69043136$

$-1.69043136$

0.79423416

0.79423416

0.79423416

0.79423416

0.90006965

0.90006965

0.90006965

0.90006965
\end{abstract}

64

\begin{tabular}{|c|c|}
\hline 0.4718 & 0.2965 \\
\hline-0.0848 & 0.1009 \\
\hline-1.70699394 & -0.23974958 \\
\hline-1.70699394 & -0.23974958 \\
\hline-1.70699394 & -0.13391411 \\
\hline-1.70699394 & -0.13391411 \\
\hline-1.60115862 & -0.23974958 \\
\hline-1.60115862 & -0.23974958 \\
\hline-1.60115862 & -0.13391411 \\
\hline-1.60115862 & -0.13391411 \\
\hline 1.14857543 & 0.57671642 \\
\hline 1.14857543 & 0.57671642 \\
\hline 1.14857543 & 0.68255186 \\
\hline 1.14857543 & 0.68255186 \\
\hline 1.25441086 & 0.57671642 \\
\hline 1.25441086 & 0.57671642 \\
\hline 1.25441086 & 0.68255186 \\
\hline 1.25441086 & 0.68255186 \\
\hline-1.09362578 & -0.46797100 \\
\hline-1.09362578 & -0.46797100 \\
\hline-1.09362578 & -0.36213556 \\
\hline-1.09362578 & -0.36213556 \\
\hline-0.98779023 & -0.46797100 \\
\hline-0.98779023 & -0.46797100 \\
\hline-0.98779023 & -0.36213556 \\
\hline-0.98779023 & -0.36213556 \\
\hline 1.78089249 & 0.98020822 \\
\hline 1.78089249 & 0.98020822 \\
\hline 1.78089249 & 1.08604372 \\
\hline 1.78089249 & 1.08604372 \\
\hline 1.88672793 & 0.98020822 \\
\hline 1.88672793 & 0.98020822 \\
\hline 1.88672793 & 1.08604372 \\
\hline 1.88672793 & 1.08604372 \\
\hline-1.20384598 & 0.58981353 \\
\hline-1.20384598 & 0.58981353 \\
\hline-1.20384598 & 0.69564903 \\
\hline-1.20384598 & 0.69564903 \\
\hline-1.09801054 & 0.58981353 \\
\hline-1.09801054 & 0.58981353 \\
\hline-1.09801054 & 0.69564903 \\
\hline-1.09801054 & 0.69564903 \\
\hline 1.52079928 & -0.50571668 \\
\hline 1.52079928 & -0.50571668 \\
\hline
\end{tabular}

$-0.39988124$

$-0.39988124$

$-0.50571668$

$-0.50571668$

$-0.39988124$

$-0.39988124$

0.82610810

0.82610810

0.93194360

0.93194360

0.82610810

0.82610810

0.93194360

0.93194360

$-0.89343792$

$-0.89343792$

$-0.78760254$

$-0.78760254$

$-0.89343792$

$-0.89343792$

$-0.78760254$

$-0.78760254$
0.66345012

0.76928562

0.66345012

0.76928562

0.66345012

0.76928562

1.52134740

1.62718296

1.52134740

1.62718296

1.52134740

1. 62718296

1.52134740

1.62718296

1. 24928117

1. 35511649

1. 24928117

1. 35511649

1. 24928117

1.35511649

1. 24928117

1. 35511649
7. 27816772

$-5.28186703$

4.57897520

$-3.60857582$

$-5.09335279$

3.71079826

0.37351412

$-0.27532947$

$-0.26200375$

0.22379915

$-0.51256001$

0.36822358

0.33027640

$-0.27070811$

0.39263919

$-0.24197260$

$-0.56969732$

0.38199854

$-0.39798999$

0.24523649

0.54832214

$-0.37547851$

$$
\begin{gathered}
1.000 \text { \# Electr. mom., scale-fac } \\
\text { \# Magnetic mom. }
\end{gathered}
$$

$-0.22430076$

$-0.11846532$

$-0.22430076$

$-0.11846532$

$-0.22430076$

$-0.11846532$

$-0.22430076$

$-0.11846532$

$-0.08068259$

0.02515285

$-0.08068259$

0.02515285

$-0.08068259$

0.02515285

$-0.08068259$

0.02515285

$-1.25737166$

$-1.15153623$

$-1.25737166$

$-1.15153623$

$-1.25737166$

$-1.15153623$

$-1.25737166$

$-1.15153623$

$-1.04271245$

$-0.93687707$

$-1.04271245$

$-0.93687707$

$-1.04271245$

$-0.93687707$

$-1.04271245$

$-0.93687707$

0.74054700

0.84638250

0.74054700

0.84638250

0.74054700

0.84638250

0.74054700

0.84638250

0.66345012

0.76928562
$-5.06159639$

5.59245920

7.19549465

$-8.12127972$

7.64420986

$-8.45502663$

$-10.27161884$

11.54633713

0.69486684

$-0.33284950$

$-1.08147120$

0.69559324

$-1.68510151$

1. 59999657

2.14973855

$-1.93110490$

1. 57289779

$-2.20665765$

$-1.46249616$

2.10886431

$-1.09922338$

1. 54822540

0.97125000

$-1.42333162$

$-0.45879701$

0.62239277

0.41620210

$-0.54544109$

0.21482147

$-0.31007892$

$-0.22922665$

0.30027267

2. 56660247

$-2.24253654$

$-3.30708480$

3.25984740

$-2.59944463$

1. 86933362

2.58585644

$-2.20945668$

6.71139526

$-6.56834936$ 
1.52079928

1. 52079928

1. 62663472

1.62663472

1. 62663472

1. 62663472

$-1.79626679$

$-1.79626679$

$-1.79626679$

$-1.79626679$

$-1.69043136$

$-1.69043136$

$-1.69043136$

$-1.69043136$

0.79423416

0.79423416

0.79423416

0.79423416

0.90006965

0.90006965

0.90006965

0.90006965

64
$-0.39988124$

$-0.39988124$

$-0.50571668$

$-0.50571668$

$-0.39988124$

$-0.39988124$

0.82610810

0.82610810

0.93194360

0.93194360

0.82610810

0.82610810

0.93194360

0.93194360

$-0.89343792$

$-0.89343792$

$-0.78760254$

$-0.78760254$

$-0.89343792$

$-0.89343792$

$-0.78760254$

$-0.78760254$

0 .

$-0.1411$

0.0245

$-1.70699394$

$-1.70699394$

$-1.70699394$

$-1.70699394$

$-1.60115862$

$-1.60115862$

$-1.60115862$

$-1.60115862$

1.14857543

1. 14857543

1. 14857543

1.14857543

1. 25441086

1. 25441086

1.25441086

1. 25441086

$-1.09362578$

$-1.09362578$

$-1.09362578$

$-1.09362578$

$-0.98779023$

$-0.98779023$

$-0.98779023$

$-0.98779023$

1. 78089249

1. 78089249

1.78089249

1. 78089249

1. 88672793

1.88672793

1.88672793

1.88672793

$-1.20384598$

$-1.20384598$

$-1.20384598$

$-1.20384598$

$-1.09801054$

$-1.09801054$

$-1.09801054$

$-1.09801054$

1.52079928

1. 52079928 $\begin{array}{ll}0.1249 & 0.0255\end{array}$

$-0.1201-0.0292$

$-0.23974958$

$-0.23974958$

$-0.13391411$

$-0.13391411$

$-0.23974958$

$-0.23974958$

$-0.13391411$

$-0.13391411$

0.57671642

0.57671642

0.68255186

0.68255186

0.57671642

0.57671642

0.68255186

0.68255186

$-0.46797100$

$-0.46797100$

$-0.36213556$

$-0.36213556$

$-0.46797100$

$-0.46797100$

$-0.36213556$

$-0.36213556$

0.98020822

0.98020822

1. 08604372

1. 08604372

0.98020822

0.98020822

1. 08604372

1.08604372

0.58981353

0.58981353

0.69564903

0.69564903

0.58981353

0.58981353

0.69564903

0.69564903

$-0.50571668$

$-0.50571668$

$\begin{array}{lr}0.66345012 & -6.47873926 \\ 0.76928562 & 5.93119383 \\ 0.66345012 & -5.51903248 \\ 0.76928562 & 5.51917362 \\ 0.66345012 & 4.93524265 \\ 0.76928562 & -4.64826536 \\ 1.52134740 & 0.18517040 \\ 1.62718296 & -0.12495344 \\ 1.52134740 & -0.13173153 \\ 1.62718296 & 0.14553070 \\ 1.52134740 & -0.33232039 \\ 1.62718296 & 0.22036780 \\ 1.52134740 & 0.16277212 \\ 1.62718296 & -0.17078283 \\ 1.24928117 & -0.83153009 \\ 1.35511649 & 0.44263050 \\ 1.24928117 & 1.07785940 \\ 1.35511649 & -0.66392243 \\ 1.24928117 & 0.81826079 \\ 1.35511649 & -0.42442429 \\ 1.24928117 & -0.97074109 \\ 1.35511649 & 0.59375745\end{array}$

1.000 \# Electr. mom., scale-fac \# Magnetic mom.

$-0.22430076$

$-0.11846532$

$-0.22430076$

$-0.11846532$

$-0.22430076$

$-0.11846532$

$-0.22430076$

$-0.11846532$

$-0.08068259$

0.02515285

$-0.08068259$

0.02515285

$-0.08068259$

0.02515285

$-0.08068259$

0.02515285

$-1.25737166$

$-1.15153623$

$-1.25737166$

$-1.15153623$

$-1.25737166$

$-1.15153623$

$-1.25737166$

$-1.15153623$

$-1.04271245$

$-0.93687707$

$-1.04271245$

$-0.93687707$

$-1.04271245$

$-0.93687707$

$-1.04271245$

$-0.93687707$

0.74054700

0.84638250

0.74054700

0.84638250

0.74054700

0.84638250

0.74054700

0.84638250

0.66345012

0.76928562
$-3.47700977$

2. 79846764

3. 32093310

$-2.78566432$

3.98660874

$-3.09884906$

$-3.50624752$

2. 77000594

$-2.36422491$

2.99899173

1.56119502

$-2.16880512$

2. 61010051

$-3.26228023$

$-1.74335694$

2. 35817385

0.10447814

$-0.20540273$

$-0.00104274$

0.06355541

0.05987301

$-0.01903739$

$-0.15049496$

0.13389136

$-1.84797752$

1. 54504371

$-2.10510278$

1. 81941724

$-2.41315794$

$-1.53330743$

2. 05026913

0.80108005

$-0.40462109$

$-0.92260605$

0.62501496

$-0.33913836$

0.04614585

0.51962721

$-0.29712966$

3. 32077646

$-3.11256266$
2. 48767495 


\begin{abstract}
1. 52079928
1.52079928

1.62663472

1.62663472

1.62663472

1.62663472

$-1.79626679$

$-1.79626679$

$-1.79626679$

$-1.79626679$

$-1.69043136$

$-1.69043136$

$-1.69043136$

$-1.69043136$

0.79423416

0.79423416

0.79423416

0.79423416

0.90006965

0.90006965

0.90006965

0.90006965
\end{abstract}

64
$-0.39988124$

$-0.39988124$

$-0.50571668$

$-0.50571668$

$-0.39988124$

$-0.39988124$

0.82610810

0.82610810

0.93194360

0.93194360

0.82610810

0.82610810

0.93194360

0.93194360

$-0.89343792$

$-0.89343792$

$-0.78760254$

$-0.78760254$

$-0.89343792$

$-0.89343792$

$-0.78760254$

$-0.78760254$

0 . \# Energy 4->9

$\begin{array}{rrr}-1.8978 & -1.2091 & -0.2007 \\ 0.0020 & -0.0347 & 0.0392\end{array}$

$-0.23974958$

$-0.23974958$

$-0.13391411$

$-0.13391411$

$-0.23974958$

$-0.23974958$

$-0.13391411$

$-0.13391411$

0.57671642

0.57671642

0.68255186

0.68255186

0.57671642

0.57671642

0.68255186

0.68255186

$-0.46797100$

$-0.46797100$

$-0.36213556$

$-0.36213556$

$-0.46797100$

$-0.46797100$

$-0.36213556$

$-0.36213556$

0.98020822

0.98020822

1. 08604372

1.08604372

0.98020822

0.98020822

1.08604372

1.08604372

0.58981353

0.58981353

0.69564903

0.69564903

0.58981353

0.58981353

0.69564903

0.69564903

$-0.50571668$

$-0.50571668$
0.66345012

0.76928562

0.66345012

0.76928562

0.66345012

0.76928562

1.52134740

1.62718296

1.52134740

1.62718296

1.52134740

1.62718296

1.52134740

1. 62718296

1. 24928117

1. 35511649

1. 24928117

1. 35511649

1. 24928117

1.35511649

1. 24928117

1. 35511649
$-3.50285125$

3. 31191778

$-3.28628230$

3. 04738617

3. 30231738

$-3.05855179$

0.25293896

$-0.20485757$

$-0.26594767$

0.22041257

$-0.29157868$

0.23265634

0.29664281

$-0.24611241$

$-0.34903798$

0.06503861

0.64551151

$-0.24510489$

0.59459633

$-0.20072798$

$-1.01360083$

0.47193041
1.000 \# Electr. mom., scale-fac \# Magnetic mom.

$-0.22430076$

$-0.11846532$

$-0.22430076$

$-0.11846532$

$-0.22430076$

$-0.11846532$

$-0.22430076$

$-0.11846532$

$-0.08068259$

0.02515285

$-0.08068259$

0.02515285

$-0.08068259$

0.02515285

$-0.08068259$

0.02515285

$-1.25737166$

$-1.15153623$

$-1.25737166$

$-1.15153623$

$-1.25737166$

$-1.15153623$

$-1.25737166$

$-1.15153623$

$-1.04271245$

$-0.93687707$

$-1.04271245$

$-0.93687707$

$-1.04271245$

$-0.93687707$

$-1.04271245$

$-0.93687707$

0.74054700

0.84638250

0.74054700

0.84638250

0.74054700

0.84638250

0.74054700

0.84638250

0.66345012

0.76928562
0.01097335

1. 70281565

$-1.52331400$

$-0.01467468$

2. 92349648

$-2.22120619$

4.59040928

10.14452744

$-15.01264668$

$-6.05768251$

10.50620556

$-8.60812378$

13.15536213

4.19377756

$-8.68208694$

1. 50851548

$-1.92217994$

$-1.62555552$

2. 20405912

$-1.41242135$

1. 86204123

1. 47122979

$-2.04849195$

1. 20125484

$-1.34315109$

$-1.72297645$

2.03366303

$-1.16267121$

1. 32384729

1.62347353

$-1.91633868$

$-27.73153496$

19.98211861

20.39747810

$-14.28955460$

24.00146866

$-18.09013557$

12.64719200

$-12.04839420$

8.86934566
$-5.13024664$

$-17.12659645$ 


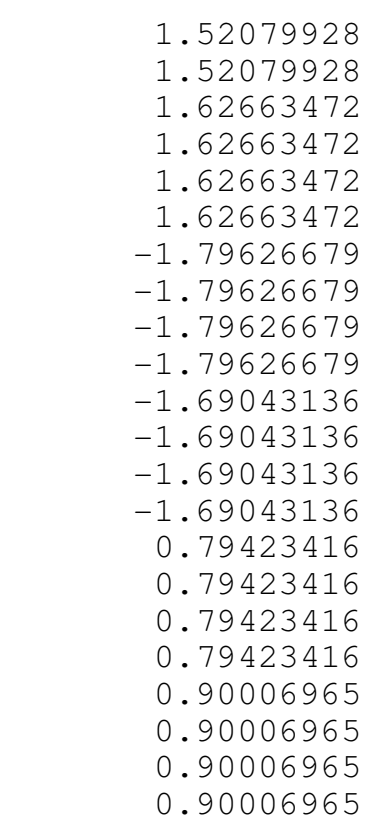

\&TRANSITION $5->\ldots$
-0.39988124
-0.39988124
-0.50571668
-0.50571668
-0.39988124
-0.39988124
0.82610810
0.82610810
0.93194360
0.93194360
0.82610810
0.82610810
0.93194360
0.93194360
-0.89343792
-0.89343792
-0.78760254
-0.78760254
-0.89343792
-0.89343792
-0.78760254
-0.78760254

0. \# Energy 5->6

$\begin{array}{lll}-0.4812 & -0.0386 & 0.5610\end{array}$

$0.0155 \quad-0.0753 \quad-0.0002$

$-1.70699394$

$-1.70699394$

$-1.70699394$

$-1.70699394$

$-1.60115862$

$-1.60115862$

$-1.60115862$

$-1.60115862$

1. 14857543

1.14857543

1.14857543

1.14857543

1.25441086

1. 25441086

1. 25441086

1. 25441086

$-1.09362578$

$-1.09362578$

$-1.09362578$

$-1.09362578$

$-0.98779023$

$-0.98779023$

$-0.98779023$

$-0.98779023$

1. 78089249

1.78089249

1.78089249

1. 78089249

1.88672793

1.88672793

1.88672793

1.88672793

$-1.20384598$

$-1.20384598$

$-1.20384598$

$-1.20384598$

$-1.09801054$

$-1.09801054$

$-1.09801054$

$-1.09801054$

1.52079928
$-0.23974958$

$-0.23974958$

$-0.13391411$

$-0.13391411$

$-0.23974958$

$-0.23974958$

$-0.13391411$

$-0.13391411$

0.57671642

0.57671642

0.68255186

0.68255186

0.57671642

0.57671642

0.68255186

0.68255186

$-0.46797100$

$-0.46797100$

$-0.36213556$

$-0.36213556$

$-0.46797100$

$-0.46797100$

$-0.36213556$

$-0.36213556$

0.98020822

0.98020822

1.08604372

1. 08604372

0.98020822

0.98020822

1.08604372

1.08604372

0.58981353

0.58981353

0.69564903

0.69564903

0.58981353

0.58981353

0.69564903

0.69564903

$-0.50571668$
0.66345012

0.76928562

0.66345012

0.76928562

0.66345012

0.76928562

1.52134740

1.62718296

1.52134740

1.62718296

1.52134740

1. 62718296

1.52134740

1.62718296

1. 24928117

1. 35511649

1. 24928117

1. 35511649

1. 24928117

1. 35511649

1. 24928117

1. 35511649
14.00825214

$-9.87392616$

10.04175186

$-7.51874828$

$-11.19387341$

7.91597843

0.48925009

$-0.17969584$

$-0.04176201$

$-0.11175880$

$-0.57748806$

0.17864417

0.07010300

0.14833315

2.35495663

$-1.74017847$

$-3.07890129$

2. 30122375

$-2.35502601$

1. 73826408

3. 04186130

$-2.28053141$
1.000 \# Electr. mom., scale-fac \# Magnetic mom.
$-0.11846532 \quad 4.65192890$

$-0.22430076 \quad 6.50399303$

$-0.11846532 \quad-6.70167351$

$\begin{array}{ll}-0.22430076 & 7.00537825\end{array}$

$-0.11846532-6.80282402$

$-0.22430076 \quad-8.82968903$

$-0.11846532 \quad 9.24289703$

$-0.08068259-6.20075846$

$0.02515285 \quad 6.99535131$

$-0.08068259$

0.02515285

$-0.08068259$

0.02515285

$-0.08068259$

0.02515285

$-1.25737166$

$-1.15153623$

$-1.25737166$

$-1.15153623$

$-1.25737166$

$-1.15153623$

$-1.25737166$

$-1.15153623$

$-1.04271245$

$-0.93687707$

$-1.04271245$

$-0.93687707$

$-1.04271245$

$-0.93687707$

$-1.04271245$

$-0.93687707$

0.74054700

0.84638250

0.74054700

0.84638250

0.74054700

0.84638250

0.74054700

0.84638250

0.66345012
6.04222965

3. 89521885

$-4.43661451$

$-3.89474726$

4.43004417

2.66918683

$-3.70815134$

$-2.64140487$

3. 66510534

$-2.51810694$

3. 43228984

2. 49934268

$-3.40795398$

$-4.30574656$

5.94649553

4.53878021

$-6.23292065$

3. 88965964

$-5.38420868$

$-3.98448563$

5.49099207

7.36276531

$-5.63450909$

$-6.44698620$

5. 01982021

$-9.21418095$

7.12449217

8.05340195

$-6.28411531$

0.39300275
$-6.78497744$ 
1.52079928

1. 52079928

1. 52079928

1.62663472

1. 62663472

1. 62663472

1.62663472

$-1.79626679$

$-1.79626679$

$-1.79626679$

$-1.79626679$

$-1.69043136$

$-1.69043136$

$-1.69043136$

0.79423416

0.79423416

0.79423416

0.79423416

0.90006965

0.90006965

0.90006965

0.90006965

64
$-1.69043136$

$-0.50571668$

$-0.39988124$

$-0.39988124$

$-0.50571668$

$-0.50571668$

$-0.39988124$

$-0.39988124$

0.82610810

0.82610810

0.93194360

0.93194360

0.82610810

0.82610810

0.93194360

0.93194360

$-0.89343792$

$-0.89343792$

$-0.78760254$

$-0.78760254$

$-0.89343792$

$-0.89343792$

$-0.78760254$

$-0.78760254$

$$
\text { 0. \# Energy 5->7 }
$$

$-0.0432$

0.0545

$-1.70699394$

$-1.70699394$

$-1.70699394$

$-1.70699394$

$-1.60115862$

$-1.60115862$

$-1.60115862$

$-1.60115862$

1.14857543

1.14857543

1.14857543

1.14857543

1. 25441086

1.25441086

1. 25441086

1. 25441086

$-1.09362578$

$-1.09362578$

$-1.09362578$

$-1.09362578$

$-0.98779023$

$-0.98779023$

$-0.98779023$

$-0.98779023$

1. 78089249

1.78089249

1.78089249

1. 78089249

1.88672793

1.88672793

1.88672793

1.88672793

$-1.20384598$

$-1.20384598$

$-1.20384598$

$-1.20384598$

$-1.09801054$

$-1.09801054$

$-1.09801054$

$-1.09801054$

1. 52079928

$$
-0.0756 \quad-0.1597
$$$$
0.0095-0.1194
$$

$-0.23974958$

$-0.23974958$

$-0.13391411$

$-0.13391411$

$-0.23974958$

$-0.23974958$

$-0.13391411$

$-0.13391411$

0.57671642

0.57671642

0.68255186

0.68255186

0.57671642

0.57671642

0.68255186

0.68255186

$-0.46797100$

$-0.46797100$

$-0.36213556$

$-0.36213556$

$-0.46797100$

$-0.46797100$

$-0.36213556$

$-0.36213556$

0.98020822

0.98020822

1.08604372

1. 08604372

0.98020822

0.98020822

1.08604372

1.08604372

0.58981353

0.58981353

0.69564903

0.69564903

0.58981353

0.58981353

0.69564903

0.69564903

$-0.50571668$
0.76928562

0.66345012

0.76928562

0.66345012

0.76928562

0.66345012

0.76928562

1.52134740

1.62718296

1.52134740

1.62718296

1.52134740

1.62718296

1.52134740

1. 62718296

1. 24928117

1. 35511649

1. 24928117

1. 35511649

1. 24928117

1. 35511649

1. 24928117

1.35511649
0.14285232

$-0.55552781$

$-0.15047684$

0.93709129

$-1.18298411$

$-0.78974980$

1.17932081

$-0.21968026$

0.25733891

0.18301775

$-0.20734502$

0.05670807

$-0.14770561$

$-0.08171837$

0.14016551

0.29730040

$-0.23795006$

$-0.13798741$

0.10942165

$-0.44368458$

0.34173515

0.24891233

$-0.18060812$

$$
\begin{aligned}
& 1.000 \text { \# Electr. mom., scale-fac } \\
& \text { \# Magnetic mom. }
\end{aligned}
$$

$-0.22430076$

$-0.11846532$

$-0.22430076$

$-0.11846532$

$-0.22430076$

$-0.11846532$

$-0.22430076$

$-0.11846532$

$-0.08068259$

0.02515285

$-0.08068259$

0.02515285

$-0.08068259$

0.02515285

$-0.08068259$

0.02515285

$-1.25737166$

$-1.15153623$

$-1.25737166$

$-1.15153623$

$-1.25737166$

$-1.15153623$

$-1.25737166$

$-1.15153623$

$-1.04271245$

$-0.93687707$

$-1.04271245$

$-0.93687707$

$-1.04271245$

$-0.93687707$

$-1.04271245$

$-0.93687707$

0.74054700

0.84638250

0.74054700

0.84638250

0.74054700

0.84638250

0.74054700

0.84638250

0.66345012
7.85463381

$-8.78400230$

$-11.36476231$

12.98322296

$-11.65750122$

13.07277393

16.18511772

$-18.38655663$

$-1.45481825$

2.99193978

1.64390945

$-3.20409656$

1. 63346338

$-3.07490396$

$-1.87820566$

3. 37824988

$-3.20995712$

4. 42125511

3.30058146

$-4.61243773$

2. 71362901

$-3.69103456$

$-2.71956468$

3. 76526070

$-0.93126512$

1. 34532452

0.98348379

$-1.35353935$

0.58934426

$-0.89919287$

$-0.65356249$

0.92412126

$-11.65419579$

11.12830734

10.86101246

$-10.58444309$

12.53209782

$-11.32458401$

$-11.17519283$

10.35470772

3. 69463468 
1.52079928

1. 52079928

1. 52079928

1.62663472

1. 62663472

1. 62663472

1.62663472

$-1.79626679$

$-1.79626679$

$-1.79626679$

$-1.79626679$

$-1.69043136$

$-1.69043136$

$-1.69043136$

$-1.69043136$

0.79423416

0.79423416

0.79423416

0.90006965

0.90006965

0.90006965

0.90006965

64
0.79423416

$-0.50571668$

$-0.39988124$

$-0.39988124$

$-0.50571668$

$-0.50571668$

$-0.39988124$

$-0.39988124$

0.82610810

0.82610810

0.93194360

0.93194360

0.82610810

0.82610810

0.93194360

0.93194360

$-0.89343792$

$-0.89343792$

$-0.78760254$

$-0.78760254$

$-0.89343792$

$-0.89343792$

$-0.78760254$

$-0.78760254$

\subsection{5}

0.0287

$-1.70699394$

$-1.70699394$

$-1.70699394$

$-1.70699394$

$-1.60115862$

$-1.60115862$

$-1.60115862$

$-1.60115862$

1.14857543

1. 14857543

1.14857543

1.14857543

1. 25441086

1.25441086

1. 25441086

1. 25441086

$-1.09362578$

$-1.09362578$

$-1.09362578$

$-1.09362578$

$-0.98779023$

$-0.98779023$

$-0.98779023$

$-0.98779023$

1. 78089249

1.78089249

1.78089249

1. 78089249

1.88672793

1.88672793

1.88672793

1.88672793

$-1.20384598$

$-1.20384598$

$-1.20384598$

$-1.20384598$

$-1.09801054$

$-1.09801054$

$-1.09801054$

$-1.09801054$

1. 52079928
0 . \# Energy 5->8

$0.3367 \quad-0.1396$

$0.1011-0.0631$

$-0.23974958$

$-0.23974958$

$-0.13391411$

$-0.13391411$

$-0.23974958$

$-0.13391411$

$-0.13391411$

0.57671642

0.57671642

0.68255186

0.68255186

0.57671642

0.57671642

0.68255186

0.68255186

$-0.46797100$

$-0.46797100$

$-0.36213556$

$-0.36213556$

$-0.46797100$

$-0.46797100$

$-0.36213556$

$-0.36213556$

0.98020822

0.98020822

1.08604372

1. 08604372

0.98020822

0.98020822

1.08604372

1.08604372

0.58981353

0.58981353

0.69564903

0.69564903

0.58981353

0.58981353

0.69564903

0.69564903

$-0.50571668$
$-0.23974958$ $\begin{array}{lr}0.76928562 & -3.47551489 \\ 0.66345012 & -4.13829994 \\ 0.76928562 & 3.87628746 \\ 0.66345012 & -3.18108392 \\ 0.76928562 & 3.07686234 \\ 0.66345012 & 3.32669187 \\ 0.76928562 & -3.19635606 \\ 1.52134740 & 1.08236980 \\ 1.62718296 & -0.78797334 \\ 1.52134740 & -0.85509062 \\ 1.62718296 & 0.60791439 \\ 1.52134740 & -1.54085183 \\ 1.62718296 & 1.11499667 \\ 1.52134740 & 1.27726746 \\ 1.62718296 & -0.89766854 \\ 1.24928117 & -0.21328403 \\ 1.35511649 & 0.02581083 \\ 1.24928117 & 0.48007190 \\ 1.35511649 & -0.18366945 \\ 1.24928117 & 0.58510590 \\ 1.35511649 & -0.25840980 \\ 1.24928117 & -0.99172586 \\ 1.35511649 & 0.52329630\end{array}$

1.000 \# Electr. mom., scale-fac \# Magnetic mom.

$-0.22430076$

$-0.11846532$

$-0.22430076$

$-0.11846532$

$-0.22430076$

$-0.11846532$

$-0.22430076$

$-0.11846532$

$-0.08068259$

0.02515285

$-0.08068259$

0.02515285

$-0.08068259$

0.02515285

$-0.08068259$

0.02515285

$-1.25737166$

$-1.15153623$

$-1.25737166$

$-1.15153623$

$-1.25737166$

$-1.15153623$

$-1.25737166$

$-1.15153623$

$-1.04271245$

$-0.93687707$

$-1.04271245$

$-0.93687707$

$-1.04271245$

$-0.93687707$

$-1.04271245$

$-0.93687707$

0.74054700

0.84638250

0.74054700

0.84638250

0.74054700

0.84638250

0.74054700

0.84638250

0.66345012
$-6.60658264$

8.17716789

9.01050854

$-11.27470016$

10.13598156

$-12.19724560$

$-13.26723671$

16.07855034 4.83078241

$-7.15128708$

$-4.11026955$

6.25993490

$-6.47007799$

9.07241154

5.76734972

$-8.05445480$

1. 41218078

$-1.82556498$

$-1.62282240$

2.11823869

$-1.19833934$

1.54398561

1.36709917

$-1.78261769$

2.65330935

$-3.91083097$

$-1.86360407$

2.89203668

$-2.54423618$

3. 66532278

1. 80224550

$-2.70962882$

10.47835159

$-9.22398090$

$-9.50655079$

8.64505386

$-10.71048069$

9.02228451

9.11400700

$-7.92938185$

0.48802510 


\begin{abstract}
1.52079928
1. 52079928

1. 52079928

1.62663472

1. 62663472

1. 62663472

1.62663472

$-1.79626679$

$-1.79626679$

$-1.79626679$

$-1.79626679$

$-1.69043136$

$-1.69043136$

$-1.69043136$

$-1.69043136$

0.79423416

0.79423416

0.79423416

0.79423416

0.90006965

0.90006965

0.90006965

0.90006965
\end{abstract}

64
0.76928562
0.66345012
0.76928562
0.66345012
0.76928562
0.66345012
0.76928562
1. 52134740
1.62718296
1.52134740
1.62718296
1. 52134740
1.62718296
1. 52134740
1. 62718296
1. 24928117
1.35511649
1. 24928117
1. 35511649
1. 24928117
1. 35511649
1. 24928117
1.35511649
-0.55442059
0.65293181
$-1.16972876$
0.95858866
$-0.67722410$
$-1.96656895$
2.06980062
$-0.48916879$
0.37702876
0.38586280
$-0.27024081$
0.60173970
$-0.45512095$
$-0.54832482$
0.37441850
$-0.38599029$
0.54874504
$-0.00240769$
$-0.43253294$
$-0.32127419$
$-0.14196685$
1. 14935541
$-0.27843738$

$\begin{array}{lrr}2.8875 & 0.4140 & 0.8117 \\ 0.0517 & -0.0553 & -0.1345\end{array}$

$-1.70699394$

$-1.70699394$

$-1.70699394$

$-1.70699394$

$-1.60115862$

$-1.60115862$

$-1.60115862$

$-1.60115862$

1.14857543

1.14857543

1.14857543

1. 14857543

1. 25441086

1.25441086

1. 25441086

1. 25441086

$-1.09362578$

$-1.09362578$

$-1.09362578$

$-1.09362578$

$-0.98779023$

$-0.98779023$

$-0.98779023$

$-0.98779023$

1. 78089249

1.78089249

1. 78089249

1. 78089249

1.88672793

1.88672793

1.88672793

1.88672793

$-1.20384598$

$-1.20384598$

$-1.20384598$

$-1.20384598$

$-1.09801054$

$-1.09801054$

$-1.09801054$

$-1.09801054$

1. 52079928
$-0.23974958$

$-0.23974958$

$-0.13391411$

$-0.13391411$

$-0.23974958$

$-0.23974958$

$-0.13391411$

$-0.13391411$

0.57671642

0.57671642

0.68255186

0.68255186

0.57671642

0.57671642

0.68255186

0.68255186

$-0.46797100$

$-0.46797100$

$-0.36213556$

$-0.36213556$

$-0.46797100$

$-0.46797100$

$-0.36213556$

$-0.36213556$

0.98020822

0.98020822

1.08604372

1.08604372

0.98020822

0.98020822

1.08604372

1.08604372

0.58981353

0.58981353

0.69564903

0.69564903

0.58981353

0.58981353

0.69564903

0.69564903

$-0.50571668$
1.000 \# Electr. mom., scale-fac \# Magnetic mom.

$-0.22430076$

$-0.11846532$

$-0.22430076$

$-0.11846532$

$-0.22430076$

$-0.11846532$

$-0.22430076$

$-0.11846532$

$-0.08068259$

0.02515285

$-0.08068259$

0.02515285

$-0.08068259$

0.02515285

$-0.08068259$

0.02515285

$-1.25737166$

$-1.15153623$

$-1.25737166$

$-1.15153623$

$-1.25737166$

$-1.15153623$

$-1.25737166$

$-1.15153623$

$-1.04271245$

$-0.93687707$

$-1.04271245$

$-0.93687707$

$-1.04271245$

$-0.93687707$

$-1.04271245$

$-0.93687707$

0.74054700

0.84638250

0.74054700

0.84638250

0.74054700

0.84638250

0.74054700

0.84638250

0.66345012 


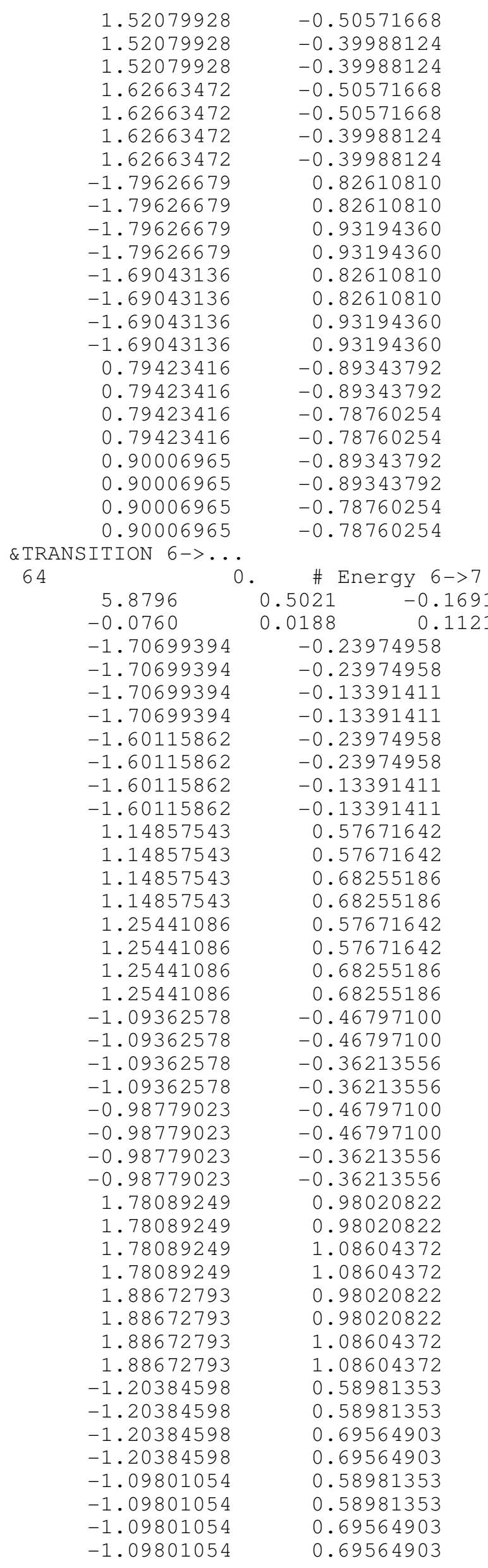

1.52079928

1.52079928

1.62663472

1.62663472

1.62663472

1.62663472

$-1.79626679$

$-1.79626679$

0.79423416

0.79423416

TRANSITION $6->$..
0.76928562

0.66345012

0.76928562

0.66345012

0.76928562

0.66345012

0.76928562

1.52134740

1.62718296

1.52134740

1.62718296

1.52134740

1.62718296

1.52134740

1. 62718296

1. 24928117

1. 35511649

1. 24928117

1.35511649

1. 24928117

1.35511649

1. 24928117

1.35511649
10.25038719

12.50577545

$-11.48166084$

12.35811329

$-10.90784168$

$-13.02942944$

11.80576038

0.19817583

$-0.40913555$

$-0.79082757$

0.77179921

$-0.09351167$

0.42762390

0.85864639

$-0.89581233$

$-1.67804253$

1. 73823595

1. 75790811

$-1.91878355$

1. 20390928

$-1.46413040$

$-1.10363078$

1. 52232063
1.000 \# Electr. mom., scale-fac \# Magnetic mom.

$-0.22430076$

$-0.11846532$

$-0.22430076$

$-0.11846532$

$-0.22430076$

$-0.11846532$

$-0.22430076$

$-0.11846532$

$-0.08068259$

0.02515285

$-0.08068259$

0.02515285

$-0.08068259$

0.02515285

$-0.08068259$

0.02515285

$-1.25737166$

$-1.15153623$

$-1.25737166$

$-1.15153623$

$-1.25737166$

$-1.15153623$

$-1.25737166$

$-1.15153623$

$-1.04271245$

$-0.93687707$

$-1.04271245$

$-0.93687707$

$-1.04271245$

$-0.93687707$

$-1.04271245$

$-0.93687707$

0.74054700

0.84638250

0.74054700

0.84638250

0.74054700

0.84638250

0.74054700

0.84638250
$-2.24538565$

3. 48262691

7.65052795

$-9.20128441$

1.74720669

$-2.89311314$

$-7.07499647$

8.18696594

$-4.91388559$

15.15182877

$-8.92774296$

8.60489464

$-20.02777100$

$-3.09790182$

13.38220978

$-3.04498887$

4.03519773

3.11743093

$-4.06252718$

4.05611610

$-5.25053406$

$-4.17877531$

5.33611059

0.85990149

$-1.32278907$

$-1.95819008$

2. 82812142

$-0.80851966$

1. 44836831

1. 37256718

$-2.25422812$

28.39835167

$-21.35543060$

$-21.88197136$

16.41474342

$-23.15627480$

16.67304611

16.98657799

$-12.12773418$
$-0.32288441$ 


\begin{abstract}
1. 52079928
1. 52079928

1. 52079928

1.52079928

1.62663472

1.62663472

1.62663472

1.62663472

$-1.79626679$

$-1.79626679$

$-1.79626679$

$-1.79626679$

$-1.69043136$

$-1.69043136$

$-1.69043136$

$-1.69043136$

0.79423416

0.79423416

0.79423416

0.79423416

0.90006965

0.90006965

0.90006965

0.90006965
\end{abstract}

64
0.3473
0 .

$-0.2698$

$-1.70699394$

$-1.70699394$

$-1.70699394$

$-1.70699394$

$-1.60115862$

$-1.60115862$

$-1.60115862$

$-1.60115862$

1.14857543

1.14857543

1. 14857543

1.14857543

1. 25441086

1. 25441086

1. 25441086

1.25441086

$-1.09362578$

$-1.09362578$

$-1.09362578$

$-1.09362578$

$-0.98779023$

$-0.98779023$

$-0.98779023$

$-0.98779023$

1. 78089249

1.78089249

1. 78089249

1. 78089249

1.88672793

1.88672793

1. 88672793

1.88672793

$-1.20384598$

$-1.20384598$

$-1.20384598$

$-1.20384598$

$-1.09801054$

$-1.09801054$

$-1.09801054$

$-1.09801054$
$-0.50571668$

$-0.50571668$

$-0.39988124$

$-0.39988124$

$-0.50571668$

$-0.50571668$

$-0.39988124$

$-0.39988124$

0.82610810

0.82610810

0.93194360

0.93194360

0.82610810

0.82610810

0.93194360

0.93194360

$-0.89343792$

$-0.89343792$

$-0.78760254$

$-0.78760254$

$-0.89343792$

$-0.89343792$

$-0.78760254$

$-0.78760254$

$$
-1.0093 \quad 1.1868
$$

$\begin{array}{ll}-0.2294 & 0.4314\end{array}$

$-0.23974958$

$-0.23974958$

$-0.13391411$

$-0.13391411$

$-0.23974958$

$-0.23974958$

$-0.13391411$

$-0.13391411$

0.57671642

0.57671642

0.68255186

0.68255186

0.57671642

0.57671642

0.68255186

0.68255186

$-0.46797100$

$-0.46797100$

$-0.36213556$

$-0.36213556$

$-0.46797100$

$-0.46797100$

$-0.36213556$

$-0.36213556$

0.98020822

0.98020822

1. 08604372

1.08604372

0.98020822

0.98020822

1. 08604372

1.08604372

0.58981353

0.58981353

0.69564903

0.69564903

0.58981353

0.58981353

0.69564903

0.69564903
0.66345012

0.76928562

0.66345012

0.76928562

0.66345012

0.76928562

0.66345012

0.76928562

1. 52134740

1.62718296

1.52134740

1. 62718296

1.52134740

1.62718296

1.52134740

1. 62718296

1. 24928117

1. 35511649

1. 24928117

1.35511649

1. 24928117

1. 35511649

1. 24928117

1. 35511649

1.000

$-0.22430076$

$-0.11846532$

$-0.22430076$

$-0.11846532$

$-0.22430076$

$-0.11846532$

$-0.22430076$

$-0.11846532$

$-0.08068259$

0.02515285

$-0.08068259$

0.02515285

$-0.08068259$

0.02515285

$-0.08068259$

0.02515285

$-1.25737166$

$-1.15153623$

$-1.25737166$

$-1.15153623$

$-1.25737166$

$-1.15153623$

$-1.25737166$

$-1.15153623$

$-1.04271245$

$-0.93687707$

$-1.04271245$

$-0.93687707$

$-1.04271245$

$-0.93687707$

$-1.04271245$

$-0.93687707$

0.74054700

0.84638250

0.74054700

0.84638250

0.74054700

0.84638250

0.74054700

0.84638250
$-9.05093002$

12.19122219

5.66163349

$-7.77585983$

5.46968269

$-8.70994568$

$-1.48741603$

4. 31889629

1.60963655

$-1.36872101$

$-1.96472490$

1. 64200437

$-1.50007510$

1.36574388

1.94647276

$-1.71625400$

1.99307299

$-1.37371230$

$-1.36067462$

1.16989529

$-1.74592340$

1. 31060827

0.42339873

$-0.67389852$

Electr: mom., scale-fac Magnetic mom.

3.60289812

$-4.61840296$

$-4.38400984$

5.88542891

$-6.02376318$

7.52627230

7.49208736

$-9.67727280$

$-15.47115040$

24.68238258

11.97301102

$-21.08919907$

14.58826065

$-25.50321770$

$-10.79058647$

21.24541283

$-0.48768163$

0.72025985

0.96997714

$-1.39282262$

0.34636891

$-0.55514354$

$-0.82267922$

1. 21144748

$-18.79616165$

26.12184143

17.09638023

$-23.86693382$

17.98930931

$-24.63224792$

$-16.21699715$

22.27659035

10.52956390

$-8.34314442$

$-8.33973789$

6.42154598

$-8.06381226$

6.63495827

6.96782541

$-5.61599350$ 


1.52079928
1.52079928
1.52079928
1.52079928
1.62663472
1.62663472
1.62663472
1.62663472
-1.79626679
-1.79626679
-1.79626679
-1.79626679
-1.69043136
-1.69043136
-1.69043136
-1.69043136
0.79423416
0.79423416
0.79423416
0.79423416
0.90006965
0.90006965
0.90006965
0.90006965

64
$-0.50571668$

$-0.50571668$

$-0.39988124$

$-0.39988124$

$-0.50571668$

$-0.50571668$

$-0.39988124$

$-0.39988124$

0.82610810

0.82610810

0.93194360

0.93194360

0.82610810

0.82610810

0.93194360

0.93194360

$-0.89343792$

$-0.89343792$

$-0.78760254$

$-0.78760254$

$-0.89343792$

$-0.89343792$

$-0.78760254$

$-0.78760254$

$-2.6880$

$-0.1099$

$-1.70699394$

$-1.70699394$

$-1.70699394$

$-1.70699394$

$-1.60115862$

$-1.60115862$

$-1.60115862$

$-1.60115862$

1.14857543

1. 14857543

1. 14857543

1.14857543

1.25441086

1. 25441086

1. 25441086

1. 25441086

$-1.09362578$

$-1.09362578$

$-1.09362578$

$-1.09362578$

$-0.98779023$

$-0.98779023$

$-0.98779023$

$-0.98779023$

1. 78089249

1. 78089249

1. 78089249

1. 78089249

1.88672793

1.88672793

1.88672793

1. 88672793

$-1.20384598$

$-1.20384598$

$-1.20384598$

$-1.20384598$

$-1.09801054$

$-1.09801054$

$-1.09801054$

$-1.09801054$

$-0.23974958$

$-0.23974958$

$-0.13391411$

$-0.13391411$

$-0.23974958$

$-0.23974958$

$-0.13391411$

$-0.13391411$

0.57671642

0.57671642

0.68255186

0.68255186

0.57671642

0.57671642

0.68255186

0.68255186

$-0.46797100$

$-0.46797100$

$-0.36213556$

$-0.36213556$

$-0.46797100$

$-0.46797100$

$-0.36213556$

$-0.36213556$

0.98020822

0.98020822

1. 08604372

1.08604372

0.98020822

0.98020822

1.08604372

1.08604372

0.58981353

0.58981353

0.69564903

0.69564903

0.58981353

0.58981353

0.69564903

0.69564903
0.66345012

0.76928562

0.66345012

0.76928562

0.66345012

0.76928562

0.66345012

0.76928562

1.52134740

1.62718296

1.52134740

1. 62718296

1.52134740

1.62718296

1. 52134740

1. 62718296

1. 24928117

1. 35511649

1. 24928117

1.35511649

1. 24928117

1. 35511649

1. 24928117

1. 35511649

$$
\text { 0. \# Energy 6->9 }
$$

$$
\begin{array}{ll}
-0.6488 & -0.1822
\end{array}
$$$$
0.0088 \quad 0.1591
$$

$-1.15153623$

$-1.25737166$

$-1.04271245$
$-13.62085629$

12.94184494

13.70358562

$-11.54305077$

12.08733082

$-11.77440739$

$-11.18526077$

10.03521061

$-0.83711994$

0.61776572

0.63306731

$-0.48223564$

0.88249356

$-0.61815894$

$-0.64507896$

0.46540999

4.13279676

$-3.18627048$

$-3.91661429$

3. 24601054

$-3.52404571$

2.89960742

2. 49392200

$-2.39681292$
\# Magnetic mom.

$-0.22430076$

$-0.11846532$

$-0.22430076$

$-0.11846532$

$-0.22430076$

$-0.11846532$

$-0.22430076$

$-0.11846532$

$-0.08068259$

0.02515285

$-0.08068259$

0.02515285

$-0.08068259$

0.02515285

$-0.08068259$

0.02515285

$-1.25737166$

$-1.15153623$

$-1.25737166$

$-1.15153623$

$-1.25737166$

$-1.15153623$

$-1.04271245$

$-0.93687707$

$-1.04271245$

$-0.93687707$

$-0.93687707$

$-1.04271245$

$-0.93687707$

0.74054700

0.84638250

0.74054700

0.84638250

0.74054700

0.84638250

0.74054700

0.84638250
2.14295435

$-1.76410043$

$-5.40145922$

5.57525778

$-2.56899214$

2. 26081204

6.00986719

$-6.10467100$

4.91293478

$-7.26282358$

$-2.38786125$

4.26540184

$-6.99974298$

9. 70309067

4.43672466

$-6.64886093$

$-1.06783342$

1. 75229800

1.17706275

$-1.89040732$

0.55718923

$-1.00147867$

$-0.68060076$

1. 16278470

$-3.17738724$

4.37638235

3. 47149396

$-4.78023863$

3. 00524688

$-4.18100691$

$-3.04456592$

4. 24761200

$-19.26126289$

14.30917549

14.89446354

$-11.03069496$

18.06108475

$-13.13863945$

$-13.81808567$

10.00306416 


1.52079928
1.52079928
1.52079928
1.52079928
1.62663472
1.62663472
1.62663472
1.62663472
-1.79626679
-1.79626679
-1.79626679
-1.79626679
-1.69043136
-1.69043136
-1.69043136
-1.69043136
0.79423416
0.79423416
0.79423416
0.79423416
0.90006965
0.90006965
0.90006965
0.90006965
\&TRANSITION $7->$.

64
0.66345012

0.76928562

0.66345012

0.76928562

0.66345012

0.76928562

0.66345012

0.76928562

1.52134740

1.62718296

1.52134740

1. 62718296

1.52134740

1.62718296

1. 52134740

1. 62718296

1. 24928117

1. 35511649

1. 24928117

1.35511649

1. 24928117

1. 35511649

1. 24928117

1. 35511649
0.08478661

$-1.67994797$

1. 44778728

0.01638349

0.96785909

0.63415664

$-2.60018110$

0.95928335

$-0.26337716$

0.26323649

0.52156574

$-0.46441975$

0.23271333

$-0.27543271$

$-0.51374525$

0.49666500

0.76329976

$-0.61660379$

$-1.23813105$

0.89799070

$-0.70393127$

0.54702747

1.31014156

$-0.90131664$
$-0.78760254$

$-0.78760254$

$$
\text { 0. \# Energy 7->8 }
$$

$\begin{array}{ll}-0.1147 & 0.2578\end{array}$ $\begin{array}{ll}-0.0437 & 0.1541\end{array}$

$-0.23974958$

$-0.23974958$

$-0.13391411$

$-0.13391411$

$-0.23974958$

$-0.23974958$

$-0.13391411$

$-0.13391411$

0.57671642

0.57671642

0.68255186

0.68255186

0.57671642

0.57671642

0.68255186

0.68255186

$-0.46797100$

$-0.46797100$

$-0.36213556$

$-0.36213556$

$-0.46797100$

$-0.46797100$

$-0.36213556$

$-0.36213556$

0.98020822

0.98020822

1.08604372

1.08604372

0.98020822

0.98020822

1.08604372

1.08604372

0.58981353

0.58981353

0.69564903

0.69564903

0.58981353

0.58981353

0.69564903
1.000 \# Electr. mom., scale-fac
\# Magnetic mom. $-0.22430076 \quad 1.05992436$

$-0.11846532 \quad-0.92576706$

$-0.22430076 \quad 0.10452705$

$-0.11846532$

$-0.22430076$

$-0.11846532$

$-0.22430076$

$-0.11846532$

$-0.08068259$

0.02515285

$-0.08068259$

0.02515285

$-0.08068259$

0.02515285

$-0.08068259$

0.02515285

$-1.25737166$

$-1.15153623$

$-1.25737166$

$-1.15153623$

$-1.25737166$

$-1.15153623$

$-1.25737166$

$-1.15153623$

$-1.04271245$

$-0.93687707$

$-1.04271245$

$-0.93687707$

$-1.04271245$

$-0.93687707$

$-1.04271245$

$-0.93687707$

0.74054700

0.84638250

0.74054700

0.84638250

0.74054700

0.84638250

0.74054700
$-0.11887044$

$-3.03475285$

3. 25790763

2. 51974511

$-3.14411235$

$-3.28338981$

8.93583870

1. 44955659

$-7.05125046$

0.46918783

$-6.18278074$

1. 45924735

4. 31701422

$-2.83556676$

3. 85528588

2. 80716276

$-3.85143518$

3. 03603721

$-4.02015591$

$-3.00294757$

3.99811530

$-5.51311111$

7.39664459

5.83719635

$-7.69286108$

5.02640486

$-6.67894602$

$-5.32799339$

6.95079184

14.24875355

$-10.02351952$

$-10.19110107$

6.93769264

$-9.99965191$

6.64884329

6.54580975 


-1.09801054
1.52079928
1.52079928
1.52079928
1.52079928
1.62663472
1.62663472
1.62663472
1.62663472
-1.79626679
-1.79626679
-1.79626679
-1.79626679
-1.69043136
-1.69043136
-1.69043136
-1.69043136
0.79423416
0.79423416
0.79423416
0.79423416
0.90006965
0.90006965
0.90006965
0.90006965

64
0.69564903

$-0.50571668$

$-0.50571668$

$-0.39988124$

$-0.39988124$

$-0.50571668$

$-0.50571668$

$-0.39988124$

$-0.39988124$

0.82610810

0.82610810

0.93194360

0.93194360

0.82610810

0.82610810

0.93194360

0.93194360

$-0.89343792$

$-0.89343792$

$-0.78760254$

$-0.78760254$

$-0.89343792$

$-0.89343792$

$-0.78760254$

$-0.78760254$

0.1879

$-1.70699394$

$-1.70699394$

$-1.70699394$

$-1.70699394$

$-1.60115862$

$-1.60115862$

$-1.60115862$

$-1.60115862$

1.14857543

1. 14857543

1.14857543

1.14857543

1. 25441086

1. 25441086

1. 25441086

1. 25441086

$-1.09362578$

$-1.09362578$

$-1.09362578$

$-1.09362578$

$-0.98779023$

$-0.98779023$

$-0.98779023$

$-0.98779023$

1. 78089249

1. 78089249

1. 78089249

1.78089249

1.88672793

1.88672793

1.88672793

1.88672793

$-1.20384598$

$-1.20384598$

$-1.20384598$

$-1.20384598$

$-1.09801054$

$-1.09801054$

$-1.09801054$

$-0.23974958$

$-0.23974958$

$-0.13391411$

$-0.23974958$

$-0.23974958$

$-0.13391411$

$-0.13391411$

0.57671642

0.57671642

0.68255186

0.68255186

0.57671642

0.57671642

0.68255186

0.68255186

$-0.46797100$

$-0.46797100$

$-0.36213556$

$-0.36213556$

$-0.46797100$

$-0.46797100$

$-0.36213556$

$-0.36213556$

0.98020822

0.98020822

1. 08604372

1.08604372

0.98020822

0.98020822

1.08604372

1.08604372

0.58981353

0.58981353

0.69564903

0.69564903

0.58981353

0.58981353

0.69564903 $\begin{array}{cc}\text { \# Energy } & 7->9 \\ 0.2441 & 0.4799\end{array}$

0.84638250

0.66345012

0.76928562

0.66345012

0.76928562

0.66345012

0.76928562

0.66345012

1.52134740

1.62718296

1.52134740

1. 62718296

1.52134740

1. 62718296

1.52134740

1.62718296

1. 24928117

1. 35511649

1. 24928117

1.35511649

1. 24928117

1. 35511649

1. 24928117

1. 35511649

$-0.1829-0.3245$

$-0.13391411$

$-0.22430076$

$-0.11846532$

$-0.22430076$

$-0.11846532$

$-0.22430076$

$-0.11846532$

$-0.22430076$

$-0.11846532$

$-0.08068259$

0.02515285

$-0.08068259$

0.02515285

$-0.08068259$

0.02515285

$-0.08068259$

0.02515285

$-1.25737166$

$-1.15153623$

$-1.25737166$

$-1.15153623$

$-1.25737166$

$-1.15153623$

$-1.25737166$

$-1.15153623$

$-1.04271245$

$-0.93687707$

$-1.04271245$

$-0.93687707$

$-1.04271245$

$-0.93687707$

$-1.04271245$

$-0.93687707$

0.74054700

0.84638250

0.74054700

0.84638250

0.74054700

0.84638250

0.74054700
0.76928562

-4.07793140
-4.75122499
5.57313299
4.19232416
-5.16261578
6.52160358
-6.86981726
-5.71948004
6.34372234
1.02179921
-0.80760157
-1.17128944
0.92826259
-1.24534118
1.01417708
1.40108657
-1.13995707
2.34500051
-1.52269948
-2.45678258
1.59206080
-2.60855579
1.71738577
2.59430933
-1.69504297

1.000 \# Electr. mom., scale-fac \# Magnetic mom.

$-10.30833149$
12.54529858

19.83295250

$-23.97207642$

19.94261169

$-23.71638680$

$-32.69310760$

38.78863907

8.51095486

$-10.73254395$

$-9.00079441$

11.41098022

$-10.58993149$

13.09287357

10.68859768

$-13.31354427$

11.08313465

$-15.91365910$

$-11.93043900$

17.32854080

$-9.64441776$

13.75993538

10.42077541

$-15.02153015$

$-0.43381515$

0.42876381

$-0.63758492$

0.35941148

$-0.50774181$

$-0.25979674$

0.43574157

21.95570183

$-20.83817101$

$-20.46982193$

19.57638550

$-30.55742264$

26.75358200

26.98207664
0.58138776 


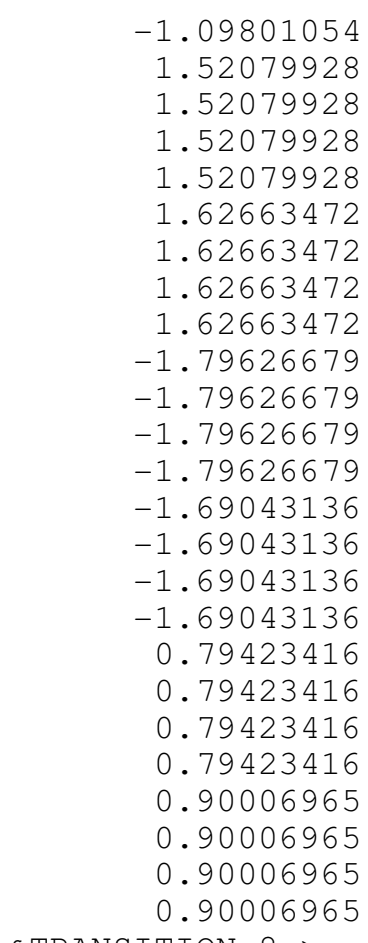

\&TRANSITION $8->\ldots$
0.69564903
-0.50571668
-0.50571668
-0.39988124
-0.39988124
-0.50571668
-0.50571668
-0.39988124
-0.39988124
0.82610810
0.82610810
0.93194360
0.93194360
0.82610810
0.82610810
0.93194360
0.93194360
-0.89343792
-0.89343792
-0.78760254
-0.78760254
-0.89343792
-0.89343792
-0.78760254
-0.78760254

0. \# Energy 8->9

1.1880

$-0.0663$

$-1.70699394$

$-1.70699394$

$-1.70699394$

$-1.70699394$

$-1.60115862$

$-1.60115862$

$-1.60115862$

$-1.60115862$

1.14857543

1.14857543

1.14857543

1. 14857543

1. 25441086

1. 25441086

1. 25441086

1. 25441086

$-1.09362578$

$-1.09362578$

$-1.09362578$

$-1.09362578$

$-0.98779023$

$-0.98779023$

$-0.98779023$

$-0.98779023$

1. 78089249

1. 78089249

1.78089249

1.78089249

1. 88672793

1.88672793

1.88672793

1.88672793

$-1.20384598$

$-1.20384598$

$-1.20384598$

$-1.20384598$

$-1.09801054$

$-1.09801054$
0.84638250

0.66345012

0.76928562

0.66345012

0.76928562

0.66345012

0.76928562

0.66345012

0.76928562

1.52134740

1.62718296

1.52134740

1.62718296

1.52134740

1. 62718296

1.52134740

1.62718296

1. 24928117

1.35511649

1.24928117

1.35511649

1.24928117

1. 35511649

1. 24928117

1. 35511649

$$
\begin{array}{r}
-23.89571953 \\
-6.81836510 \\
5.27872992 \\
9.07946014 \\
-7.79603148 \\
5.88035297 \\
-4.50121403 \\
-7.96614981 \\
6.66422033 \\
-3.08606362 \\
2.48216271 \\
2.85679269 \\
-2.30594659 \\
4.02562952 \\
-3.19325304 \\
-3.72293282 \\
2.95431232 \\
-2.79328394 \\
2.32596517 \\
2.87588930 \\
-2.52775431 \\
2.27028656 \\
-2.06211329 \\
-1.92240310 \\
1.96020389
\end{array}
$$

$$
\begin{aligned}
& 1.000 \text { \# Electr. mom., scale-fac } \\
& \text { \# Magnetic mom. }
\end{aligned}
$$

$-0.22430076 \quad 1.75893402$

$\begin{array}{ll}-0.11846532 & -2.07606840\end{array}$

$-0.22430076 \quad-3.01844478$

$-0.11846532 \quad 3.45966911$

$-0.22430076$

$-0.11846532$

$-0.22430076$

$-0.11846532$

$-0.08068259$

0.02515285

$-0.08068259$

0.02515285

$-0.08068259$

0.02515285

$-0.08068259$

0.02515285

$-1.25737166$

$-1.15153623$

$-1.25737166$

$-1.15153623$

$-1.25737166$

$-1.15153623$

$-1.25737166$

$-1.15153623$

$-1.04271245$

$-0.93687707$

$-1.04271245$

$-0.93687707$

$-1.04271245$

$-0.93687707$

$-1.04271245$

$-0.93687707$

0.74054700

0.84638250

0.74054700

0.84638250

0.74054700

0.84638250
$-3.02998018$

3.43946218

4.78204060

$-5.38262558$

0.57183182

$-1.09254003$

0.20548552

$-0.98911268$

1. 72727084

0.20239243

$-0.82046622$

$-1.70934772$

2. 47156858

1.87546670

$-2.78012681$

1. 45114386

$-2.09704995$

$-1.58086276$

2.33755493

3. 11827755

$-4.23304605$

$-2.55562711$

3. 57745481

$-3.28500867$

4.37950706

2.65707397

$-3.63281155$

$-3.96362901$

4. 72087908

3.74563432

$-4.27602768$

5. 26915121

$-5.59117460$
0.28740123 


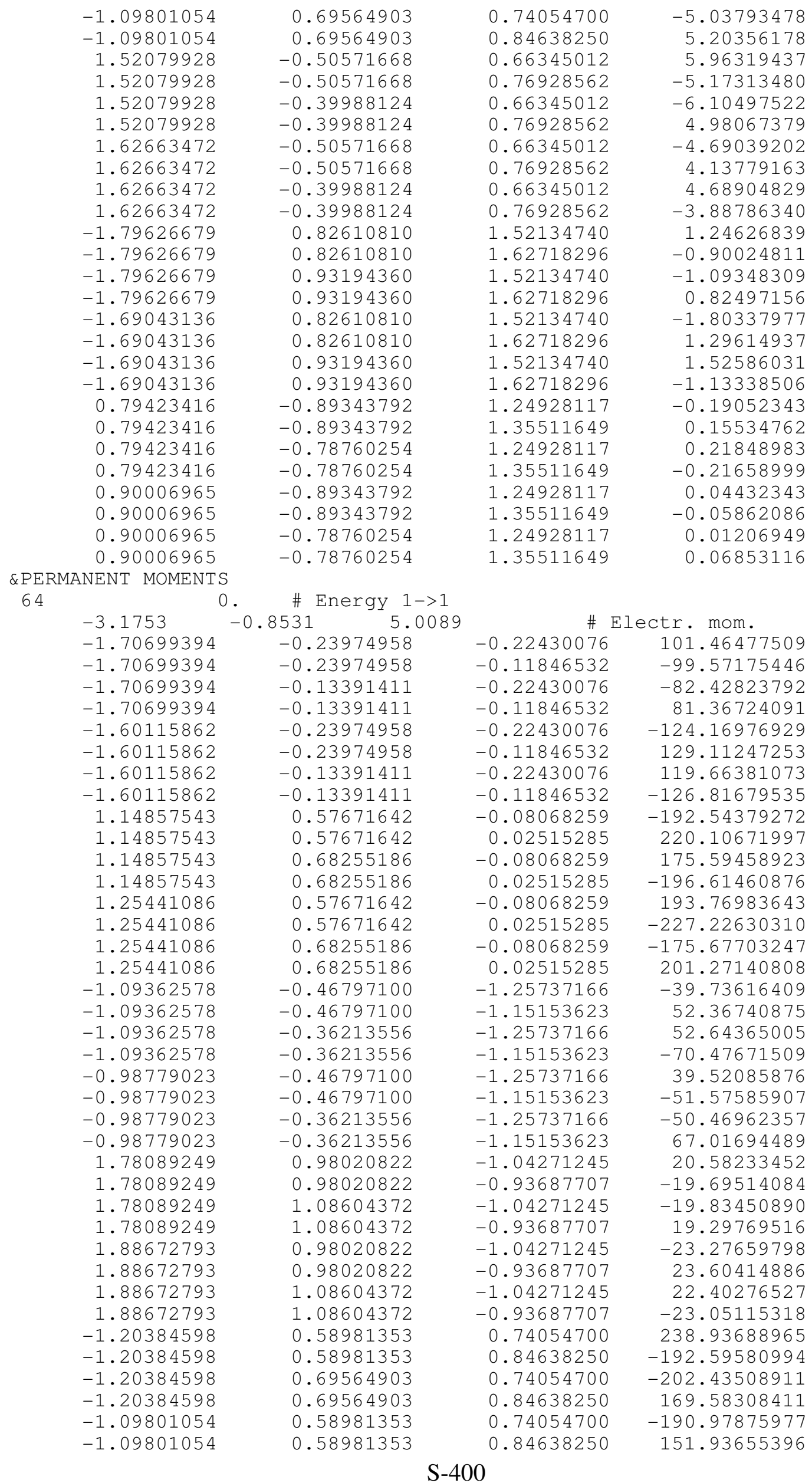


-1.09801054
-1.09801054
1.52079928
1.52079928
1.52079928
1.52079928
1.62663472
1.62663472
1.62663472
1.62663472
-1.79626679
-1.79626679
-1.79626679
-1.79626679
-1.69043136
-1.69043136
-1.69043136
-1.69043136
0.79423416
0.79423416
0.79423416
0.79423416
0.90006965
0.90006965
0.90006965
0.90006965

64
0.69564903

0.69564903

$-0.50571668$

$-0.50571668$

$-0.39988124$

$-0.39988124$

$-0.50571668$

$-0.50571668$

$-0.39988124$

$-0.39988124$

0.82610810

0.82610810

0.93194360

0.93194360

0.82610810

0.82610810

0.93194360

0.93194360

$-0.89343792$

$-0.89343792$

$-0.78760254$

$-0.78760254$

$-0.89343792$

$-0.89343792$

$-0.78760254$

$-0.78760254$

$-2.0169$

$-1.70699394$

$-1.70699394$

$-1.70699394$

$-1.70699394$

$-1.60115862$

$-1.60115862$

$-1.60115862$

$-1.60115862$

1.14857543

1.14857543

1.14857543

1.14857543

1. 25441086

1. 25441086

1. 25441086

1. 25441086

$-1.09362578$

$-1.09362578$

$-1.09362578$

$-1.09362578$

$-0.98779023$

$-0.98779023$

$-0.98779023$

$-0.98779023$

1. 78089249

1. 78089249

1. 78089249

1.78089249

1.88672793

1.88672793

1.88672793

1. 88672793

$-1.20384598$

$-1.20384598$

$-1.20384598$

$-1.20384598$

$-1.09801054$

$-1.09801054$

$-1.09801054$

0.68255186

$$
\text { . \# Energy 2->2 }
$$

$0.3188 \quad 3.0135$

$-0.23974958$

$-0.23974958$

$-0.13391411$

$-0.13391411$

$-0.23974958$

$-0.23974958$

$-0.13391411$

$-0.13391411$

0.57671642

0.57671642

0.68255186

0.68255186

0.57671642

0.57671642

0.68255186

$-0.46797100$

$-0.46797100$

$-0.36213556$

$-0.36213556$

$-0.46797100$

$-0.46797100$

$-0.36213556$

$-0.36213556$

0.98020822

0.98020822

1.08604372

1.08604372

0.98020822

0.98020822

1. 08604372

1.08604372

0.58981353

0.58981353

0.69564903

0.69564903

0.58981353

0.58981353

0.69564903
0.74054700

0.84638250

0.66345012

0.76928562

0.66345012

0.76928562

0.66345012

0.76928562

0.66345012

0.76928562

1. 52134740

1. 62718296

1.52134740

1.62718296

1. 52134740

1. 62718296

1.52134740

1. 62718296

1. 24928117

1.35511649

1. 24928117

1. 35511649

1. 24928117

1. 35511649

1. 24928117

1. 35511649
164.66084290

$-137.26234436$

$-28.25176239$

14.38099670

24.24119568

$-9.41675186$

1.84090471

3. 31124282

9. 25998974

$-14.31702328$

$-14.47190666$ 8.16936588

10.25620937

$-5.59983587$

22.05652809

$-13.58127594$

$-16.82669640$

10.14238930

$-2.43663144$

1. 96805918

0.10553713

$-0.87256652$

4.37593222

$-3.56327367$

$-0.34032857$

1.10175908

Electr. mom.

$-0.22430076$

$-0.11846532$

94.70573425

$-0.22430076$

$-0.11846532$

$-0.22430076$

$-0.11846532$

$-0.22430076$

$-0.11846532$

$-0.08068259$

0.02515285

$-0.08068259$

0.02515285

$-0.08068259$

0.02515285

$-0.08068259$

0.02515285

$-1.25737166$

$-1.15153623$

$-1.25737166$

$-1.15153623$

$-1.25737166$

$-1.15153623$

$-1.25737166$

$-1.15153623$

$-1.04271245$

$-0.93687707$

$-1.04271245$

$-0.93687707$

$-1.04271245$

$-0.93687707$

$-1.04271245$

$-0.93687707$

0.74054700

0.84638250

0.74054700

0.84638250

0.74054700

0.84638250

$-92.12420654$

$-74.28259277$

72.26390839

$-115.05747223$

118.83768463

108.23293304

$-113.88473511$

$-171.44805908$

188.93569946

154.95344543

$-167.23109436$

181.63056946

$-207.05561829$

$-161.57615662$

180.06825256

$-39.43571854$

51.83475113

51.75838852

$-69.14217377$

39.46377563

$-51.43490982$

$-49.94544601$

66.21360779

57.83938599

$-71.03713989$

$-56.42160034$

69.47484589

$-57.36172867$

70.33212280

55.18773651

$-67.85656738$

235.96989441

$-193.31515503$

$-200.66459656$

170.82884216

$-188.57588196$

152.69390869

163.27175903 


-1.09801054
1.52079928
1.52079928
1.52079928
1.52079928
1.62663472
1.62663472
1.62663472
1.62663472
-1.79626679
-1.79626679
-1.79626679
-1.79626679
-1.69043136
-1.69043136
-1.69043136
-1.69043136
0.79423416
0.79423416
0.79423416
0.79423416
0.90006965
0.90006965
0.90006965
0.90006965

64
0.69564903

$-0.50571668$

$-0.50571668$

$-0.39988124$

$-0.39988124$

$-0.50571668$

$-0.50571668$

$-0.39988124$

$-0.39988124$

0.82610810

0.82610810

0.93194360

0.93194360

0.82610810

0.82610810

0.93194360

0.93194360

$-0.89343792$

$-0.89343792$

$-0.78760254$

$-0.78760254$

$-0.89343792$

$-0.89343792$

$-0.78760254$

$-0.78760254$

0 . \# Energy 3->3

$\begin{array}{lll}-1.9801 & -1.5278 & 2.9310\end{array}$

$-1.70699394$

$-1.70699394$

$-1.70699394$

$-1.70699394$

$-1.60115862$

$-1.60115862$

$-1.60115862$

$-1.60115862$

1.14857543

1.14857543

1.14857543

1.14857543

1.25441086

1. 25441086

1. 25441086

1. 25441086

$-1.09362578$

$-1.09362578$

$-1.09362578$

$-1.09362578$

$-0.98779023$

$-0.98779023$

$-0.98779023$

$-0.98779023$

1. 78089249

1.78089249

1. 78089249

1. 78089249

1.88672793

1.88672793

1.88672793

1.88672793

$-1.20384598$

$-1.20384598$

$-1.20384598$

$-1.20384598$

$-1.09801054$

$-1.09801054$

$-1.09801054$

$-1.09801054$
$-0.23974958$

$-0.13391411$

$-0.13391411$

$-0.23974958$

$-0.23974958$

$-0.13391411$

0.57671642

0.57671642

0.68255186

0.68255186

0.57671642

0.57671642

0.68255186

0.68255186

$-0.46797100$

$-0.46797100$

$-0.36213556$

$-0.36213556$

$-0.46797100$

$-0.46797100$

$-0.36213556$

$-0.36213556$

0.98020822

0.98020822

1. 08604372

1.08604372

0.98020822

0.98020822

1.08604372

1.08604372

0.58981353

0.58981353

0.69564903

0.69564903

0.58981353

0.58981353

0.69564903

0.69564903
$-0.23974958$

$-0.13391411$
0.84638250

0.66345012

0.76928562

0.66345012

0.76928562

0.66345012

0.76928562

0.66345012

0.76928562

1.52134740

1.62718296

1.52134740

1.62718296

1.52134740

1. 62718296

1. 52134740

1.62718296

1. 24928117

1. 35511649

1. 24928117

1. 35511649

1. 24928117

1. 35511649

1. 24928117

1. 35511649
$-138.56758118$

$-40.85550308$

24.50905800

35.61973953

$-16.41559219$

5.18320608

0.45400861

8. 24868298

$-15.29816628$

$-15.15471458$

8.46847534

10.59281731

$-5.72145939$

23.84516144

$-14.67961121$

$-17.97202301$

10.82745075

$-5.27414560$

4.12203455

2.36292124

$-2.46998739$

7.03352308

$-5.57076597$

$-2.28207588$

2. 34819150

\section{\# Electr. mom.}

$-0.22430076$

$-0.11846532$

$-0.22430076$

$-0.11846532$

$-0.22430076$

$-0.11846532$

$-0.22430076$

$-0.11846532$

$-0.08068259$

0.02515285

$-0.08068259$

0.02515285

$-0.08068259$

0.02515285

$-0.08068259$

0.02515285

$-1.25737166$

$-1.15153623$

$-1.25737166$

$-1.15153623$

$-1.25737166$

$-1.15153623$

$-1.25737166$

$-1.15153623$

$-1.04271245$

$-0.93687707$

$-1.04271245$

$-0.93687707$

$-1.04271245$

$-0.93687707$

$-1.04271245$

$-0.93687707$

0.74054700

0.84638250

0.74054700

0.84638250

0.74054700

0.84638250

0.74054700

0.84638250
66.96308899

$-63.27800369$

$-47.98880768$

43.44495773

$-82.55209351$

86.94744873

80.34255219

$-85.62732697$

$-189.60688782$

224.80760193

170.81329346

$-198.51316833$

189.79055786

$-230.01712036$

$-169.88298035$

201.43264771

$-54.67188263$

73.74253845

65.52321625

$-89.02260590$

54.86023712

$-72.96931458$

$-64.46994019$

86.44982147

20.80548096

$-20.44667816$

$-19.92462730$

19.80332756

$-23.54603958$

24.36544037

22.57260513

$-23.64721680$

280.66934204

$-226.84417725$

$-234.97476196$

196.64826965

$-221.95661926$

177.24151611

188.51969910

$-157.28306580$ 
1.52079928

1. 52079928

1. 52079928

1. 52079928

1. 62663472

1. 62663472

1. 62663472

$-1.79626679$

$-1.79626679$

$-1.79626679$

$-1.79626679$

$-1.69043136$

$-1.69043136$

$-1.69043136$

$-1.69043136$

0.79423416

0.79423416

0.79423416

0.79423416

0.90006965

0.90006965

0.90006965

0.90006965

64
1. 62663472

$-0.50571668$

$-0.50571668$

$-0.39988124$

$-0.39988124$

$-0.50571668$

$-0.50571668$

$-0.39988124$

$-0.39988124$

0.82610810

0.82610810

0.93194360

0.93194360

0.82610810

0.82610810

0.93194360

0.93194360

$-0.89343792$

$-0.89343792$

$-0.78760254$

$-0.78760254$

$-0.89343792$

$-0.89343792$

$-0.78760254$

$-0.78760254$

$-1.9047$

0 .

$-1.70699394$

$-1.70699394$

$-1.70699394$

$-1.70699394$

$-1.60115862$

$-1.60115862$

$-1.60115862$

$-1.60115862$

1.14857543

1. 14857543

1.14857543

1.14857543

1. 25441086

1.25441086

1. 25441086

1. 25441086

$-1.09362578$

$-1.09362578$

$-1.09362578$

$-1.09362578$

$-0.98779023$

$-0.98779023$

$-0.98779023$

$-0.98779023$

1. 78089249

1.78089249

1.78089249

1. 78089249

1.88672793

1.88672793

1.88672793

1.88672793

$-1.20384598$

$-1.20384598$

$-1.20384598$

$-1.20384598$

$-1.09801054$

$-1.09801054$

$-1.09801054$

$-1.09801054$

1. 52079928

$-0.23974958$

$-0.23974958$

$-0.13391411$

$-0.13391411$

$-0.23974958$

$-0.23974958$

$-0.13391411$

$-0.13391411$

0.57671642

0.57671642

0.68255186

0.68255186

0.57671642

0.57671642

0.68255186

0.68255186

$-0.46797100$

$-0.46797100$

$-0.36213556$

$-0.36213556$

$-0.46797100$

$-0.46797100$

$-0.36213556$

$-0.36213556$

0.98020822

0.98020822

1.08604372

1. 08604372

0.98020822

0.98020822

1.08604372

1.08604372

0.58981353

0.58981353

0.69564903

0.69564903

0.58981353

0.69564903

0.69564903

$-0.50571668$
1. 35511649

\# Energy 4->4

$$
-1.4658 \quad 6.5906
$$

0.58981353
0.66345012

0.76928562

0.66345012

0.76928562

0.66345012

0.76928562

0.66345012

0.76928562

1.52134740

1.62718296

1.52134740

1. 62718296

1.52134740

1.62718296

1. 52134740

1. 62718296

1. 24928117

1. 35511649

1. 24928117

1.35511649

1. 24928117

1. 35511649

1. 24928117

$-27.88810921$

16.99581528

20.33811760

$-8.24623680$

0.94920588

1. 88184536

12.94320297

$-15.77259159$

$-11.30296326$

5.39761496

6.47075224

$-2.46097851$

19.51966858

$-11.11713886$

$-13.41712570$

7.10990524

$-3.56696105$

2.52610493

3. 14674020

$-2.64545941$

6.49764824

$-4.70308256$

$-5.16284418$

3.98656583

\# Electr. mom.

$-0.22430076$

$-0.11846532$

$-0.22430076$

$-0.11846532$

$-0.22430076$

$-0.11846532$

$-0.22430076$

$-0.11846532$

$-0.08068259$

0.02515285

$-0.08068259$

0.02515285

$-0.08068259$

0.02515285

$-0.08068259$

0.02515285

$-1.25737166$

$-1.15153623$

$-1.25737166$

$-1.15153623$

$-1.25737166$

$-1.15153623$

$-1.25737166$

$-1.15153623$

$-1.04271245$

$-0.93687707$

$-1.04271245$

$-0.93687707$

$-1.04271245$

$-0.93687707$

$-1.04271245$

$-0.93687707$

0.74054700

0.84638250

0.74054700

0.84638250

0.74054700

0.84638250

0.74054700

0.84638250

0.66345012
82.50487518

$-82.02684784$

$-57.93550491$

56.74236298

$-101.60073853$

108.42630768

91.61100006

$-99.39053345$

$-183.73315430$

209.64369202

168.73115540

$-188.88690186$

182.17929077

$-214.58131409$

$-165.41398621$

190.65432739

$-38.76262665$

50.37555695

49.72985458

$-65.95471954$

39.23846817

$-50.58610153$

$-48.45935059$

63.73732758

22.43048477

$-21.67634010$

$-21.21337318$

20.54261208

$-24.62642479$

24.88005447

23.61304474

$-24.06734276$

292.16824341

$-237.09387207$

$-243.29888916$

203.55892944

$-241.86257935$

194.52195740

205.18898010

$-171.25680542$

$-55.94492340$ 
1.52079928

1. 52079928

1. 52079928

1.62663472

1. 62663472

1. 62663472

1.62663472

$-1.79626679$

$-1.79626679$

$-1.79626679$

$-1.79626679$

$-1.69043136$

$-1.69043136$

$-1.69043136$

$-1.69043136$

0.79423416

0.79423416

0.79423416

0.79423416

0.90006965

0.90006965

0.90006965

64

0.90006965

$-1.8891$

$-1.70699394$

$-1.70699394$

$-1.70699394$

$-1.70699394$

$-1.60115862$

$-1.60115862$

$-1.60115862$

$-1.60115862$

1.14857543

1. 14857543

1.14857543

1.14857543

1. 25441086

1. 25441086

1. 25441086

1. 25441086

$-1.09362578$

$-1.09362578$

$-1.09362578$

$-1.09362578$

$-0.98779023$

$-0.98779023$

$-0.98779023$

$-0.98779023$

1. 78089249

1. 78089249

1.78089249

1.78089249

1. 88672793

1.88672793

1.88672793

1.88672793

$-1.20384598$

$-1.20384598$

$-1.20384598$

$-1.20384598$

$-1.09801054$

$-1.09801054$

$-1.09801054$

$-1.09801054$

1.52079928

1. 52079928
$-0.50571668$

$-0.39988124$

$-0.39988124$

$-0.50571668$

$-0.50571668$

$-0.39988124$

$-0.39988124$

0.82610810

0.82610810

0.93194360

0.93194360

0.82610810

0.82610810

0.93194360

0.93194360

$-0.89343792$

$-0.89343792$

$-0.78760254$

$-0.78760254$

$-0.89343792$

$-0.89343792$

$-0.78760254$

$-0.78760254$

. \# Energy 5->5

$-1.0050 \quad 6.5576$

$-0.23974958$

$-0.23974958$

$-0.13391411$

$-0.13391411$

$-0.23974958$

$-0.23974958$

$-0.13391411$

$-0.13391411$

0.57671642

0.57671642

0.68255186

0.68255186

0.57671642

0.57671642

0.68255186

0.68255186

$-0.46797100$

$-0.46797100$

$-0.36213556$

$-0.36213556$

$-0.46797100$

$-0.46797100$

$-0.36213556$

$-0.36213556$

0.98020822

0.98020822

1.08604372

1.08604372

0.98020822

0.98020822

1. 08604372

1.08604372

0.58981353

0.58981353

0.69564903

0.69564903

0.58981353

0.58981353

0.69564903

0.69564903

$-0.50571668$

$-0.50571668$
0.76928562

0.66345012

0.76928562

0.66345012

0.76928562

0.66345012

0.76928562

1.52134740

1.62718296

1.52134740

1.62718296

1.52134740

1.62718296

1.52134740

1. 62718296

1. 24928117

1. 35511649

1. 24928117

1. 35511649

1. 24928117

1. 35511649

1. 24928117

1.35511649
37.68807220

54.89326477

$-34.83139801$

28.98948097

$-19.82597542$

$-19.38655853$

9.74977398

$-16.56303406$

9.50829792

11.26529121

$-6.28285837$

25.49684334

$-15.77713203$

$-18.74315834$

11.36374092

$-3.36172581$

3. 25769067

1. 20429242

$-2.45338702$

4.42599154

$-4.30321836$

$-0.41034427$

1. 98984456

\# Electr. mom.

$-0.22430076$

$-0.11846532$

$-0.22430076$

$-0.11846532$

$-0.22430076$

$-0.11846532$

$-0.22430076$

$-0.11846532$

$-0.08068259$

0.02515285

$-0.08068259$

0.02515285

$-0.08068259$

0.02515285

$-0.08068259$

0.02515285

$-1.25737166$

$-1.15153623$

$-1.25737166$

$-1.15153623$

$-1.25737166$

$-1.15153623$

$-1.25737166$

$-1.15153623$

$-1.04271245$

$-0.93687707$

$-1.04271245$

$-0.93687707$

$-1.04271245$

$-0.93687707$

$-1.04271245$

$-0.93687707$

0.74054700

0.84638250

0.74054700

0.84638250

0.74054700

0.84638250

0.74054700

0.84638250

0.66345012

0.76928562
77.76054382

$-76.83435059$

$-51.21808243$

49.02061844

$-95.00337982$

101.20797729

82.71785736

$-89.31072998$

$-183.65945435$

209.61264038

167.30879211

$-187.36302185$

181.56213379

$-213.84310913$

$-163.42301941$

188.50666809

$-38.37554550$

49.78783035

49.17501450

$-65.14519501$

39.01599121

$-50.23565674$

$-48.10288620$

63.20613098

22.84384727

$-22.47956848$

$-21.45418930$

21.07437897

$-24.84259796$

25.39688873

23.70366669

$-24.38002586$

306.09225464

$-248.90150452$

$-255.07270813$

213.74104309

$-255.84161377$

206.12664795

217.01083374

$-181.26417542$

$-52.71130371$

35.04281235 
1.52079928

1. 52079928

1. 62663472

1. 62663472

1. 62663472

1. 62663472

$-1.79626679$

$-1.79626679$

$-1.79626679$

$-1.79626679$

$-1.69043136$

$-1.69043136$

$-1.69043136$

$-1.69043136$

0.79423416

0.79423416

0.79423416

0.79423416

0.90006965

0.90006965

0.90006965

0.90006965

64
$-0.39988124$

$-0.39988124$

$-0.50571668$

$-0.50571668$

$-0.39988124$

$-0.39988124$

0.82610810

0.82610810

0.93194360

0.93194360

0.82610810

0.82610810

0.93194360

0.93194360

$-0.89343792$

$-0.89343792$

$-0.78760254$

$-0.78760254$

$-0.89343792$

$-0.89343792$

$-0.78760254$

$-0.78760254$

\subsection{8}

$-1.70699394$

$-1.70699394$

$-1.70699394$

$-1.70699394$

$-1.60115862$

$-1.60115862$

$-1.60115862$

$-1.60115862$

1.14857543

1. 14857543

1.14857543

1. 14857543

1. 25441086

1. 25441086

1. 25441086

1.25441086

$-1.09362578$

$-1.09362578$

$-1.09362578$

$-1.09362578$

$-0.98779023$

$-0.98779023$

$-0.98779023$

$-0.98779023$

1. 78089249

1.78089249

1. 78089249

1.78089249

1.88672793

1.88672793

1.88672793

1.88672793

$-1.20384598$

$-1.20384598$

$-1.20384598$

$-1.20384598$

$-1.09801054$

$-1.09801054$

$-1.09801054$

$-1.09801054$

1. 52079928

1. 52079928

1. 52079928
0. \# Energy 6->6

$$
0.2789 \quad 4.2954
$$

$-0.23974958$

$-0.23974958$

$-0.13391411$

$-0.13391411$

$-0.23974958$

$-0.23974958$

$-0.13391411$

$-0.13391411$

0.57671642

0.57671642

0.68255186

0.68255186

0.57671642

0.57671642

0.68255186

0.68255186

$-0.46797100$

$-0.46797100$

$-0.36213556$

$-0.36213556$

$-0.46797100$

$-0.46797100$

$-0.36213556$

$-0.36213556$

0.98020822

0.98020822

1. 08604372

1.08604372

0.98020822

0.98020822

1. 08604372

1.08604372

0.58981353

0.58981353

0.69564903

0.69564903

0.58981353

0.58981353

0.69564903

0.69564903

$-0.50571668$

$-0.50571668$

$-0.39988124$
0.66345012

0.76928562

0.66345012

0.76928562

0.66345012

0.76928562

1. 52134740

1. 62718296

1.52134740

1.62718296

1.52134740

1. 62718296

1.52134740

1.62718296

1. 24928117

1. 35511649

1. 24928117

1. 35511649

1. 24928117

1. 35511649

1. 24928117

1. 35511649

$-0.22430076$

$-0.11846532$

$-0.22430076$

$-0.11846532$

$-0.22430076$

$-0.11846532$

$-0.22430076$

$-0.11846532$

$-0.08068259$

0.02515285

$-0.08068259$

0.02515285

$-0.08068259$

0.02515285

$-0.08068259$

0.02515285

$-1.25737166$

$-1.15153623$

$-1.25737166$

$-1.15153623$

$-1.25737166$

$-1.15153623$

$-1.25737166$

$-1.15153623$

$-1.04271245$

$-0.93687707$

$-1.04271245$

$-0.93687707$

$-1.04271245$

$-0.93687707$

$-1.04271245$

$-0.93687707$

0.74054700

0.84638250

0.74054700

0.84638250

0.74054700

0.84638250

0.74054700

0.84638250

0.66345012

0.76928562

0.66345012
51.96044922

$-32.73372650$

25.68128204

$-17.02367210$

$-16.55271912$

7. 56476831

$-17.42990875$

10.10416794

11.90989494

$-6.72580576$

26.70059586

$-16.60063171$

$-19.69147682$

12.00673199

$-4.87937927$

4. 40320492

3.06855655

$-3.89316201$

5.92597485

$-5.45844746$

$-2.16850352$

3. 37933826

Electr. mom.

69.01051331

$-66.60220337$

$-42.73340225$

39.30052948

$-87.16824341$

92.51913452

76.53372192

$-83.00629425$

$-192.54051208$

226.71914673

167.60031128

$-194.86738586$

205.27879333

$-248.75624084$

$-177.07901001$

211.70233154

$-48.60701752$

64.42877960

59.65892029

$-80.12349701$

49.76316452

$-65.30374908$

$-59.40339279$

78.95348358

39.42057037

$-45.70354843$

$-40.73824310$

47.68001175

$-39.35010529$

46.10035324

39.70217514

$-46.89540100$

317.01083374

$-257.46267700$

$-263.48178101$

220.39744568

$-256.07653809$

205.68733215

216.12208557

$-180.20935059$

$-54.10216904$

41.95885849

45.86386108 


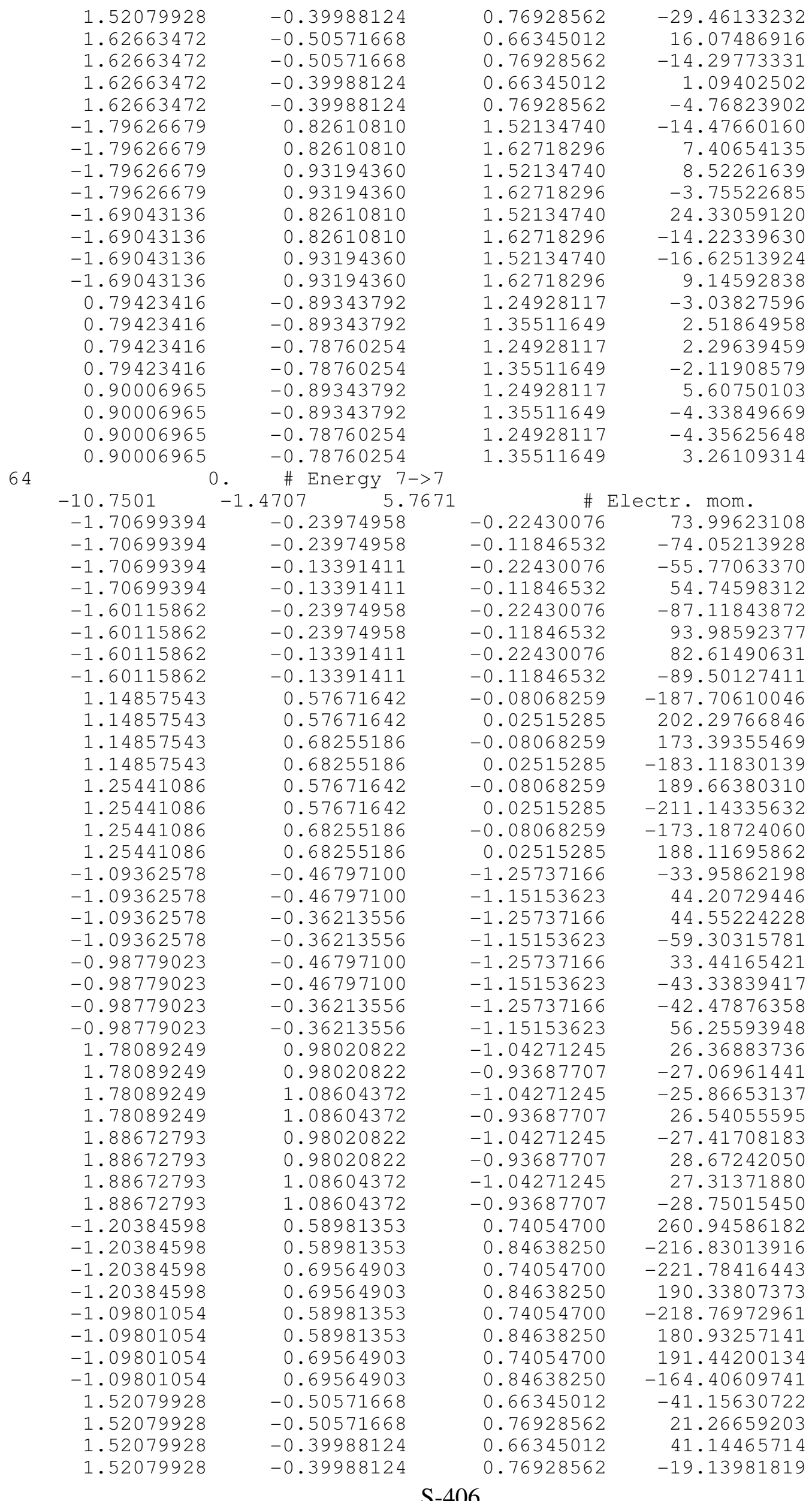




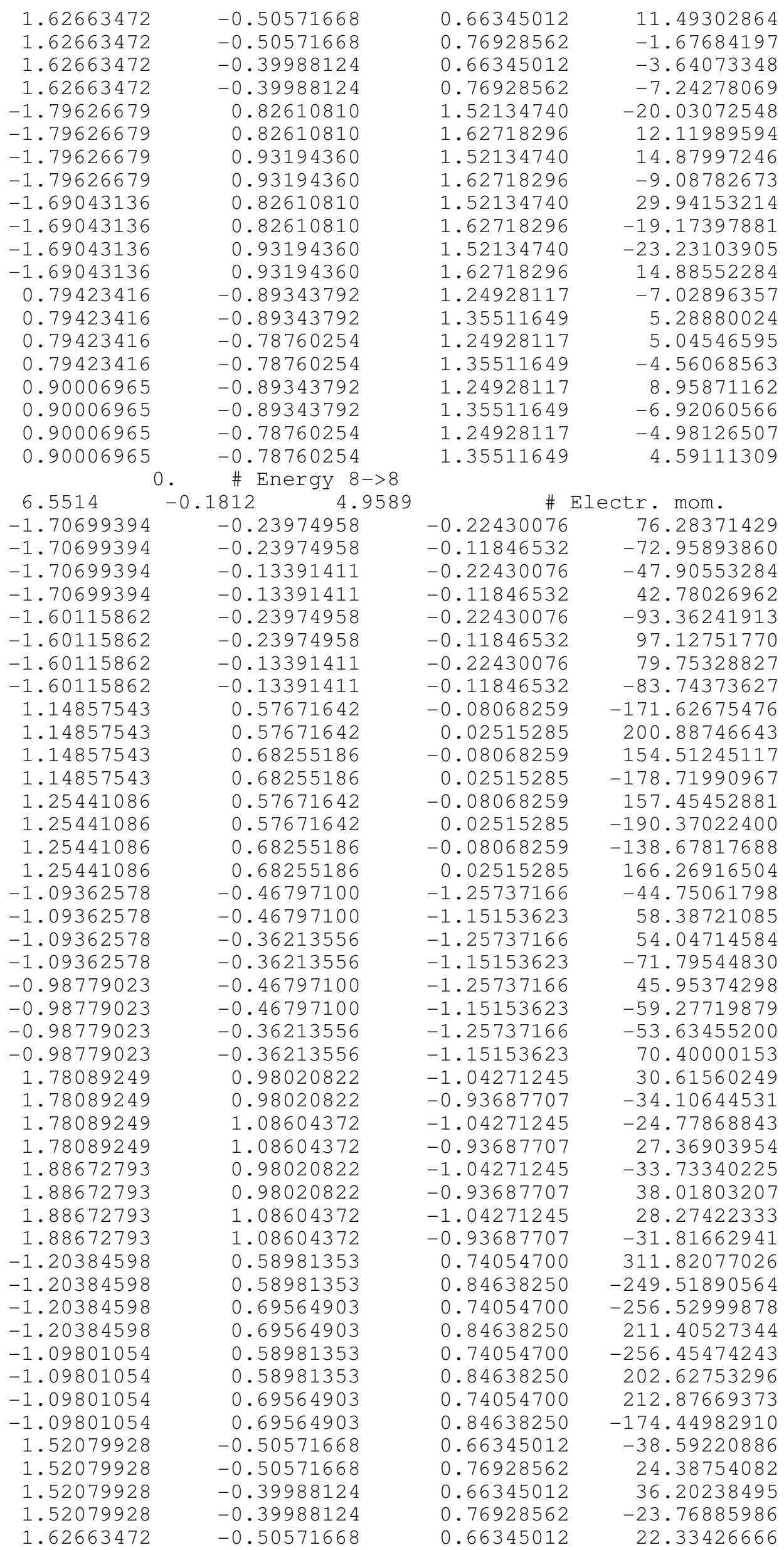




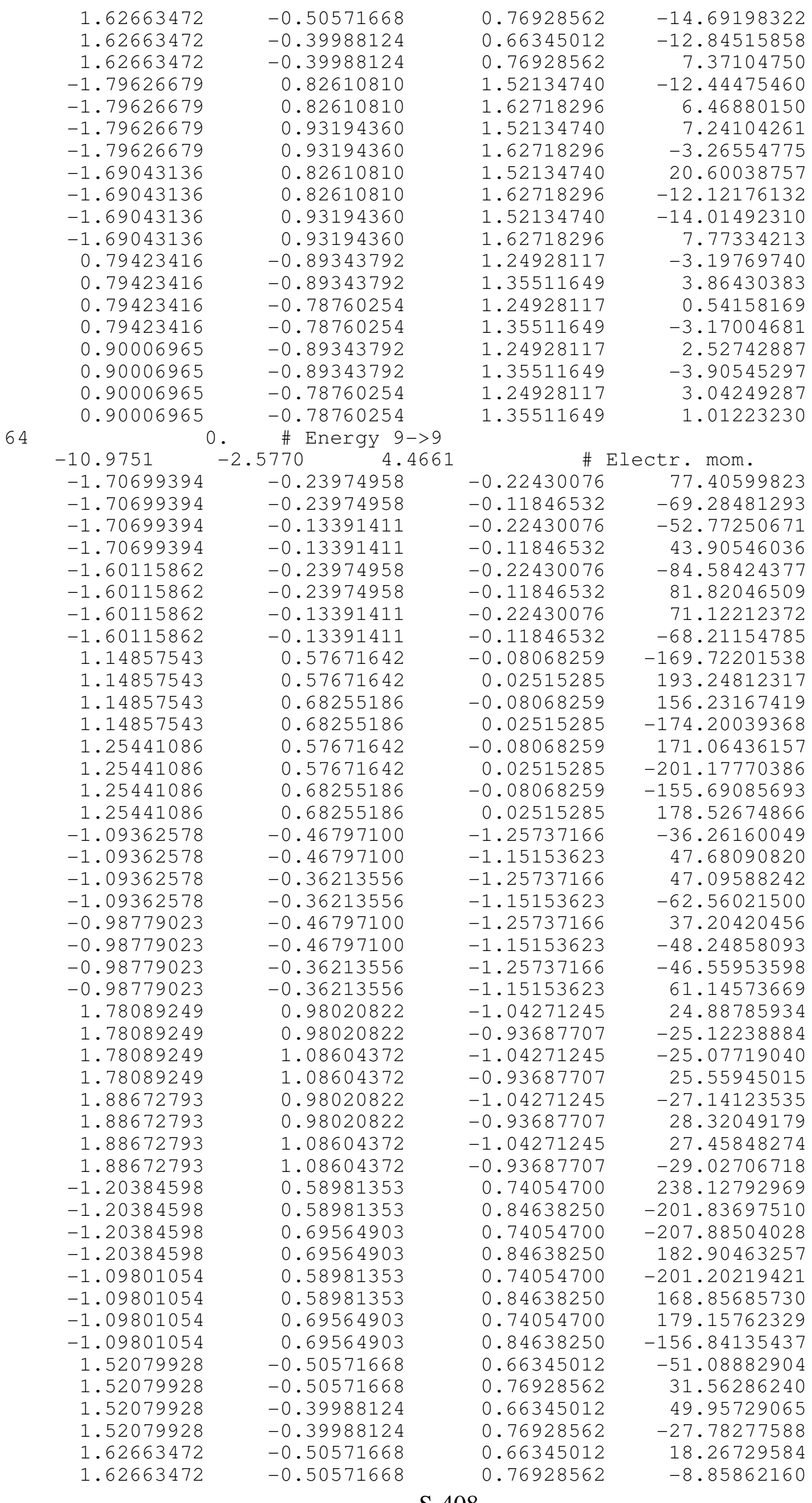

\section{S-408}




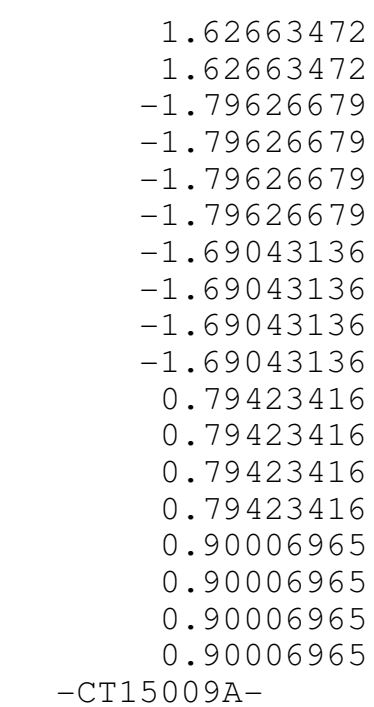

0.66345012

0.76928562

1.52134740

1.62718296

1.52134740

1.62718296

1.52134740

1.62718296

1.52134740

1.62718296

1.24928117

1. 35511649

1. 24928117

1.35511649

1. 24928117

1.35511649

1.24928117

1.35511649
-9.27267456
-1.57197022
-17.69033813
10.63568497
13.40699863
-8.10394573
27.31023979
-17.55867577
-21.71829414
13.91908360
-4.52172518
3.32059598
2.52212167
-2.43173885
6.87301970
-5.24431753
-3.22540522
2.97655153 \# phi $=-60$, psi= 6

$\begin{array}{rrrrr}1.7263 & -0.1566 & 0.1975 & 1.0000 & \# \text { C1 } \\ 1.2255 & 0.0669 & 1.3015 & 1.0000 & \text { \# } 01 \\ 1.0958 & 0.2285 & -0.9624 & 1.0000 & \text { \# N1 } \\ -1.2072 & 0.5883 & -0.0975 & 1.0000 & \text { \# C2 } \\ -1.7534 & 1.2503 & 0.7852 & 1.0000 & \text { \# N2 } \\ -1.7142 & -0.5932 & -0.5693 & 1.0000 & \text { \# O2 }\end{array}$

\&TRANSITION $1->.$.

$64 \quad 42916$.
$-0.0096$
$-0.3211$
-0.1305
0.1354
1.67341614 $-0.20954360$ 0.0480
1.67341614
$-0.20954360$
1.67341614
$-0.10370816$
1.67341614
$-0.10370816$
1.77925158
$-0.20954360$
1.77925158
$-0.20954360$
1.77925158
$-0.10370816$
1.77925158
$-1.26006997$
$-1.26006997$
$-0.10370816$
0.53541940
0.53541940
0.64125490
0.64125490
$-1.26006997$
$-1.15423441$
$-1.15423441$
$-1.15423441$
$-1.15423441$
1.17254710
1.17254710
1.17254710
1.17254710
1.27838266
1.27838266
1.27838266
1.27838266
$-1.80632699$
$-1.80632699$
$-1.80632699$
$-1.80632699$
$-1.70049155$
$-1.70049155$
$-1.70049155$
$-1.70049155$
1.04285800
1.04285800
1.04285800
0.53541940
0.53541940
0.64125490
0.64125490
0.01399621
0.01399621
0.11983165
0.11983165
0.01399621
0.01399621
0.11983165
0.11983165
1.19734502
1.19734502
1.30318046
1.30318046
1.19734502
1.19734502
1.30318046
1. 30318046
0.17553815
0.17553815
0.28137359
1.04285800
0.28137359

\# Energy 1->2
1.000 \# Electr. mom., scale-fac \# Magnetic mom.

0.14458551

0.25042096

0.14458551

0.25042096

0.14458551

0.25042096

0.14458551

0.25042096

$-0.15045778$

$-0.04462234$

$-0.15045778$

$-0.04462234$

$-0.15045778$

$-0.04462234$

$-0.15045778$

$-0.04462234$

1. 24855602

1. 35439157

1.24855602

1. 35439157

1. 24855602

1. 35439157

1.24855602

1.35439157

0.73230141

0.83813679

0.73230141

0.83813679

0.73230141

0.83813679

0.73230141

0.83813679

$-1.01531279$

$-0.90947729$

$-1.01531279$

$-0.90947729$
42.62998199

$-36.02215195$

$-54.20454788$

45.74246597

$-35.42402267$

29.98130608

43.79150391

$-36.73479843$

$-9.90854073$

3. 02193308

7.18098688

$-1.08761573$

13.44180393

$-5.69134331$

$-10.49360752$

3.73996472

11.03016090

$-8.60441875$

$-10.02477264$

7.80667782

$-9.67056084$

7.36829329

8.21226311

$-6.23066998$

1. 42900455

$-0.74689955$

$-1.28407896$

0.71541780

$-1.98747718$

1.14506519

1. 67175221

$-0.99297243$

$-18.52471733$

21.53393364

17.17946815

$-19.92828751$ 
1.14869356

1. 14869356

1. 14869356

1.14869356

$-1.76707089$

$-1.76707089$

$-1.76707089$

$-1.76707089$

$-1.66123557$

$-1.66123557$

$-1.66123557$

$-1.66123557$

1.57582414

1.57582414

1. 57582414

1.57582414

1.68165958

1.68165958

1.68165958

$-1.11259675$

$-1.11259675$

$-1.11259675$

$-1.11259675$

$-1.00676131$

$-1.00676131$

$-1.00676131$

$-1.00676131$

64
1. 68165958

0.17553815

0.17553815

0.28137359

0.28137359

$-0.64616346$

$-0.64616346$

$-0.54032809$

$-0.54032809$

$-0.64616346$

$-0.64616346$

$-0.54032809$

$-0.54032809$

0.13101105

0.13101105

0.23684651

0.23684651

0.13101105

0.13101105

0.23684651

0.23684651

$-1.22435784$

$-1.22435784$

$-1.11852252$

$-1.11852252$

$-1.22435784$

$-1.22435784$

$-1.11852252$

$-1.11852252$

\# Energy 1->3

$0.0283^{42493.0 .2560}-0.5001$

$0.3195-0.5001-0.6288$

1.67341614

1.67341614

1.67341614

1.67341614

1.77925158

1.77925158

1. 77925158

1. 77925158

$-1.26006997$

$-1.26006997$

$-1.26006997$

$-1.26006997$

$-1.15423441$

$-1.15423441$

$-1.15423441$

$-1.15423441$

1. 17254710

1. 17254710

1. 17254710

1.17254710

1. 27838266

1. 27838266

1. 27838266

1. 27838266

$-1.80632699$

$-1.80632699$

$-1.80632699$

$-1.80632699$

$-1.70049155$

$-1.70049155$

$-1.70049155$

$-1.70049155$

1. 04285800

1. 04285800

1. 04285800

1. 04285800
$-0.20954360$

$-0.20954360$

$-0.10370816$

$-0.10370816$

$-0.20954360$

$-0.20954360$

$-0.10370816$

$-0.10370816$

0.53541940

0.53541940

0.64125490

0.64125490

0.53541940

0.53541940

0.64125490

0.64125490

0.01399621

0.01399621

0.11983165

0.11983165

0.01399621

0.01399621

0.11983165

0.11983165

1. 19734502

1. 19734502

1. 30318046

1. 30318046

1. 19734502

1.19734502

1. 30318046

1. 30318046

0.17553815

0.17553815

0.28137359

0.28137359
$-1.01531279$

$-0.90947729$

$-1.01531279$

$-0.90947729$

$-0.62219280$

$-0.51635736$

$-0.62219280$

$-0.51635736$

$-0.62219280$

$-0.51635736$

$-0.62219280$

$-0.51635736$

$-1.87711871$

$-1.77128315$

$-1.87711871$

$-1.77128315$

$-1.87711871$

$-1.77128315$

$-1.87711871$

$-1.77128315$

$-1.14041448$

$-1.03457904$

$-1.14041448$

$-1.03457904$

$-1.14041448$

$-1.03457904$

$-1.14041448$

$-1.03457904$
21.46290970

$-23.87555885$

$-21.57382584$

23.98086739

$-9.24648666$

4. 77407885

7. 07611322

$-2.44099712$

11.41900063

$-5.91765070$

$-8.70765305$

3.05647135

3. 71861434

$-5.02448988$

$-3.35781097$

4.73625278

$-3.26294708$

4.27491188

2. 74468565

$-3.76390362$

2. 82670212

$-3.82408047$

$-3.38365436$

4.33497190

$-2.57769012$

3. 66652322

3. 24155974

$-4.41741467$
1.000 \# Electr. mom., scale-fac \# Magnetic mom.

0.14458551

$-2.72287846$

0.25042096

0.14458551

0.25042096

0.14458551

0.25042096

0.14458551

0.25042096

$-0.15045778$

$-0.04462234$

$-0.15045778$

$-0.04462234$

$-0.15045778$

$-0.04462234$

$-0.15045778$

$-0.04462234$

1. 24855602

1. 35439157

1. 24855602

1. 35439157

1. 24855602

1. 35439157

1. 24855602

1. 35439157

0.73230141

0.83813679

0.73230141

0.83813679

0.73230141

0.83813679

0.73230141

0.83813679

$-1.01531279$

$-0.90947729$

$-1.01531279$

$-0.90947729$
2. 04151344

4. 05126476

$-3.34305215$

0.97245604

$-0.30208534$

$-1.97471523$

1. 25110734

9.16703987

$-5.67559433$

$-5.51295996$

1. 97690821

$-3.51232409$

$-0.55085230$

$-0.82264161$

5.13448524

3. 51828051

$-2.51781201$

$-4.23150682$

3.07333565

$-3.66738033$

2. 64862585

4.39059019

$-3.20521569$

10.65922070

$-8.10030842$

$-6.66994095$

4.78228712

$-9.36095142$

6.37240410

5.01399899

$-2.84279656$

8. 25002480

$-6.93048048$

$-6.61096478$

5. 28956890 
1.14869356

1. 14869356

1. 14869356

1.14869356

$-1.76707089$

$-1.76707089$

$-1.76707089$

$-1.76707089$

$-1.66123557$

$-1.66123557$

$-1.66123557$

$-1.66123557$

1.57582414

1.57582414

1.57582414

1.57582414

1.68165958

1.68165958

1.68165958

$-1.11259675$

$-1.11259675$

$-1.11259675$

$-1.11259675$

$-1.00676131$

$-1.00676131$

$-1.00676131$

$-1.00676131$

64
1. 68165958

0.17553815

0.17553815

0.28137359

0.28137359

$-0.64616346$

$-0.64616346$

$-0.54032809$

$-0.54032809$

$-0.64616346$

$-0.64616346$

$-0.54032809$

$-0.54032809$

0.13101105

0.13101105

0.23684651

0.23684651

0.13101105

0.13101105

0.23684651

0.23684651

$-1.22435784$

$-1.22435784$

$-1.11852252$

$-1.11852252$

$-1.22435784$

$-1.22435784$

$-1.11852252$

$-1.11852252$

\# Energy 1->4

0.118753225

0.118 53225 .

$-0.3295$

1.67341614

1.67341614

1.67341614

1.67341614

1. 77925158

1. 77925158

1. 77925158

1. 77925158

$-1.26006997$

$-1.26006997$

$-1.26006997$

$-1.26006997$

$-1.15423441$

$-1.15423441$

$-1.15423441$

$-1.15423441$

1. 17254710

1. 17254710

1. 17254710

1. 17254710

1. 27838266

1. 27838266

1. 27838266

1. 27838266

$-1.80632699$

$-1.80632699$

$-1.80632699$

$-1.80632699$

$-1.70049155$

$-1.70049155$

$-1.70049155$

$-1.70049155$

1.04285800

1. 04285800

1. 04285800

1. 04285800 $\begin{array}{ll}2.9427 & 1.8597\end{array}$ $-0.4756 \quad 0.5692$

$-0.20954360$

$-0.20954360$

$-0.10370816$

$-0.10370816$

$-0.20954360$

$-0.20954360$

$-0.10370816$

$-0.10370816$

0.53541940

0.53541940

0.64125490

0.64125490

0.53541940

0.53541940

0.64125490

0.64125490

0.01399621

0.01399621

0.11983165

0.11983165

0.01399621

0.01399621

0.11983165

0.11983165

1. 19734502

1. 19734502

1. 30318046

1. 30318046

1. 19734502

1. 19734502

1. 30318046

1. 30318046

0.17553815

0.17553815

0.28137359

0.28137359
$-1.01531279$

$-0.90947729$

$-1.01531279$

$-0.90947729$

$-0.62219280$

$-0.51635736$

$-0.62219280$

$-0.51635736$

$-0.62219280$

$-0.51635736$

$-0.62219280$

$-0.51635736$

$-1.87711871$

$-1.77128315$

$-1.87711871$

$-1.77128315$

$-1.87711871$

$-1.77128315$

$-1.87711871$

$-1.77128315$

$-1.14041448$

$-1.03457904$

$-1.14041448$

$-1.03457904$

$-1.14041448$

$-1.03457904$

$-1.14041448$

$-1.03457904$$$
\begin{gathered}
1.000 \text { \# Electr. mom., scale-fac } \\
\text { \# Magnetic mom. }
\end{gathered}
$$

0.14458551

0.25042096

0.14458551

0.25042096

0.14458551

0.25042096

0.14458551

0.25042096

$-0.15045778$

$-0.04462234$

$-0.15045778$

$-0.04462234$

$-0.15045778$

$-0.04462234$

$-0.15045778$

$-0.04462234$

1. 24855602

1. 35439157

1. 24855602

1. 35439157

1. 24855602

1. 35439157

1. 24855602

1. 35439157

0.73230141

0.83813679

0.73230141

0.83813679

0.73230141

0.83813679

0.73230141

0.83813679

$-1.01531279$

$-0.90947729$

$-1.01531279$

$-0.90947729$
-8.85060215
7.77111053
6.49712753
-5.52039194
1.95523369
5.74020290
4.53147697
-11.20953846
-6.31266928
-2.93897390
-1.82373691
9.60038090
0.13369140
-0.38235143
-0.41715944
0.90129334
-0.24268968
0.50821579
0.32473245
-0.73992765
-1.01488721
-0.69343877
0.11290371
2.99795914
1.27473676
-0.36374503
-0.95731711
-0.92028427$$
3.65579820
$$

$-3.48543334$

$-5.85951328$

5.85372686

$-1.58492851$

1.38036633

3. 07934999

$-3.02690434$

$-27.70259094$

31.72212219

32.27701950

$-38.39177322$

20.66325378

$-23.08799171$

$-23.78667831$

27.97676849

$-2.79174781$

2. 15268898

3. 24607897

$-2.47455072$

2.85629654

$-2.20732760$

$-3.36662626$

2. 56073809

10.26842976

$-10.43460846$

$-12.44206524$

12.02778053

$-7.47283745$

8.38417339

10.13902187

$-10.37714672$

$-3.09583306$

3.33280826

4.89966345

$-5.41328812$ 
1.14869356

1. 14869356

1. 14869356

1.14869356

$-1.76707089$

$-1.76707089$

$-1.76707089$

$-1.76707089$

$-1.66123557$

$-1.66123557$

$-1.66123557$

1.57582414

1.57582414

1.57582414

1.57582414

1.68165958

1.68165958

1.68165958

$-1.11259675$

$-1.11259675$

$-1.11259675$

$-1.11259675$

$-1.00676131$

$-1.00676131$

$-1.00676131$

$-1.00676131$

64
$-1.66123557$

1. 68165958

$$
50033 .
$$

$\begin{array}{lll}0.2592 & 0.0738 & -2.9738\end{array}$

-0.1539
1.67341614

1.67341614

1.67341614

1.67341614

1.77925158

1. 77925158

1.77925158

1. 77925158

$-1.26006997$

$-1.26006997$

$-1.26006997$

$-1.26006997$

$-1.15423441$

$-1.15423441$

$-1.15423441$

$-1.15423441$

1. 17254710

1. 17254710

1. 17254710

1. 17254710

1. 27838266

1. 27838266

1. 27838266

1. 27838266

$-1.80632699$

$-1.80632699$

$-1.80632699$

$-1.80632699$

$-1.70049155$

$-1.70049155$

$-1.70049155$

$-1.70049155$

1. 04285800

1. 04285800

1. 04285800

1. 04285800

1. 19734502
0.17553815

0.17553815

0.28137359

0.28137359

$-0.64616346$

$-0.64616346$

$-0.54032809$

$-0.54032809$

$-0.64616346$

$-0.64616346$

$-0.54032809$

$-0.54032809$

0.13101105

0.13101105

0.23684651

0.23684651

0.13101105

0.13101105

0.23684651

0.23684651

$-1.22435784$

$-1.22435784$

$-1.11852252$

$-1.11852252$

$-1.22435784$

$-1.22435784$

$-1.11852252$

$-1.11852252$ \# Energy 1->5

$-1.01531279$

$-0.90947729$

$-1.01531279$

$-0.90947729$

$-0.62219280$

$-0.51635736$

$-0.62219280$

$-0.51635736$

$-0.62219280$

$-0.51635736$

$-0.62219280$

$-0.51635736$

$-1.87711871$

$-1.77128315$

$-1.87711871$

$-1.77128315$

$-1.87711871$

$-1.77128315$

$-1.87711871$

$-1.77128315$

$-1.14041448$

$-1.03457904$

$-1.14041448$

$-1.03457904$

$-1.14041448$

$-1.03457904$

$-1.14041448$

$-1.03457904$

$$
\begin{array}{rr}
0.0738 & -0.0172
\end{array}
$$

$-0.20954360$

$-0.20954360$

$-0.10370816$

$-0.10370816$

$-0.20954360$

$-0.20954360$

$-0.10370816$

$-0.10370816$

0.53541940

0.53541940

0.64125490

0.64125490

0.53541940

0.53541940

0.64125490

0.64125490

0.01399621

0.01399621

0.11983165

0.11983165

0.01399621

0.01399621

0.11983165

0.11983165

1. 19734502

1. 19734502

1. 30318046

1. 30318046

1.19734502

1. 30318046

1. 30318046

0.17553815

0.17553815

0.28137359

0.28137359

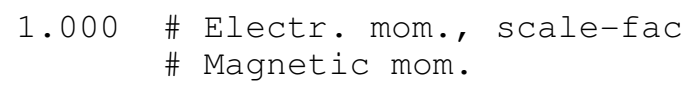

1. 76912785 
1.14869356

1. 14869356

1. 14869356

1.14869356

$-1.76707089$

$-1.76707089$

$-1.76707089$

$-1.76707089$

$-1.66123557$

$-1.66123557$

$-1.66123557$

$-1.66123557$

1.57582414

1.57582414

1. 57582414

1.57582414

1.68165958

1.68165958

1.68165958

$-1.11259675$

$-1.11259675$

$-1.11259675$

$-1.11259675$

$-1.00676131$

$-1.00676131$

$-1.00676131$

$-1.00676131$

64
1. 68165958

0.17553815

0.17553815

0.28137359

0.28137359

$-0.64616346$

$-0.64616346$

$-0.54032809$

$-0.54032809$

$-0.64616346$

$-0.64616346$

$-0.54032809$

$-0.54032809$

0.13101105

0.13101105

0.23684651

0.23684651

0.13101105

0.13101105

0.23684651

0.23684651

$-1.22435784$

$-1.22435784$

$-1.11852252$

$-1.11852252$

$-1.22435784$

$-1.22435784$

$-1.11852252$

$-1.11852252$

\# Energy 1->6

$0.6040-0.3469 \quad 0.4405$

0.0621

1.67341614

1.67341614

1.67341614

1.67341614

1.77925158

1.77925158

1. 77925158

1. 77925158

$-1.26006997$

$-1.26006997$

$-1.26006997$

$-1.26006997$

$-1.15423441$

$-1.15423441$

$-1.15423441$

$-1.15423441$

1. 17254710

1. 17254710

1. 17254710

1. 17254710

1. 27838266

1. 27838266

1. 27838266

1. 27838266

$-1.80632699$

$-1.80632699$

$-1.80632699$

$-1.80632699$

$-1.70049155$

$-1.70049155$

$-1.70049155$

$-1.70049155$

1. 04285800

1. 04285800

1. 04285800

1. 04285800
$0.3452 \quad 0.0802$

$-0.20954360$

$-0.20954360$

$-0.10370816$

$-0.10370816$

$-0.20954360$

$-0.20954360$

$-0.10370816$

$-0.10370816$

0.53541940

0.53541940

0.64125490

0.64125490

0.53541940

0.53541940

0.64125490

0.64125490

0.01399621

0.01399621

0.11983165

0.11983165

0.01399621

0.01399621

0.11983165

0.11983165

1. 19734502

1. 19734502

1. 30318046

1. 30318046

1. 19734502

1.19734502

1. 30318046

1. 30318046

0.17553815

0.17553815

0.28137359

0.28137359
$-1.01531279$

$-0.90947729$

$-1.01531279$

$-0.90947729$

$-0.62219280$

$-0.51635736$

$-0.62219280$

$-0.51635736$

$-0.62219280$

$-0.51635736$

$-0.62219280$

$-0.51635736$

$-1.87711871$

$-1.77128315$

$-1.87711871$

$-1.77128315$

$-1.87711871$

$-1.77128315$

$-1.87711871$

$-1.77128315$

$-1.14041448$

$-1.03457904$

$-1.14041448$

$-1.03457904$

$-1.14041448$

$-1.03457904$

$-1.14041448$

$-1.03457904$
2.50687218

$-5.83437014$

0.42112195

1.25310969

2. 22525215

1. 64745247

0.14033535

$-3.58490515$

$-5.02776909$

0.10713681

1.84042192

2.37675190

$-0.01383755$

0.02632847

$-0.10219709$

0.30474049

$-0.02266260$

0.00587549

$-0.04261260$

$-0.06747770$

$-0.03559816$

$-1.29590893$

$-1.08120108$

3. 36520505

0.51571941

0.26299927

0.07774347

$-1.52151215$
1.000 \# Electr. mom., scale-fac \# Magnetic mom.

0.14458551

0.25042096

0.14458551

0.25042096

0.14458551

0.25042096

0.14458551

0.25042096

$-0.15045778$

$-0.04462234$

$-0.15045778$

$-0.04462234$

$-0.15045778$

$-0.04462234$

$-0.15045778$

$-0.04462234$

1. 24855602

1. 35439157

1. 24855602

1. 35439157

1. 24855602

1. 35439157

1. 24855602

1. 35439157

0.73230141

0.83813679

0.73230141

0.83813679

0.73230141

0.83813679

0.73230141

0.83813679

$-1.01531279$

$-0.90947729$

$-1.01531279$

$-0.90947729$
$-8.88949776$

9.35978889

10.44068050

$-11.22512817$

6.88190842

$-7.16284943$

$-8.04014206$

8.56913471

1.10767019

$-1.28924561$

$-2.37771511$

2. 89225864

$-0.32195443$

$-0.24431141$

1. 42803502

$-1.17460024$

1.87560093

$-1.63469696$

$-2.37920475$

2. 01357198

$-2.09055686$

1. 78811812

2.59144473

$-2.15854502$

$-0.92614233$

1.05814910

1.70283663

$-1.67301297$

0.59557569

$-0.86724871$

$-1.54676270$

1. 62401605

2. 59855700

$-3.41406226$

$-4.22880077$

5.53319550 
1.14869356

1. 14869356

1. 14869356

1.14869356

$-1.76707089$

$-1.76707089$

$-1.76707089$

$-1.76707089$

$-1.66123557$

$-1.66123557$

$-1.66123557$

$-1.66123557$

1.57582414

1.57582414

1. 57582414

1.57582414

1.68165958

1.68165958

1.68165958

1.68165958

$-1.11259675$

$-1.11259675$

$-1.11259675$

$-1.11259675$

$-1.00676131$

$-1.00676131$

$-1.00676131$

$-1.00676131$

64

$$
64724 \text {. }
$$

0.0132

0.0387

1.67341614

1.67341614

1.67341614

1.67341614

1. 77925158

1. 77925158

1. 77925158

1. 77925158

$-1.26006997$

$-1.26006997$

$-1.26006997$

$-1.26006997$

$-1.15423441$

$-1.15423441$

$-1.15423441$

$-1.15423441$

1. 17254710

1. 17254710

1. 17254710

1.17254710

1.27838266

1.27838266

1. 27838266

1. 27838266

$-1.80632699$

$-1.80632699$

$-1.80632699$

$-1.80632699$

$-1.70049155$

$-1.70049155$

$-1.70049155$

$-1.70049155$

1. 04285800

1. 04285800

1.04285800

1. 04285800
0.17553815

0.17553815

0.28137359

0.28137359

$-0.64616346$

$-0.64616346$

$-0.54032809$

$-0.54032809$

$-0.64616346$

$-0.64616346$

$-0.54032809$

$-0.54032809$

0.13101105

0.13101105

0.23684651

0.23684651

0.13101105

0.13101105

0.23684651

0.23684651

$-1.22435784$

$-1.22435784$

$-1.11852252$

$-1.11852252$

$-1.22435784$

$-1.22435784$

$-1.11852252$

$-1.11852252$ \# Energy 1->7

$\begin{array}{ll}0.2090 & 1.2792 \\ 0.3753 & 0.0569\end{array}$

$-0.20954360$

$-0.20954360$

$-0.10370816$

$-0.10370816$

$-0.20954360$

$-0.20954360$

$-0.10370816$

$-0.10370816$

0.53541940

0.53541940

0.64125490

0.64125490

0.53541940

0.53541940

0.64125490

0.64125490

0.01399621

0.01399621

0.11983165

0.11983165

0.01399621

0.01399621

0.11983165

0.11983165

1. 19734502

1. 19734502

1. 30318046

1. 30318046

1. 19734502

1. 19734502

1. 30318046

1. 30318046

0.17553815

0.17553815

0.28137359

0.28137359
$-1.01531279$

$-0.90947729$

$-1.01531279$

$-0.90947729$

$-0.62219280$

$-0.51635736$

$-0.62219280$

$-0.51635736$

$-0.62219280$

$-0.51635736$

$-0.62219280$

$-0.51635736$

$-1.87711871$

$-1.77128315$

$-1.87711871$

$-1.77128315$

$-1.87711871$

$-1.77128315$

$-1.87711871$

$-1.77128315$

$-1.14041448$

$-1.03457904$

$-1.14041448$

$-1.03457904$

$-1.14041448$

$-1.03457904$

$-1.14041448$

$-1.03457904$
1.000 \# Electr. mom., scale-fac \# Magnetic mom.

0.14458551

0.25042096

0.14458551

0.25042096

0.14458551

0.25042096

0.14458551

0.25042096

$-0.15045778$

$-0.04462234$

$-0.15045778$

$-0.04462234$

$-0.15045778$

$-0.04462234$

$-0.15045778$

$-0.04462234$

1. 24855602

1. 35439157

1. 24855602

1. 35439157

1. 24855602

1. 35439157

1. 24855602

1. 35439157

0.73230141

0.83813679

0.73230141

0.83813679

0.73230141

0.83813679

0.73230141

0.83813679

$-1.01531279$

$-0.90947729$

$-1.01531279$

$-0.90947729$
$-1.34286284$

2.11697006

2. 61522651

$-3.83906555$

$-2.43114877$

3. 21601415

3. 01238132

$-3.74179363$

1. 74526048

$-2.78920460$

$-2.64757514$

3. 60378075

0.16296393

$-0.24693884$

$-0.14744541$

0.27207285

$-0.08244199$

0.13727906

0.05956677

$-0.14794584$

$-0.16432434$

$-0.18914720$

$-0.08316591$

0.68285435

0.29975900

$-0.08870899$

$-0.15464909$

$-0.24377549$

$-1.55155098$

1. 79581082

0.74031895

$-1.58302879$

2.04447603

$-2.02640676$

$-1.20405662$

1. 73297215

$-0.99133903$

1. 45213616

1. 56021893

$-1.98233831$

0.44583419

$-0.90908945$

$-1.19539452$

1. 59402227

$-0.48169634$

0.25014681

0.13176353

0.03740048

0.50794190

$-0.26648140$

$-0.32635340$

0.10857232

$-0.65987617$

0.41520467

0.55233443

$-0.31829700$

0.79594535

$-0.50174493$

$-0.70263249$

0.41461125

$-4.94684029$

6.23711586

3. 20828557

$-3.98184228$ 


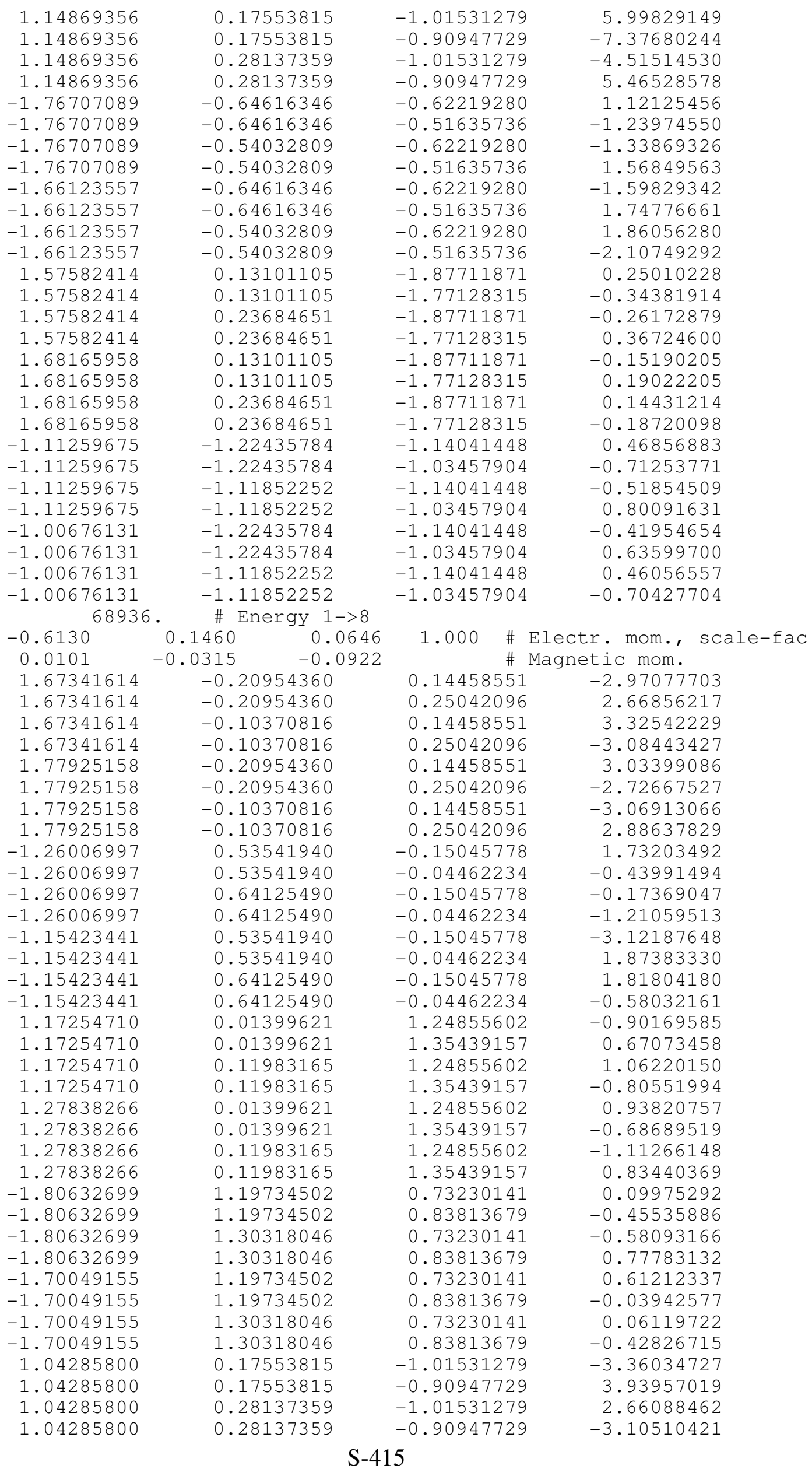


1.14869356

1. 14869356

1. 14869356

1.14869356

$-1.76707089$

$-1.76707089$

$-1.76707089$

$-1.76707089$

$-1.66123557$

$-1.66123557$

$-1.66123557$

1. 57582414

1.57582414

1. 57582414

1.57582414

1.68165958

1.68165958

1.68165958

$-1.11259675$

$-1.11259675$

$-1.11259675$

$-1.11259675$

$-1.00676131$

$-1.00676131$

$-1.00676131$

$-1.00676131$

64
$-1.66123557$

1.68165958

0.17553815

0.17553815

0.28137359

0.28137359

$-0.64616346$

$-0.64616346$

$-0.54032809$

$-0.54032809$

$-0.64616346$

$-0.64616346$

$-0.54032809$

$-0.54032809$

0.13101105

0.13101105

0.23684651

0.23684651

0.13101105

0.13101105

0.23684651

0.23684651

$-1.22435784$

$-1.22435784$

$-1.11852252$

$-1.11852252$

$-1.22435784$

$-1.22435784$

$-1.11852252$

$-1.11852252$ \# Energy 1->9

$\begin{array}{lrr}1.7622 & -0.1825 & 1.6501 \\ 0.0789 & 0.6219 & 0.2062\end{array}$

1.67341614

1.67341614

1.67341614

1.67341614

1.77925158

1.77925158

1. 77925158

1.77925158

$-1.26006997$

$-1.26006997$

$-1.26006997$

$-1.26006997$

$-1.15423441$

$-1.15423441$

$-1.15423441$

$-1.15423441$

1. 17254710

1. 17254710

1. 17254710

1.17254710

1. 27838266

1. 27838266

1. 27838266

1. 27838266

$-1.80632699$

$-1.80632699$

$-1.80632699$

$-1.80632699$

$-1.70049155$

$-1.70049155$

$-1.70049155$

$-1.70049155$

1. 04285800

1. 04285800

1. 04285800

1. 04285800
$-0.20954360$

$-0.20954360$

$-0.10370816$

$-0.10370816$

$-0.20954360$

$-0.20954360$

$-0.10370816$

$-0.10370816$

0.53541940

0.53541940

0.64125490

0.64125490

0.53541940

0.53541940

0.64125490

0.64125490

0.01399621

0.01399621

0.11983165

0.11983165

0.01399621

0.01399621

0.11983165

0.11983165

1. 19734502

1.19734502

1. 30318046

1. 30318046

1. 19734502

1. 19734502

1. 30318046

1. 30318046

0.17553815

0.17553815

0.28137359

0.28137359
-1.01531279
-0.90947729
-1.01531279
-0.90947729
-0.62219280
-0.51635736
-0.62219280
-0.51635736
-0.62219280
-0.51635736
-0.62219280
-0.51635736
-1.87711871
-1.77128315
-1.87711871
-1.77128315
-1.87711871
-1.77128315
-1.87711871
-1.77128315
-1.14041448
-1.03457904
-1.14041448
-1.03457904
-1.14041448
-1.03457904
-1.14041448
-1.03457904

3. 06173468

$-3.71190906$

$-2.17720127$

2. 67642021

1.42874193

$-0.75565249$

$-0.97145039$

0.16553745

$-1.22512817$

0.28297928

0.63024122

0.43986180

$-0.29040787$

0.45963234

0.22325894

$-0.40338668$

0.25555566

$-0.40390638$

$-0.16463973$

0.31142482

0.02573368

$-0.10569290$

$-0.21986488$

0.38771328

0.01524651

0.02826502

0.11220553

$-0.21686025$

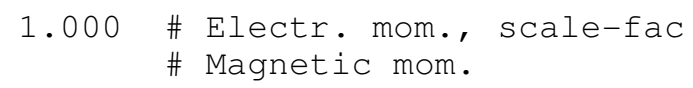

$-6.11566877$ 6.20268679

6.56492853

$-7.32466269$

5.01112747

$-4.88098001$

$-5.45010948$

5.79321527

1.10487485

$-3.08847332$

$-4.95631599$

7.39111471

2. 52299857

$-1.58204246$

0.23473781

$-1.47837722$

1.60230696

$-1.52994347$

$-1.98896790$

1.83449256

$-1.59308553$

1.51521635

1.89629602

$-1.76075983$

$-1.39034963$

1.96170461

2.59045029

$-2.72837377$

$-0.53237009$

$-0.62421179$

$-1.27576351$

1. 84519327

1.90267503

$-1.16806149$

$-3.54175806$

3.32907796 
1.14869356

1. 14869356

1. 14869356

1.14869356

$-1.76707089$

$-1.76707089$

$-1.76707089$

$-1.76707089$

$-1.66123557$

$-1.66123557$

$-1.66123557$

$-1.66123557$

1.57582414

1.57582414

1. 57582414

1.57582414

1.68165958

1.68165958

1.68165958

1.68165958

$-1.11259675$

$-1.11259675$

$-1.11259675$

$-1.11259675$

$-1.00676131$

$-1.00676131$

$-1.00676131$

$-1.00676131$

\&TRANSITION 2->...

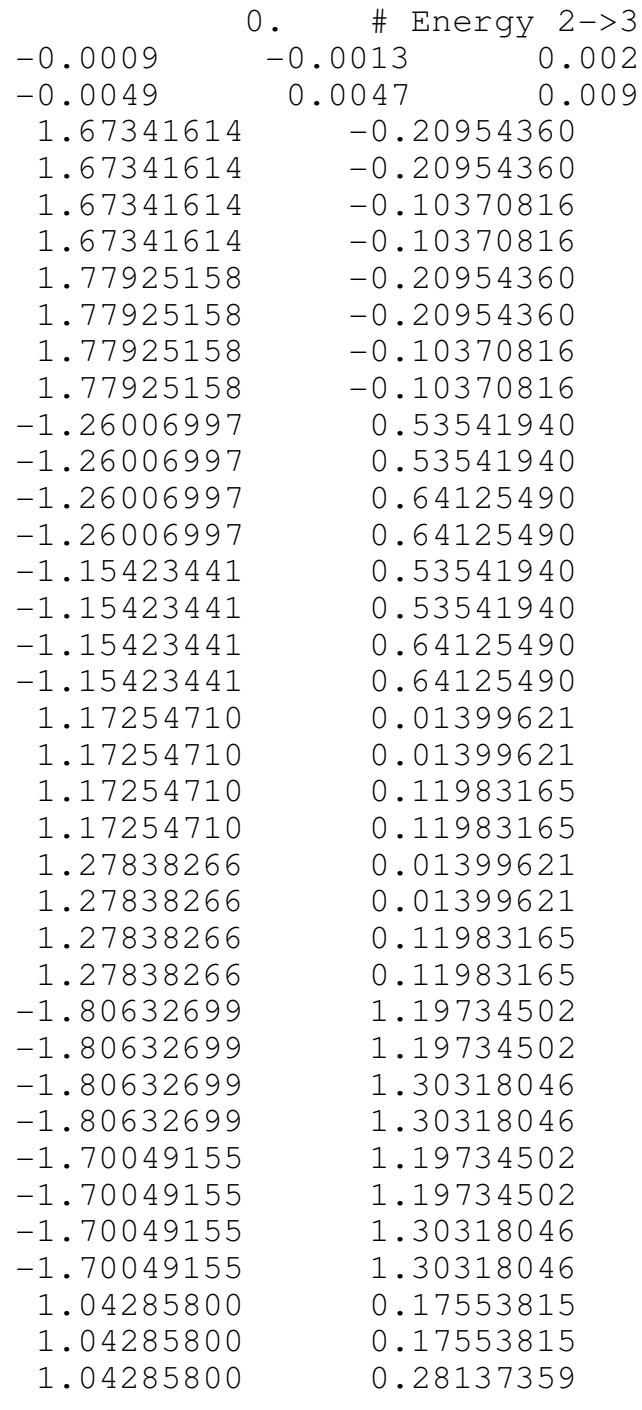

64
-1.01531279
-0.90947729
-1.01531279
-0.90947729
-0.62219280
-0.51635736
-0.62219280
-0.51635736
-0.62219280
-0.51635736
-0.62219280
-0.51635736
-1.87711871
-1.77128315
-1.87711871
-1.77128315
-1.87711871
-1.77128315
-1.87711871
-1.77128315
-1.14041448
-1.03457904
-1.14041448
-1.03457904
-1.14041448
-1.03457904
-1.14041448
-1.03457904

$-1.03517997$

0.37312248

2. 05356264

$-1.80430877$

$-4.52828932$

4. 59526110

5.01078176

$-4.95531273$

4. 26647234

$-4.28591013$

$-4.85144138$

4.83231258

0.43380684

$-0.65678358$

$-0.34365436$

0.61237472

$-0.38312149$

0.56330633

0.23320378

$-0.43126476$

0.52896315

$-1.09813523$

$-0.50038010$

1. 15855253

$-0.34507483$

0.83327711

0.28627843

$-0.85524207$
1.000 \# Electr. mom., scale-fac \# Magnetic mom.

$0.14458551 \quad-0.09493505$

$0.25042096 \quad 0.07719868$

$0.14458551 \quad 0.20887022$

$0.25042096 \quad-0.18895192$

$0.14458551 \quad 0.06550778$

$0.25042096 \quad-0.05226053$

$0.14458551 \quad-0.15504009$

$0.25042096 \quad 0.14015631$

$\begin{array}{lr}-0.15045778 & 0.19745596\end{array}$

$-0.04462234 \quad-0.15578292$

$-0.15045778 \quad-0.15038946$

$-0.04462234 \quad 0.11391505$

$-0.15045778 \quad-0.04004671$

$-0.04462234 \quad 0.00373529$

$-0.15045778 \quad-0.00905190$

$-0.04462234 \quad 0.04534275$

$\begin{array}{ll}1.24855602 & 0.07825708\end{array}$

$1.35439157 \quad-0.06011668$

$1.24855602 \quad-0.08871104$

$\begin{array}{ll}1.35439157 & 0.06694436\end{array}$

$1.24855602 \quad-0.07191649$

$\begin{array}{ll}1.35439157 & 0.05487743\end{array}$

$1.24855602 \quad 0.08603803$

$1.35439157 \quad-0.06396516$

$\begin{array}{lr}0.73230141 & 0.08632701\end{array}$

$0.83813679 \quad-0.05822815$

$0.73230141 \quad-0.03072139$

$0.83813679 \quad 0.01409045$

$0.73230141 \quad-0.06821090$

$0.83813679 \quad 0.03566124$

$0.73230141 \quad 0.00552957$

$\begin{array}{ll}0.83813679 & 0.01316918\end{array}$

$\begin{array}{ll}-1.01531279 & 0.03892572\end{array}$

$-0.90947729-0.03215195$

$-1.01531279 \quad-0.05968037$ 
1.04285800

1. 14869356

1. 14869356

1.14869356

1. 14869356

$-1.76707089$

$-1.76707089$

$-1.76707089$

$-1.76707089$

$-1.66123557$

$-1.66123557$

$-1.66123557$

$-1.66123557$

1. 57582414

1. 57582414

1.57582414

1.57582414

1.68165958

1.68165958

1.68165958

$-1.11259675$

$-1.11259675$

$-1.11259675$

$-1.11259675$

$-1.00676131$

$-1.00676131$

$-1.00676131$

64

$-1.00676131$
1. 68165958

0.28137359

0.17553815

0.17553815

0.28137359

0.28137359

$-0.64616346$

$-0.64616346$

$-0.54032809$

$-0.54032809$

$-0.64616346$

$-0.64616346$

$-0.54032809$

$-0.54032809$

0.13101105

0.13101105

0.23684651

0.23684651

0.13101105

0.13101105

0.23684651

0.23684651

$-1.22435784$

$-1.22435784$

$-1.11852252$

$-1.11852252$

$-1.22435784$

$-1.22435784$

$-1.11852252$

$-1.11852252$

0 .

0.0021

$-0.0084$

1.67341614

1.67341614

1.67341614

1.67341614

1. 77925158

1.77925158

1.77925158

1. 77925158

$-1.26006997$

$-1.26006997$

$-1.26006997$

$-1.26006997$

$-1.15423441$

$-1.15423441$

$-1.15423441$

$-1.15423441$

1.17254710

1. 17254710

1.17254710

1. 17254710

1.27838266

1. 27838266

1. 27838266

1.27838266

$-1.80632699$

$-1.80632699$

$-1.80632699$

$-1.80632699$

$-1.70049155$

$-1.70049155$

$-1.70049155$

$-1.70049155$

1. 04285800

1.04285800

1. 04285800
\# Energy 2->4

$\begin{array}{rr}-0.0217 & 0.0097 \\ 0.0039 & 0.0226\end{array}$

$-0.20954360$

$-0.20954360$

$-0.10370816$

$-0.10370816$

$-0.20954360$

$-0.20954360$

$-0.10370816$

$-0.10370816$

0.53541940

0.53541940

0.64125490

0.64125490

0.53541940

0.53541940

0.64125490

0.64125490

0.01399621

0.01399621

0.11983165

0.11983165

0.01399621

0.01399621

0.11983165

0.11983165

1.19734502

1.19734502

1.30318046

1. 30318046

1.19734502

1.19734502

1. 30318046

1. 30318046

0.17553815

0.17553815

0.28137359

$$
\begin{array}{lr}
-0.90947729 & 0.05171450 \\
-1.01531279 & -0.04073035 \\
-0.90947729 & 0.02976661 \\
-1.01531279 & 0.07619496 \\
-0.90947729 & -0.06579690 \\
-0.62219280 & -0.03841930 \\
-0.51635736 & 0.07535873 \\
-0.62219280 & 0.08893600 \\
-0.51635736 & -0.11897213 \\
-0.62219280 & 0.02570292 \\
-0.51635736 & -0.06037597 \\
-0.62219280 & -0.08649631 \\
-0.51635736 & 0.11013579 \\
-1.87711871 & -0.00408397 \\
-1.77128315 & 0.00547712 \\
-1.87711871 & 0.00833146 \\
-1.77128315 & -0.01234997 \\
-1.87711871 & 0.00108845 \\
-1.77128315 & -0.00123221 \\
-1.87711871 & -0.00385981 \\
-1.77128315 & 0.00582735 \\
-1.14041448 & -0.00722324 \\
-1.03457904 & -0.00571293 \\
-1.14041448 & 0.00645582 \\
-1.03457904 & 0.01292190 \\
-1.14041448 & 0.00548703 \\
-1.03457904 & 0.00376130 \\
-1.14041448 & -0.00600823 \\
-1.03457904 & -0.00773998
\end{array}
$$

$$
\begin{gathered}
1.000 \text { \# Electr. mom., scale-fac } \\
\text { \# Magnetic mom. }
\end{gathered}
$$

$0.14458551 \quad-0.04145377$

$0.25042096 \quad-0.06587617$

$0.14458551 \quad 0.88608980$

$0.25042096 \quad-0.77266872$

$0.14458551 \quad 0.12514400$

$0.25042096 \quad-0.05494818$

$0.14458551 \quad-0.78862590$

0.25042096

$-0.15045778$

$-0.04462234$

$-0.15045778$

$-0.04462234$

$-0.15045778$

$-0.04462234$

$-0.15045778$

$-0.04462234$

1. 24855602

1.35439157

1. 24855602

1.35439157

1. 24855602

1. 35439157

1. 24855602

1.35439157

0.73230141

0.83813679

0.73230141

0.83813679

0.73230141

0.83813679

0.73230141

0.83813679

$-1.01531279$

$-0.90947729$

$-1.01531279$

\section{S-418}

0.72395641

$-0.32342958$

0.04646447

0.20685945

0.01495228

0.36090457

$-0.02508100$

$-0.21762376$

$-0.05577683$

0.44791701

$-0.34947440$

$-0.43707246$

0.33005574

$-0.35912523$

0.27554625

0.37050813

$-0.26961851$

$-0.02990311$

0.04091497

$-0.00234870$

$-0.00812364$

$-0.02182557$

0.00498614

0.04655155

$-0.03148310$

$-0.55846804$

0.70127982

0.29801452 
1.04285800

1. 14869356

1. 14869356

1.14869356

1. 14869356

$-1.76707089$

$-1.76707089$

$-1.76707089$

$-1.76707089$

$-1.66123557$

$-1.66123557$

$-1.66123557$

$-1.66123557$

1. 57582414

1. 57582414

1.57582414

1.57582414

1. 68165958

1.68165958

1. 68165958

1.68165958

$-1.11259675$

$-1.11259675$

$-1.11259675$

$-1.11259675$

$-1.00676131$

$-1.00676131$

$-1.00676131$

64

$-1.00676131$

$-0.0036 \quad 0$.

$-0.1223$

1.67341614

1.67341614

1.67341614

1.67341614

1. 77925158

1. 77925158

1.77925158

1.77925158

$-1.26006997$

$-1.26006997$

$-1.26006997$

$-1.26006997$

$-1.15423441$

$-1.15423441$

$-1.15423441$

$-1.15423441$

1. 17254710

1. 17254710

1. 17254710

1.17254710

1.27838266

1. 27838266

1. 27838266

1. 27838266

$-1.80632699$

$-1.80632699$

$-1.80632699$

$-1.80632699$

$-1.70049155$

$-1.70049155$

$-1.70049155$

$-1.70049155$

1. 04285800

1. 04285800

1. 04285800
0.28137359

0.17553815

0.17553815

0.28137359

0.28137359

$-0.64616346$

$-0.64616346$

$-0.54032809$

$-0.54032809$

$-0.64616346$

$-0.64616346$

$-0.54032809$

$-0.54032809$

0.13101105

0.13101105

0.23684651

0.23684651

0.13101105

0.13101105

0.23684651

0.23684651

$-1.22435784$

$-1.22435784$

$-1.11852252$

$-1.11852252$

$-1.22435784$

$-1.22435784$

$-1.11852252$

$-1.11852252$

. \# Energy 2->5

$\begin{array}{ll}0.0790 & 0.1608 \\ 0.0974 & 0.3290\end{array}$

$-0.20954360$

$-0.20954360$

$-0.10370816$

$-0.10370816$

$-0.20954360$

$-0.20954360$

$-0.10370816$

$-0.10370816$

0.53541940

0.53541940

0.64125490

0.64125490

0.53541940

0.53541940

0.64125490

0.64125490

0.01399621

0.01399621

0.11983165

0.11983165

0.01399621

0.01399621

0.11983165

0.11983165

1.19734502

1.19734502

1. 30318046

1. 30318046

1.19734502

1.19734502

1. 30318046

1. 30318046

0.17553815

0.17553815

0.28137359
$-0.90947729$

$-1.01531279$

$-0.90947729$

$-1.01531279$

$-0.90947729$

$-0.62219280$

$-0.51635736$

$-0.62219280$

$-0.51635736$

$-0.62219280$

$-0.51635736$

$-0.62219280$

$-0.51635736$

$-1.87711871$

$-1.77128315$

$-1.87711871$

$-1.77128315$

$-1.87711871$

$-1.77128315$

$-1.87711871$

$-1.77128315$

$-1.14041448$

$-1.03457904$

$-1.14041448$

$-1.03457904$

$-1.14041448$

$-1.03457904$

$-1.14041448$

$-1.03457904$

$-1.03457904$
1.000 \# Electr. mom., scale-fac \# Magnetic mom.

0.14458551

0.25042096

0.14458551

0.25042096

0.14458551

0.25042096

0.14458551

0.25042096

$-0.15045778$

$-0.04462234$

$-0.15045778$

$-0.04462234$

$-0.15045778$

$-0.04462234$

$-0.15045778$

$-0.04462234$

1. 24855602

1. 35439157

1. 24855602

1. 35439157

1. 24855602

1. 35439157

1. 24855602

1.35439157

0.73230141

0.83813679

0.73230141

0.83813679

0.73230141

0.83813679

0.73230141

0.83813679

$-1.01531279$

$-0.90947729$

$-1.01531279$
$-0.44536772$

0.74458778

$-0.95299405$

$-0.32009444$

0.52041209

$-0.49268258$

0.13147929

0.25058264

0.09056347

0.69975173

$-0.23958306$

$-0.38806069$

$-0.03643463$

0.01977393

$-0.02362566$

0.03499604

$-0.06173201$

$-0.02990799$

0.03722514

$-0.00637920$

0.01979622

0.11555921

$-0.08537463$

$-0.08270732$

$-0.00115580$

$-0.12436109$

0.12366217

0.12088583

$-0.08603306$

5.04996157

$-3.44065905$

$-14.05941963$

12.20673656

$-5.08874035$

12.17746544

$-10.89394855$

$-0.04453392$

1. 14295840

0.19377790

$-1.10019481$

$-1.65694785$

0.41652989

1.65256143

$-0.71196073$

$-3.40965033$

2. 65076876

3.56751585

$-2.66892147$

2.66882277

$-2.02997684$

$-3.07767820$

2. 20506501

$-1.14114165$

0.66786087

0.55205184

$-0.23794343$

1.30601478

$-0.70877701$

$-0.58395422$

0.18552960

1.82100809

$-2.72578430$

0.41606432
3. 94472742 
1.04285800

1. 14869356

1. 14869356

1. 14869356

1. 14869356

$-1.76707089$

$-1.76707089$

$-1.76707089$

$-1.76707089$

$-1.66123557$

$-1.66123557$

$-1.66123557$

$-1.66123557$

1. 57582414

1. 57582414

1.57582414

1.57582414

1. 68165958

1.68165958

1. 68165958

1.68165958

$-1.11259675$

$-1.11259675$

$-1.11259675$

$-1.11259675$

$-1.00676131$

$-1.00676131$

$-1.00676131$

64

$-1.00676131$

-0.00710 .

$-0.0162$

1.67341614

1.67341614

1.67341614

1.67341614

1. 77925158

1. 77925158

1.77925158

1.77925158

$-1.26006997$

$-1.26006997$

$-1.26006997$

$-1.26006997$

$-1.15423441$

$-1.15423441$

$-1.15423441$

$-1.15423441$

1. 17254710

1. 17254710

1.17254710

1. 17254710

1.27838266

1. 27838266

1. 27838266

1. 27838266

$-1.80632699$

$-1.80632699$

$-1.80632699$

$-1.80632699$

$-1.70049155$

$-1.70049155$

$-1.70049155$

$-1.70049155$

1. 04285800

1. 04285800

1. 04285800
0.28137359

0.17553815

0.17553815

0.28137359

0.28137359

$-0.64616346$

$-0.64616346$

$-0.54032809$

$-0.54032809$

$-0.64616346$

$-0.64616346$

$-0.54032809$

$-0.54032809$

0.13101105

0.13101105

0.23684651

0.23684651

0.13101105

0.13101105

0.23684651

0.23684651

$-1.22435784$

$-1.22435784$

$-1.11852252$

$-1.11852252$

$-1.22435784$

$-1.22435784$

$-1.11852252$

$-1.11852252$

\# Energy 2->6

$\begin{array}{rr}-0.0019 & -0.0406 \\ 0.0237 & 0.0368\end{array}$

$-0.20954360$

$-0.20954360$

$-0.10370816$

$-0.10370816$

$-0.20954360$

$-0.20954360$

$-0.10370816$

$-0.10370816$

0.53541940

0.53541940

0.64125490

0.64125490

0.53541940

0.53541940

0.64125490

0.64125490

0.01399621

0.01399621

0.11983165

0.11983165

0.01399621

0.01399621

0.11983165

0.11983165

1.19734502

1.19734502

1. 30318046

1. 30318046

1.19734502

1.19734502

1. 30318046

1. 30318046

0.17553815

0.17553815

0.28137359
$-0.90947729$

$-1.01531279$

$-0.90947729$

$-1.01531279$

$-0.90947729$

$-0.62219280$

$-0.51635736$

$-0.62219280$

$-0.51635736$

$-0.62219280$

$-0.51635736$

$-0.62219280$

$-0.51635736$

$-1.87711871$

$-1.77128315$

$-1.87711871$

$-1.77128315$

$-1.87711871$

$-1.77128315$

$-1.87711871$

$-1.77128315$

$-1.14041448$

$-1.03457904$

$-1.14041448$

$-1.03457904$

$-1.14041448$

$-1.03457904$

$-1.14041448$

$-1.03457904$
0.64646518

$-2.62636757$

4.16611576

$-1.19108808$

$-0.37081668$

1.30070925

$-0.28021768$

$-0.86291867$

$-0.16580874$

$-1.89216638$

0.47066173

1. 22223687

0.19189753

0.18013695

$-0.27673161$

$-0.60503751$

0.98118162

0.01636840

0.00244439

0.23424533

$-0.44006073$

$-0.62260556$

0.62792605

0.54990655

$-0.41068515$

0.62656754

$-0.71360195$

$-0.64599919$

0.64205420
1.000 \# Electr. mom., scale-fac \# Magnetic mom.

0.14458551 $-0.87175155$

$0.25042096 \quad 1.02935910$

0.14458551

0.25042096

0.14458551

0.25042096

0.14458551

0.25042096

$-0.15045778$

$-0.04462234$

$-0.15045778$

$-0.04462234$

$-0.15045778$

$-0.04462234$

$-0.15045778$

$-0.04462234$

1. 24855602

1. 35439157

1. 24855602

1. 35439157

1. 24855602

1. 35439157

1. 24855602

1.35439157

0.73230141

0.83813679

0.73230141

0.83813679

0.73230141

0.83813679

0.73230141

0.83813679

$-1.01531279$

$-0.90947729$

$-1.01531279$

$-0.03310732$

$-0.12020415$

0.52207947

$-0.63781035$

0.21072957

$-0.11895607$

0.20346399

0.63265413

0.16924523

$-0.93238688$

0.46841154

$-1.41552198$

$-0.94442344$

1. 82484376

$-0.65754437$

0.48304760

0.58001930

$-0.41312751$

0.57575566

$-0.41065699$

$-0.51034951$

0.34674418

0.83448559

$-0.64733326$

$-0.48206687$

0.35830572

$-0.70439029$

0.48254675

0.31969598

$-0.17420141$

0.69717121

$-0.72840929$

$-0.45387137$ 
1.04285800

1. 14869356

1. 14869356

1.14869356

1. 14869356

$-1.76707089$

$-1.76707089$

$-1.76707089$

$-1.76707089$

$-1.66123557$

$-1.66123557$

$-1.66123557$

$-1.66123557$

1. 57582414

1. 57582414

1.57582414

1.57582414

1. 68165958

1.68165958

1. 68165958

1.68165958

$-1.11259675$

$-1.11259675$

$-1.11259675$

$-1.11259675$

$-1.00676131$

$-1.00676131$

$-1.00676131$

64

$-1.00676131$
0.28137359

0.17553815

0.17553815

0.28137359

0.28137359

$-0.64616346$

$-0.64616346$

$-0.54032809$

$-0.54032809$

$-0.64616346$

$-0.64616346$

$-0.54032809$

$-0.54032809$

0.13101105

0.13101105

0.23684651

0.23684651

0.13101105

0.13101105

0.23684651

0.23684651

$-1.22435784$

$-1.22435784$

$-1.11852252$

$-1.11852252$

$-1.22435784$

$-1.22435784$

$-1.11852252$

$-1.11852252$

0 .

\# Energy 2->7

$\begin{array}{rrr}0.0367 & -0.1387 & -0.0798 \\ -0.0530 & 0.1043 & 0.0716\end{array}$

1. 67341614

$-0.20954360$

1.67341614

1.67341614

1.67341614

1. 77925158

1.77925158

1.77925158

1. 77925158

$-1.26006997$

$-1.26006997$

$-1.26006997$

$-1.26006997$

$-1.15423441$

$-1.15423441$

$-1.15423441$

$-1.15423441$

1.17254710

1. 17254710

1. 17254710

1. 17254710

1.27838266

1. 27838266

1. 27838266

1. 27838266

$-1.80632699$

$-1.80632699$

$-1.80632699$

$-1.80632699$

$-1.70049155$

$-1.70049155$

$-1.70049155$

$-1.70049155$

1. 04285800

1. 04285800

1. 04285800

$-0.20954360$

$-0.10370816$

$-0.10370816$

$-0.20954360$

$-0.20954360$

$-0.10370816$

$-0.10370816$

0.53541940

0.53541940

0.64125490

0.64125490

0.53541940

0.53541940

0.64125490

0.64125490

0.01399621

0.01399621

0.11983165

0.11983165

0.01399621

0.01399621

0.11983165

0.11983165

1.19734502

1.19734502

1. 30318046

1. 30318046

1. 19734502

1.19734502

1. 30318046

1. 30318046

0.17553815

0.17553815

0.28137359

\section{S-421}

0.48095611

$-1.23971379$

1. 40993965

0.82081848

$-0.97166926$

0.56246924

0.36381489

0.21023564

$-1.05154383$

$-1.03141069$

$-0.10355589$

0.06903673

0.93633109

$-0.00311349$

0.01284311

$-0.07859901$

0.10533524

$-0.00358815$

$-0.00289809$

0.06447587

$-0.08386347$

$-0.10743763$

$-0.08632841$

$-0.00736374$

0.35973704

0.15823472

$-0.05302746$

$-0.11955548$

$-0.09300555$

$$
\begin{gathered}
1.000 \text { \# Electr. mom., scale-fac } \\
\text { \# Magnetic mom. }
\end{gathered}
$$

0.14458551

0.25042096

0.14458551

0.25042096

0.14458551

0.25042096

0.14458551

0.25042096

$-0.15045778$

$-0.04462234$

$-0.15045778$

$-0.04462234$

$-0.15045778$

$-0.04462234$

$-0.15045778$

$-0.04462234$

1. 24855602

1. 35439157

1. 24855602

1. 35439157

1. 24855602

1. 35439157

1. 24855602

1.35439157

0.73230141

0.83813679

0.73230141

0.83813679

0.73230141

0.83813679

0.73230141

0.83813679

$-1.01531279$

$-0.90947729$

$-1.01531279$
1.45694745

$-0.98841965$

$-5.08862114$

4.51095676

$-1.62634206$

1. 33594584

4. 45329714

$-4.10812712$

2.80915546

$-0.77042711$

$-1.36663067$

$-0.62608671$

$-1.37148261$

$-0.77314448$

$-0.16801977$

2.30250978

$-0.95800900$

0.78834975

0.91626942

$-0.71561515$

0.66442758

$-0.54266840$

$-0.72564399$

0.53567100

3.46422458

$-2.74181175$

$-2.41136217$

1.86530006

$-2.97351503$

2. 16297221

1. 84122682

$-1.24324477$

2. 95855832

$-2.91898179$

$-1.51475489$ 
1. 04285800

1. 14869356

1. 14869356

1.14869356

1. 14869356

$-1.76707089$

$-1.76707089$

$-1.76707089$

$-1.76707089$

$-1.66123557$

$-1.66123557$

$-1.66123557$

$-1.66123557$

1. 57582414

1. 57582414

1.57582414

1.57582414

1. 68165958

1.68165958

1.68165958

1.68165958

$-1.11259675$

$-1.11259675$

$-1.11259675$

$-1.11259675$

$-1.00676131$

$-1.00676131$

$-1.00676131$

64

$-1.00676131$

$-0.2567$

$-0.0206$

1. 67341614

1.67341614

1.67341614

1.67341614

1. 77925158

1.77925158

1.77925158

1.77925158

$-1.26006997$

$-1.26006997$

$-1.26006997$

$-1.26006997$

$-1.15423441$

$-1.15423441$

$-1.15423441$

$-1.15423441$

1. 17254710

1. 17254710

1. 17254710

1. 17254710

1.27838266

1. 27838266

1. 27838266

1. 27838266

$-1.80632699$

$-1.80632699$

$-1.80632699$

$-1.80632699$

$-1.70049155$

$-1.70049155$

$-1.70049155$

$-1.70049155$

1. 04285800

1.04285800

1. 04285800
0.28137359

0.17553815

0.17553815

0.28137359

0.28137359

$-0.64616346$

$-0.64616346$

$-0.54032809$

$-0.54032809$

$-0.64616346$

$-0.64616346$

$-0.54032809$

$-0.54032809$

0.13101105

0.13101105

0.23684651

0.23684651

0.13101105

0.13101105

0.23684651

0.23684651

$-1.22435784$

$-1.22435784$

$-1.11852252$

$-1.11852252$

$-1.22435784$

$-1.22435784$

$-1.11852252$

$-1.11852252$

. \# Energy 2->8

$\begin{array}{ll}0.2465 & 0.1347 \\ 0.0637 & 0.0255\end{array}$

$-0.20954360$

$-0.20954360$

$-0.10370816$

$-0.10370816$

$-0.20954360$

$-0.20954360$

$-0.10370816$

$-0.10370816$

0.53541940

0.53541940

0.64125490

0.64125490

0.53541940

0.53541940

0.64125490

0.64125490

0.01399621

0.01399621

0.11983165

0.11983165

0.01399621

0.01399621

0.11983165

0.11983165

1.19734502

1.19734502

1. 30318046

1. 30318046

1.19734502

1.19734502

1. 30318046

1. 30318046

0.17553815

0.17553815

0.28137359
$-0.90947729$

$-1.01531279$

$-0.90947729$

$-1.01531279$

$-0.90947729$

$-0.62219280$

$-0.51635736$

$-0.62219280$

$-0.51635736$

$-0.62219280$

$-0.51635736$

$-0.62219280$

$-0.51635736$

$-1.87711871$

$-1.77128315$

$-1.87711871$

$-1.77128315$

$-1.87711871$

$-1.77128315$

$-1.87711871$

$-1.77128315$

$-1.14041448$

$-1.03457904$

$-1.14041448$

$-1.03457904$

$-1.14041448$

$-1.03457904$

$-1.14041448$

$-1.03457904$
1. 48541117

$-3.66012478$

3. 96446490

1. 36131001

$-1.64648151$

0.80362105

1.47689986

1. 15046573

$-3.13012791$

$-2.20382929$

$-0.53458923$

$-0.26194081$

2. 56842375

0.10756578

$-0.19433179$

$-0.35740504$

0.62012380

$-0.07863330$

0.14764789

0.20175283

$-0.38479188$

$-0.46866870$

$-0.02283408$

0.24639986

0.65698117

0.52444452

$-0.27391034$

$-0.47457567$

$-0.05617163$
$0.14458551 \quad 2.13307667$

$0.25042096 \quad-1.91653836$

$0.14458551 \quad-3.34029651$

$0.25042096 \quad 3.07812572$

$0.14458551 \quad-2.32211852$

$0.25042096 \quad 2.18680978$

$0.14458551 \quad 3.25511074$

0.25042096

$-0.15045778$

$-0.04462234$

$-0.15045778$

$-0.04462234$

$-0.15045778$

$-0.04462234$

$-0.15045778$

$-0.04462234$

1. 24855602

1.35439157

1. 24855602

1.35439157

1. 24855602

1. 35439157

1. 24855602

1.35439157

0.73230141

0.83813679

0.73230141

0.83813679

0.73230141

0.83813679

0.73230141

0.83813679

$-1.01531279$

$-0.90947729$

$-1.01531279$

$-3.09540915$

4.91322803

$-5.52115107$

$-5.08876276$

6.01095343

$-3.90912628$

4.16059256

3.87413430

$-4.40350723$

0.77595848

$-0.48656318$

$-0.88083124$

0.59060323

$-0.97098935$

0.63590473

0.99729854

$-0.68665272$

0.05963368

0.08512998

0.50791854

$-0.57578951$

0.05099074

$-0.24605392$

$-0.64591861$

0.75131285

2.07707667

$-2.28368688$

$-1.52369237$ 
1.04285800

1. 14869356

1. 14869356

1. 14869356

1. 14869356

$-1.76707089$

$-1.76707089$

$-1.76707089$

$-1.76707089$

$-1.66123557$

$-1.66123557$

$-1.66123557$

$-1.66123557$

1. 57582414

1. 57582414

1.57582414

1.57582414

1. 68165958

1.68165958

1. 68165958

1.68165958

$-1.11259675$

$-1.11259675$

$-1.11259675$

$-1.11259675$

$-1.00676131$

$-1.00676131$

$-1.00676131$

64

$-1.00676131$

0.0178

1.67341614

1.67341614

1.67341614

1.67341614

1. 77925158

1. 77925158

1.77925158

1.77925158

$-1.26006997$

$-1.26006997$

$-1.26006997$

$-1.26006997$

$-1.15423441$

$-1.15423441$

$-1.15423441$

$-1.15423441$

1.17254710

1. 17254710

1. 17254710

1. 17254710

1.27838266

1. 27838266

1. 27838266

1.27838266

$-1.80632699$

$-1.80632699$

$-1.80632699$

$-1.80632699$

$-1.70049155$

$-1.70049155$

$-1.70049155$

$-1.70049155$

1. 04285800

1.04285800

1. 04285800
0.28137359

0.17553815

0.17553815

0.28137359

0.28137359

$-0.64616346$

$-0.64616346$

$-0.54032809$

$-0.54032809$

$-0.64616346$

$-0.64616346$

$-0.54032809$

$-0.54032809$

0.13101105

0.13101105

0.23684651

0.23684651

0.13101105

0.13101105

0.23684651

0.23684651

$-1.22435784$

$-1.22435784$

$-1.11852252$

$-1.11852252$

$-1.22435784$

$-1.22435784$

$-1.11852252$

$-1.11852252$

. \# Energy 2->9

$0.1712 \quad-0.0644$ $-0.0484 \quad-0.0220$

$-0.20954360$

$-0.20954360$

$-0.10370816$

$-0.10370816$

$-0.20954360$

$-0.20954360$

$-0.10370816$

$-0.10370816$

0.53541940

0.53541940

0.64125490

0.64125490

0.53541940

0.53541940

0.64125490

0.64125490

0.01399621

0.01399621

0.11983165

0.11983165

0.01399621

0.01399621

0.11983165

0.11983165

1.19734502

1.19734502

1. 30318046

1. 30318046

1.19734502

1.19734502

1. 30318046

1. 30318046

0.17553815

0.17553815

0.28137359
$-0.90947729$

$-1.01531279$

$-0.90947729$

$-1.01531279$

$-0.90947729$

$-0.62219280$

$-0.51635736$

$-0.62219280$

$-0.51635736$

$-0.62219280$

$-0.51635736$

$-0.62219280$

$-0.51635736$

$-1.87711871$

$-1.77128315$

$-1.87711871$

$-1.77128315$

$-1.87711871$

$-1.77128315$

$-1.87711871$

$-1.77128315$

$-1.14041448$

$-1.03457904$

$-1.14041448$

$-1.03457904$

$-1.14041448$

$-1.03457904$

$-1.14041448$

$-1.03457904$

1.000
1.000 \# Electr. mom., scale-fac \# Magnetic mom.

0.14458551

0.25042096

0.14458551

0.25042096

0.14458551

0.25042096

0.14458551

0.25042096

$-0.15045778$

$-0.04462234$

$-0.15045778$

$-0.04462234$

$-0.15045778$

$-0.04462234$

$-0.15045778$

$-0.04462234$

1. 24855602

1. 35439157

1. 24855602

1.35439157

1. 24855602

1. 35439157

1. 24855602

1.35439157

0.73230141

0.83813679

0.73230141

0.83813679

0.73230141

0.83813679

0.73230141

0.83813679

$-1.01531279$

$-0.90947729$

$-1.01531279$
1. 75527108

$-1.99548244$

2. 27908611

1.04007530

$-1.34763765$

0.79968256

0.98464555

0.42816573

$-2.10607171$

$-1.56432784$

$-0.68791372$

$-0.06117955$

2. 10527802

0.04245536

$-0.12229548$

$-0.13916489$

0.30072966

$-0.01139646$

0.07144471

0.04519903

$-0.15821086$

$-0.42244369$

0.16377617

0.18382709

0.37695217

0.45896107

$-0.35344937$

$-0.36819762$

0.05545117$$
-1.80735040
$$

2.18693066

0.63811183

$-1.01222146$

0.87604558

$-1.18245363$

0.14209892

0.12465099

$-1.60632098$

1.83078206

1.08864748

$-1.08748496$

2. 07410073

$-2.53701854$

$-1.73122847$

1. 94837439

$-0.61221749$

0.39710096

0.48145056

$-0.28091937$

0.47177413

$-0.27392012$

$-0.33942056$

0.15227076

$-1.00469542$

0.82834888

0.97432113

$-0.80090284$

0.89309406

$-0.73285460$

$-0.87330556$

0.71488482

$-0.41940176$

0.32001081

0.14431772 


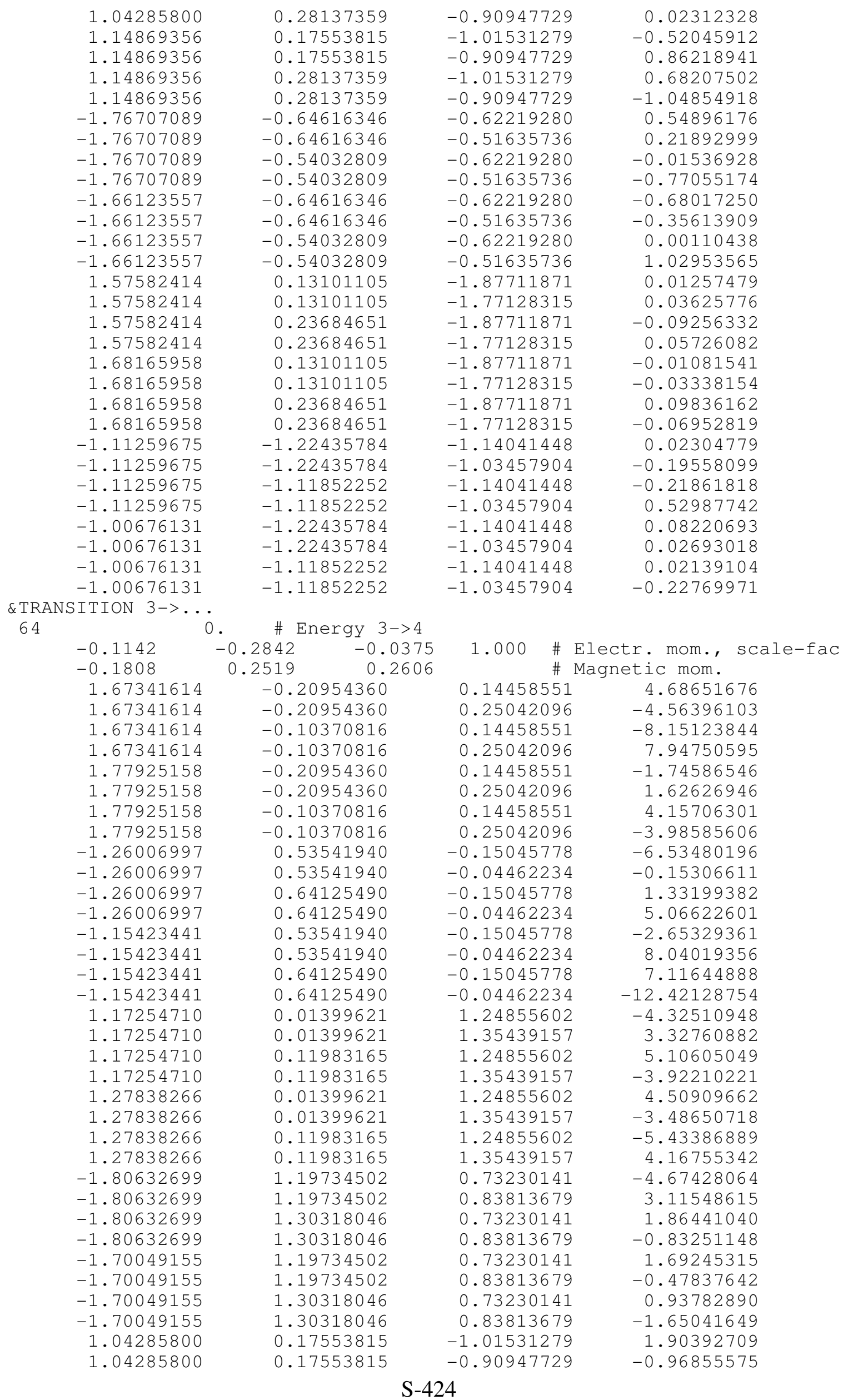


1.04285800

1. 04285800

1. 14869356

1.14869356

1. 14869356

1.14869356

$-1.76707089$

$-1.76707089$

$-1.76707089$

$-1.76707089$

$-1.66123557$

$-1.66123557$

$-1.66123557$

$-1.66123557$

1. 57582414

1. 57582414

1.57582414

1.57582414

1.68165958

1.68165958

1.68165958

1.68165958

$-1.11259675$

$-1.11259675$

$-1.11259675$

$-1.11259675$

$-1.00676131$

$-1.00676131$

$-1.00676131$

64

$-1.00676131$
0.28137359

0.28137359

0.17553815

0.17553815

0.28137359

0.28137359

$-0.64616346$

$-0.64616346$

$-0.54032809$

$-0.54032809$

$-0.64616346$

$-0.64616346$

$-0.54032809$

$-0.54032809$

0.13101105

0.13101105

0.23684651

0.23684651

0.13101105

0.13101105

0.23684651

0.23684651

$-1.22435784$

$-1.22435784$

$-1.11852252$

$-1.11852252$

$-1.22435784$

$-1.22435784$

$-1.11852252$

$-1.11852252$

$$
\text { 0. \# Energy 3->5 }
$$

$\begin{array}{lll}0.0769 & -0.0229 & -0.0817\end{array}$

$0.0531-0.0183 \quad-0.1215$

1. 67341614

1.67341614

1.67341614

1.67341614

1.77925158

1.77925158

1.77925158

1. 77925158

$-1.26006997$

$-1.26006997$

$-1.26006997$

$-1.26006997$

$-1.15423441$

$-1.15423441$

$-1.15423441$

$-1.15423441$

1. 17254710

1. 17254710

1.17254710

1.17254710

1. 27838266

1. 27838266

1.27838266

1. 27838266

$-1.80632699$

$-1.80632699$

$-1.80632699$

$-1.80632699$

$-1.70049155$

$-1.70049155$

$-1.70049155$

$-1.70049155$

1.04285800

1. 04285800
$-0.20954360$

$-0.20954360$

$-0.10370816$

$-0.10370816$

$-0.20954360$

$-0.20954360$

$-0.10370816$

$-0.10370816$

0.53541940

0.53541940

0.64125490

0.64125490

0.53541940

0.53541940

0.64125490

0.64125490

0.01399621

0.01399621

0.11983165

0.11983165

0.01399621

0.01399621

0.11983165

0.11983165

1.19734502

1.19734502

1. 30318046

1. 30318046

1.19734502

1. 19734502

1. 30318046

1.30318046

0.17553815

0.17553815
-1.01531279
-0.90947729
-1.01531279
-0.90947729
-1.01531279
-0.90947729
-0.62219280
-0.51635736
-0.62219280
-0.51635736
-0.62219280
-0.51635736
-0.62219280
-0.51635736
-1.87711871
-1.77128315
-1.87711871
-1.77128315
-1.87711871
-1.77128315
-1.87711871
-1.77128315
-1.14041448
-1.03457904
-1.14041448
-1.03457904
-1.14041448
-1.03457904
-1.14041448
-1.03457904

$-0.28704309$

$-0.83300126$

$-1.87589598$

1.07433367

$-0.52510154$

1. 51223719

3. 18871665

$-4.12707472$

$-5.42871284$

5.95041656

$-1.46841288$

1. 74813962

4.02654171

$-3.52216816$

0.42367959

$-0.65854210$

$-0.58521295$

0.99823791

$-0.43350697$

0.64715230

0.44573560

$-0.77297431$

0.66275847

0.03136904

$-0.63012046$

$-0.36552429$

$-0.43312660$

$-0.06408532$

0.48797783

0.19767343
1.000 \# Electr. mom., scale-fac \# Magnetic mom.

0.14458551

0.25042096

0.14458551

0.25042096

0.14458551

0.25042096

0.14458551

0.25042096

$-0.15045778$

$-0.04462234$

$-0.15045778$

$-0.04462234$

$-0.15045778$

$-0.04462234$

$-0.15045778$

$-0.04462234$

1. 24855602

1. 35439157

1. 24855602

1. 35439157

1. 24855602

1. 35439157

1. 24855602

1. 35439157

0.73230141

0.83813679

0.73230141

0.83813679

0.73230141

0.83813679

0.73230141

0.83813679

$-1.01531279$

$-0.90947729$
$-1.13779163$

0.67274857

1.07686055

$-0.51594794$

1. 25200021

$-0.84031719$

$-1.14359140$

0.65854037

$-1.25209582$

0.90648913

1.13365841

$-0.87430131$

$-0.65733570$

0.91397774

0.80220366

$-1.04513896$

$-1.15737176$

0.92681420

1. 21247399

$-0.95990950$

1. 05477107

$-0.83256197$

$-1.12205577$

0.87107950

$-0.27169791$

0.11736470

$-0.20439637$

0.23774676

0.27089036

$-0.03419323$

0.34689197

$-0.42153990$

0.16949753

$-0.17983168$ 
1.04285800

1. 04285800

1. 14869356

1.14869356

1. 14869356

1.14869356

$-1.76707089$

$-1.76707089$

$-1.76707089$

$-1.76707089$

$-1.66123557$

$-1.66123557$

$-1.66123557$

$-1.66123557$

1. 57582414

1.57582414

1.57582414

1.57582414

1.68165958

1.68165958

1.68165958

1.68165958

$-1.11259675$

$-1.11259675$

$-1.11259675$

$-1.11259675$

$-1.00676131$

$-1.00676131$

$-1.00676131$

64

$-1.00676131$

$-0.7820$

0.0436

1.67341614

1.67341614

1.67341614

1.67341614

1. 77925158

1.77925158

1. 77925158

1. 77925158

$-1.26006997$

$-1.26006997$

$-1.26006997$

$-1.26006997$

$-1.15423441$

$-1.15423441$

$-1.15423441$

$-1.15423441$

1. 17254710

1. 17254710

1.17254710

1.17254710

1. 27838266

1. 27838266

1.27838266

1. 27838266

$-1.80632699$

$-1.80632699$

$-1.80632699$

$-1.80632699$

$-1.70049155$

$-1.70049155$

$-1.70049155$

$-1.70049155$

1.04285800

1. 04285800
0.28137359

0.28137359

0.17553815

0.17553815

0.28137359

0.28137359

$-0.64616346$

$-0.64616346$

$-0.54032809$

$-0.54032809$

$-0.64616346$

$-0.64616346$

$-0.54032809$

$-0.54032809$

0.13101105

0.13101105

0.23684651

0.23684651

0.13101105

0.13101105

0.23684651

0.23684651

$-1.22435784$

$-1.22435784$

$-1.11852252$

$-1.11852252$

$-1.22435784$

$-1.22435784$

$-1.11852252$

$-1.11852252$

0 . \# Energy 3->6

$$
\begin{array}{ll}
0.2267 & 0.1899
\end{array}
$$

$-0.1911 \quad-0.1453$

$-0.20954360$

$-0.20954360$

$-0.10370816$

$-0.10370816$

$-0.20954360$

$-0.20954360$

$-0.10370816$

$-0.10370816$

0.53541940

0.53541940

0.64125490

0.64125490

0.53541940

0.53541940

0.64125490

0.64125490

0.01399621

0.01399621

0.11983165

0.11983165

0.01399621

0.01399621

0.11983165

0.11983165

1.19734502

1.19734502

1.30318046

1.30318046

1.19734502

1. 19734502

1.30318046

1.30318046

0.17553815

0.17553815
$-1.01531279$

$-0.90947729$

$-1.01531279$

$-0.90947729$

$-1.01531279$

$-0.90947729$

$-0.62219280$

$-0.51635736$

$-0.62219280$

$-0.51635736$

$-0.62219280$

$-0.51635736$

$-0.62219280$

$-0.51635736$

$-1.87711871$

$-1.77128315$

$-1.87711871$

$-1.77128315$

$-1.87711871$

$-1.77128315$

$-1.87711871$

$-1.77128315$

$-1.14041448$

$-1.03457904$

$-1.14041448$

$-1.03457904$

$-1.14041448$

$-1.03457904$

$-1.14041448$

$-1.03457904$

$$
\begin{gathered}
1.000 \text { \# Electr. mom., scale-fac } \\
\text { \# Magnetic mom. }
\end{gathered}
$$

0.14458551

0.25042096

0.14458551

0.25042096

0.14458551

0.25042096

0.14458551

0.25042096

$-0.15045778$

$-0.04462234$

$-0.15045778$

$-0.04462234$

$-0.15045778$

$-0.04462234$

$-0.15045778$

$-0.04462234$

1. 24855602

1. 35439157

1. 24855602

1. 35439157

1. 24855602

1. 35439157

1. 24855602

1. 35439157

0.73230141

0.83813679

0.73230141

0.83813679

0.73230141

0.83813679

0.73230141

0.83813679

$-1.01531279$

$-0.90947729$
0.14924948

$-0.15484959$

$-0.09981309$

0.06134797

$-0.17493357$

0.21024592

1.02519023

$-1.05886042$

$-1.41394770$

1.37979734

$-0.97493678$

0.88989615

1. 41390121

$-1.21836591$

$-0.12972589$

0.15838625

0.06749950

$-0.07672599$

0.13567834

$-0.16391867$

$-0.08098925$

0.09268265

$-0.09566343$

0.28789315

0.13246992

$-0.37859422$

0.08509337

$-0.24780586$

$-0.11166654$

0.31753507

7.44860601

$-7.14784288$

$-11.12274361$

10.68651295

$-4.19660044$

3.97941399

6.89512348

$-6.54602671$

$-2.84884787$

1. 38645494

4.16859198

$-2.96483803$

3. 14715171

$-1.35666287$

$-4.05763388$

2. 44631815

$-3.12957501$

2. 45569682

3. 56340432

$-2.78057075$

3.39205432

$-2.67513442$

$-4.03475475$

3.14291167

2.87653685

$-2.39949155$

$-2.88682342$

2. 31114912

$-1.41084278$

1.35159409

1.96761441

$-1.67512810$

$-8.04232693$

8.50811863 
1.04285800

1. 04285800

1. 14869356

1.14869356

1. 14869356

1.14869356

$-1.76707089$

$-1.76707089$

$-1.76707089$

$-1.76707089$

$-1.66123557$

$-1.66123557$

$-1.66123557$

$-1.66123557$

1. 57582414

1. 57582414

1.57582414

1.57582414

1.68165958

1.68165958

1.68165958

1.68165958

$-1.11259675$

$-1.11259675$

$-1.11259675$

$-1.11259675$

$-1.00676131$

$-1.00676131$

$-1.00676131$

64

$-1.00676131$
0.28137359

0.28137359

0.17553815

0.17553815

0.28137359

0.28137359

$-0.64616346$

$-0.64616346$

$-0.54032809$

$-0.54032809$

$-0.64616346$

$-0.64616346$

$-0.54032809$

$-0.54032809$

0.13101105

0.13101105

0.23684651

0.23684651

0.13101105

0.13101105

0.23684651

0.23684651

$-1.22435784$

$-1.22435784$

$-1.11852252$

$-1.11852252$

$-1.22435784$

$-1.22435784$

$-1.11852252$

$-1.11852252$

0 . \# Energy 3->7
$-1.01531279$

$-0.90947729$

$-1.01531279$

$-0.90947729$

$-1.01531279$

$-0.90947729$

$-0.62219280$

$-0.51635736$

$-0.62219280$

$-0.51635736$

$-0.62219280$

$-0.51635736$

$-0.62219280$

$-0.51635736$

$-1.87711871$

$-1.77128315$

$-1.87711871$

$-1.77128315$

$-1.87711871$

$-1.77128315$

$-1.87711871$

$-1.77128315$

$-1.14041448$

$-1.03457904$

$-1.14041448$

$-1.03457904$

$-1.14041448$

$-1.03457904$

$-1.14041448$

$-1.03457904$$$
1.000 \text { \# }
$$

0.14458551

0.25042096

0.14458551

0.25042096

0.14458551

0.25042096

0.14458551

0.25042096

$-0.15045778$

$-0.04462234$

$-0.15045778$

$-0.04462234$

$-0.15045778$

$-0.04462234$

$-0.15045778$

$-0.04462234$

1. 24855602

1. 35439157

1. 24855602

1. 35439157

1. 24855602

1. 35439157

1. 24855602

1. 35439157

0.73230141

0.83813679

0.73230141

0.83813679

0.73230141

0.83813679

0.73230141

0.83813679

$-1.01531279$

$-0.90947729$
7.94365358

$-8.61129856$

8.94573116

$-9.40730953$

$-8.62258244$

9.31958294

0.40725416

$-2.19378638$

$-1.68437338$

3. 30888724

0.95912081

1. 22745430

0.57517725

$-2.49119067$

0.91614050

$-1.23107815$

$-0.94069660$

1. 21568155

$-0.74256712$

0.96517926

0.78794402

$-0.99061048$

0.33206877

$-0.06501504$

$-0.04403423$

$-0.54905581$

$-0.49645880$

0.42142943

0.39535794

$-0.10201522$
Electr. mom., scale-fac Magnetic mom.
4. 56566668
$-3.32493186$
$-5.21138430$
3.68531775
$-4.40398502$
3. 32864571
4.77743387
$-3.47451830$
$-0.93224156$
0.33251005
0.05274719
0.53330171
2.17110634
$-1.60375547$
$-1.42952192$
0.93968588
2. 86669016
$-2.30715060$
$-2.86104083$
2. 28189754
$-2.58396840$
2. 05332351
2. 58900213
$-2.02965498$
$-0.47897720$
0.48997214
0.63959575
$-0.56602198$
$-0.05318236$
$-0.13100854$
$-0.33731037$
0.37706742
$-1.12906992$
1. 41672122 
1.04285800

1. 04285800

1. 14869356

1.14869356

1. 14869356

1.14869356

$-1.76707089$

$-1.76707089$

$-1.76707089$

$-1.76707089$

$-1.66123557$

$-1.66123557$

$-1.66123557$

$-1.66123557$

1. 57582414

1.57582414

1.57582414

1.57582414

1.68165958

1.68165958

1.68165958

1.68165958

$-1.11259675$

$-1.11259675$

$-1.11259675$

$-1.11259675$

$-1.00676131$

$-1.00676131$

$-1.00676131$

64

$-1.00676131$

$-0.1172$

0.0106

1.67341614

1.67341614

1.67341614

1.67341614

1. 77925158

1.77925158

1.77925158

1. 77925158

$-1.26006997$

$-1.26006997$

$-1.26006997$

$-1.26006997$

$-1.15423441$

$-1.15423441$

$-1.15423441$

$-1.15423441$

1. 17254710

1. 17254710

1.17254710

1.17254710

1. 27838266

1. 27838266

1.27838266

1. 27838266

$-1.80632699$

$-1.80632699$

$-1.80632699$

$-1.80632699$

$-1.70049155$

$-1.70049155$

$-1.70049155$

$-1.70049155$

1.04285800

1. 04285800
0.28137359

0.28137359

0.17553815

0.17553815

0.28137359

0.28137359

$-0.64616346$

$-0.64616346$

$-0.54032809$

$-0.54032809$

$-0.64616346$

$-0.64616346$

$-0.54032809$

$-0.54032809$

0.13101105

0.13101105

0.23684651

0.23684651

0.13101105

0.13101105

0.23684651

0.23684651

$-1.22435784$

$-1.22435784$

$-1.11852252$

$-1.11852252$

$-1.22435784$

$-1.22435784$

$-1.11852252$

$-1.11852252$

0 . \# Energy 3->8

$$
\begin{array}{ll}
0.0647 & 0.0159
\end{array}
$$

$0.0107 \quad-0.0663$

$-0.20954360$

$-0.20954360$

$-0.10370816$

$-0.10370816$

$-0.20954360$

$-0.20954360$

$-0.10370816$

$-0.10370816$

0.53541940

0.53541940

0.64125490

0.64125490

0.53541940

0.53541940

0.64125490

0.64125490

0.01399621

0.01399621

0.11983165

0.11983165

0.01399621

0.01399621

0.11983165

0.11983165

1.19734502

1.19734502

1. 30318046

1. 30318046

1.19734502

1. 19734502

1.30318046

1.30318046

0.17553815

0.17553815
$-1.01531279$

$-0.90947729$

$-1.01531279$

$-0.90947729$

$-1.01531279$

$-0.90947729$

$-0.62219280$

$-0.51635736$

$-0.62219280$

$-0.51635736$

$-0.62219280$

$-0.51635736$

$-0.62219280$

$-0.51635736$

$-1.87711871$

$-1.77128315$

$-1.87711871$

$-1.77128315$

$-1.87711871$

$-1.77128315$

$-1.87711871$

$-1.77128315$

$-1.14041448$

$-1.03457904$

$-1.14041448$

$-1.03457904$

$-1.14041448$

$-1.03457904$

$-1.14041448$

$-1.03457904$

$$
\begin{gathered}
1.000 \text { \# Electr. mom., scale-fac } \\
\text { \# Magnetic mom. }
\end{gathered}
$$

0.14458551

0.25042096

0.14458551

0.25042096

0.14458551

0.25042096

0.14458551

0.25042096

$-0.15045778$

$-0.04462234$

$-0.15045778$

$-0.04462234$

$-0.15045778$

$-0.04462234$

$-0.15045778$

$-0.04462234$

1. 24855602

1.35439157

1. 24855602

1. 35439157

1. 24855602

1. 35439157

1. 24855602

1. 35439157

0.73230141

0.83813679

0.73230141

0.83813679

0.73230141

0.83813679

0.73230141

0.83813679

$-1.01531279$

$-0.90947729$ 3. 25138474

0.54417193

$-0.75272322$

1. 02894330

$-1.18085635$

$-0.67538482$

0.79884350

$-1.80382276$

1.65342903

2.02894044

$-1.82009614$

1.97319758

$-1.78611088$

$-2.22617698$

1. 95834947

0.47292683

$-0.60116750$

$-0.35744259$

0.47407120

$-0.47277245$

0.58876961

0.34992540

$-0.44962752$

0.49838921

$-0.84066182$

$-0.72209072$

1. 17545247

$-0.36031774$

0.65186286

0.53623950

$-0.92722243$

$-2.70719957$

$-4.23004007$

3. 56828094

$-2.49086857$

2. 02294731

3.16507030

$-2.60258222$

$-3.44085336$

2. 55994725

2. 87303686

$-2.13209009$

2.73126340

$-1.86757326$

$-2.17513204$

1. 42911398

0.26119331

$-0.24197082$

$-0.16728446$

0.16443533

$-0.08637891$

0.09224844

$-0.04341189$

0.01138323

$-0.10066309$

0.05866097

$-0.17769614$

0.16146263

$-0.00271928$

0.06633004

0.34025857

$-0.32786101$

$-2.04079819$

2. 28498077 
1.04285800

1. 04285800

1. 14869356

1.14869356

1. 14869356

1.14869356

$-1.76707089$

$-1.76707089$

$-1.76707089$

$-1.76707089$

$-1.66123557$

$-1.66123557$

$-1.66123557$

$-1.66123557$

1. 57582414

1. 57582414

1.57582414

1.57582414

1.68165958

1.68165958

1.68165958

1.68165958

$-1.11259675$

$-1.11259675$

$-1.11259675$

$-1.11259675$

$-1.00676131$

$-1.00676131$

$-1.00676131$

64

$-1.00676131$
0 .

$0.3444 \quad-0.1770 \quad-0.0898$

$\begin{array}{lll}0.0608 & -0.0214 & -0.0641\end{array}$

1.67341614

1.67341614

1.67341614

1.67341614

1.77925158

1.77925158

1.77925158

1. 77925158

$-1.26006997$

$-1.26006997$

$-1.26006997$

$-1.26006997$

$-1.15423441$

$-1.15423441$

$-1.15423441$

$-1.15423441$

1.17254710

1.17254710

1.17254710

1.17254710

1. 27838266

1. 27838266

1.27838266

1. 27838266

$-1.80632699$

$-1.80632699$

$-1.80632699$

$-1.80632699$

$-1.70049155$

$-1.70049155$

$-1.70049155$

$-1.70049155$

1.04285800

1. 04285800
0.28137359

0.28137359

0.17553815

0.17553815

0.28137359

0.28137359

$-0.64616346$

$-0.64616346$

$-0.54032809$

$-0.54032809$

$-0.64616346$

$-0.64616346$

$-0.54032809$

$-0.54032809$

0.13101105

0.13101105

0.23684651

0.23684651

0.13101105

0.13101105

0.23684651

0.23684651

$-1.22435784$

$-1.22435784$

$-1.11852252$

$-1.11852252$

$-1.22435784$

$-1.22435784$

$-1.11852252$

$-1.11852252$

$-1.01531279$

$-0.90947729$

$-1.01531279$

$-0.90947729$

$-1.01531279$

$-0.90947729$

$-0.62219280$

$-0.51635736$

$-0.62219280$

$-0.51635736$

$-0.62219280$

$-0.51635736$

$-0.62219280$

$-0.51635736$

$-1.87711871$

$-1.77128315$

$-1.87711871$

$-1.77128315$

$-1.87711871$

$-1.77128315$

$-1.87711871$

$-1.77128315$

$-1.14041448$

$-1.03457904$

$-1.14041448$

$-1.03457904$

$-1.14041448$

$-1.03457904$

$-1.14041448$

$-1.03457904$

1.000

$-0.20954360$

$-0.20954360$

$-0.10370816$

$-0.10370816$

$-0.20954360$

$-0.20954360$

$-0.10370816$

$-0.10370816$

0.53541940

0.53541940

0.64125490

0.64125490

0.53541940

0.53541940

0.64125490

0.64125490

0.01399621

0.01399621

0.11983165

0.11983165

0.01399621

0.01399621

0.11983165

0.11983165

1. 19734502

1.19734502

1. 30318046

1. 30318046

1.19734502

1. 19734502

1. 30318046

1. 30318046

0.17553815

0.17553815
0.14458551

0.14458551

0.25042096

0.14458551

0.25042096

0.14458551

0.25042096

$-0.15045778$

$-0.04462234$

$-0.15045778$

$-0.04462234$

$-0.15045778$

$-0.04462234$

$-0.15045778$

$-0.04462234$

1. 24855602

1. 35439157

1. 24855602

1. 35439157

1. 24855602

1. 35439157

1. 24855602

1. 35439157

0.73230141

0.83813679

0.73230141

0.83813679

0.73230141

0.83813679

0.73230141

0.83813679

$-1.01531279$

$-0.90947729$
0.25042096
1.85107386

$-2.10891509$

2. 24028206

$-2.42886043$

$-2.10401225$

2. 33253288

$-0.36424232$

$-0.16412668$

$-0.04045165$

0.55017787

0.57811671

$-0.01696510$

$-0.07213182$

$-0.44197738$

0.41313434

$-0.55126953$

$-0.38063759$

0.51303458

$-0.37055892$

0.48053616

0.33519670

$-0.43966749$

0.36000746

$-0.40175548$

$-0.43573335$

0.45412380

$-0.29478911$

0.35523340

0.37264287

$-0.42687386$
Electr. mom., scale-fac Electr. mom.'
Magnetic mom. $-1.24192703$

1. 24954855

2.08320045

$-2.15090561$

0.17951541

$-0.16599196$

$-0.76895422$

0.81242353

8.41632748

$-7.12411213$

$-7.49961948$

6.52002811

$-4.83486652$

3.66852713

3. 90884256

$-2.92891788$

2. 04076743

$-1.53425395$

$-2.17816305$

1.63784957

$-2.18224502$

1.64068389

2. 36667490

$-1.77391815$

0.48944274

$-0.21843792$

0.52235067

$-0.54616749$

$-0.46927315$

0.02829994

$-0.85516584$

0.95855045

2.72055149

$-2.92423820$ 
1.04285800

1. 04285800

1. 14869356

1.14869356

1. 14869356

1.14869356

$-1.76707089$

$-1.76707089$

$-1.76707089$

$-1.76707089$

$-1.66123557$

$-1.66123557$

$-1.66123557$

$-1.66123557$

1. 57582414

1.57582414

1.57582414

1.57582414

1.68165958

1.68165958

1.68165958

1.68165958

$-1.11259675$

$-1.11259675$

$-1.11259675$

$-1.11259675$

$-1.00676131$

$-1.00676131$

$-1.00676131$

$-1.00676131$

\&TRANSITION $4->\ldots$ 64
$-0.2613$
$-0.0022$
1.67341614
1.67341614
1.67341614
1.67341614
1.77925158
1. 77925158
1. 77925158
1. 77925158
$-1.26006997$
$-1.26006997$
$-1.26006997$
$-1.26006997$
$-1.15423441$
$-1.15423441$
$-1.15423441$
$-1.15423441$
1.17254710
1.17254710
1.17254710
1. 17254710
1. 27838266
1.27838266
1. 27838266
1.27838266
$-1.80632699$
$-1.80632699$
$-1.80632699$
$-1.80632699$
$-1.70049155$
$-1.70049155$
$-1.70049155$
$-1.70049155$
1. 04285800

$$
\begin{array}{rr}
0.28137359 & -1.01531279 \\
0.28137359 & -0.90947729 \\
0.17553815 & -1.01531279 \\
0.17553815 & -0.90947729 \\
0.28137359 & -1.01531279 \\
0.28137359 & -0.90947729 \\
-0.64616346 & -0.62219280 \\
-0.64616346 & -0.51635736 \\
-0.54032809 & -0.62219280 \\
-0.54032809 & -0.51635736 \\
-0.64616346 & -0.62219280 \\
-0.64616346 & -0.51635736 \\
-0.54032809 & -0.62219280 \\
-0.54032809 & -0.51635736 \\
0.13101105 & -1.87711871 \\
0.13101105 & -1.77128315 \\
0.23684651 & -1.87711871 \\
0.23684651 & -1.77128315 \\
0.13101105 & -1.87711871 \\
0.13101105 & -1.77128315 \\
0.23684651 & -1.87711871 \\
0.23684651 & -1.77128315 \\
-1.22435784 & -1.14041448 \\
-1.22435784 & -1.03457904 \\
-1.11852252 & -1.14041448 \\
-1.11852252 & -1.03457904 \\
-1.22435784 & -1.14041448 \\
-1.22435784 & -1.03457904 \\
-1.11852252 & -1.14041448 \\
-1.11852252 & -1.03457904
\end{array}
$$

$$
\begin{array}{rr}
\text { 0. Energy } & 4->5 \\
0.0073 & -0.2270 \\
0.0110 & 0.0155
\end{array}
$$$$
-0.20954360
$$$$
-0.20954360
$$$$
-0.10370816
$$$$
-0.10370816
$$$$
-0.20954360
$$$$
-0.20954360
$$$$
-0.10370816
$$$$
-0.10370816
$$$$
0.53541940
$$$$
0.53541940
$$$$
0.64125490
$$$$
0.64125490
$$$$
0.53541940
$$$$
0.53541940
$$$$
0.64125490
$$$$
0.64125490
$$$$
0.01399621
$$$$
0.01399621
$$$$
0.11983165
$$$$
0.11983165
$$$$
0.01399621
$$$$
0.01399621
$$$$
0.11983165
$$$$
0.11983165
$$$$
1.19734502
$$$$
1.19734502
$$$$
\text { 1. } 30318046
$$$$
\text { 1. } 30318046
$$$$
1.19734502
$$$$
1.19734502
$$$$
1.30318046
$$$$
1.30318046
$$$$
0.17553815
$$

$$
\begin{array}{r}
-2.67835355 \\
2.97418952 \\
-3.02618051 \\
3.23497915 \\
2.83587027 \\
-3.14760041 \\
-1.10375106 \\
1.94870210 \\
2.16118956 \\
-2.91117167 \\
1.00788462 \\
-1.78385413 \\
-2.32388115 \\
2.92253137 \\
-0.39216265 \\
0.53589112 \\
0.40983158 \\
-0.53953534 \\
0.32957581 \\
-0.43821213 \\
-0.35979652 \\
0.46387035 \\
-0.40208286 \\
0.20158435 \\
0.45117399 \\
-0.14066298 \\
0.31369516 \\
-0.17511739 \\
-0.36813679 \\
0.15310359
\end{array}
$$

$$
\begin{gathered}
1.000 \text { \# Electr. mom., scale-fac } \\
\text { \# Magnetic mom. } \\
0.14458551
\end{gathered}
$$$$
0.25042096-0.08488720
$$$$
0.14458551 \quad-0.80709797
$$$$
0.25042096 \quad 1.07313228
$$$$
0.14458551 \quad 0.79465878
$$$$
0.25042096 \quad-0.32322341
$$$$
0.14458551 \quad 0.15742256
$$$$
0.25042096 \quad-0.45773801
$$$$
-0.15045778 \quad-0.85165918
$$$$
-0.04462234 \quad-0.01134747
$$$$
\begin{array}{ll}
-0.15045778 & 0.68572158
\end{array}
$$$$
-0.04462234 \quad 0.21204554
$$$$
\begin{array}{ll}
-0.15045778 & 0.59327549
\end{array}
$$$$
-0.04462234 \quad 0.14549655
$$$$
-0.15045778 \quad-0.49146688
$$$$
-0.04462234 \quad-0.29908618
$$$$
1.24855602 \quad 0.46920043
$$$$
1.35439157 \quad-0.38035551
$$$$
1.24855602 \quad-0.12536383
$$$$
1.35439157 \quad 0.10441532
$$$$
1.24855602-0.48354220
$$$$
\begin{array}{ll}
1.35439157 & 0.38309428
\end{array}
$$$$
\begin{array}{ll}
1.24855602 & 0.11615159
\end{array}
$$$$
1.35439157 \quad-0.08892129
$$$$
0.73230141 \quad 0.38398540
$$$$
0.83813679 \quad-0.38665867
$$$$
0.73230141 \quad-0.52455652
$$$$
0.83813679 \quad 0.48234889
$$$$
0.73230141 \quad-0.32094777
$$$$
0.83813679 \quad 0.35596809
$$$$
0.73230141 \quad 0.50658274
$$$$
0.83813679 \quad-0.48964915
$$$$
-1.01531279 \quad-0.43151531
$$ 


1.04285800
1.04285800
1.04285800
1.14869356
1.14869356
1.14869356
1.14869356
-1.76707089
-1.76707089
-1.76707089
-1.76707089
-1.66123557
-1.66123557
-1.66123557
-1.66123557
1.57582414
1.57582414
1.57582414
1.57582414
1.68165958
1.68165958
1.68165958
1.68165958
-1.11259675
-1.11259675
-1.11259675
-1.11259675
-1.00676131
-1.00676131
-1.00676131
-1.00676131

64
0.17553815

0.28137359

0.28137359

0.17553815

0.17553815

0.28137359

0.28137359

$-0.64616346$

$-0.64616346$

$-0.54032809$

$-0.54032809$

$-0.64616346$

$-0.64616346$

$-0.54032809$

$-0.54032809$

0.13101105

0.13101105

0.23684651

0.23684651

0.13101105

0.13101105

0.23684651

0.23684651

$-1.22435784$

$-1.22435784$

$-1.11852252$

$-1.11852252$

$-1.22435784$

$-1.22435784$

$-1.11852252$

$-1.11852252$

0 .

0.7085
0.0348

1.67341614

1.67341614

1.67341614

1.67341614

1.77925158

1.77925158

1. 77925158

1. 77925158

$-1.26006997$

$-1.26006997$

$-1.26006997$

$-1.26006997$

$-1.15423441$

$-1.15423441$

$-1.15423441$

$-1.15423441$

1. 17254710

1.17254710

1.17254710

1. 17254710

1. 27838266

1. 27838266

1. 27838266

1.27838266

$-1.80632699$

$-1.80632699$

$-1.80632699$

$-1.80632699$

$-1.70049155$

$-1.70049155$

$-1.70049155$

$-1.70049155$

1. 04285800

$$
\begin{gathered}
\text { \# Energy } 4->6 \\
-0.1789 \quad-0.1505
\end{gathered}
$$

$0.0826-0.0665$

$-0.20954360$

$-0.20954360$

$-0.10370816$

$-0.10370816$

$-0.20954360$

$-0.20954360$

$-0.10370816$

$-0.10370816$

0.53541940

0.53541940

0.64125490

0.64125490

0.53541940

0.53541940

0.64125490

0.64125490

0.01399621

0.01399621

0.11983165

0.11983165

0.01399621

0.01399621

0.11983165

0.11983165

1.19734502

1.19734502

1. 30318046

1. 30318046

1. 19734502

1. 19734502

1.30318046

1. 30318046

0.17553815
-0.90947729
-1.01531279
-0.90947729
-1.01531279
-0.90947729
-1.01531279
-0.90947729
-0.62219280
-0.51635736
-0.62219280
-0.51635736
-0.62219280
-0.51635736
-0.62219280
-0.51635736
-1.87711871
-1.77128315
-1.87711871
-1.77128315
-1.87711871
-1.77128315
-1.87711871
-1.77128315
-1.14041448
-1.03457904
-1.14041448
-1.03457904
-1.14041448
-1.03457904
-1.14041448
-1.03457904

1. 03221858

0.40141639

$-0.87392122$

$-0.24026558$

$-0.34398136$

0.03842459

0.40852749

0.66585410

$-0.46077168$

$-0.46945071$

0.30228242

$-0.88360935$

0.48767263

0.57323998

$-0.25101203$

$-0.10329729$

0.21054529

0.03928336

$-0.08965222$

0.01073832

$-0.07773260$

0.00769245

0.02435331

0.34711623

$-0.63929474$

$-0.65368617$

1. 12088156

$-0.25276276$

0.47195277

0.47354028

$-0.83076650$
1.000 \# Electr. mom., scale-fac \# Magnetic mom.

0.14458551

0.25042096

0.14458551

0.25042096

0.14458551

0.25042096

0.14458551

0.25042096

$-0.15045778$

$-0.04462234$

$-0.15045778$

$-0.04462234$

$-0.15045778$

$-0.04462234$

$-0.15045778$

$-0.04462234$

1. 24855602

1. 35439157

1. 24855602

1. 35439157

1. 24855602

1. 35439157

1. 24855602

1. 35439157

0.73230141

0.83813679

0.73230141

0.83813679

0.73230141

0.83813679

0.73230141

0.83813679

$-1.01531279$
$-2.07167816$

2.20776033

3.27859306

$-3.45688438$

0.77895170

$-0.88076556$

$-1.73365223$

1.85385191

$-3.17362952$

4.60678673

2.48087406

$-3.85988665$

3.33566737

$-4.79219294$

$-2.70030665$

4.08836031

2. 76158810

$-2.11740208$

$-2.71429801$

2.06757474

$-2.86254787$

2. 19432020

2. 91550422

$-2.21451831$

0.14830630

0.14903940

0.06274087

$-0.22777151$

$-1.04673243$

0.48318517

0.59398770

$-0.21938585$

5.34120464 


1.04285800
1.04285800
1.04285800
1.14869356
1.14869356
1.14869356
1.14869356
-1.76707089
-1.76707089
-1.76707089
-1.76707089
-1.66123557
-1.66123557
-1.66123557
-1.66123557
1.57582414
1.57582414
1.57582414
1.57582414
1.68165958
1.68165958
1.68165958
1.68165958
-1.11259675
-1.11259675
-1.11259675
-1.11259675
-1.00676131
-1.00676131
-1.00676131
-1.00676131

64
0.17553815

0.28137359

0.28137359

0.17553815

0.17553815

0.28137359

0.28137359

$-0.64616346$

$-0.64616346$

$-0.54032809$

$-0.54032809$

$-0.64616346$

$-0.64616346$

$-0.54032809$

$-0.54032809$

0.13101105

0.13101105

0.23684651

0.23684651

0.13101105

0.13101105

0.23684651

0.23684651

$-1.22435784$

$-1.22435784$

$-1.11852252$

$-1.11852252$

$-1.22435784$

$-1.22435784$

$-1.11852252$

$-1.11852252$

0 . \# Energy $4->7$

$\begin{array}{lll}-0.1504 & -0.2744 & -0.0294\end{array}$

0.0005

1.67341614

1.67341614

1.67341614

1.67341614

1.77925158

1.77925158

1.77925158

1.77925158

$-1.26006997$

$-1.26006997$

$-1.26006997$

$-1.26006997$

$-1.15423441$

$-1.15423441$

$-1.15423441$

$-1.15423441$

1.17254710

1.17254710

1.17254710

1.17254710

1.27838266

1.27838266

1.27838266

1.27838266

$-1.80632699$

$-1.80632699$

$-1.80632699$

$-1.80632699$

$-1.70049155$

$-1.70049155$

$-1.70049155$

$-1.70049155$

1.04285800
-0.90947729
-1.01531279
-0.90947729
-1.01531279
-0.90947729
-1.01531279
-0.90947729
-0.62219280
-0.51635736
-0.62219280
-0.51635736
-0.62219280
-0.51635736
-0.62219280
-0.51635736
-1.87711871
-1.77128315
-1.87711871
-1.77128315
-1.87711871
-1.77128315
-1.87711871
-1.77128315
-1.14041448
-1.03457904
-1.14041448
-1.03457904
-1.14041448
-1.03457904
-1.14041448
-1.03457904

$-5.94562197$

$-5.23253059$

5.93191671

$-5.07862806$

5.74600792

4.47631645

$-5.21873283$

$-3.28172302$

2. 98159409

2. 95041752

$-2.74003744$

3. 61553478

$-3.37256384$

$-3.37611294$

3. 24212456

$-0.19327722$

0.15473984

0.07306707

0.06913322

0.22328201

$-0.20105101$

$-0.15917419$

0.06452545

0.10274059

$-0.24669196$

$-0.29812300$

0.49263829

$-0.00987226$

0.13131869

0.19250613

$-0.37036711$
1.000 \# Electr. mom., scale-fac \# Magnetic mom.

0.14458551

1.86129856

0.25042096

0.14458551

0.25042096

0.14458551

0.25042096

0.14458551

0.25042096

$-0.15045778$

$-0.04462234$

$-0.15045778$

$-0.04462234$

$-0.15045778$

$-0.04462234$

$-0.15045778$

$-0.04462234$

1.24855602

1.35439157

1.24855602

1.35439157

1. 24855602

1.35439157

1.24855602

1.35439157

0.73230141

0.83813679

0.73230141

0.83813679

0.73230141

0.83813679

0.73230141

0.83813679

$-1.01531279$
$-1.95319033$

$-3.53153348$

3.50081348

$-0.91288835$

1.03667831

2. 27391338

$-2.27120066$

$-1.01370037$

0.33927554

1.81943250

$-1.27414727$

1.14694417

$-0.47001705$

$-1.74209952$

1.08640444

$-0.54854983$

0.45600572

0.84167969

$-0.67904347$

0.57013613

$-0.47859734$

$-0.92159826$

0.74200010

$-1.28347993$

0.56182158

0.91164565

$-0.35748011$

2. 23518443

$-1.23541200$

$-1.65990353$

0.87820870

$-3.41512322$ 


\subsection{0 \\ 1. 04285800 \\ 1. 04285800 \\ 1.14869356 \\ 1. 14869356 \\ 1. 14869356 \\ 1.14869356 \\ $-1.76707089$ \\ $-1.76707089$ \\ $-1.76707089$ \\ $-1.76707089$ \\ $-1.66123557$ \\ $-1.66123557$ \\ $-1.66123557$ \\ $-1.66123557$ \\ 1.57582414 \\ 1.57582414 \\ 1.57582414 \\ 1.57582414 \\ 1.68165958 \\ 1.68165958 \\ 1.68165958 \\ 1.68165958 \\ $-1.11259675$ \\ $-1.11259675$ \\ $-1.11259675$ \\ $-1.11259675$ \\ $-1.00676131$ \\ $-1.00676131$ \\ $-1.00676131$ \\ $-1.00676131$}

64

0 .

0.3736
-0.0328

1.67341614

1.67341614

1.67341614

1.67341614

1.77925158

1.77925158

1.77925158

1.77925158

$-1.26006997$

$-1.26006997$

$-1.26006997$

$-1.26006997$

$-1.15423441$

$-1.15423441$

$-1.15423441$

$-1.15423441$

1.17254710

1.17254710

1.17254710

1.17254710

1.27838266

1.27838266

1.27838266

1.27838266

$-1.80632699$

$-1.80632699$

$-1.80632699$

$-1.80632699$

$-1.70049155$

$-1.70049155$

$-1.70049155$

$-1.70049155$

1.04285800

0.17553815

0.28137359

0.28137359

0.17553815

0.17553815

0.28137359

0.28137359

$-0.64616346$

$-0.64616346$

$-0.54032809$

$-0.54032809$

$-0.64616346$

$-0.64616346$

$-0.54032809$

$-0.54032809$

0.13101105

0.13101105

0.23684651

0.23684651

0.13101105

0.13101105

0.23684651

0.23684651

$-1.22435784$

$-1.22435784$

$-1.11852252$

$-1.11852252$

$-1.22435784$

$-1.22435784$

$-1.11852252$

$-1.11852252$

\# Energy 4->8

$0.0238 \quad 0.0447$

$-0.0190 \quad 0.0927$

$-0.20954360$

$-0.20954360$

$-0.10370816$

$-0.10370816$

$-0.20954360$

$-0.20954360$

$-0.10370816$

$-0.10370816$

0.53541940

0.53541940

0.64125490

0.64125490

0.53541940

0.53541940

0.64125490

0.64125490

0.01399621

0.01399621

0.11983165

0.11983165

0.01399621

0.01399621

0.11983165

0.11983165

1. 19734502

1.19734502

1. 30318046

1. 30318046

1. 19734502

1. 19734502

1.30318046

1. 30318046

0.17553815
$-0.90947729$

$-1.01531279$

$-0.90947729$

$-1.01531279$

$-0.90947729$

$-1.01531279$

$-0.90947729$

$-0.62219280$

$-0.51635736$

$-0.62219280$

$-0.51635736$

$-0.62219280$

$-0.51635736$

$-0.62219280$

$-0.51635736$

$-1.87711871$

$-1.77128315$

$-1.87711871$

$-1.77128315$

$-1.87711871$

$-1.77128315$

$-1.87711871$

$-1.77128315$

$-1.14041448$

$-1.03457904$

$-1.14041448$

$-1.03457904$

$-1.14041448$

$-1.03457904$

$-1.14041448$

$-1.03457904$

1.000

\subsection{1}

0.25042096

0.14458551

0.25042096

0.14458551

0.25042096

0.14458551

0.25042096

$-0.15045778$

$-0.04462234$

$-0.15045778$

$-0.04462234$

$-0.15045778$

$-0.04462234$

$-0.15045778$

$-0.04462234$

1. 24855602

1. 35439157

1. 24855602

1. 35439157

1. 24855602

1. 35439157

1. 24855602

1. 35439157

0.73230141

0.83813679

0.73230141

0.83813679

0.73230141

0.83813679

0.73230141

0.83813679

$-1.01531279$
3. 76419401

3. 05585456

$-3.39329219$

3.08587193

$-3.44342947$

$-2.60949039$

2.97232342

$-1.83677542$

2. 21321011

1.96287417

$-2.46084809$

2.10055828

$-2.58702135$

$-2.34556913$

3. 02824330

0.09897678

$-0.05385133$

$-0.10432317$

0.02990792

$-0.11140881$

0.07342105

0.13959648

$-0.08384520$

0.49296430

$-0.70229262$

$-0.53081399$

0.75250787

$-0.44593146$

0.65943682

0.49010792

$-0.72463375$

Electr. mom., scale-fac Magnetic mom.

1.69923568
-1.81767285
-2.62565303
2.64414597
-1.27892947
1.44183278
2.06028438
-2.11498713
-1.14688480
-0.05523216
0.88204283
0.31672654
0.35738635
0.85208893

$-0.11171624$

$-1.08129787$

0.27743739

$-0.13889889$

$-0.05392893$

$-0.01944382$

$-0.39103195$

0.23065627

0.13388854

$-0.05105854$

$-0.78528327$

0.50418788

0.53996450

$-0.32406104$

0.77764767

$-0.47169790$

$-0.53088433$

0.28789207

0.02380697 


1.04285800
1.04285800
1.04285800
1.14869356
1.14869356
1.14869356
1.14869356
-1.76707089
-1.76707089
-1.76707089
-1.76707089
-1.66123557
-1.66123557
-1.66123557
-1.66123557
1.57582414
1.57582414
1.57582414
1.57582414
1.68165958
1.68165958
1.68165958
1.68165958
-1.11259675
-1.11259675
-1.11259675
-1.11259675
-1.00676131
-1.00676131
-1.00676131
-1.00676131

64
0.17553815

0.28137359

0.28137359

0.17553815

0.17553815

0.28137359

0.28137359

$-0.64616346$

$-0.64616346$

$-0.54032809$

$-0.54032809$

$-0.64616346$

$-0.64616346$

$-0.54032809$

$-0.54032809$

0.13101105

0.13101105

0.23684651

0.23684651

0.13101105

0.13101105

0.23684651

0.23684651

$-1.22435784$

$-1.22435784$

$-1.11852252$

$-1.11852252$

$-1.22435784$

$-1.22435784$

$-1.11852252$

$-1.11852252$

$0 . \quad \#$ Energy $4->9$

$\begin{array}{lll}-0.8133 & -0.6224 & -0.0882\end{array}$

$-0.0030$

1.67341614

1.67341614

1.67341614

1.67341614

1.77925158

1.77925158

1.77925158

1.77925158

$-1.26006997$

$-1.26006997$

$-1.26006997$

$-1.26006997$

$-1.15423441$

$-1.15423441$

$-1.15423441$

$-1.15423441$

1.17254710

1.17254710

1.17254710

1.17254710

1.27838266

1.27838266

1.27838266

1.27838266

$-1.80632699$

$-1.80632699$

$-1.80632699$

$-1.80632699$

$-1.70049155$

$-1.70049155$

$-1.70049155$

$-1.70049155$

1.04285800

$0.0456 \quad 0.0173$

$-0.20954360$

$-0.20954360$

$-0.10370816$

$-0.10370816$

$-0.20954360$

$-0.20954360$

$-0.10370816$

$-0.10370816$

0.53541940

0.53541940

0.64125490

0.64125490

0.53541940

0.53541940

0.64125490

0.64125490

0.01399621

0.01399621

0.11983165

0.11983165

0.01399621

0.01399621

0.11983165

0.11983165

1.19734502

1.19734502

1. 30318046

1.30318046

1.19734502

1.19734502

1.30318046

1.30318046

0.17553815
-0.90947729
-1.01531279
-0.90947729
-1.01531279
-0.90947729
-1.01531279
-0.90947729
-0.62219280
-0.51635736
-0.62219280
-0.51635736
-0.62219280
-0.51635736
-0.62219280
-0.51635736
-1.87711871
-1.77128315
-1.87711871
-1.77128315
-1.87711871
-1.77128315
-1.87711871
-1.77128315
-1.14041448
-1.03457904
-1.14041448
-1.03457904
-1.14041448
-1.03457904
-1.14041448
-1.03457904

0.13786006

0.27669081

$-0.35922980$

0.26700836

$-0.44964957$

$-0.83903027$

0.94430977

1.87503636

$-2.24732208$

$-2.13853812$

2. 54860544

$-2.01073861$

2. 41624022

2. 36624503

$-2.83288527$

$-0.08577013$

0.08741850

0.02099116

0.03735412

0.11529542

$-0.12980783$

$-0.09383855$

0.06938589

$-0.11960344$

0.25121999

0.13699484

$-0.29369920$

0.04909550

$-0.15240850$

$-0.04483723$

0.16704461

1.000

\# Electr. mom., scale-fac

0.14458551 \# Magnetic mom.

0.25042096

$-0.02895189$

0.14458551

0.25042096

$-0.37043321$

0.14458551

$-1.21601021$

1.67264843

0.25042096

0.14458551

0.25042096

$-0.15045778$

$-0.04462234$

$-0.15045778$

$-0.04462234$

$-0.15045778$

$-0.04462234$

$-0.15045778$

$-0.04462234$

1.24855602

1.35439157

1.24855602

1.35439157

1.24855602

1.35439157

1.24855602

1.35439157

0.73230141

0.83813679

0.73230141

0.83813679

0.73230141

0.83813679

0.73230141

0.83813679

$-1.01531279$

0.57867509

$-0.22035593$

0.43186384

$-0.83382154$

5.61417580

$-3.91212606$

$-4.53645802$

2.57095122

$-5.53740931$

3.82704043

4.88038826

$-3.11010122$

$-1.38597345$

1.13070095

1.59070408

$-1.28317988$

1.25669312

$-1.02546453$

$-1.52571642$

1.22085059

0.74157226

$-0.80763984$

$-1.02313256$

0.98830080

$-0.25396678$

0.48183975

0.73987269

$-0.80616766$

$-0.10676553$ 


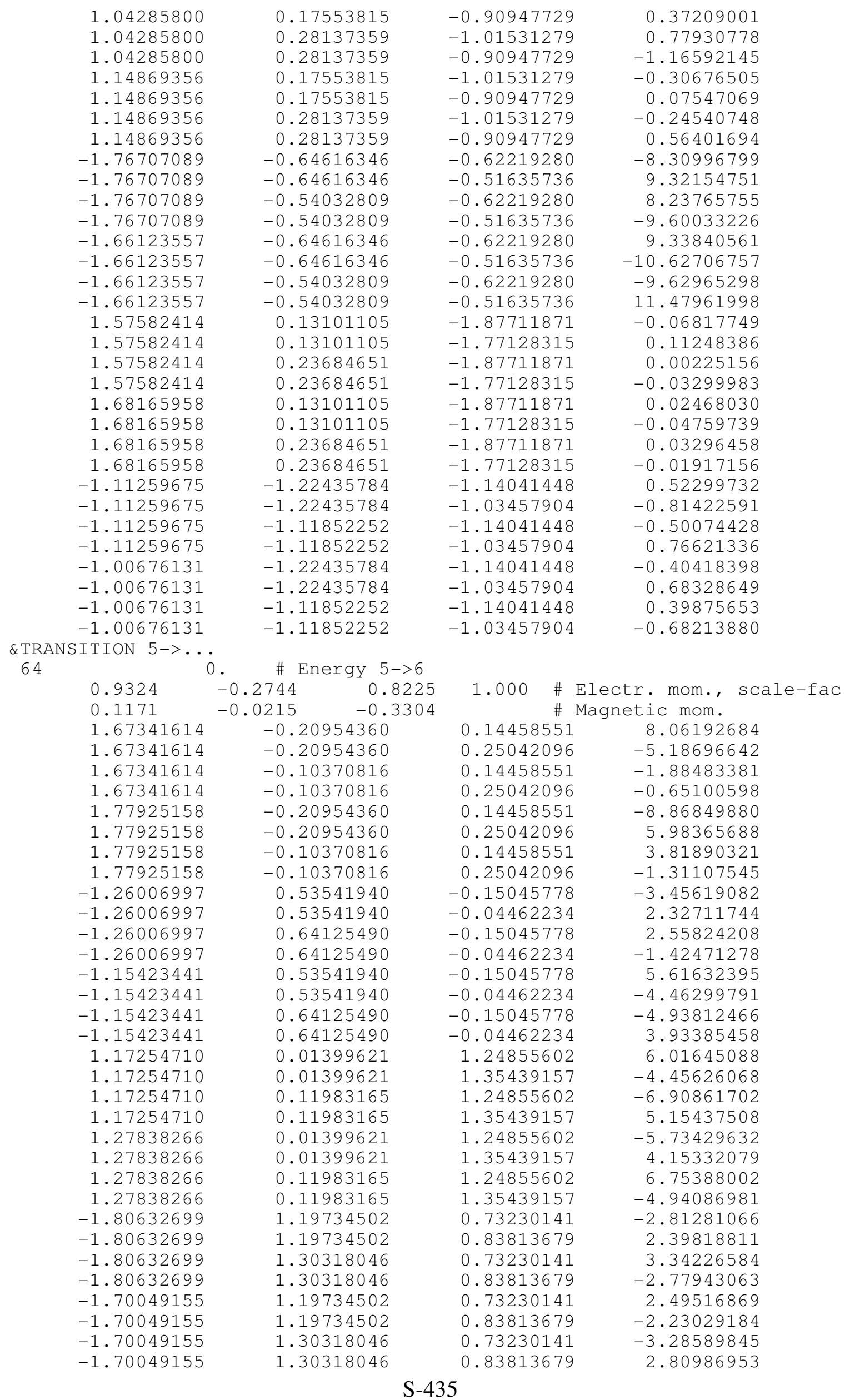


1.04285800

1. 04285800

1. 04285800

1. 04285800

1. 14869356

1. 14869356

1. 14869356

1. 14869356

$-1.76707089$

$-1.76707089$

$-1.76707089$

$-1.76707089$

$-1.66123557$

$-1.66123557$

$-1.66123557$

$-1.66123557$

1.57582414

1. 57582414

1. 57582414

1. 57582414

1.68165958

1.68165958

1.68165958

1.68165958

$-1.11259675$

$-1.11259675$

$-1.11259675$

$-1.11259675$

$-1.00676131$

$-1.00676131$

$-1.00676131$

64
0.17553815

0.17553815

0.28137359

0.28137359

0.17553815

0.17553815

0.28137359

0.28137359

$-0.64616346$

$-0.64616346$

$-0.54032809$

$-0.54032809$

$-0.64616346$

$-0.64616346$

$-0.54032809$

$-0.54032809$

0.13101105

0.13101105

0.23684651

0.23684651

0.13101105

0.13101105

0.23684651

0.23684651

$-1.22435784$

$-1.22435784$

$-1.11852252$

$-1.11852252$

$-1.22435784$

$-1.22435784$

$-1.11852252$

$-1.11852252$

0 .

$-0.5169$

$-0.0635$

1.67341614

1.67341614

1.67341614

1.67341614

1.77925158

1. 77925158

1. 77925158

1. 77925158

$-1.26006997$

$-1.26006997$

$-1.26006997$

$-1.26006997$

$-1.15423441$

$-1.15423441$

$-1.15423441$

$-1.15423441$

1. 17254710

1.17254710

1. 17254710

1. 17254710

1. 27838266

1. 27838266

1. 27838266

1.27838266

$-1.80632699$

$-1.80632699$

$-1.80632699$

$-1.80632699$

$-1.70049155$

$-1.70049155$

$-1.70049155$

$-1.70049155$

$$
\begin{aligned}
& -1 \\
& -0 \\
& -1 \\
& -0 \\
& -1 \\
& -0 \\
& -1 \\
& -0 \\
& -0 \\
& -0 \\
& -0 \\
& -0 \\
& -0 \\
& -0 \\
& -0 \\
& -0 \\
& -1 \\
& -1 \\
& -1 \\
& -1 \\
& -1 \\
& -1 \\
& -1 \\
& -1 \\
& -1 \\
& -1 \\
& -1 \\
& -1 \\
& -1 \\
& -1 \\
& -1 \\
& -1 \\
& \\
&
\end{aligned}
$$

$-1.01531279$

$-0.90947729$

$-1.01531279$

$-0.90947729$

$-1.01531279$

$-0.90947729$

$-1.01531279$

$-0.90947729$

$-0.62219280$

$-0.51635736$

$-0.62219280$

$-0.51635736$

$-0.62219280$

$-0.51635736$

$-0.62219280$

$-0.51635736$

$-1.87711871$

$-1.77128315$

$-1.87711871$

$-1.77128315$

$-1.87711871$

$-1.77128315$

$-1.87711871$

$-1.77128315$

$-1.14041448$

$-1.03457904$

$-1.14041448$

0.14458551

0.14458551
0.25042096

0.25042096
0.14458551

0.25042096

0.14458551

0.25042096

0.14458551

0.25042096

$-0.15045778$

$-0.04462234$

$-0.15045778$

$-0.04462234$

$-0.15045778$

$-0.04462234$

$-0.15045778$

$-0.04462234$

1. 24855602

1. 35439157

1. 24855602

1. 35439157

1. 24855602

1. 35439157

1. 24855602

1. 35439157

0.73230141

0.83813679

0.73230141

0.83813679

0.73230141

0.83813679

0.73230141

0.83813679

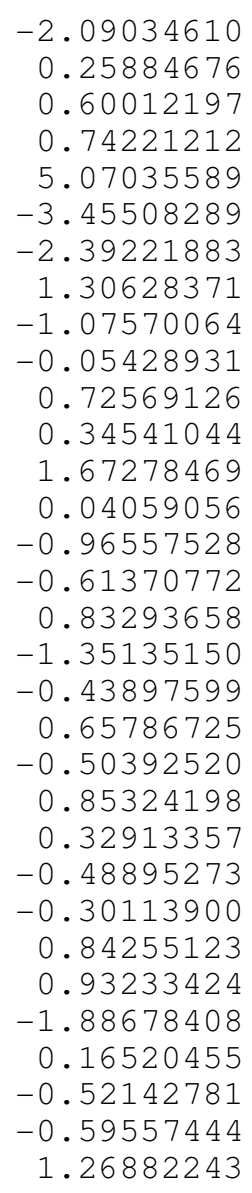


1.04285800

1. 04285800

1. 04285800

1. 04285800

1. 14869356

1. 14869356

1. 14869356

1. 14869356

$-1.76707089$

$-1.76707089$

$-1.76707089$

$-1.76707089$

$-1.66123557$

$-1.66123557$

$-1.66123557$

$-1.66123557$

1.57582414

1. 57582414

1. 57582414

1. 57582414

1.68165958

1.68165958

1.68165958

1.68165958

$-1.11259675$

$-1.11259675$

$-1.11259675$

$-1.11259675$

$-1.00676131$

$-1.00676131$

$-1.00676131$

64

$-1.00676131$

$-2.4720$

0.0428

1.67341614

1.67341614

1.67341614

1.67341614

1.77925158

1. 77925158

1. 77925158

1. 77925158

$-1.26006997$

$-1.26006997$

$-1.26006997$

$-1.26006997$

$-1.15423441$

$-1.15423441$

$-1.15423441$

$-1.15423441$

1. 17254710

1.17254710

1. 17254710

1. 17254710

1.27838266

1. 27838266

1. 27838266

1. 27838266

$-1.80632699$

$-1.80632699$

$-1.80632699$

$-1.80632699$

$-1.70049155$

$-1.70049155$

$-1.70049155$

$-1.70049155$
0.17553815

0.17553815

0.28137359

0.28137359

0.17553815

0.17553815

0.28137359

0.28137359

$-0.64616346$

$-0.64616346$

$-0.54032809$

$-0.54032809$

$-0.64616346$

$-0.64616346$

$-0.54032809$

$-0.54032809$

0.13101105

0.13101105

0.23684651

0.23684651

0.13101105

0.13101105

0.23684651

0.23684651

$-1.22435784$

$-1.22435784$

$-1.11852252$

$-1.11852252$

$-1.22435784$

$-1.22435784$

$-1.11852252$

$-1.11852252$

. \# Energy 5->8

$\begin{array}{rr}0.5549 & -0.1011 \\ -0.1659 & -0.0811\end{array}$

$-0.20954360$

$-0.20954360$

$-0.10370816$

$-0.10370816$

$-0.20954360$

$-0.20954360$

$-0.10370816$

$-0.10370816$

0.53541940

0.53541940

0.64125490

0.64125490

0.53541940

0.53541940

0.64125490

0.64125490

0.01399621

0.01399621

0.11983165

0.11983165

0.01399621

0.01399621

0.11983165

0.11983165

1. 19734502

1.19734502

1. 30318046

1. 30318046

1. 19734502

1.19734502

1. 30318046

1. 30318046
$-1.01531279$

$-0.90947729$

$-1.01531279$

$-0.90947729$

$-1.01531279$

$-0.90947729$

$-1.01531279$

$-0.90947729$

$-0.62219280$

$-0.51635736$

$-0.62219280$

$-0.51635736$

$-0.62219280$

$-0.51635736$

$-0.62219280$

$-0.51635736$

$-1.87711871$

$-1.77128315$

$-1.87711871$

$-1.77128315$

$-1.87711871$

$-1.77128315$

$-1.87711871$

$-1.77128315$

$-1.14041448$

$-1.03457904$

$-1.14041448$

$-1.03457904$

$-1.14041448$

$-1.03457904$

$-1.14041448$

$-1.03457904$
0.28552327

$-2.84103656$

$-0.14933950$

2. 53386545

2. 70317626

$-0.06465636$

$-3.16630960$

0.83680767

1.39042294

$-1.17733741$

$-0.71824002$

0.48583454

$-2.02032351$

2. 12751365

1. 46547222

$-1.51764262$

0.33353606

$-0.80663007$

$-0.51864588$

1. 01454031

0.20715170

0.01684670

0.01447497

$-0.27431613$

$-0.92779505$

1. 50780630

1. 73844802

$-2.64334393$

0.58428836

$-0.98762792$

$-1.16699600$

1. 83879781
$1.000 \#$ Electr. mom., s
\# Magnetic mom.

0.14458551

0.25042096

0.14458551

0.25042096

0.14458551

0.25042096

0.14458551

0.25042096

$-0.15045778$

$-0.04462234$

$-0.15045778$

$-0.04462234$

$-0.15045778$

$-0.04462234$

$-0.15045778$

$-0.04462234$

1. 24855602

1. 35439157

1. 24855602

1. 35439157

1. 24855602

1. 35439157

1. 24855602

1. 35439157

0.73230141

0.83813679

0.73230141

0.83813679

0.73230141

0.83813679

0.73230141

0.83813679
$-1.92402077$

1. 28364825

$-3.80253768$

3. 92070723

4.79198456

$-3.97607517$

$-0.23937233$

0.00205408

$-1.37317443$

0.05770473

0.29102093

1.07715988

$-0.73209721$

1.89315152

1.83700287

$-3.22850490$

$-1.19566798$

0.88619810

2. 23273444

$-1.69778812$

1.14478016

$-0.86988181$

$-2.33037305$

1. 78856528

$-4.91271639$

3. 02722144

3. 10971308

$-1.64726675$

4. 76961517

$-2.75485229$

$-2.99680257$

1. 40064204 
1.04285800

1. 04285800

1. 04285800

1. 04285800

1. 14869356

1. 14869356

1.14869356

$-1.76707089$

$-1.76707089$

$-1.76707089$

$-1.76707089$

$-1.66123557$

$-1.66123557$

$-1.66123557$

$-1.66123557$

1.57582414

1. 57582414

1. 57582414

1. 57582414

1.68165958

1.68165958

1.68165958

1.68165958

$-1.11259675$

$-1.11259675$

$-1.11259675$

$-1.11259675$

$-1.00676131$

$-1.00676131$

$-1.00676131$

64
1. 14869356

0.17553815

0.17553815

0.28137359

0.28137359

0.17553815

0.17553815

0.28137359

0.28137359

$-0.64616346$

$-0.64616346$

$-0.54032809$

$-0.54032809$

$-0.64616346$

$-0.64616346$

$-0.54032809$

$-0.54032809$

0.13101105

0.13101105

0.23684651

0.23684651

0.13101105

0.13101105

0.23684651

0.23684651

$-1.22435784$

$-1.22435784$

$-1.11852252$

$-1.11852252$

$-1.22435784$

$-1.22435784$

$-1.11852252$

$-1.11852252$

0 .

$$
5.6848 \quad-1.3648 \quad 1
$$

$-0.0019$

1.67341614

1.67341614

1.67341614

1.67341614

1.77925158

1. 77925158

1.77925158

1.77925158

$-1.26006997$

$-1.26006997$

$-1.26006997$

$-1.26006997$

$-1.15423441$

$-1.15423441$

$-1.15423441$

$-1.15423441$

1. 17254710

1.17254710

1. 17254710

1.17254710

1. 27838266

1. 27838266

1.27838266

1.27838266

$-1.80632699$

$-1.80632699$

$-1.80632699$

$-1.80632699$

$-1.70049155$

$-1.70049155$

$-1.70049155$

$-1.70049155$ $\begin{array}{ll}-1.3648 & 1.6068 \\ -0.0270 & 0.0918\end{array}$

$-0.20954360$

$-0.20954360$

$-0.10370816$

$-0.10370816$

$-0.20954360$

$-0.20954360$

$-0.10370816$

$-0.10370816$

0.53541940

0.53541940

0.64125490

0.64125490

0.53541940

0.53541940

0.64125490

0.64125490

0.01399621

0.01399621

0.11983165

0.11983165

0.01399621

0.01399621

0.11983165

0.11983165

1. 19734502

1.19734502

1. 30318046

1. 30318046

1.19734502

1.19734502

1. 30318046

1. 30318046
-1.01531279
-0.90947729
-1.01531279
-0.90947729
-1.01531279
-0.90947729
-1.01531279
-0.90947729
-0.62219280
-0.51635736
-0.62219280
-0.51635736
-0.62219280
-0.51635736
-0.62219280
-0.51635736
-1.87711871
-1.77128315
-1.87711871
-1.77128315
-1.87711871
-1.77128315
-1.87711871
-1.77128315
-1.14041448
-1.03457904
-1.14041448
-1.03457904
-1.14041448
-1.03457904
-1.14041448
-1.03457904

$-6.13754082$

8.56063747

5.22825623

$-7.16893196$

5.03565645

$-7.36496115$

$-4.99297714$

6.91752195

1. 81643832

$-1.58682799$

$-2.10589075$

1.66565979

$-1.14949167$

0.55194205

1. 49681175

$-0.66321093$

$-0.09603091$

0.29571980

$-0.11496272$

0.08171788

$-0.00644149$

$-0.15133591$

0.10250149

$-0.05189281$

1.03107786

$-1.28405368$

$-1.50603926$

1.87278032

$-0.85614043$

1. 12071288

1.25791252

$-1.63138926$

$$
\begin{gathered}
1.000 \text { \# Electr. mom., scale-fac } \\
\text { \# Magnetic mom. }
\end{gathered}
$$

0.14458551

0.25042096

0.14458551

0.25042096

0.14458551

0.25042096

0.14458551

0.25042096

$-0.15045778$

$-0.04462234$

$-0.15045778$

$-0.04462234$

$-0.15045778$

$-0.04462234$

$-0.15045778$

$-0.04462234$

1. 24855602

1.35439157

1. 24855602

1. 35439157

1. 24855602

1. 35439157

1. 24855602

1. 35439157

0.73230141

0.83813679

0.73230141

0.83813679

0.73230141

0.83813679

0.73230141

0.83813679

18.43928337

$-13.58934879$

$-6.29768324$

2.97930717

$-22.63347816$

17.48817825

12.79510593

$-9.33652782$

7.42854929

$-9.33778286$

$-9.10677147$

10.92099476

$-7.91580248$

9.53171921

9.08277321

$-10.26157284$

0.04554580

0.27718773

$-2.64659452$

1. 85711825

0.07148507

$-0.35470766$

2. 73270845

$-1.95544636$

$-1.85565472$

2. 49089885

2. 83718252

$-3.14459801$

0.30195042

$-1.31257105$

$-1.52292955$

2. 16905308 


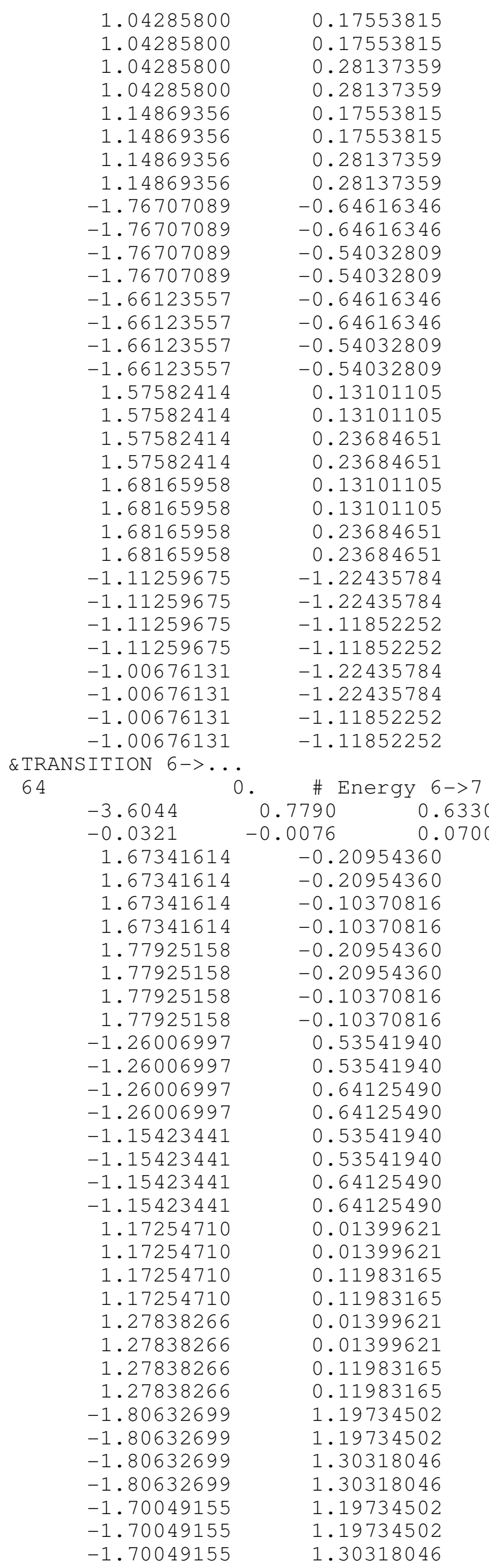

$-1.01531279$

$-0.90947729$

$-1.01531279$

$-0.90947729$

$-1.01531279$

$-0.90947729$

$-1.01531279$

$-0.90947729$

$-0.62219280$

$-0.51635736$

$-0.62219280$

$-0.51635736$

$-0.62219280$

$-0.51635736$

$-0.62219280$

$-0.51635736$

$-1.87711871$

$-1.77128315$

$-1.87711871$

$-1.77128315$

$-1.87711871$

$-1.77128315$

$-1.87711871$

$-1.77128315$

$-1.14041448$

$-1.03457904$

$-1.14041448$

$-1.03457904$

$-1.14041448$

$-1.03457904$

$-1.14041448$

$-1.03457904$

$-0.15045778$
6.83678532

$-13.67653656$

$-5.04992962$

10.26853561

$-1.48427951$

8.17209435

2. 04009914

$-7.12677908$

1.68866241

$-3.46354413$

$-2.82606554$

4. 46678305

$-0.84846437$

3.69026399

2. 76973224

$-5.19953680$

1. 04629576

$-2.04099441$

$-0.41457459$

0.86422879

$-0.30257997$

0.95547432

0.07829848

$-0.39106849$

$-3.20654106$

5.63041306

5.48609591

$-9.13709545$

2.56014872

$-4.49938869$

$-4.31900167$

7.25489855
1.000 \# Electr. mom., scale-fac \# Magnetic mom.

0.14458551

0.25042096

0.14458551

0.25042096

0.14458551

0.25042096

0.14458551

0.25042096

$-0.15045778$

$-0.04462234$

$-0.04462234$

$-0.15045778$

$-0.04462234$

$-0.15045778$

$-0.04462234$

1. 24855602

1. 35439157

1. 24855602

1.35439157

1. 24855602

1. 35439157

1. 24855602

1. 35439157

0.73230141

0.83813679

0.73230141

0.83813679

0.73230141

0.83813679

0.73230141
2.19687319

$-2.59077549$

1. 71449518

$-0.71669722$

$-1.22451711$

1. 20744133

$-2.24902582$

1.67876792

$-2.38288736$

$-0.86758715$

1.15188336

1.46484089

$-0.23896211$

3.43230104

1.37380660

$-3.87213206$

$-9.04432487$

6.61210155

8.58297825

$-6.31394243$

10.09406376

$-7.37360144$

$-9.61986828$

7.06519699

5.02235937

$-3.15955472$

$-5.03423166$

3.25163674

$-5.89357281$

3.99830055

6.29844046 


\begin{tabular}{|c|c|c|c|}
\hline-1.70049155 & 1.30318046 & 0.83813679 & -4.37093449 \\
\hline 1.04285800 & 0.17553815 & -1.01531279 & -1.93904853 \\
\hline 1.04285800 & 0.17553815 & -0.90947729 & 1.32300925 \\
\hline 1.04285800 & 0.28137359 & -1.01531279 & 2.91838026 \\
\hline 1.04285800 & 0.28137359 & -0.90947729 & -3.13538027 \\
\hline 1.14869356 & 0.17553815 & -1.01531279 & 2.17940331 \\
\hline 1.14869356 & 0.17553815 & -0.90947729 & -1.80414140 \\
\hline 1.14869356 & 0.28137359 & -1.01531279 & -1.35396898 \\
\hline 1.14869356 & 0.28137359 & -0.90947729 & 1.66537356 \\
\hline-1.76707089 & -0.64616346 & -0.62219280 & -0.02980846 \\
\hline-1.76707089 & -0.64616346 & -0.51635736 & -3.27797198 \\
\hline-1.76707089 & -0.54032809 & -0.62219280 & -2.65142202 \\
\hline-1.76707089 & -0.54032809 & -0.51635736 & 5.78913689 \\
\hline-1.66123557 & -0.64616346 & -0.62219280 & 1.72773182 \\
\hline-1.66123557 & -0.64616346 & -0.51635736 & 2.27999711 \\
\hline-1.66123557 & -0.54032809 & -0.62219280 & 1.62309182 \\
\hline-1.66123557 & -0.54032809 & -0.51635736 & -5.20170021 \\
\hline 1.57582414 & 0.13101105 & -1.87711871 & 0.68970317 \\
\hline 1.57582414 & 0.13101105 & -1.77128315 & -0.89477712 \\
\hline 1.57582414 & 0.23684651 & -1.87711871 & -0.20958564 \\
\hline 1.57582414 & 0.23684651 & -1.77128315 & 0.07728807 \\
\hline 1.68165958 & 0.13101105 & -1.87711871 & -0.76394397 \\
\hline 1.68165958 & 0.13101105 & -1.77128315 & 0.99854368 \\
\hline 1.68165958 & 0.23684651 & -1.87711871 & 0.47183532 \\
\hline 1.68165958 & 0.23684651 & -1.77128315 & -0.47773117 \\
\hline-1.11259675 & -1.22435784 & -1.14041448 & 0.07095761 \\
\hline-1.11259675 & -1.22435784 & -1.03457904 & 0.89947295 \\
\hline-1.11259675 & -1.11852252 & -1.14041448 & 0.70033419 \\
\hline-1.11259675 & -1.11852252 & -1.03457904 & -2.36118364 \\
\hline-1.00676131 & -1.22435784 & -1.14041448 & -0.23692697 \\
\hline-1.00676131 & -1.22435784 & -1.03457904 & -0.41010121 \\
\hline-1.00676131 & -1.11852252 & -1.14041448 & -0.23834430 \\
\hline-1.00676131 & -1.11852252 & -1.03457904 & 1.37890339 \\
\hline & \# Energy 6->8 & & \\
\hline-0.1392 & -0.0092 & 1.000 & Electr. mom., scale-fac \\
\hline 0.1073 & -0.1115 & $\#$ & Magnetic mom. \\
\hline 1.67341614 & -0.20954360 & 0.14458551 & 1.83511484 \\
\hline 1.67341614 & -0.20954360 & 0.25042096 & -1.27980793 \\
\hline 1.67341614 & -0.10370816 & 0.14458551 & 0.77398556 \\
\hline 1.67341614 & -0.10370816 & 0.25042096 & -1.20118093 \\
\hline 1.77925158 & -0.20954360 & 0.14458551 & -2.57772732 \\
\hline 1.77925158 & -0.20954360 & 0.25042096 & 1.98915684 \\
\hline 1.77925158 & -0.10370816 & 0.14458551 & 0.51059359 \\
\hline 1.77925158 & -0.10370816 & 0.25042096 & -0.04637519 \\
\hline-1.26006997 & 0.53541940 & -0.15045778 & 4.64868879 \\
\hline-1.26006997 & 0.53541940 & -0.04462234 & -2.03180695 \\
\hline-1.26006997 & 0.64125490 & -0.15045778 & -3.22969770 \\
\hline-1.26006997 & 0.64125490 & -0.04462234 & 0.56258631 \\
\hline-1.15423441 & 0.53541940 & -0.15045778 & -3.19149685 \\
\hline-1.15423441 & 0.53541940 & -0.04462234 & 0.99861985 \\
\hline-1.15423441 & 0.64125490 & -0.15045778 & 1.91343510 \\
\hline-1.15423441 & 0.64125490 & -0.04462234 & 0.41494432 \\
\hline 1.17254710 & 0.01399621 & 1.24855602 & 3.58485484 \\
\hline 1.17254710 & 0.01399621 & 1.35439157 & -2.68484640 \\
\hline 1.17254710 & 0.11983165 & 1.24855602 & -4.02872229 \\
\hline 1.17254710 & 0.11983165 & 1.35439157 & 3.02432799 \\
\hline 1.27838266 & 0.01399621 & 1.24855602 & -3.51130819 \\
\hline 1.27838266 & 0.01399621 & 1.35439157 & 2.59729242 \\
\hline 1.27838266 & 0.11983165 & 1.24855602 & 4.01736307 \\
\hline 1.27838266 & 0.11983165 & 1.35439157 & -2.97508907 \\
\hline-1.80632699 & 1.19734502 & 0.73230141 & 4.97840834 \\
\hline-1.80632699 & 1.19734502 & 0.83813679 & -3.43443060 \\
\hline-1.80632699 & 1.30318046 & 0.73230141 & -4.03337288 \\
\hline-1.80632699 & 1.30318046 & 0.83813679 & 2.73284221 \\
\hline-1.70049155 & 1.19734502 & 0.73230141 & -4.71228123 \\
\hline-1.70049155 & 1.19734502 & 0.83813679 & 3.11628079 \\
\hline-1.70049155 & 1.30318046 & 0.73230141 & 3.82061267 \\
\hline
\end{tabular}




\begin{abstract}
$-1.70049155$
1.04285800

1.04285800

1.04285800

1.04285800

1. 14869356

1.14869356

1.14869356

1.14869356

$-1.76707089$

$-1.76707089$

$-1.76707089$

$-1.76707089$

$-1.66123557$

$-1.66123557$

$-1.66123557$

$-1.66123557$

1. 57582414

1. 57582414

1.57582414

1. 57582414

1.68165958

1.68165958

1.68165958

1.68165958

$-1.11259675$

$-1.11259675$

$-1.11259675$

$-1.11259675$

$-1.00676131$

$-1.00676131$

$-1.00676131$

$-1.00676131$
\end{abstract}

64

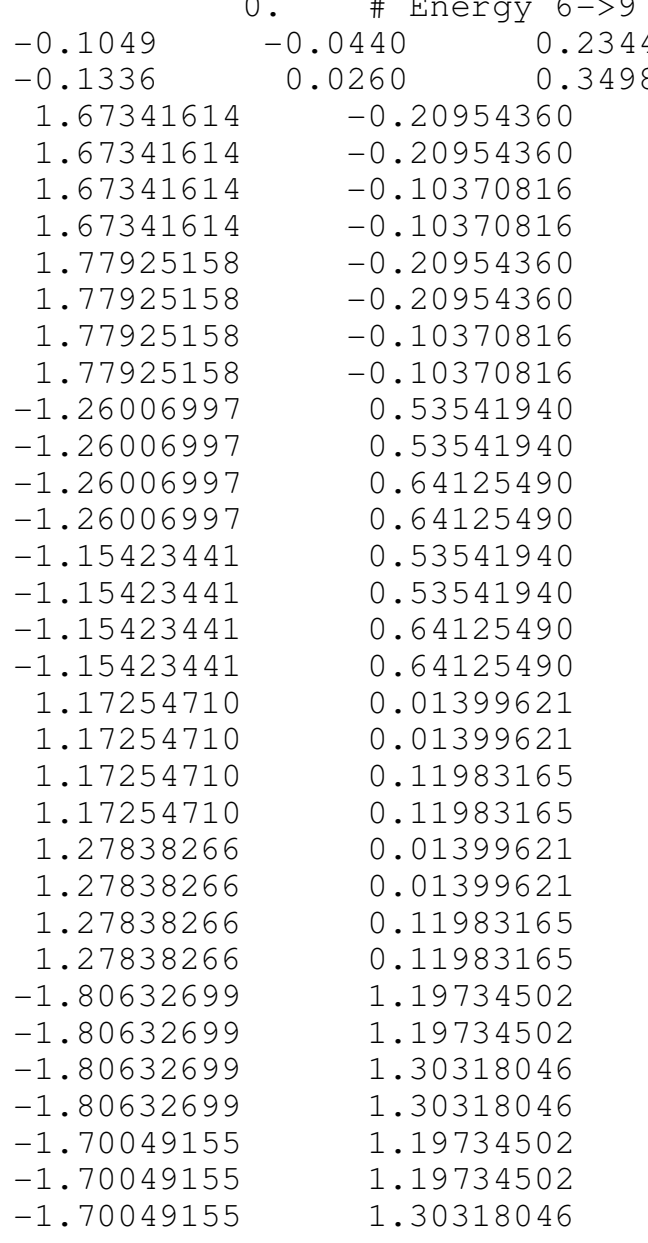

1. 30318046

0.17553815

0.17553815

0.28137359

0.28137359

0.17553815

0.17553815

0.28137359

0.28137359

$-0.64616346$

$-0.64616346$

$-0.54032809$

$-0.54032809$

$-0.64616346$

$-0.64616346$

$-0.54032809$

$-0.54032809$

0.13101105

0.13101105

0.23684651

0.23684651

0.13101105

0.13101105

0.23684651

0.23684651

$-1.22435784$

$-1.22435784$

$-1.11852252$

$-1.11852252$

$-1.22435784$

$-1.22435784$

$-1.11852252$

$-1.11852252$

0 .

\# Energy 6->9

$-0.20954360$

$-0.20954360$

$-0.20954360$

0.10370816

0.53541940

0.64125490

0.64125490

.53541940

.53541940

0.01399621

0.01399621

0.01399621

0.11983165

1.19734502

1.30318046
0.83813679
-1.01531279
-0.90947729
-1.01531279
-0.90947729
-1.01531279
-0.90947729
-1.01531279
-0.90947729
-0.62219280
-0.51635736
-0.62219280
-0.51635736
-0.62219280
-0.51635736
-0.62219280
-0.51635736
-1.87711871
-1.77128315
-1.87711871
-1.77128315
-1.87711871
-1.77128315
-1.87711871
-1.77128315
-1.14041448
-1.03457904
-1.14041448
-1.03457904
-1.14041448
-1.03457904
-1.14041448
-1.03457904

$-2.45923591$

$-0.26546299$

0.08982946

$-0.34329996$

0.44860417

1.03077722

$-0.95069194$

$-0.01940375$

$-0.00425826$

$-0.06658133$

0.19462018

0.36948678

$-0.34793827$

$-0.04886410$

0.09108967

$-0.27282995$

$-0.02839895$

0.24890003

$-0.37925890$

$-0.09881610$

0.14047743

$-0.20891853$

0.31455415

0.11476316

$-0.16046701$

$-0.39741758$

0.35975200

0.37221438

$-0.27710372$

0.36939058

$-0.39250582$

$-0.38042909$

0.37645835
1.000 \# Electr. mom., scale-fac \# Magnetic mom.

0.14458551

0.25042096

0.14458551

0.25042096

0.14458551

0.25042096

0.14458551

0.25042096

$-0.15045778$

$-0.04462234$

$-0.15045778$

$-0.04462234$

$-0.15045778$

$-0.04462234$

$-0.15045778$

$-0.04462234$

1. 24855602

1. 35439157

1. 24855602

1. 35439157

1. 24855602

1. 35439157

1. 24855602

1. 35439157

0.73230141

0.83813679

0.73230141

0.83813679

0.73230141

0.83813679

0.73230141
$-12.63364506$

10.65882683

9.83026791

$-7.86466026$

11.75876999

$-9.81984234$

$-9.35096836$

7. 43195152

$-1.15168822$

2.53349900

2.50401521

$-3.67447710$

1. 96900427

$-3.38700533$

$-3.39339423$

4.61254454

$-10.12096596$

7. 42914152

10.15266323

$-7.46683550$

10.00326443

$-7.21381807$

$-10.07387066$

7.27662086

$-0.75444877$

0.47750485

1.21366990

$-0.88445044$

1. 17275584

$-0.85721439$

$-1.65819776$ 
-1.70049155
1.04285800
1.04285800
1.04285800
1.04285800
1.14869356
1.14869356
1.14869356
1.14869356
-1.76707089
-1.76707089
-1.76707089
-1.76707089
-1.66123557
-1.66123557
-1.66123557
-1.66123557
1.57582414
1.57582414
1.57582414
1.57582414
1.68165958
1.68165958
1.68165958
1.68165958
-1.11259675
-1.11259675
-1.11259675
-1.11259675
-1.00676131
-1.00676131
-1.00676131
-1.00676131
1.06

\&TRANSITION $7->\ldots$

640 .
1.30318046

0.17553815

0.17553815

0.28137359

0.28137359

0.17553815

0.17553815

0.28137359

0.28137359

$-0.64616346$

$-0.64616346$

$-0.54032809$

$-0.54032809$

$-0.64616346$

$-0.64616346$

$-0.54032809$

$-0.54032809$

0.13101105

0.13101105

0.23684651

0.23684651

0.13101105

0.13101105

0.23684651

0.23684651

$-1.22435784$

$-1.22435784$

$-1.11852252$

$-1.11852252$

$-1.22435784$

$-1.22435784$

$-1.11852252$

$-1.11852252$

\# Energy 7->8

$\begin{array}{lll}0.2499 & 0.7974 & 0.6052\end{array}$

$0.2561-0.3625 \quad-0.4080$

1.67341614

1.67341614

1.67341614

1.67341614

1.77925158

1.77925158

1. 77925158

1. 77925158

$-1.26006997$

$-1.26006997$

$-1.26006997$

$-1.26006997$

$-1.15423441$

$-1.15423441$

$-1.15423441$

$-1.15423441$

1. 17254710

1. 17254710

1. 17254710

1.17254710

1.27838266

1. 27838266

1. 27838266

1.27838266

$-1.80632699$

$-1.80632699$

$-1.80632699$

$-1.80632699$

$-1.70049155$

$-1.70049155$
$-0.20954360$

$-0.20954360$

$-0.10370816$

$-0.10370816$

$-0.20954360$

$-0.20954360$

$-0.10370816$

$-0.10370816$

0.53541940

0.53541940

0.64125490

0.64125490

0.53541940

0.53541940

0.64125490

0.64125490

0.01399621

0.01399621

0.11983165

0.11983165

0.01399621

0.01399621

0.11983165

0.11983165

1.19734502

1. 19734502

1. 30318046

1.30318046

1. 19734502

1. 19734502
0.83813679
-1.01531279
-0.90947729
-1.01531279
-0.90947729
-1.01531279
-0.90947729
-1.01531279
-0.90947729
-0.62219280
-0.51635736
-0.62219280
-0.51635736
-0.62219280
-0.51635736
-0.62219280
-0.51635736
-1.87711871
-1.77128315
-1.87711871
-1.77128315
-1.87711871
-1.77128315
-1.87711871
-1.77128315
-1.14041448
-1.03457904
-1.14041448
-1.03457904
-1.14041448
-1.03457904
-1.14041448
-1.03457904

1. 28199220

2. 81360579

$-2.69718647$

$-1.46558487$

1. 49661887

$-5.45961857$

5.52193356

3. 74993157

$-3.99050212$

2. 45042920

$-1.17478955$

$-1.36837578$

0.26090673

$-3.72827792$

2. 19927740

2. 36143422

$-1.08255923$

$-0.89487684$

1.36721539

0.60827166

$-0.95989513$

0.68185318

$-1.02898180$

$-0.46698150$

0.73177809

$-0.17655477$

$-0.06666737$

0.11554862

0.34995130

0.13902877

$-0.00383365$

$-0.15333864$

$-0.13076966$
1.000 \# Electr. mom., scale-fac \# Magnetic mom.

0.14458551 $-8.20237160$

0.25042096

7.01862240

0.14458551

0.25042096

0.14458551

0.25042096

0.14458551

0.25042096

$-0.15045778$

$-0.04462234$

$-0.15045778$

$-0.04462234$

$-0.15045778$

$-0.04462234$

$-0.15045778$

$-0.04462234$

1. 24855602

1. 35439157

1. 24855602

1. 35439157

1. 24855602

1. 35439157

1. 24855602

1.35439157

0.73230141

0.83813679

0.73230141

0.83813679

0.73230141

0.83813679
11.58402729

$-10.09823990$

5.37683821

$-4.37639475$

$-7.68653154$

6.46627855

18.83335495

$-6.80320835$

$-11.22449970$

$-0.92627895$

$-14.42285824$

4.06475019

7.64695263

3. 00244546

2.17462754

$-1.71135938$

$-2.94843245$

2. 28960872

$-2.21438718$

1.76962090

3. 09719038

$-2.41247034$

20.27997208

$-14.55292320$

$-17.01309204$

12.04824352

$-18.09763145$

12.49929619 


-1.70049155
-1.70049155
1.04285800
1.04285800
1.04285800
1.04285800
1.14869356
1.14869356
1.14869356
1.14869356
-1.76707089
-1.76707089
-1.76707089
-1.76707089
-1.66123557
-1.66123557
-1.66123557
-1.66123557
1.57582414
1.57582414
1.57582414
1.57582414
1.68165958
1.68165958
1.68165958
1.68165958
-1.11259675
-1.11259675
-1.11259675
-1.11259675
-1.00676131
-1.00676131
-1.00676131
-1.00676131

64
1.30318046

1. 30318046

0.17553815

0.17553815

0.28137359

0.28137359

0.17553815

0.17553815

0.28137359

0.28137359

$-0.64616346$

$-0.64616346$

$-0.54032809$

$-0.54032809$

$-0.64616346$

$-0.64616346$

$-0.54032809$

$-0.54032809$

0.13101105

0.13101105

0.23684651

0.23684651

0.13101105

0.13101105

0.23684651

0.23684651

$-1.22435784$

$-1.22435784$

$-1.11852252$

$-1.11852252$

$-1.22435784$

$-1.22435784$

$-1.11852252$

$-1.11852252$

$$
0 . \quad \text { \# Energy 7->9 }
$$

$\begin{array}{lll}2.2847 & -0.2578 & -0.7497\end{array}$

$0.0927-0.0464 \quad-0.2085$

1.67341614

1.67341614

1.67341614

1.67341614

1.77925158

1. 77925158

1. 77925158

1.77925158

$-1.26006997$

$-1.26006997$

$-1.26006997$

$-1.26006997$

$-1.15423441$

$-1.15423441$

$-1.15423441$

$-1.15423441$

1.17254710

1.17254710

1. 17254710

1.17254710

1.27838266

1.27838266

1. 27838266

1.27838266

$-1.80632699$

$-1.80632699$

$-1.80632699$

$-1.80632699$

$-1.70049155$

$-1.70049155$

$-0.20954360$

$-0.20954360$

$-0.10370816$

$-0.10370816$

$-0.20954360$

$-0.20954360$

$-0.10370816$

$-0.10370816$

0.53541940

0.53541940

0.64125490

0.64125490

0.53541940

0.53541940

0.64125490

0.64125490

0.01399621

0.01399621

0.11983165

0.11983165

0.01399621

0.01399621

0.11983165

0.11983165

1. 19734502

1.19734502

1. 30318046

1. 30318046

1.19734502

1. 19734502

$$
\begin{array}{r}
0.73230141 \\
0.83813679 \\
-1.01531279 \\
-0.90947729 \\
-1.01531279 \\
-0.90947729 \\
-1.01531279 \\
-0.90947729 \\
-1.01531279 \\
-0.90947729 \\
-0.62219280 \\
-0.51635736 \\
-0.62219280 \\
-0.51635736 \\
-0.62219280 \\
-0.51635736 \\
-0.62219280 \\
-0.51635736 \\
-1.87711871 \\
-1.77128315 \\
-1.87711871 \\
-1.77128315 \\
-1.87711871 \\
-1.77128315 \\
-1.87711871 \\
-1.77128315 \\
-1.14041448 \\
-1.03457904 \\
-1.14041448 \\
-1.03457904 \\
-1.14041448 \\
-1.03457904 \\
-1.14041448 \\
-1.03457904 \\
\end{array}
$$
1.000 \# Electr. mom., scale-fac \# Magnetic mom.

0.14458551

0.25042096

0.14458551

0.25042096

0.14458551

0.25042096

0.14458551

0.25042096

$-0.15045778$

$-0.04462234$

$-0.15045778$

$-0.04462234$

$-0.15045778$

$-0.04462234$

$-0.15045778$

$-0.04462234$

1. 24855602

1. 35439157

1.24855602

1. 35439157

1. 24855602

1.35439157

1. 24855602

1.35439157

0.73230141

0.83813679

0.73230141

0.83813679

0.73230141

0.83813679
15.38337135

$-10.42804050$

$-1.59488201$

2. 44505334

0.23164944

$-0.88682872$

0.58648497

$-1.54289865$

1. 55551767

$-0.86925954$

3. 74551797

$-1.76378071$

$-1.57095218$

0.18224715

$-4.77394962$

2.84687400

2.13205981

$-1.31185865$

$-0.17889144$

0.40192997

0.29424110

$-0.63041520$

$-0.00455179$

$-0.12077686$

$-0.03296506$

0.23049185

$-1.51063788$

0.95506555

0.94659168

0.18936668

1.43399429

$-1.26019859$

$-1.17421019$

0.63348985
-2.81919122
3.73455358
5.76248884
-7.02016306
0.18812905
-0.84207243
-2.23308921
3.18469524
10.03382301
-4.93284035
-8.47334480
3.98393083
-3.66531181
-0.97435683
1.95230281
2.35010099
9.88872242
-7.49837923
-10.43518162
7.92334557
-10.28722477
7.76555920
11.00745678
-8.30126572
-0.23396324
0.59607154
2.25917745
-2.10954189
-0.06356540
-0.79764956 
$-1.70049155$

$-1.70049155$

1. 04285800

1. 04285800

1.04285800

1. 04285800

1. 14869356

1. 14869356

1. 14869356

1.14869356

$-1.76707089$

$-1.76707089$

$-1.76707089$

$-1.76707089$

$-1.66123557$

$-1.66123557$

$-1.66123557$

$-1.66123557$

1.57582414

1.57582414

1.57582414

1.57582414

1.68165958

1.68165958

1.68165958

1.68165958

$-1.11259675$

$-1.11259675$

$-1.11259675$

$-1.11259675$

$-1.00676131$

$-1.00676131$

$-1.00676131$

$-1.00676131$

\&TRANSITION $8->\ldots$ 64
$-3.3986$
0.0739
1.67341614
1.67341614
1.67341614
1.67341614
1.77925158
1. 77925158
1. 77925158
1.77925158
$-1.26006997$
$-1.26006997$
$-1.26006997$
$-1.26006997$
$-1.15423441$
$-1.15423441$
$-1.15423441$
$-1.15423441$
1. 17254710
1. 17254710
1. 17254710
1.17254710
1.27838266
1.27838266
1. 27838266
1. 27838266
$-1.80632699$
$-1.80632699$
$-1.80632699$
$-1.80632699$
$-1.70049155$

$$
0.73230141
$$

0.83813679

$-1.01531279$

$-0.90947729$

$-1.01531279$

$-0.90947729$

$-1.01531279$

$-0.90947729$

$-1.01531279$

$-0.90947729$

$-0.62219280$

$-0.51635736$

$-0.62219280$

$-0.51635736$

$-0.62219280$

$-0.51635736$

$-0.62219280$

$-0.51635736$

$-1.87711871$

$-1.77128315$

$-1.87711871$

$-1.77128315$

$-1.87711871$

$-1.77128315$

$-1.87711871$

$-1.77128315$

$-1.14041448$

$-1.03457904$

$-1.14041448$

$-1.03457904$

$-1.14041448$

$-1.03457904$

$-1.14041448$

$-1.03457904$
$-2.78286028$

2. 90878081

4.50882244

$-4.50825787$

$-5.57693195$

6.07739878

$-3.74367976$

3. 84713364

3. 90944839

$-4.45599031$

$-2.51567435$

5.40679264

5.56643009

$-8.07936859$

0.89426368

$-3.91959310$

$-4.48607969$

6.76745367

$-0.43040633$

0.42182949

0.28055429

$-0.11114641$

0.51951987

$-0.56009978$

$-0.45822671$

0.39380482

$-0.68167722$

$-0.14380360$

0.22487287

1. 18567657

0.75037295

$-0.24683097$

$-0.53767347$

$-0.36807138$
$-1.11852252$

0. \# Energy 8->9 $-0.0660 \quad-0.1307$

$-0.20954360$

$-0.20954360$

$-0.10370816$

$-0.10370816$

$-0.20954360$

$-0.20954360$

$-0.10370816$

$-0.10370816$

0.53541940

0.53541940

0.64125490

0.64125490

0.53541940

0.53541940

0.64125490

0.64125490

0.01399621

0.01399621

0.11983165

0.11983165

0.01399621

0.01399621

0.11983165

0.11983165

1. 19734502

1. 19734502

1.30318046

1. 30318046

1. 19734502

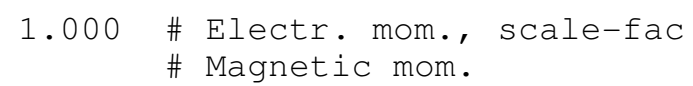

0.14458551

0.25042096

0.14458551

0.25042096

0.14458551

0.25042096

0.14458551

0.25042096

$-0.15045778$

$-0.04462234$

$-0.15045778$

$-0.04462234$

$-0.15045778$

$-0.04462234$

$-0.15045778$

$-0.04462234$

1. 24855602

1. 35439157

1. 24855602

1. 35439157

1. 24855602

1. 35439157

1. 24855602

1. 35439157

0.73230141

0.83813679

0.73230141

0.83813679

0.73230141
7.18386650

$-6.10984850$

$-4.74997854$

4.41633987

$-6.90071249$

5.60411930

4.48687315

$-3.95891523$

$-6.74917412$

$-0.33161682$

4.33186769

2. 72118974

7.33155823

$-1.08871496$

$-5.31651354$

$-0.85539794$

$-4.76600218$

3.75478649

3.94654417

$-3.09024096$

4.99265766

$-3.90728426$

$-4.20688581$

3. 25518966

$-5.93734312$

4.47591972

6.00525522

$-4.65809870$

6.18368053 


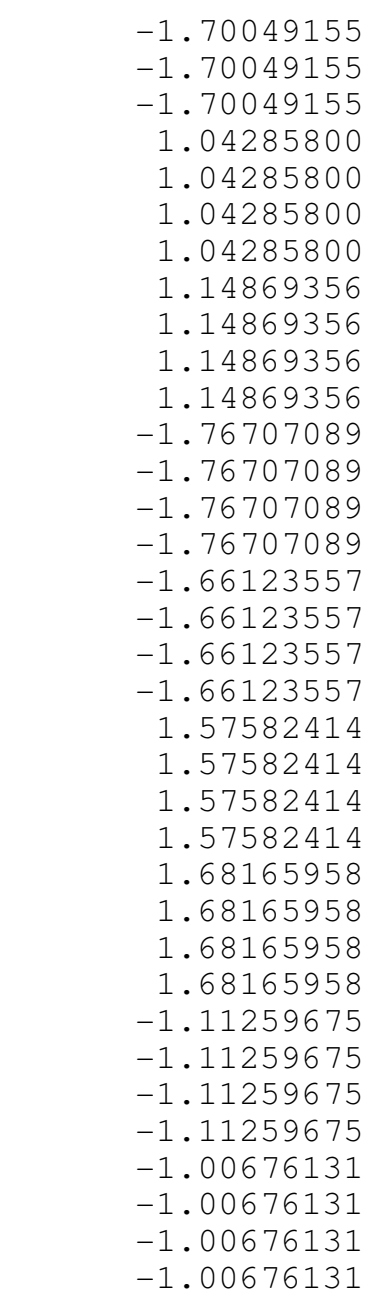

\&PERMANENT MOMENTS

64
1.19734502

1. 30318046

1. 30318046

0.17553815

0.17553815

0.28137359

0.28137359

0.17553815

0.17553815

0.28137359

0.28137359

$-0.64616346$

$-0.64616346$

$-0.54032809$

$-0.54032809$

$-0.64616346$

$-0.64616346$

$-0.54032809$

$-0.54032809$

0.13101105

0.13101105

0.23684651

0.23684651

0.13101105

0.13101105

0.23684651

0.23684651

$-1.22435784$

$-1.22435784$

$-1.11852252$

$-1.11852252$

$-1.22435784$

$-1.22435784$

$-1.11852252$

$-1.11852252$

\# Energy 1->1
0.83813679

0.73230141

0.83813679

$-1.01531279$

$-0.90947729$

$-1.01531279$

$-0.90947729$

$-1.01531279$

$-0.90947729$

$-1.01531279$

$-0.90947729$

$-0.62219280$

$-0.51635736$

$-0.62219280$

$-0.51635736$

$-0.62219280$

$-0.51635736$

$-0.62219280$

$-0.51635736$

$-1.87711871$

$-1.77128315$

$-1.87711871$

$-1.77128315$

$-1.87711871$

$-1.77128315$

$-1.87711871$

$-1.77128315$

$-1.14041448$

$-1.03457904$

$-1.14041448$

$-1.03457904$

$-1.14041448$

$-1.03457904$

$-1.14041448$

$-1.03457904$
-4.57555628
-6.16755152
4.69600058
3.16534543
-5.84908390
-0.88443291
2.60373807
-1.58535385
4.18103695
0.51729536
-2.22801733
-1.52377570
0.35188138
0.59691852
0.42469519
1.52273154
0.07145108
-0.32035372
-0.82057339
0.52958834
-0.93164068
-0.24909855
0.40797210
-0.32658169
0.63544089
0.19455937
-0.34309769
-0.43959624
1.37508416
1.64286208
-3.20675349
0.13065618
-0.76934803
-0.99674320
2.10718012
-
$2.4809-2.5446 \quad-4.8875$

1.67341614

1.67341614

1.67341614

1.67341614

1. 77925158

1. 77925158

1. 77925158

1. 77925158

$-1.26006997$

$-1.26006997$

$-1.26006997$

$-1.26006997$

$-1.15423441$

$-1.15423441$

$-1.15423441$

$-1.15423441$

1. 17254710

1. 17254710

1. 17254710

1. 17254710

1.27838266

1. 27838266

1.27838266

1.27838266

$-1.80632699$

$-1.80632699$

$-1.80632699$

$-1.80632699$

$-1.70049155$
$-0.20954360$

$-0.20954360$

$-0.10370816$

$-0.10370816$

$-0.20954360$

$-0.20954360$

$-0.10370816$

$-0.10370816$

0.53541940

0.53541940

0.64125490

0.64125490

0.53541940

0.53541940

0.64125490

0.64125490

0.01399621

0.01399621

0.11983165

0.11983165

0.01399621

0.01399621

0.11983165

0.11983165

1.19734502

1.19734502

1.30318046

1.30318046

1. 19734502
\# Electr. mom.

$0.14458551 \quad 250.89860535$

$0.25042096 \quad-210.69795227$

$0.14458551 \quad-334.51431274$

$0.25042096 \quad 286.58636475$

$0.14458551 \quad-210.29232788$

$0.25042096 \quad 174.04431152$

$0.14458551 \quad 265.57394409$

$0.25042096 \quad-223.44221497$

$-0.15045778-164.25187683$

$-0.04462234 \quad 135.49819946$

$-0.15045778 \quad 155.12239075$

$-0.04462234 \quad-132.93452454$

$-0.15045778 \quad 162.80255127$

$-0.04462234 \quad-131.65435791$

$-0.15045778 \quad-154.12556458$

$-0.04462234 \quad 129.86929321$

$1.24855602 \quad-13.79869175$

$1.35439157 \quad 12.92099857$

$1.24855602 \quad 20.34925842$

$1.35439157 \quad-17.33144379$

$1.24855602 \quad 11.36728573$

$1.35439157 \quad-11.48720169$

$1.24855602 \quad-22.05432320$

$1.35439157 \quad 18.74776459$

$0.73230141 \quad 24.94039726$

$0.83813679 \quad-20.65562057$

$0.73230141 \quad-23.24452400$

$0.83813679 \quad 19.45237541$

$0.73230141 \quad-26.14797974$ 
$-1.70049155$

$-1.70049155$

$-1.70049155$

1. 04285800

1.04285800

1. 04285800

1. 04285800

1. 14869356

1.14869356

1. 14869356

1.14869356

$-1.76707089$

$-1.76707089$

$-1.76707089$

$-1.76707089$

$-1.66123557$

$-1.66123557$

$-1.66123557$

$-1.66123557$

1. 57582414

1.57582414

1.57582414

1.57582414

1.68165958

1.68165958

1.68165958

1.68165958

$-1.11259675$

$-1.11259675$

$-1.11259675$

$-1.11259675$

$-1.00676131$

$-1.00676131$

$-1.00676131$

$-1.00676131$

64
0.83813679

0.73230141

0.83813679

$-1.01531279$

$-0.90947729$

$-1.01531279$

$-0.90947729$

$-1.01531279$

$-0.90947729$

$-1.01531279$

$-0.90947729$

$-0.62219280$

$-0.51635736$

$-0.62219280$

$-0.51635736$

$-0.62219280$

$-0.51635736$

$-0.62219280$

$-0.51635736$

$-1.87711871$

$-1.77128315$

$-1.87711871$

$-1.77128315$

$-1.87711871$

$-1.77128315$

$-1.87711871$

$-1.77128315$

$-1.14041448$

$-1.03457904$

$-1.14041448$

$-1.03457904$

$-1.14041448$

$-1.03457904$

$-1.14041448$

$-1.03457904$
21.69487381

23.50176620

$-19.80238342$

186.03903198

$-222.22128296$

$-142.95770264$

173.96083069

$-185.34403992$

224.59631348

116.85200500

$-149.77328491$

57.14879227

$-48.40115356$

$-61.53103638$

62.91403580

$-75.01940155$

56.49478149

72.59352112

$-66.72058105$

2. 40177464

$-6.85191441$

$-4.77508354$

13.69406891

$-2.82398772$

6.67140150

1.62670875

$-8.13638496$

$-9.91673660$

2. 05883431

1.09937394

14.58423424

16.64749146

$-13.75502491$

$-13.97577667$ 5.88511562
1.67341614

1.67341614

1.67341614

1.67341614

1. 77925158

1.77925158

1. 77925158

1.77925158

$-1.26006997$

$-1.26006997$

$-1.26006997$

$-1.26006997$

$-1.15423441$

$-1.15423441$

$-1.15423441$

$-1.15423441$

1. 17254710

1. 17254710

1. 17254710

1. 17254710

1.27838266

1.27838266

1. 27838266

1.27838266

$-1.80632699$

$-1.80632699$

$-1.80632699$

$-1.80632699$

$-1.70049155$

$-1.70049155$
$-2.2880 \quad-2.8177$

$-0.20954360$

$-0.20954360$

$-0.10370816$

$-0.10370816$

$-0.20954360$

$-0.20954360$

$-0.10370816$

$-0.10370816$

0.53541940

0.53541940

0.64125490

0.64125490

0.53541940

0.53541940

0.64125490

0.64125490

0.01399621

0.01399621

0.11983165

0.11983165

0.01399621

0.01399621

0.11983165

0.11983165

1. 19734502

1. 19734502

1.30318046

1.30318046

1. 19734502

1. 19734502
\# Electr. mom.

0.14458551

0.25042096

0.14458551

0.25042096

0.14458551

0.25042096

0.14458551

0.25042096

$-0.15045778$

$-0.04462234$

$-0.15045778$

$-0.04462234$

$-0.15045778$

$-0.04462234$

$-0.15045778$

$-0.04462234$

1. 24855602

1. 35439157

1. 24855602

1. 35439157

1. 24855602

1.35439157

1. 24855602

1.35439157

0.73230141

0.83813679

0.73230141

0.83813679

0.73230141

0.83813679
233.67961121

$-195.99702454$

$-303.55297852$

260.43695068

$-196.61845398$

161.46055603

239.07037354

$-200.19320679$

$-184.93420410$

151.35591125

172.96591187

$-146.74237061$

187.63575745

$-150.55238342$

$-176.29588318$

147.33091736

$-37.14940643$

29.42402458

41.58988953

$-32.52265930$

37.83063126

$-30.11312675$

$-45.65163040$

35.53381348

26.73082542

$-21.09237099$

$-23.62072182$

18.99886703

$-30.10279655$

23.63011932 


-1.70049155
-1.70049155
1.04285800
1.04285800
1.04285800
1.04285800
1.14869356
1.14869356
1.14869356
1.14869356
-1.76707089
-1.76707089
-1.76707089
-1.76707089
-1.66123557
-1.66123557
-1.66123557
-1.66123557
1.57582414
1.57582414
1.57582414
1.57582414
1.68165958
1.68165958
1.68165958
1.68165958
-1.11259675
-1.11259675
-1.11259675
-1.11259675
-1.00676131
-1.00676131
-1.00676131
-1.00676131
1

64
1.4630

1.67341614

1.67341614

1.67341614

1.67341614

1.77925158

1.77925158

1.77925158

1. 77925158

$-1.26006997$

$-1.26006997$

$-1.26006997$

$-1.26006997$

$-1.15423441$

$-1.15423441$

$-1.15423441$

$-1.15423441$

1.17254710

1.17254710

1.17254710

1.17254710

1.27838266

1.27838266

1.27838266

1.27838266

$-1.80632699$

$-1.80632699$

$-1.80632699$

$-1.80632699$

$-1.70049155$

$-1.70049155$

$-1.70049155$
1.30318046

1. 30318046

0.17553815

0.17553815

0.28137359

0.28137359

0.17553815

0.17553815

0.28137359

0.28137359

$-0.64616346$

$-0.64616346$

$-0.54032809$

$-0.54032809$

$-0.64616346$

$-0.64616346$

$-0.54032809$

$-0.54032809$

0.13101105

0.13101105

0.23684651

0.23684651

0.13101105

0.13101105

0.23684651

0.23684651

$-1.22435784$

$-1.22435784$

$-1.11852252$

$-1.11852252$

$-1.22435784$

$-1.22435784$

$-1.11852252$

$-1.11852252$ \# Energy 3->3 $\begin{array}{ll}-0.9351 & -3.1763\end{array}$

$-0.20954360$

$-0.20954360$

$-0.10370816$

$-0.10370816$

$-0.20954360$

$-0.20954360$

$-0.10370816$

$-0.10370816$

0.53541940

0.53541940

0.64125490

0.64125490

0.53541940

0.53541940

0.64125490

0.64125490

0.01399621

0.01399621

0.11983165

0.11983165

0.01399621

0.01399621

0.11983165

0.11983165

1.19734502

1.19734502

1.30318046

1.30318046

1.19734502

1.19734502

1.30318046
0.73230141

0.83813679

$-1.01531279$

$-0.90947729$

$-0.90947729$

$-1.01531279$

$-0.90947729$

$-1.01531279$

$-0.90947729$

$-0.62219280$

$-0.51635736$

$-0.62219280$

$-0.51635736$

$-0.62219280$

$-0.51635736$

$-0.62219280$

$-0.51635736$

$-1.87711871$

$-1.77128315$

$-1.87711871$

$-1.77128315$

$-1.87711871$

$-1.77128315$

$-1.87711871$

$-1.77128315$

$-1.14041448$

$-1.03457904$

$-1.14041448$

$-1.03457904$

$-1.14041448$

$-1.03457904$

$-1.14041448$

$-1.03457904$
$-1.01531279$

25.32311821

$-20.32834053$

204.85382080

$-244.47689819$

$-159.33798218$

192.23075867

$-204.77177429$

247.23146057

136.08317566

$-170.84495544$

53.12852478

$-51.57931900$

$-61.66671371$

70.01471710

$-68.09579468$

59.32974625

71.60529327

$-75.01313019$ 3.07743502

$-7.94707632$

$-4.20174694$

12.95835876

$-3.80443239$

8.23155403

1.65567660

$-8.33710575$

$-10.63216972$

5.02415228

4.08667278

7.67442036

16.81877327

$-15.37121677$

$-15.59772015$

10.14379215

\# Electr. mom.

0.14458551

0.25042096

0.14458551

0.25042096

0.14458551

0.25042096

0.14458551

0.25042096

$-0.15045778$

$-0.04462234$

$-0.15045778$

$-0.04462234$

$-0.15045778$

$-0.04462234$

$-0.15045778$

$-0.04462234$

1. 24855602

1.35439157

1. 24855602

1.35439157

1. 24855602

1.35439157

1. 24855602

1.35439157

0.73230141

0.83813679

0.73230141

0.83813679

0.73230141

0.83813679

0.73230141
237.01712036

$-197.93740845$

$-317.29315186$

270.67532349

$-199.11526489$

163.74116516

252.05796814

$-210.98141479$

$-132.91220093$

116.90099335

125.89640045

$-116.47422028$

122.05104065

$-105.23183441$

$-116.16612244$

105.81775665

$-13.33092594$

12.10540771

19.41834450

$-16.22946358$

11.02375507

$-10.80268097$

$-21.12316322$

17.68300438

67.46982574

$-50.88781357$

$-65.10694885$ 49.67959976

$-71.06864929$

54.00021744

69.20494080 


\begin{tabular}{|c|c|c|c|}
\hline-1.70049155 & 1.30318046 & 0.83813679 & -53.21070099 \\
\hline 1.04285800 & 0.17553815 & -1.01531279 & 190.56922913 \\
\hline 1.04285800 & 0.17553815 & -0.90947729 & -223.84466553 \\
\hline 1.04285800 & 0.28137359 & -1.01531279 & -147.49377441 \\
\hline 1.04285800 & 0.28137359 & -0.90947729 & 175.95219421 \\
\hline 1.14869356 & 0.17553815 & -1.01531279 & -192.53689575 \\
\hline 1.14869356 & 0.17553815 & -0.90947729 & 228.91593933 \\
\hline 1.14869356 & 0.28137359 & -1.01531279 & 123.7892913 \\
\hline 1.14869356 & 0.28137359 & -0.90947729 & -154.3291626 \\
\hline-1.76707089 & -0.64616346 & -0.62219280 & 56.5 \\
\hline-1.76707089 & -0.64616346 & -0.51635736 & -46.9 \\
\hline-1.76707089 & -0.54032809 & -0.622192 & -64.86 \\
\hline-1.76707089 & -0.54032809 & -0.516357 & 64.64 \\
\hline-1.66123557 & -0.64616346 & -0.622192 & $-71 \cdot 3$ \\
\hline-1.66 & -0.64616346 & $-0.51635^{\prime}$ & 50.3 \\
\hline-1.6612 & -0.54032809 & -0.622192 & 72.35 \\
\hline-1.66123557 & -0.54032809 & -0.51635736 & -63.266262 \\
\hline 1.57582414 & 0.13101105 & -1.877118 & 2.1 \\
\hline 1.5758241 & 0.13101 & -1.771283 & -6.2 \\
\hline 1.5758241 & 0.23684651 & -1.877118 & $-4 \cdot 5$ \\
\hline 1.57 & 0.23684651 & -1.77128 & 13 \\
\hline 1.68 & 0.13101105 & -1.8771 & -3 . \\
\hline 1.68 & 0.13101 & -1.77128 & 6.7 \\
\hline 65958 & 0.23684651 & -1.877118 & 1.7 \\
\hline 1 . & 0.23684651 & -1.77128 & -8.2 \\
\hline-1.11 & -1.22435 & -1.14041 & -10.1 \\
\hline$-1 \cdot 11$ & -1.22435 & -1.0345 & 2 \\
\hline-1.11 & -1.11852252 & -1.14041 & -0.6 \\
\hline-1.11 & -1.11852252 & -1.03457 & 16.9 \\
\hline-1 & -1.22435784 & -1.14041 & 1 \\
\hline-1.00676 & -1.22435784 & -1.03457 & -15.2 \\
\hline-1.0067613 & -1.11852252 & -1.14041 & -13.8 \\
\hline-1.006761 & -1.11852252 & -1.03457 & \\
\hline & \# Energy 4->4 & & \\
\hline 0 . & -5.7264 & & ctr. mom. \\
\hline 1.67 & -0.20954360 & 58551 & 254.3 \\
\hline 1.673 & -0.20954360 & 5042096 & $-213 \cdot 3$ \\
\hline 1.673416 & -0.10370816 & 0.14458551 & -340.98 \\
\hline 1.673 & -0.10370816 & 0.25042096 & 292.0 \\
\hline 1 . & -0.209 & 0 . & -212.3 \\
\hline 1.77925158 & -0.20954360 & 0.25042096 & 175.407 \\
\hline 1.77925158 & -0.10370816 & 0.14458551 & 269.4 \\
\hline 1.77 & -0.10370816 & 0.25042 & -226 \\
\hline-1.26 & 0.53541 & -0.15045 & -169.1 \\
\hline-1.26 & 0.53541940 & -0.04462234 & 135.3 \\
\hline-1.26 & 0.64125490 & -0.15045778 & 162.6 \\
\hline-1 & 0.64 & -0.0446 & -135 \\
\hline-1 & 0.53 & -0.15 & 16 \\
\hline-1 & 0.5354 & -0.044 & -132.5 \\
\hline-1 & 0.641254 & -0.15045778 & -160 \\
\hline-1 & 0.641 & -0.0446 & 133 \\
\hline 1 . & 0.01 & 1.24855602 & -14.6 \\
\hline 1.17 & 0.013996 & 1.354391 & 13. \\
\hline 1 . & 0.11983 & 1.2 & 21 \\
\hline 1 . & 0.11 & 1.3 & $-18 \cdot 1$ \\
\hline 1. & 0.01399621 & 1.24855602 & 12.1 \\
\hline 1. & 0.013996 & 1.35439157 & -12 \\
\hline 1 . & 0.11 & 1.248 & -23 \\
\hline 1 . & 0.11983 & 1.3 & 19.6 \\
\hline-1 & 1.19734502 & 0.732301 & 26.3 \\
\hline-1 . & 1.1 & 0 . & -24 \\
\hline-1 & 1.30318 & 0 . & -26 \\
\hline-1 & 1.30318046 & 0.83813679 & 23.912 \\
\hline-1 & 1.19734502 & 0.732301 & -2 \\
\hline-1 & 1.19734502 & 0 . & 23. \\
\hline-1 & 1.30318046 & 0.73230141 & 24.281661 \\
\hline-1 . & 1 & 0. & -2 \\
\hline
\end{tabular}

\section{S-448}


1.04285800

1. 04285800

1. 04285800

1. 04285800

1. 14869356

1. 14869356

1. 14869356

1. 14869356

$-1.76707089$

$-1.76707089$

$-1.76707089$

$-1.76707089$

$-1.66123557$

$-1.66123557$

$-1.66123557$

$-1.66123557$

1.57582414

1. 57582414

1. 57582414

1.57582414

1.68165958

1.68165958

1.68165958

1.68165958

$-1.11259675$

$-1.11259675$

$-1.11259675$

$-1.11259675$

$-1.00676131$

$-1.00676131$

$-1.00676131$

$-1.00676131$

64
0.17553815

0.17553815

0.28137359

0.28137359

0.17553815

0.17553815

0.28137359

0.28137359

$-0.64616346$

$-0.64616346$

$-0.54032809$

$-0.54032809$

$-0.64616346$

$-0.64616346$

$-0.54032809$

$-0.54032809$

0.13101105

0.13101105

0.23684651

0.23684651

0.13101105

0.13101105

0.23684651

0.23684651

$-1.22435784$

$-1.22435784$

$-1.11852252$

$-1.11852252$

$-1.22435784$

$-1.22435784$

$-1.11852252$

$-1.11852252$

\# Energy 5->5

$3.6976-2.1479 \quad-6.5603$

1.67341614

1.67341614

1.67341614

1.67341614

1.77925158

1.77925158

1. 77925158

1. 77925158

$-1.26006997$

$-1.26006997$

$-1.26006997$

$-1.26006997$

$-1.15423441$

$-1.15423441$

$-1.15423441$

$-1.15423441$

1.17254710

1.17254710

1.17254710

1. 17254710

1. 27838266

1. 27838266

1. 27838266

1. 27838266

$-1.80632699$

$-1.80632699$

$-1.80632699$

$-1.80632699$

$-1.70049155$

$-1.70049155$

$-1.70049155$

$-1.70049155$

1. 04285800
$-0.20954360$

$-0.20954360$

$-0.10370816$

$-0.10370816$

$-0.20954360$

$-0.20954360$

$-0.10370816$

$-0.10370816$

0.53541940

0.53541940

0.64125490

0.64125490

0.53541940

0.53541940

0.64125490

0.64125490

0.01399621

0.01399621

0.11983165

0.11983165

0.01399621

0.01399621

0.11983165

0.11983165

1.19734502

1.19734502

1.30318046

1. 30318046

1. 19734502

1. 19734502

1.30318046

1. 30318046

0.17553815
$-1.01531279$

$-0.90947729$

$-1.01531279$

$-0.90947729$

$-1.01531279$

$-0.90947729$

$-1.01531279$

$-0.90947729$

$-0.62219280$

$-0.51635736$

$-0.62219280$

$-0.51635736$

$-0.62219280$

$-0.51635736$

$-0.62219280$

$-0.51635736$

$-1.87711871$

$-1.77128315$

$-1.87711871$

$-1.77128315$

$-1.87711871$

$-1.77128315$

$-1.87711871$

$-1.77128315$

$-1.14041448$

$-1.03457904$

$-1.14041448$

$-1.03457904$

$-1.14041448$

$-1.03457904$

$-1.14041448$

$-1.03457904$
194.15298462

$-231.03251648$

$-147.68872070$

179.10997009

$-193.36334229$

233.53106689

120.34210205

$-153.88812256$

33.19831467

$-21.37996101$

$-38.37936020$

35.25664520

$-49.68473434$

26.69634247

46.75647354

$-34.12121582$

2.34865642

$-7.01824331$

$-5.09637117$

14.46869755

$-2.71233058$

6.73170280

1.70176327

$-8.54157162$

$-7.39747047$

$-1.48964036$

$-1.48156130$

18.16127396

14.82561588

$-10.96138668$

$-12.09360504$

2. 95140505
\# Electr. mom.

0.14458551

0.25042096

0.14458551

0.25042096

0.14458551

0.25042096

0.14458551

0.25042096

$-0.15045778$

$-0.04462234$

$-0.15045778$

$-0.04462234$

$-0.15045778$

$-0.04462234$

$-0.15045778$

$-0.04462234$

1. 24855602

1.35439157

1. 24855602

1. 35439157

1. 24855602

1.35439157

1. 24855602

1. 35439157

0.73230141

0.83813679

0.73230141

0.83813679

0.73230141

0.83813679

0.73230141

0.83813679

$-1.01531279$
230.39437866

$-186.43165588$

$-288.65103149$

238.84396362

$-202.11514282$

161.49256897

236.44996643

$-191.88252258$

$-154.51110840$

133.85157776

143.22830200

$-128.02970886$

157.28953552

$-134.74980164$

$-147.88493347$

131.60733032

$-11.78742790$

10.55682564

13.11914921

$-11.09056664$

10.57237244

$-9.96476841$

$-15.11502361$

12.71302986

31.21270943

$-23.74455452$

$-26.90118217$

20.98383713

$-36.96643829$

27.78736877

30.08665085

$-23.15989304$

214.48895264 
1.04285800

1. 04285800

1. 04285800

1. 14869356

1. 14869356

1. 14869356

1.14869356

$-1.76707089$

$-1.76707089$

$-1.76707089$

$-1.76707089$

$-1.66123557$

$-1.66123557$

$-1.66123557$

$-1.66123557$

1.57582414

1.57582414

1.57582414

1.57582414

1.68165958

1.68165958

1.68165958

1.68165958

$-1.11259675$

$-1.11259675$

$-1.11259675$

$-1.11259675$

$-1.00676131$

$-1.00676131$

$-1.00676131$

$-1.00676131$

64
0.17553815

0.28137359

0.28137359

0.17553815

0.17553815

0.28137359

0.28137359

$-0.64616346$

$-0.64616346$

$-0.54032809$

$-0.54032809$

$-0.64616346$

$-0.64616346$

$-0.54032809$

$-0.54032809$

0.13101105

0.13101105

0.23684651

0.23684651

0.13101105

0.13101105

0.23684651

0.23684651

$-1.22435784$

$-1.22435784$

$-1.11852252$

$-1.11852252$

$-1.22435784$

$-1.22435784$

$-1.11852252$

$-1.11852252$

0 . \# Energy 6->6

$11.7979-3.6069 \quad-5.6345$

1.67341614

1.67341614

1.67341614

1.67341614

1.77925158

1.77925158

1.77925158

1. 77925158

$-1.26006997$

$-1.26006997$

$-1.26006997$

$-1.26006997$

$-1.15423441$

$-1.15423441$

$-1.15423441$

$-1.15423441$

1.17254710

1. 17254710

1. 17254710

1. 17254710

1. 27838266

1. 27838266

1. 27838266

1. 27838266

$-1.80632699$

$-1.80632699$

$-1.80632699$

$-1.80632699$

$-1.70049155$

$-1.70049155$

$-1.70049155$

$-1.70049155$

1.04285800

1. 04285800
$-0.20954360$

$-0.20954360$

$-0.10370816$

$-0.10370816$

$-0.20954360$

$-0.20954360$

$-0.10370816$

$-0.10370816$

0.53541940

0.53541940

0.64125490

0.64125490

0.53541940

0.53541940

0.64125490

0.64125490

0.01399621

0.01399621

0.11983165

0.11983165

0.01399621

0.01399621

0.11983165

0.11983165

1. 19734502

1.19734502

1. 30318046

1. 30318046

1.19734502

1. 19734502

1. 30318046

1.30318046

0.17553815

0.17553815
$-0.90947729$

$-1.01531279$

$-0.90947729$

$-1.01531279$

$-0.90947729$

$-1.01531279$

$-0.90947729$

$-0.62219280$

$-0.51635736$

$-0.62219280$

$-0.51635736$

$-0.62219280$

$-0.51635736$

$-0.62219280$

$-0.51635736$

$-1.87711871$

$-1.77128315$

$-1.77128315$

$-1.87711871$

$-1.77128315$

$-1.87711871$

$-1.77128315$

$-1.14041448$

$-1.03457904$

$-1.14041448$

$-1.03457904$

$-1.14041448$

$-1.03457904$

$-1.14041448$

$-1.03457904$
$-1.87711871$

$-255.96263123$

$-172.37561035$

208.22731018

$-209.29991150$

253.72157288

142.49360657

$-180.10083008$

54.42822647

$-45.30855179$

$-58.86566925$

59.71408844

$-72.42976379$

54.30159760

70.55587769

$-65.02556610$ 2.38783264

$-7.65382433$

$-3.85358763$

13.22746563

$-2.49190211$

7. 00301313

0.61862153

$-7.55337524$

$-13.16404724$

6.93410540

5.57810307

7.85167789

19.88195992

$-18.50307465$

$-18.33671761$

12.30723953

\# Electr. mom.

0.14458551

0.25042096

0.14458551

0.25042096

0.14458551

0.25042096

0.14458551

0.25042096

$-0.15045778$

$-0.04462234$

$-0.15045778$

$-0.04462234$

$-0.15045778$

$-0.04462234$

$-0.15045778$

$-0.04462234$

1. 24855602

1. 35439157

1. 24855602

1. 35439157

1. 24855602

1. 35439157

1. 24855602

1. 35439157

0.73230141

0.83813679

0.73230141

0.83813679

0.73230141

0.83813679

0.73230141

0.83813679

$-1.01531279$

$-0.90947729$
213.38159180

$-172.82682800$

$-294.22610474$

246.24754333

$-184.25457764$

147.82318115

237.18112183

$-195.27893066$

$-147.10270691$

135.68756104

138.05894470

$-130.78117371$

148.40678406

$-136.21188354$

$-141.15773010$

133.21492004

$-5.21879911$

5.90357733

9.92191792

$-8.93444443$

1. 26621211

$-3.22809887$

$-9.41978645$

8.61586189

33.83160400

$-27.30745697$

$-31.37087250$

25.88131142

$-38.03275299$

30.18121529

33.24689484

$-27.10158730$

221.44297791

$-258.78552246$ 
1.04285800

1. 04285800

1. 14869356

1.14869356

1. 14869356

1.14869356

$-1.76707089$

$-1.76707089$

$-1.76707089$

$-1.76707089$

$-1.66123557$

$-1.66123557$

$-1.66123557$

$-1.66123557$

1. 57582414

1.57582414

1.57582414

1.57582414

1.68165958

1.68165958

1.68165958

1.68165958

$-1.11259675$

$-1.11259675$

$-1.11259675$

$-1.11259675$

$-1.00676131$

$-1.00676131$

$-1.00676131$

64

$-1.00676131$

$-1.5785$

1.67341614

1.67341614

1.67341614

1.67341614

1. 77925158

1. 77925158

1.77925158

1.77925158

$-1.26006997$

$-1.26006997$

$-1.26006997$

$-1.26006997$

$-1.15423441$

$-1.15423441$

$-1.15423441$

$-1.15423441$

1. 17254710

1. 17254710

1. 17254710

1. 17254710

1.27838266

1. 27838266

1. 27838266

1.27838266

$-1.80632699$

$-1.80632699$

$-1.80632699$

$-1.80632699$

$-1.70049155$

$-1.70049155$

$-1.70049155$

$-1.70049155$

1. 04285800

1.04285800

1. 04285800
0.28137359

0.28137359

0.17553815

0.17553815

0.28137359

0.28137359

$-0.64616346$

$-0.64616346$

$-0.54032809$

$-0.54032809$

$-0.64616346$

$-0.64616346$

$-0.54032809$

$-0.54032809$

0.13101105

0.13101105

0.23684651

0.23684651

0.13101105

0.13101105

0.23684651

0.23684651

$-1.22435784$

$-1.22435784$

$-1.11852252$

$-1.11852252$

$-1.22435784$

$-1.22435784$

$-1.11852252$

$-1.11852252$

\# Energy 7->7

$-0.9510-3.1968$

$-0.20954360$

$-0.20954360$

$-0.10370816$

$-0.10370816$

$-0.20954360$

$-0.20954360$

$-0.10370816$

$-0.10370816$

0.53541940

0.53541940

0.64125490

0.64125490

0.53541940

0.53541940

0.64125490

0.64125490

0.01399621

0.01399621

0.11983165

0.11983165

0.01399621

0.01399621

0.11983165

0.11983165

1.19734502

1.19734502

1.30318046

1. 30318046

1.19734502

1.19734502

1. 30318046

1. 30318046

0.17553815

0.17553815

0.28137359
$-1.01531279$

$-0.90947729$

$-1.01531279$

$-0.90947729$

$-1.01531279$

$-0.90947729$

$-0.62219280$

$-0.51635736$

$-0.62219280$

$-0.51635736$

$-0.62219280$

$-0.51635736$

$-0.62219280$

$-0.51635736$

$-1.87711871$

$-1.77128315$

$-1.87711871$

$-1.77128315$

$-1.87711871$

$-1.77128315$

$-1.87711871$

$-1.77128315$

$-1.14041448$

$-1.03457904$

$-1.14041448$

$-1.03457904$

$-1.14041448$

$-1.03457904$

$-1.14041448$

$-1.03457904$

$$
8.03457904
$$

0.14458551

0.25042096

0.14458551

0.25042096

0.14458551

0.25042096

0.14458551

0.25042096

$-0.15045778$

$-0.04462234$

$-0.15045778$

$-0.04462234$

$-0.15045778$

$-0.04462234$

$-0.15045778$

$-0.04462234$

1. 24855602

1.35439157

1. 24855602

1. 35439157

1. 24855602

1. 35439157

1. 24855602

1.35439157

0.73230141

0.83813679

0.73230141

0.83813679

0.73230141

0.83813679

0.73230141

0.83813679

$-1.01531279$

$-0.90947729$

$-1.01531279$
$-177.91029358$

211.70495605

$-223.94013977$

265.37442017

149.81733704

$-186.21356201$

56.49463654

$-39.32331848$

$-56.77859879$

49.41739655

$-76.64431000$

47.40903473

68.66770172

$-52.28912735$

$-0.01096766$

$-3.96523404$

$-3.26130700$

12.57934952

$-0.58954549$

4.06901550

$-0.24469098$

$-6.48109531$

$-12.08299828$

3. 12634873

1.39004433

16.78364563

19.75823212

$-16.56936073$

$-16.14755440$

6.80599308

Electr. mom.

237.76559448

$-197.87869263$

$-301.99472046$

256.83471680

$-201.83618164$

164.46961975

240.12876892

$-199.27595520$

$-162.75183105$

133.90888977

148.56294250

$-127.05696106$

151.52096558

$-121.43498993$

$-138.67098999$

116.13274384

$-28.95609856$

23.37268066

32.61818695

$-25.81470490$

29.24391365

$-23.91829300$

$-36.38192368$

28.73749161

52.45721817

$-39.54934311$

$-51.42684174$

39.45363235

$-58.71891403$

44.87578583

57.37344360

$-44.57474136$

203.06462097

$-241.51182556$

$-158.60592651$ 
1.04285800

1. 14869356

1. 14869356

1.14869356

1. 14869356

$-1.76707089$

$-1.76707089$

$-1.76707089$

$-1.76707089$

$-1.66123557$

$-1.66123557$

$-1.66123557$

$-1.66123557$

1. 57582414

1. 57582414

1.57582414

1.57582414

1. 68165958

1.68165958

1.68165958

1.68165958

$-1.11259675$

$-1.11259675$

$-1.11259675$

$-1.11259675$

$-1.00676131$

$-1.00676131$

$-1.00676131$

64

$-1.00676131$

$$
\text { 0. \# Energy 8->8 }
$$

1.67341614

1.67341614

1.67341614

1.67341614

1. 77925158

1. 77925158

1. 77925158

1. 77925158

$-1.26006997$

$-1.26006997$

$-1.26006997$

$-1.26006997$

$-1.15423441$

$-1.15423441$

$-1.15423441$

$-1.15423441$

1.17254710

1.17254710

1.17254710

1.17254710

1. 27838266

1. 27838266

1. 27838266

1.27838266

$-1.80632699$

$-1.80632699$

$-1.80632699$

$-1.80632699$

$-1.70049155$

$-1.70049155$

$-1.70049155$

$-1.70049155$

1. 04285800

1. 04285800

1. 04285800

1. 04285800

$-0.20954360$

$-0.20954360$

$-0.10370816$

$-0.10370816$

$-0.20954360$

$-0.20954360$

$-0.10370816$

$-0.10370816$

0.53541940

0.53541940

0.64125490

0.64125490

0.53541940

0.53541940

0.64125490

0.64125490

0.01399621

0.01399621

0.11983165

0.11983165

0.01399621

0.01399621

0.11983165

0.11983165

1. 19734502

1. 19734502

1. 30318046

1. 30318046

1. 19734502

1.19734502

1. 30318046

1. 30318046

0.17553815

0.17553815

0.28137359

0.28137359
0.28137359

0.17553815

0.17553815

0.28137359

0.28137359

$-0.64616346$

$-0.64616346$

$-0.54032809$

$-0.54032809$

$-0.64616346$

$-0.64616346$

$-0.54032809$

$-0.54032809$

0.13101105

0.13101105

0.23684651

0.23684651

0.13101105

0.13101105

0.23684651

0.23684651

$-1.22435784$

$-1.22435784$

$-1.11852252$

$-1.11852252$

$-1.22435784$

$-1.22435784$

$-1.11852252$

$-1.11852252$

$-2.1138-3.4290$
$-0.90947729$

$-1.01531279$

$-0.90947729$

$-1.01531279$

$-0.90947729$

$-0.62219280$

$-0.51635736$

$-0.62219280$

$-0.51635736$

$-0.62219280$

$-0.51635736$

$-0.62219280$

$-0.51635736$

$-1.87711871$

$-1.77128315$

$-1.87711871$

$-1.77128315$

$-1.87711871$

$-1.77128315$

$-1.87711871$

$-1.77128315$

$-1.14041448$

$-1.03457904$

$-1.14041448$

$-1.03457904$

$-1.14041448$

$-1.03457904$

$-1.14041448$

$-1.03457904$
190.38505554

$-200.77391052$

241.91294861

133.53833008

$-167.08685303$

52.86093521

$-51.94420624$

$-66.19093323$

74.18148041

$-64.29344177$

54.75271988

72.57151794

$-73.80078888$

3.85067439

$-9.10571671$

$-4.88060570$

13.98364639

$-4.49179697$

9. 22946358

2.25690103

$-9.23420715$

$-10.79340649$

5.91685867

3. 29527545

7.63478899

17.69739151

$-17.04656792$

$-15.85422134$

11.26635456
\# Electr. mom.

0.14458551

0.25042096

0.14458551

0.25042096

0.14458551

0.25042096

0.14458551

0.25042096

$-0.15045778$

$-0.04462234$

$-0.15045778$

$-0.04462234$

$-0.15045778$

$-0.04462234$

$-0.15045778$

$-0.04462234$

1. 24855602

1. 35439157

1. 24855602

1. 35439157

1. 24855602

1. 35439157

1. 24855602

1. 35439157

0.73230141

0.83813679

0.73230141

0.83813679

0.73230141

0.83813679

0.73230141

0.83813679

$-1.01531279$

$-0.90947729$

$-1.01531279$

$-0.90947729$
242.49316406

$-202.36558533$

$-307.85675049$

262.09710693

$-206.89576721$

169.51722717

245.96615601

$-204.81904602$

$-174.68066406$

135.51123047

163.04263306

$-130.01922607$

184.69079590

$-145.26895142$

$-174.78456116$

142.00886536

$-27.19693756$

22.31830978

30.11549759

$-24.16764450$

27.25656509

$-22.63220978$

$-33.70821762$

26.91351318

28.80105591

$-23.12460899$

$-23.28177071$

18.74174500

$-28.21476173$

22.46700096

21.72841072

$-17.46462250$

205.04972839

$-246.08087158$

$-159.27616882$

193.39859009 
1.14869356

1. 14869356

1. 14869356

1.14869356

$-1.76707089$

$-1.76707089$

$-1.76707089$

$-1.76707089$

$-1.66123557$

$-1.66123557$

$-1.66123557$

1. 57582414

1.57582414

1. 57582414

1.57582414

1.68165958

1. 68165958

1.68165958

1.68165958

$-1.11259675$

$-1.11259675$

$-1.11259675$

$-1.11259675$

$-1.00676131$

$-1.00676131$

$-1.00676131$

64
$-1.66123557$

0.17553815

0.17553815

0.28137359

0.28137359

$-0.64616346$

$-0.64616346$

$-0.54032809$

$-0.54032809$

$-0.64616346$

$-0.64616346$

$-0.54032809$

$-0.54032809$

0.13101105

0.13101105

0.23684651

0.23684651

0.13101105

0.13101105

0.23684651

0.23684651

$-1.22435784$

$-1.22435784$

$-1.11852252$

$-1.11852252$

$-1.22435784$

$-1.22435784$

$-1.11852252$

$-1.11852252$

\# Energy 9->9

$\begin{array}{lll}7.7142 & -3.5593 & -4.9598\end{array}$

1.67341614

1.67341614

1.67341614

1.67341614

1.77925158

1. 77925158

1.77925158

1. 77925158

$-1.26006997$

$-1.26006997$

$-1.26006997$

$-1.26006997$

$-1.15423441$

$-1.15423441$

$-1.15423441$

$-1.15423441$

1. 17254710

1. 17254710

1. 17254710

1. 17254710

1. 27838266

1. 27838266

1. 27838266

1. 27838266

$-1.80632699$

$-1.80632699$

$-1.80632699$

$-1.80632699$

$-1.70049155$

$-1.70049155$

$-1.70049155$

$-1.70049155$

1.04285800

1.04285800

1. 04285800

1. 04285800

1. 14869356
$-0.20954360$

$-0.20954360$

$-0.10370816$

$-0.10370816$

$-0.20954360$

$-0.20954360$

$-0.10370816$

$-0.10370816$

0.53541940

0.53541940

0.64125490

0.64125490

0.53541940

0.53541940

0.64125490

0.64125490

0.01399621

0.01399621

0.11983165

0.11983165

0.01399621

0.01399621

0.11983165

0.11983165

1. 19734502

1.19734502

1. 30318046

1. 30318046

1. 19734502

1.19734502

1. 30318046

1. 30318046

0.17553815

0.17553815

0.28137359

0.28137359

0.17553815
$-1.01531279$

$-0.90947729$

$-1.01531279$

$-0.90947729$

$-0.62219280$

$-0.51635736$

$-0.62219280$

$-0.51635736$

$-0.62219280$

$-0.51635736$

$-0.62219280$

$-0.51635736$

$-1.87711871$

$-1.77128315$

$-1.87711871$

$-1.77128315$

$-1.87711871$

$-1.77128315$

$-1.87711871$

$-1.77128315$

$-1.14041448$

$-1.03457904$

$-1.14041448$

$-1.03457904$

$-1.14041448$

$-1.03457904$

$-1.14041448$

$-1.03457904$
$-202.21644592$

245.98361206

133.79597473

$-169.72364807$

42.81203461

$-35.63128662$

$-48.22380066$

50.55472183

$-59.71880341$

43.84988403

58.47067642

$-54.12381744$ 3.48818231

$-8.81763458$

$-4.64023972$

13.75782394

$-3.89736867$

8.61508656

1.83233654

$-8.75159931$

$-10.49694252$

3. 79010797

3.84367037

9.57402039

16.94360542

$-14.75360203$

$-15.87024784$

9. 27448463

$$
\text { \# Electr. mom. }
$$

0.14458551

0.25042096

0.14458551

0.25042096

0.14458551

0.25042096

0.14458551

0.25042096

$-0.15045778$

$-0.04462234$

$-0.15045778$

$-0.04462234$

$-0.15045778$

$-0.04462234$

$-0.15045778$

$-0.04462234$

1. 24855602

1. 35439157

1. 24855602

1. 35439157

1. 24855602

1. 35439157

1. 24855602

1. 35439157

0.73230141

0.83813679

0.73230141

0.83813679

0.73230141

0.83813679

0.73230141

0.83813679

$-1.01531279$

$-0.90947729$

$-1.01531279$

$-0.90947729$

$-1.01531279$
227.55706787

$-188.54316711$

$-299.50799561$

252.70526123

$-191.97781372$

156.61300659

237.42645264

$-196.12141418$

$-167.80107117$

141.81770325

155.79653931

$-135.59147644$

169.72135925

$-141.89932251$

$-159.22581482$

137.59753418

$-12.80344963$

11.08600998

17.74454308

$-14.53942585$

11.88446808

$-10.74917221$

$-20.26289940$

16.59544182

30.08127975

$-24.05224228$

$-27.69056892$

22.66463661

$-34.57382202$

27.33405685

29.99412918

$-24.38079643$

203.37330627

$-237.97763062$

$-162.50082397$

192.90319824

$-202.48866272$ 


$\begin{array}{rrrr}1.14869356 & 0.17553815 & -0.90947729 & 240.06480408 \\ 1.14869356 & 0.28137359 & -1.01531279 & 134.50720215 \\ 1.14869356 & 0.28137359 & -0.90947729 & -166.66239929 \\ -1.76707089 & -0.64616346 & -0.62219280 & 47.17179489 \\ -1.76707089 & -0.64616346 & -0.51635736 & -38.87113190 \\ -1.76707089 & -0.54032809 & -0.62219280 & -53.13066864 \\ -1.76707089 & -0.54032809 & -0.51635736 & 54.57226944 \\ -1.66123557 & -0.64616346 & -0.62219280 & -64.03893280 \\ -1.66123557 & -0.64616346 & -0.51635736 & 46.14438629 \\ -1.66123557 & -0.54032809 & -0.62219280 & 63.39836502 \\ -1.66123557 & -0.54032809 & -0.51635736 & -57.82999802 \\ 1.57582414 & 0.13101105 & -1.87711871 & 1.88266230 \\ 1.57582414 & 0.13101105 & -1.77128315 & -6.37195683 \\ 1.57582414 & 0.23684651 & -1.87711871 & -3.92070484 \\ 1.57582414 & 0.23684651 & -1.77128315 & 13.05915451 \\ 1.68165958 & 0.13101105 & -1.87711871 & -2.60432863 \\ 1.68165958 & 0.13101105 & -1.77128315 & 6.61260891 \\ 1.68165958 & 0.23684651 & -1.87711871 & 0.88443488 \\ 1.68165958 & 0.23684651 & -1.77128315 & -7.65132713 \\ -1.11259675 & -1.22435784 & -1.14041448 & -8.80521774 \\ -1.11259675 & -1.22435784 & -1.03457904 & 0.51097047 \\ -1.11259675 & -1.1185252 & -1.14041448 & -0.59941959 \\ -1.11259675 & -1.11852252 & -1.03457904 & 16.77989388 \\ -1.00676131 & -1.22435784 & -1.14041448 & 16.11020088 \\ -1.00676131 & -1.22435784 & -1.03457904 & -12.83098984 \\ -1.00676131 & -1.11852252 & -1.14041448 & -12.96450043 \\ -1.00676131 & -1.11852252 & -1.03457904 & 4.37441778\end{array}$

